Synthesis of Subsonic Airplane Design

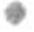




\section{Synthesis of Subsonic \\ Airplane Design}

An introduction to the preliminary design of subsonic general aviation and transport aircraft, with emphasis on layout, aerodynamic design, propulsion and performance

\section{Egbert Torenbeek}

with a foreword by

H. Wittenberg

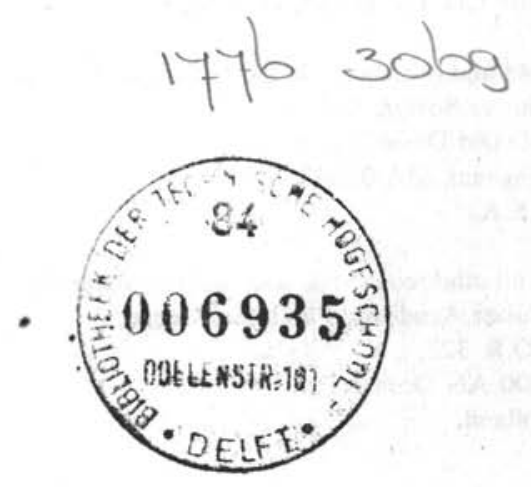

1982

Delft University Press

Martinus Nijhoff Publishers 
Torenbeek, Egbert.

Synthesis of Subsonic Airplane Design.

Includes bibliographical references and index.

1. Airplanes-Design and Construction.

I. Title.

$\begin{array}{llll}\text { TL671.2.T67 } & 1982 & 629.134 ' 1 & 82-12469\end{array}$

ISBN 90-247-2724-3

Joint edition published by

Delft University Press,

Mijnbouwplein 11,

2628 RT Delft, Holland

and by

Martinus Nijhoff Publishers,

P.O.B. 566,

$2501 \mathrm{CN}$ The Hague, Holland.

Sold and distributed in the U.S.A. and Canada by

Kluwer Boston, Inc.,

190 Old Derby Street,

Hingham, MA 02043,

U.S.A.

In all other countries, sold and distributed by

Kluwer Academic Publishers Group,

P.O.B. 322,

3300 AN Dordrecht,

Holland.

Copyright $\odot 1982$ by Delft University Press, Delft, Holland.

All rights reserved. No part of this publication may be reproduced, stored in a retrieval system, or transmitted in any form or by any means, mechanical, photocopying, recording, or otherwise, without written permission of the copyright owner, Delft University Press, Mijnbouwplein 11, 2628 RT Delft, Holland.

Printed in The Netherlands by Princo BV, Culemborg. 


\section{Contents}

FOREWORD

by Professor H. Wittenberg

xvi

AUTHOR'S PREFACE

xviii

ACKNOWLEDGEMENTS

$\mathrm{xix}$

UNITS

$\mathrm{xxi}$

CHAPTER 1. GENERAL ASPECTS OF AIRCRAFT CONFIGURATION DEVELOPMENT

1.1. Introduction

1.2. Aircraft design and development 3

1.3. Configuration development $\quad 5$

1.3.1. The design concept 6

1.3.2. Initial configuration design and configuration variations 7

1.3.3. Baseline configuration development 8

1.3.4. The preliminary design department 9

$\begin{array}{lr}\text { 1.4. The initial specification } & 10\end{array}$

$\begin{array}{ll}1.4 .1 . & 10\end{array}$

1.4.2. Transport capacity 12

1.4.3. Design cruising speed and range 13

1.4.4. Low-speed characteristics and field performance 14

$\begin{array}{ll}1.4 .5 & \text { Other requirements } \\ & 15\end{array}$

1.5. A continuous thread running through the design process 16

1.5.1. The iterative character of design 16

$\begin{array}{ll}\text { 1.5.2. Searching for the optimum } & 17\end{array}$

1.5.3. A suggested scheme for preliminary design 18

1.6. Impact of civil airworthiness requirements, and operating and flight rules 19

1.6.1. General 19

1.6.2. Federal Aviation Regulations 21

1.6.3. British Civil Airworthiness Requirements 22

1.6.4. Airworthiness standards and design 23

$\begin{array}{lr}\text { 1.7. Conclusion } & 25\end{array}$

$\begin{array}{lr}\text { CHAPTER 2. THE GENERAL ARRANGEMENT } & 27\end{array}$

$\begin{array}{lr}\text { 2.1. Introduction } & 28\end{array}$ 
2.2. Hi.gh, low or mid wing?

2.2.1. High wing $\quad 34$

2.2.2. Mid wing 36

2.2.3. Low wing $\quad 37$

2.2.4. Effects of wing location on the general arrangement 37

2.3. Location of the engines 39

2.3.1. Propeller aircraft $\quad 39$

2.3.2. Jet-propelled transport aircraft $\quad 41$

2.3.3. Single-engine subsonic jet aircraft 46

2.4. Arrangement of the tailplane 50

2.4.1. Classification of tail surface configurations $\quad 50$

2.4.2. The location of tail surfaces 51

2.5. Arrangement of the undercarriage $\quad 54$

$\begin{array}{ll}2.5 .1 . & 54\end{array}$

2.5.2. Nosewheel undercarriage $\quad 54$

$\begin{array}{lr}\text { 2.5.3. Tandem undercarriage } & 55\end{array}$

2.6. Some unconventional aircraft configurations $\quad 55$

2.6.1. The flying wing $\quad 56$

$\begin{array}{ll}\text { 2.6.2. Tailless aircraft } & 58\end{array}$

$\begin{array}{ll}\text { 2.6.3. Tail-first (or canard) layout } & 58\end{array}$

$\begin{array}{lr}\text { CHAPTER 3. FUSELAGE DESIGN } & 61\end{array}$

3.1. Introduction $r$

3.1.1. Function and design requirements $\quad 62$

3.1.2. Drag and optimization of the external shape 63

3.1.3. A design procedure for fuselages with cylindrical mid-section $\quad 67$

3.2. The fuselage of airliners and general aviation aircraft $\quad 68$

$\begin{array}{ll}3.2 .1 \text {. Importance of comfort and payload density } & 68\end{array}$

3.2.2. Cabin design $\quad 69$

3.2.3. Passenger seats $\quad 75$

3.2.4. Passenger emergency exits, doors and windows $\quad 76$

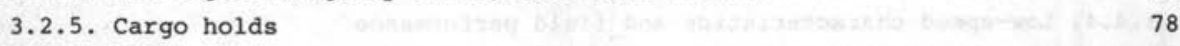

$\begin{array}{lr}3.2 .6 . & 79\end{array}$

3.3. The fuselage of cargo aircraft $\quad 81$

$\begin{array}{ll}3.3 .1 . & 81\end{array}$

3.3.2. Payload density and volume of the freight hold 83

3.3.3. Loading systems $\quad 84$

3.3.4. Accessibility of the freight hold 84

3.4. Flight deck design $\quad 88$

3.4.1. Location of the pilot's seat and the flight controls 88

3.4.2. Visibility from the cockpit $\quad 90$

3.4.3. Flight deck dimensions and layout 192

3.4.4. Emergency exits for crew members $\quad 94$

3.5. Some remarks concerning the external shape 94

3.5.1. Fuselages with a cylindrical mid-section $\quad 94$

3.5.2. Fuselages for relatively small useful loads 95 
4.1. Introductory comparison of engine types 99

4.2. Current reciprocating engines $r$

4.2.1. Some characteristics of the four stroke engine 101

4.2.2. Engine design and its influence on flight performance 106

4.2.3. Engine classification by cylinder arrangement 110

4.2.4. Two-stroke and Rotary Combustion engines . 111

4.3. Basic properties of aircraft gas turbines for subsonic speeds 112

4.3.1. The gas producer 113

4.3.2. The propulsive device 116

4.3.3. The pure jet engine 116

4.3.4. The turbofan engine 116

4.3.5. The turboprop engine 117

4.3.6. Overall efficiency, specific fuel consumption and specific thrust $\begin{array}{ll}\text { (power) } & 118\end{array}$

4.3.7. Analysis of the engine cycle 119

$\begin{array}{lr}\text { 4.4. Assessment of turbojet engines } & 120\end{array}$

$\begin{array}{lr}4.4 .1 . & 121\end{array}$

4.4.2. Turbine Entry Temperature 123

4.4.3. Bypass ratio 125

4.4.4. Engine noise 131

4.4.5. Summary and prognosis for the turbofan engine 133

4.4.6. Engine performance in non-standard atmosphere 133

4.5. Assessment of turboprop engines 134

$\begin{array}{lr}\text { 4.5.1. Performance } & 135\end{array}$

$\begin{array}{ll}\text { 4.5.2. Weight and drag } & 137\end{array}$

$\begin{array}{lr}\text { 4.5.3. Turboprop engine configurations } & 137\end{array}$

$\begin{array}{lr}\text { CHAPTER 5. DESIGN FOR PERFORMANCE } & 141\end{array}$

5.1. Introduction $r$

5.2. Initial weight prediction $\quad 144$

5.2.1. Stages in the estimation of airplane weight 144

5.2.2. Examples of weight "guesstimates" 145

$\begin{array}{lr}\text { 5.3. Initial estimation of airplane drag } & 148\end{array}$

$\begin{array}{lr}\text { 5.3.1. Drag breakdown } & 148\end{array}$

5.3.2. Low-speed drag estimation method 149

5.3.3. Compressibility drag 152

5.3.4. Retracing a drag polar from performance figures 153

5.3.5. Drag in takeoff and landing 153

5.4. Evaluation of performance requirements $\quad 155$

$\begin{array}{lr}5.4 .1 . & 155\end{array}$

$\begin{array}{lr}5.4 .2 \text {. Range performance } & 157\end{array}$

5.4.3. Climb performance 160

5.4.4. Stalling and minimum flight speeds $\quad 165$

$\begin{array}{lr}5.4 .5 . & 167\end{array}$

$\begin{array}{lr}5.4 .6 \text {. Landing } & 170\end{array}$ 
5.5. Aircraft synthesis and optimization

5.5.1. Purpose of parametric studies

5.5.2. Basic rules

5.5.3. Sizing the wing of a long-range passenger transport

5.5.4. Wing loading and thrust (power) loading diagrams

5.5.5. Optimization for low operating costs

5.5.6. Community noise considerations

CHAPTER 6. CHOICE OF THE ENGINE AND PROPELLER AND INSTALLATION OF THE POWERPLANT 181

6.1. Introduction

6.2. Choice of the number of engines and the engine type

6.2 .1 . Engine installation factors

6.2.2. Engine failure

$6 \cdot 2.3$. Engine performance and weight variations

6.2.4. Choice of the engine type

6.3. Characteristics, choice and installation of propellers

6.3.1. General aspects

6.3.2. Propeller coefficients and diagrams

6.3.3. Blade angle control

6.3.4. Propeller geometry

6.4. Installation of propeller engines

6.4 .1 . Location of the propellers

6.4.2. Tractor engines in the nose of the fuselage

6.4.3. Wing-mounted tractor engines

6.5. Installation of turbojet engines

$6 \cdot 5 \cdot 1$. General requirements

6.5 .2 . Fuselage-mounted podded engines

6.5.3. Wing-mounted podded engines

6.6. Miscellaneous aspects of powerplant installation 210

6.6.1. Thrust reversal

6.6.2. Auxiliary Power Units (APU)

7.1. Introduction and general design requirements

7.2. Wing area

7.2.1. Wing loading for optimum cruising conditions

7.2.2. Wing loading limits and structural aspects

7.3. Some considerations on low-speed stalling

7.3.1. Stall handling requirements and stall warning

7.3.2. Design for adequate stall characteristics

7.3.3. Stalling properties of airfoil sections

7.3.4. Spanwise progression of the stall

7.4. Wing design for low-subsonic aircraft

7.4.3. Thickness ratio 
7.4.4. Wing taper 236

7.4.5. Airfoil selection 237

7.4.6. Stalling characteristics and wing twist 239

7.5. Wing design for high-subsonic aircraft $\quad 241$

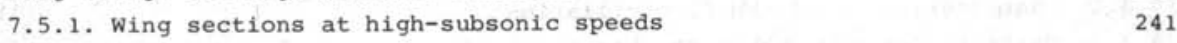

7.5.2. Wing design for high speeds 246

$\begin{array}{lr}\text { 7.5.3. Low-speed problems of high-speed wings } & 249\end{array}$

$\begin{array}{ll}\text { 7.5.4. Planform selection } & 251\end{array}$

7.6. High lift and flight control devices 252

7.6.1. General considerations 252

7.6.2. Trailing-edge flaps 253

7.6.3. Leading-edge high lift devices 256

$\begin{array}{ll}\text { 7.6.4. Flight control devices } & 257\end{array}$

7.7. Dihedral, anhedral and wing setting 258

$\begin{array}{ll}\text { 7.7.1. The angle of dihedral (anhedral) } & 258\end{array}$

$\begin{array}{lr}7.7 .2 \text {. Wing/body incidence } & 259\end{array}$

$\begin{array}{lr}\text { 7.8. The wing structure } & 259\end{array}$

$\begin{array}{lr}\text { 7.8.1. Types of wing structure } & 259\end{array}$

7.8.2. Structural arrangement in plan 261

$\begin{array}{lr}\text { CHAPTER 8. AIRPLANE WEIGHT AND BALANCE } & 263\end{array}$

8.1. Introduction; the importance of low weight 265

$\begin{array}{lr}8.2 \text {. Weight subdivision and limitations } & 268\end{array}$

8.2.1. Weight subdivision $\quad 269$

8.2.2. Weight limitations and capacities 027

8.2.3. Operational weights and the payload-range diagram 273

$\begin{array}{ll}\text { 8.2.4. The choice of weight limits } & 274\end{array}$

8.3. Methodology of empty weight prediction $\quad 275$

8.4. Weight prediction data and methods $\quad 277$

$\begin{array}{lr}\text { 8.4.1. Airframe structure } & 277\end{array}$

$\begin{array}{lr}\text { 8.4.2. The propulsion group } & 285\end{array}$

8.4.3. Airframe services and equipment 286

8.4.4. Useful Load and the All-Up Weight 293

$\begin{array}{lr}\text { 8.5. Center of gravity } & 294\end{array}$

8.5.1. The load and balance diagram 296

$\begin{array}{ll}\text { 8.5.2. Loading flexibility and restrictions } & 297\end{array}$

8.5.3. Effects of the general arrangement and layout 299

8.5.4. Design procedure to obtain a balanced aircraft 300

CHAPTER 9. PRELIMINARY TAILPLANE DESIGN 303

9.1. Introduction to tailplane design, control systems and stabilization 305

9.2. Static longitudinal stability and elevator control forces 308

9.2.1. Stick-fixed static stability and neutral point 308

9.2.2. Stick-free static stability and neutral point; the stick force
gradient

9.2,3. Stick-fixed and stick-free maneuver points and maneuver control 311 
forces

9.2.4. Reduction of control forces

9.2.5. Effects of compressibility and powerplant operation 316

9.3. Some aspects of dynamic behavior

9.3.1. Characteristics of the SP oscillation

9.3.2. Criteria for acceptable SP characteristics 319

9.3.3. A simple criterion for the tailplane size 320

9.3.4. The phugoid 323

9.4. Longitudinal control at low speeds 323

9.4.1. Control capacity required to stall the aircraft 324

9.4.2. Control capacity required for takeoff rotation and landing flareout 325

9.4.3. Out-of-trim conditions

9.5. Preliminary design of the horizontal tailplane

9.5.1. Tailplane shape and configuration

9.5.2. Design procedures

9.6. Design of the vertical tailplane

9.6.1. Control after engine failure: multi-engine aircraft 332

9.6.2. Lateral stability

9.6.3. Crosswind landings

9.6.4. The spin

9.6.5. Preliminary design of the vertical tailplane

10.2. Tailoring the undercarriage to the bearing capacity of airfields

10.2.1. Runway classification

10.2.2. The Equivalent Single Wheel Load (ESWL)

10.2.3. Multiple wheel undercarriage configurations

10.3.1. Angles of pitch and roll during takeoff and landing

10.3.2. Stability at touchdown and during taxying: tricycle undercarriages

10.3.3. Gear length, wheelbase and track: tricycle undercarriages

10.3.4. Disposition of a tailwheel undercarriage

10.4. Type, size and inflation pressure of the tires

10.4.1. Main wheel tires

10.4.2. Nosewheel tires

10.4.3. Inflation pressure

$\begin{array}{ll}\text { 10.5.1. Energy absorption on touchdown } & 360 \\ \text { 10.5.2. Dimensions of the gear } & 361\end{array}$

10.5.3. Gear retraction 362 
11.2.2. Wake drag, vortex-induced drag, and wave drag 368

11.2.3. Form drag, profile drag, and induced drag 369

11.2.4. Zero-lift drag and lift-dependent drag* 369

$\begin{array}{ll}11.2 .5 . & 370\end{array}$

$\begin{array}{ll}11.2 .6 . & 371\end{array}$

11.3. Determination of aerodynamic characteristics 371

$\begin{array}{ll}11.3 .1 . & 371\end{array}$

11.3.2. Mach number effects $\quad 372$

11.3.3. Low-speed polars $\quad 372$

11.4. The flight envelope 373

11.5. Flight profile analysis and payload-range diagram 375

11.5.1. Operational climb 375

$\begin{array}{ll}11.5 .2 . & 375\end{array}$

$\begin{array}{ll}11.5 .3 . & 377\end{array}$

11.5.4. Payload-range diagram and block time 378

$\begin{array}{ll}\text { 11.6. Climb performance } & 378\end{array}$

11.6.1. Maximum rate of climb, time to climb and ceilings 378

$\begin{array}{ll}11.6 .2 \text {. Takeoff and landing climb } & 378\end{array}$

11.7. Airfield performance 380

$\begin{array}{ll}\text { 11.7.1. Takeoff field length } & 380\end{array}$

$\begin{array}{ll}11.7 .2 \text {. Landing field length } & 381\end{array}$

11.8. Some aspects of operating economy 382

11.8.1. Economic criteria 382

11.8.2. Estimation of DOC 384

CHAPTER 12. EVALUATION AND PRESENTATION OF A PRELIMINARY DESIGN 389

12.1. Presentation of the design 390

12.2. External geometry and structural arrangement 390

12.3. Layout drawings 397

12.4. Conclusion 398

REFERENCES

APPENDIX A. DEFINITIONS RELATING TO THE GEOMETRY AND AERODYNAMIC CHARACTERISTICS OF AIRFOILS

A-1. General

$A-2$. Wing sections

A-2.1. Geometric definitions

A-2.2. Aerodynamic definitions $\quad 437$

A-2.3. Nomenclature for some NACA sections 438

A-3. Wings

A-3.1. Wing planform

A-3.2. (Wing) twist and incidence $\quad 440$

A-3.3. Aerodynamic definitions 440 
APPENDIX B. THE COMPUTATION OF CIRCUMFERENCES, AREAS AND VOLUMES OF CURVES, SECTIONS AND BODIES

$B-1$. Fuselage

B-1.1. General method

B-1,2. Quick method for bodies of revolution

B-2. Wings and tailplanes

$B-3$. Fuel tank volume

B-4. Engine nacelles and air ducts

References

APPENDIX C. PREDICTION OF WING STRUCTURAL WEIGHT

$\mathrm{C}-2$. Basic wing structure weight

C-3. High lift devices, spoilers and speedbrakes

D-1. Survey of the methodology

D-2. Gross shell weight

D-2.1. Gross skin weight

D-2.2. Gross stringer and longeron weight

D-2.3. Gross standard frame weight

D-3. Gross shell modifications

D-3.1. Removed material

D-3.2. Doors, hatches, windows and enclosures

D-4. Flooring

D-4.1. Passenger cabin and freight hold floors

D-4.2. Various other floors

D-5. Pressure bulkheads and frames

D-5.1. Pressure cabin bulkheads

D-5.2. Wheelbays for retractable undercarriages

D-6. Support structure

D-6.1. Wing/fuselage connection

D-6.2. Engine support structure

D-6.3. Other support structures

D-7. Additional weight items

APPENDIX E. PREDICTION METHODS FOR LIFT AND PITCHING MOMENT OF AIRCRAFT IN THE EN ROUTE CONFIGURATION

E-1. Applicability of the methods

$\mathrm{E}-2$. Contributions to the lift

E-3. Lifting properties of airfoil sections 
E-3.2. Lift-curve slope

E-3.3. Maximum lift

E-4. Wing lift and lift distribution

E-4.1. Lift-curve slope

E-4.2. Spanwise lift distribution

E-4.3. Zero-lift angle

475

E-4.4. Maximum lift

475

476

E-5. Pitching moment of the wing

E-5.1. Aerodynamic center

E-5.2. Pitching moment $\left(\mathrm{c}_{\mathrm{m}_{\mathrm{ac}}}\right)_{\mathrm{w}}$

477

477

$\begin{array}{lr}\text { E-6. Wing/fuselage interference effects on lift } & 477 \\ \text { E-7. Wing/fuselage pitching moment } & 479\end{array}$

E-7. Wing/fuselage pitching moment

E-7.1. Aerodynamic center

479

E-7.2. Pitching moment $\left(c_{m_{a c}}\right)_{w f}$

480

E-8. Nacelle and propeller contributions

481

E-9. Lift of the complete aircraft

481.

E-9.1. Tailplane lift

481

E-9.2. Total trimmed airplane lift

481

E-9.3. Wing/body incidence

481

E-9.4. Trimmed lift curve

482

482

E-10. Airplane pitching moment and neutral point (stick fixed)

482

E-10.1. The stick-fixed neutral point

483

E-10.2. Horizontal stabilizer incidence

483

References

APPENDIX F. PREDICTION OF THE AIRPLANE POLAR AT SUBCRITICAL SPEEDS IN THE EN ROUTE CONFIGURATION

F-2. Primary components of vortex-induced drag $\quad 492$

F-2.1. Untwisted plane wings

F-2.2. Drag due to twist

F-2.3. Wing tip correction on vortex-induced drag

F-2.4. Vortex drag induced by fuselage lift

496

F-2.5. Nacelle contribution

496

F-2.6. Horizontal tailplane contribution

496

F-3. Profile drag of smooth, isolated major components

F-3.1. The flat plate analogy

F-3.2. Wing sections

499

F-3.3. Wings

500

F-3.4. Fuselages and tail booms 501

$\begin{array}{ll}\text { F-3.5. Engine nacelles } & 505\end{array}$

$\begin{array}{ll}\text { F-3.6. Tailplane profile drag } & 508\end{array}$

F-4. Subcritical interference effects and corrections 509

$\begin{array}{ll}\text { F-4.1. Wetted area corrections } & 509\end{array}$

$\begin{array}{lr}\text { F-4.2. Wing/fuselage interference } & 509\end{array}$

$\begin{array}{ll}\text { F-4.3. Nacelle/airframe interference } & 510\end{array}$ 
F-4.4. Tailplane/airframe interference

F-5. Protuberances, surface imperfections and other extra's

F-5.1. Fixed undercarriages

F-5.2, Canopies and windshields

F-5.3. Wheel-well fairings and blisters

F-5.4. External fuel tanks

F-5.5. Streamlined struts

F-5.6. Powerplant installation drag

F-5.7. Excrescences, surface imperfections and other extra's

G-2. Effect of trailing-edge flap deflection on airfoil section lift 528

G-2.1. General aspects

G-2.2. Lift increment at zero angle of attack

G-2.3. Maximum lift coefficient

G-2.4. Lift-curve slope

G-3. Lift of aircraft with deflected trailing-edge flaps

G-3.1. Wing lift

G-3.2. Various contributions

G-3.3. Contribution of the horizontal tailplane 540

G-4. Prediction of the low-speed drag polar

G-4.1. Profile drag

G-4.2. Vortex-induced drag

G-4.3. Trim drag

$\mathrm{G}-5$. Leading-edge high-lift devices

G-5.1. Sections with plain leading-edge flaps

G-5.2. Sections with slats and Krueger flaps

G-5.3. Wing lift with leading-edge devices

G-5.4. Drag due to leading-edge devices

G-7. Ground effects

G-7.1. Ground effect on 1 ift

G-7.2. Ground effect on drag 553

G-8. Drag due to engine failure 553

G-8.1. Engine windmilling drag 553

G-8.2. Propeller drag $\quad 554$

G-8.3. Drag due to the asymmetric flight condition 555

References

APPENDIX H. PROCEDURES FOR COMPUTING TURBO-ENGINE PERFORMANCE FOR AIRCRAFT PROJECT DESIGN WORK 
H-4. Specific performance of turbofan engines

H-5. Thrust lapse rates, intake and exhaust areas of turbojet and turbofan engines

H-6. Specific performance of turboprop engines

$\mathrm{H}-7$. Cycle efficiencies and pressure losses

APPENDIX J. PRINCIPAL DATA OF THE US AND ICAO STANDARD ATMOSPHERES

APPENDIX K. THE DEFINITION AND CALCULATION OF THE TAKEOFF FIELD LENGTH REQUIRED FOR CIVIL TRANSPORT AIRCRAFT

$\mathrm{K}-1$. Reference distance definitions

$\mathrm{K}-2$. Reference speeds

$\mathrm{K}-3$. Procedure for determining the takeoff field length

$K-4$. Methods and data for the analysis of the takeoff

$\mathrm{K}-4.1$. The ground run from standstill to $\mathrm{v}_{\mathrm{x}}$

$\mathrm{K}-4.2$. The ground run from $\mathrm{v}_{\mathrm{x}}$ to $\mathrm{V}_{\mathrm{R}}$ 


\section{Foreword}

Since the education of aeronautical engineers at Delft University of Technology started in 1940 under the inspiring leadership of Professor H.J. van der Maas, much emphasis has been placed on the design of aircraft as part of the student's curriculum. Not only is aircraft design an optional subject for thesis work, but every aeronautical student has to carry out a preliminary airplane design in the course of his study. The main purpose of this preliminary design work is to enable the student to synthesize the knowledge obtained separately in courses on aerodynamics, aircraft performances, stability and control, aircraft structures, etc.

The student's exercises in preliminary design have been directed through the years by a number of staff members of the Department of Aerospace Engineering in Delft. The author of this book, Mr. E. Torenbeek, has made a large contribution to this part of the study programme for many years. Not only has he acquired vast experience in teaching airplane design at university level, but he has also been deeply involved in design-oriented research, e.g. developing rational design methods and systematizing design information. I am very pleased that this wealth of experience, methods and data is now presented in this book.

In the last twenty years of university education for engineers much attention has been devoted to the fundamental sciences such as mathematics and physics. Recent years have seen a revival of the interest in "design" and a number of general textbooks have now been published on this subject. However, very few modern textbooks on the science and the art of aircraft design, are available. It is my sincere hope that Mr. Torenbeek's book will contribute to a renewed interest in airplane design in many parts of the aeronautical world, both inside and outside universities.

In view of the immense increase of knowledge in the aeronautical sciences and engineering since the Second World War, it seems a formidable task, requiring much courage on the author's part, to write a textbook on airplane design. It is well-nigh impossible to deal with all problems of airplane design at the same depth and undoubtedly personal choice has to prevail in many areas with regard to the material to be presented. In my view, Mr. Torenbeek has made an excellent choice of his subjects, preserving a careful balance between the presentation of a design manual and a general textbook on airplane design. This volume will therefore be a most worthwhile guide to everybody who in the course of 
his professional training or career, is interested in the initial design phase of airplane projects, an activity which is very important for shaping the future of aviation.

Delft University of Technology

August 1975
H. Wittenberg

Professor of Aerospace Engineering 


\section{Author's preface}

This textbook is intended to offer readers with a professional interest in airplane design a general survey of the layout design process. It contains a large amount of data and numerous methods which will be useful for carrying out the initial design calculations associated with the dimensioning of all major airplane parts. To a certain extent it has the character of a design manual, but considerable attention is also devoted to qualitative background information.

Several of the design methodologies and procedures presented have already appeared in the literature on the subject, while others have been developed recently by the author. They have been chosen on the basis of two criteria: they are not overdependent upon the state of the art and they give reliable results with a minimum of information. Most of the procedures have been extensively tested and considerably improved during the decade for which the author was responsible for students' design courses and projects in the Department of Aerospace Engineering of the Delft University of Technology. Emphasis is laid on conventional subsonic airplane designs in the civil category, i.e., broadly speaking the airplane types to which the American FAR Parts 23 and 25 and equivalent BCAR requirements apply (light and transport-type aircraft). Although many of the aspects to be discussed are equally relevant to $\mathrm{V} / \mathrm{STOL}$ and military aircraft, other complicating factors are involved in the design of these types, resulting in a radically different approach to the design process. The large variety of design specifications and configurations in these categories prohibits a general treatment.

The author makes no apology for the fact that his approach to airplane design may be biased by a university environment, probably not the ideal one in which to carry out design studies. The teaching of design in the aeronautical departments of universities and institutes of technology has, unfortunately, not kept pace with developments in industrial design practice. Aircraft design and development have become a matter of large investments, even in the case of relatively small projects. The manhours required have increased considerably in recent years and the time is almost past when a single designer could consider himself the spiritual father of a new type.

In contrast with the increased sophistication to be observed in industrial design very few regular design courses at technological universities and institutes have been able to survive the process of continuous curriculum evaluation and revision.

Although experienced designers in the industry may possibly be the only authors qualified to write an authoritative textbook on airplane design, they are usually not in a position to devote enough of their time to a task which is not felt to be in the direct interest of their employers. The reader may therefore conclude that the present book will be most 
useful for teaching and study purposes and for people who need a general introduction to the vast field of initial aircraft design and development. Nevertheless, some of the procedures and data presented will certainly be of some assistance to design departments in industry.

A knowledge of the principles of applied aerodynamics, airplane structures, performance, stability, control and propulsion is required to derive the utmost from this book. Its usefulness for degree design courses will therefore be greatest in later stages of the course. In the presentation of the individual subjects the need to balance design considerations is frequently stressed. This is particularly the case in the second chapter, where the initial choice of the general arrangement is discussed, the basis adopted being a synthesis of many considerations of widely differing character. The main body of the book is devoted to the rationale behind layout design and although estimation methods for lift, drag, geometry, etc. are considered essential parts of the design process, they have been brought together in a separate set of appendices with a limited amount of text. Considerable attention is devoted in all the chapters to the impact of airworthiness requirements on design and to subjects that have been covered only very briefly by other authors. Particular emphasis is laid on the interior layout of the fuselage (Chapter 3), a survey of the present and future potentials of aircraft engines (Chapter 4), systematic design studies based on performance requirements (Chapter 5), and weight estimation methods (Chapter 8 ). The complex interaction of wing location, center of gravity range, and horizontal tailplane design is treated in Chapters 8 and 9. The consistent collection of prediction methods for lift, drag and pitching moment estimation will, it is thought, be useful as a general survey and as a tool for wing design (Chapter 7) and performance calculations (Chapter 11). A large collection of statistical data, illustrations and diagrams is added to this presentation, which aims at providing the individual student/ designer or the small design team with reliable guidelines. For industrial applications some of the methods may have to be refined and/or extended.

A large and systematic list of references to literature is presented, which will help the reader to find more information on the subjects specifically dealt with and on other related subjects. As he glances through these references the reader's attention may be drawn to a particular subject that interests him, possibly stimulating him to add another innovation to the design synthesis of his project and thereby contribute to the overall quality of aircraft design technology.

\section{ACKNOWLEDGEMENTS}

As is the case in the preparation of most technical books, the author of this volume is indebted to many persons who have aided in its completion.

For many years professor H. Wittenberg has been the promotor of courses in preliminary aircraft design, and the idea of writing this book came about as a direct consequence of his activities. The author wishes to express his appreciation to him for his general support, for his critical revision of the text and for his willingness to write the foreword.

I am indebted to Mr. G.H. Berenschot, who has given general and technical assistance by collecting information and data, preparing many figures and tables, compiling the index and revising the text in detail. His perseverance, friendship and the moral support he has given me for many years have been particularly invaluable.

I would like to express my appreciation to Professor J.H.D. Blom, chief aerodynamicist, and Ir. P.F.H. Clignett, preliminary design engineer, both of Fokker-VFW International 
as well as to Ir. C.H. Reede, head of the scientific department of the Royal Dutch Airlines (KLM), and to my colleagues Ir. F.W.J. van Deventer and Dr. Th. van Holten, who provided valuable and detailed suggestions together with actual text after reading parts of the book for their technical content. In addition, many students have used forerunners of the present text during their studies and their useful feedback has resulted in many improvements.

Thanks are due to the Department of Aeronautics and Space Engineering of Delft University of Technology for granting permission to prepare and publish this book, and for providing the necessary typing and duplication facilities. Many members of this Department and of the Photographic office of the Central Library have given professional help in producing the illustrative material. I am also indebted to the Delft University Press, and in particular to Ir. P.A.M. Maas and Mrs. L.M. ter Horst-Ten Wolde for their support, encouragement and assistance in editing the publication.

The author wishes to express his gratitude to Messrs. J. van Hattum, J.W. Watson M.A., and D.R. Welsh M.A., who have made admirable contributions to the translation of the Dutch text and to improving the readability of the book. I am extremely indebted to Mrs. C.G. van Niel-Wilderink, for the excellence with which she performed the formidable task of typing not only the manuscript, but also the final copy for photoprinting. I would like to thank my uncle, the Rev. E. Torenbeek, for his painstaking efforts in checking the typographic accuracy of the copy, and mr. P.K.M. De Swert for preparing the final layout and for his methodical checking.

The following individuals, companies and organizations have kindly provided data and drawings.

Advisory Group for Aerospace Research and Development: Figs. 5-24, 10-1, 10-2, 10-3, $\mathrm{F}-17$ and $\mathrm{F}-22$;

American Institute of Aeronautics and Astronautics: Figs. 2-1, 4-39, 7-20, 8-1, 9-11, $10-5, F-12$ and $\mathrm{G}-25$;

Aeronautical Research Council: Figs. 2-24, G-16, G-17 and $\mathrm{K}-6$;

Aérospatiale: Figs. $2-3,2-10$ and $7-25$;

Airbus Industries: Figs. $11-5$ and $11-9$;

Aircraft Engineering: Figs. 3-1, 3-11, 3-21, 3-26, 6-16, 7-25, 10-17, 10-16, 12-3 and $12-5$;

Alata Internazionale: Fig. 2-3;

The Architectural Press Ltd.: Fig. 3-6;

Avco Lycoming: Fig. 4-14;

Aviation Magazine: Fig. 2-8;

Avions Marcel Dassault-Bréguet Aviation: Figs. 3-3, 3-11 and 10-20,

Boeing Aircraft Company: Figs. 2-11, 2-15, 3-11, 5-18, 7-25 and 10-6,

Mr. S.F.J. Butler: Fig. F-22;

Canadair Limited: Fig. 3-21,

Canadian Aeronautics and Space Institute: Fig. 3-27;

Centre de Documentation de l'Armement: Figs. 2-5 and 10-21;

The De Havilland Aircraft of Canada Ltd.: Fig. 3-11;

Detroit Diesel Allison Division of General Motors Corporation: Fig. 4-46;

Dowty Group: Fig. 6-27;

Engineering Sciences Data Unit Ltd.: Figs. F-23, G-26 and K-9;

Flight Control Division of U.S. Air Force Flight Dynamics Laboratory: Figs. A-2, A-3, E-3 and $\mathrm{E}-9$;

Fokker-VFW International: Fig. 11-14; 
Flight International: Figs. 2-2, 2-10, 2-11, 3-8 and 7-30;

Hamilton Standard Division of United Technologies Corporation: Fig. 6-5;

Hartzell propeller Inc.: Fig. 6-7;

Hawker Siddeley Aviation: Figs. $2-20,3-21,12-2$ and $12-4$;

International Civil Aviation Organisation: Figs. 10-4 and 10-5;

De Ingenieur: Fig. 5-22;

Institute of Aerospace Sciences: Fig. 6-24;

Lockheed Aircraft Corporation: Fig. 3-17;

McDonnel1 Douglas Corp.: Figs.3-14, 6-2, 7-25;

McGraw-Hill Book Cy.: Fig. 6-19;

Messerschmitt-Bölkow-Blohm: Fig. 2-7;

(British) Ministry of Aviation: Fig. 10-6;

National Aeronautics and Space Administration: Figs. 2-25, 6-5, 7-5b, 7-22, 9-8, E-2, $\mathrm{E}-5, \mathrm{E}-6, \mathrm{G}-11, \mathrm{G}-12, \mathrm{G}-19$ and $\mathrm{G}-22$;

National Research Council of Canada: Fig. 3-27;

Pergamon Press: Fig. 5-11;

Mr. D.H. Perry: Fig. K-6;

Piper Aircraft Corporation: Fig. 4-10;

Polytechnisch Tijdschrift: Figs. 2-10 and 7-25f;

Pratt and Whitney Aircraft Division of United Technologies Corp.: Fig. 4-46;

The Royal Aeronautical Society: Figs. 1-1, 2-14, F-6, F-22 and $\mathrm{K}-8$;

Rolls Royce 1971 Ltd., Derby Engine Division: Figs. 4-15, 4-19, 4-20, 4-37, 4-46 and 6-25;

Dr. W. Schneider; Figs. $\mathrm{C}-2$ and $\mathrm{C}-3$;

Ir. G.J. Schott Jr.: Fig. 5-22;

Society of Aeronautical Weight Engineers: Figs. $11-7$ and $D-1$;

Society of Automotive Engineers, Inc.: Figs. 4-7, 4-8, 4-9, 6-2, 6-7, 6-23, 6-27, 7-5 and 11-13;

Mr. W.C. Swan: Fig. F-17;

Turboméca Bordes: Fig. 4-46;

Vereinigte Flugtechnische Werke-Fokker Gmbh: Fig. 3-11;

Mr. R.E. Wallace: Fig. 5-24;

John villey and Sons Ltd.: Fig. F-8.

Finally, I would like to thank my wife, Nel, for her unstinting help without which this book would hardly have been possible.

September 1975

Ir. E. Torenbeek

Delft University of Technology

UNITS

In accordance with the convention used in publications such as Jane's All the World's Aircraft and Flight International, all data and most of the figures have been given in the technical unit system, both in British and metric units. Hence, $1 \mathrm{~b}$ and $\mathrm{kg}$ refer to pound and kilogram forces, respectively. An exception is made in Appendix $J$, where sea level data of the Standard Atmosphere have been given both in the technical and SI systems. 


\section{Preface to the student Edition}

Textbooks on the rapidly advancing subject of aircraft design tend to become obsolete within a few years. In spite of this the first edition has proved its value up to the present time, as a reference source for design efforts and publications in many places all over the world.

It therefore pleases me that the publishers have decided to launch this new edition, aimed at an expansion of the market into the university classroom, thereby making the book affordable by many more individuals.

This has given me the opportunity to further refine some of the methods and formulations, mainly on the basis of suggestions and comments of attentive students. In spite of the reduction in size, the contents are not abbriviated.

Delft, May 1981

E. Torenbeek 


\section{Chapter 1. General aspects of aircraft configuration development}

\section{SUMMARY}

It is shown that there is an interaction between the development work for a new design and the various factors determining the need for a new type of aircraft. Preliminary aircraft design is an essential part of this development phase; its aim is to obtain the information required in order to decide whether the concept will be technically feasible and possess satisfactory economic possibilities.

Attention is paid to the impact of the design requirements laid down in the initial specification and the airworthiness regulations. General observations aimed at illustrating the aircraft design and optimization process are presented. 
1-1. INTRODUCTION

In the pioneering era of civil aviation the aircraft designer had only a very limited choice. There was practically only one category of powerplant at his disposal, namely the - nearly always aircooled - piston engine, which gave very limited power. Either there were no aerodynamic aids to augment the lift of the wing at low speeds, or those that did exist were, for various reasons, seldom used, with the result that wing loadings were kept low and high speeds were consequently unobtainable. Low wing loading favored the biplane layout which, with its high parasitic drag, formed another obstacle to high speeds. Flight was rarely above 10,000 feet $(3,000$ meters $)$, since there were no pressurized cabins. During this epoch aircraft design was generally the work of one or a very few designers in each factory and the scope of development work for each new aircraft type was 1 imited. In the twenties it was possible to design and produce a new aircraft for delivery to the customer within half a year, one of the reasons being that series were relatively small. This enabled Anthony Fokker and his staff to build fourteen entirely different commercial designs during the relatively short period of eighteen years (1918-1936). But the nature of aircraft project design has undergone a radical change since the Second World War. Development of the jet engine and subsequently the turbofan, now supplying a thrust up to about $50,000 \mathrm{lb}$ $(22,500 \mathrm{~kg})$, has greatly widened the choice of powerplants. Transport aircraft now cruise at altitudes of 30,000 to 40,000 feet $(9,000$ to $12,000 \mathrm{~m})$ at speeds not far below that of sound. The takeoff and landing speeds of the largest aircraft have, of course, risen steadily and runways have consequently become longer and longer, but means to call a halt to this trend have meanwhile proved technically feasible. Air transport has passed through an era of unprecedented growth. During the period from 1950 till 1970 the average yearly increase in the passenger-miles flown reached
148 , a growth figure which was only exceeded by the sale of plastics. The increase in transport productivity (payload times speed) of the largest transport aircraft has been equally impressive. In addition, modern aircraft have to satisfy an ever increasing number of severe safety regulations, while economic requirements, resulting from intense competition, have steadily become more exacting, with the result that the development and construction of new types of aircraft - even relatively small ones - demand a very high capital outlay and entail considerable financial risk. An aircraft industry nowadays is generally unable to produce an entirely new type of aircraft oftener than once every 12 years (roughly), quite apart from the question of whether there is any need for more rapid replacement of existing types. An exception to this "rule" must be made for the giants in the aircraft industry, e.g. Boeing and McDonnell Douglas, who are able to bring out one or two additional new designs during the same period. The sheer size and long leadtime of new projects have led various firms to share the risks by cooperation, while in Europe they have resulted in international joint ventures.

Although new concepts have been and will continue to be proposed from time to time by talented designers, the time is past when a chief designer could be regarded as the spiritual father of a new type of aircraft. A possible exception to this might be made in the case of private aircraft and small transports. Preliminary design departments nowadays have staffs numbering some dozens to several hundreds of highly trained technicians and engineers and computing facilities have increased immensely, while some preliminary design teams even have wind tunnels permanently at their disposal. More manhours are now being invested in the project design phase than were formerly spent on the entire detail design. Work in the design department has developed into a professional sccupation, carried out in teams, with regular consultations between specialists in various disciplines. 


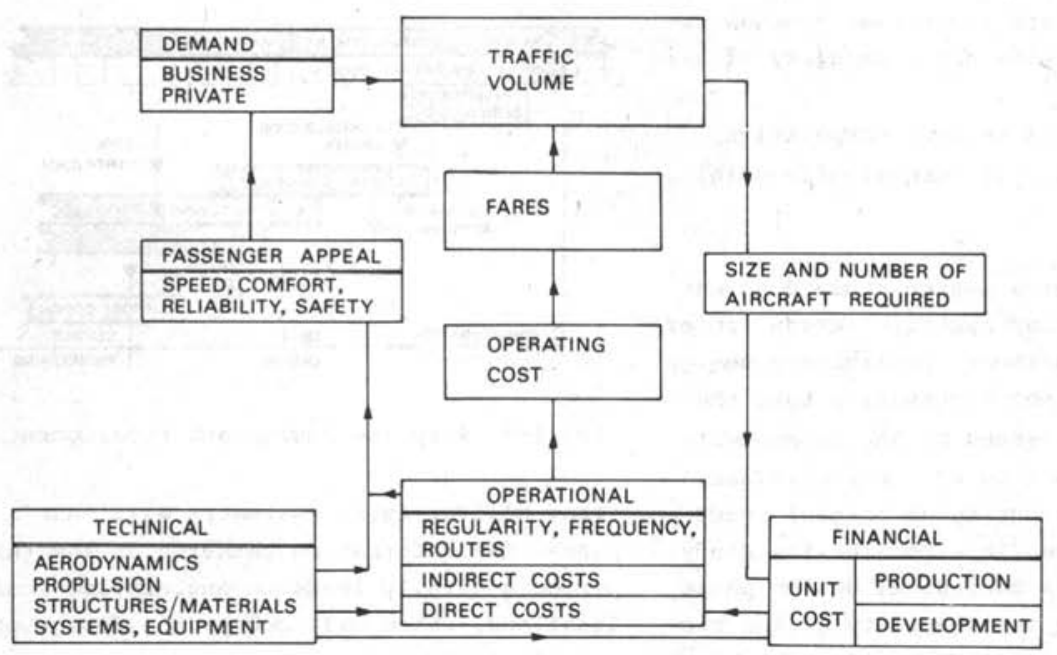

Fig. 1-1. Factors contributing to the growth of air transport, according to Morgan (Ref. 1-26)

This does not imply that the man in overall charge of preliminary design has himself to be a specialist. As will be shown further on, he must be able to take a wide interest in and have a sound insight into a great many disciplines related to design as a whole.

\subsection{AIRCRAFT DESIGN AND DEVELOPMENT}

Although the technical aspects of aircraft design form the subject of this book, it will be appreciated that this is not an activity carried on in remote offices by specialists generating designs of any kind that may occur to their imaginations. There is close interaction between the development work for a new aircraft type and the other factors which together determine the growth of and/or changes in aeronautical activities. These interrelationships being different for the various fields of aeronautics (passenger and cargo transport, business aviation, tourism, flying instruction, etc.), we will deal with the matter by quoting a single example here.

The development of new airliners has always been stimulated mainly by the growth of the traffic volume and the improvement of technical and operational standards. Fig. 1-1 is a scheme for allocating the various fac- tors contributing to the growth of air transport. Growth in air traffic stems from reduction of fares, improved quality of the aircraft (speed, comfort), increased business activity and growth of private incomes, aircraft capacity growth, increasing number of routes, increasing frequency on existing routes, and greater utilization of aircraft and ground facilities. The contribution of research and development to this process, indicated in the lower left-hand corner of the diagram, is unique because this block shows only output lines. Although the diagram is obviously a simplification of the real situation and, at the same time, must not be considered as a control system*, it does indicate that aircraft development is a primary cause of growth. As a corollary to this, it is necessary in launching a new development program to appreciate the interacting effects of the "aeronautical environment" in advance and thereby ensure that there will be no conflict with the (future) needs of operators, passengers and the general public. Moreover a number of industrial constraints set limits to the feasibility of new projects, namely:

a. the available project development organization and production capacity;

*For example, the role of the government's aeronautical activities has been omitted. 
b. the technical and industrial knowhow required for developing a new category of aircraft;

c. the prospects as regards competition;

d. the availability of adequate financial backing.

The initiative for a design study does not always stem from any specific person (chief designer) or department (preliminary design office) and does not necessarily take the form of an order issued by the management. The idea is elaborated by the preliminary design department during an initial speculative design phase in a feasibility study. The object of this conceptual design phase is to investigate the viability of the project and to obtain a first impression of its most important characteristics.

If the results seem encouraging both from a technical point of view and as regards the market prospects, a decision may be taken to develop the design further in order to initiate a new-design aircraft development program (Fig. 1-2). Comparisons will be made with some alternatives, preferably on a systematic basis. The design that has scores the highest rating will be elaborated in greater detail in the preliminary design phase. A characteristic of this phase is that modifications are made continuously until a decision can be taken to "freeze" the configuration*, and this marks the end of the preliminary design phase. If the market is considered likely to receive the design favorably and finance for the project is assured, the management may give permission for further development (goahead approval). The subsequent phases of detail design, construction and testing will lead to the granting of a Certificate of Airworthiness and some time later the

*The expression "configuration" as used in this chapter refers to the general layout, the external shape, dimensions and other relevant characteristics. It is not intended to indicate the actual airplane configuration as characterized by the position of the flaps, landing gear, etc.

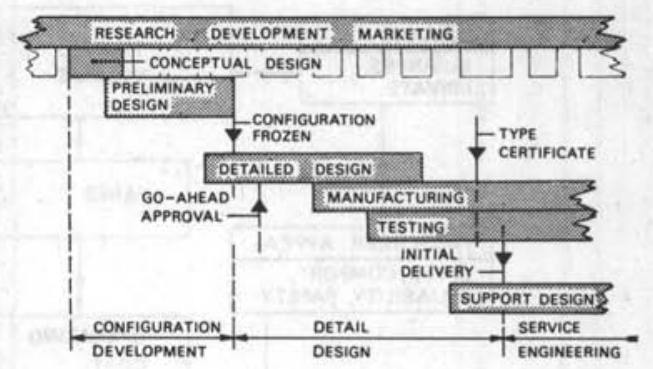

Fig. 1-2. Airplane design and development

first deliveries to customers will take place. The information gathered during this period generally leads to engineering modifications, which will occupy the design office for a long time to come.

After the first production series has left the factory, the company will continue to develop its product. These developments may take the form of an increase in the airplane's transport capacity (stretching), installation of an improved type of engine, improvement of performance by introducing aerodynamic refinements, such as "cleaning up" the aircraft, etc. Successful aircraft generally go through a process of growth which offers the customer a choice of a number of variants, each suitable for a specific transport assignment, and this considerably strengthens the company's ability to face up to competition. In Fig. 1-2 these activities have been arranged in three groups: the configuration development* phase, the detail design phase and the service engineering phase. The first two of these have been taken as separate phases, since the decisions taken during the first stage are still partly based on the statistical probabilities that specific technical aims will be achieved and the actual construction is only defined in broad general terms, whereas in the detail design phase the aircraft is designed "down to the last rivet" and the detailed production schedule is laid down. During this period the number of

*Frequently referred to as "preliminary design engineering". 


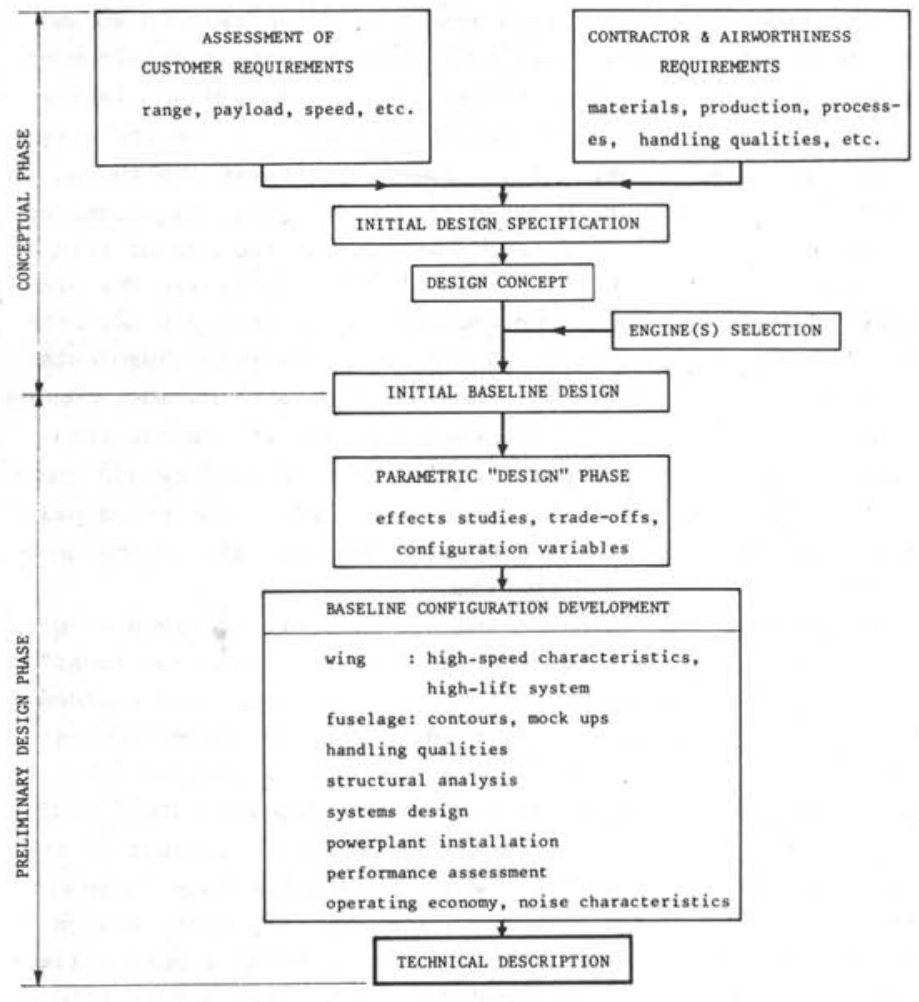

Fig. 1-3. Configuration design and development of a high-subsonic transport aircraft.

documents and drawings will increase rapidly and the development costs will show a nearly proportional rise.

\subsection{CONFIGURATION DEVELOPMENT}

The principal aim in this phase of design (Fig. 1-3) is to obtain the information required in order to decide whether the concept will be technically feasible and have satisfactory economic possibilities. In contrast to the detail design phase, neither the actual construction process nor the detailed production schedule plays a dominant part here.

An important aspect of the entire development of a new type of aircraft is that it takes place in a succession of design cycles. In the course of each of these cycles the aircraft is designed in its entirety and investigation is carried out into all the main groups and airframe systems and equipment to a similar degree of detail. The extent of this detailing steadily increases as the design cycles succeed each other, until finally the entire aircraft is defined in every detail. On the basis of the terminology given in Figs. 1-2 and 1-3, the subsequent basic design stages might be designated as follows:

a. conceptual design;

b. initial baseline design;

c. baseline configuration development;

d. detail design.

These design stages might also be referred to as the speculative design, the feasible design, the best conceivable design and the final hardware design cycles. A number of aspects of the first three of these design cycles will be further elaborated and discussed in the course of this book. The conceptual design will be the subject of the second chapter. Chapters 3 through 10 dis- 
cuss the procedure followed in evolving an initial baseline configuration and constitute the main portion of this book. Chapters 11 and 12, finally, present a survey of several items which are related to further elaboration and presentation of a design. Since the information on these items available elsewhere is abundant and exceeds the scope of our textbook, a representative choice has been made from the multitude of subjects, while some have been further elaborated in the form of appendices. In view of the fact that the author has been mainly concerned with aspects of aerodynamic design and performance, it was decided to present methods required to estimate some aerodynamic and flight characteristics.

\subsubsection{The design concept}

In the initial phase the probable demand for a new type of aircraft is further specified with the aid of market surveys, further inquiries and discussions with potential customers. Market research forms a specialized discipline for which the large aircraft companies employ a separate department or office, while in smaller factories the work is often done by the design team. In either case, it is essential that the designer or the design team is closely involved since there is no sense in starting a design before the nature of the design requirements has been studied from all angles and a clear picture has emerged on which to base the general design philosophy.

The market survey will lead to an initial specification which will mainly define the transport performance - payload and maximum range, often also the cruising speed as well as the most relevant field and climb performance, cabin arrangement, airframe services and equipment, etc. A decision will also have $L O$ be made as to which set of airworthiness and operational requirements the design will have to comply with. Fig. 1-3 oversimplifies the situation by presenting the airworthiness require- ments in a separate box whereas in actual fact they influence the entire development of the aircraft and are a dominant factor in every design decision down to the very last detail. Special attention is therefore devoted to airworthiness requirements in a separate section at the end of this chapter. Prior to and also during the project, the designer will closely study other types of aircraft or aircraft projects or certain design aspects which most closely fit the specification as regards the transport requirements. A summary and critical assessment are made of the principal data of competing designs, literature, previous experience, etc.

Brainstorming sessions are arranged in order to generate "new and wonderful ideas", most of which will generally be discarded again. The reader will find some interesting creative attempts to stimulate new conceptions in Ref. 1-13, while Ref. 1-35 presents a most fascinating account of the activities which take place in a large aircraft industry during the project design phase. Nevertheless it remains most unlike1y that procedures developed during this extremely speculative first design phase will offer any certainty of leading to a successful new conception. There is no real substitute for the originality of the engineer who is capable of forcing a breakthrough with a unique brainwave such as the monocoque construction, the sweptback wing, jet engines at the rear of the fuselage (a la Caravelle), area ruling and other inspired innovations, sometimes generated in an environment where project design is engaged in only incidentally, if at all. Design concepts are therefore being developed continuously, while only very few actually result in a preliminary design and subsequent development program (Fig. 1-2). The conception phase will result in preliminary layout sketches of the kind shown in Fig. 1-4, including a summary of the principal characteristics and basic design philosophy on which subsequent design stages will be founded. Although hardly any thought has been given at this stage to 


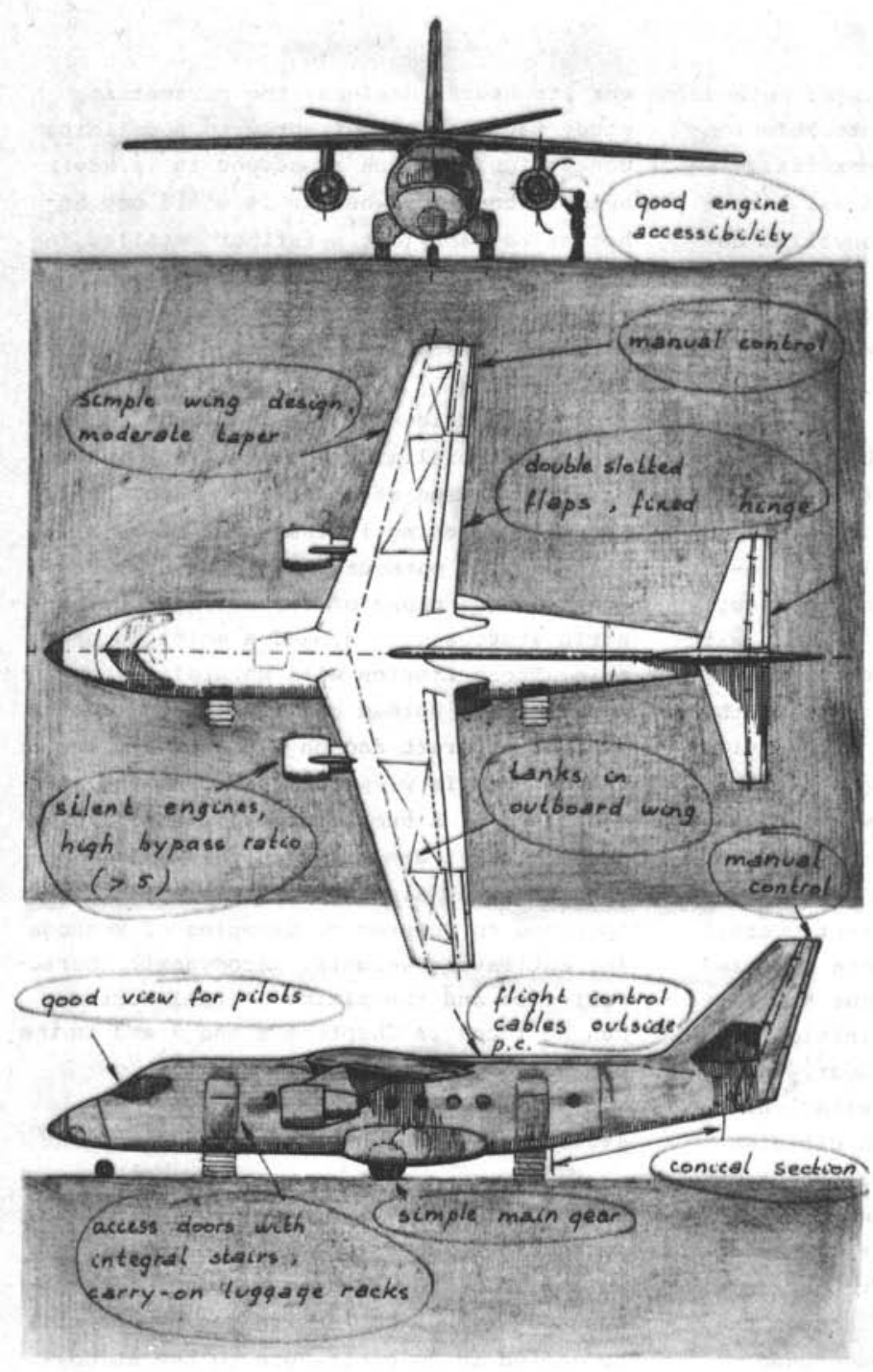

Fig. 1-4. Initial design concept of an ultra-short-haul airliner ( 30 passengers) details, a complete aircraft has already been put on paper and the designer may nave a strong feeling that it could be the answer.

\subsubsection{Initial configuration design and configuration variations}

Since design is not a deterministic process, particularly in the early stages in which the conception is realized, various solutions to attain the desired goal will present themselves. If it proves impossible to weigh up the pros and cons and arrive a realistic answer, based on the intuition and experience of the designer(s), comparative studies will have to be undertaken. Since it will generally not be found feasible to transform all likely configurations into fully developed projects, a parametric design phase, as shown in Fig. $1-3$, is often decided upon. This first entails the development of an initial baseline design (or point design), using relatively easily applied sizing methods, provided these are available. In this book the 
reader will find a number of these methods, based partly on theoretical interrelationships, partly on statistical material (see, for example, Chapters 5, 6 and 7). A complete layout is made of this baseline design, showing three views and some principal cross-sections. The next step is to check to what extent the characteristics and performance of the design will meet the design requirements.

Changes are now made in this baseline design, preferably in a systematic manner, following predetermined and clearly defined working rules. There will thus emerge a family of designs which are easily comparable with each other as well as with the baseline design. The object of this exercise is twofold: first to improve the design where it does not meet the requirements, and second to investigate the most likely possibilities and see whether other variants may prove a better proposal. It may also show that changes in the design requirements would yield a better overall balance. Although the diagram in Fig. 1-3 suggests that the type of engine has already been chosen before the initial baseline design has been put on paper, the parametric design phase may nevertheless include studies of variants with different types of engines and even a different number of engines per aircraft. When a number of variants are studied, a systematic approach is essential in order to obtain a sound basis for comparison. Although the absolute accuracy of the methods used should be as high as possible, the main objective is to differentiate between the designs. Final judgement at the end of this phase will result in a baseline configuration which, subject to approval by all concerned, will be chosen for further development. It can be presumed that this design, after detailed engineering, will probably meet the initial specification while, in addition, it will be the best conceivable design. On the other hand, in the absence of complete certainty as to specific aerodynamic characteristics of the consequences of variations in weight as between differ- ent structural designs, the parametric study may not lead directly to a definite conclusion. In such an advent it is advisable to consider whether it would not be better to carry out a further detailed investigation of, say, two alternative configurations before an irrevocable choice is made. This point is treated more fully in Ref. 1-38.

To give some idea of the magnitude of this phase of a preliminary design, the initial baseline design of a small transport will require something in the order of several thousands of manhours, whereas the subsequent design phase of variants and parametric studies will demand a multiple of this. These figures will naturally show a considerable spread and will depend on the type of aircraft and on the extent to which the company is prepared to pursue the investigation. A number of examples of parametric studies are given in this book (Section 5.5) and also in the references appended to Chapter 5. Examples of methods for estimating weights, aerodynamic characteristics and the sizing of tail surfaces can be found in Chapters 8 and 9 and in the appendices.

\subsubsection{Baseline configuration development}

During this phase the baseline design is further developed to a depth of detail which can be regarded as meaningful. Various sections of the design department will be called in to contribute to the aerodynamic design, the stressing of the main structure, design of the airframe systems and equipment, etc. At the earliest possible stage a start is made with tests in the wind tunnel, while the external lines are determined and mockups showing the internal layout of the fuselage, cable runs and installations, are built and all the remaining tasks involved in arriving at a complete definition of the project are carried out.

During the development of the baseline configuration, errors will be observed which have been made during the phases already 
described and which are usually caused by lack of data. Correction of these errors will entail corrections in the design which will have repercussions for all the disciplines involved.

Coordination during this phase will be the responsibility of the preliminary design department, for this team will be most familiar with the project and is consequently best able to visualise the consequences of the corrections. One of the most frequent jobs during this phase is the sètting-up of a weight control program, particularly for those weight components which have been estimated solely on the basis of statistical material. These rectifying programs and corrections may in some cases be covered by feedbacks in Fig. 1-3, but specific mention of them there has been omitted to preserve the clarity of the illustration.

As soon as the project can be regarded as sufficiently mature and any doubts regarding its essential characteristics have been removed, the project manager may take the decision to freeze the configuration and this means the end of the preliminary design phase. The characteristics of the design are summarized in a technical description which serves as a basis for discussions with potential customers.

Some idea of the scope of a configuration development program can be obtained from Ref. 1-36, which gives the following information concerning the Lockheed L-1011: "In the two years of the configuration development, over two million man hours were expended to investigate various configurations and approaches to determine the optimum design. More than 10,000 hours of testing have been completed in seven different wind tunnels to establish the most efficient overall configuration".

\subsubsection{The preliminary design department}

When the development of a new type of aircraft is to be undertaken, the general practice nowadays is to form a project group, containing not only preliminary de- sign engineers but also experts in other disciplines, such as:

- aerodynamicists who are directly concerned with the design of the external shape,

- structural engineers, dealing with preliminary research into the overall structural layout and carrying out the dimensioning and optimisation,

- production experts and experts in the materials field who investigate what types of production methods should be adopted, - service experts, to ensure easy maintenance and overhaul,

- weight engineers, whose job is to deal with the prediction and control of the weight (distribution) and moments of inertia,

- engineers to design the flight control system and analyse flying qualities, - designers of airframe systems and equipment, and

- financial and economic experts who are not only able to estimate the first and operating costs of the aircraft, but also keep a close check on the financing of the entire design project.

Since this book does not deal primarily with the organizational aspect of project development, but rather with the technical aspects of airplane design, we will specify only the various tasks of the preliminary design team. Unlike the other departments of the design office, this team is permanently engaged in project work and its work mainly consists of the following activities:

- market analysis and the drawing-up of initial specifications for new types of aircraft in close cooperation with the sales department;

- devising various solutions to a given design problem;

- evaluation of different design proposals using preliminary design methods in order that decisions are taken on the basis of a sound assessment of the pros and cons; - setting up and coordinating detail research oriented on aerodynamical, structural and other problem areas. These tasks 
may be of a general character, such as the development of design methods for estimating drag, weights, etc., or projectoriented, such as the aerodynamic design of a flap system;

- discussions with potential customers and (future) subcontractors for main components such as engines, landing gear, airframe services, avionics, etc.;

- assisting the sales department by supplying technical data;

- making product development studies, aimed at increasing the utility of existing aircraft.

\subsection{THE INITIAL SPECIFICATION}

There is certainly no need to prove that sufficient material on a subject such as "market analysis aimed at the development of new aircraft" exists to warrant the publication of a separate volume. The present paragraph will of necessity have to be restricted to a few general observations with civil aviation as their main background. The example used will be an initial design specification for a hypothetical short-haul airliner for 180 passengers in the all-tourist layout, referred to as "Project M-184". A design evaluation of this project can be found in Ref. $1-64$; it was intended as a highly simplified example for the purpose of illustrating the design process in a series of lectures. An apology is due for the fact that most of the considerations which follow in the present section apply to this particular design, intended for introduction into service around 1980 .

In civil aviation the specification of a new aircraft type is generally drawn up by the manufacturer. Airlines are usually more content to evaluate projects offered to them for use on their own route network, though in a few cases they themselves have taken the initiative and written the specification which they felt was required. The designer will, however, realize that a project can only be justified when it is likely to find a worldwide market. A specification issued by an airline will only be interesting provided it also appeals to competitors. It should also be realized that operators do not necessarily possess the best insight into the technical capabilities and knowhow of the airplane manufacturer. Nevertheless, there are examples of successful aircraft which have been designed to an operator's specification or a specification written with the customer's active cooperation (Viscount, Tristar and DC-10). All the same, the responsibility for the specification and the resulting project will still rest squarely on the shoulders of the aircraft manufacturer. This procedure is quite different from the case of military aircraft, where the specification will nearly always be issued by the customer: the armed forces.

The term "initial specification", as opposed to the more detailed type specification of a design, is used here to emphasize that there is an interaction between the technical design work and the development of the design requirements as a result of market analysis, engine development and various assessments during the development phase.

\subsubsection{The need for a new type of aircraft}

The following are some good reasons for initiating a new aircraft design:

a. Existing aircraft are becoming either technically or economically obsolescent, and a new type may do the job better. New standards for equipment, maintenance, operational use, noise suppression, passenger comfort, etc. may make renovation of the operator's fleet desirable.

b. Certain developments in traffic patterns have created a need for new types of air transport. For example, the growth of traffic may, as explained in Section 1.2 , result in a new class of (larger) transport aircraft, or new travel habits (home to work and back) may open up the possibility of a new class of commuter aircraft. Air transport may fulfil the needs of developing countries, where the infrastruc- 
- number of passengers in an all-tourist layout (SEAT PITCH 34 IN., .87 M): 180 OR MORE. CORRESPONDING DESIGN PAYLOAD: 20,000 KG $(44,100$ LB). AN UNDERFLOOR FREIGHTHOLD VOLUME OF AT LEAST $50 \mathrm{M}^{3}(1,762$ CU.FT) WILL BE REQUIRED. STANDARD SIZE BELLY CONTAINERS ARE PREFERRED.

- RANGE, WITH ABOVE MENTIONED PAYLOAD: 2,200 KM (1,200 NM) IN A HIGH-SPEED CRUISE, ATA DOMESTIC RESERVES. MAXIMUM RANGE (REDUCED PAYLOAD): $3,200 \mathrm{KM}(1,726 \mathrm{NM})$ AT LONG-RANGE CRUISE TECHNIQUE.

- max. CRuising SPEed at 9,150 M (30,000 FT) AL-
TITUDE: $M=.82$. DESIGN LIMITS: $M_{M O}=.85$, $v_{\text {MO }}=704 \mathrm{KMH}(380 \mathrm{KTS}) \mathrm{EAS}$.

- Field length ReQuired fOR takeoff and LaNDING, ACCORDING TO AIRWORTHINESS RULES: $1,800 \mathrm{M}$ $(5,900 \mathrm{FT})$ AT SEA LEVEL, ISA $+20^{\circ} \mathrm{C}\left(95^{\circ} \mathrm{F}\right)$, AT MAXIMUM (CERTIFICATED) TAKEOFF WEIGHT. RUNWAY LOADING: LCN $=30$, RIGID PAVBMENT, 18 CM (7 IN.) THICKNESS.

- regulations: far parts 25, 36 and 121. The noise CHARACTERISTICS MUST SHOW AN IMPROVEMENT RELATIVE TO THE 1969 VERSION OF FAR PART 36 OF 10 EPNAB.

Fig. 1-5. Initial specification of a hypothetical short-haul airliner for introduction into service around 1980

ture is inadequate for surface transport. c. A new type of aircraft is built and tested in order to give added impetus to an important new technical development, such as a V/STOL demonstrator prototype. Since experimental aircraft nearly always lead to a financial loss, at least in the first stages, there will have to be government funding, e.g. in the form of a development contract.

Manufacturers should be wary of aiming at filling the "gap in the market". That gap may well have remained unfilled for the simple reason that the need for an airplane of the kind was insufficient. Another danger which should be warned against is the adoption of a particular technical novelty which in itself may be a very clever achievement but is unlikely to contribute to profitable operation of the aircraft. Nevertheless, the design office will be continually involved in studies aimed at determining the potentialities of new technical developments and innovations. Any new type designed will have to be marketed in accordance with a properly thought-out time schedule. It is important to remember that if it is offered too early the production rate will increase too slow- 1y, resulting in a productivity loss on investments which the company has put into the project. A launching delayed too long may be equally disadvantageous, either because the market has meanwhile been saturated by competitors' products or because the production line has to expand too fast and excessive manpower has to be (temporarily) hired and additional investments made.

The initial specification shown in Fig. 1-5 was drawn up for an airliner intended to augment and replace the current class of high-subsonic short-haul passenger transports: the BAC 1-11, MCDonnell Douglas DC-9 and Boeing 737, and to some extent also aircraft designed for medium ranges: the Hawker Siddeley Trident, Aerospatiale Caravelle and Boeing 727. The category considered does not include smaller aircraft such as the Fokker F-28 or the VFW-614. The aircraft mentioned above are powered by low-bypass turbofans and have a capacity of 80-120 passengers (short-haul) or 120180 (medium-haul). The need for a new type stems from the following considerations: a. The increased traffic volume requires larger-capacity aircraft.

b. The new standard of passenger comfort 
introduced by the wide-body jets will undoubtedly be extended to short-haul traffic.

c. Reduction of noise production will be a prerequisite in the eighties.

d. Technology improvements in the fields of high-speed and low-speed wing aerodynamics, new structural materials (composite structures), lightweight avionics and improved flight control systems may be considered for application in this new aircraft category.

In view of the large volume of short-haul traffic the market seems to of fer scope for a new aircraft with smaller capacity as compared to the Airbus A-300, for example.

\subsubsection{Transport capacity}

When a new specification is being drawn up, the first step will have to be a forecast of the traffic and the transport demand over the route sector concerned during the period under review. A technique commonly used here is a statistical analysis of the yearly growth percentage of the total distance covered by passengers in terms of passenger-miles (passenger-kilometers). On the basis of an extrapolation of this growth percentage, the total transport demand for the period considered may be estimated. Assumptions will next have to be made regarding the frequency of the flights, the average load factor and yearly utilization and from these the desired productivity (number of passengers times block speed) can be deduced. A rule of the thumb sometimes used states that the most favorable time between successive flights over a particular route is about equal to the time taken to $f l y$ the route. Hence, if the block speed increases, the frequency of the service should also be stepped up. The following are some other aspects to be considered:

a. For a large capacity aircraft the operating costs per aircraft-mile will be high, but those for a seat-mile will be low, since certain costs do not rise proportion- ally to the size of the aircraft, e.g. total salaries of the flight crew and the cost of avionics and certain services, and will therefore decline with each additional seat.

b. A comparatively small aircraft will show a low cost per aircraft-mile and its critical load* will be smaller than that of a large aircraft. This does not necessarily apply to the critical load factor (critical load/maximum load).

Generally speaking, large aircraft are best suited to routes with high traffic density, provided the frequency of operations is compatible with the market requirements.

In drawing up the specification for the $M$ 184 project (Fig, 1-5), the following conclusions were arrived at:

a. During the 1960-1970 period short-haul traffic grew considerably, both in the United States and in Europe. A yearly growth of 15 percent, resulting in a doubling in five years, was no exception and the growth was even more marked during the 1965-1970 period. Charter traffic in fact underwent an explosive expansion during that same period, with growth percentages as high as 25 to 30 . Factors which contributed to this growth were: regular tariff decreases, a rising level of prosperity, and the greatly improved comfort of jet aircraft compared with other means of transport.

b. A gradual decrease in the yearly growth can be expected for the period 1975-1985 as a result of a slackening-off or decline in the economy, a certain measure of saturation of the transport market, and unavoidable increases in tariffs. The latter are a result of the rapidly increasing costs of fuel and the measures which have to be taken to meet the certification requirements regarding noise levels. Assuming an annual growth of 10 percent for the years $1973-1980$ the total yearly production

* The number of passengers required to pay the cost of the flight. 
on short routes will have to rise to 195 percent of the 1973 value, while during the first three years after the airplane's introduction the traffic demand will rise to about 250 percent.

c. On very busy routes the Airbus A-300 and possibly also the Trijets McDonnell Douglas DC-10 and Lockheed 1011 will take over a large share of the short/mediumhaul traffic. On routes where the growth will be less progressive, however, the jump in capacity from current short-haul aircraft to the A-300 will probably be too great and there will be an opening for aircraft with a capacity some 80 to 100 percent greater than that of the DC-9, provided it offers good possibilities for further growth.

d. For the M-184 a capacity of at least 180 passengers has been chosen for an alltourist layout with a possible later "stretch" to about 250 passengers, while the cargo holds require a total volume of at least $1800 \mathrm{cu}$. ft $\left(50 \mathrm{~m}^{3}\right)$. Compared to that of current airliners the passenger accommodation must show an improvement in the level of comfort, but this need not necessarily be achieved by the use of two aisles. A very close watch will have to be kept on the economical consequences of an increased level of comfort.

\subsubsection{Design cruising speed and range}

The speed factor has constituted an outstanding contribution to the development of aviation; the aircraft has proved to be the only means of transport in which increased speed does not necessarily lead to an increase in fuel consumption. Although a fast means of transport will be attractive to the passenger, the air transport companies in particular rate the speed element highly because, broadly speaking, it means that more trips can be made per day and production is increased. It is not only the cruising speed, however, that is important; equally vital is the time devoted to taxying, takeoff, climb, descent, approach and landing, which means that the block speed is a better yardstick than the cruising speed. Any new type of short-haul aircraft will have to possess a considerably higher cruising speed than the one it is intended to replace, in order to save the time needed for an extra flight. In the case of smaller general aviation aircraft the value of speed mainly depends on how the aircraft is used. A top executive whose working hours are assumed to be extremely valuable will be prepared to pay considerably more for speed than the owner of a small utility aircraft which is used for tourism or in regions with an underdeveloped infrastructure where reasonable surface transport is lacking.

In drawing up the specification for the M184 project (Fig. 1-5), it has been assumed that the design cruising speed must not be less than that of existing aircraft. In the high-subsonic speed bracket, however, any increase in speed will considerably influence the external shape (angle of sweep, airfoil shape and thickness), generally resulting in an empty weight increment, extra development costs and increased fuel consumption. The extent to which the economic advantages of the higher block speeds will outweigh these losses cannot be predicted offhand; this would have to be ascertained by a tradeoff study, which could also take into account the possibilities of recent developments in high-speed wing aerodynamics.

In the case considered here a design Mach number of .82 in high-speed cruise has been chosen on the basis of conventional section shapes, while the possible gain resulting from the use of an advanced wing shape may be either the use of a thicker airfoil - and hence a lighter structure -, a larger wing span, or a higher economical cruising speed.

As regards the choice of the design range of the M-184 it was concluded from a survey of route distributions that a peak occurs for traffic on ranges of about 280 $\mathrm{nm}(500 \mathrm{~km}), \mathrm{e} . \mathrm{g}$. Los Angeles - San Fran- 

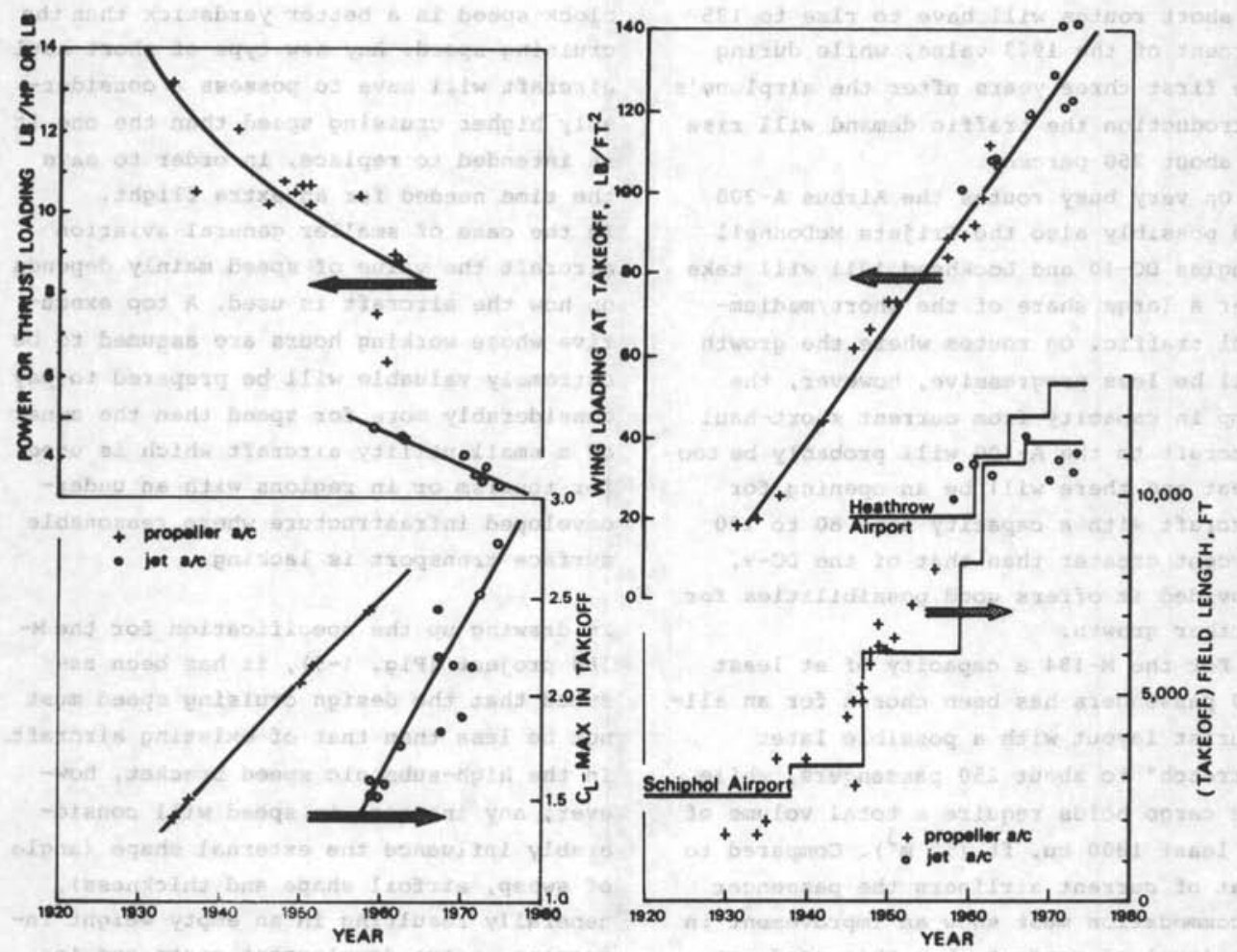

Fig. 1-6. Trends in the takeoff performance of civil aircraft (only trend-setting types have been plotted)

cisco. Another peak, although less pronounced, is observed around $500 \mathrm{~nm}(900$ $\mathrm{km})$. An aircraft designed to fly ranges between 110 and $1,200 \mathrm{~nm}$ (200 and $2,200 \mathrm{~km}$ ) will cover 87 percent of the traffic market. Although a decrease to $600 \mathrm{~nm}(1,100$ $\mathrm{km}$ ) in the range for maximum payload may lead to a slight improvement in the direct operating costs at short ranges, 25 percent of the short-haul routes are longer and a considerable number of operators would not choose the aircraft. A design range of $1,200 \mathrm{~nm}(2,200 \mathrm{~km})$ at high-speed cruise was decided for the $M-184$. In view of the specified field performance there may be an opening for a version with increased all-up weight and fuel capacity to suit operators who require a longer range version and put less emphasis on low-speed performance.
1.4.4. Low-speed characteristics and field performance

Two starting-points may be used for specifying the runway length for takeoff and landing:

a. The aircraft is optimized for cruising flight. The shape and dimensions of the wing, as well as the cruising altitude, are so chosen that the fuel consumption is a minimum for the design range flown at the design cruising speed. The thrust of the engines will be based either on the required climb performance or on the design cruising speed requirement. The takeoff and landing performance will now become more or less derived values and can be influenced only to a limited extent by the design of the flaps and the wheel brakes. The continuous growth in aircraft weight and conse- 
quent increase in wing loading (Fig. 1-6) have resulted in increased takeoff distances which have demanded a steady lengthening of the runways, in some cases and certainly at the principal international airports to as much as about 13,000 feet $(4,000 \mathrm{~m})$. The approach speeds for the landing have risen to 160 to $170 \mathrm{kts}(300$ to $315 \mathrm{~km} / \mathrm{h}$ ), although the landing distance is not critical for most long-range transports.

Any further continuation of this trend would only be justified if adaptation of the aircraft to existing runways led to a considerable increase in operating costs and, moreover, the lengthening of the runways was environmentally acceptable. If we also take into account the 1969 requirements regarding noise production (FAR 36) and a possible tightening up of these in the future, it would not appear very likely that future generations of transport aircraft will require any appreciable lengthening of runways which are now being used by aircraft like the DC-8, Boeing 707 and Boeing 747 .

b. The runway performance of the new design will be adapted to the airports from which the future customer is now operating the aircraft that the new product will have to replace. For a new short-haul aircraft this means that the runway length should not exceed that used for the category to which the DC-9, BAC $1-11$ and Boeing 737 belong and that the design of the landing gear should be adapted to the strength of these runways. Any increase in operating costs resulting from these requirements should be carefully watched and realistic data should be available when it comes to discussing the tradeoff between shorter runways and cost increase. The design study will therefore have to include an investigation into the effect of field requirements on the design characteristics, direct operating costs and noise characteristics.

with a specified runway length for the M-184 project (Fig. 1-5) of not more than $6,000 \mathrm{ft}(1,800 \mathrm{~m})$ at sea level (standard atmosphere), it is anticipated that the majority of potential customers will be able to operate the aircraft from the runways now being used, provided that the runway Load Classification Number at Maximum Takeoff Weight does not exceed 30 on a rigid pavement 7 inches $(18 \mathrm{~cm})$ thick*.

\subsubsection{Other requirements}

a. The engine constitutes an important factor in the reduction of the operating costs and its choice should be carefully matched to the aircraft. In the case of transport aircraft the design range is particularly important, while the noise level has to satisfy exacting requirements if restrictions are not to be applied to the use of the aircraft. Fuel consumption has to be carefully watched.

b. Much attention should be paid to an optimum cabin arrangement to enable the operator to use different layouts. In general the distance between fixed partitions at the front and rear of the cabin should be as great as possible.

c. Equipment and instruments. The specification will state the amount of NAV/COM equipment to be carried and its degree of duplication. This will result from discussions with customers and will be based on the mode of operation of the aircraft (VFR and/or IFR flights, category of landings), and a distinction will generally be made between standard and optional equipment.

d. Construction, inspection and maintenance. Apart from the airworthiness requirements (Section 1.6) the specification will generally also feature special requirements such as a fail-safe or safe-life design philosophy and a service life of the structure, expressed in terms of the maximum number of flight hours or flight cycles or both. The manufacturing and production processes, etc., may also be subject to special requirements which can have far-

*cf. Chapter 10.2.1. 
reaching effects when certain structural parts or even main structures are adopted from types already in use. A case in point is the Boeing 707, 727 and 737 family of aircraft all of which have almost identical fuselage cross-sections.

\section{e. Airframe services and noise level. The} principal design requirements to be met by the air-conditioning and pressurization system are related to the air supply, temperature and degree of humidity, cabin pressure differential, etc. Noise levels, both internal and external, are also decided upon. Requirements may also be written into the specification with respect to the electrical, hydraulic and pneumatic systems, anti-icing equipment and possibly also the Auxiliary Power Unit (APU).

\subsection{THE "CONTINUOUS THREAD" RUNNING THROUGH THE DESIGN PROCESS}

\subsubsection{The iterative character of design}

The creation of an airplane configuration cannot be laid down in a universal, detailed procedure. However, some general characteristics of the design process may be amplified with the help of Fig. 1-7,

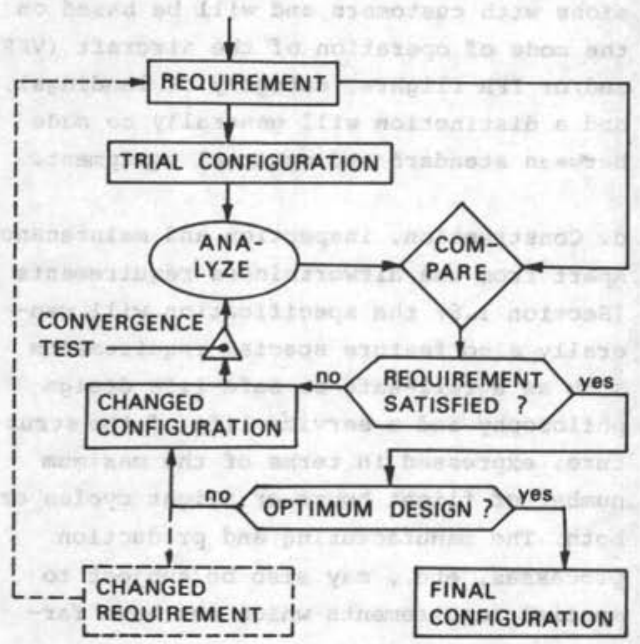

Fig. 1-7. General design procedure which shows the principal phases schematically. This diagram deals with technical and computational elements and could apply equally well to the design of other technical products, unlike Fig. 1-3 which refers specifically to an aircraft development.

An essential element of the design process is that it is always made up of iterations. After a trial configuration has been subjected to a first analysis of its characteristics (weights, mass distribution, performance, flying qualities, economy, etc.), it will be seen either that it does not meet all the requirements, or that it does comply with them but improvements in some respects are possible. Only after a number of configuration changes have been incorporated will the designer be able to determine whether the final configuration satisfies the requirements in every respect and may also be regarded as the best conceivable design, bearing in mind the inevitable uncertainties which are peculiar to the preliminary design phase. The convergence test has been incorporated in the diagram to indicate that a situation may arise in which, despite all the improvements made in the design, no configuration can be found which entirely meets all requirements simultaneously.

The reason may be that certain requirements in the specification and other constraints have proved to be contradictory or too extreme, taking into account the state of the art, or that the basic conception has not been chosen properly. For example, the designer may be confronted with a situation which, to ensure that the engines selected will supply the power required to keep the aircraft in the air after engine failure, would necessitate leaving a large part of the payload back at the airport. The convergence test in Fig. $1-7$ is therefore a general indication showing whether the attempts to improve the design have brought it closer to the requirements or not. 


\subsubsection{Searching for the optimum}

The search for the best conceivable design may be illustrated by a hypothetical case in which the quality of the design is judged on the basis of a single numerical criterium, referred to as the "merit function" or "objective function". In the case of transport aircraft this may be the Direct Operating costs (DOC, see Section 11-8) at the design range but it may also be the Maximum Takeoff Weight.

In Fig. 1-8 it has been assumed that vari-

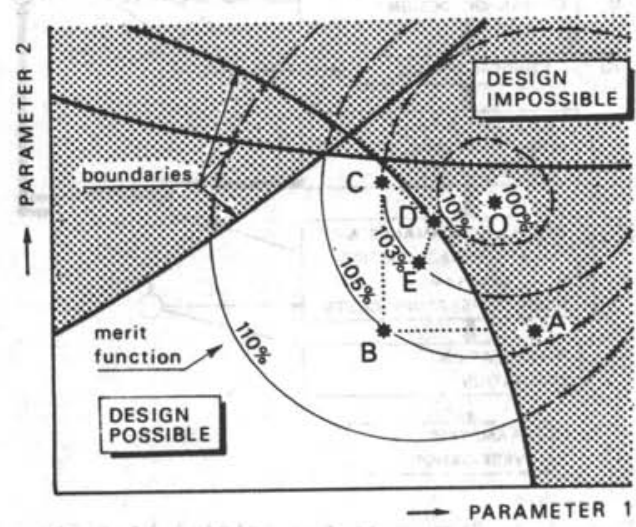

Fig. 1-8. Graphical representation of design optimization

ations in the design are limited to two independently variable parameters, such as the wing loading and the thrust loading. Each point on the diagram represents a fully defined design, the merit function of which can in principle be determined. In the case considered in Fig. 1-7 it is expressed as a percentage of the minimum value which can be obtained. The requirements of the specification have been incorporated in this overall picture as follows :

a. Requirements which sharply define the transport capacity and/or other aspects of performance should be used as a basis for the aircraft's general arrangement, layout and shape, serving as uniquely defined, explicit conditions.

b. Requirements which are put in the form of constraints in the sense of minima for flying speeds, maxima for the runway 3 a.t. length, etc. appear as boundaries of the area within which the design parameters may be chosen.

In the shaded area we find those combinations of the design variables for which it will not be possible to satisfy certain requirements laid down in the form of constraints. In the example it can be seen that the trial configuration (point A) lies in the region of unacceptable combinations. However, all requirements can be met by changing just one parameter, although at the cost of a less favorable merit function (point B). If the second parameter is now also changed (point $c$ ), we find that the merit function has been improved. Point D, where one of the limitations is tangent to the line of the constant merit function, indicates the combination with which all requirements can be met and, at the same time, the most favorable assessment obtained. Nevertheless, the designer may decide to choose point $\mathrm{E}$ for the final configuration since in general the positions of the design boundaries are still subject to some doubts and design $\mathrm{B}$ offers a certain margin which considerably reduces the risk of crossing the borderline.

Although the "absolute optimum" (point 0 ) is of no immediate importance in the case under review, it may still be useful to explore this design in somewhat greater detail, because the difference between the merit functions of designs $D$ and $O$ is an indication of the price that has to be paid for a requirement which makes it impossible to achieve the theoretical absolute optimum. If this disadvantage were to prove serious, the incorporation of more advanced techniques might be considered, enabling the designer to approach the optimum more closely. Although it is true that these will generally lead to an increase in development costs and influence the overall evaluation, the result might well be a saving in operating costs and/or an increase in productivity.

Another approach might be a certain relax- 
ation of the critical requirement in the specification, assuming this relaxation is likely to be less harmful than radical adaptation of the aircraft to the extreme requirement. In such a case a special version of the design, suitable for a particular type of operation, may be worth considering.

The example in Fig. 1-7 is essentially a highly simplified picture of the actual world of the aircraft designer. The method outlined is nothing more than a tool to arrive at a better justification for decisions. An aircraft is never evaluated on the basis of a single quantifiable criterion, while the number of variable parameters will always be much larger than two. For instance, increasing the fuselage diameter will generally lead to greater comfort and increase the passenger appeal with a possible increase in yield, but it will also increase the empty weight and the drag and hence the operating costs. It will be almost impossible to find a way out of this dilemma without relying upon the sound fudgement of the designer.

1.5.3. A suggested scheme for preliminary

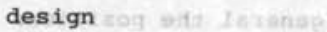

In the above we have made no reference to how a designer lays out a trial configuration and how he introduces changes. When the design problem lends 1 tself to quantification so that the survey given in Fig. 1-8 can be calculated, the problem may be tackled by means of a computer program. Routine calculation can be done very quick1y, but the designer will have to monitor the program. This procedure is gaining popularity in most large companies which undertake costly projects. In Great Britain the Royal Aircraft Establishment also has a facility of this kind at its disposal. In many other cases, however, it will be desirable to lay out a trial configuration using relatively simple procedures and statistical/analytical relationships which approach the optimum reasonably closely. Further investigation may then be limited to the introduction of relatively minor changes which do not affect the design very drastically, provided the original

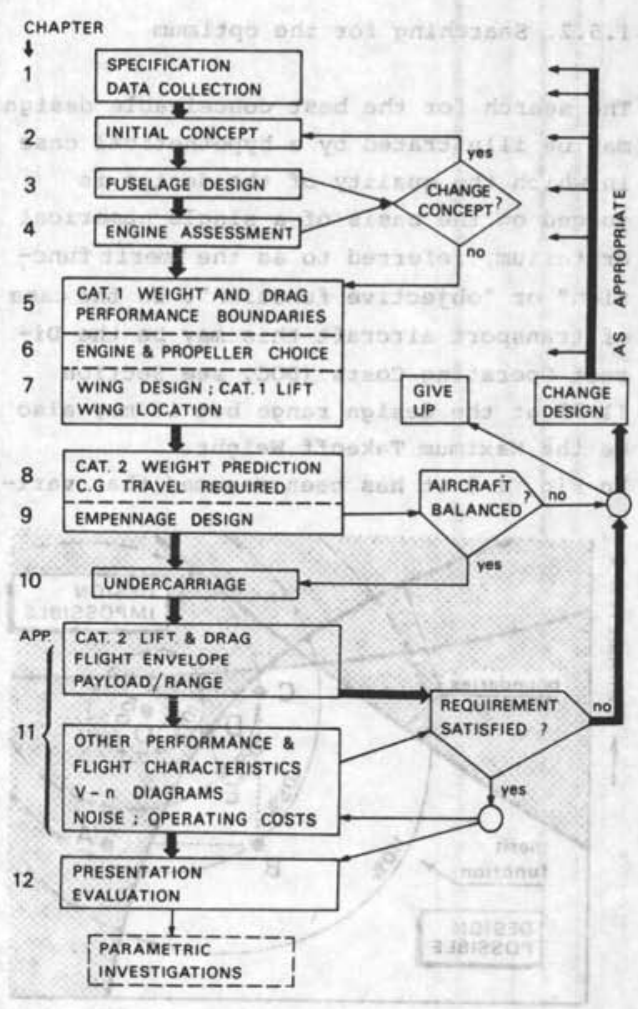

Fig. 1-9. Survey of the initial baseline configuration design $x$

concept was right.

This book has been composed in such a way that the reader will be able to use this simplified procedure with the help of the methods presented in the text. Not only will he find the necessary formulas and relationships, but his attention will also be drawn to considerations which precede decisions. No attempt has been made to streamline the design process as such, although the sequence of the chapters does show some affinity to the continuous thread that runs through the design process for a conventional subsonic transport aircraft. This is illustrated in Fig. 1-9, subject to the following reservations:

a. The diagram, although representative in character, does not possess universal validity. Designers do not always consciously work according to a set program. 
b. During the design process assumptions will repeatedly be made which will later have to be verified and, if necessary, corrected until the results agree with the assumptions. To preserve the clarity of Fig. 1-9 the number of such iterations ("feedbacks") has been drastically limited.

c. Some of the procedures indicated may not be required for a particular design. It will, for instance, be up to the designer to decide whether he will make use of the systematics to determine the wing loading and engine thrust (power) discussed in Chapter 5 .

A diagram comparable to Fig. 1-9 but compiled for use in a computer program will be found in Section 5.5.2. (Fig. 5-18).

1.6. IMPACT OF CIVIL AIRWORTHINESS REQUIREMENTS AND OPERATIONAL RULES

\subsubsection{General}

Airworthiness Requirements, Operational Rules and other regulations are framed by national governments and imposed on airplane manufacturers and operators in order to guarantee the general public a certain level of safety. These rules have a farreaching influence on the design of the structure, systems, installations, performance and flying qualities of aircraft. To begin with, the designer will have to make the correct choice of the airworthiness code to which the airplane will be designed, particularly when an international market is envisaged. He must realize that rules differ from country to country and that distinctions between various airplane categories and types of operation have to be observed. The purpose of this section is to give some insight into the most relevant criteria on which he can base his choice. Emphasis will be placed on the following rules:

a. FEDERAL AVIATION REgULATIONS (FAR), issued by the Federal Aviation Administra$t$ ion (FAA), an office of the Department of Transportation of the United States of Amer- ica :

b. BRITISH CIVIL AIRWORTHINESS REQUIREMENTS (BCAR), issued by the Civil Aviation Authority (CAA) of Great Britain. Several other countries have drawn up similar rules, but there are always more or less troublesome differences, leading to confusion and extra costs when an attempt is made to satisfy different rules simultaneously. In view of the considerable economic impact of these requirements, efforts have been made and will continue to be made to arrive at greater uniformity. In the past the International Civil Aviation organization (ICAO) has attempted to promote international requirements. For example, a well-known requirement was ICAO Circular 58-AN/53: "Provisional Acceptable Means of Compliance, Aeroplane Performance", dated 1959. These have not been generally accepted and only one type of aircraft, the Fokker F-27 (Fig, 1-10a), was certificated accord-

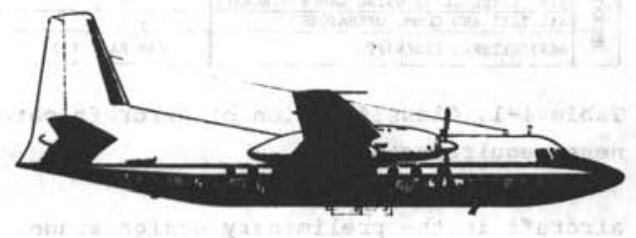

a. A "large" aircraft: the Fokker F-27 Friendship (Maximum Takeoff Weight 45,000 1b).

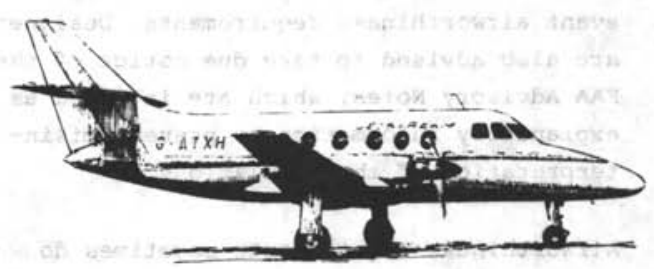

b. A "light" aircraft: the Scottish Aviation Jetstream (Maximum Takeoff Weight $12,499 \mathrm{lb}=5,670 \mathrm{~kg}$ )

Fig. 1-10. Examples of large and light aircraft

ing to these performance rules, after adoption of the code by the Dutch Civil Aviation Authority (RLD) .

In this section only those items will be reviewed which may affect the design of the 


\begin{tabular}{|c|c|c|c|c|c|c|c|}
\hline \multicolumn{3}{|c|}{ 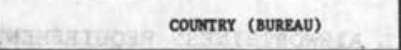 } & \multicolumn{3}{|c|}{ UNITED STATES OF NERICA (F.A.A.) } & \multicolumn{2}{|r|}{ (c.A.A.) } \\
\hline \multirow{2}{*}{\multicolumn{2}{|c|}{ GRoup }} & AIRPLANES PERFOROUNCE GROUP Y & \multicolumn{2}{|c|}{ SMALL } & \multirow{2}{*}{$\begin{array}{l}\text { GENERALLY : } \\
\text { LARGE }\end{array}$} & Whats Lichr & S LARGE \\
\hline & & MUX. TAKEOFF WEIGKT & $\leftarrow 12,500 \mathrm{LB}$ & $\leqslant 12,500 \mathrm{LB}$ & & $\begin{array}{r}\text { C, D, E } \\
\leqslant 12,500 \text { LB }\end{array}$ & $>12,500 \mathrm{LB}$ \\
\hline \multicolumn{3}{|c|}{ CATECORY } & $\begin{array}{l}\text { NOREAL, UTILITY } \\
\text { ACROBATIC AND } \\
\text { AGRICULTURAL (RESTR.) }\end{array}$ & sorrat & TRANSPORT & $\begin{array}{l}\text { NON-, SEMI- AND } \\
\text { AEROBATTC } \\
\text { AGRICULTURAL }\end{array}$ & NON-AEROBATIC \\
\hline \multirow{2}{*}{\multicolumn{2}{|c|}{ CLASS }} & NUMBER OF ENGINES & ONE OR MORE & TWO OR MDRE & TWO OR MORE & ONE OR MORE & TWO OR MORE \\
\hline & & TYPE OF ENGINE & $\begin{array}{l}\text { ALL } \\
\text { TYPES }\end{array}$ & $\begin{array}{l}\text { PROPELLER } \\
\text { ENGINES ONLY }\end{array}$ & ALL & $\begin{array}{l}\text { ALL } \\
\text { TYPES }\end{array}$ & $\frac{\text { ALL. }}{\text { TYPES* }}$ \\
\hline \multirow{2}{*}{\multicolumn{2}{|c|}{$\begin{array}{l}\text { MININUM } \\
\text { CREW }\end{array}$}} & FLIGHT & ONE OR MORE & TwO & TWO OR MORE & ONE OR MORE & TWO OR MORE \\
\hline & & CABIN ATTENDANTS & NoNE & $\begin{array}{l}\text { <20 PASS.: NONE } \\
\geqslant 20 \text { PASS.: }\end{array}$ & $\begin{array}{l}\text { <10 PASS.: NONE } \\
\geqslant 10 \text { PASS.: } \\
\text { OR MORE }\end{array}$ & 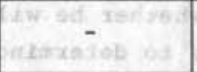 & 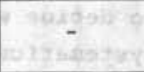 \\
\hline \multicolumn{3}{|c|}{ MUX. NURBER OF OCCUPANTS } & 10 & 11 THRU 23 & NOT RESTRICTED & 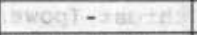 & NOT RESTRICTED \\
\hline \multicolumn{3}{|c|}{ MAX. OPERATIMG ALTITUDE } & $25,000 \mathrm{FT}$ & $25,000 \mathrm{FT}$ & NOT RESTRICTED & NOT RESTRICTED & NOT RESTRICTED \\
\hline \multicolumn{3}{|c|}{ MUX. DESIGN DIVTNG SPEED } & 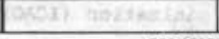 & NOT RESTRICTED & $452-1$ & $300 \mathrm{KTS} / \mathrm{M}=.6$ & NOT RESTRICTED \\
\hline \multicolumn{8}{|c|}{ 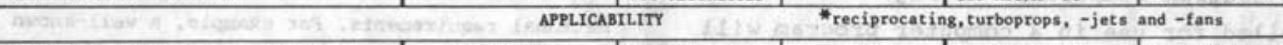 } \\
\hline \multicolumn{3}{|c|}{$\begin{array}{cll}\text { AIRWORTHINESS STANDARDS AIRPLANES } \\
* \\
* & " & \text { PNGINES } \\
\end{array}$} & $\begin{array}{ccc}\text { FAR PART } & 23 \\
11 & " & 33 \\
1 " & 1 & 35 \\
\end{array}$ & \begin{tabular}{|ccc} 
SFAR PART & 23 \\
FAR & -1 & 33 \\
- & - & 35 \\
\end{tabular} & \begin{tabular}{|ccc} 
FAR & PART & 25 \\
$\cdots$ & $*$ & 33 \\
$"$ & $*$ & 35 \\
\end{tabular} & $\begin{array}{ccc}\text { BCAR SECTION } & K \\
\pi & \text { SEC } \\
* & n & C \\
*\end{array}$ & $\begin{array}{ccc}\text { BCAR SECTION D } \\
" n \\
" & n & \mathrm{C} \\
\end{array}$ \\
\hline \multicolumn{3}{|c|}{ NOISE STANDARDS } & FAR PART 36 & $\begin{array}{l}\text { PROP. DRIVEN : } \\
\text { APPENDIX F }\end{array}$ & * $\quad 36$ & - & - \\
\hline \multicolumn{3}{|c|}{ GENERAL OPERATING AND FLICHT RULES } & FAR PAKT 91 & FAR PART 91 & FAR PART 91 & \multirow{4}{*}{\multicolumn{2}{|c|}{$\begin{array}{l}\text { 727. LATD DOWN IN } \\
\text { AIR NAVIGATION } \\
\text { REGULATIONS }\end{array}$}} \\
\hline \multirow{3}{*}{ 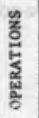 } & \multicolumn{2}{|c|}{$\begin{array}{l}\text { DONESTIC, FLAG AND SUPPLEMENTAL } \\
\text { COMM. OPERATORS OF LARGE AIRCRAFT }\end{array}$} & 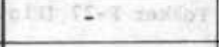 & - & FAR PART 121 & & \\
\hline & \multicolumn{2}{|c|}{\begin{tabular}{|l|} 
AIR TRAVEL CLUBS USING LARGE AIRCRAFT \\
AIR TAXI AND COMY. OPERATORS \\
\end{tabular}} & - & - & FAR PART 123 & & \\
\hline & \multicolumn{2}{|c|}{ AGRICULTURAL AIRCRAFT } & FAR PART 137 & $\frac{\text { FAR PA }}{-}$ & - & & \\
\hline
\end{tabular}

Table 1-1. Classification of aircraft categories in the American and British airworthiness requirements

\section{The reguirements}

aircraft in the preliminary design stage. Obviously, this summary is not a substitute for study and consultation of the relevant airworthiness requirements. Designers are also advised to take due notice of the FAA Advisory Notes, which are intended as explanatory information to prevent misinterpretation of the regulations.

terpretation of the regulations.

Airworthiness requirements sometimes do not cover new developments in civil aviation, while in other cases changes in the regulations are to be expected in the future. All rules are subject to continuous revision and the authorities should be consulted in connection with any particular problem areas that are not covered by current legislation. In many cases the design must incorporate provisions for (retro-)fitting changes which may be required by the regulations during development and production and sometimes even after the start of service.
A choice must be made of the group, category or class of aircraft to which the design will belong. The upper part of Table 1-1 shows the division of aircraft into groups while the lower part lists the appropriate American and British requirements. The most relevant point to note in this table is that civil aircraft are classified as "light" (U.K.) or "small" (U.S.) when their Maximum (certificated) Takeoff Weight is less than $12,500 \mathrm{lb}(5,700$ or $5,760 \mathrm{~kg})$. An important class of light aircraft is formed by the feeder liners and twin-engined business (executive) aircraft of $s 5$ 12,499 1b Maximum Takeoff Weight, an ex-d ample of which is the Scottish Aviation Jetstream (Fig. 1-10b). "Large aircraft" for the purpose of airworthiness standards have a Maximum (certificated) Takeoff Weight of more than 12,500 lb $(5,700$ or $5,760 \mathrm{~kg})$. The division into categories in Table 1-1, as used with respect to certification, indicates a grouping of aircraft 


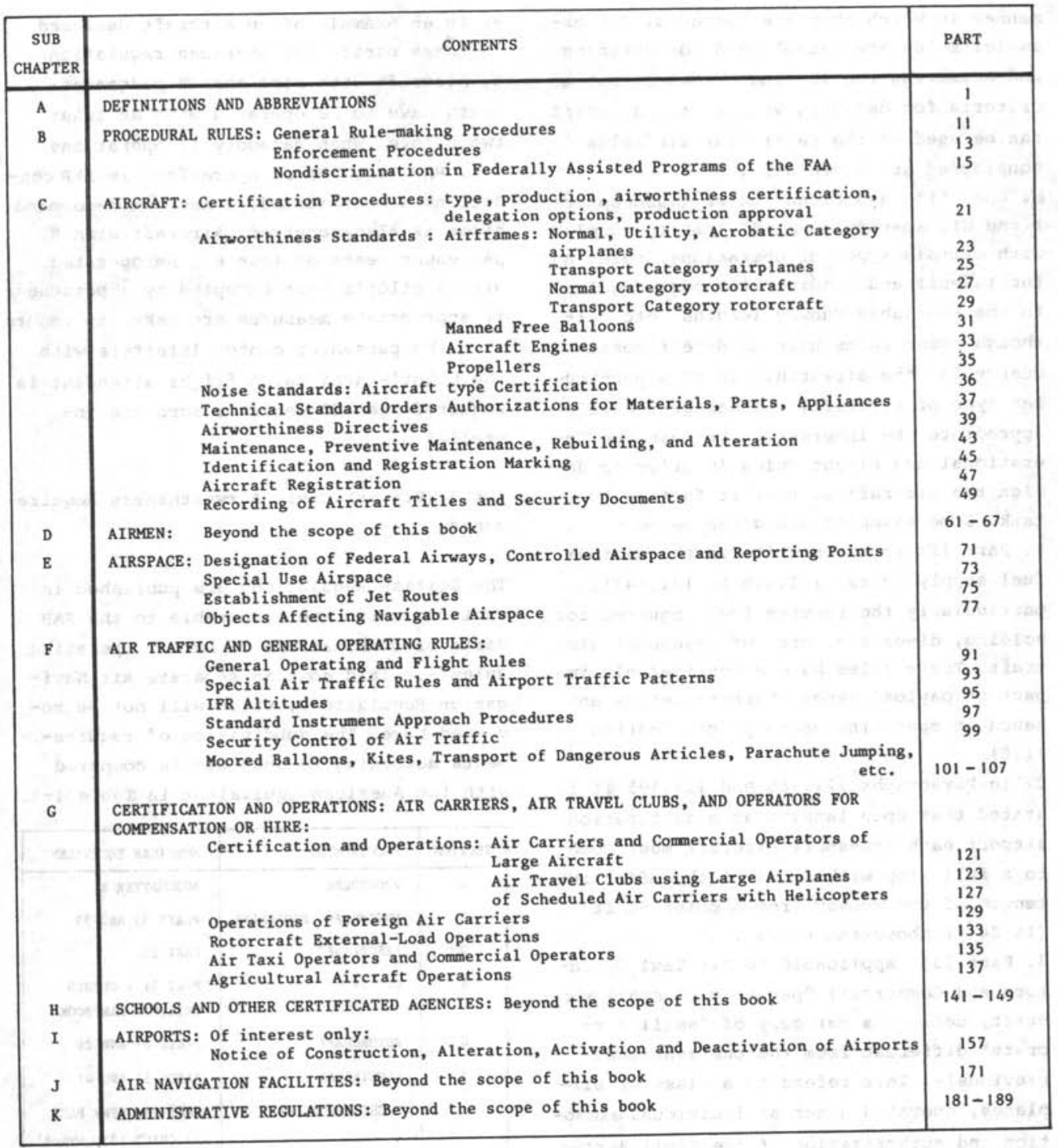

Table 1-2. Subdivision of FAR requirements

based upon intended use or operating limitations.

\subsubsection{Federal Aviation Regulations}

The FAA Regulations are divided into Subchapters, each containing one group of subjects, and these are further subdivided into Parts, as exemplified by Table 1-2. For practical purposes, the FAA issues these Parts in a Volume system, each volume containing one or more Parts. Subchapters A, C, $F$ and $G$ are of particular interest tc the designer. It is useful to note the following distinction:

a. Certification Rules and Procedures (Subchapter C), relating to the airworthiness aspects of aircraft, irrespective of the 
manner in which they are operated. For example: rules are established for defining and measuring the landing distance, but no criteria for deciding whether the aircraft can be used on the particular airfields considered are given here.

b. Operating and Flight Rules (Subchapters F and G), specifying conditions to comply with certain types of operations, relating the takeoff and landing distances required to the available runway lengths, etc. Although these rules have no direct consequence for the airworthiness of a particular type of aircraft, the designer must appreciate the interaction between the operational and Flight Rules in order to design the aircraft so that it fulfils its task. Some examples are given below.

1. Part 121 contains criteria for minimum fuel supply (Pars. 121.639 to 121.647), particularly the reserve fuel required for holding, diversion, etc. of transport aircraft. These rules have a considerable impact on payload-range characteristics and hence on operating economy (cf. Section 11.8).

2. In Paragraphs 121.185 and 121.195 it is stated that upon landing at a destination airport each transport aircraft must come to a full stop within 608 of the effective length of the runway from a point $50 \mathrm{ft}$ $(15.24 \mathrm{~m})$ above the runway.

3. Part 135, applicable to Air Taxi Operators and Commercial Operators of small aircraft, defines a category of "small aircraft" different from the one mentioned previously. This refers to a class of airplanes, operated under an individual exemption and authorization of the Civil Aeronautics Board ( $C A B)$ or under the exemption authority of the Economic Regulations of Part 298 (Ref. 1-86). In this particular context a "small transport aircraft" means a multi-engined aircraft having a maximum passenger capacity of 30 seats or less or a maximum payload of $7,500 \mathrm{lb}(3,400 \mathrm{~kg})$ or under. The operational requirements for this particular category are greatly simplified in relation to the transport category. The British Short SD 3-30 feeder lin- er is an example of an aircraft designed to these particular American regulations. 4. Aircraft with more than 9 passenger seats have to be operated with at least two pilots. When category II operations (cf. FAR Part 97) or operations in IFR conditions are conducted, a second-in-command pilot is also required. Aircraft with 8 passenger seats or less may be operated with a pilot's seat occupied by a passenger if appropriate measures are taken to ensure that the passenger cannot interfere with the pilot's actions. A flight attendant is required when 20 seats or more are installed

1.6.3. British Civil Airworthiness Requirements

The British Regulations are published in sections which are comparable to the FAR Parts or combinations of Parts. Operating Rules are laid down in separate Air Navigation Regulations, which will not be reviewed here. The subdivision of requirements according to subjects is compared with the American equivalent in Table 1-3.

\begin{tabular}{|c|l|l|}
\hline SECTION & REQUIREMENT & AMERICAN EQUIVALENT \\
\hline A & PROCEDURE & SUBCHAPTER B \\
C & ENGINE AND PROPELLER & PARTS 33 AND 35 \\
D & AEROPLANE* & PART 25 \\
E & GLIDER & PART 21 + GLIDER \\
G & ROTORCRAFT & CRITERIA HANDBOOK \\
J & ELECTRICAL & PARTS 27 AND 29 \\
K & LIGHT AEROPLANE & PARTS 37 AND 41 \\
& & PART 23, SFAR PART \\
L & LICENCING & 23 (PART 135, APP. A) \\
R & RADIO & SUBCHAPTER D \\
\hline
\end{tabular}

- large aeroplanes

Table 1-3. Subdivision of BCAR requirements

To the preliminary design engineer of British aircraft the following subdivision into performance groups is the most significant:

Group A - aircraft that, following a power- 
unit fallure, are not forced to land. Group D - aircraft that, following an engine failure, are not forced to land after takeoff, during initial climb or when $f l y-$ ing on instruments has started.

Group C - aircraft whose performance is not specified with regard to engine failure.

Group E - aircraft for which the extent of performance scheduling is limited (Maximum Takeoff Weight below $6,000 \mathrm{lb}$ or $2,730 \mathrm{~kg}$ ).

\subsubsection{Airworthiness standards and design}

The American airworthiness standards FAR Parts 23 and 25 and the BCAR Sections D and $K$ contain several subsections relating to very similar subjects. The FAR is subdivided into the following subparts:

A - general (and definitions)

B - flight

C - structures

D - design and construction

E - powerplant (installation)

F - equipment (installation)

G - operating limitations (and information)

SUB-PART B. The level of safety intended by the airworthiness regulations will only be achieved by relating the characteristics of the aircraft to those of the airport, the surroundings (obstacles) and the route. The requirements of this sub-part lay down absolute performance minima, as well as the methodology needed to define and measure flight characteristics.

SUB-PART C defines the loads on the structure, the safety factors, and the minimum strength which shall be provided in the airplane as a whole and in its components. They are directly related to the primary structure.

SUB-PARTS E AND $F$. The powerplant and equipment installations requirement must ensure safe operation within the airplane structure during all appropriate phases of the flight.

SUB-PART G. Certain limiting values are to be established, warning notices (placard speeds) displayed, and instructions made available to the flight crew.
The subdivision into "small" or "11ght" aircraft on the one hand and "large" a1rcraft on the other hand is very similar to the subdivision into the non-transport category and the transport category, except that the transport category is not restricted in Maximum Takeoff Weight. Some aircraft lighter than $12,500 \mathrm{lb}(5,670 \mathrm{~kg})$ have been certificated under FAR Part 25. The differences in airworthiness standards for transport and non-transport categories are significant. The main reason for this is that transport aircraft are intended to be operated by airlines, carrying farepaying passengers, while the bther category is primarily intended for private use by individuals and companies in general aviation. The airworthiness authorities assume that, unlike the private owner, the average passenger knows little about flying and relies upon the government to ensure the highest degree of safety in every phase of the flight. However, to reduce the effects on safety of different certification policies for the two categories, the private aircraft is normally restricted in its operations, especially above congested areas. Besides, a steady improvement in the safety of operation is aimed at for all categories. A small category of light aircraft may carry up to 15-18 passengers and for this category it has been agreed that the airworthiness standards of FAR Part 23 are too low. The arbitrariness of this subdivision may be demonstrated by Fig. 1-10b, which shows a "1ight" aircraft and Fig. 1-10a, which shows a "large" aircraft with comparable operational characteristics. Consequently, the FAA has developed a special set of regulations, Special FAR Part 23 (SFAR 23), with more stringent performance requirements, particularly after engine failure (Ref. 1-78). In order to take advantage of the future growth capacities of an airframe design, a manufacturer may decide to satisfy certain FAR 25 requirements from the outset, even though the airplane is intended primarily for FAR 23 certification. 


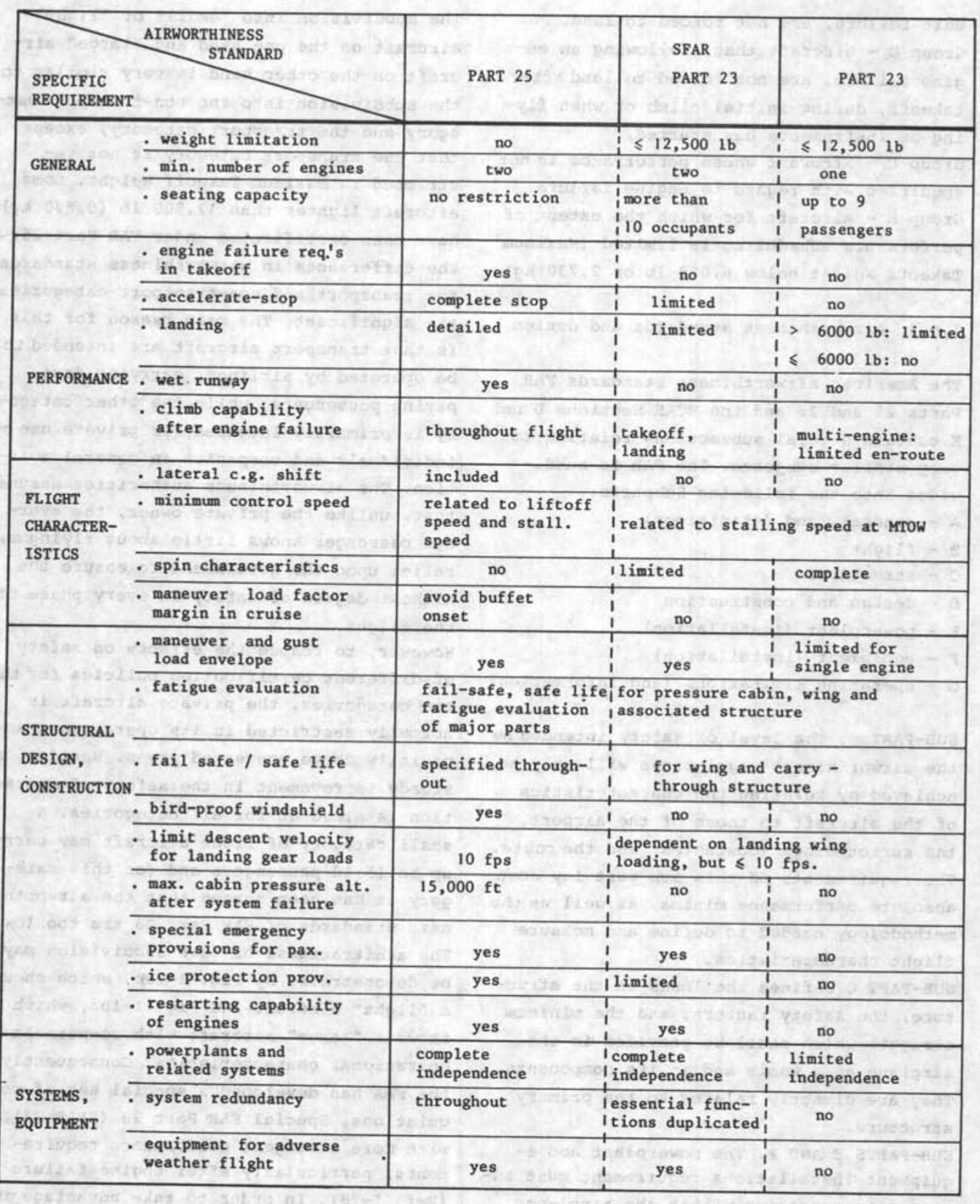

Table 1-4. Differences in FAR airworthiness standards for small and transport category airplanes

The most relevant differences between the transpprf and non-transport categories have been listed in Table 1-4. It should also be noted that transport category aircraft require an approved Flight Manual (FM), containing all the necessary information on 
performance, operating limitations and procedures, in both normal and emergency situations. Similar documentation for the non-transport category is relatively limited.

Although Table 1-4 applies to the American regulations, most of the differences are valid for British aircraft as well. Some exceptions are:

1. Section $K$ of the BCAR limits the design dive speed to $300 \mathrm{kts}(556 \mathrm{~km} / \mathrm{h})$ EAS or $M=.6$ for takeoff weights up to $12,500 \mathrm{lb}(5,670 \mathrm{~kg})$.

2. The BCAR Section $D$ is limited to aircraft with a Maximum Takeoff weight above $12,500 \mathrm{lb}(5,670 \mathrm{~kg})$, but may apply to non-transport category aircraft as well.

3. British light aircraft are divided into performance groups and weight groups, American smali aircraft into weight groups only, with some exceptions for single-engine aircraft.

4. Birdproof windshields are required for both categories of British aircraft, in contrast to American practice, where only transport-category airplanes must be provided with them.

5. British rules are more stringent and detailed. with respect to wet runway landing performance certification.

6. Special requirements for British agricultural aircraft are included in Section $K$, while American agricultural aircraft are certificated under restricted operations of FAR Parts 21 and 22.

In conclusion, the similarities between the British and American airworthiness standards far outweigh the differences, resulting in practically the same overall standard of safety, especially for the transport category. The American rules are generally more precise and the designer can apply them more easily without having to consult the authorities. On the other hand, the British rules are more flexible to accommodate new developments, avoiding special regulations. They constitute a basis for the assessment of the airworthiness of a new type of aircraft, the overall assessment being made on an engineering basis.

It will be clear that several problems have to be solved when certification is intended to comply with both the American and British regulations. For example, Ref. 1-80 shows that in the case of the Beechcraft 99, certificated for several years under FAR Part 23, many performance penalties were imposed when a British certification was applied for. After an extensive revision of the interpretation of the requirements, the performance figures were less divergent. On the other hand, the short skyvan is certificated according to both regulations and exhibits noticeable differences in performance, with the British version on the conservative side.

There are many important details of airworthiness which cannot be examined in this chapter. A number of them will be discussed in the appropriate place where they bear directly on the design. This introduction is merely intended to prove that airworthiness rules and requirements form a most important source of information for the designer and as such should belong to his daily inventory and mental toolkit.

\subsection{CONCLUSION}

We end this chapter with a summary of some of the more characteristic tasks of the staff of a preliminary design department.

a. During the development of a preliminary design and the coordination of the configuration development phase, the designer will come into contact with a number of disciplines related to aeronautical engineering: aerodynamics, flight mechanics, propulsion, the science of materials and structures, operational analysis, statistics and optimization. The designer should also know how aircraft are certificated, how flights are carried out under widely differing conditions, and how aircraft are operated. It follows that he should have a wide and up-to-date knowledge, spread over a large number of disciplines, in a profession which is characterized by its dynamic development. He should also be able to give proper attention to details.

b. Typical of almost every design is the 
use of iterations. It starts with a trial configuration which will then be analyzed and altered after comparison with the requirements. The entire cycle will then start afresh, until the result shows either that the design is not feasible or that it is reasonably well defined and may in fact be further developed with some confidence. The designer should have the courage to put something on paper to break the chickenand-egg conundrum. He will have to carry out many calculations and record the results in a clear and well-ordered manner, so that others may be able to follow the procedure. In spite of much apparently meaningless work, he has to remain motivated in order to do a professional job.

c. Particularly during the initial phase the designer should be able to anticipate on the later development and experimental results. The organisation of the project should nevertheless leave room to clear up any vital problem areas as early as possible, for instance by carrying out wind tunnel tests. As the designer's experience grows, this sense of anticipating will come to him more easily.

d. The design department must be able to deal statistically with the ever increasing flow of information on new developments and the outcome of research and make it reproducible. A well adapted documentation and library of data will be essential for permanent use, but should also be augmented for each new design (see Fig. 1-9, first box). The latest edition of Jane's All the World's Aircraft is invaluable, though the same can be said of many aviation journals and magazines. Even so, it is becoming in- creasingly difficult to evaluate the multitude of publications on the quality and reliability of their contents.

e. There will be permanent discussions with experts in other disciplines as well as with (prospective) customers, subcontractors and suppliers. Designers are generally very active in attending aviation symposia and conferences. Teamwork will be the order of the day, particulary where large projects are concerned.

f. In view of the long development period required for a modern aircraft, it will be necessary for the designer to do some crystal-gazing from time to time. A new type will only be successful if it does the job better than the obsolescent type it is intended to replace and preferably better than the designs competing with it for the same slice of the market. A careful balance should be struck between the need for technical innovations on the one hand and the desire to avold excessive financial risk on the other. Decision-making has sometimes to be based on vague and only broadly defined considerations; for this reason the experience of the design organisation is essential in making proper decisions. The designer will have to possess a faculty for judgement and a feeling for what can and what cannot be done.

In spite of the heavy demands on the designer's capacities in a modern preliminary design department, his work will still be fascinating, because it brings new challenges and offers opportunities for innovations which may have a great influence on the success of the final product. 


\section{Chapter 2. The general arrangement}

\section{SUMMARY}

A sound choice of the general arrangement of a new aircraft design should be based on a proper investigation into and interpretation or the transport function and a translation of the most pertinent requirements into a suitable positioning of the major parts in relation to each other. The result of this synthetic exercise is of decisive importance to the success of the aircraft to be built. However, no clear-cut design procedure can be followed and the task of devising the configuration is therefore a highly challenging one to the resourceful designer.

Considerations, arguments and some background information are presented here in order to provide the reader with a reasonably complete picture of the possibilities. The differences between a high wing and a low wing layout, and the location of the engines either on the wing or fuselage or elsewhere, are discussed on the basis of various cases from actual practice. Examples of unconventional layouts and many references to relevant 11 terature are given to stimulate further study and may possibly generate ideas for new conceptions.

The study of possible configurations should result in one or more sketches of feasible layouts. They serve as a basis for more detailed design efforts, to be discussed in later chapters, and they can therefore be regarded as a first design phase. 


\subsection{INTRODUCTION}

Before a general arrangement drawing of a new design can be put on paper, a choice will have to be made as to the relative location of the main components: wings, fuselage, engines, tail surfaces and landing gear.

A specific configuration is often inspired by a trend or line of evolution which may have its origin somewhere in the past. It may be that previous experience with aircraft in a similar category has established a tradition which cannot be easily discarded. But even when a company tackles an entirely new type, it is generally found that designers fall back on research work done years before by the company's research department or aeronautical laboratories. One example is the Boeing 707 - or its imme- diate predecessor, the $\mathrm{KC}-135$ Stratotanker - in which certain design features can be traced back as far as the 1945 Stratocruiser design, which itself was developed from the B-29 Superfortress (Fig. 2-1). At first sight the final version shows practically no similarity to any aircraft the Boeing company had previously built. Even so, the 367-60 and 367-64 preliminary designs have much in common with the Stratocruiser Model 377, particularly as regards the fuselage, while an obvious similarity also exists with the B-47 with respect to the location of the engines (Ref. 2-2). Although the Model 707 pioneered the new era of long-range high-subsonic transport aircraft with jet propulsion, its general shape still had its origin in previous designs. It follows that a sound evaluation of practical solutions incorporated in ex-

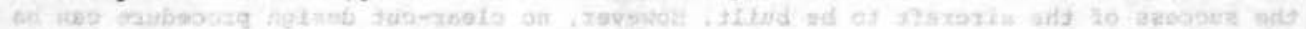

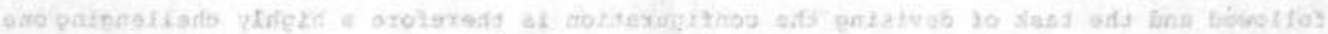

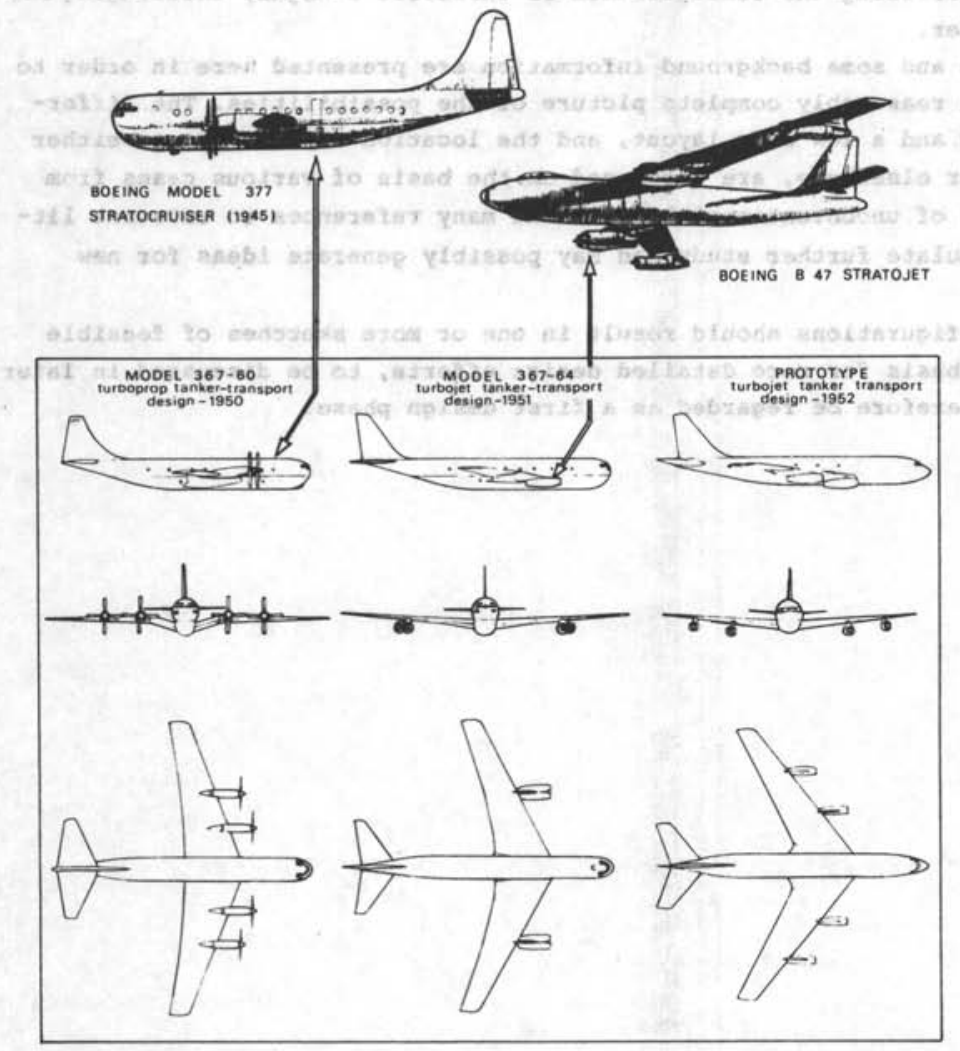

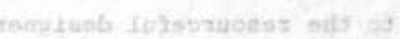

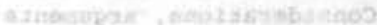

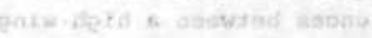

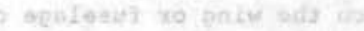

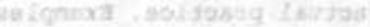

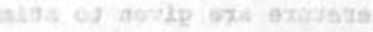
inglussonos for stertarog to ybuga with

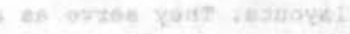

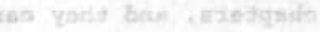

Fig. 2-1. Similarity between various designs by Boeing (Ref. 2-2) 

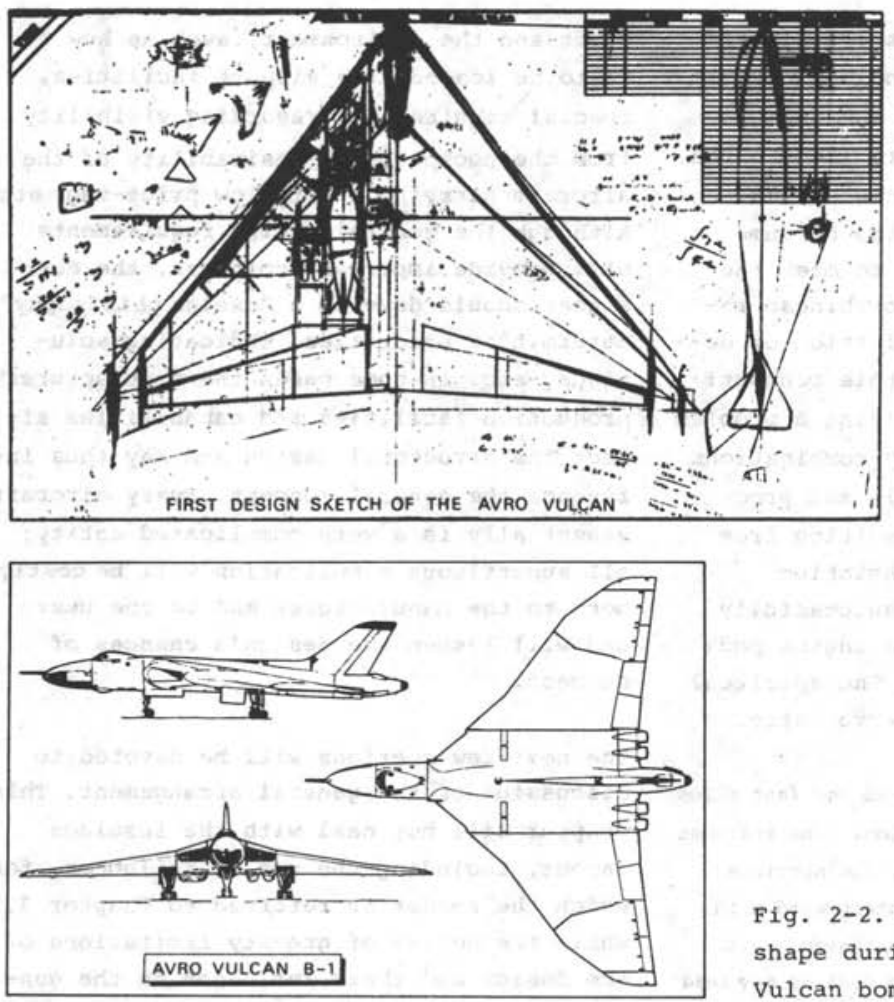

Fig. 2-2. Development of the external shape during the design of the Avro Vulcan bomber. (Ref.: Flight, 31 Jan. 1950)

isting successful designs, should be the first step in the conceptual phase.

A successful first choice of the configuration does not mean that no major changes will be required as development proceeds. This is illustrated in Fig. 2-2 in which an early design sketch of the AVRO Model 698 is compared with the final layout of the B-1 Vulcan bomber (approximately 19451948). Though these versions exhibit considerable differences, a gradual evolution of the initial baseline configuration took place in the course of the project development (Ref. 2-7). If this design is compared with that of the Handley Page Victor and the Vickers Valiant, which were both based on the same specification, it can be concluded that various solutions, each with its own particular merits, are possible. This will be discussed in more detail in Section 2.3. Unfortunately, for various reasons few examples of design evolutions have been published, and it is therefore difficult to draw general conclusions from which recommendations can be deduced. The list of references includes one publication, Ref. 2-6, which is particularly interesting in this connection since it presents some very unusual arrangements dating from the introduction of jet propulsion.

The general arrangement adopted can, in fact, only be properly justified once the design has been finalized. A satisfactory comparison of two different solutions for the same specification will not always be possible, as many design details add up to determine the characteristics of an aircraft and the design considerations published by the manufacturers are as a rule insufficiently detailed.

Competition forces manufacturers to explore new solutions, which is one of the 
reasons why competition has the long-term effect of advancing the technology. Excessively large departures from the existing state of the art may, however, lead to the taking of unwarranted commercial risks. Another restraining factor is the circumstance that all designs have to meet the existing or anticipated airworthiness requirements. Hawthorne's ${ }^{*}$ definition of design may be aptly quoted in this context: "Design is the process of solving a problem by bringing together unlikely combinations of known principles, materials and processes". A typical example resulting from such a procedure is the Sud-Aviation Caravelle (Fig. 2-3), which successfully pioneered the location of the engine pods at the tall of the fuselage. The spiritual father of this design was Pierre Satre.

It is scarcely possible to give hard and fast rules for arriving at a sound configuration. Some relevant considerations will be presented in the sections which follow but these should be interpreted with caution, as it sometimes happens that even small dimensional differences between the designs may lead to completely different conclusions. Sketches that are reasonably accurate with respect to dimensions are indispensable in the design stage. Without a correct representation of the relative size of the major components, the design drawing might perhaps result in a good artist's impression of the designer's ideas but it is likely to be useless as a basis for further engineering. Engine dimensions, especially in the case of high bypass ratio engines, are often of particular importance in view of their relation to duct sizes, landing gear height, etc. Certain dimensions needed for these drawings may be deduced from data of similar aircraft, preferably using parameters such as wing loading, aspect ratio, relative airfoil thickness, etc. The statistical data presented in other chapters of this book may also be used as a source of information.

During the configuration study the designer should have a clear picture in his mind of the operational requirements of the air-

\footnotetext{
* Engineering Laboratory, Cambridge University
}

craft and the environment, such as how it is to be loaded, the airport facilities, special requirements regarding visibility from the cockpit, the desirability of the aircraft carrying a very low price-tag, etc. Although the general design requirements will provide important pointers, the designer should develop a "design philisophy", determining priorities, indicating solutions, etc. In some cases the manufacturer's production facilities and capabilities affect the structural design and may thus influence the general concept. Every aircraft essentially is a very complicated entity; all superfluous complication will be costly both to the manufacturer and to the user and will lessen the design's chances of success.

The next few sections will be devoted to discussion of the general arrangement. This chapter will not deal with the fuselage layout, including the use of tallbooms, for which the reader is referred to Chapter 3 , while the center of gravity limitations of the design and their influence on the general configuration will be discussed in Chapter 8 . When engine location is dealt with (Para. 2.3), 1t will be assumed that the number and type of engines have already been decided. If this is not so, the reader should turn to Section 6.2 for more information.

\subsection{HIGH, LOW OR MID WING?}

The vertical location of the wing relative to the fuselage must be considered first. Fig. 2-4 shows three layouts of aircraft design projects in different categories. It will be obvious that the wing location relative to the fuselage is to a very large extent determined by the operational requirements. Although the aerodynamic and structural differences are not without importance, they can only be deciding factors when the choice between high, low and mid wing is not dictated by considerations of maximum operational flexibility. 

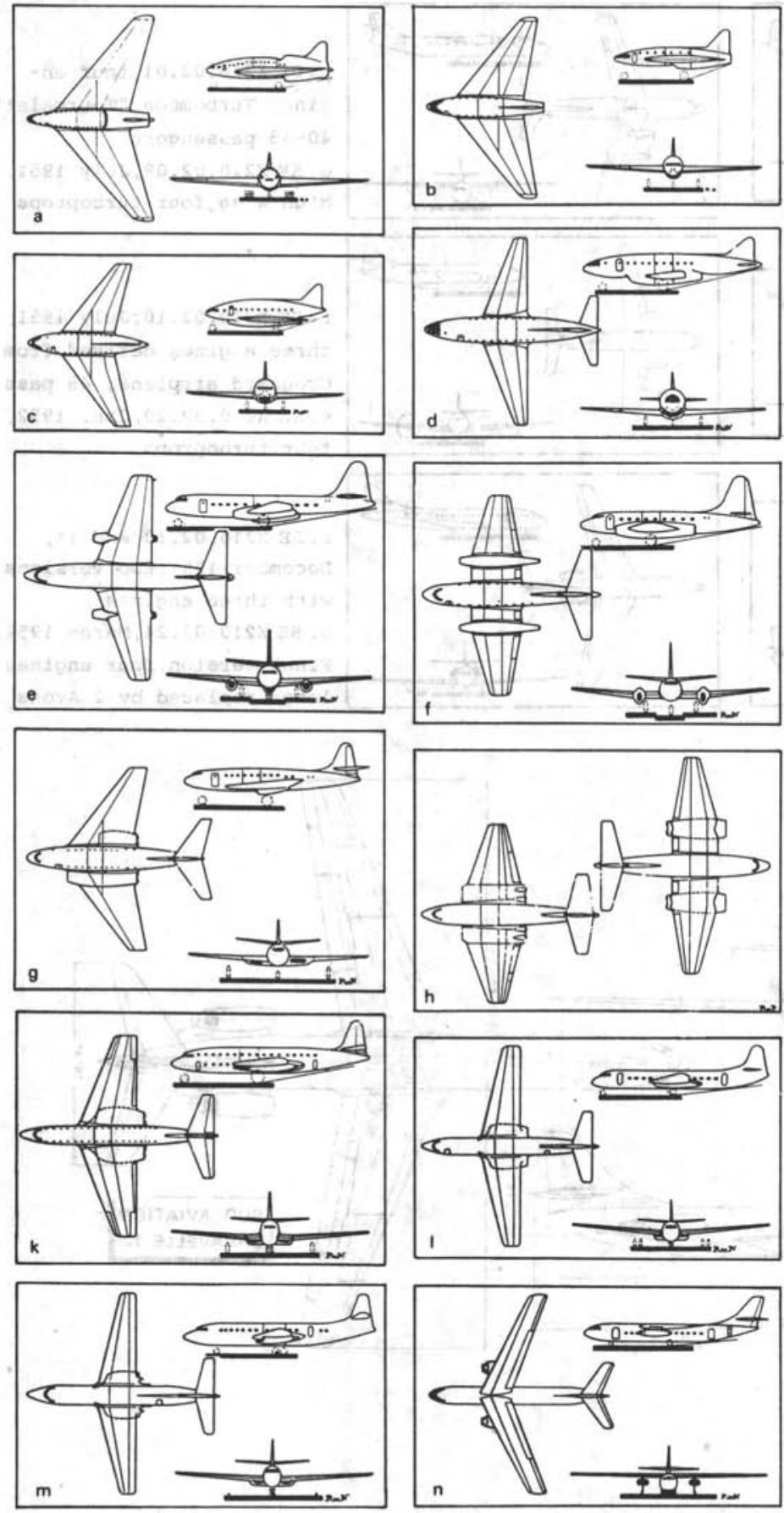

a. SE X200，200-tons a11 wing for transatlantic ranges b. SE X202, Feb. 1947

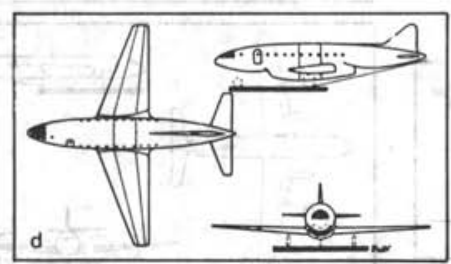

c. SE X203, March 1947, 40 passengers d. SE X204, two engines, 30 passengers

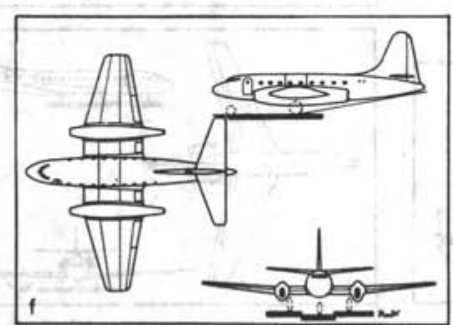

e. SE X205, July 1947 , three engines f.SE X206, four engines, 40 passengers

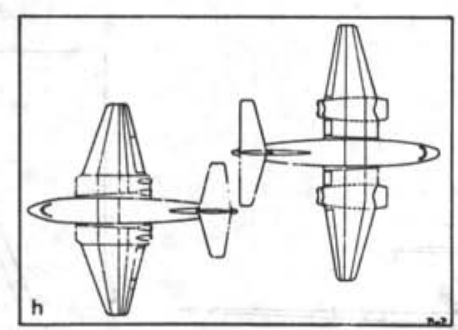

g. SE X206.02.03, different version from $f$.

h. SE X206.02.03, two other versions

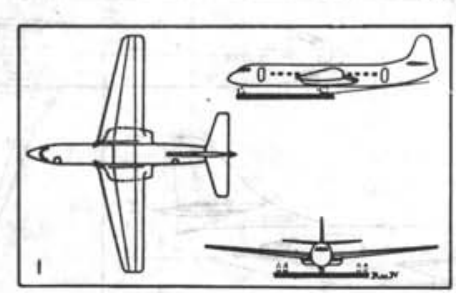

k. SE X206.02.04, September 1947, four engines, two decks

1. SE X206.02.09, Jan. 1948, four engines, 40 passengers

m. SE X206-02-17, May 1948

four engines (Nene)

n. SE X206-02-21, June 1951, four engines (ATAR D), 48-60 passengers

Fig. 2-3. Design projects by Sud-Aviation (Ref. ALATA, Febr. 1959) 

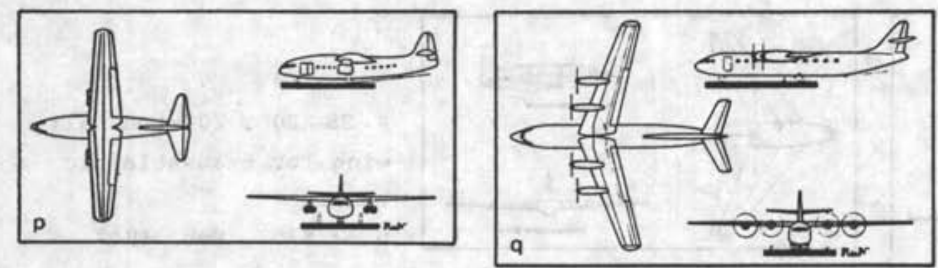

p. SE X210.02.01, four en-

gines Turboméca "Tourmalet", 40-58 passengers

q. SE X210.02.09, July 1951, high wing, four turboprops
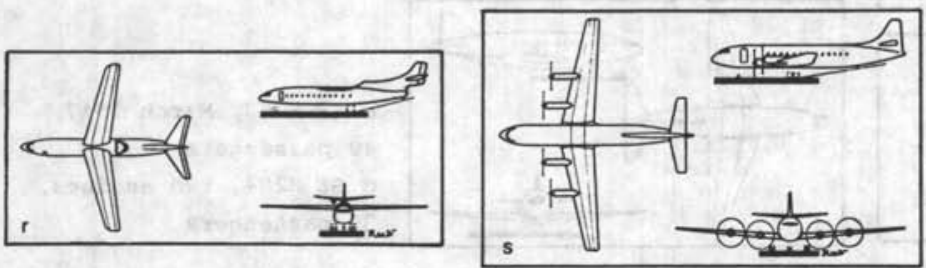

r. SE X210.02.10, July 1951, three engines derived from Grognard airplane, 48 pass. s. SE X210.02.20, Jan. 1952, four turboprops
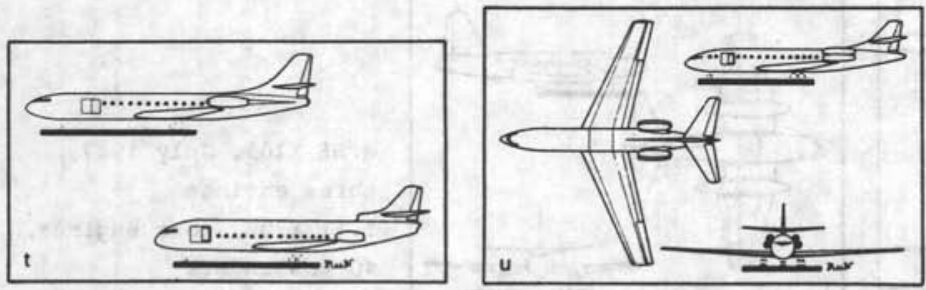

t. SE X210.02,10 and 14 , December 1951, two versions with three engines

u. SE X210.02.24, March 1952. Final version, four engines later replaced by 2 Avons

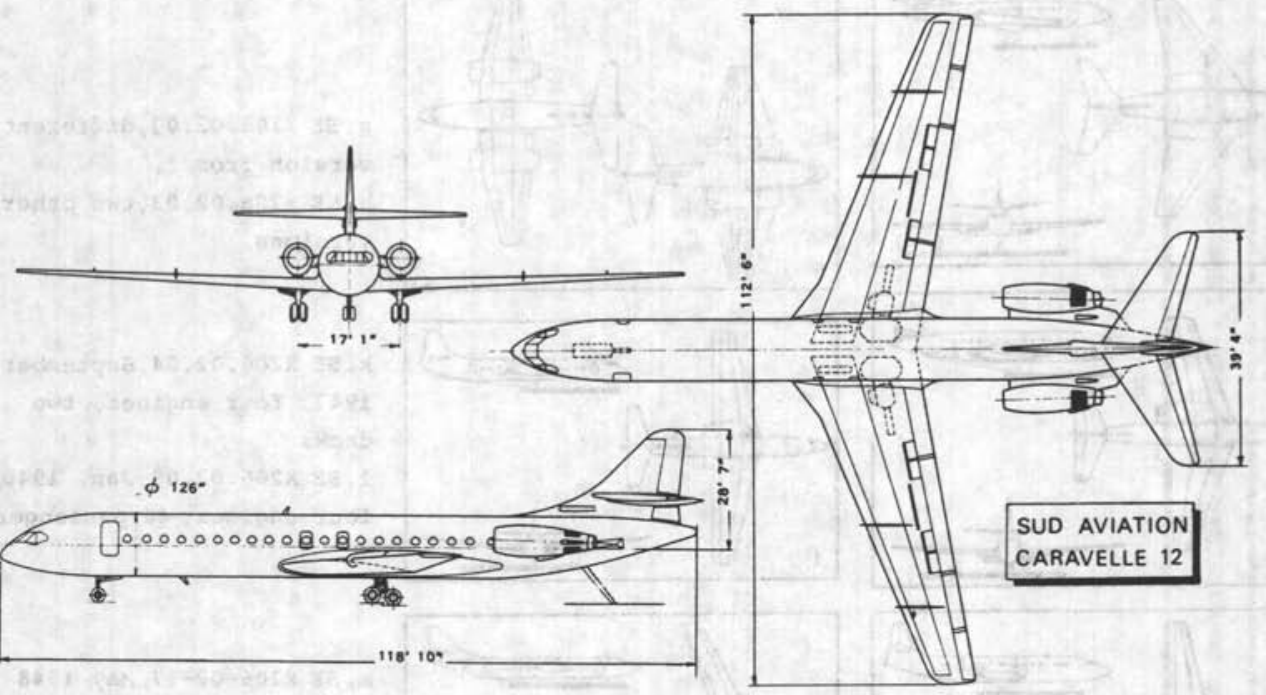

Fig. 2-3. (continued) 

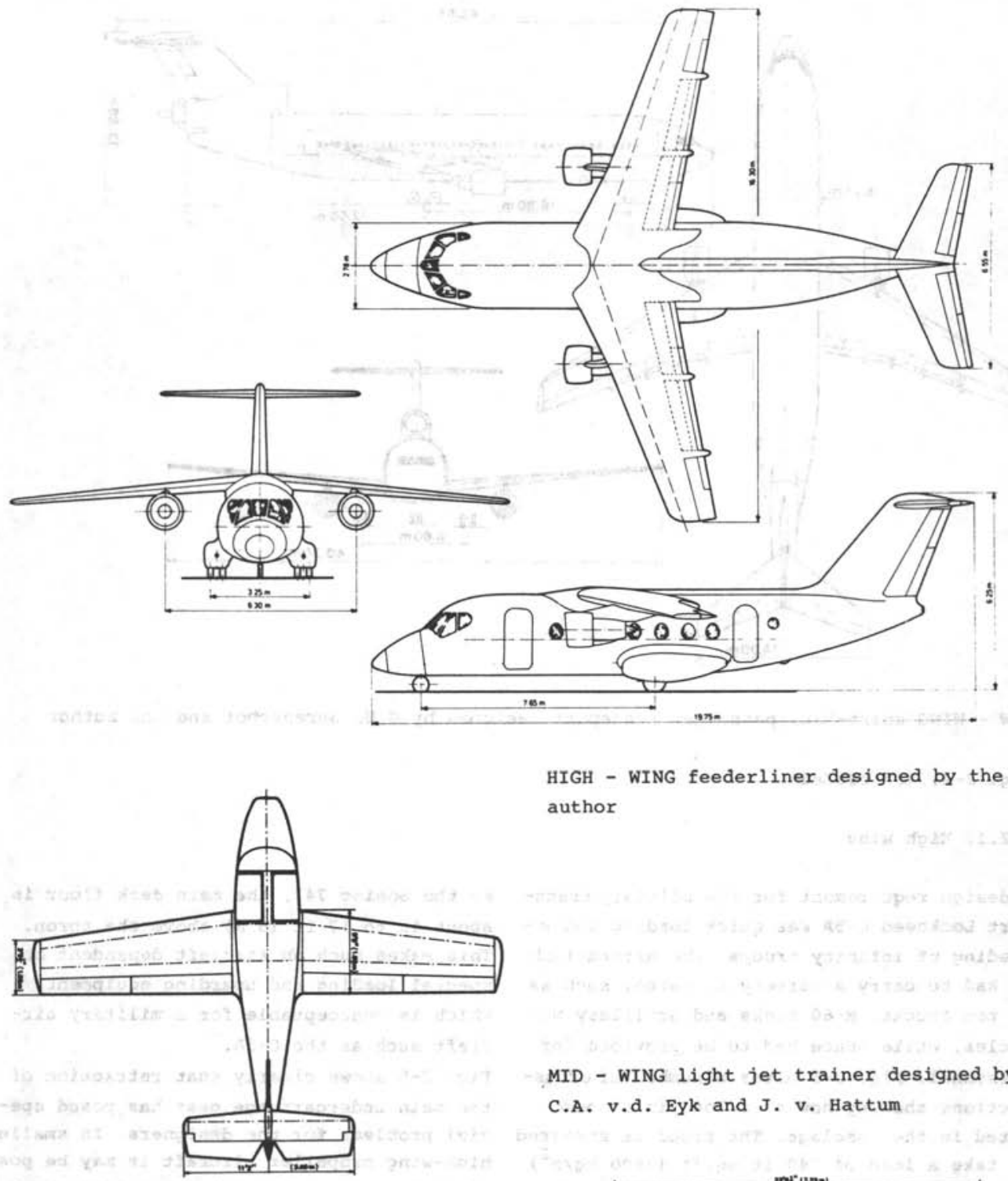

HIGH - WING feederliner designed by the author

MID - WING light jet trainer designed by C.A. v.d. Eyk and J. v. Hattum

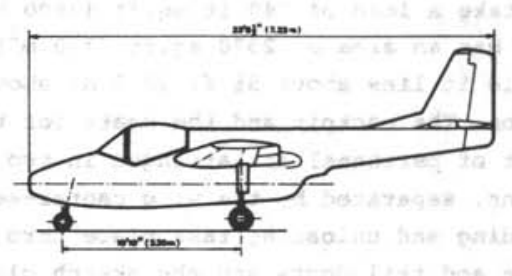

Fig. 2-4. Examples of high-wing, mid-wing and low-wing layouts (preliminary designs) 


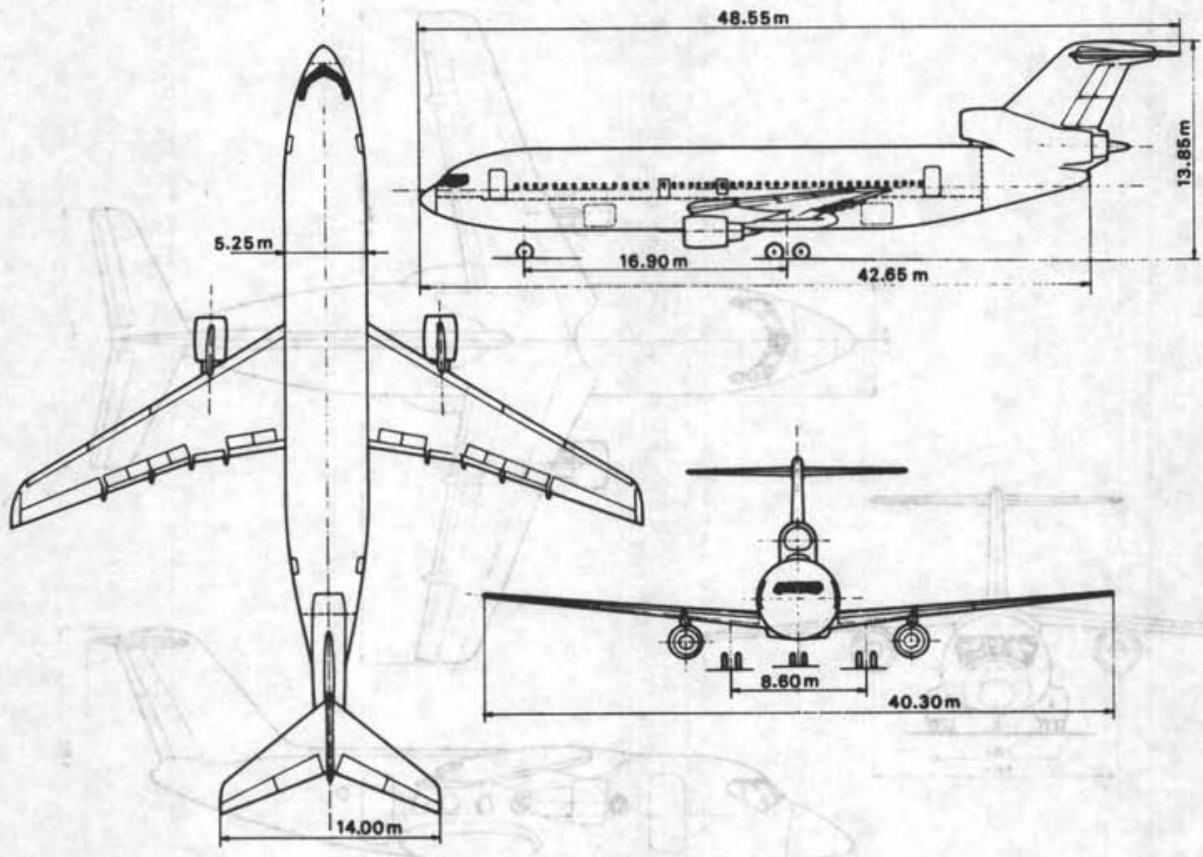

LOW - WING short-haul passenger transport designed by G.H. Berenschot and the author

Fig. 2-4. (concluded)

\subsubsection{High wing}

A design requirement for the military transport Lockheed C-5A was quick loading and unloading of infantry troops. The aircraft also had to carry a variety of cargo, such as $2 \frac{1}{2}$ ton trucks, M-60 tanks and artillery vehicles, while space had to be provided for personnel. Fig. 2-5 gives a number of crosssections showing how this load is accommodated in the fuselage. The floor is stressed to take a load of $740 \mathrm{lb} / \mathrm{sq}$. ft $\left(3600 \mathrm{~kg} / \mathrm{m}^{2}\right)$ and has an area of $2370 \mathrm{sq} . \mathrm{ft}\left(220 \mathrm{~m}^{2}\right)$, while it lies about $8 \frac{1}{2} \mathrm{ft}(2.5 \mathrm{~m})$ above the apron. The cockpit and the seats for transport of personnel are arranged in two sections, separated by the wing center-section Loading and unloading take place through nose and tail doors and the sketch clearly shows the importance of a low floor level for this very large aircraft. In the case of a low wing aircraft of comparable size, such as the Boeing 747, the main deck floor is about 16 to $17 \mathrm{ft}(5 \mathrm{~m})$ above the apron. This makes such an aircraft dependent on special loading and boarding equipment, which is unacceptable for a military aircraft such as the C-5A.

Fig. 2-5 shows clearly that retraction of the main undercarriage gear has posed special problems for the designers. In smaller high-wing propeller aircraft it may be possible to retract the main gear into the engine nacelles (Fokker F-27) or in the tail booms (Hawker Siddeley Argosy), but in the case of very large aircraft doing so would make it too tall and too heavy. This will unavoidably lead to mounting the gear to the fuselage, but the strengthening of the fuselage structure required for the transmission of the landing impact loads will result in a weight increase. This is only 

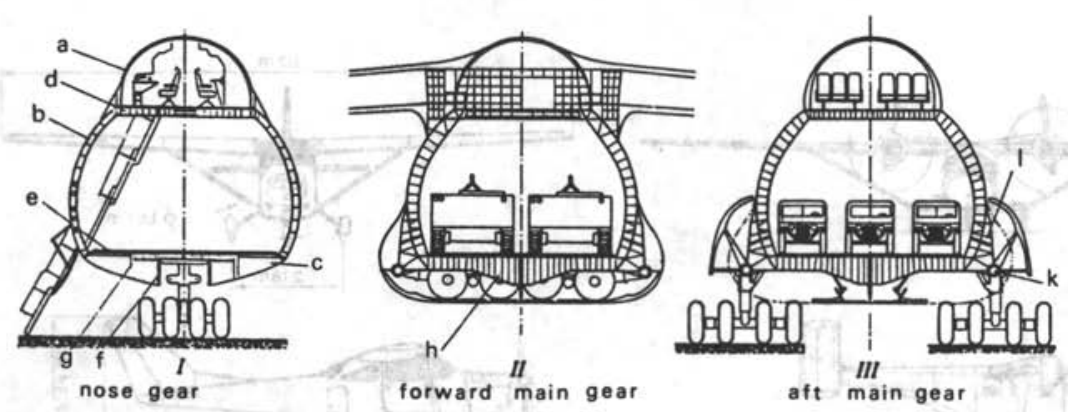

a: upper lobe; b: central lobe; c: lower lobe; d: upper deck; e: main deck; f,g: longitudinal supports; h: main fuselage frames; $k$ : main gear shock strut; 1 : external mounting frame
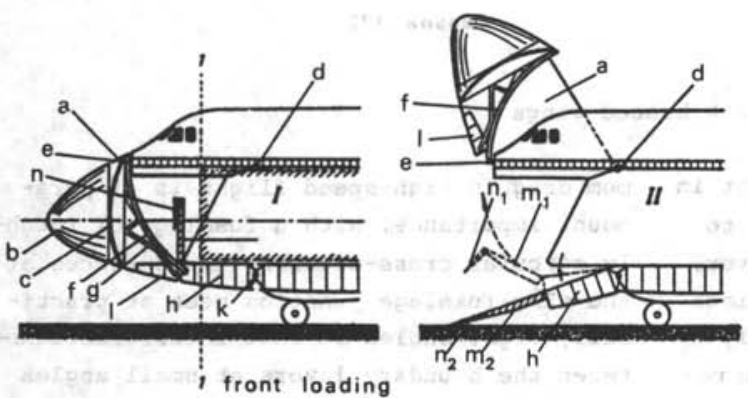

a: fuselage nose; b: radome; c: pressure bulkhead; d: door hinges; e: guide; $f$ : slide; g: post; h: adjustable floor element; $\mathrm{k}$ : articulation; 1: nose floor element; m: ramp; $\mathrm{n}$ : flap

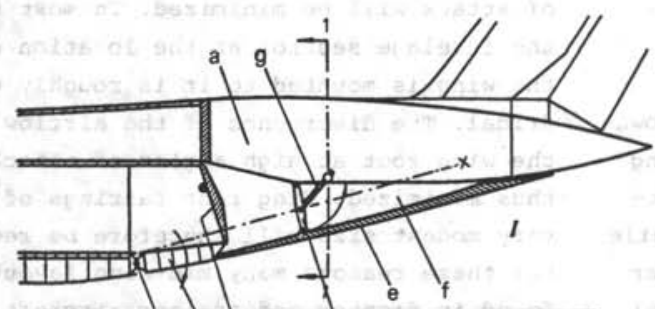

aft loading
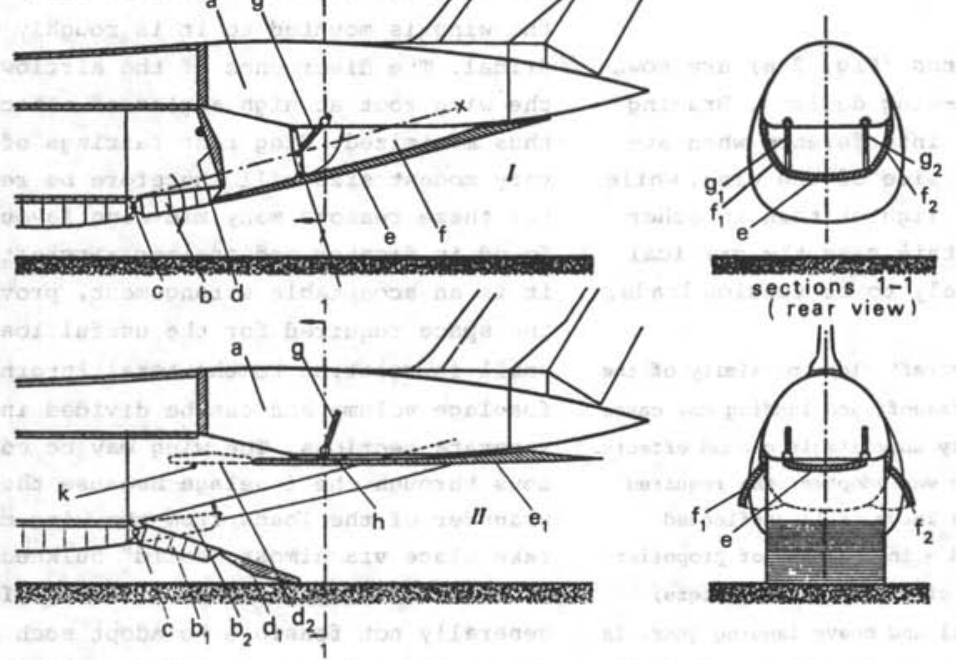

a: load-carrying structure; b: adjustable floor element; c: articulation; $d, k$ : flap; e: central loading door; $f_{1}, f_{2}$ : lateral doors; $g_{1}, g_{2}$ : screwjacks; $h$ : levers

Fig. 2-5. Loading provisions and undercarriages supports for the Lockheed C-5A (Ref.: DOC-AIR-ESPACE No 113 - Nov. 1968) 


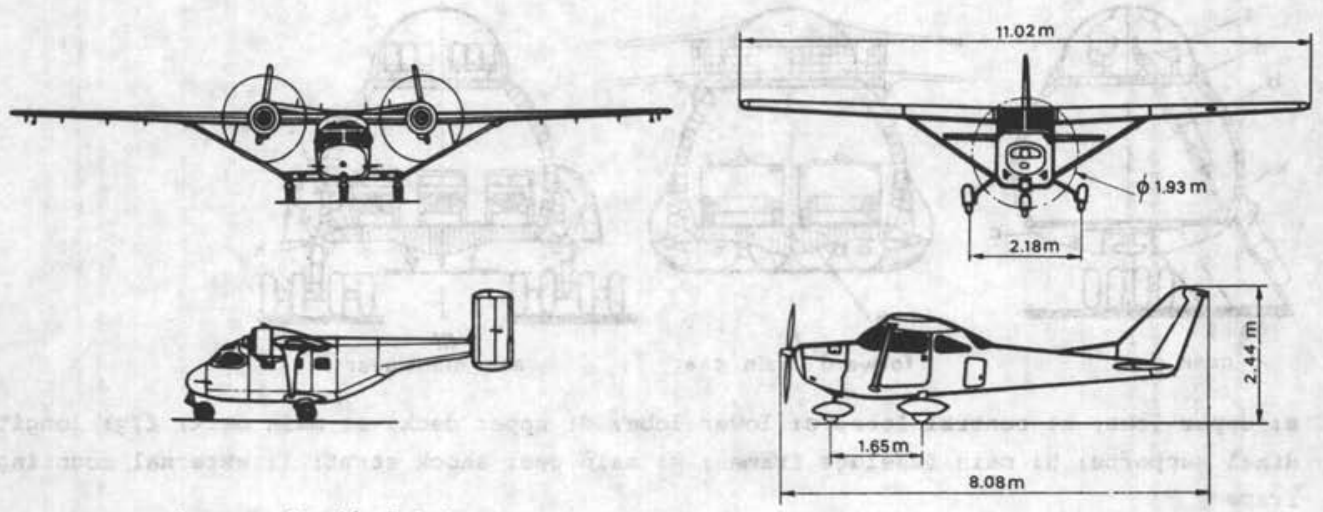

Antonov An-14 Pchelka

Fig. 2-6. Examples of airplane types with braced wings

partially offset by the saving in weight in comparison with a low-wing design, due to the shorter landing gear struts. Moreover, with a fuselage-mounted main undercarriage it is difficult to obtain a sufficiently wide track. These considerations may be regarded as having favored the use of lowwing monoplanes.

Braced-wing monoplanes (Fig. 2-6) are nowadays generally high-wing designs. Bracing struts cause little interference when attached to the lower side of the wing, while they usually can be lighter than in other positions since in this case the critical strut loads are likely to be tension loads.

In the case of STOL aircraft close proximity of the wing to the ground in takeoff and landing may cause pronounced and generally undesirable ground effects. Moreover, if a low wing was adopted, the required ground clearance of the large, fully deflected trailing-edge flaps and - in the case of propellerdriven STOL aircraft - of the large propellers, would entail a very tall and heavy landing gear. In this case a high-wing design generally has more to recommend it.

\subsubsection{Mid wing}

Thls layout is generally, chosen when mini- mum drag in high-speed flight is of paramount importance. With a fuselage of rough1y circular cross-section, the surfaces at the wing-fuselage junction meet at practically right angles so that interference between the boundary layers at small angles of attack will be minimized. In most cases the fuselage section at the location where the wing is mounted to it is roughly cylindrical. The divergence of the airflow over the wing root at high angles of attack is thus minimized. Wing root fairings of only very modest size will therefore be required. For these reasons many mid-wing layouts are found in fighter and trainer aircraft, where it is an acceptable arrangement, provided the space required for the useful load is small in relation to the total internal fuselage volume and can be divided into separate sections. The wing may be continuous through the fuselage because the transfer of the loads from the wing may take place via almost "solid" bulkheads to which each winghalf is attached. It is generally not feasible to adopt such a scheme for transport aircraft, and very few mid-wing monoplanes are to be found in this category. However, it is worth noting that on large transport aircraft the wing arrangement adopted approaches the mid-wing position, the reason being that the cabin 
floor, which is located just above the wing center section, is positioned relatively high, in the fuselage cross section. Another exception is the HFB 320 Hansa (Fig. 2-7), which features a negative angle

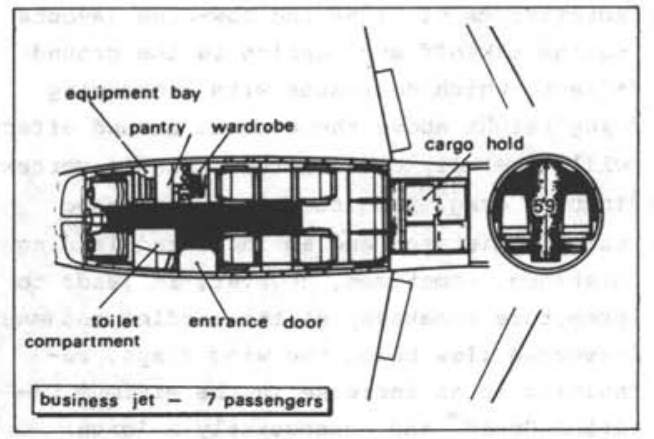

Fig. 2-7. Fuselage layout of the HFB Hansa jet

of sweep, with the engines at the tail balancing the cabin ahead of the wing center section. However, with this layout it is difficult to avoid considerable shift of the center of gravity for different loading conditions unless serious loading restrictions are accepted. The swept-forward wing presents certain aeroelastic problems which are difficult to solve without the use of tip tanks. Although the manufacturer claims that the aircraft possesses low drag characteristics, maximum cabin height for. a given fuselage diameter, and a good view for all the occupants, it remains doubtful whether these outweigh the disadvantages.

\section{$2 \cdot 2 \cdot 3$. Low wing}

The low wing position frequently offers many advantages. It is true that light aircraft still account for a fair number of highwing monoplanes, but this may be more a matter of company tradition than an obvious technical advantage. The low cargo floor height is of benefit to small freighters designed for operating into secondary air-

\footnotetext{
*See Section 8.5.4
}

ports and from airfields and airstrips where special loading equipment is not available. In the case of most passenger aircraft, the height of the cabin floor above the ground is of lesser importance as use can be made of steps of loading bridges.

Efficient use of the underfloor space in the fuselage for the stowage of cargo is possible only if the fuselage is at a suitable height above the apron. Without resorting to a tall undercarriage, this is more easily achieved in $a_{1}$ low-wing design. The generally larger fuselage height above ground level on a low-wing configuration may also offer advantages when, after a fuselage stretch, the tail angle available is still sufficiently large to allow for optimum rotation during the takeoff, without creating an unacceptable geometrical pitch angle limitation (Fig. 2-8).

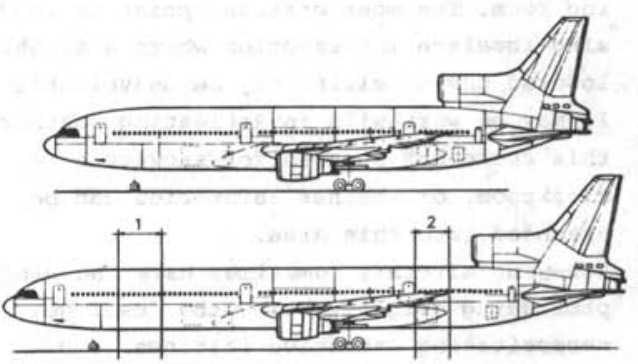

Fig. 2-8. A stretched version of the Lockheed Tristar is envisaged in the design stage. The fuselage will be stretched by $3.56 \mathrm{~m} \mathrm{(1)} \mathrm{and} 4.57 \mathrm{~m} \mathrm{(2)} \mathrm{in} \mathrm{front} \mathrm{of} \mathrm{and}$ behind the wing resp. The undercarriage is sufficiently long to allow rotation over $12^{\circ}$ after stretching (Ref.: Aviation Mag. No. 550,30 Nov. 1970)

2.2.4. Effect of the wing location on the general arrangement

a. Interior arrangement.

On a high-wing aircraft the fuselage section below the floor is generally flattened in order to reduce the undercarriage height and to keep the floor at the desired level 
above the ground, truckbed height being generally 4 to $4 \frac{1}{2} \mathrm{ft}(1.20$ to $1.40 \mathrm{~m})$. A flat fuselage belly leaves little or no room to carry underfloor cargo and this may necessitate a longer cargo hold in the cabin, as compared to aircraft with a circular fuselage cross-section. This in turn may lengthen the fuselage, particularly when, in addition, most equipment and services will have to be located above the floor. A large center of gravity travel may result. On low-wing aircraft the landing gear may be retracted into (propeller) engine nacelles or into the fuselage just behind the center-section of a swept-back wing. Retraction of the main undercarriage between the main wing spars is more easily achieved with a non-stressed lightly loaded skin than with a stressed-skin structure.

In small high-wing transports the aisle is sometimes sunk in relation to the rest of the cabin floor to provide adequate standing room. The most critical point is at the wing-fuselage intersection where a slightly lowered cabin ceiling may be unavoidable. It may be worthwile investigating whether this space can be used for stowage or a cloakroom, or whether lavatories can be extended into this area.

Low-wing aircraft sometimes have the wing protruding slightly below the fuselage, necessitating extensive fairings (e.g. Hawker Siddeley 125). In some small touring aircraft the front seats may be mounted directly on the wing box, thus saving space.

b. Safety.

The low wing and possibly the engines will form a large energy-absorbing mass during a forced landing, although they also present potential fire hazards upon contact with the ground. The wing generally contains fuel and the tanks are 1 ikely to be damaged, particularly if they are of the integral type. If the impact is not too heavy, damage and fire risk in a high-wing aircraft may be limited. When an aircraft is forced down on water, the fuselage of a high-wing monoplane will be submerged; provisions must therefore be made for escape through the cabin roof. It should be noted, however, that not all aircraft have to be certificated for overwater operation.

\section{c. Performance and flying qualities.}

The principal difference between the characteristics of high- and low-wing layouts during takeoff and landing is the ground effect, which decreases with increasing wing height above the ground. Ground effect will generally cause a reduction in vortexinduced drag, resulting in a decreased takeoff distance and an increased landing distance. Sometimes, however, it leads to premature breakaway of the airflow and even reversed flow below the wing flaps, resulting in an increase in the Minimum unstick speed ${ }^{*}$ and consequently a longer takeoff run.

Probably more important is the decrease in downwash at the horizontal tail, leading to a nose-down pitching moment. This will require greater elevator deflection for the takeoff rotation and the landing flare-out and this may be a determining factor for the elevator power required. The proximity of the ground may have an opposite effect, causing the aircraft "to land itself". This means that after a properly executed final approach little or no elevator movement is required for the flare-out. This can be the case when the wing is placed in such a low position that ground effect causes a marked lift increment, while the nose-down pitching moment mentioned above is approximately compensated by a nose-up pitching moment due to the wing lift. Though this characteristic may in itself be advantageous, it is practically impossible to design the aircraft from the outset in such a way as to achieve it.

With respect to maximum 1 ift and minimum drag, there are admittedly differences between the high and low wing locations, but these may be minimized by proper use of fillets and fairings (Fig. 2-9). Even so, the high wing is superior in this respect to the low wing, particularly where induced

\footnotetext{
* See Appendix K, Section K-2
} 


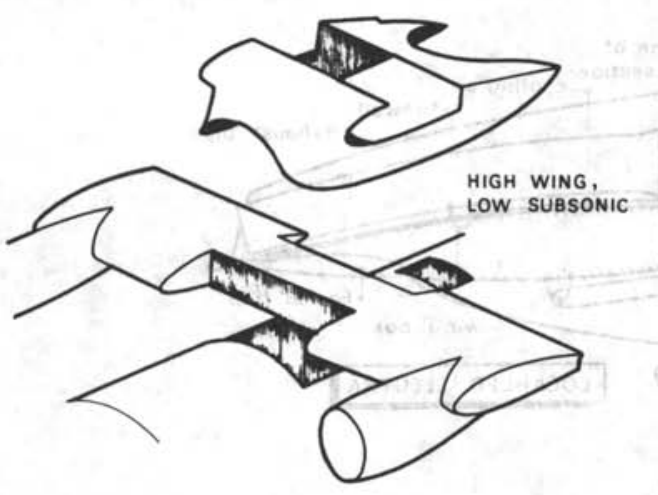

a. fillets mainly on lower wing surface

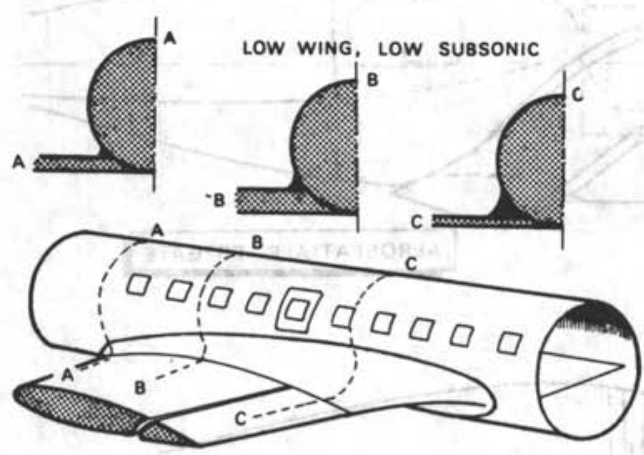

b. fillets mainly on upper wing surface

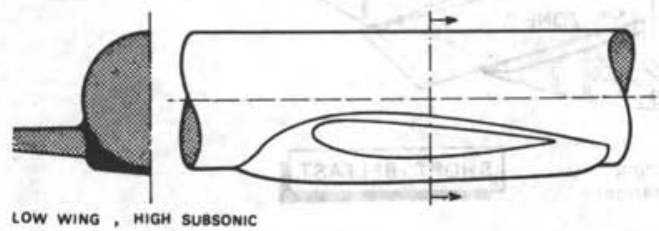

c. fillets act to obey the area rule and to house the main landing gear

Fig. 2-9. Several types of fillets to reduce unfavorable wing-fuselage interference drag at high lift is concerned. The potential differences in damping the Dutch roll may be largely suppressed by good design, particularly proper choice of the wing dihedral angle and fin area. Negative wing dihedral, desirable on swept-back wings, can easily be incorporated in a high-wing design without resorting to a tall undercarriage. However, both configurations may possess comparable flying qualities, except for fast maneuvers in aerobatics, which are favored by the mid- and low-wing layout. High-wing aircraft will generally require roughly 20 percent more vertical tail area than low wing types.

\section{d. Structural aspects.}

The Lockheed $\mathrm{C}-5 \mathrm{~A}$ has already been cited as an example of the difficulties encountered when designing the undercarriage of a highwing aircraft. Although the weight penalty in the fuselage structure is partly of fset by a lighter wing and a shorter and lighter nosewheel gear, on balance the high-wing layout will be at a disadvantage with respect to empty weight and complication of the structural design.

\subsection{LOCATION OF THE ENGINES}

\subsubsection{Propeller aircraft}

Aircraft powered by piston engines are generally seen in two layouts: the single tractor engine type with the powerplant in the fuselage nose and the twin tractor type with both engines fitted to the wing. New aircraft types with four piston engines are not being built any more since any rating over, say, $500 \mathrm{hp}$ is produced more efficiently by the turboprop engine, and piston engines in that class are practically obsolete. Configurations are occasionally observed which differ from the generally accepted solutions described above, but in such cases the choice must have been influenced by special considerations, such as the desirability of creating a high thrustline (amphibians) or avoiding asymmetry in 


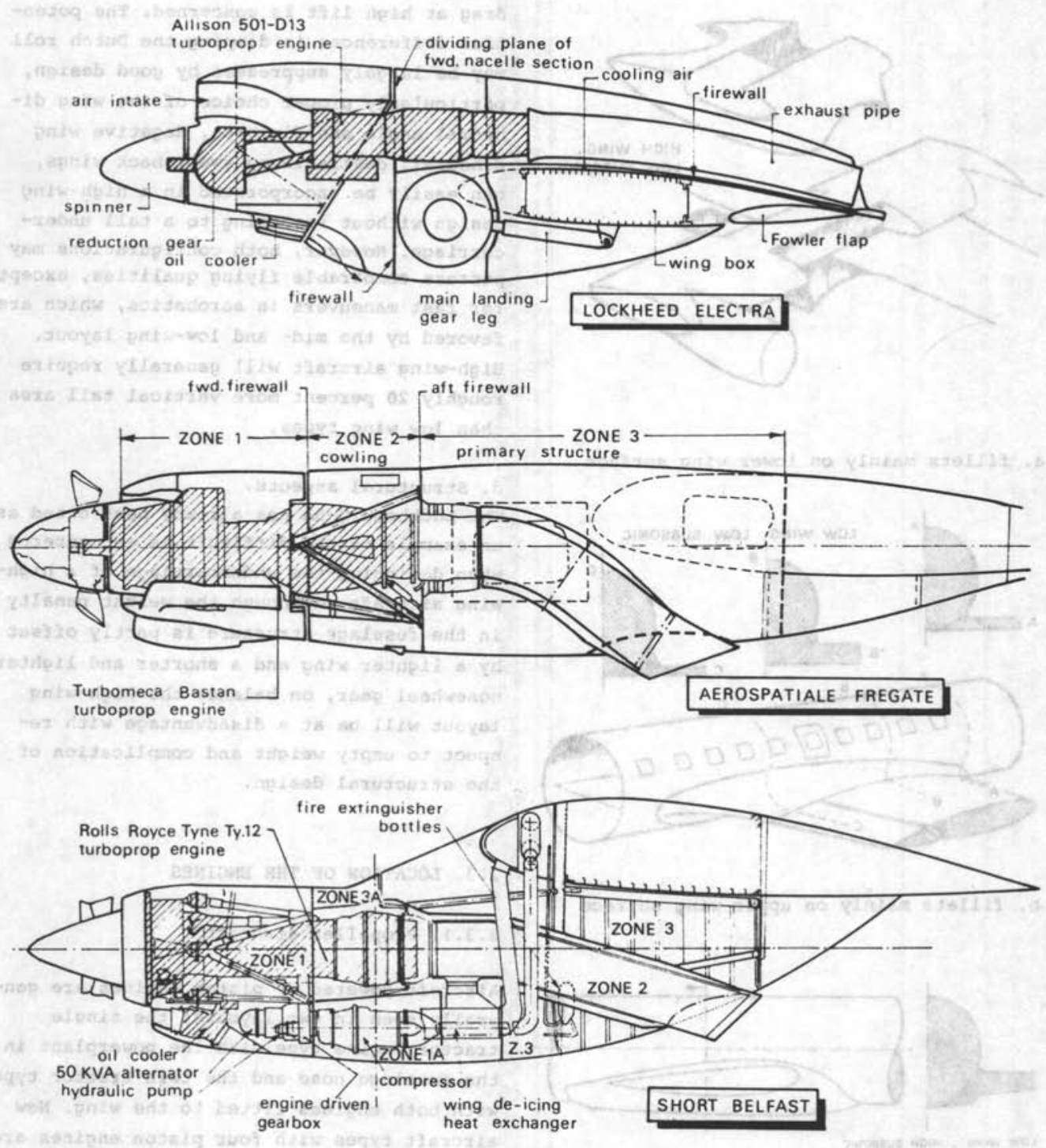

Fig. 2-10. Three types of turbopropeller engine installation

the event of engine failure by using one tractor and one pusher engine in the plane of symmetry (e.g. Cessna skymaster).

Positioning the propeller engines in front of the wing generally results in the most attractive configuration from the aerodynamic and structural point of view. The propeller slipstream of operating engines generally has a favorable effect on the stall and increases the wing lift, in particular when trailing-edge flaps are extended, thus forming a kind of built-in safeguard against stalling. On the other hand, an engine failure may cause considerable windmilling drag before the propeller 
is feathered and while the flow over the wing is still disturbed. The yawing and rolling moments induced by engine failure present control problems and downgrade the flight performance, in particular when the engine fails in the takeoff. Variation of the engine power will change the downwash behind the wing and this is of particular influence on the stabilizing contribution of the tail surfaces (Section 2.4.2). The location and installation of the engines in nacelles mounted to the wing leading edge is illustrated in Fig. 2-10 for several aircraft types. As will be shown in Chapter 6 , the engine configuration and the propeller design have considerable influence on this. In the case of a high-wing layout there is generally more freedom with respect to the vertical position of the engines relative to the airfoil as compared to low-wing aircraft, since propeller clearance over the ground is relatively easily provided.

When turboprop engines are used, an engine nacelle which is placed low relative to the wing is to be favored, both for its light supporting structure and for effective discharge of the hot gases, requiring only a short exhaust pipe. On a low-wing aircraft designers are often forced to adopt a relatively high position for the engine nacelles in order to ensure sufficient propeller to ground clearance. This may lead to unfavorable interference effects between the nacelle and the wing, causing premature breakaway of the airflow and additional induced drag.

\subsubsection{Jet-propelled transport aircraft}

When the jet engine became an acceptable prime mover for both transport and large military aircraft (about 1947-1950), the traditional piston engine layout was discarded and.a new configuration was sought which would suit the specific characteristics and demands of the jet engine. Smaller jet aircraft were generally designed for military purposes and had a single engine in the fuselage; in the case of transport

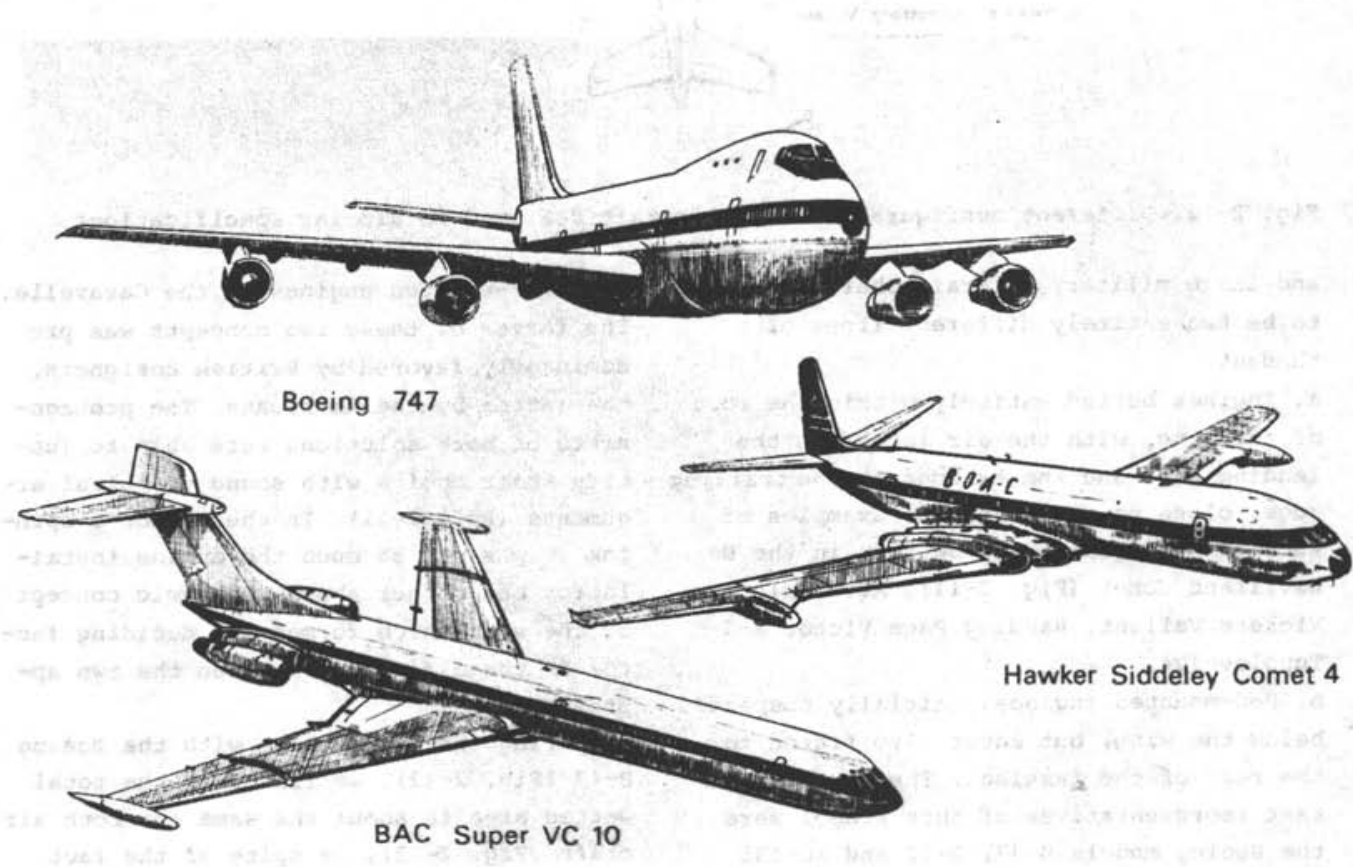

Fig. 2-11. Examples of powerplant installation on subsonic jet transports 


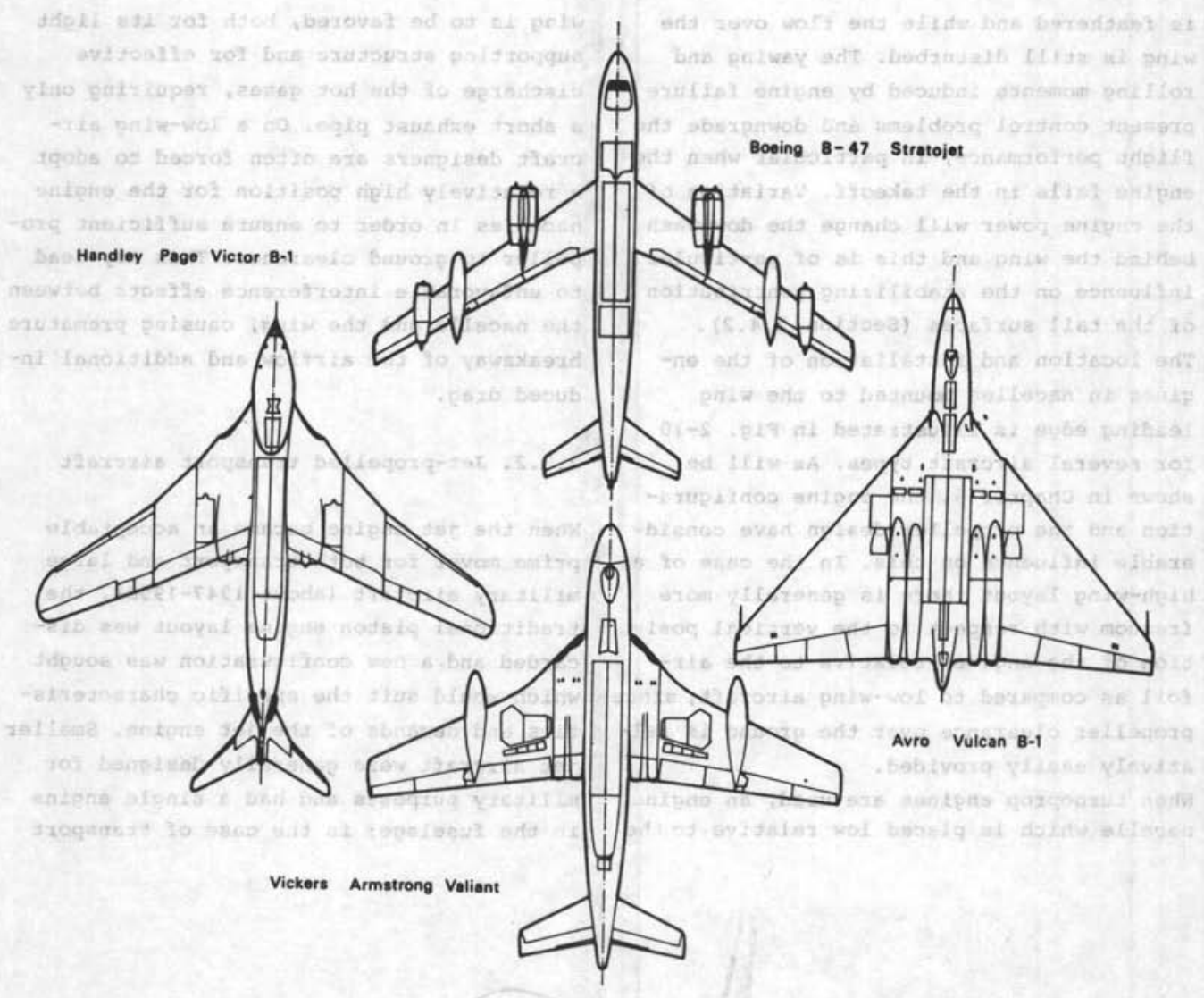

Fig. 2-12. Different configurations for aircraft designed to similar specifications

and large military aircraft there appeared to be two entirely different lines of thought:

a. Engines buried entirely within the root of the wing, with the air intake in the leading edge and the exhaust at the trailing edge, close to the fuselage. Examples of such an arrangement may be seen in the De Havilland Comet (Fig. 2-11), Avro Vulcan, Vickers Valiant, Handley Page Victor and Tupolev 104.

b. Pod-mounted engines, initially suspended below the wing, but later also fitted to the rear of the fuselage. The first important representatives of this school were the Boeing models B-47, B-52 and KC-135 (Fig. 2-1), while Sud-Aviation originated the rear-mounted engines in the caravelle. The former of these two concepts was predominantly favored by British designers, the latter by the Americans. The protagonists of both solutions were able to justify their choice with sound technical arguments (Ref. 2-11). In the author's opinion it was not so much the engine installation but rather the aerodynamic concept of the wing which formed the deciding factor in the difference between the two approaches.

Comparing the Avro Vulcan with the Boeing B-47 (Fig. 2-12), we find that the total wetted area is about the same for both aircraft (Fig. 2-13), in spite of the fact that the wing area of the Vulcan is nearly 


\begin{tabular}{|c|c|c|}
\hline & DOEING B-47 & AVhO VULCAN \\
\hline CMOSS WING AMEA $\sim \mathrm{ft}^{2}\left(\mathrm{~m}^{2}\right)$ & 1430 (133) & $3440(320)$ \\
\hline TOTAL WETTEO AREA $\sim \mathrm{ft}^{2}\left(\mathrm{~m}^{2}\right)$ & 11300 (1050) & 9500 (885) \\
\hline$\sim t(m)$ & $116 \quad(354)$ & $99 \quad(302)$ \\
\hline MAX. WING LOAOING $\sim 1 \mathrm{lb} / \mathrm{tr}^{2}\left(\mathrm{~kg} / \mathrm{m}^{2}\right)$ & $140 \quad(690)$ & $435(212)$ \\
\hline MaX. SPAN LOAOING - $1 \mathrm{~b} / \mathrm{ht}(\mathrm{kg} / \mathrm{m})$ & $1750(2590)$ & $1520(2250)$ \\
\hline ASPECT matio $=4$ ta & 2.43 & 2.84 \\
\hline $\begin{array}{l}c_{g_{\text {}}} \text { (ESTIMATEO) } \\
\text { y/maO (0-OSWALO FACTOR) }\end{array}$ & $\begin{array}{c}.0190 \\
.0425(-8)\end{array}$ & $\begin{array}{c}.0069 \\
.125 \quad(.9)\end{array}$ \\
\hline$L / D_{\max } ; c_{L_{\text {opt }}}$ & $17.25 ; .682$ & $12.0 ; .235$ \\
\hline
\end{tabular}

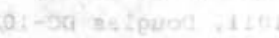

Fig. 2-13. Similarity in $\max$. lift/drag ratios for two widely different configurations

three times that of the $B-47$ (wing area*). In contrast to this, there is the difference between the aspect ratios**, namely a figure in excess of 3 . However, both aircraft have nearly the same span loading (weight/span). The remarkable conclusion can be drawn that for a given dynamic pressure both the profile and vortex-induced $\operatorname{arag}^{* * *}$ will be roughly equal for these aircraft. Although the comparison shown in Fig. 2-13 is based on estimated values, it clearly shows that it is possible to achieve a comparable range performance with both wing layouts. There are, all the same, considerable differences between the two types:

a. The maximum 1 ift/drag ratio occurs at $C_{L}=.235$ for the Vulcan and at $C_{L}=.68$ for the B-47. When cruising at high altitude the Vulcan had more freedom to maneuver without experiencing serious buffeting due to compressibility.

b. The structural height at the wing root of the Vulcan, namely about 6 feet 8 inches (2 meters), proved ample to house the engines internally; in the case of the B-47 the height available was only about 26 inches $(.66 \mathrm{~m})$.

* The gross wing area used in Fig. 2-13 is as defined in Appendix A Section A-3.1.

* Aspect ratio $=\operatorname{span}^{2} /$ area $=$ span/geometric mean chord. See Appendix A.

*** Definitions in Section 11.2.
In a sense this means that the design philosophy with regard to the engine installation was mainly decided by the shape of the wing. Although this example is predominantly of historical interest, it shows that there is a close connection with decisions in other fields.

The protagonists of podded engines attached to the wings by means of pylons use the following arguments to support their views:

a. Separately spaced engines are well placed from the safety point of view. In the event of fire in one of the pods the likelihood that fire will spread to the fuel in the wing is limited. In fact this was the main argument for the choice of the B-47 configuration.

b. The short intake and exhaust ducts enable the engine to run under optimal conditions.

c. The mass of the engines and the pylons lead to a reduction in the bending moment of the inner wing, thus lightening part of the wing structure. When they are located ahead of the flexural axis, they constitute a mass balance against flutter. d. The engines can be made easily accessible at the cost of very little increase in structure weight since the pods do not form part of the stressed structure. Access to engines buried in the wing roots has to be provided by detachable skin panels at a location where the wing is highly stressed.

e. The engine pylons appear to have a favorable effect on the airflow at large angles of attack and tend to counteract pitch-up of sweptback wings (Fig. 2-14). The pylons act in a manner similar to

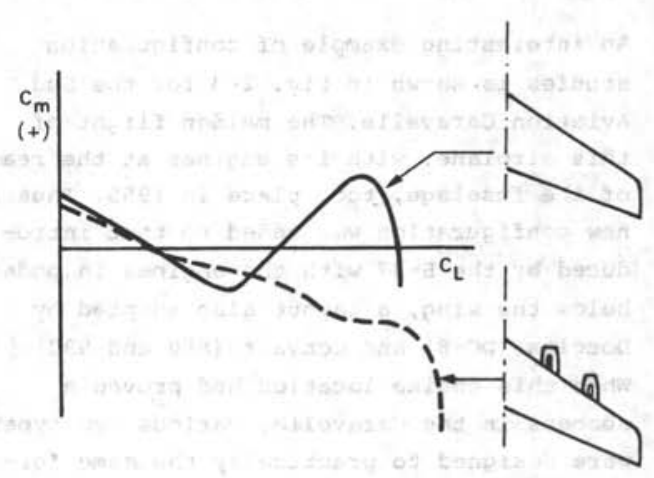

Fig. 2-14. Effect of wing-mounted pods on longitudinal stability (Ref. 2-11) 
fences, which are often used on "clean" wings. Against this the protagonists of completely buried engines list the following arguments:

a. The extra drag resulting from the buried engine installation is only a few percent as against about 158 of the total arag in the case of a configuration similar to the B-47. Incidentally, the current generation of turbofans show a value of about 8 to 108.

b. As a result of the low wing loading and low value of $\mathrm{C}_{\mathrm{L}}$ in cruising flight, maneuvering is possible without compressibility problems such as buffeting.

c. The pitch-up problem of swept wings is less significant for $10 \mathrm{w}$ aspect ratio wings.

a. As a result of the low wing loading, the lowspeed performance will be better.

e. The relatively low aspect ratio wing box structure will lead to greater stiffness and aeroelasticity will be less of a problem.

Many of these arguments are only valid up to a point, and in particular the progress in engine technology towards high bypass ratio engines, together with the development of more efficient highift devices in 1950-1970, has settled the case in favor of high wing loadings and pod-mounted engines. This does not mean that buried engines will not return to favor again in the future. For example, the application of laminar flow control by suction of the boundary layer to reduce drag might eventually lead to a totally different design approach, such as a combination of low wing loading and engines of relatively low thrust in cruising flight, integrated into the wing or fuselage.

An interesting example of configuration studies is shown in Fig. 2-3 for the Sud Aviation Caravelle. The maiden flight of this airplane, with its engines at the rear of the fuselage, took place in 1955. Thus a new configuration was added to that introduced by the B-47 with the engines in pods below the wing, a layout also adopted by Douglas (DC-8) and Convair (880 and 990). When this engine location had proved a success in the Caravelle, various new types were designed to practically the same formula: the BAC $1-11$, Vickers VC. 10 , Hawker Siddeley Trident, Douglas DC-9, Boeing 727 ,
Fokker F-28 and all executive turbojet aircraft. Though this layout has the obvious advantages of a "clean" wing, low door sill height and little asymmetric thrust after engine failure, it also has a large center of gravity variation with variation in loading condition and it has to be carefully designed to avoid the superstall problem. Therefore, after 1965, a new trend towards engines on the wings occurred (Boeing 737, Lockheed L-1011, Douglas DC-10, Dassault Mercure).

It would serve little purpose to express a general verdict in favor of either of these two configurations. Each specification, as well as every new type of engine, will require renewed study to support a particular choice and the outcome can only be properly assessed when various configurations have been designed according to the same ground rules. This procedure was followed by the Boeing Company for the development of their Model 737 (Fig. 2-15), when two competing
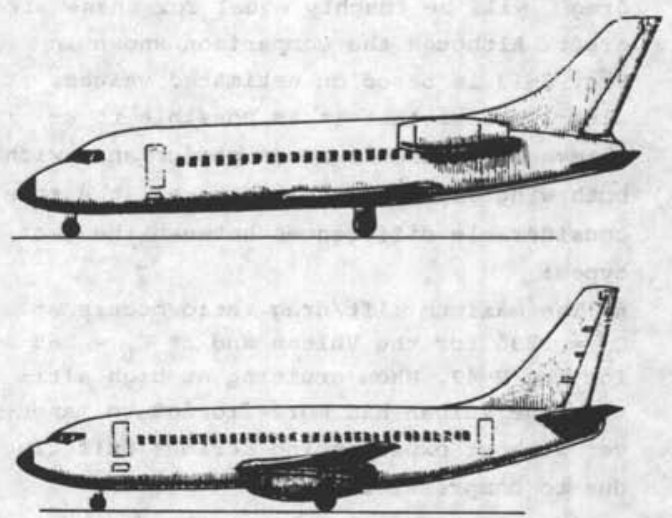

Fig. 2-15. Two configurations of the Boeing Model 737, resulting from a competition of two design teams

design teams were put on the same exercise. Factors which should be investigated in the case of a transport aircraft are indicated in Fig. 2-16. The principal differences between the Caravelle and the Boeing 737 engine location will be briefly discussed with reference to that 1 ist. 


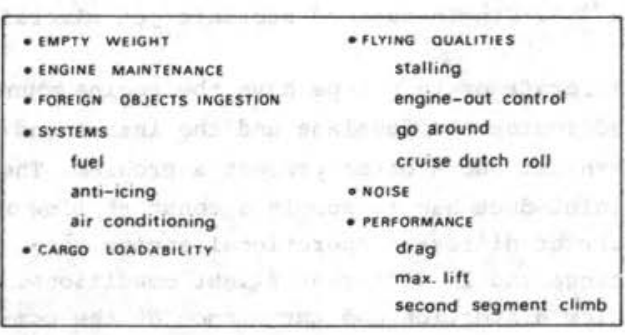

Fig. 2-16. Engine location factors (Ref.: ATA Engng and Maint. Conf., Oct. 27, 1964)

a. Empty weight.

The following factors have to be considered: - A wing structure weight saving is possible with wing-mounted engines due to the mass relief effect on the bending moment on the inner wing.

- Engines placed too far outboard increase the landing impact loads and necessitate a large vertical tailplane.

- Engines at the rear of the fuselage require local "beef-up" and lead to loss of useful space in the tail, resulting in added structure weight and a larger fuselage for the same payload.

- Differences in weight of the tail surfaces depend on various factors which do not permit a general conclusion.

Suming up, we may say without too much emphasis that the empty weight of a Caravelle-type layout will typically be 2 to 48 more than that of a comparable design with the engines on the wing.

b. Engine maintenance.

Although the size of the aircraft comes into it, engines below the wings are generalIy better accessible from the ground than in any other layout.

\section{c. Flexibility of loading.}

This depends primarily on the location of the load relative to the center of gravity of the empty aircraft, a subject that is treated more fully in Chapter 9. Both configurations may be designed for good loading characteristics, although a greater c.g. travel must be catered for in the case of rear-mounted engines. At full payload the download on the tail, with consequent loss in the lift to drag ratio, will be considerable with engines mounted to the rear fuselage. Besides, the layout with wing-mounted engines will have a larger underfloor cargo-hold behind the wing, which is generally more easily used.

\section{d. Performance.}

Regarding drag in cruising flight there is little to choose between the two layouts, assuming that both have been well designed aerodynamically. Douglas, however, claims that in the case of the DC-9 the drag of the wing plus nacelles at high subsonic speeds is reduced as a result of favorable aerodynamic interference, (Ref. 2-30). Generally speaking, a layout with wing-mounted engines will lead to an increase in induced drag and a slight reduction in the dragcritical Mach number. The drag resulting from the asymmetrical flight condition following engine failure, rapidly increases with the lateral distance of the failed engine to the aircraft centerline and will, therefore, be greater with engines mounted on the wing. The protagonists of the Caravelle layout claim that their clean wing gives a gain of 208 in maximum lift. The Boeing Cy. does not agree, basing its opinion on test data and arguing that in the case of the clean wing the useful lift is reduced by gadgetry to ensure a favorable pitching behaviour at the stall. And indeed, looking at maximum $C_{L}$ values for a number of aircraft with different layouts, it is not possible to discern a clear-cut tendency either way.

\section{e. Flying qualities.}

Engines mounted to the rear of the fuselage are often combined with a tailplane on top of the fin (T-tail). This particular layout has a potential problem in the high incidence range, namely the "deep stall"*

\footnotetext{
"Also referred to as "superstall" or
} "locked-in stall"; see Section 2.4.2 
(Section 2.4.2). In the case of engines on the wings, the yawing moment resulting from engine failure will be more pronounced.

f. Mounting of a central engine. Three-engined aircraft generally have one engine mounted centrally at the rear of the fuselage. The problem which will have to be faced here is whether to bury this engine in the fuselage, which will require a fair1y long and curved inlet (Boeing 727, Hawker Siddeley Trident, Lockheed L-1011) with consequent loss of intake efficiency and extra weight. Alternatively, the engine can be installed in a pod on top of the fuselage, but in that case the vertical tail surface forms an obstruction. Fig. 2-17 depicts some possible solutions, al1

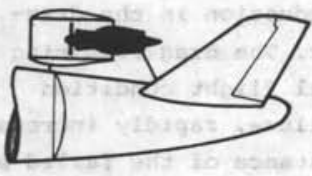

BUTTERFLY TAIL

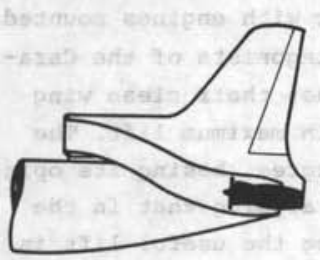

$\mathrm{L}-1011$

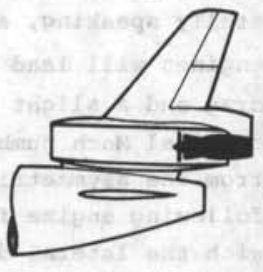

DC -10

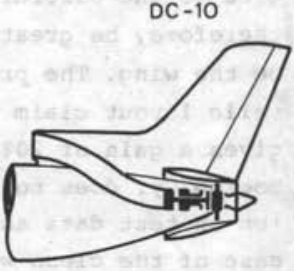

AFT-FAN
Fig. 2-17. Installation of the central engine on a three-engined jet aircraft

of which present particular design problems. The thing to do here is to optimize the chosen solution in such a way that the disadvantages will be 1 imited. Ref. 2-23 shows that a purely objective comparison of two solutions is very difficult. Manufacturers' data for structure weight, fuel consumption and economy for both the L-1011 and the DC10 are used to show that both solutions are best.

\subsubsection{Single-engined subsonic jet aircraft}

Aircraft of this type have the engine mounted inside the fuselage and the intake and exhaust ducts often present a problem. The inlet duct has to supply a constant flow of air at different operational engine settings and in different flight conditions. Flow distortion and turbulence at the compressor face must remain within the limits

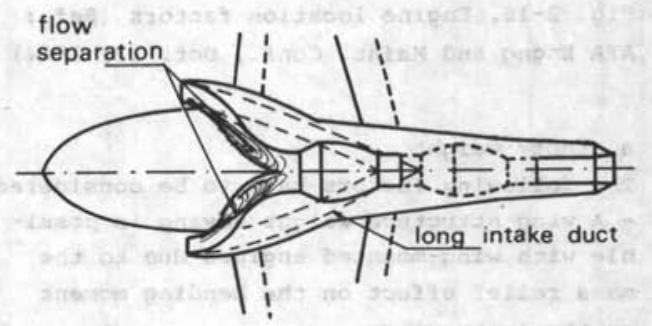

Fig. 2-18. Intake problem on a stubby fuselage with side intakes

laid down by the engine manufacturer. Pronounced curvature in the inlet duct should therefore be avoided. This is not very easy to comply with in the case of a fuselage which is relatively wide at the location of the air inlets unless a long inlet duct is acceptable (Fig. 2-18). The latter is generally undesirable for reasons of space or balancing. It also costs weight and results in inlet pressure loss.

At different angles of attack variations in the direction of the incoming air should

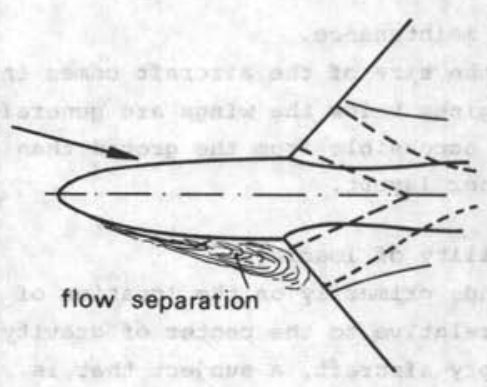

Fig. 2-19. Asymmetric intake condition in a sideslip 


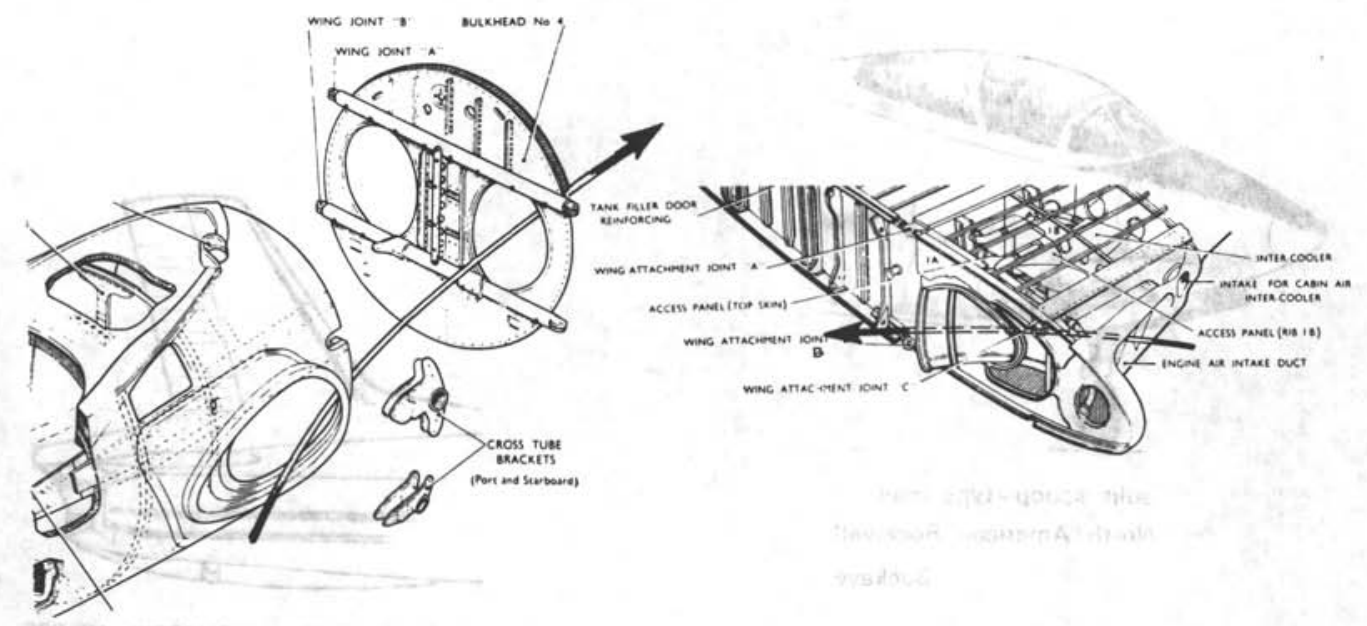

Fig. 2-20. Structural arrangement of wing-root air intake (De Havilland Vampire)

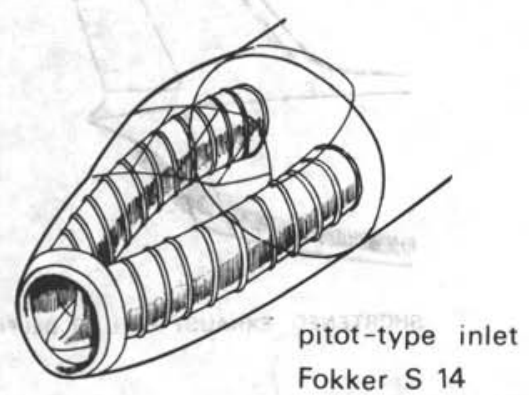

Fokker S 14

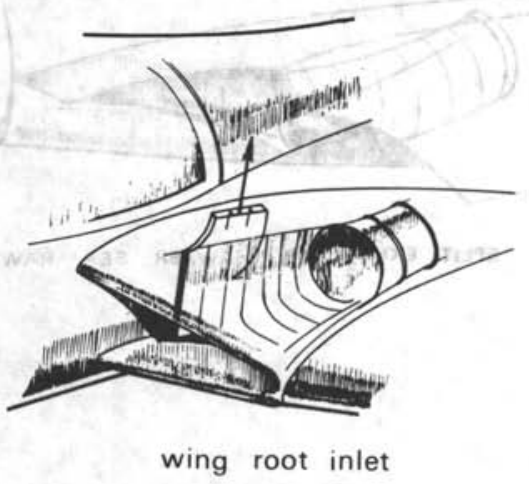

Hawker Hunter
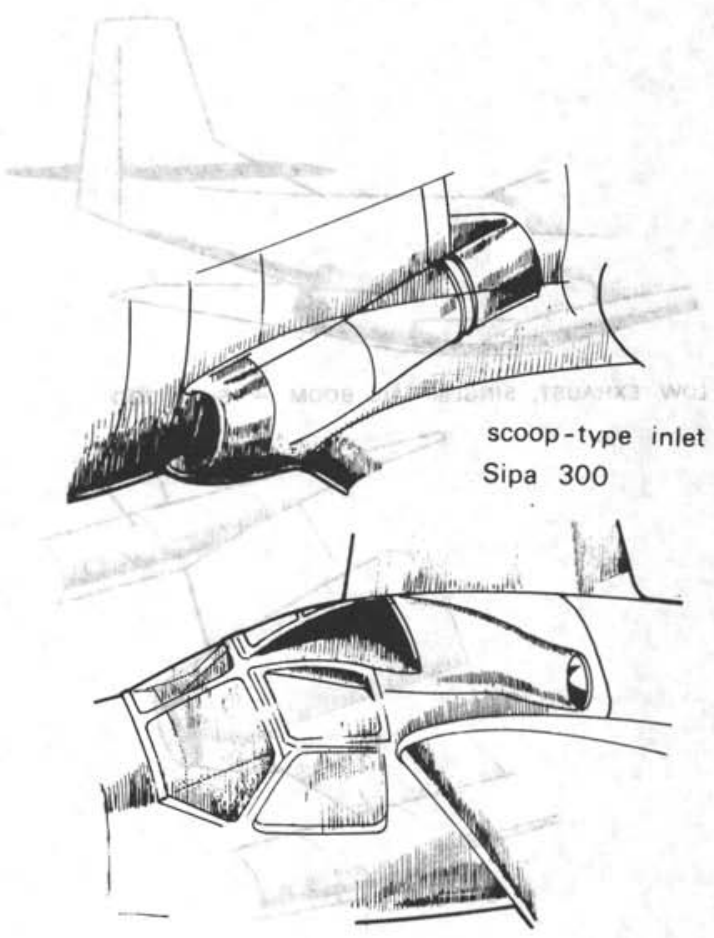

NACA inlet Miles Student

Fig. 2-21. Intakes on subsonic aircraft with engine(s) buried in the fuselage 


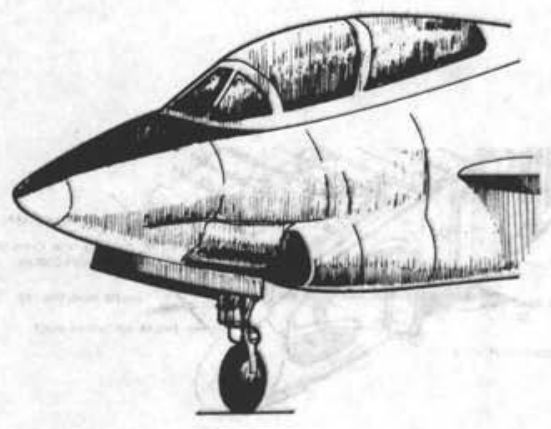

split scoop-type inlet

North American Rockweil

\section{Buckeye}

Fig. 2-21. (Concluded)

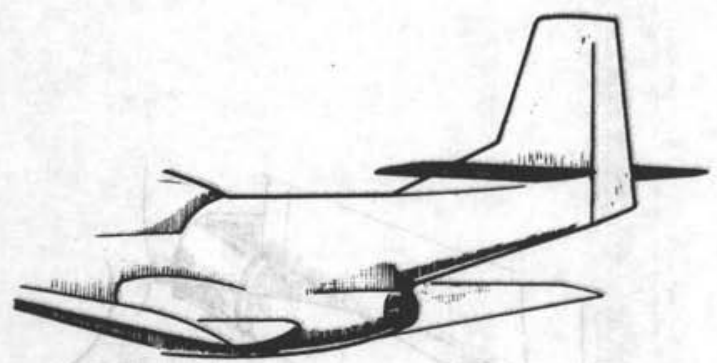

LOW EXHAUST. SINGLE TAIL BOOM - SIPA 300

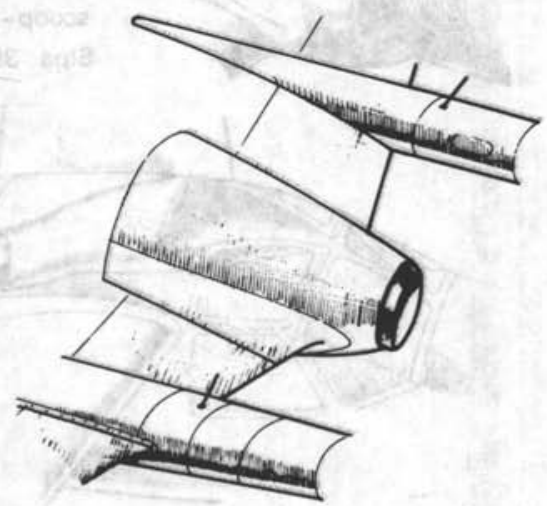

SHORT EXHAUST PIPE

DE HAVILLAND VAMPIRE

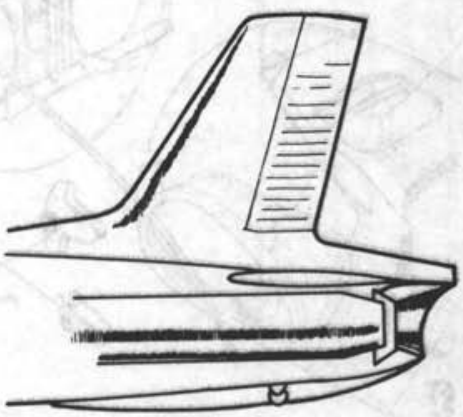

LONG EXHAUST - AERMACCHI MB 326

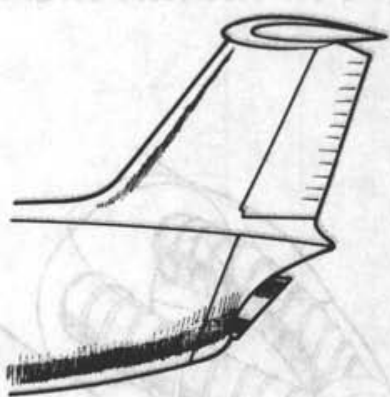

SHORTENED EXHAUST - L 29 DELFIN

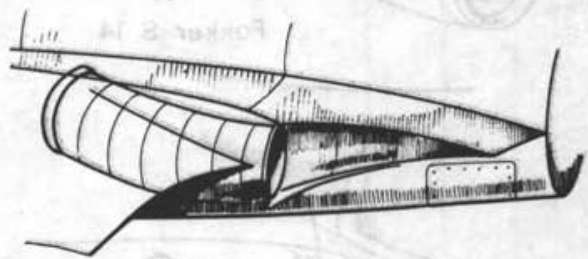

SPLIT EXHAUST - HAWKER SEA HAWK

Fig. 2-22. Exhaust locations of single-engined subsonic jet aircraft 
not be excessive. The wake of the partly stalled wing must not enter the inlet duct, which means that the leading edge of the wing would be an unsuitable location unless special measures were taken. When split intakes are used, a sideslipping condition will cause dissimilar flow patterns, which may lead to unstable flow and even to air oscillating instead of entering the duct as shown in Fig. 2-19.

When the engine is installed in the fuselage, the designer has to decide whether it is desirable to continue the wing structure through the fuselage without interruption. on a highly maneuverable aircraft, designed for high normal load factors, such a continuous structure is very attractive. It will then depend upon the relative proportions of the inlet duct and the thickness of the wing whether it is feasible to lead the inlet ducts through the spar webs. Fig. 2-20 shows that this was possible in the case of the De Havilland Vampire, but in other cases it may prove desirable to lead the inlet either over or under the wing. Fig. 2-21 shows different types of inlet which will be briefly discussed here.

The pitot type (a) provides the engine with undisturbed airflow for all flight conditions. It requires a long inlet duct, which generally will have to be divided at the level of the cockpit, and intake efficiency is low. This type is now rarely used on subsonic aircraft.

An intake in the wing-root (b) is difficult to realize as the intake opening must be able to supply the required airflow at different intake velocities and also cope with changes in the angle of attack and angle of sideslip. At the same time the local airfoil shape must not be modified more than is strictly necessary.

Side inlets on either side of the fuselage form scoops and thus cause additional drag. To keep this drag low, the airscoops must not be kept too short and must be well faired. A divertor is needed to prevent the fuselage boundary layer from entering the duct but this also adds to the drag. The inlet opening should be located sufficient- 1y far ahead of the wing in order to avoid interference with the wing and excessive variations in the intake conditions.

An air inlet on top of the fuselage has sometimes been used in experimental aircraft and was adopted for the Miles student. The opening has to be raised sufficiently far above the fuselage to avoid boundary layer and wake ingestion at large angles of incidence.

A split inlet at the bottom of the fuselage may be regarded in some ways as a compromise between the pitot inlet and side inlets. When measures are taken to avoid the ingestion of debris during takeoff and taxying, this layout may be particularly attractive for mid-wing and high-wing aircraft.

The exhaust nozzles should be so positioned and directed that the (hot) jet efflux will not impinge on the structure. At subsonic speeds in a parallel flow, the expanding gases of a pure jet may be assumed to expand within a cone with half the top angle equal to 6 degrees. Exhaust nozzles are manufactured from stainless steel sheet and are fairly heavy; on pure jet engines they will weigh from 1 to 1.58 of the engine weight per foot of length ( 3 to 58 per meter). The weight will be even greater in the case of bypass engines. Moreover, exhaust nozzles cause a thrust loss of about .38 per foot (18 per meter). They should therefore be kept as short as possible. Some examples shown in Fig. 2-22 will be discussed.

When the exhaust nozzle is located in the rear end of the fuselage, it is possible to keep the efflux away from the aircraft without having to take any special precautions. A single tail boom is sometime's adopted in order to shorten the exhaust. Another solution consists of a split exhaust with two openings on either side of the fuselage. Unfortunately, both configurations lead to structural problems, while complicated fair ings must be used around the exhausts. Another way to shorten the length of the ex- 
(A) Fuselage- or FINMOUNTED STABILIZER
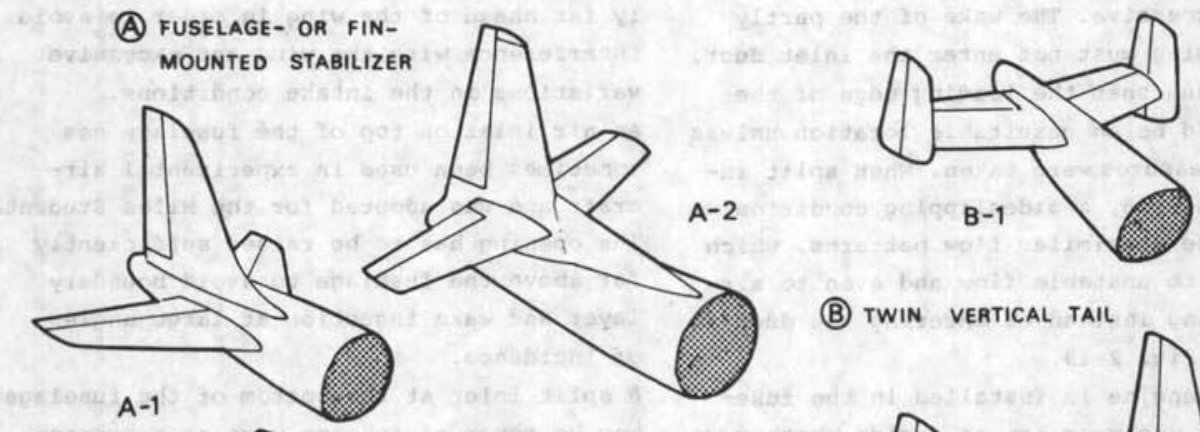

(B) TWIN VERTICAL TAIL

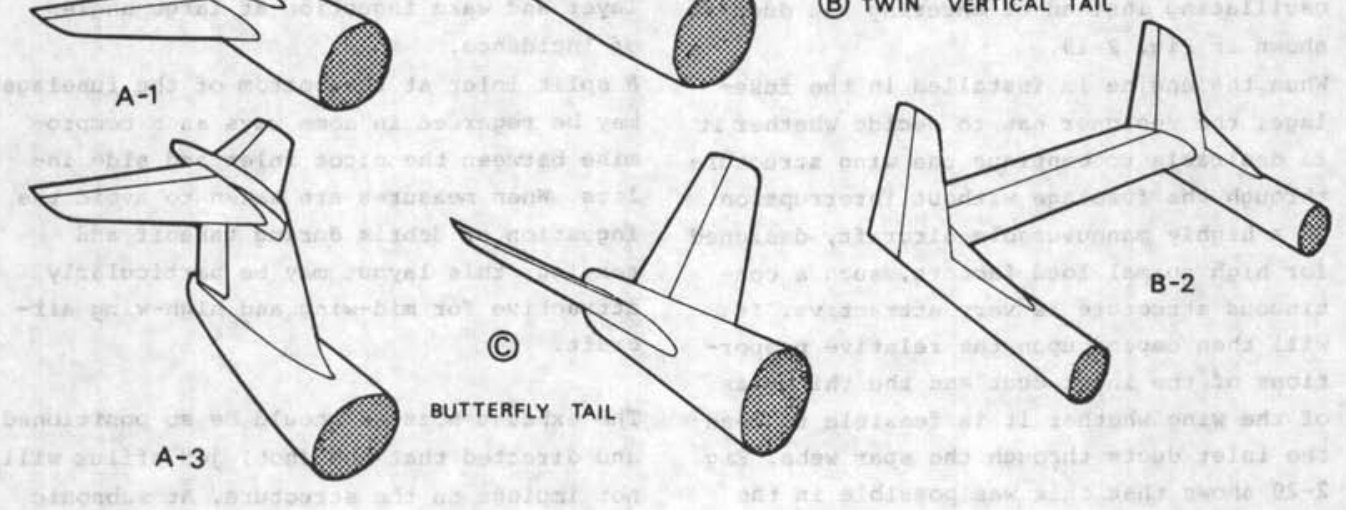

Fig. 2-23. Classification of tailplane configurations

haust pipe is to use two tail booms. This has the added advantage that it provides excellent accessibility to the engine.

\subsection{ARRANGEMENT OF THE TAILPLANE}

The design of the tail surfaces probably depends more on the general arrangement and the detail layout of the aircraft than any other major part. Because of their location, their effectiveness is influenced by the wing and the operation of the engines, particularly in the case of propellerdriven aircraft. The way in which the empennage is mounted to the fuselage, or possibly to tail booms, affects the structural layout of the tail surfaces and that of the fuselage. General instructions applicable in the preliminary design stage are therefore very difficult to lay down.

2.4.1. Classification of tail surface configurations
Examples are given in Fig. 2-23 of the principal configurations seen in practice. Although there are many intermediate solutions, these will not be discussed here.

Group A: A single fin with the stabilizer mounted either on the fuselage or on the fin represents the most common current layout. It also ensures structural simplicity and stiffness, although in the case of the $\mathrm{T}-\mathrm{tail}(\mathrm{A}-3)$ attention must be devoted to preventing tailplane flutter. Aerodynamic considerations leading to this choice are discussed in Section 2.4.2.

Group B: The considerable height of a large fin will cause a rolling moment due to rudder deflection as a consequence of the large distance of the fin aerodynamic center from the longitudinal axis of the aircraft. If this is considered to be objectionable, a twin fin may be well worth investigating as a means of minimizing this effect. When twin tail booms are used (group B-2), such a layout is the fairly obvious choice. 
Group C: The V- or butterfly tail is often adopted for sailplanes, with the object of avoiding damage to the tail when landing on overgrown terrain. The V-tail is sometimes also used on powered aircraft, e.g. the Fouga Magister where it served to keep the tail surface clear of the jet efflux of the engines, without having to resort to a Ttail. Another classical example is the Beechcraft Bonanza. The $\mathrm{V}$-tail has never become popular, mainly because the moving surfaces have to serve both as rudders (differential deflection) and as elevators (simultaneous deflection), which leads to a complication in the control system design.

\subsubsection{The location of tail surfaces}

a. Jet efflux effects.

The tail surfaces must never be in the jet efflux. Assuming that the efflux of a pure jet spreads out conewise with half the top angle equal to 6 degrees, this defines a region which may be regarded as "out of bounds" so far as the tail surfaces are concerned. If necessary, the centerline of the jet efflux may be diverted a few degrees in any desired direction. Another possibility is to apply a moderate dihedral to the horizontal tailplane. It is advisable to have as great a distance as possible between the noise generating regions and the tail surfaces, since otherwise the very high intensity of the engine noise may cause acoustic fatigue in the relatively flat skin panels of the tail. Any special measures to prevent this will entail a weight penalty. A jet efflux close to the stabilizer will affect the direction of the airflow and diminish its stabilizing contribution due to the jet pumping effect.

\section{b. slipstream effects,}

In symmetrical flight, the lift distribution of the wing with deflected flaps depends on the engine speed. The same applies to the downwash and the local velocity distribution at the tail. When the airspeed and the angle of attack are changed, the stabilizer moves in a vertical direction relative to the slipstream, which causes variations in the longitudinal stability. These depend partly on the location of the stabilizer, measured in the vertical direction. Fig. 2-24 shows

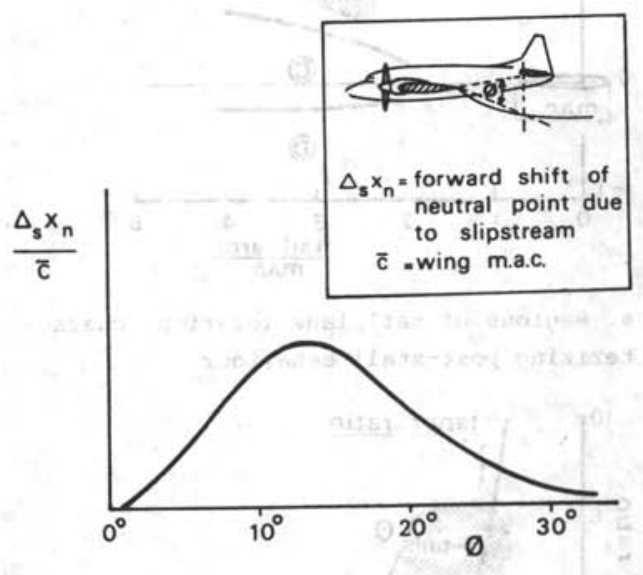

Fig. 2-24. Forward shift of the neutral point due to slipstream (Ref.: ARC R \& M 2701)

that loss of static stability is small with the stabilizer placed very high or very low, but this cannot always be realised in practice. As the power to weight ratio and the maximum lift coefficient increase, the slipstream effects will also become more pronounced and generally the tail size will have to be increased.

In flight with one engine inoperative there will be a yawing moment which has to be counteracted mainly by rudder deflection. There will also be a non-symmetrical lift distribution over the wing and this will cause a sidewash at the fin, effectively resulting in an increase in the yawing moment. This condition of flight provides a criterion for the size of the $f$ in and rudder in the case where the engines are mounted on the wing.

c. Stability and control in the stall and post-stall condition.

Although in normal operating conditions a wing stall is avoided by applying adequate safety margins relative to the minimum fly- 


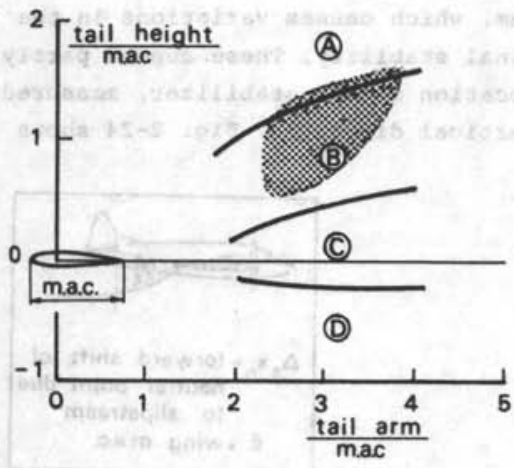

a. Regions of tailplane location, characterizing post-stall behaviour

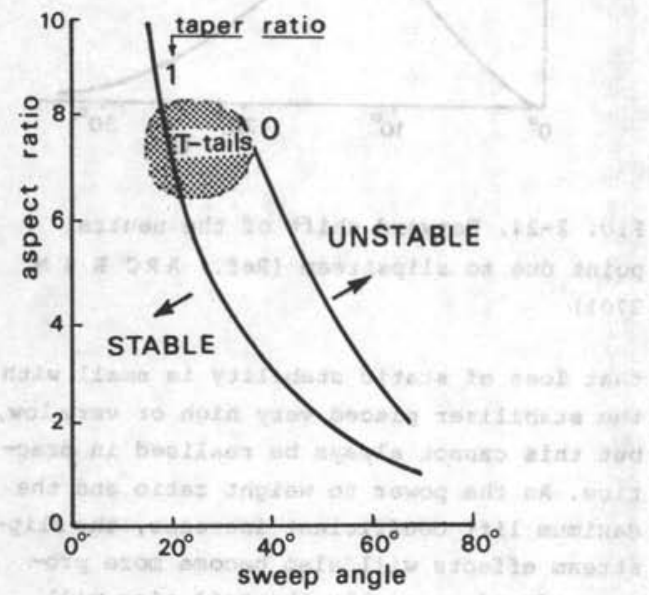

b. Stability boundary for the wing alone

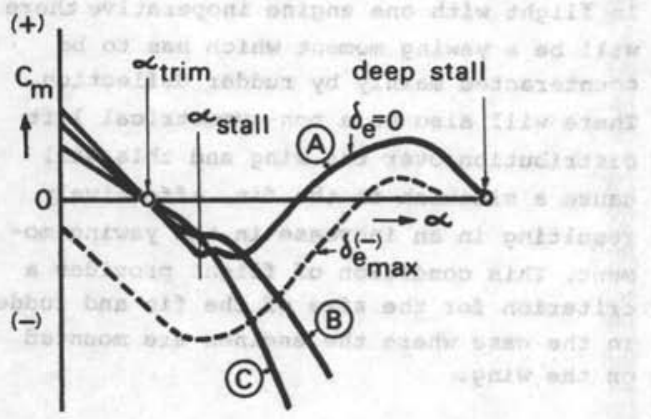

c. Post stall stability for the complete airplane

Fig. 2-25. Static stability at high angles of attack as affected by wing shape and tailplane location (Ref.: NASA TM-X-26) able speed, a stall may be encountered occasionally. The stall speeds must be demonstrated during certification testing as they form the baseline for most performance figures in takeoff and landing. Safe recov ery from a stall is therefore a requirement. The longitudinal flight characteristics are affected primarily by the "stiffness in pitch", represented by the slope of the $\mathrm{C}_{\mathrm{m}}-\alpha$ curve (Fig. 2-25c). A negative slope corresponds to positive static stability, while the trimmed condition is equivalent to $c_{m}=0$, to be obtained by elevator and/ or stabilizer deflection. The wing and horizontal tailplane are the main contributing components, the tailplane location being of prime importance. Fig. 2-25b shows combinations of wing sweep angles and aspect ratios which ensure a stable wing pitching moment slope at the stall. The stable region marked is only approximate and may be influenced by airfoil variation, wing twist, boundary layer fences, engine pylons and leading edge high-lift devices. The boundary of the stable region, as derived from windtunnel tests, indicates a reason why highly swept wings generally are of low aspect ratio. A slightly unstable wing pitch-up may be acceptable, provided the horizontal stabilizer is sufficiently effective. The effect of the vertical location of the stabilizer is illustrated in Fig. 2-25c for several cases, defined in Fig. 2-25a. In region $A$, which covers most T-tails, instability at large angles of incidence is generally preceded by a less pronounced instability at the stall. In region $B$ the stabilizer only enters the wake of the wing when the latter becomes unstable. Region $C$ does not show these phenomena at low speeds, but pitch-up may occur on maneuvering flight at high subsonic speeds. Region D is a location which may be regarded as satisfactory for all angles of incidence. This arrangement may sometimes be possible in the case of high-wing aircraft, but attention should be paid to the location of the wake, particularly when flaps are deflected.

Most tailplanes designed for normal opera- 
ting conditions will be sufficiently effective to provide stability at high angles of attack as well. However, if the wing wake is augmented by the wake of a wide fuselage and pod-mounted engines on either side of the rear fuselage tail, conditions may exist such that a T-tail aircraft encounters extended regions of post-stall instability. At very high angles of attack the tailplane contribution to longitudinal stability will be reduced to 10-20s of its normal value. At angles of attack between 30 and 40 degrees the tailplane itself stalls and the slope of the $\mathrm{C}_{\mathrm{m}}{ }^{-\alpha}$ curve is once more reversed into a stable one. At $c_{m}=0$ the aircraft is trimmed in a "deep stall". In that condition the pitching moment due to elevator deflection may be insufficient to restore the normal attitude and the airplane is locked in this condition. A very fast descent at low forward speed is unavoidable and recovery from it is very doubtful. There are various methods of curing such unacceptable behavior, e.g. increasing the tailplane span and modifying the wing shape. For added safety a stick shaker can be installed to warn the pilot at a preset angle of attack, while a stick pusher is frequently used to force the steering column forward when the stalling angle of attack is approached.

Adoption of a T-tail does not necessarily face the designer with disadvantages only.

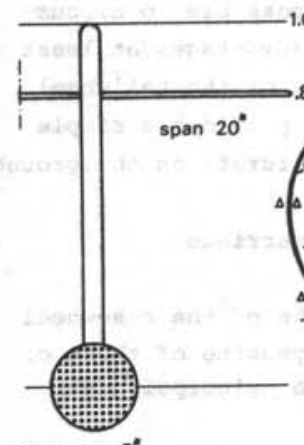

diameter $5^{\circ}$ length $20^{\circ}$

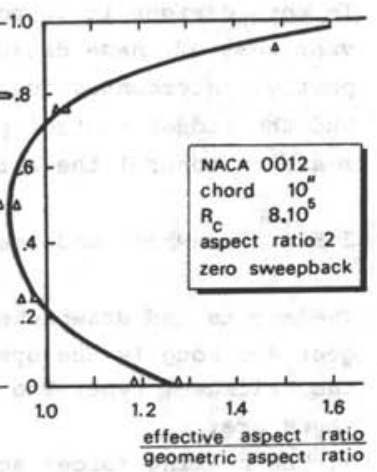

Fig. 2-26. Effective aspect ratio of the fin in combination with a horizontal tailplane (Ref.: NACA TN 2907)
Fig, 2-26 shows that placing one tail surface at the tip of another leads to an increase of about 50 percent in the aerodynamic aspect ratio, so that the stabilizer may increase the lift curve slope of the fin by roughly 15 percent. A similar improvement in the effectiveness of the horizontal stabilizer may be obtained by the use of two fins at the tips. Another point is that the downwash at moderate angles of incidence decreases with increased verticality of the stabilizer, which in the case of a $\mathrm{T}$-tail may sometimes justify reducing the area. The same effect is achieved by placing the stabilizer on top of a sweptback fin, thus increasing its moment arm.

a. Recovery from spins.

In the case of aircraft designed for aerobatics (e.g. trainers), recovery from a spin must be possible. In small aircraft this involves use of the rudder, which must therefore be effective even at very large angles of incidence. It will be seen from Fig. 2-27 that the indicated location of

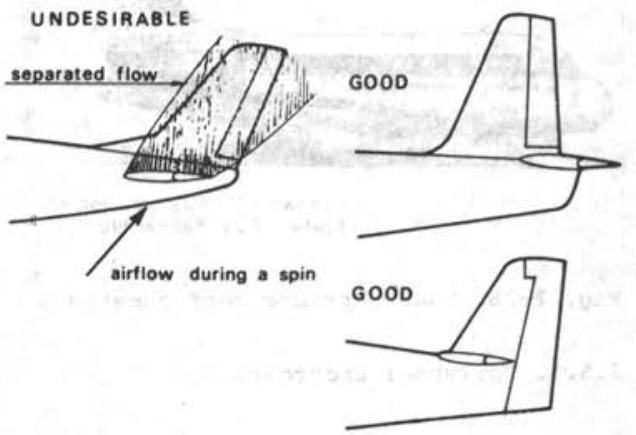

Fig. 2-27. Effectiveness of the rudder during a spin

the stabilizer will cause the greater part of the rudder to be shielded. Some layouts for avoiding this are indicated. V-tails and fins at the tips of the stabilizer are favorable in this respect. 


\subsection{ARRANGEMENT OF THE UNDERCARRIAGE}

Various configurations for the undercarriage have been adopted in the past, but many of them were designed for special purposes. Only three of these need be discussed in the present context. The discussion is further amplified by Fig. $2-28$ and Refs. 2-35 through 2-38.

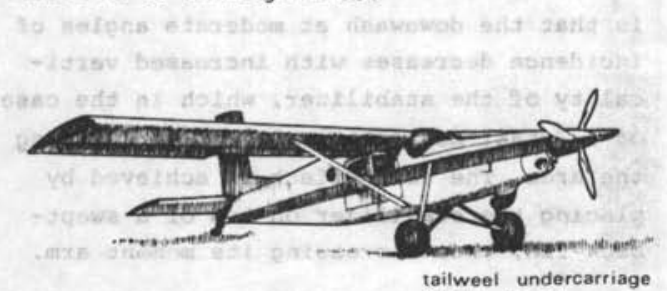
Pilatus Porter

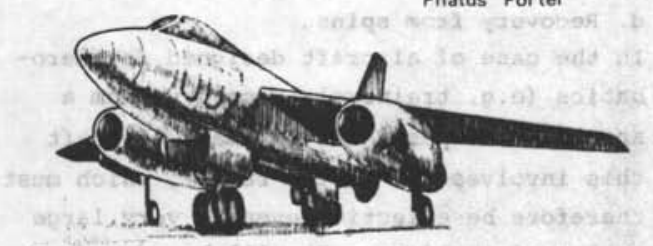

tandem undercarriage

Sud Ouest 4050 Vautour

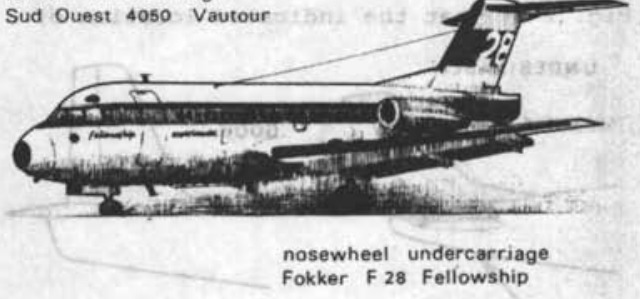

Fig. 2-28. Undercarriage configurations

\subsubsection{Tailwheel undercarriage}

Although this type of undercarriage was in general use during the first three decades of aviation, it must now be regarded as obsolete for most designs. Its advantages should nevertheless be mentioned: diaje of a. The tailwheel is small, light and simple to design.

b. The location of the main gear legs makes attachment to the wings an easy matter.

c. A three-point landing can be carried out by bringing the aircraft to a stalled condition. The aerodynamic drag will provide a retarding force, which is particularly needed when the airfield is unsuitable for full application of brakes (e.g. wet grass). d. When brakes are applied the vertical load on the main gear will increase, thereby reducing the risk of skidaing.

The reason why the tailwheel undercarriage has been almost completely superseded by the nosewheel or tricycle gear is that it also possesses the following drawbacks: a. Violent braking tends to tip the aircraft onto its nose.

b. The braking force acts ahead of the center of gravity and thus has a destabilising effect when the aircraft is moving at an angle of yaw relative to its track. This may cause a ground loop.

c. In a two-point landing a tail-down moment will be created by the impact force on the main landing gear, resulting in an increase in lift which makes the aircraft. bounce.

d. The attitude of the wing makes taxying difficult in a strong wind.

e. In the case of transport aircraft the inclined cabin floor will be uncomfortable for the passengers and inconvenient for loading and unloading.

f. In the tail-down attitude the inclination of the fuselage will limit the pilot's view over the nose of the aircraft. g. During the initial takeoff run drag is high until the tail can be raised.

In some designs it is possible to circumvent some of these disadvantages at least partly. Interconnection of the tailwheel and the rudder control provides a simple means to control the aircraft on the ground.

2.5.2. Nosewheel undercarriage

The merits and drawbacks of the nosewheel gear are roughly the opposite of those of the tallwheel type. The principal advantages are:

a. The braking forces act behind the c.g. and have a stabilizing effect, thus enabling the pilot to make full use of the brakes.

b. With the aircraft on the ground, the 
fuselage and consequently the cabin floor are practically level.

c. The pilot's view is good.

d. The nosewheel is a safeguard against the aircraft turning over and so protects the propeller(s) when used.

e. During the initial part of the takeoff the drag is low.

f. In a two-point landing the main gear creates a nosedown pitching moment.

The steady increase in landing speeds of modern aircraft has accentuated these advantages, so that they carry more weight than the following disadvantages:

a. The nose unit must take 20 to 30 of of the aircraft's weight in a steady braked condition and it is therefore relatively heavy. b. The landing gear will probably have to be fitted at a location where special

structural provisions will be required. In the case of a retractable nosegear on light aircraft it may also prove difficult to find stowage space inside the external contours of the aircraft.

Although there is still a measure of choice during the preliminary design stage, this constitutes one of the most difficult problems to be solved.

Summing up, we may state that the nosewheel undercarriage has gained favor because it greatly facilitates the landing maneuver and enables the brakes to be used more efficiently.

\subsubsection{Tandem undercarriage}

Here the main wheels are arranged practically in the plane of symmetry of the aircraft and the front and rear wheels absorb landing impact forces of the same magnitude. Use of the tandem gear is justified when much emphasis has to be placed on the following advantages:

a. Both main legs are placed at nearly equal distances ahead of and behind the center of gravity, thus locally creating space for payload close to it.

b. The wheels may be retracted inside the fuselage without interrupting the wing structure. The increase if any in fuselage weight will depend on other factors.

Against these we have to set the following disadvantages:

a. Outrigger wheels will be required to stabilize the aircraft on the ground and these may increase the all-up weight by approximately 18 . However, by using two pairs of main legs instead of single ones, a certain amount of track may be obtained, resulting in a reduction of the load on the outriggers (Boeing B-52).

b. The pilot must carefully maintain'the proper touchdown attitude in order to avoid overstraining the gear. Care has also to be taken to limit the angle of bank during the landing to avoid overstraining the outriggers. It may sometimes be possible to 10cate the rear legs close to the center of gravity of the aircraft, and so reduce this disadvantage, but that also means losing the opportunity to have an unobstructed space.

c. A larye tail download is required to rotate the aircraft. It will therefore be desirable to chose the attitude of the aircraft at rest so that it will fly itself off, but this may lead either to an increase in drag during the takeoff roll or to a high liftoff speed.

Generally speaking, the arguments against the tandem gear are of such a nature that its adoption should only be considered when no other solution meets the case.

\subsection{SOME UNCONVENTIONAL AIRCRAFT CONFIGU-} RATIONS

The characteristics of different general arrangements discussed in the preceding sections mainly apply to the classic airplane layout for which a clear distinction can be made between lifting, nonlifting and stabilizing major components. It was assumed that the tailplane was mounted at the rear of the aircraft. The payload is carried inside the fuselage while the fuel is mainly stored in the wings and, if 
necessary, the fuselage. The fuselage is basically designed for optimum transport and rapid loading and unloading, and contributes little to the lift.

A radical departure from the classic layout is the integrated configuration of which the flying wing is the purest representative. The wing is designed to produce lift as well as to contain the entire payload while it also provides stability and control. Less radical is the tailless aircraft which does have a fuselage but no horizontal tail surfaces. A third unusual layout is the tail-first or canard.

When the choice of one of these types is considered, there have to be obvious points which indicate that materially better performance, a considerably lighter structure or improved flying qualities will be achieved. For example, the flying wing layout would most probably be considered only in the case of a sailplane or a long-range aircraft, both of which make full use of the potential improvement in lift drag ratio. Practical experience with this type indicates that a new design will require extensive research before a reliable product can be put on the market. Many examples illustrating this point are known in the history of aviation.

\subsubsection{The flying wing}

During the period around the second World War, several designers in various countries regarded the flying wing as the ideal layout, promising large reductions in drag and structure weight. They included A. Lippisch and the Horten brothers in Germany, J.K. Northrop in the U.S.A. and G.H. Lee in Great Britain. Round about 1965 Lee attempted to draw attention to a flying wing design for a short-haul airliner (Fig. $2-30$, Ref. $2-45$ ).

Since a pure flying wing possesses no fuselage and no horizontal tail surface, it may be possible to achieve a very low zero-lift drag coefficient. This may be of the order of .008 to .011 as compared to .015 to .020 for conventional aircraft. The maximum lift/ drag ratio being inversely proportional to the square root of this figure, a theoretical improvement of about 40 s may be obtained for a given aspect ratio (Fig. 2-29). Assuming similar fuel weights, takeoff weights and cruising speeds, the same improvement applies to the range. Alternatively, this gain may be taken in the form of a reduction in fuel consumption, engine power and takeoff weight for a specified payload and range.

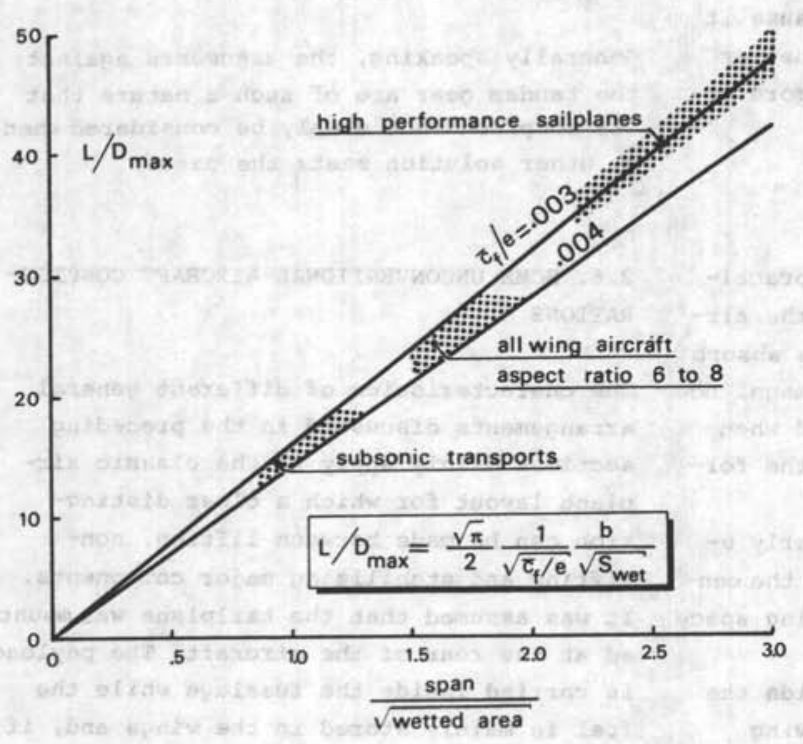

$$
\begin{aligned}
b & =\text { wing span } \\
\bar{c}_{f}= & \text { mean skin friction coeffi- } \\
& \text { cient, based on } S_{\text {wet }} \\
e \quad= & \text { Oswald's span efficiency } \\
& \text { factor } \\
S_{\text {wet }}= & \text { total airplane wetted area } \\
D= & \text { drag } \\
L \quad= & 11 \mathrm{ft}
\end{aligned}
$$

Fig. 2-29. Maximum lift/drag ratio at subsonic speeds 
The empty weight of the flying wing could be less, mainly as a result of the favorable mass distribution within the wing, which reduces the bending moment at the root. Supposing the mass to be distributed along the span in a similar manner to the lift, it would even be theoretically possible to reduce the bending moment to zero in $1-g$ flight. Hence the bending moment will predominate in the landing and the torsion moments in flight and a large part of the wing structure will be designed on the basis of stiffness requirements. By and large, a reduction in structure weight relative to the conventional layout is likely to be possible. To ensure stability in a trimmed condition, the following requirements must be fulfilled:

a. The aerodynamic pitching moment at zero lift and zero control deflection must be positive (i.e. nose-up). This condition can be met by using a special wing section with a bent-up trailing edge or a sweptback wing with washout at the tip, or aileron deflec tion. Both measures tend to increase the vortex-induced drag.

b. The center of gravity must be ahead of the aerodynamic center, but this condition is difficult to fulfill in the case of a straight wing, as it implies that the entire load must be concentrated in the forward part of the wing. With a sweptback wing there is less trouble, first because the aerodynamic center is situated further back and second because more space is available in the plane of symmetry ahead of it.

A high aspect ratio sweptback wing is longitudinally unstable at large angles of incidence (Fig. 2-25b). In the case of a flying wing this instability cannot be corrected by means of a horizontal tailplane and a high aspect ratio is therefore detrimental to stability. Consequently, part of the aerodynamic gain is lost and in the case of Lee's flying wing (Fig. 2-30) its aerodynamic superiority over the conventional layout has largely disappeared.

A low aspect ratio wing enables the design-

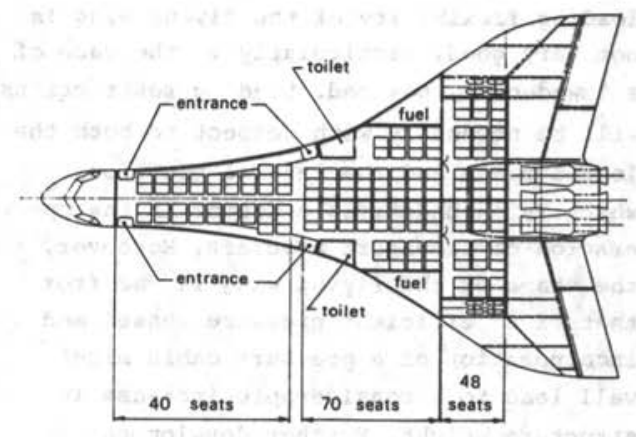

Fig. 2-30. Proposal for a short-range allwing aircraft

er to get a sufficiently thick wing to accommodate the load to be carried. In addition to comparing a conventional aircraft and a flying wing on the basis of equal aspect ratios, we can also do so on that of equal volumes. With a given payload density an optimum design will generally be one in which the space within the external contours is fully utilized. For equal volumes both configurations have roughly the same wetted area and the flying wing can only gain through a greater span and the use of buried engines, creating less drag. Another drawback of the flying wing is that it is incapable of achieving a high maximum lift coefficient. Effective flaps at the trailing edge cause a nose-down pitching moment which cannot be trimmed. A low wing loading is not just a secondary effect here but an absolute lis must. However, high load factors in turbulent air will be the inevitable result; these will be objectionable less from the structural viewpoint than from that of the occupant's comfort and the pilot's workload. The flying wing can be made longitudinally stable but its response to control surfaces deflections and bumps will always be accompanied by a poorly damped phugoid and an oscillatory short period motion, both annoying characteristics to the pilot, although this might be improved by some form of artificial stability augmentation.

It should finally be pointed out that the 
loading flexibility of the flying wing is not very good, particularly in the case of a low-density payload. Loading restrictions will be necessary with respect to both the longitudinal and the lateral position, which is an undesirable factor in the operation of transport aircraft. Moreover, the shape of the flying wing is far from that of an efficient pressure vessel and incorporation of a pressure cabin might well lead to a considerable increase in structure weight. Further development to increase the payload is not feasible as the stretch potential of the flying wing is almost nil.

Summing up the case for the flying wing, we may say that this configuration is potentially capable of reaching a high lift to drag ratio with a low structure weight but the flying and operational characteristics are troublemakers. Since the control function is integrated in the wing, there will be additional trim drag. As a passenger transport aircraft the flying wing does not appear to be a suitable proposition but it may be considered for special purposes, such as sailplanes, long-distance reconnaissance or very large, special-purpose cargo aircraft.

\subsubsection{Tailless aircraft}

Although the flying wing and the tailless aircraft share the characteristic of not possessing a horizontal tailplane, the latter type has a conventional fuselage which carries a large part of the load. The tail of the fuselage is relatively short and carries only a vertical tail surface. The tailless aircraft is generally designed for supersonic speeds and utilises a slender delta wing. The movable parts at the trailing edge act as elevators when deflected in the same direction and as ailerons when deflected in different directions. Like the flying wing the tailless aircraft is unable to carry effective landing flaps and sufficient lift for landing is obtained by choosing a low aspect ratio wing of large area, resulting in large approach angles. Some of the disadvantages mentioned in connection with the flying wing also apply to this type, although they generally weigh less heavily on account of the lower aspect ratio and larger mean chord.

Since the tailless delta is of a much less radical nature than the flying wing, various successful aircraft have been built to this formula and have reached series production. The best known are: Avro Vulcan (almost a flying wing, Fig. 2-12), Convair B-58, Convair F-102, Lockheed YF-12A, Douglas F-4D, Dassault Mirage, SAAB Draken and the BAC-SUD Concorde. These aircraft all operate in the transonic or supersonic speed region, the tailless delta being one of the best configurations for supersonic cruise. Ref. 2-44 gives general information concerning the design of such aircraft.

\subsubsection{Tail-first or canard aircraft}

Canard aircraft have attracted the interest of designers from time to time on account of several particular characteristics. After all, the Wright brothers' aircraft was a canard and it appears an attractive idea to place the longitudinal control surface in front of the wing and out of the wing downwash to where it can never be in its wake. The aircraft's equilibrium is preserved by means of an upward force on the forward plane, which contributes to the lift in a positive sense. In contrast to the conventional layout, this will increase maximum lift and reduce the trim arag, a characteristic which is of particular advantage in the case of high-speed, highly maneuverable aircraft.

A distinction can be made between a longcoupled and a close-coupled canard (Fig. 2-31). In the former category emphasis is laid on the reduction of drag in cruising flight, which is obtained by placing the forward plane far ahead of the wing, thereby reducing the mutual interference. Dynamic stability may be assured by keeping the area of the forward plane below 108 of that of the mainplane. Design problems re- 


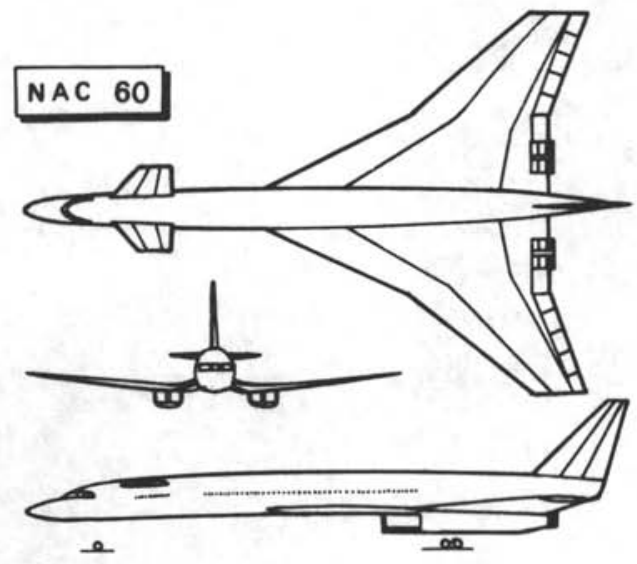

a. Long-coupled canard (North American design for an SST, 1964)

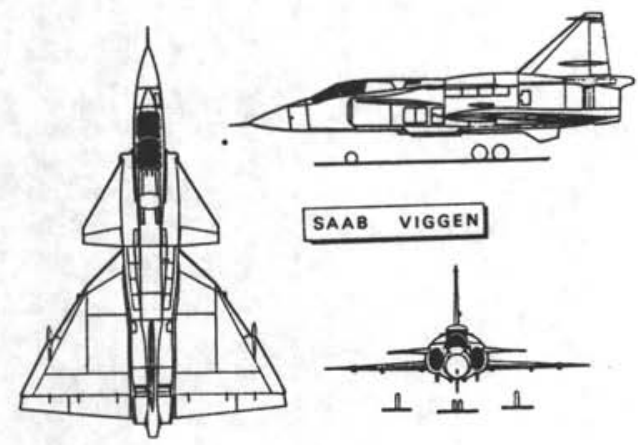

b. Short-coupled canard

Fig. 2-31. Tail-first airplane configurations lating to this configuration are:

a. To achieve an acceptable range for the center of gravity, the forward plane has to be capable of producing a higher maximum lift coefficient than the main wing. Generally speaking, this'can only be achieved when the main wing possesses a low aspect ratio. The forward plane has to be provided with a sophisticated flap system.

b. The trailing vortices of the forward plane affect the flow over the wing and will set up a rolling moment in a sideslip. The vortices may also strike the fin. In the case of the short-coupled canard the mutual interference between the two planes is deliberately used to achieve a high maximum lift. This effect is obtained at large angles of attack on surfaces of low aspect ratio with sharply sweptback leading edges. The large drag which now occurs will only be acceptable for aircraft with sufficiently powerful engines.

In short, the canard layout appears to be suitable for transonic or supersonic and highly maneuverable aircraft, in the latter case if sufficient thrust reserve is available. 



\section{Chapter 3. Fuselage design}

\section{SUMMARY}

This chapter starts with an introductory section dealing with the design requirements, the possibility of achieving an optimum external shape and suggestions for a design procedure.

The second section presents detailed instructions for the design of the passenger cabin, stressing the desirability of achieving efficient arrangement. This is important in order to ensure that, for a given level of passenger comfort, the fuselage makes the maximum possible contribution to the operation of the aircraft.

Some attention is given to freight aircraft where the choice of specified density, the use of containers and pallets, and the loading and unloading provisions are of considerable influence on the design. The final sections contain directives relating to the design of the flight deck and the external shape. 


\subsection{INTRODUCTION}

\subsubsection{Function and design requirements}

The preliminary general arrangement of the aircraft is closely tied up with the fuselage, the main dimensions of which should be laid down in some detail. In fact, the fuselage represents such an important item in the total concept that its design might well be started before the overall configuration is settled.

The main characteristics of the fuselage are as follows:

a. It constitutes the shell containing the payload which must be carried a certain distance at a specified speed. It must permit rapid loading before the flight and rapid unloading after it. The fuselage structure also offers protection against climatic factors (cold, low pressure, a very high wind velocity) and against external noise, provided suitable measures have been taken. b. The fuselage is the most suitable part for housing the cockpit, the most functional location generally being in the nose. c. The fuselage may be regarded as the central structural member to which the other main parts are joined (wings, tail unit and in some cases the engines) on the one hand, and as the link between the payload and the aircraft on the other. In some aircraft a number of these duties are assigned to tail booms.

a. Most of the aircraft systems are generally housed in the fuselage, which sometimes also carries the engines, fuel and/or the retractable undercarriage.

Although the installation of aircraft systems will not be dealt with in this chapter, the reader may refer to Fig. 3-1 which shows how the Auxiliary Power Unit (A.P.U.) and the air-conditioning equipment can be installed in the fuselage.

Many of the requirements laid down in relation to the fuselage limit the designer's range of choice. The 1ist below - though far from complete - enumerates the factors which should be given serious attention as they affect most designs.

a. The drag of the fuselage should be low, since it represents 20 to 408 of the zerolift drag. At a given dynamic pressure the

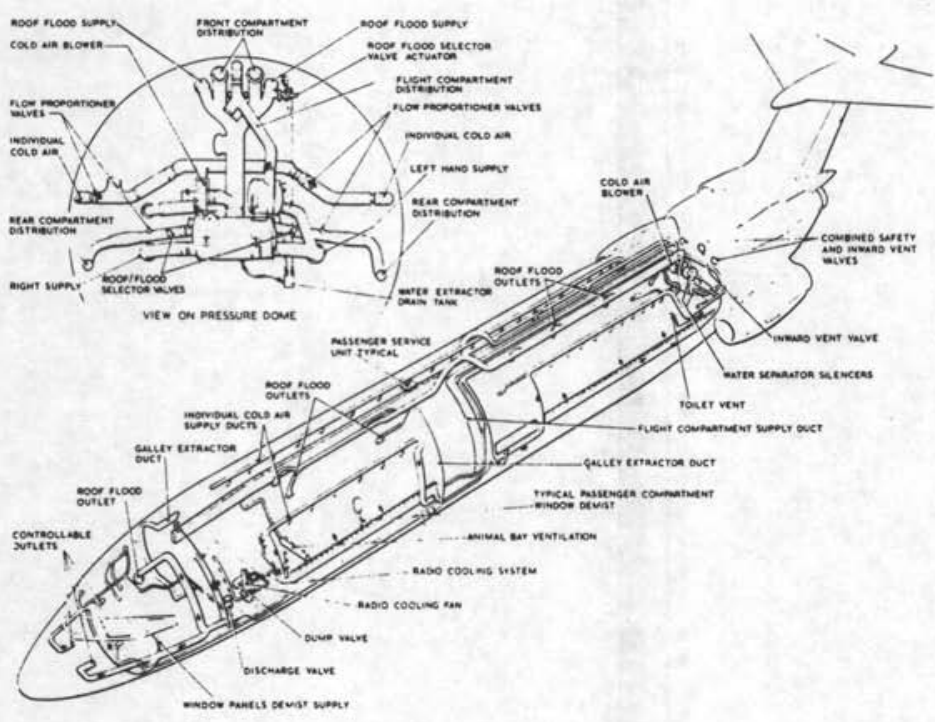

$-$
Fig. 3-1. The air conditioning system of the Hawker Siddeley Trident 
drag is mainly determined by the shape and the wetted area. If we choose a fuselage diameter 108 larger than strictly necessary, the direct result will be a 1.5-38 increase in total drag. This will mean a higher fuel consumption or decreased range, increased takeoff weight, hence another arag increment, etc. This "snowball effect" in weight growth depends on the type of operation for which the aircraft is to be used, and this, in turn, determines how much effort should be made to achieve minimum drag. In the case of a freight aircraft designed for low speeds and modest yearly utilization, such as the Short "Skyvan", a good aerodynamic shape has been sacrificed to easy loading by means of a readily accessible rear loading door.

b. The structure must be sufficiently strong, rigid and light, possess a fixed useful life and be easy to inspect and maintain. In order to avoid fatigue failure of the pressure cabin, a relatively low stress level should be chosen for the skin, e.g. 12,000 p.s.i. $\left(850 \mathrm{~kg} / \mathrm{cm}^{2}\right)$ which is about 308 of the $\sigma_{.2}$ limit for A1 2024-T3. Pressure cabins have a circular cross-section, or a cross-section built up of segments of a circle.

c. Operating costs are influenced by the effect of the fuselage design on fuel consumption and by manufacturing costs. Generally speaking, we gain by keeping the fuselage as small and compact as possible within acceptable limits. On the other hand, it must be remembered that the design and dimensions of the fuselage are decisive factors with regard to the aircraft's earning capacity. In aiming for a compact design, the designer should never go so far that potential customers will reject the aircraft because it lacks comfort as a result of cramped accommodation.

d. The fuselage does not merely serve to carry the empennage, but also affects the tailplane configuration. It will generally contribute a destabilizing effect to the aerodynamic moments in pitch and yaw which is approximately proportional to its volume, while the stabilizing contribution of the tail surfaces is mainly dependent on the length of the fuselage tail.

3.1.2. Drag and optimization of the external shape

Surprising though it' may be, the fuselage of transport afrcraft - a category which is particularly suited for optimization are seldom ideally streamlined in shape. Amongst subsonic aircraft, the Lockheed Constellation and Airspeed Ambassador were the last to be developed and a more recent example, although in a different category, is the HFB Hansa. We may ask to what extent aerodynamic optimization of the fuselage's external shape is both desirable and possible. Apart from the question of optimization in a broader sense, which constantly occupies the designer - what is the best arrangement for the seats, where is the best location for the freight hold, etc. the dominant questions to be answered in the preliminary design stage are:

a. Should the aim be to achieve the ideal streamline shape with minimum drag, or is a cylindrical mid-section to be preferred? b. Should a long, slender shape be adopted or would a short, squat fuselage be better?

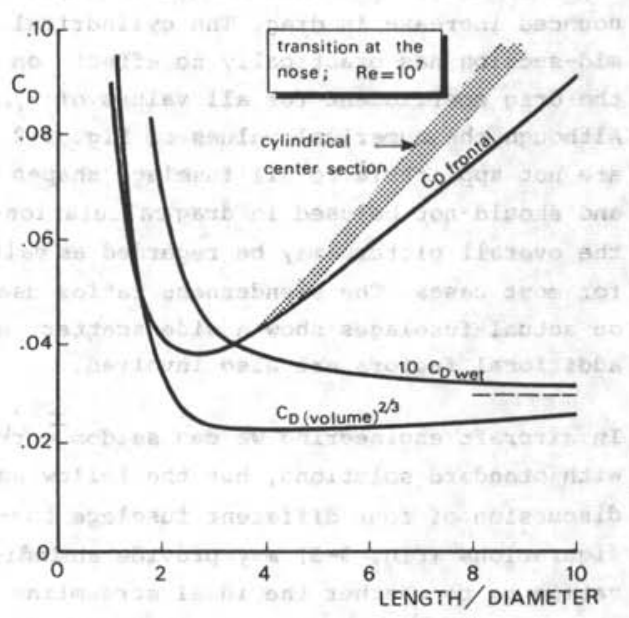

Fig. 3-2. Drag coefficient of streamline bodies of revolution at low speeds (Curves calculated with Section F-3.4.) 
Fig, 3-2 illustrates the influence of the fuselage slenderness ratio $\lambda_{f}$ (length/diameter) on drag, by showing the drag coefficient of a fuselage related to several reference areas. These are: the frontal area, the wetted area and the (volume) ${ }^{2 / 3}$. The figures refer to a fully streamlined body of revolution for slenderness ratios higher than 4. The same coefficients are also given for a fuselage with a cylindrical section. It is assumed here that for these values of $\lambda_{f}$ the drag coefficient, based on the wetted area, is essentially equal to that of the pure streamline body. We may now draw the following conclusions from the figure.

a. The drag coefficient, based on the wetted area, approaches the flat plate value for very slender shapes. When $\lambda_{f}$ is low, there is a considerable pressure drag.

b. The coefficient based on the frontal area shows a pronounced minimum at $\lambda_{f}=2.5$ to 3. When a cylindrical mid-section is used, the drag rises considerably, particularly in the case of high values of $\lambda_{f}$. c. On the basis of (volume) ${ }^{2 / 3}$ the arag coefficient shows a shallow minimum for $\lambda_{f}=4$ to 6 , but rises only slightly for higher values of the slenderness ratio. Slenderness ratios of less than 3 lead to a pronounced increase in drag. The cylindrical mid-section has practically no effect on the arag coefficient for all values of $\lambda_{f}$. Although the numerical values of Fig. 3-2 are not applicable to all fuselage shapes and should not be used in drag calculations, the overall picture may be regarded as valid for most cases. The slenderness ratios used on actual fuselages show a wide scatter as additional factors are also involved.

In aircraft engineering we can seldom work with standard solutions, but the following discussion of four different fuselage configurations (Fig. 3-3) may provide an indication as to whether the ideal streamline shape, or rather the minimum of one of the curves of Fig. 3-2, might have been the designer's aim. In the case of transport aircraft (Fig. 3-3a), the space allotted to the load takes up to between 60 and 70 \% of the fuselage volume. The shape of the fuselage here is derived from an efficient arrangement of passengers or freight.

A cylindrical mid-section is used for the following reasons:

a. Structural design and manufacture are considerably simplified.

b. It is possible to obtain an efficient internal layout with little loss of space. c. The flexibility of the seating arrangement is improved.

d. Further development by increasing the length of the fuselage (stretching)is facilitated.

As the length of the fuselage increases, the areas of the tail surfaces will be reduced, but this is only true up to a point. slender fuselages in large aircraft with a fineness ratio of 12 to 15 ; may well involve stiffness problems. The analytical approach used in Ref. $3-3$ indicates that it is not so much the fuselage drag but more particularly the weight which is the deciding factor where the optimum shape is concerned. This is confirmed by the small variation in the drag coefficient based on (volume) $^{2 / 3}$ with the slenderness ratio (Fig. $3-2)$. For slender fuselages there is also a favorable influence of the Reynolds number on friction drag.

In the case of passenger as well as freight aircraft, the possibilities of varying the shape of the fuselage are limited by practical considerations relating to the load. The fuselage should be designed "from the inside outwards", and the skin should envelop the load in such a way that the wetted area is minimum, thus avoiding breakaway of the airflow as far as possible.

In the case of freight aircraft (Fig. 3-3b), loading and unloading in the longitudinal direction will be the aim. A door in the nose is unsuitable for relatively small freighters as the cockpit would have to be extended to an unreasonable extent on top of the fuselage. Nor is it an easy matter to design a freight door in the tail where stresses are introduced by the tail unit 


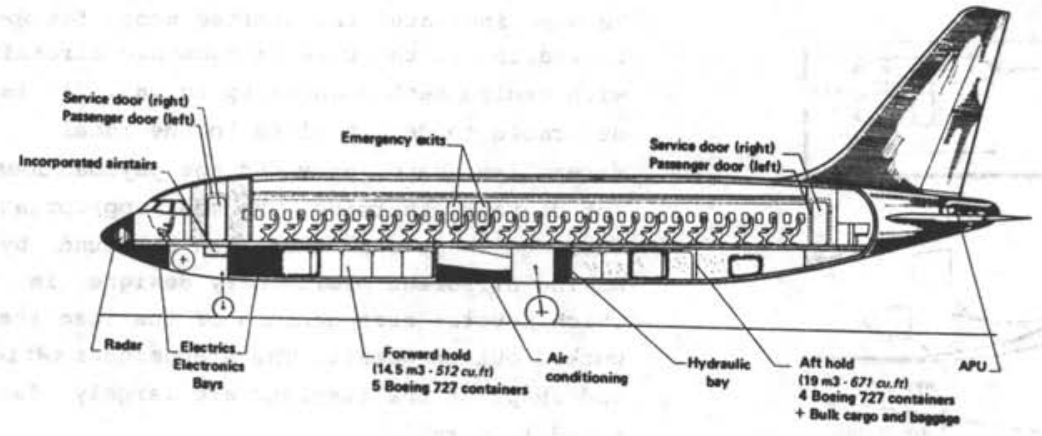

a. Fuselage with relatively large payload volume and efficient internal arrangement (Dassault Mercure)

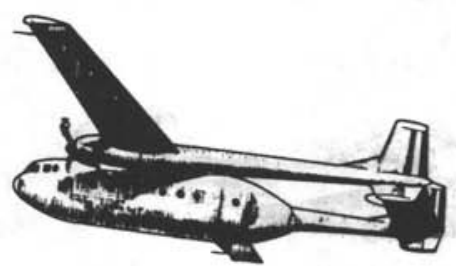

b. Freighter aircraft (Nord 2508) with tail booms and short fuselage afterbody

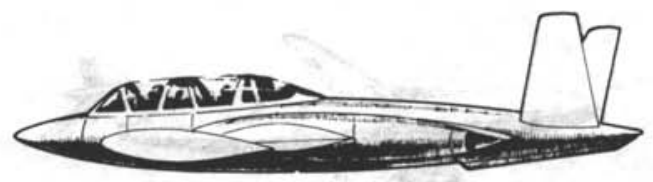

c. Jet trainer (Fouga Magister) with small useful load volume

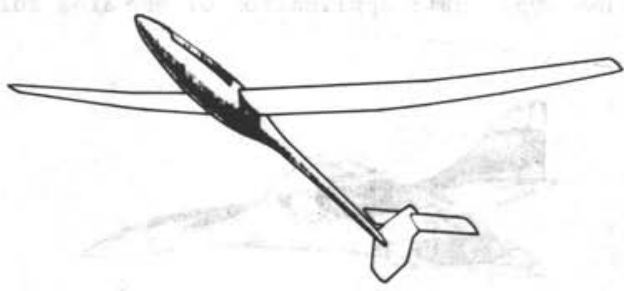

d.Sailplane (Sigma Glider) with fuselage forebody and afterbody designed to different aerodynamic·criteria

Fig. 3-3. Categories of fuselage shapes and by pressurization. In such a case the use of tail booms may be considered (Section 3.3.3), although other solutions are also possible. The length of the fuselage is chosen as short as aerodynamic considerations permit, resulting in a short, squat body. Although this may not approach the optimum streamline shape, it may come close to the minimum drag value for a given frontal area, as shown in Fig. 3-2 (e.g. H.S. Argosy $\lambda_{f}=4.85$; IAI Arava: $\lambda_{f}=3.75$ ) Fig. 3-4 shows that the afterbody slenderness ratio may be quite small without giving $r i s e$ to a large increase in arag. It should, however, be remembered that the flow induced by the wing $11 \mathrm{ft}$ will generally alter this picture in an unfavorable sense. The tail booms, including the engine nacelles of the Arava, have a slenderness ratio of 14 , which shows that these parts have been designed with the object of keeping the wetted area to a minimum.

Trainers and small touring aircraft (Fig. 3-3c) carry a relatively small useful load in relation to the size of the fuselage and a certain measure of freedom is present in choosing the disposition of the occupants, the engine or engines, equipment and possibly the fuel. The length of the fuselage tail will mainly be decided as a function of the operation of the tail surfaces. When two occupants are seated side by side a slenderness ratio of about 6 is a common 


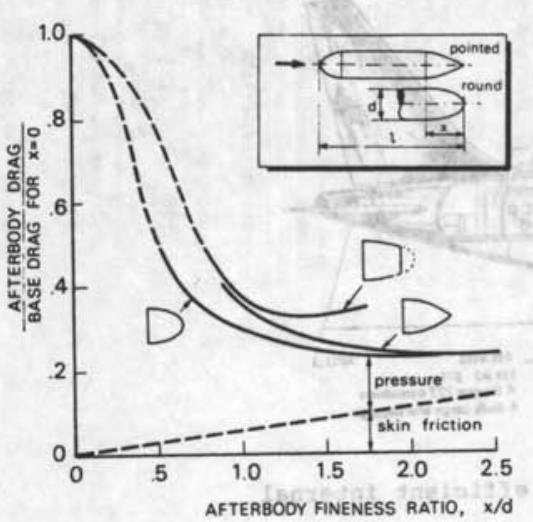

Fig. 3-4. Afterbody drag of a fuselage tail, when added to a cylindrical shape yd bis

value; in a tandem arrangement this figure should be at least 8 . An interesting exception is the Sipa Minijet dating from 1952 (Fig. 3-5a). This had a fineness ratio of 3.3, which obviously showed that the aim was to achieve minimum drag for a given frontal area by using tail booms to carry the empennage.

In the design of sailplanes (Fig. 3-3d) great emphasis is laid on minimum drag. For the forward part of the fuselage, there is a tendency to choose a shape which results in minimum drag for a given crosssectional area of the cockpit. From Fig. 3-2 it can be seen that in such a case $\lambda_{f}=2.5$ to 3 would lead to a low drag figure. Contrary to the examples already discussed, the assumption that the boundary layer is fully turbulent does not apply to gliders. When a favorable shape is chosen, the body shape may be compared to a laminar airfoll section revolved about an axis and the boundary layer will become turbulent some distance behind the nose. In that case the optimum slenderness ratio would be 3 to 4. The length of the tail boom should be decided by the moment arm of the tail surfaces The extremely slender boom will have a small wetted area as well as a low drag coefficient. Attention should also be paid to the required stiffness and weight.
We have indicated the limited scope for optimization in the case of subsonic aircraft with cruise Mach numbers up to .85 . It is desirable to design close to the ideal streamline shape, provided the payload does not dictate otherwise. The most appropriate fuselage shape will therefore be found by making different preliminary designs in which several arrangements of the load are worked out in detail. The slenderness ratio and shape of the fuselage are largely derived factors.

At flying speeds above $M=.85$, fuselages having conventional slenderness ratios will generally create wave draq. As this chapter will not deal with

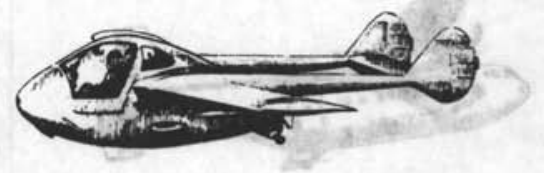

a. Sipa Minijet (1952); fuselage length/diameter $=3.3$

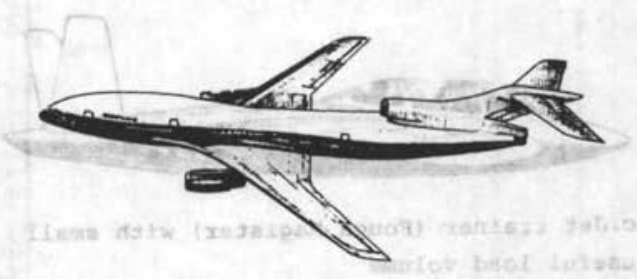

b. Boeing design for a transonic airliner $(M=.95-.98)$; application of the area rule

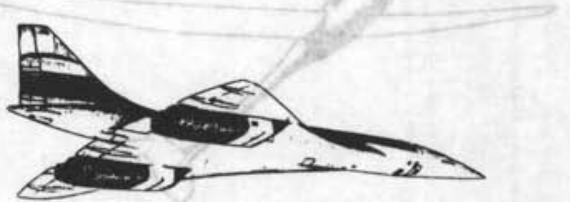

c. Aérospatiale/BAC Concorde supersonic passenger transport; fuselage length/diameter $\sim 20$

Fig. 3-5. Examples of special fuselage shapes 
the aerodynamic problems occurring at transonic and supersonic speeds we shall confine ourselves to discussing a few brief examples. Boeing's design for a transonic transport aircraft (Fig. 3-5b) shows how a satisfactory arag at $\mathrm{M}=.95$ to .98 can be obtained by applying Whitcomb's area rule, combined with supercritical aerofoils. The central portion of the fuselage has a kind of waist, which is unlikely to present many problems with regard to interior planning in the case of aircraft designed for a relatively small payload. Detailed studies, however, will be required to show to what extent a gain in cruising speed of 10 to 158 outweighs the practical drawbacks of this fuselage shape.

Flight at supersonic speeds demands a very slender fuselage in order to keep the wave-drag down to an acceptable value. For the Aérospatiale/BAC Concorde, the Tupolev 144 and the Boeing 2707 SST project, slenderness ratios of about $20,18.5$ and 18 to 25 , respectively were chosen. Incidentally, it is interesting to note that both the concorde (Fig. 3-5c) and the Tu-144 have a cylindrical mid-section.

3.1.3. A design procedure for fuselages with cylindrical mid-section

The following design procedure, derived from Ref. $3-1$, is applicable to fuselages of transport aircraft with a cylindrical mid-section. It applies particularly to the large wide-body category transports. In the case of smaller transports catering for, say, less than 120 passengers, a somewhat simplified procedure may be used by assuming that the passenger cabin is almost entirely cylindrical. Some of the steps to be discussed below are also applicable to cargo aircraft, but in many cases the 10cation of the freight doors will be the deciding factor in the design (Section 3.3.3).

a. Choose the number of seats abreast in a cross-section and/or the dimensions of the cargo load, selecting a section which will most likely determine the diameter of the central part of the fuselage. The remaining principal dimensions of the fuselage will be largely dependent on this parameter. b. Design the shape of the cross-section on the basis of certain predetermined rules (seat dimensions, seat pitch, safety provisions, etc.). If more than 150 to 200 passengers are to be accommodated the use of two aisles should be considered; if more than 500 passengers are carried, it becomes feasible to think of using two decks. Inside contour points are laid down which limit the internal dimensions of the cabin and the freight hold. Around this a contour is drawn as tightly as possible. This will generally be circular in shape, but may be built up of circular segments (double bubble, flattened belly).

c. The external shape may be determined by assuming the minimum thickness of the fuselage walls (skin, formers, upholstering, etc.).

d. It will now be possible to draw in the planview of the fuselage nose and tail, including the cockpit. These are parts which do not possess a cylindrical contour. Since they generally contain fewer passengers per unit of volume than the cylindrical part, they should be kept as short as possible. Some guidelines can be found in section 3.5.1.

e. The capacity of the fuselage nose and tail portions is subtracted from the total payload. For the remainder, essentially the major part of the payload, a prismatic portion is chosen.

$f$. On the basis of the plans of side and front views, the following details may be decided:

- The main dimensions of the cockpit.

- The dimensions and location of doors, windows and emergency exits, spaces required for embarcation and disembarcation or evacuation in case of emergency.

- The tail of the fuselage. This must be planned in such a way that it will not create an unacceptable geometrical limit to rotation in takeoff and landing.

- The indication of spaces for the wing centre-section, attachment frames of the engines, retraction of the landing gear, pressurization and air-conditioning, electrical and electronic systems, etc., insofar as they are present.

- The presence of adequate space below the 
cabin floor for cargo and passenger baggage. If this is not available, consideration may be given to altering the shape of the cross-section (e.g. double bubble), increasing the diameter, raising the cabin floor, changing the arrangement of the seats, etc.

g. If the first assumption does not lead to a satisfactory design, or if various possible solutions are to be evaluated, the procedure must be started again at a. and repeated as often as necessary. It will generally be necessary to check whether alternative planning schemes are possible within the fuselage as it stands, e.g. different seating arrangements in various classes, combiplane*, freighter version . etc.

The work scheduled above will lead to a provisional design when a number of data, such as those regarding the wing root 10cation, are not yet available. In particular the position of the center of gravity may necessitate a fundamental re-arrangement of the payload at a later stage. The preliminary design of the fuselage is made almost entirely on the drawing board since very few analytical studies are available in the existing literature. Provided the outcome of these investigations is limited to the influence of the fuselage shape on empty weight and drag, the results sometimes prove reliable, though they are rarely accurate. Apart from this it is important to consider such factors as the comfort of the occupants, easy access for the passengers, embarcation and servicing and the influence which the shape of the fuselage has on the general configuration of the aircraft. These aspects all have an important bearing on operating economy, although their influence cannot be evaluated quantitatively. Operating economy is of such vital importance that manufacturers generally build one or more mockups of the fuselage before deciding on the

*an airplane with combined transportation of passengers and cargo on the main deck. final design. A separate group is responsible for the details of the interior design, but a useful starting point for this should be established in the preliminary design.

\subsection{THE FUSELAGE OF AIRLINERS AND GENERAL AVIATION AIRCRAFT}

\subsubsection{Importance of comfort and payload density}

Although passengers judge the accommodation in an aircraft according to many yardsticks, there are a number of minimum requirements which should be met. Comfort is mainly dependent on the following factors:

a. The design and arrangement of the seats. This applies particularly to the adjustability of the seat and the legroom available.

b. The general aesthetic impression created by the interior, especially the suggestion of spaciousness within the limited dimen sions of the cabin.

c. The room available for the passenger to move about in the cabin.

d. The climate in the cabin: temperature, moisture, freedom from draughts and the provision of an adjustable supply of air. It is important to keep the rate of pressure variations during climb and descent within acceptable limits.

e. Noise in the cabin, or more specifically Speech Interference Level (SIL) and the presence of resonances.

f. Accelerations, mainly normal to the flight path but also in the direction of roll during braking. Apart from external factors such as the weather, comfort is largely influenced by wing design and the flexibility of the fuselage structure.

g. The aircraft's attitude during climb and descent.

h. The duration of the trip.

1. The number and accessibility of lavatories, washrooms, lounges (if provided) and suchlike amenities.

j. Stewardess service, in-flight entertainment, meal service, snacks, etc. 
The aircraft designer has, at least to some extent, some direct influence with respect to these factors: the space in the fuselage and the influence of wing design on accelerations (wing loading, aspect ratio, angle of sweep). Other factors, such as air-conditioning and pressurization, sound proofing etc. will be dealt with at a later stage of the design. The designer has no direct say in the in-flight services provided for the passengers, although he should allow for the weight and locations of toilet facilities, pantries and cloakrooms.

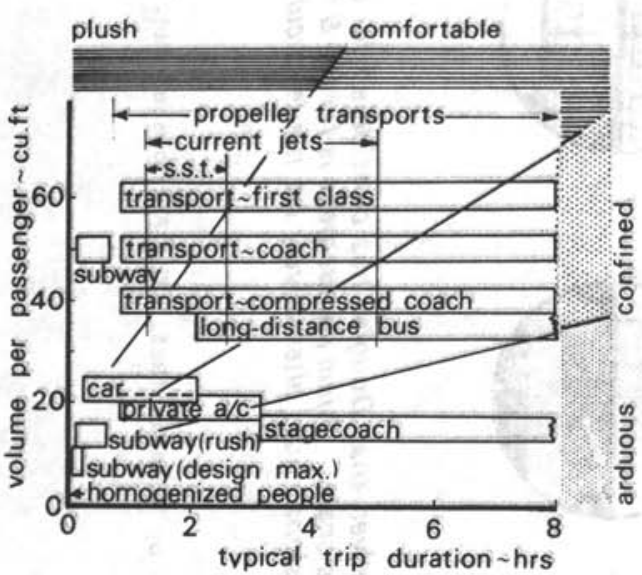

Fig. 3-6. Volume per passenger and trip duration (Ref.: The Architectural Review)

Fig. 3-6 illustratès the space available in an aircraft as compared with a number of other vehicles. It shows the relationship between the available volume per passenger and the average trip duration with respect to comfort. In the case of aircraft, a distinction is made between different classes of fares. Any attempt to increase the level of comfort by choosing a large cabin volume, will result in a growth in fuselage dimensions which will have a considerable effect on operating costs. International agreements and competition generally make it impossible to offset this by increasing fares. On profitable routes where there is keen competition, however, an increase in space will

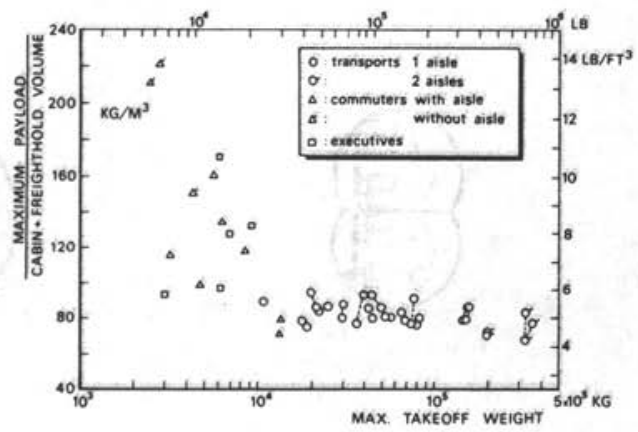

Fig. 3-7. Equivalent payload density

pay dividends. Statistical data (Fig. 3-7) clearly show that for takeoff weights in excess of 25,000 ib $(11,000 \mathrm{~kg})$ approximately, the "density" of the load varies little with the size of the aircraft. For most of the current fleet of transport aircraft the average density is 5.0 to 5.5 $1 \mathrm{~b} / \mathrm{cu}$. ft $\left(80\right.$ to $\left.90 \mathrm{~kg} / \mathrm{m}^{3}\right)$ while it comes to about $4.51 \mathrm{~b} / \mathrm{cu}$. ft $\left(70 \mathrm{~kg} / \mathrm{m}^{3}\right)$ in the case of the new category of large widebody jets (Boeing 747, Douglas DC-10, Lockheed $\mathrm{L}-1011, \mathrm{~A}$ irbus $\mathrm{A}-300 \mathrm{~B}$ ). In the case of small short-haul aircraft with an allup weight of up to $25,000 \mathrm{ib}$ ( $11,000 \mathrm{~kg}$ ) approximately, the design specifications vary so much that the load densities lie between 5.0 to $12.5 \mathrm{lb} / \mathrm{cu}$. ft $(80-200$ $\left.\mathrm{kg} / \mathrm{m}^{3}\right)$ even rising to $14 \mathrm{ib} / \mathrm{cu}$. ft $(220$ $\mathrm{kg} / \mathrm{m}^{3}$ ) in the Britten Norman $\mathrm{BN}-2 \mathrm{~A}$.

In the above an average has been taken for the payload density and no allowance has been made for a distinction between the weights of passengers, luggage and cargo. This factor will be discussed in more detail in section 3.2 .5 , when dealing with the dimensions of the cargo holds.

\subsubsection{Cabin design}

\section{a. Cross-section.}

The first step is to decide upon the number of seats to be placed abreast in a crosssection. Fig. 3-8 shows several cross-sections investigated by the McDonnell Douglas Aircraft Corp., in connection with the de- 

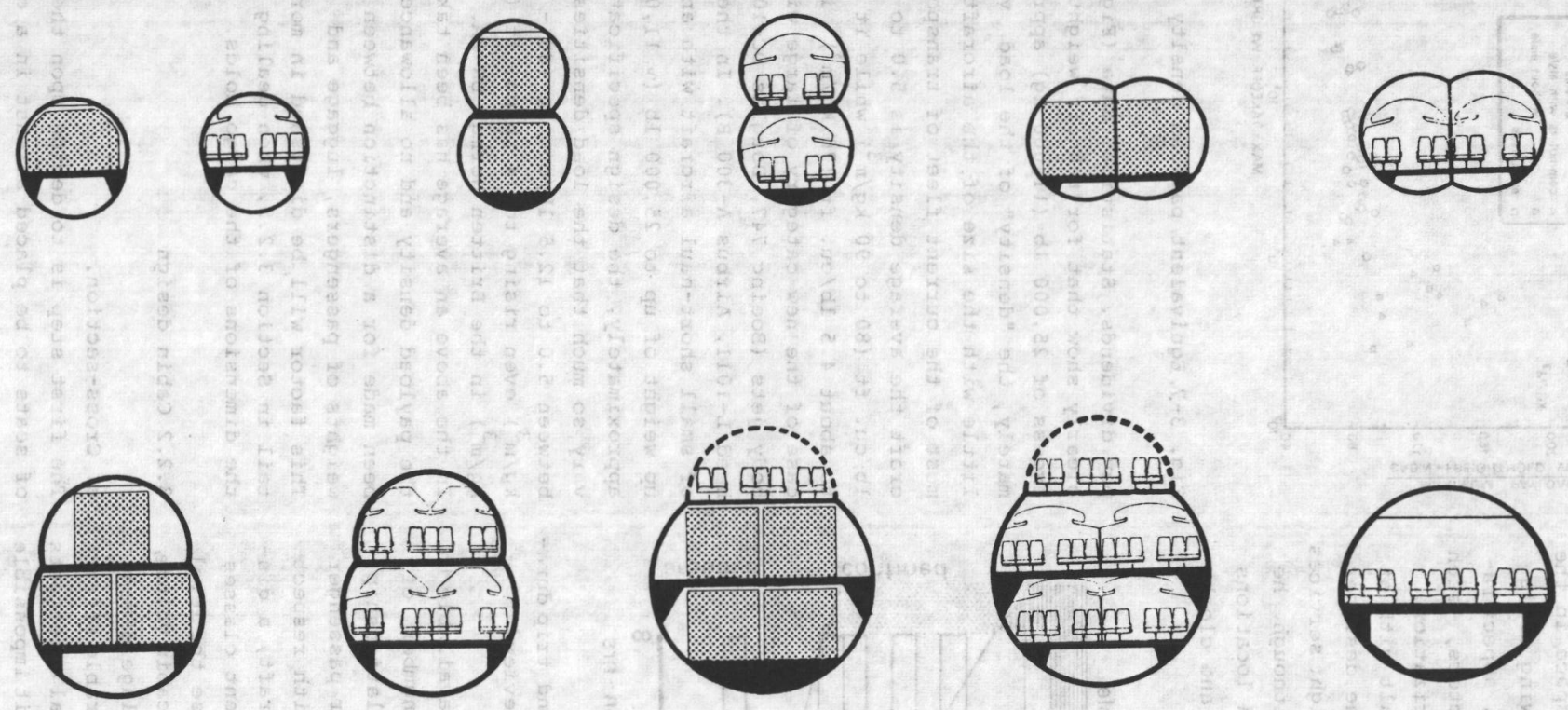

Some of the basic cross-sectional alternatives that Douglas has considered for the very large aircraft project, with a present-day cross section to provide scale. Prime requirement is for the $8 \mathrm{ft} \times \mathbf{8} \mathrm{ft}$ container to be carried efficiently. The bottom-row (middle) designs could alternatively be made circular, dispensing with the upper (dotted) lobe. 
sign of a very large transport which had also to be operated as a freighter. When studying large aircraft of this kind various configurations are projected.

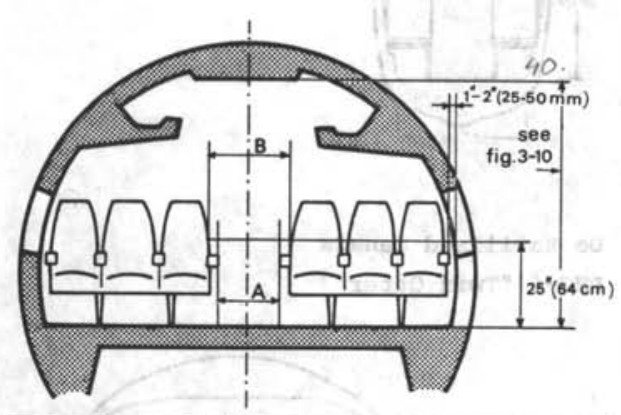

\begin{tabular}{|c|c|c|}
\hline UMBER OF SEA & $A n$ & B minimu \\
\hline 100 & & \\
\hline $\begin{array}{l}11 \text { THROUGH } 19 \\
20 \text { OR MORE }\end{array}$ & $\begin{array}{l}12 \text { in. } \\
15 \text { in. } 3\end{array}$ & $\begin{array}{l}20 \text { in. }, 508 \mathrm{~mm} \\
20 \mathrm{in} ., 508 \mathrm{~mm}\end{array}$ \\
\hline
\end{tabular}

Fig. 3-9. Minimum aisle width for passenger transports (Ref.: FAR 25.815 and BCAR D4-3 par. 5.2 .6 )
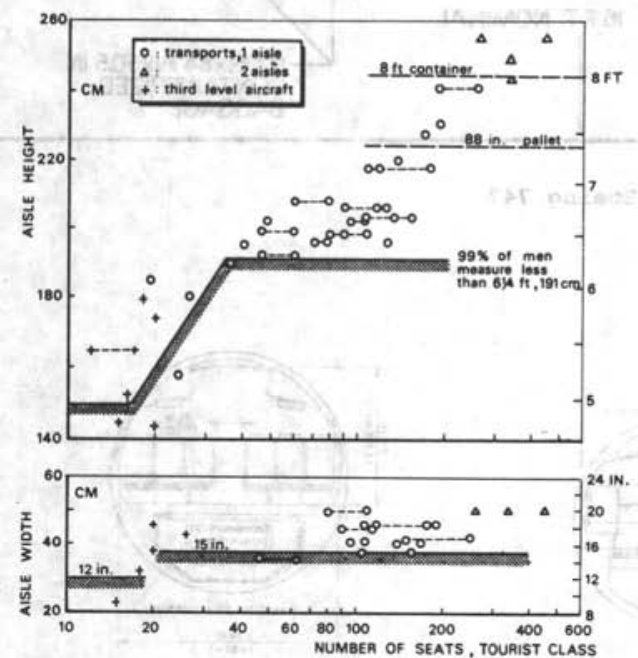

Fig. 3-10. Statistical data on dimensions for the aisle

Details such as the dimensions of the seats (Section 3.2.3) and the aisle(s) (Figs. 3-9 and $3-10)$ are entered in the cross-section.
FAR 25.817 limits the number of seats on each side of the aisle to three, so if more than six passengers are planned in a crosssection the designer will have to allow for two aisles. The minimum permissible width of the aisle in transport aircraft is laid down in FAR 25.817 (Fig. 3-9). All passengers must be able to move their heads freely without touching the cabin walls. This requires a free space with a radius of at least 8 to 10 inches (.20 to $.25 \mathrm{~m})$, measured from the eyes. The cabin wall can then be drawn accordingly. In the case of pressure cabins the cross-section will generally be a circle, or it may be built up from segments with different radii (see examples in Fig. 3-11). If no luggage can be carried under the cabin floor, the fuselage belly contour may be flattened. This is sometimes done in the case of high wing monoplanes (e.g. the Fokker F-27) and has the advantage that the undercarriage may be shortened. The external fuselage diameter can be found from the internal dimensions by adding about 4 inches $(10 \mathrm{~cm})$ for the thickness of the cabin wall. Remarkably enough statistics show that aircraft size has hardly any effect on the wall thickness of pressure cabins, though wide variations do, of course, exist.

In aircraft where the pressure cabin is limited to the cockpit, or in unpressurized fuselages, a rounded rectangular, elliptical or oval cross-section is a common choice. With the internal dimensions specified, this generally leads to a minimum frontal area. In this category of aircraft we may assume the wall thickness to be 28 of the fuselage width plus approximately 1 inch (25 mm).

The result of this design procedure may be compared with Fig. 3-12 which is based on existing aircraft and shows the fuselage width as a function of "total seat width" in the cross-section.

b. Location of seats and dimensions of the cabin.

In order to increase the flexibility of the cabin interior, the seats are mounted on 


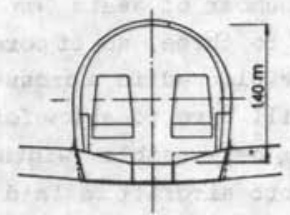

Dassault "Falcon" 10

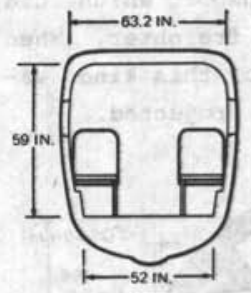

De Havilland Canada DHC-6 "Twin Otter"

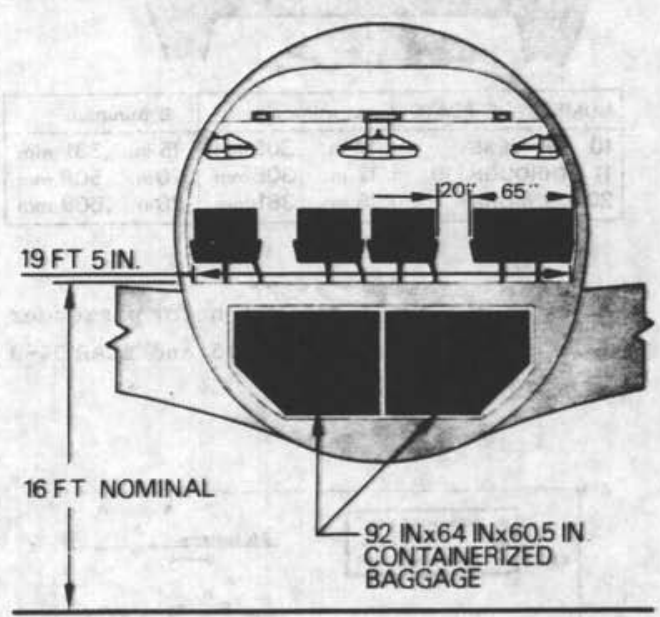

Boeing 747

De Havilland Canada DHC-7

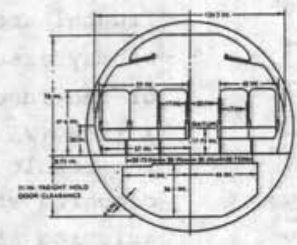

BAC- 111

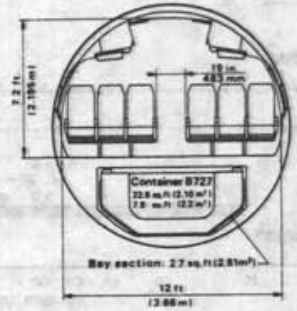

Dassault Bréguet Mercure

VFW-Fokker 614

Fig. 3-11. Examples of some typical fuselage cross-sections of transport aircraft 


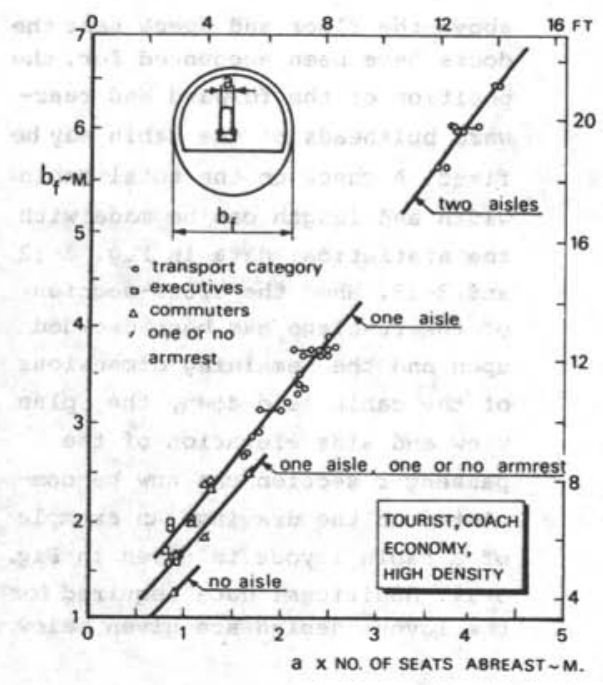

Fig. 3-12. Fuselage width vs. "total seat width"

rails sunk into the floor. Standard seat rails allow the seat pitch (i.e. the longitudinal distance between corresponding points on the two nearest seats in a row) to be adjusted by increments of one inch.

Seat pitch is generally associated with the class of service. At present, however, the terminology in the comfort standards is somewhat confured due to the historical developments in passenger service.

Up to about 1950 the level of seating comfort on normal journeys could be compared to the current first class standard which offers a seat pitch of 40 inches. As a result of pressure by airlines which charged lower fares for special flights ("air (coach"), the tourist class was introduced around 1952. This differed from first class not only in that seats were more closely spaced (pitch about 38 inches), but in some cases the number of seats abreast was also increased. Another factor was the type of meal served. Around 1959 the official tourist class was replaced by the economy class with a typical seat pitch of 34 inches. Nowadays the expressions "tourist", "coach" and "economy" are used without any explanation of the exact distinction between them. There is also "high density" seating with a pitch down to 29 or 30 inches. To avoid this somewhat unattractive term, some companies prefer to use the expression "economy class" in order to avoid adverse passenger reactions.

As a guideline the following figures may be used for typical seat pitch values:

first class : 38 to 40 inches tourist/coach/economy: 34 to 36 inches high density/economy: 30 to 32 inches. Since comfort is not only a matter of seat pitch, the choice of pitch may also be influenced by the trip duration and the width of the seat. Another important point is the maximum number of seats abreast: passengers tend to dislike three seats in a row. This can be improved by choosing a greater.pitch or by using a wider center seat.

If there is a wall or partition in front of a row of seats some space should be left to allow sufficient leg room and permit limited adjustment of the seat back. A distance of about 40 inches between the seat backrest and the partition should be adequate. Extra space is also required at the emergency exits (see Section 3.2.4).

The cabin floor should preferably be kept level in the normal cruise attitude, although this may not always be possible nor necessary in a small aircraft. It is particularly important to have a level floor in large aircraft where food and drinks are served from carts. The floor should be sufficiently strong to support the maximum number of passengers in a high density layout. The permissible floor loading should generally be at least 75 to $1001 \mathrm{~b} / \mathrm{sq}$. $\mathrm{ft}$ $\left(350-500 \mathrm{~kg} / \mathrm{m}^{2}\right)$, but $200 \mathrm{lb} / \mathrm{sq}$. ft $(1000$ $\mathrm{kg} / \mathrm{m}^{2}$ ) is required when the floor has to carry freight. The thickness of a cantilever floor will be about 58 of the fuselage diameter. The total floor area can be found from the fuselage design drawing. Statistically average values are $6.5 \mathrm{sq}$. $\mathrm{ft}$ $\left(.6 \mathrm{~m}^{2}\right)$ per passenger for normal aircraft and $7.5 \mathrm{sq} . \mathrm{ft}\left(.7 \mathrm{~m}^{2}\right)$ for wide-body jets, both in an all-tourist configuration.

When the seats have been arranged and lavatories, pantries, wardrobe(s), freight holds 


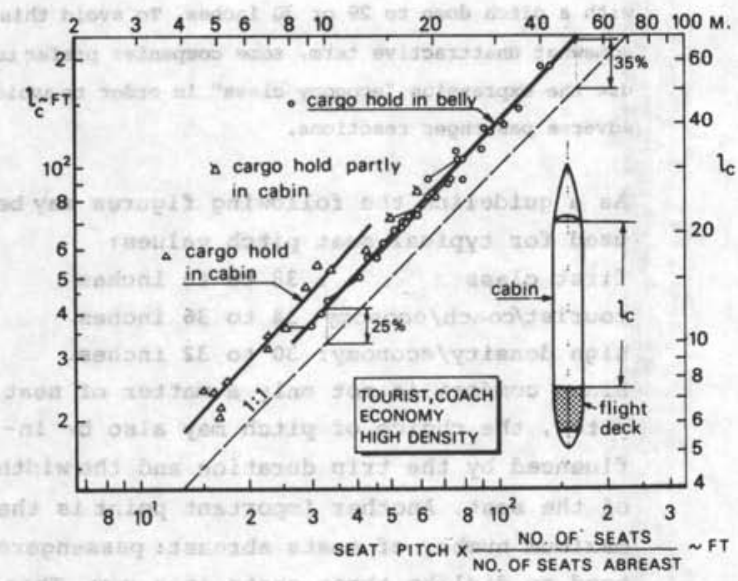

Fig. 3-13. Statistical correlation at the cabin length above the floor and space near the doors have been accounted for, the position of the forward and rearward bulkheads of the cabin may be fixed. A check on the total cabin width and length can be made with the statistical data in Fig. 3-12 and 3-13. When the cross-section of the fuselage has been decided upon and the remaining dimensions of the cabin laid down, the plan view and side elevation of the passenger section can now be completed on the drawing. An example of a cabin layout is given in Fig. 3-14. Additional data required for the layout design are given below.

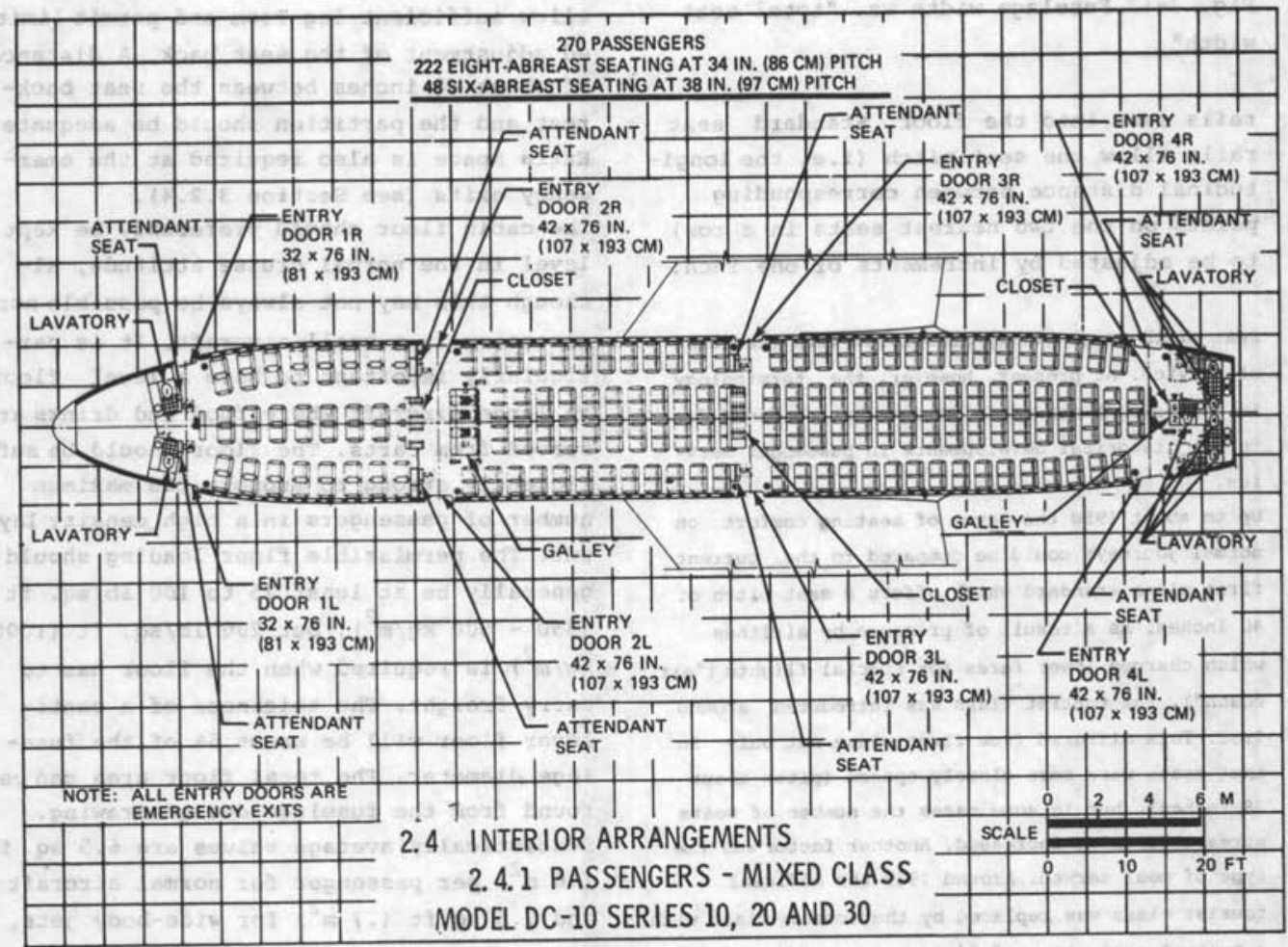

Fig. 3-14. Cabin arrangement of the McDonnell Douglas DC-10 


\subsubsection{Passenger seats}

Although the preliminary design will be based on a certain type of seat, due allowance should be made for the fact that airlines tend to lay down their own specifications for the cabin furnishings. The passenger seats on which data are given in Fig, 3-15 and Table 3-1 are representative
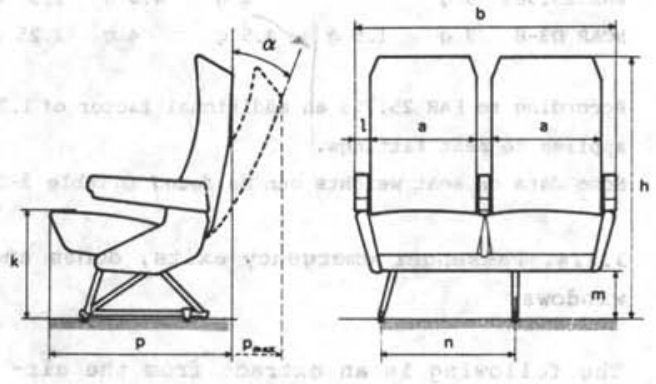

Fig. 3-15. Definitions of seat dimensions

of the types in normal use at the time of writing. They are classified as follows: de luxe type: seat pitch 37-42 inches normal type: seat pitch 32-36 inches economy type: seat pitch 28-31, inches The de luxe type is used in the first class section, while economy-type seats are fitted in the high-density class. Apart from this there is no clear-cut relationship between the comfort classes and the classification given above. Seats may also be listed according to the width between the armrests (distance "a" in Fig. 3-15), as follows:

de luxe: $a=19$ inches

normal : $a=17$ inches

narrow : $a=16$ inches

A seat width of 19 inches $(48 \mathrm{~cm})$ corresponds approximately to first class in most passenger aircraft, but is also used in the tourist class of wide-body jets. In the latter type of aircraft it is also possible to use a layout with normal seat width, thus enabling one more seat to be added to a cross-section. Ref. 3-9 employs a somewhat more detailed distinction according to the standards used in international and domestic

\begin{tabular}{|c|c|c|c|c|}
\hline \multicolumn{2}{|c|}{ 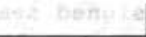 } & \multicolumn{3}{|c|}{ SEAT CLASSIYICATION } \\
\hline smoot* & UNIT & DE LUXE & NoRgaL & Econoery \\
\hline \multirow{4}{*}{$b_{2}^{m}$} & inch & $20(18\}-21)$ & $\left.17(16\}-17 \frac{1}{2}\right)$ & $16.5(16-17)$ \\
\hline & cal & $50(47-53)$ & $43,5(42,5-45)$ & $42(40,5-43,5)$ \\
\hline & inch & $47(46-48 i)$ & $40(39-41)$ & $39(38-40)$ \\
\hline & $\mathrm{cm}$ & $120(117-123)$ & $102(100-105)$ & $99(47-102)$ \\
\hline \multirow{2}{*}{$\mathrm{b}_{3}$} & inch & 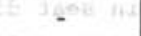 & $60(59-63)$ & 57 \\
\hline & $\mathrm{cm}$ & $-r^{4} b$ & $152(150-160)$ & 145 \\
\hline \multirow[t]{2}{*}{1} & inch & 21 & $2 !$ & 2 \\
\hline & $\mathrm{cm}$ & 7 & 5.5 & 5 \\
\hline \multirow{2}{*}{ h } & inch & $42(41-46)$ & $42(41-44)$ & $39(36-41)$ \\
\hline & $\mathrm{cm}$ & $107(104-112)$ & $107(104-112)$ & $99(92-104)$ \\
\hline \multirow[t]{2}{*}{ k } & inch & 17.77 est? & Q45. 17? & 171 \\
\hline & $\mathrm{cm}$ & 43 & 45 & 45 \\
\hline \multirow[t]{2}{*}{ m } & inch & 71 & 81 & 81 \\
\hline & $\mathrm{cm}$ & 20 & 22 & 22 \\
\hline \multirow{2}{*}{$\mathrm{n}$} & inch & \multirow{2}{*}{\multicolumn{3}{|c|}{$\begin{array}{lll} & 32 & (24-34) \\
\text { usually } & 81 & (61-86)\end{array}$}} \\
\hline & $\mathrm{cm}$ & & & \\
\hline \multirow[t]{3}{*}{$p / p_{\max }$} & inch & $28 / 40$ & $27 / 37$ & $26 / 351$ \\
\hline & $\mathrm{cm}$ & $71 / 102$ & $69 / 95$ & $66 / 90$ \\
\hline & deg & $15 / 45$ & $15 / 38$ & $15 / 38$ \\
\hline
\end{tabular}

*definitions in Fig. 3-15

** the index denotes the number of seats per block

NOTES

1. The data represent normal values and are not standard values. A statistical range is indicated in brackets.

2. In wide-body aircraft, seats are used in the tourist/coach class with $a=19^{\prime \prime}(48 \mathrm{~cm}), b_{3}=66^{\prime \prime}$ $(168 \mathrm{~cm}), \mathrm{h}=43^{\prime \prime}(109 \mathrm{~cm})$. In high-density arrangments the "normal" type seat is used.

3. In third-level aircraft it is customary to install seats with only one or no armrest, with typical dimensions: wiath $16 \zeta^{\prime \prime}(42 \mathrm{~cm}), \mathrm{h}=35^{\prime \prime}$ $(89 \mathrm{~cm}), \mathrm{p}=26^{\prime \prime}(66 \mathrm{~cm})$.

Table 3-1.Seat dimensions (Ref.: Seat manufacturers brochures, Flight Int., July 8 , 1965)

transport. The seats used in long-range aircraft are often finished more luxuriously, with a resultant effect on weight.

The above applies to aircraft used for normal transport routes. The following data apply to other types:

a. Small passenger aircraft for low density traffic: third level, commuters, feederliners. These only make short flights and this 
justifies the use of simply designed seats without armrests. The seat pitch would be 30 - 36 inches $(76-81 \mathrm{~cm})$.

b. Business aircraft are generally furnished in lavish style and there is a considerable variation in seat dimensions. One finds seats with a width of 24 inches $(60$ cm) across the armrests and a pitch of 34 36 inches $(86-92 \mathrm{~cm})$. When the aircraft is used for passenger transport the pitch is reduced to 30 inches $(76 \mathrm{~cm})$ and a bench seat for three passengers may be provided at the rear of the cabin.

c. Private aircraft generally have no aisle between the seats and it is not so much the seat width as the cabin width which matters. Taking the average shoulder width of an occupant as 20 inches $(51 \mathrm{~cm})$ and allowing for 2 inches $(5 \mathrm{~cm})$ clearance on either side, the minimum internal width will be 21 inches $(61 \mathrm{~cm})$ for a single tandem arrangement and 46 inches $(117 \mathrm{~cm})$ for sideby-side seating. A narrower cabin will give most occupants a cramped feeling.

\begin{tabular}{|c|c|c|c|c|}
\hline \multirow{2}{*}{ SEAT CLASSIFICATION } & \multicolumn{2}{|c|}{$\begin{array}{l}\text { MEDIUM/ } \\
\text { LONG HAUL }\end{array}$} & \multicolumn{2}{|c|}{$\begin{array}{l}\text { SHORT } \\
\text { HAUL }\end{array}$} \\
\hline & LB & KG & LB & KG \\
\hline de luxe -single & 47 & 21.3 & 40 & 18.1 \\
\hline -double & 70 & 31.8 & 60 & 27.2 \\
\hline normal -single & 30 & 13.6 & 22 & 10.0 \\
\hline -double & 56 & 25.4 & 42 & 19.0 \\
\hline -triple & 78 & 35.4 & 64 & 29.0 \\
\hline economy -single & 24 & 10.9 & 20 & 9.1 \\
\hline -double & 47 & 21.3 & 39 & 17.7 \\
\hline \multirow{2}{*}{$\begin{array}{r}\text {-triple } \\
\text { commuter-single }\end{array}$} & 66 & 29.9 & 60 & 27.2 \\
\hline & - & - & 17 & $\cdot 7.7$ \\
\hline -double & - & - & 29 & 13.2 \\
\hline lightweight seats & - & - & 14 & 6.4 \\
\hline attendant s' seats & 18 & 8.2 & 14 & 6.4 \\
\hline
\end{tabular}

executive seats,

single - VIP : $50 \mathrm{lb}(22.7 \mathrm{~kg})$

- normal $=40 \mathrm{lb}(18.1 \mathrm{~kg})$

- small a/c: 32 lb $(14.5 \mathrm{~kg})$

ejection seats - trainers: 150 lb (installed)

Table 3-2. Typical seat weights for civil aircraft
Seats and seat mounts are designed for passenger weights of $170 \mathrm{lb}(77 \mathrm{~kg})$. Normal loads must be absorbed during flight and on the ground, but the loads occurring during an emergency constitute a more critical case. These are laid down in the airworthiness regulations as follows:

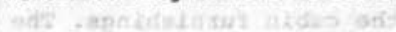

$\begin{array}{lrcrrr} & \text { for- } & \text { rear- } & \text { up- } & \text { down- } & \text { side- } \\ & \text { wards } & \text { wards } & \text { wards } & \text { wards } & \text { ways } \\ \text { FAR } 25.561 & 9 \mathrm{~g} & - & 2 \mathrm{~g} & 4.5 \mathrm{~g} & 1.5 \mathrm{~g} \\ \text { BCAR D3-8 } & 9 \mathrm{~g} & 1.5 \mathrm{~g} & 4.5 \mathrm{~g} & 4 \mathrm{~g} & 2.25 \mathrm{~g}\end{array}$

According to FAR 25.785 an additional factor of 1.33 applies to seat fittings.

Some data on seat weights can be found in Table 3-2.

3.2.4. Passenger emergency exits, doors and windows

The following is an extract from the airworthiness requirements which contain the most relevant points for a preliminary design. This extract has no legal validity and the actual requirements should be consulted for more detailed particulars.

a. Passenger aircraft, to be certificated according to FAR 25 and BCAR section D (see FAR 25.807 through 813 and BCAR Section D para. 5 of chapter D4-3).

Emergency exits are generally grouped in four classes, the particulars of which are given in Fig. 3-16 and Table 3-3. Types I and II are located at cabin floor level, unless type II is placed above the wing. Types III and IV are located above the wing. Apart from these, FAR 25.807 also describes ventral emergency exits and escapes through the tail cone.

The minimum number of exits required is shown in Table 3-4. Aircraft carrying more passengers than are given in this table must comply with special conditions. The FAR requirements demand exits of type $A$, not less than 42 inches wide and 72 inches high $\left(107 \times 183 \mathrm{~cm}^{2}\right)$. The reader should refer to FAR 25.807 for details regarding location, accessibility, escape chutes, etc. for this class of aircraft. 

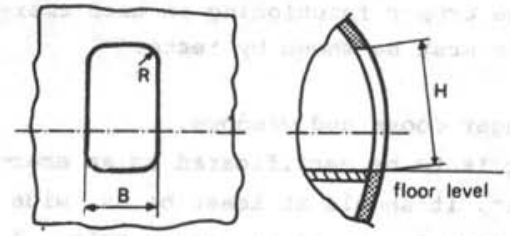

TYPE I AND II
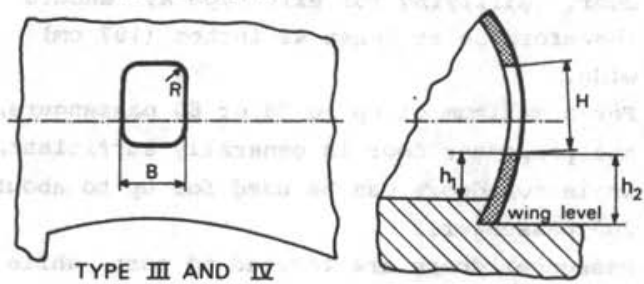

Fig. 3-16.Classification of emergency exits

\begin{tabular}{|c|c|c|c|c|c|c|}
\hline \multirow{2}{*}{\multicolumn{2}{|c|}{$\begin{array}{l}\text { Eeracescr ExIT } \\
\text { CLASsirtCation } \\
\text { AND LOCATton }\end{array}$}} & \multirow{2}{*}{$\begin{array}{c}8 \\
\text { (nin) } \\
\text { inches } \\
(\mathrm{m})\end{array}$} & \multirow{2}{*}{$\begin{array}{c}\text { H } \\
\text { (min) } \\
\text { inches } \\
\text { (m) }\end{array}$} & \multirow{2}{*}{$\begin{array}{c}\text { R } \\
\text { (nax) } \\
\text { inches } \\
\text { (m) }\end{array}$} & \multicolumn{2}{|c|}{$\begin{array}{c}\text { mux. HEIChT } \\
\text { of sTEP }\end{array}$} \\
\hline & & & & & $\begin{array}{l}\text { inside } \\
\left(\begin{array}{l}\text { (h) } \\
\text { inche: } \\
\text { (min) }\end{array}\right.\end{array}$ & $\begin{array}{l}\text { outside } \\
\text { (h2) } \\
\text { inches } \\
\text { (m) }\end{array}$ \\
\hline 1 & nOOR LEVE & $\begin{array}{c}24 \\
(610)\end{array}$ & $\begin{array}{c}48 \\
(1219)\end{array}$ & $\frac{1}{3} \mathrm{~A}$ & - & - \\
\hline \multirow{2}{*}{ II } & nLOR LEVEL. & \multirow{2}{*}{$\begin{array}{c}20 \\
(508)\end{array}$} & \multirow{2}{*}{ (1118) } & \multirow{2}{*}{$\frac{1}{3}=$} & - & - \\
\hline & ABOVE UINC & & & & $\begin{array}{l}10 \\
\text { (254) }\end{array}$ & $\begin{array}{l}17 \\
(432)\end{array}$ \\
\hline III & ABOVE VINC & $\begin{array}{c}20 \\
\text { (508) }\end{array}$ & $\begin{array}{c}36 \\
\text { (915) }\end{array}$ & $\frac{1}{3} B$ & $\begin{array}{l}20 \\
\text { (50s) }\end{array}$ & $\begin{array}{l}27 \\
\text { (686) }\end{array}$ \\
\hline IV & ABOVE UIRE & $\begin{array}{c}19 \\
(483)\end{array}$ & $\begin{array}{c}26 \\
(661)\end{array}$ & $\frac{1}{3} 8$ & (73) & $\begin{array}{l}36 \\
(914)\end{array}$ \\
\hline
\end{tabular}

NOTE

dimensions defined in Fig. $3-16$, according to FAR 25.807 .

Table 3-3. Classification of emergency exits

When it is impossible to place exits above the wing, as in the case of high wing aircraft, an exit having at least the dimensions of type III should replace each exit III and IV as shown in Table 3-4.

When these requirements are difficult to fulfil, the exceptions listed in FAR 25 should be consulted. Additional requirements apply to aircraft which are to be certificated for making emergency descents on water (FAR 25.807 para. d). There should be unobstructed access to emergency exits;

\begin{tabular}{|c|c|c|c|c|}
\hline \multirow{2}{*}{$\begin{array}{c}\text { SEATIMG CAPACITY } \\
\text { (EXCL. CABIN STAFF) }\end{array}$} & \multicolumn{4}{|c|}{$\begin{array}{c}\text { NUMBER OF EXITS REQUIRED } \\
\text { ON EACH SIDE OF THE FUSELAGE }\end{array}$} \\
\cline { 2 - 5 } & TYPE I & TYPE II & TYPE III & TYPE TV \\
\hline 1 through 10 & - & - & - & 1 \\
11 through 19 & - & - & 1 & - \\
20 through 39 & - & 1 & - & 1 \\
40 through 59 & 1 & - & - & 1 \\
60 through 79 & 1 & - & 1 & - \\
80 through 109 & 1 & - & 1 & $1+$ \\
110 through 139 & 2 & - & 1 & - \\
140 through 179 & 2 & - & 2 & - \\
\hline
\end{tabular}

NOTE

1. BCAR requirements are slightly different for 1-10 passengers; for this case an temergency exit of type III is required on both sides of the fuselage. Two exits of type $I$ and II are required for a seating capacity of 180 up to 219 .

2. The relevant rules should be consulted where passenger seats exceed this number and for special regulations.

3. Exits need not be at locations diametrically opposite each other. They should be located in accordance with the passenger seating distribution. 4. Two exits of type IV may be used instead of each type III exit.

5. The classification of emergency exits is defined in Table 3-3 and Fig. 3-16.

Table 3-4. Minimum number of passenger emergency exits according to the FAR Part 25 requirements

the width is laid down in the BCAR requirements as 20 inches $(51 \mathrm{~cm})$ for types $I$ and II.

Reference 3-4 recommends the following standards :

Type I : 36 inches.

Type II : 20 inches.

TYpe III and IV: 18 inches.

These distances determine the seat pitch next to emergency exits and will therefore affect the total cabin length. 
b. Aircraft to be certificated according to FAR Part 23 .

The following is quoted from FAR Part 23.807

"Number and location: Emergency exits must be located to allow escape in any probable crash attitude. The airplane must have at least the following emergency exits:

(1) For all airplanes, except airplanes with all engines mounted on the approximate centerline of the fuselage that have a seating capacity of five or less, at least one emergency exit on the opposite side of the cabin from the main door specified in para. 23.783 .

(2) Reserved.

(3) If the pilot compartment is separated from the cabin by a door that is 1ikely to block the pilot's escape in a minor crash, there must be an exit in the pilot's compartment. The number of exits required by subparagraph (1) of this paragraph must then be separately determined for the passenger compartment, using the seating capacity of that compartment.

Type and operation: Emergency exits must. be movable windows, panels, or external doors, that provide a clear and unobstructed opening large enough to admit a 19-by-26 inch $(483 \times 660 \mathrm{~mm})$ ellipse. In addition, each emergency exit must

(1) be readily accessible, requiring no exceptional agility to be used in emergencies,

(2) have a method of opening that is simple and obvious,

(3) be arranged and marked for easy location and operation, even in darkness,

(4) have reasonable provisions against jamming by fuselage deformation, and

(5) in the case of acrobatic category airplanes, allow each occupant to bail out quickly with parachutes at any speed between $\mathrm{v}_{\mathrm{S}_{\mathrm{O}}^{*}}$ and $\mathrm{v}_{\mathrm{D}}$.

* $\mathrm{V}_{\mathrm{S}_{\mathrm{O}}}=$ stalling speed, flaps down; $b$ asedis

$\mathrm{V}_{\mathrm{D}}=$ design diving speed.
Tests: The proper functioning of each emergency exit must be shown by tests."

c. Passenger doors and windows.

If a door is to be certificated as an emergency exit, it should at least be as wide as the relevant type of emergency exit. A door, qualifying for exit type A, should therefore be at least 42 inches $(107 \mathrm{~cm})$ wide.

For a maximum of up to 70 or 80 passengers, one passenger door is generally sufficient, while two doors can be used for up to about 200 passengers.

Passenger doors are located to port while service doors are fitted to starboard. Widebody jets are an exception as these can be boarded from both sides.

Doors should preferably be $6 \mathrm{ft}$ high and $3 \mathrm{ft}$ wide $\left(1.80 \times .90 \mathrm{~m}^{2}\right)$ but these dimensions are difficult to achieve in the case of smaller aircraft.

The window pitch is not always decided by the seat pitch, but frequently by the optimum distance between the fuselage formers. An average figure is 20 inches $(.50 \mathrm{~m}$ ) for the former and window pitch. In pressure cabins the windows are circular, rectangular with rounded corners, elliptical or oval in shape. The top of the window is roughly at the passenger's eye level. In the case of smaller aircraft the main frames will have to be installed at the wing attachment points and these will influence the location of doors and windows Access to passenger doors, service doors and panels should be unobstructed fromboth the outside and the inside. For example, the space between the wing and the fuselage-mounted engines should permit sufficient freedom for maneuvring food carts and cargo loaders.

\subsubsection{Cargo holds}

The design specification does not always stipulate the amount of cargo to be carried Airlines may have radically different requirements, depending on the kind of traffic they carry, so the best way is to con- 
duct an inquiry among potential customers. If there is no time for this, the method given below may provide a quick answer. This is based on the following assumptions: 1. Volume-limited and structure-limited payload are equal.

2. Weight of a passenger is $170 \mathrm{lb}(77 \mathrm{~kg})$, see BCAR Section D, ch. D 3.1 para. 3.4 .

3. Luggage weight is $351 \mathrm{~b}(16 \mathrm{~kg})$ per passenger on short-haul flights and 40 lb (18 $\mathrm{kg}$ ) on long-haul flights.

4. Loading efficiency is $85 \%$, i.e. $15 \%$ of the space is lost.

5. Average density of cargo is $10 \mathrm{lb} / \mathrm{cu} . \mathrm{ft}$ $\left(160 \mathrm{~kg} / \mathrm{m}^{3}\right)$ and of luggage $12.51 \mathrm{~b} / \mathrm{cu} . \mathrm{ft}$ $\left(200 \mathrm{~kg} / \mathrm{m}^{3}\right)$.

Ignoring storage losses at the freight doors, the following expression can be derived:

Freight hold volume $=.118 \mathrm{cu} . \mathrm{ft}$ per $1 \mathrm{~b}$ $\left(.0074 \mathrm{~m}^{3}\right.$ per $\left.\mathrm{kg}\right)$ of max. payload minus 20.8 cu.ft $\left(.59 \mathrm{~m}^{3}\right)$ per passenger.

Alternatively, this expression can be used to obtain the volume-limited payload ${ }^{*}$ :

Max. payload $=8.5 \mathrm{lb}$ per cu.ft $(136 \mathrm{~kg}$ per $\mathrm{m}^{3}$ ) of freight hold volume plus $177 \mathrm{lb}(80 \mathrm{~kg})$ per passenger.

This yardstick may be used in the preliminary design stage but it cannot be applied to all aircraft. There is sometimes a space limit to the load, while the structure is strong enough to carry greater loading weights. In other cases, a limit is set by the difference between the Maximum Zero Fuel Weight and the Operational Empty Weight. This is generally an undesirable condition on civil aircraft. Belly freight holds should have an effective height of at least 20 inches $(50 \mathrm{~cm})$ but a height of more than 35 inches $(90 \mathrm{~cm})$ is to be preferred, particularly when it is necessary for staff to work in the hold. This condition cannot be

*This term is explained in Section 8.2.2. satisfied for fuselage diameters of less than about $10 \mathrm{ft}(3 \mathrm{~m})$, so either a doublebubble fuselage cross-section will have to be adopted or the freight holds must be located above the floor.

To control the center of gravity travel it might be of advantage to keep the underfloor holds both ahead and behind the wing. Small twin-engined aircraft sometimes have a luggage hold in the (fuselage) nose ahead of the cockpit or in the engine nacelles. In pressurized airliners the freight and baggage holds are pressurized as well, though the temperature may be lower than in the cabin. They must be easily accessible by means of hatches or be located close to a door. When determining the volume required, allowance should be made for possible loss of space near the hatches.

In the case of very large aircraft, it is recommended that freight holds be designed to take the universal containers used in other wide-body jets; the relevant dimensions are given in Fig. 3-20.

\subsubsection{Services}

Although the airliners belonging to the IATA have come to certain arrangements regarding the service to be offered to the passenger, individual companies have varying ideas about this. Before starting the design of the cabin, the outcome of a separate study devoted to this subject should be obtained and be incorporated in the specification. An example of one of these studies is provided by Ref. 3-9.

a. Pantries, lavatories and wardrobes. The number and dimensions of the above facilities are shown in Table $3-5$. The data are derived from standard type specifications and do not necessarily apply to individual users. Some flexibility in layout design should be incorporated.

Location: For aesthetic reasons toilets should preferably be located so that they are not directly visible from the pantry. They should be easily accessible, and when 


\begin{tabular}{|c|c|c|c|c|c|c|c|c|c|}
\hline & & & & galleys & & toilets & & & wardrobes \\
\hline $\begin{array}{l}\text { Aircraft type: } \\
\text { Airats } \\
\end{array}$ & 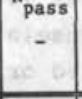 & N M & 皆 & $1 \times b$ (inch) & 占 & $1 \times b($ inch $)$ & $\begin{array}{c}\text { pass } \\
\text { toilet }\end{array}$ & 崖 & $1 \times b$ (incl \\
\hline Aérospatiale N-262 Frégate & 29 & 400 & 1 & $23 \times 20$ & 1 & $41 \times 28$ & 29 & 1 & $40 \times 24$ \\
\hline Grumman Gulf stream I & 19 & 2100 & 1 & $34 \times 25$ & 1 & $67 \times 37$ & 19 & 1 & $36 \times 32$ \\
\hline Hawker Siddeley 748 srs 200 & 44 & 1000 & 1 & $37 \times 14$ & 1 & $53 \times 35$ & 44 & - & \\
\hline Fokker-VFW F.27 Friendship srs 200 & 48 & 1100 & 1 & $43 \times 35$ & 1 & $47 \times 46$ & 48 & 1 & $31 \times 16$ \\
\hline De Havilland Canada DHC-7 & 44 & 800 & 1 & $26 \times 24$ & 1 & $46 \times 30$ & 44 & 1 & $26 \times 24$ \\
\hline Lockheed L-188 Electra & 95 & 2300 & 2 & $46 \times 26$ & 4 & $46 \times 41$ & 24 & 2 & $46 \times 34$ \\
\hline HFB 320 Hansajet & 7 & 1000 & 1 & $24 \times 24$ & 1 & $30 \times 26$ & 7 & 1 & $24 \times 15$ \\
\hline Hawker Siddeley HS-125 srs 400 & 8 & 1450 & - & & 1 & $35 \times 28$ & 8 & 1 & $24 \times 12$ \\
\hline Dassault Falcon 20.F & 10 & 1500 & 1 & $27 \times 18$ & 1 & $44 \times 30$ & 10 & 1 & $51 \times 25$ \\
\hline Dassault Falcon $30 /$ Mystère 40 & 34 & 750 & - & & 1 & $41 \times 31$ & 34 & - & \\
\hline VFW-Fokker 614 & 40 & 700 & 1 & $35 \times 28$ & 1 & $55 \times 32$ & 40 & & $65 \times 40$ \\
\hline Fokker VFW-F. 28 Mk 1000 & 60 & 1025 & 1 & $44 \times 25$ & 1 & $58 \times 25$ & 60 & 1 & $25 \times 21$ \\
\hline$B A C-111$ srs $200 / 400$ & 74 & 900 & 2 & $49 \times 22$ & 2 & $65 \times 35$ & 37 & 1 & $49 \times 22$ \\
\hline Mc Donnell Douglas DC-9 srs $10 / 20$ & 80 & 1100 & 1 & $48 \times 33$ & 2 & $48 \times 48$ & 40 & 2 & $48 \times 21$ \\
\hline Boeing 737 srs 200 & 115 & 1800 & 1 & $55 \times 43$ & 2 & $43 \times 34$ & 58 & 1 & $55 \times 43$ \\
\hline Aérospatiale Caravelle 12 & 118 & 1000 & 1 & $51 \times 43$ & 2 & $55 \times 43$ & 59 & 2 & $24 \times 17$ \\
\hline Dassault Mercure & 140 & 800 & - & & 2 & $47 \times 34$ & 70 & 2 & $49 \times 16$ \\
\hline Boeing 727 series 200 & 163 & 1150 & 2 & $51 \times 32$ & 3 & $43 \times 39$ & 55 & - & \\
\hline Europlane & 191 & 1400 & 3 & $42 \times 42$ & 4 & $42 \times 42$ & 48 & 1 & $52 \times 26$ \\
\hline $\mathrm{A}-300 \mathrm{~B} / 4$ & 295 & 1600 & 3 & -4 & 5 & $59 \times 35$ & 59 & - & \\
\hline Lockheed L-1011 & 330 & 2700 & 1 & $20 \times 13.5 \mathrm{ft}^{2}$ & 7 & $45 \times 36$ & 47 & - & head racks \\
\hline Mc Donnell Douglas DC-10 & 380 & 3000 & 1 & $\begin{array}{l}\text { under troor } \\
\text { galley }\end{array}$ & 9 & $40 \times 40$ & 42 & 2 & $6.3 \times 1.8 \mathrm{ft}^{2}$ \\
\hline$B A C-V C-10$ & 135 & 4200 & 1 & $49 \times 32$ & 5 & $47 \times 41$ & 27 & 2 & $42 \times 24$ \\
\hline Boeing 707-320 B & 189 & 5000 & 2 & $79 \times 47$ & 4 & $40 \times 37$ & 48 & 1 & $79 \times 43$ \\
\hline Mc Donnell Douglas DC- 8 srs 63 & 251 & 4000 & 2 & $48 \times 34$ & 5 & $42 \times 42$ & 50 & 4 & $34 \times 20$ \\
\hline Boeing 747 & 490 & 5000 & 4 & $6.6 \times 2.1 \mathrm{ft}^{2}$ & 12 & $40 \times 40$ & 41 & 2 & $5.9 \times 2.3 \mathrm{ft}^{2}$ \\
\hline
\end{tabular}

NOTES:

$\mathrm{N}_{\text {pass }}=$ maximum number of passengers, tourist class, approx. $34^{n}$ seat pitch Range at about $\mathrm{N}_{\text {pass }} \times 205 \mathrm{lb}$ payload and including normal fuel reserves Dimensions are approximate average length $x$ width; toilets are not always rectangular.

Table 3-5.: Number and dimensions of galleys, lavatories and wardrobes of some airliners

the cabin arrangement includes a separate first-class section it is desirable to provide toilet facilities in that part too. Toilets are generally not movable since they form an integral part of the aircraft structure and require special provisions. Only limited flexibility is available with regard to the location and arrangement of galleys. It is advisable to locate these facilities at the forward and/or rearward end of the cabin, thus allowing for different cabin layouts. It is permitted to locate them in the plane of the propellers. In the case of wide-body jets, space may be saved by placing the pantry below the floor. When servicing, loading and unloading the aircraft between flights, the following operations are performed:

- replenish potable water,

- remove left-over food, drinks and waste 


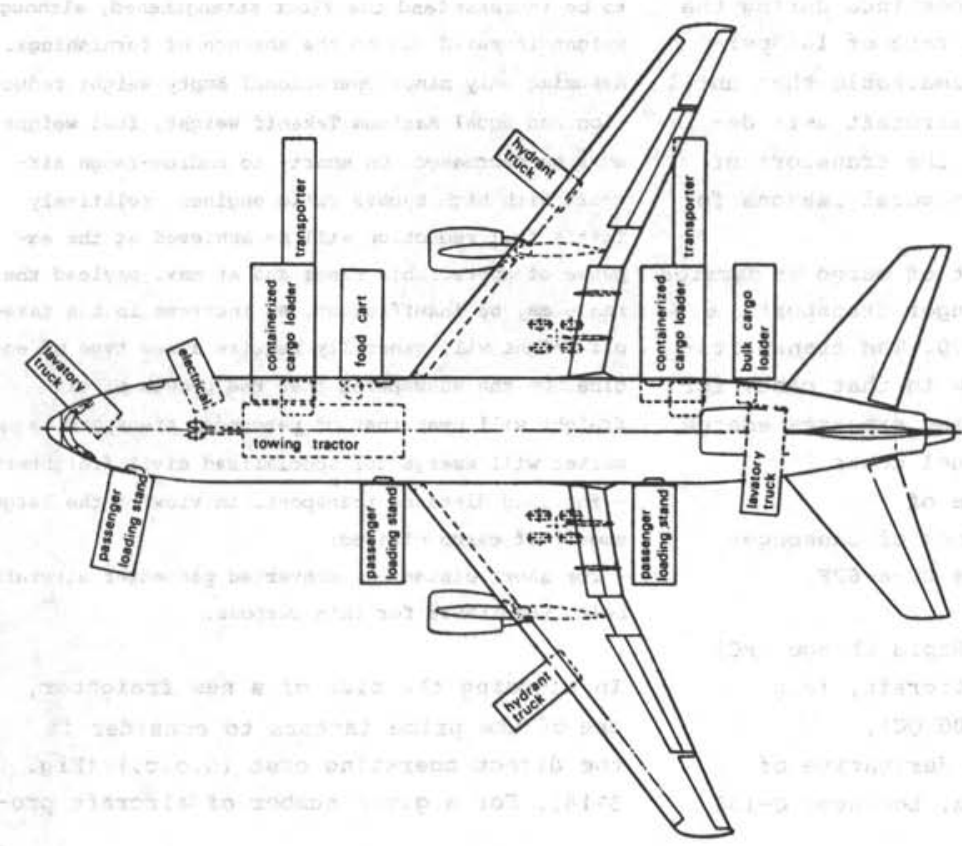

Fig. 3-17. Ground handiling of the Lockheed L-1011 Tristar

from the pantries and take on fres supplies,

- service toilets,

- clean cabin.

- unload and load passengers freight and luggage.

The trucks, stairs, carts, loaders, etc. needed for these operations should not obstruct each other, which means that careful planning of door locations and service points is required, particularly in the case of large aircraft. An example is given in Fig. 3-17.

\section{b. Cabin systems.}

Among the facilities which should be available in every passenger aircraft are a public address system, lighting, cooling air (operated by the passenger), hatracks over the seats and space for hand luggage. A supplementary oxygen supply is compulsory when cruising height is above $25,000 \mathrm{ft}$ (7620 meters), cf. FAR 91.32.

c. Cabin staff.

The minimum number of flight attendants is specified by the airworthiness regulations (e.g. FAR 91.215); the actual number of cabin staff is fixed by the company. The following data from Ref. 3-9 give the average number of passengers per member of cabin staff:

$\begin{array}{lccc} & \begin{array}{l}\text { first } \\ \text { class }\end{array} & \text { mixed } & \begin{array}{c}\text { tourist } \\ \text { class }\end{array} \\ \begin{array}{l}\text { International sched- } \\ \text { uled flights }\end{array} & 16 & 21 & 31 \\ \begin{array}{l}\text { U.S.A. domestic } \\ \text { flights } \\ \text { Other domestic }\end{array} & 20^{*} & 29 & 36 \\ \text { flights } & 21 & - & 39\end{array}$

At least one folding chair is placed at each exit for members of the cabin staff. This should permit a good view into the passenger cabin.

* revised number

\section{3. THE FUSELAGE OF CARGO AIRCRAFT}

\subsubsection{The case for the civil freighter}

During the sixties the transport of air freight has shown a very rapid annual growth of the order of 198 in terms of ton-miles carried per year. According to ICAO projec- 
tions this growth will continue during the seventies at an average rate of 16 \% per year. It is therefore remarkable that until recently only very few aircraft were designed specifically for the transport of air freight. There are several reasons for this.

a. A considerable amount of cargo is carried in the bellies of passenger transports, e.g. approximately 608 in 1970. The transportation costs are quite low in that case, for the extra direct operating expenses emerge mainly in the form of fuel costs.

b. Extensive use is made of

- special freight versions of passenger transports (e.g. Douglas DC-8-62F, Boeing $707 / 320 \mathrm{C})$,

- Quick Change (QC) or Rapid Change (RC) versions of passenger aircraft, (e.g. DC-9-30RC, Boeing 727-200 QC),

- civil freighters as a derivative of military freighters (e.g. Lockheed $\mathrm{C}-130$ and $\mathrm{Li} 00 / \mathrm{L} 200$ ),

- obsolete passenger transports, converted into freighters (Douglas DC-6, Lockheed L-1049).

Provided the growth of air freight continues on the same lines in the coming years, an expanding market can be expected for new freighter aircraft. The following arguments favor such a development.

a. Most converted passenger transports have loading doors in the side of the fuselage. In view of the increasing popularity of $8 \mathrm{ft} \times 8 \mathrm{ft}$ containers, very large doors might be required in the future, while most passenger cabins, except on the widebody jets, are not suitable for these sizes. It may prove prohibitive to design the cabins of short-tomedium haul aircraft especially for container transport in view of the cost penalty involved.

b. The average density of freight is considerably higher than the payload density of a passenger cabin. The difference is 1 ikely to increase in the future, as passenger comfort will be improved, while freight densities are tending to increase. Consequently. the passenger transport converted into a freighter will have a payload that is 1.5 to two times as large. The Max, Zero Fuel Weight* will have

*This term is explained in section 8.2.2. to be increased and the floor strengthened, although weight is saved due to the absence of furnishings. Assuming only minor Operational Empty Weight reduction and equal Maximum Takeoff Weight, fuel weight must be decreased. On short- to medium-range aircraft with high bypass ratio engines, relatively little fuel reduction will be achieved at the expense of appreciable range and at max. payload the range may be insufficient. An increase in the takeoff weight will generally require a new type of engine. On the assumption that the growth of air freight will beat that of passenger transport, a new market will emerge for specialized civil freighters, - for long distance transport, in view of the large amount of cargo offered;

- for short distances, converted passenger aircraft being unsuitable for this purpose.

In choosing the size of a new freighter, one of the prime factors to consider is the direct operating cost (d.o.c.) (Fig. 3-18). For a given number of aircraft pro-

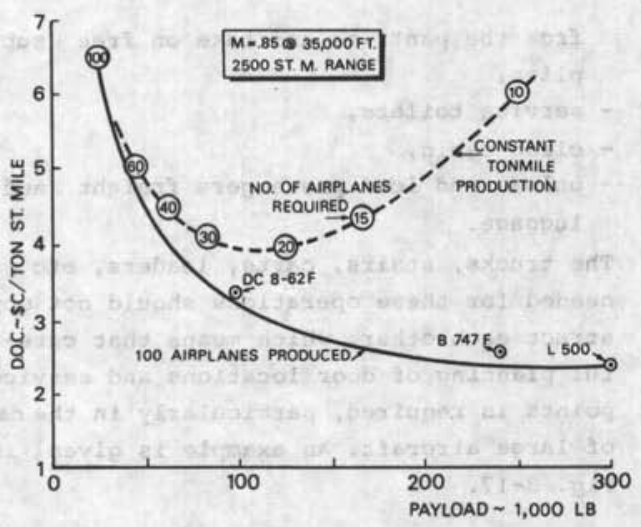

Fig. 3-18. Effect of freighter size on direct operating costs

duced, the d.o.c. will decrease with airplane size. The reasons are mainly the lower fuel cost per $1 \mathrm{~b}$ of air freight, the decreasing cost of flying staff, systems and maintenance. On the other hand, it will be extremely aifficult, if not impossible, to sell 100 aircraft of the size of the L-500*.

*A civil version of the C-5A (project). 
Therefore, assuming a market share in the form of a constant ton-mile production, the result will be an optimum size. Incidental1y, in the example presented here, the specialized freighter cannot compete with freighter versions of passenger transports.

\subsubsection{Payload density and volume of the freight hold}

The following factors may play a part in deciding whether to ship goods by air or by surface transport:

a. Fast transport may prevent decay or depreciation. Examples are: foodstuffs, fresh vegetables, fruit, cut flowers, certain types of animals. Some highly valuable goods and expensive instruments are ideal items for air cargo.

b. Rapid distribution of an article may increase its sales potential (e.g. newspapers) or enhance a service (airmail).

c. Air transport may lead to a reduction in storage space and capital investment in spares. It is more advantageous to ship certain goods by sea, the ship sometimes also serving as storage space. These are generally goods with a high specific density.

d. Transport of spare parts, modified products or new models may be important in the case of a hold-up in a production line. Goods whose value depends on fashion will generally be sent by air.

e. Packing costs for air freight are sometimes considerably lower than those for surface transport. Less damage is incurred during loading and unloading, particularly when containers are used. This results in lower insurance rates.

f. Isolated regions will be difficult to reach owing to time-consuming surface transport. In such cases air transport will be the only means of meeting their requirements with regard to medical supplies and perishable foods, etc.

From histograms giving dimensions and densities (examples in Refs. 3-11 to 3-14, see also Fig. 3-19) it can be seen that freight presents a wide variety of characteristics. processing on the airfield as well as in the aircraft demands more equipment and manpower and is considerably more costly than the transport of passengers.

It is possible to imagine the optimum case when the freight hold is completely filled, while at the same time the payload is maximum. However, when loads of a typically high density are carried, the hold will be only partly filled, with the result that the unused empty space will increase the drag and weight. With low density loads, however, there will be less than the permissible weight in freight and the aircraft will in some way be excessively strong. A proper mean can only be found on the basis of detailed data concerning the dimensions and weights of the goods to be carried and these should be supplied by potential customers. In some cases a few preliminary layouts are made, using fuselages suited to

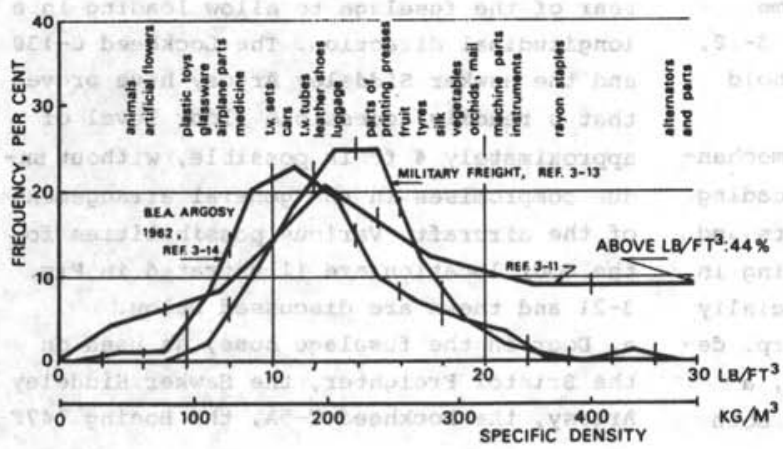

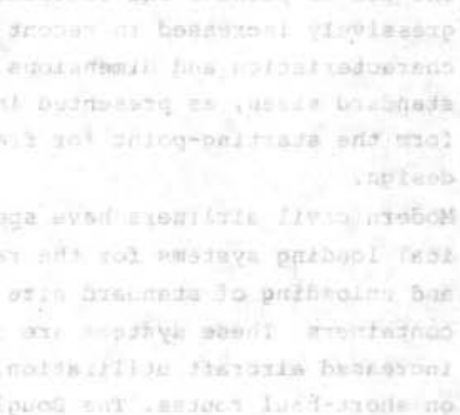

Fig. 3-19. Histograms of specific density of freight 
different load densities in order to see which choice will maximize the revenue. Although most histograms show a peak at 10 to $12 \mathrm{lb} / \mathrm{cu}$.ft $\left(190 \mathrm{~kg} / \mathrm{m}^{3}\right)$, goods of higher density are offered at widely varying frequency. Ref. 3-12 recommends that the volume of the hold should be determined by means of the following data which apply to bulk transportation of goods.

Net volume $=$ density reserve $x \frac{\max \text {. payload } \mathrm{x} \text { average freight percentage }}{\text { stowage factor }}$

The average freight percentage, comparable to the average load factor for passenger transports, may be put at .65 . The reserve density magnitude 1.20 to 1.30 is required to allow for freight having a lower density than the average.

The stowage factor represents the percentage of usable space in relation to the net volume. This allows for space losses for storage nets, clearance between cargo and structure, room for inspection, etc. Depending on the fuselage shape and the particular nature of the freight, this factor may vary between .70 and .85 . Combining these data, the result will be a spacelimited payload in the case of average and low densities. For a density of $15-25$ per cent higher than the average, the maximum payload capacity will be obtained.

\subsubsection{Loading systems}

The use of pallets and containers has progressively increased in recent years. The characteristics and dimensions of some standard sizes, as presented in Fig. 3-20, form the starting-point for freight hold design.

Modern civil airliners have special mechanical loading systems for the rapid loading and unloading of standard size pallets and containers. These systems are resulting in increased aircraft utilization, especially on short-haul routes. The Douglas Corp. developed the $463 \mathrm{~L}$ system for the USAF, a complete system for handling freight both on the ground and in the aircraft itself. It is being used in the Lockheed $c-130$, $\mathrm{C}-133, \mathrm{C}-135, \mathrm{C}-141$ and $\mathrm{C}-5 \mathrm{~A}$ aircraft. The system utilizes $88 \times 108$ inch pallets and trailers with a platform, $20 \mathrm{ft}$ wide and $48 \mathrm{ft}$ long, provided with rollers and adjustable to heights between 40 inches and 156 inches in order to fit various aircraft floor levels. Roller conveyor strips are mounted on the fuselage floor and the rails used to guide the pallets can be adjusted to various standard sizes. Similar to this is the Rolamat system used in the Hawker Siddeley Argosy (Fig. 3-21), which is designed to increase the loading capacity to $4,000 \mathrm{lb}$ per minute. Latching points in the floor are present to secure the load by means of nets and ties. When these nets are designed for normal loads only, a strong net is required in front of the freight hold to catch the load in case of a $9 \mathrm{~g}$ deceleration. Frequently, however, containers and pallet nets are designed to cope with this load and the catching net is not required.

In the case of combined transportation of passengers and freight on the same floor, it is advisable to locate the freight hold in front of the passenger cabin. A passageway between the cockpit and the passenger cabin is then required.

\subsubsection{Accessibility of the freight hold}

Although many passenger aircraft which have been converted into freighters have side doors, a pure freighter should have better accessibility via doors in the front or rear of the fuselage to allow loading in a longitudinal direction. The Lockheed c-130 and the Hawker Siddeley Argosy have proved that a readily accessible floor level of approximately $4 \mathrm{ft}$ is possible, without undue compromises in the general arrangement of the aircraft. Various possibilities for the door location are illustrated in Fig. 3-21 and these are discussed below. a. Door in the fuselage nose, as used on the Bristol Freighter, the Hawker Siddeley Argosy, the Lockheed C-5A, the Boeing $747 \mathrm{~F}$ 
I.A.T.A, CONTAINERS
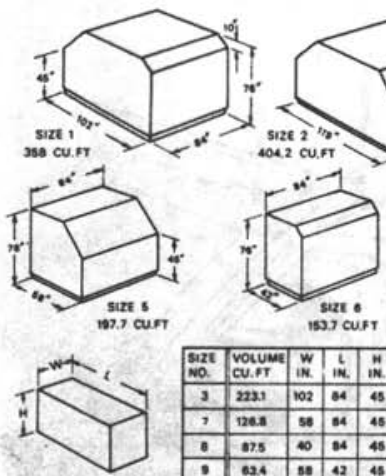

PALLETS
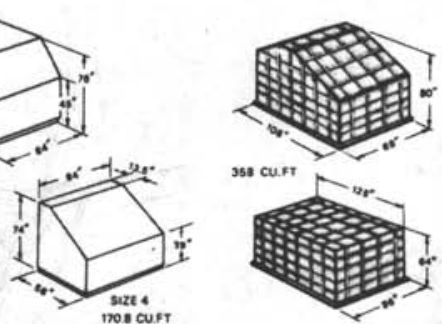

498 cu.nt
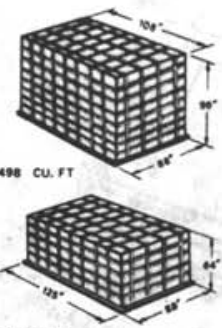

s90 CU.T

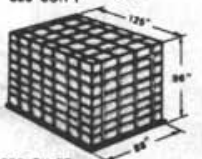

$580 \mathrm{CU} . \mathrm{FT}$

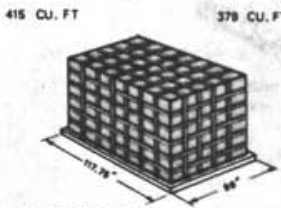

As. mI Tre I NET

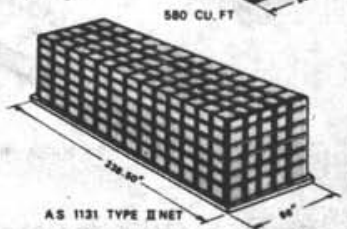

A.T.A. CONTAINERS

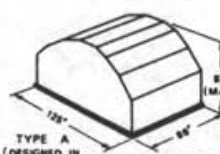

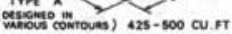
BELLY CONTAINERS

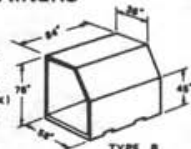

197.7 cu.f

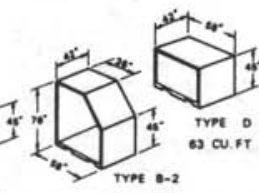

90 CU.FT
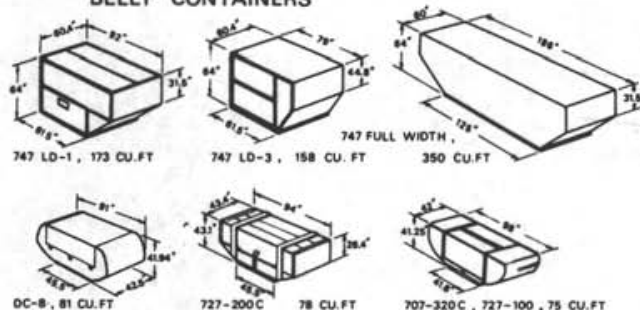

707-220C, 277-100,75 CU.

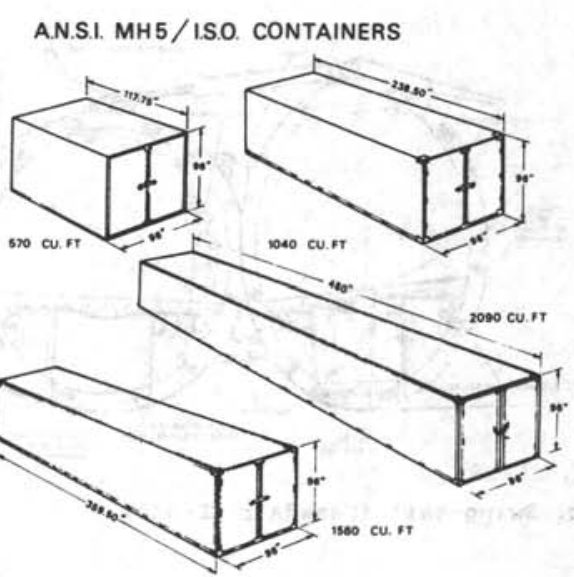

S.A.E. CONTAINERS

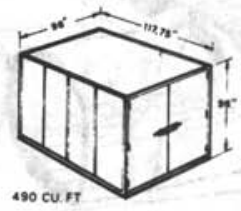

TrPe 1, hoLlen CONVEYANCE

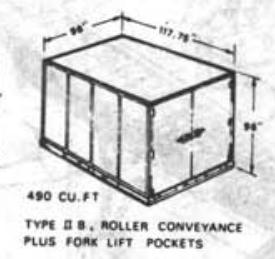

TrPes II A, IIC ANO II O Mave COANER FITTINGS 


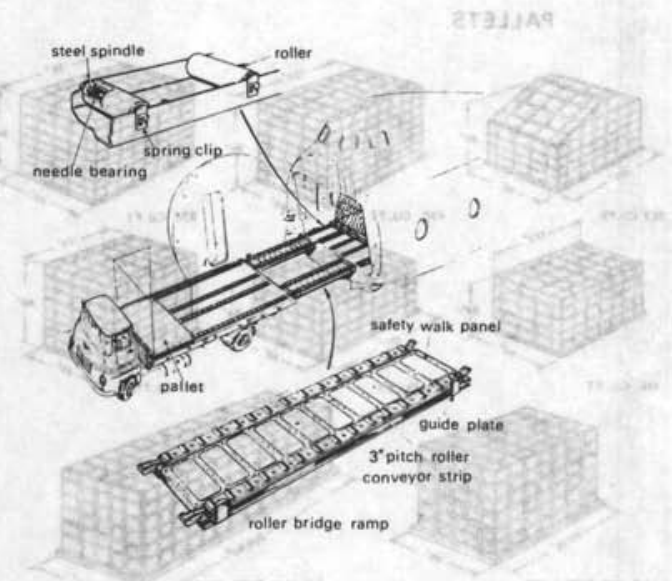

a. Front loading and the use of the Rolamat system (Hawker Siddeley Argosy)

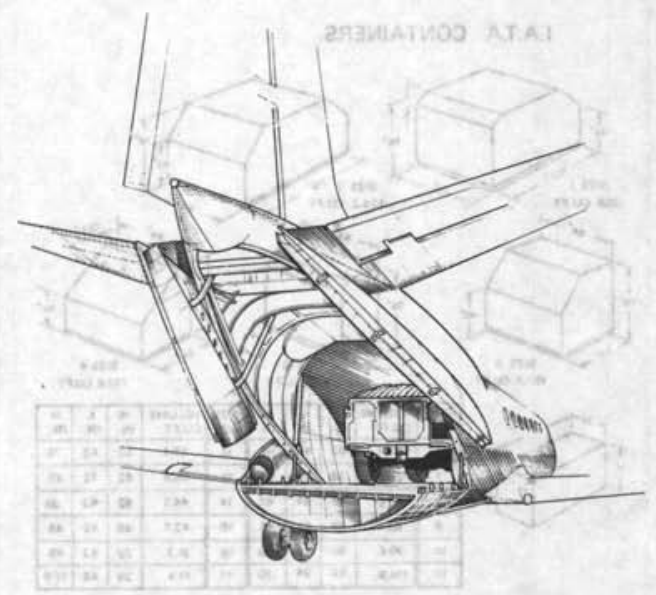

b. Rear loading door and ramps (Hawker Siddeley Andover)

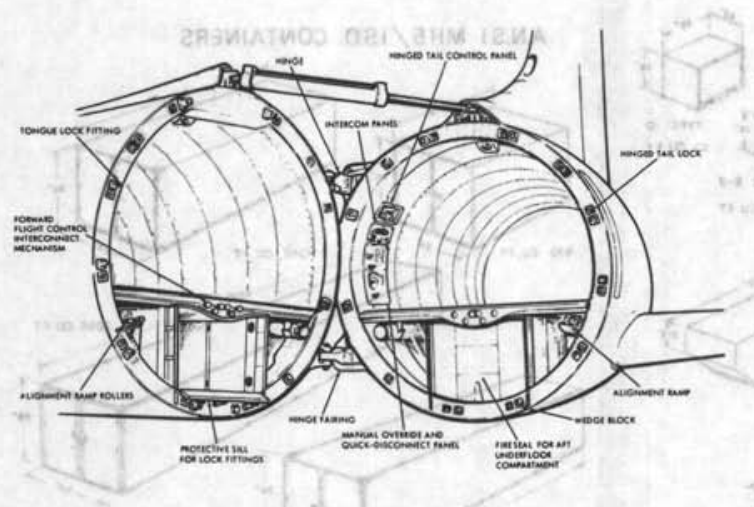

c. Swing-tail (Canadair CL-44D)

29ahiRTVG2 A, K, A
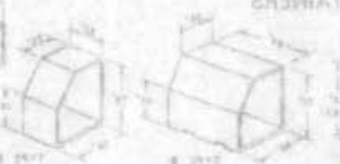

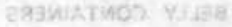
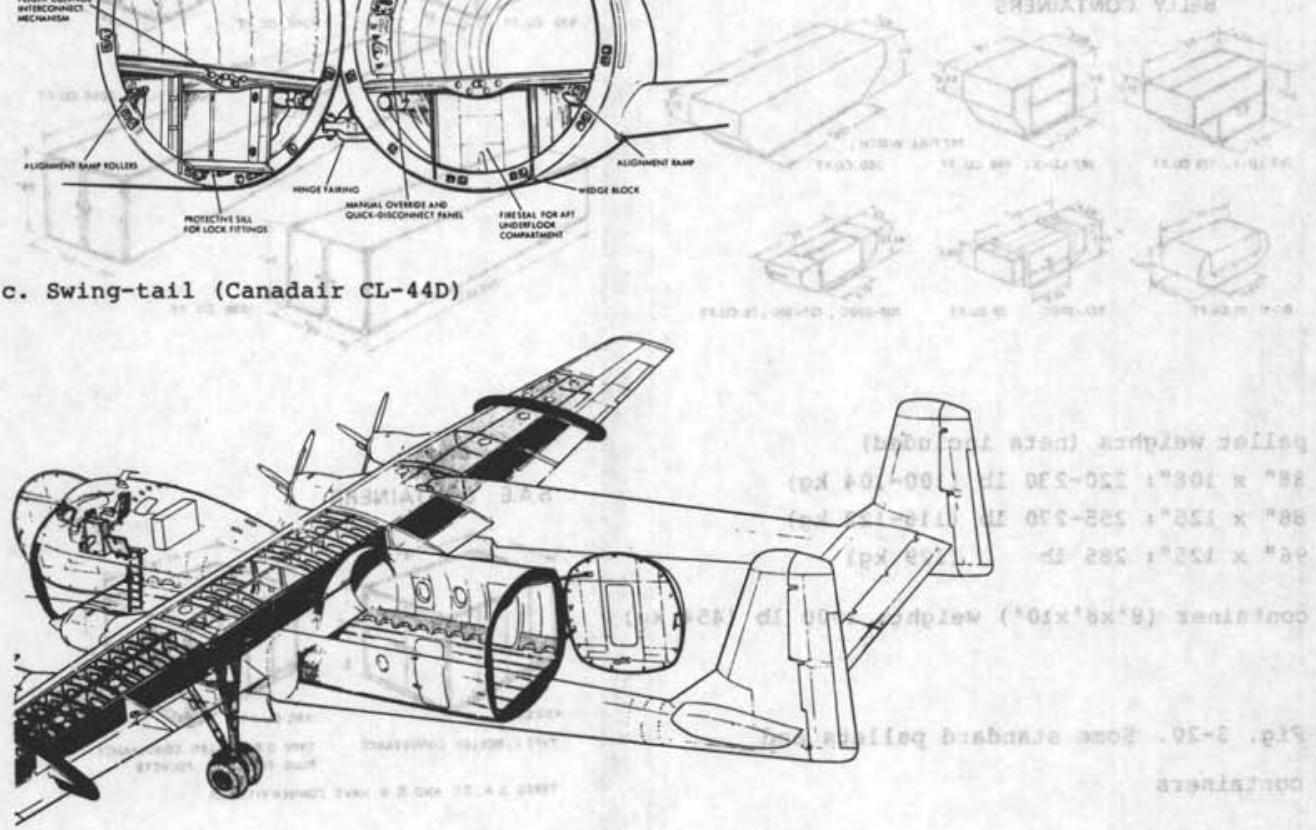

d. Rear loading door on aircraft with tailbooms

Fig. 3-21. Accessibility and loading of freighter aircraft 
and the Aviation Traders Carvair. The major problem is to avoid the considerable drag caused by the high cockpit, which is difficult to avoid on relatively small aircraft. b. Door in the fuselage tail, as used on the Short Skyvan, Transall C-160, Hawker Siddeley Andover, De Havilland Caribou and Buffalo, Short Belfast, Breguet 941, Lockheed $\mathrm{C}-130, \mathrm{C}-141$ and $\mathrm{C}-5 \mathrm{~A}$. For easy access, especially in small freighters, it is essential to camber the fuselage tail upwards, thus creating an aerodynamic problem (Section 3.5.1). The fuselage weight penalty is of the order of 6 to 108 , depending on the structural details. The zero-lift drag increment is of a similar order of magnitude, but may equally well be much higher. The door size is relatively large and it may become difficult to seal the pressure cabin.

c. Tail boom layout, in combination with a door in the rear part of a stubby fuselage. This configuration, occasionally seen on freighter aircraft (H.S. Argosy, Noratlas, IAI Arava, Fairchild C-82 and (-119), offers a readily accessible freight hold and permits the use of a beaver tail for dropping purposes, if required. The high aerodynamic drag is a disadvantage.

d. Swing-tail, a layout proposed by Folland in a freighter project as far back as 1922 . To date, the swing-tail has been implemented only on the Canadair CL-44, at the expense of a penalty of some $1,000 \mathrm{lb}(450 \mathrm{~kg})$ structure weight relative to a side door, i.e. about $6 \frac{1}{2} 8$ of the fuselage structure weight. From an aerodynamic standpoint, however, the swing-tail is ideal and the structural complexity may be outweighed by a considerable reduction in fuel comsumption. e. A swinging fuselage nose (including the flight deck) creates considerable difficulties in carrying through cables, wires, plumbing, etc. The weight penalty is of the order of 128 of the fuselage structure weight. Its use may be considered in very special cases (e.g. the Guppy family).

The maximum floor loading of freighters for an evenly distributed load must be at least:
- civil : 125-300 lb/sq:ft-6600-1,500 $\mathrm{kg} / \mathrm{m}^{2}$ )

- military: 225-1, $200 \mathrm{lb} / \mathrm{sq}$.ft $(1,100-6,000$

$$
\mathrm{kg} / \mathrm{m}^{2} \text { ) }
$$

Design criteria for local loads are:

- civil : $3,500-9,000 \mathrm{lb}(1,600-4,000 \mathrm{~kg})$

- military: 3,000-10,000 lb (1,300-4,500 kg)

Door sizes must be adapted to the type of freight to be loaded and unloaded, and in the case of loading in the longitudinal direction a clearance of at least one inch $(2.5 \mathrm{~cm})$ must be present on both sides. The freight hold ceiling must be at least 6 inches $(15 \mathrm{~cm})$ above the freight for ease of loading. The need for a passageway through the loaded freight hold depends on the type of freight carried. Inspection during the flight is not always necessary in the case of containerized freight. For preference the freight hold should be prismatic in shape; steps in the floor are not acceptable, except in very special cases. A separate door for the cockpit crew is necessary. Several windows are usually incorporated in the fuselage walls. For civil freighters it may be useful to consider a passenger version (convertible freighters) and in that case more windows and passenger doors are required.

\subsection{FLIGHT DECK DESIGN}

3.4.1. Location of the pilot's seat and the flight controls

On light aircraft the cockpit may, to some extent, be arranged in line with the particular design requirements. This applies particularly to the location of foot pedals in the vertical direction, as this factor affects the cross-sectional height and hence the fuselage frontal and wetted areas. The pedals must be placed below the level of the seat bottom to avoid tiring the pilot.

Instructions for the location of the seat and stick controls are presented in Fig.3-22. 


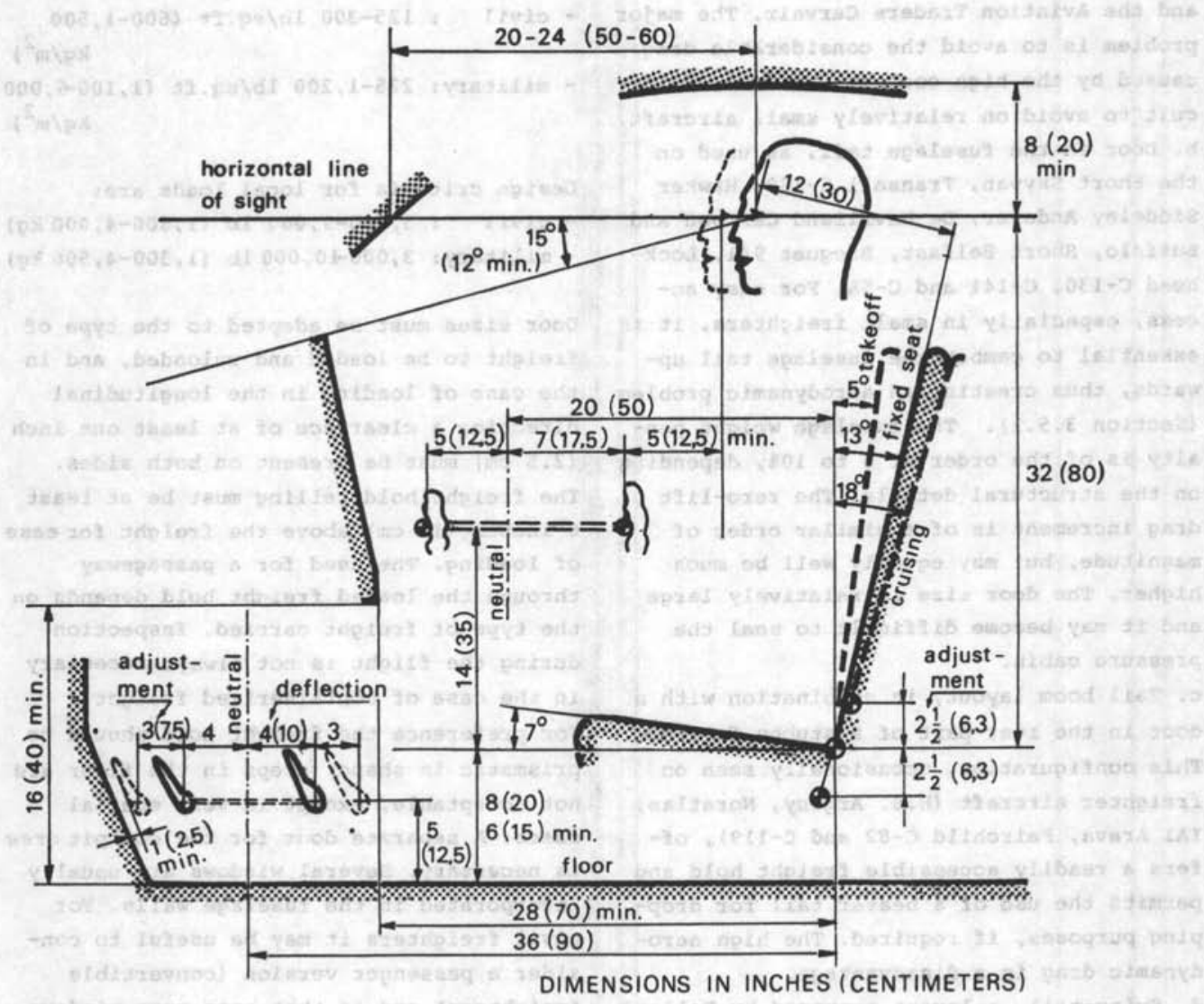

NOTES :

1. Distance between foot pedal centerlines: 8 inches $(20 \mathrm{~cm})$ minimum, 12 inches $(30 \mathrm{~cm})$ maximum.

2. The indicated floor is a reference line; the actual floor need not be horizontal. Only the local height of the foot pedal relative to the floor is of importance.

3. For many light aircraft the seatback has a fixed position. The recommended setting relative to the vertical is $13^{\circ}$.

REFERENCES :

1. F. Maccabee: "Light aircraft design handbook". Loughborough University of Technology, Feb. 1969 .

2. Draft ISO recommendation No. $1558,1973$.

3. Mil. Standard MS 33574 .

Fig. 3-22. Recommended dimensions for the cockpit of a light aircraft with stick control

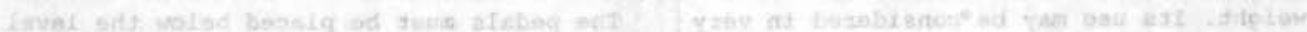

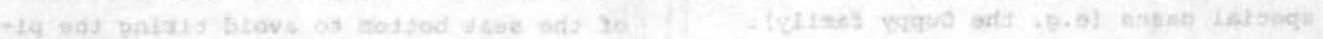


For a control-wheel layout it is advisable to use the data for transport aircraft. Generally speaking, the outside view from the cockpit is only obstructed by the wing and no special measures are necessary. The downward view forward is determined by the instrument panel, the glare shield, the fuselage nose or the engine cowling. Part of the cockpit roof of light aircraft can be of a light-alloy construction to improve stiffness and strength and to provide protection against sunlight.

Particularly on transport aircraft, more is required than merely the convenient location of the flight controls and instruments. The position of the pilot relative to the cockpit windows, and the window shape, are equally important. Pilots of varying body dimensions must feel at ease in the cockpit and be able to take up a position from which a clear outside view is possible. A design aid which is usually employed here is the reference eye point. This is a fixed point chosen by the designer in the aircraft, which serves as a reference for defining both the outside view and the seat position. It is defined as follows:

(1) The reference eye point must be located not less than five inches aft of the rearmost extremity of the primary longitudinal control column when the control is in its most rearward position (i.e. against the elevator up stops).

(2) The reference eye point must be located between two vertical longitudinal planes which are one inch to either side of the seat centerline.

(3) Any person from $5.4 \%(1.63 \mathrm{~m})$ to $63^{\prime \prime}$ ' $(1.91 \mathrm{~m})$ tall, sitting in the seat must be able to adjust the seat with the seat back in the upright position, so as to locate the midpoint between his eyes at the reference eye position. With the seat belts fastened, he must also be able to operate the aircraft controls with lap strap and shoulder harness fastened.

In the proposed para. FAR 25.777 dated Jan. 12 , 1971, a requirement is laid down with respect to the seat adjustment relative to a position of the seat bottom located $31 \frac{1}{2}$ inches below the reference eye point. The Society of Automotive Engineers (SAE) and the International Standardization Organization (ISO) have made recommendations (e.g. Ref. 3-24) for the standardization of other dimensions of the cockpit. A condensed version of the various proposals is presented in Fig. 3-23, which may serve as a startingpoint for the cockpit design or mock-up design.

On most transport aircraft the crew seat position can be adjusted horizontally and vertically, while the seat back is reclinable. In some cases seat rails extend far back and/or allow sideways displacement to facilitate easy access/egress or to permit crew members to take up a position where other controls can be handled or panels read off. The seatback in its upright position is used for takeoff and landing; on short trips this position is generally not changed. The cruise position is used when the autopilot is operative.

\subsubsection{Visibility from the cockpit}

During VFR flights the pilot must have a clear view of such a part of the air space that he has adequate information to control the flight path and avoid collisions with other aircraft or obstacles. For design purposes this general requirement can be evaluated in the form of minimum angles of vision during cruising flight, takeoff, landing and taxying.

a. Horizontal flight (Fig. 3-24).

To define clear areas of vision, binocular vision and azimuthal movement of the head and eyes are assumed to take place about a radius, the center of which is the central axis. The areas of vision are measured from the eye position with the airplane longitudinal axis horizontal. For example, in level flight, with the pilot looking straight ahead from the reference eye position, clear vision must be possible up to $17^{\circ}$ downward and $20^{\circ}$ upward. The complete envelope of the clear areas of vision is given 


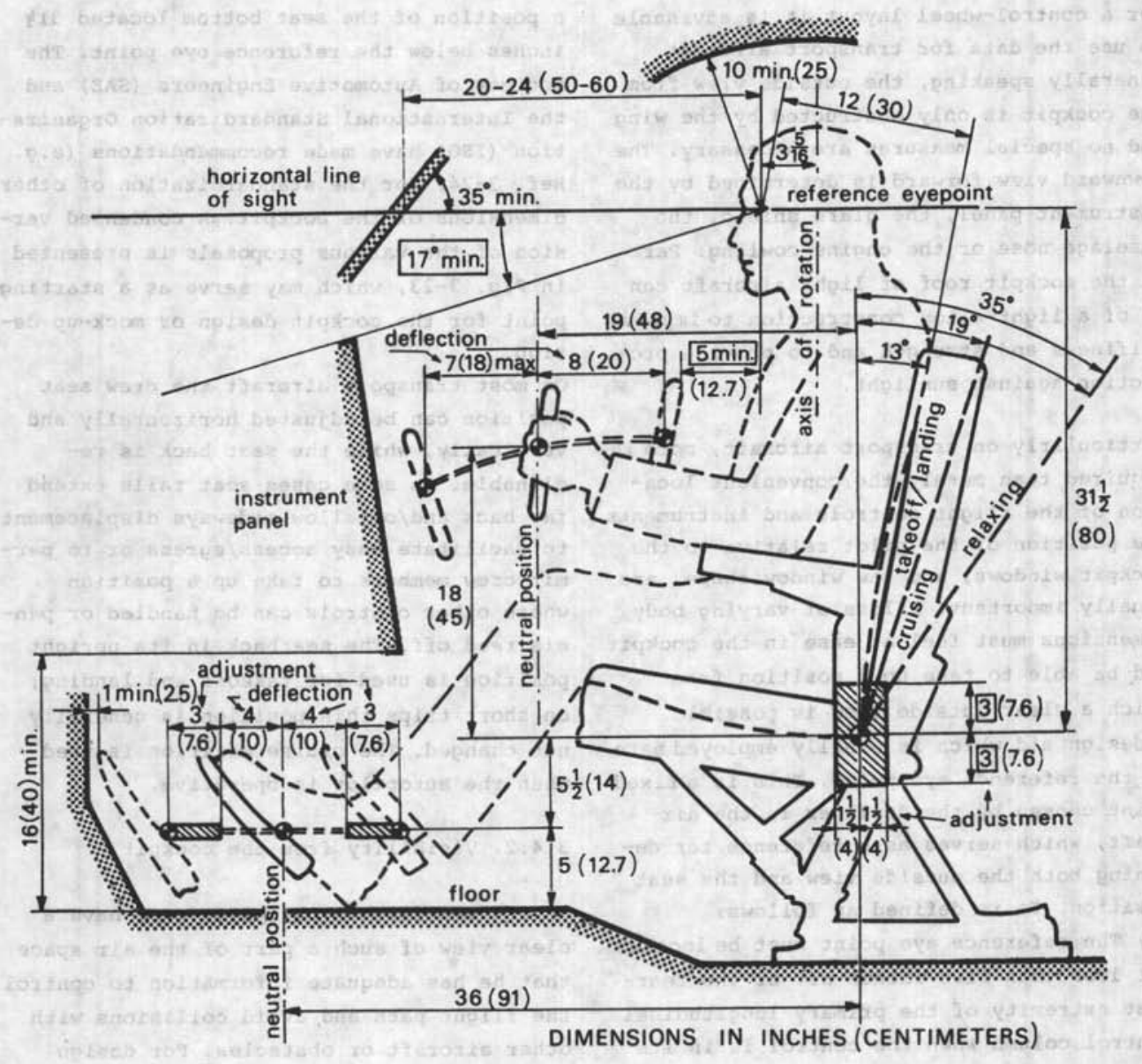

NOTES:

1. Distance between the centerlines of both seats: see Table 3-6.

2. Distance between the centerlines of the foot pedals: 14 inches $(35 \mathrm{~cm})$.

3. Most dimensions can be chosen within wide ranges, except the framed ones: these are specified in the rule proposed in FAR 25.772 .

4. The indicated floor is a reference line; frequently a footrest is used.

\section{REFERENCES :}

1. FAR 25.772 (proposed), dated Jan. 12, 1971.

2. Draft ISO recommendation $1558,1973$.

3. Mil. Standard MS 33576.

Fig. 3-23. Recommended flight deck dimensions for transport aircraft with wheel controls. 


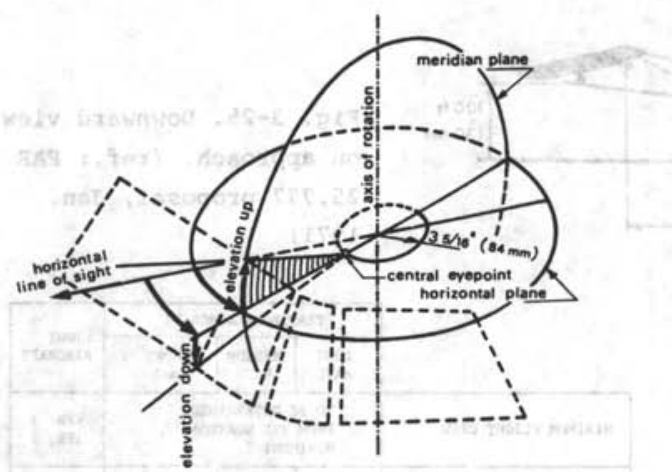

a. Definition of the pilot's view

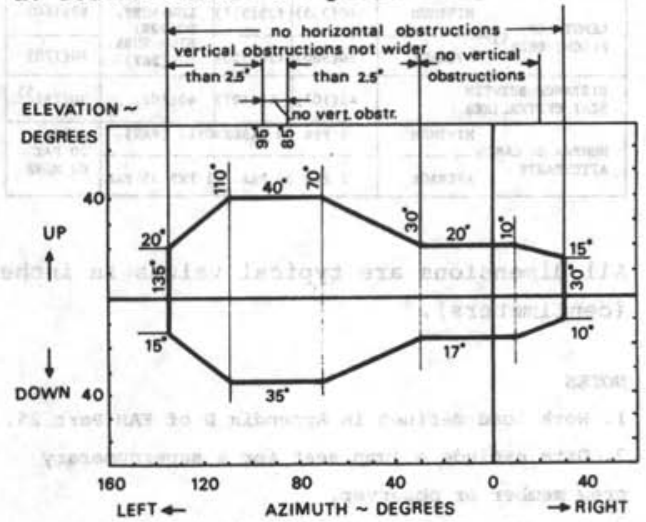

b. Minimum required clear areas of vision

Fig. 3-24. Vision from the pilot's (port) seat in horizontal flight (Ref.: FAR Part 25.777 proposal, Jan. 1971)

in Fig. 3-24b, where areas are also indicated where no obstructions may impair the pilot's vision. This determines the location of windshield posts, instruments and other cockpit equipment. Areas are also indicated where windshield posts of limited width are considered to be acceptable.

\section{b. Visibility during approach (transport category).}

In the case of modern transport aircraft, considerable variations can be observed in the airplane attitude during low-speed

flight. These are caused by great differences in wing aspect ratio, angle of sweep and type of high-lift devices. Accordingly, standards must be evolved for this category of aircraft to ensure clear areas of vision during the approach. The angle of view forward and downward must be sufficient to allow the pilot to see the approach and/or touchdown zone lights over a distance equal to the distance covered in 3 seconds at the landing speed when the aircraft is

(1) on a $2 \frac{1}{2}^{\circ}$ glide slope,

(2) at a decision height which places the lowest part of the aircraft at a height of 100 feet above the touchdown zone (see Fig. 3-25),

(3) yawing $\pm 10^{\circ}$,

(4) making an approach with 1,200 feet Runway Visual Range, and

(5) loaded to the most critical weight and center of gravity location.

In the British requirement BCAR Appendix No. 2 to Chapter D4-2 some additional stipulations are made:

(1) When taxying, the pilot should be able to see the ground at a maximum of $130 \mathrm{ft}$ from the airplane, but preferably this distance should be $50 \mathrm{ft}$ or less

(2) When climbing, the pilot should be able to see at least $10^{\circ}$ below the horizon and preferably $15-20^{\circ}$ below it.

(3) When landing, the pilot should be able to see below the horizontal when the airplane is in the tail-down attitude.

Another desirable feature is that during taxying the pilot should be able to see the wingtip on his side of the airplane.

When all these requirements have to be incorporated in cockpit design, the designer of high-speed aircraft with a pressurized fuselage may run into considerable trouble. Unacceptable deformation of the fuselage contour, high drag penalties and unacceptable noise levels may be the result. Therefore most transport aircraft do not completely meet all requirements, but nevertheless these should be used as a startingpoint for crew compartment design.

\subsubsection{Flight deck dimensions and layout}

The minimum number of flight crew is based on the total work load, consisting of the 


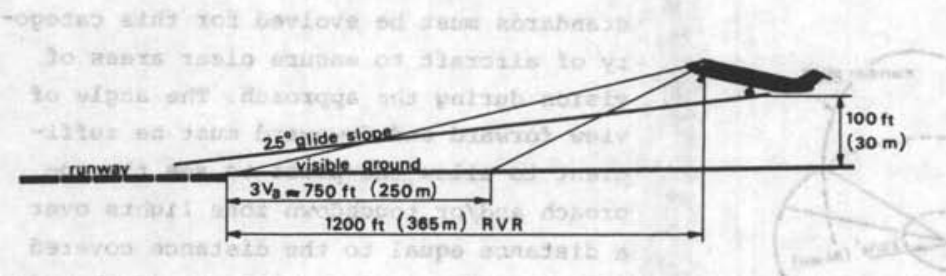

Fig. 3-25. Downward view on approach. (ref.: FAR 25.777 proposal, Jan. 1971)

\section{following activities:}

(1) Flight path control.

(2) Collision avoidance.

(3) Navigation.

(4) Maintaining contact with Air Traffic Control Centers.

(5) Operation and supervision of systems.

(6) Taking decisions concerning the execution of the flight.

The total work load is affected by the duration of the flight, the degree of automation and complication of the systems and the operational limitations. Accordingly, the data in Fig. $3-26$ and Table 3-6 relating

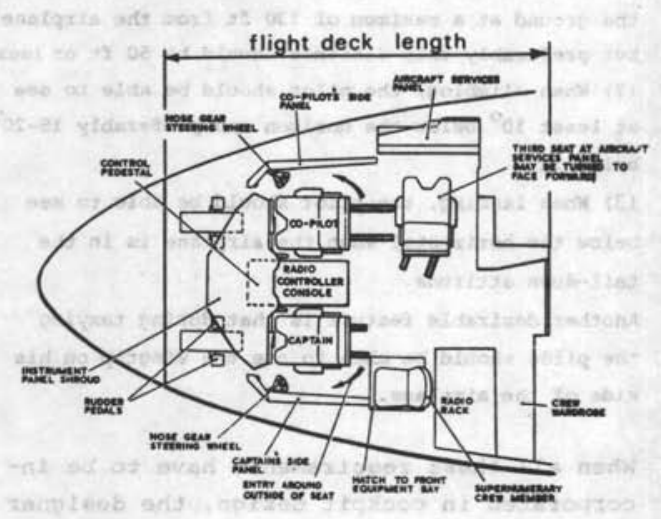

Fig. 3-26. Crew cabin layout for a mediumrange transport

to the number of seats are statistical figures and obviously not standard requirements.

Transport aircraft must have duplicated flight controls and must be operated by at least two pilots. Short-to medium-range aircraft can be operated by two crew members. However, long-range and several mediumrange aircraft require a third crew member because of the generally long duration of

\begin{tabular}{|c|c|c|c|c|}
\hline & \multicolumn{3}{|c|}{ TRANSPORT AIRCRAPT } & \multirow{2}{*}{$\begin{array}{l}\text { LiGHT } \\
\text { AIRCRAFT }\end{array}$} \\
\hline & $\begin{array}{l}\text { LONG } \\
\text { HUVL }\end{array}$ & $\begin{array}{c}\text { MEDTUM } \\
\text { HAUL }\end{array}$ & $\begin{array}{l}\text { SHORT } \\
\text { HUUL }\end{array}$ & \\
\hline MININUM FLICHT CREW & \multicolumn{3}{|c|}{$\begin{array}{l}\text { TO BE DETERINED } \\
\text { FROM THE WORKLOND1), } \\
\text { MINIMUM: } 2\end{array}$} & $\begin{array}{ll}\text { VFR: } & 1 \\
\text { IFR: } & 2\end{array}$ \\
\hline $\begin{array}{l}\text { NUKBER OF FLIGHT } \\
\text { DECK SEATS }\end{array}$ & 4 & 3 or 4 & 2 or $3^{3}$ & 2 \\
\hline \multirow{2}{*}{$\begin{array}{l}\text { LENGTH OF } \\
\text { FLIGHT DECK }\end{array}$} & $140(355)$ & $125(317)$ & \multirow{2}{*}{$\begin{array}{l}\text { LOW-suBs. } \\
90(228) \\
\text { HIGH-SUSS. } \\
105(267)\end{array}$} & $63(160)$ \\
\hline & $150(380)$ & $130(330)$ & & $70(178)$ \\
\hline $\begin{array}{l}\text { DISTANCE BETNEEN } \\
\text { SEAT CERTERLINES }\end{array}$ & $42(107)$ & $42(107)$ & $40(102)$ & $30(76)^{5)}$ \\
\hline NUMBER OF CABIN MINTMUM & \multicolumn{3}{|c|}{ I PER SO FASSENGERS (PAX) } & \multirow{2}{*}{$\begin{array}{l}\text { I FOR } \\
20 \text { PAX } \\
\text { OR WORE }\end{array}$} \\
\hline ATTENDANTS & & 30 PAX & PER 35 PAX & \\
\hline
\end{tabular}

All dimensions are typical values in inches (centimeters).

NOTES

1. Work load defined in Appendix D of FAR Part 25 . 2. Data exclude a jump seat for a supernumerary crew member or observer.

3. According to an old rule a flight engineer must be present for a max. takeoff weight above $80,000 \mathrm{lb}(36,300 \mathrm{~kg})$.

4. Definition in Fig. 3-26. Space for electronics rack included on transport aircraft, excluded on light aircraft.

5. This figure varies widely; it is affected primarily by the external fuselage wiath.

References

FAR Part 23.1523 and 25.1523 ; Appendix D to Part 25;

FAR Operating Rules $91.211,91.213,91.215$ and 121.385 through 121.391.

Table 3-6. Statistical data on number of crew members and flight deck dimensions

the flights and the complexity of the systems installed. A control panel and a seat are then provided for a flight engineer or 
third pilot (systems operator). Fig. 3-26 shows that a fairly large flight deck is required in long-range airplanes. Adequate space must be provided so that crew members can stow their baggage, coats, etc. in or adjacent to the flight deck.

The flight deck accommodation in general aviation aircraft is generally limited, so that the length of the flight deck is not more than $5 \mathrm{ft}(1.5 \mathrm{~m})$ for small touring aircraft, and up to about $6 \mathrm{ft}(1.8 \mathrm{~m})$ for business aircraft.

\subsubsection{Emergency exits for crew members} (Ref. FAR 25.805 and BCAR Section D para. 5.2.1 of chapter D 4-3)

The following requirements are quoted from the FAR-regulations:

"Except for airplanes with a passenger capacity of 20 or less, in which the proximity of passenger emergency exits to the flight crew area offers a convenient and readily accessible means of evacuation for the flight crew, the following apply:

(a) There must be either one exit on each side of the airplane or a top hatch, in the flight crew area.

(b) Each exit must be of sufficient size and must be located so as to allow rapid evacuation of the crew. An exit size and shape of other than at least 19 by 20 inches (482 x $508 \mathrm{~mm})$ unobstructed rectangular opening may be used only if exit utility is satisfactorily shown, by a typical flight crewmember, to the Administrator".

\subsection{SOME REMARKS CONCERNING THE EXTERNAL} SHAPE

3.5.1. Fuselages with a cylindrical midsection

The following applies to the fuselage nose, i.e. the non-cylindrical front part of the fuselage.

a. A frequently used value for the length/ diameter ratio is 1.5 to 2.0 . A lower value may be used on freighters provided that this lightens the door and door support structure to such an extent that it outweighs the extra drag.

b. All passenger transports and many highspeed general aviation aircraft have a radar installation, for which a reflector must be planned in the nose section.

c. It may be advantageous to locate the nose gear in front of the forward pressure bulkhead: in that case the wheelbay has no pressure walls.

d. On small aircraft the fuselage nose can be used to contain Nav/Com equipment and/or luggage. In the case of piston engines this may lead to a forward location of the center of gravity and the wing can be so located that the propellers are in front of the cockpit. The accessibility of.'such a nose bay on the ground is generally quite satisfactory.

The following hints are pertinent to the fuselage tail, i.e. the non-cylindrical rear part.

a. To avoid large regions of boundary layer separation and the associated drag increments, the tail length is usually 2.5 to 3 times the diameter of the cylindrical section. For a tail boom configuration a slenderness ratio of 1.2 to 1.5 may be acceptable, provided that the weight of the fuselage and door structure can be reduced. b. For ease of production, part of the fuselage tail may be conical; half the top angle of this cone should be $10^{\circ}$ to $11^{\circ}$, or at most $12^{\circ}$. The transition between the cone and the cylinder ought to be smooth with sufficiently large radii of curvature. c. Tail cross-sections may approximate circles or standing ellipses in shape. Beavertails have unfavorable drag characteristics and should be avoided on civil aircraft. d. During takeoff and landing the fuselage tail must clear the ground under normal operating conditions.

e. There is usually plenty of space in the fuselage tail to contain the A.P.U. and/or the air conditioning system, provided that the position of the center of gravity will permit this location. If a central engine 


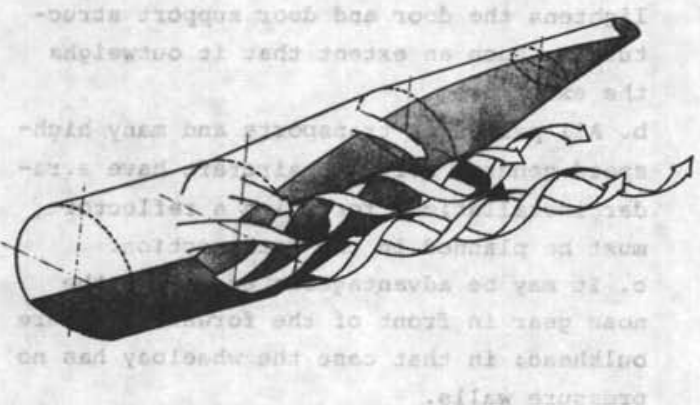

a. Schematic drawing of flow separation and vortex shedding from a rear-loading fuselage (Ref.: NCR Aeron. Report LR-395)

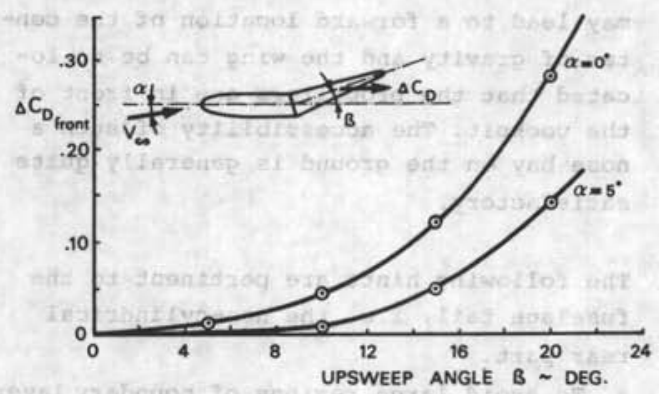

b. Drag increment vs. upsweep angle (Ref. 3-26)

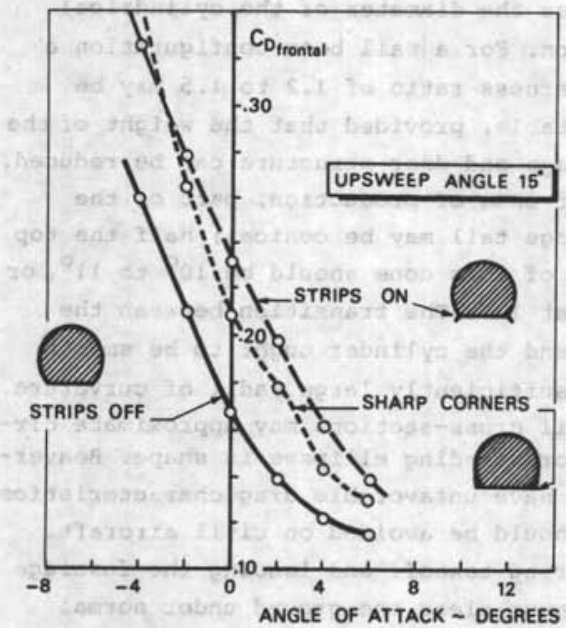

c. Effect of cross-sectional shape on drag (Ref. 3-27)

Fig. 3-27. Flow phenomena around cambered rear, fuselages is present, the minimum tail length may be determined by the allowable curvature of the intake duct. This situation may be improved by locating the engine fairly high.

\section{Fillets}

Where the wing is connected to the fuselage, too much divergence in the airflow must be avoided, Some form of filleting is required but its exact shape must be determined by means of windtunnel experiments. Some examples of fillets are shown in Fig. 2-9.

\section{Cambered fuselage tail}

The rear part of the fuselage is often slightly upswept in order to obtain the required rotation angle during takeoff or landing. The drag resulting from this slight camber is negligible. However, on freight aircraft with a rear loading door the fuselage must be swept up over a considerable angle, especially on small freighters like the De Havilland Caribou and Buffalo. Adverse interference may occur in the flow fields induced by the wing (downwash), the wheel fairings and the rear fuselage. The formation of vortices below the rear part of the fuselage is shown in Fig. 3-27a. These vortices are unstable and can cause lateral oscillation, especially at low speeds, high power, and high flap deflection angles. A considerable arag penalty in cruising flight is also caused by a large fuselage camber (Fig. 3-27b). Sharp corners on the lower part of the fuselage may relieve the problem by generating stable vortices, inducing upwash below the fuselage and thereby creating attached flow. Measurements (Fig. 3-27c) have shown that the drag penalty can be limited to reasonable values. References $3-26$ and 3-27 give more detailed descriptions of the aerodynamic phenomena involved.

\subsubsection{Fuselages for relatively small use-} ful loads

Several considerations outlined in the previous section also apply to this category, together with the following: 
a. If an engine is mounted in the fuselage nose, the required downward view of the pilot (Fig. 3-22) and the required propellerto-ground clearance determine the vertical level of the engine.

b. Allow sufficient width in the fuselage around the rudder pedals and for the pilot's shoulders and elbows.

c. Avoid sharp changes of cross-sectional area, as well as discontinuities in the radius of curvature in the longitudinal direction. The fuselage should not be tapered in the region where the wing is attached, for this will entail the use of large fillets.

d. The fuselage tail length is determined by the tailplane moment arm required. A reasonable value for the distance between the wing and horizontal tailplane quarter- chord points* is 2.5 to 3 times the wing MAC**.

e. The details of the external lines are affected by the type of structure. A design sketch of the structural concept should be made at an early stage. In the case of welded frames, the fuselage sides will be flat and not curved like the panels used on semi-monococque fuselages.

f. For ease of production, a substantial part of the fuselage should have single curvature.

g. In the case of a jet engine (or engines) buried in the fuselage, attention must be paid to the possibility of removing the engine(s) for major overhauls and to ensuring their accessibility for inspection.

*Definitions in Appendix A Section A-3.3. 



\section{Chapter 4. An appreciation of subsonic engine technology}

SUMMARY

This survey presents some background information which is required when an engine has to be chosen for a new subsonic aircraft design.

The first chapter compares piston and turbo-engines and their principal applications. The second chapter presents a survey of the characteristics, possible applications and performance of piston engines with a power rating of up to about $500 \cdot \mathrm{h} . \mathrm{p}$. Various engine configurations are discussed and a generalized method is given for estimating the takeoff power and weight of the piston engine.

Single flow, bypass turbojet engines and turboprop engines are compared, taking a division between the gas generator and the propulsive device as the point of departure. An explanation of the significance of the thermal efficiency and propulsive efficiency, as well as specific fuel consumption, specific thrust and power rating, is also given. The influence of the compressor pressure ratio, turbine inlet temperature and bypass ratio on engine performance, general configuration, weight, drag and external engine noise is discussed on the basis of generalized data. Some attention is paid to possible future developments. 


\begin{tabular}{|c|c|}
\hline $\mathrm{A}_{\mathrm{e}}$ & - exhaust nozzle area \\
\hline BHP & - Brake Horse Power ( $\left.\mathrm{P}_{\mathrm{br}}\right)$ \\
\hline MEP & - Brake Mean Effective Power \\
\hline PR & - By Pass Ratio \\
\hline & $\begin{array}{l}\text { - specific fuel consumption of tur- } \\
\text { bojet engine }\end{array}$ \\
\hline & $\begin{array}{l}\text { - specific fuel consumption of pro- } \\
\text { peller engine }\end{array}$ \\
\hline & $\begin{array}{l}\text { - specific heat of engine air at } \\
\text { constant pressure }\end{array}$ \\
\hline D & - drag \\
\hline
\end{tabular}
engine)

$v_{0}$ - flight speed

$v_{\text {cyl }}$ - total swept cylinder volume per engine

v - velocity of fully expanded exhaust flow

$w_{e}$ - dry engine weight

$\dot{W}$ - weight flow per unit time (no index: engine air)

$\gamma$ - ratio of specific heats of air

$\delta_{\mathrm{m}}$ - relative static pressure = static pressure/ambient pressure

FPR - Fan Pressure Ratio $\left(=\varepsilon_{f}\right)$

G - gas generator function

g - acceleration due to gravity

H - heating value of fuel

h - enthalpy; altitude

IMEP - Indicated Mean Effective Pressure

ISA - ICAO Standard Atmosphere

K - ratio for estimating engine weight

$k$ - constant of proportionality

Mo - flight Mach number
METO - Maximum Except Take-off (power)

m - mass flow per unit time

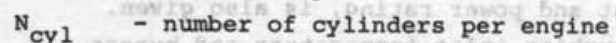

$\mathrm{n}$ - engine rotation speed; exponent of $\mathrm{V}$ in polytropic process; exponent of $\mathrm{v}_{\text {cy } 1}$

OPR - Overall Pressure Ratio $\left(=\varepsilon_{c}\right)$

P - power

$P_{b r} \quad$ - brake horsepower

${ }^{P} g_{i s} \quad-$ convertible energy, generated by gasifier

$P_{\text {to }} \quad$ static takeoff power

p - (static) pressure

po - ambient (static) pressure

$p_{t} \quad-$ total (stagnation) pressure

R - gas constant; ratio for estimating piston engine takeoff power

rpm - revolutions per minute

SFC - Specific Fuel Consumption $\left(C_{T}\right.$ or $\mathrm{C}_{\mathrm{p}}$ )

shp - shaft horsepower

T $\quad$ - (static) temperature; thrust

To - ambient temperature

$\mathrm{T}_{\mathrm{s}} \quad$ - standard ambient temperature

$\mathrm{T}_{\mathrm{t}} \quad$ - total (stagnation) temperature

TET, TIT - Turbine Entry (Intake) total

Temperature $\left(\mathrm{T}_{\mathrm{t}_{4}}\right)$

- increment

- compression ratio (piston engine); pressure ratio (gasturbine engine)

- efficiency

- bypass ratio

- atmospheric density

- relative (atmospheric) density

- non-dimensional TET

\section{INDICES}

B - combustion chamber

c - (high pressure) compressor

cr - cruising flight

cyl - cylinder(s)

d - intake duct

e - engine (installation); exhaust opening

- fuel

f - fan

g - gas generator; gearing

1 - fuel injection

id - ideal (thrust definition)

j - jet

m - manifold inlet

mech - mechanical transmission (gearbox)

n - rotational speed

p - propulsive; pressure

prop - propeller

r - ram effect

s - supercharging

sn - standard net (thrust definition)

$t$ - turbine

tf - combination turbine-fan

to - takeoff

tot - overall (efficiency definition) 
4.1. INTRODUCTORY COMPARISON OF ENGINE TYPES

Engine types suitable for use on subsonic aircraft are:

- piston engines

- turboprop engines

- single-flow jet engines

(straight jet engines)

- bypass jet engines

(turbofans)

propeller-driven aircraft

jet-propelled aircraft

As far as subsonic aircraft are concerned, rocket engines and ramjets can only be regarded as suitable for particular applications, e.g. when additional thrust is required for a short period of time. These engines will not be discussed in this chapter, but the interested reader can find relevant information in the various textbooks mentioned in the list of references.

The type of engine suitable for a particular aircraft design is mainly determined by the following considerations:

a. Flight envelope.

The range of normal flying speeds at which the aircraft will operate (the flight envelope). The propeller operates at a high propulsive efficiency up to $M=.5$ to .6 , after which compressibility phenomena at the tips will cause a considerable loss in efficiency. For higher speeds only the jet engine may be regarded as a suitable means of propulsion.

b. Fuel consumption.

Fig. 4-1 shows the quantity of fuel used per hour by some representative examples in the categories mentioned above, the figures being for cruising flight at a given thrust which is equal to the drag. In the case of jet engines, this specific fuel consumption is referred to as Thrust Specific Fuel Consumption (TSFC, $C_{T}$ ). For propeller aircraft, it should be compared with $\mathrm{c}_{\mathrm{P}} \mathrm{V} / \mathrm{n}_{\text {prop, }}$, where $\mathrm{v}$ is the flying speed, $\mathrm{c}_{\mathrm{P}}$ the specific fuel consumption related to the shaft horsepower and nprop the effi-

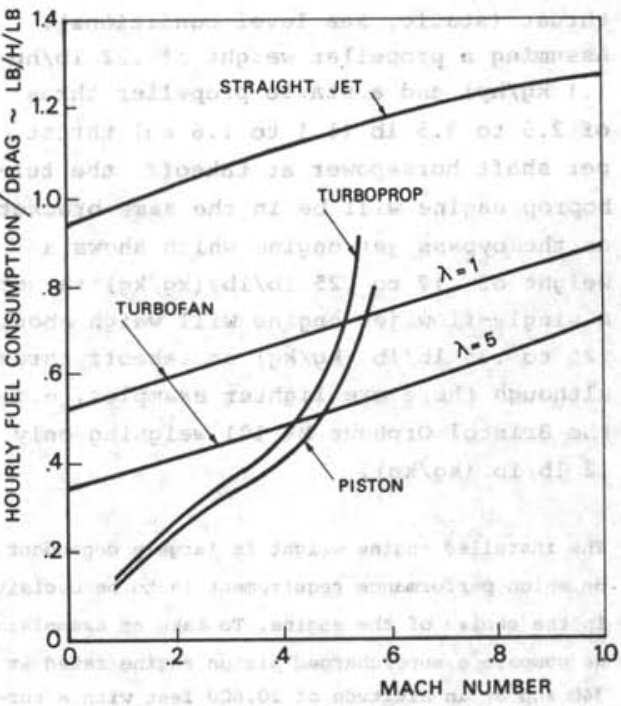

Fig. 4-1. Engine comparison based on fuel consumption to overcome drag

ciency of the propeller*. Fig. 4-1 shows that up to about $\mathrm{M}=.4$ to .5 the piston engine has the lowest fuel consumption. Generally speaking, the turboprop engine has a slightly higher fuel consumption than the piston engine, but it burns kerosine which is cheaper than gasoline. Over the entire speed range the single-flow jet engine is the thirstiest type, while at high subsonic speeds the bypass engine is the most economical with regard to fuel consumption.

\section{c. Engine weight.}

Piston engines generally weigh about 1.1 to $1.75 \mathrm{lb}(.5$ to $.8 \mathrm{~kg})$ per takeoff shaft horsepower, while for turboprop engines the equivalent figure is between .35 and $.55 \mathrm{lb} / \mathrm{hp}(.15$ to $.25 \mathrm{~kg} / \mathrm{hp})$. In order to arrive at a fair comparison with jet engines, the specific weight may be expressed as the weight of the engine with installed propeller per unit of propeller

* The terms used will be explained in Section $4 \cdot 3 \cdot 7$. 
thrust (static, sea level conditions). Assuming a propeller weight of $.22 \mathrm{lb} / \mathrm{hp}$ $(.1 \mathrm{~kg} / \mathrm{hp})$ and a static propeller thrust of 2.5 to $3.5 \mathrm{lb}(1.1$ to $1.6 \mathrm{~kg})$ thrust per shaft horsepower at takeoff, the turboprop engine will be in the same bracket as the bypass jet engine which shows a weight of .17 to, $25 \mathrm{lb} / \mathrm{lb}(\mathrm{kg} / \mathrm{kg})$ thrust. A single-flow jet engine will weigh about .25 to $.35 \mathrm{lb} / \mathrm{lb}(\mathrm{kg} / \mathrm{kg})$ at takeoff thrust, although there are lighter examples, e.g. the Bristol Orpheus Mk 101 weighing only $.2 \mathrm{lb} / \mathrm{lb}(\mathrm{kg} / \mathrm{kg})$.

The installed engine weight is largely dependent on which performance requirement is to be decisive in the choice of the engine. To take an example: if we compare a supercharged piston engine rated at 340 shp at an altitude of 20,000 feet with a turboprop supplying the same power at that height, it is clear that their specific weights, based on this condition, are much closer as compared with the takeoff condition. This is because the turboprop has a rating of 550 shp at sea level as compared with 450 bhp supplied by the piston engine. Whether or not the excess takeoff power of the turboprop can be used efficiently will depend on the type of aircraft. In this particular case, however, the piston engine will still weigh between 2 and 2.5 times more than the turboprop. Broadly speaking, this means that by choosing the turboprop it will be possible to carry more useful load for the same takeoff weight.

It is not possible to give a generally valid conclusion, but Table $4-1$ shows that the piston engine is at a disadvantage in weight when compared to the other types. Aircraft with turbojet engines differ very little with regard to the powerplant installation weight.

d. Dimensions.

When the engines are installed in separate nacelles, their dimensions become important with regard to parasite drag. Here only a comparison between piston engines and turboprop engines will be meaningful. The following comparison shows the dimensions of the nacelles of two propeller-driven aircraft with engines of comparable rating. Cessna 414: two Continental TSIO 520-J engines of 310 takeoff/bhp each, nacelle width: $3 \mathrm{ft} 4 \mathrm{in}$. $(1 \mathrm{~m})$; height $2 \mathrm{ft}(.6 \mathrm{~m})$; length: $13 \mathrm{ft}(3.9 \mathrm{~m})$; frontal area: .016 sq.ft $\left(.0015 \mathrm{~m}^{2}\right)$ per hp.

Government Aircraft Factories $\mathrm{N}-22$ two Al1ison 250-B17 of 400 takeoff bhp each, nacelle width and height: $2 \mathrm{ft} 2 \mathrm{in} .(.65 \mathrm{~m})$; length: $8 \mathrm{f}(2.4 \mathrm{~m})$; frontal area: .009 sq.ft $\left(.00083 \mathrm{~m}^{2}\right)$ per hp.

Here, too, the piston engine is seen to be at a disadvantage, although in the case of the Cessna 414 the nacelle contains a small luggage/baggage hold.

e. First cost.

At a first cost of some $\$ 25$ to $\$ 50$ per hp the piston engine is the cheapest type of powerplant. The price of a turboprop amounts to approximately $\$ 60$ to $\$ 100$ per shp (price levels 1974). Turbojet engines cost about $\$ 20$ to $\$ 40$ per 1 b takeoff thrust,

\begin{tabular}{|c|c|c|c|}
\hline Type of aircraft & \begin{tabular}{|c|} 
MTOW \\
Ib
\end{tabular} & Number and type of engines & $\begin{array}{l}\text { Powerplant weight of Bas- } \\
\text { ic Empty Weight }\end{array}$ \\
\hline Convair 340 & 47,000 & $\begin{array}{l}2 \text { Pratt and Whitney R-2800 } \\
\text { of } 2400 \text { BHP each }\end{array}$ & 31.0 \\
\hline Fokker F-27 Mk. 500 & 45,000 & $\begin{array}{l}2 \text { Rolls-Royce Dart R.Da7 } \\
\text { of } 2100 \text { ESHP each }\end{array}$ & 20.0 \\
\hline Sud Aviation Caravelle VIR & 110,230 & $\begin{array}{l}2 \text { Rolls-Royce Avon 533R } \\
\text { of } 12,600 \text { lb each }\end{array}$ & 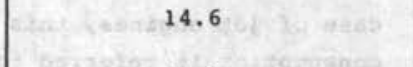 \\
\hline VFW 614 & 41,000 & $\begin{array}{l}2 \text { Bristol Siddeley } \mathrm{M} 45 \mathrm{H} \\
\text { of } 7,4801 \mathrm{~b} \text { each }\end{array}$ & 14.9 \\
\hline
\end{tabular}

NOTES

1. Basic Empty Weight is defined in Section 8.2

2. Thrust and power refer to sea leavel static (dry) takeoff rating

Table 4-1. Weight of powerplant installation as a fraction of the Basic Bmpty Weight 
dependent on size.

\section{f. Engine overhaul.}

Time between two major overhauls (Time Between Overhaul, TBO), will be about 1,500 to 2,000 hours in the case of a normally aspirated piston engine; for supercharged types this time amounts to 1,000 to 1,500 hours. In the case of a good turboprop engine the time between two revisions may be anything up to 3,000 to 4,000 hours.

\section{g. Engine noise and vibration.}

Engine noise and vibration, which largely result from the recoprocating movement of the pistons, are the principal drawback of the piston engine, in spite of the attention paid to balancing and noise suppression. The turboprop engine makes less machinery noise, but the propeller noise is predominant. For the occupants of the aircraft the jet engine is the most silent, but the observer on the ground will regard the jet aircraft as the most annoying of the three, particularly during the takeoff and approach phases. As a result of the low exhaust velocity of the engine gases the bypass engine is much quieter than the single-flow jet engine, provided suitable measures are taken to suppress the noise of the fan. The external noise of the propeller engine can only be reduced drastically by adopting a slowly rotating propeller (low tip speed).

h. Passenger appeal.

It is generally accepted that jet aircraft possess more passenger appeal than propeller aircraft.

A final assessment of the factors enumerated above can only be made by means of detailed design studies with proper attention to the installation of the engines in the aircraft and their influence on the general layout of the aircraft. It is, however, possible to draw some general conclusions:

1. For high-speed aircraft only jet propulsion can be considered. The relatively sim- ple and less expensive single-flow engine is suitable today for a limited category of aircraft where price is the dominant factor and the higher fuel consumption is regarded as less important.

2. For low-speed aircraft with a power of more than, say, 500 hp per engine, the propeller turbine will be the best choice. 3. When power ratings of less than about $500 \mathrm{hp}$ are used, the piston engine will be a competitor of the propeller turbine, due to the relatively low cost of the power unit. In the case of aircraft designed for high utilization (small passenger aircraft, some business aircraft, commuters), the cost of the powerplant will be spread out over a large number of flying hours and the propeller turbine will be at an advantage because of the low maintenance costs and cheaper fuel.

4. Small aircraft for private use (sports and touring aircraft, club trainers), are nearly always fitted with piston engines.

The various engine types will be discussed more fully in the following sections. Methods for calculating engine performance will not be presented here since these can be found in the publications mentioned in the list of references and in Appendix $\mathrm{H}$, nor will an attempt be made to explain how engines are designed. The discussion will be confined to some of the most important characteristics with which the aircraft designer should be familiar when he has to choose an engine for his design. Some background information is supplied in connection with the future technological development of engines.

\subsection{CURRENT RECIPROCATING ENGINES}

4.2.1. Some characteristics of the four stroke engine

A short description is given here of the cycle of the four stroke otto-type engine, explaining the $\mathrm{p}-\mathrm{V}$ diagram presented in Pig. 4-2a. 


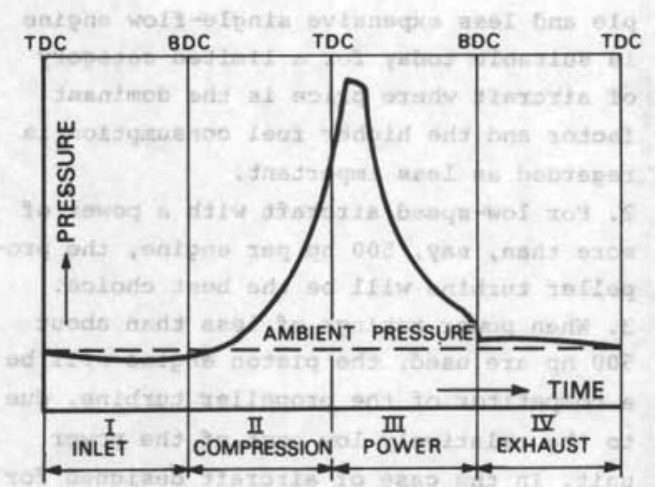

a. Cylinder pressure vs time

TDC-Top Dead Center

BDC-Bottom Dead Center

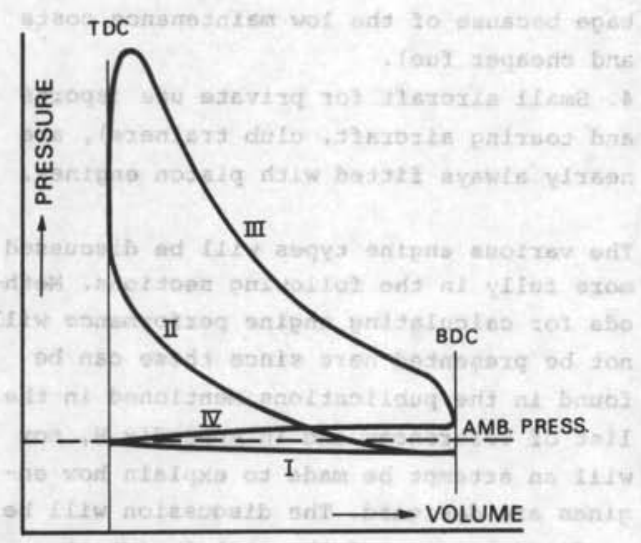

b. Indicator diagram

Fig. 4-2. Cylinder pressure of a four stroke engine

a. During the induction stroke the inlet valve is open and the gas mixture (carburetor) or the air (injection) is drawn from the inlet manifold into the cylinder. b. During the compression stroke the gas mixture is compressed, following the polytropic relationship $\mathrm{pv}^{\mathrm{n}}=$ constant, in which $\mathrm{n}$ is mainly dependent on the composition of the gas mixture ( $n \sim 1.2$ to 1.35 ). Apart from the inlet pressure, the final pressure is determined by the compression rat10*, that is, the ratio between the volumes contained in the cylinder above the piston crown at the beginning and at the end of the compression stroke.

The compressed gas mixture' is ignited 'af ter being compressed and combustion takes place at a practically constant volume. Combustion temperature and gas pressure are very high since the mixture ratio is nearly stoichiometric, with the result that the thermal efficiency of the cycle is relatively high. Thermal efficiency for a given mixture ratio is a function of the compression ratio and, contrary to the jet engine, is not affected by the rotation speed of the engine.

c. During the power stroke the burning and expanding charge transmits power to the piston. This power is transferred to the propeller by means of the connecting rod and the crankshaft. The process of expansion again follows the polytropic relationship $\mathrm{pv}^{\mathrm{n}}=$ constant.

d. After the exhaust valve is opened, the gas is expelled into the exhaust manifold during the exhaust stroke at approximately constant volume.

The total energy imparted to the piston may be determined by adding the work done during these processes algebraically and multiplying the sum by the number of power strokes per unit of time $(n / 2)$ and the number of cylinders per engine. The power determined in this way can be derived from an indicator diagram (Fig. 4-2b) by integrating the pressure versus the volume swept by the piston, resulting in the Indicated Mean Effective Power. This power is often associated with the Indicated Mean Effective Pressure (IMEP**), defined as follows:

* Note that the compression ratio is a ratio of volumes and not pressures ** In $(4-1)$ and $(4-2)$ the total cylinder volume in cu.inch must be divided by 396,000 to obtain BMEP in psi, when the engine speed is in rpm 


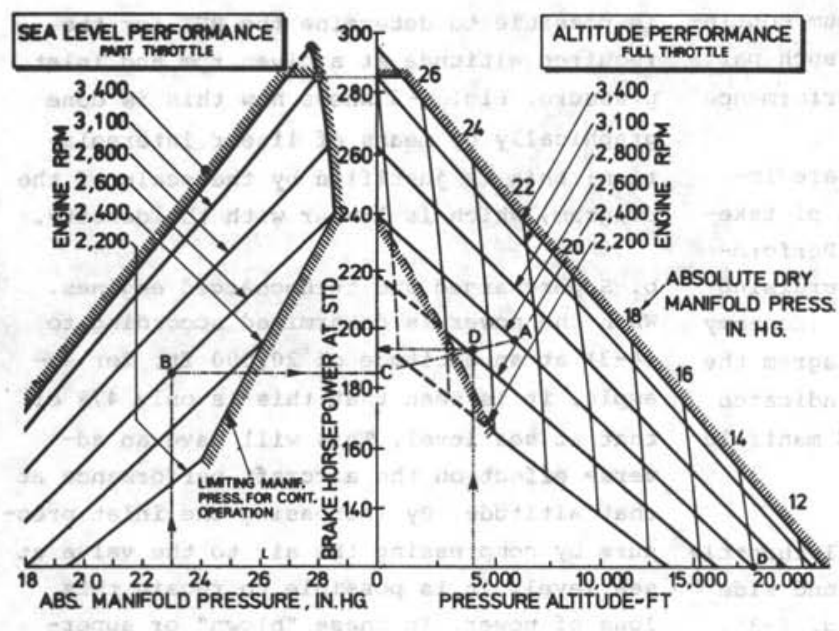

NOTE:

To find the actual horsepower from altitude, rpm, manifold pressure and air inlet temperature:

1. Locate $A$ on full throttle altitude curve for given rpm and manifold pressure

2. Locate $B$ on sea level curve for rpm and manifold pressure and transfer to $C$ 3. Connect $\mathrm{A}$ and $\mathrm{C}$ by a straight line and read horsepower at given altitude: D 4. Modify horsepower at D for variation of air inlet temperature $T$ from standard altitude temperature $\mathrm{T}_{\mathrm{S}}$ by the formula: Actual hp $=h p$ at $D \times \sqrt{\frac{T}{T}}$ where $T$ and $T_{S}$ are absolute temperatures

Fig. 4-3. Example of a standard power diagram of a normally aspirated engine

IMEP $=\frac{\text { Indicated Mean Effective Power }}{\text { Cylinder Volume } x(n / 2)}(4-1)$

The Brake Horse Power (BHP) is the product of the Indicated Mean Effective Power and the mechanical efficiency. Allowing for power losses due to friction and driving accessories, this efficiency declines with increasing engine speed.

The specific engine performance is often expressed as Brake Mean Effective Pressure (BMEP *):

BMEP $=\frac{\text { Brake Horse Power }}{\text { Cylinder Volume } \times(n / 2)}$

The standard engine diagram, an example of which is given in Fig. 4-3, shows BHP as a function of the manifold pressure, rpm and altitude in the standard atmosphere, generally with a mixture control which leads to maximum power. Sometimes, however, the takeoff power of continuous power is given for a rich mixture. Corrections are given for non-standard atmospheric conditions. Engines can be fed with air of roughly at-

*In $(4-1)$ and $(4-2)$ the total cylinder volume in cu.inch must be divided by 396,000 to obtain BMEP in psi, when the engine speed is in rpm mospheric pressure (normally aspirated) or with air compressed by a blower (blown, sal supercharged or turbocharged engines), resulting in standard diagrams of different shape.

a. Normally aspirated engines.

The full throttle inlet pressure differs from atmospheric pressure by only about 28 to 58 . On the left hand section of the diagram the power at sea level is given as a function of the (inlet) Manifold Absolute Pressure, MAP, and the rpm. Power limits may be dictated by:

- the maximum permissible rpm,

- the manifold pressure corresponding to the full throttle condition (throttle valve fully open),

- an inlet pressure limit in the case of a continuously running engine.

The following engine ratings are distinguished:

a. Takeoff Power*, which is the maximum power permitted for a limited period of time during the takeoff of the aircraft, e.g. 1 to 5 minutes.

*Takeoff power is sometimes called "Rated Power" and the maximum continuous rating is known as METO power (Maximum Except Take off) 
b. Normal Rated Power*, the maximum continuous power which may be used for such parts of the flight as maximum climb performance and maximum level speed.

c. Cruising flight, where limits are imposed for fast cruising, e.g. 75 of of takeoff power at 908 of maximum rpm (Performance (ruise), and for economical cruising flight, e.g. 658 of takeoff power (Economy Cruise). In the standard power diagram the permissible region is sometimes indicated in the form of limits for rpm and manifold pressure.

The effect of altitude in the full-throttle condition is shown on the right hand side of the standard power diagram (Fig. 4-3). Since the power is directly proportional to the mass of the charge per unit of time, the brake horsepower will diminish for a given rpm and fully opened throttle with increasing altitude as a result of decreasing density of the air. An expression often used to calculate this relationship reads :

$\frac{\text { BHP at altitude }}{\text { BHP at sea level }}=(1+c) \sigma-c$

The factor $\mathrm{c}$ is often taken as . 132 , although this number is based on limited experimental evidence with several radial engines.

When determining the BHP for part throttle working conditions at increasing altitude, we can make use of the characteristic that for a given rpm and inlet pressure, power will increase slightly as a result of :

- a decrease in air temperature, which increases the mass of air aspirated by the engine,

- a decrease of back pressure in the exhaust manifold, which leads to better filling of the cylinders.

It has been observed that the relationship between power and density is also linear in nature. By using this characteristic it

\section{* Takeoff power is sometimes called "Rated} Power" and the maximum continuous rating is known as METO power (Maximum Except Take off

is possible to determine the BHP for the required altitude at a given $\mathrm{rpm}$ and inlet pressure. Fig. $4-3$ shows how this is done graphically by means of linear interpolation; this is justified by the scale of the diagram, which is linear with the density.

b. Supercharged and turbocharged engines. When the power is determined according to (4-3) at an altitude of $20,000 \mathrm{ft}$, for example, it is seen that this is only 478 of that at sea level. This will have an adverse effect on the aircraft performance at that altitude. By increasing the inlet pressure by compressing the air to the value at sea level, it is possible to regain this loss of power. In these "blown" or supercharged engines (Fig. 4-4), one generally

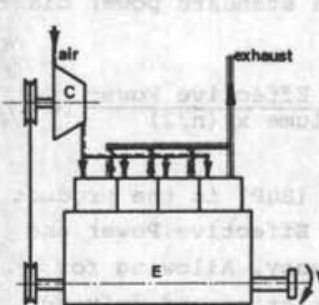

MECHANICALIY SUPEACHARGED

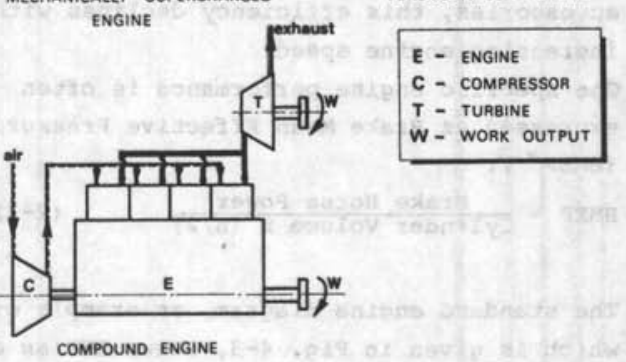

Fig. 4-4. Methods of boosting piston engine performance

uses a centrifugal compressor which is driven by either the crankshaft or by a turbine placed in the exhaust gases.

Mechanical superchargers are driven through gears, sometimes with multiple gear ratios. This means, however, that power is used from the crankshaft (about 6 to 108 ), thus reducing the output and slightly increasing the fuel consumption in relation to the work output of the engine. 


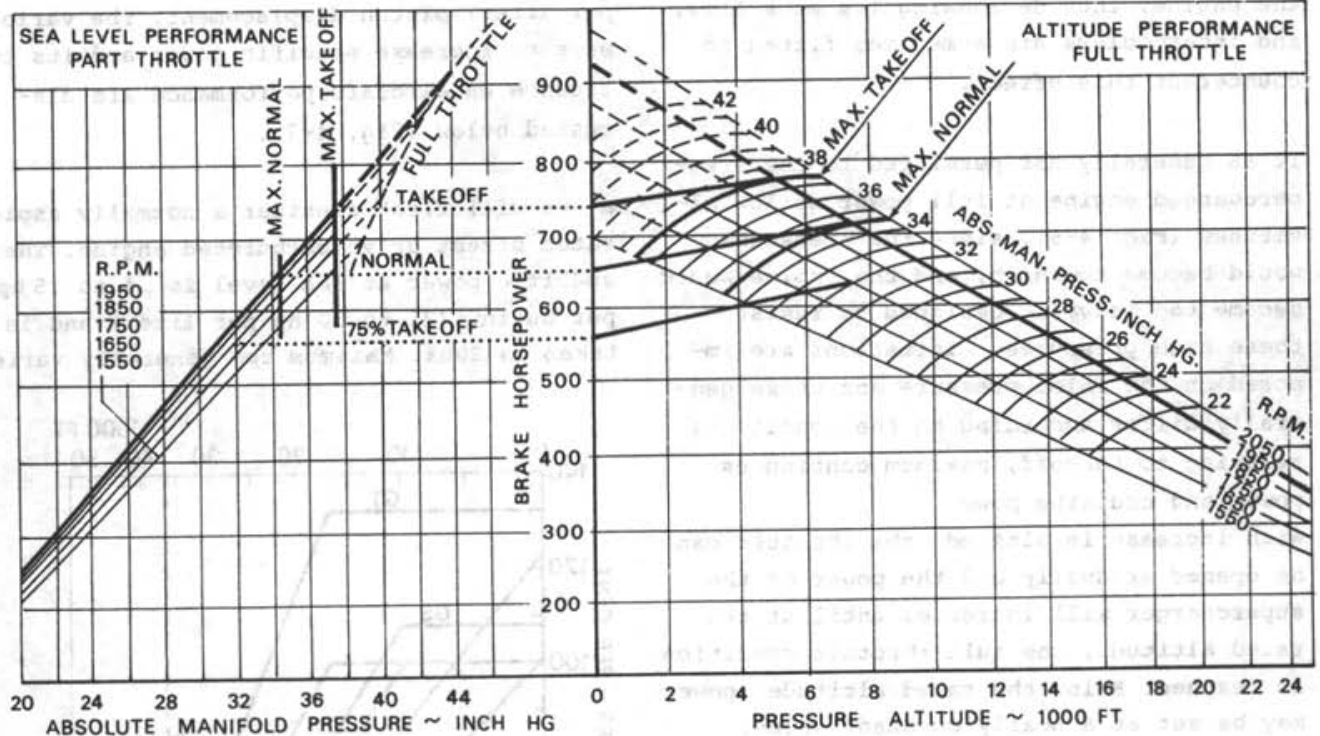

Fig. 4-5. Performance diagram of a supercharged engine

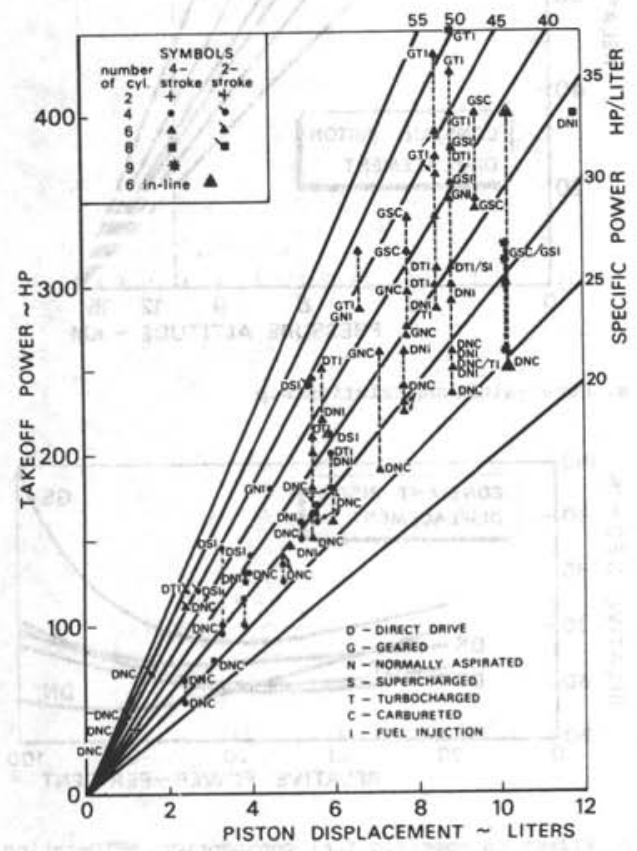

Fig. 4-6. Takeoff power of reciprocating engines vs total piston displacement (Ref. Jane's All the World's Aircraft 1972-'73)
The turbo-supercharger not only increases the inlet pressure but the back pressure in the exhaust as well, with the result that during the working stroke more work has to be done and the cylinders will not be completely filled. Part of the energy present in the exhaust gases, which is of the order of one-third of the energy supplied by the fuel, is absorbed by the turbine and the exhaust thrust will diminish. Incidentally, this thrust is small in any case and is generally disregarded when considering engine performance, unless special devices, like nozzles, are incorporated to utilize some of the energy present.

Compound engines may have different configurations. The one shown in Fig. 4-4 is mechanically supercharged and utilizes the energy of the exhaust gases to drive a turbine, thus contributing directly to the effective work output. This system may be considered as intermediate between the piston engine and the gas turbine. Due to its complexity, however, the compound engine has never been very successful. Superchargers will heat the air admitted to 
the engine, thus decreasing its mass flow, and intercoolers are sometimes fitted to counteract this effect.

It is generally not permitted to use a supercharged engine at full power at low altitudes (Fig. 4-5), since the pressures would become too high, and the engine would become too heavy if designed to resist these high pressures. Limitations are imposed on the inlet pressure and these generally differ according to the conditions relating to takeoff, maximum continuous power and cruising power.

With increase in altitude the throttle can be opened gradually and the power of the supercharger will increase, until at the rated altitude, the full-throttle condition is reached. Below the rated altitude, power may be set at a nearly constant value.

Above this it decreases almost linearly with density, as in the normally aspirated engine.

c. Engine control and fuel consumption in cruising flight.

A lean mixture will basically result in low fuel consumption. This is seldom used, however, particularly in the case of engines with high compression ratios, where there is a risk of detonation. The engine manufacturer will generally supply information regarding the relationship between the (specific) fuel consumption, rpm and power, and sometimes also for various mixture ratios. By means of these data and the relationship between speed and the shaft power required to propel the aircraft, it will be possible to determine the most favorable cruising condition where the fuel consumption per mile is a minimum.

\subsubsection{Engine design and its influence on flight performance}

The takeoff power of various engines in the 50 to $450 \mathrm{hp}$ power bracket has been plotted against piston displacement in Fig. 4-6. Specific BHP is observed to lie between about .40 to $.80 \mathrm{hp}$ per cu. in. $(25$ to $50 \mathrm{hp}$ per liter) piston displacement. The various ways to increase specific power and its influence on aircraft performance are discussed below (Fig. 4-7).

a. We will first consider a normally aspirated direct drive carbureted engine. The specific power at sea level is .4 to $.5 \mathrm{hp}$ per cu.in. ( 25 to $30 \mathrm{hp}$ per liter) and is taken as 1008 . Maximum rpm generally varies

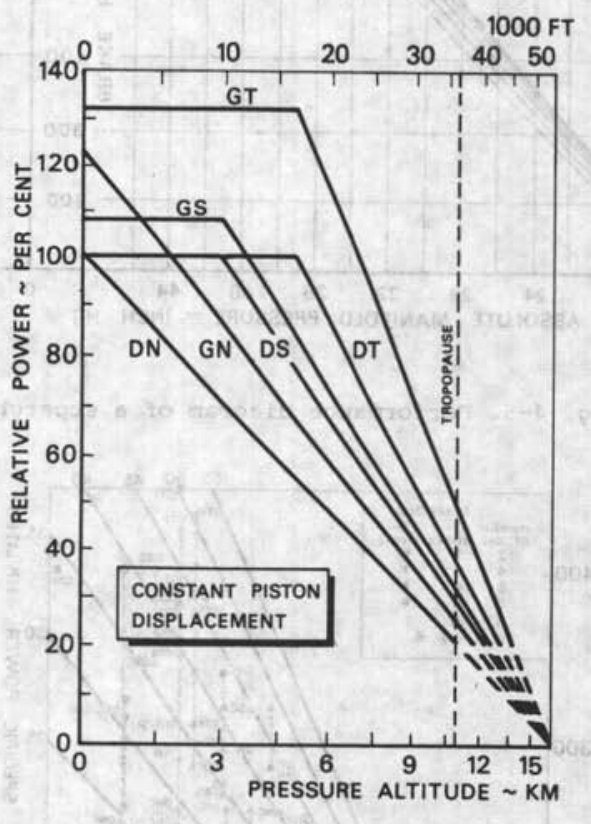

a. Power-altitude relationship

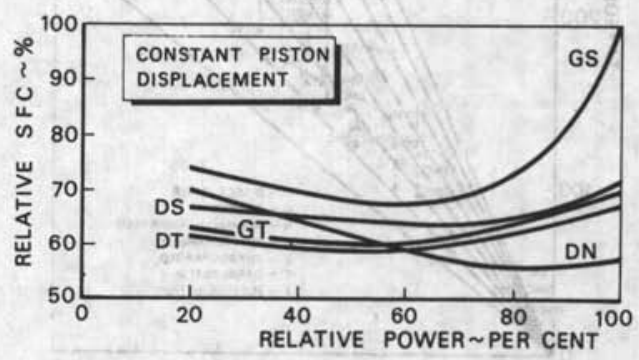

b. Effect on specific fuel consumption, METO-rating

Fig. 4-7. Reciprocating engine design improvements (Note: symbols defined in Fig. 4-6. Ref. 4-16) 


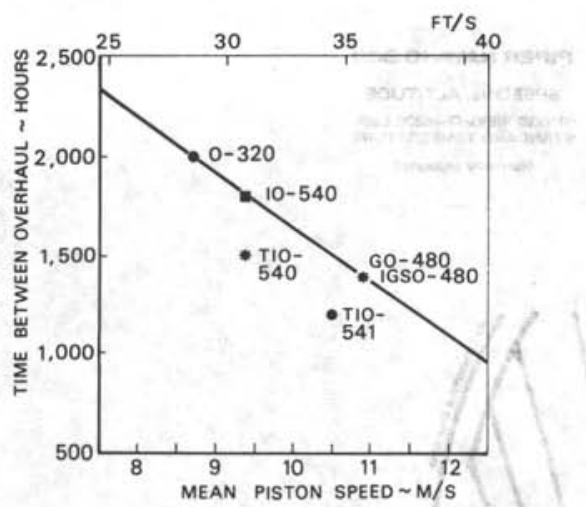

Fig. 4-8. The effect of piston speed on TBO (Ref. SAE Paper 710381)

between 2500 and 2800 . When a higher value is taken, either the tip speed of the propeller will become too high or the propeller diameter will be too small, resulting in a low efficiency.

b. Higher engine speeds are possible with the use of reduction gearing between the crankshaft and the propeller, raising the $\mathrm{rpm}$ to 3,200 or 3,400 . In the case of the
Continental Tiara engine it is even possible to obtain $4,000-4,400 \mathrm{rpm}$. For a given BMEP and cylinder volume, the brake horsepower increases with $\mathrm{rpm}$, as demonstrated by $(4-2)$. Gearing will cost a few per cent of the engine power and raise the weight and cost by approximately 128 and 508 respectively (Ref. 4-13). Specific powers of .53 to $.56 \mathrm{hp} / \mathrm{cu} . \mathrm{in}$. ( 33 to $35 \mathrm{hp} / 1 \mathrm{iter}$ ) are obtained from geared engines.

The following limiting factors should be taken into consideration:

- Engine vibration results from the torsional fluctuations in the crankshaft.

- High piston speeds have an adverse influence on the $t+m e$ between overhauls (Fig. 4-8).

c. Effect of cylinder geometry.

With a given piston speed and total swept cylinder volume (piston displacement), the power developed may be increased by:

- choosing a relatively large cylinder bore with respect to the stroke;

- reducing the piston displacement by increasing the number of cylinders ( $\left.\mathrm{N}_{\mathrm{cy} 1}\right)$, which leads to higher rpm. It can be shown

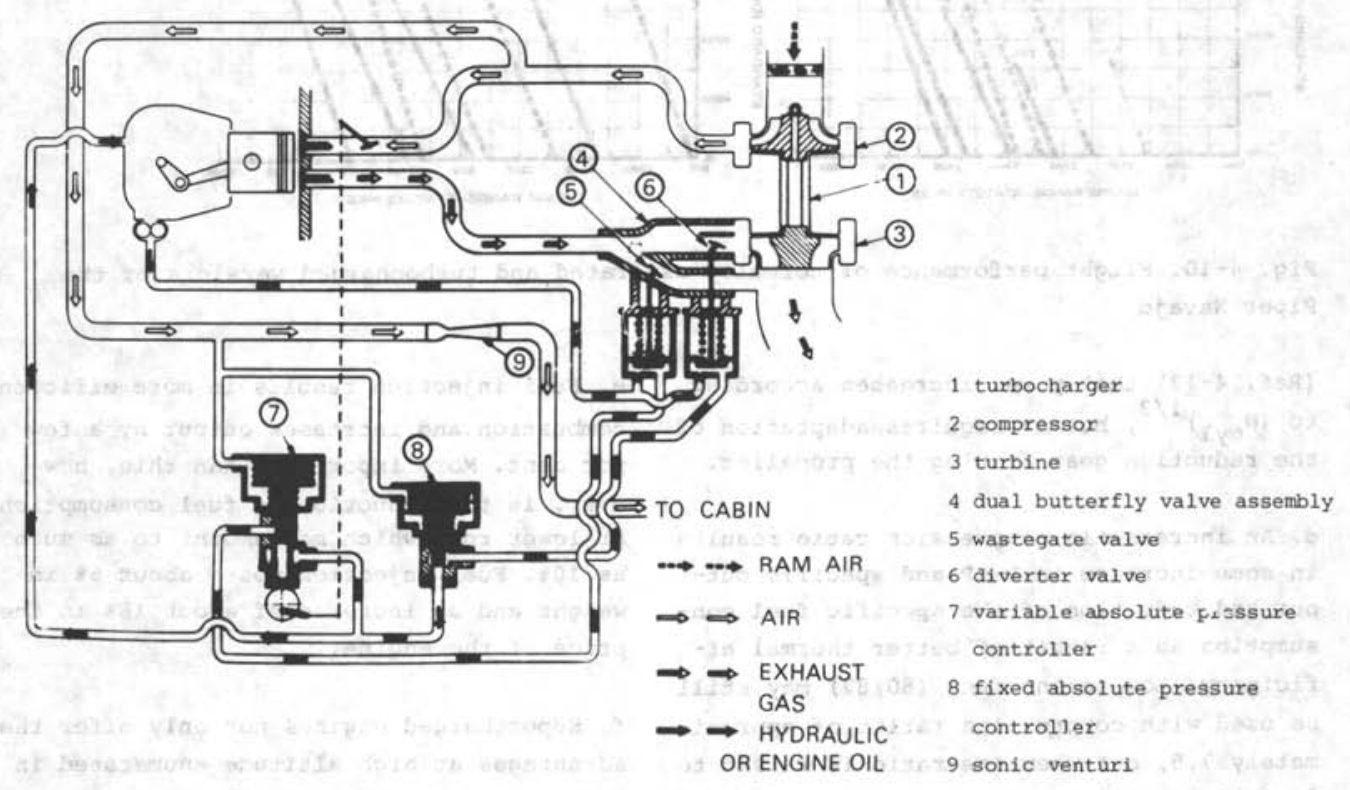

Fig. 4-9, Turbocharging engine and cabin system 

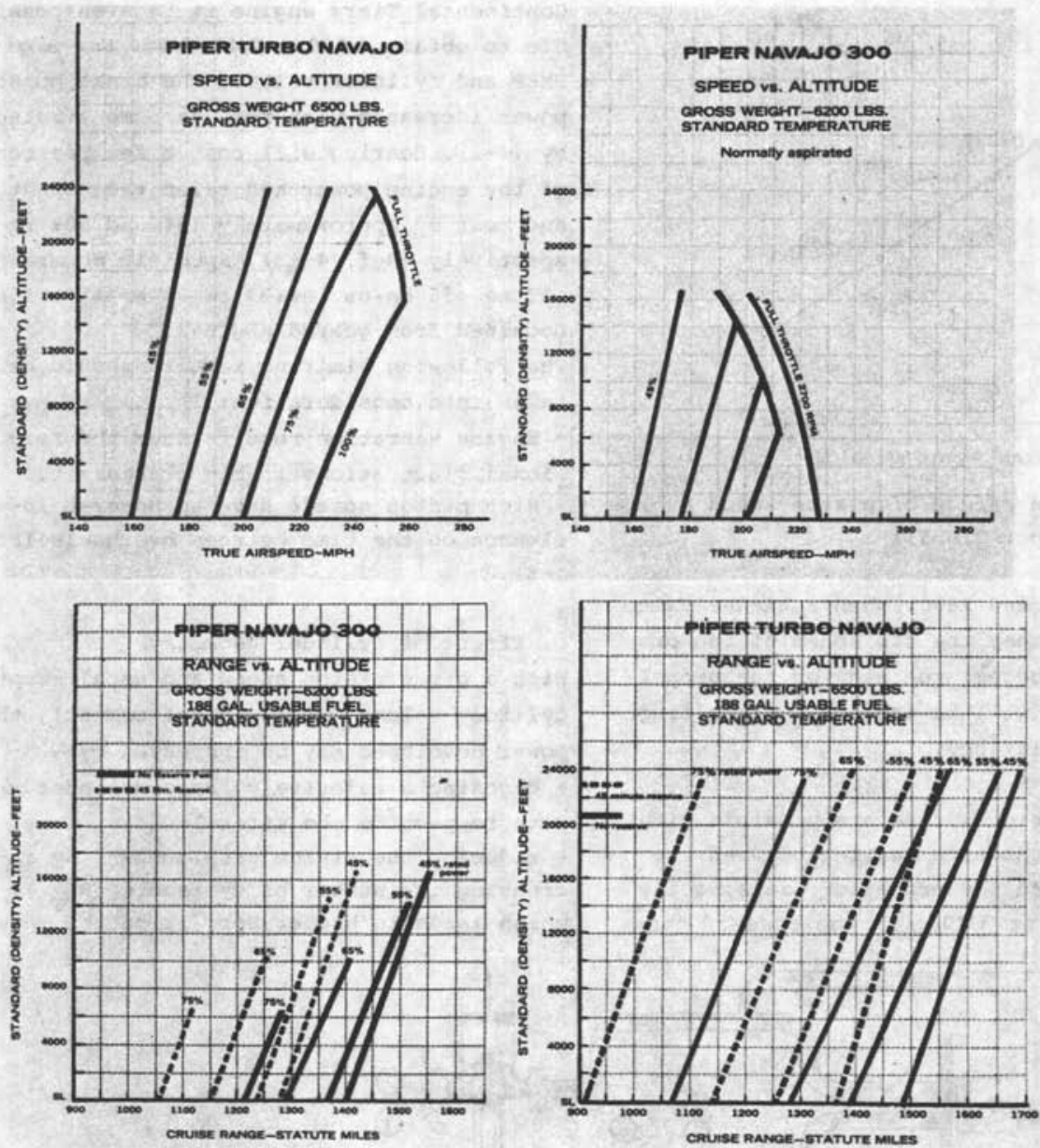

Fig. 4-10. Flight performance of normally aspirated and turbocharged versions of the Piper Navajo

(Ref. 4-13) that power increases according to $\left(\mathrm{N}_{\mathrm{cy}}\right)^{1 / 3}$, but it requires adaptation of the reduction gear ariving the propelier.

d. An increase in compression ratio results in some increase in BMEP and specific output and reduction of the specific fuel consumption as a result of better thermal efficiency. Low octane fuel $(80 / 87)$ may still be used with compression ratios of approximately 7.5 , but when the ratio is raised to 8 or 10 it will be necessary to use $100 / 130$ octane fuel. e. Fuej injection results in more efficient combustion and increases output by a few per cent. More important than this, however, is the reduction in fuel consumption at lower rpm, which may amount to as much as 108 . Fuel injection costs about $6 \%$ in weight and an increase of about 188 in the price of the engine.

f. Supercharged engines not only offer the advantages at high altitude enumerated in Section 4.2 .2 , but also the secondary advantage that they are able to supply pres- 


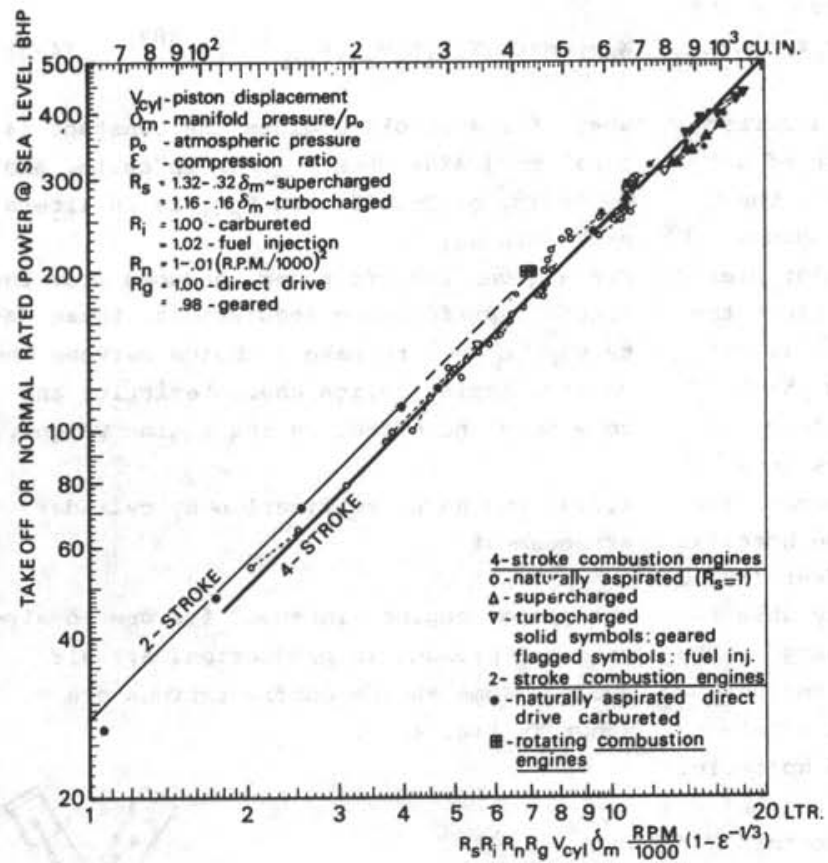

Fig. 4-11. Estimation of takeoff horsepower of reciprocating engines at SL/ISA

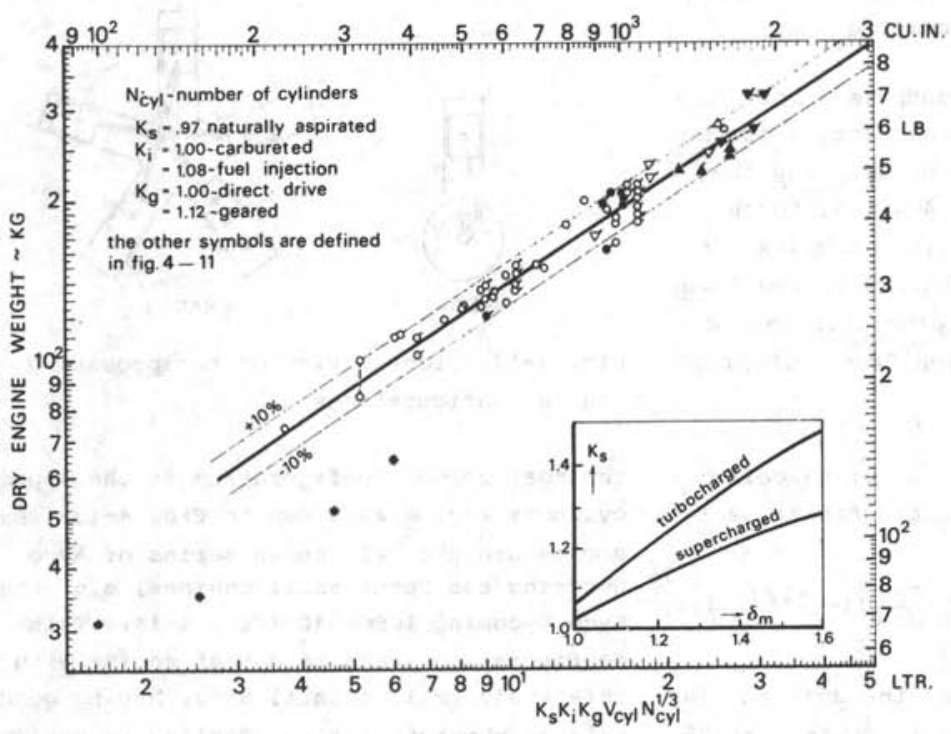

Fig. 4-12. Estimation of dry weight of reciprocating engines 
sure to the air conditioning system of the cabin. A diagrammatic layout of a typical system is shown in Fig. 4-9.

An example of the improvement in aircraft performance resulting from the use of a supercharger is given in Fig. 4-10. The supercharger used in this case produces practically no increase in the inlet pressure at sea level. In order to improve the takeoff and climb performance as well, it is possible to use a supercharger which raises the inlet pressure at sea level to, say, 1.5 atmospheres. This results in a marked improvement in the performance. The example in Fig. $4-7$ shows that the specific power amounts to 1108 at 20,000 feet $(6,150$ m) with this engine, which is only able to supply 478 output without supercharger. The specific takeoff power of piston engines with propeller reduction gear and supercharger may lie between .7 and $.8 \mathrm{hp} / \mathrm{cu}$.in. (45-50 hp/liter) or even higher. The increase in engine weight may be something like 188 for a crankshaft-driven supercharger and $30 \%$ for a turbo-supercharger, but will depend on the desired increase in performance. In order to avold detonation, the compression ratio should be low.

f. In the case of large outputs (about 3000 hp) of the type used in the past, a further increase may be achieved by adopting the compound principle (Fig. 4-4). Although this type of engine is fairly complex, it is not impossible that engines in the lower power brackets may be developed in this direction to meet competition from turboprop engines.

A semi-empirical correlation of takeoff power and engine design characteristics is given in Fig. 4-11:

$\mathrm{P}_{\text {to }}=$ const $\times \mathrm{R}_{\mathrm{S}} \mathrm{R}_{1} \mathrm{R}_{n} \mathrm{R}_{\mathrm{g}} \mathrm{V}_{\text {cy } 1}{ }^{\delta} \mathrm{m} \frac{\mathrm{RPM}}{1000}\left(1-\varepsilon^{-1 / 3}\right)(4-4)$

a hORIZONTALYY OPPOSED CYLINDER ENGINE

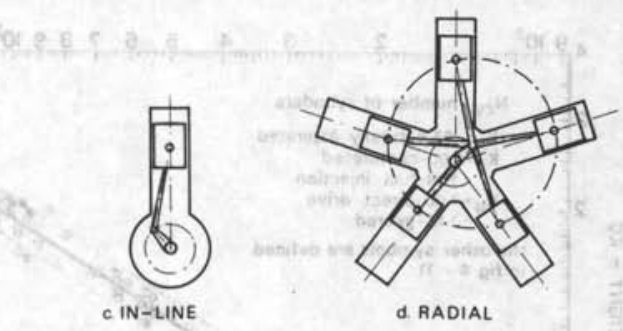

Fig. 4-13. Frontal view of reciprocating engine configurations

The most common configuration is the opposed cylinder engine as shown in Fig. 4-13a. Examples are the well known series of Avco Lycoming and Continental engines, e.g. the Avco Lycoming IGSO-540 (Fig. 4-14). This configuration leads to a flat engine with a relatively small frontal area, having gond balance characteristics. Cooling is satisfactory with up to four cylinders on either side.

A type which is no longer produced is the 


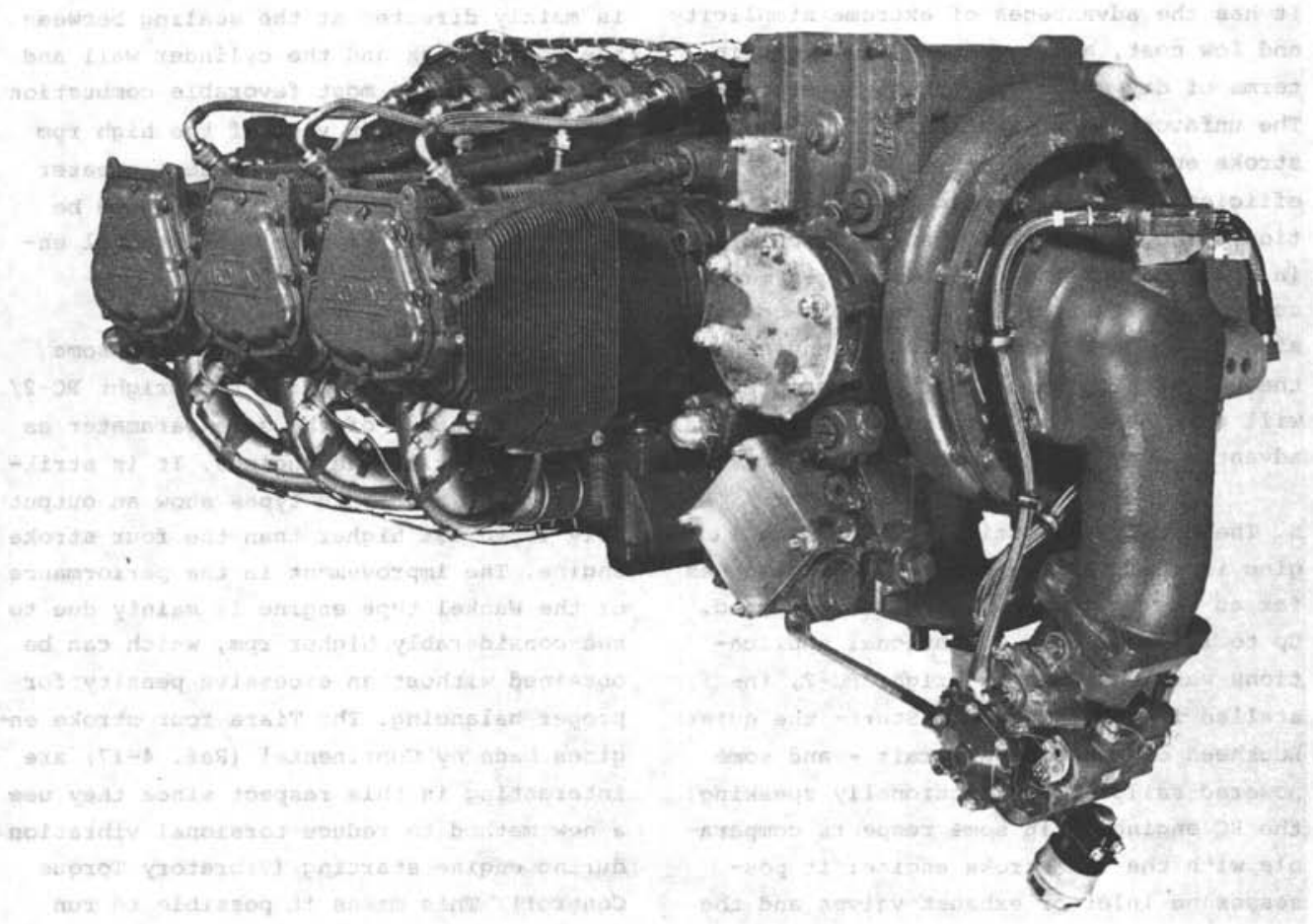

bey)

Fig. 4-14. Avco Lycoming IGSO-540-A series; 380 horsepower: $\frac{3}{4}$ left rear view gat a the 3

"Vee" engine (Fig. 4-13b), which is narrower than the horizontally opposed cylinder arrangement and has a smaller frontal area. In-line engines (Fig. $4-13 c$ ) have all cylinders placed one behind the other with their axes parallel, resulting in a very small frontal area. When more than four cylinders are required, air cooling will present a problem, particularly when the engine is used in a pusher installation. The in-line arrangement has frequently been used in the past for water cooled engines. When the engine is installed in nacelles on the wings, both the drag and the adverse influence on the lift will be minimum when a vertical in-line engine is adopted. With a tractor engine in the nose of the aircraft, however, the frontal area is generally dictated by the cross-section of the passenger cabin. In the case of side-byside seating the only advantage of the inline engine is that it improves the view of the occupants of the forward seats. When two seats are arranged in tandem, the inline engine is well suited for the narrow fuselage.

The radial engine (Fig. 4-13d) possesses a large frontal area, resulting in high drag, but the excellent cooling makes it very suitable for aircraft which have to fly at low speeds for long periods (helicopters, agricultural aircraft). Radial engines with two rows of 7-9 cylinders have been built and used for airplane propulsion in large numbers, e.g. in the Lockheed Constellation and Douglas DC-6. They are now only built in the East European countries. te ib wa wor

4.2.4. Two stroke and Rotary Combustion engines

a. The two stroke engine is only offered in low power outputs and is suitable for powerassisted sailplanes and unmanned aircraft. 
It has the advantages of extreme simplicity and low cost, and low specific weight in terms of dry weight per shaft horsepower. The unfavorable reputation of the twostroke engine is largely caused by its inefficient combustion, high fuel consumption, irregular ignition and difficulties in starting up. These disadvantages may be cured by using fuel injection, pre-compression of the air admitted to the engine and the use of high rpm, but these measures will to some extent eliminate the essential advantages.

b. The Rotary Combustion (RC) or Wankel engine is still in the experimental stage as far as its role in aviation is concerned. Up to 1972 the only operational applications were the Curtiss Wright RC-2, installed in the Lockheed Q-Star - the quiet Lockheed observation aircraft - and some powered sailplanes. Functionally speaking, the RC engine is in some respects comparable with the two stroke engine: it possesses no inlet or exhaust valves and the engine does not act for half the time as an air compressor, as in the case of the four stroke engine. It is, therefore, possible to obtain more power per unit of cylinder volume which makes the RC engine lighter and more compact for a given output. This is confirmed in practice in the case of the RC-2/60, which has an output of $200 \mathrm{hp}$ with a cylinder volume of only some $120 \mathrm{cu} . i n$. (two liters), running at $5,000 \mathrm{rpm}$. A more recent project is the German RFB Fantrainer A WI-2, a two-seat training aircraft powered by a $300 \mathrm{hp}$ Wankel four-disk RC engine. It features a ducted propeller of small diameter to cater for the high rpm.

A second important advantage is that the rotary disk does not follow a reciprocating movement. This makes it possible to transmit the disk movement directly to the shaft, resulting in less noise and vibration. It also leads to lighter balancing, a lighter propeller, engine mounting and installation, together with a more compact unit. Development work still being carried out is mainly directed at the sealing between the rotary disk and the cylinder wall and at obtaining the most favorable combustion characteristics. In view of the high $\mathrm{rpm}$ it will be necessary to choose a greater propeller reduction ratio than would be used in the case of more conventional engines.

Fig. 4-11 shows the performance of some two-stroke engines and of the Wright RC-2/ 60 as a function of the same parameter as used for four stroke engines. It is striking to note that both types show an output only 10 to 158 higher than the four stroke engine. The improvement in the performance of the Wankel type engine is mainly due to the considerably higher rpm, which can be obtained without an excessive penalty for proper balancing. The Tiara four stroke engines made by Continental (Ref. 4-17) are interesting in this respect since they use a new method to reduce torsional vibration during engine starting (Vibratory Torque control). This makes it possible to run these engines at relatively high rpm (up to 4,400 ), and these engines also have a high specific power output, running at low noise levels.

4.3. BASIC PROPERTIES OF AIRCRAFT GAS TURBINES FOR SUBSONIC SPEEDS

In spite of the fact that at first sight gas turbine engines show considerable mutual differences in general configuration (Fig. 4-15), it is possible to compare them on a common basis with respect to their thermodynamic properties.

The majority of the gas turbine power plants designed for use in civil aviation can be classified according to one of the following categories:

- straight jet (simple jet) or single-flow turbojet engines,

- bypass engines (turbofans) or double-flow engines,

- gas turbines driving a propeller or rotor: turboprop and turboshaft engines, re- 


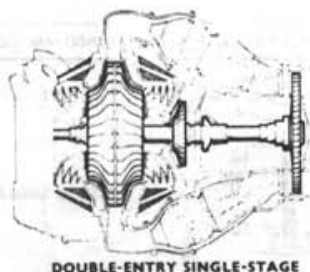

DOUBLE-ENTRY SINGLE-STAGE CENTRIFUGAL TURBO-JET

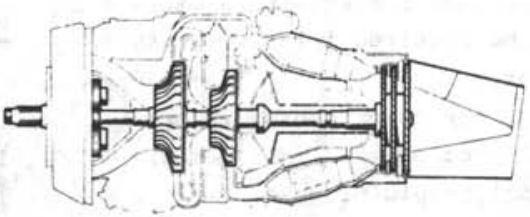

SINGLE-ENTRY TWO-STAGE CENTRIFUGAL TURBO-PROPELLER
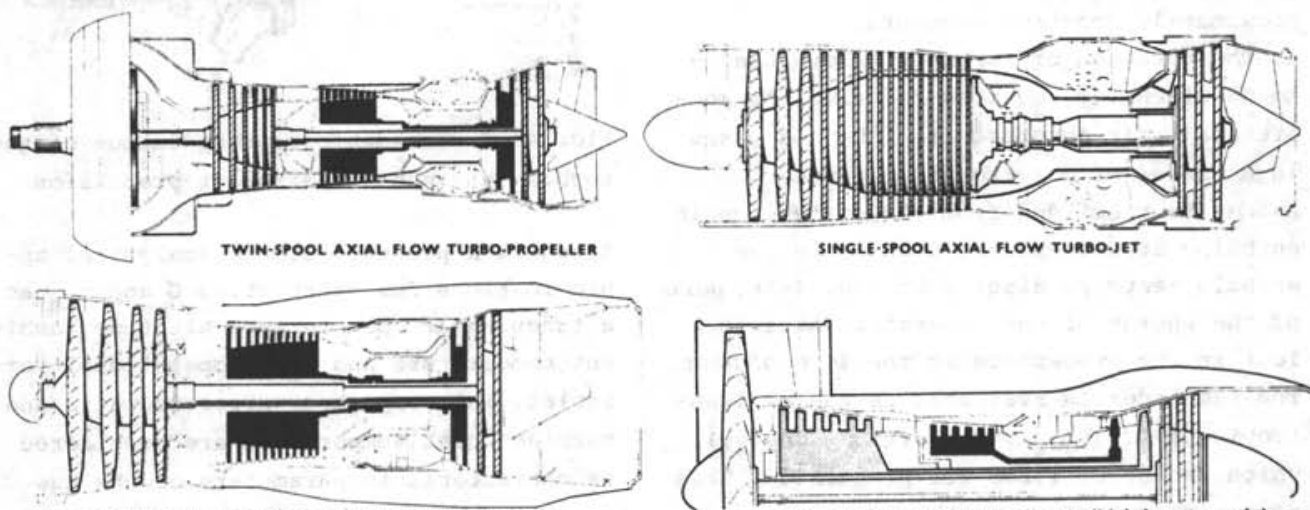

TWIN-SPOOL BY-pAss TURBo-jET (low by-pass ratio)

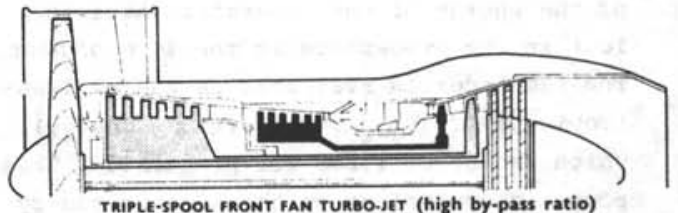

TRIPLE-SPOOL FRONT FAN TURBO-JET (high by-pass ratio)

Fig. 4-15. Aircraft gas turbine engine configurations

spectively. The engine category which only supplies power from the shaft (turboshaft engines) will here be regarded as belonging to the turboprop category. Engines with afterburning are mainly installed in aircraft designed for supersonic cruising speeds and military aircraft which must be capable of short bursts of speed. These types will not be dealt with here.

In comparing gas turbine engines it is useful to split them up as follows:

1. the gas producer or gasifier (gas generator) which is the source of gases with high energy,

2. the propulsive device which is the means to transform the energy of the gases into useful propulsive power (thrust times speed).

\subsubsection{The gas producer}

The gas producer is the essential element of the engine in which a continuous self- sustaining process takes place in the production of hot gases according to the Brayton cycle (Fig. 4-16). The component cycles take place in the following main elements :

1. The entry duct in which atmospheric air

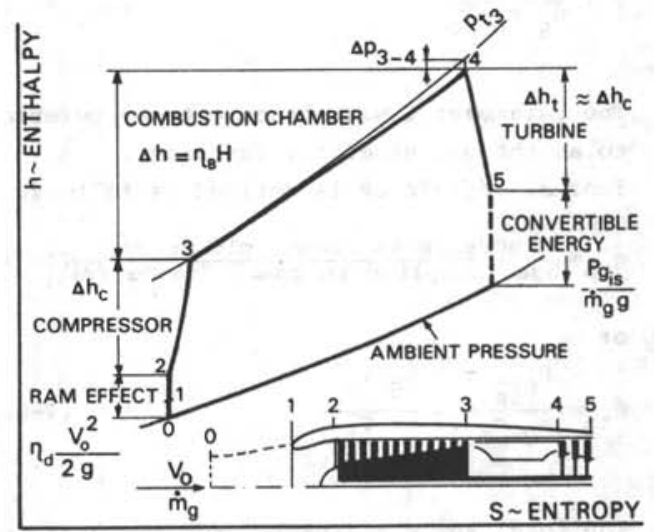

Fig. 4-16. Gas generator thermodynamic cycle 
is accelerated or decelerated with a minimum of loss to the required inlet velocity of the compressor.

2. The compressor (or compressors) in which the air is usually compressed in a number of stages in a polytropic process.

3. The combustion chamber (or chambers) in which fuel is injected and ignited at approximately constant pressure.

4. The turbine (or turbines) where the amount of energy required to drive the compressor(s) is absorbed from the hot gases in a number of polytropic processes.

As may be concluded from the difference in enthalpy between points 0 and 5 in the enthalpy-entropy diagram in Fig. 4-16, part of the energy of the generator gases is lost in the atmosphere in the form of heat. The remainder is available as useful isentropic power $\left(\mathrm{P}_{\mathrm{g}_{\text {is }}}\right.$, convertible energy), which can be utilized for propulsion. This power is equivalent to the kinetic energy which the generator gases would obtain when expanding isentropically to atmospheric pressure.

The thermodynamic performance of the gas generator may be characterized by the specific convertible energy and the thermal efficiency. The specific convertible energy, $\mathrm{P}_{\mathrm{g}_{\text {is }} / \mathrm{m}_{\mathrm{g}}}$, is written in non-dimensional form:

$\mathrm{G}=\frac{{ }_{\mathrm{g}_{\text {is }}}}{\mathrm{m}_{\mathrm{g}}{ }_{\mathrm{p}} \mathrm{T}_{\mathrm{o}}}$

The parameter $\mathrm{G}$ will henceforth be referred to as the gas generator function.

Thermal efficiency is defined as follows: usinativop $\eta_{\mathrm{th}}=\frac{\text { increase in isentropic energy }}{\text { heat supplied in comb. chamber }(\mathrm{s})}(4-7)$

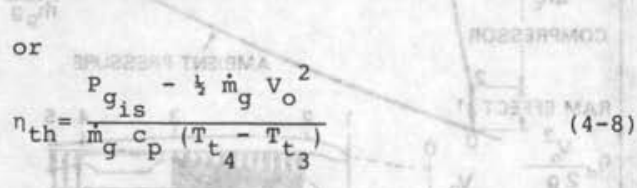

The total temperatures $\mathrm{T}_{\mathrm{t}_{3}}$ and $\mathrm{T}_{\mathrm{t}_{4}}$ refer to the entry to the combustion chambers and that of the turbine (Fig. 4-17).

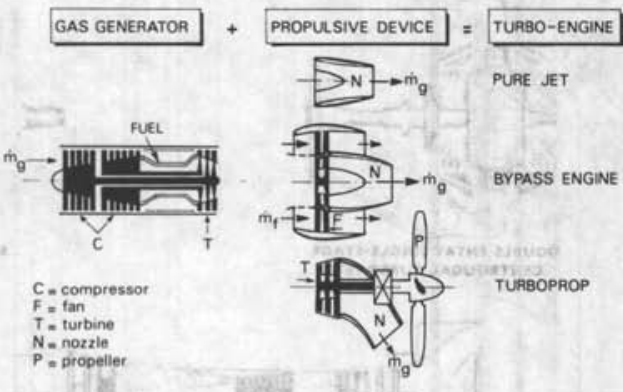

Fig. 4-17. Schematic representation of gas turbine engines for aircraft propulsion

Appendix $H$ presents simple analytical approximations for calculating $G$ and $n_{t h}$ as a function of the pressure altitude, ambient temperature and $M_{0}$ as operational variables. The compressor pressure ratio and turbine inlet temperature are considered as characteristic parameters of the gas generator. Reasonable assumptions have to be made for cycle efficiencies and pressure losses. The thermodynamic calculations which form the basis of these expressions can be found in various publications, e.g. Refs. $4-30$ and $4-39$.

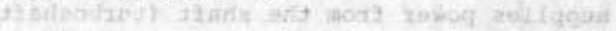

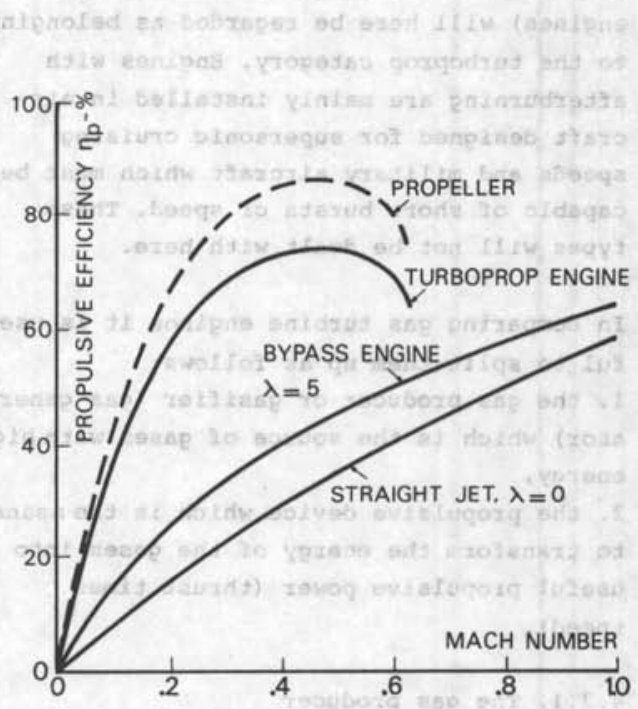

Fig. 4-18. Propulsive efficiency of subsonic turbo-engines 


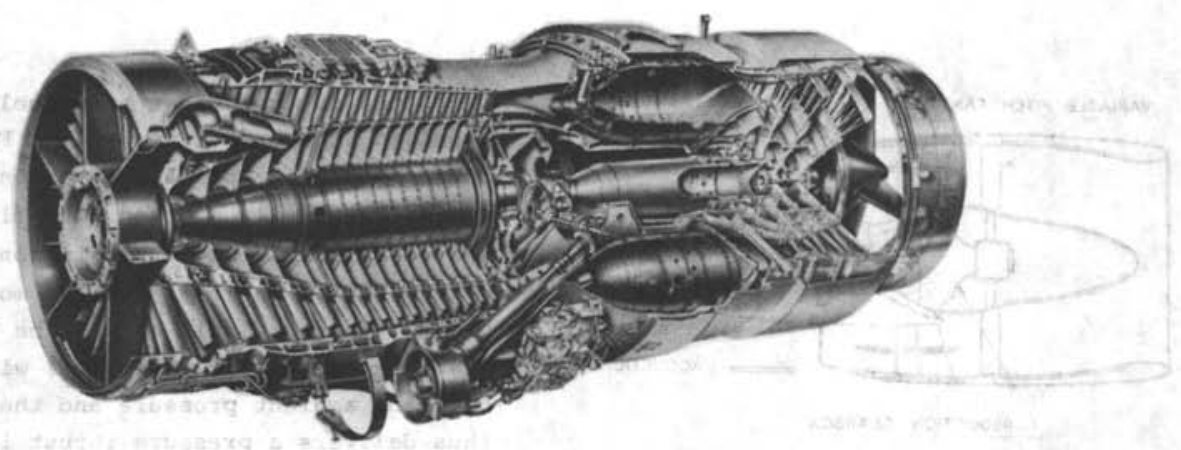

a. Rolls-Royce Avon - straight jet engine

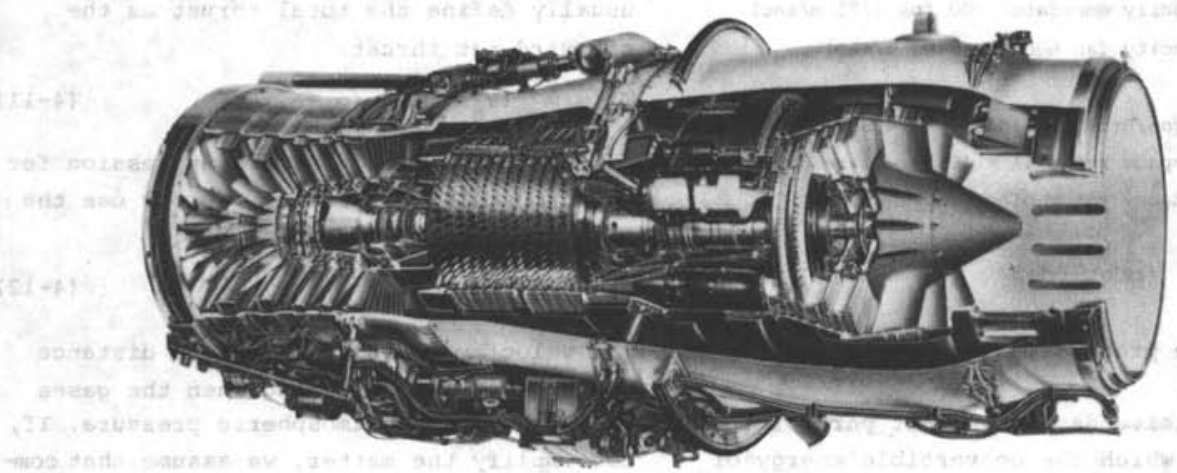

b. Rolls-Royce Spey - low bypass engine $(\lambda=1)$

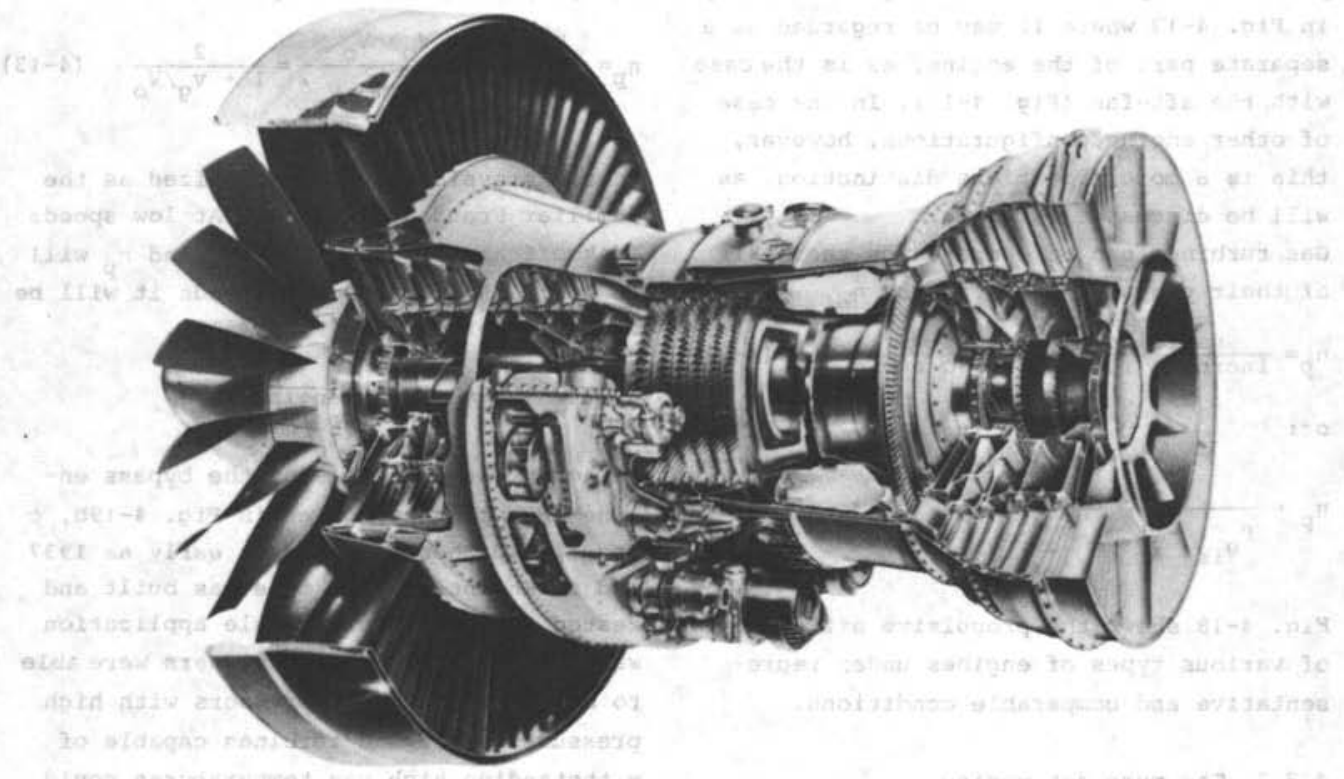

c. Rolls-Royce RB-211 - high bypass engine $(\lambda=5)$

Fig. 4-19. Examples of turbojet engine configurations (data can be found in Table 6-1) 


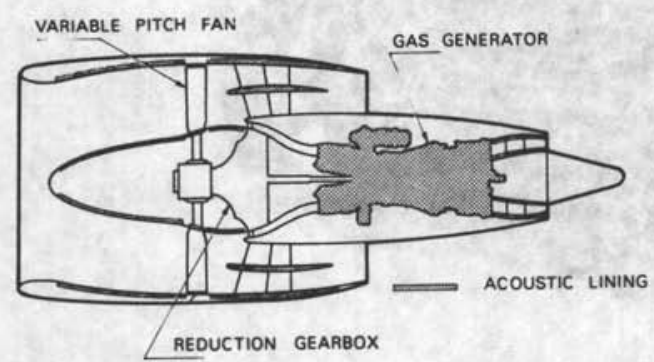

fan tip speed 1,050 fps $(320 \mathrm{~m} / \mathrm{sec})$

exhaust velocity generator $900 \mathrm{fps}(275 \mathrm{~m} / \mathrm{sec})$ exhaust velocity fan $600 \mathrm{fps}(180 \mathrm{~m} / \mathrm{sec})$

d. Ro11s-Royce/Bristol Siddeley M-45S (project)very high bypass ratio $(\lambda=10.5)$; geared fan; variable incidence rotor blades

Fig. 4-19. (concluded)

\subsubsection{The propulsive device}

The propulsive device is that part of the engine in which the convertible energy of the gases is transformed into propulsive power. This part is shown diagrammatically in Fig. 4-17 where it may be regarded as a separate part of the engine, as is the case with the aft-fan (Fig. 4-15). In the case of other engine configurations, however, this is a more fictitious distinction, as will be discussed later on.

Gas turbines can be compared on the basis of their propulsive efficiency ' $p$ '

$\eta_{p}=\frac{\text { useful propulsive power }}{\text { increase in isentropic energy }}$

or:

$\eta_{p}=\frac{T v_{0}}{P_{g_{i s}}-\frac{1}{2} \dot{m}_{g} v_{o}^{2}}$

Fig. 4-18 shows the propulsive efficiency of various types of engines under representative and comparable conditions.

\subsubsection{The pure jet engine}

The propulsive device of a straight jet (example in Fig. 4-19a) is the nozzle in which the exhaust gases are accelerated and expelled rearwards (Fig. 4-17). For subsonic aircraft applications a converging nozzle is usually adopted. The flow in the exit opening of the nozzle is sonic in the design condition (high rpm) of modern turbojets: the nozzle is said to be choked. The local static pressure $\left(\mathrm{p}_{e}\right)$ will be above the ambient pressure and the engine thus delivers a pressure thrust in addition to the impulse thrust. Engine manufacturers usually define the total thrust as the standard net thrust:

$\mathrm{T}_{\text {sn }}=\dot{\mathrm{m}}_{\mathrm{g}}\left(\mathrm{v}_{\mathrm{e}}-\mathrm{v}_{\mathrm{o}}\right)+\mathrm{A}_{\mathrm{e}}\left(\mathrm{p}_{\mathrm{e}}-\mathrm{p}_{\mathrm{o}}\right)$

In order to obtain a simple expression for the propulsive efficiency, we may use the ideal thrust instead:

$\mathrm{T}_{\text {id }}=\dot{\mathrm{m}}_{\mathrm{g}}\left(\mathrm{v}_{\mathrm{g}}-\mathrm{v}_{\mathrm{o}}\right)$

The velocity $v_{g}$ is reached some distance behind the nozzle opening when the gases have expanded to atmospheric pressure. If, to simplify the matter, we assume that complete expansion is an isentropic process, the propulsive efficiency will be:

$n_{p}=\frac{\dot{m}_{g}\left(v_{g}-v_{o}\right) v_{o}}{\frac{1}{2} \dot{m}_{g} v_{g}{ }^{2}-\frac{1}{2} \dot{m}_{g} v_{o}{ }^{2}}=\frac{2}{1+v_{g} / v_{o}}$

This expression may be recognized as the familiar Froude efficiency. At low speeds (takeoff and climb), $v_{g} \gg v_{0}$ and $\eta_{p}$ will be low; at high subsonic speeds it will be of the order of .50 (Fig. 4-18).

\subsubsection{The turbofan engine}

Although the principle of the bypass engine (examples are given in Fig. 4-19b, c and d) had been patented as early as 1937 and an engine of this type was built and tested in $1946^{*}$, large-scale application was only possible when designers were able to build efficient compressors with high pressure ratios and turbines capable of withstanding high gas temperatures could

* The Metropolitan Vickers F 2/3 aft-fan engine 
be designed. The pure jet engine was the only turbojet engine available for transport aircraft up to around 1960 , but in later years it has been entirely replaced by the bypass engine as far as civil aviation is concerned.

The propulsive device of the bypass engine (Fig. 4-17) consists of the following elements:

1. a low pressure turbine which absorbs the energy from the generator gases and transmits it through a shaft or directly to 2. a low pressure compressor (fan) which compresses cold bypass air,

3. jet nozzles, both for the generator exhaust (hot flow) and for the bypass air (cold flow). A modest gain in thrust can be obtained for bypass engines by mixing the hot and cold flows in a special mixing device.

Since in the turbofan the convertible energy is spread over a greater quantity of air than in the case of the pure jet engine, the mean exhaust veloclty will be lower and, according to $(4-13)$, the propulsive efficiency higher (Fig. 4-18). However, the transmission of energy from the hot to the cold flow will entail losses, so that an improvement will only be achieved when the gas generator specific output is sufficiently high. It can be shown that useful application of the bypass principle is dependent on the fundamental condition that:

$G>\frac{y-1}{2} m_{0}^{2} \frac{n_{d}}{n_{t f}{ }^{2}}$

Here $\eta_{d}$ is the isentropic inlet efficiency of the fan (cf. Fig. 4-16) and $n_{t f}$ is the efficiency of the transmission of energy from the tot to the cold flow $\left(n_{t f}=n_{t} \times n_{f}\right)$. With modern gas generators this condition is easily met at subsonic speeds, but at su ersonic speeds this will not always be the case.

The most important parameter of the bypass engine is the ratio of the mass flows per unit time through the fan $\left(\dot{m}_{f}\right)$ and through the gas generator $\left(\dot{\mathrm{m}}_{\mathrm{g}}\right)$; the bypass ratio is $\lambda=\frac{\dot{\mathrm{m}}_{\mathrm{f}}}{\dot{\mathrm{m}}_{\mathrm{g}}}$
MEDIUM EYPASS RATIO
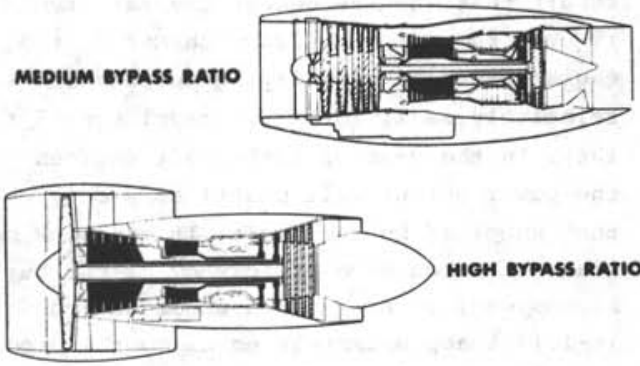

Fig. 4-20. Configuration differences of low/medium and high bypass engines

Obviously, for straight jet engines $\lambda=0$. The following distinction is often made (Fig. 4-20)

a. Engines with a low bypass ratio $(\lambda<$ about 2): low to medium bypass engines. The bypass air is completely ducted around the core engine and is sometimes also mixed with the hot gases.

b. Engines with a high bypass ratio $(\lambda>$ about 2). Engines of this type are generally fitted with a relatively short fan cowling, though the desirability of installing noise suppression material (acoustic lining) favors the use of a longer duct.

The standard net thrust of a bypass engine is defined as:

$\mathrm{T}_{\mathrm{sn}}=\sum_{\mathrm{g}, \mathrm{f}}\left\{\dot{\mathrm{m}}\left(\mathrm{v}_{\mathrm{e}}-\mathrm{v}_{\mathrm{o}}\right)+\mathrm{A}_{\mathrm{e}}\left(\mathrm{p}_{\mathrm{e}}-\mathrm{p}_{\mathrm{o}}\right)\right\}$

in which the mass flows and exhaust conditions refer to those of the gas generator (g) and fan air (f).

\subsubsection{The turboprop engine}

In this type of engine the propulsive device (Fig. 4-17) consists of:

1. the power turbine,

2. the propeller shaft plus reduction gear,

3. the propeller,

4. the exhaust nozzle for the engine gases. Most of the energy derived from the gases is used to drive the propeller. The propulsive power from the exhaust gases is about 10 to 208 of that of the shaft, with the 
result that when we deduct the ram drag ( $\dot{m}_{\mathrm{g}} \mathrm{V}_{\mathrm{o}}$ ) the net propulsive thrust of the engine gases at high flying/speeds will be relatively small (order of magnitude: 5 to $10 \%)$. In the case of turboshaft engines the power output will practically only be that supplied by the shaft. It can be shown that the propulsive efficiency of the turboprop engine, as defined according to $(4-10)$ is approximately equal to the product of the turbine efficiency, the mechanical efficiency of the reduction gear and the propeller efficiency:

$n_{p}=n_{t} n_{\text {mech }} n_{\text {prop }}$

At flying speeds up to $M_{0}=.5$ to .6 the propulsive efficiency of the turboprop engine will be superior to that of turbojet engines (Fig. 4-18). With increasing speeds, however, critical compressibility phenomena will occur at the propeller tips and propeller efficiency will deteriorate progressively.

The total power of the turboprop engine is often expressed as Equivalent Shaft Horse Power (ESHP):

$P_{\text {eq }}=P_{b r}+\frac{T_{j} V_{o}}{n_{\text {prop }}}$

Since in the static condition $\mathrm{V}_{0}=n_{\text {prop }}=$ 0 , the second term is indeterminate, and the ESHP is sometimes expressed as follows: $\mathrm{P}_{\mathrm{eq}}=\mathrm{P}_{\mathrm{br}}+\frac{\mathrm{T}_{\mathrm{j}}}{2 \cdot 5}\left(\mathrm{~T}_{\mathrm{j}}\right.$ in $1 \mathrm{~b}, \mathrm{P}_{\text {eq }}$ in $\left.\mathrm{hp}\right)$

Here the thrust of the engine gases is compared to that of a propeller which has a static thrust of $2.5 \mathrm{lb}$ per shaft horsepower.

\subsubsection{Overall efficiency, specific fuel} consumption and specific thrust (power)

The definitions given above of the thermal efficiency and propulsive efficiency are logical as they enable us to compare different types of engine with the same gas generator on the basis of their propulsive. devices.

The distinction between the gas generator and the propulsive device may, however, be fictitious where the actual engine hardware is concerned. The outer part of the fan - which compresses cold air - forms part of the propulsive device, while the inner part should be regarded as part of the gas generator. Also, the low pressure turbine supplies power to both the fan and the gas generator. The total pressure ratio of the bypass engine, therefore, is the product of the pressure ratios across the fan and across the high-pressure compressor when the fan is mounted ahead of the compressor.

In some publications dealing with the bypass engine the propulsive efficiency is put equal to the Froude efficiency, assuming one (average) exhaust. velocity. In that case, however, the thermal efficiency will have to be corrected for losses in the fan and the low-pressure turbine.

The overall or total efficiency is defined as follows:

$\eta_{\text {tot }}=\frac{\text { useful propulsive power }}{\text { heat content of the fuel }}=\frac{T V_{O}}{\dot{W}_{F} H}(4-20)$ where $\mathrm{H}$ is the heating value of the fuel. The overall efficiency may be split up into combustion efficiency,

$n_{B}=\frac{\text { heat supplied in combustion chamber(s) }}{\text { heat content of the fuel }}$

and the thermal and propulsive efficiencies given by $(4-7)$ and $(4-9)$. Hence the following will apply to the product:

$n_{\text {tot }}=n_{B} n_{\text {th }} n_{p}$

The specific fuel consumption is often used to represent the efficiency of the engine. For turbojet engines the Thrust spe. cific Fuel Consumption (TSFC) is defined as follows:

$C_{T}=\frac{\text { fuel consumption per hour }}{\text { net thrust }}$ 
while the Power Specific Fuel Consumption (PSFC) is used for turboprop engines:

$C_{p}=\frac{\text { fuel consumption per hour }}{\text { shaft (or equivalent) power }}$

Since-fuel consumption is a direct indication of the quantity of heat supplied to the engine, we may deduce from $(4-20)$ and $(4-23)$ :

$C_{T}=$ constant $\frac{V_{0}}{n_{\text {tot }}}$

where $\mathrm{C}_{\mathrm{T}}$ is in $1 \mathrm{~b} / 1 \mathrm{~b} / \mathrm{h}$ (or $\mathrm{kg} / \mathrm{kg} / \mathrm{h}$ ) and the conversion constant is equal to 7.820 when $\mathrm{V}_{\mathrm{O}}$ is in $\mathrm{kts}$ and $\mathrm{H}$ in $\mathrm{BTU} / 1 \mathrm{~b}$ or equal to 8.435 when $V_{0}$ is in $\mathrm{m} / \mathrm{s}$ and $H$ in $\mathrm{kcal} / \mathrm{kg}$. Knowing that the speed of sound at sea level is equal to $661 \mathrm{kts}(340.43 \mathrm{~m} / \mathrm{s})$ and assuming a heating value of $18,550 \mathrm{BTU} / \mathrm{lb}$ $(10,300 \mathrm{kcal} / \mathrm{kg})$, the corrected $\mathrm{TSFC}\left(\mathrm{C}_{\mathrm{T}} /\right.$ $\sqrt{\theta}$ ) can be shown to be given by:

$\frac{C_{T}}{\sqrt{\theta}}=.2788 \frac{M_{0}}{n_{\text {tot }}}$

where $\theta=$ the relative ambient air temperature. The specific fuel consumption in cruising flight is a figure of merit of the engine which determines the quantity of fuel required to overcome the drag. In the case of the straight turbojet the use of $\mathrm{C}_{\mathrm{T}}$ has the advantage that it varies but little with speed. As opposed to this, for turbofan engines the variation with speed will be greater with increasing bypass ratio. Turbofan engines may be regarded as being situated midway between the straight jet engine and the turboprop engine, as the fuel consumption of the latter is related to the power output and also varies little with the speed.

The specific thrust of a jet engine indicates how much thrust the engine develops for a given quantity of air flowing through it per unit of time:

$\frac{T}{\dot{W}}=\frac{\text { net thrust }}{\text { weight of airflow per unit of time }}$
The specific thrust is also equal to the product of the fuel/air ratio divided by the specific fuel consumption. When the specific thrust is high, a relatively. small inlet diameter will be needed in order to produce the required thrust and the weight and drag of the powerplant will generally also be low. This conclusion only applies, however, when engines are compared which have a comparable mechanical configuration.

For a single-flow engine it is possible to derive a relation between the specific thrust and the propulsive efficiency, by combining $(4-12),(4-13)$ and $(4-27)$ :

$\frac{T}{\dot{W}}=\frac{2 v_{0}}{g}\left(\frac{1}{n_{p}}-1\right)$ for $\lambda=0$

Jet engines with a relatively high propulsive efficiency will therefore have a low specific thrust at a given flying speed. The same conclusion is also valid for turbofan engines. It follows that when an engine is being designed or selected for a specific aircraft, a compromise between contradictory requirements will always be unavoidable. In the case of long-range aircraft a low fuel consumption (i.e. a high value of $\eta_{p}$ ' will be regarded as essential. When a high momentary performance is desired, however, a high thrust for a limited engine size must be achieved and it will be necessary to choose a high value of the specific thrust.

In the case of turboprop engines, the power may be related to the air mass flow through the engine. The specific power is maximum when $G^{*}$ is maximum, but this condition does not result in a minimum for the specific fuel consumption, so here again a compromise will have to be sought.

4.3.7. Analysis of the engine cycle

When an off-the-shelf engine is not available for a particular aircraft design, or when parametric studies are being carried out, it may be desirable to make a cycle

*Section 4.3 .1 
analysis of the engine, taking various engine design parameters as variables. Engine manufacturers have computer programs for performing these studies, and the same applies to some aircraft companies. Methods are also presented in the relevant $11 \mathrm{t}-$ erature (Refs. 4-25, 4-26, 4-29 and 4-50). In Appendix $\mathrm{H}$ a summary is given of analytical expressions which, in spite of the simplifications introduced, are suitable for making an initial estimate of engine performance in preliminary aircraft design. This method has been used for the generalized calculations which follow. When engine performance is calculated a distinction can be made between:

1. performance at the design point,

2. off-design performance.

Design point is intended to indicate the working condition at high rpm where the efficiencies of the compressor and turbine are optimum. The design point may be chosen for a representative situation, such as the takeoff, climb or cruise. In this way the engine can be adapted as far as possible to its use in the aircraft to match the most critical performance requirement. In the case of the straight jet engine, the design point is generally assumed as the takeoff, whereas the most critical condition for high bypass engines will often be the cruising flight.

\subsection{ASSESSMENT OF TURBOJET ENGINES}

The most important design parameters determining engine performance are:

1. Cycle efficiencies and pressure losses

2. Overall Pressure Ratio of the gas generator-compressor (OPR, $\varepsilon_{c}=p_{t_{3}} / p_{t_{2}}$ )

3. Turbine Entry (Intake) Temperature (TET, TIT, $\mathrm{Tt}_{4}$ )

4. Bypass ratio $(\lambda)$

5. Fan Pressure Ratio (FPR, $\varepsilon_{f}$ )

As shown by Fig. 4-21, an improvement in the process efficiencies is an effective method of enhancing engine performance. In the simplified example given, a 5 i improve-

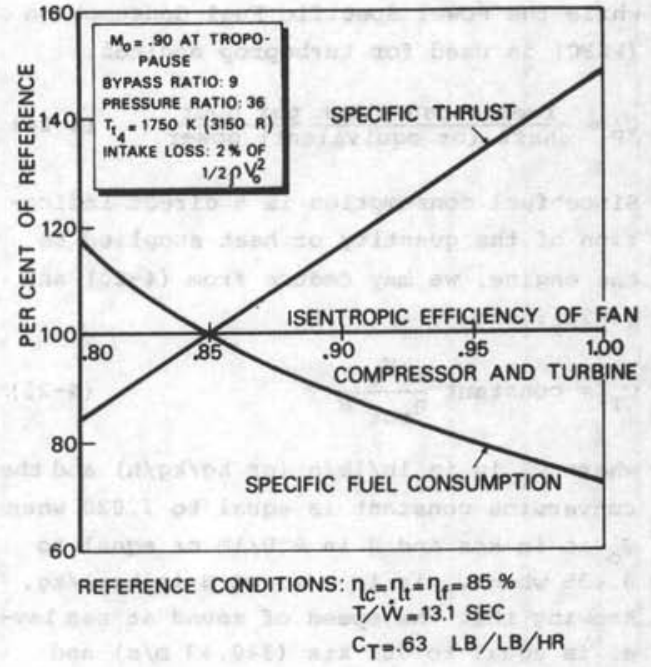

Fig. 4-21. Effect of cycle efficiency variation on turbofan performance

ment in all efficiencies (e.g. 858 to 908 ) will result in an 118 decrease in fuel consumption and a $16 \%$ increase in specific thrust. A high bypass engine will be more sensitive to increase in efficiency than a low bypass engine. It should be noted, however, that this 58 improvement in efficiency is equivalent to a reduction in losses by one-third. The ways in which the engine manufacturer can reduce these losses fall outside the scope of this book. We shall now explain the influence of the other parameters relating to

1. the fuel consumption in cruising flight, 2. the specific thrust under various conditions and the decrease of thrust with altitude and speed (thrust lapse rates), 3. the weight and drag of the powerplant as installed in the aircraft,

4. the noise production of the engine. The initial cost, maintenance and service life are very important factors influencing the choice of an engine, but these will on$1 y$ be dealt with incidentally, as insufficient data are available to enable us to determine conclusively how far they are affected by the engine design. The only general rule is that engine price and mainte- 


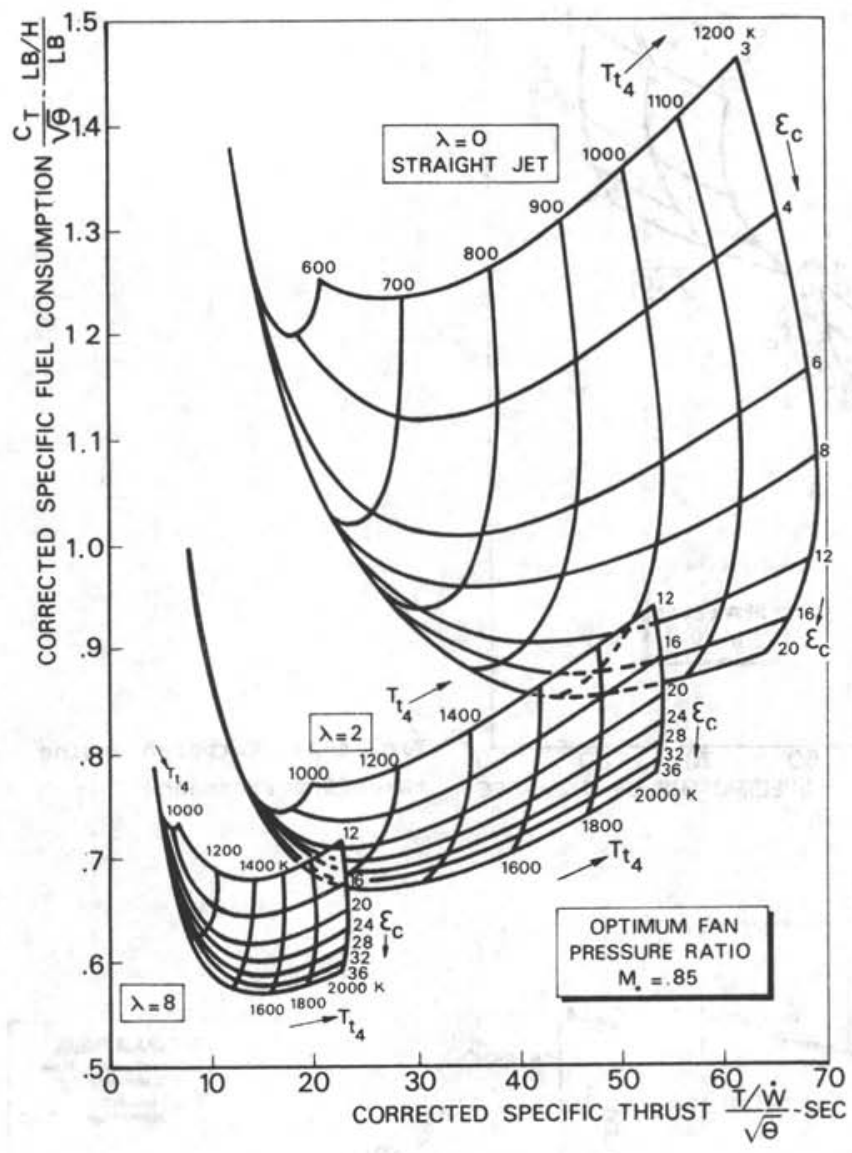

NOTE: This diagram is drawn for the tropopause, but is equally valid for other altitudes, provided $\mathrm{T}_{\mathrm{t}_{4}}$ is chosen so that the same value of $\mathrm{T}_{\mathrm{t}_{4}} / \mathrm{T}_{\mathrm{o}}$ is obtained

Fig. 4-22. Effect of $O P R$ and TET at cruising conditions nance cost increase with complexity.

\subsubsection{Overall Pressure Ratio}

When a cycle analysis is carried out on turbofan engines with varying working pressures and temperatures at the design condition, a convenient representation of the results can be given in the form of Figs. 4-22 and 4-23. The corrected specific thrust and fuel consumption have been used in order to make the result valid for different altitudes. This figure shows that in all configurations an increase in OPR leads to a reduction in the specific fuel consumption in cruising flight.

In order to obtain a high specific thrust, either in cruising flight (Fig. 4-22) or during takeoff (Fig. 4-23), a high value for the OPR is not required at the given TET. In the case of the pure jet engine, for example, with TET $=1,000$ to $1,200 \mathrm{~K}$ in cruising flight, the condition for maximum specific thrust is $\varepsilon_{c}=7$ to 9 and in the takeoff between 9 and 11. In the case of turbofan engines these values will be slightly higher, say 12 to 16 at the generally acceptable turbine temperatures (Section 4.4 .2 ). In view of the fact that - within certain limits - an increase of OPR over these optimum values has little influence on the specific thrust, the designer generally aims at higher pressure ratios which lead to a better fuel con- 


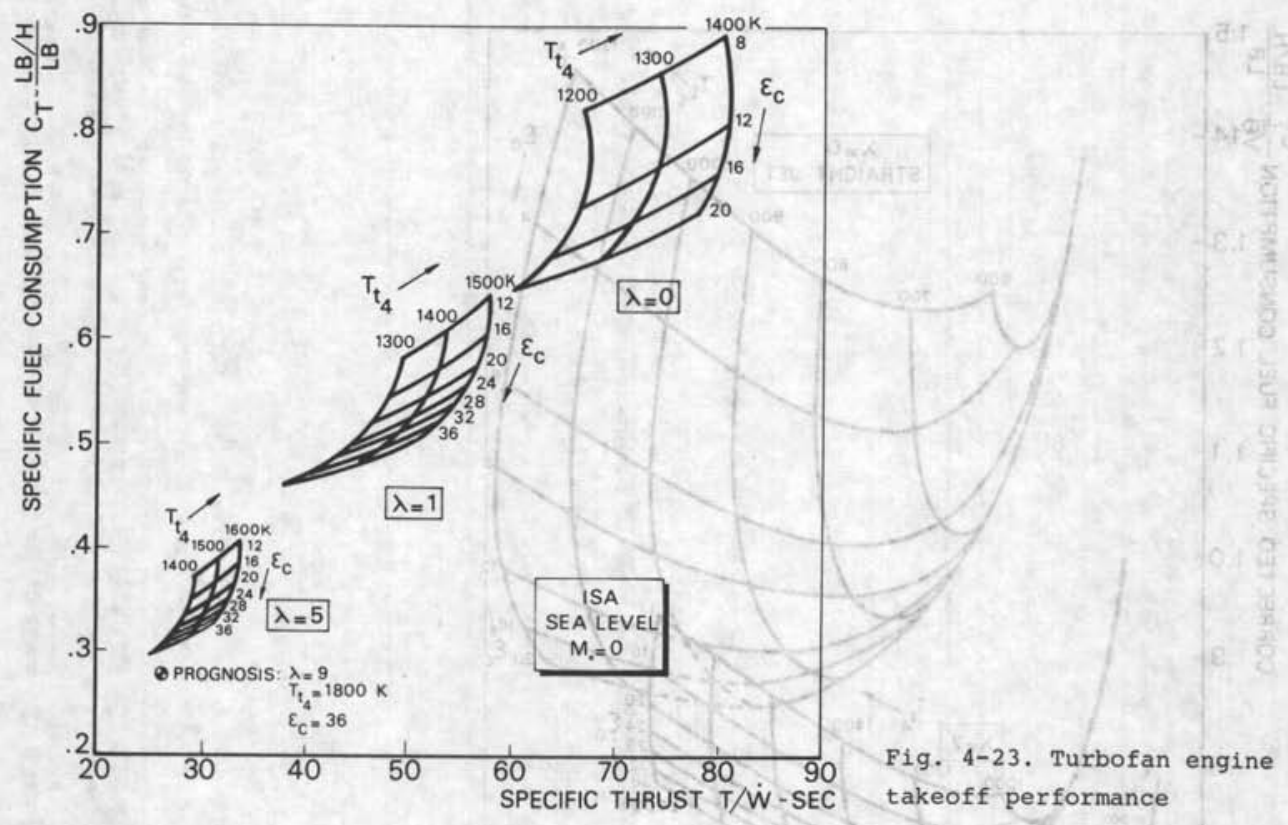

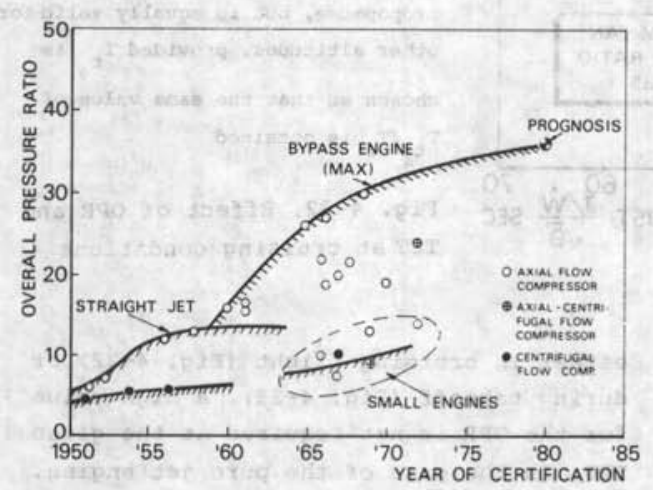

Fig. 4-24. Historic development of the OPR (References: Jane's All the World's Aircraft, various engine manufacturers' data) sumption in the design point and also at reduced $\mathrm{rpm}$. Up to about 1953 jet engines were designed with a single stage centrifugal compressor (Fig. 4-15) which gave a maximum OPR of around four. As these engines had a high fuel consumption and large frontal area, they have been superseded by engines with an axial compressor (Figs.

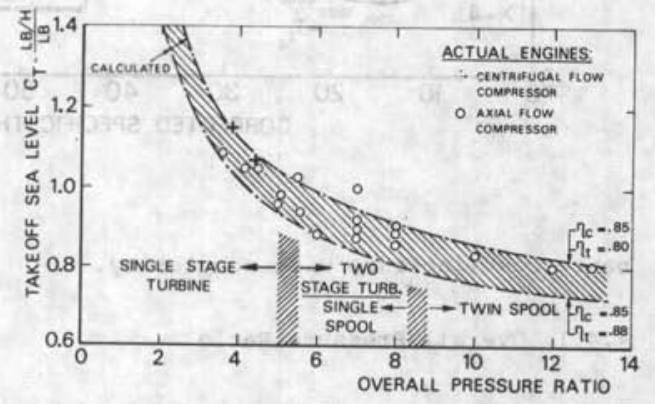

Fig. 4-25. Specific fuel consumption of straight jet engines at sea level

4-15 and 4-19a). There are some recent applications of combined axial and centrifugal compressors on relatively small engines, such as the Garret Airesearch ATF-3, which have quite acceptable pressure ratios and a small frontal area.

Axial compressors make it possible to obtain large pressure ratios and a small 
frontal area. A value of $\varepsilon_{c}=8.5$ is still possible with a single compressor and single spool. When this value is exceeded, the design conditions for the foremost and rearmost compressor stages are so far apart, that it will be necessary to employ two compressors, running at different rpm: twin spool engines.

The highest value for the OPR actually used in straight jet engines is about thirteen. Fig. 4-24 shows how the pressures have risen during the fifties. Fig. $4-25$ gives an impression of the influence of OPR on $\mathrm{C}_{\mathrm{T}}$ during takeoff. The actual fuel consump$t i o n$ of a number of engines is compared with calculated values.

The tendency towards high pressure ratios has been continued in the turbofan engine up to values of 25 to 30 in the case of the latest generation for large transport aircraft. Fig. 4-26 gives a schematic repre-

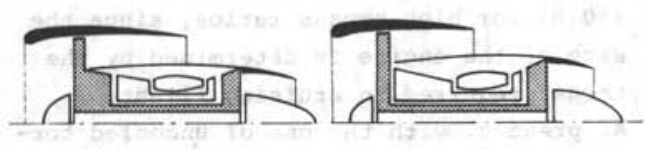

A. Twin spool; fan com- B. Twin spool; separate bined with low pressure fan section of compressor

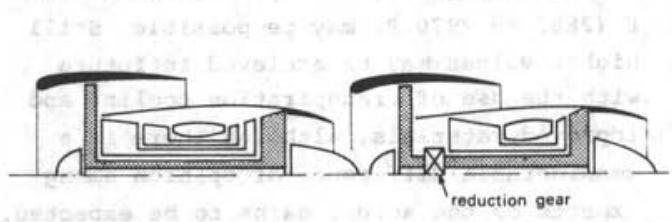

C. Triple spool

D. Twin spool; geared fan

Fig. 4-26. Principle of various fan-compressor configurations of high bypass engines

sentation of some engine configurations in which these high pressure levels can be achieved.

Layout $A$ has the disadvantage that the last stages of the low pressure compressor contribute little to the increase in pressure, as they operate at relatively low tip speeds.
Layout B does not have that disadvantage, but demands a very high pressure ratio from the high pressure part, which entails the use of variable incidence guide vanes. Layout C, employing three shafts, is attractive for various applications, since a relatively small pressure ratio is demanded from each of the compressors (Ref. 4-34). The specific weight is lower than that of the others.

Layout $D$, the two-shaft engine with geared fan (see also Fig. 4-19d) appears to be particularly suitable for relatively small engines where the power to be transmitted by the reduction gear is limited. The reduction results in a low fan tip speed, while the turbine is still working at a relatively high tip speed, resulting in a compact turbine. For several existing or projected geared fan engines the gear ratio amounts to between .25 and .50 .

From Fig. 4-22 it is seen that for an engine with $\lambda=8$, for example, a pressure ratio increase of up to 36 will only give a modest gain in the specific fuel consumption. At these high pressures the temperature at the rearmost compressor blades becomes so high that the material properties will deteriorate. For a future generation of large engines for long-range transport aircraft $\varepsilon_{c}=36$ may possibly prove to be the limit (Fig. 4-24), but there is also a notable tendency towards considerably lower values in order to achieve simplicity coupled with low cost. In the future we may expect a range of values between $\varepsilon_{c}=12$ and 40 , depending upon the use for which the engine is designed. The author's forecast is as follows:

- small executive aircraft, feederliners:

12 to 14

- short-range transport aircraft: 20 to 25

- long-range transport aircraft: 35 to 40

\subsubsection{Turbine Entry Temperature}

Returning to Fig. 4-22, the following may be observed: 
a. Eor a given bypass ratio a TET value which gives a minimum $C_{T}$ can be found for every OPR. This is henceforth indicated as the "optimum" TET.

b. The optimum TET increases with increasing bypass ratio, the reason being that for high $\lambda$ the propulsive efficiency is not so sensitive to increasing TET as it is in the case of low bypass engines (Fig. 4-27).

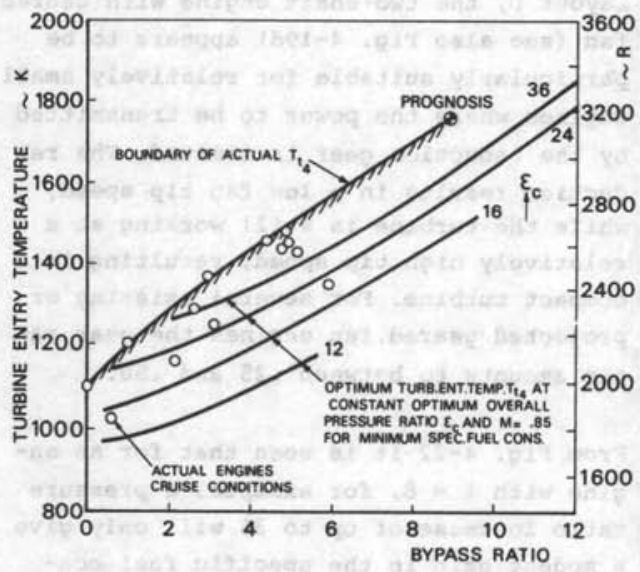

Fig. 4-27. Actual and optimum TET in cruising flight (civil engines)

c. At high bypass ratios, TET may be varied within fairly wide limits above or below the optimum value without greatly affecting $C_{T}$. At $\lambda=8$ and $\varepsilon_{C}=28$, the TET will be optimum at $1500 \mathrm{~K}(2 / 00 \mathrm{R})$ in the example presented. However, when the TET is chosen 108 lower $(1350 \mathrm{~K}, 2430 \mathrm{R})$ it will cost only about 18 in $\mathrm{C}_{\mathrm{T}}$.

d. For all combinations considered, a high TET is favorable for obtaining a high specific thrust.

The maximum TET values used in actual engines for cruising conditions (Fig. 4-27) are generally higher than the "optimum" values. On the one hand, a high specific thrust has the advantage of low engine weight and installed drag, while, on the other, it will mean that in normal longrange cruise the engine will not operate at the maximum permissible cruise TET, but at a lower value which is closer to the op- timum for low fuel consumption and long operating $11 \mathrm{fe}$.

Maximum values for TET are higher during takeoff than in cruise (Fig. 4-28) as they

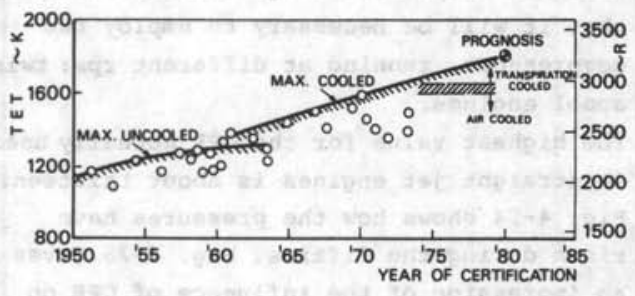

Fig. 4-28. Historic development of maximum TET during takeoff (References: engine manufacturers' data)

will generally be required only for a $11 \mathrm{~m}-$ ited period of time. In the case of low bypass engines, the difference will be in the region of 150 to $200 \mathrm{~K}$ ( 270 to $360 \mathrm{R})$, but they will be less ( 50 to $100 \mathrm{~K}, 90$ to $180 \mathrm{R})$ for high bypass ratios, since the size of the engine is determined by the thrust required in cruising flight.

At present, with the use of uncooled turbine blades, temperatures of about $1200 \mathrm{~K}$ $(1260 \mathrm{R})$ may be regarded as permissible for short periods. When they are aircooled temperatures of up to 1600 to 1650 K ( 2880 to 2970 R) may be possible. Still higher values may be achieved in future with the use of transpiration cooling and improved materials, although there is a considerable difference of opinion among experts on the actual gains to be expected. Looking at the values used in practice over the past years (Fig. i-28), it may be noted that the average increase comes to about $20 \mathrm{~K}(36 \mathrm{R})$ per year. If this trend continues in the future we may expect a generation of large, high bypass engines in the eighties with a TET of $1800 \mathrm{~K}$ ( $3240 \mathrm{R})$ during takeoff and $1750 \mathrm{~K}$ (3150 R) duriny cruise. The data in Ref. 4-48 indicate that these temperatures are feasible, with only moderate quantities of air required for cooling. 


\subsubsection{Bypass ratio}

The choice of this parameter has such a far reaching effect on the design of the engine and its installatioh, that the optimization of $\lambda$ cannot be fully dealt with here, in view of the complicated nature of the problem. Accordingly, we shall confine ourselves to summarizing some of the more important aspects.

a. Design of the fan and low pressure turbine.

The power supplied by the gas generator may be divided in various ways over the hot and cold flows. The division of power can be characterized by the work ratio,

work ratio $=\frac{\text { power transferred to fan }}{\text { convertible energy }}(4-29)$

This ratio is controlled by the pressure ratio of the low pressure turbine and the Fan Pressure Ratio and determines the exhaust velocities imparted to the hot and cold gases. It can be shown (Ref. 4-30) that the propulsive (and overall) efficiency will be maximum when:

$\frac{v_{f}}{v_{g}}=n_{t f}=n_{t} \cdot n_{f} \quad \begin{aligned} & (.70 \text { to } .75 \text { are } \\ & \text { typical values })\end{aligned}$

where $n_{t}$ and $n_{f}$ are the turbine and fan efficiencies respectively. Fig. 4-29 shows

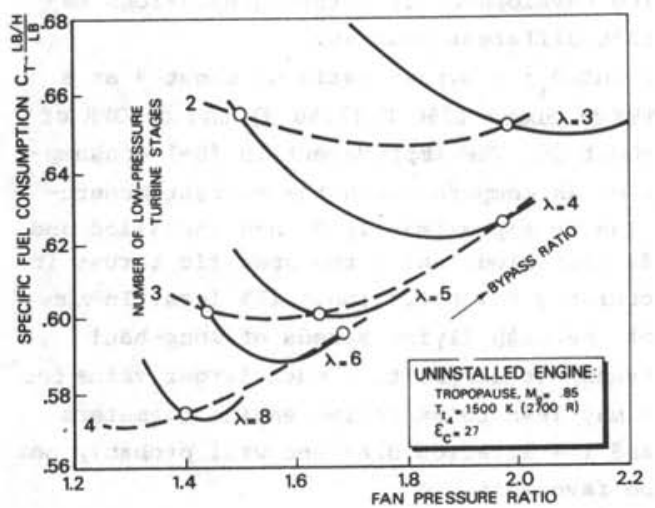

Fig. 4-29. Effect of fan pressure ratio on SFC and number of low-pressure turbine stages that for moderate values of $\lambda$, the Fan Pressure Ratio (FPR) has no great influence on the specific fuel consumption, at any rate within certain limits. If, say, for $\lambda=5$ we take an FPR of 1.55 instead of the thermodynamic optimum of 1.66 , this will result in an increase of only 18 in $C_{T}$. The variation in the power absorbed by the fan, however, is closely related to that of the turbine. In view of the limited pressure ratio per stage, the number of turbine stages will have to be adapted for certain combinations of $\lambda$ and FPR. If we now vary the FPR with a given number of turbine stages, the optimum will be found to lie at another value than in the previous case.

In Fig. 4-30 the optimum FPR is given as a

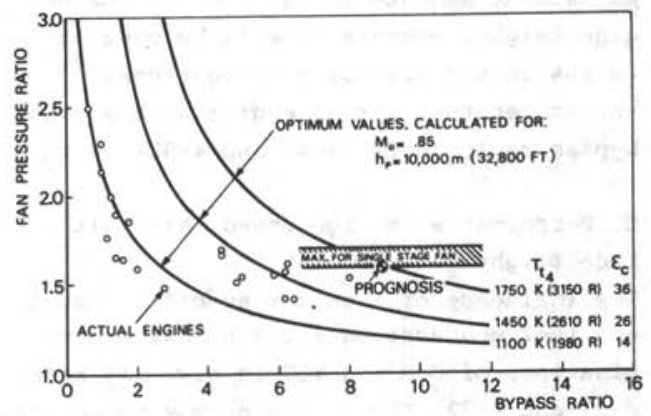

Fig. 4-30. Calculated and actual FPR

function of $\lambda$ for some combinations of OPR and TET, calculated for $M=.80$ with the analytical method given in Appendix $H$. The points plotted in this figure represent values for the takeoff applicable to actual engines; corresponding values for cruising flight are higher (Section 4.4.2). The graph shows that for a given gas generator cycle the optimum value for the FPR decreases with increasing $\lambda$. For high bypass engines this effect will be partly compensated by the higher temperature and pressure levels which will generally be chosen in such cases, resulting in a greater specific convertible power. The maximum value for the FPR with a single stage fan will be about 1.60 to 1.70 . 
When the bypass ratio is increased at a given rpm, the fan diameter and tip speed will also increase. In the case of many modern angines, part of the fan will work at transonic speeds which, amongst other things, will cause an increase in noise level (sub-para. f). This may be improved by using three engine shafts, thus reducing the $\mathrm{rpm}$ of the fan, or reducing fan speed by means of a reduction gear (geared fan, see Fig. 4-19d).

The Dowty-Rotol Company has developed a geared fan with rotor blades which can be adjusted in flight, so that an optimum adjustment may be obtained for various engine settings. Thrust reversal can be achieved by giving the rotor blades a negative pitch. The extra weight of the blade adjustment amounts to about 88 of the engine weight, whereas it will be over 15 to $20 \%$ in the case of a conventional thrust reverser for an engine with a high bypass ratio (Refs. 4-47 and 4-52)

b. Performance in high speed, high altitude flight.

The influence of $\lambda$ on the specific thrust and fuel consumption for a number of combinations of OPR and TET is given in Figs. $4-31$ and $4-32$. The following may be noted:

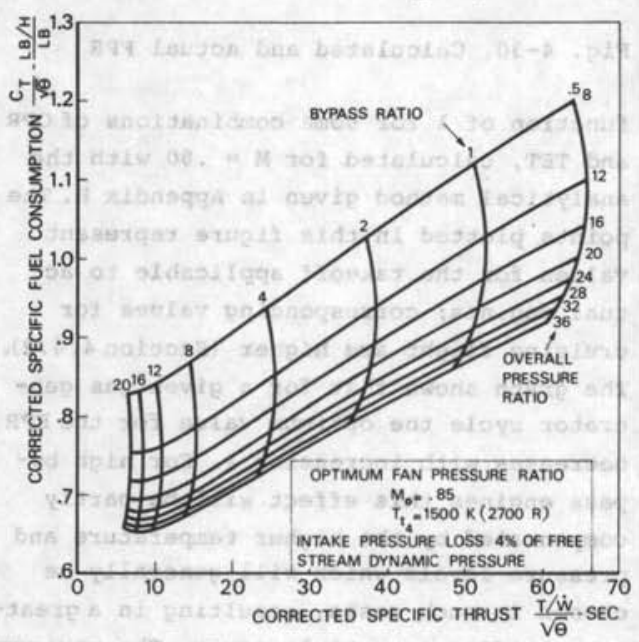

Fig. 4-31. Turbofan performance in cruising flight as affected by $O P R$ and bypass ratio at constant TET
1. In the case of engines without intake and exhaust nozzle losses, $C_{\mathrm{T}}$ will continue to decrease with increasing $\lambda$, provided the optimum FPR is chosen for each configuration. A theoretical minimum will be reached with $\lambda \rightarrow \infty$, corresponding to a propulsive efficiency $n_{t f}=n_{t} \cdot n_{f}$.

2. The specific thrust decreases rapidly with increasing $\lambda$, although this effect may be partly compensated by choosing higher values for the TET.

3. When intake and exhaust nozzle losses are accounted for, there will be a bypass ratio for which $C_{T}$ is minimum. The effect of these losses is greatest when $\lambda$ is large and will even be accentuated under operational conditions by the power extraction required to drive aircraft systems and bleed air to the de-icing and air conditioning systems. The minimum installed $C_{T}$ which may be achieved, and the corresponding value for $\lambda$, are largely dependent on the magnitude of these losses (Fig. 4-33).

4. Fig. 4-32 shows three combinations (points A, B and C), which are representative of different generations of engines, both recent and present, for transport aircraft. A radical improvement in $C_{T}$ has been achieved in the present generation of large engines like the Rolls-Royce RB-211, Pratt and Whitney JT-9D and General Electric CF-6 high bypass ratio turbofan engines.

The development of future generations may take different courses.

point $D_{1}$ : A bypass ratio of about 9 at a TET of about $1750 \mathrm{~K}(3150 \mathrm{R})$ and an OPR of about 36. The improvement in fuel consumption as compared with the current generation is approximately 98 non-installed and 88 installed, while the specific thrust in cruising flight is about 158 less. In view of the high flying speeds of long-haul transport aircraft, a much larger value for $\lambda$ may lead to excessive engine diameters and installation drag and will probably not be favorable.

Point $\mathrm{D}_{2}$ : Very large bypass ratios of, say, 20 to 30 at a TET of $1600 \mathrm{~K}(2880 \mathrm{R})$ and an OPR of 25 . This configuration will imply a 


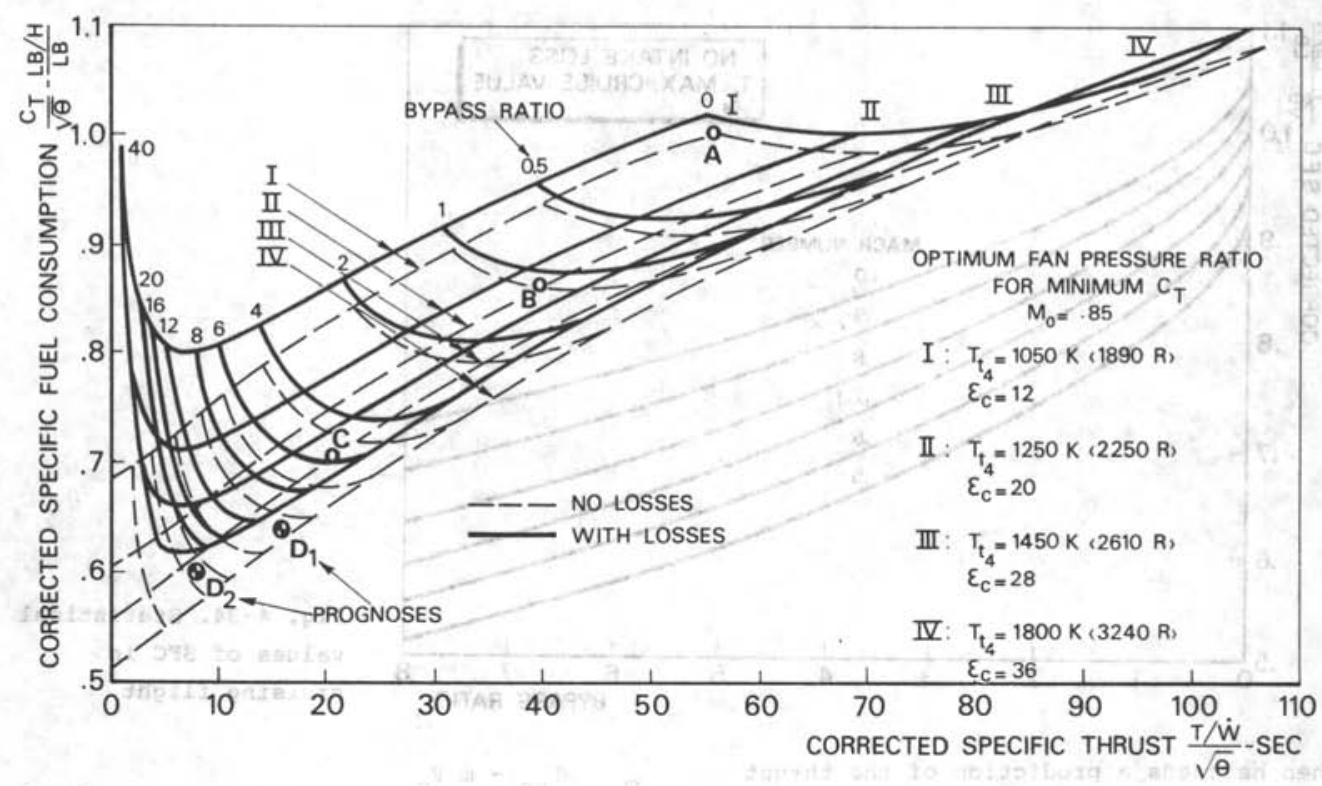

Fig. 4-32. Effect of bypass ratio and intake/exhaust losses on performance in cruising flight:

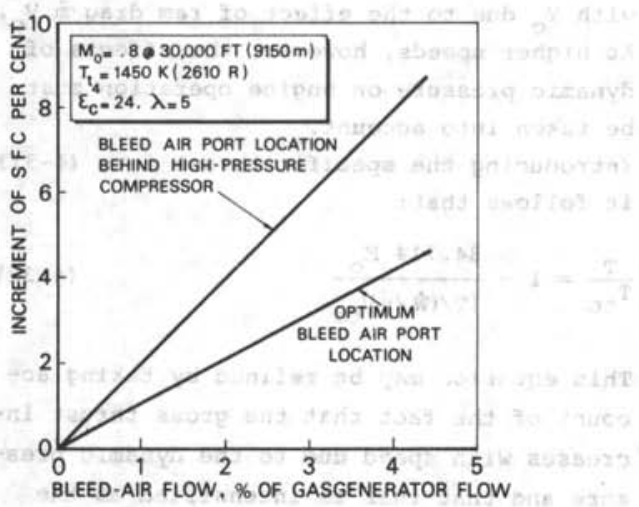

Fig. 4-33. Effect of bleed air takeoff on SFC

very large fan diameter ("prop-fan") and entails the use of a reduction gear. Fuel consumption may be about 158 less at the flying speed chosen for the case in Fig. 4-32, but the gain will be greater as the cruising speed decreases. The drag of these engines increases rapidly with cruising speed.

The engines in this forecast are suitable for different types of aircraft. Type $D_{1}$ may be particularly useful for fast longrange transport aircraft, while type $D_{2}$ may possibly be used on smaller and less rapid short-haul aircraft which should have good takeoff and landing performance and a very low noise level (sub-para. f).

The effect of altitude on engine performance is dependent on many factors. An approximation for the thrust lapse with altitude is occasionally found in the literature, for example:

$\frac{\text { thrust at altitude }}{\text { thrust at sea level }}=\sigma^{n} \quad(n<1) \quad(4-30)$

where both values of the thrust have been defined at the same Mach number and engine rating. Equation 4-30 should be considered as an interpolation method for calculating engine performance at altitudes where the engine manufacturer has not specified the thrust, rather than as a prediction method. The designer should refer to Appendix $\mathrm{B}$ or employ any other suitable method available 


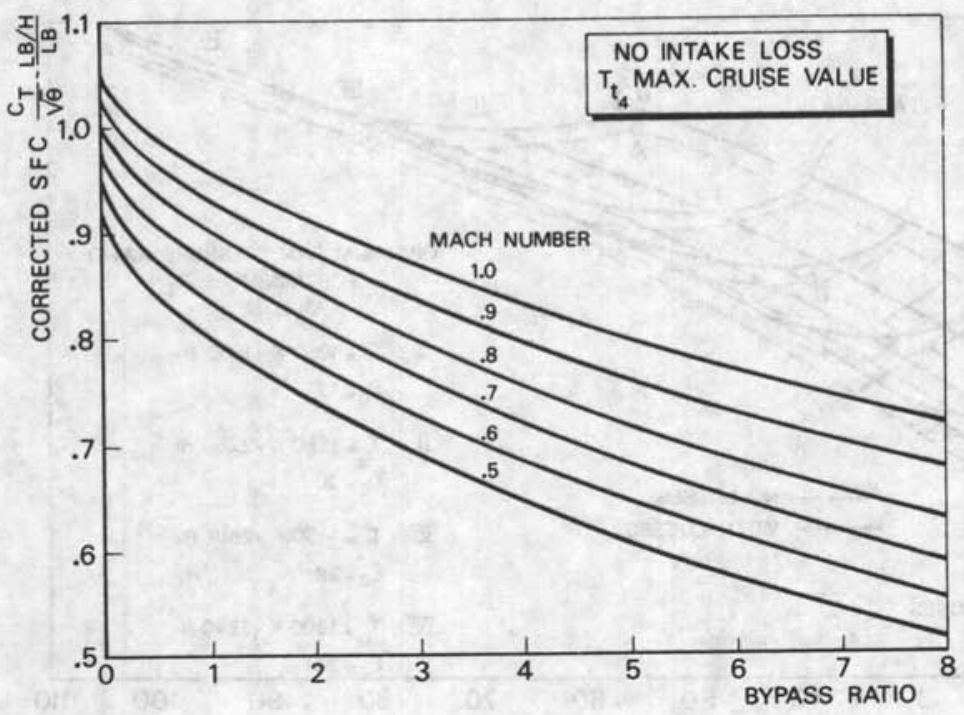

Fig. 4-34. Statistical values of SFC in cruising flight when he needs a prediction of the thrust lapse with altitude.

Although engine performance can easily be predicted by using the method given in Appendix $\mathrm{H}$, the working pressures and temperatures of the engine should either be known or chosen. When the designer is prepared to accept statistical averages for cruising flight, reasonabiy accurate figures can be found from Fig. 4-34 for efficient engines with high OPR. As against this, a comparison with Fig. 4-31 shows that for low OPR values the SFC will be considerably higher.

\section{c. Takeoff performance and other flight} conditions.

The specific fuel consumption is of less importance for performance in takeoff and climb, and we shall therefore pay rather more attention to the thrust lapse with speed, altitudie and engine setting.

If $\mathrm{T}_{\text {to }}$ represents the static thrust at sea level, and if at the same time we assume that the gross thrust and mass flow through the engine do not change appreciably over a speed ranqe of up to about $M=.15$, we may write:
$\frac{T}{T_{\text {to }}}=\frac{T_{\text {to }}-\dot{m} V_{0}}{T_{\text {to }}}$

In this expression $T$ decreases linearly with $v_{0}$ due to the effect of ram drag $\dot{m} v_{o}$. At higher speeds, however, the effects of dynamic pressure on engine operation must be taken into account.

Introducing the specific thrust into (4-31) it follows that:

$\frac{T}{T_{\text {to }}}=1-\frac{34.714 \mathrm{M}_{0}}{(\mathrm{~T} /(\dot{\mathrm{w}} \sqrt{\theta}))_{\text {to }}}$

This equation may be refined by taking account of the fact that the gross thrust increases with speed due to the dynamic pressure and that this is intensified as the bypass ratio increases. Assuming the mass flow through the engine to be constant, we may deduce (Appendix $\mathrm{H}$ ) :

$\frac{T}{T_{\text {to }}}=1-\frac{.45 M_{0}(1+\lambda)}{\sqrt{(1+.75 \lambda) G}}+\left(.6+\frac{.11 \lambda}{G}\right) M_{0}{ }^{2}(4-33)$

Here $G$ is the gas generator function, defined in Section 4.3.1, which will typical$1 y$ be of the order of .9 to 1.2 during takeoff.

From inspection of $(4-32)$, it will be clear that since the specific thrust decreases 
with the bypass ratio (Fig. 4-23), the takeoff thrust of a high bypass engine will decrease more rapidly with speed than is the case with a low bypass engine. Fig. 4-35, which is based on empirical data,

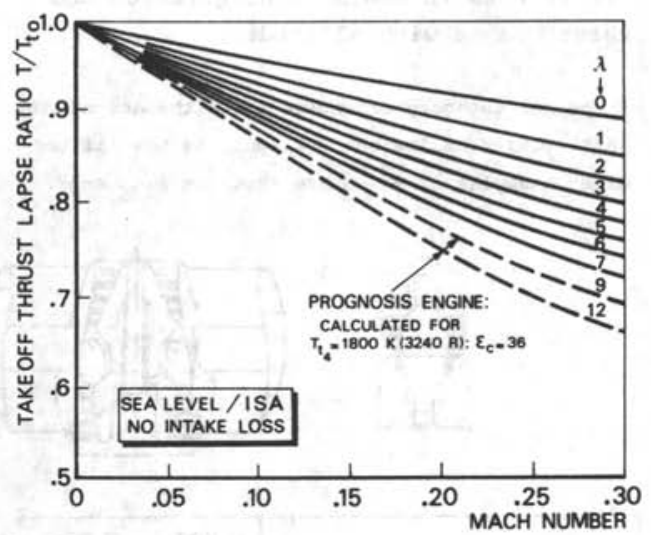

Fig. 4-35. Statistical curves of thrust lapse during takeoff

may be used for performance calculations in preliminary design. It shows the variation in thrust, deduced from engines with $\lambda$ ranging from 0 to 7 . Both curves for $\lambda=9$ and 12 apply to a TET of $1800 \mathrm{~K}(3240$ R) and an OPR of 36 , and are computed with 4-33.

Some of the consequences of the above are as follows:

1. If, for a particular aircraft design, the thrust level is based on a requirement relating to the runway length or climb performance at low flying speed, the aircraft which is fitted with low bypass engines will have more thrust in cruising flight than one with high bypass engines, since the thrust decreases less rapidly with speed and altitude.

2. If two aircraft designs, which in other respects are entirely similar, have the same maximum cruising speed at a certain height, while their engines have the same installed cruise thrust, the high bypass engine will generally result in better takeoff and climb performance.
For given aircraft design requirements for cruise and takeoff, it is possible to find a bypass ratio where the thrust required in both conditions will be matched and this might form a basis for choosing $\lambda$. In actual practice there are still other factors to be considered:

1. Cruising altitude is often a fairly arbitrarily chosen parameter which may still be varied within certain limits.

2. The engine manufacturer can influence the engine thrust lapse to some extent by adaptation of the compressor design point. When, for instance, the emphasis lies on the takeoff requirement, the design point of the compressor is chosen accordingly so that the compressor efficiency will be maximum during takeoff, which implies that it will be lower during cruising flight. When, however, the cruising flight is taken as the sizing condition, the design point will be chosen with this requirement in mind. 3. The engine manufacturer will design the engine for use in several types of aircraft. Once the engine configuration has been chosen it is still possible to influence performance to a limited extent - for example by increasing the TET, which will increase the quantity of air flowing through the gas generator, though the engine will work slightly less efficiently with regard to fuel consumption.

\section{d. Engine weight.}

From the decrease in the specific thrust with $\lambda$ (Fig. 4-23) one might tend to conclude that both weight and drag due to the powerplant installation will increase. It will be seen from Fig. 4-36 that this rea-

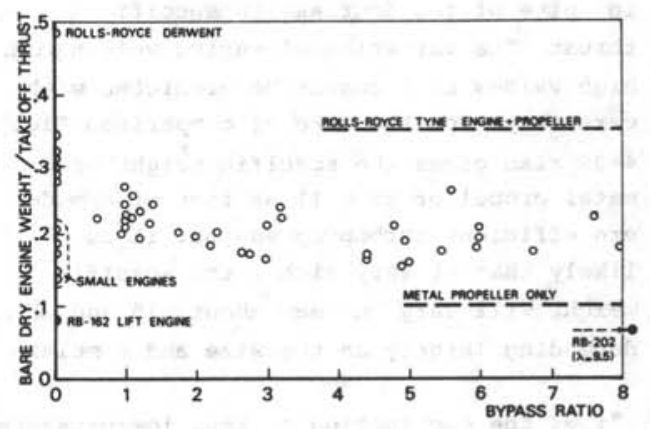

Fig. 4-36. Specific dry weight of turbojet engines. (References Jane's A11 the World's Aircraft, engine manufacturers' data) 
soning is not entirely correct, at any rate where the specific engine weight for takeoff conditions is concerned. This may be explained by assuming that the dry engine weight comprises that of the gas generator and the propulsive device*. Assuming the first component proportional to $\dot{\mathrm{w}}_{\mathrm{g}}$ and the second proportional to the fan thrust, we may write:

$w_{e}=c_{1} w_{g}+c_{2} T_{f}$

The static fan thrust as a fraction of the total takeoff static thrust can be derived from the analytical expressions in Appendix $\mathrm{H}$ as follows:

$\frac{T_{f}}{T_{t 0}}=1-\frac{1}{\sqrt{1+n_{t f} \lambda}}\left(\right.$ at $\left.v_{0}=0\right)$

Elaborating $(4-34)$ further and applying it to actual types of engines, we find $c_{1}=$ $10 \varepsilon_{c}^{1 / 4}$ and $c_{2}=.12$. This results in an approximation for the specific dry engine weight**:

$\frac{W_{e}}{T_{\text {to }}}=\frac{10 \varepsilon_{c}^{1 / 4}}{(T / W)_{\text {to }}(1+\lambda)}+.12\left(1-\frac{1}{\sqrt{1+.75 \lambda}}\right)(4-36)$

The second contribution is about 208 higher for geared turbofans and another 208 should be added to it for variable pitch fans. Extra weight for acoustic treatment - if present - should be added (Table 8-8). In general it may be concluded that the specific weight decreases with increasing $\lambda$, in spite of the decrease in specific thrust. The variation of engine weight with high values of $\lambda$ cannot be predicted with certainty. For the sake of comparison Fig. 4-36 also gives the specific weight of a metal propeller as well as that of a modern efficient turboprop engine. It is likely that at very high $\lambda$ the specific weight will vary between about, .15 and .25 , depending largely on the size and complex-

*1.e. the combination of fan, low-pressure turbine and nozzle(s), see Fig. 4-17

**A more detailed weight prediction method will be found in AIAA Paper No. 70-669 ity of the engine.

When the specific weight is related to cruising conditions there is no clear tendency to rise or drop with $\lambda$. The wide variation observed is mainly caused by differences in engine configuration and thrust lapse with altitude.

A special category of engine where the accent lies particularly on low speed flight, is the lift engine, examples of which are shown in Fig. 4-37.

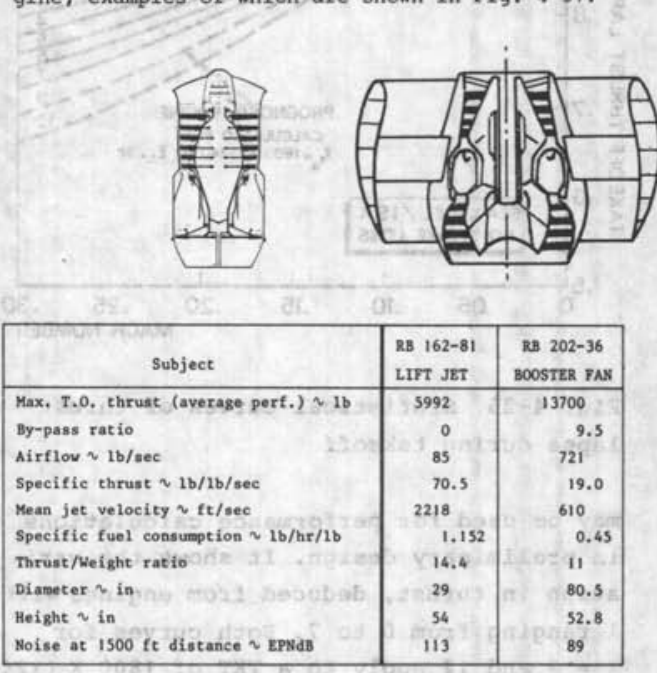

Fig. 4-37. Principal characteristics of Rolls Royce lift engines

These engines are intended for use on V/STOL aircraft and have found commercial application in the Hawker Siddeley Trident 3 , where they only operate as a booster during takeoff. Their main characteristics are:

1. high thrust at low installed weight and small volume,

2. relatively short operational iffe.

These features permit a specific weight of only .08 to .10 to be achieved. In the Rolls-Royce RB-202 project the objectives include a low velocity of the exhausted engine gases, a low noise level and low specific fuel consumption. As a result of these requirements the general configuration and the engine cycle are very different from those of normal engines designed primarily for high speed propulsion. 
e. Installation drag.

This is the drag of the engine mounted in a nacelle on the aircraft. In accordance with Fig. 4-38 the following contributions to the total drag must be taken into account:

1. gas generator nacelle drag,

2. drag of the plug in the hot flow,

3. fan nacelle drag,

4. pylon drag, and

5. powerplant/airframe interference arag. When the jet effluxes are separated, the gas generator nacelle will be located in the fan exhaust flow, which has a higher velocity than the flight speed. The same applies to the plug in the hot gases, which, however, have a still higher velocity. The extra drag is usually termed the scrubbing drag $^{*}$ and it decreases with $\lambda$ since the velocities of the effluxes decrease. Curve VIII in Fig. 4-38 has been calculated by assuming optimum values for the FPR and a gas generator of constant design. With a given gas generator an increase in $\lambda$ will result in a larger fan diameter, a greater wetted area of the fan nacelle and the pylon. This increase is approximately proportional to the static thrust. Because of various counteracting effects, the total installation drag - except interference drag - $\left(D_{1}+D_{2}+D_{3}+D_{4}\right)$ does not vary appreciably within a variation of bypass ratios between 3 and 10 , especially when expressed in relation to the thrust (curve VI in Fig. 4-38).

Although the data in Fig. 4-38 only apply to a given gas generator, they present a good impression of the trends. It should be observed that the frontal area of the engine is not a sound reference for weight and drag.

Installation drag increases in proportion to $\mathrm{v}_{0}{ }^{2}$. This increase may be reduced by lowering $\lambda$, though the result will be an

*Due to the interrelationship between scrubbing drag and the engine operating conditions, this arag contribution is frequently considered as a thrust loss

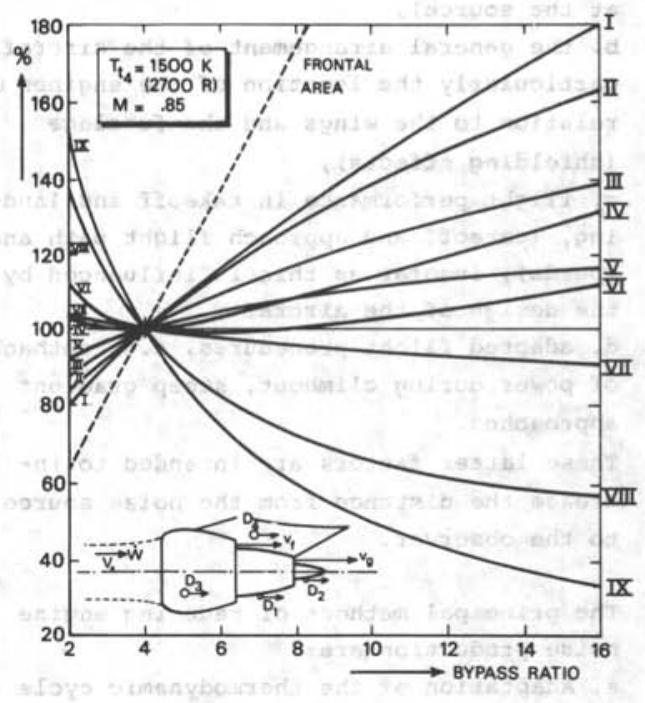

I static thrust ' $T$ to' fan diameter; $D_{3}+D_{4}$

II Basic ary weight $w_{e}$

III Specific weight, cruise condition, $\mathrm{w}_{\mathrm{e}} / \mathrm{T}$ cr

IV Drag of installed engine in cruise condition

$$
\left(D_{1}+D_{2}+D_{3}+D_{4}=D_{e}\right)
$$

$\mathrm{V}$ Cruise thrust $\mathrm{T}$ cr

VI Specific arag, $D_{e} / T_{c r}$

VII Specific weight at takeoff $\mathrm{w}_{\mathrm{e}} / \mathrm{T}$ to

VIrI Drag $\mathrm{D}_{1}+\mathrm{D}_{2}$

IX Specific thrust in cruise $\mathrm{T}_{\mathrm{Cr}} / \mathrm{W}$

Fig. 4-38. Effect of bypass ratio on thrust, drag and weight for a given gas generator

(Refs. $4-33,4-36,4-38$ and $4-53$ )

increase in $\mathrm{C}_{\mathrm{T}}$. In general it can be said that the optimum value of $\lambda$ for cruising performance will decrease with the design cruising speed.

\subsubsection{Engine noise}

In view of the fact that very few systematic design rules have as yet been established to reduce engine noise, we shall have to confine ourselves to a qualitative summary of the most important factors. The external noise production of an aircraft will be largely influenced by: a. the design of the engine and its installation in a nacelle (noise reduction 
at the source),

b. the general arrangement of the aircraft, particularly the location of the engines in relation to the wings and the fuselage (shielding effects),

c. flight performance in takeoff and landing, (takeoff and approach flight path and speeds), insofar as this is influenced by the design of the aircraft,

d. adapted flight procedures, e.g. cutback of power during climbout, steep gradient approaches .

These latter factors are intended to increase the distance from the noise source to the observer.

The principal methods of reducing engine noise production are:

a. Adaptation of the thermodynamic cycle to reduce the aerodynamic noise, the most important single factor being the bypass ratio.

b. Measures to avoid the creation of machinery noise.

c. Measures to suppress noise that has been generated.

Fig. 4-39 gives a general impression of the variation in different noise sources with bypass ratio. In the straight turbojet the dominant source is the violent jet mixing process aft of the engine, together with the exhaust system. Jet noise suppressors reduce the noise level by some $5 \mathrm{PNaB}$. The reduced exhaust velocity of low bypass ratio engines lessens the tailpipe and jet noise, but at the same time the fan compressor and turbine noise are not far below this level. Turbomachinery noise frequently becomes dominant at reduced engine rpm. In the case of high bypass engines the fan noise is particularly predominant at all relevant power settings.

Practical measures which will lead to a low noise level are indicated in Fig. $4-40$ and enumerated below:

1. Use of low efflux velocities will decrease the jet noise, particularly at low bypass ratios. Since an acceptable specific thrust is desirable, a fairly high TET will be chosen on high bypass engines.

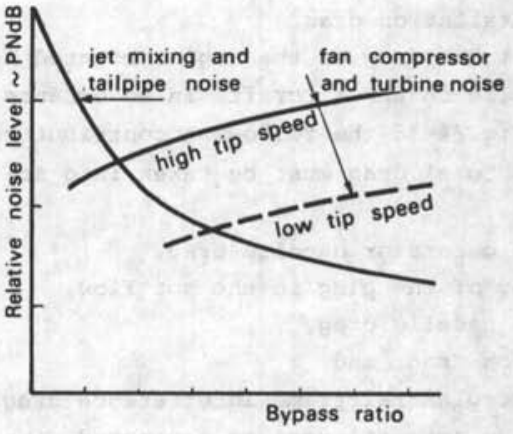

a. Variation of critical noise sources

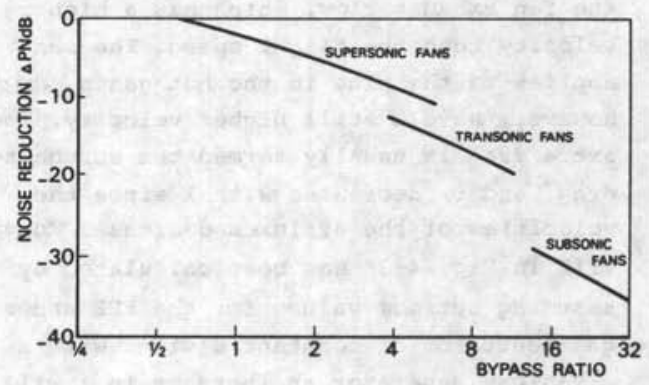

b. Potential noise reduction trend (Ref. 4-47)

Fig. 4-39. Effect of bypass ratio on noise production

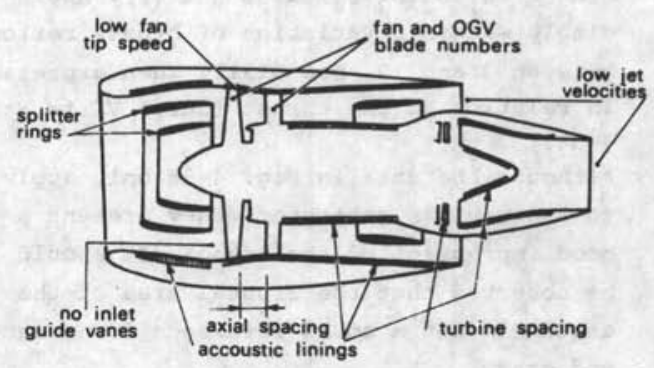

Fig. 4-40. Practical measures to reduce the noise of a turbofan installation

2. Use of jet noise suppressors on low bypass engines.

3. Readjustment of the ratio of the efflux velocities of the cold and hot gases relative to the theoretically optimum 
value*. For a given $\lambda$, this will bring the combined jet and fan noise to a minimum.

4. Adjustable fan rotor blades, which will have the optimum setting with reyard to noise during various flight conditions (variable pitch fans).

5. Low fan tip speed, which is made possible by adopting the three-shaft layout

(Fig. 4-19C) or the twin spool layour with reduction gear (Fig. 4-190).

6. Low pressure ratios of both the fan and the low pressure turbine.

7. Elimination of the inlet stator biades and an increase in the distance between the fan rotor blades and the stators.

8. Optimization of the number of rotor and stator blades of the fan.

9. Use of acoustic lining in the engine housing and/or sound suppressing rings (splitter plates) in the inlet and exhaust. This may reduce the noise by 5 to 10 PNdB. 10. Reduction of machinery noise, particularly turbine and tailpipe noise.

4.4.5. Summary and prognosis for the turbofan engine

a. For each bypass ratio there is a "thermodynamic" optimum for the fan pressure ratio. As $\lambda$ increases this optimum becomes more marked and it will be more important to choose the optimum Fan Pressure Ratio. b. The present bypass engine with $\lambda=5$ to 8 shows a considerable improvement in specific fuel consumption as compared with the pure jet engine and engines with a low $\lambda$.

A further improvement of about 158 will only be possible if $\lambda$ is chosen equal to 20 to 30 , combined with a high overall Pressure Ratio and Turbine Entry Temperature.

c. Sensitivity to inlet and exhaust losses increases with bypass ratio, while for $\lambda>10$ to 12 there will be a great increase in the total installed drag. These values of $\lambda$ require another design conception for both the engine (fan reduction gear) and

*i.e. in accordance with the optimum work ratio; see Section $4 \cdot 4 \cdot 3$ a. the aircraft, due to the very large engine diameter.

d. The bypass ratio plays an important role in matching the takeoff, climb and cruise performance. When the accent is on low speed performance a high $\lambda$ will be preferred, but when cruising performance is regarded as the critical requirement, an optimum will have to be found with respect to specific fuel consumption, installed weight â: $A$ drag. The optimum $\lambda$ decreases as crlizing speed goes up.

e. An increase of $\lambda$ results in a reduction in noise, provided the tip speed of the fan does not become too high. With bypass ratios larger than about 9 to $10 \mathrm{it}$ will be necessary to adopt a fan reduction gear. f. From extrapolations of TET and OPR we may expect a generation of engines in the eighties, with the main characteristics summarized in Table 4-2.

\begin{tabular}{|l|c|c|c|c|}
\hline Application & \multicolumn{2}{|c|}{ Long-haul } & \multicolumn{2}{|c|}{$\begin{array}{c}\text { short-haul, } \\
\text { short runways }\end{array}$} \\
\hline \hline Bypass Ratio & \multicolumn{2}{|c|}{9} & \multicolumn{2}{|c|}{20 to 30} \\
\hline OPR & \multicolumn{2}{|c|}{36} & \multicolumn{2}{|c|}{25} \\
\hline FPR & \multicolumn{2}{|c|}{1.6} & 1.4 to $1.3^{\star}$ \\
\hline Condition & cruise & takeoff & cruise & takeoff \\
\hline Mach number & .9 & 0 & .7 & 0 \\
\hline TET & $1750 \mathrm{~K}$ & $1800 \mathrm{~K}$ & $1500 \mathrm{~K}$ & $1600 \mathrm{~K}$ \\
$(3150 \mathrm{R})$ & $(3240 \mathrm{R})$ & $(2700 \mathrm{R})$ & $(2880 \mathrm{R})$ \\
\hline $\begin{array}{l}\text { Spec.thrust } \\
\text { (sec) }\end{array}$ & 14 & 27.5 & 8 to 5.5 & 18 to 13.5 \\
\hline $\begin{array}{l}\text { Corrected SFC } \\
\text { C }_{\mathrm{T}} / \sqrt{\theta} \text { (h }^{-1} \text { ) }\end{array}$ & .64 & .27 & .56 to & .19 to \\
\hline
\end{tabular}

*geared fan

Table 4-2. Forecast of principal characteristics for two new generations of turbojet engines (all data apply to the uninstalled engine)

\subsubsection{Engine performance in non-standard} atmosphere

The thrust is found to be highly dependent. on the ratio $\phi$, 


\section{$\phi=\frac{\text { Turbine Entry Temperature }}{\text { Atmospheric Temperature }}$}

as well as on the temperature correction

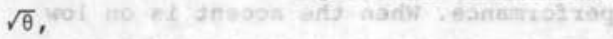
$\theta=\frac{\text { ambient temperature }}{\text { standard ambient temp., sea level }}(4-38)$

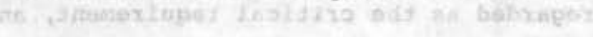

When the influence of the atmospheric temperature is determined on the assumption that the TET remains constant (curve I in Fig. 4-41), it is seen that at an air tem-

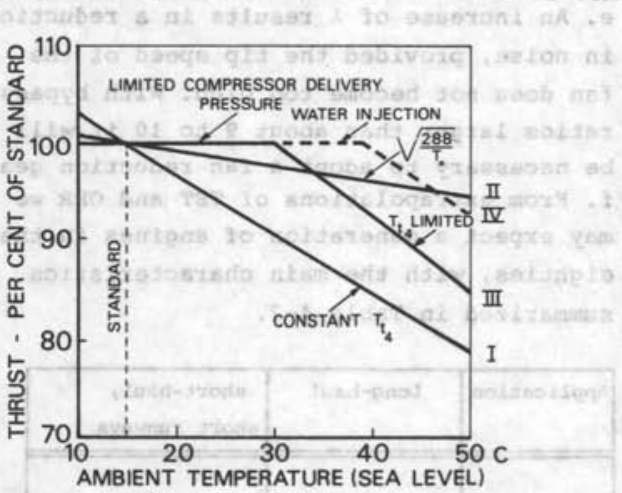

Fig. 4-41. Thrust in non-standard conditions

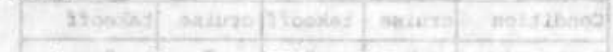

perature of, say, $95 \mathrm{~F}(35 \mathrm{C})$ at sea level the thrust will be $12 \frac{1}{2}$ 8 below the value for standard conditions. If $\phi$ is taken constant, the thrust will be approximately proportional to $1 / \sqrt{\theta}$ (curve II). In this case the loss in thrust will be considerably less, but at high atmospheric temperatures the TET will rise too high, with a resultant detrimental effect on the engine's service 1 ife.

The thrust of most modern engines is controlled according to curve III, where two regions can be distinguished:

a. A temperature region where the exit pressure of the compressor is limited. As the temperature increases the supply of fuel will be increased in such a way that the thrust will remain practically con 3 tant and the TET will also increase.

b. A region where tive IS' is limited so that the hrust will decrease as the am- bient temperature rises.

Known as the method of flat rating, this ensures that the engine will deliver a practically constant thrust within a large range of ambient conditions encountered in operational practice.

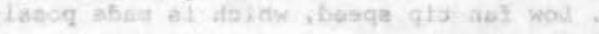

Another method of reducing thrust losses at high atmospheric temperatures is to use water injection. A mixture of water and methanol is sprayed into the compressor inlet or injected at the combustion chamber inlet. As this evaporates it will cool the air and the mass flow through the engine will increase. The Turbine Entry Temperature is restored by the burning of methanol. With the use of water injection the standard thrust may be maintained up to about $95 \mathrm{~F}(35 \mathrm{c})$ and, if required, can be increased by about 3 to 78 . A water injection system is required, but this does not entail any drastic changes in the design of the engine, so that the engine manufacturer will offer it as an optional item.

Intang as?

\subsection{ASSESSMENT OF TURBOPROP ENGINES}

Contrary to expectations in the fifties, the development and use of turboprop engines in civil aviation has not been so widespread as that of turbojets. This is mainly due to: a. the relatively great complexity of the combination of a gas, turbine engine with propeller reduction gear and constant speed propeller, as compared with the straight jet engine, b. the higher flying speeds which can be attained with the jet engine, as compared with the propeller engine.

In the competition with the piston engine, the turboproo was only able to take the lead when technological progress enabled compressor pressure ratios to be raised to about 5 or 8 and Turbine Entry Temperatures of about 1200 to $1300 \mathrm{~K}(2160$ to $2340 \mathrm{R})$ became feasible. This has resulted in the turboprop unit completely replacing the piston in the 500 to $3000 \mathrm{hp}$ class of civil 
engines. Where engine first price is an important factor, however, the turboprop unit is still very much at a disadvantage.

\subsubsection{Performance}

The power developed by the gas generator is distributed partly to the propeller shaft and partly to the engine exhaust gases. It can be shown that the propulsive efficiency and the flying speed are the deciding factors in the optimum distribution of energy. The following optimum jet efflux velocity is found (Ref. 4-7):

$v_{j_{\text {opt }}}=\frac{v_{o}}{n_{\text {prop }} n_{t} n_{\text {mech }}}$

It is concluded that when the flight speed in the design conditions increases, the optimum gas efflux velocity will also increase. Consequently, when a turboprop engine is designed for relatively high speed operation, the thrust of the exhaust gases will be considerable at low speeds. If, on the contrary, the main emphasis is on high power output at low speeds, a low efflux velocity will be desirable (turboshaft engines).

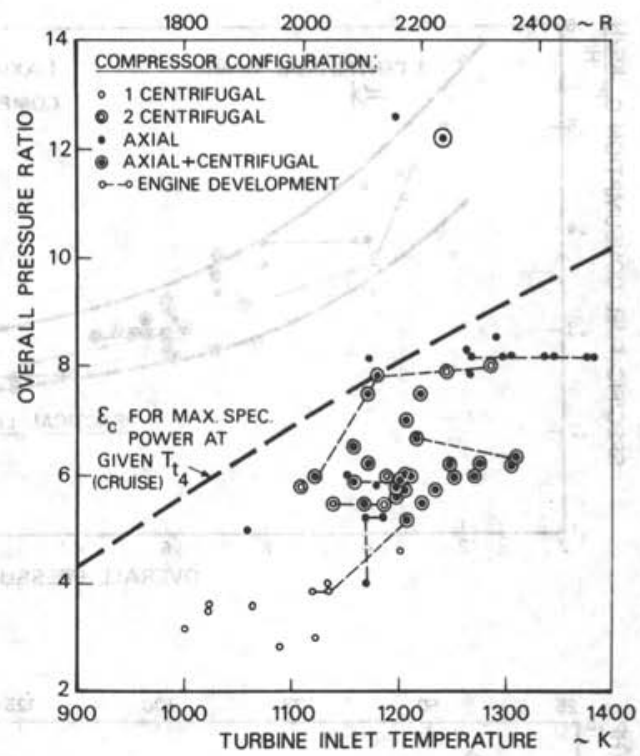

Fig. 4-43. 'I peratures and pressure ratios of turboprop engines (data from Ref. 4-27)

When (4-39) is applied to a family of turboprop engines, the specific fuel consumption and power may be computed with the method explained in Appendix $\mathrm{H}$. This has been carried out for a cruising speed of

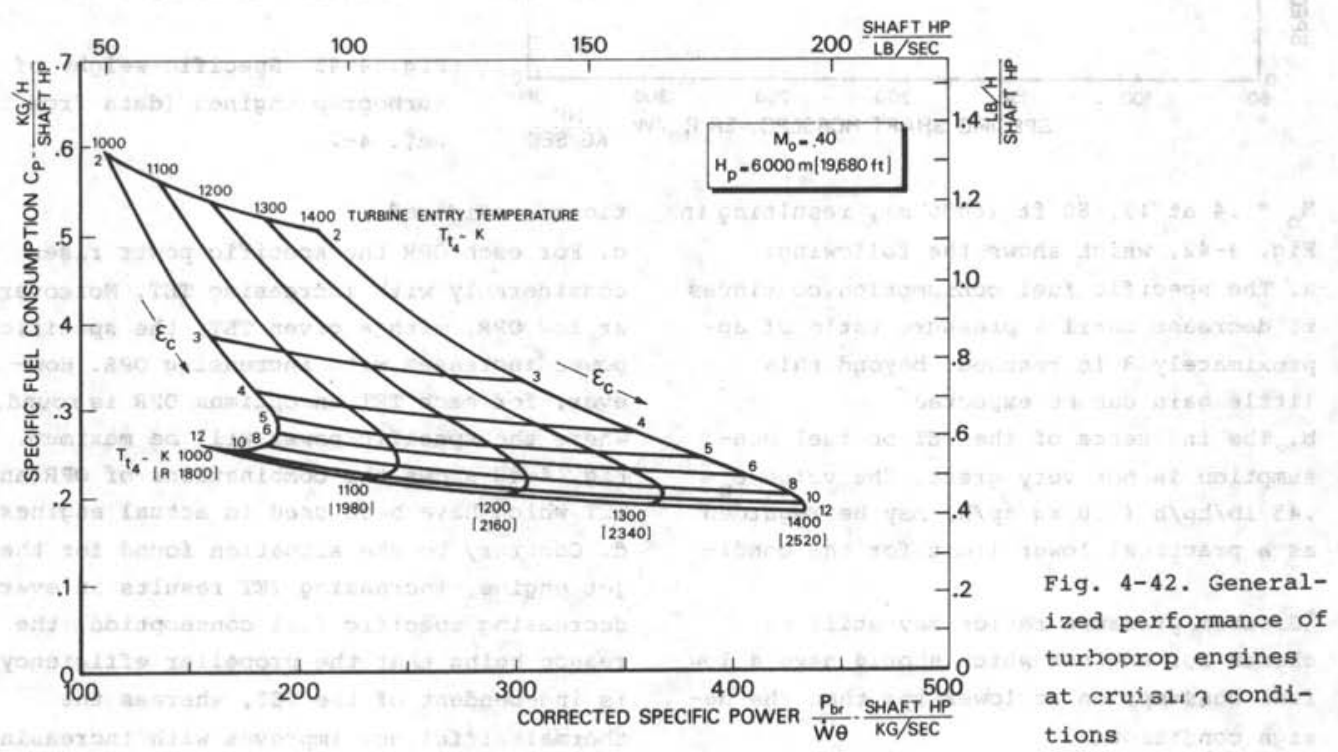




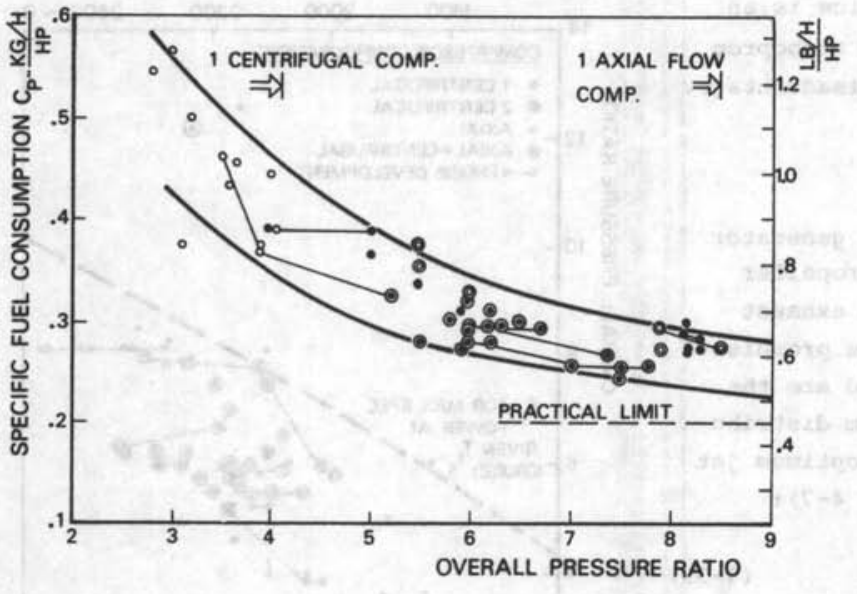

NOTE: Symbols as in Fig. 4-43

Fig. 4-44. Specific fuel consumption at sea level static conditions

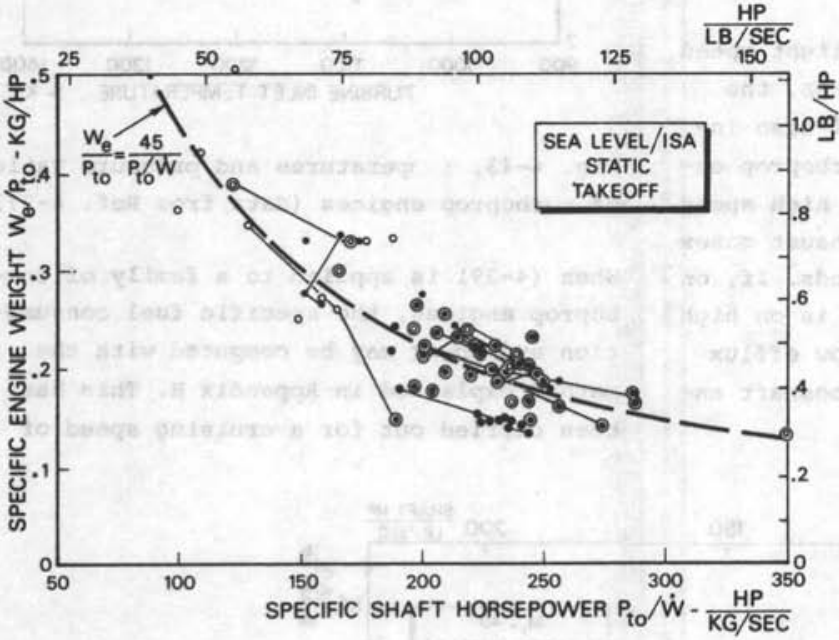

NOTE: Symbols as in Fig. $4-43$

Fig. 4-45. Specific weight of turboprop engines (data from

Ref. 4-27)

$M_{0}=.4$ at $19,680 \mathrm{ft}(6000 \mathrm{~m})$, resulting in Fig. 4-42, which shows the following:

a. The specific fuel consumption continues to decrease until a pressure ratio of approximately 8 is reached; beyond this little gain can be expected.

b. The influence of the TET on fuel consumption is not very great. The value $c_{p}=$ $.45 \mathrm{lb} / \mathrm{hp} / \mathrm{h}(.20 \mathrm{~kg} / \mathrm{hp} / \mathrm{h})$ may be regarded as a practical lower limit for the condi-

* Greater pressure ratios may still be chosen for engines which should have a low fuel consumption at lower rpm than the design condition. tions considered.

c. For each OPR the specific power rises considerably with increasing TET. Moreover, at low OPR, with a given TET, the specific power increases with increasing OPR. However, for each TET an optimum OPR is found, where the specific power will be maximum. Fig. 4-43 shows the combinations of OPR and TET which have been used in actual engines. d. Contrary to the situation found for the jet engine, increasing TET results in ever decreasing specific fuel consumption, the reason being that the propeller efficiency is independent of the TET, whereas the thermal efficiency improves with increasing 
TET.

Fig. 4-43 shows that for a number of engines the values adopted for the OPR do not depart to any great extent from the calculated optimum. This leads us to think that further development of turboprop engines will not prove spectacular as far as specific fuel consumption and specific power are concerned. There are, however, still possibilities of achieving a low fuel consumption for engines which have to run at low power during very long flights, e.g. regenerative engines, for which the reader should consult Ref. 4-6.

The effect of the OPR on specific fuel consumption is shown statistically in Fig. 4-44.

\subsubsection{Weight and drag}

The weight of the turboprop engine is largely decided by the mass of air flowing through it and amounts to about $45 \mathrm{lb}$ per $1 \mathrm{~b} / \mathrm{s}$ of airflow. Therefore:

$\frac{W_{e}}{P_{\text {to }}}=\frac{45}{(P / \dot{W})_{\text {to }}}(1 \mathrm{~b} / \mathrm{hp}$ or $\mathrm{kg} / \mathrm{hp})$

From this expression, which is compared with actual examples in Fig. 4-45, it is concluded that a high specific power is important for low weight. To this we may add that the frontal area of the engine is also proportional to $\dot{w}$, so for the nacelle drag per hp it is possible to derive a similar relationship, as in $(4-40)$.

\subsubsection{Turboprop engine configurations}

older types of turboprop engines are fitted with a single centrifugal compressor. (See Fig. 4-46). Since OPR values of about 4 have been achieved, these engines show a specific fuel consumption of at least .65 to .75 $\mathrm{lb} / \mathrm{hp} / \mathrm{h}(.30$ to $.35 \mathrm{~kg} / \mathrm{hp} / \mathrm{h})$. For this reason the air is often pre-compressed by means of an axial compressor and with this combination it will be possible to reach pressure ratios of the order of 10 . These pressure ratios may also be obtained with a single axial compressor or with two centrifugal compressors in series. Some engines are fitted with two axial compressors on separate shafts and this makes a high pressure ratio possible (e.g. Rolls-Royce Tyne, OPR $=13.5$, see Fig. $4-46 \mathrm{~b})$.

Many turboprop engines are fitted with a free turbine. This is a low pressure turbine which is not coupled mechanically to the gas generator, but drives the propeller through a separate shaft. With these engines it is often possible for the pilot to adjust the power in certain phases of the flight by selecting the propeller pitch ( $\beta$-control). This demands a special device which governs the quantity of fuel supplied to the engine and controls the power output in such a way that the propeller rpm remains constant (cf. Section 6.3.3.). The following features are offered by the free turbine:

a. When output has to be rapidly increased in a flight phase with low power, it will only be necessary to increase the rpm of the gas generator. The high-inertia propeller already revolves at the required rpm, thus making it possible to obtain a quick response.

b. In flight conditions which require control of the aircraft relative to a given glide path the use of $\beta$-control will give improved speed stability, particularly in the low speed regime.

c. Due to the aerodynamic coupling with the gas generator, the free turbine runs close to the optimum rpm under different working conditions, resulting in a high efficiency.

d. In the case of engine failure, the freely revolving propeller will only have to drive the free turbine and propeller drag immediately after the failure will be small.

There are various possible engine cunfigurations which can be adapted to feature a free turbine, without resorting to the coaxial layout. Examples are the Allison 250 (Fig. 4-46c) and the Pratt and Whitney PT6A, 


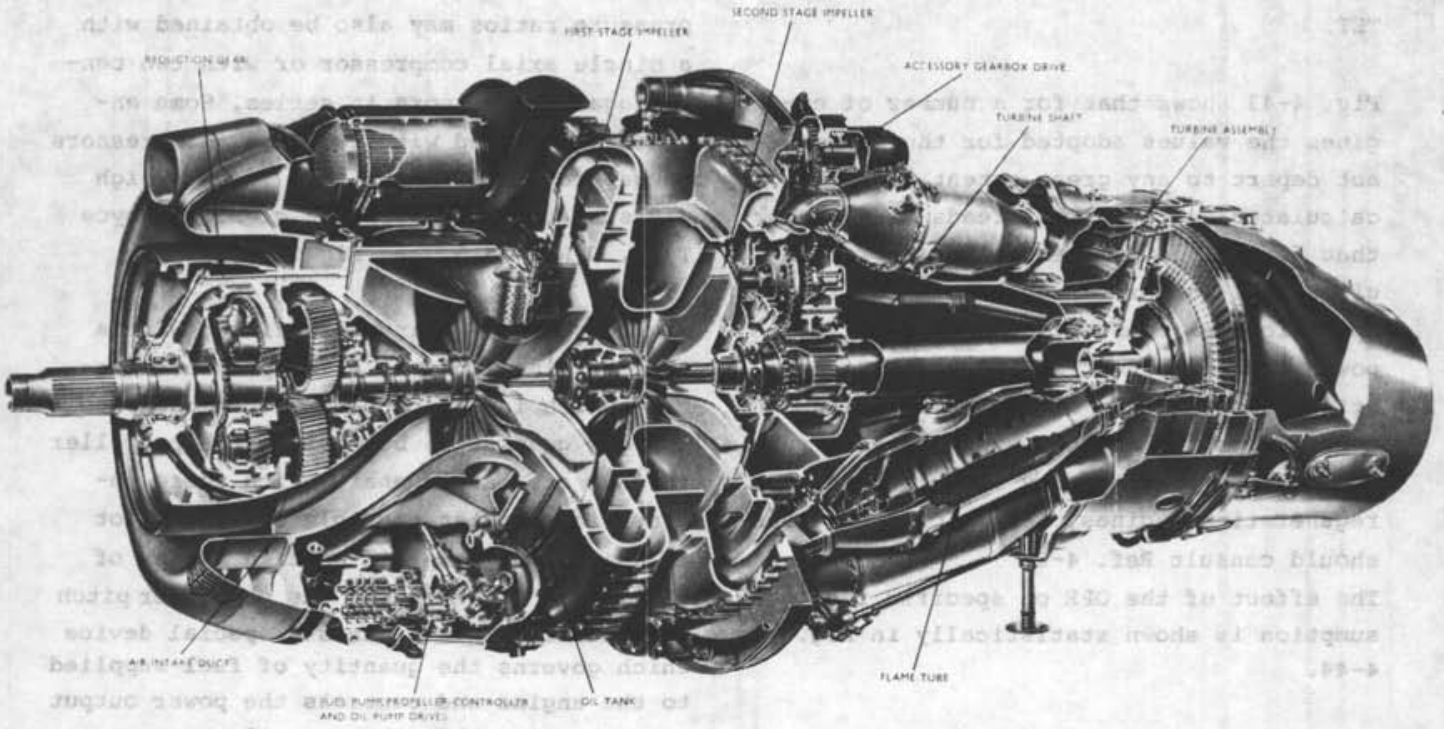

a. Rolls-Royce DART-Mk. 510

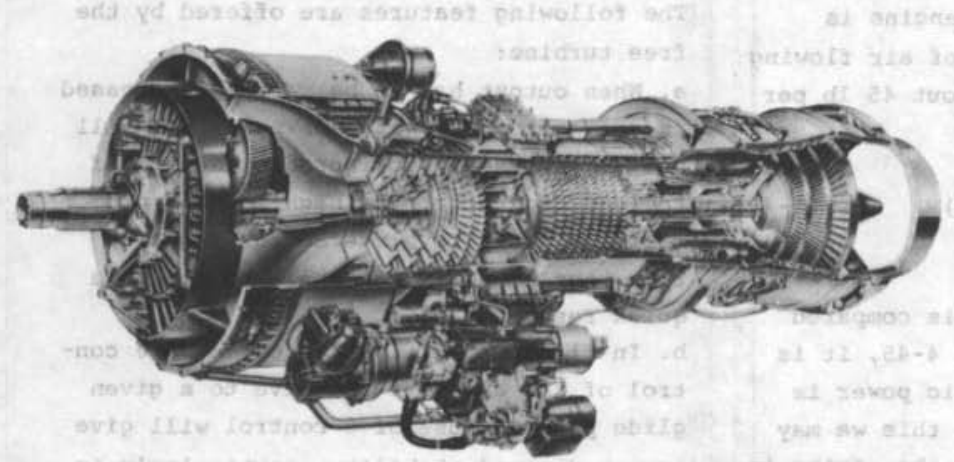

Fig. 4-46. Examples of turboprop engine configurations (data can be found in Table 6-2)

b. Ro11s-Royce TYNE 12

\section{c. Allison Model 250}

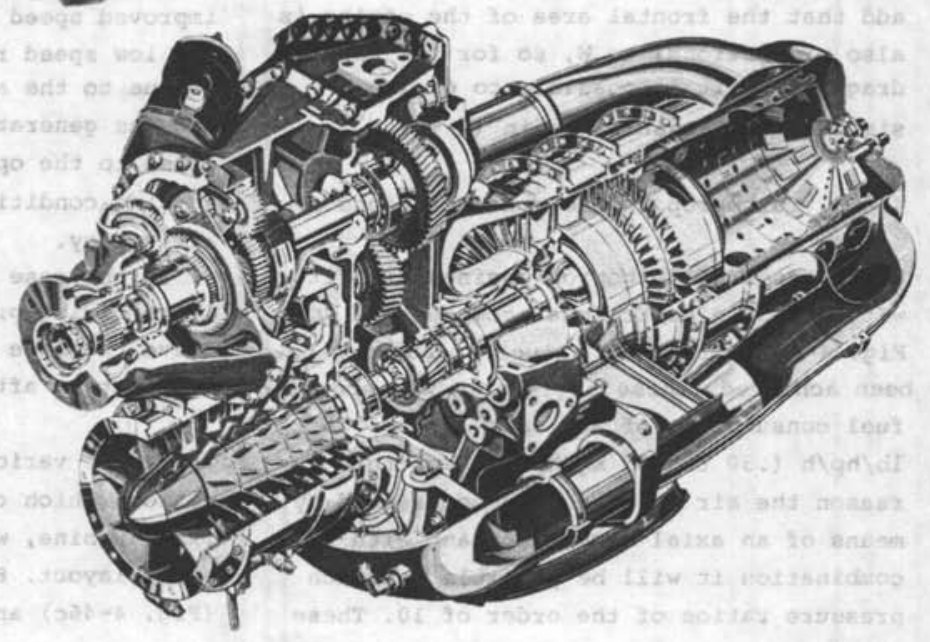




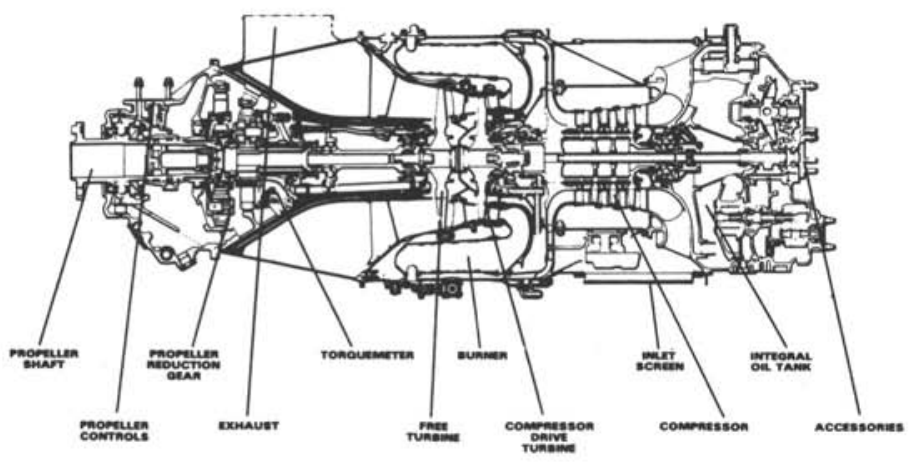

d. Pratt and Whitney PT-6A

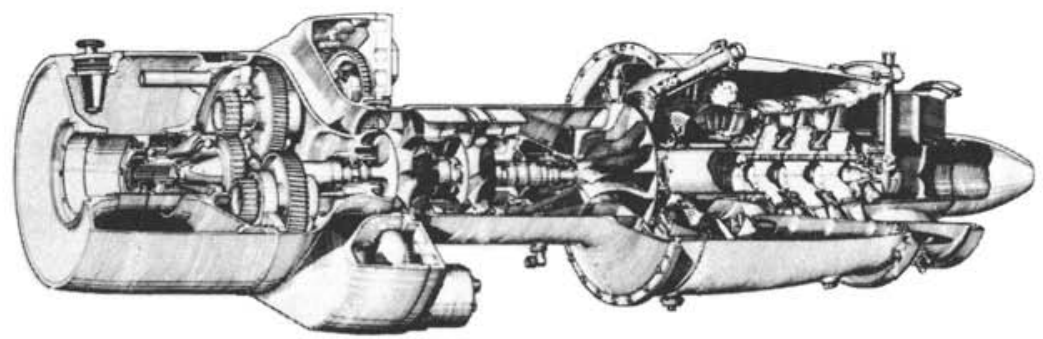

e. Turboméca Astazou XIV

Fig. 4-46. Examples of turboprop engine configurations. (data can be found in Table 6-2)

(Fig. 4-46d), which work on the "reverse flow" principle with reversal of the flow within the engine. The gases are ejected either in front or in the centre of the engine at the sides. The advantage lies in the very compact layout, demanding little space to accommodate the engine. This may be compared with the Turbomeca Astazou (Fig. 4-46e) with the jet pipe at the rear of the engine. However, with the jet pipes at the sides it is not possible to take full advantage of the thrust of the engine gases, unless one or more curved jet pipes are used. This will lead to an increase in drag and a loss of thrust. 


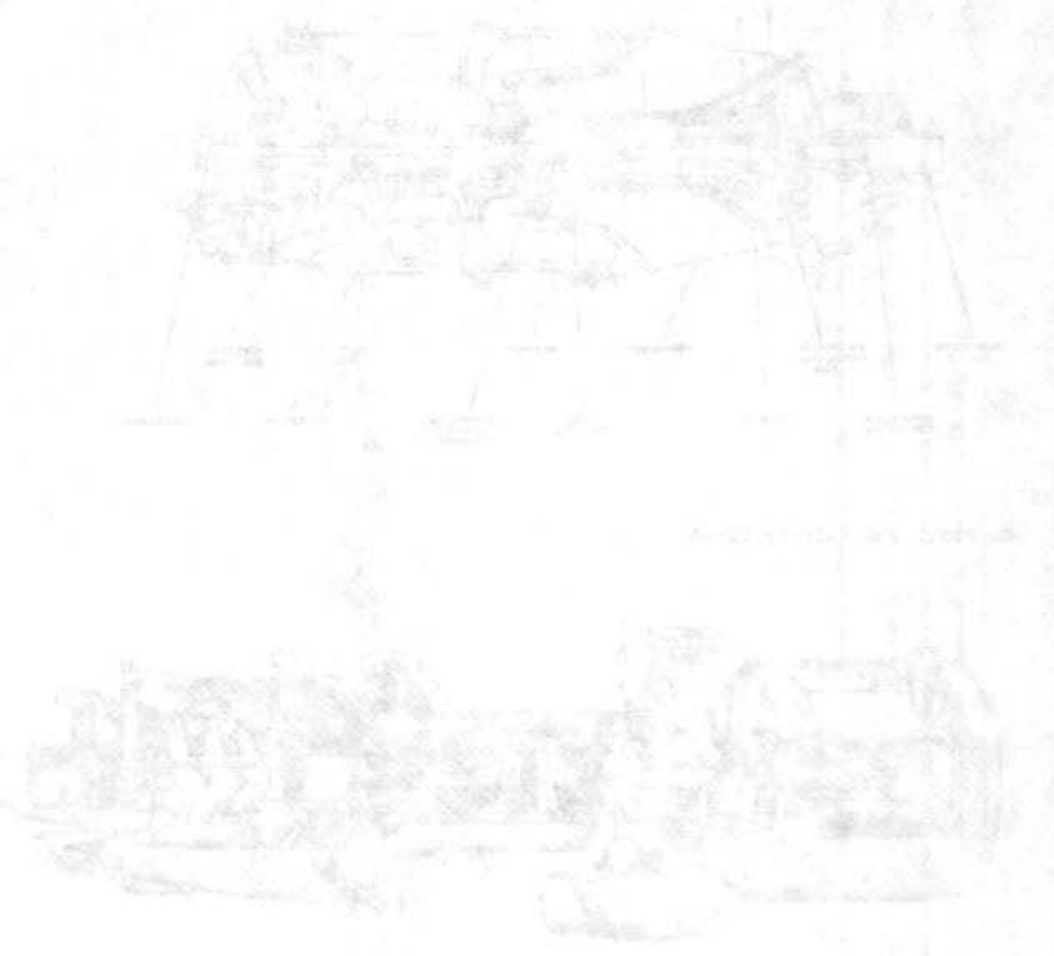




\section{Chapter 5. Design for performance}

SUMMARY

After laying down the broad outlines of the general arrangement and finalizing the design of the fuselage, the designer's next step will be to decide on the type of engine to be installed and the size of the wing. Both have a direct effect on performance and operating costs.

Basic relationships are presented for the performance of systematic design studies. Initial weight and drag estimation methods are given and boundary values are derived for aircraft parameters such as wing loading, thrust or power loading and aspect ratio. Some examples of the use of parametric studies and synthesis diagrams are discussed. Finally performance aspects related to noise reduction are dealt with on a qualitative basis. 


\begin{tabular}{|c|c|}
\hline $\mathbf{A}$ & $\begin{array}{l}\text { - wing aspect ratio, } A=b^{2} / S \\
\text { - mean deceleration or acceleration }\end{array}$ \\
\hline$\left(a_{0}\right)$ & $\begin{array}{l}\text { - speed of sound (at sea level ISA) } \\
\text { - Balanced Field Length }\end{array}$ \\
\hline$n$ & - span or width; no index: wing span \\
\hline C & - rate of climb \\
\hline$c_{0}$ & $\begin{array}{l}\text { - coefficient in generalized expres } \\
\text { sion for } L / D \text { at } V_{2}\end{array}$ \\
\hline - & $\begin{array}{l}\text { - airplane drag coefficient, } C_{D}= \\
\text { D } / \frac{1}{2} \rho V^{2} S\end{array}$ \\
\hline
\end{tabular}

$\mathrm{C}_{\mathrm{D}_{1}} \quad$ - induced or 1ift-dependent drag SEP coefficient

$C_{D_{0}} \quad$ - zero-lift drag coefficient

C F - mean airplane zero-lift drag coefficient, based on wetted area

$C_{L} \quad-$ lift coefficient, $C_{L}=L / \frac{1}{2} \rho V^{2} S$

$\mathrm{C}_{\mathrm{L}_{\max }}-\operatorname{maximum}_{\mathrm{W} / \frac{1}{2} \rho \mathrm{V}_{\mathrm{S}}{ }^{2} \mathrm{~S}}$ ifft coefficient, $\mathrm{C}_{\mathrm{L}_{\max }}=$

$C_{P} \quad-$ specific fuel consumption, propeller engines

$\mathrm{C}_{\mathrm{T}} \quad$ - specific fuel consumption, turbojet engines

c - chord length

$c_{F} \quad$ - friction coefficient of boundary layer

D - drag

$d_{1}, a_{2}, d_{3}-$ factors defining the zero-1ift drag coefficient

$\mathrm{E}$ - coefficient in generalized expression for $L / D$ at $V_{2}$

EPNAB - equivalent, perceived noise, dB

e - Oswald's induced drag factor, $1 / \mathrm{e}=\pi \mathrm{A}\left(\mathrm{dC}_{\mathrm{D}_{i}} / \mathrm{dC}_{\mathrm{L}}{ }^{2}\right)$

- field length factor for takeoff ( $f_{\text {to }}$ ) or landing ( $f_{\text {land }}$ )

- acceleration due to gravity

- altitude; height; screen height in takeoff ( $\left.\mathrm{h}_{t o}\right)$ or landing $\left(\mathrm{h}_{50}\right)$

ISA - ICAO Standard Atmosphere

$k_{W} \quad-$ ratio of takeoff weight to actual weight

- $11 \mathrm{ft}$

- length

- Mach number

- number of engines

- load factor ( $n=L / W)$; exponent
- power

- total (equivalent) takeoff horsepower of all engines

- static atmospheric pressure (at sea level ISA)

- range

- Reynolds number, $\mathrm{Re}=\mathrm{V} 1 / \mathrm{v}$

- correction factor or ratio for drag estimation

- distance; area (no index: wing area)

- Specific Excess Power

- Sea Level, Static condition

- thrust; atmospheric temperature $\left(\mathrm{T}_{0}=\mathrm{T}\right.$ at sea level ISA)

$T_{j} \quad$ - total jet thrust (turboprop engines only)

$T_{\text {to }}$

- total static takeoff thrust, all engines operating, uninstalled engines

- mean thrust in takeoff run

- time; section thickness

- equivalent inertia time

- speed

- approach speed

- rotation speed

- stalling speed; takeoff config-

- uration: $\mathrm{V}_{\mathrm{S}_{1}}$; landing configuration: $v_{S_{0}}$

$v_{t d} \quad-$ touchdown speed

$v_{x} \quad$ - engine failure speed

$\mathrm{v}_{1} \quad$ - speed at the decision point

$v_{2} \quad-$ takeoff safety speed

$\mathrm{V}_{3} \quad$ - initial climbout speed, all engines operating

- weight

w

- operational empty weight; weight empty equipped

- dry weight of engines

- fixed part of $w_{e}$ (i.e. not determined by $w_{\text {to }}$ )

- (maximum) takeoff weight

- variable part of $\mathrm{w}_{\mathrm{e}}$ (i.e. determined by $\mathrm{w}_{\text {to }}$ )

- angle of climb or descent; ratio of specific heats

- equivalent angle of climb or descent 


\begin{tabular}{|c|c|}
\hline$\gamma_{2}$ & $\begin{array}{l}\text { - second segment climb angle } \\
\text { - increment }\end{array}$ \\
\hline & - relative static pressure; $\delta=p / p_{0}$ \\
\hline $\mathrm{n}$ & $\begin{array}{l}\text { - nacelle frontal area/cylinder } \\
\text { volume per engine }\end{array}$ \\
\hline & - efficiency \\
\hline & $\begin{array}{l}\text { - relative atmospheric temperature; } \\
\theta=\mathrm{T} / \mathrm{T}_{0}\end{array}$ \\
\hline & - sweep angle \\
\hline & - bypass ratio \\
\hline & $\begin{array}{l}\text { - friction coefficient; } \mu=\text { retar- } \\
\text { dation force/normal force }\end{array}$ \\
\hline ' & $\begin{array}{l}\text { - equivalent friction coefficient, } \\
\text { including aerodynamic forces }\end{array}$ \\
\hline & - coefficient of kinematic viscosity \\
\hline$\rho\left(p_{0}\right)$ & - air density (at sea level ISA) \\
\hline & - relative density; $\sigma=\rho / \rho_{0}$ \\
\hline & - power/engine frontal area \\
\hline & $\begin{array}{l}\text { - specific thrust (jet thrust/air- } \\
\text { flow); specific power (bhp/cyl- } \\
\text { inder volume per engine) }\end{array}$ \\
\hline
\end{tabular}

Subscripts

\subsection{INTRODUCTION}

After deciding on the general arrangement (Ch. 2), incorporating this in initial design sketches, and finalizing the layout drawings of the fuselage (Ch. 3), the designer's next step will be to decide on the type of engine to be installed and the size of the wing. At this stage of the design the specified mission and flight performance will play an important part. In Chapter 4 it was concluded that engine performance is affected by many parameters, such as cycle temperatures and pressure ratios, bypass ratio, etc. The most important properties of the wing are wing sections and area, aspect ratio and high-lift devices. In a well-balanced design the various parameters and shape factors are combined so as to minimize both the initial costs and operating costs, while meeting all performance requirements.

In view of the limited availability of engines, the type of engine to be installed

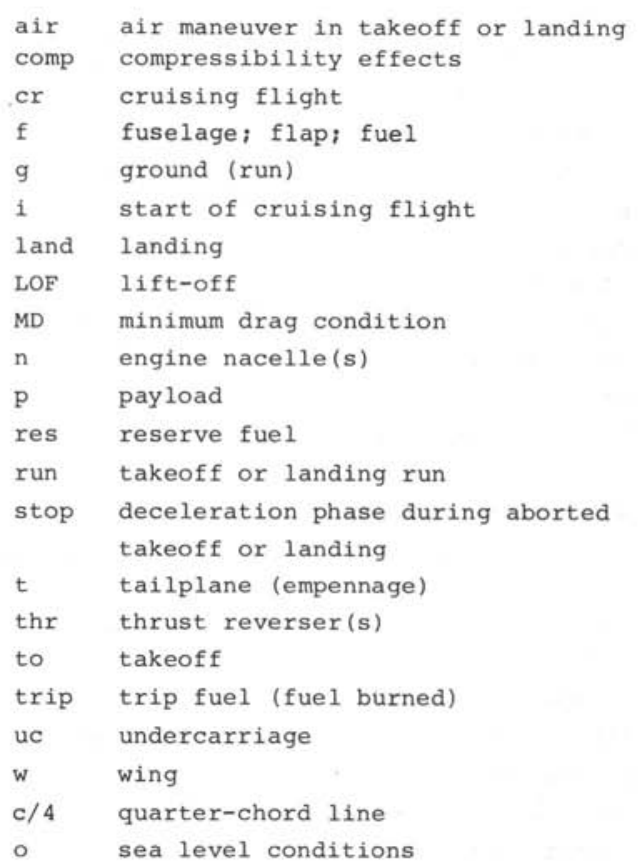

is occasionally chosen on the basis of limited performance studies. In such a case the wing design procedure is considerably simplified but the result may be unsatisfactory if the number of suitable engines is too restricted. If several engine types are available, a systematic study is required to find optimum combinations of wing design and types of engine. In this Chapter the basic relationships for the performance of such studies will be developed and elementary examples will be given. A complete evaluation of the effect of the design choices on the complete aircraft is virtually equivalent to carrying out as many design studies as there are parametric variations and many details must therefore remain undiscussed.

Simplified illustrations are presented to cover the more complex computational system and the reader may refine all relationships insofar as he thinks fit. The procedure is divided into three major parts:

a. Initial estimation of the empty weight, fuel weight, all-up weight and drag polars 
(Sections 5.2 and 5.3).

b. Derivation of boundary values of the design parameters, based on "reversed" performance calculations (Section 5.4).

c. Some examples of performance optimizations (Section 5.5).

The actual choice of the type of engine and wing shape is based not only on performance calculations but also on other factors to be considered in Chapters 6 and 7 .

In recent years, low noise production has become a requirement of major importance in aircraft design. Although the methods for achieving a low-noise design have not yet been settled, some aspects will be mentioned in this chapter.

It is emphasized that most of the methods presented here are intended as illustrative examples, applicable to an early stage of the design; they should be refined and improved as soon as more data become available. Chapter 8 and the appendices to this book contain more detailed data and many references to the relevant literature.

\subsection{INITIAL WEIGHT PREDICTION}

\subsubsection{Stages in the estimation of airplane} weight

In the early days of aviation, the design engineer was responsible for the design, stress analysis and weight control for the components of the aircraft. As design problems became more complex, specialized fields of engineering were developed. One of these fields is weight determination, control and coordination. Today, weight prediction and control are part and parcel of every phase in the design and development of every type of aircraft.

To be effective, weight prediction and control must be carried out during the early stages of the preliminary design, before a design configuration becomes "frozen". As soon as weight figures are distributed to the various departments of a design office as a starting point for further design evaluation, they will strongly resist any attempt to alter design weights at a later stage. The weight data issued by the preliminary design department serve as goals for other engineering departments.

Weight prediction is necessary not only for stress and performance computations, but also for design optimization, as reflected in the following categories of estimating methodology:

1. Pre-configuration selection methods.

2. Configuration selection methods.

3. Post-configuration selection methods. The general requirements to be satisfied by estimating methods are given below.

a. Pre-configuration selection methods. "Weight guesstimates" are used during the period in the design where the mission requirements are practically the only objectives which are definitely known. The airplane size and structural arrangement cannot be finalized until systematic studies have been made to determine the optimum design. The prediction methods to be used should be elementary in nature, and the use of statistics is appropriate when it produces rapid answers. Some examples are given in Section 5.2.2. and in References 5-6 to 5-10.

b. Configuration selection methods . The methods normally employed are semi-empirical and seek to account for weight variations due to changes in major design parameters. Such methods are valuable in parametric studies aimed at finding out which combination of parameters yields the best compromise of all the input variables. Not only must the methods used provide accurate answers in an absolute sense, but the predicted effect of the variation of each parameter should be equally correct. The computation methodology is iterative in nature, i.e. starting values for the design weights must be assumed or based on previous estimates, and after completion of the weight breakdown the new values must be used to start a further computation cycle, continuing in this way until the process has con- 
verged to within the required accuracy. Examples of these types of methods will be presented in Chapter 8.

c. Post-configuration selection methods. In this phase a baseline configuration has been selected and it is necessary to analyse the loads and weight in greater detail. Major elements of such a method are a detailed weight breakdown and the use of preliminary stress analysis to determine the amount of structural material required to resist the applied loads and provide adequate stiffness. Weights must be added for joints, cut-outs, splices and other features which complicate the structural arrangement. Design specifications of all systems must be available to estimate their configuration, power requirements and weight. Due to the very complex character of the type of work involved, comprehensive methods covering all conceivable aircraft types are not readily available. However, many examples of rational methods applicable to detail weights can be found in the literature. The Society of Allied Weight Engineers (SAWE) has published many of these and a selection of papers appears in the list of references to Chapter 8 .

\subsubsection{Examples of waight "guesstimates"}

The takeoff weight is the sum of operating empty weight, payload and fuel weight:

$w_{t o}=w_{e}+w_{p}+w_{f}$

The empty weight can be considered as the sum of a fixed weight and a variable weight:

$w_{e}=w_{f i x}+w_{\text {var }}$

The actual subdivision can be adapted to the case under consideration. By way of example, if the engine to be used is known at this stage, it will be considered as a fixed weight. Like the fuel load, the variable weight can be considered as a fraction of the takeoff weight, resulting in:

$$
w_{\text {to }}=\frac{w_{p_{p}}+w_{f i x}}{1-\frac{w_{\text {var }}}{w_{\text {to }}}-\frac{w_{f}}{w_{\text {to }}}}
$$

This equation will now be evaluated for several airplane categoriés.

a. Light aircraft with piston engines. One important contribution is the engine weight, which is frequently a known factor. Alternatively, it can be estimated from Fig. 4-12. We then write:

$w_{\text {to }}=\frac{w_{p}+w_{\text {eng }}}{1-\frac{w_{\text {var }}}{w_{\text {to }}}-\frac{w_{f}}{w_{\text {to }}}}$

The following averages were found from data relating to some 100 light aircraft:

$$
\begin{aligned}
\frac{\mathrm{w}_{\text {var }}}{\mathrm{W}_{\text {to }}}=\frac{\mathrm{W}_{\mathrm{e}}-\mathrm{W}_{\text {eng }}}{\mathrm{W}_{\text {to }}} & =.45-\text { fixed gear } \\
& =.47-\begin{array}{l}
\text { retractable } \\
\text { gear }
\end{array} \mid \begin{array}{l}
\text { nor- } \\
\text { mal } \\
\text { cate- } \\
\text { gory }
\end{array} \\
& =.50-\begin{array}{l}
\text { utility } \\
\text { category }
\end{array} \\
& =.55-\begin{array}{l}
\text { acrobatic } \\
\text { category }
\end{array}
\end{aligned}
$$

$\frac{\mathrm{w}_{\mathrm{f}}}{\mathrm{w}_{\text {to }}}=$ constant $\frac{\mathrm{R}}{1000} \mathrm{r}_{\mathrm{uc}} \mathrm{A}^{-.5}+.035(5-6)$

where the constant is .31 when $R$ is in $\mathrm{n} . \mathrm{m}$. or .17 when $\mathrm{R}$ in $\mathrm{km}$.

The factor $r_{u c}$ accounts for undercarriage drag (see section 5.3.2.).

b. Turbojet and turbopropeller aircraft. In principle, cruise fuel can be determined with the well-known Breguet range equation. However, this is complicated by the fact that extra fuel is required for takeoff, climb, descent, reserves, etc. To avoid lengthy computations an estimate can be made as follows:

Turboprop aircraft: the total fuel quantity is obtained from Fig. 5-1.

Turbojet aircraft: the fuel is split up into trip fuel and reserve fuel. Trip fuel is given by Fig. 5-2, explained in Section 5.4.2., and reserve fuel is given by eq. 5-46 or 5-47. 


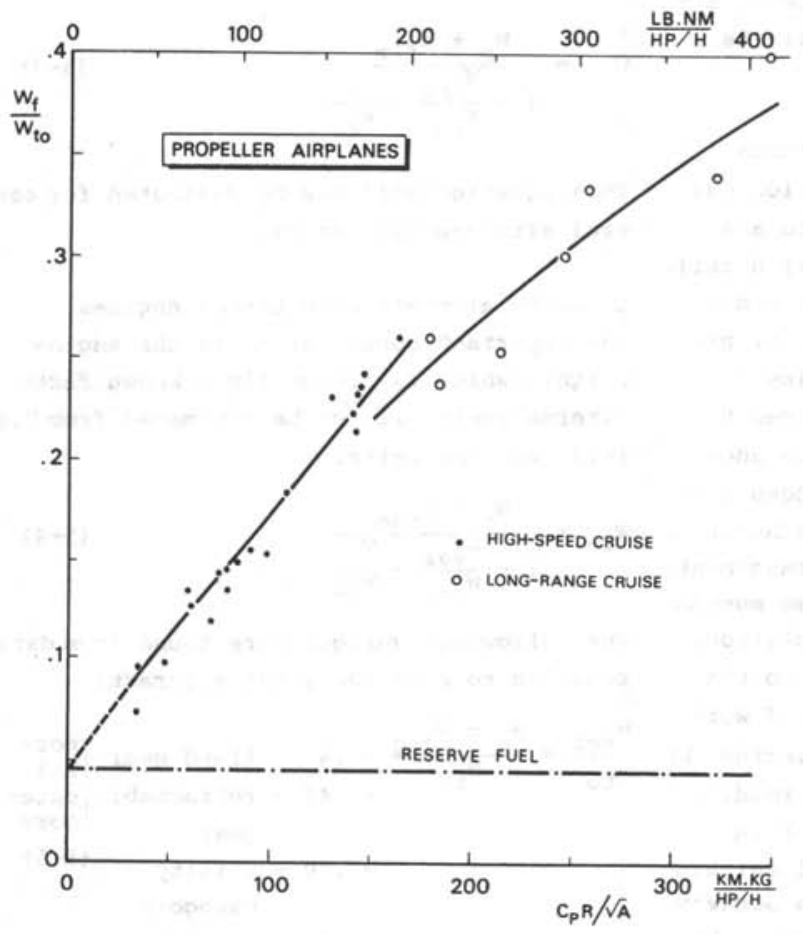

Fig. 5-1. Estimation of fuel weight fraction for turboprop aircraft (small and transport category)

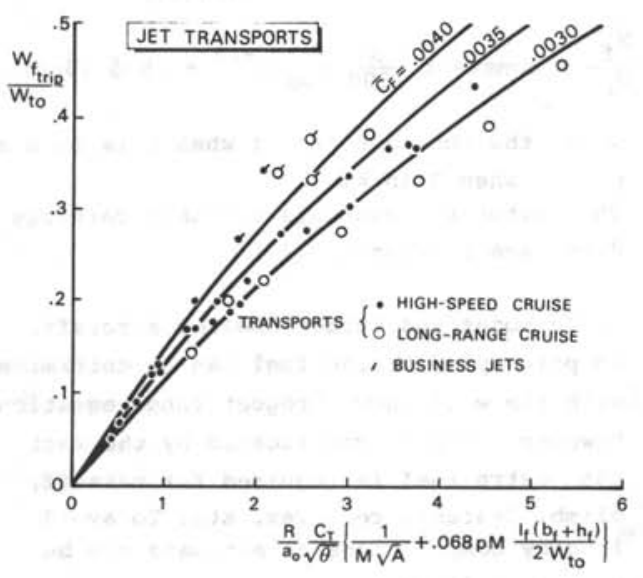

Explanation:

$\mathrm{p}$ and $\mathrm{M}$ : ambient pressure and cruise Mach number

$\mathrm{C}_{\mathrm{T}} / \sqrt{\theta}:$ corrected s.f.c. tions $b_{f}, h_{f}, l_{f}: \max$. width, height and length resp. of fuselage

R : range

a : speed of sound at SL, ISA

$\overline{\mathrm{C}}_{\mathrm{F}} \quad$ : mean skin friction coefficient, based on wetted area

Typical values:

$\begin{aligned} \overline{\mathrm{C}}_{\mathrm{F}} \quad= & .0030- \\ & \begin{array}{l}\text { large, long-range trans- } \\ \text { ports }\end{array} \\ .0035- & \text { smal1, short-range } \\ & \text { transports } \\ .0040- & \text { business and executive } \\ & \text { jets }\end{aligned}$

Fig. 5-2. Estimation of trip fuel weight fraction for jet airliners and executive aircraft 


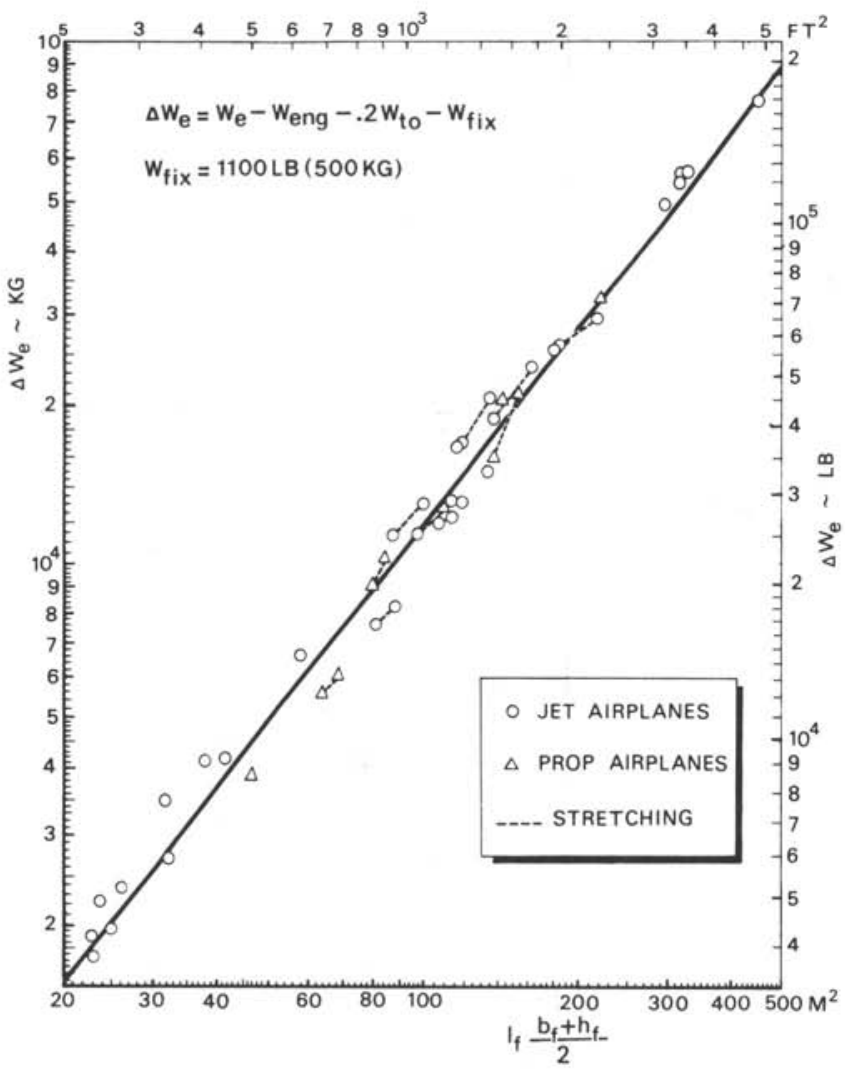

Fig. 5-3. Chart to estimate the empty weight of transport and executive airplanes

The empty weight of light aircraft ( $W_{\text {to }}$ * $12,5001 \mathrm{~b}, 5670 \mathrm{~kg}$ ) is roughly 608 of the takeoff weight, hence:

$w_{\text {to }}=\frac{w_{p}}{.4-w_{f} / w_{\text {to }}}$

For transport aircraft with $\mathrm{w}_{\text {to }}>12,5001 \mathrm{~b}$ $(5,670 \mathrm{~kg})$, the accuracy of the empty weight prediction can be improved by splitting it up into:

- the weight of the dry engines,

- a fixed weight of approximately 1,100 1b $(500 \mathrm{~kg})$,

- a constant fraction of the takeoff weight, mainly associated with wing and undercarriage structure,

- a weight group dependent upon the fuselage size.
A justification for this approach is given in Fig. 5-3. The 208 fraction of $w_{t o}$ is derived from data relating to several developed aircraft types, leading to takeoff weight growth, without changes in fuselage dimensions. The importance of the fuselage size is obvious not only from the direct contribution of the fuselage structure, but also from the fact that the weight of such items as sound insulation, wall trim, floor covering and the airconditioning system, etc. is closely related to the fuselage dimensions as well.

The result of this approach is:

$w_{t o}=\frac{w_{p}+w_{\text {eng }}+w_{f i x}+\Delta w_{e}}{.8-\frac{w_{f}}{w_{\text {to }}}-\frac{w_{\text {eng }}}{w_{\text {bo }}}}$

where $\Delta w_{e}$ is derived from Fig. 5-3 and $w_{f i x}$ is equal to $1,100 \mathrm{lb}(500 \mathrm{~kg})$. 
At this stage, the fuselage dimensions $\ell_{f}$, $b_{f}$ and $h_{f}$ will generally be known from a layout drawing, or may be estimated from statistical data (e.g. from Figs. 3-12 and 3-13). The fuel weight fraction can be estimated from comparable aircraft types or from Fig. 5-1 for turbopropeller aircraft and Fig. 5-2 for turbojet aircraft. The engine weight will be known once the engine is chosen. Otherwise 5 to 6 of of the takeoff weight may be assumed as a typical value.

\subsection{INITIAL ESTIMATION OF AIRPLANE DRAG}

\subsubsection{Drag breakdown}

In the cruise configuration (flaps and urdercarriage retracted) and for low-subsonic flight speeds, the drag coefficient $C_{D}$ is usually expressed as a unique function of the lift coefficient $C_{L}$, referred to as the airplane drag polar. Most low-speed polars are approximated by a parabola,

$C_{D}=C_{D_{0}}+C_{D_{i}}=C_{D_{0}}+\frac{C_{L}{ }^{2}}{\pi A e}$

The drag coefficient, based on the wing area, is frequently expressed in counts; one count $=.0001$. The symbol $\mathrm{A}=\mathrm{b}^{2} / \mathrm{s} \mathrm{de}-$ notes the aspect ratio of a wing with span $\mathrm{b}$ and gross planfcrm area $S$ (definitions in Appendix A-2).

When the actual drag polar, as determined from measurements in test flights or a wind tunnel, is compared with eq. 5-9, it is found that in a practical region of $C_{L}$ values the straight line approximation of $C_{D}$ vs. $C_{L}{ }^{2}$ is acceptable (Fig. 5-4).

Contrary to the exact polar curve, having a minimum at some small positive value of $C_{L}$, eq. 5-9 indicates that $C_{D}$ is minimum for $C_{L}=0$. The zero-lift drag coefficient $C_{D_{0}}$ is a fictitious quantity for the general case of a non-symmetrical aircraft, the actual $C_{D}$ for $C_{L}=0$ being slightly higher. The induced or lift-dependent drag coefficient can be compared to the theoretical induced drag of a wing with elliptic spanwise lift distribution,

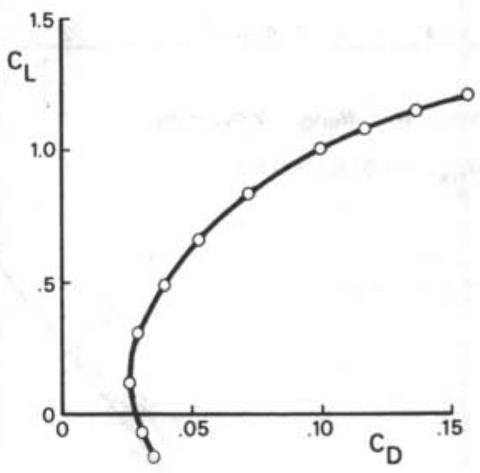

a. $C_{D}$ vs. $C_{L}$

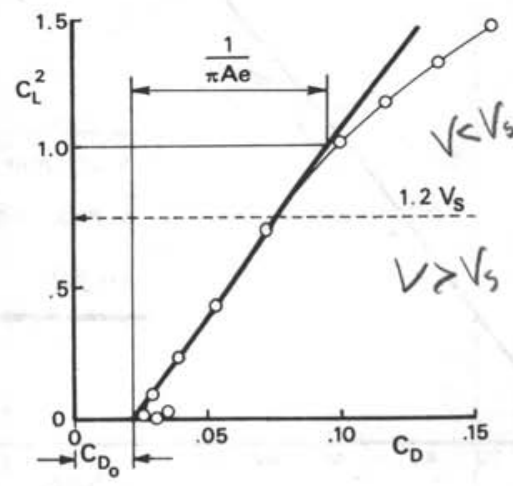

b. $C_{D}$ vs. $C_{L}{ }^{2}$

Fig. 5-4. Typical low-speed polar curve $C_{D_{i}}=\frac{C_{L}{ }^{2}}{\pi A}$

The Oswald efficiency factor e in eq. 5-9 accounts for the non-ellipticity of the lift distribution, the increase of profile drag of the wing, fuselage, tailplane, nacelles and various interference effects with the angle of attack.

A preliminary stage of drag estimation may be accomplished by adding the individual drag contributions of the various components of the airplane:

$C_{D_{0}}=\frac{\sum C_{D_{j}} s_{j}}{S}$

The product $C_{D_{j}} S_{j}$ is the drag area of each 
component. For wings with thickness/chord ratios of up to $20 \%$ and slender fuselages (length/diameter ratio greater than about 4), skin friction drag is predominant and it is customary to deduce the drag coefficient of the components from their wetted area. Table 5-1 shows typical low-speed drag figures for various classes of airplanes in the cruise configuration.

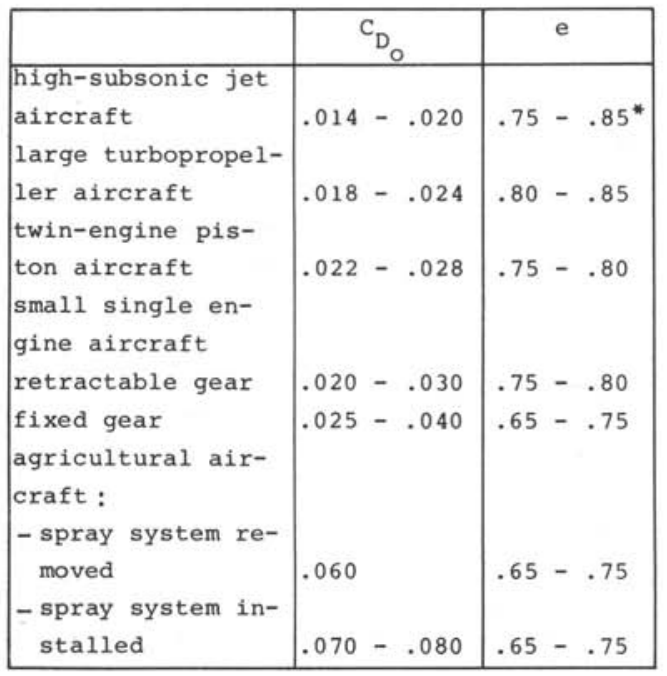

* The higher the sweep angle, the lower the e-factor

Table 5-1. Drag figures for various aircraft types

\subsubsection{Low-speed drag estimation method}

The example of a drag prediction method presented in this section, is a very elementary approach, which can be refined if desired. A more detailed drag prediction method is given in Appendix F.

The drag of an aircraft component may be estimated by comparing it to the friction drag of an equivalent flat plate having the same wetted area and length. For this comparison to be valid, the boundary layer must develop in a similar manner for both cases and therefore the Reynolds number, with respect to the length, must be equal, while the transition from laminar to tur- bulent flow is assumed to occur at the same distance from the nose or leading edge.

A second condition which must be fulfilled for the flat plate analogy to be valid is that there should be no appreciable regions of separation, and the aircraft components should therefore be well-streamlined, moderately cambered and smooth in shape. With regard to sharp corners, rear fuselage upsweep or short stubby fuselage tails, for example, the flat plate analogy yields no realistic answers. In such cases reference should be made to experimental data, a valuable collection of which is to be found in Ref. 5-12.

To account for distributed surface irregularities and roughness drag, the boundary layer is sometimes assumed to be fully turbulent. In this case the friction coefficient according to Prandt-Schlichting, based on the wetted area, is given by:

$C_{F}=\frac{.455}{(\log R e)^{2.58}}$

This equation is depicted in Fig. 5-5 in a normalized form, i.e. the value of $\mathrm{C}_{\mathrm{F}}$ for $\operatorname{Re}=10^{8}$ is taken equal to 1 . To account for the thickness of the body, shape factors representing the ratio of actual drag to flat plate drag are given in literature for wing sections and circular streamline bodies (e.g. in Ref. 5-12). For each aircraft the exposed (wetted) area must be calculated, interference effects estimated and extras added to account for protuberances, flap and control surface slots, cockpit windows and the like (Appendix F). A detailed drag estimation is usually a very elaborate exercise, for which most airplane manufacturers have developed their own procedures. For the purpose of sizing the airplane and the engine, a somewhat simplified approach based on statistical data is suggested here. In the present method, the zero-lift drag will be calculated according to the following basic equation:

$C_{D_{0}} S=r_{\operatorname{Re}}{ }_{u C}\left[r_{t}\left\{\left(C_{D} S\right)_{w}+\left(C_{D} S\right)_{f}\right\}+\left(C_{D} S\right)_{n}\right](5-13)$

The various contributions will be explained 


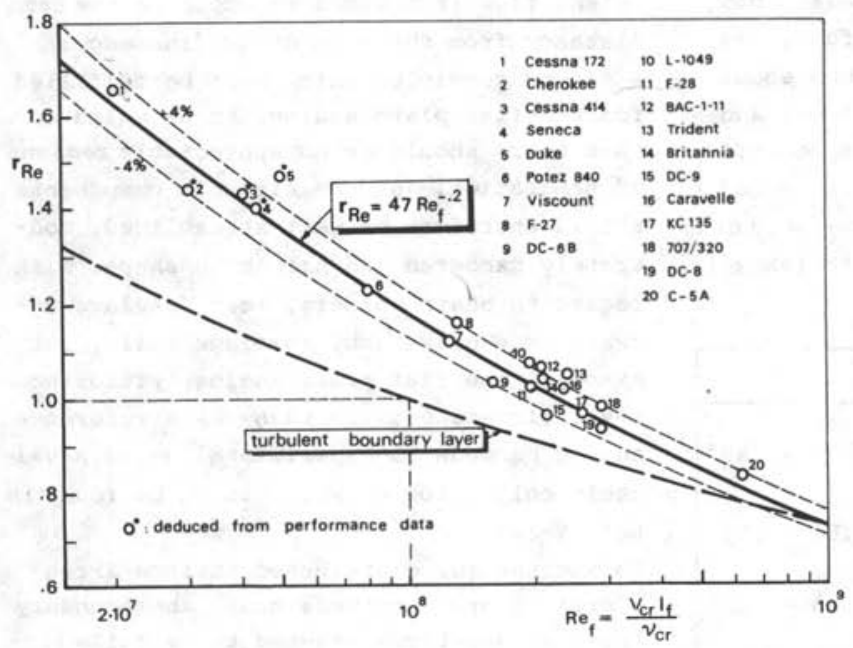

Fig. 5-5. Correction factor on $\mathrm{C}_{\mathrm{D}_{\mathrm{O}}}$ for scale effects, roughness, etc

below. Base* drag is not included but may be accounted for, if present, by assuming $\Delta\left(C_{D} S\right)=.13$ times the projection of the base area on a plane normal to the flow.

\section{a. wing.}

Uncorrected drag area for smooth wings:

$$
\left(C_{D} S\right)_{w}=.0054 r_{w}\left\{1+3(t / c) \cos ^{2} \Lambda_{.25}\right\} S
$$

where $r_{w}=1.0$ for cantilever and 1.1 for braced wings

$t / c=$ mean thickness/chord ratio

$\Lambda_{.25}=$ sweep angle at the quarter-chord line

$\mathrm{S}=$ gross planform area (Appendix $\mathrm{A}-2$ )

Eq. 5-14 is derived from the flat plate analogy and a typical thickness correction for $t / c$ up to .20 . The "uncorrected" drag figure applies to a smooth wing with the transition region at approximately 108 of the chord, for a Reynolds number of 12.5 million, based on the geometric mean chord length.

* Base: a surface, usually at the rear end of the fuselage, more or less normal to the flow. b. Fuselage.

Uncorrected parasite drag for streamline shapes:

$$
\left(C_{D} S\right)_{f}=.0031 \mathrm{r}_{f} l_{f}\left(b_{f}+h_{f}\right)
$$

$1_{f}=$ fuselage length, including propeller spinner or jet engine outlet, if present,

$\mathrm{b}_{\mathrm{f}}, \mathrm{h}_{\mathrm{f}}=\max$. width and height of the major cross section, including canopy,

$r_{f}=$ shape factor, i.e. the ratio of actual wetted area to that of a fuselage with elliptical or circular cross-section and cylindrical midsection, for which $r_{f}=1.0$,

$r_{f}=1.30$ - rectangular cross-section,

$=1.15$ - one side of cross-section rectangular, other side rounded off, $=.65+1.5 \frac{\text { diameter }}{\text { length }}$ fully streamlined fuselages without cylindrical mid-section.

The "uncorrected" drag figure applies to a fuselage with fully turbulent boundary layer, for a Reynolds number equal to 100 million, based on $\ell_{f}$. Calculations have shown that the thickness correction, multiplied by the ratio of gross wetted area to the cylinder area $.5 \pi l_{f}\left(b_{f}+h_{f}\right), y i e l d s$ an almost constant value of approximately .93 for most practical length/diameter ra- 
t1os. Great care should be taken when applying eq. 5-15 to aircraft with rear fuselage upsweep, bluff canopies, etc., for which considerably higher drag figures must be expected (cf. Section 3.5.1. and Fig. 3-27).

c. Tailplane.

By analyzing tailplane drag in the same way as the wing drag, it was found that in practical cases the tailplane contribution amounts to roughly 248 of fuselage plus wing drag; hence $r_{t}=1.24$ is a good average. On STOL-type aircraft $r_{t}$ may be as high as 1.30 due to the relatively large tailplane area.

d. Engine installation and nacelles. The parasite areas will be related to the installed thrust or power, in order to account for variations of nacelle or intake scoop size and shape with engine thrust or power.

\section{TURBOJET ENGINES}

The isolated engine pod is taken as the reference case; its wetted area can be related to the engine mass flow, taking due account of differences in pod design for variation in bypass ratio. On the basis of actual pod shapes and wetted areas, an uncorrected friction coefficient of .003 and typical scrubbing and supervelocity drag values for cruising flight, the following expression was found:

$\left(C_{D} S\right)_{n}=1.72 r_{n} r_{t h r}\left(\frac{5+\lambda}{1+\lambda}\right) \frac{T_{\text {to }}}{\psi_{\text {to }} \mathrm{p}_{0}}$

where $T_{\text {to }}$ and $\psi_{\text {to }}$ refer to standard SLS conditions and

$r_{\text {thr }}=1.0$ - thrust reversers installed,

$$
=.82 \text { - no thrust reversers. }
$$

The factor $r_{n}$ is an installation factor to account for pylon and interference drag in the case of podded engines. For buried engines $r_{n}$ represents drag due to intake scoops, exhaust pipes, etc.

The following figures are suggested as typical: $r_{n}=1.50-$ all engines podded

$=1.65$ - two engines podded, one buried in fuselage tail (this figure includes internal drag of the central inlet)

$=1.25$ - engines buried in nacelles, attached on the side of the fuselage

$=1.00$ - engines fully buried, intake scoops on fuselage

$=.30$ - engines fully buried, wing root intakes

It should be noted that the factor $(5+\lambda) /$ $(1+\lambda)$ in eq. 5-16 has no physical meaning; it has been derived on a statistical basis from the ratio of wetted area to frontal area for actual engines with varying bypass ratios.

\section{TURBOPROP ENGINES}

The uncorrected nacelle drag is:

$\left(C_{D} s\right)_{n}=.1 r_{n} \frac{P_{\text {to }}}{\phi_{\text {to }}}$

where $\mathrm{P}_{\text {to }}$ and $\phi_{\text {to }}$ refer to standard SLS conditions and

$r_{n}=1.0$ - ring-type inlets

$=1.6$ - scoop-type inlets, increasing the frontal area

Eq. 5-17 is based on a typical drag coefficient .065 based on the nacelle frontal area, while for ring-type inlets the engine frontal area is approximately 658 of the nacelle frontal area.

\section{PISTON ENGINES}

Wing-mounted:

$\left(C_{D}\right)_{n}=.07 \zeta_{n} \frac{P_{\text {to }}}{\psi_{\text {to }}}$

where $\mathrm{P}_{\text {to }}$ and $\psi_{\text {to }}$ refer to SLS-ISA conditions and

$\zeta_{\mathrm{n}}=\frac{\text { nacelle frontal area }}{\text { cylinder volume }}$

Typical values for $\psi_{\text {to }}$ may be obtained from Section 4.2 or from engine manufacturers' data. A favorable effect of size is reflected in the factor ' ${ } n$ ' which is of the order of .012 to $.015 \mathrm{sq}$.ft per cu.in. $\left(.07-.09 \mathrm{~m}^{2} / 1\right.$ iter $)$ for engine powers up to 
$500 \mathrm{hp}$, but may go down to .05 to $.07 \mathrm{sq} . \mathrm{ft}$ per cu.in. (.03 to $.04 \mathrm{~m}^{2} / 1$ iter) for the 2,000-4,000 hp class.

Fuselage-mounted tractor engines:

$$
\left(C_{D} s\right)_{n}=.015 b_{f} h_{f}
$$

e. Undercarriage.

For undercarriages which are fully retracted within the airplane external lines* a drag penalty is not necessary $\left(r_{u c}=1.0\right)$. other values can be derived by retracing differences in measured performance of aircraft with fixed and retractable gear versions and from published data on drag:

$r_{\text {uc }}=1.35$ - fixed gear, no streamlined wheel fairings

$=1.25$ - fixed gear, streamlined wheel fairings and struts

$=1.08$ - main gear retracted in streamlined fairings on the fuselage (like C- 130 and $C-5 A$ )

$=1.03$ - main gear retracted in nacelles of turbopropeller engines

f. Wing tip tanks.

A typical figure, such as $\Delta\left(C_{D} S\right)=.055$ times the tank's frontal area, should be added to eq. 5-13. To compute the effective aspect ratio, the effective wing span and area should be taken as the distance and area between the tank centerlines (Ref. 5-12).

g. Corrections for Reynolds number and miscellaneous drag.

It can be argued that interference effects, surface irregularities, excrescences, air scoops, aerials, slots, etc. generally affect the boundary layer more on small lowspeed aircraft than they do on large highspeed aircraft, due to differences in relative size and emphasis on pure aerodynamic design. In evaluating the present method, the ratio of actual measured to basic zero lift drag was therefore plotted versus the Reynolds number, based on fuselage length (Fig. 5-5).

\footnotetext{
*i.e. no wheel fairings are required
}

The following approximation accounts for the effect of the Reynolds number on turbulent skin friction drag and miscellaneous drag items:

$\mathrm{r}_{\mathrm{Re}}=\frac{\text { actual zero-1ift drag coefficient }}{}{ }^{*}=$

$$
=47 \operatorname{Re}_{\mathrm{f}}^{-.2}
$$

where

$$
\operatorname{Re}_{f}=\frac{v_{c r}{ }^{1} f}{v_{c r}}
$$

The subscript "cr" refers to the design cruising altitude and speed. Figure 5-5 shows that the miscellaneous drag contributions amount to 25-308 for light aircraft and 10-158 or less on large transport aircraft. The rms error of the method is 48 , but this figure also allows for inaccuracy in the available data on drag polars.

\subsubsection{Compressibility drag}

Compressibility effects on drag are generally ignored at Mach numbers below .5. The drag polars of a high-subsonic transport aircraft in Fig. 5-6 illustrate that for

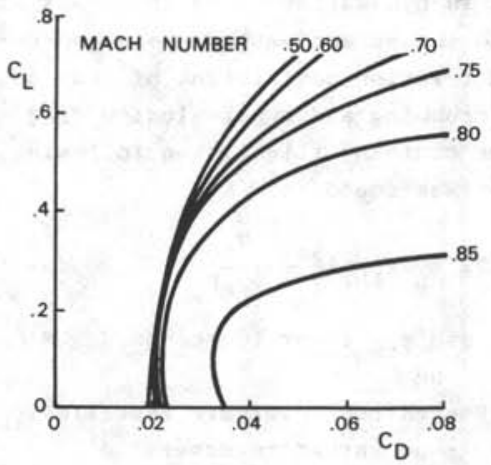

Fig. 5-6. Effect of compressibility on the drag polar

\footnotetext{
*including excrescences, protuberances, roughness, etc.
} 
low $C_{L}$ values and Mach numbers up to .70 the effects of compressibility on drag are of secondary nature. Between $\mathrm{M}=.70$ and .80 a steady increase in the drag is observed ("drag creep") and at a critical Mach number of approximately .85 a rapid rise is experienced in both the zero-lift drag and the induced drag. This drag rise is caused by shock waves and boundary layer separation induced by these shock waves. The endeavor to achieve low compressibility drag, or rather a high drag-critical Mach number, is one of the most comprehensive tasks in aerodynamic design. This subject is dealt with more fully in Section 7.2. For the purpose of initial design calculations, it is fair to assume that the aerodynamicists will achieve acceptable drag figures at the design cruising speed, provided that wing sweep and thickness ratio are chosen appropriately. We may therefore assume that:

$$
\begin{gathered}
\Delta \mathrm{C}_{\mathrm{D}_{\text {comp }}=.0005-} \begin{array}{l}
\text { long-range cruise condi- } \\
\text { tions }
\end{array} \\
\Delta \mathrm{C}_{\mathrm{D}_{\text {comp }}=.0020-} \begin{array}{l}
\text { high-speed cruise condi- } \\
\text { tions }
\end{array}
\end{gathered}
$$

\subsubsection{Retracing a drag polar from perform-} ance figures

In order to compare an estimated drag polar with the drag figures of existing airplanes, drag coefficients may be deduced from performance data as supplied by the airplane manufacturer. To do this for a given type of aircraft, the lift coefficient in cruising flight is calculated first,

$\mathrm{C}_{\mathrm{L}_{\mathrm{Cr}}}=\left.\frac{\mathrm{W} / \mathrm{S}}{\frac{1}{2} \rho \mathrm{V}^{2}}\right|_{\mathrm{Cr}}$

Cruising altitude and speed are obtained from the manufacturer's brochure or other appropriate publications. The drag due to lift,

$C_{D_{i_{c r}}}=\frac{C_{L_{c r}} 2}{\pi A e}$ is estimated by assuming a typical value for e (cf. Table 5-1) and taking a condition with a fairly low $C_{L}$. As thrust equals drag in cruising flight, we may write:

$\left.\mathrm{C}_{\mathrm{Dr}}=\frac{\mathrm{T}}{\frac{1}{2} \mathrm{PpM}^{2} \mathrm{~S}}\right]_{\mathrm{cr}}$

(jet airplanes)

or

$\left.C_{D_{c r}}=\frac{n_{p} P_{b r}}{\frac{1}{2} \rho v^{3} s}\right]_{c r} \quad \begin{gathered}\text { (propeller } \\ \text { airplanes) }\end{gathered}$

For jet-propelled aircraft, $T$ is the installed thrust, which is lower than the uninstalled thrust due to bleed air, power offtakes and inlet pressure loss. A typical figure may be 48 reduction, but for high bypass engines it may well be as high as 88 . For propeller aircraft $\eta_{p}$ represents the combined effect of installed propeller efficiency, extra drag of aircraft components in the slipstream, intake losses, power and bleed air offtakes and cooling drag. Typical figures are:

$n_{p}=.85$ - turbopropeller engines (including jet thrust)

$=.80-$ wing-mounted piston engines

$=.78$ - tractor piston engine in fuselage nose.

Cruise power or thrust may be obtained from the engine brochure for the appropriate rating, altitude and flight speed. In the case of piston engines, ratings are usually 758 or 658 rated power for performance and economic cruising respectiveiy. Finally, the zero-lift drag is calculated:

$\left.C_{D_{0}}=C_{D_{C r}}-C_{D_{i}}\right]_{c r}$

A more accurate determination can be made by analyzing several flight conditions in a similar way. By plotting the values of $C_{D}$ vs $C_{L}{ }^{2}, C_{D}$ and $e$ can be found by a straight-line approximation, in the manner indicated in Fig. 5-4.

\subsubsection{Drag in takeoff and landing}

Drag in the en route configuration determines the cruise thrust and hourly fuel 


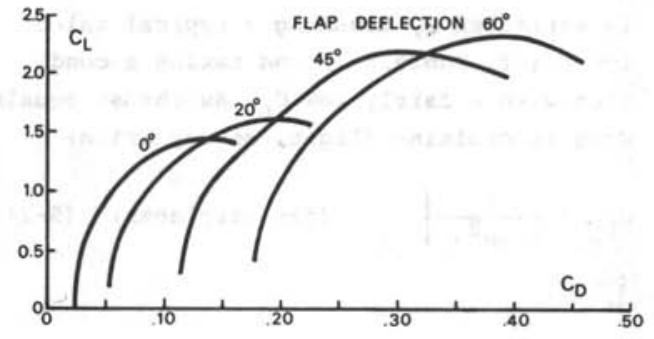

Fig. 5-7. Low-speed polars for a light transport

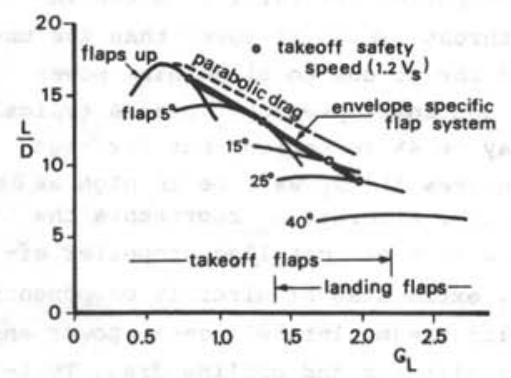

Fig. 5-8. Typical locus of $11 \mathrm{ft} / \mathrm{drag}$ ratios for the takeoff safety speed

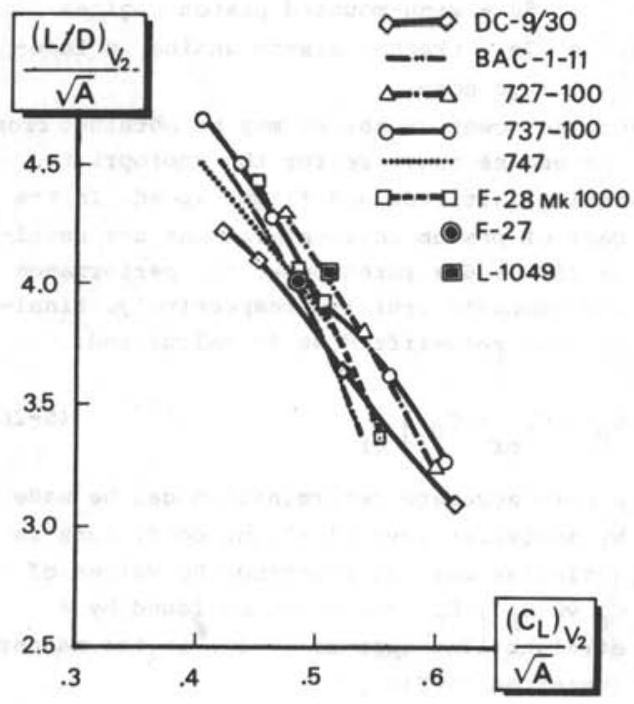

Fig. 5-9. Generalized takeoff 1 ift/drag ratios consumption. Alternatively, drag in the low-speed configuration (flaps deflected) determines the permissible takeoff weight for a particular flight of a passenger alrliner and hence the maximum useful load. On a hot and high airfield, a limitation on takeoff weight may result in a reduced pay load or a restricted fuel weight and range. In such a case a drag deterioration of 108 may result in 308 less payload to be carried.

Typical polars for several flap deflections for takeoff and landing are shown in Fig. 5-7. As the available climb gradient,

$\gamma=\frac{T}{W}-\frac{C_{D}}{C_{L}}$

depends on the 1 ift/drag ratio, the aerodynamic characteristics are sometimes plotted accordingly (Fig. 5-8) ${ }^{* *}$ For each flap angle, the L/D ratio at the takeoff safety speed $v_{2}$ are indicated on the curves. A line connecting these points forms an envelope or locus, which is useful in comparing one flap system with another.

Since accurate prediction of the results of a flap design program is obviously impossible, it is useful to compare the $v_{2}$ locus on a basis of generalized parameters, by writing the drag polar as follows:

$\frac{C_{L} / C_{D}}{\sqrt{\AA}}=\left(\frac{C_{D_{O}} \sqrt{\AA}}{C_{L}}+\frac{C_{L}}{\pi e \sqrt{\bar{A}}}\right)^{-1}$

The values of $C_{D_{0}}$ and $e$ refer to the configurations with ${ }^{\circ}$ flaps deflected. Fig. 5-9 presents a collection of some published $\mathrm{v}_{2}$ loci. The following approximation is suggested for preliminary design purposes:

$\mathrm{C}_{\mathrm{D}_{\mathrm{V}_{2}}}=\mathrm{c}_{\mathrm{o}}+\frac{\left(\mathrm{C}_{\mathrm{L}_{\mathrm{V}}}\right)^{2}}{\pi \mathrm{AE}}$

where $\mathrm{C}_{0}=.018, \mathrm{E}=\cdot .70$ slats extended $c_{0}=.005, E=.61$ no slats or slats retracted.

* Definition in Section 8.2.1.

** A more complete figure is shown on Fig. $11-4$ 
Note that this equation does not represent an actual aircraft polar; it refers to the initial climb-out after takeoff. To include drag due to engine failure at low thrust/ weight ratios, E may be reduced by approximately 4 응 for wing-mounted engines and $2 \%$ for engines mounted on either side of the fuselage tail.

Although the accuracy of eq. 5-29 for the approach and landing configurations is probably not so good, due to the higher ratio of flight speed to stalling speed, it still remains. a useful first-order approximation.

\subsection{EVALUATION OF PERFORMANCE REQUIREMENTS}

The objective of performance analysis is to predict the performance of an airplane type of given design and geometry. The designer, however, is confronted with the reverse problem: knowing the performance objectives, he must find combinations of design characteristics which will result in a design that satisfies or exceeds all requirements.

The intention of this section is to translate the performance requirements into boundary values for those major aircraft parameters and characteristics, which have a first-order effect on performance:

\section{a. Powerplant.}

1. Total SLS takeoff thrust (power) of all engines, uninstalled, usually combined with the takeoff weight in the thrust/weight ratio $\left(\mathrm{T}_{\text {to }} / \mathrm{W}_{\text {to }}\right)$ or power/weight ratio $\left(\mathrm{P}_{t o} /\right.$ $\left.\mathrm{w}_{\text {to }}\right)$. Thrust loading or power loading is the reciprocal value of this: $\mathrm{W}_{\text {to }} / \mathrm{T}_{\text {to }}$ or $\mathrm{w}_{\text {to }} / \mathrm{P}_{\text {to }}$.

2. The number of engines, $\mathrm{N}_{e}$.

3. Engine type or configuration. The thrust or power lapse ratios with speed, altitude and ambient temperature ( $\mathrm{T} / \mathrm{T}_{\text {to }}$ or $\left.\mathrm{P} / \mathrm{P}_{\text {to }}\right)$, and the specific fuel consumption at cruising conditions are particularly relevant. It is not customary to derive boundary values for these characteristics, as they can- not be considered as independent variables (cf. Chapter 4, Section 4.4.).

b. Wing.

1. Gross wing area $S$, usually combined with the all-up weight into the wing loading at takeoff $\mathrm{w}_{\text {to }} / \mathrm{s}$.

2. Aspect ratio $A=b^{2} / S$. Instead of $A$, the span loading $\mathrm{w}_{\text {to }} / \mathrm{b}^{2}$ is occasionally used.* 3. High-lift devices, in particular the available $C_{L}$-max and lift/drag ratios in takeoff and landing configurations.

The wing section shape, the taper ratio and the sweep angle are not considered here; these will be discussed in Chapter 7 .

The various performance equations will be presented in a way which permits their ready application. For climb and high-speec performance it is generally convenient to express the engine thrust (power) in terms of the other parameters, for low-speed performance the wing loading limitations are most easily derived explicitly, particularly in the case of a fixed engine and given aircraft weight.

The performance aims may not always be realized. For example, with regard to imaginary solutions for the wing area. Even in the case of a fixed engine it is therefore useful to consider possible engine growth, or alternatively to use the equations to deduce the permissible takeoff weight from the most critical performance item.

\subsubsection{High-speed performance}

a. Jet-propelled aircraft.

The thrust required to fly at a given speed and predetermined altitude is given by :

$T=D=\frac{1}{2} \gamma p M^{2} C_{D} S$

* span loading is sometimes defined as $\mathrm{w}_{\text {to }} / \mathrm{b}$ 
where $T$ is the installed thrust, as derived from the engine manufacturers' brochure for a given engine, or assumed equal to a certain percentage of the uninstalled thrust when the engine has not yet been sized (cf. Section 5.3.4.).

The drag coeficient, as given by eq. 5-9, is dependent on the lift coefficient,

$c_{L}=\frac{w / S}{\frac{1}{2} \gamma \mathrm{pM}^{2}}$

and eq. 5-30 can be rewritten as follows:

$\frac{T}{W}=\frac{\frac{1}{2} \gamma M^{2} C_{D_{0}}}{W / p S}+\frac{W}{p S} \frac{1}{\frac{1}{2} \gamma M^{2} \pi A e}$

The zero-lift drag coefficient may be elaborated in terms of the wing area and the installed thrust. Any drag prediction method available to the designer may be used for this purpose. In this text the semistatistical method of section 5.3. is used to derive the following result:

$\frac{\mathrm{T}_{\text {to }}}{\mathrm{w}_{\text {to }}}=\frac{\frac{.7 \delta \mathrm{M}^{2} \mathrm{~d}_{1}}{\mathrm{w}_{t o} / \mathrm{P}_{\mathrm{o}} \mathrm{S}}+\frac{\mathrm{w}_{t o} / \mathrm{p}_{\mathrm{o}} \mathrm{s}}{.7 \mathrm{k}_{\mathrm{W}}{ }^{2} \delta \mathrm{M}^{2} \pi \mathrm{Ae}}+.7 \delta \mathrm{M}^{2} \mathrm{~d}_{2}}{\mathrm{~T} / \mathrm{T}_{\mathrm{to}}-.7 \delta \mathrm{M}^{2} \mathrm{~d}_{3}}(5-33)$

where

$k_{W}=\frac{w_{t o}}{W}$

$d_{1}=r_{R e} r_{u c} r_{t} \frac{\left(C_{D} S\right) w}{S}+\Delta C_{D_{c o m p}}$

$d_{2}=r_{R e} r_{u c} r_{t} \frac{\left(C_{D} s_{f} p_{o}\right.}{w_{t o}}$

$d_{3}=r_{\operatorname{Re}} r_{u c} \quad \frac{\left(C_{D} s\right)_{n} p_{0}}{T_{\text {to }}}$

These terms are explained in Sections 5.3.2. and $5 \cdot 3 \cdot 3$.

In equation 5-33 the three terms in the numerator are associated with wing profile drag, induced drag, fuselage drag and empennage drag. The denominator may be interpreted as an effective lapse ratio of the engines installed in nacelles, including internal and external nacelle or intake scoop drag. The value for $T / T_{\text {to }}$ can be ob- tained from non-dimensional thrust curves or generalized data in Appendix $\mathrm{H}$.

The minimum thrust at a given airspeed and altitude is found when the wing loading is equal to:

$\frac{W}{S}=\frac{1}{2} \gamma \mathrm{pM}^{2} \quad \sqrt{\mathrm{d}_{1} \pi \mathrm{Ae}}$

This condition is identical to the condition for minimum drag (or L/D-max) of the wing plus that part of the tailplane contribution which is proportional to the wing drag. The factor $d_{1}$ is thus proportional to the wing profile arag coefficient; the order of magnitude is $.008-.010$, for aircraft with retractable undercarriage.

b. Propeller aircraft.

The equivalent horsepower required to fly at a given speed and altitude is given by:

$n_{p} P=D V=\frac{1}{2} \rho v^{3} C_{D} S$

The power/weight ratio in the sea level static (SLS) condition is derived in the same way as for jet aircraft:

$\frac{1}{v} \frac{P_{t o}}{W_{t o}}=\frac{\frac{1}{2} \rho v^{2}\left\{\frac{d_{1}}{W_{t o} / S}+\frac{d_{2}}{P_{o}}\right\}+\frac{2 w_{t o} / S}{k_{W}{ }^{2} \rho v^{2} \pi A e}}{n_{p} P / P_{t o}-\frac{1}{2} \rho v^{3} d_{3}}$

For wing-mounted engines, the factors $d_{1}$ and $d_{2}$ are defined analogous to eq. 5-34, while

$d_{3}=r_{\operatorname{Re}} r_{\text {uc }} \frac{\left(C_{D} S\right)_{n}}{P_{\text {to }}}$

This effect of compressibility on the drag can generally be ignored. For a tractor engine in the fuselage nose, $d_{3}=0$ and $d_{1}$ is equal to:

$d_{1}=r_{R e} r_{u c}\left\{r_{t}\left(C_{D} s\right)_{w}+\left(C_{D} s\right)_{n}\right\} / s$

The power lapse ratio $\mathrm{P} / \mathrm{P}_{\text {to }}$ must be derived from the engine manufacturer's data. For the installed efficiency $\eta_{p}$ typical values may be assumed as presented in Section 5.3.4. 


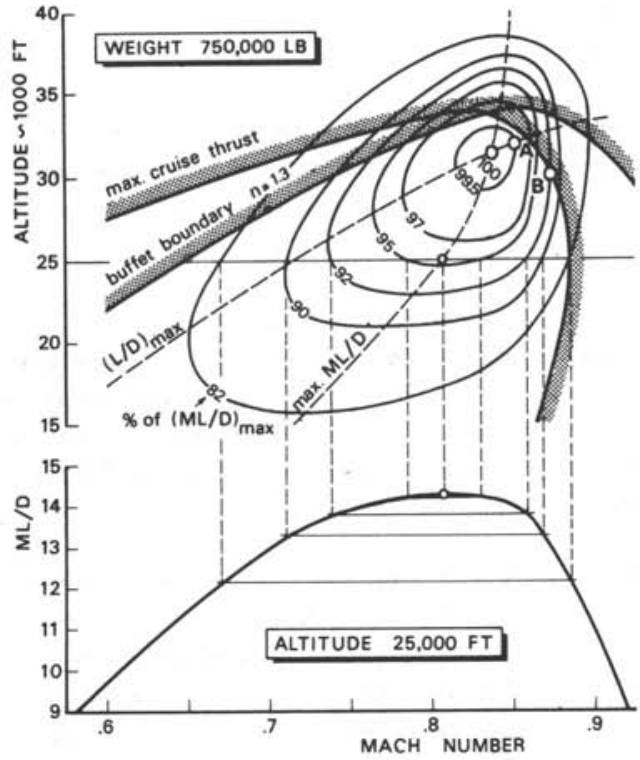

a. With compressibility effects on drag; $1008(\mathrm{ML} / \mathrm{D})_{\max }=14.8$

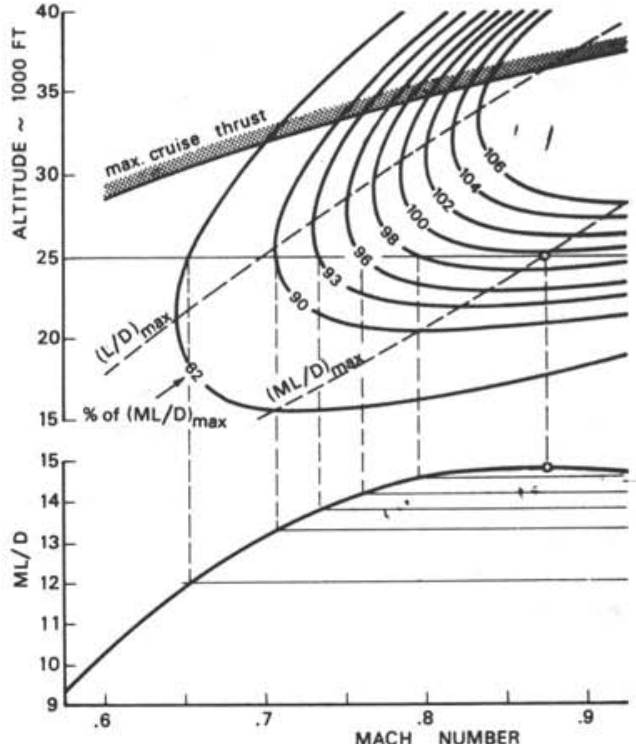

b. No compressibility effects on drag; $1008(\mathrm{ML} / \mathrm{D})_{\max }=14.8$

Fig. 5-10. Range performance of a high-subsonic long-range jet transport

\subsubsection{Range performance}

A specified range or radius of action must be achieved with a given payload or maximum fuel capacity, taking into account fuel reserves for holding and diversion. An indication of the cruise procedure will generally be mentioned: (initial) cruise altitude, long-range or high-speed condition.

\section{a. Jet aircraft.}

Although range performance depends upon the cruise procedure, the Bréguet equation is useful as a basis for an initial prediction of cruise fuel. On the conditions of constant angle of attack, airspeed and specific fuel consumption (climb cruise), the range is:

$R=\frac{V}{C_{T}} \frac{L}{D} \ln \frac{W_{i}}{W_{1}-W_{f}}$

or:

$$
\frac{R}{a_{0}}=\frac{M L / D}{C_{T} / \sqrt{\theta}} \ln \frac{W_{i}}{W_{1}-W_{f}}
$$

where $w_{i}$ is the initial weight and $w_{f}$ the cruise fuel weight. For a given fuel fraction and engine type, the primary parameter in this equation is $M L / D$, the range parameter. The operational variables (cruise altitude, Mach number), the wing loading and the drag polar are the primary variables. For medium- and long-range aircraft, the amount of fuel consumed is large and it is necessary to aim at optimum flight conditions.

For a given drag polar and wing loading, lines of constant $\mathrm{M} \mathrm{L} / \mathrm{D}$ can be plotted on a speed-altitude diagram: Fig. 5-10; the lower part of this figure is an intersection for one altitude. The inclination of the tangent from the origin to the $C_{D} / C_{L}$-curve represents the condition for $\operatorname{maximum} M L / D$. Considering flight at a specified sub-critical Mach 
number and variable altitude first, the maximum range is obtained at an altitude where $L / D$ is maximum, hence

$c_{L}=\sqrt{c_{D_{0}} \pi A e}$

corresponding to the minimum drag speed $\mathrm{M}_{\mathrm{MD}}$. The locus of this condition for each altitude is indicated in the upper part of Fig. 5-10.

For a specified altitude and variable Mach number, ignoring compressibility effects on the drag, the conaition for maximum $M L / D$ is:

$\frac{d}{d C_{L}}\left(\sqrt{\frac{W}{S} \frac{2}{\gamma p} \frac{C_{L}}{C_{D}^{2}}}\right)=0$

resulting in:

$C_{L}=\sqrt{\frac{1}{3} C_{D_{0}} \text { TAe }}$

corresponding to a Mach number equal to $\sqrt[4]{3} \mathrm{MD}$. The conditions for $C_{L}$ according to (5-42) and (5-44) are incompatible and no absolute optimum combination of $M$ and altitude can be obtained. This is confirmed in Fig. 5-10, indicating that in the absence of compressibility $M$ L/D continues to increase with altitude.

For high-subsonic speeds, a rapid rise in drag and subsequent deterioration of the range is observed beyond the drag-critical Mach number. Although the complex character of the flow does not allow an analytical treatment of compressibility effects, Fig. 5-10 indicates that a definite condition for maximum $M L / D$ is now present. The locus of $C_{L}$ for this condition is correspondingly modified and intersects the locus of (L/D) $\max _{\text {at }}$ the optimum combination of $M$ and altitude.

The condition for maximum specific range (i.e. the distance travelled per pound of fuel consumed) is slightly different from (M L/D) $\max$ due to the effects of altitude and speed on engine s.f.c. In operational practice the flight speed will always be some 10-208 above $M_{M D}$ in horizontal flight in order to obtain positive speed stability and to avoid buffet during maneuvers. A typical long-range cruise condition results in 988 of the maximum specific range (point A in Fig. 5-10a). In operations where fuel consumption is not a dominant factor, the high-speed cruise at a somewhat lower cruise alti- tude is significant (point B). The maximum cruise rating of the engines determines the flight speed, and a typical extra drag of some 20 counts is acceptable. An intermediate condition is the costeconomical cruise, resulting in a favorable combination of fuel costs and block time effects on operating costs (Section 11-8).

The designer's problem with respect to range performance is to choose a favorable combination of speed, altitude and airplane geometry, to obtain the best - or at least a satisfactory - range performance and to estimate the amount of fuel.

Flight speed variation has a major effect on the fuel required, but also on the design of the wing (sweep angle, section shape), the structural weight, engine s.f.c., and problems of stability and control. Optimization of the design - Mach number is a very complex study and this parameter is usually specified in a rather arbitrary manner in the design requirements.

Cruising altitude has a direct effect on fuel weight. When the installed engine thrust is based on the cruise condition, the engine size required increases with altitude, as the density decreases, and for a given specific thrust the inlet diameter must increase as well. The weight of engine plus fuel is a minimum for some altitude below the altitude for maximum L/D. This case is elaborated analytically by Küchemann in Ref. 5-4, resulting in an optimum condition for $C_{L}$ depicted in Fig. 5-11. For longrange aircraft the minimum fuel requirement dominates while on short hauls the amount of fuel consumed is less and the engine weight is the main factor, leading to a relatively low optimum for $C_{L}$ in cruising flight.

Obviously the matter is more complicated in real life:

- Cruise fuel is only part of the total fuel load.

- Fuel weight and engine weight do not have the same significance from the point of view of achieving minimum operating costs. 


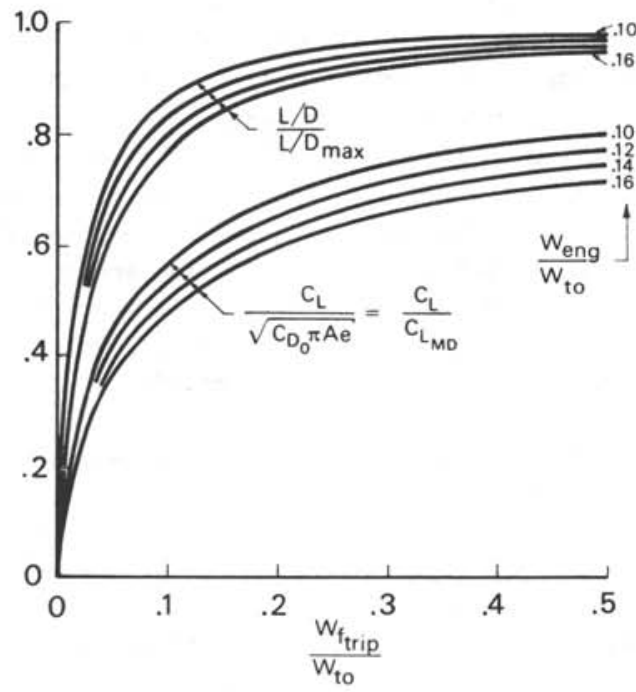

Fig. 5-11. Optimum cruise conditions according to the criterion of Küchemann and Weber (Ref. 5-4)

- For short-haul aircraft the engine thrust is frequently determined by the takeoff field length or an engine-out climb requirement.

- Oxygen system requirements are dependent on cruising altitude (e.g. FAR 121.327-333), which may be a deciding factor.

- Air Traffic Control considerations affect the choice of cruising altitude.

For an initial estimation of the amount of trip fuel required, the fuel consumed for cruising is derived from eq. 5-41 :

$\frac{w_{f_{C r}}}{w_{\text {to }}}=1-\exp \left(-\frac{R C_{T} / \sqrt{\theta}}{a_{0} M} \frac{C_{D}}{C_{L}}\right)$

where the initial weight is assumed to be approximately equal to $w_{\text {to }}$. Additional fuel is also used during takeoff, climb to and descent from cruise altitude, approach and landing. Fig. 5-2 is based on the following assumptions :

a. The cruise fuel according to eq. 5-45 is the dominant factor in the fuel contribution.

b. For transport aircraft the wing loading given by eq. 5-35 can be used to find a first-order approximation for the L/Dratio of the wing.

c. The wetted area of the fuselage is the primary parameter for the fuselage drag area; other drag contributions are assumed proportional to wing plus fuselage drag. Fig. 5-2 can be used, provided the fuselage dimensions are known, for example from a fuselage layout drawing or the data from Figures $3-11$ and $3-12$. Engine s.f.c. is deduced from the engine manufacturer's brochure or from the data listed in Chapter 4 .

Reserve fuel consists of various contributions (cf. Table 11-2). One important item is holding fuel, which is proportional to the airplane minimum drag, hence inversely proportional to $\sqrt{\mathrm{A}}$. The following empirical correlation has been found to give a good approximation for transport aircraft:

$\frac{\mathrm{W}_{f_{\text {res }}}}{\mathrm{W}_{\text {to }}}=.18 \frac{\mathrm{C}_{\mathrm{T}} / \sqrt{\theta}}{\sqrt{\AA}} \quad($ transport $\mathrm{a} / \mathrm{c})$

where $C_{T} / \sqrt{\theta}$ is the same quantity as used in Fig. 5-2. A fuel reserve for $3 / 4$ hour extra flying time should be allowed for business jets and executive aircraft. This can simply be translated into an equivalent range increment:

$\Delta \mathrm{R}=.75 \mathrm{~V}_{\mathrm{cr}} \quad \begin{aligned} & \text { (business and } \\ & \text { executive jets) }\end{aligned}$

Fig. 5-2 is thus valid, provided an equivalent range is used equal to $R+\Delta R$.

If the effects of varying parameters like wing loading and aspect ratio are to be assessed, the aircraft weight distribution must be computed in terms of these parameters. The details of these calculations will not be dealt with here; a simplified example in Section 5.5.3 shows the effects of wing area variation on the weight distribution of a long-range aircraft.

b. Propeller aircraft.

The Breguet range equation for propeller 
aircraft is :

$R=\frac{n_{p}}{C_{p}} \frac{L}{D} \ln \frac{w_{i}}{W_{i}-w_{f}}$

A similar picture to Fig. 5-10 can be drawn up. If s.f.c. and propeller efficiency variations are ignored, the specific range can be shown to be a maximum

- for minimum airplane drag, if the altitude is fixed and flight speed variable, - for minimum power, if the flight speed is $f i x e d$ and altitude variable.

As in the case of jet aircraft, these conditions are incompatible and no absolute optimum exists on the basis of flight mechanics. In general, range performance continues to improve with altitude until the available engine power becomes the $1 \mathrm{im}$ iting factor.

Fig. 5-1 can be used for an initial estimation of fuel weight. The wing aspect ratio is the primary factor in obtaining a high $L / D$ ratio and good range performance. It is the only parameter used in this figure to characterize the aerodynamic performance, as no considerable improvement was found when fuselage dimensions were introduced.

\subsubsection{Climb performance}

Climb performance may be specified in the form of :

a. Operational requirements, derived from desired performance capabilities in normal operating conditions, e.g.

- Rate of climb at sea level, clean configuration, all engines operating.

- Service ceiling altitude for max. rate of climb $=100 \mathrm{ft} / \mathrm{min}, .5 \mathrm{~m} / \mathrm{sec})$, clean configuration, all engines operating or one engine inoperative.

b. Airworthiness requirements, to ensure adequate performance for safety in normal and critical conditions, e.g.

- Minimum climb gradient in various configurations (takeoff, en route, landing), one engine inoperative or all engines operating; flaps deflected or retracted, flying at or above a specified flight speed. This item of performance is of particular interest for jet-propelled transports and will be dealt with in more detail in Chapter 11.6. - Rate of climb at a specified altitude, one engine inoperative. Frequently the rate of climb is related to the stalling speed (flaps down). This item of performance is of particular interest to piston-powered transports and all light aircraft.

Climb requirements may be categorized as follows.

a. Rate of climb at a fixed flight speed

b. Rate of climb at optimum flight speed

c. Climb gradient at a fixed flight speed

d. Climb gradient at optimum flight speed In actual practice cases, b. and c. are by far the most important ones; case a. can be found in airworthiness requirements for transport aircraft with reciprocating engines. Case d. may incidentally occur where a takeoff climb gradient cannot be fulfilled at $v_{2 \min }$ for transport category aircraft ("overspeed").

A special case of operational climb performance - the time to climb to a given altitude - will not be discussed here as no analytical procedure is available to convert such a requirement into combinations of design variables.

The various cases will first be dealt with as a general performance problem. Some examples and applications will then be presented in order to illustrate the procedure.

A useful general term for specifying climb performance is Specific Excess Power, SEP:

SEP $=\frac{T-D}{W} V=\frac{V}{g} \frac{d V}{d t}+\frac{d h}{d t}$

Excess power is available for climbing, accelerating and making turns. In the case of a steady climb at $\mathrm{n}=\mathrm{L} / \mathrm{W} \sim 1$, SEP is identical to the rate of climb $C$, provided the angle of climb $\gamma$ is not too large (approximation: $\cos \gamma=1$ ).

For horizontal flight at $\mathrm{n}=1$, the SEP is 
equivalent to the rate of increase of $\mathrm{ki-}$ netic energy and is therefore a measure of the time required to accelerate from one speed to another. In a horizontal turn at a specified rate of turn or load factor, the SEP represents the maneuvering and acceleration capability. Note that the dimension of SEP is length per unit time and not power.

\section{a. Jet aircraft.}

The thrust required is derived from eq. 5-48:

$\frac{T}{W}=\frac{S E P}{V}+n \frac{C_{D}}{C_{L}}$

1. In the case of a specified climb gradient for $n=1$, steady flight:

$\gamma=\frac{\mathrm{dh} / \mathrm{dt}}{\mathrm{V}}=\frac{\mathrm{SEP}}{\mathrm{V}}$

and eq. 5-49 yields :

$\frac{T}{W}=\gamma+\frac{C_{D}}{C_{L}}$

The lift and drag coefficients are given by eqs. 5-31 and 5-9. Hence:

$\frac{T}{W}=\gamma+\frac{\frac{1}{2} \gamma M^{2} C_{D_{0}}}{W /(p S)}+\frac{W /(p S)}{\frac{1}{2} \gamma M^{2} \pi A e}$

The minimum value for $\mathrm{T} / \mathrm{W}$ corresponds to the minimum $C_{D} / C_{L}$ ratio with

$C_{L}=\sqrt{C_{D_{0}} \pi A e}$ at $M=\left\{\frac{W /(p S)}{\frac{1}{2} \gamma \sqrt{C_{D_{0}} \pi A e}}\right\}^{\frac{1}{2}}=$

$$
=M_{(L / D)} \text { max }
$$

and consequently:

$$
\left(\frac{T}{W}\right)_{\text {min }}=\gamma+2 \sqrt{C_{D_{0}} / \pi A e}
$$

2. For a specified rate of climb for $d V / d h=$ $0, n=1$ and arbitrary $v$, eq. 5-49 is modified to:

$\frac{T}{W^{\prime}}=\frac{\operatorname{SEP} / a}{M^{\prime}}\left(\frac{M^{\prime}}{M}\right)+n \sqrt{\frac{C_{D_{O}}}{\pi A e}}\left\{\left(\frac{M}{M^{\prime}}\right)^{2}+\left(\frac{M^{\prime}}{M}\right)^{2}\right\}$ where

$M^{\prime}=\left\{\frac{n W /(p S)}{\frac{1}{2} \gamma \sqrt{C_{D_{0}}^{\pi A e}}}\right\}^{\frac{1}{2}}=\sqrt{n} M_{(L / D)} \max$

If the Mach number is specified, this equation can be used directly to obtain $T / W$. However, in most cases a condition for $M$ is sought for which the $\mathrm{T} / \mathrm{W}$ ratio is a minimum. A plot of $T / W$ vs. $M$, together with a typical thrust lapse curve (Fig. 5-12) in-

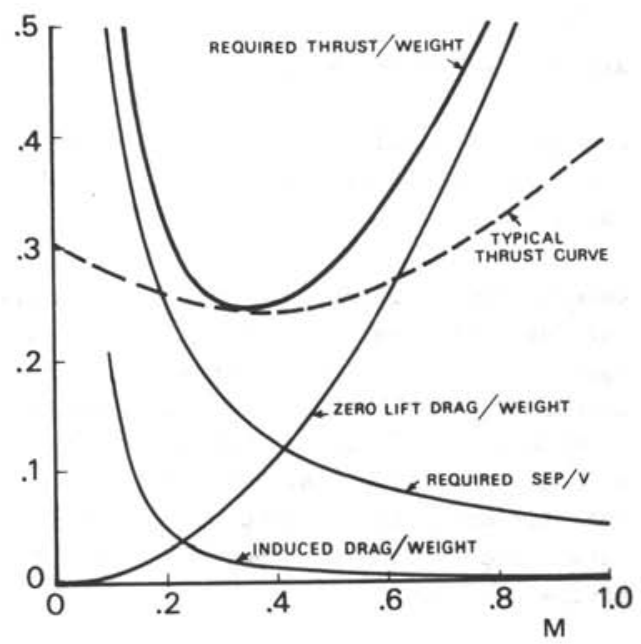

Fig. 5-12. Required thrust in a climb with specified SEP

dicates that an acceptable approximation for $T / W$ is found at the Mach number for which eq. 5-55 has a minimum value. This condition is:

$\left(\frac{M}{M^{\prime}}\right)^{3}-\left(\frac{M^{\prime}}{M}\right)=\frac{S E P / a}{2 n M^{\prime}} \sqrt{\frac{\pi A e}{C_{D_{0}}}}$

The solution is presented in graphical form in Fig. 5-13. In general, it is acceptable to calculate $C_{D_{0}}$ as follows:

$\mathrm{C}_{\mathrm{D}_{\mathrm{O}}}=1.1\left(\mathrm{~d}_{1}+\mathrm{d}_{2} \frac{\mathrm{w}_{\text {to }}}{\mathrm{S} \mathrm{p}_{\mathrm{o}}}\right)$

where the drag due to powerplant installation is assumed at 108 of $C_{D_{0}}$ and $d_{1}$ and $d_{2}$ 


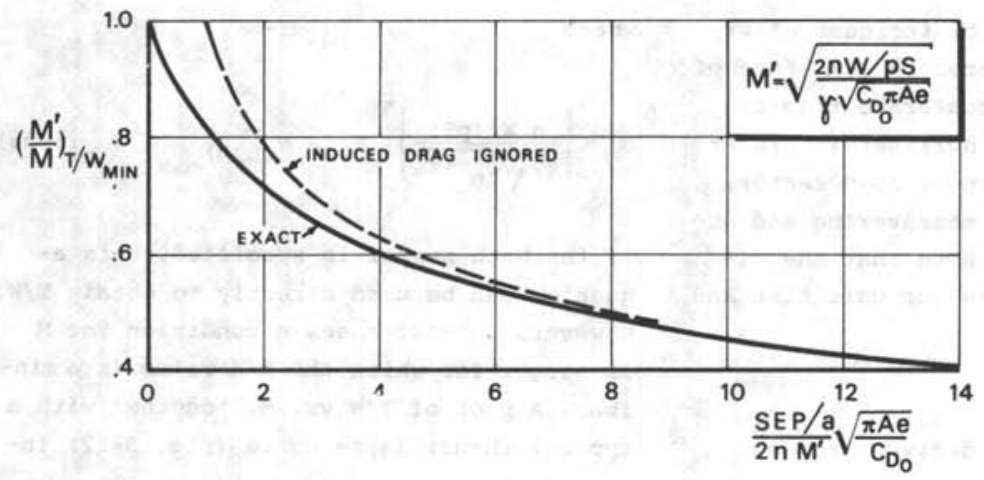

Fig. 5-13. Condition for the minimum thrust Mach number in a climb with specified SEP (jet aircraft) are defined by eq. 5-34.

The general result for a specified rate of climb may be simplified for two special cases.

Case A: steady flight at low altitude, all engines operating, in order to achieve a specified rate of climb $c$ at $n=1$. The contribution of the induced drag (second term in eq. 5-57) to the optimum $T / W$ can be neglected. From Fig. $5-13$ it is obvious that for sufficiently large values of $C$ this approximation is acceptable. The condition for $M$ is:

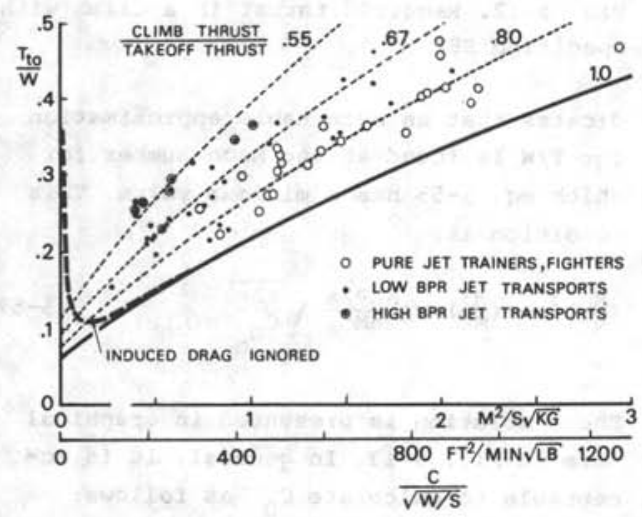

Fig. 5-14. Correlation of thrust/weight ratio and maximum rate of climb
$\mathrm{M}^{3}=\frac{\mathrm{C} / \mathrm{a}}{\mathrm{rC}_{\mathrm{D}_{0}}} \frac{\mathrm{w}}{\mathrm{pS}}$

Substitution into eq. 5-55 yields:

$$
\begin{aligned}
& \frac{T}{W}=\frac{3}{2} \gamma^{1 / 3}\left\{\frac{\frac{C}{a} \sqrt{C_{D_{O}}}}{\sqrt{W /(p S)}}\right\}^{2 / 3}+ \\
& +\frac{2}{\gamma^{1 / 3}} \frac{C_{D_{0}}}{\pi A e}\left\{\frac{\sqrt{W /(p S)}}{\frac{C}{a} \sqrt{C_{D_{0}}}}\right\}^{2 / 3}
\end{aligned}
$$

In most cases this expression is quite accurate, provided C corresponds to low altitude performance with all engines operating. In the derivation of $T / w, 1 t$ was assumed that $a v / d t=0$. In operational practice, however, a rate of climb is usually established in a flight with constant EAS or CAS. It can be shown that the acceleration necessary for flying at constant EAS at low altitude is given by:

$\frac{\mathrm{v}}{\mathrm{g}} \frac{\mathrm{dV}}{\mathrm{dt}}=\frac{\mathrm{v}}{\mathrm{g}} \frac{\mathrm{dV}}{\mathrm{dh}} \frac{\mathrm{dh}}{\mathrm{dt}}=.567 \mathrm{M}^{2} \mathrm{C}$

This may be translated into an additional engine thrust required for a given $C$ :

$\frac{\Delta T}{w}=.567 \frac{c}{a}\left(\frac{\frac{c}{a} \times \frac{w}{p S}}{\gamma C_{D_{0}}}\right)^{1 / 3}$

Instead of a detailed calculation; for which many data must be available, a statistical correlation of $T / W$ and $c / \sqrt{W / S}$ at sea level may be used, as given in Fig. 5-14.

Case B: flight at high altitude with low rate of climb, in order to achieve a specified service ceiling. The contribution of 
SEP in eq. 5-57 can be neglected and the Mach number for minimum $T / W$ is equal to $M^{\prime}$. At the service ceiling, $C=100 \mathrm{ft} / \mathrm{min}$ $(.5 \mathrm{~m} / \mathrm{sec})$, hence

$\frac{\mathrm{SEP}}{\mathrm{a}_{\mathrm{O}}}=.00147$

and the thrust required at the ceiling is:

$\frac{T}{\mathrm{~W}}=2 \mathrm{n} \sqrt{\frac{\mathrm{C}_{\mathrm{D}_{0}}}{\pi \mathrm{Ae}}}+\frac{.00123}{\sqrt{\theta}} \frac{\sqrt[4]{\mathrm{C}_{\mathrm{D}_{0}} \pi \mathrm{Ae}}}{\sqrt{\mathrm{nW} /(\mathrm{pS})}}$

where the relative temperature $\theta$ and the ambient pressure p refer to the ceiling. The ratio of thrust at the ceiling to static thrust must be used to convert this $\mathrm{T} / \mathrm{W}$ value into $\mathrm{T}_{\text {to }} / \mathrm{W}_{\text {to }}$.

b. Propeller aircraft.

For $\mathrm{n}=1$ the available power is equal to the power required to climb plus the power necessary to balance the drag. Assuming steady flight, $\operatorname{SEP}=\mathrm{dh} / \mathrm{dt}=\mathrm{C}$, and eq. 5-48 is modified into:

$\eta_{p} \frac{P}{W}=C+\frac{C_{D}}{C_{L}} v$

If $\mathrm{C}$ is defined at a given flight speed, the lift and drag coefficients are known and eq. 5-65 can be used directly.

To find the flight speed for which the power required to climb reaches a minimum, assuming a parabolic drag polar, the $\mathrm{P} / \mathrm{W}$ ratio for given rate of climb is modified to:

$\frac{P}{\mathrm{~W}}=\frac{1}{n_{p}}\left\{\mathrm{C}+\left(\frac{\mathrm{C}_{\mathrm{D}_{\mathrm{O}}}}{\mathrm{C}_{\mathrm{L}}{ }^{3 / 2}}+\frac{\mathrm{C}_{\mathrm{L}}{ }^{1 / 2}}{\pi \mathrm{Ae}}\right) \sqrt{\frac{2 \mathrm{~W}}{\rho S}}\right\}$

For a given altitude and engine rating, $P / W$ is affected by $\eta_{p}$ and $c_{L}$. For a parabolic polar, the value of the term:

$\frac{C_{D_{O}}}{C_{L}^{3 / 2}}+\frac{C_{L}^{1 / 2}}{\pi A e}$

is minimum for $C_{L}=\sqrt{3 C_{D_{O}} \pi A e}$. However, propeller efficiency generally improves with increasing airspeed and in practice the most favorable speed is roughly 208 higher than the speed for the minimum power required to balance the drag. The result is:

$$
\frac{p}{a W}=\frac{1}{n_{p}}\left|\frac{\mathrm{C}}{\mathrm{a}}+2.217 \frac{\mathrm{C}_{\mathrm{D}_{\mathrm{O}}} 1 / 4}{(\pi \mathrm{Ae})^{3 / 4}} \sqrt{\frac{\mathrm{W}}{\mathrm{pS}}}\right|
$$

at $M=1.09 \frac{\sqrt{\mathrm{W} /(\mathrm{pS})}}{\sqrt[4]{\mathrm{C}_{\mathrm{D}_{0}} \pi \mathrm{Ae}}}$

The engine power* is generally the maximum continous (equivalent) power for turboprop engines or the rated (METO) power for reciprocating engines, at the ambient conditions for which $\mathrm{C}$ is specified.

In the case of a service ceiling, eq. 5-67 can be used by taking $C=100 \mathrm{ft} / \mathrm{min}$

$(0.5 \mathrm{~m} / \mathrm{s})$, hence

$\frac{c}{a_{0}}=.00147$

while in (5-67) the speed of sound and the static pressure refer to the service ceiling.

\section{c. Applications.}

Airworthiness climb requirements are to be found in:

FAR 23.65 and 67

SFAR 23. Amendment $1 \mathrm{ch}$. 6

FAR 25.65 and $67,25.117-119-121$

BCAR Chapter D2-4.

A survey of the most pertinent data for transport aircraft will be given in Chapter 11 Section 11.5. It is not intended to deal with all possible requirements in this section. Instead, some examples will be presented to illustrate applications of the formulas already derived and the importance of climb requirements, with particular reference to civil aircraft engine sizing.

Example 1

The rate of climb specified at sea level for a subsonic jet trainer is $4500 \mathrm{ft} / \mathrm{min}(23 \mathrm{~m} / \mathrm{s})$, corresponding to $\mathrm{c} / \mathrm{a}=.0672$. The weight is $7000 \mathrm{lb}$ $(3170 \mathrm{~kg})$, wing area $\mathrm{S}=210 \mathrm{sq} . \mathrm{ft}\left(19.5 \mathrm{~m}^{2}\right)$ and

* divide $(5-67)$ by 550 if $P$ is in hp, $W$ in ib and $c$ in $\mathrm{ft} / \mathrm{sec}$ 
$A=5.5$, hence $W /\left(p_{0} S\right)=.0156$ and $\pi A e=13.8$ for $\mathrm{e}=.8$. Furthermore $\mathrm{C}_{\mathrm{D}}=.019$.

To use Fig. 5-13, we calculate

$M^{\prime}=\left\{\left.\frac{W /\left(p_{0} s\right)}{\zeta \gamma \sqrt{C_{D_{0}} \pi A e}}\right|^{h / 2}=.209\right.$ and $\frac{C / a_{0}}{2 M^{\prime}} \sqrt{\frac{\pi A e}{C_{D_{0}}}}=4.33$

The figure indicates $M^{*} / M=.59$; hence $M=.35$ for minimum $T / w$. Using eqs. 5-55 and 5-62, we calculate:

$$
\begin{aligned}
& \frac{T}{W}=.309 \text { for steady flight } \\
& \frac{\Delta T}{W}=013 \text { for acceleration (constant EAS) }
\end{aligned}
$$

total $\mathrm{T} / \mathrm{W}=.322$

For a thrust lapse rate of .85 at $M=.35$, the takeoff SLS thrust/weight ratio must be at least .38. The approximate equation 5-60 for steady flight yields $\mathrm{T} / \mathrm{W}=.314$, as compared with .309 for the "exact" solution.

\section{Example 2}

For a twin-engined subsonic jet passenger transport, the service ceiling with one engine failed is specified at $15,000 \mathrm{ft}(4570 \mathrm{~m})$. The following data are pertinent to the aircraft.

$w=75,000 \mathrm{lb}(34,000 \mathrm{~kg}) ; \mathrm{s}=850 \mathrm{sq} \cdot \mathrm{ft}\left(79 \mathrm{~m}^{2}\right)$; $C_{D}=.018 ; A=8.5$ and $\mathrm{e}=.85$, hence $\pi \mathrm{Ae}=22.7$. For engine failure, an 88 decrease of $e$ is assumed: $\pi$ Ae $=20.9$. At an altitude of $15,000 \mathrm{ft}, \mathrm{w} /(\mathrm{ps})=$ .072 and $\theta=.897$. According to eq. 5-56,

$M^{\prime}=\left\{\frac{.072}{.7 \sqrt{.018 \times 20.9}}\right\}^{\frac{1}{2}}=.41$

We now calculate from eq. 5-55:

$\frac{\mathrm{SEP} / \mathrm{a}}{2 \mathrm{M}^{\prime}} \sqrt{\frac{\pi \mathrm{Ae}}{\mathrm{C}_{\mathrm{D}_{0}}}}=.0645$

From Fig. 5-13 we find that $M^{\prime} / M=.975$, and therefore the speed for minimum thrust at this altitude is $\mathrm{M}=.42$. Bquation $5-56$ yields: $T / W=.0624$. The engine thrust lapse at an altitude of $15,000 \mathrm{ft}$ and with $M=.42$ is .47 . The thrust/weight ratio required at SLS is: $T_{\text {to }} / W_{\text {to }}=.265$. The effect of flying at constant EAS is neglected in view of the low rate of climb at the service ceiling.

Example 3

An important airworthiness requirement for civi] aircraft is the so-called second-segment climb gra- dient, laid down for example in FAR 25.121 (b). It states that with one engine inoperative, flaps in takeoff position, landing gear retracted, engines in the takeoff rating and out of ground effect, a specified minimum climb gradient is to be obtained at the takeoff safety speed $v_{2}$. This requirement must be met for all operational ambient conditions and may, especially on hot and high airfields, limit the authorized takeoff weight.

In the case of a subsonic passenger transport equipped with 3 engines, for example, the required climb gradient is 2.78 . The ambient conditions are: sea level, temperature 95 F (i.e. 35 C, ISA + 20 C). Other data:

$w_{\text {to }}=210,000$ ib $(95,000 \mathrm{~kg}), s=2,060 \mathrm{sq} \cdot \mathrm{ft}$ $\left(192 \mathrm{~m}^{2}\right): \mathrm{v}_{2}=1.2 \mathrm{v}_{\mathrm{s}}$ with $\mathrm{C}_{\mathrm{L}}-\max =2.40$ for the takeoff. Aspect ratio: $A=7.5$; section 5.3.5. is used to estimate the $1 \mathrm{ift} / \mathrm{drag}$ ratio in symmetrical flight with flaps deflected at $C_{L}=C_{L}-\max /(1.2)^{2}=$ 1.67 .

We find: $C_{D} / C_{L}=.094$ for a slatted wing, hence $C_{D} / C_{L}=.10$, assuming an increment of 58 for arag due to engine failure. The Mach number at $v_{2}$ is:

$M_{2}=\frac{v_{2}}{a}=1.2 \sqrt{\frac{w /(p s)}{b \gamma C_{L_{\max }}}}=.2$

From eq. $5-51$ we now find: $T / W=.027+.10=.127$ for two engines operating at $\mathrm{M}=.2$ and $95 \mathrm{~F}(35 \mathrm{C})$. For a thrust lapse rate of .75 , including the effect of non-standard temperature, the total takeoff thrust (SLS, ISA) must be at least:

$\mathrm{T}_{\text {to }} / \mathrm{W}_{\text {to }}=\frac{\mathrm{N}_{\mathrm{e}}}{\mathrm{N}_{\mathrm{e}}-1} \cdot \frac{\mathrm{T} / \mathrm{W}}{\mathrm{T} / \mathrm{T}_{\text {to }}}=.254$

For $w_{\text {to }}=210,000 \mathrm{lb}(95,000 \mathrm{~kg})$, the thrust per engine must be at least $17,780 \mathrm{lb}(8,054 \mathrm{~kg})$.

\section{Example 4}

Light aircraft with $\mathrm{w}<6,000 \mathrm{lb}(2720 \mathrm{~kg})$ must comply with the requirement FAR 23.67. With takeoff power at sea level, undercarriage down and flaps in the takeoff position:

$c \geqslant 300 \mathrm{ft} / \mathrm{min}$, or $\mathrm{c} / \mathrm{a} 2.0045$

and

$c \geqslant 11.5 \mathrm{v}_{\mathrm{s}} \mathrm{ft} / \mathrm{min}$;

where $v_{S_{1}}=$ equivalent stalling speed (knots).

The first requirement can be substituted directly into eq. 5-67. The second can be evaluated as follows: 
$\frac{\mathrm{P}_{\text {to }}}{\mathrm{a}_{0} \mathrm{~W}_{\text {to }}} \geqslant \frac{1}{\eta_{\mathrm{p}}}\left(\frac{\mathrm{w}_{\text {to }}}{\mathrm{P}_{\mathrm{O}} \mathrm{S}}\right)^{\frac{1}{2}}\left\{\frac{.136}{\left(\mathrm{C}_{\mathrm{L}_{\max }}\right)^{\frac{1}{2}}}+\frac{2.21 \mathrm{c}_{\mathrm{D}_{0}}^{1 / 4}}{(\pi \mathrm{Ae})^{3 / 4}}\right\}$

By way of example, the following data may be applied to a light aircraft: $w_{\text {to }}=3,300 \mathrm{lb}(1,500 \mathrm{~kg}) ; \mathrm{s}=$ 130 sq.ft $\left(12 \mathrm{~m}^{2}\right)$, hence $\mathrm{w}_{\text {to }} /\left(\mathrm{p}_{\mathrm{O}} \mathrm{s}\right)=.0121 ; \mathrm{C}_{\mathrm{L}_{\max }}=$ 1.8; $\pi A e=15.3$ and $C_{D}=.055$ with flaps de- $\max$ flected and undercarriage down. Effective propeller efficiency: $\eta_{p}=.65$. Substitution of $\mathrm{c} / \mathrm{a}>.0045$ into eq. 5-67 yields: $\mathrm{P}_{\text {to }} / \mathrm{a}_{\mathrm{o}} \mathrm{W}_{\text {to }} \geqslant .0305$, while eq. 5-69 yields $P_{\text {to }} / a_{0} w_{\text {to }} \geqslant .0408$. The latter being the most critical requirement, it is concluded that $\mathrm{P}_{\text {to }}$ must be at least $275 \mathrm{hp}$.

d. Design data.

If no better information is available, the following data may be useful in working out climb performance requirements.

Propeller efficiency during climb at sea level:

tractor propeller in fuselage nose,

fixed pitch : $n_{p}=.61 \quad( \pm .052)$

constant speed: $n_{p}=.665( \pm .059)$

tractor propellers, wing-mounted,

constant speed: $n_{p}=.73 \quad( \pm .058)$

These data were found by application of the present method to the performance data of a large number of aircraft. The second number gives the rms error. All figures include slipstream effects, cooling drag, power offtakes and intake losses.

For the effect of engine failure on drag, 48 may be added to $C_{D}$ for the drag of feathering propellers, while the Oswald factor may be reduced by approximately 10 \% for wing-mounted engines.

The airplane drag polar may be estimated by the method explained in section 5.3. For the effect of undercarriage extension, it is reasonable to take $\Delta C_{D_{0}}=.015$ to .020 as a typical value. It should be noted that in most equations for climb performance small variations in $C_{D_{0}}$ are of minor importance, unless the flaps are deflected. Hence, it may be assumed that the drag due to powerplant installation adds roughly 88 to $C_{D_{0}}$ on turbine-powered aircraft and 128 with piston engines.
The engine thrust or power lapse rate may be determined from engine manufacturer's brochures. For jet engines the effect of $M$ on the thrust is important, while lapse rates are very sensitive to the bypass ratio as we11. Curves of $\mathrm{T} / \delta \mathrm{T}_{\text {to }}$ vs. $\mathrm{M}$ may serve the purpose. An example is shown in Fig. 6-3. In the case of turboprop engines the shaft power increases noticeably with $M$ due to the ram effect, unless there is a structural or thermal engine limitation up to some specified altitude. If this is not the case, the following approximation may be used for a given rating and Mach number:

$\frac{\text { power at altitude }}{\text { power at sea level }}=\sigma^{n}$

where $\mathrm{n}$ is generally between .7 and .8 . For naturally aspirated piston engines at constant rpm:

$\frac{\text { full throttle power at altitude }}{\text { full throttle power at sea level }}=$

$=1.132 \sigma-.132$

Supercharged engines maintain constant power up to the rated altitude. Above this the power decreases linearly with $\sigma$ in the same way as in eq. 5-71. With many supercharged engines a cruising power of 658 to 758 of rated power can be maintained up to the cruising altitude.

\subsubsection{Stalling and minimum flight speeds}

In establishing low speed performance, operational flight speeds must have a specified safety margin relative to the stalling speed, in order to provide the pilot with some measure of freedom to maneuver and in order to avoid stalling due to vertical gusts. The required field length being roughly proportional to the kinetic energy at the screen height, and hence to (velocity) ${ }^{2}, a$ low stalling speed provides a powerful method of obtaining good field performance. On the other hand, a decrease in stalling speed generally entails a cost penalty as a more sophisticated flap system must be developed or the wing loading decreased, or 


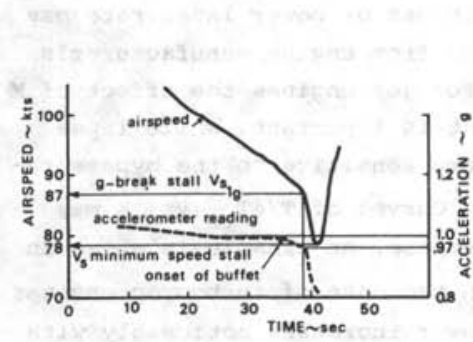

Fig. 5-15. Time history of the airspeed during a stall maneuver

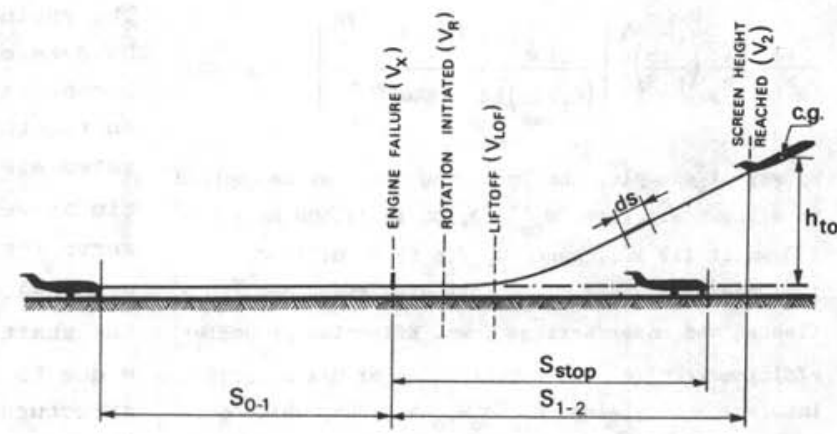

Fig. 5-16. Phases during the takeoff with engine failure both.

With regard to the definition of stalling speeds, reference is made to Fig. 5-15, which depicts a time history of the airspeed during a stalling maneuver. In principle, several flight procedures can be chosen for this - for example, flight at constant longitudinal deceleration, constant flight path angle (or horizontal flight) or constant normal acceleration. The FAR certification procedure is a stall maneuver at constant dV/dt. Several tests are carried out at different values of $\mathrm{dV} / \mathrm{dt}$ and the stalling speed is defined by interpolation at $\mathrm{dv} / \mathrm{dt}=-1 \mathrm{kt} / \mathrm{s}$.

In approaching the stall, the normal load factor initially remains approximately constant until a break occurs, indicating that wing stalling is progressing rapidly. The corresponding airspeed is referred to as the $1-g$ stalling speed or g-break stalling speed $\mathrm{v}_{\mathrm{S}-1 \mathrm{~g}}$. Immediately after the g-break the airspeed continue $V_{S}$ to decrease, and the sink speed increases rapidly until the pilot takes corrective action by pitching down the nose, resulting in a positive dv/dt. The minimum airspeed measured in this procedure, $\mathrm{V}_{\mathrm{S}^{\prime}}$, is noticeably lower than $\mathrm{V}_{\mathrm{S}-1 \mathrm{~g}}$. The FAR25 regulations allow $v_{S}$ to be used in scheduling various reference speeds for definition of the performance, whereas the British requirements do not allow the stalling speed to be less than $94 \%$ of $\mathrm{V}_{\mathrm{S}-1 \mathrm{~g}^{\circ}}$.
In view of this reasoning, it must be noted that a definition of $\mathrm{C}_{L}$-max according to

$\mathrm{C}_{\mathrm{L}_{\max }}=\frac{\mathrm{w} / \mathrm{S}}{\frac{1}{2} \rho \mathrm{v}_{\mathrm{S}}{ }^{2}}$

does not result in a "physical" $C_{L}-\max$ as obtained in the wind tunnel, but produces a value which may be some 10 to 20 o higher. Alternatively, when calculating the stalling speed from $C_{L}$-max obtained by theoretical methods or wind tunnel experiments, it is generally appropriate to assume an apparent $C_{L}$-max increment of, say, 138. All values of $\mathrm{C}_{\mathrm{L}}$-max mentioned in the following sections are corrected in this manner.

Occasionally a limit is imposed on the stalling speed and the corresponding wing loading is limited by:

$\frac{w}{s} \leqslant \frac{1}{2} \rho v_{s}{ }^{2} c_{L_{\text {max }}}$

For example, in the BCAR requirement Ch. D2-11, an upper limit applies to the stalling speed: $70 \mathrm{mph}(112.5 \mathrm{kmh})$ - group C $60 \mathrm{mph}$ ( $96.5 \mathrm{kmh}$ ) - group D These values can be substituted into eq. 5-71 to find a limiting wing loading for these classes of aircraft. The reader should also refer to FAR 23.49 (b) for aircraft with a takeoff weight of $6,000 \mathrm{lb}$ $(2720 \mathrm{~kg})$ or less. 


\subsubsection{Takeoff}

The following cases are distinguished:

a. All-engine takeoff distance requirements

b. one-engine-out takeoff distance requirements

c. Accelerate-stop distance requirements For aircraft to be certificated under the FAR Part 23 regulations, no explicit requirements need to be met with regard to the event of engine failure during takeoff. The all-engine takeoff distance applies to airplanes with a takeoff weight of $6,000 \mathrm{lb}$ $(2720 \mathrm{~kg})$ or more and is generally defined as the distance required to pass the screen height of $50 \mathrm{ft}(15.3 \mathrm{~m})$ at a speed of 1.3 $\mathrm{v}_{\mathrm{S}}$. For a particular class of aircraft operating under the FAR Part 135 operational rules, the FAR Part 23 performance standards are considered to be inadequate. The performance standards laid down in SFAR 23 and NRPM 68-37 are intended as intermediate steps towards improving safety in the operation of small passenger airplanes and air taxis capable of carrying more than 10 persons (Ref. 5-22). Accelerate-stop distances are introduced in these requirements. Under SFAR 23 this is the distance required to accelerate to the critical engine-failure speed $v_{1}$ and then to decelerate to 35 knots, while NRPM 68-37 covers the distance needed to come to a full stop. These regulations contain no specifications for a one-engine inoperative takeoff distance.

The takeoff performance of transport category airplanes is a fairly complicated matter, which is dealt with in more detail in Appendix $K$. It is generally found that the most critical item of performance is determined by the case of a one-engine-out takeoff. The condition that the airplane must be brought to standstill after engine failure at the critical engine failure speed leads to the concept of the Balanced Field Length (BFL), which is usually considered as the most important design criterion as far as field performance of transport category aircraft is concerned. The simplified methods presented in this section are intended to serve as a first approximation of field length for the purpose of sizing the engine thrust or power and wing design. They can be refined if more detailed information is available to the designer; an example is given in Appendix $\mathrm{K}$.

a. All-engines takeoff.

Since the takeoff consists of a takeoff run and an airborne phase, we may write:

$s_{\text {to }}=s_{\text {run }}+s_{\text {air }}$

The expression for $\mathrm{S}_{\text {run }}$ is

$S_{\text {run }}=\frac{1}{2 g} \int_{0}^{v_{\text {LOF }}{ }^{2}} \frac{\mathrm{dv}^{2}}{\mathrm{a} / \mathrm{g}}$

where the momentary acceleration,

$a / g=\frac{T}{W_{t o}}-\mu-\left(C_{D}-\mu C_{L}\right) \frac{\frac{1}{2} \rho V^{2} S}{W}$

can be approximated by

$\mathrm{s}_{\text {run }}=\frac{\mathrm{v}_{\text {LOF }}{ }^{2 / 2 \mathrm{~g}}}{\overline{\mathrm{T}} / \mathrm{w}_{\text {to }}-\mu^{\prime}}$

where $\bar{T}$ is a mean value of the thrust during the takeoff run. Assuming the lift-off speed to be approximately $1.2 \mathrm{~V}_{\mathrm{S}}$ and the lift coefficient during the takeoff run to be equal to twice the value for minimum $\left(C_{D}-\mu C_{L}\right)$, it is found that

$\overline{\mathrm{T}}=$ thrust at $\mathrm{v}_{\mathrm{LOF}} / \sqrt{2}$

$\mathrm{C}_{\mathrm{L}}=\mu \pi \mathrm{Ae}$

$\mu^{\prime}=\mu+.72 \mathrm{C}_{\mathrm{D}_{0}} / \mathrm{C}_{\mathrm{L}_{\max }}$

Assuming an air maneuver after lift-off with $C_{L}=C_{L-L O F}=$ constant and $T-D=$ constant, the following result can be derived from the AGARD Flight Test Manual, Vol. 1:

$S_{\text {air }}=\frac{v_{\text {LOF }}{ }^{2}}{g \sqrt{2}}+\frac{h_{\text {to }}}{\gamma_{\text {LOF }}}$

and 
$\frac{v_{3}}{v_{L O F}}=\sqrt{1+\gamma_{L O F} \sqrt{2}}$

where $\gamma_{\text {LOF }}=(T-D) / W$ at liftoff and $v_{3}=$ velocity at the takeoff height ( 30 or 50 ft).

The liftoff speed corresponding to a given $v_{3}$ is:

$v_{\text {LOF }}=v_{3}\left(\frac{1}{1+\gamma_{\text {LOF }} \sqrt{2}}\right)^{\frac{1}{2}}$

From eqs. 5-75, 5-76 and 5-79 the takeoff distance is now:

$\frac{s_{\text {to }}}{f_{\text {to }} h_{\text {to }}}=\left(\frac{v_{3}}{v_{S}}\right)^{2} \frac{w_{t o} / S\left\{\left(\bar{T} / w_{t o}-\mu^{\prime}\right)^{-1}+\sqrt{2}\right\}}{h_{t o} \rho g c_{L_{\max }}\left(1+\gamma_{L O F} \sqrt{2}\right)}+$

$+\frac{1}{\gamma_{\text {LOF }}}$

\begin{tabular}{|r|l|l|l|}
\hline $\begin{array}{l}\text { Regula- } \\
\text { tions }\end{array}$ & \multicolumn{1}{|c|}{$\mathrm{v}_{3} / \mathrm{v}_{\mathrm{S}}$} & $\mathrm{f}_{\text {to }}$ & \multicolumn{1}{|c|}{$\mathrm{h}_{\text {to }}$} \\
\hline $\begin{array}{r}\text { (S) FAR 23 } \\
\text { FAR 25 }\end{array}$ & $\begin{array}{l}1.325 \text { to } 1.30 \\
\text { (no require- } \\
\text { ment) }\end{array}$ & 1.15 & $35 \mathrm{ft}(10.7 \mathrm{~m})$ \\
\hline
\end{tabular}

Table 5-2. Characteristic values for the all-engines takeoff according to FAR 23 and 25 .

In the absence of better information, the following assumptions and approximations may be made in appıying eq. 5-80.

1. In calculating $\mu^{\prime}$ according to eq. 5-76, it is reasonable to assume: $.72 \mathrm{C}_{\mathrm{D}_{\mathrm{O}}} / \mathrm{C}_{\mathrm{L}_{\max }}=.010 \mathrm{C}_{\mathrm{L}_{\max }}$, $\mu=.02$ for concrete and $\mu=.04-.05$ for short grass.

2. $\gamma_{\text {LOF }}=.9 \frac{\overline{\mathrm{T}}}{\mathrm{w}_{\text {to }}}-\frac{.3}{\sqrt{\mathrm{A}}}$

3. The mean thrust/weight ratio at mean velocity $\mathrm{v}_{\text {LOF }} / \sqrt{2}$, allowing for slipstream effects and power offtakes, is as follows:

jet aircraft:

$\bar{T}=.75 \frac{5+\lambda}{4+\lambda} T_{\text {to }}$

aircraft with constant speed propellers:

$\overline{\mathrm{T}}=\mathrm{k}_{\mathrm{p}} \mathrm{P}_{\text {to }}\left(\frac{\sigma \mathrm{N}_{\mathrm{e}} \mathrm{D}_{\mathrm{p}}{ }^{2}}{\mathrm{P}_{\text {to }}}\right)^{1 / 3}$ where $\mathrm{P}_{\text {to }} /\left(\mathrm{N}_{\mathrm{e}} \mathrm{D}_{\mathrm{p}}{ }^{2}\right)$ is the propeller disc loading; see Fig. 6-9, for example.

$k_{p}=5.75$ when $\bar{T}$ is in $1 b, P_{\text {to }}$ in $h p, D_{p}$ in $\mathrm{ft}$ $k_{p}=.321$ when $\bar{T}$ is in $\mathrm{kg}, \mathrm{P}_{\text {to }}$ in $\mathrm{kgm} / \mathrm{s}, \mathrm{D}_{\mathrm{p}}$ in $\mathrm{m}$. For fixed-pitch propellers the mean thrust is roughly 15-208 below the value given by eq. 5-82. From eq. 5-77 it follows that for a specified takeoff distance, the wing loading is limited to:

$\frac{W_{\text {to }}}{S} \leqslant\left\{\frac{S_{\text {to }}}{\mathrm{f}_{\text {to }}}-\frac{\mathrm{h}_{\text {to }}}{\gamma_{\text {LOF }}}\right\} \frac{\rho g \mathrm{C}_{L_{\max }}\left(1+\gamma_{\text {LOF }} \sqrt{2}\right)}{\left(\mathrm{V}_{3} / \mathrm{V}_{\mathrm{S}}\right)^{2}\left\{\left(\overline{\mathrm{T}} / \mathrm{w}_{\text {to }}-\mu^{\prime}\right)^{-1}+\sqrt{2}\right\}}$

b. Takeoff with engine failure and accelerate-stop distance.

A critical decision speed $v_{1}$ is defined so that, with a single engine failure, the total accelerate-stop distance required becomes identical with the total takeoff distance to reach screen height safely. A simple analytical method for determining the BFL in the preliminary design stage, devised by the author and presented in Ref. 5-27, will be summarized below.

As opposed to the usual subdivision (takeoff run to liftoff, transition and climb distance), the continued takeoff is split up into 2 phases (Fig. 5-16):

- Phase 0-1: acceleration from standstill to engine failure speed $V_{x}$,

- Phase 1-2: the motion after engine failure, up to the moment of attaining the screen height at takeoff safety speed $\mathrm{V}_{2}$. The distance travelled during phase $0-1$ is:

$s_{0-1}=\frac{v_{x}^{2}}{2 \bar{a}_{0-1}}$

where $\overline{\mathbf{a}}_{0-1}$ is calculated in the same way as for the all-engines takeoff.

The energy equation is applied to phase 1-2 (Fig. 5-16), resulting in

$S_{1-2}=\frac{1}{\bar{\gamma}}\left(\frac{v_{2}{ }^{2}-v_{x}^{2}}{2 g}+h_{t o}\right)$

where the equivalent climb gradient $\bar{\gamma}$ is defined as follows: 
$\bar{\gamma}=\frac{\int_{1}^{2}\left(T-D_{a i r}-D_{g}\right) d s}{w_{t o} s_{1-2}}$

The distance required to come to a standstill after engine failure can be represented by:

$\mathrm{s}_{\text {stop }}=\frac{\mathrm{v}_{\mathrm{x}}{ }^{2}}{2 \overline{\mathrm{a}}_{\text {stop }}}+\mathrm{v}_{\mathrm{x}} \Delta \mathrm{t}$

where $\Delta t$ is referred to as an equivalent inertia time, affected in principle by the thrust/weight, ratio at $\mathrm{v}_{\mathrm{x}}$ (Fig. $\left.\mathrm{K}-6\right)$. The condition for balancing the field length is $\mathrm{s}_{1-2}=\mathrm{s}_{\text {stop }}$ and Ref. 5-27 gives the following expression for the critical engine failure speed $v_{1}$ :

$\frac{v_{1}}{v_{2}}=\left\{\frac{1+2 g h_{t o} / v_{2}^{2}}{1+\bar{\gamma} /(\bar{a} / g)_{s t o p}}\right\}^{\frac{1}{2}}-\frac{\bar{\gamma} g(\Delta t-1)}{v_{2}}$

The condition that $v_{1}<v_{R}$ must be satisfied. To check this, a more detailed anal$y$ sis of the rotation and flare maneuver is necessary (Appendix $K$ ). In the case that $v_{1}=v_{R^{\prime}}$ the field length is generally no longer balanced.

Combination of eqs. 5-84 through 5-88 results in the expression

$\mathrm{BFL}=\frac{\mathrm{v}_{2}{ }^{2}}{2 \mathrm{~g}\left\{1+\bar{\gamma} /(\overline{\mathrm{a}} / \mathrm{g})_{\text {stop }}\right\}}\left\{\frac{1}{(\bar{a} / g)_{0-1}}+\frac{1}{(\bar{a} / g)_{\text {stop }}}\right\} \times$

$\left(1+\frac{2 \mathrm{~g} \mathrm{~h} \text { to }}{\mathrm{v}_{2}{ }^{2}}\right)+\frac{\Delta \mathrm{S}_{\text {to }}}{\sqrt{\sigma}}$

In this expression the inertia distance $\Delta \mathrm{S}_{\text {to }}$ may be assumed equal to $655 \mathrm{ft}(200 \mathrm{~m})$ for $\Delta t=4 \frac{1}{2}$ seconds, a value derived for typical combinations of wing and thrust (power) loadings. This result is valid for both propeller and jet aircraft.

To make eq. 5-89 readily applicable for preliminary design, some further simplifications can be introduced.

1. On the basis of several realistic assumptions regarding undercarriage drag, ground effect, etc.. it was found (cf. Ref. 5-27) that the following ap- proximation can be made:

$\bar{\gamma}=.06+\Delta \gamma_{2}$

where $\Delta \gamma_{2}$ is the difference between the second segment climb gradient $\gamma_{2}$ and the minimum value of $\gamma_{2}$ permitted by the airworthiness regulations.

2. An average value of $\overline{\mathrm{a}}_{\text {stop }}=.37 \mathrm{~g}$ has been found from application of the method to 15 jet transports, although with optimum brake pressure control, lift dumpers and nosewheel braking, decelerations as high as $.45 \mathrm{~g}$ to $.55 \mathrm{~g}$ can be achieved on dry concrete. For very high decelerations the balancing condition may not be satisfied.

Using these simplifications, we find the following expression:

$\left.\mathrm{BFL}=\frac{.863}{1+2.3 \Delta \gamma_{2}}\left(\frac{\mathrm{w}_{t \mathrm{O}} / \mathrm{s}}{\rho \mathrm{g} \mathrm{C}_{\mathrm{L}_{2}}}+\mathrm{h}_{\mathrm{to}}\right) \mid \frac{1}{\overline{\mathrm{T}} / \mathrm{w}_{\mathrm{to}}-\mu^{\prime}}+2.7\right\}+$

$+\frac{\Delta \mathrm{s}_{\text {to }}}{\sqrt{\sigma}}$

where

$\mathrm{h}_{\text {to }}=35 \mathrm{ft}(10.7 \mathrm{~m})$ and $\Delta \mathrm{s}_{\text {to }}=655 \mathrm{ft}(200 \mathrm{~m})$

$\mu^{\prime}=.010 \mathrm{C}_{L}+.02$ for flaps in takeoff position

$C_{L_{2}}=C_{L}$ at $v_{2}{ }^{i}$ normally $v_{2}=1.2 v_{S^{\prime}}$, hence $C_{L_{2}}=.694 x$ $C_{L}{ }^{2}$ max

$\overline{\mathrm{T}}=$ mean thrust for the takeoff run, given by eqs. 5-81 and 5-82

$\Delta \gamma_{2}=\gamma_{2}-\gamma_{2}$ min

$\gamma_{2}$ is the second segment climb gradient calculated from eq. 5-51 at airfield altitude, one engine out (cf. example 3 of Section 5.4.3)

$\gamma_{2}=.024, .027$ or .030 for $\mathrm{N}_{\mathrm{e}}=2,3$ or 4 respectively.

For project design, the case of $\Delta \gamma_{2}=0$ presents most interest, as the corresponding weight is $1 \mathrm{im}-$ ited by the second segment climb requirement and the BFL is a maximum for the particular flap setting, disregarding the case of overspeed (Appendix K). obviously, when $\Delta \gamma_{2}=0$ is substituted into eq. 5-91, the thrust/weight ratio must be chosen accordingly so that the thrust is sufficient to obtain

$\gamma_{2}=\gamma_{2 \min }$.

For a given BFL, eq. 5-91 may be used to find the following limitation to the wing loading:

$\left.\frac{w_{\text {to }}}{\mathrm{S}} \leqslant \rho g \mathrm{C}_{\mathrm{L}_{2}} \mid \frac{1.159\left(\mathrm{BFL}-\Delta \mathrm{S}_{\text {to }} / \sqrt{\sigma}\right)\left(1+2.3 \Delta \gamma_{2}\right)}{\left(\overline{\mathrm{T}} / \mathrm{w}_{\text {to }}-\mu^{\prime}\right)^{-1}+2.7}-\mathrm{h}_{\text {to }}\right\}(5-92)$ 


\subsubsection{Landing}

The landing is split up into two phases: the air distance from passing the screen to touchdown and the landing run from touchdown to standstill. The air distance can be derived from conservation of total energy:

$\mathrm{s}_{\mathrm{air}}=\frac{1}{\bar{\gamma}}\left(\frac{\mathrm{v}_{\mathrm{a}}{ }^{2}-\mathrm{v}_{\mathrm{td}}{ }^{2}}{2 \mathrm{~g}}+\mathrm{h}_{\text {land }}\right)$

where $\bar{\gamma}$ is the mean value of $(D-T) / W$. The length of the landing run from touchdown to standstill is:

$s_{\text {run }}=\frac{v_{t d^{2}}}{2 \bar{a}}$

where $\overline{\mathrm{a}}$ is the mean deceleration, taking into account an equivalent inertia time, similar to the case of the accelerate-stop distance. Expressing the minimum speed in the stall in terms of the wing loading, the density and $C_{L}-\max$, and adding eqs. 5-93 and 5-94, we may find:

$\frac{s_{1 \text { and }}}{h_{\text {land }}}=\frac{1}{\gamma}+\frac{\mathrm{w}_{1 \text { and }} / \mathrm{s}}{\mathrm{h}_{\text {land }} \rho \mathrm{g} \mathrm{C}_{\mathrm{L}_{\text {max }}}} \times$

$\left\{\left(\frac{v_{t a}}{v_{a}}\right)^{2}\left(\frac{1}{\bar{a} / g}-\frac{1}{\bar{\gamma}}\right)+\frac{1}{\bar{\gamma}}\right\}\left(\frac{v_{a}}{v_{s_{0}}}\right)^{2}$

The landing distance is affected by variations in the touchdown speed which is largely dependent upon piloting technique. A reasonable estimate can be obtained by assuming that the landing flare is approximately a circular arc, flown with a constant incremental load factor $\Delta \mathrm{n}$. In that case it can be shown that a first-order approximation for the touchdown speed is given by

$\frac{v_{t d}^{2}}{v_{a}^{2}}=1-\frac{\bar{\gamma}^{2}}{\Delta n}$

Substitution into eq. (5-95) yields:

$\frac{\mathrm{S}_{1 \text { and }}}{\mathrm{h}_{\text {land }}}=\frac{1}{\bar{\gamma}}+1.69 \frac{\mathrm{w}_{1 \text { and }} / \mathrm{S}}{\mathrm{h}_{\text {land }} \rho \mathrm{g}} \mathrm{C}_{\mathrm{L}_{\max }} \times$ $\left|\frac{1}{\bar{a} / g}\left(1-\frac{\bar{\gamma}^{2}}{\Delta \mathrm{n}}\right)+\frac{\bar{y}}{\Delta \mathrm{n}}\right|$

The approach speed has been assumed as 1.3 $\mathrm{v}_{\mathrm{S}}$, in accordance with most airworthiness regulations.

The following factors have an effect upon landing performance:

a. The mean value of thrust minus drag. The initial value of $\gamma$ at the threshold is generally .05 for transport category aircraft, corresponding to an approach angle of $3^{\circ}$.

The final value is equal to $C_{D} / C_{L}$ in ground effect, the thrust being approximately zero at touchdown. It is difficult to calculate an accurate value of $\bar{\gamma}$, but an average of .10 may be taken as reasonable. The screen height is $\mathrm{h}_{\text {land }}=50 \mathrm{ft}(15.3 \mathrm{~m})$.

b. The load factor increment during the flare is normally of the order of $\Delta n=.10$, although large variations can be observed, due to differences in atmospheric conditions, piloting technique and airplane response.

c. The average deceleration during the landing run $(\bar{a})$ is affected by the same factors as discussed with regard to the emergency distance in Section $\mathrm{K}-4$ of Appendix $\mathrm{K}$. For design purposes, the so-called arbitrary landing distance is frequently used. This is established on a dry concrete runway from which the required landing field length is found by multiplying by a factor $f_{1 \text { and }}=5 / 3$, according to FAR Part 91. In Fig. 5-17 the unfactored landing distance is plotted for actual aircraft and compared with eq. 5-97, in order to estimate typical values for the mean deceleration. For pre-design purposes the following values may be assumed for $\bar{a} / g$ :

.30 to .35 light aircraft, simple brakes, .35 to .45 turboprop aircraft, propeller reverse thrust inoperative, .40 to .50 jets with ground spoilers, antiskid devices, speed brakes,

.50 to .60 as above, with nosewheel braking. The limiting wing loading for a given (factored) field length can be approximated by using the mean values for $\bar{\gamma}$ and $\Delta n$ stated 


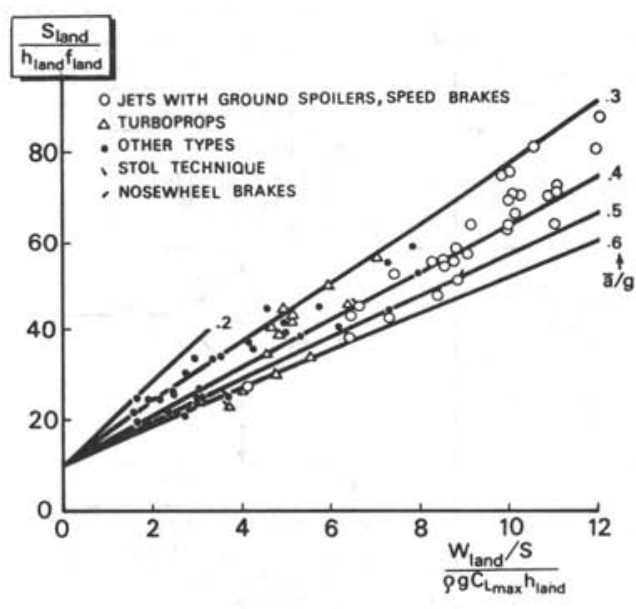

Fig. 5-17. Statistical correlation of the unfactored landing distance

previously:

$\frac{\mathrm{w}_{1 \text { and }}}{\mathrm{s}} \leqslant\left(\frac{\mathrm{S}_{\text {land }}}{\mathrm{f}_{\text {land }} \mathrm{h}_{\text {land }}}-10\right) \frac{{ }^{\mathrm{h}_{\text {land }}} \rho \mathrm{g} \mathrm{C_{L_{ \text {max } } }}}{\frac{1.52}{\overline{\mathrm{a}} / \mathrm{g}}+1.69}$

For light aircraft the calculated or measured landing distance is unfactored, i.e. $\mathrm{f}_{\text {land }}=1.0$.

According to eq. 5-98 the wing loading limit is independent of the engine thrust, provided reverse thrust is not used. However, the usable value of $C_{L}-\max$ can be limited by climb requirements in the approach or landing configuration. Provided the airplane polar is known, these limitations can be worked out in the same way as example 3 of Section 5.4.2.

\subsection{AIRCRAFT SYNTHESIS AND OPTIMIZATION}

From the previous chapters it will have become clear that the performance aims may be realized in various ways, by choosing suitable combinations of wing loading, aspect ratio and thrust loading. A frequently used systematic approach to this problem is the parametric investigation. A parametric investigation is a design study based on generalized calculation methods and sizing procedures, making it possible to vary configuration parameters and to quantify their effect on the design. The most relevant aircraft parameters to be studied are mentioned in the introduction to section 5.4. Engine cycle parameters like design Turbine Entry Temperature, Overall Pressure Ratio and bypass ratio, may be subjected to parametric investigations in order to gain an insight into the most suitable type of engine to be installed. As there are many types of parametric design studies, depending on the accuracy required and the phase of the design, some simplified examples to demonstrate the principle will be given below. The list of references relating to this chapter contains a number of more complete studies.

\subsubsection{Purpose of parametric studies}

Parametric studies can be useful in many types of design problems, provided the design criteria can be quantified in terms of minimum weight, cost and/or noise figures. However, in airplane design they require a considerable amount of computational work, as all variations will have farreaching consequences. For this reason, computerized design studies are increasingly becoming a prerequisite for advanced and complex aircraft. However, the system must be devised, monitored and utilized by experienced designers in order to define the design problems and the interfaces between various technical disciplines in the design an essential for avoiding unrealistic results.

Although the optimum choice of aircraft design parameters has been a major design problem since the early days of aviation, the generalized approaches described in the published literature are scanty and have a very limited validity due to the necessary simplifications and lack of flexibility. The following design goals can be achieved with a computerized system: 


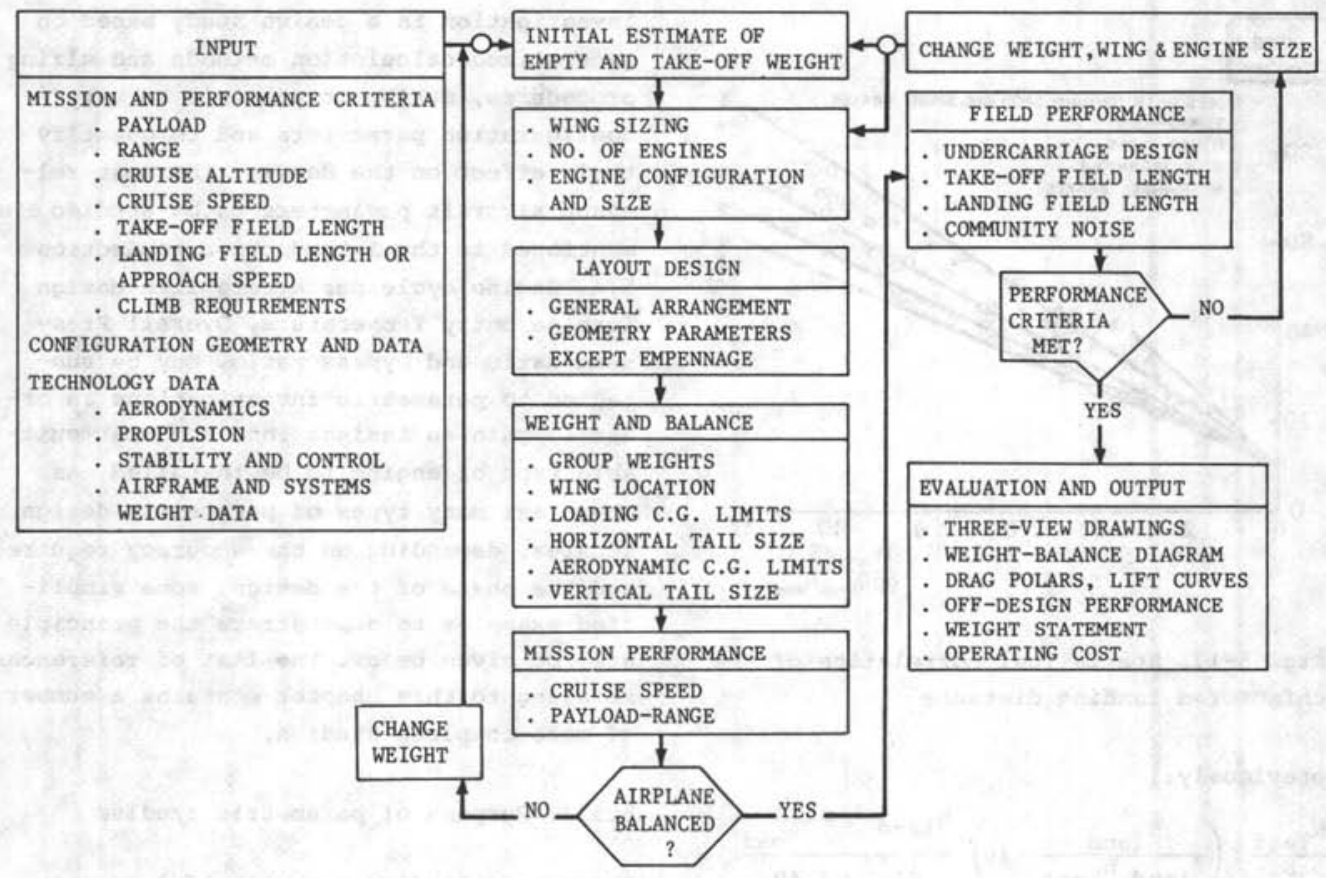

Fig. 5-18. Example of a generalized design procedure (Ref. 5-45, modified)

a. Determination of combinations of parameters, characterizing designs that satisfy specified operational requirements.

b. Calculation of values for the configuration parameters already mentioned, resulting in the most favorable objective function, e.g. takeoff weight or operating costs.

c. Sensitivity studies to assess the effects of minor changes in the shape or geometry, material properties, drag coefficients, etc.

d. Mission/performance analysis and tradeoff studies to investigate the effect of variations in the performance requirements. e. The effect of certain technological constraints in terms of weight and cost penalties. It should be realized that the validity of computerized studies is determined entirely by the accuracy of the input data and design methods available. In that respect they are no better than manual computations. However, improved data can easily be incorporated in a computerized system. On the other hand, the design of the program and evaluation of the output may be time-consuming and costly.

\subsubsection{Basic rules}

Variation of design parameters in effect means designing many aircraft layouts. It is therefore necessary to lay down basic rules governing the design process for each variant. A generalized procedure as depicted in Fig. 5-18 will be explained. Input data are performance criteria and technological constraints. Performance data are generally mission data (payload and range) and performance constraints (field length, approach speed). Cruise performance may be considered as a constraint or as a fixed mission requirement, depending on the application.

The first design step will be to generate initial configuration characteristics such 
as takeoff weight, wing loading and thrust loading, on the basis of semi-statistical formulas of the type presented in this chapter. Configuration and layout design activities generally refer to the work on the drawing board; for computerized design this must be translated into a mathematical procedure for defining the external geometry of the major aircraft components. This is a difficult part of the program and in many cases it will, for example, be acceptable to use the geometry of a fuselage design generated outside the numerical program.

The next step is to calculate group weights and the empty weight, desirable loading center of gravity limits and empennage size*. Sufficient data are then available to calculate the maximum range for the design payload and a conclusion can be drawn about whether the fuel weight is sufficient or not. In the first attempt, this will not be the case and the airplane is not balanced with respect to the all-up weight. The fuel and takeoff weights must be changed and the procedure repeated, until the design characteristics have converged sufficiently. Field performance and noise characteristics may now be calculated and if there is some deficiency, the wing size must be changed. Except in the case of a fixed engine, some scaling of the engine size may be feasible as well. The design procedure is repeated again, until all performance requirements are met. The output is presented in the form of drawings, diagrams and characteristic data. Excellent examples of such a program are described in Refs. 5-40, 5-43 and 5-45.

Two comments must be made on the type of studies discussed.

1. The prediction methods for weight and aerodynamic characteristics must not only be accurate in absolute values, but must also predict the effect of design changes accurately. More accurate data than those presented in this chapter are generally

* These subjects will be dealt with in Chapter 8 required.

2. The evaluation of computer output is a time-consuming problem. The computer graphics facility is a useful tool here (cf. Ref. 5-41).

\subsubsection{Sizing the wing of a long-range passenger transport}

This section is concerned with investigating the effect of wing area variation on the takeoff weight (Fig. 5-19) of a large, long-range passenger transport, equipped with turbofan engines with a total thrust of $176,000 \mathrm{lb}(80,000 \mathrm{~kg})$ at SLS (ISA)4. The airplane will be designed to fly a payload of $126,000 \mathrm{lb}(57,000 \mathrm{~kg})$ over a range of $4,000 \mathrm{n} . \mathrm{m}$. $(7,400 \mathrm{~km}) ; M=.85$ at $35,000 \mathrm{ft}(10,700 \mathrm{~m})$. Fuel reserves must be available for two hours holding at 958 of $(L / D)_{\text {max }}$ plus 58 tolerance on the total fuel weight. The field length is limited to $3,000 \mathrm{~m}(10,000 \mathrm{ft})$ and $2,000 \mathrm{~m}(6,500 \mathrm{ft})$ for takeoff and landing respectively. For simplicity, climb performance requirements are summarized in. the condition that at 85 s of the ISA-SLS thrust the second segment climb gradient (FAR 25.121 b) must be achieved.

The all-up weight at takeoff is initially estimated at $705,000 \mathrm{lb}(320,000 \mathrm{~kg})$, with a wing area of $5,900 \mathrm{sq}$. ft $\left(550 \mathrm{~m}^{2}\right)$. A subdivision is made as follows:

a. A fixed weight of $390,000 \mathrm{lb}(177,000$ $\mathrm{kg}$ ) for the payload and payload service items, the fuselage group, the propulsion group, the undercarriage, fixed equipment and systems.

b. A variable weight affected by the wing and tailplane size and fuel quantity required.

The wing plus tailplane structure weight is estimated after several iterations by a simple formula given in Fig. 5-19. Reserve fuel for 2 hours holding is estimated as follows:

$\mathrm{w}_{\mathrm{f}}=\frac{2}{.95} \mathrm{C}_{\mathrm{T}}\left(\mathrm{C}_{\mathrm{D}} / \mathrm{C}_{\mathrm{L}}\right)_{\text {min }} \mathrm{w}_{\text {land }}$

while 


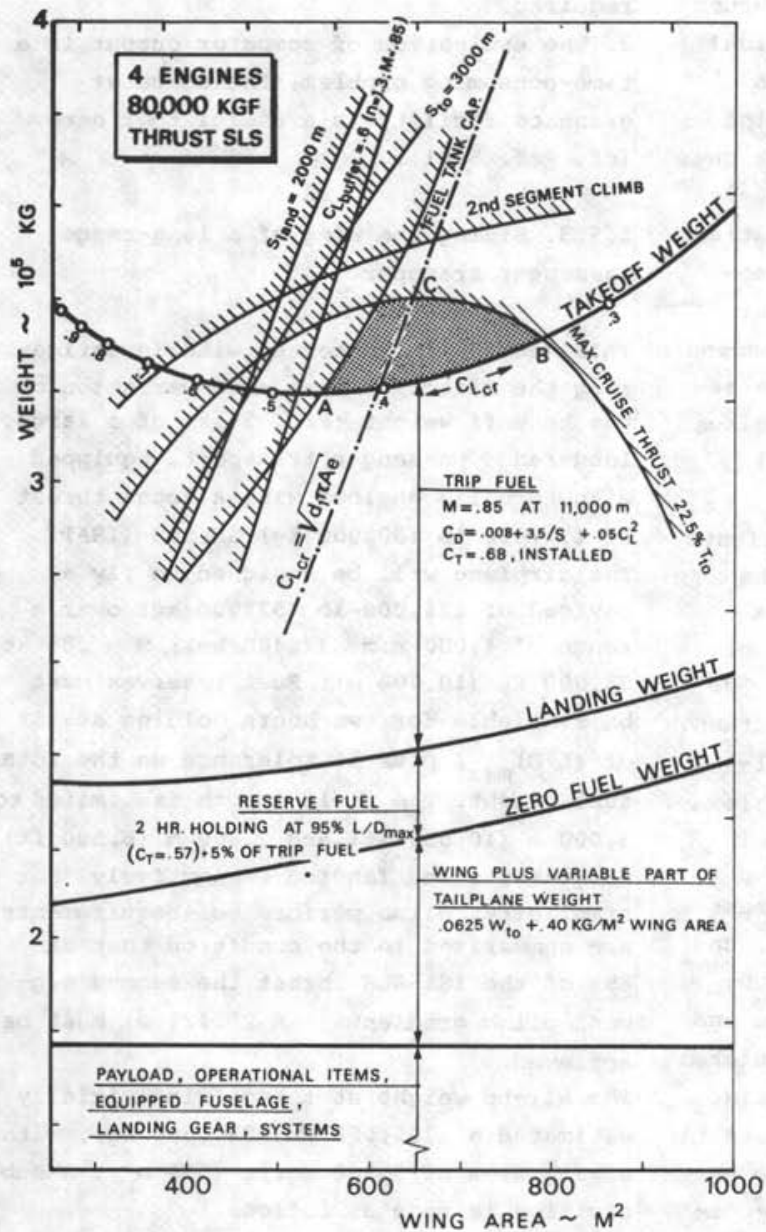

Fig. 5-19. Effect of wing area on the weight breakdown of a large passenger transport (project)

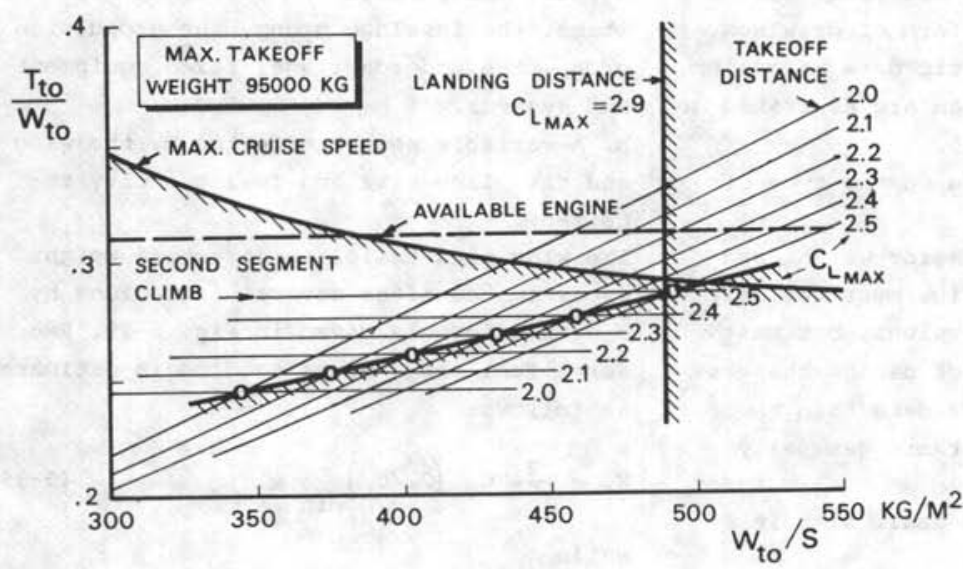

Fig. 5-20. Wing loading vs. thrust loading diagram of the three-engine airliner in Fig. 12-1 
$C_{D} / C_{L}=2 \sqrt{C_{D_{0}} / \pi A e}$

$(5-100)$

It is noted that $C_{D}$ is a function of the wing area (cf. Section 5.3.2.). Cruise fuel is derived from the Bréguet equation:

$\mathrm{w}_{\mathrm{f}} / \mathrm{w}_{\mathrm{to}}=1-\exp \left\{-\frac{\mathrm{M} \mathrm{C}_{\mathrm{L}} / \mathrm{C}_{\mathrm{D}}}{\mathrm{R} \mathrm{C}_{\mathrm{T}} / \sqrt{\theta}}\right\}$

For an initial cruise altitude of 35,000 ft $(10,700 \mathrm{~m})$ the dynamic pressure at $M=.85$ is $2671 \mathrm{~b} / \mathrm{ft}^{2}\left(1,300 \mathrm{~kg} / \mathrm{m}^{2}\right)$. The effects of climb and descent, flying horizontally at constant engine rating, intake losses, power offtakes and bleed air, etc., are summarized in an equivalent $C_{T}=.68$, which is $10 \%$ above the uninstalled $\mathrm{C}_{\mathrm{T}}$.

The wing loading for minimum thrust in cruising flight according to eq. 5-35 is $108 \mathrm{lb} / \mathrm{ft}^{2}\left(525 \mathrm{~kg} / \mathrm{m}^{2}\right)$. According to Fig. 5-19 this value is quite close to the wing loading for minimum all-up weight.

The limitations to the takeoff weight corresponding to each performance requirement can be found from the equations derived in the previous sections. For example, the condition that $M=.85$ must be achieved with max. cruise thrust can be converted into a limitation of the all-up weight, derived from eq. 5-32:

$\left.\mathrm{W} \leqslant \frac{1}{2} \gamma \mathrm{pM}^{2} \mid \pi \mathrm{Ae} s\left(\frac{\mathrm{T}}{\frac{1}{2} \gamma \mathrm{pM}^{2}}-\mathrm{C}_{\mathrm{D}_{\mathrm{O}}} \mathrm{s}\right)\right\}^{\frac{1}{2}}$

Substitution of $C_{D} S$ according to the expression in Fig. $5-19$ results in the weight limit indicated in this figure. Other boundaries are derived in the same way from the second segment climb requirement and the field length limitation.

In addition, the wing must have sufficient volume to contain the fuel. It can be shown (Appendix B, Section B-3) that the maximum wing tank volume is proportional to the thickness/chord ratio, the wing area squared and inversely proportional to the span. For a given aspect ratio and section shape it follows that $\mathrm{s}^{3 / 2}$ is the factor of proportionality. The available tank volume restricts the fuel load and consequently the all-up weight. In the case of $t / c=10$ \% (mean value) this requirement appears to set a lower limit to the wing area. If $t / c$ is increased to 128 , to be achieved for the same $M$ with advanced wing sections or more sweep, the tank volume is no longer the limiting factor. Another operational limit originates from the requirement that it must be possible to maneuver the airplane at cruise altitude with $n=1.3$ without entering the buffet condition* at an assumed conservative $C_{L}=.6$ at $M=.85$. This leads to a maximum cruise lift coefficient of .462 , or a wing loading limit of $600 \mathrm{~kg} / \mathrm{m}^{2}$ (123 lb/sq. $\left.\mathrm{ft}\right)$. As in the previous case this value can be improved by suitable wing design.

The resulting diagram indicates a zone of acceptable combinations for $S$ and $w_{\text {to }}(A B C)$. In point $A$ the takeoff weight is a minimum and for a long-range aircraft this is also an approximation for the condition of minimum direct operating costs (d.o.c.). Point $B$ results in the most favorable field performance, but decreased ride comfort and increased costs. Point $\mathrm{C}$ is the maximum range condition, provided the extra weight is converted into fuel.

Additional factors in the final choice of the wing area will be discussed in Sect. 7.2.

5.5.4. Wing loading and thrust (power) loading diagrams

A convenient way of illustrating how the powerplant and wing size may be chosen is /the wing loading vs thrust (power) loading diagram. An example is shown in Fig. 5-21,20? pertaining to the short haul passenger aircraft project (185 pax.), referred to in Fig. 1-5. Instead of the thrust loading we have used the $\mathrm{T} / \mathrm{W}$-ratio.

Each point in the diagram in effect represents a different airplane design, with different weight distribution. In the case of a constant design payload and range,

*See Section 7.2.2. of Chapter 7 
the fuel weight and takeoff weight are different for each combination. Alternatively, for a constant all-up weight and payload, the fuel weight and range vary with the empty weight. Lines of constant takeoff weight, constant range or constant d.o.c. can be plotted, using the design procedure described in Section 5.5.2. The diagram is then suitable for simplifying the final choice of wing size and engine thrust (power). In many cases, the trend is to take the highest practicable value of the wing loading and the minimum-size engine within the region of possible combinations.

Inspection of the performance equations derived in the previous sections reveals that in all formulas except $C_{D}$ the wing area and engine thrust (power) ${ }^{\circ}$ are combined with the weight ${ }^{*}$. The exception is eq. 5-13 for the zero-lift drag coefficient. In a first-order analysis Fig. 5-20 is therefore valid for different values of the takeoff weight, except the boundary derived from the high-speed performance requirement.

The following comments can be made on the limitations in the diagram:

a. The max. cruise speed requirement (given $M$, given cruise altitude) limits the thrust loading to a value which appears to be relatively insensitive to $\mathrm{W} / \mathrm{s}$. The wing loading for minimum $T / W$ is 670 $\mathrm{kg} / \mathrm{m}^{2}(137.4 \mathrm{lb} / \mathrm{sq} . \mathrm{ft})$ but the gain in $\mathrm{T} / \mathrm{W}$ for wing loadings above $500 \mathrm{~kg} / \mathrm{m}^{2}(102.5$ $\mathrm{lb} / \mathrm{sq} . \mathrm{ft}$ ) is small.

b. The second segment climb requirement is derived from FAR Part 25.121, using eqs. 5-51 and 5-29. The lift/drag ratio with flaps in the takeoff position (see Fig. 5-9) and the thrust lapse rate with speed are assumed to be independent of the wing loading, but for large $W / S$ variations this is no longer true. The required $\mathrm{T} / \mathrm{W}$ is calculated for different values of $C_{L}$-max,

* Although in a more detailed analysis this is no longer true, the conclusion that one diagram can be used for different takeoff weights remains acceptable. i.e. different flap deflections. c. The takeoff balanced field length of $1,800 \mathrm{~m}(5,900 \mathrm{ft})$ is calculated according to eq. 5-91, to find a wing loading limitation for each $C_{L}$-max. The intersections of these lines with those of the climb requirement define the limiting combinations of wing and thrust loading for variable (critical) flap settings. Calculation of the all-engine takeoff with eq. 5-80 indicates that this case is not critical for the case considered.

d. The $w / S$ limit for landing was derived from eq. 5-98, assuming an average deceleration of $.38 \mathrm{~g}$ during the landing run. Although for the landing and approach conditions certain climb requirements must be met, it was found that these were not critical and they have therefor been omitted from the diagram to improve its clarity.

The actual choice of wing area and engine thrust is subject to several considerations outside the field of performance (cf. Chapters 5 and 7). In the case of Fig. 5-20 the available engine type resulted in a takeoff $\mathrm{T} / \mathrm{W}$ ratio of .31 for the initially estimated takeoff weight of $95,000 \mathrm{~kg}$ $(209,440 \mathrm{lb})$. A weight increase of 58 to $100,000 \mathrm{~kg}(220,460 \mathrm{lb})$ is feasible for a constant wing loading of $490 \mathrm{~kg} / \mathrm{m}^{2}(100 \mathrm{lb} /$ sq.ft) without running into a cruise speed limitation. The extra weight might be used in the form of fuel or payload, provided there is no space limitation.

As already stated, the fuel weight and range for the different airplane designs, represented by Fig. 5-20, will vary as a consequence of fluctuations in empty weight. This makes an evaluation of the design parameters more difficult, in spite of the simplicity of the representation. The result of a more detailed design procedure for a very large passenger transport is presented in Fig. 5-21. In this example the design payload and range are constant, and the weight distribution is calculated for each combination of wing loading and thrust loading, resulting in the indicated 


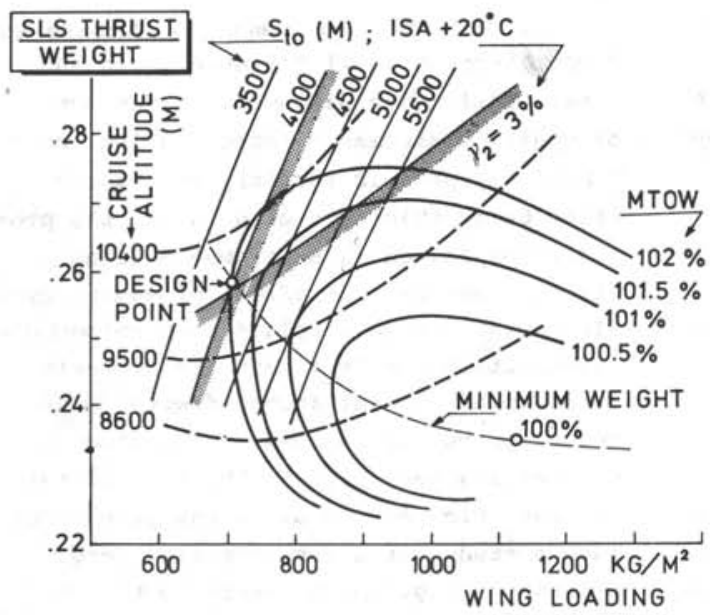

Design data: cruise $\mathrm{M}=.9$; payload: $145,000 \mathrm{~kg}(320,000 \mathrm{lb})$; range: 7600 km ( $4100 \mathrm{n} . \mathrm{m}) ;$.4 engines of bypass ratio 12; wing aspect ratio 7

Fig. 5-21. Wing loading vs. thrust/ weight ratio for a very large longhaul passenger transport
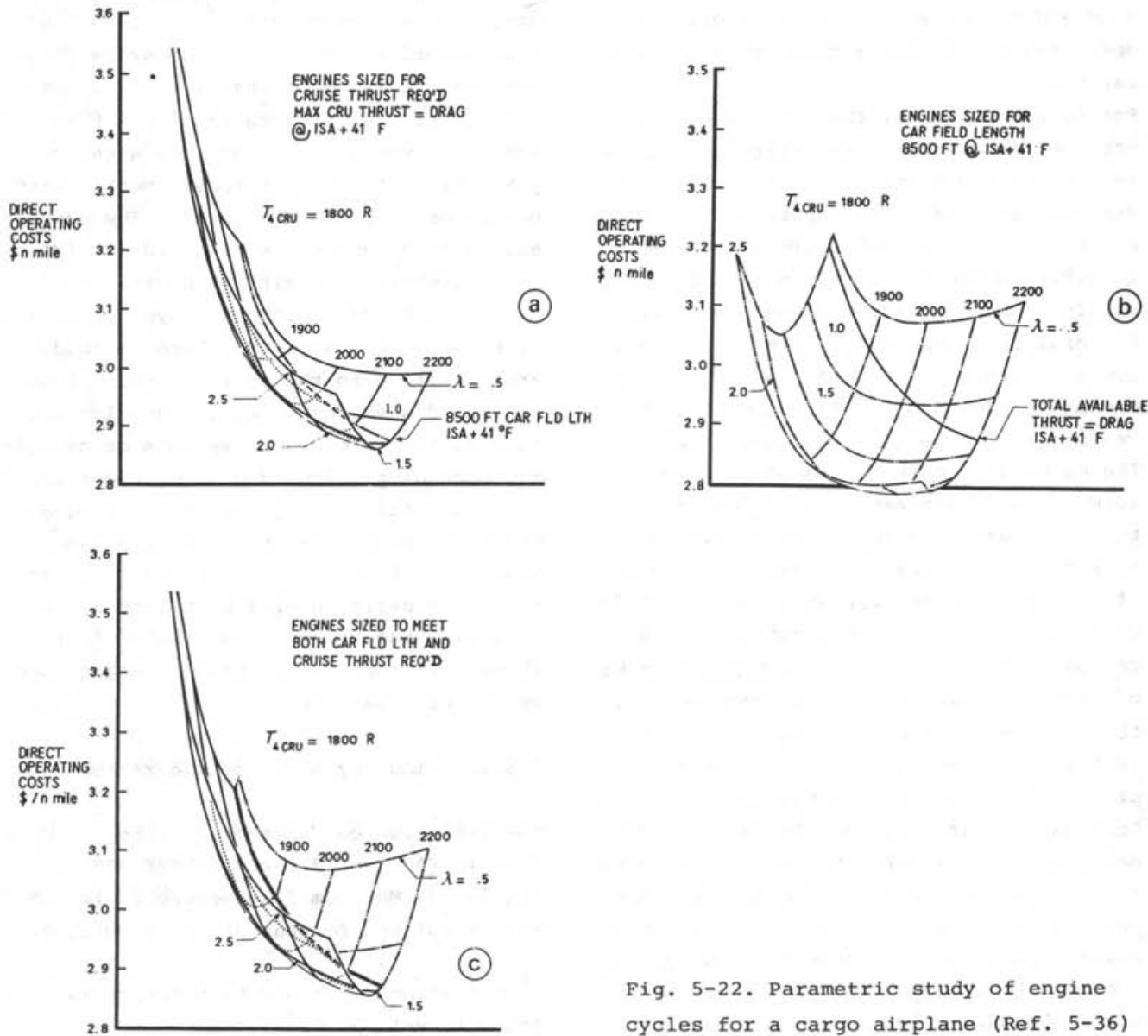

Fig. 5-22. Parametric study of engine cycles for a cargo airplane (Ref. 5-36) 
lines of constant takeoff weight. The minimum all-up weight is achieved at the clearly impracticable wing loading of 1125 $\mathrm{kg} / \mathrm{m}^{2}$ (231 lb/sq. $\mathrm{ft}$ ). The indicated design point is based on only two performance limitations, again for the sake of simplicity. The figure illustrates that decreasing the wing loading to $700 \mathrm{~kg} / \mathrm{m}^{2}$ (144 $\mathrm{lb} / \mathrm{sq} . \mathrm{ft}$ ) results in a weight penalty of 28 , provided the thrust is chosen near the value for minimum takeoff weight for specified wing loading.

\subsubsection{Optimization for low operating costs}

In the previous examples the takeoff weight was considered as an important factor in judging a preliminary design. For the airline and the private user, however, the operating costs form a more relevant criterion.

For design purposes, the total operating costs of an airplane are split up into direct operating ${ }^{\circ}$ costs (d.o.c.), which are directly related to the operation of the aircraft, and indirect operating costs (i.o.c.), which comprise general administrative costs and advertising costs, etc. A useful tool for categorizing the various d.o.c. factors is the ATA 1967 method for estimating the d.o.c., to which more attention will be paid in Section 11.8 . The major difficulty in using the d.o.c. formula for optimization purposes is to generate realistic estimates of such data as aircraft initial costs and the precise effect of parameter variation. For example, when the effect of aspect ratio variation on costs is estimated from a typical value of airplane price per $1 \mathrm{~b}$ of empty weight, the fact is ignored that systems, equipment and engines have a relatively higher price per $1 \mathrm{~b}$ than structural material.

Cost aspects are involved in each design decision, and if definite answers could be given a discussion on economics would appear in each section. Since this is obviously impossible, the results of a typical d.o.c. optimization study are presented here instead.
In Sect. 4.4. the importance of Turbine Entry Temperature, Overall Pressure Ratio and bypass ratio were discussed in the context of engine performance (specific fuel consumption; specific thrust), weight and size. Using this type of data and the procedure explained in Section 5.5.2. as starting points, the effect of engine cycle variations on airplane drag and weight distribution can be investigated, taking into account installation effects. A number of propulsion system optimization studies are mentioned in the list of references. Fig. 5-22 depicts the results of such a study for a jet-propelled cargo transport. Fig. 5-22a. refers to the case where the engines are sized for the specified cruise speed and in Fig. 5-22b. engine size is determined by the length of the takeoff field length. Comparing these figures, it is found that for low bypass ratios the field length requirement is dominant, while for relatively high bypass ratios the cruise requirement determines the size of the engines. The combination of a. and b. is depicted in Fig. 5-22c., where the critical performance criterion is given for each type of engine. The results of this kind of study are sensitive to the state of the art and disregard advances in engine development such as the three-shaft systems or gearedfan technology. Provided conclusions are drawn carefully, they give the airplane designer yet another tool to help him choose the right type of engine. In practice, the decision will be biased by factors like the prices of available (offthe-shelf) engines and project engine development schedules.

\subsubsection{Community noise considerations *}

The FAA noise certification criteria, laid down in FAR Part 36, are summarized in Fig. 5-23. Maximum fly-over noise levels are stipulated for takeoff at a point 3.5

* Definitions of noise terminology is found in Ref. 5-50 


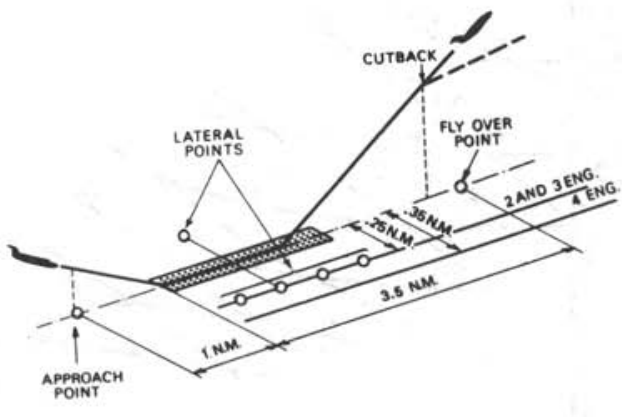

a. Reference points for noise measurements

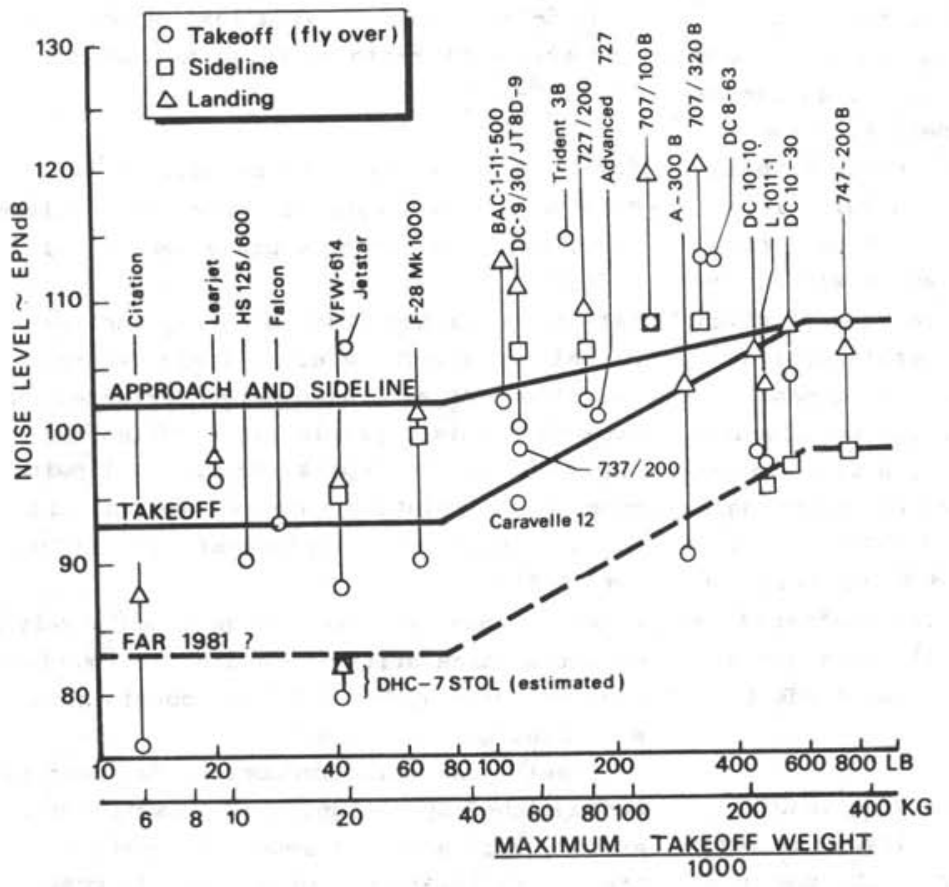

b. Noise levels: regulations and measurements (Ref. NASA SP-265, Ref. 5-55, Flight, Nov. 1972, F11ght Manuals F-28, DC- $10 / 30,8747 / 200 B$, A-300B brochure)

Fig. 5-23. Noise certification according to the FAR 36 airworthiness regulations

$n . m$. from the start of the takeoff roll and for landing at a point $1 \mathrm{n} . \mathrm{m}$. from the threshold. A limit is also imposed on the sideline noise, which is measured on a line to the side of, and parallel to, the runway. The noise limits are compared with figures for some current airplane types. The basic methods of obtaining desirable noise levels were dealt with in Section 4.4.3.: engine design, installation and noise attenuation (acoustic lining), gen- eral arrangement of the airplane, performance and special flight procedures. The performance aspects will be considered from two points of view.

a. Aircraft with Short Take-off and Landing (STOL) field lengths making steep gradient approaches may exhibit a considerable community noise reduction in comparison to current conventional subsonic transports. However, the effects of reducing the field length on airplane configuration are far- 
reaching and generally a considerable direct operating cost penalty must be expected.

The present line of thought (cf. Ref. 5-55) tends to favour increased climb and descent angles, without necessarily aiming at very short field lengths. The result may be a relatively conventional airplane with Reduced Take-off and Landing field lengths (RTOL) of the order of 3,000 to $5,000 \mathrm{ft}$ (900 to $1500 \mathrm{~m})$.

b. Aircraft performance may be optimized so as to achieve minimum noise for a fixed field length requirement. As in the case of performance optimization, the wing loading, thrust (power) loading and aspect ratio can be varied. In general, an increment in the aspect ratio decreases the drag and thrust level for a given airplane lift and therefore leads to a noise reduction. On the other hand, an aspect ratio increment will lead to an empty weight increment and both landing and takeoff weight will be affected. The net result will generally be a thrust and noise increment. Similar arguments apply to the wing loading For a given aircraft weight, a wing loading reduction results in increased climb angles after takeoff and decreased thrust level during approach. As against this, operational speeds are lower and noise exposure time increases. The increased all-up weight due to the increased wing area also tends to increase the thrust level.

An example of the effect of wing loading and thrust loading on noise levels is presented in a diagram similar to the one discussed in Section 5.5.4.: Fig. 5-24. The relative reduction in EPNAB relative to the FAR 36 requirement is shown for a specific

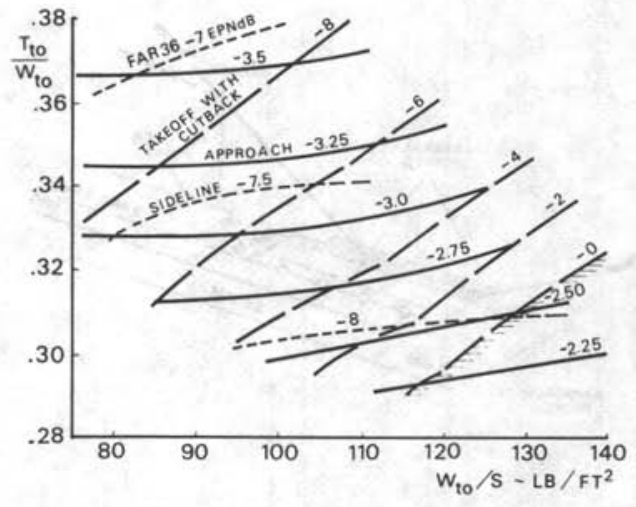

Fig. 5-24. Effect of wing loading and thrust/weight ratio on noise production (Ref. 5-43)

design example. No standard calculation methods for this type of curve can be given here, but an example is presented in Ref. 5-56.

Various published studies convey the impression that, for a given field length, the effect of airframe design variables on community noise, particularly during the approach, is not impressive. The following conclusions relating to a medium-haul highsubsonic transport airplane are quoted from Ref. 5-51:

1. An optimum airplane design based purely on performance criteria is also optimum for minimum noise within 1 EPNAB, considering all three noise sources.

2. Significant noise reductions can best be accomplished by designing the powerplant so as to permit adequate sound attenuation treatment. Regardless of weight and cost, the noise attenuation available by this method is limited to approximately 10 to 12 EPNaB. 


\section{Chapter 6. Choice of the engine and propeller and installation of the powerplant}

\section{SUMMARY}

In this chapter it will be assumed that the total thrust (power) to be supplied by the engines is approximately known. The various considerations which govern the choice of the number and type of engines are discussed. A short survey is given of propeller coefficients, diagrams and methods for controlling the propeller blade angle. Methods are presented for choosing the diameter, shape and number of blades, and some attention is paid to the location and installation of propeller and jet engines, thrust reversers and Auxiliary Power Units. 
NOMENCLATURE

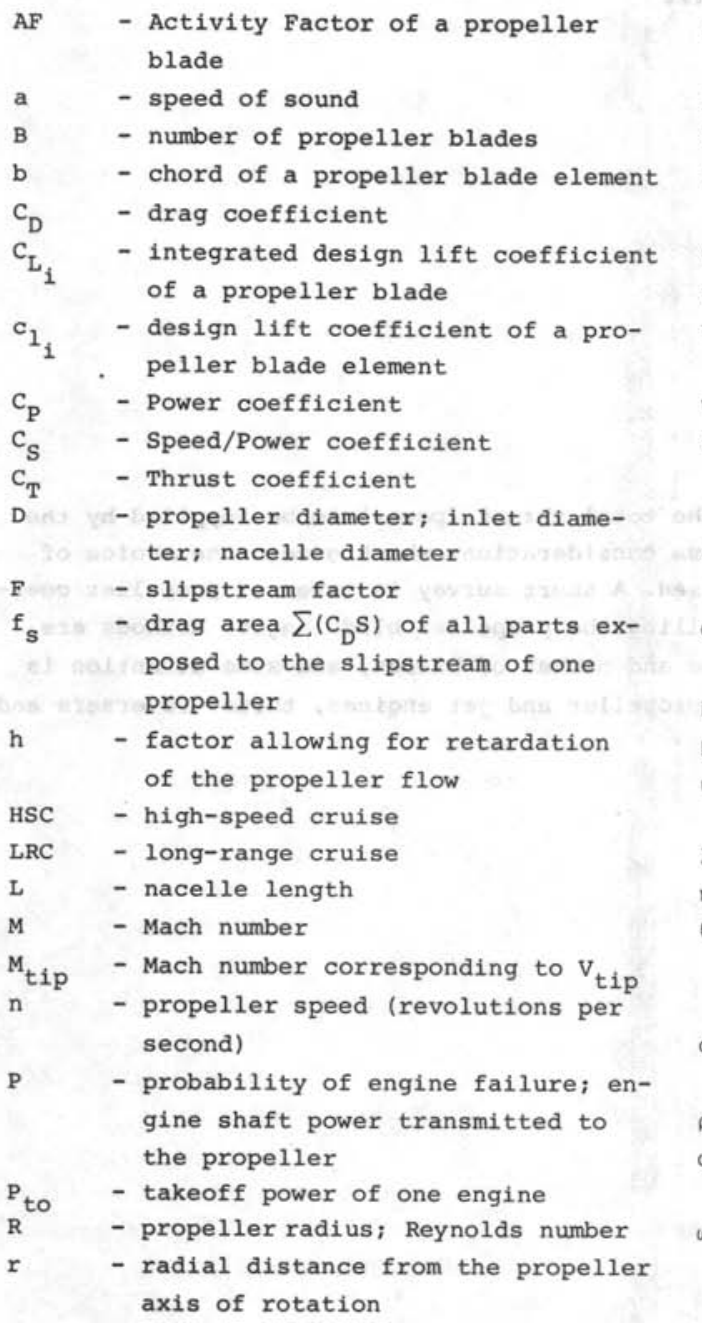

- propeller speed (revolutions per minute)

s $\{071$ - area

s c - cross-sectional area of the body in the slipstream of a propeller

T - thrust

Teff - effective propeller thrust

TAF - Total Activity Factor of a propeller

- flight speed

- cruising speed

- helical tip speed of the propelier blade

- aircraft weight

- fore-and-aft location of nacelle leading edge relative to the wing leading edge

- vertical distance of thrust line to the chord

- angle of attack of a propeller blade element

B, B.70-geometric pitch or blade angle

B. 75 (at 70 or 758 R)

$\varepsilon$ - tilt-down angle of engine thrust line

$\lambda$ - engine scaling factor

$\eta \quad$ - propeller efficiency

$\theta$ - relative ambient temperature (temperature/ambient temperature at sea level ISA)

$\phi \quad$ - effective pitch angle; toe-in angle of nacelle

- air density

- relative air density (air density/air density at sea level ISA)

- rotational speed of propeller

\subsection{INTRODUCTION}

In order to be able to choose the appropriate type of engine, the total thrust (power) required must be known first, the deciding factor here being the specified performance. As explained in the previous chapter, the engine thrust (power) will also be affected up to a certain extent by the geometry and aerodynamic character-

istics of the wing, which may possibly not yet have been finalized at the stage of the design when the engine type is chosen. If the number of engines has in fact been chosen, the approximate thrust (power) to be supplied per engine will already be known. Most designers will prefer to choose an engine which has already been developed and tested, but in some cases the airplane design will be based on an engine project 
for which certain characteristics are still subject to variation. The choice is generally limited to only a few types and in some cases there may even be only one engine which is regarded as suitable. Aircraft design studies are sometimes intended to investigate the possibilities of a new type of engine and that implies that there is no choice at $a 11^{*}$. When two or more engine types are considered, an assessment may be made on the basis of a rough comparison of technological and economical factors. If this comparison does not provide a clear basis for choice, various designs should be developed to the extent that all the important consequences which the choice of engine has on the overall design will be clearly shown.

In the case of a propeller aircraft, the design of the propeller should fit both the engine characteristics and the performance of the aircraft. The optimum propeller must do full justice to the performance of the engine and for this reason a standard propeller is not used on highperformance aircraft. The geometry of the propeller is also important in view of the clearance between the propeller and the airframe or the ground. Jet aircraft offer a relatively high degree of freedom in the location of the engines. Although the final answer regarding the details of the location will have to come from aerodynamic research, even the preliminary design should already be aimed at achieving the right basic configuration. Although the thrust reversal system will generally not be designed by the aircraft manufacturer, its presence should be taken into account at an early stage. This also applies to the Auxiliary Power Unit (APU), which is generally used in modern transport aircraft.

\subsection{CHOICE OF THE NUMBER OF ENGINES AND THE ENGINE TYPE}

*cf. also Section $6 \cdot 2 \cdot 3$. : rubberizing of turbo engines

\subsubsection{Engine installation factors}

Several general aspects of engine location in relation to the number of engines were mentioned in Section 2.3. We shall now focus our attention on several considerations on which the final choice of the number of engines will have to be based. Fig. 6-1 shows the values generally used

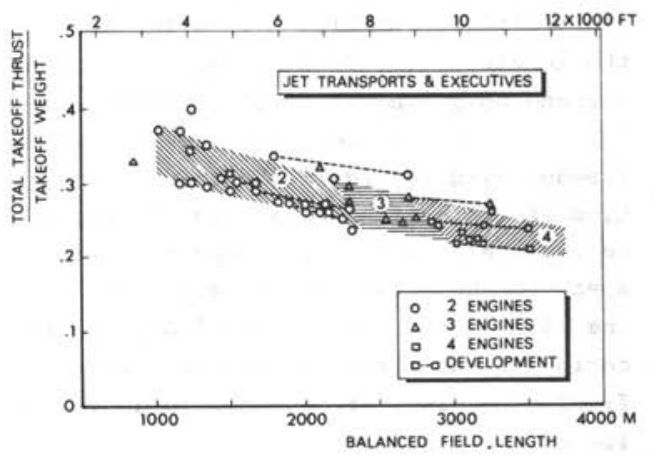

Fig. 6-1. Thrust loading, number of engines and takeoff field length required

for the takeoff thrust/weight ratio in the case of civil jet aircraft. The length of the takeoff runway has proved to be the most useful parameter for comparison purposes. Takeoff performance will also be influenced by the wing loading, flap design and, to a lesser degree, the bypass ratio. The shaded regions show the combinations of thrust loading and the number of engines usually employed for different design requirements as regards takeoff field length. It is also generally found that two engines are used for short-haul aircraft and three or four for medium and long ranges. However, it should be pointed out that Fig. 6-1 should not be regarded as in any way mandatory with regard to the choice of the number of engines. For propeller aircraft a simple correlation of the type shown in Fig. $6-1$ is not readily obtainable.

The limited availability of suitable engine types may sometimes be a decisive factor, forcing the designer to choose an aircraft configuration which is 
possibly not ideal. In such a case it will be worthwhile to consider a variant based on a hypothetical engine, delivering a thrust in the desired order of magnitude. A comparative study of both versions provides a good indication of the price that will have to be paid for the choice one is "forced" to make.

Mounting four jet engines on the wing generally presents minor problems. The engines are relatively small in diameter, so that the length of the landing gear will be correspondingly limited, even in the case of bypass ratios of about six. There is some freedom with regard to the lateral location of the engines, enabling the designer to reach a satisfactory compromise with respect to the weight of the wing structure, the size of the vertical tail and the 10cation of the centre of gravity. This configuration makes it possible to achieve a low empty weight.

When three engines are used, there is always the problem of installing the third engine, for this will have to be placed in the plane of symmetry (cf. Section 2.3.2. and Fig. 2-17). Whichever location is chosen, it is almost certain that the weight penalty caused by installing the central engine will be increased relative to that of the other two engines.

If only two engines are installed the fan diameter will be relatively large, and in order to avoid a high landing gear, location on the rear of the fuselage may be worth investigating. If this is not a favorable location, a high wing may solve the problem of engine-to-ground clearance, though this will result in increased structural weight.

\section{$6.2 \cdot 2$. Engine failure}

\section{a. Probability of engine failure.}

Although the modern turbine engine is very reliable, the possibility of an engine malfunction must never be ignored. In unfavorable circumstances the engine may have to be stopped or may be unable to operate. This will not only lead to a considerable decrement in thrust, but also to yawing and rolling moments, as well as extra drag. of the nacelle of the dead engine and drag resulting from the asymmetrical flight condition. The airworthiness authorities have therefore laid down a number of airworthiness criteria relating to takeoff speeds, takeoff distances, climb performance, etc., which must be met when engine failure has occurred. In addition, regulations also require that the aircraft should be capable of being stopped safely if failure occurs below the decision speed $v_{1}$. Although only 50 percent of the thrust will be available after failure of one engine out of two, the airplane must be designed to provide an acceptable level of safety. If the probability of malfunction per flying hour is taken as $\mathrm{P}$, then the probability that no malfunction will occur will be $1-P$. Since $P$ is extremely small in relation to 1 - the order of magnitude comes to $.5 \times 10^{-3}$ to $10^{-4}$ - an approximation may be introduced for aircraft with two, three and four engines, resulting in the figures in Table 6-1.

\begin{tabular}{|l|c|c|c|}
\hline & \multicolumn{2}{|c|}{$\begin{array}{r}\text { Probability of engine failure } \\
\text { (per flying hour) }\end{array}$} \\
\hline Failure of & $\begin{array}{c}1 \\
\text { engine }\end{array}$ & $\begin{array}{c}2 \\
\text { engines }\end{array}$ & $\begin{array}{c}3 \\
\text { engines }\end{array}$ \\
\hline $\begin{array}{l}\text { twin-engine } \\
\text { aircraft } \\
\text { three-engine } \\
\text { aircraft } \\
\text { four-engine } \\
\text { aircraft }\end{array}$ & $3 \mathrm{P}$ & $3 \mathrm{P}^{2}$ & $\mathrm{P}^{2}$ \\
\hline
\end{tabular}

Table 6-1. Probability of engine failure related to the number of engines.

Table 6-1 shows that during a given period of time, the probability of malfunction in one engine is twice as great in the case of a four-engined aircraft as in the case of a twin-engined aircraft, and the likelihood that two engines may fail is even six times as great. Although engine malfunction in itself need not have fatal con- 
sequences, failure of both engines in a twin-engined aircraft will obviously be extremely critical. However, the reliability of today's engines is such that a probability of $\mathrm{P}^{2}$ may be practically ignored for a short time period such as the takeoff phase and the safety level of a twin-engined passenger aircraft is now generally considered to be acceptable for passenger transports which are not intended for extended overwater flights. On the other hand, $6 \mathrm{P}^{2}$ is not a negligible quantity and this is why a four-engined aircraft must meet certain requirements regarding performance and flying characteristics with two engines inoperative in the en route configuration. The three-engined aircraft is an intermediate case. If two engines should fail, say during a transatlantic flight, the DC-10 will be able to continue on one engine by flying low and dumping fuel. Failure of two engines out of three or four during takeoff is extremely unlikely and need not be considered, provided the failure of any one engine does not entail a situation in which a second engine is likely to fail.

\section{b. Engine failure during or shortly after takeoff.}

Under these circumstances, a transport aircraft should always have a sufficiently large reserve of thrust to enable it to continue the takeoff safely, and for this reason it must be equipped with at least two engines.

The requirements for small aircraft (FAR Part 23) are less severe. Although the aircraft should remain controllable in case of failure, no requirements are laid down for its performance during takeoff. Safety is ensured by allowing for a generous margin in the takeoff safety speed. There are requirements with regard to climb performance, but aircraft with a marginal performance do not have to comply with these, provided the stalling speed does not exceed 61 knots $(113 \mathrm{~km} / \mathrm{h})$; see FAR Part 23.67 and section 5.4.3. of this textbook. c. Engine failure during cruising flight. This case may lead to a forced descent to a lower altitude, which will depend on the thrust still available. In the case of three- and four-engined aircraft the service ceiling after engine failure will be adequate, so that mountains can be crossed with a wide margin. This is not always the case with twin-engined aircraft and the route to be flown will be the main factor in determining whether a service ceiling of, say, 12,000 feet $(3600 \mathrm{~m})$ would still be acceptable. During development of the Lockheed L-101: and the Douglas DC-10 Trijets, this consideration contributed to the choice of the three-engined layout. Fig. 6-2 shows the influence of the alti-

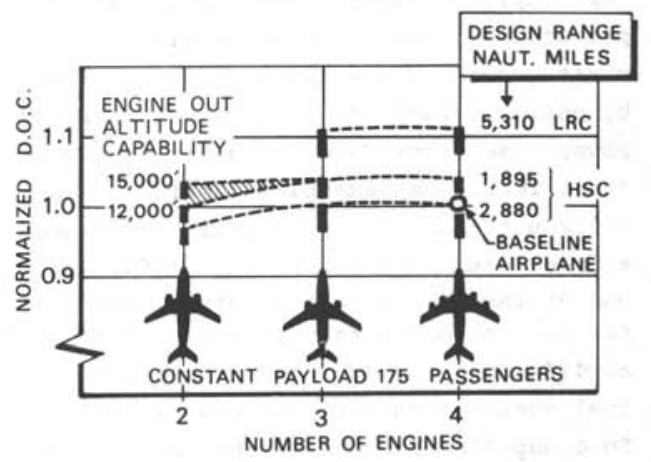

Fig. 6-2. Direct operating costs and number of engines (Ref. SAE Paper No. 710423)

tude requirement on the direct operating costs of an aircraft project. When a cruising level of $15,000 \mathrm{ft}(4575 \mathrm{~m})$ after engine failure is stipulated, the thrust will have to be adapted accordingly. In that case the difference in direct operating costs (d.o.c.) for all versions is very smal1. However, when the acceptable limit is put at $12,000 \mathrm{ft}(3660 \mathrm{~m})$, the choice of the engines will be based on other criteria and the initial engine costs will be reduced, resulting in lower depreciation. Twin-engined aircraft may not be operated on routes where more than one hour's flying from an airport would be involved at 
any point. This implies that a twin-engined alrcraft cannot be considered for extended overwater flights.

d. Flying qualities after engine failure. In the case of failure of a wing-mounted engine, a yawing moment of such magnitude will be created that the area of the $f$ in and rudder will have to be largely designed to cope with it. This will be discussed more fully in Chapter 9.

6.2.3. Engine performance and weight variations

a. Engine thrust (power) growth. Transport aircraft shows a steady rise in capacity, both during the development and production phase and after delivery. Aircraft growth is frequently made possible by engine growth, for which the thrust or power rises considerably in the course of time. To cite an example: the Turboméca Astazou turboprop engine started life with a power rating of 562 ESHP in 1963, while one of the most recent versions, the Astazou XX, has a takeoff power of as much as 1442 ESHP. In addition, the specific fuel consumption has gone down slightly. Some aspects of boosting the performance of piston engines have been dealt with in Section $4 \cdot 2.2$. For gas turbines the growth is obtained by raising the Turbine Entry Temperature, improving cycle efficiencies, increasing the diameter of the air intake and sometimes by increasing the overall Pressure Ratio. None of these leads to any appreciable increase in engine weight, if anything, they result in a considerable decrease in specific weight. In the case of propeller aircraft, it is recommended that this growth element be anticipated by making allowances for it in propeller design and location so as to ensure efficient absorption of the increased power.

\section{b. Rubberizing of turbo engines}

Engine development is usually a long-term process, but as against this the airplane designer may sometimes have to consider engines which are still in the project phase. This, in turn, may imply that the size has not yet been frozen. In order to adjust the thrust level desired to the specific airplane project application, the engine geometry may be scaled up or down identically, without changing the major thermodynamic characteristics like design Turbine Entry Temperature and Overall Pressure Ratio. This technique is referred to as "rubberizing" the engine for a constant technology.

When the size of engine components is increased, various cycle efficiencies will improve slightly as a result of the increased Reynolds number. Provided the thrust (power) variation is of the order of, say, 10 to 20 percent, the influence of rubberizing on specific thrust and specific fuel consumption is hardly noticeable and is frequently ignored. The growth of engine weight with size is much more difficult to assess. If the well-known squarecube law held good for the turbojet, we would find that an inlet diameter growth with a factor $\lambda$ would entail growth factors $\lambda^{2}$ for the airflow and thrust and $\lambda^{3}$ for engine mass. As a consequence, the engine weight would be proportional to $\mathrm{T}^{1.5}$ and the specific engine weight would vary in proportion to $\mathrm{T}^{\cdot 5}$. However, a more detailed

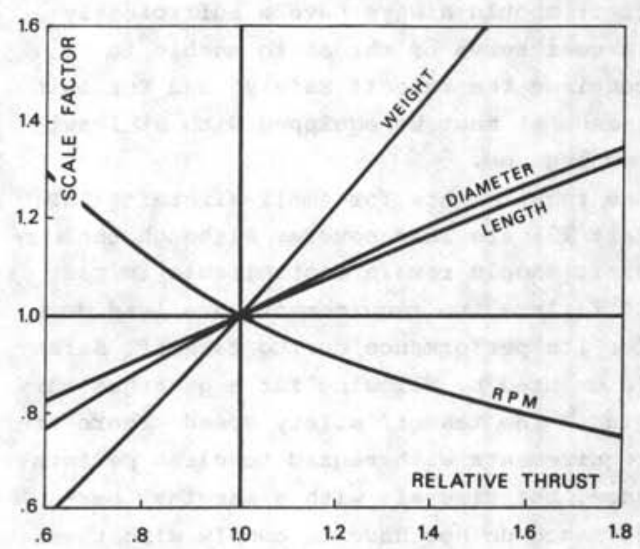

a. Rubberizing effects for a turbojet aircraft (example)

Fig. 6-3. Engine scaling effects 


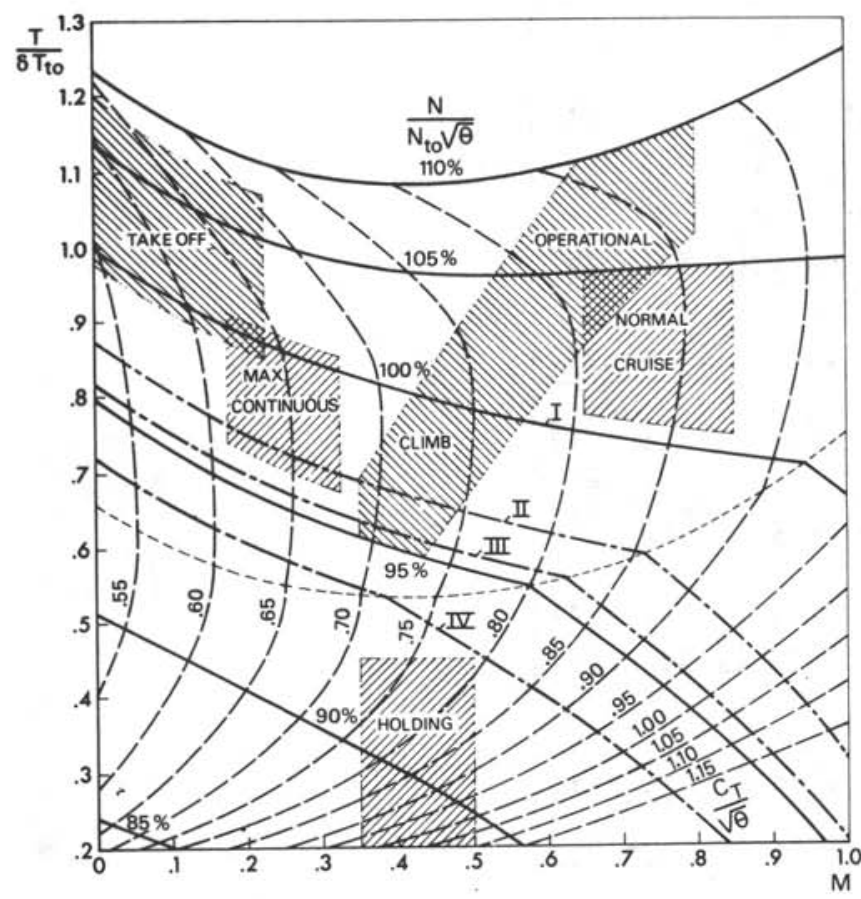

NOTE

1. The following ratings apply to Sea Level ISA conditions:

I: Maximum takeoff

II: Maximum continuous

III: Maximum recommended climb IV: Maximum recommended cruise 2. The subscript "to" refers to Sea Level static takeoff conditions

b. Non-dimensional presentation of engine performance of a low-bypass jet engine

Fig. 6-3. (continued)

breakdown of the engine weight into component weights shows that in actual practice the growth of weight with size is considerably overestimated by the square-cube law (e.g. Refs. 6-4 and 6-5). A more realistic relationship is therefore:

engine weight $\doteqdot(\text { thrust })^{\mathrm{n}}$

in which $\mathrm{n}$ has a typical value of 1.07 to 1.14. The example in Fig. 6-3a shows that one should also be aware of the change in engine rotational speed. Reasoning on the basis of the theoretical result of squarecube law, or the more moderate sensitivity given by $(6-1)$, it may be concluded that from the point of view of minimum engine weight it would be advantageous to generate a specified total thrust with a large number of small engines instead of a small number of larger engines, but the advantage in installed engine weight is then much less or may even disappear. Moreover, if real engines of different thrust level are compared (Table 6-2), a systematic trend cannot be observed in the specific weight for various sizes. It is therefore justifiable to conclude that in practice the number of engines to be chosen cannot be based on simplified theoretical considerations. There is no clear evidence to show that the cost of engines on a basis of dollars per pound of thrust is significantly affected by the number of engines required to develop that thrust. Although for a given number of engines to be produced the engine costs per $1 \mathrm{~b}$ of thrust (or per hp) will decrease with increasing engine size, this effect is compensated for by the fact that the smaller engine will probably be produced in larger numbers, resulting in lower development and production costs per engine.

\subsubsection{Choice of the engine type}

After preliminary performance calculations have been carried out and the number of engines has been decided upon, the thrust (power) per engine will be roughly known. The choice between engines satisfying the requirements as regards required thrust 


\begin{tabular}{|c|c|c|c|c|c|c|c|c|c|c|c|c|c|c|c|c|c|c|c|c|}
\hline \multirow{2}{*}{$\begin{array}{l}\text { Engine } \\
\text { Manufacturer }\end{array}$} & \multirow{2}{*}{ Engine Type } & \multicolumn{2}{|c|}{$\begin{array}{l}\text { Net Thrust } \\
\mathrm{kg}\end{array}$} & \multirow{2}{*}{$\begin{array}{c}\text { Cruise } \\
\text { SFC } \\
\mathrm{kg} / \mathrm{kg} / \mathrm{h}\end{array}$} & \multirow{2}{*}{$\begin{array}{l}\text { Bypass } \\
\text { ratio } \\
\text { SL/ISA } \\
\text { static }\end{array}$} & \multirow{2}{*}{\begin{tabular}{l|l} 
Air \\
flow \\
SL/ISA \\
static \\
T.0. \\
$\mathrm{kg} / \mathrm{sec}$
\end{tabular}} & \multicolumn{2}{|c|}{$\begin{array}{c}\text { Pressure } \\
\text { ratio }\end{array}$} & \multirow{2}{*}{\begin{tabular}{|l|} 
Turbine \\
Entry \\
Temp. \\
SL/ISA \\
T.0. \\
K
\end{tabular}} & \multirow{2}{*}{ 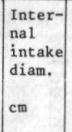 } & \multirow{2}{*}{\begin{tabular}{|c|} 
Length \\
m
\end{tabular}} & \multirow{2}{*}{\begin{tabular}{|c|}
$\begin{array}{l}\text { Dry } \\
\text { weight }\end{array}$ \\
kg
\end{tabular}} & \multicolumn{4}{|c|}{ Engine assembly } & \multirow{2}{*}{$\begin{array}{l}\text { Comb. } \\
\text { Chamber }\end{array}$} & \multirow{2}{*}{$\begin{array}{l}\text { Turbine } \\
\text { Cooling }\end{array}$} & \multirow{2}{*}{$\begin{array}{l}\text { Date } \\
\text { first } \\
\text { run }\end{array}$} & \multirow[b]{2}{*}{ Remarks } \\
\hline & & $\begin{array}{l}\text { Takeoff } \\
\text { SL/ISA } \\
\text { static } \\
\end{array}$ & $\begin{array}{l}\text { Cruise } \\
11,000 \mathrm{~m} \\
\mathrm{M}=.80\end{array}$ & & & & tota1 & fan & & & & & 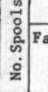 & & $\begin{array}{l}\text { Number of } \\
\text { Compressor }\end{array}$ & $\begin{array}{l}\text { tages } \\
\text { Turbine }\end{array}$ & & & & \\
\hline 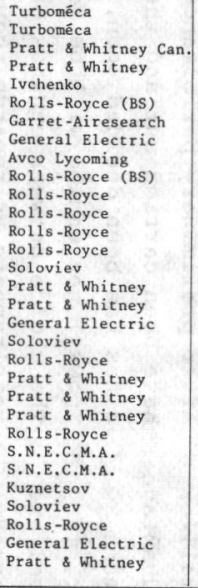 & 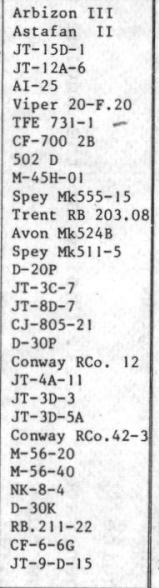 & \begin{tabular}{|l|}
400 \\
710 Dry \\
1000 \\
1360 \\
1500 \\
1545 \\
$1585-$ \\
1950 \\
2800 \\
3520 \\
4470 \\
4530 \\
4760 \\
5170 \\
5400 \\
5440 \\
6350 \\
6800 \\
6800 \\
7940 \\
7940 \\
8170 \\
9920 \\
9900 \\
10000 \\
10000 \\
1000 \\
11500 \\
1900 \\
19500 \\
20500 \\
\end{tabular} & $\begin{array}{c}133 \\
230^{*} \\
223 \\
363 \\
443^{*} \\
6.67 \\
408 \\
428 \\
609 \\
1245^{*} \\
1680^{*} \\
1315^{*} \\
1975^{*} \\
1100 \\
1540 \\
1540 \\
\approx 1300 \\
1300^{*} \\
2075 \\
2130\end{array}$ & $\begin{array}{l}1.14 \\
.63^{*} \\
.88 \\
1.06 \\
.84^{*} \\
1.26 \\
.83- \\
.93 \\
.78 \\
.73^{*} \\
.79^{*} \\
.72^{*} \\
.79^{*} \\
.78^{*} \\
.90 \\
.81 \\
.76 \\
.77 \\
.89 \\
.92\end{array}$ & \begin{tabular}{|l}
0 \\
6.5 \\
3.2 \\
0 \\
2 \\
0 \\
$2.7-$ \\
1.9 \\
6.1 \\
2.8 \\
1 \\
3 \\
0 \\
.64 \\
1. \\
0 \\
1.1 \\
1.5 \\
1 \\
.3 \\
0 \\
1.4 \\
0.6 \\
4.0 \\
4.8 \\
1 \\
2.3 \\
4.8 \\
5.6 \\
4.4
\end{tabular} & \begin{tabular}{|l}
6 \\
31 \\
33.4 \\
22 \\
42 \\
20 \\
51.3 \\
38 \\
18 \\
108 \\
104 \\
91 \\
136 \\
83 \\
92 \\
92 \\
113 \\
91 \\
141 \\
188 \\
185 \\
127 \\
115 \\
204 \\
165 \\
1623 \\
333 \\
334 \\
232 \\
272 \\
602 \\
655 \\
687
\end{tabular} & \begin{tabular}{|c|}
5.5 \\
9 \\
10.2 \\
6.5 \\
9 \\
5.6 \\
19. \\
6.8 \\
10.3 \\
18 \\
16 \\
16 \\
8.7 \\
19 \\
14 \\
13 \\
13 \\
$12^{*}$ \\
18 \\
15.6 \\
12 \\
13 \\
16 \\
15.1 \\
18 \\
25 \\
23.2 \\
20 \\
25 \\
29 \\
22 \\
22
\end{tabular} & \begin{tabular}{|l|}
1.32 \\
1.52 \\
1.7 \\
-
\end{tabular} & $\begin{array}{c}1135 \\
1285 \\
1285 \\
1330 \\
990 \\
1100 \\
1350\end{array}$ & 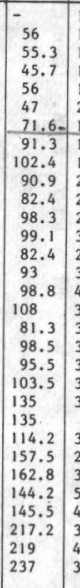 & 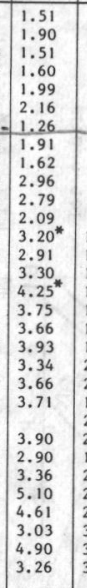 & \begin{tabular}{|l|}
115 \\
195 \\
229 \\
203 \\
290 \\
270 \\
272 \\
272 \\
334 \\
526 \\
673 \\
990 \\
805 \\
1516 \\
1050 \\
1470 \\
1585 \\
1431 \\
1680 \\
1520 \\
2060 \\
2310 \\
1890 \\
2073 \\
2310 \\
1940 \\
2120 \\
2200 \\
2150 \\
3267 \\
3540 \\
3970 \\
\end{tabular} & 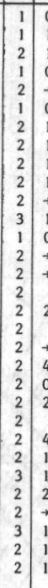 & 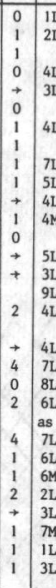 & 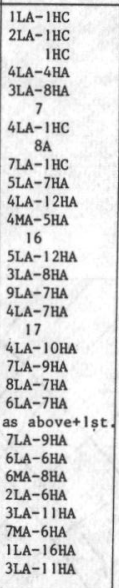 & \begin{tabular}{c|c}
1 \\
3 \\
$1 H-2 \mathrm{~L}$ \\
$2 \mathrm{~L}$ \\
$1 \mathrm{H}-2 \mathrm{~L}$ \\
$1 \mathrm{~L}$ \\
$1 \mathrm{H}-3 \mathrm{~L}$ \\
$2 \mathrm{~L}$ \\
$2 \mathrm{H}-2 \mathrm{~L}$ \\
$1 \mathrm{H}-3 \mathrm{~L}$ \\
$2 \mathrm{H}-2 \mathrm{~L}$ \\
$1 \mathrm{H}-1 \mathrm{M}-1 \mathrm{~L}$ \\
8 \\
$2 \mathrm{H}-2 \mathrm{~L}$ \\
$1 \mathrm{H}-2 \mathrm{~L}$ \\
$1 \mathrm{H}-2 \mathrm{~L}$ \\
$1 \mathrm{H}-3 \mathrm{~L}$ \\
3 \\
$2 \mathrm{H}-2 \mathrm{~L}$ \\
$1 \mathrm{H}-2 \mathrm{~L}$ \\
$1 \mathrm{H}-2 \mathrm{~L}$ \\
$1 \mathrm{H}-3 \mathrm{~L}$ \\
$1 \mathrm{H}-3 \mathrm{~L}$ \\
$1 \mathrm{H}-2 \mathrm{~L}$ \\
$1 \mathrm{H}-4 \mathrm{~L}$ \\
$2 \mathrm{H}-1 \mathrm{M}-4 \mathrm{~L}$ \\
$1 \mathrm{H}-2 \mathrm{~L}$ \\
$2 \mathrm{H}-4 \mathrm{~L}$ \\
$1 \mathrm{H}-1 \mathrm{M}-3 \mathrm{~L}$ \\
$2 \mathrm{H}-\mathrm{L}$ \\
$2 \mathrm{H}-4 \mathrm{~L}$ \\
\end{tabular} & \begin{tabular}{|l} 
ann. \\
ann. \\
ann.rev. \\
8 can. \\
ann. \\
ann. \\
ann. \\
ann. \\
ann. \\
can. \\
ann. \\
8 can. \\
10 can. \\
12 can. \\
8 can. \\
9 can. \\
10 can. \\
12 can. \\
10 can. \\
8 can. \\
8 can. \\
8 can. \\
10 can. \\
ann. \\
ann. \\
12 can. \\
ann. \\
ann. \\
ann. \\
\end{tabular} & \begin{tabular}{|l} 
no \\
air \\
no \\
no \\
no \\
air \\
air \\
air \\
no \\
air \\
no \\
air
\end{tabular} & $\begin{array}{l}1970 \\
1969 \\
1967 \\
1958 \\
1966 \\
1965 \\
1969 \\
1962 \\
1970 \\
1968 \\
1965 \\
1967 \\
1957 \\
1960 \\
1960 \\
1957 \\
1961 \\
1960 \\
1965 \\
1957 \\
1956 \\
1958 \\
1960 \\
\text { proj. } \\
\text { proj. }\end{array}$ & 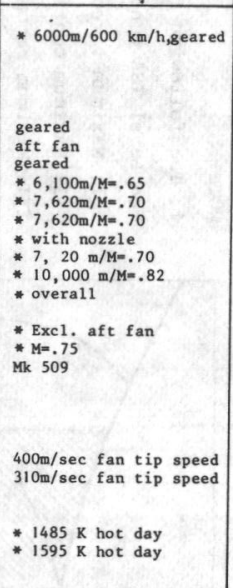 \\
\hline
\end{tabular}

Table 6-2. Principal characteristics of turbine engines 


\begin{tabular}{|c|c|c|c|c|c|c|c|c|c|c|c|c|c|c|c|c|c|c|c|}
\hline \multirow{3}{*}{$\begin{array}{c}\text { Engine } \\
\text { Manufacturer }\end{array}$} & \multirow{3}{*}{$\begin{array}{c}\text { Engine } \\
\text { Type }\end{array}$} & \multicolumn{3}{|c|}{ Performance, IS A } & \multicolumn{3}{|c|}{ Engine cycle } & \multicolumn{3}{|c|}{ Propeller } & \multicolumn{2}{|c|}{ Dimensions } & \multirow{3}{*}{\begin{tabular}{|c|} 
Dry \\
weight \\
$\mathrm{kg}$
\end{tabular}} & \multicolumn{4}{|c|}{ Engine-assembly } & \multirow{3}{*}{$\begin{array}{l}\text { Date } \\
\text { first } \\
\text { run }\end{array}$} & \multirow[t]{3}{*}{ Remarks } \\
\hline & & \multicolumn{2}{|l|}{ Power } & \multirow{2}{*}{\begin{tabular}{|c|} 
ESFC \\
cruise \\
$\mathrm{kgf} / \mathrm{pk} / \mathrm{h}$ \\
\end{tabular}} & \multirow{2}{*}{$\begin{array}{l}\text { Air- } \\
\text { flow } \\
\mathrm{kg} / \mathrm{s}\end{array}$} & \multirow[t]{2}{*}{ OPR } & \multirow{2}{*}{$\begin{array}{l}\text { TET } \\
(\mathrm{K})\end{array}$} & \multirow{2}{*}{$\begin{array}{l}\text { Max. } \\
\text { RPM }\end{array}$} & \multirow{2}{*}{$\begin{array}{l}\text { Cear } \\
\text { ratio }\end{array}$} & \multirow[t]{2}{*}{ RPM } & \multirow[t]{2}{*}{ length } & \multirow{2}{*}{$\begin{array}{c}\text { width/ } \\
\text { diam. } \\
\mathrm{m}\end{array}$} & & \multirow{2}{*}{\begin{tabular}{|l|}
$n$ \\
0 \\
0 \\
0 \\
$\vdots$ \\
0 \\
0 \\
$\vdots$ \\
$z$ \\
\end{tabular}} & \multirow{2}{*}{\begin{tabular}{|c|} 
Number of \\
Compressor \\
\end{tabular}} & \multirow{2}{*}{$\begin{array}{l}\text { stages } \\
\text { Turbine }\end{array}$} & \multirow{2}{*}{$\begin{array}{l}\text { Comb. } \\
\text { Chamb. }\end{array}$} & & \\
\hline & & \begin{tabular}{|l|} 
Takeoff \\
SL/static
\end{tabular} & \begin{tabular}{|c|} 
Cruise \\
$20,000 \mathrm{ft}$ \\
$245 \mathrm{kts}$ \\
\end{tabular} & & & & & & & & & & & & & & & & \\
\hline Rover & Mode1 TP-90 & 110 & 49 & .609 & .85 & 2.8 & - & 47,000 & .0540 & $2538 \mathrm{~A}$ & .90 & .52 & 91 & 1 & IC & 1 & 1 can & 1960 & NS \\
\hline Allison & Mode1 250(T-63) & 250 & 149 & .295 & 1.35 & 6 & 1170 & 48,950 & - & $-c$ & .95 & .40 & 62 & 2 & $6 \mathrm{LA}-\mathrm{IHC}$ & $2 \mathrm{H}-2 \mathrm{LF}$ & ann. & 1959 & turboshaft \\
\hline Turboméca & Orédon III & E 350 & 190 & .242 & 1.35 & 7.5 & & 59,100 & .1024 & - & 1.09 & .37 & 82 & 1 & $2 \mathrm{LA}-1 \mathrm{HC}$ & 3 & ann. & 1964 & turboshaft \\
\hline Turboméca & Astazou II & E 555 & 307 & .361 & 2.50 & 5.7 & & 43,500 & .0558 & $2425 \mathrm{C}$ & $1.91^{*}$ & .46 & 122 & 1 & ILA- $1 \mathrm{HC}$ & 3 & ann. & 1960 & *incl, prop. \\
\hline Pratt \& Whitney & PT $6 \mathrm{~A}-6$ & E 578 & 332 & .279 & 2.25 & 5.7 & & 33,000 & .0663 & $2200 \mathrm{C}$ & 1.58 & .48 & 122 & 2 & $3 \mathrm{LA}-1 \mathrm{HC}$ & $1 \mathrm{H}-1 \mathrm{LF}$ & ann. & 1963 & turboshaft \\
\hline Turboméca & Astazou XIV A & E 600 & 321 & .251 & 2.50 & 7.5 & & 43,000 & .0415 & $1783 \mathrm{C}$ & 1.43 & .68 & 160 & 1 & $2 \mathrm{LA}-1 \mathrm{HC}$ & 3 & ann. & 1966 & R \\
\hline Turboméca & Bastan IV & E 935 & 552 & .251 & 4.50 & 5.8 & 1143 & 33,500 & & & 1.55 & .75 & 222 & 1 & ILA- $1 \mathrm{HC}$ & 3 & ann. & 1959 & $* 15,000 \mathrm{ft}$ \\
\hline AVCO Lycoming & $T-53-L-7$ & E 1150 & $730^{*}$ & $.261^{*}$ & 5.00 & 6.0 & & 25,240 & & 1700 & 1.49 & .58 & 245 & 2 & $5 \mathrm{LA}-1 \mathrm{HC}$ & $2 \mathrm{H}+2 \mathrm{LF}$ & ann. & 1959 & aircooled \\
\hline AiResearch & TPE $331-20$ & 600 & 355 & .255 & 2.50 & 7.82 & 1240 & 41,730 & .0479 & $2000 \mathrm{C}$ & 1.18 & .55 & 129 & 1 & $2 \mathrm{C}$ & 3 & ann. & 1963 & $\beta$-control \\
\hline General Electric & $T-58-G E-8$ & 1250 & 780 & .247 & 5.62 & 8.30 & 1150 & 19,500 & & $2000 \mathrm{C}$ & 1,39 & .41 & 130 & 2 & $10 \mathrm{~A}$ & $2 \mathrm{H}+1 \mathrm{LF}$ & can & 1959 & turboshaft \\
\hline AVCO Lycoming & $\mathrm{T}-5313 \mathrm{~A}$ & 1400 & 800 & .240 & 5.50 & 7.40 & 1210 & 25,240 & & $1680 \mathrm{C}$ & 1.48 & .58 & 274 & 2 & $5 \mathrm{LA}-1 \mathrm{HC}$ & $2 \mathrm{H}+2 \mathrm{LF}$ & ann. & 1967 & $\mathrm{R}$ \\
\hline AVCO Lycoming & $\mathrm{T}-5313 \mathrm{~B}$ & 1400 & 800 & .240 & 5.50 & & 1210 & & & $1680 \mathrm{C}$ & 1.48 & .58 & 312 & 2 & $5 \mathrm{LA}-1 \mathrm{HC}$ & $2 \mathrm{H}+2 \mathrm{LF}$ & ann. & 1967 & $\mathrm{R}$ \\
\hline Ro11s-Royce & Dart Mk511-7E & E 1740 & 930 & .308 & 9.30 & 5.50 & 1130 & 14,500 & .086 & & 2.43 & .96 & 494 & 1 & $1 \mathrm{LC}-1 \mathrm{HC}$ & 2 & $7 \mathrm{can}$ & 1953 & \\
\hline AVCO Lycoming & $\mathrm{T}-5321 \mathrm{~A}$ & 1800 & 920 & .229 & 5.80 & & 1325 & 21,300 & & c & & & 306 & 2 & $5 \mathrm{LA}-1 \mathrm{HC}$ & $2 \mathrm{H}+2 \mathrm{LF}$ & ann. & 1969 & aircooled, R \\
\hline Rolls-Royce & Dart Mk 528 & E 2105 & 1170 & .266 & 10.70 & 5.75 & 1160 & 15,000 & .0929 & & 2.50 & .96 & 560 & 1 & $1 \mathrm{LC}-1 \mathrm{HC}$ & 3 & $7 \mathrm{can}$ & 1956 & \\
\hline & & &, $000 \mathrm{ft}$ & $300 \mathrm{kts}$ & & & & & & & & & & & & & & & \\
\hline General Electric & $T-64-G E-6$ & 2650 & 1350 & .200 & 11.10 & 12.60 & 1200 & $13,640 \mid$ & & & 2.80 & .74 & 490 & 2 & $14 \mathrm{~A}$ & $2 \mathrm{H}+2 \mathrm{LF}$ & ann. & 1959 & \\
\hline Napier & El and N.E1. 1 & 3000 & 1230 & .229 & 14.10 & 7.00 & & 12,500 & .0714 & $893 \mathrm{~A}$ & 3.10 & .92 & 715 & 1 & $10 \mathrm{~A}$ & 3 & $6 \mathrm{can}$ & 1952 & \\
\hline Rol1s-Royce & Dart Mk542-10 & E 3025 & (2) & & 12.25 & 6.35 & 1225 & 15,000 & .0775 & & 2.53 & .96 & 625 & 1 . & $1 \mathrm{LC}-1 \mathrm{HC}$ & 3 & $7 \mathrm{can}$ & 1960 & \\
\hline Allison & $501-D-13$ & E 3750 & 1450 & .213 & 14.75 & 9.25 & 1249 & 13,820 & & A & 3.69 & .69 & 797 & 1 & $14 \mathrm{~A}$ & 4 & $6 \mathrm{can}$ & 1954 & \\
\hline Allison & T-56-A-10W & 4050 & 1450 & .215 & 14.65 & 9.25 & 1249 & & & $1021 \mathrm{C}$ & 3.71 & .69 & 838 & 1 & $14 \mathrm{~A}$ & 4 & $6 \mathrm{can}$ & 1953 & \\
\hline Bristol Siddeley & Proteus Mk765 & E 4400 & 2155 & .224 & 20.90 & 7.10 & 1150 & & .0863 & & 3.13 & 1.10 & 1315 & 2 & $12 \mathrm{LA}-1 \mathrm{HC}$ & $2 \mathrm{H}-2 \mathrm{~L}$ & $8 \mathrm{can}$ & 1958 & \\
\hline Rolls-Royce & Tyne R.Ty. 12 & E 5505 & 2400 & .185 & 21.20 & 13.50 & 1242 & 15,250 & .064 & $976 \mathrm{~A}$ & 2.77 & 1.03 & 1000 & 2 & 6LA-9HA & $.1 \mathrm{H}-3 \mathrm{~L}$ & $10 \mathrm{can}$ & 1959 & \\
\hline Rolls-Royce & Tyne R.Ty. 20 & E $6100^{\circ}$ & 2500 & .193 & 21.20 & 13.50 & 1273 & 15,250 & .064 & $976 \mathrm{~A}$ & 2.76 & 1,40 & 1085 & 2 & 6LA-9HA & $1 \mathrm{H}-3 \mathrm{~L}$ & $10 \mathrm{can}$ & 1962 & aircooled \\
\hline
\end{tabular}

Table 6-2. (Continued) 
(power), fuel consumption and noise production, etc., is usually very limited. Table 6-2 presents a survey of the most important characteristics of some types of engines.

Engines, like aircraft, are subjected to extensive airworthiness tests, cf. for instance ICAO Circular 51-AN/4612. The designer must consider whether the engine chosen has been designed for civil or military use, when testing and certification are planned, which regulations will apply, and so on. To ensure smooth progress of the aircraft project, it is desirable to use an engine which is sufficiently far developed; there is generally a preference for a type which has already been built and tested (off-the-shelve engines).

Even a preliminary design requires a complete engine type specification or brochure, which should preferably contain at least the following data:

- limits regarding the engine output (ratings) and the operational conditions (temperatures, altitudes, speeds),

- thrust and fuel consumption for various engine ratings, altitude and airspeed,

- influence of airbleed and power off-take on engine performance,

- installation data: weight, dimensions, location of centre of gravity,

- noise levels, particularly for civil jet aircraft.

Engine performance is sometimes presented in the form of non-dimensional curves; an example is given in Fig. 6-3b. These curves not only represent the thrust lapse with altitude, airspeed and engine rating, but may also be used for the purpose of rubberizing the engine.

The choice of the engine and the design of the aircraft are so closely interrelated that it is difficult to make an appropriate choice. The designer may obtain a fair picture by an overall comparison of specific fuel consumption, thrust variation with altitude, specific weight and dimensions, engine configuration, initial cost and average time between major overhauls, etc. Sometimes an important part is played by contacts with the engine manufacturer, or economic and political factors affect- ing aviation. The survey of engine technology in Chapter 4 may possibly help the designer to make up his mind.

Summing up the arguments in the previous sections we may conclude that:

a. the number of engines may be decided entirely by the limited choice from competitive types of power plant;

b. the minimum number of engines with which the specified performance can be obtained will, as a rule, also be the most favorable number;

c. three-engined aircraft will be at a slight disadvantage technically as compared with four-engined aircraft;

d. a design study can only be started when sufficiently detailed engine performance and installation data are known.

The best engine for a particular application can only be arrived at by long and close collaboration between aircraft and engine designers.

6.3. CHARACTERISTICS, CHOICE AND INSTALLATION OF PROPELLERS

\subsubsection{General aspects}

The design and production of modern propellers is so specialized in nature that there are only a few manufacturers in this field. The aircraft designer draws up the propeller specification and has a number of possibilities to choose from. He should be aware of :

- the airplane's performance

- the engine characteristics and engine control

- propeller noise and vibrations

- the installed weight of the propellers

- the influence of the propellers on flying qualities

- the structural limits imposed by the layout of the aircraft.

In the preliminary design stage the choice of the propeller is mainly associated with the following characteristics:

a. The blade angle control system: fixed or 
constant speed propeller, B-control, etc. b. The blade shape: the planform of the blades, aerofoil shape and, incidentally, the twist along the blade

c. The number of blades per propeller d. The propeller diameter and rotational speed (gearing).

The losses incurred by transmitting (crank) shaft power into useful thrust horsepower, is expressed in the propeller efficiency,

$n=\frac{\text { useful power output }}{\text { shaft power input }}=\frac{T V}{P}$

Propeller efficiency is zero under static conditions; its maximum value is obtained in high-speed flight and amounts to between approximately 85 and $92 \%$ depending upon the blade shape.

Since a high propeller efficiency is desired in all the important phases of flight, the efficiency constitutes the most important design criterion for the propeller. It is essential to realize that cruising flight is not the only decisive factor, for the takeoff and climb performance may be equally important, particularly in case of engine failure.

This section will not deal with propeller theory, and the mechanical design of propellers will only be referred to very briefly; the list of references may be consulted for literature on these subjects. The application of propeller theory to various airplane design aspects is covered very adequately, for example, in Ref. 6-20.

\subsubsection{Propeller coefficients and diagrams}

The characteristic angles relating to the propeller blade element are shown in Fig. 6-4. The propeller rotates with an angular velocity $\omega$, while the airspeed is $v$. When the plane of the propeller is normal to the airspeed, the resulting speed at the blade element at a distance $r$ from the axis of the propeller will have an effective pitch angle $\phi$ with the circumferential velocity wr

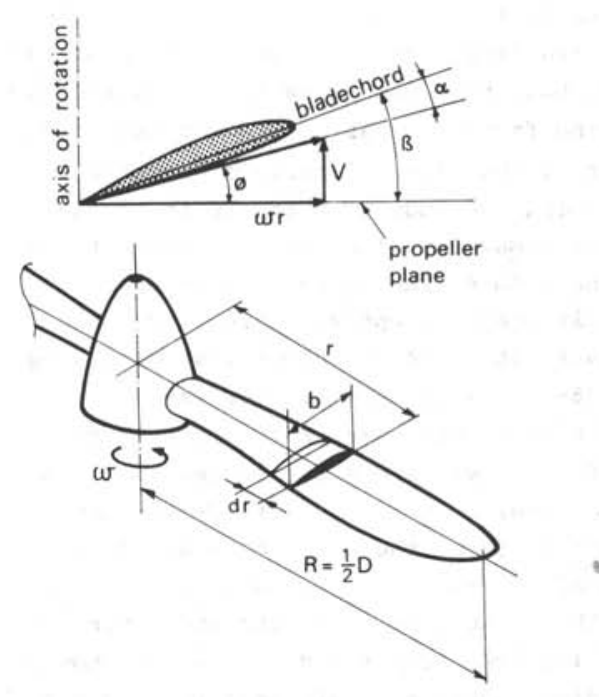

Fig. 6-4. Propeller blade element and local direction of flow

$\phi=\arctan \frac{\mathrm{V}}{\omega \mathrm{r}}$

In terms of the rotational speed and the propeller diameter,

$\phi=\arctan \frac{\mathrm{V}}{\mathrm{nD}} \times \frac{1}{\pi \mathrm{r} / \mathrm{R}}$

For $r=R, \phi$ represents the pitch angle of the helical path described by the propeller tip. The decisive factor here is the advance ratio,

$\mathrm{J}=\frac{\mathrm{V}}{\mathrm{nD}}$

The angle between the blade chord and the propeller plane is the geometric pitch or blade angle $B$. The angle of incidence of the blade element relative to the airstream is

$\alpha=\beta-\phi$

or, in combination with $(6-4)$ and $(6-5)$ :

$\alpha=\beta-\arctan \frac{\mathrm{J}}{\pi \mathrm{r} / \mathrm{R}}$

The angle $\alpha$ is not equal to the local effective angle of attack, since an extra 
speed is induced at the blade element by the trailing vortex system of the propeller, but $(6-7)$ does show that $J$ is the deciding factor as far as the working condition of the blade element is concerned. Assuming the induced velocity to be constant along the blade and noting that each blade element should operate at approximately the same optimum effective angle of attack, it is easily shown that according to $(6-7) \quad B$ will have to decrease with increasing $r$. For this reason propeller blades always have a pronounced built-in twist angle. It is usual to define the blade pitch by the value of $\beta$ at 708 or $758^{\circ}$ of the radial distance: $\beta, 70$ or $\beta, 75^{\circ}$ If the effects of viscosity and compressibility are disregarded, it can be shown by dimensional analysis that for a given blade geometry the following propeller coefficients are determined only by $J$ and
B. $75^{\circ}$

the Power Coefficient: $C_{P}=\frac{P}{\rho n^{3} D^{5}}$

the Thrust Coefficient: $C_{T}=\frac{T}{\rho n^{2} D^{4}}$

or in British units:

$$
\begin{aligned}
& C_{\mathrm{P}}=50 \frac{\mathrm{Bhp}}{\sigma(\mathrm{rpm} / 1000)^{3}(\mathrm{D}, \mathrm{ft})^{5}} \\
& C_{\mathrm{T}}=1.515 \frac{\mathrm{T}, 1 \mathrm{~b}}{\sigma(\mathrm{rpm} / 1000)^{2}(\mathrm{D}, \mathrm{ft})^{4}}
\end{aligned}
$$

Provided the tip speed is sufficiently far below the speed of sound and the blades are not stalled, the influence of the Mach and Reynolds number will be negligible. From this it follows that the power and thrust coefficients of geometrically similar propellers, within a certain region

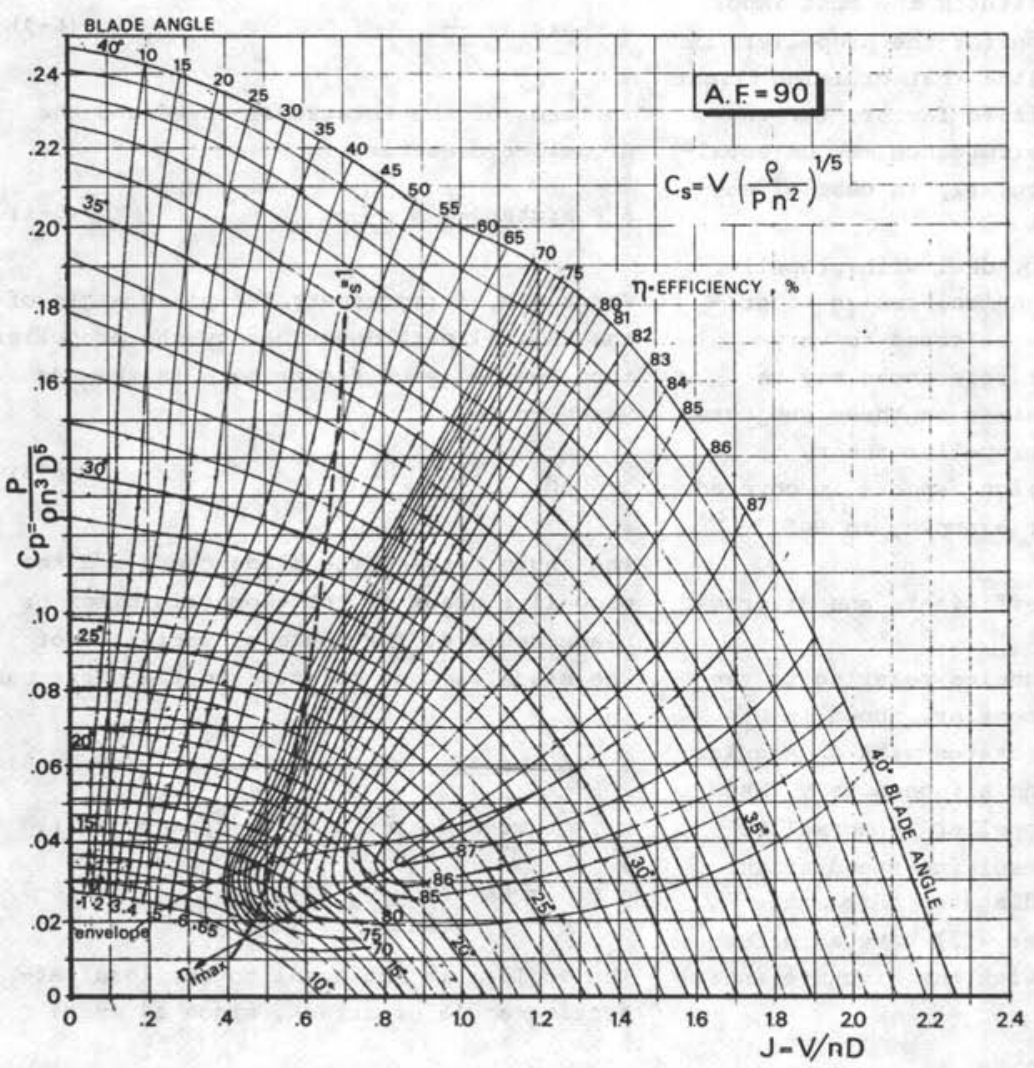

a. Two-blade propeller (Ref.: NACA WR 286)

Fig. 6-5. Performance diagrams of isolated propellers 


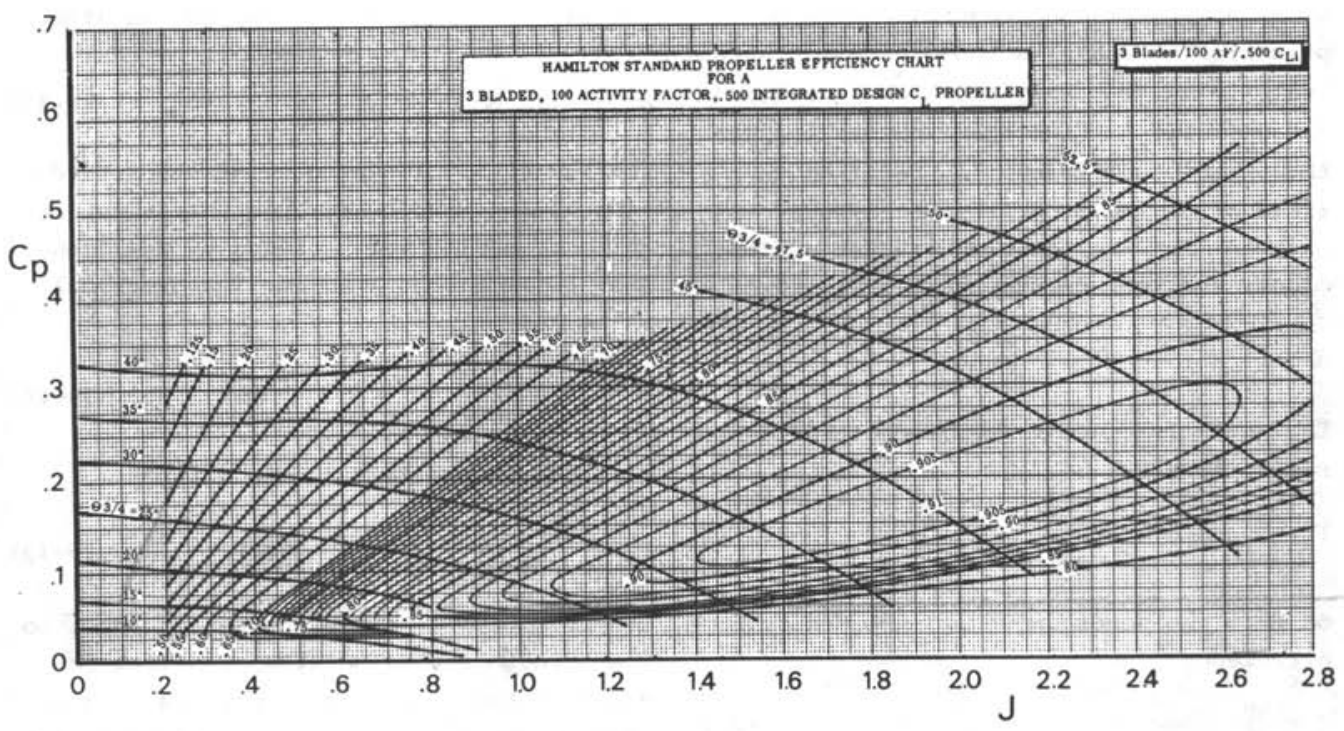

b. Three-blade propeller (Ref. Hamilton Standard PDB 6101)

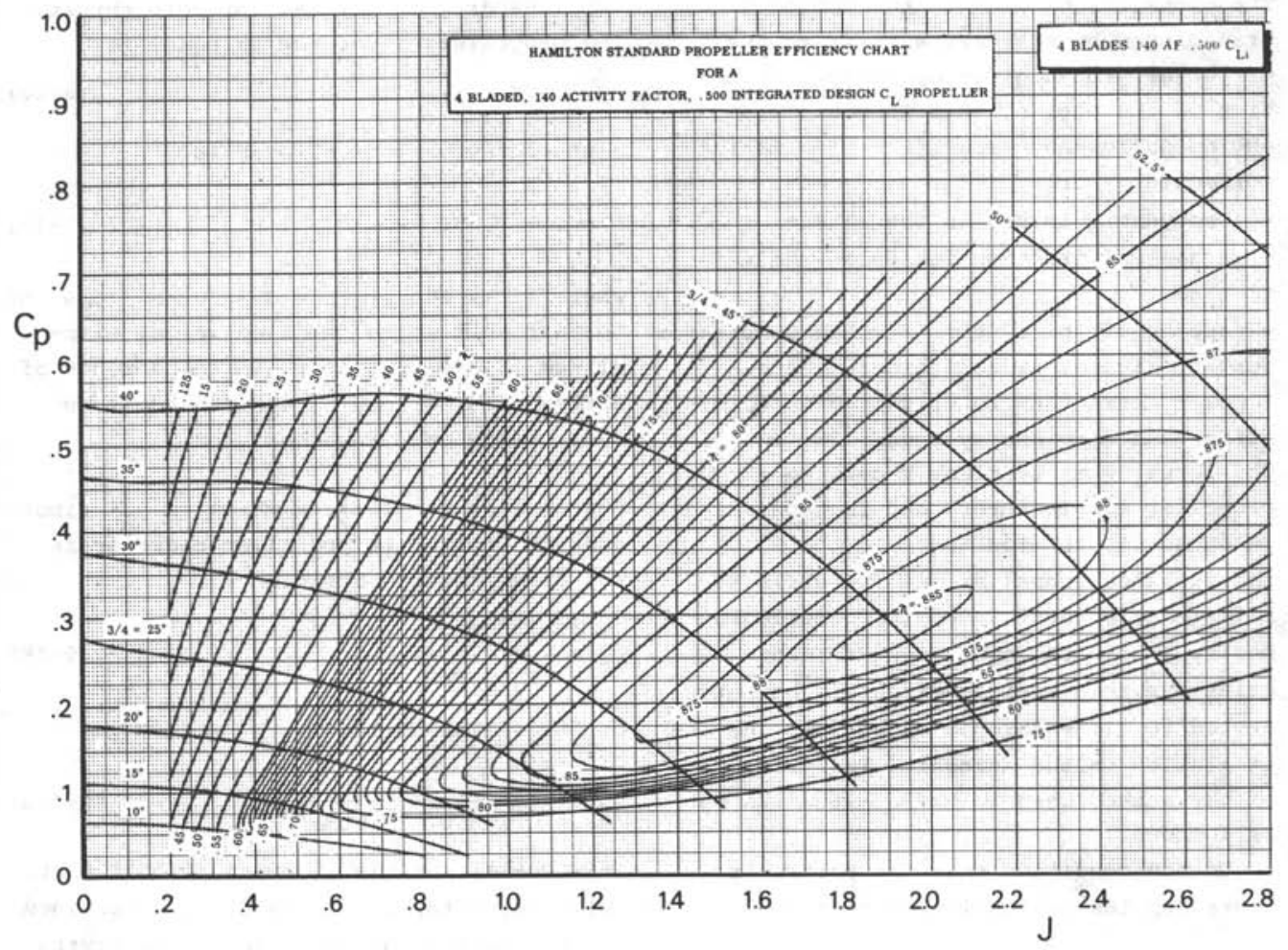

c. Four-blade propeller (Ref. Hamilton Standard PDB 6101)

Fig. 6-5 (concluded) 
of $M$ and $R$ (per unit length) can be represented in a single non-dimensional propeller diagram. Examples of such diagrams are depicted in Fig. 6-5. In some diagrams the thrust coefficient is specified instead of $n$. The propeller efficiency is derived from the power and thrust coefficients and the advance ratio as follows:

$\eta=\frac{\mathrm{C}_{\mathrm{T}}}{\mathrm{C}_{\mathrm{P}}} \mathrm{J}$

The propeller thrust may be obtained directly from $\mathrm{C}_{\mathrm{T}}$ :

$T=C_{T} \rho n^{2} D^{4}$

or from the power input and the propeller efficiency

$T=\frac{n P}{V}$

The propeller power $\mathrm{P}$ is the net power transmitted by the engine shaft to the propeller. For a given operational condition of the engine, this is found from the engine performance specification, making corrections for:

- aerodynamic losses in the intake

- mechanical friction losses in the reduction gear*

- tapping off bleed air from the compressor for anti-icing, airconditioning, etc.

- power extraction to drive engine accessories and airplane systems.

The thrust must be clearly defined (by the propeller manufacturer) and is generally taken as the tension force in the shaft of the isolated propeller $\left(n_{i s}\right)$ that is the efficiency without an engine nacelleplaced behind $i t$. In the case of performance calculations this efficiency should be corrected to allow for the effects of the installation in the aircraft. The principle of the method used in Ref. 6-16 works out as follows.

1. On the condition that the propeller chart applies to the isolated propeller,

*Frequently included in the specified engine perfọrmance the efficiency is determined for an effective advance ratio, which is given by:

$J_{\text {eff }}=(1-h) \mathrm{J}$

The factor $h$ takes account of the retardation of the airflow through the propeller disk, caused by the presence of the fuselage or nacelle behind it. Its numerical value is:

$h=.329 \frac{S_{c}}{D^{2}}$

2. A slipstream factor $F$ is defined, $F=\frac{\text { effective propeller thrust }}{\text { isolated propeller thrust }}=\frac{T_{\text {eff }}}{T_{\text {is }}} \quad(6-15)$ The effective propeller thrust is equal to the thrust of the isolated propeller reduced by the increment in profile drag of those parts that are exposed to the slipstream of the propeller. Provided that the airplane drag is defined for zero thrust, the propeller efficiency is equal to:

$n_{\text {eff }}=F n_{\text {is }}$

A usual approximation for $\mathrm{F}$ is:

$F=1-1.558 \frac{\sigma f_{s}}{D^{2}}$

where $f_{s}$ is the profile arag area $\sum\left(C_{D} S\right)$ of the aircraft parts immersed in the slipstream of one propeller. In the absence of better data, $C_{D}$ may be assumed equal to .004 , based on the wetted area.

A propeller coefficient which is sometimes used to choose the propeller diameter is the Speed/Power Coefficient:

$c_{S}=v \sqrt[5]{\frac{\rho}{\mathrm{Pn}^{2}}}$

or in British units:

$\mathrm{C}_{\mathrm{S}}=.638 \frac{\mathrm{mph} \sigma^{1 / 5}}{\mathrm{Bhp}^{1 / 5}(\mathrm{rpm})^{2 / 5}}$

This coefficient is independent of the propeller diameter and is entirely determined by the operational conditions: the flying speed, altitude, engine power and rpm. In some outdated propeller diagrams $\mathrm{C}_{\mathrm{S}}$ will be 
found as a parameter and these can be used directly for the choice of $\mathrm{J}$ and hence $\mathrm{D}$. Manipulating $(6-5),(6-8)$ and $(6-18)$, we find:

$c_{p}=\left(\frac{J}{c_{S}}\right)^{5}=\frac{p n^{2}}{\rho v^{5}} J^{5}$

and for given flight conditions we may draw the corresponding line of $\mathrm{C}_{\mathrm{S}}=$ constant in the propeller diagram as in Fig. $6-5 a$; in the example this has been done for $\mathrm{C}_{\mathrm{S}}=1$. From a comparison of this line with those for constant $n$ it may be concluded that in this case the maximum efficiency ( 77.5 percent) is obtained at $\mathrm{J}=.51, B=15^{\circ}$ and $\mathrm{C}_{\mathrm{p}}=.033$. The diameter which will give the maximum efficiency can be deduced from this condition. The usefulness of the coefficient $C_{S}$ is limited, since various operational conditions have to be considered when choosing a propeller. In such a case it will be desirable to have the propeller work under the best possible conditions, both in cruising flight and during takeoff and climb. A procedure which may be followed for choosing the propeller geometry is given in Section 6.3.4.

Propeller performance may be determined in various ways:

a. By using propeller diagrams supplied by the manufacturer, for example, Ref. 6-21. Propeller diagrams may also be found in various NACA Reports and handbooks. This method is generally satisfactory for a preliminary choice.

b. By calculation, using generalized methods, such as:

- the approximations given in Ref. 6-15

(light aircraft) or Ref. 6-17 (transport aircraft)

- the SBAC standard method (Ref. 6-16), which also allows for the installation of the propeller

- the method given in Ref. 6-26, which also makes it possible to calculate propeller noise characteristics.

An advantage of generalized methods is that systematic variations in blade shape may be accounted for, thus making opti- mization possible.

The way in which the propeller data - and more particularly the propeller diagrams should be used depends on the particular application and the blade angle control, as will be explained in the next section.

\subsubsection{Blade angle control}

The following distinctions are made with regard to propeller control:

a. Propellers with constant pitch during flight:

- fixed pitch propellers, with the pitch built in during manufacture

- adjustable propellers, with the blade angle adjustable on the ground but fixed in flight.

b. Propellers with variable blade angle during flight:

- adjustable propellers, for which the blade angle may, within given limits, be set at some pre-determined values, e.g. fine and coarse pitch

- controllable pitch propellers, for which the blade angle, within given limits, may be set by the pilot at any desired value - constant speed propellers, for which the rate of revolution is kept constant at a value set by the pilot. The blade angle is controlled automatically, so that there will be equilibrium between the power supplied by the engine and the power absorbed by the propeller

- constant speed propellers with blade angle control ( $\beta$-control), for which the blade angle may be set and controlled directly by the pilot, while the rate of revolution during flight remains constant. Engine power is governed by an automatic fuel control system.

Adjustable and controllable pitch propellers are hardly ever used nowadays. Fixed pitch propellers are still fitted to small aircraft used for flying instruction, touring and business flights. Controllable pitch propellers are in use on some powered sailplanes, the pilot being able to feather the blades in order to reduce the drag when the engine is switched off. Propeller- 

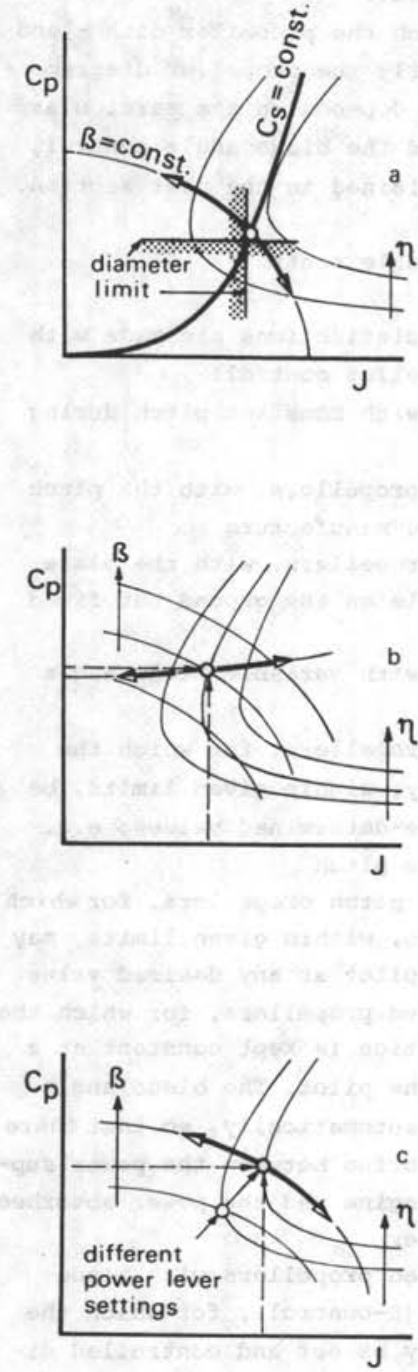

UNITS:

$V$ in $\mathrm{m} / \mathrm{s} \quad(1 \mathrm{~m} / \mathrm{s}=1.942 \mathrm{kts}) ; \mathrm{D}$ in $\mathrm{m}(1 \mathrm{~m}=3.28 \mathrm{ft})$

P in $\mathrm{kgm} / \mathrm{s}(1 \mathrm{kgm} / \mathrm{s}=.01316 \mathrm{hp}) ; \rho$ in $\mathrm{kg} \mathrm{sec} / \mathrm{m}^{4}$

$$
\text { (1 } \mathrm{kg} \mathrm{sec} / \mathrm{m}^{4}=.019 \mathrm{slug} / \mathrm{ft}^{3} \text { ) }
$$
ficiency. plane polar (horizontal flight). are calculated. peller power are matched.

Effect of flight speed and engine control on power and propeller ef-

1. For specified $v$ the power required is determined by using the air-

2. Assuming $n$, the engine power for equilibrium is found and $J$ and $c_{P}$

3. $n$ is read from diagram and if necessary the procedure is repeated. 4. For variable speed $\beta$ remains constant. The engine power is controlled automatically to keep $n$ constant until the engine and the pro-

Fig. 6-6. Applications of the propeller diagram

powered transport aircraft are always fitted with constant speed propellers; these are often equipped with blade angle control. Turbopropeller engines have to be fitted with constant speed propellers.

a. Fixed pitch propellers.

The advantage of fixed pitch propellers is 
obviously that they are simple to produce, hence cheap, while they are also light and require no maintenance. For these reasons they are used on small aircraft with engines of up to about $200 \mathrm{hp}$ and even more in the case of agricultural aircraft. An aircraft with a fixed pitch propeller, however, is very much at a disadvantage where flexibility in performance is concerned. During the takeoff and subsequent climb the rpm of the engine is limited by the power which the propeller is able to absorb, and consequently the power cannot be fully utilized. Decreasing the blade angle will improve the situation during takeoff and low-speed flight, but in highspeed flight the engine will be overspeeded and must be throttled back.

When a preliminary choice of the blade angle and diameter has been made, it may be assumed that the aircraft will fly at maximum cruising speed with the rpm and power values recommended by the engine manufacturer. The equations required to estimate the cruising speed are presented in Sections 5.3.4. and 5.4.1. If at this stage the drag polar is still unknown, the cruising speed may be estimated by comparison with similar aircraft or it can be deduced from the design specification. The Speed/ Power Coefficient $\mathrm{C}_{\mathrm{S}}$ can then be determined by $(6-18)$ and the efficiency will be dependent only on the diameter. When this is chosen as large as possible, the propeller blade angle will be small and at low speeds it will be possible to obtain a high rpm, and consequently high engine power. The propeller diameter is limited by the permissible Mach number at the tip $\left(M_{t i p}<.80\right.$ to .85), or by practical limits, such as the clearance from the ground. Fig. 6-6a illustrates the choice by means of a propeller diagram.

For a more definite propeller design, the aerodynamic characteristics of the aircraft should be known, but these will only be determined at an advanced stage. It will be necessary to carry out performance calculations under varying flight conditions in order to find the most favorable compromise. Once the angle of incidence of the blade has been chosen, the only remaining important factor in the propeller diagram will be the line related to the fixed propellex pitch angle $\beta$.

b. Constant-speed propellers.

As far back as the 19th century the French pioneer Alphonse Pénaud proposed a propeller with adjustable blades, but it was not until about 1925 that the constant-speed propeller proved to be a practical proposition and it came into service around 1935 . The performance of aircraft improved to such an extent that since that time the constant-speed propeller has come into general use. The pilot sets the rpm of the engine to the value desired and for each flight speed the propeller blade angle is automatically adjusted in such a way that the propeller is able to absorb the power of the engine. The main difference between this and the fixed pitch propeller is that the engine power available can be fully utilized at each airspeed. There are various methods for obtaining an automatic system of adjustment. For example, oil pressure can be used to twist the blade in one direction, while a counterweight at the hub of the blade permits movement in the opposite direction. Fig. 6-7a shows a simple system but many variants have been derived from this. The sketch in Fig. 6-7b gives an example of the mechanism of a pitch-control system. Electrical systems

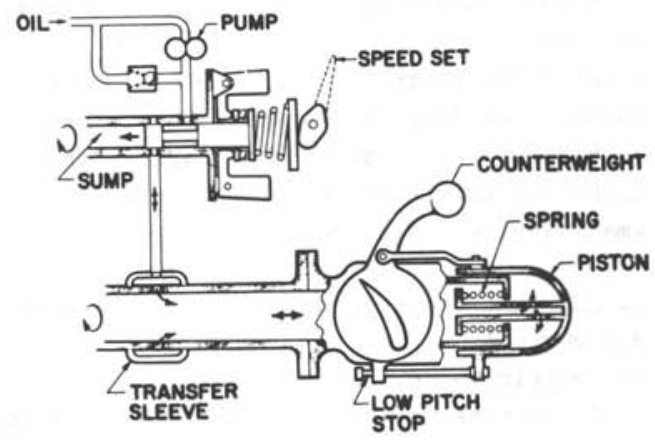

a. Non-reversing propeller and governor

Fig. 6-7. Mechanical principle of constantspeed propellers 


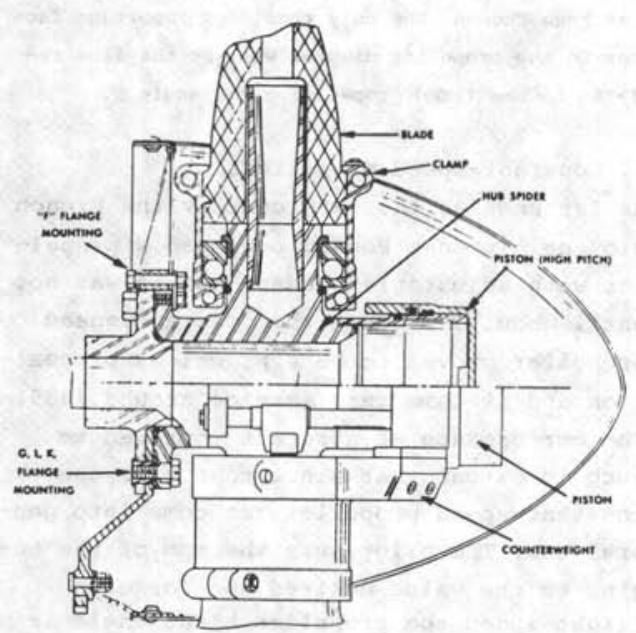

Hartzell Model HC.82X-1 Constant Speed

b. Blade angle control

Fig. 6-7 (concluded)

are used as well. Constant-speed propellers are often provided with:

- a feathering device, which allows the blade to take up an angle of attack of 80 to 90 degrees when the engine is inoperative, thus considerably reducing the drag of the propeller, while engine damage due to windmilling is avoided;

- a facility for turning the blade to a negative pitch angle, which is used to obtain reverse thrust and so reduce the landing run;

- automatic restriction of the blade angle which comes into action when the oil pressure drops, in order to safeguard against high revolutions of the propeller with small angles of attack.

c. Constant-speed propellers with blade angle control.

Two versions are known:

1. The $\beta$-control system, where the pilot is able to control the blade pitch angle both in flight and on the ground

2. A system in which control of the propeller blade is only possible during maneuvering on the ground.
When the $\beta$-control is in operation during the flight phase and the pilot wants to increase the engine power, the direct result of moving the power level will be an increase in the blade pitch. Since the propeller torque will then increase, the rpm will tend to drop. A "speed set" will now transmit a signal to the fuel control system and more fuel will be fed to the engine. Engine power will increase until the $\mathrm{rpm}$ again reaches the pre-set value.

Fig. 6-8 illustrates how this method of en-

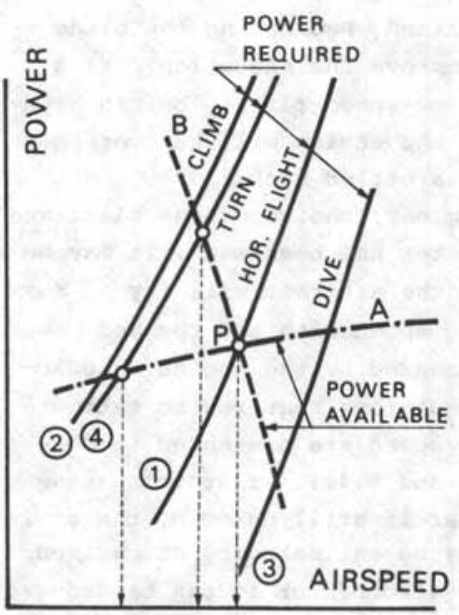

A: Power control mode

B: Blade angle control mode ( $B$ constant)

Fig. 6-8. Comparison of normal power control and $B$ control (Ref.: SAE Paper No. 670244)

gine control works in practice. It should be noted that it is only applicable to turboprop engines where the propeller rpm and power can be varied independently. Curve 1 shows the power required for steady horizontal flight. Curves 2,3 and 4 represent the total power required during stationary climb and descent and in a stationary turn. The speed in horizontal flight is determined by the equilibrium between the power available and the power required (point B). The difference between the lines of power available with the conventional 
method of adjustment (line A) and with Bcontrol (line B) results from the automatic adaptation of the power to the flying speed in the case of $\beta$-control. To take an example of the $B$-control system: when the flying speed in a stationary condition is reduced, the propeller blade angle of attack will increase for constant blade setting and so will the propeller-torque (equation 6-7). The engine control system will react to this change and will cause the power to increase to the extent that the rpm remains constant. On the other hand, when the speed is increased, engine power will automatically drop. Fig. 6-8 shows that with the use of $\beta$-control the loss in speed resulting from a transition to a climb or turn is small as compared with that which occurs with power control by means of a conventional constant speed propeller. Consequently, with a pre-set flight condition the margins relative to the stalling speed will be greater and the pilot will have to make fewer speed corrections. This will increase flying comfort and safety. In descents there will be a lower speed buildup, which reduces the danger of overloading the structure.

The $\beta$-control mode is only operational when the engine is running within the predetermined limits. When, during some phase of the flight, the permissible Turbine Entry Temperature is reached, another control system is activated and the engine-propeller combination will function on the lines of the conventional control system. The $\beta-$ control facility will therefore not affect the ultimate performance in conditions where the engine is operating at its $1 \mathrm{im}-$ its, e.g. during takeoff or maximum continuous operation.

The differences which exist between the conventional control and $\beta$-control system are illustrated once more in the propeller diagrams in Figs. $6-6 b$ and $6-6 c$.

\subsubsection{Propeller geometry}

Apart from the considerations which will follow, there may be certain overriding factors which govern the choice of a propeller, such as the low costs of spare parts for existing type of propeller, or the possibility of varying the gear ratio between the engine and the propeller, etc. For a final propeller design specification it will always be necessary to consult the propeller manufacturer.

a. Propeller diameter and tip speed. The diameter is undoubtedly the most important design parameter of the propeller and in a sense comparable to the span of the wing, albeit with definite limitations. It can be shown theoretically that propeller efficiency grows with increasing propeller diameter, provided it is possible to vary propeller rpm and blade shape freely (Ref. 6-14). In actual practice, however, the propeller speed is determined by the operating regime of the engine and the propeller reduction which cannot, as a rule, be chosen arbitrarily. The most important factors governing the choice of the propeller diameter are:

- the propeller performance (efficiency) under varying conditions,

- the permissible tip speed in connection with propeller noise and performance, - practical limits such as ground clearance and the clearance between the tips and the aircraft structure (Section 6.4.1.), and

- the weight of the propeller installation. The propeller tip speed is the resulting velocity of the propeller tip relative to the airflow. If the induced velocity is ignored, Fig. 6-4 shows that:

$v_{t i p}=\sqrt{v^{2}+(\pi n D)^{2}}$

Using $(6-5)$, this expression may be rewritten as:

$M_{\text {tip }}=M \sqrt{1+\left(\frac{\pi}{J}\right)^{2}}$

Although in the case of propellers with thin blades (thickness/chord ratios about 6 per cent) and small camber, the efficiency only shows an appreciable decline at 


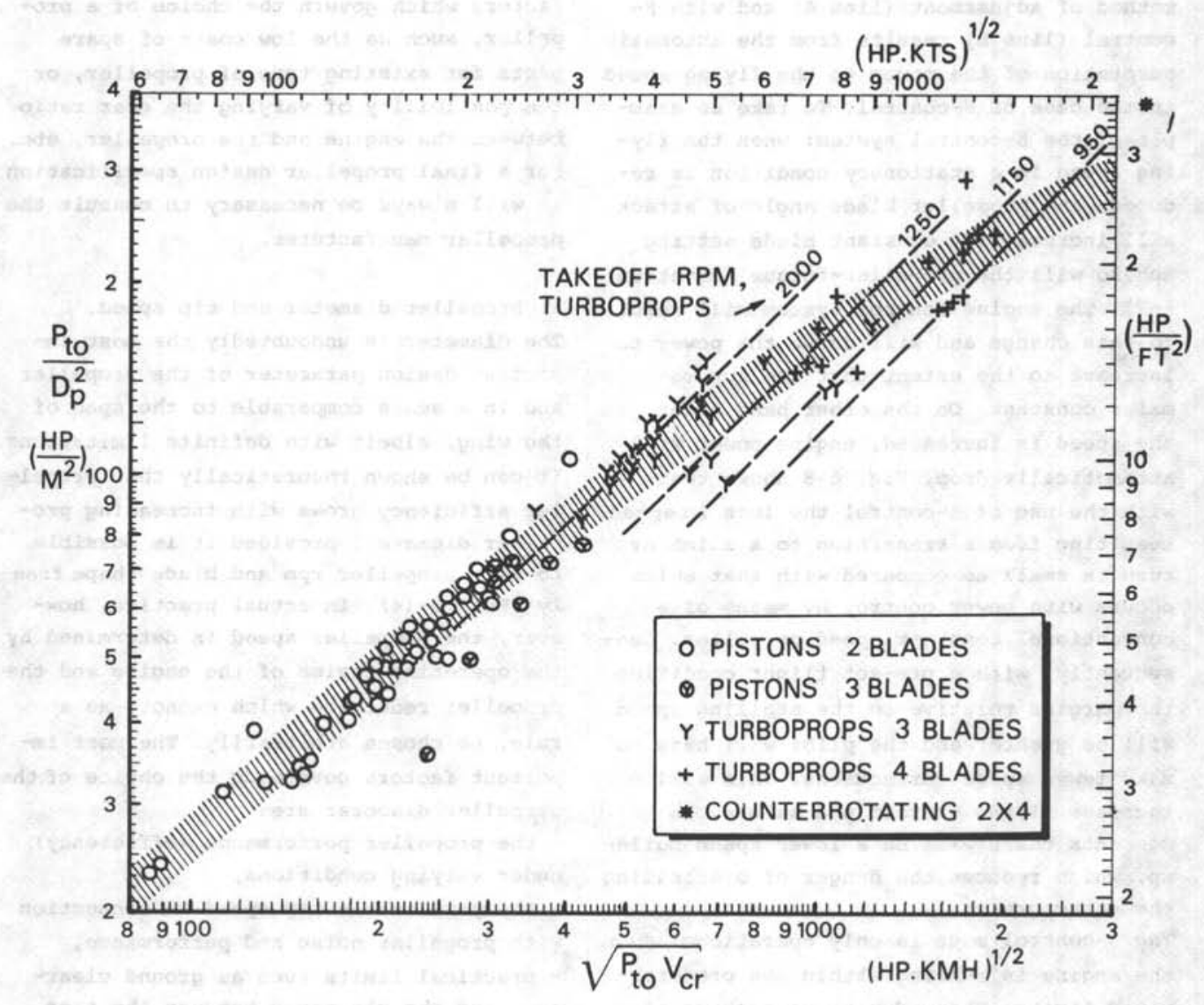

NOTE: $\mathrm{P}_{\text {to }}=$ takeoff $\mathrm{bhp}$ per engine

Fig. 6-9. Diagram to facilitate the choice of the disk loading

$M_{t i p}>.90$ to .92 , a practical limit of .85.9 .0 must be observed in order to keep the propeller noise down to an acceptable level.

b. Static tip speeds $\left(V_{t i p}=\pi n D\right)$ of some 800 to $1000 \mathrm{ft} / \mathrm{s}(250$ to $300 \mathrm{~m} / \mathrm{s})$ have long been regarded as normal values. Now that considerable attention is being paid to reducing the airplane noise level, tip speeds of 500 to $700 \mathrm{ft} / \mathrm{s}(150$ to $200 \mathrm{~m} / \mathrm{s})$ are being aimed at, while $800 \mathrm{ft} / \mathrm{s}(250 \mathrm{~m} / \mathrm{s})$ is regarded as the upper limit. Tip speed is the most important factor in the suppression of noise. A low tip speed, however, will only give acceptable performance when the diameter is sufficiently large. Accordingly, in the design of propeller aircraft for short runways, the accent lies on the development of slowly revolving propellers with a large diameter and special shape of blade (cf. References $6-24$ and 6-25).

c. A preliminary choice of propeller diameter may be made on the basis of one or more of the following methods: Method $A$ : the propeller disk loading $P / D^{2}$ at takeoff is used as a parameter. In Fig. 6-9 this quantity is plotted vs. the parameter $\sqrt{\mathrm{P}_{\text {to }} \mathrm{V}_{\mathrm{cr}}}$ for a large number of aircraft. This correlation is based on the ob- 
servation that during cruising flight $\mathrm{J} / \mathrm{C}_{\mathrm{P}}$ does not vary appreciably, and for given altitude and engine speed the disk loading should be proportional to the parameter $\sqrt{\text { PV. }}$

Method B: The diameter is deduced from a specified M tip' which is considered to be acceptable. For this purpose we can derive from $(6-21)$ :

$D=\frac{a}{\pi n} \sqrt{M_{t i p}^{2}-M^{2}}$

Assume $M_{\text {tip }} 6.80$ for low-speed propellers with relatively thick aerofoils with thickness/chord ratios of about 10 per cent, and $M_{\text {tip }} \leqslant .85$ to .90 for high-speed propellers with relatively thin blades (thickness/ chord ratios about 6 per cent). The speed of sound is taken at the appropriate design (cruising) altitude.

For low-speed aircraft with piston engines the rpm during takeoff will be considerably higher than in cruising flight, so the former will be the determining factor. In the case of high-speed propeller aircraft the cruising flight will generally be decisive.

Method C: The influence of the diameter on efficiency under various flight conditions is calculated on the basis of the propeller diagram for one or more blade shapes and number of blades per propeller, as appropriate. It will be necessary to estimate the flight speed of the aircraft in the flight phases chosen. Although these calculations are more involved than those used in the approximate methods mentioned above, a better picture of the performance trade-off is obtained.

Fig. 6-10 gives an example of a small commercial aircraft with two turboprop engines of $650 \mathrm{shp}$ each. The influence of the propeller diameter on efficiency was investigated and it was found that $D=7 \mathrm{ft} 7 \mathrm{in}$. $(2.33 \mathrm{~m})$ is the optimum value for the cruise and $D=8 \mathrm{ft} 5 \mathrm{in.}(2.57 \mathrm{~m})$ for the climb. Admittedly, the static thrust improves rapidly with increasing diameter, but at 88 knots $(45 \mathrm{~m} / \mathrm{s})$ there will be little gain with $D>8 \mathrm{ft}(2.44 \mathrm{~m})$. With
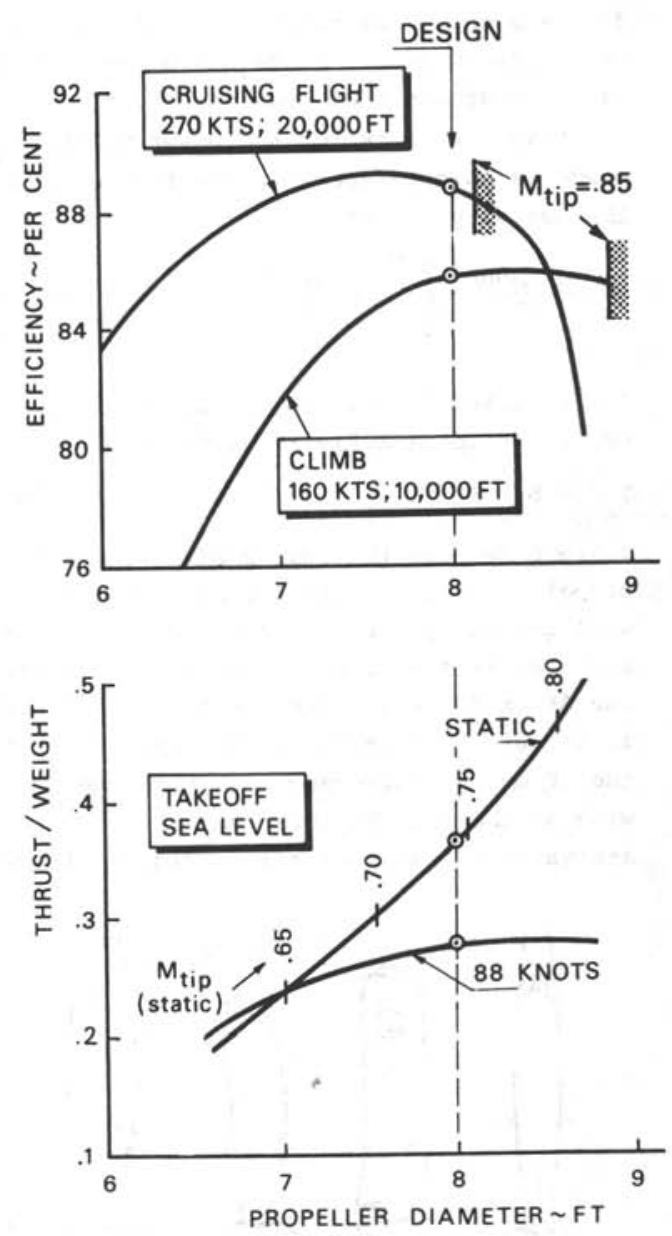

Fig. 6-10. Propeller diameter optimization ( 3 blades, $\mathrm{AF}=140, \mathrm{C}_{L_{i}}=.5$ )

this diameter the tip speed at takeoff will be $830 \mathrm{ft} / \mathrm{s}(254 \mathrm{~m} / \mathrm{s})$, which is considered an acceptable value for this type of aircraft. As indicated in the figure, D was chosen as $8 \mathrm{ft}$ in this case.

It should be noted that the slipstream correction, approximated by $(6-13)$ through $(6-17)$, is dependent on the diameter. The result of this will be that, from the performance point of view, the optimum diameter of the propeller as installed on the aircraft will be greater than for the isolated propeller. 
d. Blade shape and number of blades The planform of the blade may be expressed as a non-dimensional quantity which is a relative yardstick for the power which the blade is able to absorb. This is known as the Activity Factor:

$A F=\frac{100,000}{16} \int_{.15}^{1.0}\left(\frac{b}{D}\right)\left(\frac{r}{R}\right)^{3} d\left(\frac{r}{R}\right)$

The notation is defined in Fig. 6-4. The propeller Total Activity Factor is:

$\mathrm{TAF}=\mathrm{B} \times \mathrm{AF}$

where $B$ denotes the number of blades per propeller. For a propeller blade the AF will generally lie between 80 and 180 . For example, in the case of the Helio Courier, the AF is 90 , while for the Lockheed C-130 it is 162. From $(6-23)$ it is apparent that the AF may be increased by making the blade wide at the tip. In this connection there are various shapes of blade (Fig. 6-11) and

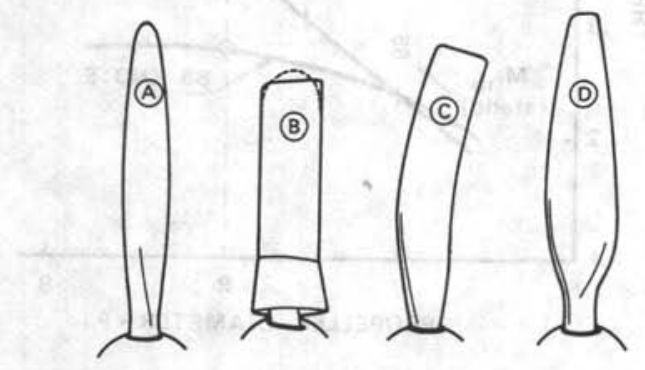

LOW SPEED M=.5-.7 TRANSONIC LOW NOISE

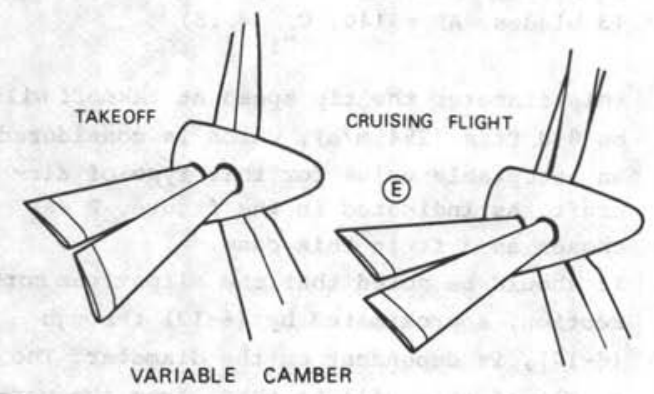

Fig. 6-11. Some propeller blade shapes

their application depends on the operational conditions and the engine power.
Type A is used on low-speed aircraft with engines in the lower power bracket. For an AF of up to about 90 a tapered blade form is chosen; a blunt tip is generally adopted for an AF between 90 and 115. The shank of the blade has a section which is nearly circular or elliptical, evolving to an aerofoil with a thickness/chord ratio of 8 to 12 per cent at the tip. The resulting Mach number at the tip is limited to about .80 and the flying speed to about $M=.4$. Type $B$ is a practical shape for speeds between $M=.4$ and $\cdot 6$. Examples of aircraft using this type are the Lockheed Electra and $\mathrm{C}-130$. For an $\mathrm{AF}$ of 115 to 140 almost prismatic blades with rectangular tips are used, while for AF values greater than 140 , blades are designed with inverted taper, the tip chord being larger than the root chord. As a result of the relatively large local chord the lift coefficient at the tip will be low and the critical flight Mach number high. This is also achieved by the use of a thin blade section (about 6 per cent) and the relatively low rpm. Streamline fairings (cuffs), which improve the inlet conditions of the turboprop engine particularly with reverse thrust, may be fitted at the shank of the blades.

Type $\mathrm{C}$ is an example of the types of blade evolved around 1950-1960, intended for high-subsonic flight speeds of up to $M=$ .95. The tip speeds are transonic and the blades are very thin: about 6 per cent at the root down to 2 per cent at the tip. Sections of this type are now of historical interest only, since the turbofan engine is a more efficient prime mover for speeds at the high-subsonic level.

Type $D$ is a special design for a quiet, slowly revolving propeller (Ref. 6-25). Here the chord is changed in such a way that in the static condition the region of the blade which is stalled is kept as small as possible.

Type E is a propeller with variable blade camber which has been the subject of research by Hamilton Standard* and may be of

*cf. Av.Week \& Space Techn.11/24/69,pp.56-65 


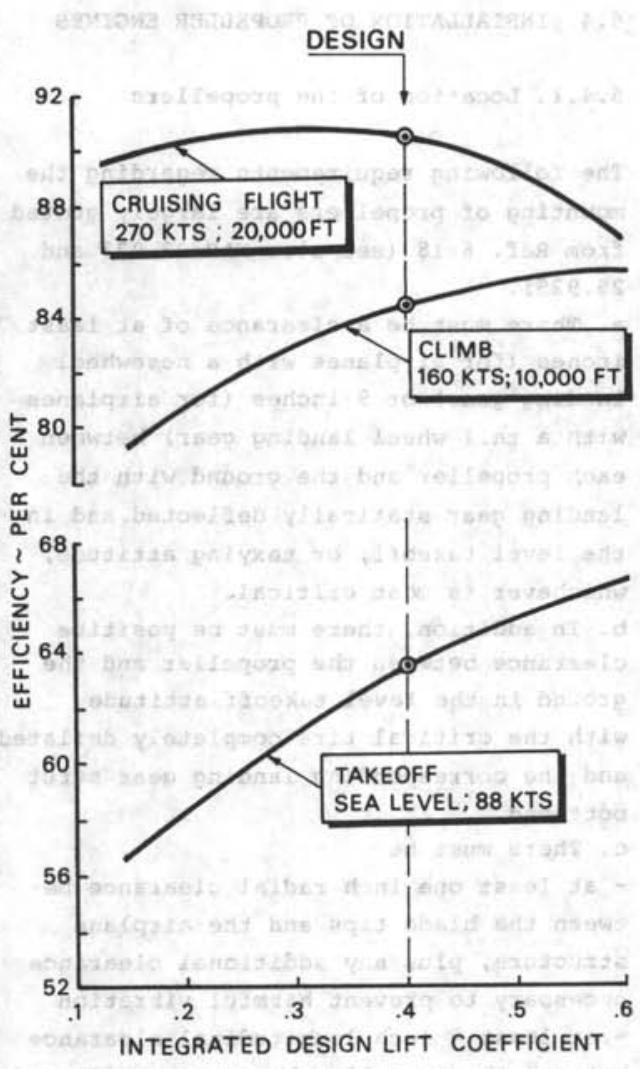

Fig. 6-12. Effect of $\mathrm{C}_{L_{1}}$ on propeller performance $(\mathrm{TAF}=350 ; \mathrm{B}=3 ; \mathrm{D}=8 \mathrm{ft}, 2.44$ m)

interest for propeller-powered V/STOL aircraft designs. The variable angle of incidence of the pairs of blades relative to each other is optimum both for the takeoff and cruising flight. The slot action between the blades may be compared to that of wing trailing edge flaps.

e. The camber of a blade element is represented by the design-lift coefficient $c_{l_{i}}$. This is the value of $c_{l}$ for the airfoil which gives the lowest profile drag. The integrated design-lift coefficient is used for the propeller blade. This is defined as follows:
$C_{L_{i}}=4 \int_{.15}^{1.0} C_{l_{i}}\left(\frac{r}{R}\right)^{3} d\left(\frac{r}{R}\right)$

For the notations, see Fig. 6-4.

The blade camber $C_{L_{i}}$ is generally chosen between .4 and $.6{ }^{{ }^{1}}$ For optimum cruise a fairly low value of $C_{L_{i}}$ has been found to be advantageous, but $a^{i}$ higher value of $C_{L_{i}}$ leads to higher thrust at low speeds.

Fig. 6-12 shows the effect of blade camber on etficiency for the airplane design mentioned previously.

Although the TAF and the $C_{L_{4}}$ do not entirely define the shape of the blade profile - thickness, taper and twist are also important - these parameters will suffice for analyzing the airplane performance at the preliminary design stage. Even when the aerodynamic characteristics of the aircraft are not yet accurately known, it is still possible to check the influence of the TAF and $C_{L_{1}}$ on the performance of the propeller for some of the flight conditions chosen or laid down in the specification. Such a comparison will only be of any significance when data on propellers with geometrically comparable blade shapes are available. Useful sources in this respect are mentioned in References $6-21$ and $6-26$. The propeller diagrams shown in Fig. $6-5 b$ and $c$ have been taken from Ref. 6-21. This publication presents diagrams for three $A F$ and four $C_{L_{i}}$ values for both three- and four-bladed propellers.

Fig. 6-13 shows how propeller efficiency is influenced by the number of blades and the blade AF in the case of the aircraft mentioned. As can be seen, the four-bladed propeller is at a slight advantage under all conditions so far as performance is concerned. If the TAF is chosen slightıy larger in the case of the three-bladed propeller, however, the disadvantage in the takeoff will be eliminated, while the propeller will be both simpler and lighter. The four-bladed version shows 1 to $1 \frac{1}{2}$ percent improvement in efficiency in cruising flight and climb. From Fig. 6-9 it will be observed that the transition from three to four blades occurs at about $1500 \mathrm{hp}$ engine power. A four-bladed propeller will also be 

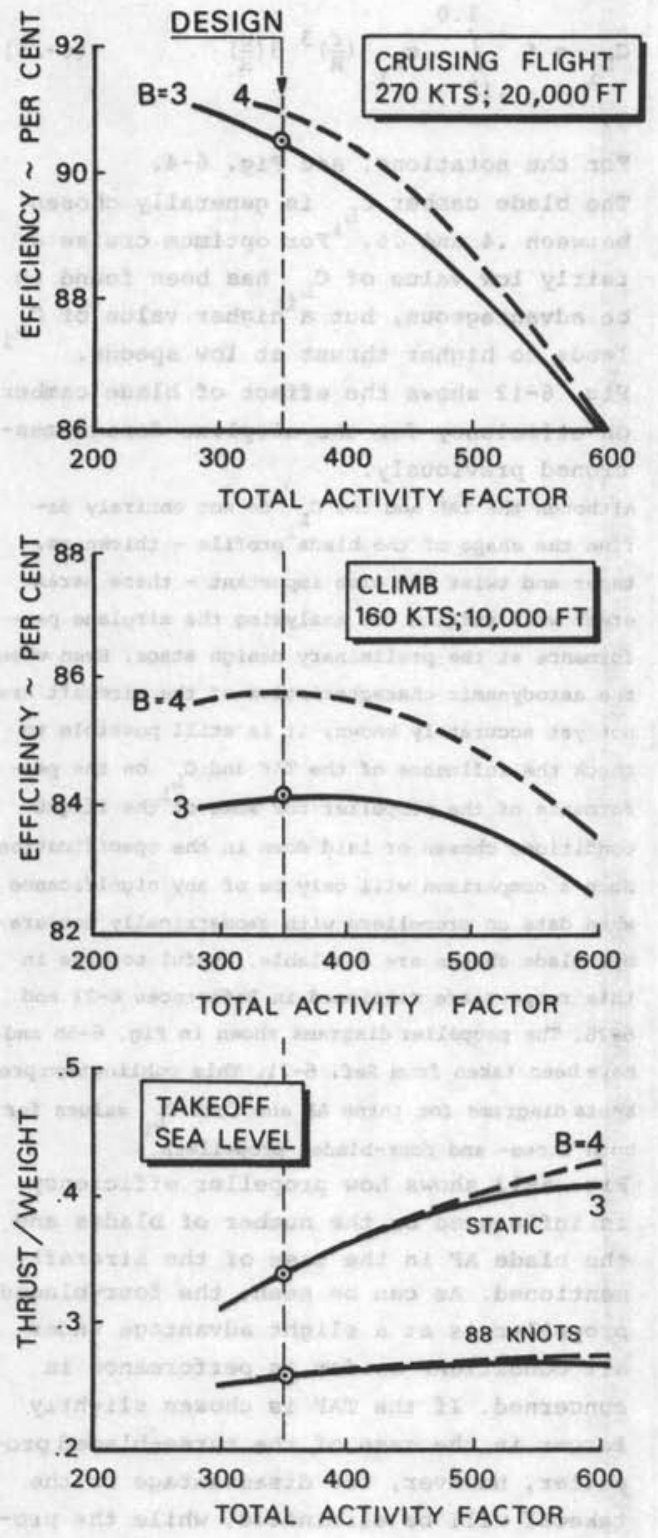

Fig. 6-13. Effects of $\mathrm{AF}$ and number of blades on propeller performance $\left(C_{L_{i}}=.4\right.$,
$D=8 \mathrm{ft}, 2.44 \mathrm{~m})$

chosen when the diameter is restricted for structural reasons, because in this case the efficiency of the three-bladed propeller would be too low.

\subsection{INSTALLATION OF PROPELLER ENGINES}

\subsubsection{Location of the propellers}

The following requirements regarding the mounting of propellers are largely quoted from Ref. 6-18 (see also FAR 23.925 and 25.925).

a. There must be a clearance of at least 7 inches (for airplanes with a nosewheel landing gear) or 9 inches (for airplanes with a tail wheel landing gear) between each propeller and the ground with the landing gear statically deflected and in the level takeoff, or taxying attitude, whichever is most critical.

b. In addition, there must be positive clearance between the propeller and the ground in the level takeoff attitude with the critical tire completely deflated and the corresponding landing gear strut bottomed.

c. There must be

- at least one inch radial clearance between the blade tips and the airplane structure, plus any additional clearance necessary to prevent harmful vibration - at least $\frac{1}{2}$ inch longitudinal clearance between the propeller blades or cuffs and stationary parts of the airplane - positive clearance between other rotating parts of the propeller or spinner and stationary parts of the airplane.

The values stated above are absolute minima; in order to limit cabin noise level it is advisable to provide a propeller tip / fuselage clearance of at least 4 inches $(10 \mathrm{~cm})$ plus .65 inch $(1.65 \mathrm{~cm})$ per $100 \mathrm{hp}$ of one engine. It should also be kept in mind that in the case of engine power growth the optimum diameter of the propeller is likely to increase.

d. In the case of seaplanes and flying boats there must be a clearance of at least 18 inches $(46 \mathrm{~cm})$ between each propeller and the water, but it is recommended that this clearance should be at least 40 per cent of the propeller diameter.

e. When two propeller disks are adjacent to each other, the relative distance between the two, as seen in front view, should be at least 9 inches $(23 \mathrm{~cm})$. Over- 
lapping of propeller disks is discouraged, although this will probably not apply to STOL aircraft with deflected slipstream.

f. Another important ruling (FAR 23.771 and 25:771) stipulates that the primary controls, excluding cables and control rods, must be located with respect to the propellers, so that no member of the minimum flight crew, or part of the flight deck controls, lies in the region between the plane of rotation of any inboard propeller and the area generated by lines passing through the center of the propeller hub making an angle of 5 degrees forward and aft of the plane of rotation of the propeller.

This latter requirement is depicted in Fig. 6-14 and is in practice more decisive

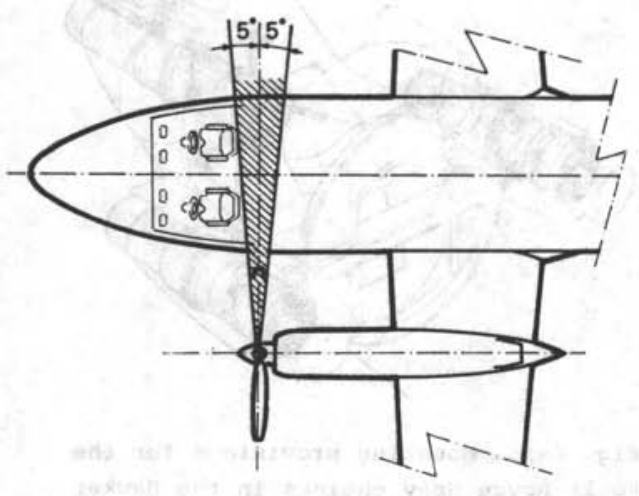

Fig. 6-14. Region in which flight crew members, flying controls and instruments may not be located

for the propeller location than for the layout of the flight crew compartment. It is also advisable to avoid siting passenger seats in this region, although this will not always be possible. In addition, it is preferable to locate the propellers in such a way that cargo holds, tollets and suchlike will not be in the plane of the propellers. It will be obvious that they should not be placed too close to cabin doors. The fuselage will have to be reinforced locally in view of the possibility that lumps of ice may be thrown from the propellers.

\subsubsection{Tractor engines in the nose of the} fuselage

In the vertical direction the position of the engine will be dictated by the downward view of the pilot (Section 3.4.1.), as well as by the required clearance between the propeller blade tips and the ground. It may sometimes be desirable to adapt the length of the nosewheel strut in order to give the aircraft a slight taildown attitude.

When piston engines are used there should be a proper supply and exit of cooling air to and from the engine and oil cooler, as well as an adequate supply of engine air to the carburetor.

The exhaust gases of turboprop engines should not be blown against any part of the aircraft, nor penetrate into the cockpit or cabin compartments. Tilting down the engine thrust line may have a favorable effect on the longitudinal stability (Fig. 6-15), but this should be limited to a few degrees in order to avold excessive variation in the angle of attack between the airflow and the propeller axis.

\subsubsection{Wing-mounted tractor engines}

Examples of some engine arrangements are shown in Fig. 2-10.

High-wing monoplanes will allow a certain measure of freedom with regard to the vertical location of the engine before a limit is imposed by the ground clearance of the propeller. If the landing gear has to be retracted into the engine nacelle this will largely determine the position of the en-

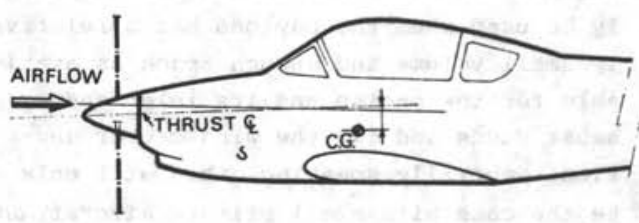

Fig. 6-15. Propeller thrust line tilt-down for improved power-on longitudinal stability 
gine and its jet-exhaust pipe. Where this is not the case, it is preferable to place the thrustline at about the level of the wing-chord or a little below it. The exhaust gases must be directed in such a way that they do not impinge on the wing flaps in the extended position.

Low-wing monoplanes will often present a problem when it comes to obtaining sufficient ground clearance for the propeller. The engine nacelle, on the other hand, should not be placed too high on the wing since that would create excessive drag. An acceptable compromise may be created by placing the thrustline in such a position that its extension would be at a tangent to the top of the local aerofoil. In the case of turboprop engines the exhaust gases will be directed over the wing through extended exhaust pipes.

When the direction of the airflow is not perpendicular to the propeller disks the blades will be subject to alternating loads with a period equal to the time of revolution of the propellers (1P). This periodic loading may be reduced by a correct choice of the angle of incidence of the propeller axis in relation to the wing. It will be advisable to contact the propeller manufacturer at an early stage.

\subsection{INSTALLATION OF TURBOJET ENGINES}

\subsubsection{General requirements}

A number of aspects relating to the location of the engines has already been discussed in Section 2.3.

Engines buried within the fuselage can on$1 y$ be used when the payload has a relative1y small volume and enough space is available for the engine and its inlet and exhaust ducts and for the wing center section. Generally speaking, this will only be the case with small private aircraft and trainers, as far as civil aviation is concerned. The relatively large diameter of modern high bypass engines virtually prohibits the installation of engines buried in the wing roots, as in the Hawker Siddeley Comet and Tupolev 104. These engines are generally installed in pods, which are provided with detachable or hinged panels for ease of maintenance. The engines and their pods are connected to the airframe by means of pylons. The pylons of fuselage-mounted engine pods are more heavily loaded as a consequence of bending and - unlike wing-mounted podded installations - contain heavy forgings (Fig. 6-16).

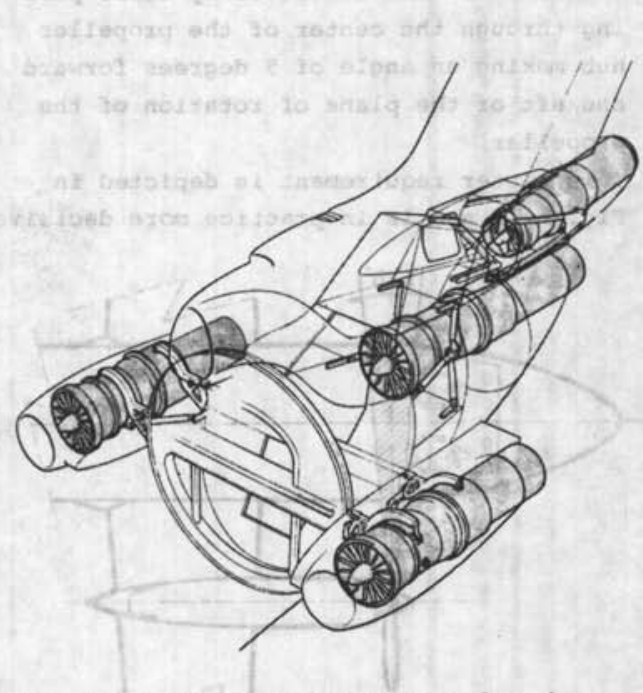

Fig. 6-16. Mounting provisions for the Rolls-Royce Spey engines in the Hawker Siddeley Trident $3 \mathrm{E}$ rear fuselage (Ref. Aircraft Englneering, April 1969)

in order to ensure favorable working conditions for the engine the air should reach the compressor with a minimum of pressure losses and fluctuations. The velocity of the incoming air will be about $M=.4$ to .5 in the plane of the compressor and it follows that at high-subsonic speeds the inlet air should be decelerated in a diffusor. When the shape of the inlet is optimized for this condition, there will be a possibility that during takeoff the air may separate inside the inlet duct, resulting in inlet pressure fluctuations and thrust loss. Possible solutions are a relatively blunt and long inlet lip or the 


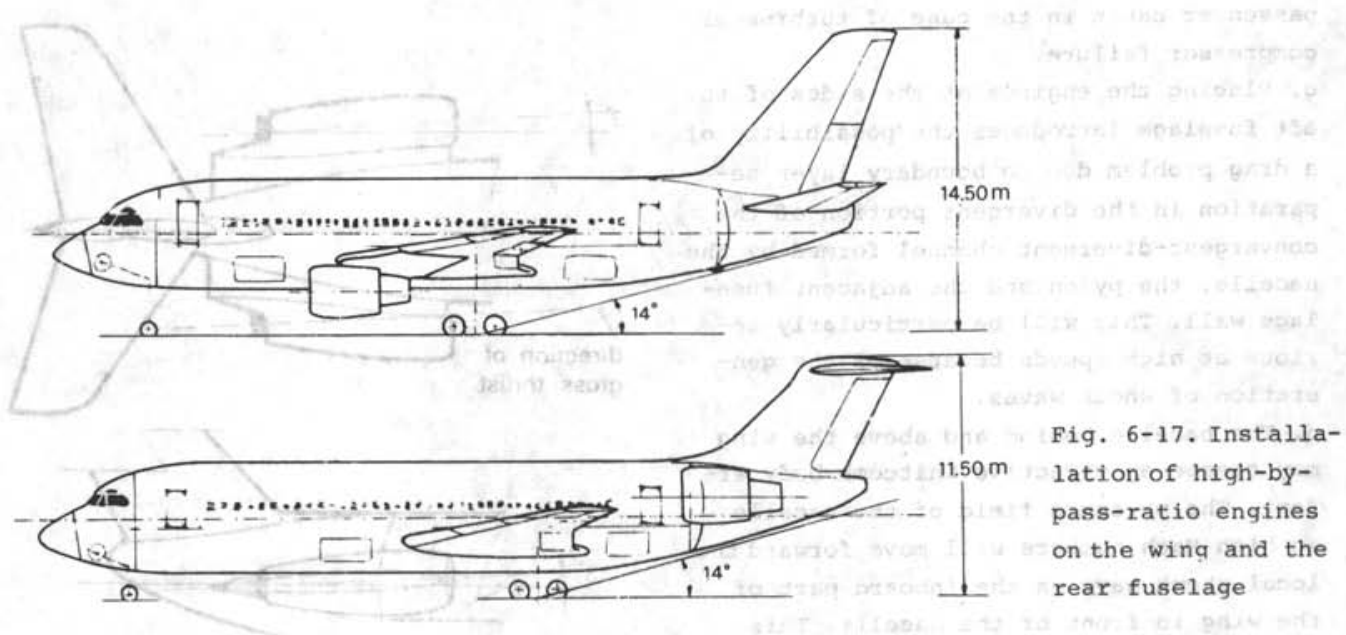

provision of auxiliary inlet doors which admit air only at low speeds.

The absence of large propellers, the relatively good accessibility and the compactness of podded jet engines allow the designer some measure of freedom in locating the nacelles in a favorable position, the bypass ratio being an important parameter here. Fig. 6-17 shows two desian sketches of a short-range airliner powered by two engines with a bypass ratio of 6.5 . The figure shows that when the engines are mounted below the wing this will lead to a fairly high undercarriage and a large vertical tailplane. In this case it would be preferable to place the encines at the tail of the fuselaqe, provided this configuration did not result in an unacceptably large c.g. travel (cf. Section 8.5.3.). An alternative solution was souqht on the Fokker-VFw 614 short-haul aircraft, where the engines are located above the wing. Some problems associated with this layout are discussed in Ref. 6-41.

With respect to the location of the engine inlet we quote the following requirement from FAR 25.1091:

"The airplane must be designed to prevent water or slush on the runway, taxiway, or other airport operating surfaces from being directed into the engine air inlet ducts in hazardous quantities, and the air inlet ducts must be located or protected so as to minimize the ingestion of foreign matter during takeoff, landing, and taxying".

\subsubsection{Fuselage-mounted podded engines}

The following points will have to be considered when choosing the location of the engines:

a. A good transfer of the loads acting on the engine - approximately 10 times the installed weight, forwards as well as downwards - necessitates the adoption of the type of construction shown in Fig. 6-16. Another solution would be to use an almost solid bulkhead at the location of the fittings which pick up the outriggers carrying the engine. An opening the size of a door might still be acceptable. Such a frame would generally serve as the rearmost limit of the passenger cabin, thereby fixing the maximum forward location of the engines.

b. When there is only a thin-walled cabin structure between the engine and the cabin interior, it will be undesirable to have passengers seated in the plane of either the turbines or the compressor, although this may be acceptable in the case of engine with a very high bypass ratio, provided there are sufficient safeguards against any engine parts penetrating the 
passenger cabin in the case of turbine or compressor failure.

c. Placing the engines at the sides of the aft fuselage introduces the possibility of a drag problem due to boundary layer separation in the divergent portion of the convergent-divergent channel formed by the nacelle, the pylon and the adjacent fuselage wall. This will be particularly serious at high speeds because of the generation of shock waves.

d. The nacelle behind and above the wing may create an effective Whitcomb-body effect. The pressure field of the nacelle at high Mach numbers will move forward the local shock wave on the inboard part of the wing in front of the nacelle. This will postpone the breakaway of the wing boundary layer induced by the shock waves. For an optimized design this effect may increase the drag-critical Mach number of the aircraft (Ref. 6-32).

e. Since the nacelles are located behind the aerodynamic center of the wing, they will cause the aerodynamic center to move backwards. This stabilizing effect is partly compensated by an increment in the downwash at the horizontal tailplane due to the low effective aspect ratio of the nacelle/pylon combination.

f. At large angles of attack, particularly when the airflow over the wing has separated, the wake created by the nacelles and the pylons may greatly reduce the effectiveness of the horizontal tailplane. For this reason the location of the engines will be an important factor in connection with the deep stall problem (cf. Section 2.4.2.).

The aerodynamic design group's task is to take maximum advantage of the favorable effects and suppress the unfavorable aspects as far as possible. Fig. 6-18 shows that this may result in a complexly shaped nacelle. In this example, the nacelle forms an angle of 3 degrees with the fuselage datum line, while in plan view the shape has been adapted to the local contour of the fuselage. If just any nacellẹ is taken
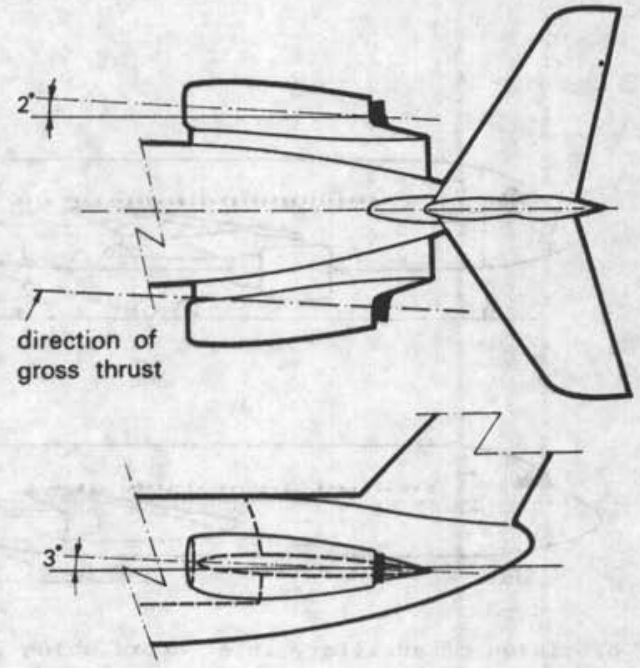

Fig. 6-18. Example of the shape of engine nacelles mounted to the rear sides of the fuselage

at random, the interference arag may amount to 40-50 per cent of that of the nacelle plus pylon, but this value can be reduced to $10-20$ per cent by appropriate aerodynamic development. As to the distance between the centerline of the nacelle and the local fuselage contour, something like 75 to 80 percent of the maximum diameter of the nacelle will be an adequate value. In the example given the jet pipe is toed slightly outwards in order to reduce the moment arm of the thrust about the center of gravity. This reduces the yawing moment created in the event of engine failure.

For speeds in excess of $M=.90$, it will be necessary to adapt the shape of the fuselage in accordance with Whitcomb's "area rule" (example in Ref. 6-39). In the case of aircraft with a long fuselage and a relatively small nacelle diameter, the requirement that the fuselage bcundary layer should not enter the duct is decisive in determining the location of the inlet. At large angles of attack or yaw the wake of the fuselage, wing or other parts of the aircraft may enter the inlet 
duct. The designer should be aware that even in this case the fluctuations in inlet pressure must not exceed the limit laid down by the engine manufacturer.

\subsubsection{Wing-mounted podded engines}

The following considerations will influence the location of the engines:

a. The reduction in the bending moment at the wing root increases proportionally as the engines are placed further outwards until the taxi loads become predominant and the trend is reversed. In addition, when the spanwise coordinate exceeds a certain value, the area of the vertical tail surfaces must be increased in order to compensate for the adverse yawing moment in the case of engine failure.

b. With swept-back wings it is possible to effect corrections in the location of the c.g. by changing the position of the engines in a spanwise direction, since for given chordwise location that will also entail a shift in the longitudinal direction.

c. The shape of the flow channel between the nacelle and the wing will be the decisive factor as far as the interference drag between the two is concerned. Fig. 6-19 shows that when an unfavorable position is chosen, the drag penalty may be considerable. As a general rule, the front and back of the nacelle should not coincide with or lie near the leading and trailing edge of the wing. Incidentally, by designing a suitable shape for the nacelle and the pylon the result shown in Fig. 6-19 can be considerably improved. d. The hot jet blast is generally not allowed to impinge on any part of the aircraft. This necessitates a local interruption of the flaps, resulting in a typical $C_{L}-\max$ penalty of about .07 relative to the uninterrupted flap. However, the efflux of high bypass engines is fairly cool and may not be harmful to the structure.

e. When the horizontal tail surfaces are mounted to the fuselage, the jet efflux

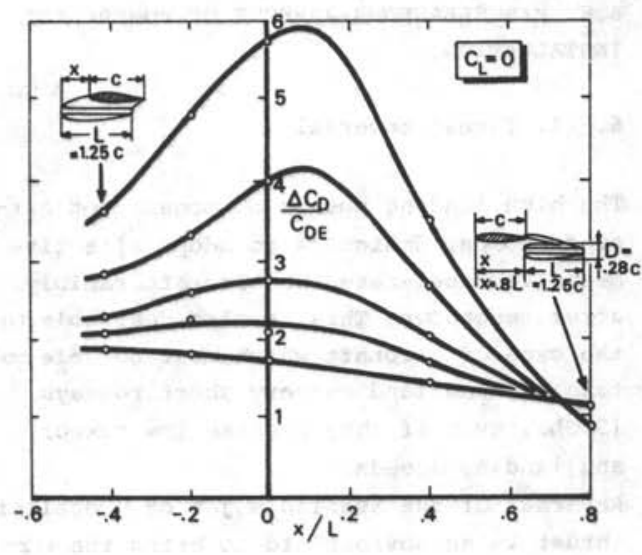

$C_{D E}=$ external drag of isolated nacelle

$\Delta C_{D}=$ installed drag of nacelle, including interference

wing sweep angle $=27^{\circ}$

inlet velocity $=50$ of airspeed

Fig. 6-19. Location effects on interference drag of a wing-mounted nacelle (Ref. 6-28)

will be close to the stabilizer at large angles of attack. The pumping effect of the jet will induce a downwash which will decrease the effectiveness of the stabilizer. This effect can be reduced by locating the engine or engines further outboard.

Reference 6-35 contains a survey of the results obtained by Lockheed during investigation of the most favorable location of the engine on the wing, using various configurations. Fig. $6-20$ shows a location which may be regarded as one of the most suitable solutions, although it should be noted that this may not apply when a different type of engine is chosen. The structural arrangement of a typical podded installation of a high bypass engine is depicted in Fig. 6-21. A comprehensive discussion on engine installation considerations and the aerodynamic development of configurations with rear fuselage- and wing-mounted engines can be found in Refs. $6-32$ and $6-33$. 
6.6. MISCELLANEOUS ASPECTS OF POWERPLANT INSTALLATION

\subsubsection{Thrust reversal}

The high landing speeds of modern jet aircraft compel designers to adopt effective means to decelerate the aircraft rapidly after touchdown. This is also desirable in the case of aircraft which must be able to take off and land on very short runways (STOL), even if they possess low takeoff and landing speeds.

Reversal of the avallable jet or propeller thrust is an obvious aid to bring the aircraft to a stop. The main advantages are: a. When the aircraft is landed on wet or snow-covered runways it is only possibie to obtain a considerably reduced deceleration by the use of wheelbrakes and aerodynamic drag. Fig. $6-22$ shows the ratio of the landing run length with thrust reversers to the distance required in the case that no reversing is used. As can be seen, the use of thrust reversers leads to a considerable reduction in the landing run. In such cases they contribute to the safety of operation*

b. Thrust reversers are also used during day-to-day landings on dry runways although, as shown in Fig. 6-22, the reduction in the landing run is less pronounced. One advantage is claimed to be that there will be less wear on the wheelbrakes as well as on the tires.

c. On some types of aircraft it is possible to operate the thrust reversers in flight as well as on the yround, thus providing the pilot with an effective means for controlling the glide-path.

Two types of thrust reversers for jet engines are shown in Fig. 6-24. In the case of engines with a high bypass ratio and separate hot. and cold nozzles, weight can be saved by merely reversing the fan stream.

* The use of thrust reversers is generally not allowed in defining the certificated field performance, cf. Section 11.7.
The thrust of the hot gases will thus be eliminated, which makes for a simple system (Fig. 6-25).

Fig. 6-23 shows the maximum percentage of engine thrust which may be reversed when using different systems. A typical value would be 45 per cent. Thrust reversers are designed by the engine manufacturer or by specialist firms. The following points are of importance to the airplane designer: a. The weight of the reverser system installation is considerable and increases with the bypass ratio. It is generally of the order of 15 to 20 per cent of the bare engine weight.

b. There should be the necessary space to fit the system and this will often entail a lengthening of the nacelle.

c. The loss in thrust and increase in fuel consumption resulting from the use of the system may amount to 1 or 2 per cent in cruising flight.

d. Precautions should be taken against overheating and excitation of parts of the aircraft by the diverted hot gases.

e. At low speeds the gases which are diverted in a forward direction may enter the inlet duct, resulting in overheating of the engine. The likelihood that this will take place increases with the effectiveness of the reverser system. When four engines are mounted on the wings there is also a possibility that the gases of one engine will enter the inlet duct of the adjacent engine. For this reason, thrust reversers are only activated above a certain critical speed of about 40 to 70 knots (cancellation or cut-off speed). The cancellation speed will generally be lower for engines at the sides of the rear fuselage.

With the very high bypass ratios which may be used in the future, the conventional thrust reverser system will be inadequate and too heavy. Several firms have designed fans with variable blade adjustment (Fig. 6-26). Apart from other advantages (cf. Section 4.4.3.a.), it is possible to reverse about 60 per cent of the thrust by turning the rotor blades to a negative 


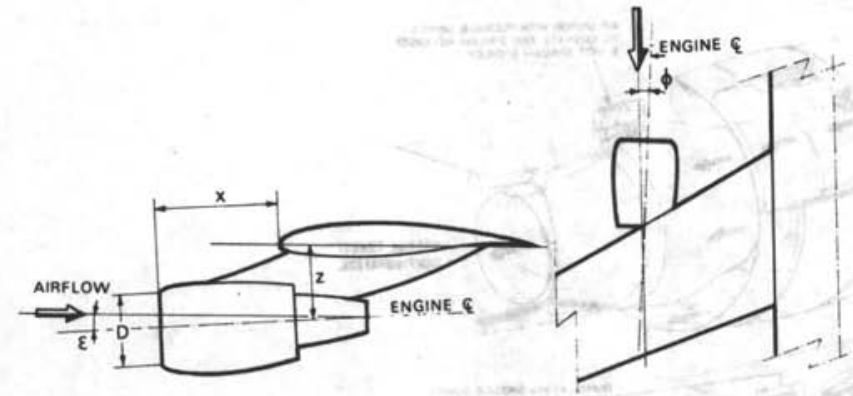

OPTIMUM FOR THE LOCKHEED 1011, ACCORDING TO SAE PAPER NO. 680688 : $\varepsilon=4^{\circ}, \phi=2^{\circ}, x=1,85 \mathrm{D}, z=.95 \mathrm{D}$
Fig. 6-20. Typical installation of a wing-mounted, highbypass-ratio engine pod

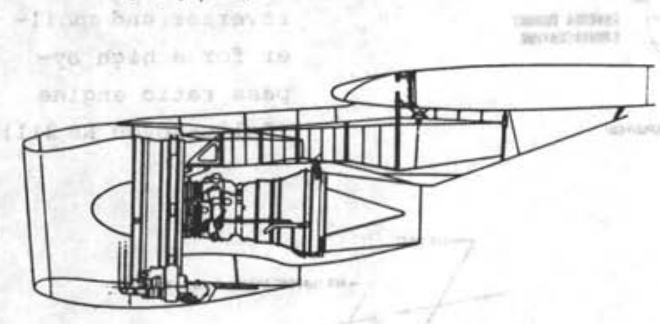

Fig. 6-21. General Electric CF-6 engine installation on the McDonnell Douglas DC-10 wing

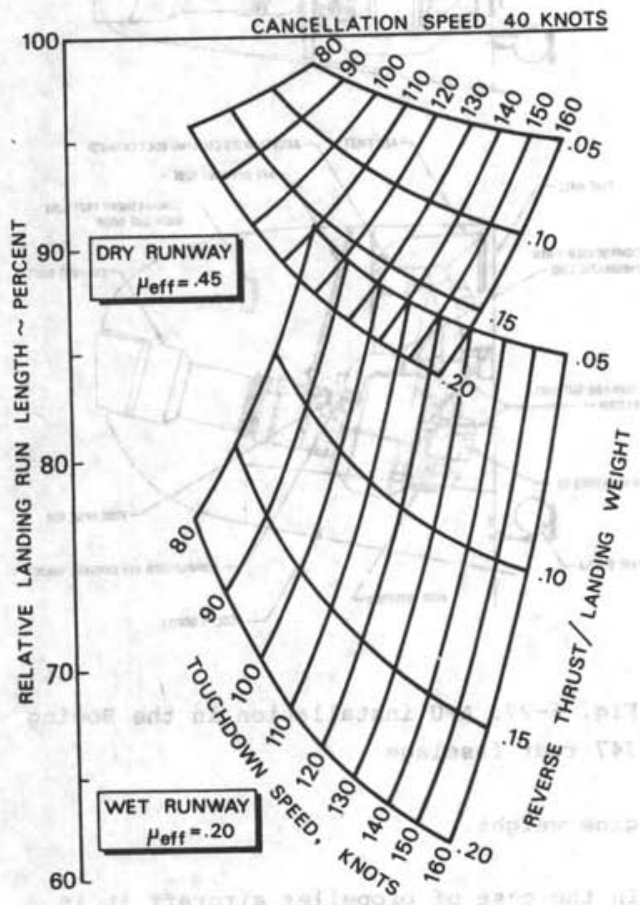

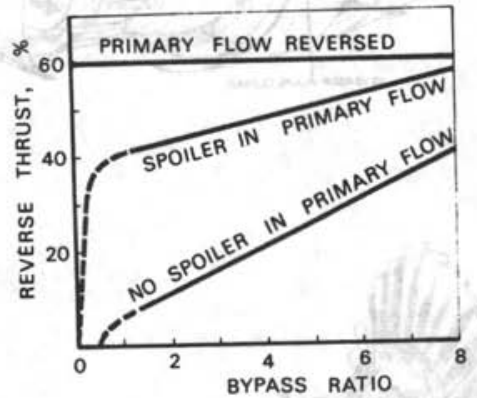

Fan reverser effectiveness: 608

Fig. 6-23. Reverse thrust of bypass engines (Ref. 6-45)

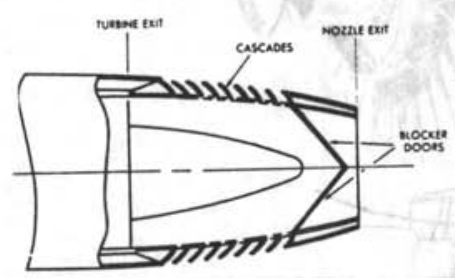

A. Cascade Type Thrust Reverser

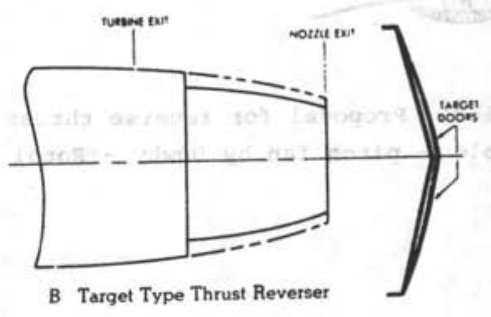

Fig. 6-24. Thrust reverser configurations for single-flow jet engines (Ref. 6-43)

Fig. 6-22. Reduction of the landing run due to thrust reverser action 


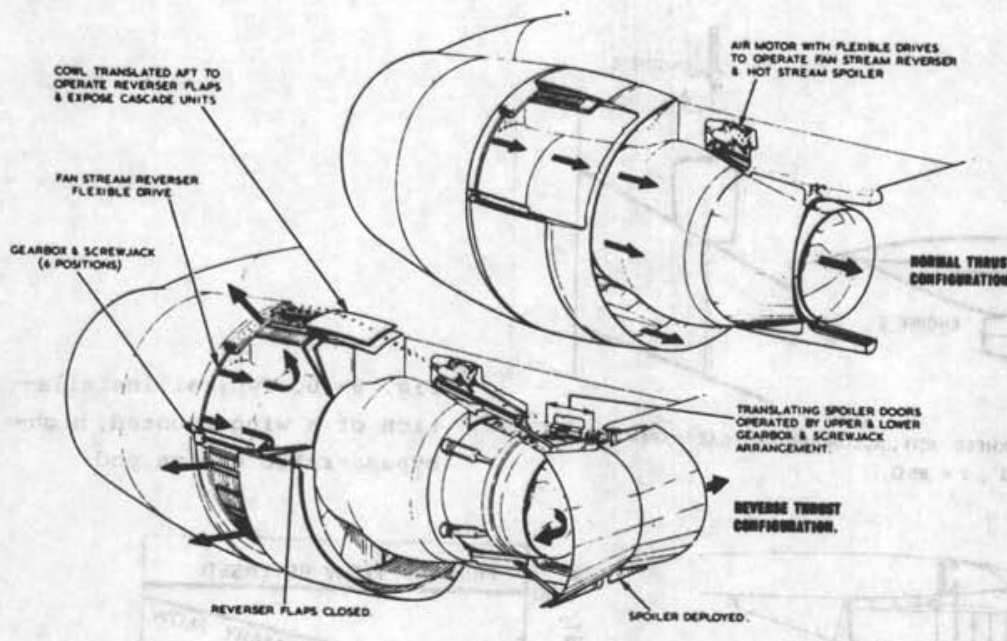

Fig. 6-25. Thrust reverser and spoiler for a high bypass ratio engine (Rolls-Royce RB 211)

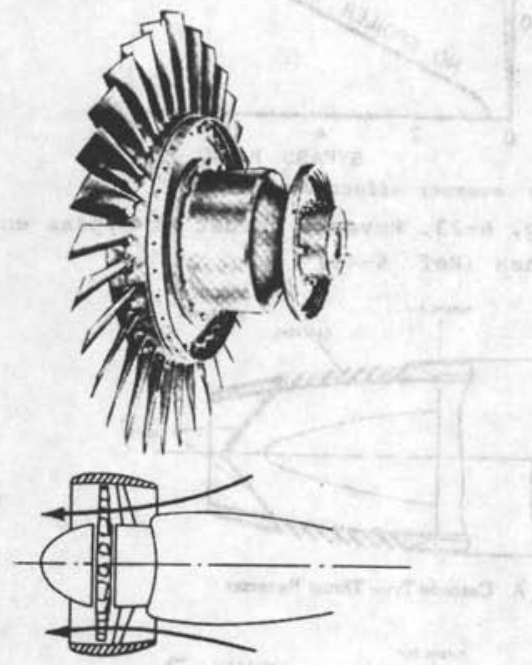

Fig. 6-26. Proposal for reverse thrust on a variable - pitch fan by Dowty - Rotol

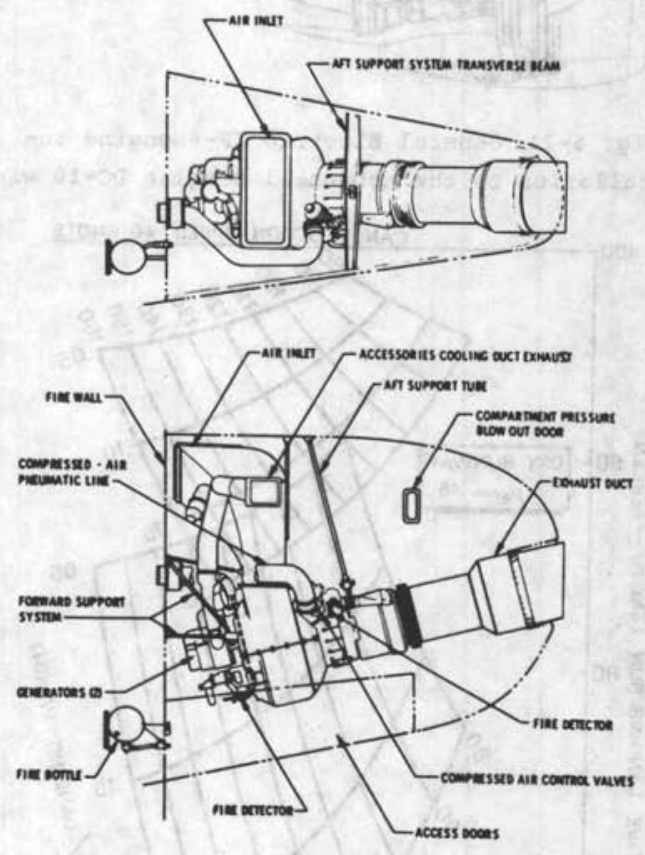

Fig. 6-27. APU installation in the Boeing 747 rear fuselage

gine weight.

it will also be possible to reverse the thrust at very low speeds. This system accounts for only 8 per cent additional en-

In the case of propeller aircraft it is possible to obtain negative thrust by 
changing the angle of pitch of the propeller blades from positive to negative. This requires a hydromechanical system which is operated by means of the throttle and ensures that the fuel supply is adapted to the blade angle. Negative thrust may also be used for reverse travel on the ground. The braking thrust of propellers (on piston engines) can be calculated by using the data given in Ref. 6-47.

\subsubsection{Auxiliary Power Units (APU)}

Almost every new transport aircraft is equipped with a relatively small gas turbine, which supplements the main engines for the following purposes.

a. Supplying pressurized air and power for operating the air conditioning system while the aircraft is on the ground.

b. Supplying power for the electrical system.

c. Starting the main engines.

d. Supplying for maintenance work outside the hangar.

The APU may, in principle, also be used for the pressurization and air conditioning systems during the takeoff, or as an emergency power supply for the electrical system during flight. This requires separate certification.

The principle advantage of the APU is that it makes the aircraft independent of power supplies at airports and so increases its flexibility. Since no auxiliary ground equipment is required, the aircraft is more easily accessible for embarking and disembarking passengers, as well as for servicing purposes, and comfort during stops is also improved. The APU will inevitably increase the empty weight, complexity and initial price, but in spite of this even small business jets are also optionally equipped with an APU.

Up to a certain point the choice of the APU is subject to the same factors as those which apply to the main engines. An assessment can be made from the few standard types which are offered, but in many cases the requirements are such that a new unit will have to be designed by a specialized (engine) manufacturer to fit the project. Table 6-3 presents a survey of the main

\begin{tabular}{|l|l|l|}
\hline AIRPLANE TYPE & BOEING 727 & BOEING 747 \\
\hline APU MODEL & GTCP 85-98 & GTCP 660-4 \\
MANUFACTURER & AiResearch & AiResearch \\
WEIGHT - DRY & $290 \mathrm{lb}$ & $543 \mathrm{lb}$ \\
INSTALLED & $(132 \mathrm{~kg})$ & $(246 \mathrm{~kg})$ \\
& $600 \mathrm{lb}$ & $1110 \mathrm{lb}$ \\
& $(272 \mathrm{~kg})$ & $(503 \mathrm{~kg})$ \\
POWER & $450 \mathrm{hp}$ & $2500 \mathrm{hp}$ \\
AIR FLOW CA- & $110 \mathrm{lb} / \mathrm{min}$ & $527 \mathrm{lb} / \mathrm{min}$ \\
PACITY & $(50 \mathrm{~kg} / \mathrm{min})$ & $(248 \mathrm{~kg} / \mathrm{min})$ \\
MTBUR* & $1955 \mathrm{~h}$ & $1980 \mathrm{~h}$ \\
\hline
\end{tabular}

* Mean Time Between Unscheduled Removals, time period Jan.-Sept. 1970

Table 6-3. Main characteristics of two APU installations (Ref. 6-54)

characteristics of two units. From this we may deduce that the installed weight of the APU is fairly high. This is mainly due to the sound-proofing required and to the inlet and outlet ducts. Although the weight only amounts to roughly .5 per cent of the empty weight, its effect on the location of the center of gravity should not be underestimated, because the tail of the fuselage is generally the best location for the APU for the following reasons:

a. As a rule, there will be sufficient space in the tail of the fuselage and a drastic adaptation of the rear fuselage can be avoided.

b. The APU should be isolated from the aircraft by means of a firewall; in the tail of the fuselage this can be done at little expense in weight.

c. The engine gases can easily be exhausted into the open air.

d. The location of the installation in the rear of the aircraft gives the designer some freedom in the choice on inlet and exhaust, which leads to a reduction of the noise level of the APU.

e. Accessibility is good, particularly when 
the APU is mounted inside the rear extremity of the fuselage.

This location may have certain disadvantages:

a. With the engines mounted on the wing a separate fuel feed to the rear fuselage will be required.

b. Noise levels are high near passenger doors located in the rear fuselage.

Fig. 6-27 shows an example of an APU installation.

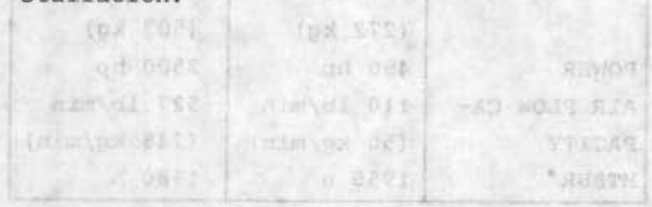

Possible alternative locations are as follows: a. In the case of a high-wing ai rcraft with the landing gear attached to the fuselage, the streamline fairings housing the retracted gear.

b. Any appropriate space near the centre-section of the wing and the main landing gear wheelbay. In the case of large aircraft it may become feasible to install two APUs in order to increase the regularity of the service. In this case it would appear suitable to place them in streamline bodies at the wing trailing edge, since these would act as "Whitcomb bodies" and so increase the arag-rise Mach number, as on the General Dynamics CV-990.

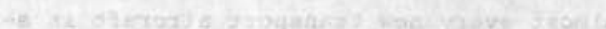

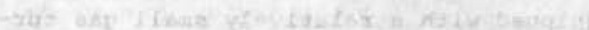

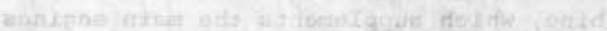
6.

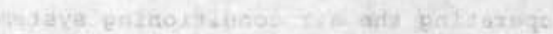

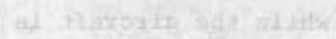

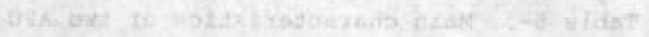

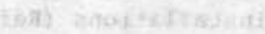

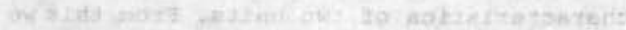

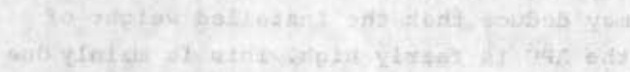

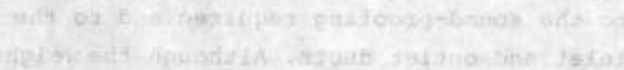

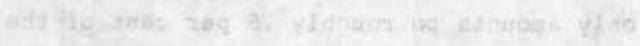

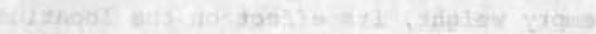

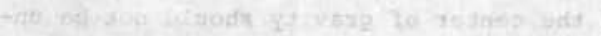

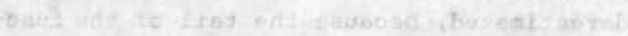

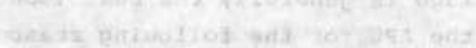

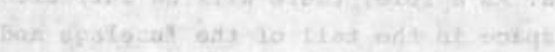

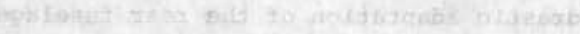

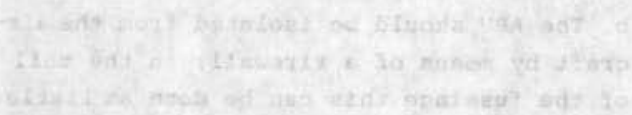

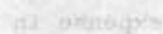

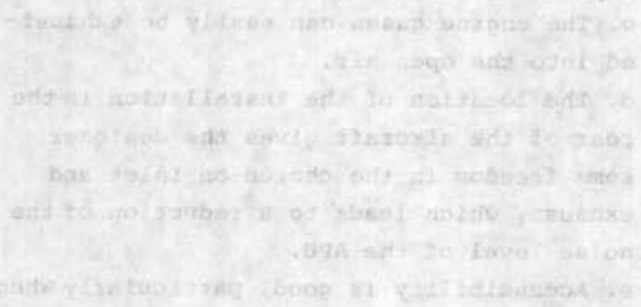




\section{Chapter 7. An introduction to wing design}

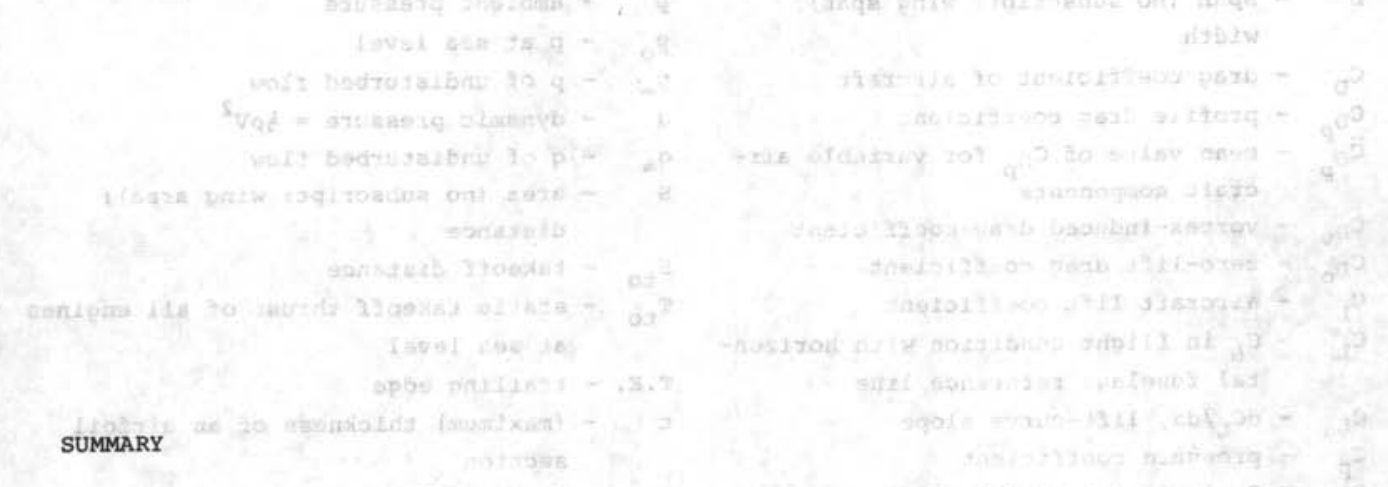

The basic requirements for wing design are associated with performance and operational aspects, flying characteristics and handing, structural design and considerations of general layout design.

Conditions are derived for optimizing the wing loading of long-range aircraft and compared with constraints on the wing loading imposed by low-speed performance requirements, available tank volume and buffet margins for high-speed aircraft.

The information on stall handling requirements, stall characteristics of airfoil sections and stall progression on wings is applicable to all conventional wing designs. Radical differences are shown between low-speed and high-subsonic aircraft with respect to planform shape and airfoil section design.

Definitions of critical Mach numbers are discussed and an approximate method is presented to find combinations of wing sweep and thickness ratio to attain a specified high-speed Mach number. Low-speed problems of swept-wing aircraft are dealt with qualitatively. An assessment of high-lift technology is followed by recommendations regarding the arrangement of ailerons and spoilers and the choice of the dihedral and the wing/fuselage incidence, together with some considerations relating to structural design. 


\section{NOMENCLATURE}

A - aspect ratio of wing

b - span (no subscript: wing span); width

$C_{D}$ - drag coefficient of aircraft

$\mathrm{C}_{\mathrm{D}_{\mathrm{p}}}$ - profile drag coefficient

$\bar{C}_{D_{p}}$ - mean value of $C_{D_{p}}$ for variable aircraft components

$\mathrm{C}_{\mathrm{D}_{\mathrm{v}}}$ - vortex-induced drag coefficient

$\mathrm{C}_{\mathrm{D}_{\mathrm{O}}}$ - zero-lift drag coefficient

$C_{L}$ - aircraft lift coefficient

$C_{L}^{*}-C_{L}$ in flight condition with horizontal fuselage reference line

$\mathrm{C}_{\mathrm{L}_{\alpha}}-\mathrm{dC}_{\mathrm{L}} / \mathrm{d} \alpha$, lift-curve slope

$c_{p}$ - pressure coefficient

$c_{p_{1}}-c_{p}$ in incompressible flow

$C_{T}$ - thrust specific fuel consumption

c - chord (no subscript: wing chord)

$c_{d_{p}}$ - two-dimensional profile drag coefficient

$c_{\ell}$ - two-dimensional lift coefficient

$c_{m}$ - two-dimensional pitching moment coefficient

D - drag

$f_{f i x}$ - drag area of fixed aircraft components

g - acceleration due to gravity

$\mathrm{h}_{\text {to }}$ - takeoff height

$i_{w}$ - angle of incidence of wing relative to the fuselage reference line

$\mathrm{K}_{\mathrm{g}}$ - gust alleviation factor

$k_{\text {to }}$ - factor of proportionality for the takeoff distance

L $\quad-11 \mathrm{ft}$

L.E. - leading edge

M - Mach number

$M_{c r}$ - critical Mach number

$\mathrm{M}_{\mathrm{Cr}}$ - drag-divergence Mach number

$\mathrm{M}_{\mathrm{Cr}}$ - lift-divergence Mach number

$M_{D}$ - Design Diving Mach number

MMO - Maximum Operating Mach number

$M_{n}$ - component of $M$ normal to the leading edge

$M_{\infty} \quad$ - $M$ referred to undisturbed flow conditions

$M^{*}$ - equivalent Mach number characterizing the extent of supercritical flow in the design condition

$\mathrm{N}_{\mathrm{e}}$ - number of engines
- load factor $=\mathrm{L} / \mathrm{W}$

- total static (equivalent) engine power of all engines at, sea level

- ambient pressure

- p at sea level

$p_{\infty} \quad-p$ of undisturbed flow

$q \quad$ - dynamic pressure $=\frac{1}{2} \rho v^{2}$

$q_{\infty} \quad-q$ of undisturbed flow

S - area (no subscript: wing area); distance

$S_{\text {to }}$ - takeoff distance

$\mathrm{T}_{\text {to }}$ - static takeoff thrust of all engines at sea level

T.E. - trailing edge

$t \quad-$ (maximum) thickness of an airfoil section

$\mathrm{V}$ - flight speed

$\mathrm{V}_{\mathrm{D}}$ - Design Diving Speed

$\mathrm{V}_{\mathrm{E}}$ - Equivalent Air speed (EAS)

$\mathrm{v}_{\text {MO }}$ - Maximum Operating Airspeed

$\mathrm{V}_{\mathrm{NE}}$ - Never-Exceed Speed

$\mathrm{V}_{\mathrm{S}}$ - minimum speed in a stall

$\mathrm{V}_{2} \quad$ - takeoff safety speed

w - aircraft weight

$W_{E}$ - design gust speed (EAS)

$\alpha$ - angle of attack

$\alpha_{\ell_{0}}$ - zero-lift angle of attack of a section

- change in wing zero-lift angle of attack per degree of positive twist

- wing dihedral

- ratio of specific heats of air.

- increment

- relative ambient pressure $=\mathrm{p} / \mathrm{p}_{0}$

- wing twist

- relative ambient temperature = (static) temperature divided by temperature at sea level

- angle of sweep

- wing taper ratio

- density of air

- $\rho$ at sea level

- relative density $=\rho / \rho_{0}$

- vortex-induced drag factor; scaling factor

Subscripts

BO - buffet onset

BP - buffet penetration 


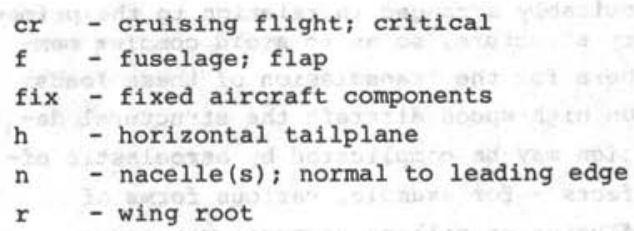

7.1. INTRODUCTION AND GENERAL DESIGN REQUIREMENTS

The following basic requirements form the point of departure for wing design.

a. The aircraft must satisfy the performance figures laid down in the design specification and within these limits it must achieve the best economic yield and operational flexibility.

b. Flight characteristics must be satisfactory both at high and low flying speeds, at high and low altitudes and in the various configurations (flap angles, power settings).

c. It must be possible to design a structure within the external lines and the general arrangement which satisfies demands regarding strength, rigidity, weight, service life, accessibility, development and manufacturing costs.

d. Sufficient space must be provided for fuel and to permit the attachment and retraction of the main undercarriage.

Whether or not all requirements can be satisfied also depends on various other factors, such as the engine thrust (power) and fuel consumption, the design of the empennage, the weight distribution, etc. Each project will differ in the degree of freedom available to the designer to make a chuice from the opportunities available to him. It is, for example, common practice to choose the type of powerplant at a fairly early stage of the design, but it may be necessary to compromise on the wing design as a result. As against this, it is very likely that the design of the tailplane will not be started until the wing design has been established.

$$
\begin{aligned}
& \text { t - wing tip } \\
& \text { to - takeoff } \\
& \text { v - vertical tailplane } \\
& \text { var - variable aircraft components } \\
& \text { w - wing }
\end{aligned}
$$

a. Performance requirements. These may be subdivided as follows. 1. Minimum requirements and rules for establishing the performance relating to the safe operational use of the aircraft. Most of these are laid down in the airworthiness regulations and any concessions in this field are generally not permitted.

2. Design requirements laid down in the design specification, relating to the transport capacity and such aspects of economic operation as cruising speeds and a range of cruising altitudes, maximum range with full payload and airfield performance (runway length and elevation). When it appears to be impossible to meet all requirements simultaneously, it may be necessary to reconsider the choice of the powerplant or revise certain demands.

Chapter 5 presents a methodology for finding combinations of wing loading, aspect ratio and maximum lift coefficients with flaps deflected, which permit the performance goals to be achieved. The results still have to be evaluated and a choice must be made from a large number of possibilities.

This chapter is intended to form a link between the initial performance feasibility study and the final stage where a fairly complete performance assessment is carried out fur one or more configurations. The choice will be narrowed down by considerations of optimization, available high-lift technology and structural weight, etc. The use of statistics (see for example the data in Table 7-1) is probably not essential if enough time is available to investigate many possibilities. However, designing under the constraint of insufficient time available for a thorough job may greatly 
simplify the designer's task in the more usual case where he is expected to be finished even before he has had an opportunity to recognize the important problems.

\section{b. Flying qualities.}

The following flight characteristics are particularly affected by wing design:

1. Stalling speeds and handling of the aircraft prior to and during the stall. The stalling speed is determined by the wing loading and the maximum lift coefficient, the stalling behavior by the planform, airfoil section(s) and twist.

2. The phenomenon of buffet on high-speed civil aircraft, which should be experienced only occasionally, 1.e. during maneuvers or in gusty weather.

3. High-subsonic aircraft may inherently suffer from several types of longitudinal instability (tuck under, speed instability), lateral-directional stability problems (poor Dutch roll damping, wing drop or wing rocking), and lateral control deficiencies (aeroelastic deformation at high EAs, aircraft dynamics at high lift).

In the case of low-speed aircraft the proposals drawn up by the preliminary design engineer may be quite adequate to obtain inherently good flight characteristics. However, opportunities to provide highspeed aircraft with good low-speed flight characteristics are often conflicting, while in addition theoretical methods to ensure accurate prediction of flight characteristics are lacking. In this case the final wing design will of necessity be made at a stage where the wind tunnel can be used, while the completion may even take place during the period of flight testing. Artificial devices such as stick pushers, Mach trimmers and yaw dampers are usually indispensable on high-speed aircraft.

\section{c. The wing structure.}

The prerequisites for the good structural design of a wing for low-speed aircraft are usually present if the main members which introduce high loads (engine mountings, undercarriage supports, wing tanks) are suitably arranged in relation to the primary structure, so as to avold complex members for the transmission of these loads. On high-speed aircraft the structural design may be complicated by aeroelastic effects - for example, various forms of flutter or aileron reversal may occur, wing twist induced by bending of the sweptback wing may cause reduced longitudinal stability. These snags can only be prevented by careful analysis and by such measures as shifting the aeroelastic axis, repositioning the powerplant and using high-speed ailerons and spoilers. The objective of preliminary structural wing design is to provide a good point of departure for the detailed design.

Small variations in the wing shape may sometimes have far-reaching effects in all areas. Design requirements aiffer from project to project and they are frequently conflicting. Since most aspects of wing design are closely related, a good syntnesis is only arrived at after consultation with various specialists. No attempt has therefore been made to present this chapter as a kind of "universal design procedure". Wing design is a highly iterative process, particularly in the preliminary stage; the following comments may help to speea it up. 1. It is often convenient to make a distinction between:

- wing size (area),

- basic wing shape (planform, sections, twist), and - high-1ift devices.

Wing size and high-lift performance are closely related to performance, while shape parameters primarily affect the stalling properties. The aspect ratio spoils the simplification: it is a shape parameter affecting performance.

2. In the case of low-speed aircraft it is probably best to determine the aspect ratio first. The wing loading and type of high-1ift devices are dealt with next, and the basic shape is finally evaluated mainly on the basis of the stalling characteristics. Small variations in the wing size have only a minor effect on the stalling characteristics. 3. In the case of high-speed jet aircraft the span loading and wing loading may be tackled first, using Sections 7.2. and 7.5.4. as background information; the aspect ratio will be found from this. Short-haul transports usually have a wing loading 
based on field performance and the type of wing flaps must be decided at an early stage. The emphasis lies on cruise performance in the case of long-range aircraft and the criterion in section 7.2.1. is fairly decisive in this respect.

4. The wing sweep and mean thickness/chord ratio of high-subsonic aircraft are based primarily on the Mach number in high-speed flight. Various combinations are possible, and the combination of wing span, root thickness ratio, sweep and taper should be checked against statistical data on cantilever ratios.

5. On high-subsonic, long-range aircraft the highift configuration is 1ikely to be decided after a satisfactory wing shape for high-speed flight has been obtained.

6. A final check on low-speed performance, fuel tank volume and buffet margins may lead to corrections of the wing area which have only minor effects on high-speed performance.

The data in Table 7-1 may be useful at every stage of the design.

\subsection{WING AREA}

The choice of the wing area is mainly based on performance requirements, although structural (weight) aspects are by no means unimportant. In performance considerations the wing area usually appears in combination with the All-Up Weight (wing loading, w/s).

The choice of the wing area is important for laying down the cruise conditions on which the choice of the wing shape will primarily be based. The wing loading is subject to optimization from the point of view of minimum fuel consumption and to constraints imposed by other criteria. The final choice of the wing area will be decided by the aerodynamic performance of the high-lift system (Section 7.6.).

\subsubsection{Wing loading for optimum cruising conditions}

The primary variables affecting cruise performance for a given aircraft geometry are the cruising speed and altitude. Conditions have been derived in section 5.4.2. for which the parameter $M L / D^{*}$ is maximum, corresponding to an optimum cruising speed and altitude (see Fig. 5-10). In the case of a specified cruising speed, the designer will try to find the best combination of wing loading and cruise altitude so that minimum operating costs can be achieved. A simplified example of a representative wing sizing study has been discussed in Section 5.5.3., where it was concluded that for the long-range aircraft project considered there the condition for minimum drag is representative of the minimum takeoff weight (see Fig. 5-19). As the direct operating costs are approximately proportional to the MTOW, it may be argued that the lift/drag ratio at a given cruising speed is a fair indication of the optimum design for long-range aircraft.

In order to get some insight into the major factors involved, simple generalized results may be obtained by assuming the aircraft's wetted components to be composed of two major groups:

a. "Fixed" components, which are assumed to be unaffected by variations in the wing size. The fuselage is an example of this group and, in the case of a fixed engine type, this also applies to the engine installation and the nacelles. The total profile drag area of these items will be denoted as $\mathrm{f}_{\mathrm{fix}}$ :

$f_{f i x}=\sum\left(C_{D_{p}} s\right)_{f i x}$

b. Variable components, the size of which is affected directly by variations in wing size, e.g. the wing itself and the horizontal tailplane. Their profile drag coefficient will be assumed constant:

$\overline{\mathrm{C}}_{\mathrm{D}_{\mathrm{p}}}=\frac{\sum\left(\mathrm{C}_{\mathrm{D}_{\mathrm{p}}} \mathrm{s}\right)_{\operatorname{var}}}{\mathrm{s}}$

* This parameter is a fair indication of the cruise fuel required, provided the corrected specific fuel consumption $C_{T} / \sqrt{\theta}$ is approximately independent of the altitude and Mach number. 


\begin{tabular}{|c|c|c|c|c|c|c|c|c|c|c|c|c|c|c|c|c|c|c|c|c|c|}
\hline & \multirow[t]{2}{*}{\begin{tabular}{|l|} 
Ist flight \\
protoetype
\end{tabular}} & \multirow{2}{*}{\begin{tabular}{c|c} 
Aspect \\
ratio \\
$\AA$
\end{tabular}} & \multirow{2}{*}{ 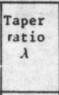 } & \multirow{2}{*}{\begin{tabular}{|l|} 
Sueep \\
angle \\
$A_{\text {eles. }}$ \\
deg. \\
\end{tabular}} & \multirow{2}{*}{ 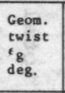 } & \multirow{2}{*}{\begin{tabular}{|c|} 
Dihedral \\
$r$ \\
deg. \\
\end{tabular}} & \multicolumn{2}{|c|}{$\begin{array}{l}\text { Profilie type and } \\
\text { streamise } \\
\text { thickness }\end{array}$} & \multirow{2}{*}{$\begin{array}{c}(\overline{E T C}) \\
z\end{array}$} & \multirow{2}{*}{$\left|\begin{array}{l}v_{M O}=\cdots \\
\mathrm{km} / \mathrm{h} \text { EAS }\end{array}\right|$} & \multirow[t]{2}{*}{$M_{400}$} & \multirow{2}{*}{\begin{tabular}{|c|}
$v_{\mathrm{D}}$ \\
$\mathrm{ka} / \mathrm{h} \mathrm{BAS}$
\end{tabular}} & \multirow[t]{2}{*}{$M_{D}$} & \multirow{2}{*}{$\begin{array}{l}\text { Plap type" } \\
\text { T.E. /L.E. }\end{array}$} & \multirow{2}{*}{ 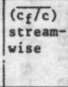 } & \multirow[t]{2}{*}{$\mathrm{b}_{\mathrm{f} / \mathrm{b}}$} & \multicolumn{2}{|c|}{ Flap angle } & \multicolumn{2}{|c|}{$\begin{array}{c}C_{\text {Canax }} \\
\text { (flight test) }\end{array}$} \\
\hline & & & & & & & & 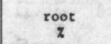 & ${ }_{2}^{\text {tip }}$ & & & & & & & & & keos. & $\begin{array}{l}\text { Landing } \\
\text { deg. }\end{array}$ & akeoft & landing \\
\hline & 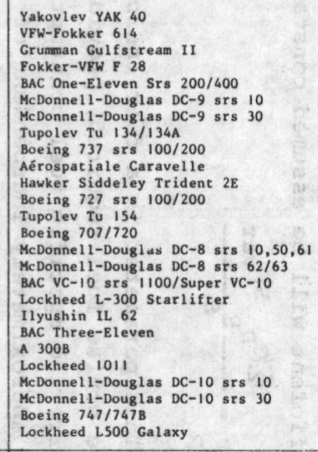 & 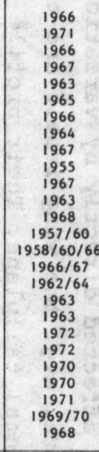 & 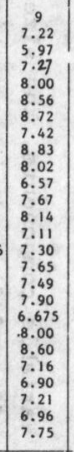 & 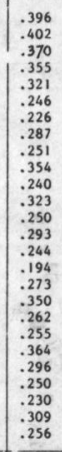 & $\begin{array}{c}0 \\
15 \\
25 \\
16 \\
20 \\
24 \\
24 \\
35 \\
25 \\
25 \\
20 \\
35 \\
35 \\
35 \\
35 \\
30 \\
30 \\
32030 \\
25 \\
35 \\
25 \\
28 \\
35 \\
35 \\
35 \\
35030 \\
25\end{array}$ & $\begin{array}{c}-4 \\
-4 \\
\vdots \\
-2 \\
-5 \\
\vdots \\
\vdots\end{array}$ & $\begin{array}{r}7 \\
6030 \\
6030 \\
3\end{array}$ & 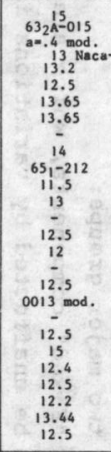 & $\begin{array}{c}11.5 \\
651-21 \\
9 \\
9 \\
90.16 \\
10.16 \\
9.75 \\
0010 \text { mod } \\
10 \\
10 \\
10 \\
10 \\
8.4 \\
9.7 \\
9.7\end{array}$ & 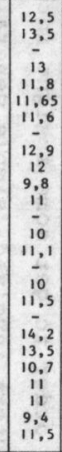 & 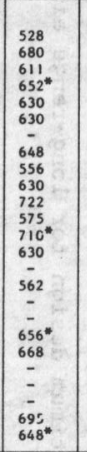 & $\begin{array}{l}.650 \\
.860 \\
.750 \\
.780 \\
.840 \\
.840 \\
.847 \\
.840 \\
.880 \\
.900 \\
.900 \\
.900 \\
.880 \\
.860 \\
.860 \\
.840 \\
.840 \\
.858 \\
.880\end{array}$ & $\begin{array}{c}615 \\
\\
760 \\
\vdots \\
\vdots \\
722 \\
732 \\
73 \\
787 \\
751 \\
770 \\
700 \\
760^{\circ} \\
\vdots \\
\end{array}$ & $\begin{array}{l}.740 \\
.830 \\
.860 \\
.890 \\
.890 \\
.890 \\
.890 \\
.850 \\
.950 \\
.950 \\
.950 \\
.950 \\
.940 \\
.890 \\
- \\
.950 \\
.950 \\
.970 \\
.875\end{array}$ & 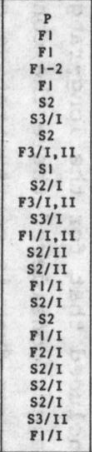 & \begin{tabular}{|l}
31.5 \\
31 \\
30 \\
32 \\
30 \\
30 \\
36 \\
36 \\
23 \\
29 \\
29 \\
27 \\
28 \\
30 \\
31 \\
32 \\
30 \\
30 \\
39 \\
32 \\
32 \\
32 \\
32.5 \\
22 \\
252 \\
257 \\
30 \\
24
\end{tabular} & $\begin{array}{l}67 \\
69 \\
73 \\
69.5 \\
75 \\
67 \\
67 \\
67.5 \\
74 \\
66 \\
69 \\
69 \\
75 \\
67 \\
76 \\
73 \\
62 \\
66 \\
72 \\
75 \\
82 \\
77 \\
71.5\end{array}$ & $\begin{array}{l}20 \\
20 \\
25 \\
18 \\
15 \\
15 \\
25 \\
159 \\
10 \\
20 \\
25 \\
15 \\
25 \\
23\end{array}$ & $\begin{array}{l}40 \\
40 \\
42 \\
40 \\
50 \\
50 \\
30 \\
40 \\
55 \\
40 \\
40 \\
50 \\
50\end{array}$ & $\begin{array}{l}2.12 \\
2.12 \\
2.16 \\
1.86 \\
2.45 \\
1.45 \\
2.250 \\
1.20 \\
1.93 \\
2.35 \\
2.35 \\
1.07 \\
1.87 \\
1.87 \\
1.87\end{array}$ & 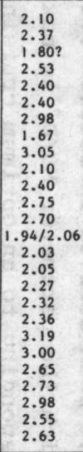 \\
\hline$y$ & 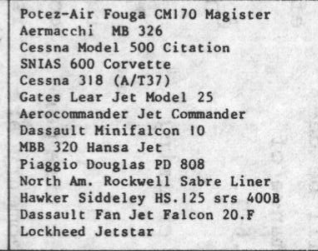 & 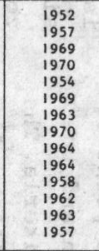 & \begin{tabular}{|l|}
7.42 \\
5.26 \\
7.45 \\
7.45 \\
6.20 \\
5.02 \\
6.19 \\
7.11 \\
6.00 \\
6.25 \\
5.77 \\
6.25 \\
6.40 \\
5.47 \\
.47
\end{tabular} & $\begin{array}{l}.400 \\
.600 \\
.390 \\
.468 \\
.511 \\
.313 \\
.233 \\
.333 \\
.4110 \\
.313 \\
.300 \\
.312 \\
.335\end{array}$ & 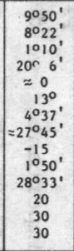 & $\begin{array}{c}-10^{\circ} 13 \\
-2038^{\prime} \\
-2 \\
-2\end{array}$ & 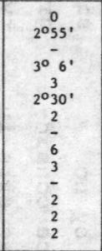 & 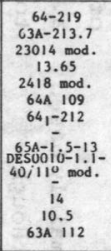 & 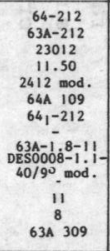 & $\begin{array}{c}15,5 \\
12,9 \\
13 \\
12,6 \\
16 \\
9 \\
12 \\
12 \\
12 \\
-9\end{array}$ & 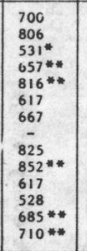 & $\begin{array}{c}.700 \\
.770 \\
.705 * * \\
.765 \\
.800 \\
.87\end{array}$ & $\begin{array}{l}740 \\
- \\
695 \\
843 \\
833 \\
833 \\
700 \\
788 \\
685 \\
68 \\
787\end{array}$ & \begin{tabular}{|l|}
.850 \\
.890 \\
.830 \\
.850 \\
.850 \\
850
\end{tabular} & $\begin{array}{l}51 \\
s 1 \\
s 1 \\
s 2 \\
s 2 \\
s 1 \\
s 1 \\
s 2 / 1 \\
s 2 \\
s 1 \\
s 1 / 1 \\
s 2 \\
s 1 / 11 \\
\text { s2/II }\end{array}$ & \begin{tabular}{|l|}
25 \\
25 \\
25 \\
31 \\
24 \\
28 \\
25 \\
31.5 \\
22.5 \\
30
\end{tabular} & $\begin{array}{c}59.5 \\
61\end{array}$ & $\begin{array}{l}20 \\
20 \\
15\end{array}$ & $\begin{array}{l}40 \\
5 \\
40 \\
40 \\
60 \\
55\end{array}$ & $\begin{array}{c}1 . \overline{55} \\
\vdots \\
1.37 \\
2.04 \\
1.61 \\
\vdots \\
1.84 \\
\vdots\end{array}$ & $\begin{array}{l}1.711 \\
2.37 \\
2.19 \\
2.32 \\
1.67\end{array}$ \\
\hline
\end{tabular}

Table 7-1. Wing design data
* Cas or IAS

**maximum level

flight speed

*** $\mathrm{V}_{\mathrm{NE}}$ is taken

equal to $\mathrm{v}_{\text {мо }}$

\author{
*P - plain or split flap \\ S - slotted flap; 1,2 or 3 : \\ number of slots \\ F - Fowler flap \\ I - slat \\ II - droop nose, leading
}

edge flap or slot 


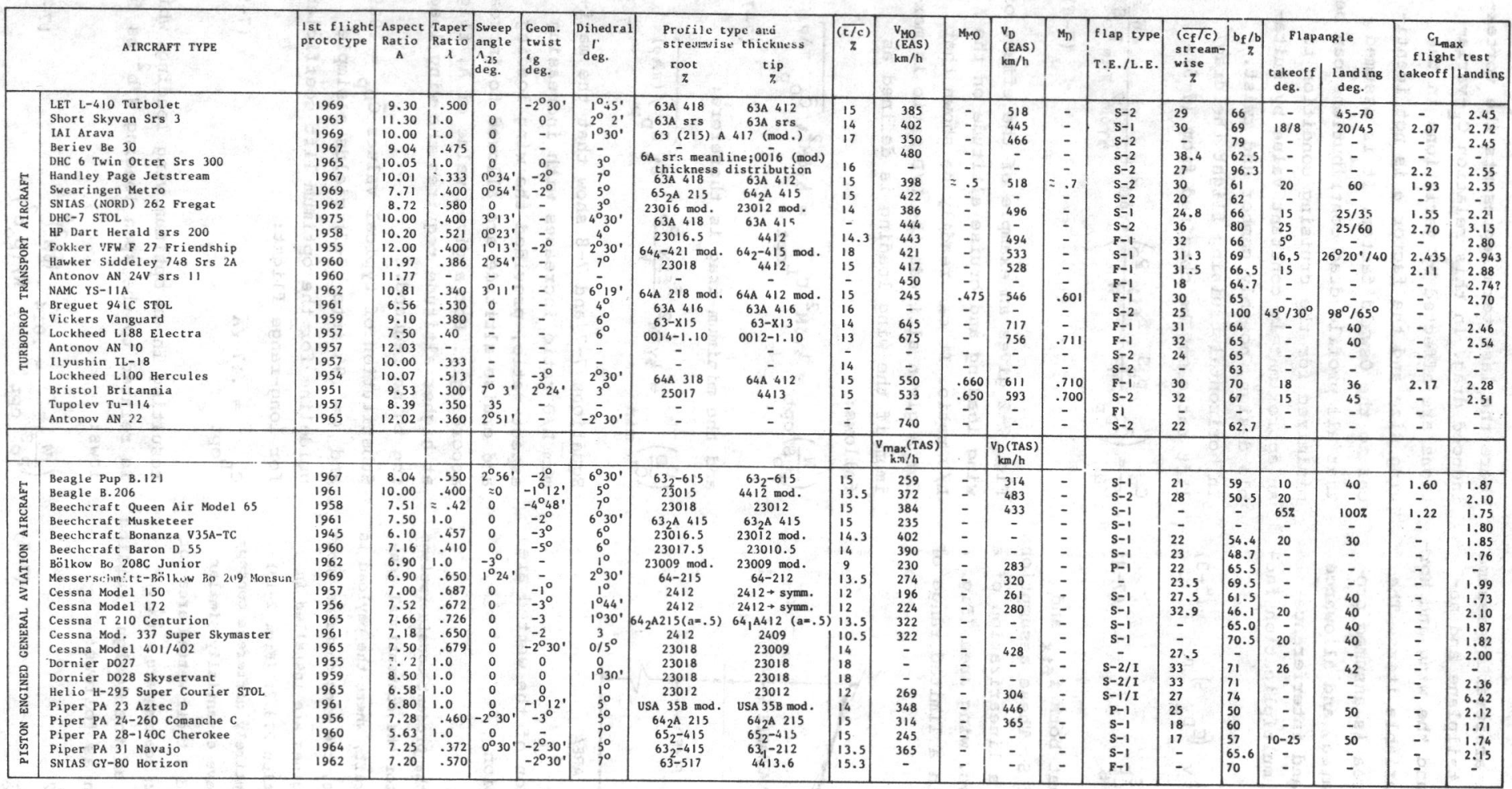

Table 7-1 (continued) 
In what follows we will arbitrarily assume the fuselage; vertical tailplane and nacelles as fixed items and the wing and horizontal tailplane as variable items. The horizontal tailplane area is assumed proportional to the wing area, and allowance is made for roughness and interference drag by using suitable multiplication factors. Thus we have:

$$
\begin{aligned}
& f_{f i x}=\left(C_{D_{p}} s\right)_{f}+\left(C_{D_{p}} s\right)_{v}+\left(C_{D_{p}} s\right)_{n} \\
& \bar{C}_{D_{p}}=\left(C_{D_{p} w}\right)+\left(C_{D_{p} h}\right)^{\frac{s}{s}}
\end{aligned}
$$

while it is assumed that both $f_{f i x}$ and $\bar{C}_{D_{p}}$ are independent of $S$. These assumptions can be interpreted as a linearization of a curve of wetted area vs. wing area (Fig. $7-1$ ), which is valid in a limited range of wing area variations.

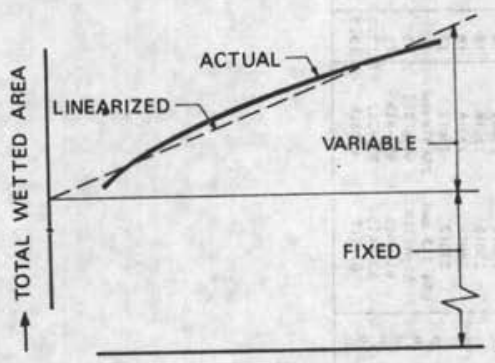

$\rightarrow$ WING AREA

Fig. 7-1. Linearization of the wetted area with wing area variation

It should be noted that the present considerations are strictly valid only for the conventional general arrangement of aircraft, where the payload is contained by the fuselage, the wing contains (most of) the fuel, and the engines are installed in pods. The example in Section 2.3.2. (Fig. 2-13) shows that aircraft of entirely different conceptions may nevertheless have essentially similar range performance, provided their wetted area (or internal volume) and span loading are equal. The drag coefficient at the design cruising $C_{L}$ may now be written as follows:

$C_{D}=\bar{C}_{D_{p}}+\frac{f_{f i x}}{S}+\frac{C_{L}{ }^{2}}{\pi A_{\theta}}$ where the last term represents the vortexinduced drag. In this relation $C_{L}$-variations are effected by variations in the wing size and the factor $\phi$ is not identical to the oswald factor. It is assumed that all profile drag contributions can be minimized for the cruising condition to an approximately constant value by suitable design, notably camber and twist. In horizontal cruising flight the drag/ lift ratio may be obtained from $(7-5)$ :

$\frac{C_{D}}{C_{L}}=\left(\bar{C}_{D_{p}} \frac{P_{O} S}{W}+\frac{f_{f i x} P_{o}}{W}\right) \frac{1}{2} \gamma \delta M^{2}+\frac{1}{\frac{1}{2} \gamma \delta M^{2} \pi A \varphi} \frac{W}{P_{O} S}$

Fig. 7-2 gives an example of the effect of wing loading and cruise altitude on the $L / D$ ratio. It can readily be shown that for a given altitude the $L / D$ ratio is maximum if the wing loading is defined as follows :

$$
\left(\frac{\mathrm{W}}{\mathrm{p}_{\mathrm{O}} \mathrm{S}}\right)_{\text {opt }}=\frac{1}{2} \gamma \delta \mathrm{M}^{2} \mathrm{C}_{\mathrm{L}_{\text {opt }}}=\frac{1}{2} \gamma \delta \mathrm{M}^{2} \sqrt{\overline{\mathrm{C}}_{\mathrm{D}_{\mathrm{p}}} \pi \mathrm{A} \varphi}
$$

and the minimum drag is therefore:

$\left(\frac{C_{D}}{C_{L}}\right)_{\text {min }}=\frac{1}{2} \gamma \delta M^{2} \frac{f_{f i x} P_{O}}{W}+2 \sqrt{\bar{C}_{D_{p}} /(\pi A \varphi)}$

Equations $7-7$ and $7-8$ show that the maximum L/D ratio increases with increasing aspect ratio, provided the wing loading and cruise altitude are allowed to increase accordingly. For a given value of $\mathrm{A}$, flight at higher altitude requires the wing loading to be reduced.

Substitution of typical values $\bar{C}_{D_{p}}=.0095$ and $\varphi=.95$ into $(7-7)$ yields a simple guideline for the optimum lift coefficient for long-range flight:

$\mathrm{C}_{\mathrm{L}_{\text {opt }}}=.17 \sqrt{\AA}$

resulting in an optimum wing loading, which is related to the span loading $\mathrm{w} / \mathrm{b}^{2}$ as follows:

$\left(\frac{w}{p_{o} S}\right)_{o p t}=.014 \frac{\left(\delta M^{2}\right)^{2}}{w /\left(p_{o} b^{2}\right)}$ 


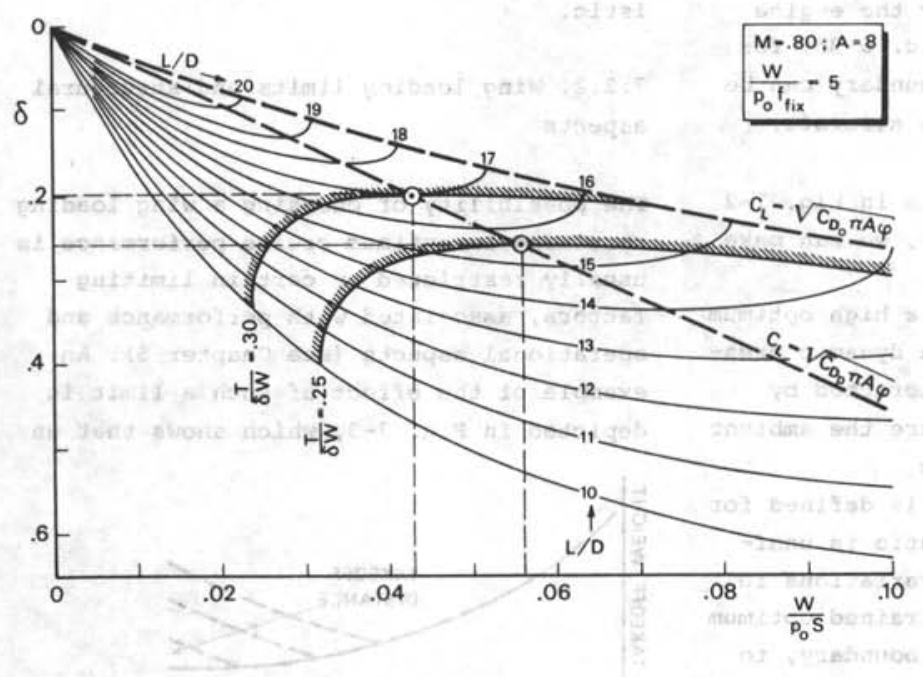

Fig. 7-2. Example of the effect of wing loading and cruise altitude on the lift/drag ratio for a highsubsonic long-range jet transport aircraft

Note that this condition applies to a representative mean All-Up Weight.

The well-known condition for minimum drag (constant wing loading):

$C_{L}=\sqrt{C_{D_{0}} \pi A \varphi}$

resulting in

$\left(\frac{C D}{C_{L}}\right)_{\min }=21 \frac{\overline{C_{D_{O}}}}{\pi A \omega}=21 \frac{\overline{\bar{C}_{D_{D}}+f_{f i x} / S}}{\pi A \varphi}$

is obtained at a cruising altitude defined by:

$$
\delta=\frac{w / S}{h_{\gamma_{0}} M^{2} \sqrt{C_{D_{0}} \pi A \varphi}}
$$

The curves for constant L/D in Fig. 7-2 have not been drawn for higher altitudes as in this region $C_{L}$ is too high and the same performance can be obtained for lower $C_{L}$ at a lower altitude.

An absolute optimum for the wing loading and altitude cannot be obtained from these considerations as conditions (7-7) and (7-11) are incompatible and L/D continues to increase with altitude. However, in the case of a fixed powerplant, the cruising altitude is limited by the available thrust:
$\delta>\left(\frac{L}{D} \cdot \frac{T}{\delta W}\right)^{-1}$

Since we know from gas turbine theory that in an isothermal atmosphere ${ }^{*} \mathrm{~T} / \delta$ is constant for a given engine rating and Mach number, we obtain a thrust boundary in the $W / S-\delta$ diagram as indicated in Fig. 7-2. The following "optimum" wing loading and cruise a).titude are now obtained from $(7-7),(7-8)$ and $(7-14)$ :

$$
\begin{aligned}
& \left(\frac{\mathrm{W}}{\mathrm{p}_{\mathrm{O}} \mathrm{S}}\right)_{\text {opt }}=\frac{2 \overline{\mathrm{C}}_{\mathrm{D}_{\mathrm{p}}}}{\frac{\mathrm{T}}{\delta \mathrm{T}_{\text {to }}} \frac{\mathrm{T}_{\text {to }}}{\mathrm{W}} \frac{2}{\gamma \mathrm{M}^{2}}-\frac{\mathrm{f}_{\mathrm{fix}} \mathrm{P}_{\mathrm{o}}}{\mathrm{W}}} \\
& \delta_{\text {opt }}=\frac{2 \sqrt{\overline{\mathrm{C}}_{\mathrm{D}_{\mathrm{p}}} /\left(\pi \mathrm{A}_{\varphi}\right)}}{\frac{\mathrm{T}}{\delta \mathrm{T}_{\text {to }}} \frac{\mathrm{T}_{\text {to }}}{\mathrm{W}}-\frac{1}{2} \gamma \mathrm{M}^{2} \frac{\mathrm{f}_{\mathrm{fix}} \mathrm{P}_{\mathrm{o}}}{\mathrm{W}}}
\end{aligned}
$$

and the highest obtainable L/D ratio for a given powerplant is:

$$
(L / D)_{\max }=\frac{1}{2} \sqrt{\frac{\pi A \varphi}{\bar{C}_{D_{p}}}}\left\{1-\frac{1}{2} \gamma^{2}{ }^{2} \frac{f_{f i x} P_{0}}{W} /\left(\frac{T}{\delta T_{\text {to }}} \frac{T_{t o}}{W}\right)\right\}
$$

* This condition simplifies the analysis, but the results are essentially similar for other atmospheric models. 
where $T /\left(\delta T_{\text {to }}\right)$ is defined by the engine rating and $M$, as shown in Fig. $6-3 b$, for example. A similar thrust boundary can be derived for propeller-driven aircraft.

Referring back to the example in Fig. 7-2 and to $(7-15)$ through $(7-17)$, we can make the following observations:

1. High-speed aircraft have a high optimum wing loading due to the high dynamic pressure. This tendency is counteracted by flying at high altitudes where the ambient pressure and density are low.

2. If the optimum condition is defined for a given altitude, the L/D ratio is unaffected by relatively large variations in the wing loading. If a constrained optimum is defined along the thrust boundary, to be obtained by optimizing the altitude for each wing loading, the L/D ratio is much more sensitive to wing loading variations. 3. For a given cruise altitude the optimum wing loading increases with the aspect ratio, whereas according to $(7-15)$ the "absolute" optimum is not affected by this. Both the best cruising altitude and the maximum L/D ratio increase sensitively with increasing aspect ratio.

4. For a given cruise altitude the size of the aircraft, represented by $w / f_{f i x}$, does not affect the optimum wing loading, contrary to the "absolute" optimum, which is definitely affected. For example, if the aircraft is stretched in such a way that $w / f_{f i x}$ decreases, the optimum wing loading increases and the altitude decreases. The opposite is true if the fuselage is shortened, as demonstrated by the Boeing 747 SP variant.

5. Aircraft with high bypass engines have relatively low values of $\mathrm{T} /\left(\delta \mathrm{T}_{\text {to }}\right)$ due to the thrust decay with speed. Their optimum cruise altitude is low and the wing loading high, although this effect is partly cancelled by the relatively low thrust loading required for adequate low-speed performance.

Obviously, all conclusions stated are valid on the condition that the simplifying assumptions mentioned previously are real- istic.

\subsubsection{Wing loading limits and structural} aspects

The possibility of choosing a wing loading resulting in optimum cruise performance is usually restricted by certain limiting factors, associated with performance and operational aspects (see Chapter 5). An example of the effect of such a limit is depicted in Fig. 7-3, which shows that an

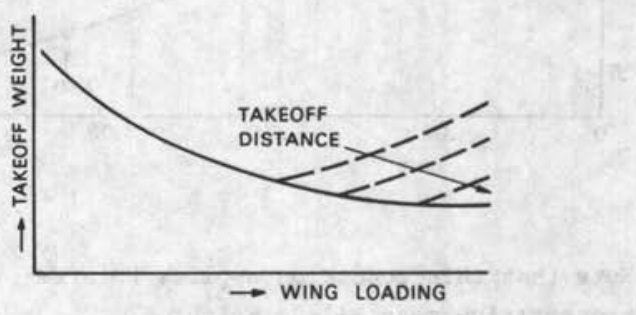

Fig. 7-3. Effect of a performance constraint on the takeoff weight

increase in the wing loading results in a decreasing takeoff weight up to a point where the takeoff field length requirement becomes critical. A larger engine must be chosen or a more complicated flap system designed if the wing loading continues to increase, resulting in a progressive takeoff weight increment. In addition, considerations associated with structural weight reduction may bias the designer's decision.

\section{a. Takeoff field length.}

The takeoff distance required is approximately proportional to the sum of the $\mathrm{ki}$ netic and the potential energy at the takeoff height and to the thrust loading. For jet aircraft:

$s_{\text {to }}=k_{\text {to }} \frac{w_{\text {to }}}{\mathrm{T}_{\text {to }}}\left(\frac{\mathrm{v}_{2}{ }^{2}}{2 \mathrm{~g}}+\mathrm{h}_{\text {to }}\right)$

The constant of proportionality $k_{\text {to }}$ is primarily dependent on the type of operation - reflected in the airworthiness rules - the number of engines, the bypass ratio and the size of the aircraft. The 
designer may derive $k_{\text {to }}$ from statistical data on existing aircraft or from calculations of the type discussed in section 5.4.5. A typical result is $k_{\text {to }}=2.2,2.0$ and 1.8 for twin-, three- and four-engine transport aircraft, respectively. For propeller aircraft the power loading must be used instead of the thrust loading. The limitation in the takeoff safety speed $\mathrm{V}_{2}$ can be translated into a wing loading limit as follows:

$\mathrm{w}_{\mathrm{to}} / \mathrm{S}=\frac{1}{2} \rho \mathrm{V}_{2}{ }^{2} \mathrm{C}_{\mathrm{L}_{\max }}\left(\mathrm{V}_{\mathrm{S}} / \mathrm{V}_{2}\right)^{2}$

where minima for $V_{2} / V_{S}$ are specified in the regulations.

It is noted that the performance of the high-lift system* has an appreciable effect on the wing loading limit (see section 7.6.).

b. Landing field length.

A semi-empirical method for estimating the wing loading limit to cope with a specified landing distance is derived in Section 5.4.6. Suitable design of the highlift system is again a powerful means for attaining the optimum wing loading. For long-range aircraft this requirement is usually not critical in view of the relatively low landing weight (see section 8.2.4.).

\section{c. Fuel tank volume.}

The internal volume of a wing of given shape is proportional to $\mathrm{s}^{3 / 2}$ and the available fuel tank volume thus decreases rapidly with increasing wing loading. In the example in Fig. 5-19 it was shown that for the long-range aircraft considered this condition determines the wing area.

An approximate wing area limit for a given fuel quantity may be obtained with Appendix B, Section B-3. Preliminary estimation of the fuel weight has been discussed in Section 5.4.2., while data on the specific gravity of fuel are given in Table 8-14. It should be noted that the internal wing volume

${ }^{*} \mathrm{C}_{\mathrm{L}_{\max }}$ in (7-19) refers to the flap deflection angle for takeoff can be increased by increasing the sweepback angle, resulting in a thicker wing for a given highspeed design condition (see Section 7.5.4a.).

d. High-speed buffet boundaries: transport aircraft.

Limitations to the wing loading can be derived from the requirements in section 7.5.2b., provided the lift coefficients for buffet onset $C_{L_{B O}}$ and for maximum buffet penetration $C_{L_{B P}}$ are known. The maneuver requirements will limit the wing loading or the cruise altitude as follows:

$\frac{W / S}{P_{O}} \leqslant .538 \delta M^{2} C_{L_{B O}}(n=1.3)$

and:

$\frac{\mathrm{W} / \mathrm{S}}{\mathrm{P}_{\mathrm{O}}} \leqslant .438 \delta \mathrm{M}^{2} \mathrm{C}_{\mathrm{L}_{\mathrm{BP}}}(\mathrm{n}=1.6)$

Assuming a gust alleviation factor of .8 and using (7-23), it can readily be shown that a specified gust speed of $41 \mathrm{ft} / \mathrm{s}$ $(12.5 \mathrm{~m} / \mathrm{s})$ can be coped with if the wing loading is limited as follows:

$\frac{W / S}{P_{O}} 6.78 M^{2}\left(C_{L_{B P}}-.03 \frac{{ }_{L} \frac{L_{\alpha}}{M \sqrt{\theta}}}{M}\right)$

For a given wing shape, these buffet boundaries are a function of the Mach number on$1 y$. The lift-curve slope is a function of the Mach number, the aspect ratio and the sweepback angle (see Appendix E, Section $\mathrm{E}-4.1$.$) . It is obvious that the various$ limits can be determined only after design of the wing shape and prediction of the buffet boundaries.

e. Some structural aspects.

Variation of the wing area will obviously entail a variation of its structural weight. It is obvious that the wing loading resulting in a minimum All-Up Weight will therefore be higher than that for the best range performance, provided other design considerations do not impose constraints on the optimum. This influence of the wing structure weight is particularly pronounced in the case of short-range atr- 
craft, where the emphasis lies on reducing the empty weight and fuel economy is of secondary importance.

The size and takeoff weight of the largest transport aircraft have increased steadily since the dawn of aviation. Whereas the aircraft with which the Wright brothers made their first powered flight had a wing loading of only $1.5 \mathrm{lb} / \mathrm{sq} . \mathrm{ft}\left(7.3 \mathrm{~kg} / \mathrm{m}^{2}\right)$, the loading amounts to a hundred times this figure for modern transports like the McDonnell Douglas DC-10 (see also Fig. 1-6). The effect of aircraft growth on the empty weight has been the subject of many theoretical considerations, based on the squarecube law. Applying this law to the aircraft structure, it is concluded that when its linear dimensions are enlarged by a scale factor $\%$, the wing area will be increased proportional to $\varphi^{2}$, but the volume - and hence the structure weight - will increase by ${ }^{3}$, provided the specific density and stress level of the structural material are constant. If the wing loading is assumed constant, the All-Up Weight will increase proportional to $\varphi^{2}$ and consequently the structure weight grows more rapidly than the All-Up Weight. A point will be reached where the aircraft is unable to carry more than the structure and no margin is left for useful load, engines or equipment.F.w. Lanchester (1868-1946) drew attention to this difficulty in the early 1900 s and several others have made (widely divergent) predictions of a practical limit to the size of aircraft.

The square-cube law is based on many simplifying assumptions and has been defeated by the ingenuity of designers. Aircraft will not be scaled up according to a geometric similarity and stress levels have increased considerably. Nevertheless, the actual wing structural weight fraction will tend to increase with the size of the aircraft, unless the wing loading is increased (Section 8.4.1.). A statistical plot (Fig. 7-4) shows that for propeller aircraft the wing loading trend increases in proportion to $\mathrm{w}^{1 / 3}$, while for jet aircraft it is approximately proportional to $\mathrm{w}^{1 / 5}$. From other statistical material it can also be observed that the cruising speed of propeller aircraft increases considerably with size, as opposed to jet transports which usually operate at highsubsonic speeds where the flight Mach number is limited by compressibility effects.

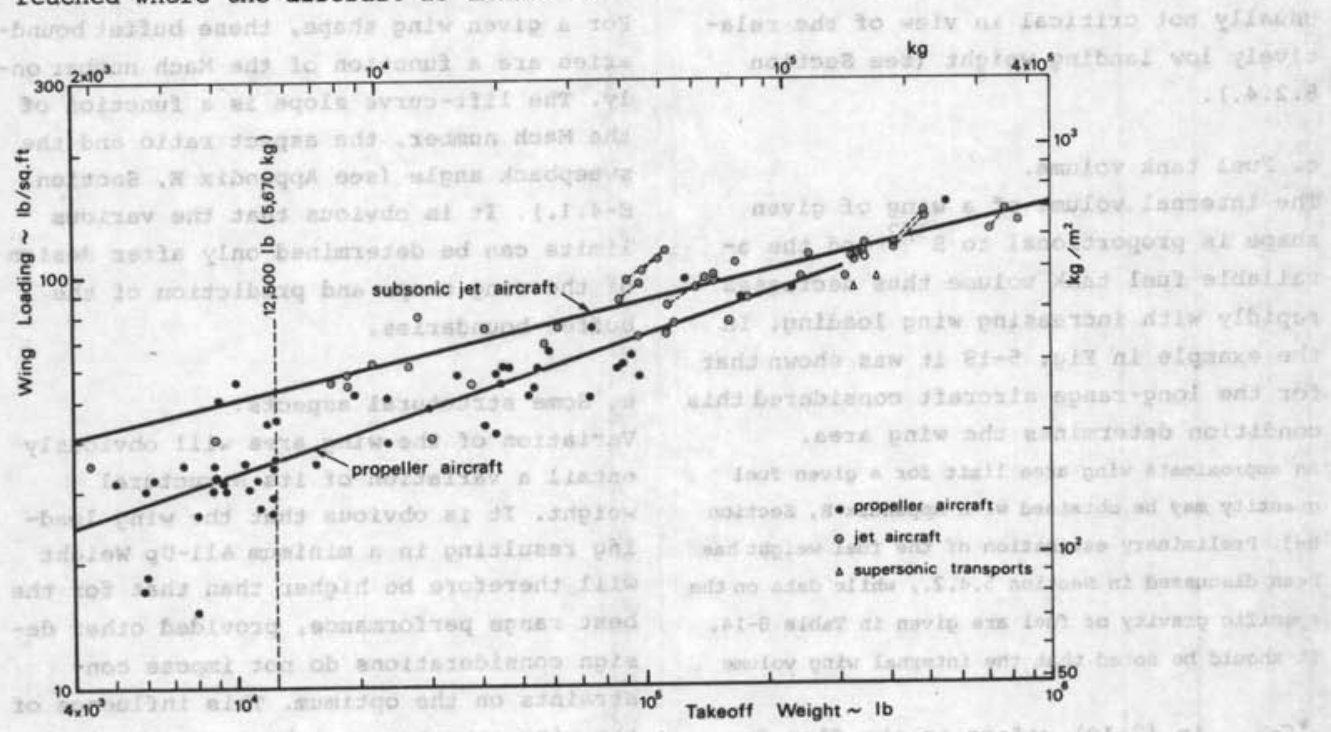

Fig. 7-4. Wing loading trends. 
The wing size not only has a direct influence on the structural weight, it also affects the gust loads. This is observed from the normal load factor resulting from a sharp-edged gust velocity:

$\mathrm{n}=1+\mathrm{K}_{\mathrm{g}} \mathrm{C}_{\mathrm{L}_{\alpha}} \frac{1}{\frac{1}{2} \rho_{O}} \mathrm{w}_{\mathrm{E}} \mathrm{V}_{\mathrm{E}} \frac{\mathrm{S}}{\mathrm{W}}$

where $K_{g}$ is a gust alleviation factor which is mainly dependent on the altitude and the wing loading. Since the weight of the wing is approximately proportional to $\sqrt{n}$, a high wing loading is favorable for achieving a low structural weight, provided the gust load is the deciding factor for a critical load. Apart from the weight aspect a reduction of gust loads offers the occupants a smooth ride when flying through turbulent air.

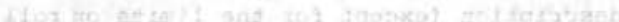

\subsection{SOME CONSIDERATIONS ON LOW-SPEED} STALLING

Although the stall is outside the normal pattern of transport flight, stall incidents occur from time to time. It appears that the probability of the occurrence of stall is one in $10^{5}$, but the probability of reaching the stall warning is between one in $10^{2}$ to $10^{3}$ (Ref. 7-103). Acceptable handiing characteristics at the stall must therefore be shown during certification tests, while the outcome of flight tests also forms the basis for establishing the minimum flight speed(s). The regulatory requirements for stalling behavior are stated in FAR 23.201 through 23.207 (BCAR Section K Ch. K 2-11) for light aircraft, and FAR 25.201 through 25.207 (BCAR Section D Ch. D 2-11) for transport aircraft. These regulations stipulate that acceptable stalling behavior must be demonstrated in straight flight and in a coordinated turn, for the operational flap settings, center of gravity positions, undercarriage up and down and specified power. In addition, satisfactory stall behavior must be shown during powered flight with one engine inoperative.
The aim of this section is to provide some generalized guidelines for achieving a wing design with inherently acceptable stalling characteristics. It is emphasized that in the preliminary design stage the available methods to achieve this are rather limited, particularly for sweptwing aircraft. The discussion is confined to the "conventional" type of stalling at angles of attack between about 10 and 20 degrees, the wing shape being the major factor affecting this stall. Some remarks on post-stall behavior and deep-stall, relevant to a restricted category of aircraft, are given in section 2.4.2c. In that context the shape of the wing is of limited interest only.

\subsubsection{Stall handling requirements and stall warning}

The purpose of specifying certain acceptable stall characteristics is to minimize the chance of an inadvertent entry into a stall and to ensure recovery from it if the pilot stalls the aircraft intentional1y. During flight testing the aircraft will also be deliberately stalled with fully throttled engines in order to establish the stalling speed, which constitutes the most important basis for the low-speed performance.

\section{a. Stalling behavior.}

During the standard stalling maneuver the engines are fully throttled and the elevator control is pulled back so that the aircraft is decelerating at approximately $1 \mathrm{knot} / \mathrm{sec}\left(.5 \mathrm{~m} / \mathrm{sec}^{2}\right)$. With the usual three control system it must be possible to produce and correct angles of roll and yaw by the normal (unreversed) use of the controls up to the moment when the stall becomes apparent. The stall should preferably be characterized by a distinct and initially uncontrollable nose-down pitching motion, and during recovery to level flight it must be possible to prevent angles of roll and yaw of more than approximately 15 degrees (light aircraft) or 20 
degrees (transport aircraft). In addition, dynamic stall tests must be executed at higher rates of deceleration - up to 4 knots/sec, $2 \mathrm{~m} / \mathrm{sec}^{2}$ - with engines operating at specified power levels and with an inoperative engine.

It is generally conceded that a "good stall" is non-existent and many attempts have been made to prevent stalling altogether by limiting the elevator control power in such a way that the angle of attack for maximum lift can never be attained ("stall proofing"). This approach is usually rejected as the limited control power conflicts with other maneuverability requirements in unstalled flight, and is difficult to achieve in practice in view of the differences in aircraft configuration. In addition, it results in degraded performance, and the conventional approach is therefore to minimize the consequences of a stall rather than prevent it altogether.

\section{b. Stall warning.}

The occurrence of a stall must be preceded by an appropriate stall warning in a range of speeds of about 108 EAS above the stalling speed, i.e. in a range of angles of attack between about 808 and 1008 of the stalling angle. Large increases in elevator control forces or control stick movements are generally unmistakable warnings. A gradually increasing, small amplitude oscillation in roll and pitch may also be acceptable, provided it is initially controllable by the pilot. Vibrations or buffeting of the aircraft and the control stick on approaching the stall are also considered adequate stall warnings, provided they cause no danger of structural damage.

Mechanical and electronic devices (stick shakers, warning lights, audible alarms) have been developed to provide stall warning when adequate characteristics cannot be made inherent to the design. A survey of the most common devices is presented in Ref, 7-75.
7.3.2. Design for adequate stall characteristics

The stalling characteristics of an aircraft may be markedly different in varying conditions of flap setting and engine power, depending on the engine location relative to the wing. Small variations in the external shape may have a great effect on stalling, as illustrated by Ref. 7-100, for example. Stalling characteristics cannot be predicted accurately by theoretical methods, while wind tunnel experiments have limited value in view of the effects of differences in the Reynolds number, surface roughness and structural details. An additional complication is that a design criterion cannot be easily defined, as the requirements give a qualitative description (except for the limits on roll and yaw angles) and are to some extent open to differences in interpretation.

Nevertheless, a few general principles can be given which permit the achievement of a basically good design by suitably shaping the wing, which is the primary element affecting the stalling characteristics, and carefully locating the horizontal tailplane. The wing loading sets the stalling speed, while its planform, section shape and twist determine the initial occurrence and progression of the separation and the violence of the motions associated with wing stalling. The horizontal tail load will contribute to the desirable pitchdown behavior in a way which is determined primarily by its location relative to the wing (cf. Section 2.4.2.). Finally, the type of flow around the control surfaces in the condition of a stalled wing determines the effectiveness of control deflections while the pilot is trying to keep the aircraft on course.

The progression of the stall can usually be predicted for straight wings of moder- 
ate to high aspect ratio and the wing shape can be chosen so that its behavior will be satisfactory. These aims are much more difficult to achieve on swept wings, not only because of the marked three-dimensional character of the flow, which is difficult to predict, but also because the shape of swept wings is usually dictated primarily by considerations of high-speed flight, with the result that the stalling properties of the basic wing may tend to be compromised. Where inherently acceptable characteristics cannot be achieved, some form of stall protection system may be adopted. These systems limit the maximum usable angle of incidence by a large nose-down control input of short duration, counteracting the action taken by the pilot to produce higher angles of incidence ("stick-pusher"). These devices are generally incorporated on the basis of investigations outside the field of preliminary aerodynamic design.

\subsubsection{Stalling properties of airfoil sec- tions}

The airfoil section is fundamental to wing design and much research has been done and will continue to be done - to correlate the geometric properties of wing sections to the stalling properties and the shape of the lift and pitching moment curves. Following the classical exposition of McCullough and Gault (Ref. 7-74), three representative types of airfoil stall are considered: trailing edge stall, leading edge stall and thin airfoil stall. The course of events which determines the type of flow and the resulting lift and pitching moment curves is illustrated in Fig. 7-5. Although every airfoil cannot be classified uniquely in one of these stalling categories, the following description of the aerodynamic phenomena is generally valid for most commonly used airfoils.

TYPE I: TRAILING EDGE STALL. This type of stall is characteristic of most airfoil sections with thickness/chord ratios* of approximately 158 and above. The flow at large angles of attack is characterized by a progressive thickening of the turbulent boundary layer on the upper surface. As the angle of attack is increased to about 10 degrees (B) flow separation starts at the trailing edge and moves gradually forward. This is associated with a decreasing lift curve slope, although initially the increasing lift near the leading edge is predominant and the lift gradient remains positive. A maximum lift coefficient of approximately 1.5 is obtained on a symmetrical airfoil of 188 thickness when the separation region reaches the mid-chord point (C). Beyond maximum lift (D) the forward progression of separation continues at about the same rate as prior to the stall, and the peak of the lift curve is rounded. The variation of the pitching moment with lift is smooth; there is no sudden break at the stall.

TYPE II: LEADING EDGE STALL. Airfoils with thickness/chord ratios of about 9 to 12 percent experience an abrupt separation of the flow near the leading edge. On these sections separation of the laminar boundary layer may occur well before the attainment of maximum lift and prior to transition to a turbulent boundary layer. Transition occurs in the shear layer thus formed, and the expansion of the turbulent motion spreads at such an angle that reattachment of the flow quickly occurs, enclosing a "short bubble" and subsequently forming a turbulent boundary layer (B). The pressure distribution is affected by the short bubble only locally and the peak suction is not greatly altered by the short bubble, which is very small in comparison with the wing chord. An increase of the angle of attack (C) moves the separation point in a region of sharp airfoil curvature, so that the turbulent shear layer fails to reattach. At this critical incidence a complete disruption of the

* Defined in. Appendix A, Section A-2.1. 

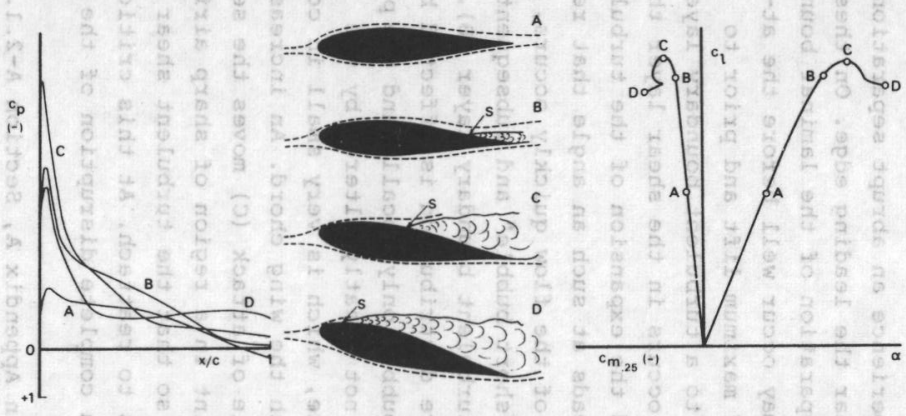

TYPE III THIN AIRFOIL STALL
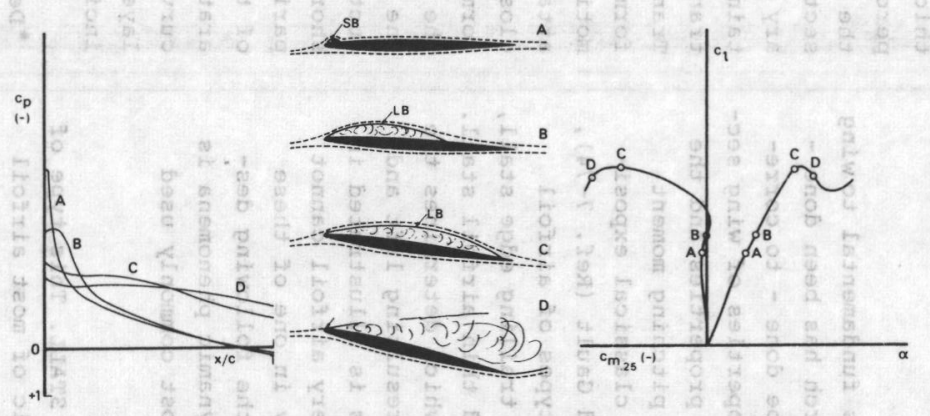

a. Upper surface pressure distributions, growth of the boundary layer and separation regions and lift and pitching moment curves.
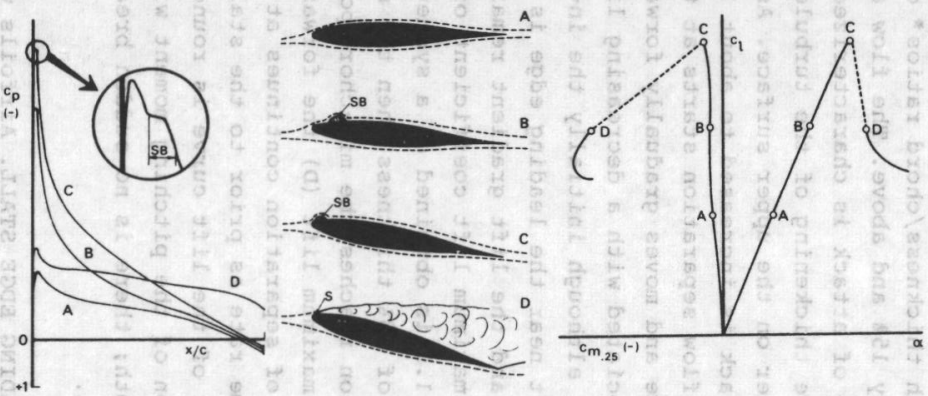

NOTES: 1. Drawings are not exactly to scale

2. $\mathbf{S}=$ separation, $\mathrm{SB}=$ short bubble, $\mathrm{LB}=$ long bubble

Fig. 7-5. Representative types of low-speed airfoil stall 
flow occurs over the entire upper surface; the short bubble is said to burst (D). The leading edge pressure peak collapses and the pressure is subsequently redistributed along the chord into a more or less

flattened form which is characteristic of complete separation.

The lift and pitching moment curves exhibit abrupt changes when the angle of attack for maximum lift is exceeded. There is little or no rounding of the lift curve and a sudden negative shift of the pitching mothent resulting from the rearward shift of the centre of pressure is observed.

TYPE III: THIN AIRFOIL STALL. On very thin sections of thickness/chord ratios of less than about 6 percent and on round noses a small separation bubble occurs at very small angles of attack (S). At a certain critical angle of attack the short bubble breaks down, but the flow subsequently reattaches downstream, forming a "long bubble" which causes a slight reduction in the lift-curve slope (B). With increasing angle of attack the point of flow reattachment progressively moves backward until it coincides with the trailing edge and maximum lift is reached at this condition (C). The lift curve is characterized by a rounded peak, while the pitching moment curve shows a pronounced negative trend near maximum lift.

The pressure distribution associated with a long bubble has a reduced level suction peak which extends over the length of the bubble.

In addition to the three types of stall, various forms of combinations of trailing and leading edge stall can be observed. These are characterized by a semirounded or relatively sharp peak of the lift curve. The various types of stall should not be too closely allied to the thickness ratio. It has been shown by Gault (Ref. 7-81) that there is a relationship between stalling characteristics, airfoil nose geometry and Reynolds number. In Fig. $7-5 b$ the upper surface ordinate at 1.25 percent chord has been used to characterize the leading edge shape. The correlation shows that distinct areas are present where any of the three types of stalling dominates, but in addition certain airfoils show a combination of trailing edge and leading edge types of stall. The stalling characteristics are difficult to predict and very sensitive to minor variations in airfoil geometry.

The flow field around an airfoil with leading-edge and/or trailing-eage high-1ift devices ("multielement" airfoils) is considerably more complex than that of a basic section. Boundary layer separation may occur on any of the airfoil components, there is a strong interaction of the flow around these components and the resulting flow fields are very difficult to analyze. A theoretical prediction of the stalling characteristics of wings with flaps deflected has a very limited value in the preliminary design stage. Some comments on the effect of trailing-edge flap derlection on the type of stall are made in Appendix G, Section G-2.3.

7.3.4. Spanwise progression of the stall

If flow separation starts at the wing tip and progresses inboard, the stall is likely to be characterized by a violent roll without warning, as the stalled region exerts a large rolling moment. Adequate aileron control power may also be lost because of the separation of the flow on the aileron. Nevertheless, a stall starting at the tip may be acceptable, provided the tip sections have a flat-top lift curve while the inboard sections have a sharp lift curve. In this case the wing tip maintains enough lift beyond the stall and large rolling moments are not generated.

An initial stalling region near the wing root may result in a large wake hitting the horizontal tailplane - depending upon its location relative to the wing - thus. producing tailplane buffet. This may be considered as a desirable feature - if limited - on small aircraft, but it will be unacceptable if excessively violent 
buffeting is $11 \mathrm{kely}$ to cause structural damage. A stall confined to a limited inboard area will also be accompanied by large regions of unstalled flow, and this will generally result in an appreciable loss in maximum lift. In view of the extra wing area required, the early root stall may not be an efficient solution for transport aircraft. In addition, the wake of the stalled inner wing may blanket the vertical tail and as a result rudder control may be lost, resulting in a directionally uncontrollable aircraft. A point in favor of root stalling is the resulting nose-down pitching moment which is caused by a decrease in downwash at the tail and the loss in lift over the forward part of a sweptback wing.

A wing stall starting at about 40 percent semi-span is probably a desirable feature for transport aircraft. An increment of several degrees in the angle of attack should be required for the stall to progress from the root to the tip in order to prevent the whole wing from stalling simultaneously and aileron control being abruptly lost.

The effects of wing/fuselage interference, the presence of outboard nacelles and the effects of slipstream may have a large, unpredictable influence on the stalling properties, as shown by several examples mentioned in the references.

\subsection{WING DESIGN FOR LOW-SUBSONIC AIRCRAFT}

The class of aircraft referred to as "lowsubsonic" is interpreted as aircraft with an operational flight envelope which does not allow critical compressibility effects to occur up to the Design Diving speed or Mach number. These dircraft have maximum level flight Mach numbers of less than about $M=.6$ and dive Mach numbers of up to about $M=.7$, depending primarily on the wing thickness ratio. Straight wings can be used and the airfoil thickness / chord ratio may be varied within certain limits in order to obtain a favorable interplay between 'aerodynamic and structura requirements.

The term "straight wing" may be interpreted as a wing with zero sweepback of a spanwise' 1 ihe interconnecting corresponding points at the tip and root sections, and must not be confused with the term "untapered wing". The aerodynamicist will favor a wing with zero sweepback of the quarter-chord line, as this considerably simplifies the aeroaynamic analysis. However, the location of the wing root cannot always be freely chosen in view of considerations pertaining to the general layout or structural design. In order to get the airplane balanced it is necessary to find an optimum location of the center of gravity relative to the aerodynamic center. To this end, the aerodynamic center may be shifted backwards or forwards by applying sweepback or sweep forward, respectively, while the center of gravity is shifted over a smaller distance.

\subsubsection{Planform}

The planform of a wing is defined as the shape of the wing when viewed from direct1y above, as shown in Fig. 7-6. Planform is directly related to aspect ratio and
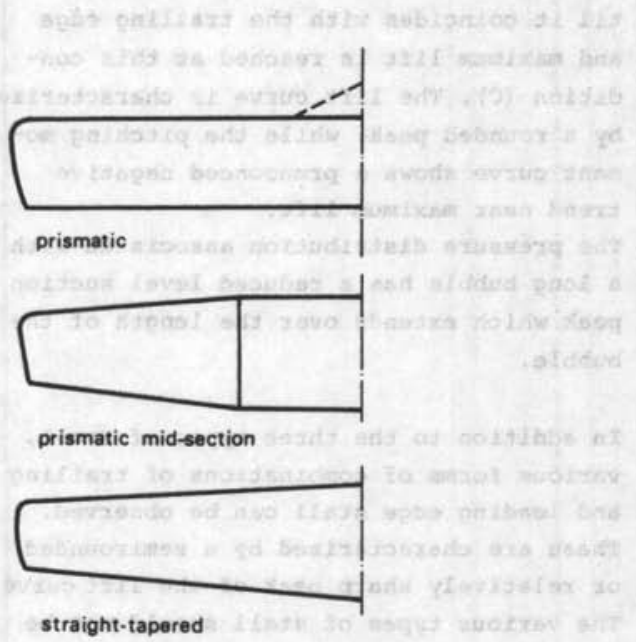

STRAIGHT WINGS

Fig. 7-6. Basic planform shapes for straight wings 
taper* and the main aerodynamic characteristics influenced by planform are the induced drag coefficient and the stalling characteristics.

Great variations in the planform can sometimes be observed in the final design compromise adopted by different airplane designers, even though the design specifications may be almost the same. The choice of the basic wing shape, however, to be made by the individual designer or design team, does not offer quite so much scope as might be expected after observing all the shapes which have been actually adopted on aircraft. Prior to a preliminary design effort, various design offices have gained experience with certain shapes, or may have carried out test programs to investigate aspects of aerodynamic performance, stability and control and structural design. One or more acceptable concepts will emerge from such a program, to be further evaluated during preliminary design.

It is observed that nowadays there are basically three forms of straight wings: the tapered wing, the untapered (rectangular) wing and the wing with a prismatic inner portion and a tapered outboard portion (Fig. 7-6). Tapered wings have been adopted for the majority of aircraft since they offer an efficient solution on account of their low induced drag, high maximum lift, low structural weight and good stowing provisions for the undercarriage. Acceptable stalling characteristics can be obtained, provided the wing is not too sharply tapered.

The untapered wing is attractive from the point of view of manufacture, since only one airfoil contour is involved; this simplifies jigging as there are no compound curvatures. It is aerodynamically inferior to the tapered wing, but may nevertheless be the logical choice for inexpensive private aircraft, where the utilization factor is low and initial cost and cheapness

*The terminology used in this section is explained in Appendix A. of components are important. Untapered wings are well suited for the application of efficient full-span flaps, where the structural complication is outweighed by the relative simplicity of constant-chord flap segments. Untapered cantilever wings are generally of relatively low aspect ratio to save weight, but braced wings may have a high aspect ratio in spite of the absence of any taper (e.g. Short skyvan). Wings with a prismatic inboard section have good aerodynamic characteristics and offer some advantages for the structural design and manufacturing of the mid-section, particularly in the case of twin-engine aircraft with wing-mounted nacelles.

\subsubsection{Aspect ratio}

The aspect ratio denotes the ratio of the wing span to the mean geometric chord. For a given wing area, it provides a direct measure for the wing span:

$\mathrm{b}=\sqrt{\mathrm{AS}}$

Instead of the aspect ratio, which determines the vortex-induced drag coefficient, use is sometimes made of the span loading, which is related to $\mathrm{A}$ and the wing loading:

$\frac{W}{b^{2}}=\frac{W / S}{A}$

It can readily be shown that the span loading is a direct measure for the vortexinduced drag as a fraction of the weight, if the dynamic pressure is fixed. The span loading will therefore be a good criterion in design studies where the restricted field length imposes a limit on the stalling speed and the aim is to $11 \mathrm{ft}$ as high a takeoff weight as possible under the adverse condition of engine failure. This criterion will be used in Section 7.5.4. to make an initial choice of $\mathrm{A}$ and the span for jet-propelled transports.

The climb requirements for propeller transports work out slightly differently. Using the flight mechanics of Section 5.4.3., it can be shown that the parameter 


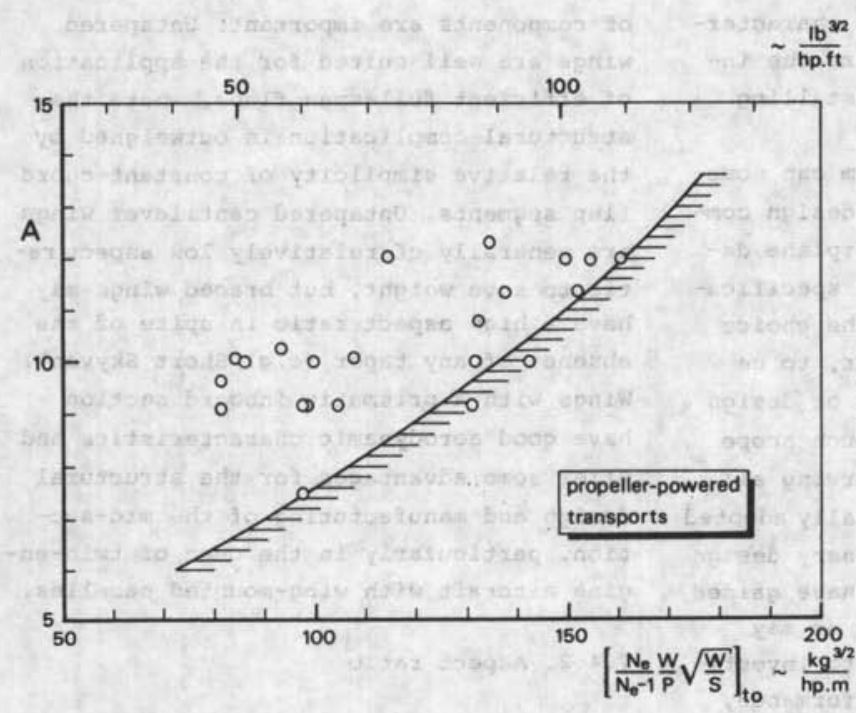

Fig. 7-7. Recommended lower limit for the aspect ratio of propeller transports.

$$
\frac{N_{e}-1}{N_{e}} \frac{P_{t o}}{W_{t o}} / \sqrt{\frac{W_{t_{0}}}{C_{L_{\max }} S}}
$$

is of primary importance here. The reciprocal value of this factor is shown in Fig. 7-7 - where variations in $C_{L}-\max$ are ignored - which may be used as an indication for the minimum acceptable value of

A.

It is recognized that for wing loadings satisfying $(7-7)$ the maximum $L / D$ ratio and the range for given cruising speed are quite sensitive to A. Good range performance may thus be obtained for a highly loaded, high aspect ratio wing, but an efficient flap system will be required to ensure acceptable stalling speeds in this case. The concept of a high aspect ratio wing is therefore a logical one for transport aircraft where the emphasis lies on high cruising efficiency; however, sophisticated high-lift devices are inherent to this design concept.

For light aircraft, the wing loading is usually fairly low, the complication of a sophisticated high-lift system generally being considered as undesirable by most manufacturers. Consequently, the optimum cruising speed for long-range flight may be too slow to make it an attractive speed, particularly when the altitude is limited to some $10,000 \mathrm{ft}(3,000 \mathrm{~m})$. These aircraft are usually flown at the maximum cruise rating and it can readily be shown that a large increase in A results in a relatively modest gain in speed and range. For example, increasing the aspect ratio of an aircraft with $w=3,300 \mathrm{lb}(1,500$ $\mathrm{kg}), \mathrm{S}=160 \mathrm{sq} \cdot \mathrm{ft}\left(15 \mathrm{~m}^{2}\right), P=180 \mathrm{hp}$ and $\mathrm{C}_{\mathrm{D}_{\mathrm{O}}}=.025$ from 6 to 10 results in a speed increment at sea level from 128 knots (238 $\mathrm{km} / \mathrm{h})$ to $134 \mathrm{knots}(248 \mathrm{~km} / \mathrm{h})$, a gain of only 48 .

A high aspect ratio may result in a low drag in the landing configuration, which tends to flatten the approach glide, makes judgment of the landing point more difficult to the pilot and gives the aircraft a tendency to "float" after the landing flare. In addition, a high aspect ratio wing does not favor good maneuverability in roll due to its large damping and the reduced effectiveness of the small-chord ailerons (Ref. 7-96).

In conclusion, moderate aspect ratios between 7 and 9 are usually applied for twinengine general aviation aircraft; for single-engine aircraft these values are usually somewhat lower, e.g. between 5.5 
and 8

\subsubsection{Thickness ratio}

The desirable high aspect ratio for lowspeed transport aircraft can be achieved only if sufficient structure height is available at the wing root, where the bending moment during flight is maximum. In this connection use is often made of the cantilever or overhang ratio, which is defined as the structural wing semi-span, divided by the maximum root thickness. The expression for the wing weight in section 8.4.1. shows that the structural wing weight fraction increases linearly with increasing cantilever ratio, provided all other parameters remain constant.

The cantilever ratio is plotted in Fig. 7-8 for aircraft of various weight categories. Transport aircraft usually have values of between 18 and 22 ; ratios in excess of 25 are rare, even in the case of supersonic transport aircraft. A rather lower value is found for trainers, probably because of the high maneuver load fac- tors for which their wings must be designed.

The favorable trend of low induced drag of a high aspect ratio wing is partly cancelled out by a profile drag increment if the thickness ratio is allowed to increase in proportion to A. Maximum lift is also affected by the thickness ratio, as shown by Fig. 7-9. The trend for basic wing sections is readily explained by recalling that for thin wings the leading edge type of stall dominates, while for thick sections the trailing edge stall is predominant (see Fig. 7-5). The highest maximum $1 i f t$ of conventional, standard NACA airfoil sections is achieved for thickness ratios of 12 to 15 percent chord, where a combined stall will be observed. Recent developments in sections for low-speed aircraft show that higher maximum lift coefficients can be obtained with special sections having a thickness ratio of about 17 percent (Ref. 7-55). The maximum $11 \mathrm{ft}$ coefficient with trailing-edge flaps deflected has a relatively flat maximum for

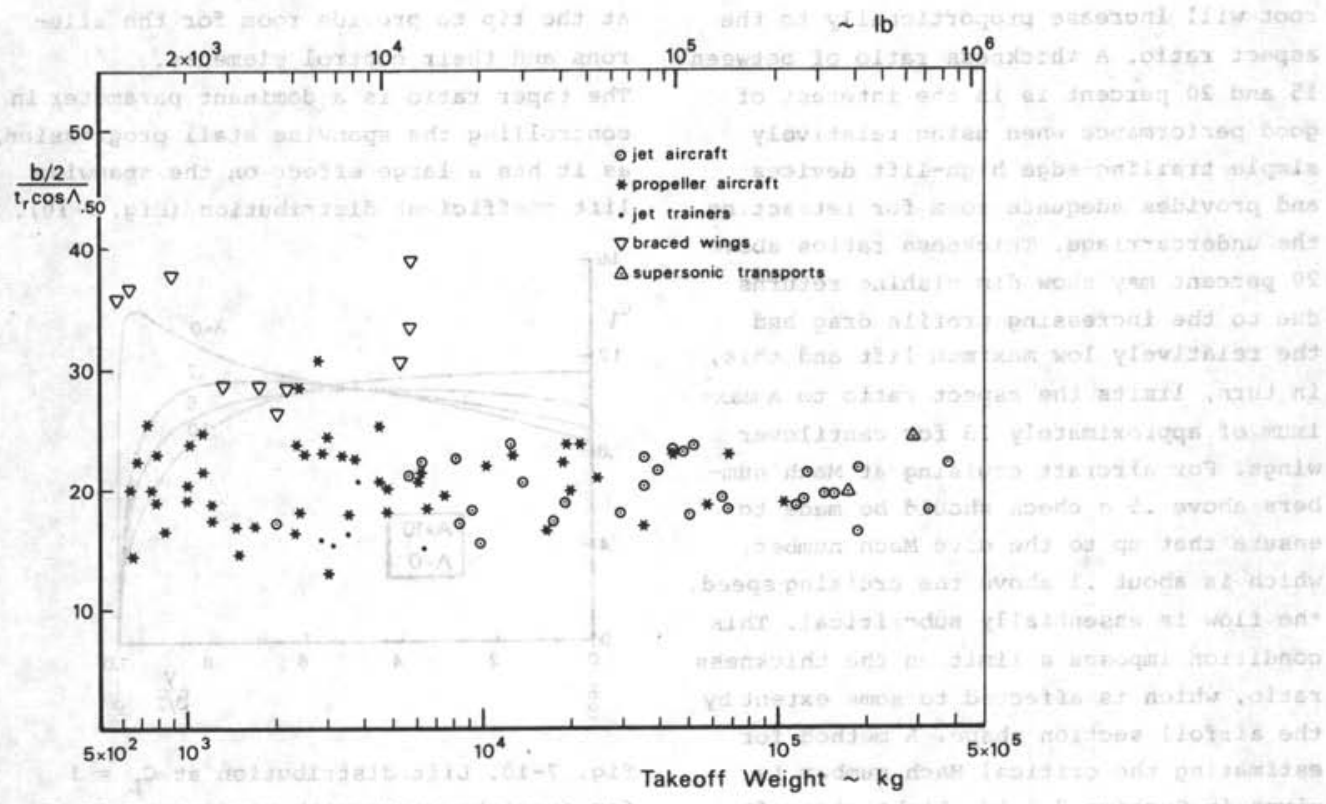

Fig. 7-8. Cantilever ratio vs, aircraft size 


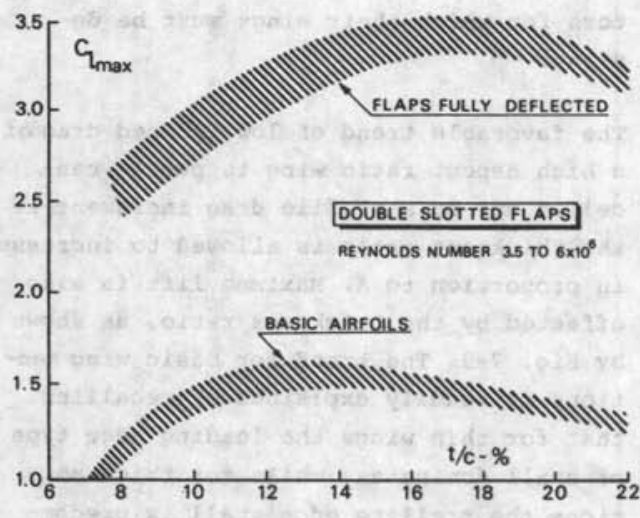

Fig. 7-9. Trends of best maximum lift values of NACA sections vs. thickniess ratio.

thickness ratios between 15 and 20 percent. The reasons for this are explained in Appendix G, Section G-2.3.

It is concluded that the root section thickness of transport aircraft should be chosen such that a good cantilever ratio is obtained. For a given taper ratio this implies that the thickness ratio at the root will increase proportionally to the aspect ratio. A thickness ratio of between 15 and 20 percent is in the interest of good performance when using relatively simple trailing-edge high-lift devices and provides adequate room for retracting the undercarriage. Thickness ratios above 20 percent may show diminishing returns due to the increasing profile drag and the relatively low maximum lift and this, in turn, limits the aspect ratio to a maximum of approximately 13 for cantilever wings. For aircraft cruising at Mach numbers above .5 a check should be made to ensure that up to the dive Mach number, which is about .1 above the cruising speed, the flow is essentially subcritical. This condition imposes a limit on the thickness ratio, which is affected to some extent by the airfoil section shape. A method for estimating the critical Mach number is given in Section 7.5.1d. Light aircraft wings have lower aspect ratios and the best root sections are approximately 15 percent thick.

Tip sections (without flaps) should be between 10 and 15 percent in order to attain a high maximum lift. This reduction relative to the root is also in favor of low structural weight. A minimum practicable thickness should be present on light aircraft in order to provide adequate room for control system elements.

\subsubsection{Wing taper}

The taper ratio $\lambda$ has a great effect on the spanwise lift distribution. The spanwise position of the center of pressure of a half wing moves in the direction of the wing root as $\lambda$ decreases and the root bending moment due to lift decreases accordingly. Since the structural height of the wing root also increases - for a given wing area, span and section shape - a highly tapered wing can be built lighter and with much more torsional rigidity than a rectangular wing. In the case of small aircraft, a practical lower limit to $\lambda$ is imposed by the structural height required at the tip to provide room for the ailerons and their control elements.

The taper ratio is a dominant parameter in controlling the spanwise stall progression, as it has a large effect on the spanwise lift coefficient distribution (Fig. 7-10).

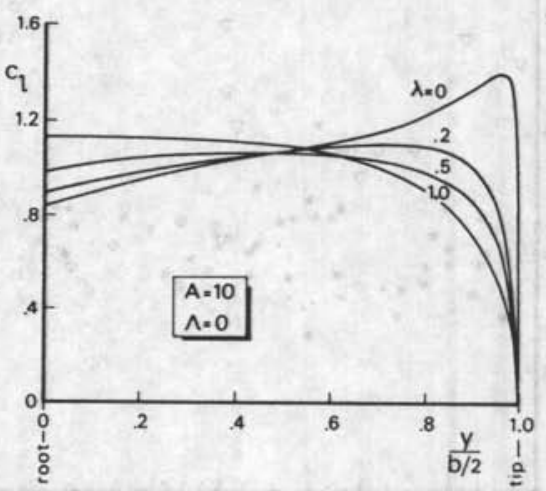

Fig. 7-10. Lift distribution at $C_{L}=1$ for straight wings with various taper ratios 
A first-order approximation of the location where the $c_{\ell}$-distribution is at its peak is:

$n=1-\lambda$

for untwisted, straight-tapered wings. Hence, a wing with constant section properties and a taper ratio of .4 will tend to stall first at 60 percent semi-span, fairly close to the inner part of the aileron. If a wing is tapered sharply, there will also be a notable reduction in the maximum lift coefficient near the tip due to the locally reduced Reynolds number, thus aggravating the tendency towards ear1y tip stall. Although precautions can be taken to shift the initial separation point inboard by means of section shape variation and twist, the amount of taper has a definite limit. Realizing that the vortex-induced drag of a tapered wing is minimum for a taper ratio of about .4 and is insensitive to relatively large deviations from this value, it is concluded that for straight wings taper ratios appreciably below .4 are of little use (Fig. 7-11).

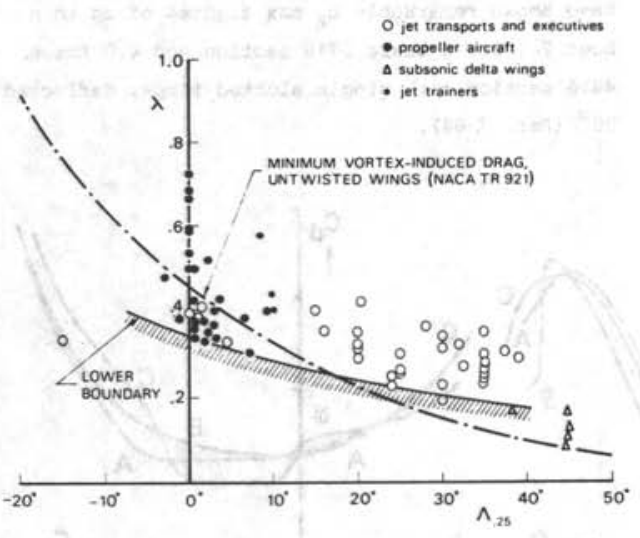

Fig. 7-11. Taper ratics of straight, swept and delta wings.

Once the taper is chosen, the wing geometry is known, provided the area and aspect ratio have also been selected. For straight-tapered wings the tip and root chords are given by: $c_{r}=\frac{2}{1+\lambda} \frac{S}{b}$

$c_{t}=\lambda c_{r}$

and the plan view of the wing may be provisionally drawn.

It will be noted that wings with taper both in planform and in the sectional thickness ratio, airfoil sections between fairing stations will be slighly distorted when linear lofting is used for structural simplicity. A tapered wing with respectively 18 percent and 12 percent thickness ratios at the root and tip does not have a 15 percent thickness ratio at the section midway between root and tip, but at a station closer to the "ip.

\subsubsection{Airfoil selection}

In selecting the airfoil sections the designer must give consideration to several general requirements.

1. The basic airfoil must have a low profile drag coefficient for the range of $11 \mathrm{ft}$ coefficients used in cruising flight.

2. For the inboard sections with flaps extended, the drag must be low in high lift conditions, particularly during the takeoff climb.

3. The tip section should have a fairly high maximum lift coefficient and gradual stalling characteristics.

4. The inboard wing section should have high maximum lift with flaps extended. 5. The critical Mach number should be sufficiently high to ensure that critical compressibility effects are avoided in the case of aircraft reaching dive Mach numbers of approximately .65.

6. The pitching moment coefficient should be of low to moderate magnitude to prevent a high trim drag and torsional moments at maximum dynamic pressure.

7. The aerodynamic characteristics should not be extremely sensitive to manufacturing variations in the wing shape, contaminations and dirt, etc.

8. The wing sections should have the largest possible thickness ratio in the interest of low structural weight. Sufficient internal space must be provided for 
fuel, main gear, mechanical controls and possibly other components.

Al1 these requirements cannot be satisfied by one single airfoil. Spanwise variation of the sectional shape and some measure of compromise will therefore generally be accepted.

For low-subsonic aircraft the selection is usually made from NACA standard sections, to which modifications may be made if necessary, usually during the stage of detailed aerodynamic design. The effect of systematic variations in the profile shape has been the subject of thorough investigations by the NACA, resulting in many series of satisfactory airfoil sections. The relevant findings have been presented in the form of very complete information. Refs. $7-5$ and $7-51$ in particular are very useful tools for the designer, and the use of this data has greatly simplified the choice of a suitable airfoil for conventional aircraft. The observations made in Ref. 7-5 (Chapter 7) are fairly complete as far as wing sections are concerned, while Ref. 7-96 gives a systematic treatment of the effect of airfoil variation on stalling characteristics, using the successful method or R.F. Andersen (Ref. 7-70). A survey will follow of the most commonly used NACA sections*, examples of which are shown in Fig. 7-12.

a. The NACA four-digit wing sections basically constitute a synthesis of early Göttingen and clark $\mathrm{Y}$ sections, empirically developed on a basis of pre-war experimental data. Both the thickness distributions and the mean lines are defined in the form of polynomials; the sections have a near-elliptic shape. The maximum camber is at approximately the mid-chord posit1on.

Although the sections in the 4-digit series are by no means low-drag profiles, the drag increase with lift is fairly gradual. The cambered sections have relatively high maximum lift and the stalling is fairly docile. These properties have marked the 2412 and 4412 sections, for example, as being suitable tip sections for the wings of light aircraft and tailplanes. In view of the gradual changes in drag and pitching moment with lift, the 4-digit sections are frequently used for light trainers, which often $f l y$ in different conditions.

Recent experiments with 16 percent thick sections have shown remarkable $c_{\ell}$-max figures of up to about 2.1 for a basic 6716 section and 4.0 for a 4416 section with single slotted flaps, deflected $30^{\circ}$ (Ref. 7-64).

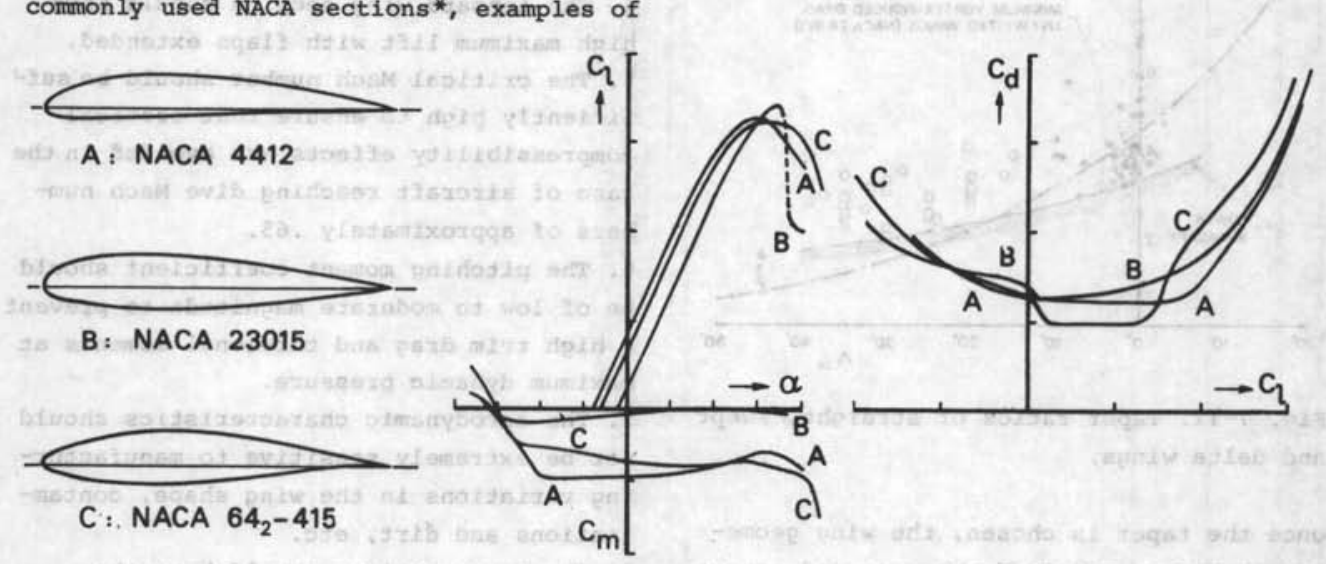

Fig. 7-12. Characteristics of NACA standard series airfoils at $R=6 \times 10^{6}$ (Ref. $\left.7-5\right)$.

* Terminology explained in Appendix A, Section A-2.3. 
b. The NACA 5-digit wing sections have the same thickness distribution as the 4-digit series, but the mean lines are different, having their maximum ordinate further forward. The well-known 230-series airfoils have the maximum camber at the .15 chord point. These sections have the highest maximum lift of the standard NACA sections, but the stalling behavior is not particularly favorable and rather sensitive to scale effects. For wings where high lift performance is a prerequisite, the 230-series sections have been frequent1y used, sometimes combined with a 4-digit section at the tip.

\section{c. The NACA 6-series ("laminar flow") of} wing sections is the outcome of a succession of attempts to design airfoils by (approximate) theoretical methods, aimed at achieving low profile arag in a limited range of lift coefficients: the "sag" or "bucket" in the low-drag range. The laminar boundary layer over the forward part of the section is stabilized by avoiding pressure peaks, keeping the local velocities low and applying a favorable pressure gradient over the forward part of the upper surface. The extent of laminar layer is limited by the separation of the turbulent boundary layer over the rear part. The low supervelocities on these airfoils also favor the attainment of a high critical Mach number.

Due to the relatively sharp nose of thin laminar flow sections, their maximum lift is notably below that of the $4-$ and 5digit series, although the difference for the thicker cambered sections is negligible; these sections also exhibit a docile stall. The profile drag, although very low under ideal conditions, is sensitive to surface roughness, excrescences and contaminations. Special structures are therefore needed to maintain the laminar flow, and on practical wing constructions of transport aircraft the potentially large extent of laminar layer will not normally be realized.

A great advantage of the 6 -series is that sectional properties have been tested extensively and reported systematically. The designer is thus provided with a tool for establishing the best sectional shape by systematically varying the shape parameters. A modification to the standard series is the A-series (Ref. 7-52) in which the sharp trailing edge angle is replaced by a larger one, resulting from straight contours which run from 80 percent chord backwards.

Having decided on the series of sections to be used, the designer will have to choose the various shape parameters. THICKNESS/CHORD RATIO: see Section 7.4.3. LOCATION OF MAXIMUM THICKNESS: the further back this point is chosen, the lower the minimum profile drag (of smooth profiles) and the higher the critical Mach number at the design $c_{\ell}$. This works out at the expense of $\mathrm{c}_{\ell}$-max and profile drag at high lift. For these reasons, the 63- and 64sections are the most popular amongst the 6-series airfoils.

(MAXIMUM) CAMBER: determines the angle of attack for zero lift, the pitching moment coefficient, the lift coefficient for minimum profile drag and $c_{\ell}$-max. A large camber is in the interest of high $c_{\ell}$-max, but the tail load required to trim the aerodynamic pitching moment may cause toomuch extra drag. The camber is usually chosen so that in normal cruising flight the section operates at its design $c_{\ell}$. Little camber is used on trainers, where a requirement exists for acceptable characteristics in inverted flight.

SHAPE OF THE MEAN LINE: a forward location of the point of maximum camber results in a high $c_{\ell}$-max with a leading-edge type of stall at normal thickness ratios. A lower $\mathrm{c}_{\ell}{ }^{-\max }$ and a more gratual stall are obtained when the maximum camber is further back.

7.4.6. Stalling characteristics and wing twist

The following observations, originally 
made in Ref. 7-71, are considered as a good starting point for achieving the desirable stalling characteristics of straight wings, specified in Section 7.3.: 1. The point of initial stalling should be sufficiently far inboard, the best location being at about 40 percent semi-span from the root.

2. Stall progression should be more rapid to the inboard than to the outboard sections.

3. The margin in $c_{\ell}$ at 70 percent of the semi-span from the root - approximately at the inboard end of the aileron - should be at least. 1 in the condition where separation occurs first.

Considering the practical measures available to produce the desirable characteristics, it is possible on straight wings of moderate to high aspect ratios to choose a suitable spanwise variation of the local and naximum lift coefficient. An example is shown in Fig. 7-13. The designer is also

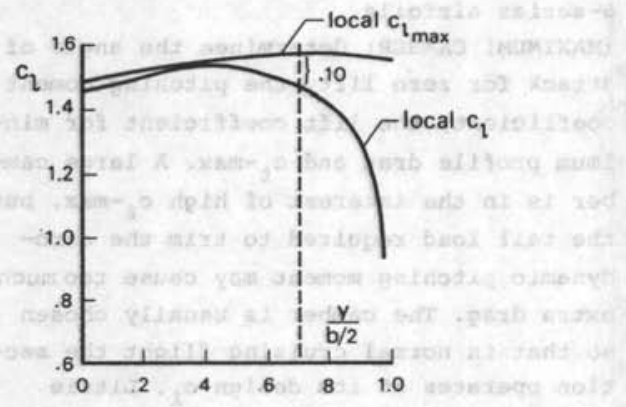

Fig. 7-13. Calculated spanwise lift distribution at high incidence (flaps ups)

concerned with the abruptness of the stall progression at slightly higher angles of attack and he may try to gain an impression of the chordwise stall progression by using Fig. 7-5 and the measured sectional characteristics.

The spanwise lift distribution* is influenced primarily by the wing planform and

"A survey of prediction methods is,given in Appendix E, Section E-4.2. wing twist (washout). The type of airfoil section has little effect in the linear range of incidences in view of the generally small spanwise variations in the liftcurve slope. The curve of local $c_{\ell}$-max is determined exclusively by the local sectional shape. The effect of variations in the taper ratio, aspect ratio and washout on stalling characteristics, $C_{L}-\max$ and induced drag may therefore be investigated without making any decision on the section to be used, which saves a lot of work. The large number of wing shapes to be studied may be reduced by making several restrictions, for example:

1. Although the aspect ratio is important from aircraft performance considerations, its effect on the stalling characteristics is generally small.

2. An aerodynamic washout of more than about five degrees results in unacceptably large induced drag increments of the order of 5 to 10 counts.

3. Low taper ratios may be used only on wings with thick root sections of the 4and 5-digit series and tip thickness ratios of about 12 percent.

4. The effect of wing taper on $C_{L}-\max$ may be much larger for 5-digit series airfoils than for 4- and 6-series, particularly if the Reynolds number at the tip is below 2 million.

There is no chain of logic to show how the designer will arrive at a (provisional) solution. The basic wing shape will finally be developed after many hours of wind tunnel work. Modifications to the basic standard section may appear desirable and during flight tests stall control devices may prove unavoidable, even though theoretical predictions and wind tunnel tests have not shown any deficiencies.

It should also be noted that most theoretical methods do not take account of any wing/fuselage interference effects. For high-wing aircraft, where the flow interaction is confined mainly to the less critical lower surface, these effects are small. The low-wing position introduces the largest interference effects, but these can usually be controlled by local fuselage contour modifications 
and/or adequate root fairings. Established theoretical methods for predicting the characteristics of faired wing/fuselage combinations are not available and the final geometry of these fairings is determined by wind tunnel or flight experiments. Propeller slipstream effects near maximum lift generally result in unstalling the wing in the areas directly influenced by the slipstream. In flight tests aimed at establishing stalling speeds these effects are small as a power-off condition is then required. Power-on stalls may, however, cause a completely different type of behavior, depending on the configuration.

\subsection{WING DESIGN FOR HIGH-SUBSONIC AIR-} CRAFT

In the aerodynamic design of high-performance aircraft, emphasis is placed on speed as a major factor contributing to the economy and operational suitability of the conceptual design. If we assume for the moment that the type of powerplant to be installed and its rating are fixed, the main factor in determining the speed is the drag area in the case of relatively slow transports and light aircraft, as shown in Fig. 7-14. For a given flying al-
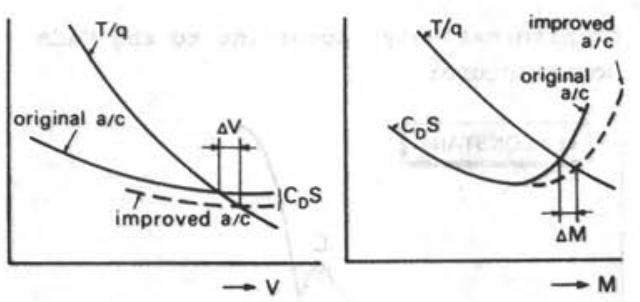

a. LOW-SUBSONIC AIRCRAFT

Fig. 7-14. The basic high-speed design problems of improving low-subsonic and high-subsonic aircraft.

titude any improvement in the speed $\Delta \mathrm{V}$ may be obtained by a reduction of the drag area $C_{D} S$ - for example by minimizing the wetted area, streamlining, reducing interference, optimizing the wing loading and aspect ratio, etc.
For high-subsonic aircraft these aims are still present but, contrary to the situation with low-speed aircraft, the region of compressible flow is penetrated intentionally in order to attain as high a cruising speed as possible. The problems of aerodynamic design in these aircraft mainly relate to attaining a high critical Mach number, avoiding undesirable flight characteristics at off-design conditions (maneuvering, gustiness, speed overshoots) and providing good low-speed characteristics of the sweptback wing.

The basic opportunities for attaining a high cruise Mach number are the adoption of sweepback (or sweep forward), reduction of the thickness/chord ratio, design of improved airfoil sections and optimum distribution of spanwise camber and twist. The use of a moderate wing loading and aspect ratio is of some help, but may conflict with other objectives of performance optimization. The application of wing/ fuselage blending, fairings and anti-shock bodies may be considered, provided they do not conflict with the structural and layout design.

The aerodynamic design problems are by no means different in the case of a new aircraft which is not required to fly faster than the aircraft to be replaced. The advanced technology may then be used to increase the thickness and/or aspect ratio, or to reduce the angle of sweepback.

This section will be confined to the contribution of the wing only; compressibility effects in other areas, such as the nacelle/airframe junction, may have an appreciable effect (see Section 6.5.).

7.5.1. Wing sections at high-subsonic speeds

a. Subcritical speeds.

In the subcritical region the flow around an airfoil in two-dimensional flow is subsonic throughout. The effects of compressibility on the pressure distribution are well described by potential flow methods, known as the Prandtl-Glauert correction, 
$c_{p}=\frac{C_{p_{1}}}{11-M_{\infty}^{2}}$

or more accurate approximations, e.g. the von Karman-Tsien relation. In $(7-29) C_{p}$ is the pressure coefficient,

$C_{p}=\frac{p-p_{\infty}}{q_{\infty}}$

and the subscript $i$ refers to the incompressible situation $\left(M_{\infty} \ll 1\right)$.

Fig. 7-15 shows both the effect of sub-

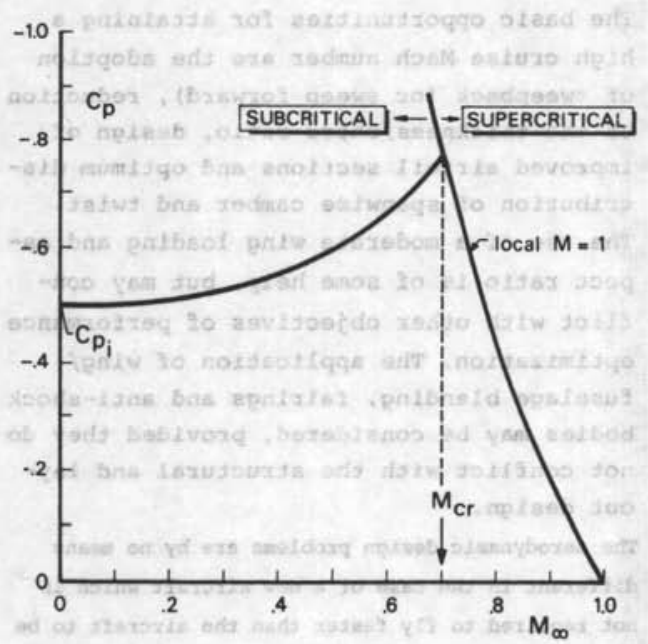

Fig. 7-15. Variation of the pressure coefficient with Mach number and determination of the critical Mach number

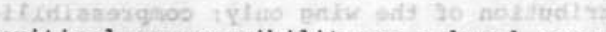
critical compressibility on a local pressure coefficient and the boundary of the subcritical region, which is defined by

$c_{p_{c r}}=\frac{2}{\gamma M_{\infty}{ }^{2}}\left[\left\{\frac{2+(\gamma-1) \cdot M_{\infty}{ }^{2}}{\gamma+1}\right\}^{\gamma /(\gamma-1)}-1\right](7-31)$

This equation can be derived by using the Bernoulli equation for compressible flow and substituting $\mathrm{M}=1$ for the local velocity (see, for example, Ref. 7-7).

The critical Mach number of an airfoil sec$t i o n$ is defined as the free stream Mach number for which sonic flow is reached at the point of minimum pressure. Provided the value of $c_{p}$ in Fig. 7-15 refers to this pressure, the critical Mach number is defined by the intersection of both curves, assuming a constant angle of attack. The critical Mach number is thus easily determined from the low-speed pressure distribution, and for NACA standard airfoils this method is used to compute $\mathrm{M}_{\mathrm{Cr}}$ (Ref. 7-51).

Fig. 7-16 shows that the effects of subcritical compressibility on the profile drag and pitching moment coefficients at constant angle of attack are small, while the lift coefficient - and hence the liftcurve slope - are affected in a similar fashion to $\mathrm{C}_{\mathrm{p}}$.

b. Supercritical speeds.

In the case of positive $\alpha$, regions of supersonic flow will appear on the upper surface when $M_{C r}$ is exceeded. As soon as this region terminates in a shock wave of appreciable strength, which thickens the boundary layer, the drag increases noticeably. The drag-divergence (or drag-critical) Mach number is defined as $M_{\infty}$ for which:

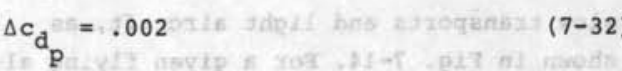

or alternatively, according to the NACA nomenclature:

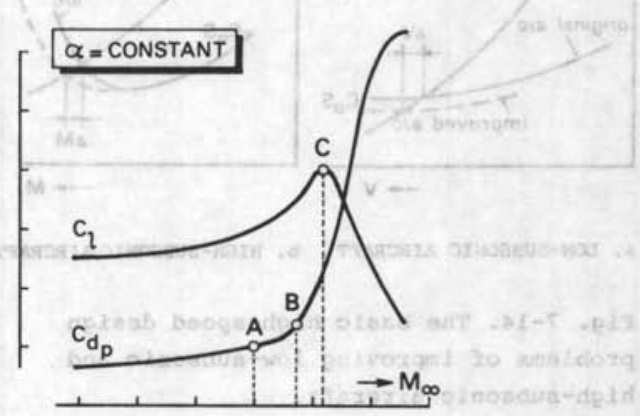
A: critical pressure - $\mathrm{M}_{\mathrm{cr}}$
B: drag-divergence - $\mathrm{M}_{\mathrm{Cr}}$
C: lift-divergence- $\mathrm{M}_{\mathrm{Cr}_{\mathrm{L}}}$

Fig. 7-16. Section lift and drag coefficients vs. Mach number 
$\mathrm{dc}_{\mathrm{p}} / \mathrm{dm}_{\infty}=.10$

An increase in $M_{\infty}$ above this speed (point B in Fig. 7-16) results in a progressive rise in drag. The lift continues to increase until - at point C - a shock appears on the lower surface of the section. At this point - the lift-critical or liftdivergence Mach number - the lift coefficient diverges from its previous trend. As from this point onwards, $d c_{\ell} / d M_{\infty}$ is negative, a flight regime of longitudinal instability ("tuck under") becomes manifest. This must be neutralized by an artificial stabilization system ("Mach trimmer") or by aerodynamic means.

The presence of strong shock waves may lead to separation of the flow and large pressure fluctuations, experienced as an excitation of the wing. This phenomenon, referred to as buffet, constitutes a limitation to the operational flight regime.

c. Trends in high-speed section design. Much work is being devoted to the development of airfoil sections, the objective being to increase the thickness ratio for given design conditions (drag-divergence Mach number and lift coefficient) and to improve the off-design characteristics. Early attempts in this direction were based on designing for low supervelocities in order to postpone supercritical flow to high Mach numbers. They were followed by designs where regions of local supersonic flow were admitted on the forward part of the airfoil, terminating in near-isentropic and shock free compression. These designs allow greater thickness ratios to be used for the same design Mach number and $c_{\ell}$.

The aerodynamic analysis of mixed (subsonic and supersonic) flows is only possible with sophisticated methods, and a certain amount of empiricism is still common practice here. Several aspects of existing aerodynamic design concepts will be further explained, using the examples in Fig. 7-17. It is not suggested here that in modern section design a choice must be

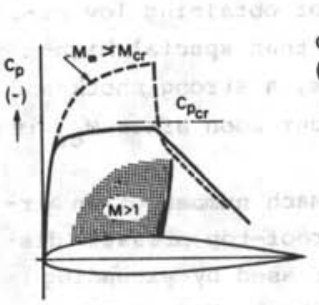

a. Conventional section with roof-top pressure distribution

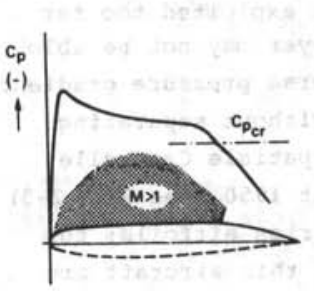

c. Supercritical upper surface pressure distribution

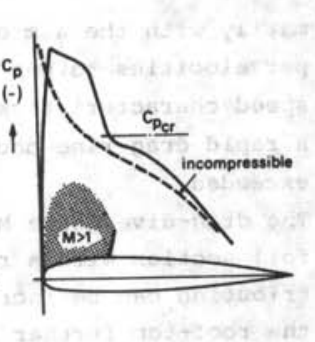

b. Peaky upper surface pressure distribution

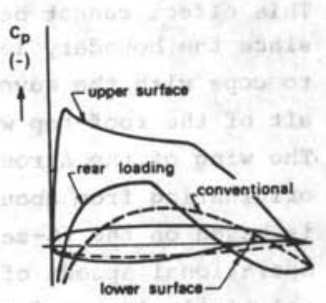

d. Rear loading airfoil compared with conventional airfoil (lower surface)

Fig. 7-17. Aerodynamic design concepts for high-subsonic airfoil sections in the design condition

made from the various concepts; a mixture of these will be present in a practical wing.

ROOF-TOP PRESSURE DISTRIBUTIONS have a gradually changing or approximately constant upper surface pressure over the forward part of the section, which delays the critical Mach number by virtue of a uniform velocity at the design condition (F1g. 7-17a). Slightly above this speed large regions of supersonic flow will appear and the associated suction forces then occur near the crest* of the airfoll or behind it. The NACA 6-series have this type of pressure distribution at subcritical speed for a limited region of $c_{\ell}$ values. Since the pressure distribution is designed pri-

* The highest point of the airfoil relative to the free flow direction. 
marily with the aim of obtaining low supervelocities rather than special highspeed characteristics, a strong shock and a rapid drag rise occur soon after $\mathrm{M}_{\mathrm{cr}}$ is exceeded.

The drag-divergence Mach number of an airfoil section with a roof-top pressure distribution can be increased by extending the roof-top further back. For example, a crest at about 60 percent chord results in a $\Delta M_{C r_{D}}$ value of about .04 relative to a section with the crest at 30 percent. This effect cannot be exploited too far since the boundary layer may not be able to cope with the adverse pressure gradient aft of the roof-top without separating. The wing of the Aerospatiale Caravelle, originating from about 1950 (see Fig. 2-3) is based on the 65-series airfoils; the operational speeds of this aircraft are relatively low, and allow a reasonable thickness ratio of 12 percent.

A PEAKY PRESSURE DISTRIBUTION (Fig. 7-17b), pioneered by Piercy at the NPL, and by others, intentionally creates supersonic velocities and suction forces close to the leading edge. The airfoil nose is carefully designed so that near-isentropic compression and a weak shock are obtained. The suction forces have a large forward component and the drag rise is postponed to high speeds. As compared with conventional sections of the same thickness ratio, the value of $\mathrm{M}_{\mathrm{Cr}}$ is approximately .03 to .05 higher and the off-design behavior is improved. This type of airfoil has been used on the BAC 1-11, VC-10 and DC-9 aircraft. The technique employed in designing peaky airfoils was highly empirical.

Recent advances in high-speed airfoil development have resulted in SUPERCRITICAL SECTIONS - first proposed by R.T. Whitcomb which have a relatively flat upper surface contour (Fig. 7-17c). With these sections a much greater extent of shock-free supersonic flow can be created than in the peaky design. The amount of flattening of the upper surface is limited by the pressure rise which the boundary layer can accept without separation. An extensive NASA program has been conducted to investigate the potentials of this wing technology (Ref. 7-39). Large gains in dragdivergence Mach number and off-design performance are claimed if rear loading is also used in designing supercritical airfoils.

REAR LOADING (Fig. 7-17d) is a method for improving high-speed performance by generating lift at the rear part of the airfoil, mainly by pronounced camber of the lower surface.

The effect of rear loading may be explained in different ways. For a given thickness ratio and $c_{\ell}$ the supervelocities at the upper surface can be reduced, and $\mathrm{M}_{\mathrm{Cr}}$ can be increased, by generating higher pressures at the lower surface. Alternatively, if an airfoil is considered for which the upper surface has a critical flow condition, the rear part is contoured in such a way that a higher lift is generated, maintaining the same thickness ratio and $M_{C r_{D}}$. Finally, for a given $M_{C r_{D}}$ and $c_{\ell}$ the front part of the airfoil may be beefed up until near-sonic flow is created at the lower surface, while the associated suction forces are cancelled out by high pressures near the trailing edge. Sections of high thickness ratio can thus be obtained for given design conditions.

The extent to which the rear loading can be accommodated is limited by the nosedown pitching moment and trim drag associated with the rear location of the center of pressure. It may also prove difficult to install an effective flap system in the sharp, cambered rear part of such a section. The European Airbus A-300 wing is an example of a wing design with a limited amount of rear loading (Ref. 7-28).

d. Criteria for section characteristics in design and off-design conditions. 
The conditions to be considered in selecting airfoil sections are illustrated in Fig. 7-18. The sections should be selected

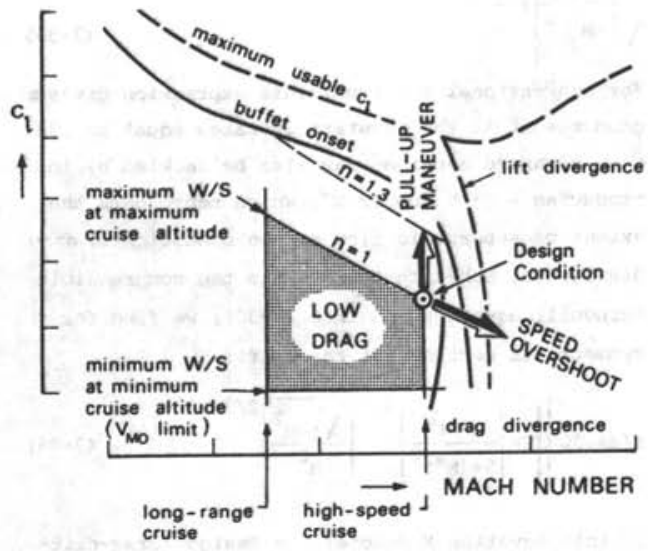

Fig. 7-18. Criteria for the selection of high-speed sections

primarily in order to achieve low drag at the high-speed cruise Mach number and the highest lift coefficient relevant to this speed. This point is labeled Design Condition in the figure. The lift coefficient in this condition corresponds with the maximum cruise altitude specified for the aircraft and the highest wing loading anticipated for cruising flight at that altitude.

The drag characteristics of an airfoil section selected on this basis will generally also be satisfactory at lower $11 \mathrm{ft}$ coefficients and reduced Mach numbers (shaded area in the figure) which correspond to lower cruising altitudes and speeds, down to the long-range cruise Mach number. This region of cruising conditions must be specified for any highspeed aircraft design in order to check the drag of the profile sections. Offdesign conditions outside this region result from aircraft maneuvering and gustiness and may be associated with alleviated requirements relating to drag. A distinction may be made here between two types of maneuvers: overshoots in speed to increased
Mach numbers, without significant change in $c_{\ell}$, and pull-ups or turning flights. with increased $c_{\ell}$ at constant Mach number. The overshoot in speed has to be demonstrated in certification flight testing to show compliance with requirements relating to stability and maneuvering characteristics up to the Dive Mach number $M_{D}$. In this Mach number region the interaction between shocks of increasing strength and the boundary layer will result in flow separations. A rapid rise in drag beyond the design Mach number caused by these effects may be conducive to smaller overshoots in speed, but a rapid lift divergence may be unacceptable as it can adversely affect stability. Shock-induced separations and shock waves hitting the tailplane cause buffeting vibrations, which should remain sufficiently mild in the dive and the ensuing pull-up maneuver to restore the normal flight condition. Buffet will also occur if $c_{\ell}$ is increased for constant $M$ during pull-up or turning maneuvers and in gusty weather. Buffet onset will be experienced as a light vibration when the boundary layer starts to separate. The violence of the excitation will subsequently increase with the angle of attack through conditions of light and moderate to heavy buffet - a condition which defines the upper limit to the range of useful lift coefficients at high speeds. It is generally acknowledged, though not considered as a formal requirement, that for transport aircraft during cruising flight a maneuver load factor of at least $1.3 \mathrm{~g}$ must be available without any buffeting vibrations occurring. It is sometimes additionally laid down that load factors of up to 1.6 or normal gust velocities up to $12.5 \mathrm{~m} / \mathrm{s}(41 \mathrm{ft} / \mathrm{s})$ and a wavelength of $33 \mathrm{~m}$ (110 ft) must be covered with only a moderate amount of buffet, this condition being taken as the maximum penetration of the buffet regime acceptable for civil operations.

An important additional requirement for the airfoil section is therefore that it should be capable of producing lift with- 
out flow separation up to 1.3 times the design $c_{\ell}$. Cruising performance may be seriously impaired if the buffet boundaries do not permit this reserve in $c_{l}$ in the design condition. A reduced cruising altitude is generally unfavorable and since the optimum cruise wing loading is high for high-speed transports (Section 7.2.1.), much attention is devoted in the design stage to the development of section and wing shapes which are suitable both at design and off-design lift coefficients providing generous buffet margins.

It should be noted that the criteria presented here are basically applicable to the three-dimensional wing, or rather the complete aircraft. It will be shown, however, that for high aspect ratio wings these requirements can be transformed into airfoil selection criteria.

\section{e. Thickness ratio and drag-divergence}

Mach number.

An objective of high-speed airfoil design is to obtain a section with the highest possible thickness for a specified combination of $M_{\infty}$ and $c_{\ell}$. In view of the very complex character of mixed flow, concise methods for making predictions of aerodynamic characteristics, such as drag- and lift-divergence Mach numbers, are not available. Faced with this problem, the preliminary design engineer will have to consult an aeronautical department or establishment equipped with facilities to tackle the problems of supercritical flow. An estimation of the permissible thickness ratio of a section for a given value of $M_{C r_{D}}$ may be obtained from available design charts or from the following suggested approach. Sectional data at low speeds $\left(M_{\infty} \ll 1\right)$ indicate that the lowest pressure coefficient of symmetrical sections at zero lift is approximately:

$$
\left(\mathrm{c}_{\mathrm{p}_{1}}\right)_{\min }=\text { constant } \cdot\left(\frac{\mathrm{t}}{\mathrm{c}}\right)^{1.5}
$$

where the constant is determined by the thickness distribution. Using $(7-29)$ and $(7-31)$ it is easily found that for a given critical Mach number the permissible thickness is represented by: $t / c=$ constant $\left\{\frac{2}{\gamma M_{c x}^{2}} \mid 1-\left\{\frac{2+(\gamma-1) M_{c r}^{2}}{\gamma+1}\right\}^{\gamma /(\gamma-1)}\right]$

$\left.\sqrt{1-M_{c r}^{2}}\right|^{2 / 3}$

For conventional sections, this expression gives a good result if the constant is taken equal to .24 . More advanced sections may also be tackled by introducing a Mach number $\mathrm{M}^{*}$, which represents the extent of supersonic flow at the condition of drag divergence. Substituting $M^{*}$ into the compressible Bernoulli equation and into $(7-30)$, we find for symetrical sections at zero lift:

$t / c=.30\left\{\left[1-\left\{\frac{5+M^{2}}{5+\left(M^{*}\right)^{2}}\right\}^{3.5}\right] \frac{\sqrt{1-M^{2}}}{M^{2}}\right\}^{2 / 3} \quad(7-36$

In this equation $M$ denotes the design (drag-critical) Mach number for which the airfoil is to be designed.

The factor $M^{*}$ in $(7-36)$ has no physical meaning and is merely a figure defining the aerodynamic sophistication employed to obtain supercritical flow at the design condition. Good results are obtained by taking:

$M^{*}=1.0$, conventional airfoils; maximum

$t / c$ at about $.30 \mathrm{c}$

$M^{*}=1,05$, high-speed (peaky) airfoils, 1960-1970 technology

$M^{*}=1.12$ to 1.15 , supercritical airfoils

$(7-37)$

It is difficult to make adequate allowance for the effects of airfoil camber and $11 \mathrm{ft}$. Provided the airfoil operates at the design $c_{\ell}$, it is possible to use an approximation by reducing $M^{*}$ in $(7-36)$ by, 25 times the design $c_{\ell}$ for $c_{\ell}$ up to .7 .

\subsubsection{Wing design for high speeds}

a. Simple wing sweep theory and its $11 \mathrm{mi-}$ tations.

Sweeping back the wing postpones the effects of critical compressibility to a certain extent, an effect which can be explained by the "simple sweep concept". This assumes an infinitely long sheared wing (Fig. 7-19a), for which the supervelocities and the pressure distribution are determined solely by the velocity com- 


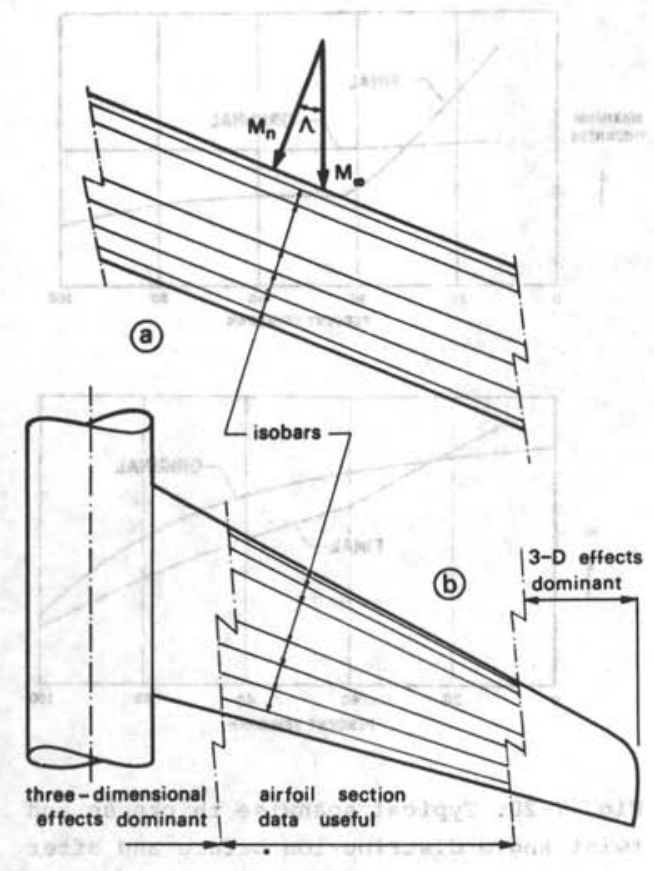

Fig. 7-19. The simple wing sweep theory for infinitely long and high aspect ratio wings

ponent normal to the leading edge, $M_{n}=M_{\infty} \cos \Lambda$. The section normal to the leading edge is thus the relevant shape to be considered. The simple sweep concept yields the following relationships. 1. The thickness ratio and effective angle of attack of the normal section are greater than those of the streamwise section by an amount equal to $1 / \cos \Lambda$.

2. The wing lift coefficient $C_{L}$ is equal to $c_{\ell}(\cos \Lambda)^{2}$, where $c_{\ell}$ is the normal section lift coefficient.

3. The critical Mach number of the wing is $M_{c r} / \cos \Lambda$, where $M_{c r}$ is the normal section's critical Mach number at the normal section's $c_{\ell}$.

These relationships show that potentially the critical compressibility effects may be postponed to a free stream Mach number which is increased relative to that for a straight wing by a factor $(\cos \Lambda)^{-1}$. The normal section shape, however, has to be designed to cope with a design value of $c_{\ell}$ which is greater than the wing lift coefficient by a factor $(\cos \Lambda)^{-2}$. For example, a wing sweptback by 35 degrees, and operating at $M=.85$ and $C_{L}=.4$, will have normal sections designed for operation at $\mathrm{M}=.7$ and $c_{\ell}=.6$.

In early applications of sweptback wings it became apparent that the gain actually obtained in $\mathrm{M}_{\mathrm{cr}}$ was smaller than predictions based on the simple sweep theory had indicated; in effect a factor $(\cos \Lambda)^{-1 / 2}$ was achieved, rather than $(\cos \Lambda)^{-1}$. The reasons for this observation stem from the finite span and the detrimental effects of fuselage and nacelles. For an untwisted sweptback wing with constant section shapes the lift is concentrated towards the tip and the outboard sections work at relatively high $c_{\ell}$ values, resulting in a local reduction in $\mathrm{M}_{\mathrm{cr}}$. In addition, root and tip effects decline the isobars in a direction normal to the flow, so that the sweep effect is, in fact, reduced. The points of lowest pressure will be shifted backwards near the root and forwards near the tip. The natural curved path of the flow over a swept wing is hampered by the fuselage and nacelles, if any, and this results in a system of expansion waves, terminating in a spanwise shock. The various effects combine to form a complex pattern of shocks and expansion waves, appearing first on the outboard wing and next on the rear and front part of the inner wing. The complete picture is sometimes referred to as a $\lambda$-shock. The techniques developed to restore the full effect of wing sweep are therefore based on eliminating these detrimental effects by reshaping the wing and bodies in the areas affected (Fig. 7-19b). The mid-section of a high aspect ratio wing, however, is only slightly affected by three-dimensional effects, provided suitable measures have been taken to counteract the root and tip effects. Two-dimensional section shapes and data may therefore be used to define this part of the wing. 
b. Aerodynamic optimization of the wing and drag-divergence Mach number.

The objective of high-speed wing design is to obtain a pattern of approximately straight isobars swept back at an angle at least equal to the wing sweepback angle, the upper surface generally being critical for the drag divergence*. If this aim is achieved, the flow will be approximately two-dimensional and the drag-divergence will occur at the same Mach number everywhere along the span. A detailed examination of the very complex optimization procedures of this type is outside the scope of this book, but it is considered appropriate to mention some of the measures which may be taken, although not all of them are required for each design.

1. The sweep angle and thickness ratio between approximately 30 and 80 percent semi-span from the root are based on a pressure distribution obtained from the simple sweep concept.

2. The points of maximum thickness at the root and tip are shifted forwards and backwards, respectively. Streamwise tips are used on the BAC VC-10.

3. The lift on the inboard wing is increased by a negative twist (washout). The example in Fig. 7-20 shows a linear lofted twist compared with the more complex twist distribution required to increase the critical Mach number.

4. For low-wing designs the pressures tend to be increased at the lower surface. This may be turned to advantage by locally thickening the lower part of the section and bending the nose of the root section slightly upwards. This results in a root section with negative camber which is a few percent thicker than the outboard wing (Fig. 7-20).

5. The sweep angle near the root section may be increased by introducing a kink in the leading edge (Fig. 7-21). Incidental1y, the kink in the trailing edge, observed

* Wings with rear loading sections may form an exception in view of the nearcritical conditions at the lower surface
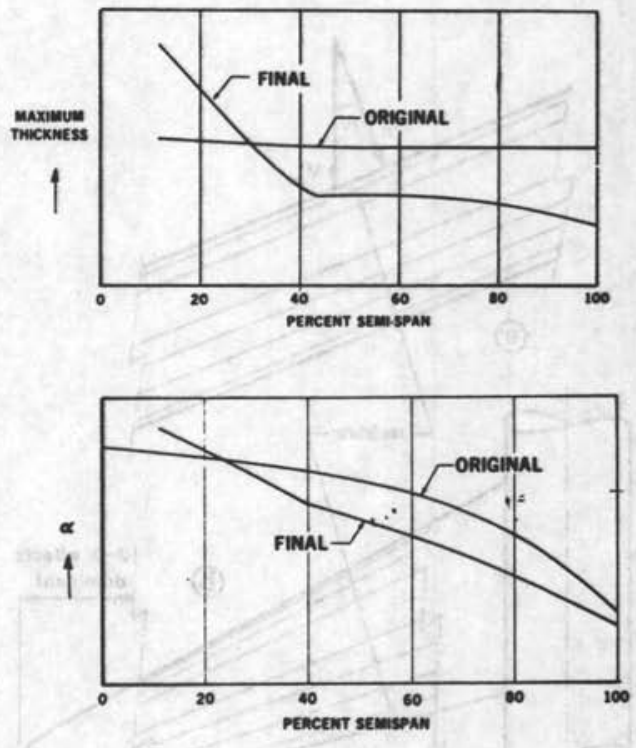

Fig. 7-20. Typical spanwise thickness and twist angle distribution before and after aerodynamic optimization (Ref. 7-24).

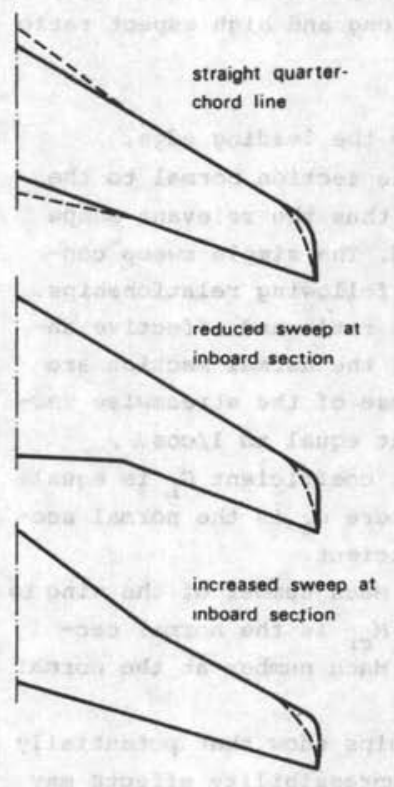

SWEPTBACK WINGS

Fig. 7-21. Sweptback wing planforms. 
in many high-subsonic transport, is used to provide internal space for retracting the undercarriage (see Section 7.8.2.).

6. Adequate wing/fuselage fairings must be incorporated as a means of obtaining a good area distribution (see Fig. 2-9c). The cylindrical fuselage is generally maintained in the interest of interior layout but if the speed is increased above, say, $M_{\infty}=.90$, wing/fuselage blending is generally regarded as unavoidable (Fig. 3-5b).

Some examples of swept wing planforms are shown in Fig. 7-21. These wings are usually composed of at least two sections, with linear lofted contours between the intermediate profiles. This sophistication in aerodynamic design results in compound curvatures, giving rise to complications in the structural design and the manufacturing process. An early example of an aerodynamically efficient design, the crescent wing (Handley Page Victor, see Fig. 2-12 and Ref. 7-137), has never been widely adopted, probably due to its structural complexity.

For the purpose of preliminary wing optimization it may be desirable to derive the combinations of $\overline{t / c}$ and $\Lambda_{.25}$ required to achieve a specified dragdivergence Mach number. Equation 7-36 may be modified by substitution of $M \cos \Lambda .25$ and $\overline{t / c} x$ $(\cos \Lambda)^{-1}$ where $M$ and $t / c$ are used. For wings with symmetrical sections at zero lift we then find:

$$
\begin{aligned}
& \frac{\overline{\mathrm{t}}}{c}=\frac{.30}{M}\left\{\left(M \cos \Lambda_{.25}\right)^{-1}-M \cos \Lambda_{.25}\right\}^{1 / 3} \times \\
& {\left[1-\left.\left\{\frac{5+\left(M \cos \Lambda_{.25}\right)^{2}}{5+\left(M^{*}\right)^{2}}\right\}^{3.5}\right|^{2 / 3}\right.}
\end{aligned}
$$

Here $M$ refers to the drag-divergence Mach number, defined by $\Delta C_{D}=.002$, and $\overline{t / c}$ is the thickness ratio at about ${ } 50$ percent semi-span from the root. The values for $M$ * are given by $(7-37)$ for zero iift and should be reduced by $.25 \mathrm{C}_{\mathrm{L}}(\cos \Lambda .25)^{-2}$ to account for lift and camber at the design condition.

The drag-critical Mach number to be selected depends on the amount of compressibility drag the designer is prepared to accept during cruising flight. It is fair to assume that in high-speed flight over relatively short distances a penalty of up to some 20 to 30 counts will be acceptable. In this case the drag-divergence Mach number may be taken as being equal to or slightly below the high-speed Mach number.

For example, if a high-speed cruising flight Mach number of .8 is specified at $C_{L}=.3$, we may assume for a straight wing with a peaky-type airfoil $M^{*}=1.05-.25(.3)=.975$. Substitution of this value, together with $M=.8$ and $\Lambda=0$ into $(7-38)$ results in a permissible thickness ratio of only 9.7 percent. For a sweep angle of $30^{\circ}$ we obtain $M^{*}=.95$, and a permissible thickness ratio of 12 percent. This would be increased to 15.3 percent if an advanced airfoil could be designed with $M^{*}=1.20$ at zero 1 ift. This may be compared with the allowable 11 percent thickness for a conventional airfoil with the maximum thickness at about 30 percent chord.

The permissible thickness of a wing may well be determined by the requirement of freedom from buffet. As the prediction of buffet boundaries is as yet based on wind tunnel measurements; theoretical design methods for matching a wing to a specified buffet boundary cannot be presented in this book. The designer should probably follow an intuitive approach, based on the available information relating to buffet boundaries of existing aircraft types or (new) wing sections.

\subsubsection{Low-speed problems of high-speed} wings

In the same way as straight wings, the flow over swept wings may separate first at the leading edge or at the trailing edge. Leading edge separation results in a leading edge vortex, which cleans up the flow over the inner wing, but stalls the outer wing. At the lift coefficient where this stalling occurs, the forces and pitching moment on the wing are characterized by a break (Fig. 7-22), indicating that the tip stall causes an undesirable pitch-up, for which the remote position of the tip is mainly responsible. The primary causes of this stalling behavior are: 


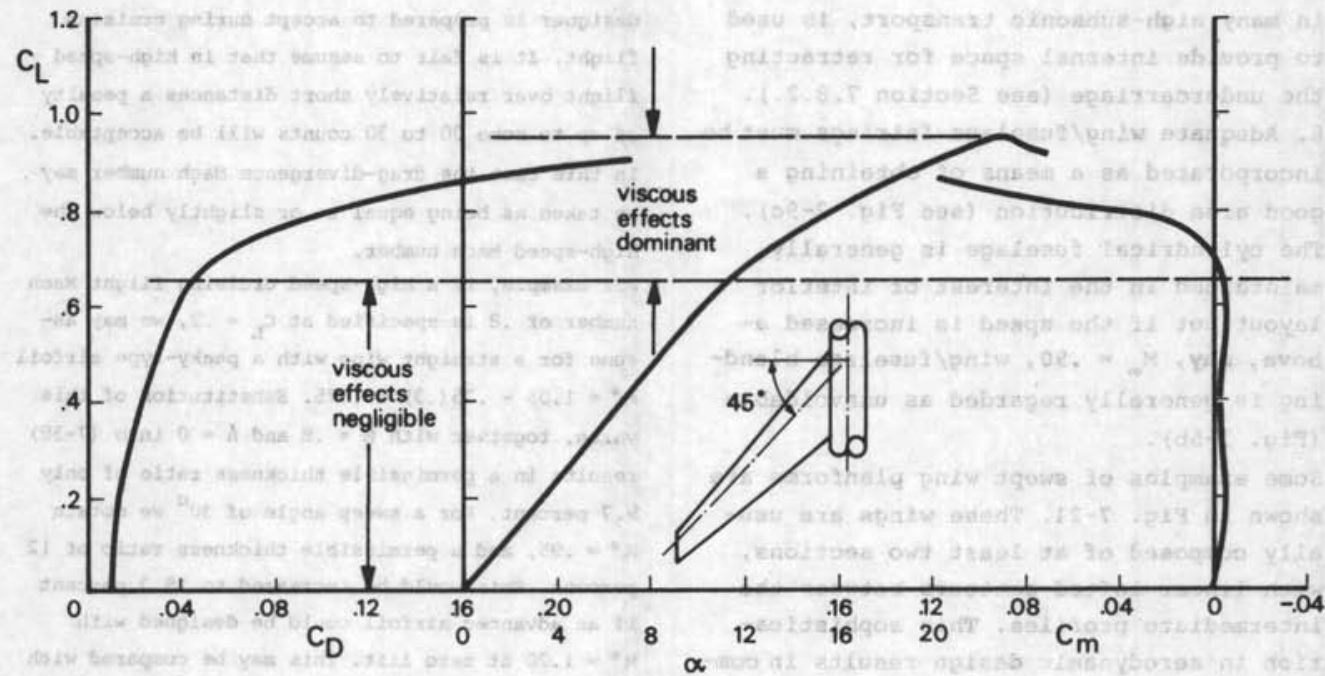

Fig. 7-22. Aerodynamic characteristics at high lift of a typical sweptback wing configuration (Ref. 7-89)

1. the highly loaded tip of swept wings,

2. changes in the chordwise pressure distribution due to sweep, and

3. a spanwise flow of the boundary towards the tip, cleaning up the flow over the inboard wing and making it difficult to stall.

Several investigators have made attempts to correlate the type of separation with the angle of sweepback, the nose sharpness and the Reynolds number (Refs. 7-82 and 7-92). Others have derived conditions for a stable pitching moment curve at the stall (Refs. 7-82 and 7-84), a typical outcome of which is shown in Fig. 2-25b. The highest aspect ratio for a longitudinally stable wing at the stall is found to increase with increasing taper (1.e. decreasing $\lambda$ ) and to decrease with the angle of sweepback. The location of the horizontal tailplane is another factor, determining whether the longitudinal stability improves or deteriorates if the tail contribution is taken into account (see Section 2.4.2b.).

The objective of low-speed design for transport aircraft is to avoid separated flow and to increase the range of useful lift coefficients. The obstacles to achieving this goal are the three-dimensional nature of the viscous flow and the incompatibility of the measures to be taken with high-speed design requirements. As theoretical methods are not avallable, an experimental program is required, where a cholce will have to be made from various methods of triggering the flow. For example:

1. Washout and increasing camber towards the tip.

2. Leading edge modifications, such as nose droop or an increased nose radius on the outer wing.

3. Leading edge devices postponing the stall. The inboard end of a slat almost invariably fixes the separation.

4. Fences, acting as barriers to the cross flow.

5. Pylons for wing-mounted engines, shedding a vortex at high incidences. The "vortilons" on the DC-9 wing have the same effect (Ref. 7-13).

6. Discontinuities in the leading edge: saw tooth, chord extensions.

7. Vortex generators on the outboard wing, in front of the aileron.

It will be clear that these opportunities for design render a simple pitch-up criterion almost useless. 
In spite of the potentially unfavorable stalling characteristics of swept wings, reasonably high aspect ratios can be accepted if suitable measures are taken. In view of the small thickness at the root, the span is in any case limited by considerations of structural design, and the swept wings of transport aircraft have a cantilever ratio of the same order as that used for propeller aircraft (Fig. 7-8). The maximum usable lift (flaps retracted) is reduced relative to straight wing designs when trailing edge separation occurs. A theoretical reduction factor of $\cos ^{2} \Lambda$ might be expected according to the simple sweep theory, but in reality the trend is more nearly approximated by a $\cos \Lambda$ effect due to the postponed stalling of the inner wing.

The stalling behavior of swept wings with high$11 \mathrm{ft}$ devices depends to a great extent on the sophistication of these devices. For simple trailing-edge devices (plain and split flaps) the maximum lift increment is greatly reduced, but the performance of optimized, multi-element airfoils on swept wings is quite good. The stalling behavfor is generally not very different from that of the basic wing, or slightly better. Leading-edge devices have a powerful effect on the longitudinal stability at the stall and can be used to advantage.

\subsubsection{Planform selection}

a. Angle of sweepback.

The previous section has made it clear that the wing sweepback should not be more than the minimum required. For cruising speeds of up to about $\mathrm{M}=.65$ to $.70 \mathrm{com}-$ pressibility effects can be catered for with a straight wing of acceptable thickness ratio. Increasing the Mach number makes it desirable to use sweepback (or sweep forward) in order to avoid severe compressibility problems in a dive. At cruising speeds of $\mathrm{M}=.75$ to $.80 \mathrm{a}$ straight wing may only be acceptable if it is very thin and this requires a low aspect ratio (e.g. Learjet 24 ). The angle of sweepback increases progressively for cruising speeds in excess of $\mathrm{M}=.80$ (see Table 7-1).

It should be noted that different combinations of $\Lambda, 25$ and $t / c$ are possible for $a$ given drag-divergence Mach number. Increasing the angle of sweep allows a thicker wing for the same design condition, permitting more fuel to be carried if required. In addition, sweepback reduces the lift-curve slope and causes the wing to be twisted if it is bent upwards by a $11 \mathrm{ft}$ load. As a result the gust loads are reduced and sweptback wings take the bumps in gusty weather more smoothly. The best choice is the result of a tradeoff between structural weight, maximum usable lift and high-speed performance at design and off-design conditions. It may also be affected by considerations of layout design - for example, if the loca$t i o n$ of the retractable main undercarriage conflicts with that of the rear spar (see section 7.8.2.).

\section{b. Taper ratio.}

The considerations relating to straight wings in section 7.4.4. are equally valid for swept wings. In addition we may note that for untwisted wings the taper ratio for minimum induced drag decreases with the sweepback angle (see Fig. 7-11). The trend shows that actual designs have higher taper ratios, probably to avold excessive washout and the associated drag penalty.

A highly tapered wing is favorable for reducing the pitch-up tendency and has a high torsional rigidity. This is a desirable feature, particularly for high-speed wings as it minimizes aeroelastic problems.

\section{c. Aspect ratio and span.}

As in the case of straight wings, an optimum aspect ratio can be determined which results in a good balance between fuel weight (fuel cost) and empty weight (initial cost). The aspect ratio is structurally limited by the range of acceptable cantilever ratios (Fig. 7-8), while a performance limit stems from the climb gradi- 
ent requirement with one engine inoperative.

Realizing that high-subsonic aircraft are jet-propelled, an approximate range for the span loading for this category is derived from statistical data, as follows:

$\frac{\mathrm{W} / \mathrm{b}^{2}}{\frac{1}{2} \mathrm{pV}_{2}{ }^{2}}=.18$ to .20

It can be shown * that this parameter is proportional to the ratio of induced drag to weight at the takeoff safety speed $v_{2}$, which is related to the takeoff field length by $(7-19)$. Hence, even without considering the flap effectiveness, a reasonable choice of the span loading can be made with $(7-39)$.

For twin-engine transport aircraft the thrust available after engine failure imposes a rather sharp limit on the span loading. The following recommended limit is derived from statistical data:

$\mathrm{w} / \mathrm{b}^{2}<\frac{1}{2} \rho \mathrm{v}_{2}{ }^{2}\left(1.45 \frac{\mathrm{T}_{\text {to }}}{\mathrm{w}_{\text {to }}}-.215\right)$

where $T_{\text {to }}$ refers to standard sea level conditions. Aircraft with a higher span loading are likely to be penalized in takeoffs from hot and high airfields.

\subsection{HIGH-LIFT AND FLIGHT CONTROL DEVICES}

\subsubsection{General considerations}

In Section 7.2. 1t was argued that in most designs an attempt will be made to achieve a favorable wing loading, based on considerations of fuel usage, structural weight and acceptable comfort in turbulent weather. High-lift devices are required to prevent the flight speeds from reaching unacceptable values during takeoff, approach and landing. These devices have proved to be of vital importance to an aircraft's operational characteristics and economic yield and much work has been

* See Chapter 11, equation 11-2. done to foster their development. Although the improvement in $C_{L}-\max$ since the DC-6, for example, may not appear excessive at first sight, the application of swept wings with thin sections has obscured the actual progress made. The multi-element systems at present in use permit a sectional $c_{\ell}$-max of about 5.5 to be obtained during the landing, and this appears to be about the limit of passive systems*. The designer may choose from a large collection of feasible high-lift systems, although in the case of a specific project this freedom will be limited, since incremental drag, mechanical complexity, development and maintenance costs and structural weight are all factors to be considered.

The additional wing lift contributed by high-lift devices is basically obtained through:

- increased airfoil camber,

- boundary layer control resulting from improved pressure distributions, reenergizing or removing low energy boundary layers,

- an increment of the effective wing area in the case of flaps extending the chord when deflected.

Not all existing configurations combine these actions simultaneously. Trailingedge flaps increase the camber and improve the flow at the trailing edge, but tend to promote leading edge stall on thin sections and may cause a reduction in the stalling angle of attack. Leading-edge high-lift devices postpone or eliminate leading edge stall, but they have little effect on the airfoil camber as a whole, although locally the camber is increased. Fig. 7-23 shows that these differences result in large variations in the range of operational angles of incidence at low speeds.

Most flap systems are offered with a

" "passive" means that no boundary layer control by suction or blowing is used. 


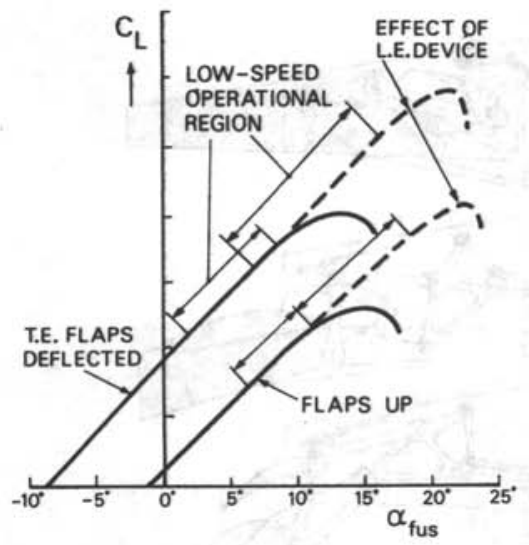

Fig. 7-23. Lift curves with and without high-lift devices.

choice of several discrete deflection angles. Deflections of up to about 25 degrees are used on takeoff, while higher values are used during the approach and landing. For each item of performance there is an optimum flap angle which constitutes a tradeoff between high lift and low drag - see Section 11.7.1. Though the operational flexibility of the aircraft is improved by increasing the freedom of choice, the cost and time involved with. the certification tests and data handing may become prohibitive.

Typical deflection angles are shown in Table 7-2 for several commonly used highlift configurations, together with a range of $C_{L}$-max values, defined as follows:

\begin{tabular}{|c|c|c|c|c|c|}
\hline \multicolumn{2}{|c|}{ HICH-LIFT DEVICE } & \multicolumn{2}{|c|}{ TYPICAL FLAP ANCLE } & \multicolumn{2}{|c|}{$/ \cos A_{.25}$} \\
\hline TRAILISG EDGE & $\begin{array}{l}\text { LEADINC } \\
\text { EDGE }\end{array}$ & TAKEOFF & LANDING & TAKEOFF & IASDING \\
\hline PLAIN & - & $20^{\circ}$ & $60^{\circ}$ & $1.40-1.60$ & $1.70-2.00$ \\
\hline SINGLE SLOTTED & - & $20^{\circ}$ & $40^{\circ}$ & $1.50-1.70$ & $1.80-2.20$ \\
\hline FONLER* & - & $15^{\circ}$ & $40^{\circ}$ & $2.00-2.20$ & $2.50-2.90$ \\
\hline DOUBLE SLOTTED $* *$ & - & $20^{\circ}$ & $50^{\circ}$ & $1.70-1.95$ & $2.30-2.70$ \\
\hline & SLAT & & & $2.30-7.60$ & $2.80-3.20$ \\
\hline TRIPLE SLOTTED & SLAT & $20^{\circ}$ & $40^{\circ}$ & $2.40-2.70$ & $3.20-3.50$ \\
\hline
\end{tabular}

\section{- single slotted}

.. UITH VARYING amotsts OF CHORD EXTENSION (FONLER MOTION)

Table 7-2. Typical maximum lift coefficients for wings with high lift devices.
$c_{L_{\max }}=\frac{\mathrm{w} / \mathrm{s}}{\frac{1}{2} \rho v_{S}{ }^{2}}$

Generally speaking, $C_{L}-\max$ is less for small airplanes than for large ones, as a result of a less sophisticated wing design and lower Reynolds numbers. There are obviously many other details which may lead to departures from the quoted figures, such as differences in the relative flap chord, flap span, airfoll type and interference with engine nacelles, etc It should also be noted that the values stated apply to the trimmed aircraft, and hence they include the "tailplane load required for longitudinal equilibrium. A detailed aerodynamic prediction method, taking account of the most relevant geometric design features, is presented in Appendix G-2.

\subsubsection{Trailing-edge flaps}

Typical lift and profile drag increments caused by flaps on airfoil sections are compared in Fig. 7-24.

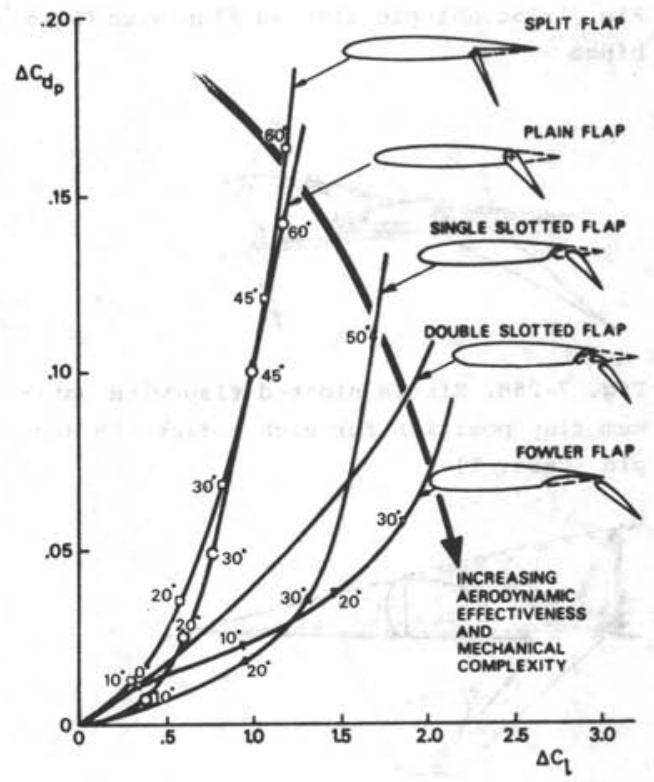

Fig. 7-24. Trends in performance of trailing-edge flaps. 


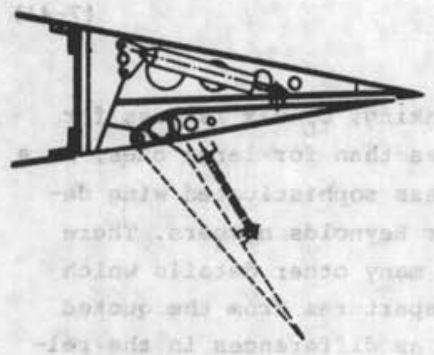

Fig. 25a. Split flap
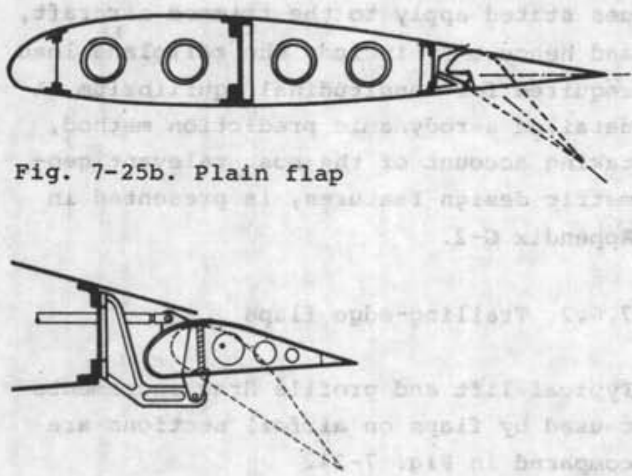

Fig. 7-25c. Single slotted flap with fixed hinge

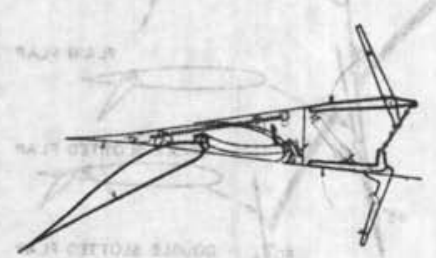

Fig. 7-25d. Single slotted flap with optimum flap position for each deflection angle (Caravelle)

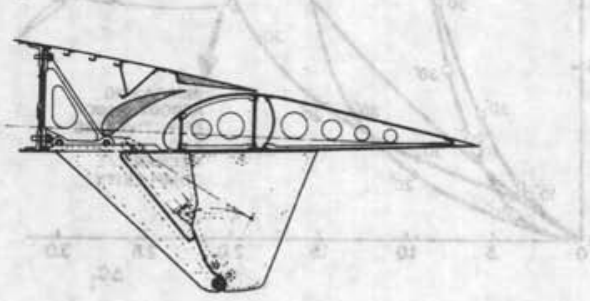

Fig. 7-25e. Double slotted flap with fixed hinge and fixed vane (Douglas DC-9)

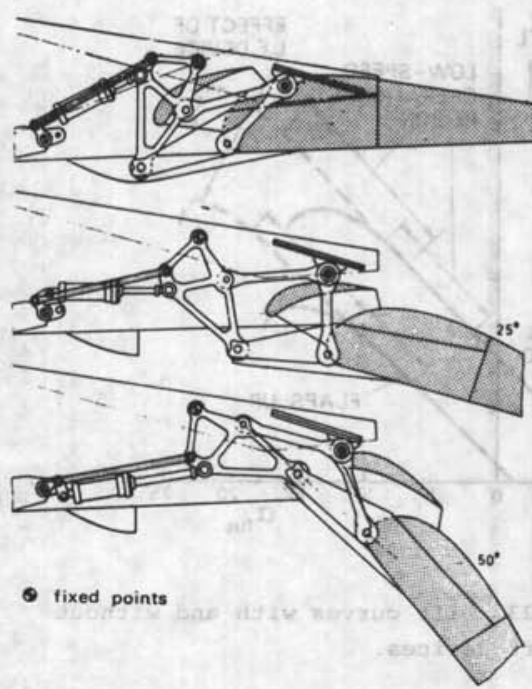

Fig. 7-25f. Double slotted flap with fourbar motion (Douglas DC-8)
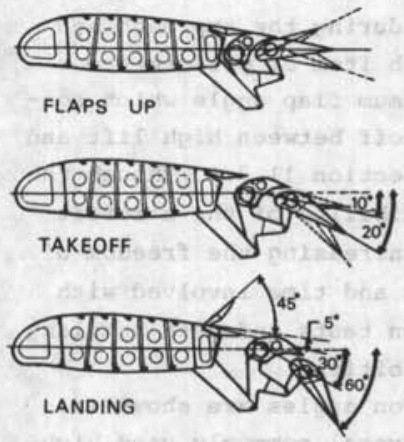

Fig. 7-25g. Double slotted flaps with individual adjustment of flap segments and drooped aileron (GAF N-22 Nomad)

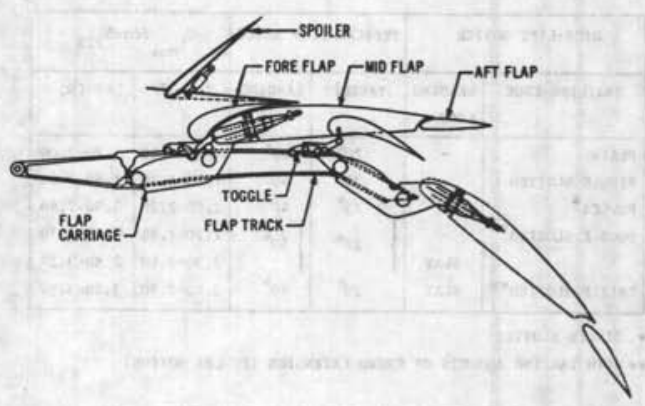

Fig. 7-25h. Triple slotted flap (Boeing 727) 


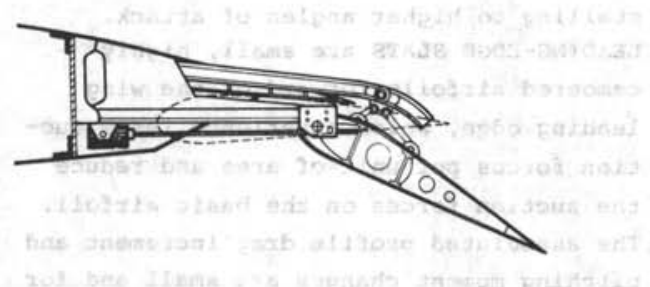

Fig. 7-25j. Single slotted Fowler flap

SPLIT FLAPS consist of a stiffened plate on the lower surface, hinged just aft of the rear spar, by means of a piano hinge (Fig. 7-25a). The drag due to flap deflection is large, particularly in the case of small deflections, thus making the split flap less suitable for takeoff. Although its structural simplicity and low weight are attractive, this type of flap must now be considered as obsolete.

PLAIN FLAPS are hardly more than a hinged part of the trailing edge (Fig. 7-25b). The best performance is obtained with a sealed gap. If the deflection exceeds 10 to 15 degrees, the flow separates immediately after the knuckle, the lift effectiveness drops progressively and the drag increases to values comparable to those of the split flap.

SINGLE SLOTTED FLAPS derive their favorable action from a specially contoured slot through which air is admitted from below the wing. The upper surface boundary layer is stabilized by the suction on the flap's leading adge and diverted at the trailing edge of the basic wing. A new boundary layer is formed over the flap which permits an effective deflection of up to 40 degrees. The performance is sensitive to the shape of the slot, which is determined by the kinematics of deflection. A simple fixed hinge (Fig. 7-25c) is not very effective, but the achievement of an optimum slot shape requires a system which can only be realized by means of a track and flap carriage assembly (Fig. 7-25d). Single slotted flaps with fixed hinge are commonly used on light aircraft.
DOUBLE SLOTTED FLAPS are markedly superior to the previous type at large deflections, because separation of the flow over the flap is postponed by the more favorable pressure distribution. Various degrees of mechanical sophistication are possible: a. Flaps with a fixed hinge and a fixed vane of relatively small dimensions (Fig. 7-25e) are structurally simple, but may have high profile drag during takeoff. If the vane is made retractable for small flap deflections, the flap is effectively single-slotted during the takeoff, which improves the drag and hence the climb performance. The external flap supports cause a noticeable parasite drag, which may be acceptable only on short ranges.

b. Double slotted flaps may be supported on a four-bar mechanism (Fig. 7-24f). During extension the slot shape closely approximates to the aerodynamic optimum, but the flap supports cause a disturbance in the flow through the slot. The parasite drag penalty in the en route configuration being negligible, this configuration is probably most suitable for application on three- or four-engine long-range transports.

c. If the two flap elements are independently adjustable, the maximum deflection may be increased up to 70 degrees. This rather complex system (Fig. 7-25g) is occasionally used on STOL aircraft, such as the $\mathrm{DHC}-7$. The figure shows the system used on the GAF N-22 Nomad full span flaps with aileron control at high speeds, augmented by spoiler ailerons at large flap deflections.

TRIPLE SLOTTED FLAPS are used on several transport aircraft with very high wing loadings. In combination with leading-edge devices, this system represents almost the ultimate achievement in passive high-lift technology, but Fig. 7-25h shows that complicated flap supports and controls are required.

THE FOWLER FLAP is a slotted flap travelling aft on tracks over almost its 
entire chord and subsequently deflecting to its maximum angle (Fig. $7-25 j$ ). The upper wing skin continues to approximately 90 to 95 percent of the chord. The favorable performance is derived from the effective wing area extension, yielding a gain in lift for very little extra drag which makes the Fowler flap particularly suitable for twin-engine aircraft.

Configurations have been devised with one, two and even three slots, depending upon the magnitude of the required lift. These systems not only have considerable chord extension due to the basic flap motion, but the flap sections move relative to each other and the extended length of the chord is therefore in excess of the nested length. The structural complications of these multi-track supports and their weight penalty are usually the limiting factor here. The shape of the slots must be very carefully optimized to obtain good perforormance.

\subsubsection{Leading-edge high-1ift devices}

The increased circulation associated with the deflection of an effective trailingedge device induces an upwash at the nose. The local suction peak increases and on airfoils which are liable to leading edge stall the flow will separate at an angle of attack which is below that of the basic wing. Leading-edge high-lift devices (Fig. 7-26) are intended primarily to delay the

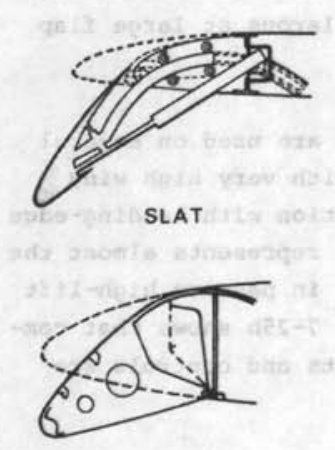

HINGED NOSE

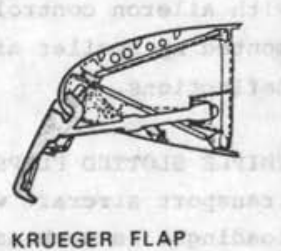

KRUEGER FLAP

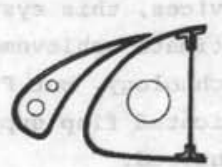

FIXED SLOT

Fig. 7-26. Leading-edge high-1ift devices. stalling to higher angles of attack. LEADING-EDGE SLATS are small, highly cambered airfoils forward of the wing leading edge, which experience large suction forces per unit of area and reduce the suction forces on the basic airfoil. The associated profile drag increment and pitching moment changes are small and for optimized configurations a $C_{L}$-max increment due to a full-span slat between .5 and .9 can be obtained without any appreciable increase in tail load and trim drag. In view of the large stalling angle of attack, the angles of pitch during takeoff and landing are large and proper attention must therefore be paid to the visibility from the cockpit, particularly if the angle of sweep is large and the aspect ratio low.

Slats may be used to advantage by varying their effectiveness in spanwise direction in order to delay the onset of the tip stall and the associated pitch-up of sweptback wings. This may be achieved by varying the gap width, the slat deflection angle or the relative slat chord in spanwise direction.

KRUEGER FLAPS perform in the same way as slats, but they are thinner and more suitable for installation on thin wings.

Krueger flaps are often used on the inboard part of wings, in combination with outboard slats, to obtain positive longitudinal stability in the stall.

(PLAIN) LEADING-EDGE FLAPS (hinged noses) are less effective than slats. They are mechanically simple and rigid and particularly suitable for thin airfoil sections. FIXED SLOTS are the simplest devices for postponing leading edge stall, but their profile drag penalty is generally prohibitive for effective cruising, except on some low-speed STOL aircraft.

Leading-edge devices are beneficial if there is any possibility of leading edge stall occurring, but with the comparatively thick sections used on light aircraft and small propeller transports, slats or leading-edge flaps are not normally con- 
sidered necessary. Stall prevention on the outboard wing should be obtained by a proper choice of the sectional shape, moderate taper ratio and washout. If necessary, an increased leading-edge radius, drooped nose or a part-span slot may be considered.

\section{7,6,4. Flight control devices}

A typical arrangement of high lift and flight control devices on a swept wing is shown in Fig. 7-27, to which the following comments can be added.

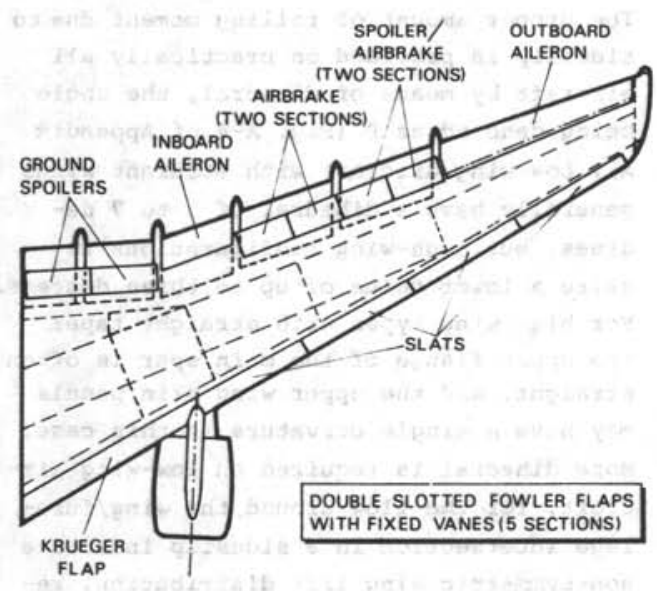

Fig. 7-27. Arrangement of high-lift devices and flight control surfaces in plan view.

\section{a. Ailerons.}

The aileron span is chosen as small as possible in order to obtain the largest possible flap span, within the limits imposed by roll control requirements. Slotted ailerons, which are drooped with the flaps, are sometimes used on straight wings, where the object is to attain the highest possible lift with the additional advantage of reducing the induced drag. Their maximum symmetrical deflection is generally limited to some 20 degrees, unless roll control is augmented by means of spoilers. Drooped ailerons on highly swept wings are likely to be fairly ineffective, because they introduce a large pitching moment and the gain in lift is largely reduced by the associated trim load.

If the aileron of a thin wing is deflected at a high EAS, the load on it causes the wing to twist in the opposite direction. This could, in some cases, offset the load produced by the aileron, resulting in a roll in the wrong direction (aileron reversal). For this reason some high-speed aircraft employ ailerons located closer to the wing root, where adequate torsional rigidity is present. The outboard ailerons operate only at low speeds, while the small-span "high-speed" ailerons are operative both at low and high speeds. A suitable location for the high-speed aileron is behind the (inboard) engines, since the flap will have to be interrupted there to keep it free from the jet efflux. In addition, to reduce the size of the ailerons on high-speed aircraft, these are frequently assisted by spoiler-ailerons.

The area of conventional ailerons may be estimated in the preliminary design stage from statistical data of the parameter $S_{a_{a}} a / S b$ (Fig. $7-28$ ), which is a measure of the rolling moment, for a given aileron deflection.

b. Spoilers may be fitted for various reasons; they often combine more than one function and usually occupy a substantial part of the flap span, just behind the rear spar. As shown in Fig. 7-27, the upper skin consists of stiffened panels which can be deflected in almost upright position. Immediately after landing, or in the event of an aborted takeoff, they may be activated either by the pilot or auto-

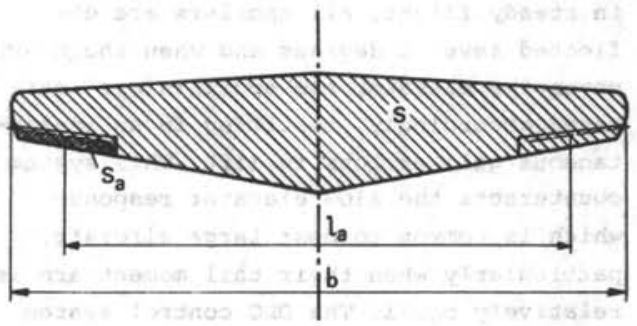

Fig. 7-28. Dimensions related to the aileron geometry. 
matically (pre-armed). As a result of the interrupted airflow over the flaps, the wing loses a large part of its lift, which increases the normal force on the tires and makes braking more effective. In addition, they create considerable drag and these combined effects increase the deceleration by some 20 percent.

Outboard spollers may be used in flight when an appreciable increment in drag is required to obtain a high rate of descent or improved speed stability with a constant angle of descent. Inboard spoilers are not deflected in this case to avoid disturbing the flow over the empennage and prevent buffeting. For this reason the inboard spoilers are only installed to decrease the lift on the ground and are referred to as ground spoilers (11ftdumpers), while the others are known as (in-)flight spoilers. When acting as drag-producing devices, these spoilers are referred to as airbrakes (speedbrakes) which may be installed both on the upper and lower surfaces of the wing (Fig. 7-25d).

The effectiveness of roll control may be increased by operating the outboard spoilers simultaneously with the upwardmoving aileron. Their motion is proportional to the control wheel movement when it is turned beyond a predetermined angle of rotation. These devices, referred to as lateral spoilers, may be used as airbrakes by deflecting them on both sides.

A recent development is the use of spoilers as elements of a system known as Direct Lift Control (DLC), which has been adopted on the Lockheed 1011. During the approach, in steady flight, all spoilers are deflected several degrees and when the pilot moves the elevator the spollers are activated accordingly, resulting in an instantaneous gain or loss in lift. This system counteracts the slow elevator response which is common to most large aircraft, particularly when their tail moment arm is relatively small. The DLC control system is most effective when the flaps are deflected and it is therefore used only in the low-speed configuration.

\subsection{DIHEDRAL, ANHEDRAL AND WING SETTING}

Once the wing shape and the high-lift system have been selected, the position of the wing relative to the fuselage can be laid down. Its vertical position has been dealt with in Section 2.2., while the longitudinal position will depend on considerations of center of gravity position (Chapter 8 ) and horizontal tail size (Chapter 9).

\subsubsection{The angle of dihedral (anhedral)}

The proper amount of rolling moment due to sideslip is provided on practically all aircraft by means of dihedral, the angle being denoted as $\Gamma$ (Fig. A-4 of Appendix A). Low-wing aircraft with straight wings generally have a dihedral of 5 to 7 degrees, but high-wing configurations require a lower value of up to three degrees. For high-wing types with straight taper the upper flange of the main spar is often straight, and the upper wing skin panels may have a single curvature in this case. More dihedral is required on low-wing aircraft, for the flow around the wing/fuselage intersection in a sideslip induces a non-symmetric wing lift distribution, resulting in a rolling moment which counteracts the effect of the dihedral. Similarly, the wing/fuselage interaction contributes to the desired rolling moment on high-wing configurations. The air forces on a swept wing are affected by a sideslip to the extent that the same effect is obtained as that exerted by the dihedral on a straight wing. This results in an effective dihedral which increases with the lift coefficient and may become too large at high lift coefficients. The yawing motion, following a side gust, is accompanied by a rolling motion (Dutch roll) which may be poorly damped or even unstable and consequently objectionable to pilots. This is one of the reasons that for most swept wing aircraft it is necessary to resort to the use of a device, known as a yaw damper, which essentially consists of a rate gyro 
transmitting a signal to a servomechanism. The rudder is then operated in a direction so as to oppose the yawing motion. The yaw damper is incorporated in the autopilot, and on aircraft with wing-mounted engines it has the additional function of suppressing the yawing motion following engine failure.

An alternative method to combating the lateral-directional stability problems with swept wings is to reduce the dihedral, or even to use anhedral. This can be realized without any layout problem on highwing aircraft (Fig. 2-4); the application of anhedral on some Russian low-wing designs by Tupolev necessitated a special undercarriage configuration to ensure a minimum wing tip clearance with respect to the runway in takeoff and during landing in crosswind conditions (see also Section 10.3.1.).

\subsubsection{Wing/body incidence}

The wing setting relative to the fuselage is usually chosen such that during cruising flight with a representative All-Up Weight at a representative altitude the fuselage axis or cabin floor will be horizontal. An expression for the wing incidence is derived in Appendix E (Section E-9.3.), taking into account the effects of wing/ fuselage interference, body lift and trim load. If these effects are ignored, the following expression is found:

$i_{w}=\frac{c_{L}^{*}}{c_{L_{\alpha}}}+\alpha_{o_{1}} \varepsilon_{t}+\left(\alpha_{\ell_{0}}\right)_{r}$

where $C_{L}^{*}$ is the lift coefficient for which the fuselage reference line is horizontal, $\alpha_{o_{1}}$ the change in zero-lift angle of the wing per degree of positive twist at the tip and $\left(\alpha_{\ell_{0}}\right)_{r}$ the zero-lift angle of the root section. The lift-curve slope can be obtained from section E-4.1., while $\alpha_{O_{1}}$ is approximately -.4 for straighttapered wings with linear twist. The wing/ body incidence may be affected to some extent by considerations of tail clearance during takeoff and landing (Section 10.3.1.). Deviations of more than about 2 degrees from a level cabin in cruising flight should, however, be avoided.

\subsection{THE WING STRUCTURE}

The outline of the wing, both in planform and in the cross-sectional shape, must be suitable for housing a structure which is capable of doing its job. As soon as the basic wing shape has been decided, a preliminary layout of the wing structure must be indicated, which is expected to lead, after further refinement and detail design, to a sufficiently strong, stiff and light solution, with a minimum of manufacturing problems.

\subsubsection{Types of wing structure}

Three basic types of primary wing structure can be distinguished (Fig. 7-29):

MASS BOOM types of wing structure, where the flanges of one or two spars take the normal forces resulting from bending, while the torsional load is carried by either the spar webs (differential bending) or the combination of the spars and skin covers (shear). The single spar wing uses the spar web and the nose section or the section aft of the spar to combine into a box which provides torsional stiffness. Mass boom structures are mainly used on slow aircraft with thick wings ( $t / c$ above 15 percent) and low wing loadings (up to $30 \mathrm{lb} / \mathrm{sq} . \mathrm{ft}, 150 \mathrm{~kg} / \mathrm{m}^{2}$ ). Advantages of this arrangement are as follows.

a. Tapered booms are easy to produce and may be adapted to the local stress level desired. If the booms are stabilized against buckling by means of closely spaced ribs, high stress levels are attainable. b. The skin is subject only to shear forces and with the use of closely spaced ribs stringers may be dispensed with, which simplifies the manufacturing of the ribs. c. Attachment to the fuselage is a simple 


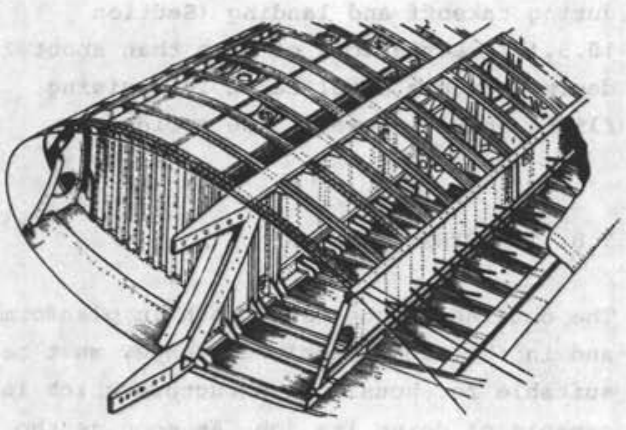

a. TYpical single spar mass boom structure

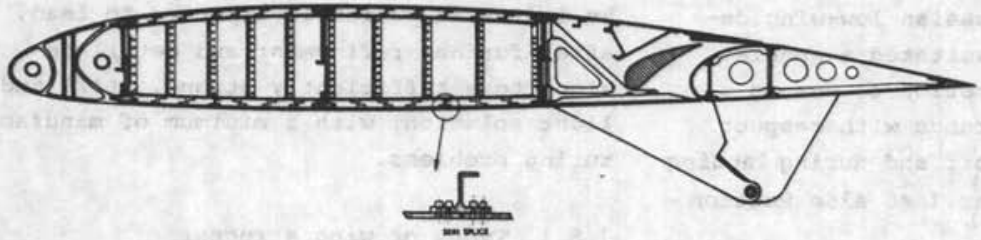

b. Typical wing section of a box beam structure

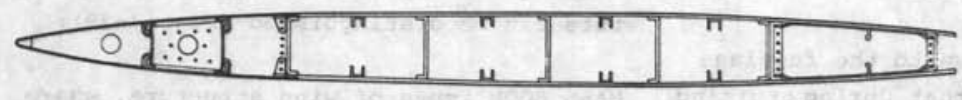

c. Example of a multispar structure

Fig. 7-29. Examples of basic types of wing structure

matter: only two main frames are required. d. Weight penalties for openings and inspection doors will be modest.

The disadvantages, however, may be of decisive importance:

a. Failure of a spar boom is catastrophic. Due to the absence of fail-safe characteristics, the mass boom wing structure is no longer used in new transport aircraft designs.

b. Due to the high stresses in the spar booms the deflections under bending loads are large.

c. The skin plays no part in absorbing the bending moment so that it is not used very efficiently.

d. If a two-spar configuration is used, the spar height is less than the airfoil thickness. The forces in the spar booms due to bending are thus increased and more material will be required.

e. Many ribs are required to stabilize the spar booms.

f. The skin will buckle when loaded if no stringers are used; this will adversely affect the aerodynamic cleanness.

BOX BEAM structures incorporate skin panels, which are stressed not only to take shear forces, but also the end load due to bending. The example in Fig. $7-29 \mathrm{~b}$ shows a three-spar box beam, but various alternatives are known, such as the twospar box beam with or without a stiffened nose section.

From the point of view of fail-safe design the stressed skin structure is much better than the mass boom type. The skin can be divided into several planks (multi-load path) coupled with spanwise splice members. 


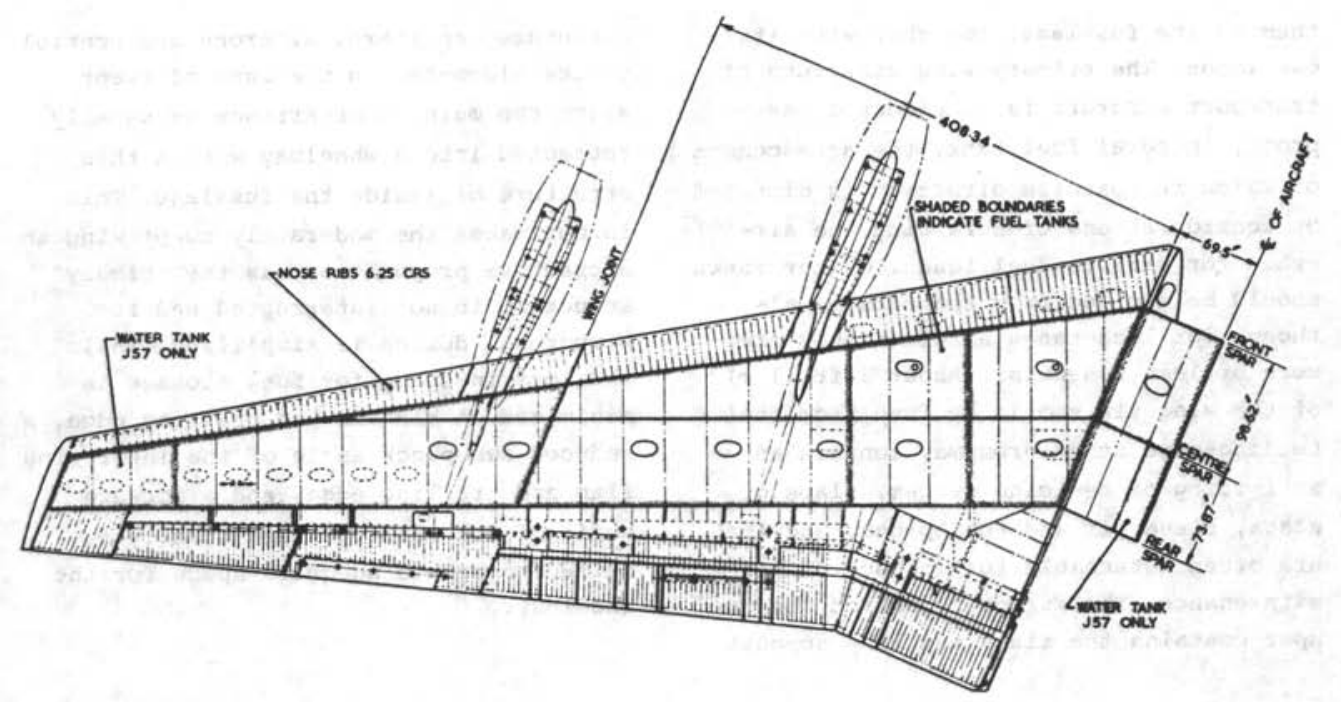

Fig. 7-30. DC-8 wing structure in plan (Ref.: The Aeroplane, 15 Aug. 1958, page 230)

The web can be designed to accommodate an in-service crack, as indicated in the figure.

By and large, the pros and cons of the box beam structure contrast with those of the mass boom type. The advantages of the box beam will be evident when considerable skin thickness is required to obtain sufficient torsional rigidity on wings designed for high speeds and/or thin, high aspect ratio wings. In lightly loaded wings, however, the stress levels in the upper skin will be kept fairly low to avoid buckling and the difference in weight will be small as compared with the mass boom type. Integral construction is often employed on highly loaded wings.

MULTI-SPAR structures are used on very thin wings, sometimes in conjunction with closely spaced ribs. This type of structure is most unsuitable for the provision of cutouts, such as would be required for stowing the landing gear or for inspection facilities.

\subsubsection{Structural arrangement in plan}

Fig. 7-30 shows the plan view of a typical wing of a high-subsonic transport aircraft (DC-8: wing loading approximately 100 $\mathrm{lb} / \mathrm{sq} . \mathrm{ft}, 500 \mathrm{~kg} / \mathrm{m}^{2}$; thickness ratio 12 percent).

The rear spar must be located at a suitable chordwise station, leaving sufficient space for the flaps and for housing the controls to operate the flaps, ailerons and spoilers. A rearward shift of this spar increases the cross-sectional area of the torsion box - and incidentally the fuel storage space - but the reduction in the sectional height will make it less efficient in bending. Similar criteria apply to the front spar when it is moved forward. It is noted that the best flap chord for simple plain and split flaps is about 25 percent wing chord, but highly efficient slotted and Fowler flap systems are more effective with flap chords of up to 35 or even 40 percent of the wing chord. The front spar is located at about 15 percent chord, the rear spar at 55 to 60 percent. About 5 to 10 percent chord should be available between the nested flap and the rear spar for control system elements. The central part of the wing, bounded by the front and rear spars, takes the loads from the nose and rear sections and carries 
them to the fuselage, together with its own loads. The primary wing structure of transport aircraft is in effect a leakproof, integral fuel tank, the arrangement of which in spanwise direction is dictated by considerations of balancing the aircraft for various fuel loads. Center tanks should be avoided from the outset, although for long-range aircraft they are more or less essential. About $3 \mathrm{ft}(1 \mathrm{~m})$ of the wing tip should be free from fuel. Leading-edge structures may contain an anti-icing or de-icing system, flaps or slats, bleed air and fuel pipes, and they are often detachable for inspection and maintenance. The structure behind the rear spar contains the flaps and flap support structure, spoilers, ailerons and control system elements. In the case of swept wings the main undercarriage is usually retracted into a wheelbay within this structure or inside the fuselage. This layout makes the moderately swept wing an attractive proposition, as the primary structure is not interrupted and its structural design is simplified, while the loss in space for fuel storage is minimized. A kink in the trailing edge, a reduced sweepback angle of the inner wing flap and trailing edge, and a forward shift of the rear spar are usually required to create adequate space for the wheelbay. thayorila jrogenexy surbadus-rets a lo pate

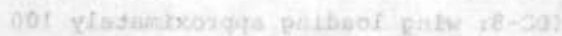

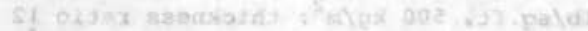

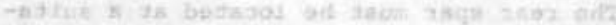

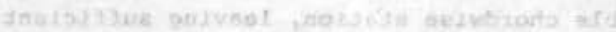

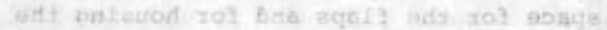

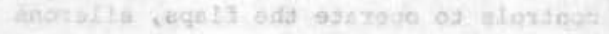

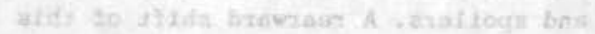

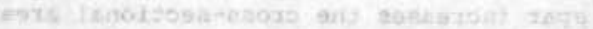

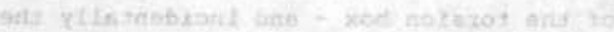

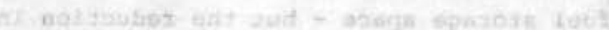

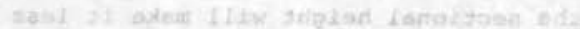

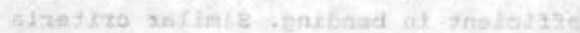

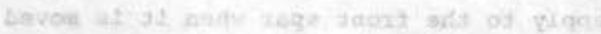

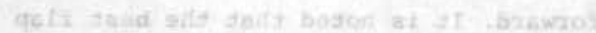

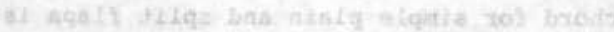

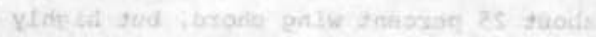

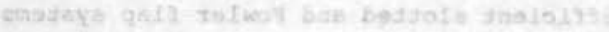

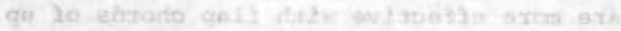

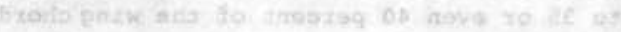

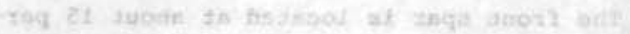

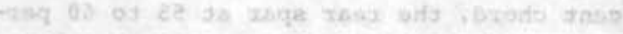

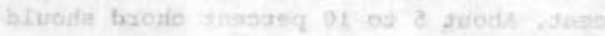

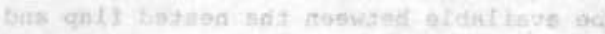

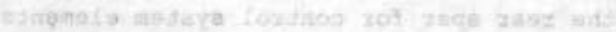

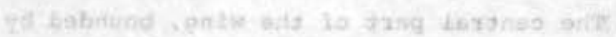

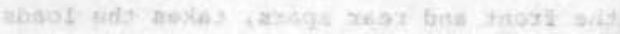

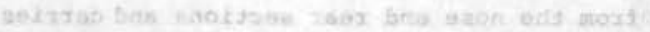

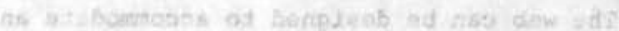

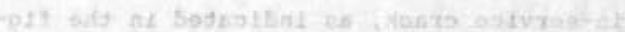

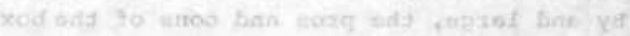

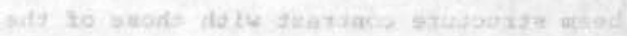

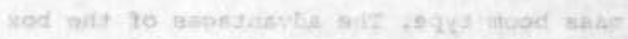

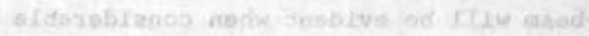

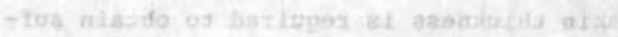

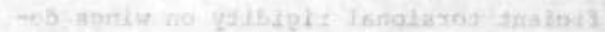

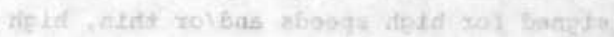

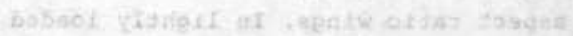

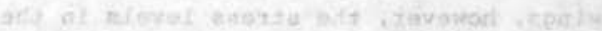

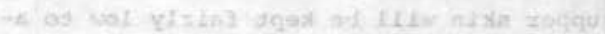

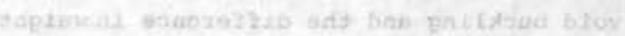

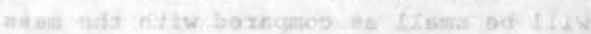

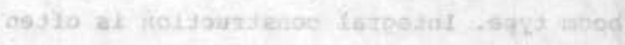

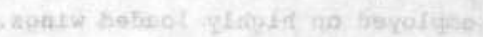

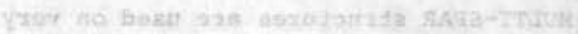

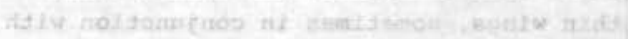

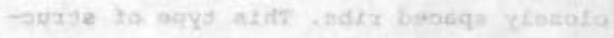

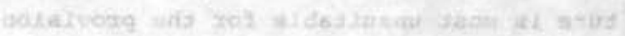

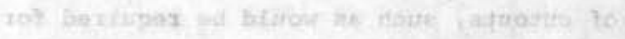

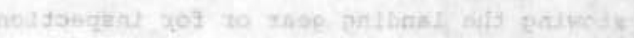
wetinisat 


\section{Chapter 8. Airplane weight and balance}

\section{SUMMARY}

The sensitivity of airplane performance and operating economy to the empty weight is discussed and the value of weight-saving is demonstrated.

An accurate weight prediction in the preliminary design stage is a most effective way to control the weight; it begins with a consistent scheme for weight subdivision and limitations. Considerations are presented for making a sound choice of the operational weight limitations.

Some general remarks on weight prediction methods are followed by a comprehensive collection of available and consistent methods, useful for most categories of modern civil aircraft. Attention is paid both to simple approximate methods and to more detailed procedures, for which detailed design information must be available.

The load and balance diagram is introduced to illustrate the flexibility of loading an airplane. The effect of the general arrangement and layout of the aircraft on the problem of obtaining adequate balance in all likely flight conditions is discussed and a procedure suggested for establishing a suitable longitudinal wing location and center of gravity range.

Many references to literature are given, as well as a large collection of data on weights and center of gravity ranges of airplane types in present service. 
AC

$A_{1}$

APS

APU

AUW

a

BOW

${ }_{\mathrm{p}}$

b

$b_{\text {ref }}$

$\bar{c}^{\mathrm{b}}$

c.g.

D

DC

$\mathrm{D}_{\mathrm{p}}$

ESHP

h

$\mathrm{h}_{\mathrm{h}}$

k

$k_{w}$

1

$1_{h}$

$1_{t}$

MAC

MLW (MRLW) Maximum (Regular) Landing Weight

MTOW(MRTOW) Maximum (Regular) Takeoff Weight

MZFW

$\mathrm{m}_{1}$

N

Maximum Zero Fuel Weight

ratio of actual to estimated

weight for a sample point

number of an item present in

the airplane

$\mathrm{n}_{1}, \mathrm{n}_{2}, \ldots, \mathrm{n}_{\mathrm{m}}$ exponent of a weight parameter

nult

OEW

$P_{\text {el }}$ ultimate load factor

Operational Empty Weight

total electrical generator

power (kVA)
$\mathrm{R}_{\mathrm{B}}$

$\mathrm{R}_{\mathrm{D}}$

$\mathrm{R}_{\text {ref }}$

$\mathrm{s}$

.

$\mathrm{s}_{1}, \mathrm{~s}_{2}$

$S_{G}$

$S_{\text {he }}$

SIV

SMC

$S_{\text {wet }}$

$T_{\text {to }}$

$t_{r}$

U

u.c.

$\mathrm{v}$

$v_{b}$

$v_{D}$

$\mathrm{V}_{\text {max }}$

$w$

$\dot{\mathrm{w}}_{\mathrm{ba}}$

$\dot{\hat{W}}_{\text {to }}$

$w_{D E}$

$w_{O E}$

$w_{G}$

$\mathrm{w}_{\mathrm{zF}}$

$\mathrm{X}$

$x_{\text {LEMAC }}$

$\mathrm{x}$

$\Delta \mathrm{x}$

$\mathrm{x}_{\mathrm{OE}}$

Y

$\mathrm{Y}_{1}$

$\delta$ takeoff horsepower per engine

(sea level, static)

maximum range with maximum payload (Fig. 8-3)

maximum range with maximum fuel

(Fig. 8-3)

reference range

(projected) area of a surface

(no index: wing area); standard

error of prediction

areas of parallel end faces of

a prismoid

gross shell area of the fuselage

exposed horizontal tailplane

area

Standard Item Variations

Standard Mean Chord

wetted area

takeoff thrust per engine (sea

level, static)

(absolute) maximum thickness

of root chord

annual airplane utilisation

undercarriage

speed; volume

blockspeed

Design Dive speed

maximum horizontal flight speed weight

rated bleed airflow of APU

fuel flow per engine, corre-

sponding to $P_{\text {to }}$ or $T_{\text {to }}$

Delivery Empty Weight

Operating Empty Weight

Gross Weight

Maximum zero Fuel Weight

$\mathrm{X}$-axis; parameter for wing

weight estimation example

coordinate of MAC leading edge

coordinate of weight contribu-

tion; sample value of $x$

range of $x$-coordinates for the

c.g.

airplane c.g. position for the OEW

weight of an airplane part actual (measured) value of $Y$ for a sample

maximum deflection angle;

incidence variation 


$\begin{array}{ll}\text { APU } & \text { Auxiliary Power Unit } \\ \text { APUG } & \text { APU Group } \\ \text { ba } & \text { (APU) bleed airflow } \\ \text { cc } & \text { cabin crew } \\ \text { cf } & \text { cabin floor } \\ \text { cg } & \text { center of gravity } \\ \text { ch } & \text { cargo hold } \\ \text { d } & \text { intake duct } \\ \text { e } & \text { engine(s) } \\ \text { el } & \text { electrical system } \\ \text { er } & \text { bending moment relief due to } \\ & \text { engine(s) } \\ \text { f } & \text { fuselage; flaps; fuel } \\ \text { fc } & \text { flight crew } \\ \text { fg } & \text { fuselage group } \\ \text { ft } & \text { fuel tank }\end{array}$

8.1. INTRODUCTION; THE IMPORTANCE OF LOW WEIGHT

Weight minimization of an airplane design is a subject of the utmost importance. Although reduction of weight is generally obtained only at some initial cost penalty, the effects on total operating costs are paramount for most high-performance designs, particularly for large and complex airplanes. In many cases the increased weight of one component means added weight elsewhere, leading to the well-known snowball effect of weight growth. The opportunities to achieve a weight reduction and the associated costs depend upon the phase of the design process.

a. During the initial conceptual design the choice of the airplane layout, geometry and detailed configuration affects weight. The design layout should be carefully optimized and high accuracy of the initial weight prediction is a prerequisite. Weight pre-

$\begin{array}{ll}\text { geo } & \text { geometric shape } \\ \text { h } & \text { horizontal tailplane } \\ \text { hc } & \text { horizontal tail controls } \\ \text { i } & \text { inlet; installation; sample } \\ \text { ieg } & \text { instruments and electronics } \\ \text { ld } & \text { group } \\ \text { LEMAC } & \text { lift dumper } \\ \text { n } & \text { Leading Edge of MAC } \\ \text { p } & \text { nacelle (group) } \\ \text { pax } & \text { propeller } \\ \text { pc } & \text { passengers } \\ \text { pg } & \text { passenger cabin } \\ \text { s } & \text { propulsion group } \\ \text { sb } & \text { structure; slat } \\ \text { sc } & \text { speed brake } \\ \text { tail } & \text { surface controls group } \\ \text { thr } & \text { horizontal plus vertical tail } \\ \text { to } & \text { thrust reverser } \\ \text { uc } & \text { takeoff } \\ \text { v } & \text { undercarriage } \\ \text { w } & \text { vertical tailplane } \\ \text { wc } & \text { wing; weight } \\ \text { wg } & \text { toilet/watercloset compartment } \\ \text { wt } & \text { wing group } \\ & \text { water tank for injection fluid }\end{array}$

diction is necessary not only to make an assessment of the design qualities, but also to set a goal for the structural and systems design offices. The initial weight prediction must be a realistic challenge to both. This type of work, being the normal task of the preliminary design office, involves virtually no extra costs.

Weight reductions or increments are generally evaluated for constant design performance, unless 1 imited engine performance does not permit this. Any component weight increase is therefore associated with a takeoff weight increase. If, however, the component weight growth is caused by a design change to improve performance - e.g. improved high-lift devices, increased wing span - the final result may well be a takeoff weight reduction.

Sensitivities to structure weight increments are shown in Table $8-1$ for some typical missions. In the case considered, a 108 structure weight growth was followed by a re- 


\begin{tabular}{|l|c|c|}
\hline AIRPLANE CATEGORY & DESIGN RANGE & EFFECT ON MTOW \\
\hline subsonic transport & $250 \mathrm{~nm}$ & $6.5 \%$ \\
& $1000 \mathrm{~nm}$ & $6.9 \%$ \\
& $3000 \mathrm{~nm}$ & $7.0 \%$ \\
supersonic transport & $3000 \mathrm{am}$ & $9.4 \%$ \\
transport VTOL & $250 \mathrm{am}$ & $6.9 \%$ \\
military VTOL & $250 \mathrm{~nm}$ & $9.5 \%$ \\
\hline
\end{tabular}

Table 8-1. Effect of a 108 increase in structure weight on maximum takeoff weight for a constant mission (Reference: ICAS Paper No. 66-1)

sizing process in order to maintain the specified payload-range and other performance. The data presented are 1llustrative only, the sensitivity also depending on where the weight growth occurs.

An even more sensitive case is that of a fixed takeoff weight. A typical weight breakdown* of a conventional design of a medium subsonic propeller turbine transport is as follows:

OEW : 618 MTOW

Payload: 228 MTOW

Fuel : 178 MTOW (58 reserve, 128 trip fuel)

Increasing the OEW by 58 MTOW to 668 MTOW initially reduces the payload by 228 for the same range or the range by 428 for the same payload. Obviously, the final result may be quite different if the airplane is redesigned to accommodate the reduced payload.

b. During detail design it is essential to save every small item of weight that can possibly be saved, in order to ensure a high standard of weight prediction accuracy and to continuously monitor the weight, using an effective weight control system. In most airplane development programs weight reduction programs must be started occasionally in order to redress unfavorable weight creep which may have become apparent. Some cost may be involved in the form of additional manpower, but this is often a small

* Definitions in Section 8.2.2. portion of the penalties incurred by an overweight design.

In order to save weight, the use of advanced materials and sophisticated manufacturing techniques may be considered, resulting in a reduction of the amount of material required. The weight saving may be used to reduce the takeoff weight or to increase the payload or fuel load. However, the cost involved may lead to a noticeable increase in the price of the airplane and an assessment of the value of the weight saving should be made.

c. During negotiations with potential customers the question will arise whether or not the design is subject to special requirements, resulting in a weight penalty. For a civil transport aircraft the weight/ cost tradeoff can be based on the value of payload to the operator, which in turn depends upon the productivity of the aircraft and the frequency with which the payload availability can be sold. The revenue on a pound of capacity payload on a critical

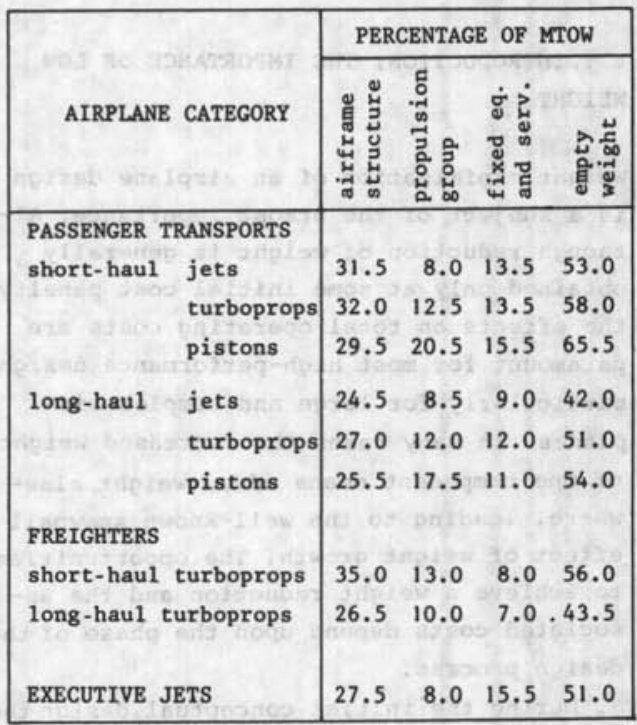

Table 8-2. Typical average empty weight fractions for several categories of transport aireraft 


\begin{tabular}{|c|c|c|c|c|}
\hline \multirow{2}{*}{\multicolumn{2}{|c|}{$\begin{array}{l}\text { AIRPLANE TYPE: } \\
\text { ENGINE TYPE: }\end{array}$}} & \multicolumn{3}{|l|}{ DATE: } \\
\hline & & \multicolumn{3}{|l|}{ MAME: } \\
\hline GROUP INDICATION Laje & WEIGHT ( ) & \multicolumn{3}{|c|}{$\mathrm{x}()^{\text {MOHENT ARM }} \mathrm{z}(\mathrm{l})$} \\
\hline AIRFRAME STRUCTURE & $(1+19)$ & $(12$ & ) & $(20)$ \\
\hline H: & 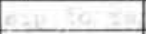 & 80 & & $x+3$ \\
\hline \multicolumn{5}{|l|}{ TAIL GROUP } \\
\hline \multicolumn{5}{|l|}{ BODY GROUP } \\
\hline \multicolumn{5}{|l|}{ ALIGHTING GEAR GROUP } \\
\hline SURFACE CONTROLS GROUP & 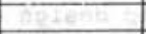 & 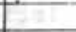 & - & 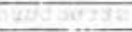 \\
\hline \multicolumn{5}{|l|}{ EMGINE SECTION OR NACELLE GROUP } \\
\hline PROPULSION GROUP & ) & ( & ) & \\
\hline \multicolumn{5}{|l|}{ ENG INE INSTALLATION AND AFTERBURNERS } \\
\hline ACCESSORY GEAR BOXES AND DRIVES & 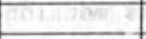 & $0+1$ & & Cotno \\
\hline SUPERCHARGERS (FOR TURBO TYPES) & 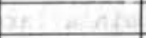 & was & & 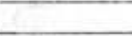 \\
\hline \multicolumn{5}{|l|}{ AIR INDUCTION SYSTEM } \\
\hline \multicolumn{5}{|l|}{ EXHAUST SYSTEA } \\
\hline \multicolumn{5}{|l|}{ OIL SYSTEM AND COOLER } \\
\hline \multicolumn{5}{|c|}{\begin{tabular}{|l|l|l|} 
LUBRICATING SYSTEM & & \\
FUEL. SYSTEM & & \\
\end{tabular}} \\
\hline FUEL. SYSTEM & \begin{tabular}{|r|r|}
+2 \\
\end{tabular} & antan & & $\cos ^{2}$ \\
\hline \multicolumn{5}{|l|}{ WATER INUECTION SYSTEM } \\
\hline \multicolumn{5}{|l|}{ ENGINE CONTROLS } \\
\hline \multicolumn{5}{|l|}{ STARTING SYSTEM } \\
\hline \multicolumn{5}{|l|}{ PROPELLER INSTALLATION } \\
\hline \multicolumn{5}{|l|}{ THRUST REVERSERS } \\
\hline \multirow{2}{*}{\multicolumn{5}{|c|}{$\begin{array}{c}\text { AIRFRAME SERVICES AND EQUIPMENT } \\
\text { AUXILIARY POWER PLANT GROUP }\end{array}$}} \\
\hline & & & & \\
\hline \multicolumn{5}{|l|}{ INSTRUMENTS AND NAV. EQPT. GROUP } \\
\hline \multicolumn{5}{|l|}{ HYDRAULIC AND PNEUMATIC GROUP } \\
\hline \multicolumn{5}{|l|}{ ELECTRICAL GROUP } \\
\hline \multicolumn{5}{|l|}{ ELECTRONICS GROUP } \\
\hline \multicolumn{5}{|l|}{ FURNISHINC AND EQUIPMENT GROUP } \\
\hline \multicolumn{5}{|l|}{ AIRCONDITIONING AND ANTI-TCING GROUP } \\
\hline \multicolumn{5}{|l|}{ 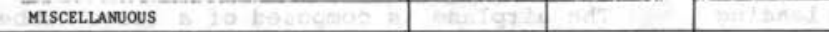 } \\
\hline BASIC (EMPTY) WEIGHT & $(\quad)$ & 6 & ) & 1 \\
\hline OPERATIONAL ITEAS & 1 & ( & ) & 1 \\
\hline CREW PROVISIONS & & & & \\
\hline PASSENGER CABIN SUPPLIES & 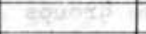 & Fa & & \\
\hline POTABLE HATER AND TOTLET CHEMICALS & $x^{-2}$ & yan & & \\
\hline SAFETY EQUIPMENT & $a$ & 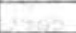 & & \\
\hline OIL, RESIDUAL FUEL, HATER/METHANOL & & & & \\
\hline CARGO HUNDLING EQUIPMENT, MISC. & & & & \\
\hline OPERATIONAL EMPTY WEIGHT & 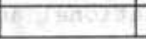 & 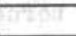 & & \\
\hline
\end{tabular}

Table 8-3. Weight breakdown for civil conventional airplanes (AN 9103 D, modified)

sector ${ }^{*}$ is given by:

revenue $=$ constant $V_{b}$ U D $\$$ /annum $(8-1)$

For a utilization $\mathrm{U}=3000$ hours per annum, a load factor $\phi=.55$ and a passenger revenue $D=.45 \$$ per $\mathrm{sh}$. ton $\mathrm{nm}$, the revenue is $\$ 167$ per $1 \mathrm{~b}$ per annum for an average block speed $\mathrm{V}_{\mathrm{b}}=450 \mathrm{kts}(835 \mathrm{kmh})$. During a 12year service period this amounts to $\$ 2000$

*i.e. a sector where the MTOW is restricted by field length or other operational limitations. per $1 \mathrm{~b}$. In practice, the money lost by the operator will be a mixture of lost passenger revenue, cargo revenue or extra fuel. Moreover, not all sectors are critical all the time and therefore the value of a pound of empty weight will be a fraction of the stated value, e.g. 10-158. However, the lesson to be learnt from these figures is that the empty weight must not increase to such an extent that the capacity payload cannot be achieved.

Basic Empty Weight is composed of airframe 
structure weight, propulsion group weight and the weight of airframe equipment and services (see Table 8-3). Many contribuilons to these weight groups are affected to some extent by the airplane layout; this applies to the structure weight in particular. A survey of empty weight fractions for several airplane categories is given in Table 8-2. In comparing the structure weight fraction of modern airplane types with oldtimers, it is sometimes noted that this fraction has not improved, probably for the following reasons:

a. The relative fuel and propulsion group weights must be taken into account. Due to the improved engines of recent years, these weight fractions have decreased and, for the same payload, the structure weight fraction tends to increase.

b. The wings of modern high-subsonic airplanes are swept and relatively thin. More emphasis has to be laid on stiffness requirements, leading to weight penalties. c. Improved high-lift devices have resulted in higher unit structure weights for the wings.

d. Fatigue life requirements are critical for modern transport airplanes, leading to limitations in the stress levels, e.g. in pressurized cabins. An example of the empty weight penalty to improve the airframe lifetime is shown in Fig. 8-1.

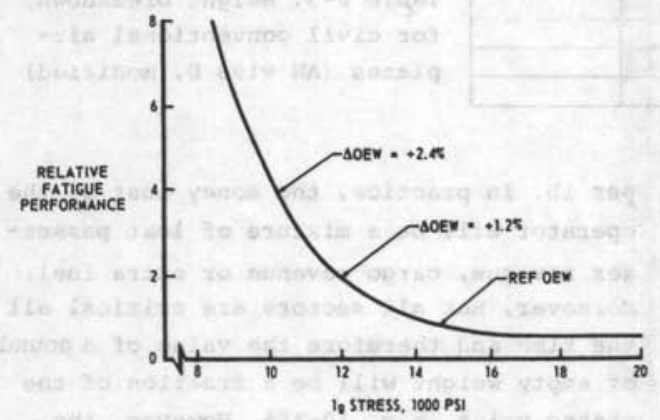

Fig. 8-1. Effect of level flight stress level on OEW and fatigue performance (Reference: AIAA Paper No. 66-882)

e. More stringent airworthiness requirements concerned with the safety and comfort level have resulted in more complex and heavier structure and airframe systems.

Weight prediction in the preliminary design is necessary to performance prediction, center of gravity determination and design of the undercarriage, and also to provide the various design departments with realistic design weights and weight limits. The following sections demonstrate how a weight prediction may be obtained. The publications mentioned in the list of references contain a large amount of information, although in many cases the methods presented are based on a particular category of aircraft and state of the art and do not take changes in technology into account; for these reasons their value is very limited. A good weight estimate starts, however, with clear definitions and effective subdivision of the items to be considered and that is the subject dealt with in the following section.

\subsection{WEIGHT SUBDIVISION AND LIMITATIONS}

The airplane is composed of a large number of parts which can be combined into groups according to several schemes. The weight of these groups and several combinations of groups are of importance in the design, certification and operation of the airplane. Unfortunately, there is little international agreement in the matter of weight terminology, leading to some confusion with regard to the actual meaning of terms. In this section the most commonly used weight terminology is explained and various relationships demonstrated. Not only the weight subdivision is dealt with, but also various limitations on several characteristic weights. The proposed scheme is representative of a wide range of commercial airplane designs. Some considerations are offered to enable a reasonable first choice of the various limits to be made. 


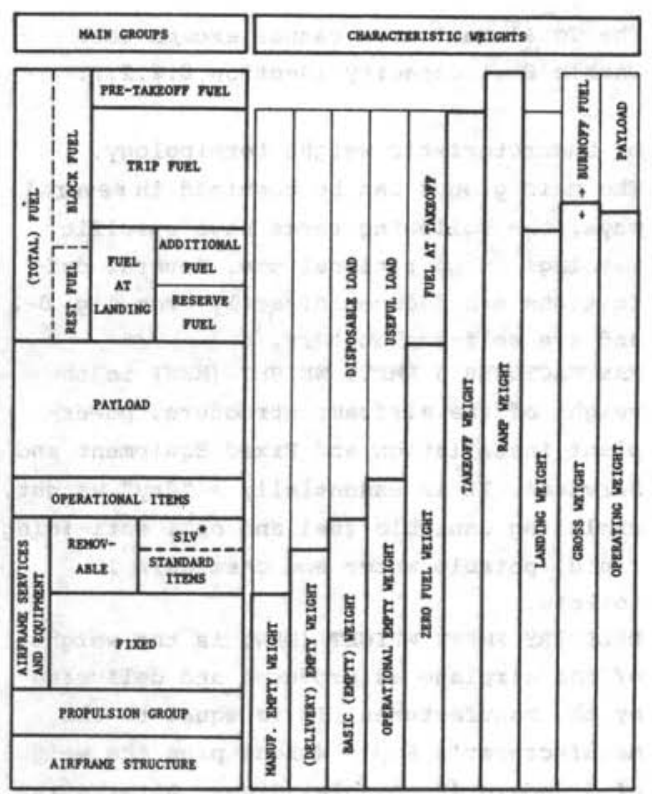

- standare tтes vasutross

Fig. 8-2. Weight groups and characteristic weight terminology (not to scale)

\subsubsection{Weight subdivision}

Figure 8-2 gives a subdivision into main groups and combinations of these in characteristic weight terms.

a. Main groups.

Various terms are defined below and in Table 8-3. More detailed weight breakdowns will be presented in Section 3.4. AIRFRAME STRUCTURE: the wing group, the tail group, the body group, the alighting gear group and the engine nacelle group. The surface controls group may be classified as airframe structure or as a part of the airframe services.

PROPULSION GROUP: the engine(s), items associated with engine installation and operation, the fuel system, and thrust reversing provisions.

AIRFRAME EQUIPMENT AND SERVICES: APU(s) instruments, the hydraulic, electric and electronic systems, furnishings and equipment, air-conditioning, anti-icing systems and other equipment. A further subdivision into fixed and removable equipment is useful for obtaining an accurate and repeatable empty weight definition.

FIXED EQUIPMENT AND SERVICES are considered an integral part of a particular aircraft configuration. These include the weight of fixed ballast (if present) and the fluids which are contained in a closed system (such as hydraulic fluid).

REMOVABLE EQUIPMENT AND SERVICES are those items of equipment or system fluids that are not considered an integral part of a particular aircraft configuration. Removable separating walls, passenger seats*, floor covering, basic emergency equipment. and the like are typical examples:"

Aircraft of a given type are usually ordered by different operators, each of whom has particular requirements with regard to equipment and services. Various items of equipment are not delivered by the airplane manufacturer (government- or buyer-furnished equipment). Consequently, a standard or basic airplane configuration is usually defined with items of equipment or systems fluid which do not vary for aircraft of the same type: Standard Items. Those items which operators choose to add to, omit from or change in the Removable Equipment are referred to as Standard Item Variations (SIV). These items are the Operator's responsibility.

OPERATIONAL ITEMS are those items of personnel, equipment and supplies that are necessary on a particular operation, unless already included in the Basic Empty Weight**, i.e. crew, manuals, crew bags, catering supplies, water supplies, oil, unusable fuel $1^{* *}$, additional emergency equipment, tool kits, etc. These items may vary for a particular airplane configuration according to the operator's allowances for the service intended. However, a minimum crew is defined for each airplane by government regulations. Alternative terms: Operators Items, A (ircraft) $P$ (repared for) S(ervice) Items.

PAYLOAD: all commercial load which is

* Passenger seats are occasionally considered as Operational Items

** See Para. b of this section and Fig. 8-3 *** Unusable fuel is occasionally included in the Removable Fquipment 


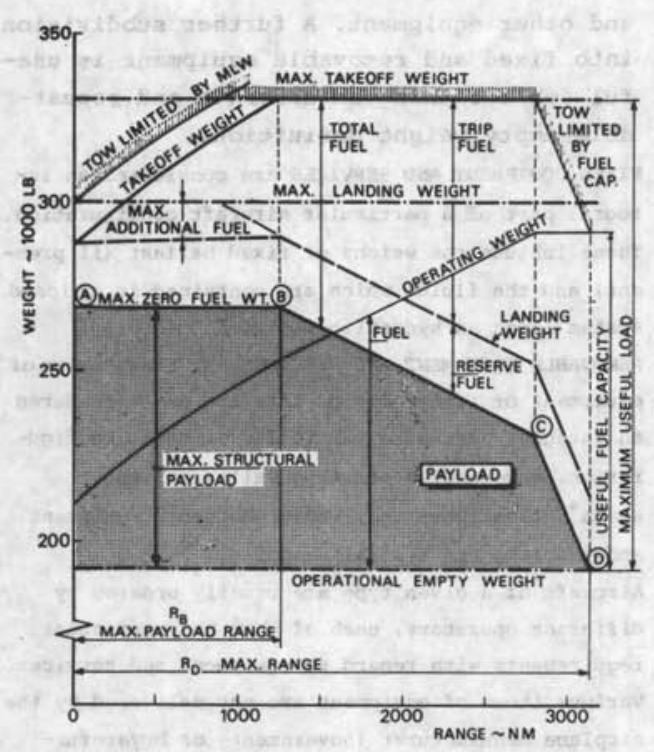

Fig. 8-3. Derivation of the payload-range diagram

carried: passengers and their baggage, cargo and mail. Alternative terms: traffic load, transport load. The payload must never exceed the maximum volumetric payload capacity or the maximum structural payload (Section 8.2.2.). The Estimated Normal Payload is a payload selected by an operator according to the seating pattern and for the passenger and cargo unit weights allowable, and is used for statistical and other related purposis.

TOTAL FUEL: all usable fuel, engine injection fluid and other consumable propulsion agents. A subdivision is made into:

- fuel consumed during engine runup and taxying prior to takeoff,

- trip fuel, i.e. the fuel consumed during flight up to the moment of touchdown in the landing,

- reserve fue ${ }^{*}$, determined by the Operator in accordance with the relevant operational Rules, and

- additional fuel (available for further flights, for example).

* Reserve fuel may be burnt during taxying after landing
The Total Fuel load cannot exceed the Usable Fuel Capacity (Section 8.2.2.).

b. Characteristic weight terminology. The main groups can be combined in several ways; the following terms have specific meanings in operational use. Several definitions are deduced directly from Fig. 8-2 and are self-explanatory.

MANUFACTURER'S - EMPTY WEIGHT (MEW) is the weight of the airframe structure, powerplant installation and Fixed Equipment and Services. It is essentially a "dry" weight, excluding unusable fuel and oil, anti-icing fluid, potable water and chemicals in toilets.

DELIVERY EMPTY WEIGHT (DEW) is the weight of the airplane as produced and delivered by the manufacturer. It is equal to the Manufacturer's Empty Weight plus the weight of Standard (Removable) Items. Alternative term: Empty Weight Dry.

The condition at the time of determining the MEW must be one that is well defined and easily repeatable. The Empty Weight is established experimentally by weighing the delivered airplane and is laid down in the certification documents.

The Tare Weight is the nominal (calculated) Bmpty Weight; it differs from the experimentally determined Empty Weight due to the Manufacturing Variation.

BASIC EMPTY WEIGHT (BEW) is the Delivery Empty Weight plus or minus the net weight of the SIV. It is also equal to the Manufacturer's Empty Weight plus the weight of the Removable Items. Alternative term: Basic Weight.

The Fleet Empty Weight is an average value of the Basic Empty Weight of a fleet or group of airplanes of the same model and configuration, with similar Services and Equipment, operated by the same airliner.

OPERATIONAL EMPTY WEIGHT (OEW): the weight of the airplane without Payload and Fuel. Alternative terms: APS Weight, Basic Operational Weight (BOW), Weight Empty Equipped. ZERO FUEL WEIGHT: the OEW plus payload. It must not exceed the Maximum Zero Fuel Weight (Section 8.2.2.). Alternative term: Empty Tank Weight. Occasionally the term 
Zero wing Fuel weight is used to denote the airplane weight less fuel in the wing. This concept is important in the case of airplanes with fuel tanks both in the wing and in the fuselage or elsewhere.

TAKEOFF WEIGHT: the total weight of a dispatch-loaded aircraft at the moment of brake release or start of the takeoff run. It depends upon the loading condition and must not exceed the Maximum Takeoff Weight and the operational weight limitations in relation to the available performance (Sections 8.2 .2 . and 8.2 .3$.$) .$

RAMP WEIGHT: the Takeoff Weight plus the weight of fuel required for engine run-up and taxying prior to takeoff. It must not exceed the Maximum Ramp Weight (Section 8.2.2.). Alternative term: Taxying weight. LANDING WEIGHT: the airplane weight at the moment of touchdown in the landing. The Landing Weight must not exceed the Maximum Landing Weight or the operational weight limitations referred to in Sections 8.2.2. and 8.2 .3 .

GROSS WEIGHT: the total airplane weight at any moment during the flight. It decreases during the flight due to fuel and oil consumption and may also vary due to payload dropping or refuelling during flight. Alternative term: All-Up Weight (AUW). At the moment of brake release, the Gross Weight is equal to the Takeoff Weight; during flight it is referred to as EnRoute Weight or In-Flight Weight. It is limited by several weight restrictions mentioned in Section 8.2.2.

OPERATING WEIGHT (the author recommends the term Zero Payload Weight): the weight of the laden airplane, but without payload. Consequently, the Operating Weight is equal to the OEW plus the Total Fuel. It cannot exceed the Usable Fuel Capacity (Section 8.2.2.).

DISPOSABLE LOAD AND USEFUL LOAD: the variable load (Payload and Fuel) that can be taken on a particular flight operation. Both items are determining factors for the payload-range capabilities and the economic yield of the airplane, as explained in Section 8.2 .3 . These loads are limited by the Permissible Load, i.e. the difference between the Maximum Takeoff Weight and the Basic Empty Weight.

\subsubsection{Weight limitations and capacities}

Restrictions must be imposed on several variable characteristic weights in order to avoid overloading of the structure or unacceptable performance or handling qualities during any phase of day-to-day operation. Weight limits are therefore established during the process of design and certification for airworthiness and laid down in the Flight Manual (FM) and other documents associated with the Certificate of Airworthiness (C of A).

Although definite weight limits are not established until certification tests, reasonable values must be anticipated during preliminary design in order to ensure satisfactory operational flexibility.

a. Maximum and Minimum Weights.

Maximum weights must be established for each airplane operating condition (ramp or taxying, takeoff, en-route flight, approach and landing) and loading condition (zero fuel condition, center of gravity position, weight distribution). They should not exceed the least of the following: 1. The greatest weight for which compliance with the relevant structural and engineering requirements has been shown. This weight limit is usually designated as a Design Weight, e.g. the Design Take-off Weight.

2. The greatest weight for which compliance with the relevant handling requirements in flight has been demonstrated, a weight restriction which is particularly important for aerobatic airplanes, where certain aerobatic maneuvers can only be executed with a limited Gross Weight.

3. The greatest weight for which performance data have been established.

4. A weight selected by the Applicant* These maximum weights are determined by the

* generally the airplane manufacturer 
design and are invariable during service. As a rule, the four criteria mentioned above will not differ greatly, except in the case of an airplane type for which certification is requested under several different regulations or airworthiness categories. The following maximum weights must be established on most commercial airplane types. MAXIMUM RAMP WEIGHT: the maximum weight authorized* for ground maneuvering prior to brake release in the takeoff. Alternative term: Maximum Design Taxying Weight. MAXIMUM TAKEOFF WEIGHT (MTOW): the maximum weight authorized at takeoff brake release. It is frequently fixed by structural requirements in the takeoff and occasionally by the Maximum En Route Weight. The Useful Load corresponding to the MTOW is the Permissible Load or Maximum Useful Load. MAXIMUM LANDING WEIGHT (MLW): the maximum weight authorized * at the landing. It generally depends on the landing gear strength or the landing impact loads on certain parts of the wing structure. The MLW must not exceed the MTOW.

MAXIMUM (IN-) FLIGHT WEIGHT (MFW): the maximum weight at which flight other than takeoff and landing is permitted. The Maximum Flight Weight and the MTOW are generally equal, except when provisions for refueling in flight exist. The "flaps-up" condition is assumed, unless otherwise stated. Alternative term: Maximum En Route Weight.

MAXIMUM WEIGHT: the greater of the MTOW and the MFW. The Maximum Design Weight is the maximum of the corresponding Design Weights. MAXIMUM ZERO FUEL WEIGHT (MZFW): the maximum weight of an aircraft less the weight of the Total Fuel Load and other consumable propulsive agents in particular sections of the aircraft that are structurally limited by this condition. At this weight the subsequent addition of fuel will not result in the aircraft design strength being exceeded. The weight difference between the MTOW or MLW and the MZFW may be utilized only for the addition of fuel.

*by relevant government regulations
The empty tank case can be a critical loading case in certain critical areas of the structure at positive load factors, as there is no relieving load due to the fuel mass. For some aircraft there may be a limit to the weight for initiating fuel transfer between tanks (Maximum Design Fuel Transfer Weight, MFTW).

b. Loading restrictions and minimum weight. Mass distribution: for each airplane weight the mass distribution must be such that:

1. the center of gravity remains within the appropriate limits for ensuring satisfactory handling qualities (Section 8.5.2. and Chapter 9);

2. compliance with structural requirements such as allowable floor loads, external loads, braking loads, deceleration loads in emergency landings, etc. can be shown. Minimum Weight: flight is prohibited if not clearly impracticable at weights below the Minimum Weight, which is not less than the greatest of the following weights:

1. the Design Minimum Weight, i.e. the lowest Gross Weight at which compliance with the structural loading conditions can be demonstrated;

2. the lowest Gross Weight for which compliance with the relevant handling requirements can be demonstrated;

3. a weight selected by the Applicant.

c. Capacities.

SPACE LIMITED PAYLOAD (SLP): the maximum payload available for passengers, passengers' baggage and/or cargo when restricted by seating limitations and/or volumetric limitations in the cabin, cargo and/or baggage compartments. It must not exceed the Maximum Payload.

Alternative terms: Volumetric Payload Capacity, Maximum Volumetric Capacity Payload.

MAXIMUM (STRUCTURAL) PAYLOAD: the maximum weight of passengers and their baggage, cargo and/or mail that can be loaded in the Aircraft Prepared for Service without exceeding the MFZW.

MAXIMUM SEATING CAPACITY: the maximum number of passengers anticipated for certifi- 
cation. This limit may, for example, correspond to the number of available emergency exits.

MAXIMUM CARGO VOLUME: the maximum space available in the cargo compartment(s). USABLE FUEL CAPACITY: the maximum volume of fuel that can be carried for a particular operation, less drainable and trapped unusable fuel remaining after a fuel runout test has been effected.

Alternative term: Standard Fuel Capacity. The OEW plus the (weight corresponding to the) Usable Fuel Capacity is the Maximum Operating Weight. The author recommends the term: Maximum Zero Payload Weight.

d. Weight restrictions based on available performance.

For each flight the highest operational weights must be determined for which all performance requirements can be met, taking off from the field of departure and landing at the scheduled field of destination or alternate. Appropriate atmospheric conditions (pressure altitude, temperature, wind speed and direction) and field characteristics (runway length, inclination, clearways, stopways) must be accounted for. The wing flap angle(s) may be chosen such that the most favorable condition is obtained.

The weight permitted for takeoff and landing is the lowest of the following:

1. the weight restricted by the available takeoff and landing field length;

2. the weight restricted by available climb performance as laid down in the W(eight) A(1titude) $\mathrm{T}$ (emperature) diagrams*;

3. the weight limited by obstacle clearance requirements :

4. the weight limited by airport runway or platform loading restrictions;

5 . the weight limited by local noise regulations (if any).

8.2.3. Operational weights and the payload-

*cf. Section 11.6.2. range diagram

For each regular flight operation, the operator must guarantee that all limitations applying to the weights and capacities mentioned previously have been respected. The interaction of the various weight criteria results in limitations to the operational weights determined in accordance with the Operational Rules (Operational Weights, Regular Weights). The actual Takeoff Weight, Landing Weight and Payload for a particular flight must never exceed the limiting weights defined below.

OPERATIONAL LANDING WEIGHT (OLW) is the maximum weight authorized for landing and is the lowest of:

1. the Maximum Landing Weight;

2. the permissible Landing Weight based on available performance;

3. the Maximum Zero Fuel Weight plus the fuel load on landing.

Alternative (British) term: Maximum Regular Landing Weight (MRLW) .

OPERATIONAL TAKEOFF WEIGHT (OTOW) is the maximum weight authorized for takeoff and is the lowest of:

1. the Maximum Take-off Weight (MTOW);

2. the permissible Takeoff Weight based on available performance;

3. the Operational Landing Weight plus trip fuel;

4. the MzFW plus the fuel un takeoff;

5. the Takeoff weight restricted by the Maximum Operating Weight (Useful Fuel Capacity).

Alternative (British) term: Maximum Regular Take-off Weight (MRTOW).

WEIGHT-LIMITED PAYLOAD is the weight remaining after the Operating weight is deducted from the orow.

The payload that can be carried is shown in Fig. 8-3 in relation to the flight range and can be computed from the interrelationships between the previous operational weights and other limitations.

For short stages (line $\mathrm{AB}$ ) the Payload must not exceed the Space Limited Payload or Maximum Structural Payload or is limited by the allowable floor loading. In the 
example given the Maximum Structural Payload is assumed to be the limiting criterion.

For flight ranges below $R_{B}$, additional fuel can be carried up to the orow determined by the MLW, as indicated. This is possible only if the MzFW plus fuel reserve is less than the MLW, as is usually the case. For intermediate and long stages the payload is weight-limited and thus equal to the difference between the OTOW and the Operating Weight. Between points B and C, the MTOW is assumed to limit the takeoff weight in the case considered. Point B corresponds to the maximum flight range $\left(R_{B}\right)$ with maximum payload appropriate to the relevant cruising conditions and reserve fuel policy. Increasing the range beyond $\mathrm{R}_{\mathrm{B}}$ is possible on $\mathrm{l}_{\mathrm{Y}}$ by an exchange of payload against fuel.

At point $C$ (range $R_{C}$ ) the usable fuel load is limited by the fuel tank capacity and the Operating Weight reaches its limit. Further increase of the range is possible only by reducing the takeoff weight - and thus the hourly fuel consumption - resulting in a progressive payload reduction with increasing range. At point $D$ there is no payload left and $R_{D}$ is the maximum flight range (without payload), usually specified for a long-range cruise technique.

For normal commercial use the region $C D$ is of minor importance and $R_{C}$ is frequently referred to as the maximum range. If sufficient space is available, both $R_{C}$ and $R_{D}$ may be increased by additional internal fuel tank capacity for relatively low structural weight and drag penalties. A range increment may also be achieved with external fuel tanks.

\subsubsection{The choice of weight limits}

A compromise must be made between various conflicting requirements in order to design an airplane with adequate operational flexibility, satisfying the requirements of several operators on a variety of routes, under different atmospheric and field con- ditions. Discussions with operators may lead to incompatible requirements, in which case several versions of basically the same airplane may be considered, with differences in MZFW and Useful Fuel Capacity, for example. Fig. 8-4 illustrates the flexibility of a particular airplane design. A discussion on this subject can also be found in Ref. 8-11.

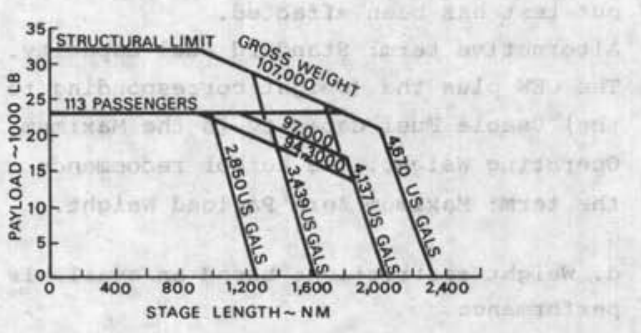

Fig. 8-4. Payload-range diagrams for several versions of the same airplane type

The Maximum Take-off Weight is generally determined by structural design, and occasionally by handling criteria. In the pre-design stage the MTOW is calculated by adding together the OEW, the Estimated Normal Payload and the Total Fuel Load required at the design range*. Empty weight predictions (Section 8.4.) and performance calculations (Section 11.5.) are required to accomplish this. The airplane layout, geometry and engine thrust must be balanced in such a way that for the airport elevations and atmospheric temperatures most frequently encountered the takeoff weight is not unduly limited by restrictions based on available performance. An increment in MTOW might be envisaged for operators whose low-speed performance requirements are not so stringent, in order to provide greater ranges and/or payload capability. In such a case increments in the MZFW, the MLW and the DEW will be desirable to cater for the increased loads on certain critical regions of the structure.

*The word "design" here refers to the values laid down in the design specification 
Certain light airplane categories are defined by limitations on the MTOW, e.g. airplanes with MTOW up to $6,000 \mathrm{lb}$ or $12,500 \mathrm{lb}(2,722 \mathrm{~kg}$ or $5,670 \mathrm{~kg})$. The associated differences in airworthiness requirements have far-reaching effects on the design and operation of such airplanes (cf. Section 5.4.).

The Maximum Zero Fuel Weight must be sufficiently high to ensure that this structural limit will not create a payload restriction for the usual payload specific densities and loading conditions. An increment in MZFW increases the loading flexibility at the cost of a structure weight penalty, mainly in the wing and wing-support structure. Usually this also entails an increase in the MLW. For preliminary design the Space limited Payload can be estimated with the data in Sections 3.2.5. and 8.4.4. The MZFW is thus assumed equal to the OEW plus the payload corresponding to a high-density version of the passenger seating arrangement.

The Maximum Landing Weight should always be higher than the MzFW plus the regular fuel reserve, otherwise the payload will frequently be limited by the MLW. For certain categories of light airplanes the MLW and the MTOW are equal (BCAR group $K$ ); for others the MLW may be slightly lower, namely down to 958 MTOW (cf. FAR 23.473). The difference between the OTOW and the actual takeoff weight required for a specified flight may be used for additional fuel (Fig. 8-3). This is of particular concern to operators flying short route sectors without refueling. For short-haul aircraft this type of flight execution is common practice and a fairly high MLW is desirable, namely of the order of 90 to 958 of the MTOW. Sufficient fuel tank capacity should accordingly be provided. A fuel jettisoning system is required on most multi-engine aircraft unless the airplane meets certain landing and approach climb requirements at a weight equal to the MTOW minus the fuel consumed during a specified 15-minute flight (FAR 25.1001). Choosing the MLW above this characteristic weight makes a fuel dump system superfluous. In earlier regulations this characteristic (landing) weight was equal to the MTOW divided by 1.05 .

The position may be summarized by saying that for commercial use the MLW is usually between the MZFW plus a normal fuel reserve and approximately 958 of the Mrow. The following statistical relationship can be used to make a reasonable choice of the MLW:

$$
\begin{aligned}
\frac{M L W-M Z F}{\text { MTOW }- \text { MZFW }}= & .2+.9 \exp \left(-R_{B} / R_{\text {ref }}\right) \\
& \text { for } R_{B}>172 \mathrm{~nm}(320 \mathrm{~km})
\end{aligned}
$$

where $R_{B}$ is the design range for max. payload (cf. Fig. 8-3) and

$R_{\text {ref }}$ is $1000 \mathrm{~nm}$ if $R_{B}$ is in $\mathrm{nm}$, or $1854 \mathrm{~km}$ if $R_{B}$ is in $\mathrm{km}$.

\section{3 METHODOLOGY OF EMPTY WEIGHT PREDICTION}

Most airplane manufacturers develop their own methods of empty weight prediction, usually basing them on extensive experience with a limited and well-defined category of airplanes. The more generalized and simpler methods presented in this textbook have a predictive accuracy of the order of 5 to 108 standard error for the major groups. In the terminology of Section 5.2.1., they may be classified as methods for configuration selection as far as the structure weight is concerned. If the method is applied judiciously, the result will be quite realistic. Part of the discrepancy between the predicted weight of a particular aircraft part and the actual weight can be ascribed to different definitions of such weight items. Typical sources of accounting differences can be observed in the wingfuselage interconnection and carry-through structure and fillets, in retractable undercarriage structures such as wheel doors, and in several items of furnishing and equipment. If a consistent weight predic- 
tion method is used, the final addition of all items to the empty weight will be not greatly in error. Although the accuracy of prediction does not necessarily improve when more details or parameters are introduced, the designer generally aims at a fairly detailed knowledge of weight penalties and contributions for the purpose of center of gravity determination, optimization and weight control. It is shown in several reports of the Society of Allied Weight Engineers (SAWE) that there is at this stage of the design an optimum number of parameters which yields a minimum prediction error (cf. Ref. 8-38).

The subdivision of the empty weight as presented in Table $8-3$ is based on the $\mathrm{AN}^{*}$ 9103-D Group Weight statement, slightly adapted for civil use. For center of gravity determination (Section 8.5.) the moment arm and static moment of each item can be substituted. More detailed weight breakdowns are frequently required; for several weight groups these will be given in Section 8.4.

Although a wealth of detailed published information on weight engineering can be found in various Technical Papers of the SAWE, consistent and up-to-date methods for calculating the empty weight of the various categories of modern civil airplanes are very scanty indeed. Several examples in the list of references (Ref. 8-47) 8-63) may be used, but in most cases the designer will have to develop ad hoc methods. The information contained in Table 8-5 may be helpful for checking the accuracy of these methods by comparing the results with data on several existing airplanes.

In preliminary aircraft design weight prediction is always a mixture of rational analysis and statistical methods, the reason being that many design details are still not known at that moment. Statistical weight equations for many components are usually written in the exponential form,

\footnotetext{
*American Normalisation
}

as follows:

Weight=constant. $\phi_{1}{ }^{n_{1}} \phi_{2}{ }^{n_{2}} \phi_{3}{ }^{n_{3}} \ldots \phi_{m}{ }^{n_{m}}$

The constant of proportionality and the exponents $\mathrm{n}_{1}, \mathrm{n}_{2}, \ldots \mathrm{n}_{\mathrm{m}}$ of the design parameters $\phi_{1}, \phi_{2}, \ldots \phi_{\mathrm{m}}$ are determined by standard regression analysis techniques, using weight and geometric data of actual components, and subject to the condition of minimum standard deviation. The problem may arise that the magnitude and/or algebraic sign of the exponents is unacceptable for use in design analysis, the results being clearly irrational. Staton explains in Ref. 8-41 how constrained regression analysis can be used to solve this problem. A much better approach, though not always feasible owing to lack of verificatory data, is to break the item considered down into the sum of several other contributions. For each contribution relevant parameters must be chosen on a rational basis. If a weight contribution $\mathrm{Y}$ is expressed as a linear function of a parameter $\mathrm{X}$, assumed to be known without error, linear regression analysis can then be used to obtain the best curve fit.

For example, on the assumption of a constant average stress level, the material required to resist the wing bending moment is proportional to the root bending moment times the structural span divided by the root chord thickness. For a large proportion of the wing structural weight the following parameter is therefore relevant:

$x=\frac{n_{u l t} w_{G} b^{2}}{t_{r} \cos ^{2} \Lambda_{\frac{1}{2}}}$

The parameter $\mathrm{X}$ is calculated for $\mathrm{N}$ sample airplanes (values $x_{i}$ ), for which the wing weight has known values $y_{i}$. The regression line of $\mathrm{Y}$ upon $\mathrm{X}$ is fitted by the method of least squares, according to the equation:

$Y=a+b x$ 
where

$a=\frac{\sum x_{i} \sum x_{i} y_{i}-\sum y_{i} \sum x_{i}{ }^{2}}{\left(\sum x_{i}\right)^{2}-N \sum x_{i}{ }^{2}}$

and

$b=\frac{\sum x_{i} \sum y_{i}-N \sum x_{i} y_{i}}{\left(\sum x_{i}\right)^{2}-N \sum x_{i}^{2}}$

For limited ranges of $\mathrm{X}$ and $\mathrm{Y}$ a linear function may be satisfactory, but if a considerable variation in the actual size of the item exists, a better result is usually obtained with:

$\mathrm{Y}=k \mathrm{x}^{\mathrm{n}}$

On a $\log -\log$ scale this relation is linear: $\log \mathrm{Y}=\log \mathrm{k}+\mathrm{n} \log \mathrm{X}$

and again linear regression analysis can be used.

The standard error of a prediction method is :

$S=\sqrt{\frac{1}{N-1}\left[\sum m_{i}{ }^{2}-\frac{\left(\sum m_{i}\right)^{2}}{N}\right]}$

where $m_{i}$ is the ratio of actual to estimated weight of the sample.

References $8-41$ and $8-44$ give more information on the use of statistics and various types of regression analysis. A certain amount of care should always be taken when using statistical methods. A check must be made to see if the airplane being analyzed falls within the range of data points that were used to develop the method. The choice of parameters to be used is always somewhat arbitrary and due attention must be paid to data points that are far from the regression line. They may indicate that alternative correlations should be investigated. Finally, all parameters used in weight prediction must be well defined and not give rise to misinterpretation or vagueness.

Finally, it should be realized that many weight prediction methods apply to a limited category of airplanes. Occasionally they may be adapted to other categories simply by modifying the factor of proportionality, provided that the basic expression has a rational background and derivation.

\subsection{WEIGHT PREDICTION DATA AND METHODS}

\subsubsection{Airframe structure}

a. Structure weight prediction based on the aircraft specific density. An intriguing approach to structure weight estimation, applicable to conventional configurations, is made by Caddell in Ref. 8-39, who uses the aircraft density, i.e. the design gross weight divided by the total airplane volume. If his line of thought is adopted, the structural weight fraction of transport-type turbine-powered airplanes can be expressed in terms of the ultimate load factor, the fuselage dimensions and the MTOW:

$\frac{w_{s}}{w_{t o}}=k_{s} \sqrt{n_{u l t}}\left(\frac{b_{f} h_{f} l_{f}}{w_{t o}}\right)^{.24}$

where

$k_{s}=.230$ for $b_{f}, h_{f}$ and $l_{f}$ in $\mathrm{ft}$ and $w_{s}$ and $w_{\text {to }}$ in $1 b$,

$k_{s}=.447$ for $b_{f}, h_{f}$ and $l_{f}$ in $m$ and $w_{s}$ and $w_{\text {to }}$ in $\mathrm{kg}$, and

nult corresponds to the MrOW.

Although this simple expression yields a reasonably accurate prediction, it is useless for design optimization, as the effects of the airplane layout are not accounted for. The only alternative is a detailed assessment of the contributions of all structural components or groups. The subdivision in Table $8-4$ can be used to collect structural weight data. A compilation of structure weight data for existing aircraft is presented in Table $8-5$. According to (8-11) the ultimate load factor affects structural weight to a considerable extent. The rules for establishing the ultimate load factor ( 1.5 times the limit load factor) are laid down in the various airworthiness regulations. It should be 


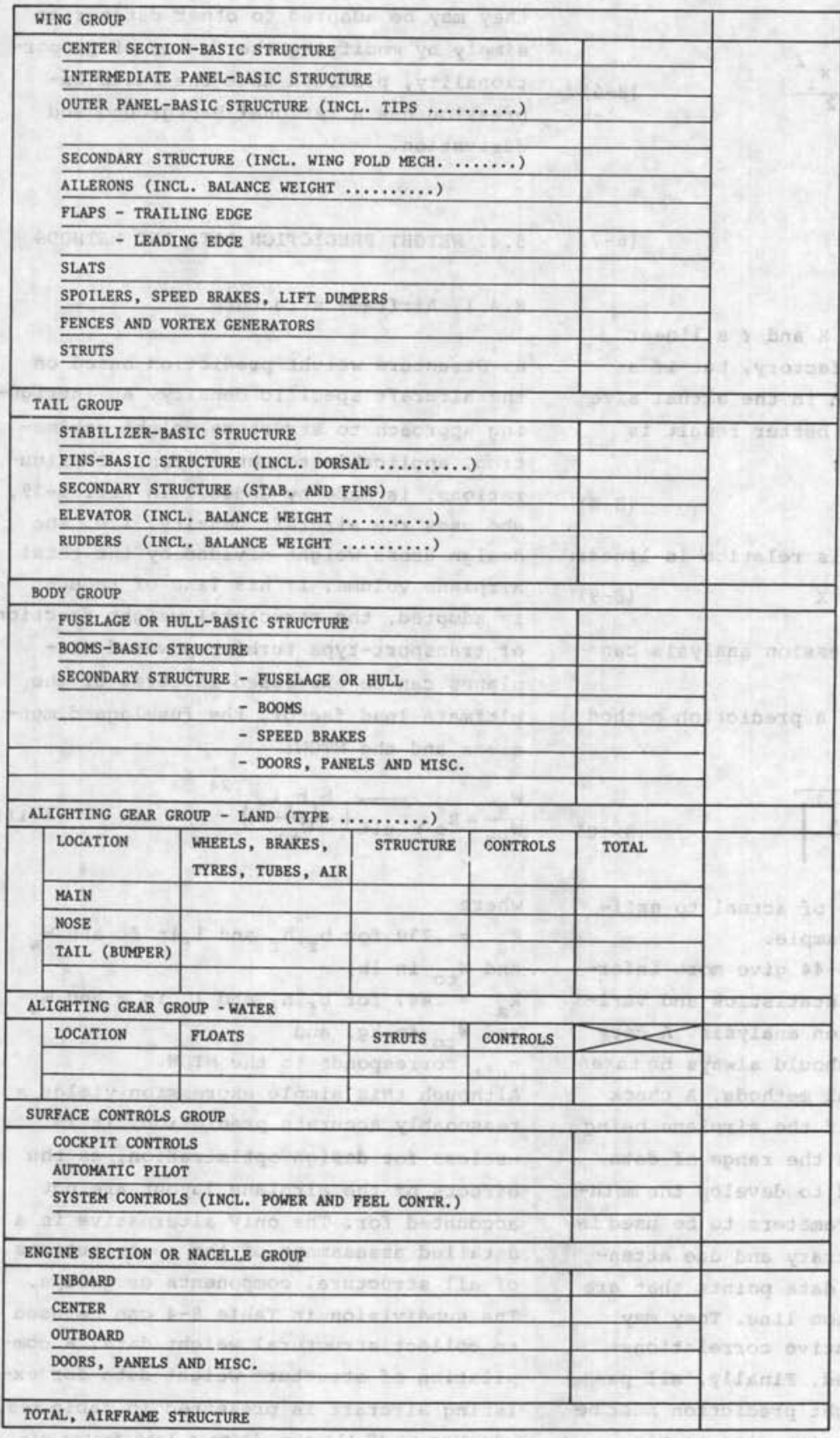

Table 8-4. Airframe Structure Group weight breakdown according to AN-9103-D (modified) 


\begin{tabular}{|c|c|c|c|c|c|c|c|c|c|c|c|c|c|c|}
\hline \multirow{2}{*}{\multicolumn{2}{|c|}{$\begin{array}{l}\text { AIRPLANE CATEGORY } \\
\text { AND TYPE }\end{array}$}} & \multirow{2}{*}{\begin{tabular}{|c|} 
MTOW \\
$10^{3} 1 \mathrm{~b}$ \\
\end{tabular}} & \multicolumn{2}{|c|}{\begin{tabular}{l|} 
WING \\
GROUP \\
\end{tabular}} & \multicolumn{2}{|c|}{$\begin{array}{l}\text { TAIL } \\
\text { GROUP }\end{array}$} & \multicolumn{2}{|c|}{$\begin{array}{c}\text { FUSELAGE } \\
\text { GROUP }\end{array}$} & \multicolumn{2}{|c|}{$\begin{array}{l}\text { LANDING } \\
\text { GEAR }\end{array}$} & \multicolumn{2}{|c|}{$\begin{array}{l}\text { SURFACE } \\
\text { CONTROLS }\end{array}$} & \multicolumn{2}{|c|}{$\begin{array}{l}\text { NACELLE } \\
\text { GROUP }\end{array}$} \\
\hline & & & $10^{3} 1 \mathrm{~b}$ & $z$ & $10^{3} 1 \mathrm{~b}$ & $z$ & $10^{3} 1 \mathrm{~b}$ & $z$ & $10^{3} 1 \mathrm{~b}$ & $z$ & $10^{3} 1 \mathrm{~b}$ & 2 & $10^{3} 1 \mathrm{~b}$ & $z$ \\
\hline 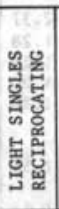 & $\begin{array}{l}\begin{aligned} & \text { Cessna }-150 \mathrm{~A} \\
&-172 \mathrm{~B} \\
&-180 \mathrm{D} \\
&-182 \mathrm{D} \\
&-185 \\
&-210 \\
& \\
& \text { Beechcraft } \mathrm{J}-3 \mathrm{~S}\end{aligned} \\
\text { Saab Saf ir }\end{array}$ & $\begin{array}{l}1.50 \\
2.20 \\
2.65 \\
2.65 \\
3.20 \\
2.90 \\
2.90 \\
2.66\end{array}$ & $\begin{array}{l}0.213 \\
0.236 \\
0.254 \\
0.254 \\
0.266 \\
0.261 \\
0.379 \\
0.276\end{array}$ & $\begin{array}{c}14.2 \\
10.7 \\
9.58 \\
9.58 \\
8.31 \\
9.0 \\
13.1 \\
10.4\end{array}$ & $\begin{array}{l}0.041 \\
0.061 \\
0.059 \\
0.061 \\
0.071 \\
0.071 \\
0.058 \\
0.060\end{array}$ & $\begin{array}{l}2.73 \\
2.77 \\
2.23 \\
2.30 \\
2.22 \\
2.45 \\
2.00 \\
2.26\end{array}$ & $\begin{array}{l}0.166 \\
0.253 \\
0.270 \\
0.273 \\
0.290 \\
0.316 \\
0.200 \\
0.386\end{array}$ & $\begin{array}{c}11.1 \\
11.5 \\
10.2 \\
10.3 \\
9.06 \\
10.90 \\
6.90 \\
14.5\end{array}$ & $\begin{array}{l}0.106 \\
0.122 \\
0.119 \\
0.136 \\
0.132 \\
0.207 \\
0.205 \\
0.119\end{array}$ & $\begin{array}{l}7.07 \\
5.55 \\
4.49 \\
5.13 \\
4.13 \\
7.14 \\
7.07 \\
4.47\end{array}$ & $\begin{array}{l}0.031 \\
0.031 \\
0.036 \\
0.036 \\
0.036 \\
0.044 \\
0.056 \\
*\end{array}$ & $\begin{array}{l}2.07 \\
1.41 \\
1.36 \\
1.36 \\
1.13 \\
1.52 \\
1.93 \\
-\end{array}$ & $\begin{array}{l}0.024 \\
0.031 \\
0.037 \\
0.036 \\
0.041 \\
0.031 \\
0.062 \\
* *\end{array}$ & $\begin{array}{l}1.60 \\
1.41 \\
1.40 \\
1.36 \\
1.28 \\
1.07 \\
2.14 \\
-\end{array}$ \\
\hline 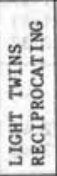 & $\begin{array}{l}\text { Cessna C-310 } \\
\text { Beecheraft G-50 } \\
-65 \\
-95 \\
D-18 S \\
\text { E-18S } \\
\text { De Havilland Dove }\end{array}$ & $\begin{array}{l}4.83 \\
7.15 \\
7.37 \\
4.00 \\
8.75 \\
9.70 \\
8.80\end{array}$ & $\begin{array}{l}0.454 \\
0.656 \\
0.670 \\
0.458 \\
0.858 \\
0.874 \\
0.930\end{array}$ & $\begin{array}{c}9.40 \\
9.17 \\
9.09 \\
11.5 \\
9.81 \\
9.01 \\
10.6\end{array}$ & $\begin{array}{l}0.118 \\
0.156 \\
0.153 \\
0.079 \\
0.177 \\
0.180 \\
0.196\end{array}$ & $\begin{array}{l}2.44 \\
2.18 \\
2.08 \\
1.98 \\
2.02 \\
1.86 \\
2.23\end{array}$ & $\begin{array}{l}0.319 \\
0.495 \\
0.601 \\
0.276 \\
0.733 \\
0.768 \\
0.745\end{array}$ & $\begin{array}{l}6.60 \\
6.92 \\
8.15 \\
6.90 \\
8.38 \\
7.92 \\
8.47\end{array}$ & $\begin{array}{l}0.263 \\
0.447 \\
0.444 \\
0.218 \\
0.560 \\
0.585 \\
0.391\end{array}$ & $\begin{array}{l}5.45 \\
6.25 \\
6.02 \\
5.45 \\
6.40 \\
6.03 \\
4.44\end{array}$ & $\begin{array}{l}0.066 \\
0.120 \\
0.132 \\
0.073 \\
0.115 \\
0.115 \\
*\end{array}$ & $\begin{array}{l}1.37 \\
1.68 \\
1.79 \\
1.83 \\
1.31 \\
1.19 \\
-\end{array}$ & $\begin{array}{l}0.129 \\
0.261 \\
0.285 \\
0.180 \\
0.311 \\
0.331 \\
0.220\end{array}$ & $\begin{array}{l}2.67 \\
3.65 \\
3.87 \\
4.50 \\
3.55 \\
3.41 \\
2.50\end{array}$ \\
\hline 占热 & $\begin{array}{l}\text { Cessna } \mathrm{T}-37 \\
\text { Fouga Magister } \\
\text { Canadair CL- } 41\end{array}$ & $\begin{array}{l}6.44 \\
6.28 \\
6.50\end{array}$ & $\begin{array}{l}0.531 \\
1.089 \\
0.892\end{array}$ & $\begin{array}{l}8.24 \\
17.3 \\
13.7\end{array}$ & $\begin{array}{l}0.128 \\
0.165 \\
0.201\end{array}$ & $\begin{array}{l}1.99 \\
2.63 \\
3.09\end{array}$ & $\begin{array}{l}0.839 \\
0.743 \\
0.955\end{array}$ & $\begin{array}{l}13.0 \\
11.8 \\
14.7\end{array}$ & $\begin{array}{l}0.330 \\
0.459 \\
0.318\end{array}$ & $\begin{array}{l}5.12 \\
7.31 \\
4.89\end{array}$ & $\begin{array}{l}0.154 \\
0.260 \\
0.172\end{array}$ & $\begin{array}{l}2.39 \\
4.14 \\
2.65\end{array}$ & $\begin{array}{c}- \\
\overline{0} 040\end{array}$ & $\overline{-}-$ \\
\hline 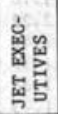 & $\begin{array}{l}\text { H. Siddeley - } 125 \\
\text { Jet Commander } 1121 \\
\text { N. An. Sabreliner } \\
\text { Lockheed Jetstar }\end{array}$ & $\begin{array}{l}21.200 \\
16.000 \\
16.700 \\
30.680\end{array}$ & $\begin{array}{l}1.968 \\
1.322 \\
1.753 \\
2.827\end{array}$ & $\begin{array}{c}9.28 \\
8.26 \\
10.5 \\
9.21\end{array}$ & $\begin{array}{l}0.608 \\
0.425 \\
0.297 \\
0.879\end{array}$ & $\begin{array}{l}2.87 \\
2.66 \\
1.78 \\
2.87\end{array}$ & $\begin{array}{l}1.628 \\
1.622 \\
2.014 \\
3.491\end{array}$ & $\begin{array}{l}7.68 \\
10.1 \\
12.1 \\
11.4\end{array}$ & $\begin{array}{l}0.659 \\
0.443 \\
0.728 \\
1.061\end{array}$ & $\begin{array}{l}3.11 \\
2.76 \\
4.36 \\
3.46\end{array}$ & $\begin{array}{l}0.217 \\
0.223 \\
0.344 \\
0.768\end{array}$ & $\begin{array}{l}1.02 \\
1.39 \\
2.06 \\
2.50\end{array}$ & $\begin{array}{l}* 4 \\
0.35 \\
0.315 \\
0.792\end{array}$ & $\begin{array}{l}- \\
2.19 \\
1.89 \\
2.58\end{array}$ \\
\hline
\end{tabular}

* estimated

** included in other items

Table 8-5. Weight breakdown of the structure group weight

PROPELLER TRANSPORTS

\begin{tabular}{|c|c|c|c|c|c|c|c|c|c|c|c|c|c|c|c|}
\hline \multirow{2}{*}{\multicolumn{3}{|c|}{$\begin{array}{l}\text { AIRPLANE CATEGORY } \\
\text { AND TYPE }\end{array}$}} & \multirow{2}{*}{\begin{tabular}{|c|} 
MTOW \\
$10^{3} 1 \mathrm{~b}$ \\
\end{tabular}} & \multicolumn{2}{|c|}{$\begin{array}{l}\text { WING } \\
\text { GROUP }\end{array}$} & \multicolumn{2}{|c|}{$\begin{array}{l}\text { TAIL } \\
\text { GROUP }\end{array}$} & \multicolumn{2}{|c|}{$\begin{array}{l}\text { FUSELAGE } \\
\text { GROUP }\end{array}$} & \multicolumn{2}{|c|}{$\begin{array}{c}\text { LANDING } \\
\text { GEAR }\end{array}$} & \multicolumn{2}{|c|}{$\begin{array}{l}\text { SURFACE } \\
\text { CONTROLS }\end{array}$} & \multicolumn{2}{|c|}{$\begin{array}{c}\text { NACELLE } \\
\text { GROUP }\end{array}$} \\
\hline & & & & $10^{3} 1 \mathrm{~b}$ & $z$ & $10^{3} \mathrm{ib}$ & $z$ & $10^{3} 1 \mathrm{~b}$ & $\pi$ & $10^{3} 1 \mathrm{~b}$ & $z$ & $10^{3} 1 \mathrm{~b}$ & $z$ & $10^{3} 1 \mathrm{~b}$ & $z$ \\
\hline \multirow{2}{*}{ 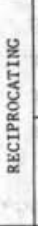 } & 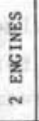 & $\begin{array}{l}\text { De Havilland DHC-4 } \\
\text { Saab Scandia } \\
\text { H. Page Herald } \\
\text { S.A. Twin Pioneer } \\
\text { Canadair CL-21 }\end{array}$ & $\begin{array}{l}24.000 \\
35.273 \\
37.500 \\
14.600 \\
32.500\end{array}$ & $\begin{array}{l}2.925 \\
4.195 \\
4.365 \\
2.121 \\
3.99\end{array}$ & $\begin{array}{l}12.2 \\
11.9 \\
11.6 \\
14.5 \\
12.3\end{array}$ & $\begin{array}{l}0.790 \\
0.584 \\
0.987 \\
0.576 \\
1.055\end{array}$ & $\begin{array}{l}3.29 \\
1.66 \\
2.63 \\
3.95 \\
3.25\end{array}$ & $\begin{array}{l}2.849 \\
2.773 \\
2.986 \\
1.381 \\
3.260\end{array}$ & $\begin{array}{c}11.9 \\
7.86 \\
7.96 \\
9.46 \\
10.0\end{array}$ & $\begin{array}{l}1.23 \\
1.841 \\
1.625 \\
0.703 \\
1.609\end{array}$ & $\begin{array}{l}5.13 \\
5.22 \\
4.33 \\
4.82 \\
4.95\end{array}$ & $\begin{array}{l}0.326 \\
0.369 \\
0.364 \\
0.300 \\
0.371\end{array}$ & $\begin{array}{l}1.36 \\
1.05 \\
0.97 \\
2.05 \\
1.14\end{array}$ & $\begin{array}{l}0.781 \\
1.479 \\
0.830 \\
0.230 \\
1.29\end{array}$ & $\begin{array}{l}3.25 \\
4.19 \\
2.21 \\
1.58 \\
3.97\end{array}$ \\
\hline & 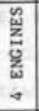 & $\begin{aligned} & \text { Douglas } D C-6 B \\
& \text { DC }-7 C \\
& \text { Lockheed } L-749 \\
& L-1049\end{aligned}$ & $\begin{array}{r}81.500 \\
143.000 \\
102.072 \\
137.500\end{array}$ & $\begin{array}{r}7.506 \\
11.100 \\
11.102 \\
11.542\end{array}$ & $\begin{array}{l}9.21 \\
7.76 \\
10.9 \\
8.39\end{array}$ & $\begin{array}{l}1.406 \\
1.900 \\
2.059 \\
2.604\end{array}$ & $\begin{array}{l}1.73 \\
1.33 \\
2.02 \\
1.89\end{array}$ & $\begin{array}{r}5.471 \\
8.450 \\
7.407 \\
12.839\end{array}$ & $\begin{array}{l}6.71 \\
5.91 \\
7.26 \\
9.34\end{array}$ & $\begin{array}{l}4.165 \\
5.130 \\
4.782 \\
5.422\end{array}$ & $\begin{array}{l}5.11 \\
3.59 \\
4.68 \\
3.94\end{array}$ & $\begin{array}{l}1.052 \\
1.215 \\
1.488 \\
1.685\end{array}$ & $\begin{array}{l}1.29 \\
0.85 \\
1.46 \\
1.23\end{array}$ & $\begin{array}{l}2.871 \\
4.130 \\
3.869 \\
4.420\end{array}$ & $\begin{array}{l}3.52 \\
2.89 \\
3.79 \\
3.21\end{array}$ \\
\hline \multirow{2}{*}{ 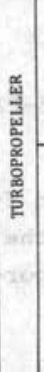 } & 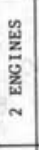 & $\begin{array}{l}\text { Nord } 262 \\
\text { Fokker F-27/100 } \\
\text { F-27/200 } \\
\text { F-27/500 } \\
\text { Grumman Gulf stream } \\
\text { Short Skyvan }\end{array}$ & $\begin{array}{l}23.050 \\
39.000 \\
43.500 \\
45.000 \\
33.600 \\
12.500\end{array}$ & $\begin{array}{l}2.698 \\
4.408 \\
4.505 \\
4.510 \\
3.735 \\
1.220\end{array}$ & $\begin{array}{l}11.7 \\
11.3 \\
10.4 \\
10.0 \\
11.2 \\
9.76\end{array}$ & $\begin{array}{l}0.805 \\
0.977 \\
1.501 \\
1.060 \\
0.874 \\
0.374\end{array}$ & $\begin{array}{l}3.49 \\
2.51 \\
2.42 \\
2.35 \\
2.60 \\
2.99\end{array}$ & $\begin{array}{l}3.675 \\
4.122 \\
4.303 \\
5.142 \\
3.718 \\
2.154\end{array}$ & $\begin{array}{c}15.9 \\
10.6 \\
9.89 \\
11.4 \\
11.1 \\
17.2\end{array}$ & $\begin{array}{l}1.085 \\
1.940 \\
1.825 \\
1.865 \\
1.207 \\
0.466\end{array}$ & $\begin{array}{l}4.71 \\
4.97 \\
4.20 \\
4.14 \\
3.59 \\
3.73\end{array}$ & $\begin{array}{l}0.408 \\
0.613 \\
0.620 \\
0.626 \\
0.461 \\
0.265\end{array}$ & $\begin{array}{l}1.77 \\
1.57 \\
1.43 \\
1.39 \\
1.37 \\
2.12\end{array}$ & $\begin{array}{l}0.236 \\
0.628 \\
0.667 \\
0.668 \\
1.136 \\
0.254\end{array}$ & $\begin{array}{l}1.02 \\
1.61 \\
1.53 \\
1.48 \\
3.38 \\
2.03\end{array}$ \\
\hline & 鵕 & $\begin{array}{l}\text { Bréguet } 941 \\
\text { H.S. Argosy } \\
\text { Vickers Viscount } 810 \\
\text { Bristo1 Brit, } 300 \\
\text { Brit. } 320 \\
\text { Canadair CL-44C } \\
\text { CL-44D } \\
\text { Lockheed Electra } \\
\text { C-130E } \\
\text { C-133A }\end{array}$ & $\begin{array}{r}58.421 \\
82.000 \\
69.000 \\
155.000 \\
184.523 \\
205.000 \\
205.000 \\
106.700 \\
151.522 \\
275.000\end{array}$ & $\begin{array}{c}4.096 \\
10.800 \\
6.25 \\
13.433 \\
14.199 \\
15.710 \\
15.588 \\
7.670 \\
11.697 \\
27.403\end{array}$ & $\begin{array}{l}7.01 \\
13.2 \\
9.06 \\
8.67 \\
7.69 \\
7.66 \\
7.60 \\
7.19 \\
7.72 \\
9.96\end{array}$ & $\begin{array}{l}1.387 \\
1.300 \\
1.245 \\
3.202 \\
3.221 \\
3.749 \\
3.540 \\
1.924 \\
3.425 \\
6.011\end{array}$ & $\begin{array}{l}2.37 \\
1.59 \\
1.80 \\
2.07 \\
1.75 \\
1.83 \\
1.73 \\
1.80 \\
2.26 \\
2.19\end{array}$ & $\begin{array}{r}6.481 \\
11.100^{*} \\
6.900 \\
11.100 \\
11.750 \\
20.524 \\
16.047 \\
9.954 \\
14.340 \\
30.940\end{array}$ & $\begin{array}{c}11.1 \\
13.5 \\
10.0 \\
7.16 \\
6.38 \\
10.0 \\
7.83 \\
9.33 \\
9.46 \\
11.3\end{array}$ & \begin{tabular}{|l|}
2.626 \\
3.180 \\
2.469 \\
5.785 \\
6.500 \\
7.083 \\
7.300 \\
3.817 \\
5.341 \\
10.635 \\
\end{tabular} & $\begin{array}{l}4.94 \\
3.88 \\
3.58 \\
3.73 \\
3.52 \\
3.46 \\
3.56 \\
3.58 \\
3.53 \\
3.87\end{array}$ & $\begin{array}{c}1.056 \\
* * \\
0.824 \\
1.221 \\
2.048 \\
2.146 \\
1.830 \\
* * * \\
1.702 \\
1.804\end{array}$ & $\begin{array}{c}1.81 \\
- \\
1.19 \\
0.79 \\
1.11 \\
1.05 \\
0.89 \\
- \\
1.12 \\
0.66\end{array}$ & $\begin{array}{l}.04 \\
1.200 \\
1.810 \\
4.930 \\
7.350 \\
6.834 \\
6.043 \\
4.417 \\
2.675 \\
3.512\end{array}$ & $\begin{array}{l}- \\
1.46 \\
2.62 \\
3.18 \\
3.98 \\
3.33 \\
2.95 \\
4.14 \\
1.77 \\
1.28\end{array}$ \\
\hline
\end{tabular}

- tail booms $(2,360 \mathrm{lb})$ included $\quad \ldots$ included in other items

*.* no data available

Table 8-5. (Continued) 


\begin{tabular}{|c|c|c|c|c|c|c|c|c|c|c|c|c|c|c|}
\hline & \multirow{2}{*}{$\begin{array}{l}\text { AIRPLANE CATECORY } \\
\text { ARD TYPE }\end{array}$} & \multirow{2}{*}{ MTOW } & \multicolumn{2}{|c|}{$\begin{array}{l}\text { WTMG } \\
\text { GROUP }\end{array}$} & \multicolumn{2}{|c|}{$\begin{array}{l}\text { IAIL } \\
\text { GROUP }\end{array}$} & \multicolumn{2}{|c|}{$\begin{array}{c}\text { FUSELAGE } \\
\text { GROUP }\end{array}$} & \multicolumn{2}{|c|}{$\begin{array}{c}\text { LANDING } \\
\text { GEAR }\end{array}$} & \multicolumn{2}{|c|}{$\begin{array}{l}\text { SURYACE } \\
\text { CONTROLS }\end{array}$} & \multicolumn{2}{|c|}{$\begin{array}{c}\text { NACELLE } \\
\text { GROUP }\end{array}$} \\
\hline & & & $10^{3} 1 \mathrm{~b}$ & 7 & $10^{3} 1 \mathrm{~b}$ & 7 & $10^{3} 1 \mathrm{~b}$ & $\pi$ & $10^{3} 1 \mathrm{~b}$ & $z$ & $10^{3} 16$ & $z$ & $10^{3} 1 \mathrm{~b}$ & $z$ \\
\hline 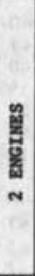 & $\begin{array}{c}\text { VFW-Fokker } 614 \\
\text { Fokker-VFW } \mathrm{F}-28 / 1000 \\
\mathrm{~F}-28 / 2000 \\
\mathrm{~F}-28 / 5000 \\
\mathrm{~F}-28 / 6000 \\
\text { BAC } 1-11 / 300 \\
1-11 / 400 \\
\text { Kc D. Douglas DC-9/10 } \\
\text { Boeing } 737-100 \mathrm{M} \\
737-200 \\
\text { Aerospat. Carave11e YIR } \\
\text { Airbus A30nB/2 }\end{array}$ & $\begin{array}{r}40.981 \\
65.000 \\
65.000 \\
70.800 \\
70.800 \\
87.000 \\
87.000 \\
91.500 \\
97.800 \\
100.000 \\
110.230 \\
304.000\end{array}$ & $\begin{array}{l}5.767 \\
7.330 \\
7.347 \\
8.223 \\
8.244 \\
9.643 \\
9.670 \\
9.470 \\
9.968 \\
10.613 \\
14.735 \\
44.131 \\
\end{array}$ & $\begin{array}{l}14.1 \\
11.3 \\
11.3 \\
11.6 \\
11.6 \\
11.1 \\
11.1 \\
10.3 \\
10.2 \\
10.6 \\
13.4 \\
14.5\end{array}$ & $\begin{array}{l}1.121 \\
1.632 \\
1.632 \\
1.632 \\
1.632 \\
2.369 \\
2.419 \\
2.630 \\
2.700 \\
2.718 \\
1.957 \\
5.941\end{array}$ & $\begin{array}{l}2.74 \\
2.46 \\
2.46 \\
2.31 \\
2.31 \\
2.72 \\
2.78 \\
2.87 \\
2.76 \\
2.72 \\
1.77 \\
1.95\end{array}$ & $\begin{array}{r}5.233 \\
7.043 \\
7.649 \\
7.043 \\
7.649 \\
9.713 \\
9.743 \\
11.206 \\
12.380 \\
12.108 \\
11.570 \\
35.820\end{array}$ & $\begin{array}{l}12.8 \\
10.8 \\
11.8 \\
9.95 \\
10.8 \\
11.2 \\
11.3 \\
12.2 \\
12.7 \\
12.1 \\
10.5 \\
11.8\end{array}$ & $\begin{array}{l}1.620 \\
2.759 \\
2.759 \\
2.759 \\
2.789 \\
2.865 \\
2.899 \\
3.660 \\
3.687 \\
4.354 \\
5.110 \\
13.611\end{array}$ & $\begin{array}{l}3.45 \\
4.24 \\
4.24 \\
3.90 \\
3.94 \\
3.29 \\
3.33 \\
4.00 \\
3.77 \\
4.35 \\
4.63 \\
4.47\end{array}$ & $\begin{array}{l}0.745 \\
1.387 \\
1.400 \\
1.665 \\
1.674 \\
1.481 \\
1.207 \\
1.264 \\
1.589 \\
2.348 \\
2.063 \\
5.808\end{array}$ & $\begin{array}{l}1.82 \\
2.13 \\
2.15 \\
2.35 \\
2.36 \\
1.76 \\
1.39 \\
1.38 \\
1.62 \\
2.35 \\
1.87 \\
1.94\end{array}$ & $\begin{array}{l}0.971 \\
0.834 \\
0.834 \\
0.849 \\
0.849 \\
1.417 \\
1.392 \\
1.581 \\
7.039\end{array}$ & $\begin{array}{l}2.37 \\
1.28 \\
1.28 \\
1.20 \\
1.20 \\
- \\
- \\
1.55 \\
1.39 \\
1.43 \\
2.32\end{array}$ \\
\hline$\frac{\mathrm{y}}{\mathrm{C}}$ & $\begin{array}{c}\text { H. Siddeley } 121-\mathrm{IC} \\
121-\mathrm{IE} \\
\text { Boeing } \begin{array}{c}727-100 \\
727-100 \mathrm{C}\end{array}\end{array}$ & $\begin{array}{l}115.000 \\
134.000 \\
161.000 \\
160.000\end{array}$ & $\begin{array}{l}12.600 \\
13.462 \\
17.764 \\
17.492 \\
\end{array}$ & $\begin{array}{l}11.0 \\
10.0 \\
11.0 \\
10.9\end{array}$ & $\begin{array}{l}3.225 \\
3.341 \\
4.133 \\
4.142 \\
\end{array}$ & $\begin{array}{l}2.80 \\
2.49 \\
2.57 \\
2.59 \\
\end{array}$ & $\begin{array}{l}12.469 \\
13.328 \\
17.681 \\
20.044\end{array}$ & $\begin{array}{l}10.8 \\
9.95 \\
10.9 \\
12.5\end{array}$ & $\begin{array}{l}4.413 \\
5.073 \\
7.211 \\
6.860\end{array}$ & $\begin{array}{l}3.84 \\
3.79 \\
4.48 \\
4.29 \\
\end{array}$ & $\begin{array}{l}1.792 \\
1.689 \\
2.996 \\
2.957\end{array}$ & $\begin{array}{l}1.56 \\
1.26 \\
1.86 \\
1.85 \\
\end{array}$ & $\begin{array}{l}* * \\
3.864 * \\
3.839\end{array}$ & $\begin{array}{l}- \\
2.40 \\
2.40\end{array}$ \\
\hline 离 & $\begin{array}{c}\text { Boeing } \mathrm{KC}-135 \\
707-121 \\
707-320 \\
707-320 \mathrm{C} \\
707-321 \\
720-022 \\
747-100 \\
747-200 \mathrm{~B} \\
\text { DC-8-10 } \\
\text { Me D. Douglas DC-55 } \\
\text { BAC VC-10-1101 } \\
\text { G. Dynamics } 880 \\
990\end{array}$ & $\begin{array}{l}297.000 \\
246.000 \\
311.000 \\
330.000 \\
301.000 \\
203.000 \\
710.000 \\
775.000 \\
273.000 \\
328.000 \\
312.000 . \\
184.500 \\
253.000\end{array}$ & $\begin{array}{l}25.251 \\
24.024 \\
29.762 \\
32.255 \\
28.647 \\
22.850 \\
86.402 \\
92.542 \\
26.235 \\
34.759 \\
34.672 \\
17.669 \\
26.871\end{array}$ & $\begin{array}{c}8.50 \\
9.76 \\
9.57 \\
9.77 \\
9.52 \\
11.3 \\
12.2 \\
11.9 \\
9.61 \\
10.6 \\
11.1 \\
9.58 \\
10.6\end{array}$ & $\begin{array}{r}5.074 \\
5.151 \\
5.511 \\
6.165 \\
6.004 \\
5.230 \\
11.850 \\
11.842 \\
4.740 \\
4.889 \\
6.958 \\
4.247 \\
5.326\end{array}$ & $\begin{array}{l}1.71 \\
2.09 \\
1.77 \\
1.87 \\
1.99 \\
2.58 \\
1.67 \\
1.53 \\
1.74 \\
1.49 \\
2.23 \\
2.30 \\
2.11\end{array}$ & $\begin{array}{l}18.867 \\
20.061 \\
21.650 \\
26.937 \\
22.129 \\
19.035 \\
71.845 \\
72.053 \\
21.495 \\
22.248 \\
25.113 \\
13.699 \\
16.673\end{array}$ & $\begin{array}{c}6.35 \\
8.15 \\
6.96 \\
8.16 \\
7.35 \\
9.38 \\
10.1 \\
9.30 \\
7.87 \\
6.78 \\
8.05 \\
7.42 \\
6.59\end{array}$ & $\begin{array}{r}10.180 \\
9.763 \\
12.700 \\
12.737 \\
11.122 \\
8.110 \\
31.427 \\
32.693 \\
10.185 \\
11.255 \\
10.489 \\
6.203 \\
8.718\end{array}$ & $\begin{array}{l}3.43 \\
3.97 \\
4.08 \\
3.86 \\
3.70 \\
4.00 \\
4.43 \\
4.22 \\
3.73 \\
3.43 \\
3.36 \\
3.36 \\
3.44\end{array}$ & $\begin{array}{l}2.044 \\
2.044 \\
2.400 \\
3.052 \\
2.408 \\
2.430 \\
6.982 \\
7.073 \\
2.000^{*} \\
2.253 \\
. * * \\
. * \\
. *\end{array}$ & $\begin{array}{l}0.69 \\
0.83 \\
0.77 \\
0.92 \\
0.80 \\
1.21 \\
0.98 \\
0.91 \\
0.73 \\
0.69 \\
= \\
= \\
=\end{array}$ & $\begin{array}{c}2.575 \\
4.639 \\
4.497 \\
4.183 \\
5.119 \\
4.510 \\
10.031 \\
10.136 \\
3.505 \\
4.685 \\
* \\
3.685 \\
6.772\end{array}$ & $\begin{array}{l}0.87 \\
1.89 \\
1.45 \\
1.27 \\
1.70 \\
2.22 \\
1.41 \\
1.31 \\
1.28 \\
1.43 \\
- \\
2.00 \\
2.68\end{array}$ \\
\hline
\end{tabular}

- estimated * included in other items

Table 8-5. (Continued)

taken as the larger of the maximum positive gust or the maneuver load factor for the applicable weight at the most critical flight altitude (approximately 20,000 ft for pressurized transports). For further details see Appendix C.

\section{b. Wing group.}

A reasonably accurate wing weight estimate can be made in preliminary design as the loads on the wing are fairly well known at the design stage. Usually the bending moment in flight is assumed to be decisive for most of the primary structure. For a curtain category of high-speed aircraft, however, torsional stiffness requirements may become dominant and the extra structure weight required to safeguard against flutter may amount to as much as 208 of the wing weight. The location of the inertia axis of the wing plus wing-mounted engines is of importance. A fairly large portion is also made up of secondary structure and non-optimum penalties, such as joints, non-tapered skin, undercarriage attachments, etc.

The derivation of a typical wing weight prediction method is explained in Ref. 8-101, the results of which are summarized in Appendix $C$. If sufficient data are not available to apply this method, the following simplified approximation can be used for civil airplanes with Al-alloy cantilever wings. The following basic expression is valid for the case of a wing-mounted retractable undercarriage, but not for wingmounted engines:

$\frac{w_{w}}{w_{G}}=k_{w_{s}} b_{s} \cdot 75\left|1+\sqrt{\frac{b_{\text {ref }}}{b_{s}}}\right| n_{\text {ult }} \cdot .55\left(\frac{b_{s} / t_{r}}{w_{G} / s}\right)^{.30}$

where $b_{\text {ref }}=6.25 \mathrm{ft}$ or $1.905 \mathrm{~m}$ for $b_{s}$ in $\mathrm{ft}$ or $\mathrm{m}$, respectively, while $\mathrm{b}_{\mathrm{s}}=\mathrm{b} / \cos \Lambda_{\frac{1}{2}}$, the structural wing span. The factor of proportionality is as follows:

Light aircraft, $W_{\text {to }} 612,500 \mathrm{lb}(5670 \mathrm{~kg})$ : $k_{w_{2}}=1.25 \times 10^{-3} ; w_{G}=$ MTOW in $1 \mathrm{~b}, b_{s}$ in $\mathrm{ft}, \mathrm{s}$ in $\mathrm{ft}^{2}, \mathrm{w}_{\mathrm{w}}$ in $1 \mathrm{~b}$.

$\mathrm{k}_{\mathrm{w}}=4.90 \times 10^{-3} ; \mathrm{w}_{\mathrm{G}}$ =MTOW in $\mathrm{kg}, \mathrm{b}_{\mathrm{s}}$ in $\mathrm{m}, \mathrm{s}$ in $\mathrm{m}^{2}, \mathrm{w}_{\mathrm{w}}$ in $\mathrm{kg}$. 
Transport category aircraft $\mathrm{w}_{\text {to }}>12,500 \mathrm{lb}$ $(5670 \mathrm{~kg})$ :

$k_{w}=1.70 \times 10^{-3} ; w_{G} \equiv M Z F W$ in $1 b, b_{s}$ in $\mathrm{ft}, \mathrm{s}$ in $\mathrm{ft}^{2}, \mathrm{w}_{\mathrm{w}}$ in $\mathrm{lb}$.

$\mathrm{k}_{\mathrm{w}}=6.67 \times 10^{-3} ; \mathrm{w}_{\mathrm{G}} \equiv$ MZFW in $\mathrm{kg}, \mathrm{b}_{\mathrm{s}}$ in $\mathrm{m}, \mathrm{s}$ in $\mathrm{m}^{2}, \mathrm{w}_{\mathrm{w}}$ in $\mathrm{kg}$.

The weight given by $(8-12)$ includes highlift devices and ailerons. For spoilers and speed brakes, if incorporated, 28 should be added. Reduce $w_{w}$ by 58 or 108 for 2 or 4 wing-mounted engines, respectively and by 58 if the main undercarriage is not mounted to the wing. For braced wings a reduction of approximately $30 \%$ relative to $(8-12)$ can be assumed. This figure includes the strut, contributing about $1 \mathrm{C}$. of the total wing group weight.

Wing optimization studies must be sensitive to variations in the external geometry, configuration and operational characteristics. It is generally recognized that for modern wing designs the weight of high-lift devices should be determined separately. The method in Appendix $\mathrm{C}$ meets these requirements and predicts the wing weights with a standard prediction error of $9.64 \%$.

On inspection of $(8-12)$, the observation can be made that the structural weight fraction, for a given cantilever ratio $b_{S} / t_{Y}$ and wing loading $w_{G} / s$, increases with the wing span. This unfavorable scale effect, associated with the square-cube law (cf. Section 7.2 .2 .), can be counteracted by increasing the wing loading. This is one of the reasons why large aircraft usually have high wing loadings. Decreasing the cantilever ratio is unfavorable as it results in a drag increment; its value is usually between 35 and 45 (see Fig. 7-8.).

c. Tail group.

This weight is only a small part - about 2 to 38 - of the MTOW but on account of its remote location it has an appreciable effect on the position of the airplane's center of gravity. Accurate weight prediction is difficult due to the wide variety of tailplane configurations and the limited knowledge of strength, stiffness and other conditions which will govern the design.
For relatively low-speed, 1ight aircraft ( $V_{D}$ up to $\left.250 \mathrm{kts} E A S\right)$, the maneuvering loads are most important and the specific tailplane weight is affected by the load factor as follows:

$\mathrm{w}_{\text {tail }}=\mathrm{k}_{\text {wt }}\left\{\mathrm{n}_{\text {ult }} \mathrm{s}_{\text {tail }}{ }^{2}\right\}^{.75}$

where $\begin{aligned} k_{w t} & =.04 ; w_{t a i l} \text { in } 1 b \text { and } s_{t a i l} \text { in } \mathrm{ft}^{2} \\ k_{w t} & =.64 ; w_{\text {tail }} \text { in } \mathrm{kg} \text { and } s_{\text {tail }} \text { in } \mathrm{m}^{2}\end{aligned}$ It is interesting to note that for this category the specific tailplane weight obeys the square-cube law, the weight being proportional to the cube while the area is proportional to the square of the linear dimension. If the tailplane area is not (yet) known, the total tailplane weight may be assumed between $3 \frac{1}{2}$ and 48 of the empty weight. For transport category aircraft and executive jets the Design Dive speed appears to have a dominant effect (Fig. 8-5):

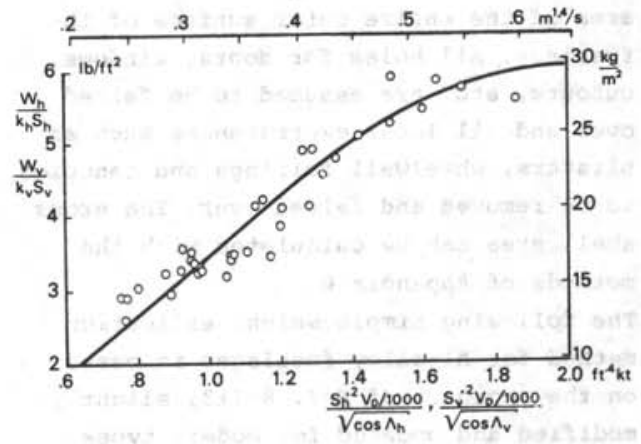

Fig. 8-5. Normalized specific horizontal tailplane weight

$\frac{w_{h}}{s_{h}}=k_{h} \cdot f\left(\frac{s_{h} \cdot 2 v_{D}}{\sqrt{\cos \Lambda_{h}}}\right)$

$\frac{w_{v}}{S_{v}}=k_{v} \cdot f\left(\frac{S_{v} \cdot 2 \text { v }}{\sqrt{\cos \Lambda_{v}}}\right)$

where $V_{D}$ is expressed in terms of EAS and $k_{h}$ and $k_{v}$ are correction factors for the tailplane configuration:

$k_{h}=1.0$ - fixed stabilizer, 1.1 for variable-incidence tails; for a bullet of ap- 
preciable size 8 s should be added. $k_{v}=1.0$ - fuselage-mounted horizontal tailplanes

$\mathrm{k}_{\mathrm{v}}=1+.15 \frac{\mathrm{s}_{\mathrm{h}} \mathrm{h}_{\mathrm{h}}}{\mathrm{S}_{\mathrm{b}} \mathrm{b}_{\mathrm{v}}}-\mathrm{fin-mounted} \mathrm{stabilizers}$ (e.g. T-tails) ${ }^{v^{2}} \quad b_{v}$ defined in Fig. 9-20. Fig. 8-5 demonstrates that the scale effect on specific tailplane weight $\left(\mathrm{S}^{2}\right)$ applies to medium-sized airplanes, but disappears for very large aircraft.

\section{d. Body group.}

The fuselage makes a large contribution to the structural weight, but it is much more difficult to predict by a generalized method than the wing weight. The reason is the large number of local weight penalties in the form of floors, cutouts, attachment and support structure, bulkheads, doors, windows and other special structural features.

Fuselage weight is affected primarily by the gross shell area $S_{G}$, defined as the area of the entire outer surface of the fuselage. All holes for doors, windows, cutouts, etc. are assumed to be faired over and all local excrescences such as blisters, wheelwell fairings and canopies to be removed and faired over. The gross shell area can be calculated with the methods of Appendix B.

The following simple weight estimation method for Al-alloy fuselages is based on the approach of Ref. 8-113, slightly modified and updated for modern types. The basic fuselage weight is:

$w_{f}=k_{w f} \sqrt{v_{D} \frac{1_{t}}{b_{f}+h_{f}}} s_{G}^{1.2}$

The Design Dive speed $v_{D}$ is expressed in terms of EAS. For definitions of $1_{t}, b_{f}$ and $h_{f}$ see Appendix D (Fig. D-2). The constant of proportionality is:

$k_{w f}=.021-w_{f}$ in $1 b, v_{D}$ in kts and $s_{G}$ in $\mathrm{ft}^{2}$ $k_{w f}=.23-w_{f}$ in $k g, v_{D}$ in $m / s$ and $s_{G}$ in $m^{2}$ To the basic weight given by $(8-16), 88$ should be added for pressurized cabins, 48 for rear fuselage-mounted engines, 78 if the main landing gear is attached to the fuselage, and an extra 10 s for freighter aircraft. If there is no attachment structure for the landing gear nor a wheelbay, 48 may be subtracted from the basic weight. Most of the more detailed prediction methods are based on the approach in Ref. 8-115 applicable to semi-monocoque structures. The calculation of the shell weight according to this method, supplemented with some recent data to estimate various weight penalties, is given in Appendix D.

For tail booms $(8-16)$ can be used for each boom separately. In this case $1_{t}$ is defined as the distance between the quarter-chord points of the local wing chord and the horizontal tailplane. Add 78 for a main landing gear wheelbay and undercarriage attachment.

\section{e. Alighting gear group*.}

The undercarriage has a well-defined set of loading conditions and weight prediction can therefore be dealt with on a analytical basis. To this end the weight of each gear must be subdivided into:

- wheels, brakes, tires, tubes and air

- main structure, i.e. legs and struts

- items such as the retraction mechanism, bogies, dampers, controls, etc.

The first part of the weight prediction process is to decide upon tire and wheel size, inflation pressure, location of the gears, length of the legs, etc. This subject will be treated in Chapter 10 , an example of a weight prediction method is given in Ref. 8-125.

The weight of conventional undercarriages may be found by sumnation of the main gear and the nose gear, each predicted separately with the following expression:

$$
\mathrm{w}_{\mathrm{uc}}=\mathrm{k}_{\mathrm{uc}}\left\{\mathrm{A}+\mathrm{B} \cdot \mathrm{w}_{\mathrm{to}}^{3 / 4}+\mathrm{C} \cdot \mathrm{w}_{\mathrm{to}}+\mathrm{D} \cdot \mathrm{w}_{\mathrm{to}}^{3 / 2}\right\}
$$

where $k_{u c}=1.0$ for low-wing airplanes and $k_{u c}=1.08$ for high-wing airplanes Table 8-6 gives suggested values of the factors A, B, C and D, based on a statistical evaluation of data on undercar-

* Only conventional undercarriages will be dealt with 


\begin{tabular}{|c|c|c|c|c|c|c|c|c|}
\hline A/C CATECOAY & \multicolumn{2}{|c|}{ v.c. conviculurion } & & A & & $B$ & c & D \\
\hline \multirow{2}{*}{ 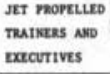 } & \multirow{2}{*}{ ЕETRACTABLE } & $\min$ & & (15.0) & .04 & $(.033)$ & .021 & - \\
\hline & & Mose & 12 & (5.4) & .06 & $(.049)$ & - & - \\
\hline \multirow{6}{*}{$\begin{array}{l}\text { ALL OTIER } \\
\text { CIVIL TrPes }\end{array}$} & \multirow{3}{*}{ ruxed } & $\operatorname{mans}$ & 20 & $(9.1)$ & .10 & $(.082)$ & .019 & - \\
\hline & & Mose & 25 & (11.3) & & - & .0024 & - \\
\hline & & TAIL & & $(4.1)$ & & - & .0024 & - \\
\hline & \multirow{3}{*}{ nетиставие } & mats & 40 & $(18.1)$ & .16 & $(.131)$ & .019 & $1.5(2.23) \cdot 10^{-5}$ \\
\hline & & MOSE & 20 & $(9.1)$ & .10 & $(.082)$ & - & $2 \quad(2.97) \cdot 10^{-6}$ \\
\hline & & TAIL & 3 & $(2,3)$ & & - & .0031 & - \\
\hline
\end{tabular}

CORPICIESTS CORRESPOND TO VEICWTS IS LA(KG)

Table 8-6. Coefficients for the calculation of the landing gear weight

riage weights of existing airplanes.

Fig. 8-6 compares the result of $(8-17)$ with data for existing airplanes. Up to 100,000 Ib $(45,000 \mathrm{~kg})$ takeoff weight the weight fraction decreases with increasing airplane size. The main reasons are that for large airplanes a larger part of the gear structure can be highly stressed, while the use of higher inflation pressures on large aircraft saves some weight as well. For main landing gears the weight fraction does not appreciably decrease at takeoff weights above $100,000 \mathrm{lb}(45,000 \mathrm{~kg})$, but for nose gears there is still a reduction of the weight fraction up to very large airplane sizes like the B-747 and C-5A.

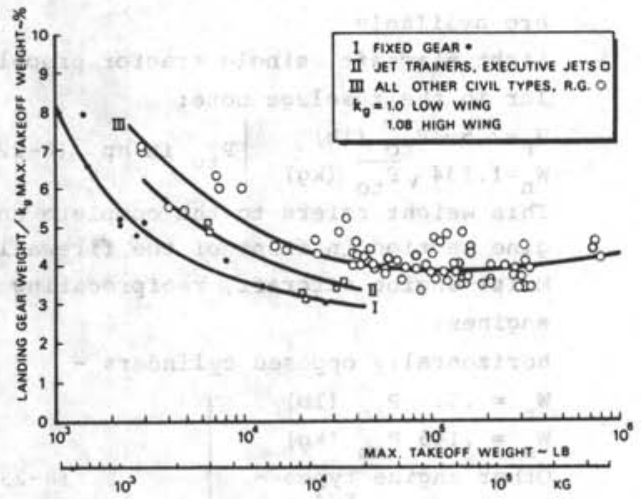

Fig. 8-6. Landing gear weight fraction

It can be argued that in many aircraft the critical load is formed by the landing impact load and that the MLW should therefore be used to predict the undercarriage weight. A reasonable approximation for the weight of retractable undercarriages is 4.78 of the MLw.

f. Surface controls group.

The weight of surface controls is generally of the order of .8 to 28 of the takeoff weight. An approximation is:

$$
w_{s c}=k_{s c} w_{t o}^{2 / 3}
$$

The factor $k_{\text {sc }}$ can be determined from known weights of airplanes in the same category with a similar flight control system. Alternatively, for $w_{s c}$ and $w_{\text {to }}$ in $1 b$, we have:

$\mathrm{k}_{\mathrm{sc}}=.23-$ light airplanes without duplicated system controls,

$k_{\text {sc }}=.44-$ transport airplanes and trainers, manually controlled, and $\mathrm{k}_{\mathrm{sc}}=.64-$ transport airplanes, with powered controls and trailing-edge highlift devices only.

Multiply these values by .768 when $w_{s c}$ and $\mathrm{w}_{\text {to }}$ are in $\mathrm{kg}$. Add 208 for leading-edge flap or slat controls and $15 \%$ for 1 ift dumper controls, if used.

If sufficient data are available, a more detailed analysis can be made. To this end the surface controls group weight is subdivided into:

1. cockpit controls:

$$
\begin{aligned}
& .056 \mathrm{w}_{t o} 3 / 41 \mathrm{~b}\left(.046 \mathrm{w}_{\mathrm{to}} 3 / 4 \mathrm{~kg}\right) \\
& \text { for } \mathrm{w}_{\mathrm{to}} \leq 25,000 \mathrm{lb}(11,340 \mathrm{~kg}) \\
& 110 \mathrm{lb}(50 \mathrm{~kg}) \\
& \text { for } \mathrm{w}_{t o}>25,0001 \mathrm{~b}(11,340 \mathrm{~kg})
\end{aligned}
$$

2. automatic pilot:
$17 \mathrm{w}_{\text {to }} 1 / 5$
(9. $\mathrm{w}_{\text {to }}{ }^{1 / 5} \mathrm{~kg}$ )

for transport and executive aircraft

3. system controls:

$.008 \mathrm{w}_{\text {to }}$ for light airplanes with single flight control systems For transport aircraft a prediction of the system controls weight can be made with the aid of Table $8-7$. In the absence of better data these formulas may be also used for trainers and executive airplanes.

g. Engine section or nacelle group.

The following statistical data may be used if no details of the engine installation 


\begin{tabular}{|c|c|c|c|}
\hline SYSTEM COMPONENT & \multicolumn{2}{|l|}{ (2) METHOD } & \begin{tabular}{|c|} 
NoneschutuRg \\
uto $_{\text {to }}-$ Max. Takeoff \\
Weight $\sim 1 \mathrm{~b}(\mathrm{~kg})$
\end{tabular} \\
\hline \multirow{3}{*}{$\begin{array}{l}\text { MUNEUVERING CONTROL } \\
\text { SYSTEM } \\
\text { (elevator, rudder, } \\
\text { ailerons, spoilers) }\end{array}$} & $\begin{array}{l}\text { manually operated } \\
\text { duplicated controls }\end{array}$ & $\underset{(.154)}{.2} \times x_{t 0}^{.67}$ & $\begin{array}{l}\text { Wto } \\
\text { Weight } \sim 1 \mathrm{~b}(\mathrm{~kg})\end{array}$ \\
\hline & $\begin{array}{l}\text { duplicated povered controls, } \\
\text { single hydr. pover system }\end{array}$ & $\frac{.42}{(.318)} \times 16.0 .65$ & $x_{-1}^{-1}$ \\
\hline & $\begin{array}{l}\text { duplicated powered controls, } \\
\text { dual hydr. pover system }\end{array}$ & $\frac{1.06}{(.773)} \times 1 t_{t 0} .60$ & inting \\
\hline \multirow{2}{*}{$\begin{array}{l}\text { TRAILING EDGE FLAP } \\
\text { CONTROL. SYSTEM }\end{array}$} & $\begin{array}{l}\text { rotating flaps (cylinder } \\
\text { actuation) }\end{array}$ & $\frac{1.38}{(5.569)} \times\left(s_{f} \sin \delta_{f}\right)^{.92}$ & \multirow{2}{*}{$\begin{array}{l}S_{f}=- \text { total projected } \\
\text { flap area } \sim \mathrm{ft}^{2}\left(\mathrm{~m}^{2}\right) \\
\delta_{\mathrm{f}}=\operatorname{maximum} \text { flap } \\
\text { deflection angle }\end{array}$} \\
\hline & $\begin{array}{l}\text { translating (Fowler) flaps } \\
\text { (screvjack actuation) }\end{array}$ & $(11.02) \times\left(s_{f} \sin \delta_{f}\right)^{.92}$ & \\
\hline $\begin{array}{l}\text { LEADING EDGE FLAP OR } \\
\text { SLAT CONTROL SYSTEM }\end{array}$ & \multicolumn{2}{|c|}{$\begin{array}{c}3.53 \\
(11.23)\end{array} \times \mathrm{s}_{\mathrm{s}}^{.82}$} & $\begin{array}{r}s_{n}=- \text { total projected } \\
\text { slat area } \sim \mathrm{ft}^{2}\left(\mathrm{~m}^{2}\right)\end{array}$ \\
\hline $\begin{array}{l}\text { VARIABLE INCIDENCE } \\
\text { STABILIZER CONTROLS }\end{array}$ & \multicolumn{2}{|c|}{$\begin{array}{l}k_{h e}\left(s_{h e} v_{\max } .5 \sin \delta_{h}, .88 \begin{array}{l}\text { single povered: } k_{h c}=.31 \\
\text { dual povered: } k_{h c}=.44(2.52)\end{array}\right. \\
\delta_{h}=\text { total range of hor. tailplane incidence variation }\end{array}$} & $\begin{array}{l}S_{\text {he }}^{-} \text {exposed hor. tail } \\
\text { area } \sim \mathrm{ft}^{2}\left(\mathrm{~m}^{2}\right) \\
V_{\max }^{-} \text {max. hor. flight } \\
\text { speed } \sim \text { kts }(\mathrm{m} / \mathrm{s}) \text { TAS) }\end{array}$ \\
\hline SPEED BRUKE CONTROLS & \multicolumn{2}{|c|}{$\operatorname{lag}_{(40.4)}^{10} \times \mathrm{s}_{\mathrm{sb}}^{.92}$} & $\begin{array}{l}\mathrm{S}_{\mathrm{sb}}=\text { speed brake } \\
\text { vetted area } \sim \mathrm{ft}^{2}\left(\mathrm{~m}^{2}\right)\end{array}$ \\
\hline LIFT DUMPER CONTROLS & \multicolumn{2}{|c|}{$\frac{5}{(20.2)} \times\left(s_{\ell d} \sin \delta_{\ell d}\right)^{.92}$} & \multirow{2}{*}{$\begin{array}{l}S_{\ell d^{-}} \text {total area of lift } \\
\text { dumpers } \sim \mathrm{ft}^{2}\left(\mathrm{~m}^{2}\right) \\
{ }^{6} \mathrm{Qd}^{-} \text {maximum lift dumper } \\
\text { deflection angle }\end{array}$} \\
\hline$C T$ & SYSTEM: no & & \\
\hline
\end{tabular}

ALL COMPONENT WEIGHTS IN LB (KG)

NOTES:

1. Most formulas are approximations of the curves in SAWE Technical Paper No 812

2. Coefficients in brackets refer to the metric system

Table 8-7. Weight of system controls (transport aircraft)

\begin{tabular}{|c|c|}
\hline wetcat contrisurion & 5. \\
\hline $\begin{array}{l}\text { ENCINE nOUSTS NSD } \\
\text { VIBRTITOS ABSORBERS }\end{array}$ & $\begin{array}{l}5 \pi \text { of engine plus propeller installation } \\
\text { veight }\end{array}$ \\
\hline $\begin{array}{l}\text { MACELLE STRUCTURE, } \\
\text { PYLONS AND STRUTS, } \\
\text { ENGINE CONLTNCS, } \\
\text { TLAPS AND BAFTLES }\end{array}$ & $\begin{array}{l}.03 \sqrt{V_{D}} s_{\text {vet }} 1.3 \text { (1b): } v_{D}-k t s \text { EAS; } s_{\text {wet }} \sim \text { sq.ft } \\
.405 \sqrt{v_{D}} s_{\text {vet }} 1,3(\mathrm{~kg}): v_{D} \sim=/ s \text { EAS; } s_{\text {vet }} \sim \mathrm{m}^{2} \\
s_{\text {wet }} \sim \text { total area per nacelle vetted by } \\
\text { the cold airflow, both internally and } \\
\text { externally }\end{array}$ \\
\hline $\begin{array}{l}\text { CAS GENERTOR COULTNG } \\
\text { AND RUVG }\end{array}$ & $3 \mathrm{sb} / \mathrm{sq} . \mathrm{ft}\left(16.6 \mathrm{~kg} / \mathrm{m}^{2}\right)$ of vetted area \\
\hline $\begin{array}{l}\text { SOISE SUPPRESSION } \\
\text { MATERIAL (EXTRA VETGT) }\end{array}$ & $\begin{array}{l}.35 \mathrm{lb} / \mathrm{sq} . \mathrm{ft}\left(1.71 \mathrm{~kg} / \mathrm{m}^{2}\right) \text { - nacelle valls } \\
1.75 \mathrm{bb} / \mathrm{sq} . \mathrm{ft}\left(8.53 \mathrm{~kg} / \mathrm{m}^{2}\right) \text { - splitter plates }\end{array}$ \\
\hline $\begin{array}{l}\text { FIREWALLS NSD SHROUDS } \\
\text { POR YIRE PROTECTION }\end{array}$ & $1.13 \mathrm{Ib} / \mathrm{sq} . \mathrm{ft}\left(5.51 \mathrm{~kg} / \mathrm{m}^{2}\right)$ \\
\hline
\end{tabular}

* for straight jet engines the external nacelle area plus the inlet duct area

Table 8-8. Data for estimating the nacelle group weight are available.

Light aircraft, single tractor propeller in the fuselage nose:

$\begin{aligned} & w_{n}=2.5 \sqrt{P_{\text {to }}}(1 \mathrm{~b}) \\ & w_{n}=1.134 \sqrt{P_{\text {to }}}(\mathrm{kg})\end{aligned} \mid P_{\text {to }}$ in $\mathrm{hp}$ This weight refers to the complete en. gine section in front of the firewall. Multi-engine aircraft, reciprocating engines:

horizontally opposed cylinders -

$\mathrm{w}_{\mathrm{n}}=.32 \quad \mathrm{P}_{\text {to }}$ (1b)

$\mathrm{W}_{\mathrm{n}}=.145 \mathrm{P}_{\text {to }}(\mathrm{kg})$

Other engine types -

$w_{n}=.045 \mathrm{p}_{\text {to }} 5 / 4$ (1b)

$w_{\mathrm{n}}=.0204 \mathrm{P}_{\text {to }} 5 / 4(\mathrm{~kg})$

All weights per nacelle

$P_{\text {to }}$ : takeoff bhp per engine

Aircraft with turboprop engines:

$w_{n}=.141 \mathrm{~b}(.0635 \mathrm{~kg})$ per

takeoff ESHP 
Add $.04 \mathrm{lb}(.018 \mathrm{~kg})$ per ESHP if the main landing gear is retractable into the nacelle and $.11 \mathrm{lb}(.05 \mathrm{~kg})$ per ESHP for overwing exhausts (cf. Fig. 2-10, Lockheed Electra). Aircraft with pod-mounted turbojet or turbofan engines:

$\mathrm{w}_{\mathrm{n}}=.055 \mathrm{~T}_{\text {to }}$

high bypass turbofans with

short fan duct -

$\mathrm{w}_{\mathrm{n}}=.065 \mathrm{~T}_{\text {to }}$

This value includes the pylon weight and extended nacelle structure for a thrust reverser installation. In the absence of thrust reversing a reduction of 108 may be assumed.

If a more detailed weight analysis taking into account the configuration and geometry of the nacelle and engine mounting is desirable, some degree of structural design must be attempted first. The subdivision and weight data in Table 8-8 may then be used to calculate the weight. The weight penalty due to noise suppression material obviously depends upon the amount of suppression desired; the engine manufacturer should be consulted for detailed data. For a typical "quiet" turbofan pod, acoustic lining may be required over $50 \%$ of the nacelle area. A typical weight penalty is 208 of the nacelle weight, apart from the extra weight of the engine itself.

\subsubsection{The propulsion group}

Project designs are normally based on existing engine types or paper studies of engines in an advanced state of development. Thus a specification of the definitive engine weight $w_{e}$ is usually available comprising:

1. engine weight, bare and dry,

2. standard engine accessories and

3. additional weight contributions such as gas generator cowling and/or noise suppression material.

During parametric investigations it may be convenient to employ more general information and the engine weight data in Chapter 4 may be used:

- reciprocating engines: Section 4.2.2. and
Fig. 4-12,

- turbojet and turbofan engines: section

4.4.3. and equation 4-36,

- turboprop engines: Section 4.5.2. and equation $4-40$.

Detailed methods for the computation of turbojet engine weights will be found in References 8-129 through 8-136.

If sufficient details of the powerplant installation are not available, a first approximation for the propulsion group weight is obtained by assuming that part of this weight contribution is proportional to the engine weight, while propeller weight is proportional to the power to be absorbed:

propeller aircraft -

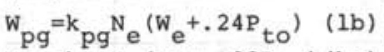

$\left.\mathrm{w}_{\mathrm{pg}}=\mathrm{k}_{\mathrm{pg}^{\mathrm{N}} \mathrm{e}} \mathrm{W}_{\mathrm{e}}+.109 \mathrm{P}_{\text {to }}\right)(\mathrm{kg})$

$\mathrm{P}_{\text {to }}$ : takeoff hp per engine

jet aircraft -

$\mathrm{w}_{\mathrm{pg}}=\mathrm{k}_{\mathrm{pg}} \mathrm{k}_{\text {thr }} \mathrm{N}_{\mathrm{e}} \mathrm{W}_{\mathrm{e}}$

where

$k_{p g}=1.16$ for single tractor propeller in fuselage

$=1.35$ for multi-engine propeller airplanes

$=1.15$ for jet transports, podded engines

$=1.40$ for light jet airplanes, buried engines

$k_{\text {thr }}=1.00$ with no thrust reversers

$=1.18$ with thrust reversers installed

Add 1.58 for jets and 38 for propeller aircraft with a water injection system. The term .24 (.109) $\mathrm{P}_{\text {to }}$ in $(8-26)$ for propeller aircraft represents the propeller installation weight in $1 \mathrm{~b}(\mathrm{~kg})$.

Instead of the simple approximation given above, Table $8-9$ can be used to analyze the powerplant weight in more detail. Weight data for some present-day aircraft are presented in Table 8-10.

A large contribution to the powerplant group included in Table $8-9$ is made by the fuel system, comprising:

1. fuel tanks and sealing,

2. pumps, collector tanks and plumbing,

3. distribution and filling system, and

4. fuel dump system (if used). 


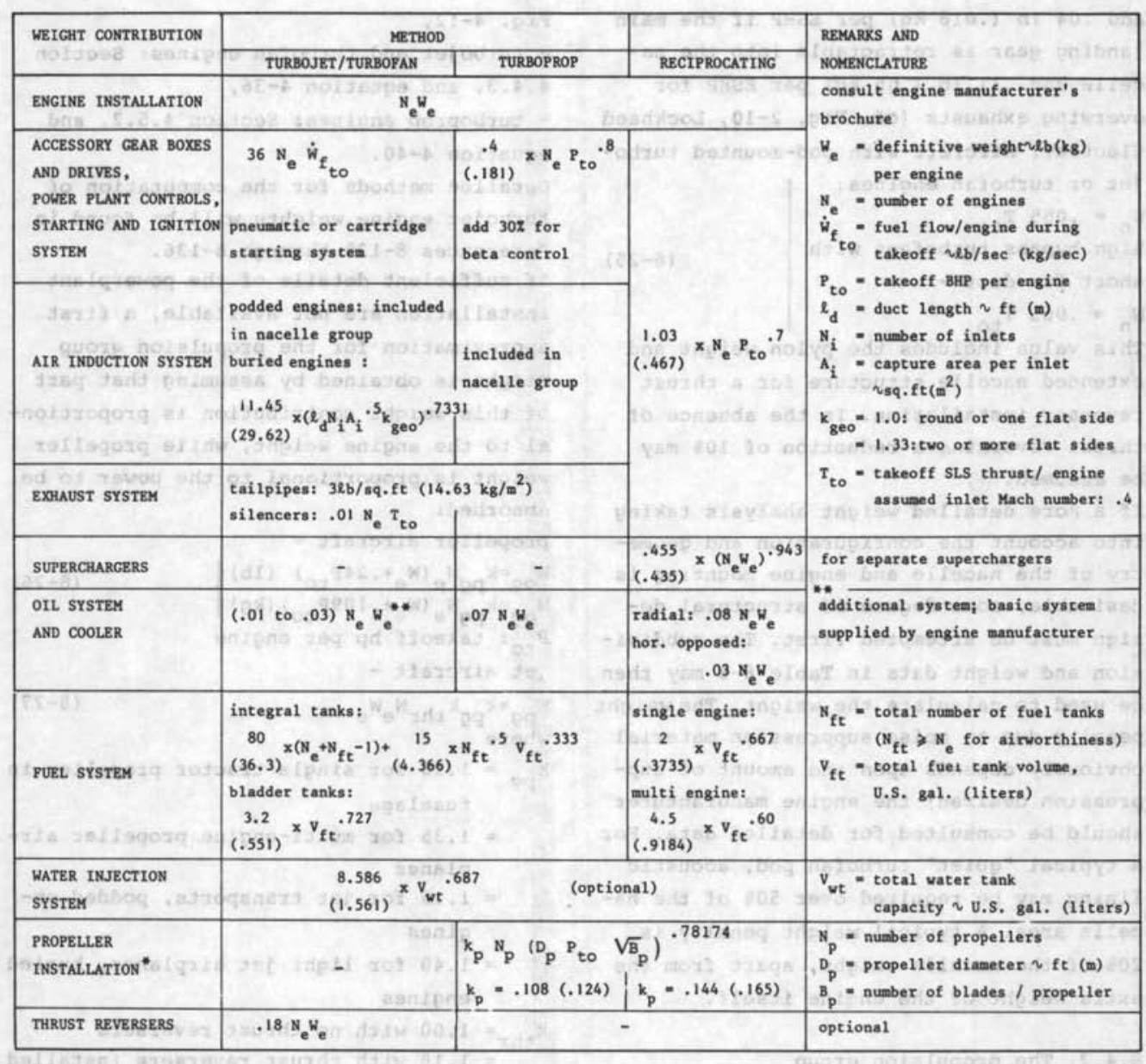

ALL WEIGHTS IN LB (KG)

sFrom SAWE Technical Paper No, 790

Note: coefficients in brackets refer to the metric system

Table 8-9. Weight analysis of the propulsion group

It will be observed that, for a given integral fuel tank capacity, the number of fuel tanks and the number of engines are primary parameters for determining the fuel system weight.

\subsubsection{Airframe services and equipment}

In the pre-design phase, with few details of the design of the airframe services and equipment, their weight is very difficult to predict. The initial prediction error may be very large, as demonstrated by the examples quoted in Ref. 8-151. As soon as preliminary discussions with system (component) manufacturers have been held, the initial weight prediction must be revised.

* A subdivision is shown in Table 8-3. 


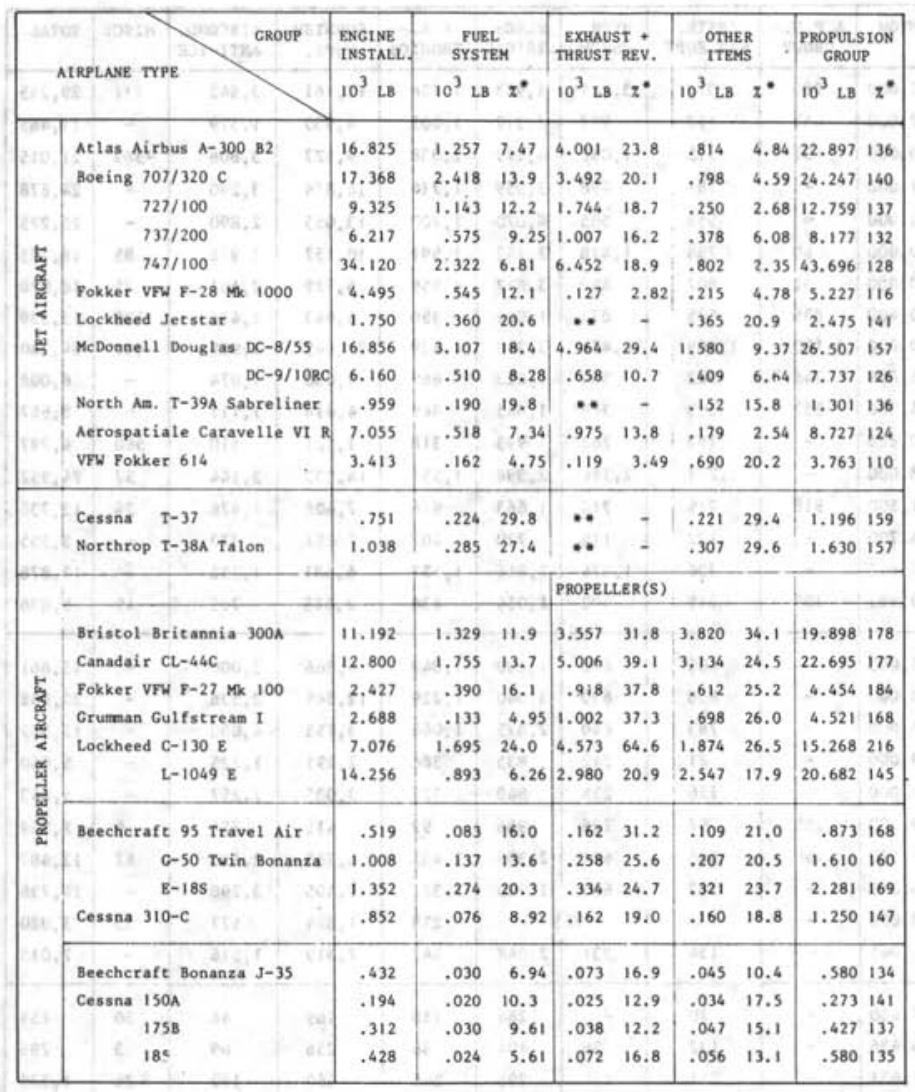

- percent of engine installation veight
. not specified; included in other iteas

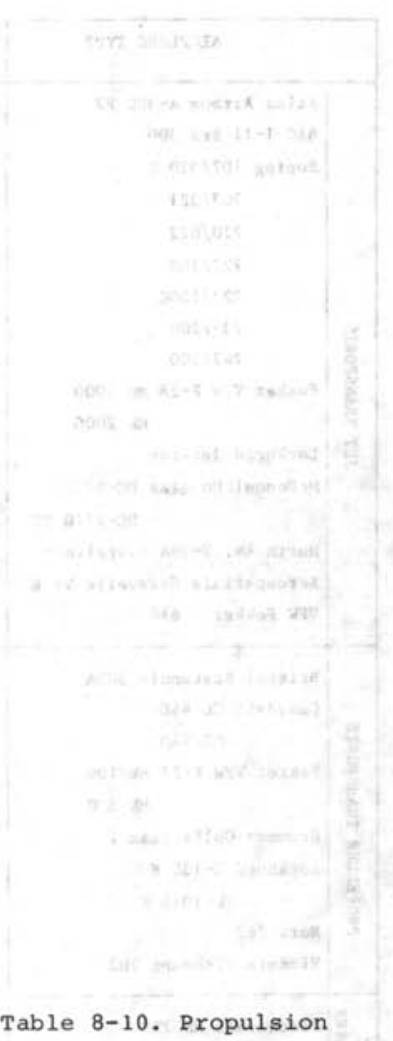
group weight breakdown for existing aircraft types
The data and methods in this section are based primarily on statistical correlations. There is, however, not always a functional relationship between the parameter on which the correlation is based and the actual weight contribution. Consequent$1 y$, if some weight item is related to the takeoff weight or the empty weight and the first and second estimation of these characteristic weights are different, it may be unnecessary to reiterate the complete weight estimation, provided the estimates do not differ greatly.

It should be noted that for several individual

weight contributions a marked discrepancy between the calculated value according to the present methods and the actual value for existing aircraft may be observed. This will be caused to a large extent by differences in de definitions of these items. However, the total estimated systems and equipment weight will be reasonably representative of the actual weight of the operational airplane. In some cases, particularly for wide-body aircraft, the weight estimate may be somewhat conservative due to recent improvements in systems design technology. Typical averages for the total airframe services and equipment weight are:

light single-engine private airplane: $88 \mathrm{~W}_{\text {to }}$ light twin-engined airplanes jet trainers short-range transports medium-range transports long-range transports

$$
\begin{aligned}
& : 118 \mathrm{~W}_{\text {to }} \\
& : 138 \mathrm{~W}_{\text {to }} \\
& : 148 \mathrm{~W}_{\text {to }} \\
& : 118 \mathrm{~W}_{\text {to }} \\
& : 88 \mathrm{~W}_{\text {to }}
\end{aligned}
$$

A collection of weight data is presented in 


\begin{tabular}{|c|c|c|c|c|c|c|c|c|c|c|c|}
\hline & AIRPLANE TYPE & Mrow & $\begin{array}{c}\text { A.P.U.- } \\
\text { GROUP }\end{array}$ & $\begin{array}{c}\text { INSTR. } \\
\text { NAV.EQPT. }\end{array}$ & $\begin{array}{l}\text { HYDR. } \\
\text { PNEUM. }\end{array}$ & $\begin{array}{l}\text { ELEC- } \\
\text { TRICAL }\end{array}$ & $\begin{array}{l}\text { ELEC- } \\
\text { TRONICS }\end{array}$ & $\begin{array}{c}\text { FURNISH. } \\
\text { EQPT. }\end{array}$ & $\begin{array}{l}\text { AIRCOND. } \\
\text { ANTI-ICE }\end{array}$ & MIsc. & TOTAL. \\
\hline \multirow{17}{*}{$\begin{array}{l}\frac{n}{2} \\
\frac{0}{0} \\
\frac{5}{2} \\
\frac{2}{3} \\
\frac{5}{2} \\
\frac{5}{2}\end{array}$} & At las Airbus A-300 B2 & 302,000 & 983 & 377 & 3,701 & 4,923 & 1,726 & 13,161 & 3,642 & 732 & 29,245 \\
\hline & BAC $\mid-11$ Srs 300 & 87,000 & 457 & 182 & 997 & 2,317 & 1,005 & 4,933 & 1,579 & - & 11,465 \\
\hline & Boeing $707 / 320 \mathrm{C}$ & 330,000 & 151 & sis & 1,086 & 4,179 & 2,338 & 9,527 & 3,608 & -389 & 21,015 \\
\hline & $707 / 321$ & 301,000 & - & 561 & 498 & 3,959 & 1,716 & 14,854 & 3,290 & - & 24,878 \\
\hline & $720 / 022$ & 203,000 & - & 555 & sos & 4,070 & 1,200 & 13,055 & 2,890 & - & 22,275 \\
\hline & $727 / 100$ & 160,000 & 60 & 756 & 1,418 & 2,142 & 1,591 & 10,257 & 1,976 & 85 & 18,285 \\
\hline & $727 / 100 \mathrm{C}$ & 160,000 & 52 & 802 & 843 & 3,617 & 1,559 & 6,729 & 2,401 & is & 16,078 \\
\hline & $737 / 200$ & 100,400 & 836 & 625 & 873 & 1,066 & 956 & 6,643 & 1,416 & 124 & 13,539 \\
\hline & $747 / 100$ & 710,000 & 1,130 & 1,909 & 4,471 & 3,348 & 4,429 & 37,245 & 3,969 & -421 & 54,380 \\
\hline & Fokker VFW F-28 Mk 1000 & 65,000 & 346 & 302 & 364 & 1,023 & 869 & 4,030 & 1,074 & - & 8,008 \\
\hline & Mk 2000 & 65,000 & 353 & 309 & 366 & 1,045 & 869 & 4,614 & 1,111 & - & 8,667 \\
\hline & Lockheed Jetstar & 30,680 & - & 153 & 262 & 973 & 318 & 1,521 & 510 & 560 & 4,297 \\
\hline & MeDonnell Douglas DC- $8 / 55$ & 328,000 & - & 1,271 & 2,196 & 2,398 & 1,551 & 14,335 & 3,144 & 57 & 24,952 \\
\hline & $\mathrm{DC}-9 / 10 \mathrm{RC}$ & 91,500 & 818 & 719 & 714 & 1,663 & 914 & 7,408 & 1,476 & 24 & 13,736 \\
\hline & North Am. T-39A Sabreliner & 16,700 & - & 122 & 116 & 720 & 407 & 857 & 333 & - & 2,555 \\
\hline & Aerospatiale Caravelle VI R & 114,640 & - & 236 & 1,376 & 2,846 & 1,187 & 6,481 & 1,752 & - & 13,878 \\
\hline & VFW Fokker - 614 & 40,981 & 305 & 215 & 403 & 1,054 & 436 & 2,655 & 719 & 49 & 5,836 \\
\hline \multirow{10}{*}{ 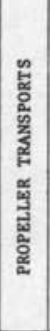 } & Bristol Britannia $300 \mathrm{~A}$ & 155,000 & - & sos & 650 & 1,800 & 1,040 & 6,866 & 3,000 & - & 13,861 \\
\hline & Canadair CL- $44 \mathrm{C}$ & 205,000 & - & 858 & 630 & 3,040 & 1,229 & 12,349 & 2,536 & - & 20,662 \\
\hline & $\mathrm{CL}-44 \mathrm{D}$ & 205,000 & - & 783 & 640 & 2,875 & 1,046 & 3,155 & 4,090 & - & 12,589 \\
\hline & Fokker VFW F-27 Mk 100 & 39,000 & - & 81 & 242 & 835 & 386 & 2,291 & 1,225 & - & 5,060 \\
\hline & Mk 500 & 45,000 & - & 126 & 256 & 840 & 329 & 3,035 & 1,257 & - & 5,843 \\
\hline & Grumman Gulf stream I & 33,600 & 355 & 97 & 235 & 966 & 99 & 415 & 755 & 6 & 2,929 \\
\hline & Lockheed C-130 E & 151,522 & 466 & 665 & 671 & 2,300 & 2,432 & 4,765 & 2,126 & 62 & 13,487 \\
\hline & $\mathrm{L}-1049 \mathrm{E}$ & 133,000 & - & 503 & 654 & 1,505 & 1,371 & 7,405 & 3,298 & - & 14,736 \\
\hline & Nord 262 & 23,050 & - & 133 & 765 & & 238 & 1,324 & 527 & 33 & 3,020 \\
\hline & Vickers Viscount 702 & 50,044 & - & 154 & 331 & 2,048 & 447 & 2,519 & 1,516 & - & 7,015 \\
\hline \multirow{3}{*}{ 齿㟥 } & Beecheraft MS 760 & 7,650 & - & 70 & - & 284 & 158 & 169 & 48 & 30 & 759 \\
\hline & Cessna $\mathrm{T}-37$ & 6,436 & - & 132 & 56 & 194 & 86 & 256 & 69 & 3 & 796 \\
\hline & Northrop T-38A Talon & 11,651 & - & 211 & 154 & 296 & 246 & 460 & 142 & 24 & 1,539 \\
\hline \multirow{4}{*}{ 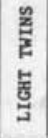 } & Beecheraft 95 Travel Air & 2,900 & - & 49 & - & 96 & 26 & 194 & 48 & 25 & 438 \\
\hline & $\mathrm{G}-50$ & 7,150 & - & 80 & - & 184 & 9 & 333 & 81 & 27 & 834 \\
\hline & $E-18 \mathrm{~s}$ & 9,700 & - & 100 & - & 295 & 63 & 524 & 144 & 58 & $1 ; 184$ \\
\hline & Cessna $310 \mathrm{c}$ & 4,830 & - & 46 & - & 121 & - & 154 & 46 & 65 & 498 \\
\hline \multirow{5}{*}{ 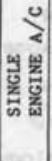 } & Beechcraft Bonanza $\mathrm{J}-35$ & 2,900 & - & 16 & - & 72 & - & 174 & 12 & 7 & 281 \\
\hline & Cessna $150 \mathrm{~A}$ & 1,500 & - & 7 & 2 & 41 & - & 42 & 4 & - & 96 \\
\hline & $172 B$ & 2,200 & - & 7 & a 3 & 41 & $-19=$ & 99 & 4 & - & 154 \\
\hline & 180D & 2,650 & - & 8 & 3 & 59 & - & 105 & 6 & - & 181 \\
\hline & $210 A$ & 2,900 & - & 16 & 4 & 60 & - & 116 & 12 & 20 & 228 \\
\hline
\end{tabular}

ALL WEIGHTS IN LB

Table 8-11. Airframe services and equipment group weight breakdown

Table 8-11. Several items will be discussed in greater detail in the paragraphs below.

\section{a. APU group.}

An APU is installed in most modern transport aircraft and also in some jet execu- tives. The installed weight may be based on the dry weight of the APU:

$$
\text { Weight }=k_{\text {APU }} w_{\text {APU }}
$$

The installation factor accounts for the inlet and exhaust ducting mounting frames, 
silencers, fire protection and accessories, and is generally of the order of 2.0 to 2.5 . The APU engine weight is mainly a function of the airflow capacity and power delivery. The bleed airflow requirement is approximately $.025 \mathrm{lb} / \mathrm{min}$ per cu. ft $(.4 \mathrm{~kg} / \mathrm{min}$ per $\mathrm{m}^{3}$ ) of passenger cabin volume or 1.1 $1 \mathrm{~b} / \mathrm{min}(.5 \mathrm{~kg} / \mathrm{min})$ per passenger in the high-density layout.

The APU engine weight can be obtained from the APU specification once the engine has been chosen. The following relationship may be used instead:

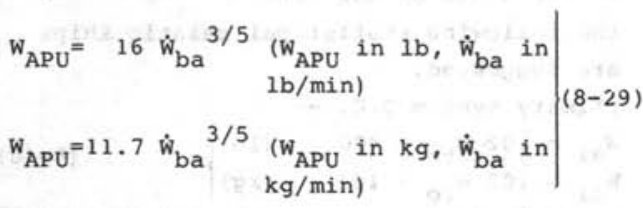

Recent APU engines used on wide-body transports have a specific weight of only $65 \%$ of this value, due to improved materials and cycle efficiencies and increased cycle pressures and turbine temperatures.

b. Instruments, navigational equipment and electronics groups.

Requirements for the instruments and NAV/ COM equipment (avionics) are usually listed in the design specification. The minimum equipment required for safe operation is supplemented by a choice of optional equipment to improve the operational flexibility. The effects of airplane size are found mainly in the weight of wiring and the flight control system, which increases in size and complexity when the aircraft is scaled up. NAV/COM equipment is partly or fully duplicated on modern transports and even iriplicated on recent large transports. A weight estimate may be based on the unit weight of each item of equipment, as obtained from manufacturers, as well as on data for airplanes designed for similar operational capabilities. If these data are not available, the following statistical correlations may be used for the combined weight of instruments and avionics.

Single-engine propeller aircraft: 8 lb $(3.6$ $\mathrm{kg)}$ per pilot, for instruments and 20-30 lb $(9-13.6 \mathrm{~kg})$ for radio, which is optional on private aircraft but compulsory on trainers, commuters and taxi aircraft. Propeller-powered utility airplanes up to $12,500 \mathrm{lb}(5,670 \mathrm{~kg})$ takeoff weight, VFR operations:
$40+.008 \mathrm{w}_{\text {to }}$
(1b)
$18.1+.008 \mathrm{w}_{\text {to }}$

Low-subsonic transports with manual flight control system, intended for IFR operations and equipped with single NAV/COM equipment:
$120+20$
$\mathrm{N}_{\mathrm{e}}+.006 \mathrm{w}_{\text {to }}$
(1b)
$54.4+9.1 \mathrm{~N}_{\mathrm{e}}+.006 \mathrm{w}_{\text {to }}$
(kg)

where $\mathrm{N}_{e}$ is the number of engines per aircraft. This equation also gives reasonable results for low-subsonic jet trainers.

For high-subsonic jet transports with predominantly duplicated NAV/COM equipment, jet executives and high-subsonic trainers, the weight of the instruments and electronics group is:

$\mathrm{w}_{\text {ieg }}=\mathrm{k}_{\text {ieg }} \mathrm{w}_{\mathrm{DE}}^{5 / 9} \mathrm{R}_{\mathrm{D}}^{1 / 4}$ where $W_{D E}$ is the Delivery Empty Weight and $R_{D}$ the maximum range (Fig. 8-3)

$k_{\text {ieg }}=.575$ for $w_{D E}$ and $w_{\text {ieg }}$ in $1 b, R_{D}$ in $n m$ $k_{\text {ieg }}=.347$ for $w_{D E}$ and $w_{\text {ieg }}$ in $k g, R_{D}$ in $k m$ These data do not include the autopilot weight, which is considered part of the surface control system weight in the present subdivision.

\section{c. Hydraulic, pneumatic and electrical} groups.

On light aircraft (MTOW up to 12,500 lb or $5,670 \mathrm{~kg}$ ) the hydraulic system is generally restricted to a brake system and flap and undercarriage operation. For some categories a good correlation was found for the combined weight of hydraulic and electrical systems:

utility aircraft -

weight $=.00780 \mathrm{w}_{\mathrm{E}}^{6 / 5} \mathrm{lb}\left(\mathrm{w}_{\mathrm{E}}\right.$ in $\left.1 \mathrm{~b}\right)$ $(8-33)$ 
jet trainers -

weight $=.064 \mathrm{w}_{\mathrm{E}}$

propeller transports -

$\left.\begin{array}{llll}\text { weight }=.325 \mathrm{w}_{e^{4 / 5}} \mathrm{lb} & \left(\mathrm{w}_{E} \text { in } \mathrm{lb}\right) \\ \text { weight }=.277 \mathrm{w}_{\mathrm{e}}^{4 / 5} \mathrm{~kg}\left(\mathrm{w}_{E} \text { in } \mathrm{kg}\right)\end{array}\right\}$

A subdivision for jet transports and jet executives appears desirable. The hydrauiic and pneumatic power system weight is mainly affected by:

1. the number of functions to be powered, i.e. powered or non-powered controls, operation of spoilers, etc.,

2 . the extent of duplication or even triplication,

3. the operating hydraulic or pneumatic pressure, as well as other details of the system design,

4. the airplane size and geometry as related to the length of the plumbing,

5. the relative quantity of pneumatic functions, if any, and

6. the state of the art.

The combined weight of the hydraulic plus pneumatic system may be assumed to be $1 \frac{1}{2} 8$ of the DEW or, alternatively:

no powered controls -

weight $=.004 \mathrm{~W}_{\mathrm{DE}}+100$

weight $=.004 \mathrm{w}_{\mathrm{DE}}+45$

$(\mathrm{kg})$

boosted controls, only some essential

functions duplicated -

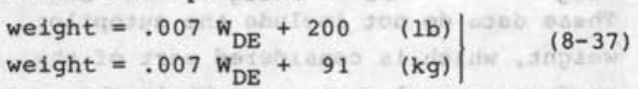

powered controls, fully duplicated system -

$\left.\begin{array}{ll}\text { weight }=.011 \mathrm{w}_{\mathrm{DE}}+400 & (1 \mathrm{~b}) \\ \text { weight }=.011 \mathrm{w}_{\mathrm{DE}}+181 & (\mathrm{~kg})\end{array}\right\} \quad(8-38)$

powered controls, triplex system -

weight $=.015 \mathrm{w}_{\mathrm{DE}}+600 \quad(1 \mathrm{~b})$

weight $=.015 \mathrm{w}_{\mathrm{DE}}+272 \quad(\mathrm{~kg})$

For jet freighters these figures are roughly 308 higher, due partly to the somewhat lower empty weight and partly to the extra services required for loading and unloading. Some weight reduction is possible for an increasing number of pneumatic system functions.

The electrical system weight is affected mainly by:
1. the total electrical power required, which is primaliry determined by the galley power, electronic equipment and fuel system power,

2. whether or not the primary system is an A.C. or D.C. system",

3. the size of the airplane, in view of the length of wiring,

4. the amount of system duplication and the standby systems,

5. whether or not electrical power is generated by the A.P.U., and

6. the state of the art.

the following statistical relationships are suggested:

primary system D.C. -

$\mathrm{w}_{\mathrm{el}}=.02 \cdot \mathrm{w}_{\mathrm{to}}+400 \quad(1 \mathrm{~b})$

$\mathrm{w}_{\mathrm{el}}=.02 \mathrm{w}_{\text {to }}+181 \quad(\mathrm{~kg})$

primary system A.C., total electrical power generated up to $400 \mathrm{kVA}$ -

$w_{e l}=36 \quad P_{e l}\left(1-.033 \sqrt{P_{e l}}\right) \quad(1 b)$

$w_{e l}=16.3 \mathrm{P}_{\mathrm{el}}\left(1-.033 \sqrt{\mathrm{P}_{\mathrm{el}}}\right)(\mathrm{kg})$

In the absence of better information the electrical power generation may be obtained from statistical data in publications like Jane's All the World's Aircraft or from correlations with the passenger cabin volume $\mathrm{V}_{\mathrm{pc}}$ :

if no electrical power is generated by the APU, $v_{p c}$ up to $8,000 \mathrm{cu} . \mathrm{ft}\left(227 \mathrm{~m}^{3}\right)$

$\mathrm{P}_{\mathrm{el}}=.016 \mathrm{v}_{\mathrm{pc}}\left(\mathrm{v}_{\mathrm{pc}}\right.$ in cu.ft $)$ $\mathrm{P}_{\mathrm{el}}=.565 \mathrm{v}_{\mathrm{pc}}^{\mathrm{pc}}\left(\mathrm{v}_{\mathrm{pc}}\right.$ in $\left.\mathrm{m}^{3}\right)$

if electrical power generation by the APU is included -

$\begin{array}{lll}\mathrm{P}_{\mathrm{el}}=.3 & \mathrm{v}_{\mathrm{pc}} \cdot 7 & \left(\mathrm{v}_{\mathrm{pc}} \text { in cu.ft }\right) \\ \mathrm{P}_{\mathrm{el}}=3.64 & \mathrm{v}_{\mathrm{pc}} \cdot 7 & \left(\mathrm{v}_{\mathrm{pc}} \text { in } \mathrm{m}^{3}\right)\end{array} \mid$

These figures on electrical systems are based on 1950-1965 technology. Recent developments have indicated that considerable improvements in system weights are possible by applying advanced techniques like multiplexing** and high-speed generators.

* Most present-day transport aircraft feature A.C. primary systems

**Aviation Week of October 28, 1968, pp. 157-161: a weight reduction of $400 \mathrm{lb}$ (181 $\mathrm{kg}$ ) was achieved on the Boeing 747 


\begin{tabular}{|c|c|c|c|}
\hline GROUP & DESCRIPTION & METHOD & REMARKS \\
\hline ACCOMODATIONS & $\begin{array}{l}\text { flight crew seats, instrument panels, } \\
\text { control stands, sound proofing, insu- } \\
\text { lation, trim, floor covering, lighting } \\
\text { and viring, miscellaneous equipment }\end{array}$ & $\begin{array}{ll}\text { jet a/c }: & \begin{array}{c}29 \\
(16.5) \times W_{D E} .285 \\
16\end{array} \\
\text { propeller a/c: } & (9.1) \times W_{D E}^{.285}\end{array}$ & $\begin{array}{l}\text { WE - Delivery } \\
\text { Empty Weightulb }(k g)\end{array}$ \\
\hline \multirow{3}{*}{$\begin{array}{l}\text { PASSENGER } \\
\text { CABIN } \\
\text { ACCOMMODATIONS }\end{array}$} & passenger and attendants' seats & Table 3-2 & che \\
\hline & $\begin{array}{l}\text { galley (pantry) structure } \\
\text { and provisions } \\
\text { lavatory and toilet provisions, } \\
\text { vater system (dry) }\end{array}$ & 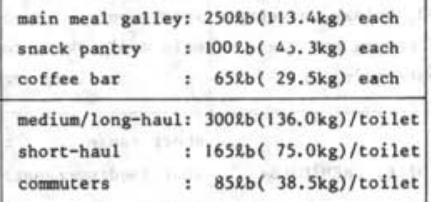 & $\begin{array}{l}\text { galley inserts, } \\
\text { potable water } \\
\text { and toilet } \\
\text { chemicals not } \\
\text { included }\end{array}$ \\
\hline & floor covering & \begin{tabular}{c|l} 
jet aircraft: & propeller aircraft: \\
$.18 \quad 1.15$ & $\begin{array}{l}.135 \\
(1.15\end{array}$ \\
$(1.25) \times \mathrm{S}_{\mathrm{cf}}$ & $(.94) \times \mathrm{S}_{\mathrm{ef}}$
\end{tabular} & \multirow{2}{*}{ 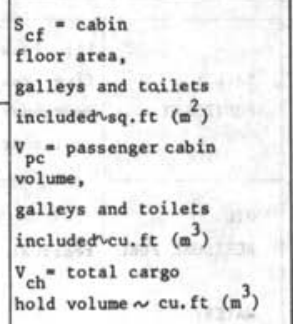 } \\
\hline \multirow{3}{*}{$\begin{array}{l}\text { CARGO } \\
\text { ACCOMMODATIONS }\end{array}$} & $\begin{array}{l}\text { soundproofing and insulation, wall } \\
\text { covering, curtains, screens, window } \\
\text { shades, ceiling, lighting panels, } \\
\text { hatracks, partitions and doors; ward- } \\
\text { robe and stowage provisions, freight } \\
\text { hold linings and partitions }\end{array}$ & 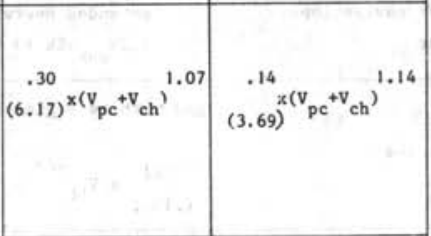 & \\
\hline & $\begin{array}{l}\text { cargo restraints and handling } \\
\text { provisions }\end{array}$ & $.08 \mathrm{lb} / \mathrm{cu} . \mathrm{ft}\left(1.28 \mathrm{~kg} / \mathrm{m}^{3}\right)$ of $\mathrm{v}_{\mathrm{ch}}$ & 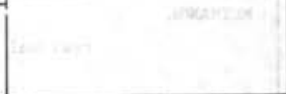 \\
\hline & $\begin{array}{l}\text { container or pallet cargo } \\
\text { handling provisions }\end{array}$ & $\begin{array}{r}2.8 \mathrm{\ell b} / \mathrm{sq} \cdot \mathrm{ft}\left(13.67 \mathrm{~kg} / \mathrm{m}^{2}\right) \text { of freight } \\
\text { convertible }\end{array}$ & $\begin{array}{l}\text { floor area for } \\
\text { passenger/cargo versions }\end{array}$ \\
\hline \multirow{3}{*}{$\begin{array}{l}\text { (STA } \\
\text { (STANDARD) } \\
\text { EMERGENCY } \\
\text { EQUIPMENT }\end{array}$} & $\begin{array}{l}\text { fixed oxygen system, } \\
\text { portable oxygen sets }\end{array}$ & \multicolumn{2}{|c|}{ 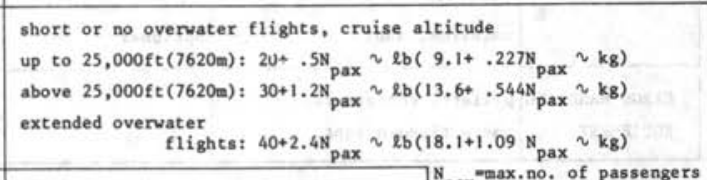 } \\
\hline & $\begin{array}{l}\text { fire detection and extinguishing } \\
\text { system, portable extínguishers }\end{array}$ & $\begin{array}{l}\text { jet a/c : }: .0012 \mathrm{w}_{\mathrm{to}} \\
\text { turboprop a/c: } .0030 \mathrm{w}_{\text {to }} \\
\text { reciproc. a/c: } .0060 \mathrm{w}_{\text {to }}\end{array}$ & $\begin{array}{l}\text { Nax } \max \text {.no. of passengers } \\
\text { for certification } \\
\text { (pressure cabins) }\end{array}$ \\
\hline & $\begin{array}{l}\text { escape provisions } \\
\text { (evacuation slides and ropes) }\end{array}$ & $1 \mathrm{lb}(.453 \mathrm{~kg})$ per occupant & $\begin{array}{l}\text { Other provisions in } \\
\text { Operational Items }\end{array}$ \\
\hline
\end{tabular}

ALL. WEIGHTS IN LB (KG)

NOTE: coefficients in brackets refer to the metric system

Table 8-12. Furnishing and equipment group weight for transport and executive aircraft

d. Furnishing and equipment group.

Light single-engine aircraft: this weight group consists mainly of the weight of seats, wall and floor covering, and some miscellaneous contributions. The weight is approximately. $13 \mathrm{lb}(5.9 \mathrm{~kg})$ per seat, plus $25 \mathrm{lb}(11.3 \mathrm{~kg})$ per row of two seats, plus an additional $5 \mathrm{lb}(2.3 \mathrm{~kg})$.

Light twin-engine aircraft: $15 \mathrm{lb}(6.3 \mathrm{~kg})$ per seat, plus $1 \mathrm{lb}$ per cu.ft $(16 \mathrm{~kg}$ per $\mathrm{m}^{3}$ ) of cabin plus cargo compartment volume. Jet trainers, equipped with two ejection seats: 6.58 of the Delivery Empty Weight. Civil freighters: $3 \mathrm{lb}$ per sq.ft $(14.7 \mathrm{~kg} /$ 


\begin{tabular}{|c|c|c|c|}
\hline ITEM & SUBDIVIStoN & METHOD & REMARKS, SYMBOLS \\
\hline $\begin{array}{l}\text { CREW } \\
\text { PROVISIONS }\end{array}$ & $\begin{array}{l}\text { flight and cabin crew with } \\
\text { baggage, flight equipment }\end{array}$ & ${ }_{(93)}^{205} \times \mathrm{N}_{\mathrm{fc}}+\underset{(68)}{150} \times \mathrm{N}_{\mathrm{cc}}$ & $\begin{array}{l}\mathrm{N}_{\mathrm{fc}}, \mathrm{N}_{\mathrm{cc}} \text { number of } \mathrm{flight/} \\
\text { cabin crew members respectively }\end{array}$ \\
\hline $\begin{array}{l}\text { PASSENGER } \\
\text { CABIN } \\
\text { SUPPLIES }\end{array}$ & $\begin{array}{l}\text { removable galley bar equip- } \\
\text { ment, meal service, consum- } \\
\text { able food, drinks, beverages } \\
\text { pillows, papers and maga- } \\
\text { zines, entertainment }\end{array}$ & $\begin{array}{l}\text { commuters: } 1 \mathrm{lb}(.453 \mathrm{~kg}) \times \mathrm{N}_{\text {pax }} \\
\text { transport aircraft, } \\
\text { snacks only } \quad 5 \mathrm{lb}(2.27 \mathrm{~kg}) \cdot \mathrm{N}_{\text {pax }} \\
\text { main meal, short-range: } 14 \mathrm{lb}(6.35 \mathrm{~kg}) \cdot \mathrm{N}_{\text {pax }} \\
\qquad \text { long -range: } 19 \mathrm{lb}(8.62 \mathrm{~kg}) \cdot \mathrm{N}_{\text {pax }}\end{array}$ & $\begin{array}{l}N_{\text {pax }}=\text { number of passengers, } \\
\text { all-tourist. } \\
\text { First class: all data } \\
5 \text { bb }(2.27 \mathrm{~kg}) \text { per passenger } \\
\text { higher }\end{array}$ \\
\hline \multicolumn{2}{|c|}{ POTABLE WATER AND TOILET CHEMICALS } & \multicolumn{2}{|c|}{ 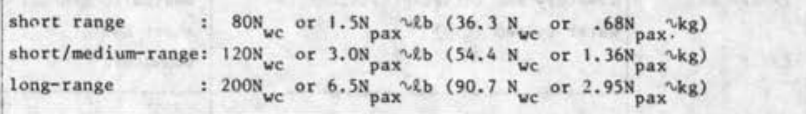 } \\
\hline $\begin{array}{l}\text { SAFETY } \\
\text { EQUIPMENT }\end{array}$ & $\begin{array}{l}\text { life jackets, } \\
\text { fire axes, } \\
\text { emergency navigational } \\
\text { equipment }\end{array}$ & $\begin{array}{l}\text { short or no overwater sectors: } \\
2 \mathrm{~N}_{\text {pax }}{ }^{2} \mathrm{~b}\left(.907 \mathrm{~N}_{\text {pax }}{ }^{2 \mathrm{~kg}}\right) \\
\text { extended overwater flights: } \\
7.5 \mathrm{~N}_{\text {pax }} 2 \mathrm{lb}\left(3.4 \mathrm{~N}_{\text {pax }}{ }^{2 \mathrm{~kg})}\right.\end{array}$ & $\begin{array}{l}\text { Ne number of } \\
\text { toilets/water closets: } \\
\text { data based on all- tourist } \\
\text { layout }\end{array}$ \\
\hline \multirow[t]{4}{*}{$\begin{array}{l}\text { OIL. } \\
\text { RESIDUAL FUEL. } \\
\text { WATER/ } \\
\text { METHANOL }\end{array}$} & residual fuel & \multirow{3}{*}{$\begin{array}{l}\text {. siprocating: } \\
.008 \mathrm{w}_{\text {to }} \\
.045 \mathrm{w}_{\mathrm{f}}\end{array}$} & \multirow{4}{*}{$\begin{aligned} v_{f t}= & \text { total fuel tank } \\
& \text { capacity } \sim \text { U.S. gal. } \\
& \text { (liters) } \\
W_{t o}= & \text { Max. Takeoff } \\
& \text { Weight } \approx \ell b(\mathrm{~kg}) \\
W_{f}= & \text { fuel weight } \\
& \sim \ell b \quad(\mathrm{~kg})\end{aligned}$} \\
\hline & residual oil & & \\
\hline & engine oil consumed & & \\
\hline & water/methanol & optional & \\
\hline $\begin{array}{l}\text { CARGO HANDLING } \\
\text { EQUIPNENT }\end{array}$ & $\begin{array}{l}\text { pallets, containers, } \\
\text { cargo tiedown eqpt. }\end{array}$ & Fig. $3-20$ & ALL. WEIGHTS IN LB (KG) \\
\hline
\end{tabular}

NOTE: coefficients in brackets refer to the metric system

Table 8-13. Data for estimating the weight of Operational Items (transport aircraft)

$\mathrm{m}^{2}$ ) of main freightfloor area.

Passenger transports and jet executives: a rough approximation is obtained with the statistical expression:

weight $=.211 \mathrm{w}_{\mathrm{ZF}} .91$
weight $=.196 \mathrm{w}_{\mathrm{ZF}} .91$

where $w_{\mathrm{ZF}}$ is the Maximum Zero Fuel Weight. The furnishing and equipment weight forms a very substantial contribution, of the order of half the fuselage structure weight. Instead of using $(8-44)$ the designer may prefer to use a more detailed estimation by breaking down the weight into several individual contributions. A proposed subdivision and calculation methods are presented in Tables $8-12$ and $3-2$. It should be noted that several items such as the weight of seats depend on the required standard of comfort and the type of interior; these may be subject to customer requirements (Standard Items Variation).

e. Air-conditioning and anti-icing group. The weight of the air-conditioning and pressurization system depends on many factors: 
1. the type of system used: air cycle or vapor cycle, use of ram air or engine bleed air, etc.,

2. design requirements, in terms of airconditioning airflow per unit of time, air temperature, humidity and cabin pressure differential, cargo compartment air-conditioning,

3. the amount of system duplication,

4. the airplane size, or more specifically the cabin volume and length, and the subdivision into zones,

5. the state of the art.

Factors affecting the anti-icing and deicing system weight are:

1. type of system (electrical, hot-air, rubber boots),

2. dimensions, mainly the length or span of the airplane parts concerned, and

3. the type of operation, viz. IFR or VFR flights.

For the combined system, the following data can be used:

Light single-engine aircraft - 2.5 lb (1.1 $\mathrm{kg}$ ) per seat. Multi-engine unpressurized aircraft and jet trainers - 1.8 of the Delivery Empty Weight

pressurized transports and executive aircraft -

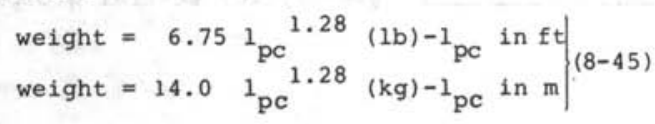

\section{f. Miscellaneous.}

This item refers to auxiliary gears, photographic equipment, external paint, manufacturing variation, unaccounted items, unexpected weight growth, etc. No systematic data are available, but in general a figure of up to 18 of the Delivery Empty Weight is typical for existing aircraft.

\subsubsection{Useful Load and the All-Up Weight}

\section{a. Operational Items.}

Due to the large variation in operational conditions and requirements applying to passenger service, considerable variations in the weight of operational items can be observed. The data in Table $8-13$ are re- presentative of but by no means mandatory for the transport aircraft category. For private aircraft and jet trainers the only item of interest is the residual fuel and oil.

It should be noted that the data of Table 8-13 are generally applicable to modern, gas turbine powered aircraft. Considerably higher weight values are applicable to older piston-engine powered transport aircraft.

b. Payload and fuel.

Some data on specific gravity of fuels and civil payload will be found in Table $8-14$.

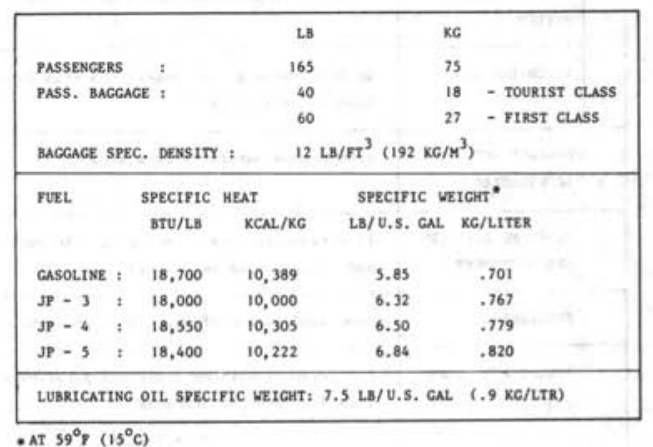

Table 8-14. Standard weights of payload, fuel and oil

The data presented in this section 8.4 and Appendices C and D are sufficiently complete to enable the designer to make a fairly accurate prediction of the OEW of a civil airplane. Of necessity, the procedure is based on an initial estimate of the various characteristic weights, as obtained, for example, with Sections 5.2 and 8.2.

The more detailed weight prediction will result in a value for the OEW that is essentially different from the first "guesstimate". The designer must therefore decide whether he should modify the Useful Load (i.e. fuel and/or payload) or the MTOW. Fresh calculations of the weight distribution will then be necessary until the designer is satisfied with the convergence. 


\begin{tabular}{|c|c|c|}
\hline & COMPONENT & 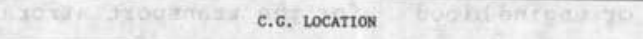 \\
\hline \multirow{6}{*}{ 宸 } & WTNG (HAL.F) & $\begin{array}{l}\text { straight ving: } 38-42 \pi \text { chord from } L E \text { at } 40 \mathrm{z} \text { seai-span from centerline } \\
\text { swept wing: } 70 \mathrm{z} \text { local distance between front and rear spar, measured } \\
\text { from front spar, at } 35 \mathrm{z} \text { seai-span from centerline }\end{array}$ \\
\hline & 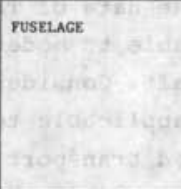 & 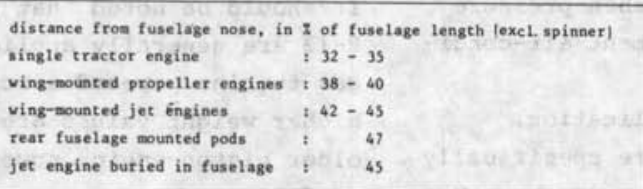 \\
\hline & $\begin{array}{l}\text { TATLPLASE } \\
\text { (HALF) }\end{array}$ & $\begin{array}{l}42 \pi \text { chord froe LE at } 38 \pi \text { seni-span from root chord. } \\
\text { Fin, T-tail conf iguration: } \\
42 \pi \text { chord from } L E \text { at } 55 \pi \text { of height from root chord }\end{array}$ \\
\hline & SACELLES & $40 z$ of nacelle length from nose, spinner excluded \\
\hline & $\begin{array}{l}\text { SURFACE CONTROL } \\
\text { SYSTEM }\end{array}$ & $100 z$ MaC from LEMC, autopilot exeluded \\
\hline & ALTGHTING GEAR & $\begin{array}{l}\text { at airplane c.g., or determined from location and veight of main and } \\
\text { nose undercarriage }\end{array}$ \\
\hline & $\begin{array}{l}\text { ENGINES AND } \\
\text { ACCESSORIES }\end{array}$ & froe engine nanufacturer's data \\
\hline & $\begin{array}{l}\text { AIRFRUNE SERVICES } \\
\text { AND EQUIPMENT }\end{array}$ & $\begin{array}{l}\text { from educated guess, taking into account location of main elements } \\
\text { and functions to be povered }\end{array}$ \\
\hline & FURNISHING & from subdivision of Table $8-11$ and cabin layout \\
\hline & FILLED FUEL. TANK & 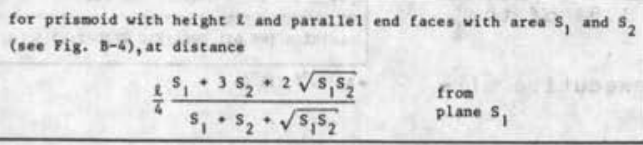 \\
\hline
\end{tabular}

NOTE: more accurate estimates can be made by further breakdown of each item into several contributions

Table 8-15. Approximate location of the center of gravity for several groups

If the designer decides to alter the Useful Load, the specified transport performance (payload-range) may no longer be achieved, while in the second eventuality the takeoff weight may become too high for acceptable takeoff, landing and/or climb performance. Sufficient aerodynamic data must be available to evaluate the design performance (Chapter 11).

In this textbook we will proceed with the layout design, assuming that the previous weight prediction has not entailed major difficulties and that only minor changes in the weight distribution are required. However, there may be occasions when weight evaluation and changes result in a considerable increase of the takeoff weight.

\subsection{CENTER OF GRAVITY}

Each airplane must be designed in such a way that good stability and control properties and adequate flexibility in loading conditions are obtained. By suitable arrangement of the design layout and acceptable tailplane size, acceptable fore-andaft limits of the center of gravity must be established, taking into account the following aspects:

1. fore-and-aft position of the wing relative to the fuselage,

2. provision of suitable locations for payload and fuel,

3. design of the horizontal tailplane, the elevator and the longitudinal flight con- 


\begin{tabular}{|c|c|c|c|c|c|c|c|c|c|c|c|}
\hline \multirow{3}{*}{\multicolumn{2}{|c|}{ AIRPLANE TYPE }} & \multicolumn{6}{|c|}{ C.G. LIMITS, PER CENT M.A.C. } & \multirow{4}{*}{\begin{tabular}{|l} 
PAY- \\
LOAD \\
$Z$ OEW \\
28.3
\end{tabular}} & \multirow{4}{*}{$\frac{\frac{s_{h^{2} h}^{2}}{s^{-}}}{.64}$} & \multirow{4}{*}{$\begin{array}{c}\text { hor. } \\
\text { tail } \\
\text { type } \\
* *\end{array}$} & \multirow{4}{*}{$\begin{array}{l}\mathrm{C}_{\mathrm{L}_{\max }} \\
\stackrel{* *}{2.40}\end{array}$} \\
\hline & & \multicolumn{2}{|c|}{ FORWARD } & \multicolumn{2}{|c|}{ REAR } & \multicolumn{2}{|c|}{ RANGE } & & & & \\
\hline & & \begin{tabular}{|l|} 
takeoff \\
landing \\
\end{tabular} & flight & \begin{tabular}{|l|} 
takeoff \\
landing \\
\end{tabular} & flight & $\begin{array}{l}\text { takeoff } \\
\text { landing }\end{array}$ & flight & & & & \\
\hline \multirow{13}{*}{ 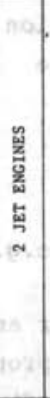 } & Aerospatiale Corvette SN60I & $\overline{6}$ & 20.0 & - & $36: 0$ & - & 16.0 & & & & \\
\hline & A.C. Jet Commander 1121 & 20.0 & 20.0 & 36.0 & 36.0 & 16.0 & 16.0 & 20.6 & .64 & $\mathbf{F}$ & 1.66 \\
\hline & Lear Jet 25 & 9.0 & 9.0 & 30.0 & 30.0 & 21.0 & 21.0 & 35.6 & .64 & $\mathbf{F}$ & 1.39 \\
\hline & H. Siddeley HS- $125 \mathrm{IA} / \mathrm{BB}$ & 18.0. & $18.0 *$ & $37.5 *$ & $37.5 \cdot$ & $19.5 *$ & $19.5 \cdot$ & 14.0 & .69 & $\mathbf{F}$ & 2.44 \\
\hline & Dassault Mystère 20F & 14.0 & 16.0 & 28.5 & 28.5 & 14.5 & 12.5 & 23.1 & .66 & $\mathbf{v}$ & 2.30 \\
\hline & H.F.B. Hansa & 13.0 & 11.7 & 23.0 & 21.7 & 10.0 & 10.0 & 30.9 & .71 & $\mathbf{F}$ & 2.00 \\
\hline & Fokker VFW F-28 Mk 1000 & 18.0 & 17.0 & 35.0 & 37.0 & 17.0 & 20.0 & 42.0 & .97 & $\mathbf{v}$ & 2.53 \\
\hline & BAC 1-11 Srs. 400 & $15.0 *$ & $14.0 \cdot$ & $39.0 *$ & $41.0 \cdot$ & $24.0 *$ & $27.0 \cdot$ & 35.3 & .85 & v & 2.38 \\
\hline & Sud. Av. Caravelle IOR & 25.0 & 25.0 & 41.5 & 41.5 & 16.5 & 16.5 & 32.3 & .56 & $\mathbf{F}$ & 2.10 \\
\hline & MeD. Douglas $D C-9 / 10$ & 16.3 & 15.0 & 39.0 & 40.0 & 22.7 & 25.0 & 42.4 & 1.15 & v & 2.40 \\
\hline & (9) $\quad D C-9 / 33 F$ & 5.9 & 3.1 & 34.7 & 34.7 & 28.8 & 31.6 & 70.8 & 1.18 & v & 2.98 \\
\hline & Boeing $737 / 100$ & 15.0 & 15.0 & 35.0 & 35.0 & 20.0 & 20.0 & 49.4 & 1.14 & v & 3.10 \\
\hline & Airbus $\mathrm{A}-300 \mathrm{B2}$ & 11.0 & 11.0 & 31.0 & 31.0 & 20.0 & 20.0 & 37.4 & 1.07 & $v$ & 2.65 \\
\hline \multirow{7}{*}{ 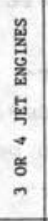 } & Lockheed 1011 Tristar & - & 12.0 & - & 32.0 & - & 20.0 & 36.1 & .93 & $A$ & 2.57 \\
\hline & Boeing $707 / 120$ & 16.0 & 16.0 & 34.0 & 34.0 & 18.0 & 18.0 & 38.2 & .61 & $\mathbf{v}$ & 1.86 \\
\hline & $720 / 022$ & 15.0 & 15.0 & 31.0 & 31.0 & 16.0 & 16.0 & 31.9 & .59 & $v$ & 2.26 \\
\hline & $747 / 200 B$ & - & 12.5 & - & 32.0 & - & 19.5 & 45.8 & 1.00 & $v$ & 2.55 \\
\hline & MeD. Douglas DC-8/21 & 16.5 & 16.5 & 32.0 & 32.0 & 15.5 & 15.5 & 27.0 & .58 & v & 2.10 \\
\hline & Lockheed C-141A & 19.0 & 19.0 & 32.0 & 32.0 & 13.0 & 13.0 & 50.3 & .51 & v & 2.32 \\
\hline & Lockheed $C-5 A$ & 19.0 & 19.0 & 41.0 & 41.0 & 22.0 & 22.0 & 67.9 & .64 & $\mathbf{v}$ & 2.60 \\
\hline \multirow{10}{*}{ 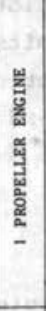 } & Fokker $\mathrm{S}-11$ Inst ructor & 21.5 & 21.5 & 27.0 & 27.0 & 5.5 & 5.5 & 22.2 & .43 & $\mathrm{~F}$ & 1.25 \\
\hline & Cessna 172, Nornal Cat. & 15.6 & 15.6 & 36.5 & 36.5 & 20.9 & 20.9 & 64.3 & .59 & $\mathbf{F}$ & 2.14 \\
\hline & 177, Norsal Cat. & 5.0 & 5.0 & 28.0 & 28,0 & 23.0 & 23.0 & 58.6 & .60 & $\mathbf{A}$ & 1.86 \\
\hline & 177, Utility Cat. & 5.0 & 5.0 & 18.5 & 18.5 & 13.5 & 13.5 & 58.6 & .60 & $\mathbf{A}$ & 1.86 \\
\hline & 206 Skywagon & 12.2 & 12.2 & 39.4 & 39.4 & 27.2 & 27.2 & 67.3 & .77 & $\mathbf{F}$ & 2.16 \\
\hline & Beecheraft B- 45 Mentor & 20.1 & 19.0 & 28.0 & 28.0 & 7.9 & 9.0 & 17.4 & .54 & $\mathbf{F}$ & 2.01 \\
\hline & Piaggio P-148 ( 3 seater) & 22.3 & 22.3 & 30.7 & 30.7 & 8.4 & 8.4 & 26.3 & .43 & $\mathbf{F}$ & 1.90 \\
\hline & Pilatus PC-6-M2 Porter & 11.0 & 11.0 & 34.0 & 34,0 & 23.0 & 23.0 & 79.9 & .67 & $\mathbf{A}$ & 2.28 \\
\hline & Saab 91-B Safir & 17.9 & 17.9 & 27.1 & 27.1 & 9.2 & 9.2 & 23.9 & .64 & $\mathbf{F}$ & - \\
\hline & De Havilland $\mathrm{DHC}-2$ Beaver & 17.4 & 17.4 & 40.3 & 40.3 & 22.9 & 22.9 & 49.3 & .76 & $\mathbf{F}$ & - \\
\hline \multirow{11}{*}{ 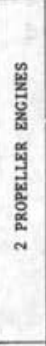 } & Cessna Mode1 337 & 17.3 & 17.3 & 30.9 & 30.9 & 13.6 & 13.6 & 37.1 & .51 & $\mathbf{F}$ & 1.78 \\
\hline & Piper PA $30 \mathrm{C}$ Twin Comanche & 12.0 & 12.0 & 27.8 & 27.8 & 15.8 & 15.8 & 40.7 & .44 & $\mathbf{A}$ & 1.66 \\
\hline & Beecheraft Queen Air M. 80 & 16.0 & 16.0 & 29.9 & 29.9 & 13.9 & 13.9 & 44.9 & .73 & $\mathbf{F}$ & 1.88 \\
\hline & Dornier Do 28-D-1 & 10.7 & 10.7 & 30.8 & 30.8 & 20.1 & 20.1 & 34.7 & .67 & $A$ & 2.36 \\
\hline & DHC-6 Twin otter & 20.0 & 20.0 & 36.0 & 36.0 & 16.0 & 16.0 & 74.0 & .93 & $\mathbf{F}$ & 2.37 \\
\hline & Nord 262 & 16.0 & 16.0 & 30.0 & 30.0 & 14.0 & 14.0 & 48.0 & .96 & $\mathbf{F}$ & 2.23 \\
\hline & Fokker VFW F-27 Mhe 200 & 20.0 & 18.7 & 38.0 & 40.7 & 18.0 & 22.0 & 55.8 & .96 & $\mathbf{F}$ & 2.94 \\
\hline & Hurel Dubois HD 32 & 23.5 & 23.5 & 46.5 & 46.5 & 23.0 & 23.0 & 36.5 & 1.32 & $\mathbf{F}$ & 2.70 \\
\hline & Convaír 240 & 15.0 & 8.5 & 31.0 & 33.0 & 16.0 & 24.5 & 33.9 & 1.07 & $\mathbf{F}$ & 2.33 \\
\hline & 340 & 13.0 & 8.5 & 34.0 & 35.0 & 21.0 & 26.5 & 56.0 & 1.03 & $\mathbf{F}$ & 2.61 \\
\hline & H. Siddeley Andover C.Mk 1 & $13.3 *$ & $13.3 \cdot$ & $36.0 *$ & $36.0 *$ & $22.7 \bullet$ & $22.7 \cdot$ & 53.6 & 1.09 & $\mathbf{F}$ & 2.88 \\
\hline \multirow{8}{*}{ 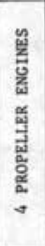 } & Bréguet 941 & 23.0 & 23.0 & 32.0 & 32.0 & 9.0 & 9.0 & 70.0 & 1.05 & v & 7.19 \\
\hline & Douglas DC- 6 & 16.0 & 12.0 & 33.0 & 35.0 & 17.0 & 23.0 & 29.4 & 1.04 & $\mathbf{F}$ & 2.77 \\
\hline & Lockheed $188 \mathrm{C}$ Electra & 15.0 & 13.0 & 32.0 & 33.0 & 17.0 & 20.0 & $\$ 3.6$ & .80 & $F$ & 2.54 \\
\hline & Bristol 175 Britannia & $13.0 *$ & $12.0 \cdot$ & $34.5 *$ & $35.5 \cdot$ & $21.5 *$ & $23.5 *$ & 37.3 & .97 & $\mathbf{F}$ & 2.56 \\
\hline & Lockheed L- 1049 H & 18.0 & 15.0 & 32.0 & 34.0 & 14.0 & 19.0 & 44.9 & 1.15 & $\mathbf{F}$ & 2.60 \\
\hline & $1-1649 A$ & 15.0 & 12.0 & 32.0 & 34.0 & 17.0 & 22.0 & 17.5 & 1.12 & $\boldsymbol{F}$ & 2.53 \\
\hline & $\mathrm{C}-130 \mathrm{E}$ & 15.0 & 15.0 & 30.0 & 30.0 & 15.0 & 15.0 & 51.4 & 1.00 & $\mathbf{F}$ & 2.28 \\
\hline & Canadaír $\mathrm{CL}-44 \mathrm{C}$ & 12.2 & 12.2 & 30.5 & 31.4 & 18.3 & 19.2 & 22.8 & 1.14 & $\mathbf{F}$ & 2.56 \\
\hline
\end{tabular}

- per cent SMC

*. F - fixed stabilizer, V - variable incidence stabilizer, A = all-movable tail

... flap angle for landing

Uaflap angle for landing

Table 8-16. Center of gravity limits for several types of aircraft 
trol system, and

4. location of the undercarriage legs. The designer's freedom of choice is greatly limited by the conditions imposed upon the c.g. location. This applies in particular to the location of fixed items like engines and cargo holds, both having a considerable bearing on the balance of the airplane.

The c.g. must be established in both the longitudinal and the vertical direction. Having obtained the weight distribution, the designer can produce a sideview with a suitable system of coordinate axes and the centers of gravity plotted on it for each individual item (Table 8-15). Tables similar to Table $8-3$ are filled out and the final result of this tabulation yields the horizontal and vertical location of the c.g. for the Operating Empty Weight condition:

$x_{c g}=\frac{\sum x_{i} w_{i}}{\sum w_{1}}$

$z_{c g}=\frac{\sum z_{i} w_{i}}{\sum w_{i}}$

Fore- and -aft shift of limits for the c.g. due to different loading conditions must be estimated and indicated in the drawing. The designer must demonstrate that in all likely loading conditions the actual c.g. will remain inside the fore-and-aft $11 \mathrm{~m}-$ itations, without undue penalties in the form of loading restrictions. Some procedures used to obtain a good wing location and c.g. range will be discussed in the following sections.

\subsubsection{The load and balance diagram}

It is not sufficient to determine the c.g. for only one condition, eig. the 'ffully loaded condition. What is important for an analysis of the stability and control properties are the most critical fore-and-aft locations.

Assuming that the general (airplane) arrangement and layout design have been decided upon, the loading flexibility can be illustrated in a weight and balance diagram (Fig. 8-7). Both the likely operational c.g. shift and the "aerodynamic" limits are indicated in this diagram as a function of the weight. The Mean Aerodynamic Chord $(M A C)^{*}$ or the Standard Mean Chord (SMC)* are used to define the c.g. position. The Alrcraft Prepared for Service, but without fuel and payload (OEW), is represented by point $\mathrm{A}$ in Fig. 8-7. A margin of a few per cent MAC is usually assumed

\section{* Definitions are given in Appendix A.}

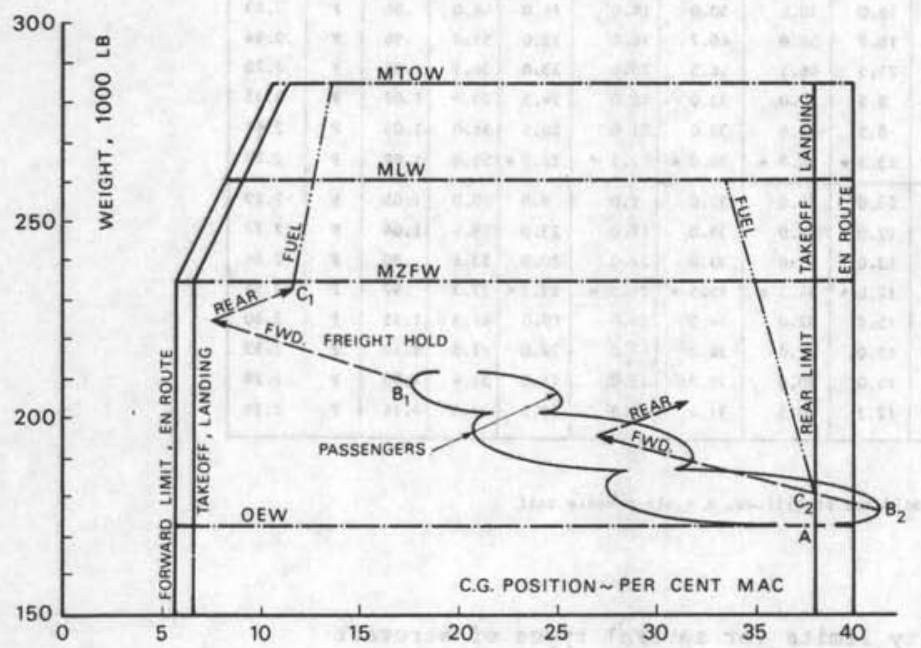

Fig. 8-7. Load and balance diagram for a short-haul wide-body airliner 
on both sides of the standard location to allow for variation in the weights and 10cations of the airframe services, equipment and Operational Items (cf. Section 8.2.1.), undercarriage retraction, etc. The loading of passengers in the cabin and baggage or cargo in the compartments increases the weight and causes a shift of the c.g. which will depend upon the location of the variable load relative to point $A$.

For passenger transports it is not usual to assume that the passenger seating is completely arbitrary. The procedure to be described in Section 8.5.2. is a statistical prediction of the most practicable envelope of passenger loading distributions, assuming a free choice of the seats. In view of its shape the passenger loading envelope is sometimes referred to as "loading potato" or "passengers loop". The effects of loading the cargo compartments is indicated by shifting the most extreme points of the loading envelope, points $B_{1}$ and $B_{2}$. In the example $B_{2}$ is located outside the allowable c.g. limits and hence for low payloads there must always be some load in the forward cargo hold. At a later stage of the design loading restrictions of this type are translated into load sheets used by the operator's personnel to control the c.g. Assuming that points $c_{1}$ and $c_{2}$ represent the most extreme locations, the fuel load can be inserted by further envelopes, originating at $c_{1}$ and $c_{2}$. In the example shown it is assumed that a fuel management procedure has been developed to minimize the c.g. shift, resulting in approximately straight lines in the diagram. On a swept wing this is not always entirely feasible; the use of reserve tanks to minimize the c.g. shift can then be considered. As opposed to this, the BAC-Sud Concorde features a system for transferring fuel from the wing tanks to a rear fuselage tank, in order to cope with the a.c. shift of the airplane caused by the transition from subsonic to supersonic flight.

Variations in the c.g. have an effect not only on stability and control characteristics, but also on the tail maneuver loads and the ground loads acting on the nose undercarriage. A loading envelope must therefore be established, defining possible combinations of c.g. and Gross Weight, for which certification is requested. The principal aim during preliminary design is to ensure that the most likely operational loadings are not unduly penalized by complicated and/or stringent loading restrictions.

\subsubsection{Loading flexibility and restrictions}

The type of operation envisaged for the airplane determines to what extent special loading restrictions are acceptable. Though a generous allowable c.g. travel improves the operational flexibility, there are some pitfalls as well:

1. A large c.g. shift entails large variations in the stability and maneuver margins and generally makes the design of the tailplane and control system more difficult. Added complexity, weight and skin friction drag will be the inevitable result.

2. A large stability margin entails appreciable trim drag.

3. A c.g. location that is well forward of the airplane's neutral point must be balanced by a download on the tail. The maximum lift coefficient (flaps down) may be considerably reduced by this.

A collection of data on c.g. locations is presented in Table $8-16$. The following comments will make them easier to interpret. For passenger transports a generous c.g. travel of the order of 20-25\% MAC is desirable. Different seating arrangements and cabin layouts, payload growth, alternate conversions into Quick Change (QC) or combined passenger/freight transport, and increased fuel tank capacity on later versions are all aspects to be considered. Airlines object strenuously to assigning passengers definite seats, but balancing with freight is acceptable up to a certain point. Fuel management procedures are ac- 
ceptable, but fixed ballast should be avoided altogether.

For light aircraft it is desirable to analyze all likely loading conditions. For passenger transports the following "window seating rule" is observed (example in Fig. 8-8).

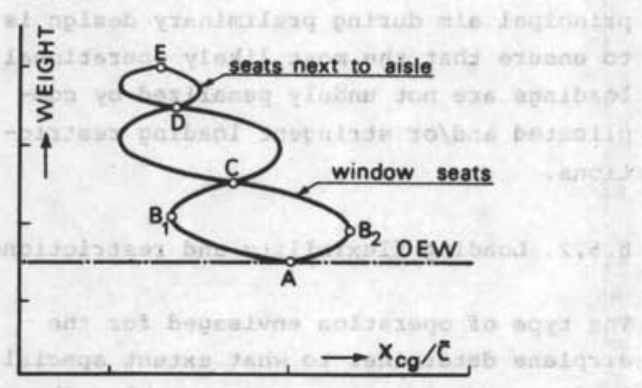

Fig. 8-8. The window seating procedure

In the empty aircraft the passengers are assumed to occupy the seats nearest to the window first. When these are filled $(A \rightarrow C)$, the rows next to the window seats are occupied $(C \rightarrow D)$, followed finally by those nearest to the aisle $(D+E)$. The window seats are occupied in two different ways: - starting from the front $\left(A+B_{1}+C\right)$ - starting from the rear $\left(A+B_{2} \rightarrow C\right)$ In this way a loop is formed. Here the most forward and rearward points $\left(B_{1}\right.$ and $B_{2}$ ) correspond to the situation where all window seats in front of or behind $A$ respectively, are occupied. The loops $C \rightarrow D$ and $\mathrm{D} \rightarrow \mathrm{E}$ are computed in a similar manner. The example applies to a cabin layout with 5-abreast seating, resulting in a small upper loop for a single third row of seats. other aircraft arrangements may give quite different results (Section 8.5.3.).

General aviation aircraft (commuters, utility aircraft, air taxis) usually need a fairly large c.g. travel for versatility in operation. For example, the Pilatus Porter has a travel of $238 \mathrm{MAC}$, the Scottish Aviation Jetstream as much as $30 \%$ SMC. A large travel is not required on light private aircraft as the payload is relatively low and located close to the c.g. of the empty aircraft. Only simple loading procedures will be acceptable. Light aircraft for 2 persons need a c.g. travel of only 5 to 108 MAC. Statistical variation of the occupant weights, and variations in equipment and furnishing should be accounted for.

In the case of freighter aircraft the large variety in payload characteristics means that there are too many conceivable loading conditions for all of them to be considered in the preliminary design stage. It is suggested that reasonable fore-and-aft c.g. limits be chosen on the basis of statistical evidence or stability and acceptable control characteristics, assuming the horizontal tailplane design to be fixed. A balance diagram can be drawn to limit the cargo c.g. as a function of its weight (Fig. $8-9$ ). The relevant equation is:

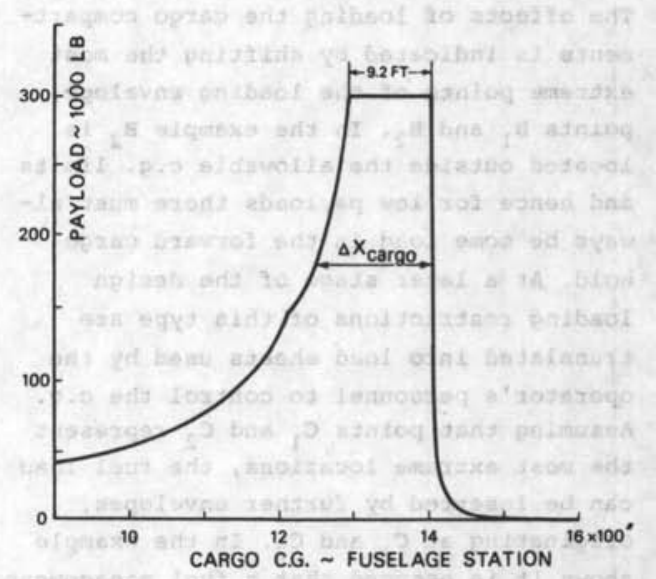

Fig. 8-9. Typical loading limits for a large freighter

$x_{\text {cargo }}=\frac{w_{O E}}{w_{\text {cargo }}}\left(x_{\operatorname{cg}_{1 \text { imit }}}-x_{O E}\right)+x_{\operatorname{cg}_{1 \text { imit }}}(8-48)$ where $\mathrm{W}_{\mathrm{OE}}$ and $\mathrm{x}_{\mathrm{OE}}$ are the OEW and the associated c.g. position, while $x_{c g_{1 i m i t}}$ represents the fore-and-aft c.g. limits, corresponding to the weight $\mathrm{w}_{\mathrm{OE}}+\mathrm{w}_{\text {cargo }}$. The cargo loading flexibility,

$\Delta \mathrm{x}_{\text {cargo }}=\Delta \mathrm{x}_{\mathrm{cg}}\left(1+\frac{\mathrm{w}_{\mathrm{OE}}}{\mathrm{w}_{\text {cargo }}}\right)$ 
is therefore proportional to the allowable c.g. travel. The asymmetric shape of the diagram is caused by the c.g. location of the empty airplane, which is very near the aft limit in this particular example. On a fully loaded airplane the freight must always be unloaded from the aft section of the freighthold. Whether or not this is acceptable depends upon the location of the loading doors and the type of operation.

A noticeable c.g. shift will also occur during ground loading and unloading of transport aircraft. To avoid an unexpected turnover, the c.g. must always be inside the triangle interconnecting the wheel contact points. Passenger access and freighthold doors must be located not too far to the rear end of the fuselage if a nosewheel undercarriage is envisaged.

\subsubsection{Effects of the general arrangement} and layout

The effect of engine location on the balance is illustrated in Fig. 8-10 for three typical general arrangements. To give the comparison point, the designs have approximately equal weights, wing area, payload and fuel. The differences in engine location for Configurations 2 and 3 have resulted in a shift of the wing backwards or forwards relative to configuration 1 .

Configuration 1 is generally the easiest. to balance as the cabin volumetric center is close to the c.g. of the empty aircraft. The c.g. travel is large for a mixed class layout if one of the cabin compartments is empty. Cargo can be used to balance the aircraft by suitable distribution between the forward and rear cargo holds, which are of roughly equal volume. The c.g. corresponding to the OEW is usually located at 25-308 MAC.

Configuration 2 is generally the most difficult layout for which to obtain a satisfactory balance, particularly on short-haul aircraft, as both the engines and the payload masses are relatively large.

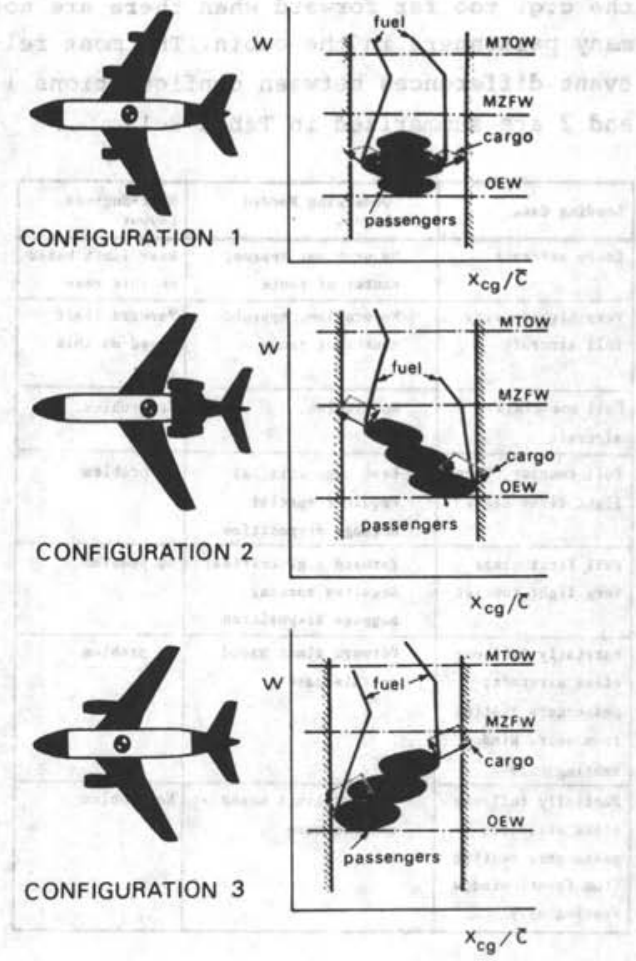

Fig. 8-10. Effect of the general arrangement on load and balance

The c.g. of the empty aircraft is very near the rear limit (approximately 35-408 MAC)* . Most of the payload is ahead of the c.g. and the most forward location corresponds to the MZFW.

The c.g. shift of Configuration 2 is 3 to 58 MAC larger than for an-otherwise comparable Configuration 1, but an acceptable loading flexibility is nevertheless feasible in most cases, in particular when a $\mathrm{T}$-tail is adopted, being very effective under normal flight conditions. For combined passenger/cargo transport, however, this configuration is generally considered undesirable, because the cargo, when located in front of the passenger cabin, will bring

* It is known that the Tupolev 154 has its c.g. at 508 MAC in the empty condition (cf. The Aeroplane, Oct. 1966, page 18) 
the c.g. too far forward when there are not many passengers in the cabin. The most relevant differences between configurations 1 and 2 are summarized in Table 8-17.

\begin{tabular}{|c|c|c|}
\hline Loading Case & $\begin{array}{l}\text { Underving Podded } \\
\text { Layout }\end{array}$ & $\begin{array}{l}\text { Rear-Engined } \\
\text { Layout }\end{array}$ \\
\hline Empty aireraft & $\begin{array}{l}\text { No problen. Approx. } \\
\text { center of range }\end{array}$ & $\begin{array}{l}\text { Rear linit based } \\
\text { on this case }\end{array}$ \\
\hline $\begin{array}{l}\text { Very high-density } \\
\text { full aireraft }\end{array}$ & $\begin{array}{l}\text { No problea. Approx. } \\
\text { center of range }\end{array}$ & $\begin{array}{l}\text { Forvard limit } \\
\text { based on this } \\
\text { case }\end{array}$ \\
\hline $\begin{array}{l}\text { Full one-class } \\
\text { aireraft }\end{array}$ & No problea. & No problea \\
\hline $\begin{array}{l}\text { Full tourist. Very } \\
\text { light first class }\end{array}$ & $\begin{array}{l}\text { Rear c. } 8 \text {. critical } \\
\text { Requires special } \\
\text { baggage disposition }\end{array}$ & No problea \\
\hline $\begin{array}{l}\text { Fu11 first class } \\
\text { Very light tourist }\end{array}$ & $\begin{array}{l}\text { Porvard c.g. critical } \\
\text { Requires special } \\
\text { baggage disposition }\end{array}$ & No problem \\
\hline $\begin{array}{l}\text { Partially full one - } \\
\text { class aircraft; } \\
\text { passengers seating } \\
\text { froe rear. Window } \\
\text { seating only }\end{array}$ & $\begin{array}{l}\text { Yorvard linit based } \\
\text { on this case }\end{array}$ & No problem \\
\hline $\begin{array}{l}\text { Partially full one- } \\
\text { class aircraft; } \\
\text { passengers seating } \\
\text { fros front. Window } \\
\text { seating only }\end{array}$ & $\begin{array}{l}\text { Forvard limit based } \\
\text { on this case }\end{array}$ & raniugrat \\
\hline
\end{tabular}

Table 8-17. Summary of critical loading conditions for two airplane configurations (Reference: Aerospace Engineering, October 1960, page 74)

Configuration 3. Although the example assumes jet engines, this layout is chosen more frequently in propeller aircraft, particularly if the propellers are in front of the flight deck. The situation is to some extent the reverse of Configuration 2, but the balancing problems are generally less difficult. The empty aircraft has its c.g. at about 20-258 MAC, the critical loading case being the high-density layout, resulting in the rear c.g. position. The relatively large tail arm aids in solving stability and control problems and the horizontal tailplane can be kept relatiyely small, particularly for $\mathrm{T}$-tail designs. The provision of a cargo compartment in the fuselage nose in front of the cockpit will improve the balance.
8.5.4. Design procedure to obtain a balanced aircraft

There may be various ways in which the airplane can be balanced, depending upon the degree to which the design has been frozen: 1. The c.g. of the empty aircraft can be optimized by locating the wing appropriately in the longitudinal direction.

2. The c.g. range can be reduced by a favorable cabin layout and suitable location of the engines, cargo compartments, fuel tanks and certain items of airframe services and equipment.

3. Suitable tailplane and control system design and undercarriage location should provide acceptable fore-and-aft c.g. limits.

The first step in c.g. calculation is usually made in the stage when the wing location has to be determined. Wing location may be fiked in some cases by consideration of wing attachment provisions.

For example, in the case of the HFB Hansa business jet, a mia-wing layout, the condition was imposed that the wing box had to be located aft of the passenger cabin. This could be solved only by using a swept-forward wing. For low-subsonic aircraft, however, wing sweep should be limited to some 5 or 10 degrees to avoid undesirable aerodynamic effects.

A proposed design procedure to balance the wing location, tail size and c.g. travel will be discussed in section 9.5.2. In a first approach it is generally sufficient to aim at a reasonable c.g. travel and to choose the wing location accordingly. This simple procedure is illustrated by Fig. 8-11.

\section{Step 1.}

The airplane is subdivided into at least the following groups:

1. The fuselage group* - containing parts whose location is fixed relative to the fuselage, e.g. the fully furnished and

"Note that the terms "fuselage group" and "wing group" as used here have different meanings from those in Section 8.4.2. 


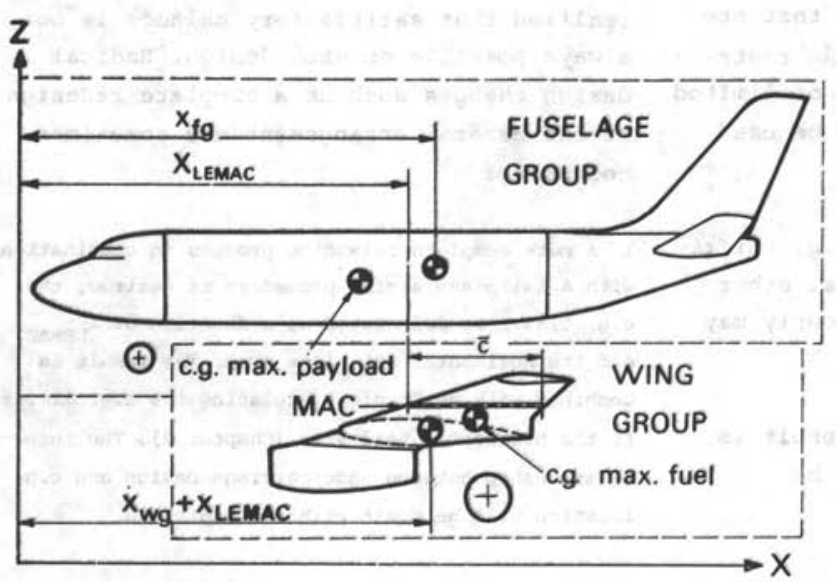

Fig. 8-11. Determination of the wing location

equipped fuselage, several airframe services, fuselage-mounted engines, the horizontal and vertical tailplane, the nosewheel u.c., but not the payload nor the main u.c., as the latter is more or less fixed relative to the $\mathrm{MAC}^{*}$ (assume 10-15\% behind the aft c.g. 1imit or 45-508 MAC approximately for a nosewheel u.c.). 2. The wing group* - parts whose location is fixed relative to the wing: the wing structure, fuel system, main u.c. legs (even if attached to the fuselage) and wing-mounted jet engines. Occasionally, wing-mounted engines should be considered as part of the fuselage group if, for example, the propeller plane has a fixed location next to a cargo compartment (cf. Section 6.4.5.).

3. The (variable) payload.

4. The (variable) fuel load.

Step 2

The fuselage group is drawn and the $\mathrm{X}$-axis usually assumed parallel to the cabinfloor or the propeller axis. Using the data of Section 8.4. and Table 8-15, the c.g. of the complete group is computed in both the $\mathrm{X}$ - and the $\mathrm{z}$-direction.

step 3

The empty wing group is drawn on a separate

"Note that the terms "fuselage group" and "wing group" as used here have different meanings from those in section 8.4.2. (transparent) sheet and the root chord, the tip chord and the MAC* or SMC* are indicated. The c.g. is computed and expressed as a fraction of the MAC relative to the MAC Leading Edge (LEMAC).

Step 4

Assume a reasonable location for the c.g. of the empty aircraft $\left(\mathrm{x}_{\mathrm{OE}}\right)$ relative to the MAC, using statistical data and the considerations presented in Section 8.5.3. Step 5

Calculate the coordinate of the wing leading edge ( $\left.\mathrm{X}_{\text {LEMAC }}\right)$ relative to the fuselage coordinate system:

$x_{\text {LEMAC }}=x_{f g}{ }^{-x_{O E}}+\frac{w_{w g}}{w_{f g}}\left(x_{w g}-x_{O E}\right)$

where "fg" and "wg" respectively denote the fuselage and wing groups.

Step 6

Compute a load and balance diagram of the type illustrated in Fig. 8-7 by considering various possible combinations of payload and fuel loading. The window seating rule (Section 8.5.2.) is applied to passenger transports.

Step 7

Allowing for weight growth, various cabin layouts, fuel capacity growth, Standard Items Variations and design tolerances,

*Defined in Appendix A. 
estimate the fore-and-aft limits that are 1ikely to yield acceptable loading restrictions and a horizontal tailplane of limited size. The data in Table 8-16 may be used for comparison.

\section{Step 8}

In the case of an unacceptable c.g. shift, a revised choice of $x_{O E}$ or several other design revisions discussed previously may improve the situation.

\section{Step 9}

Repeat the procedure until the result is considered satisfactory. It must be realized that satisfactory balance is not always possible on each design. Radical design changes such as a complete redesign of the general arrangement are sometimes necessary.

If a more complete balancing process in combination with a tailplane sizing procedure is desired, the c.g. travel is calculated as a function of $X_{\text {LEMAC }}$ and the horizontal tailplane area. The result is combined with an "X-plot", relating the c.g. limits to the horizontal tail size (Chapter 9). The interrelationship between undercarriage design and c.g. location will be dealt with in Chapter 10 . 


\section{Chapter 9. Preliminary tailplane design}

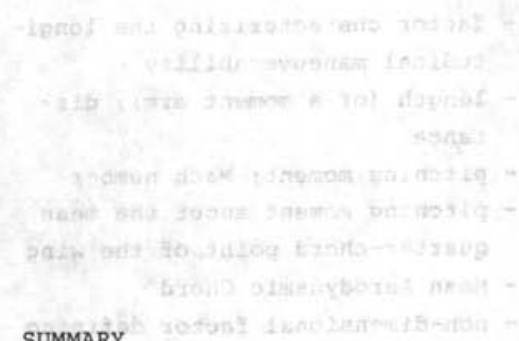

SUMMARY

The aerodynamic design of the tailplane is based on many specific requirements regarding its functions, which are to provide equilibrium in steady flight (trim), to ensure that this condition is stable and that disturbances are well damped, and to generate aerodynamic forces for maneuvering the aircraft. The control forces involved must be acceptable to pilots, with the airplane both in trimmed and out-of-trim conditions.

Design requirements for longitudinal stability and control characteristics - basically those specified in the airworthiness regulations - form the starting point for the derivation of limits to the location of the center of gravity in connection with the size of the horizontal tailplane. Attention is paid to the reduction of control forces by aerodynamic balancing and to some aspects of dynamic behavior, the latter affecting the tailplane design of large aircraft with irreversible control systems. A detailed procedure is presented, together with design data, for obtaining a balance between the required and available c.g. range, and the wing location.

Design criteria, considerations and methods are presented for estimating the minimumsize of the vertical tailplane and the rudder control capacity. Control after failure of an engine on multi-engine transports, directional stability and landings in crosswind are considered as the most pertinent aspects. Design recommendations relevant to light aircraft are made in order to ensure recovery from spins. 
NOMENCLATURE

A

- aspect ratio (no subscript: wing aspect ratio)

a.c. - aerodynamic center

$\mathrm{B}_{\mathrm{p}} \quad$ - number of blades per propeller

$b^{2}$ - span (no index: wing span); width

$\mathrm{C}_{\mathrm{h}}$ - hinge moment coefficient of a control surface = hinge moment/ (local dynamic pressure $x$ control surface area $x$ mean control surface chord)

$\mathrm{C}_{\mathrm{h}} ; \mathrm{C}_{\mathrm{h}_{\delta}}-\partial \mathrm{C}_{\mathrm{h}} / \partial \alpha$ and $\partial \mathrm{C}_{\mathrm{h}} / \partial \delta$, respectively

$\mathrm{C}_{\mathrm{L}}$ - lift coefficient, based on local dynamic pressure and gross area of airfoil

$\mathrm{C}_{\mathrm{L}} \quad$ - lift-curve slope $=\mathrm{aC}_{\mathrm{L}} / \mathrm{d} \alpha$ $\mathrm{C}_{\mathrm{L}_{\alpha}}^{\alpha} ; \mathrm{C}_{\mathrm{L}_{\mathrm{\delta}}}-\mathrm{C}_{\text {tively }} / \partial \alpha_{\mathrm{h}}$ and $\partial \mathrm{C}_{\mathrm{L}_{\mathrm{h}}} / \partial \delta_{\mathrm{e}^{\prime}}$ respec-

$c_{m} \quad-$ pitching moment coefficient, $\mathrm{C}_{\mathrm{m}}=\mathrm{M} /\left(\frac{1}{2} \rho \mathrm{V}^{2} \mathrm{~s} \overline{\mathrm{c}}\right)$

$\mathrm{C}_{\mathrm{m}_{\alpha}} ; \mathrm{C}_{\mathrm{m}_{\dot{\alpha}}}-\partial \mathrm{C}_{\mathrm{m}} / \partial \alpha$ and $\partial \mathrm{C}_{\mathrm{m}} / \partial\left(\frac{\dot{\alpha} \bar{c}}{2 \mathrm{~V}}\right)$

$\tau_{\mathrm{m}}$ - pitching moment coefficient about the aerodynamic center

$c_{m}-\partial c_{m} / \partial\left(\frac{g \bar{c}}{2 V}\right)$

$\mathrm{C}_{\mathrm{N}}$ - normal force coefficient $=$ normal force/(local dynamic pressure $x$ reference area)

$c_{n} \quad-$ yawing moment coefficient $=$ $\mathrm{N} /\left(\frac{1}{2} \rho \mathrm{V}^{2} \mathrm{Sb}\right)$

$\mathrm{C}_{\mathrm{n}_{\beta}} \quad$ - coefficient of directional stability $=\partial C_{n} / \partial \beta$

$c_{y}, C_{y_{v}}$ - side force coefficient $=$ $\mathrm{Y} /\left(\frac{1}{2} \rho \mathrm{V}^{2} \mathrm{~S}\right)$ and $\mathrm{Y}_{\mathrm{V}} /\left(\frac{1}{2} \rho \mathrm{V}_{\mathrm{v}}{ }^{2} \mathrm{~S}_{\mathrm{v}}\right)$, respectively

$c_{y_{v_{\alpha}}} \quad-1 i f t$-curve slope of vertical tailplane $=\partial \mathrm{c}_{\mathrm{yv}_{\mathrm{v}}} / \partial \alpha_{\mathrm{v}}$

$\mathrm{C}_{\frac{1}{2}}$ - cyclic damping of an oscillation $=\mathrm{T}_{\frac{1}{2}} / \mathrm{P}$

$\bar{c}$ - length of the MAC

c.g. - center of gravity

D p - propeller diameter

$\mathrm{F}_{\mathrm{e}}$ - elevator control force, positive forwards (push)

- frequency (sec ${ }^{-1}$ )

f

G

9

h

$\mathbf{I}_{\mathrm{Y}}$ ih 1-horizontal tailplane incidence

$\mathrm{k}_{\mathrm{y}}$ - non-dimensional radius of inertia in pitch, $\mathrm{K}_{\mathrm{y}}^{2}=\mathrm{I}_{\mathrm{y}} \mathrm{g} /\left(\mathrm{wc}^{-2}\right)$

k - correction factor

L $\quad-1 i f t$

$\mathrm{L}_{\alpha} \quad$ - factor characterizing the longitudinal maneuverability

l - length (of a moment arm); distance

- pitching moment; Mach number

- pitching moment about the mean quarter-chord point of the wing

- Mean Aerodynamic Chord

- non-dimensional factor defining the vertical position of the horizontal tailplane

- yawing moment

$\begin{array}{lll}\mathrm{N} & -\mathrm{N} / \partial \mathrm{B}\end{array}$

$\mathrm{N}_{\mathrm{e}}$ - yawing moment caused by asymmetrical engine thrust

$\mathrm{n}$ - normal load factor $=\mathrm{L} / \mathrm{W}$

$\mathrm{n}_{\mathrm{L}} \quad-$ limit load factor

$\mathrm{n}_{\mathrm{z}}$ - normal load factor gradient $=\partial \mathrm{n} / \partial \alpha$

$P$ - engine power; period of an oscillation

$P_{\text {br }} \quad$ - brake horsepower

q - angular pitching velocity; dynamic pressure

$r$ - factor defining the longitudinal position of the horizontal tailplane $=2 l_{\mathrm{h}} / \mathrm{b}$

- area (no subscript: gross wing area)

SMC - Standard Mean Chord

T - thrust

$\mathrm{T}_{\frac{1}{2}}$ - time to half amplitude of an oscillation

$\Delta \mathrm{T}_{\mathrm{e}} \quad$ - asymmetry in the thrust caused by engine failure

$\mathrm{V} \quad$ - (true) airspeed

$V_{\text {a }} \quad$ - approach speed

$\mathrm{V}_{\mathrm{cr}} \quad-$ cruising speed

$V_{F E} \quad-$ design speed with flaps extended (EAS)

$\mathrm{V}_{\max }-\operatorname{maximum}$ level flight speed

$\mathrm{V}_{\mathrm{MC}_{\mathrm{A}}}$ - minimum control speed in the air (EAS)

- rotation speed

- minimum speed in a stall

- trim speed 


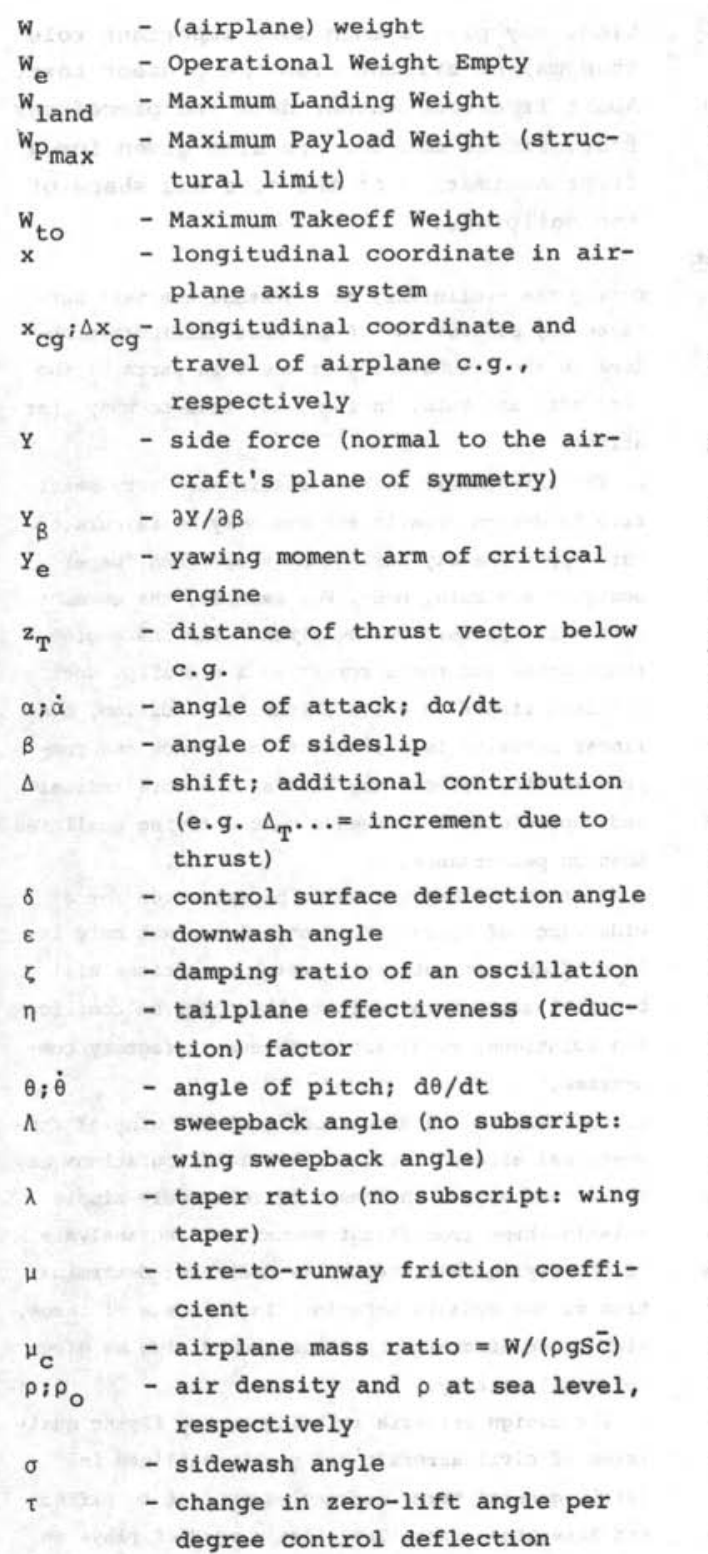

A prime denotes the stick-free condition or a special definition of the vertical tailplane
- angle defining the location of the horizontal tailplane relative to the wing root chord

- angle of bank

- angular frequency of an oscillation

- undamped natural frequency of an oscillation

Subscripts

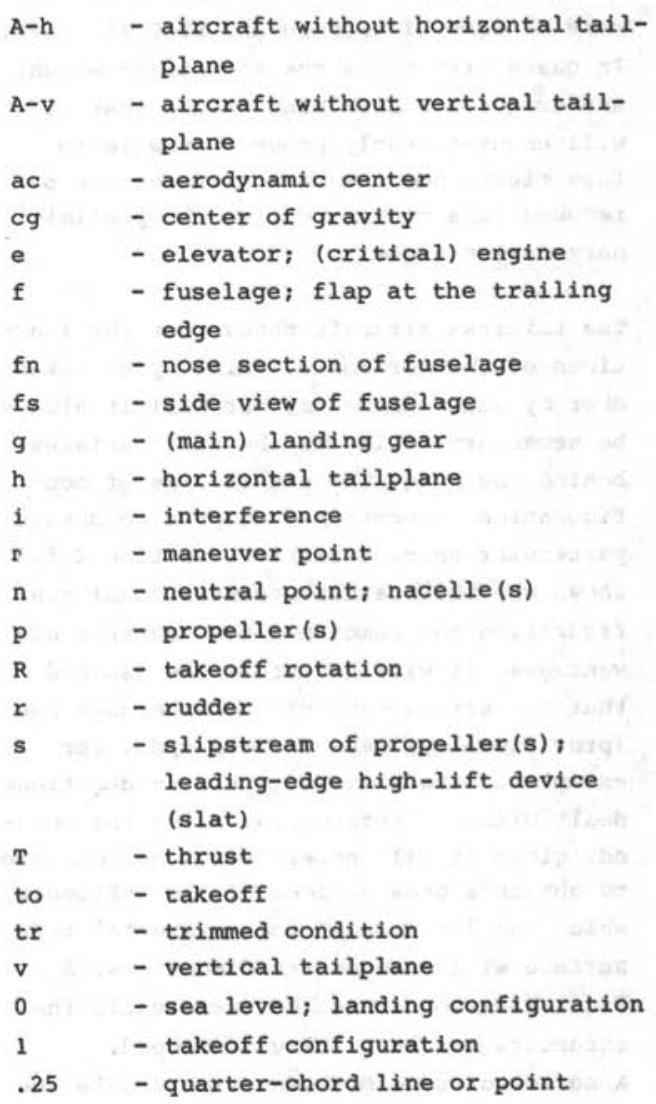

9.1. INTRODUCTION TO TAILPLANE DESIGN, CONTROL SYSTEMS AND STABILIZATION

The functions allotted to the fixed and movable tail surfaces are as follows: a. To ensure equilibrium of moments in steady flight by exercising a force at a given distance from the center of gravity. b. To ensure that this equilibrium is stable, which implies that arter a disturbance the equilibrium is restored and that there is adequate damping for the 
rapid suppression of oscillations.

c. To generate forces for maneuvering the aircraft: rotation during takeoff, control of the flight path, flareout during landing and taxying.

These useful attributes are counteracted to some extent by a large item on the debit side. Although the total tail surface area of small aircraft will not exceed some 25 to 30 percent of the wing area, ratios of 40 to 50 percent are no exception in the case of some high-speed and STOL aircraft. In cases like these the structural weight and drag reach such high values that it will unquestionably prove worthwile to investigate how the tail surfaces can be reduced to a minimum during the preliminary design stage.

The tailless aircraft shows that the functions of the horizontal tail may be taken over by other elements, nor will it always be necessary to locate the tail surfaces behind the wing. The canard type of configuration is sometimes adopted to obtain particular characteristics. Section 2,6.3. shows why this rather unconventional configuration may sometimes have certain advantages. It will henceforth be assumed that the arrangement of the empennage has (provisionally) been decided upon, for example on the basis of the considerations dealt with in Section 2,4. Using the methods given it will nevertheless be possible to obtain a general idea of the influence which the location of the horizontal tail surface will have on its dimensions. A final decision cannot be taken until the structure has been fully developed.

A survey of some methods which enable the designer to size the tail surfaces and determine their principal characteristics will be presented here, taking a number of important design criteria as point of departure. The survey makes no claim to completeness, and the approach recommended is not necessarily representative of that chosen in most design departments. In many cases the knowledge gained through experience, as well as the designer's own intui- tion, may play a much more important role than may be evident from the present text. Apart from some rather detailed procedures fast, simple methods are also given for a first estimation of the size and shape of the tailplane.

During the preliminary design stage the tail surfaces may present one of the most difficult problems in the dimensioning of the main parts of the aircraft, and this, in turn, may lead to many iterations.

a. The aerodynamic characteristics are very sensitive to design details and can only be calculated very approximately during the stage when "paper designs" are being made. For example, the asymetrical flow pattern during flight with one engine inoperative and the aircraft in a sideslip, does not lend itself to calculations. In addition, nonlinear behavior is a frequent occurrence and compressibility effects may have a much more radical and unpredictable influence on the flying qualities than on performance.

b. Most requirements have to pe satisfied for a wide range of operational conditions. Not only is it difficult to anticipate which conditions will be critical, but in addition there may be confilicting solutions, resulting in an unsatisfactory compromise.

c. Contrary to the dimensioning of the wing of conventional aircraft, where reliable calculations may be carried out on the basis of relatively simple relationships from flight mechanics, the analysis of the flying characteristics calls for determination of the dynamic behavior. In the case of large, high-speed aircraft attention should also be given to aeroelasticity.

d. The design criteria relating to the flying qualities of civil aircraft are mostly outlined in fairly general terms and may be subject to different interpretations. The pilot's verdict plays an important part and for this reason a flight simulator is sometimes introduced dưring the design stage.

e. The design of the empennage is closely tied up with that of the surface control system. The distribution of masses and its variations - e.g. center of gravity locations, moments of inertia - for various loading conditions will also have to be known. Proposals for further modifications may 
neverthesess follow from the dimensioning of the tail surface.

Several attempts have been made to combine existing knowledge on aerodynamic characteristics relating to tailplane design into a consistent set of handbook-type methods. The most important of these are the USAF Stability and Control Datcom (Ref. 9-39) and the sheets prepared by the Engineering Sciences Data Unit (ESDU), formerly by the Royal Aeronautical Society (Ref. 9-41). The Royal Aircraft Establishment (RAE) has recently drawn up a computer program based on these procedures (Ref. 9-46). A more concise collection of prediction methods can be found in Appendices $\mathrm{E}$ and $\mathrm{G}$ of the present textbook.

The detailed design of the surface control system is generally not regarded as part of the work of the preliminary design engineer, with the usual exception of light aircraft which have relatively simple mechanical systems. Nevertheless, it is essential to decide how the aerodynamic loads exerted on the movable portion of the tailplane will be felt by the pilot.

a. When manual control is adopted there will be a direct mechanical transmission of forces from the control surfaces to the flight deck controls and back by means of rods and/or cables (reversible system). The aerodynamic forces acting on the control surface will be felt by the pilot directly or indirectly (via tabs), although friction in the system will obscure his perception.

For a given type of control, the stick forces increase with the size, equivalent airspeed and load factor. Methods are required to reduce these forces, e.g. overhanging balance, balancing tabs and spring tabs.

b. Power-assisted controls may be used when the desired control forces and system linearity cannot be satisfactorily obtained by aerodynamic means. The rudder or elevator is operated by means of a pneumatic or hydraulic ram which exerts a multiple of the force applied by the pilot (boost ratio). Control feel will still be provided without excessive control forces.

c. Power(-operated) controls are used on many large and high-speed transports and even on relatively small high-subsonic executive aircraft. In these systems the control surfaces are moved by electrical, hydraulic or pneumatic means without direct physical effort by the pilot. Control feel is obtained by artificial means. A survey of the advantages and disadvantages of the various systems can be found in Ref. 9-6.

The majority of present-day high-speed aircraft are controlled by power-assisted or power-operated control systems, because aerodynamic balancing of the control surfaces demands considerable time in development, while the result is often sensitive to manufacturing tolerances, and modifications are sometimes required during flight evaluation. In the case of very large, fast aircraft with highly sweptback wings and effective flaps, it will not always be possible to achieve satisfactory characteristics for all operational conditions and aircraft configurations. Electronic systems have been developed - and are in operational use - which improve the stability (Stability Augmentation Systems, SAS) and provide more effective response to control wheel movements as compared with the natural behavior of the aircraft. Development work is also being carried out to find out whether a worthwile gain can be obtained from a fully automatic stabi1ization system. An appreciable reduction in tail area may be feasible if the natural "aerodynamic" stability is dispensed with and if the whole aircraft is designed from the outset with this approach in mind (Control Configured Vehicle). The actual gain from such a system will be determined by the tail design requirements which stem from conditions other than stabilization (maneuvering, takeoff rotation and landing flareout) and may thus vary widely for different types of aircraft.

Although these aspects are closely tied up with the design of the tail surfaces, they 
will only be referred to as occasion demands. Ref. 9-11 investigates this subject more fully on the basis of a practical approach.

\subsection{STATIC LONGITUDINAL STABILITY AND ELEVATOR CONTROL FORCES}

A condensed account will be given of the major requirements relating to static longitudinal stability and control and to the influence of tail surface design on these aspects. For more detailed information the reader should consult the relevant literature. For the sake of clarity the derivation of most of the formulas has been omitted since they can be found in practically every textbook on the present subject.

\subsubsection{Stick-fixed static stability and} neutral points

In flight conditions which may persist for any length of time, as well as during the takeoff, approach and landing phases, the aircraft will have to be statically stable with fixed as well as free elevator controls. Under these circumstances a state of equilibrium is achieved by adjusting the elevator or the stabilizer, if the latter is adjustable. The aircraft will possess static longitudinal stability if, from this trimmed state, a disturbance in the angle of attack results in a pitching moment which tends to restore the aircraft to the state of equilibrium. In Fig. 9-1 the trimmed condition is defined by the equilibrium of forces*. Ignoring the contribution of drag forces, we have for the total lift:

* Departing from convention, we have taken lift forces instead of normal forces, since this is acceptable for small angles of attack. The reference line is taken through the leading edge of the MAC (LBMAC), parallel to the fuselage reference line (e.g. the cabin floor)

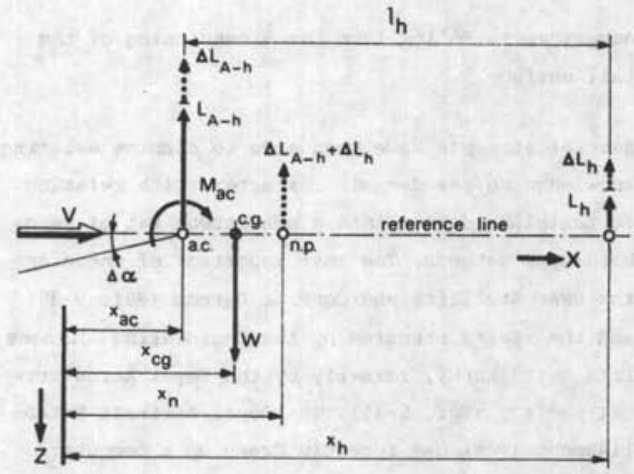

Fig. 9-1. Geometric definitions, forces and moments

$L=L_{A-h}+L_{h}=W$

and the moment about an axis through the aerodynamic center, normal to the $\mathrm{x}-\mathrm{z}$ plane:

$M=M_{a c}+W\left(x_{c g}-x_{a c}\right)-L_{h}{ }_{h}$

or, in nondimensional form:

$\mathrm{c}_{\mathrm{m}}=\mathrm{C}_{\mathrm{m}_{\mathrm{ac}}}+\mathrm{c}_{\mathrm{L}} \frac{\mathrm{x}_{\mathrm{cg}}{ }^{-\mathrm{x}_{\mathrm{ac}}}}{\overline{\mathrm{c}}}-\mathrm{c}_{\mathrm{L}_{\mathrm{h}}} \frac{\mathrm{s}_{\mathrm{h}^{\mathrm{l}} \mathrm{h}}}{\mathrm{s} \overline{\mathrm{c}}}\left(\frac{\mathrm{v}_{\mathrm{h}}}{\mathrm{v}}\right)^{2}$

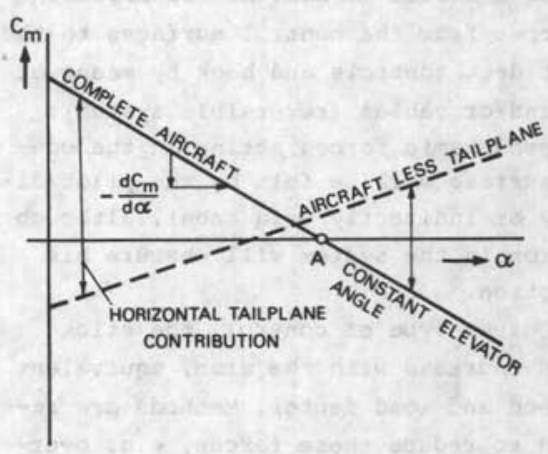

Fig. 9-2. Pitching moment curve and trimmed condition

Fig. 9-2 shows the pitching moment coefficient of the aircraft without the horizontal tail, the tailplane contribution and the aircraft pitching moment coefficient. The trimmed condition is represented by point $A$, where $C_{m}=0$. A disturbance by an 
instantaneous change in the angle of attack $\Delta \alpha$ causes incremental lift forces on the aircraft less the horizontal tail, and the horizontal tailplane, whose resultant acts through the neutral point. By definition the stick-fixed neutral point is the position of the center of gravity for which $\mathrm{dc}_{\mathrm{m}} / \mathrm{d} \alpha=0$, for constant elevator angle. The degree of static stability is normally defined as the distance of the center of gravity ahead of the neutral point, expressed as a fraction of the Mean Aerodynamic Chord (MAC) or the Standard Mean Chord (SMC), referred to as the static margin:

$\frac{\mathrm{x}_{\mathrm{n}}-\mathrm{x}_{\mathrm{cg}}}{\overline{\mathrm{c}}}=-\frac{\mathrm{dc}_{\mathrm{m}}}{\mathrm{dC}_{\mathrm{N}}}=-\frac{\mathrm{dc}_{\mathrm{m}} / \mathrm{d} \alpha}{\mathrm{dC}_{\mathrm{N}} / \mathrm{d \alpha}} \approx-\frac{\mathrm{dc}_{\mathrm{m}} / \mathrm{d} \alpha}{\mathrm{dc}_{\mathrm{L}} / \mathrm{d \alpha}}$

The condition for static stability is $\mathrm{dc}_{\mathrm{m}} / \mathrm{d} \alpha<0$ and the center of gravity must therefore be in front of the neutral point to achieve this.

It can be shown that the neutral point is related to the aerodynamic center of the aircraft, less tail, as follows:

$\frac{x_{n}-x_{a c}}{\bar{c}}=\frac{c_{L_{h}}}{C_{L_{\alpha}}}\left(1-\frac{d \varepsilon}{d \alpha}\right) \frac{s_{h}{ }^{l} h}{s \bar{c}}\left(\frac{v_{h}}{v}\right)^{2}$

where

$C_{L_{\alpha}}=\left(C_{L_{\alpha}}\right)_{A-h}+C_{L_{h}} \frac{S_{h}}{S}\left(1-\frac{d \varepsilon}{d \alpha}\right)\left(\frac{V_{h}}{V}\right)^{2}$

This expression is valid only in the absence of compressibility effects and effects due to powerplant operation. Some additional details will be given in Section 9.2.5.

A simple criterion for the size of the horizontal tail may be obtained by assuming that the stick-fixed static margin should at least have a certain specified minimum value, from which a condition for the aft c.g. location is found:

$\frac{x_{c g}}{\bar{c}}=\frac{x_{a c}}{\bar{c}}+\frac{x_{n}-x_{a c}}{\bar{c}}-\frac{x_{n}-x_{c g}}{\bar{c}}$

The aft c.g. location is depicted in Fig. 9-3 as a function of the horizontal tail

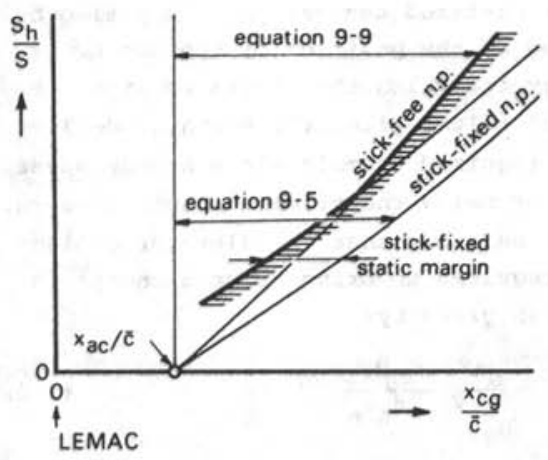

Fig. 9-3. Stick-fixed and stick-free neutral points and aft c.g, limits vs. tail volume

size.

The aerodynamic center (aircraft less tail) is mainly determined by the wing shape, but important contributions due to the fuselage and nacelles must be taken into account. Appendix $\mathrm{E}$ provides methods and data for estimating its location. The term $x_{n}-x_{a c}$ in (9-7) is obtained from $(9-5)$ and $(9-6)$, for which the required coefficients can be obtained from Appendix E.

A minimum required value of 5 percent of $\bar{c}$ is frequently used for $x_{n}-x_{c g}$, but it should be realized that for most aircraft the effects of compressibility and power result in large variations. In addition, an analysis of static margins at the aft c.g. of existing aircraft shows a considerable spread, which must be attributed to the following points.

a. The stick-fixed static margin is not really a significant criterion, but rather an outcome of the tail sizing process according to other criteria (stick forces, dynamic stability, etc.).

b. The use of the MAC in static stability considerations is arbitrary. It has been found that aircraft with a relatively small MAC - or a large value of $l_{h} / \bar{c}-$ have large static margins. A better result would be obtained by relating the static margin to the tail moment arm $l_{h}$, which is more significant for c.g. shifts due to different loading conditions. A static margin of 1.5 to 2 percent of $l_{h}$ at the aft c.g. appears to be a good assumption, if, in spite of the previous objections, the tail size is based on static stability. 
The stick-fixed neutral point may also be defined as the position of the center of gravity for which the displacement of the control column (i.e. the elevator deflection) required to maintain a steady speed, above or below the trimmed speed, is zero. It can be shown that the elevator deflection required to bring about a change in speed is given by:

$\frac{d \delta}{d V}=\frac{2 C_{L}\left(x_{n}-x_{c g}\right) s}{c_{L_{h_{\delta}}} v_{t r} S_{h} l_{h}}$

where $v_{t r}$ is the speed in trimmed flight. The elevator and stick movements required to change the trimmed speed by a small amount are thus proportional to the stickfixed static margin. Since the pilot regards the stick forces as more important than the control displacements, no limits are imposed on $\mathrm{d} \delta \mathrm{e}_{\mathrm{e}} / \mathrm{dV}$.

\subsubsection{Stick-free static stability and} neutral point; the stick force gradient

In the case of a manual (reversible) control system the free elevator will assume a position where the aerodynamic hinge moment will be zero, provided any friction in the system is neglected. This will result in a different position for each angle of attack, and consequently the stabilizing action of the tail surface will be changed. There is an analogy with the case where the controls are fixed, so for the stick-free neutral point we have:

$\frac{x_{n}{ }^{\prime}-x_{a c}}{\bar{c}}=\frac{c_{L_{h}}}{c_{L_{\alpha}^{\prime}}}\left(1-\frac{d \varepsilon}{d \alpha}\right) \frac{s_{h^{l} h}}{s \bar{c}}\left(\frac{v_{h}}{V}\right)^{2}$

where

$c_{L_{h_{\alpha}}}^{\prime}=c_{L_{h_{\alpha}}}-c_{L_{h_{\delta}}} \frac{c_{h_{\alpha}}}{c_{h_{\delta}}}$

and

$C_{L_{\alpha}}^{\prime}=\left(C_{L_{\alpha}}\right)_{A-h}+C_{L_{h}}, \frac{S_{h}}{S}\left(1-\frac{d \varepsilon}{d \alpha}\right)$
Hence the stick-free stability is identical to the stick-fixed stability, except. that the tailplane lift gradient is effectively reduced by the factor $\mathrm{C}_{\mathrm{L}_{\mathrm{h}}} \mathrm{C}_{\mathrm{h}_{\alpha}} / \mathrm{C}_{\mathrm{h}_{\delta}}$. Since for a normally balanced elevator $\mathrm{C}_{\mathrm{h}_{\delta}}<0$ and $\mathrm{C}_{\mathrm{L}_{\mathrm{h}_{\delta}}}$ is always positive, the sign of $c_{h_{\alpha}}$ will decide whether or not the stability will be increased when the control is left free. If $\mathrm{C}_{\alpha}>0$ the control will trail against the wind, stability will be increased and the stick-free neutral point will be further back than it would be with the stick fixed. If $\mathrm{c}_{\mathrm{h}_{\alpha}}<0$ the control trails with the wind and the stick-free stability is less than in the stick-fixed situation.

It can be shown'that the stick-free stability is related to the stick force gradient, i,e, the change in stick force required for a small change in forward speed is given by the following relationship:

$\frac{d F_{e}}{d V}=-2 \frac{W}{v_{t r}} \frac{S_{e} \bar{c}_{e}}{S_{h^{l} h}} \frac{\bar{c} G c_{h_{\delta}}}{C_{L_{h_{\delta}}}} \frac{x_{n}{ }^{\prime}-x_{c g}}{\bar{c}} \quad(9-12)$ where the stick gearing $G$ is defined as the ratio of the elevator rotation to the control column displacement. Normal values of $\mathrm{G}$ will be .6 to .9 rads $/ \mathrm{ft}(2$ to 3 rads $/ \mathrm{m}$ ).

It can be seen that the stick force gradient is positive if the pilot has to push the control dolumn forward $\left(\mathrm{dF}_{\mathrm{e}}>0\right)$ to increase the flight speed from a trimmed condition $(\mathrm{dV}>0)$.

For transport aircraft the FAR 25.173 regulation stipulates that for all weights between the MLW and the MTOW the stick force gradient must amount to at least one lb per six knots $(.15 \mathrm{~kg}$ per $\mathrm{m} / \mathrm{s})$ of speed increment from the trimmed speed. Assuming that the elevator span is equal to that of the horizontal stabilizer, we may assume:

$\frac{\mathrm{C}_{\mathrm{L}_{\mathrm{h}}}}{\mathrm{C}_{\mathrm{L}_{\mathrm{h}}}} \approx V \frac{\overline{\mathrm{S}_{\mathrm{e}}}}{\mathrm{S}_{\mathrm{h}}}$ 
Substituting this in $(9-12)$, we obtain the following condition for the stick-free static margin:

$\frac{x_{n}{ }^{\prime}-x_{c g}}{\bar{c}} \geqslant-\frac{\left(d F_{e} / d V\right)_{\min } V_{\max } \sqrt{A_{h}} \ell_{h} c_{L_{h}}}{2 w_{\text {land }} \sqrt{S_{h}}\left(S_{e} / S_{h}\right)^{3 / 2} \bar{c} G C_{h_{\delta}}}$

where the maximum level flight speed and the MLW are assumed to form the most demanding situation. The aft c.g. limit corresponding to $(9-14)$ has been plotted in Fig. 9-4.

Another requirement is formulated as a 1imitation of the control force (FAR 25.175) within a limited region of approach speeds (1.1 $\mathrm{v}_{\mathrm{S}_{\mathrm{O}}}<\mathrm{V}<1.8 \mathrm{v}_{\mathrm{S}_{\mathrm{O}}}$ ). This should not exceed $80 \mathrm{lb}(36 \mathrm{~kg})$ at a trimmed speed of $1.4 \mathrm{~V}_{\mathrm{S}_{0}}$. Then, by approximation, we have: $\frac{d P_{e}}{d\left(V / v_{S_{0}}\right)} \leqslant 2001 \mathrm{~b}(91 \mathrm{~kg})$

This requirement is equivalent to a forward limit of the c.g. position:

$\frac{x_{n}{ }^{\prime}-x_{c g}}{\bar{c}}<-.7\left[\frac{d F_{e}}{d\left(v / v_{s_{0}}\right)}\right]_{\max } x$

$$
\frac{\sqrt{\mathrm{A}_{h}} e_{h} C_{L_{h}}}{\mathrm{w}_{\text {land }} \sqrt{\mathrm{S}_{\mathrm{h}}}\left(\mathrm{S}_{\mathrm{e}} / \mathrm{S}_{\mathrm{h}}\right)^{3 / 2} \overline{\mathrm{c}} \mathrm{GC} \mathrm{h}_{\delta}}
$$

This requirement is generally less critical than the limits imposed by the maximum permissible stick force per $g$, which will be dealt with in the following section.

9.2.3. Stick-fixed and stick-free maneuver points and maneuver control forces

The maneuver stability of a conventional aircraft must satisfy the condition:

$\frac{d \delta}{d n}<0$

which implies that, in order to pull the aircraft out of a dive at a steady speed, the pilot must pull the control column towards him. The maneuver stability is made up of two components, one resulting from the change in incidence involved and one from the angular rotation in pitch as the aircraft follows a curved flight path. The stick-fixed maneuver point is defined as the position of the center of gravity when the stick displacement per $g$ is zero. This point is related to the stick-fixed neutral point by the following relationship:

$\frac{x_{m}-x_{n}}{\bar{c}}=-\frac{c_{m_{q}} \rho g s \bar{c}}{4 W}$

where the stability derivative $\mathrm{C}_{\mathrm{m}_{\mathrm{q}}}$,

$c_{m_{q}}=\frac{\partial c_{m}}{\partial\left(\frac{q \bar{c}}{2 v}\right)}$

represents the aerodynamic damping in pitch, primarily due to the change in horizontal tailplane angle of attack. The following equation can be used to estimate the stick-fixed maneuver point:

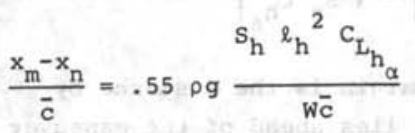

This equation shows that the stick-fixed maneuver point lies behind the stick-fixed neutral point (Fig. 9-4) and that the dis-

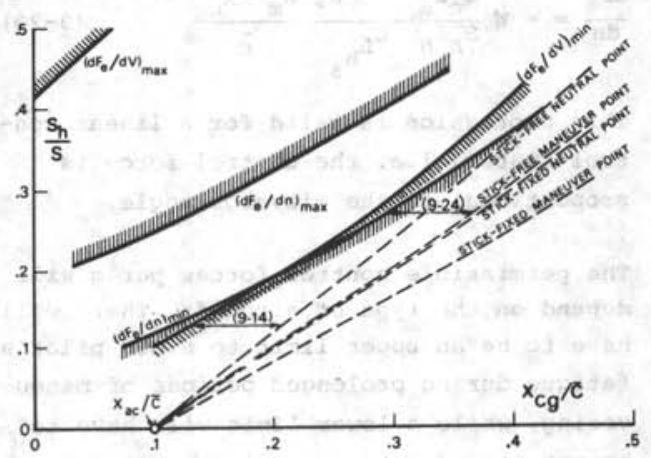

Fig. 9-4. Limitations for the horizontal tail area and c.g. position for aircraft with manual control, based on stick force requirements 
tance between the two points decreases with altitude.

There are no civil requirements which stipulate limits regarding $d \hat{f} e^{/ d n}$. However, as will be seen from section 9.3.2., the maneuver stability is important in relation to the longitudinal short period oscillatory response of an aircraft to control movements.

The stick-free maneuver point is defined as the position of the center of gravity with free elevator control - for which the stick force per $g$ is zero in the case of a steady pull-out of an airplane, trimmed for level flight at the same speed. Again using $(9-13)$, the location of this point can be related to the stick-free neutral point:

$\frac{x_{m}{ }^{\prime}-x_{n}{ }^{\prime}}{\bar{c}}=.55 \rho g \frac{s_{h^{l} h^{2}} c_{L_{h}}{ }^{\prime}{ }^{\bar{c}}}{w^{\prime}}$

where

$\left.c_{L_{h_{\alpha}}}=c_{L_{h_{\alpha}}} \mid 1-1 \frac{\overline{s_{e}}}{s_{h}} \frac{c_{h_{\alpha}}}{c_{h_{\delta}}}\right\}$

The maneuver margin is the distance by which the c.g. lies ahead of the maneuver point, and it represents a direct measure of the stick force per $g$ :

$\frac{d F_{e}}{d n}=-w \frac{s_{e} \bar{c}_{e}}{s_{h^{l} h}} \frac{\bar{c} G c_{h_{\delta}}}{C_{L_{h_{\delta}}}} \frac{x_{m}^{\prime}-x_{c g}}{\bar{c}}$

This expression is valid for a linear control system, i.e. the control force is proportional to the elevator angle.

The permissible control forces per $g$ will depend on the type of aircraft. There will have to be an upper limit to avoid pilot's fatigue during prolonged periods of maneuvering, while a lower limit will have to be set in order to prevent excessive stresses-in the structure and over-sensitivity in rough air. The limits taken from the military requirement MIL-F-8785 (Ref. 9-18) may also be usefully applied to

\begin{tabular}{|c|c|c|c|}
\hline if & Unit & Control theel & Control seick \\
\hline Maximun $d F_{e} / d n$ & $1 \mathrm{~b} / \mathrm{s}$ & $\begin{array}{l}\frac{120}{n_{L}-1} \text { but } \leqslant 120 \\
\frac{54.6}{n_{L}-1} \text { but } 654.4\end{array}$ & $\frac{\frac{56}{a_{L}-1} \text { but } 628}{\frac{25.6}{n_{L}-1} \text { but } 612.7}$ \\
\hline Mininus $d F_{e} / d n$ & $1 \mathrm{~b} / \mathrm{g}$ & $\begin{array}{l}\frac{45}{n_{L}-1} \text { but } 26 \\
\frac{20.4}{n_{L}-1} \text { but } 22.7\end{array}$ & $\begin{array}{l}\frac{21}{n_{L}-1} \text { but } 23 \\
\frac{9.5}{n_{L}-1} \text { but } \geqslant 1.4\end{array}$ \\
\hline
\end{tabular}

Table 9-1. Longitudinal stick force limits according to MIL-F-8785 B (ASG), Ref. 9-18

transport aircraft; seeitiable 9-1. The factor $\mathrm{n}_{\mathrm{L}}$ in this table is the limit load factor, specified in the airworthiness regulations for maneuver loads. For transport aircraft weighing more than $50,000 \mathrm{lb}$ $(22,680 \mathrm{~kg})$ this load factor is equal to 2.5 , while for lighter aircraft it is a function of the weight.

The control force limits may be used to supply corresponding limits to the stickfree maneuver margin. Substituting (9-13) into (9-22), and rearranging, we obtain:

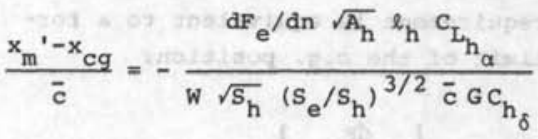

Flight at sea level will be the deciding case for the forward limit, while the aft. limit will be determined by flight at high altitude. An example of the permissible range of c.g. positions is shown in Fig. 9-4 as a function of the relative tailplane area.

The range of allowable c.g. locations for transport aircraft with manual control systems should not be unduly limited by the permissible stick forces per g. In particular, the forward limit is determined by considerations of controllability in the lowspeed configuration (Section 9.4.). If, therefore, we assume that for this c.g. limit the stick force per $g$ reaches its maximum value, we may derive a condition for the aft c.g. limit from the value of minimum stick force per $g$ by subtracting the maximum and minimum values obtained for $(9-23)$ within the flight envelope, with the following result for 
the aft c.g. limit:

$$
\frac{x_{\mathrm{cg}}}{\bar{c}}=\frac{x_{m}^{\prime}}{\bar{c}}-.6 \frac{\Delta x_{c g}}{\bar{c}}-.88 \frac{\left(\rho_{0}-\rho_{\min }\right) g_{h_{h}{ }^{2}{ }^{2} c_{L_{h}}{ }^{\prime}}^{w \bar{c}}}{w}
$$

where $x_{m}$ ' is the stick-free maneuver point at sea level. The forward limit is located at a distance $\Delta x_{c g}$ ahead of the aft 1 imit.

Equation 9-25 shows clearly that, in order to obtain acceptable maneuvering stick forces, the aft c.g. limit should be forward of the stick-free maneuver point by an amount which is directly related to the c.g. travel. For aircraft with a large c.g. travel we conclude that the maneuver margin at the aft c.g. will also be relatively large, and this is one of the reasons why a generalized minimum for the static margin is not relevant, as already stated in section 9.2 .1 .

\subsubsection{Reduction of control forces}

The control forces for a manual control system are dependent on the hinge moment coefficients $c_{h_{\alpha}}$ and $c_{h_{\delta}}$ and the amount of stick-free stability provided by the aircraft. For given values of $c_{h_{\alpha}}$ and $\mathrm{C}_{\mathrm{h}_{6}}$ the stick force per $\mathrm{g}$ increases proportionally to the All-Up Weight of the aircraft and the linear dimensions, as shown by (9-23), although even in the case of light aircraft it will be necessary to reduce the control forces. Incidentally, aerodynamic balancing is applied not only to manually controlled surfaces, but also to power-assisted or power-operated surfaces, in order to reduce the power requirements.

Various methods of balancing are available, but the reduction of $\mathrm{c}_{\mathrm{h}_{\delta}}$, for example, must not be carried too far, because if $c_{h_{\delta}}$ becomes positive the control surface is overbalanced. The degree of control force reduction is limited by non-linearities in the control force/deflection relationship, by the variation in characteristics between aircraft of the same production batch due to production imperfections, and by small changes in the shape of the control surface due to the formation of ice.
In addition, certain tab systems exhibit a slow response to the pilot's commands.

The hinge moment coefficient $\mathrm{c}_{\mathrm{h}_{\delta}}$ - the control "heaviness" parameter - is of primary importance in reducing the control forces. In order to get some idea of the reduction in $\mathrm{C}_{\mathrm{h}_{\delta}}$ required to meet the stick force requirements for various airplane sizes, it may be useful to impose the condition that the maximum and minimum permitted values of the stick force per $g$ should be obtained at the forward and aft c.g. limit, respectively. The corresponding value of $\mathrm{C}_{\mathrm{h}_{\delta}}$ is obtained from $(9-23)$ by ignorjing the effect of altitude variation on the stick-free maneuver point:

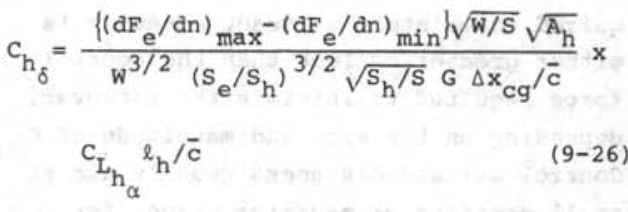

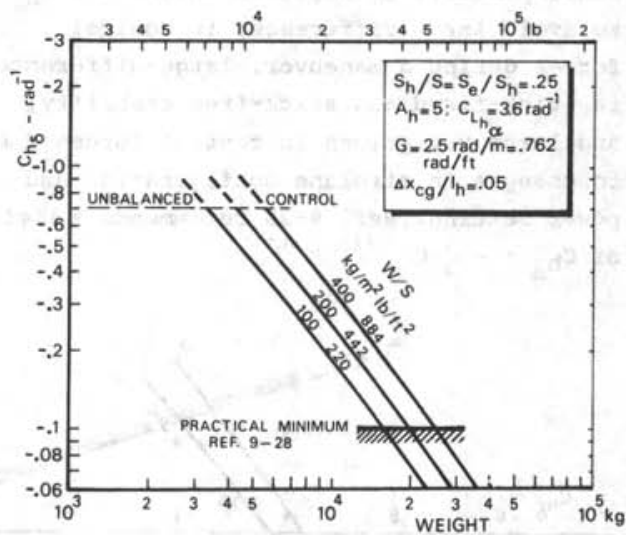

Fig. 9-5. Typical hinge moment coefficient required to obtain maximum and minimum stick force per $g$ at forward and aft c.g. limit for transport aircraft with control wheels

This result has been plotted in Fig. 9-5 for a typical combination of parameters vs. the All-Up Weight of transport aircraft, for various wing loadings. The figure suggests that for weights in excess of about 
$50,000 \mathrm{lb}(23,000 \mathrm{~kg})$ the reduction of $\mathrm{C}_{\mathrm{h}_{\delta}}$ becomes problematic in view of the minimum value recommended in Ref. 9-28. However, various servo-tab and spring-tab systems are available, which may extend the range of airplane sizes for which manual control can be applied up to the Douglas DC -8 class of high-speed transports, though this will entail an increasing number of control design problems.

The hinge coefficient $\mathrm{C}_{\mathrm{h}_{\alpha}}$ - the control "floating" parameter - is decisive for the change in stick force resulting from the response of the aircraft to the control movement. When the aircraft responds to the elevator deflection, its angle of attack is changed and the control force required to maintain a steady maneuver is either greater or less than the control force required to initiate the maneuver, depending on the sign and magnitude of $c_{h_{\alpha}}$. Control surface designers usually aim at small positive or negative values for $c_{h_{\alpha}}$ to avoid large differences in control forces during a maneuver, large differences in stick-fixed and stick-free stability, and large variations in control forces due to changes in airplane configuration and power setting. Ref. 9-28 recommends a limit of $c_{h_{\alpha}}<-\frac{1}{3} c_{h_{\delta}}$.
Various methods for obtaining satisfactory values of $c_{h_{\delta}}$ and $c_{h_{\alpha}}$ will now be reviewed briefly (see Figs. $9-6$ and 9-7). Methods for estimating these coefficients can be found in Refs. 9-24 and 9-41, but high prediction accuracies must not be expected. THE PLAIN (UNBALANCED) CONTROL SURFACE has an increase in linear lift with deflections of up to 10 to 15 degrees. It has values of $c_{h_{\delta}}$ and $c_{h_{\alpha}}$ that are too large to give acceptable forces on most aircraft. OVERHANGING (OR SET-BACK HINGE) BALANCE

- A blunt-nose balance has a greater effect on hinge moments than a sharp-nose balance, but it may produce non-linear characteristics at relatively small deflections. Unsealing the gap between the control and the airfoil tends to increase the balance.

- Small values of $\mathrm{c}_{\mathrm{h}_{\alpha}}$ cannot always be obtained without overbalancing.

- Set-back hinge balance is commonly used on aircraft with manual controls, sometimes in combination with other methods. SEALED INTERNAL BALANCE

- It is structurally complicated to install a flexible, airtight seal and to provide enough deflection without the balance interfering with the inside part of the fixed structure.

- Balancing effectiveness is comparable to

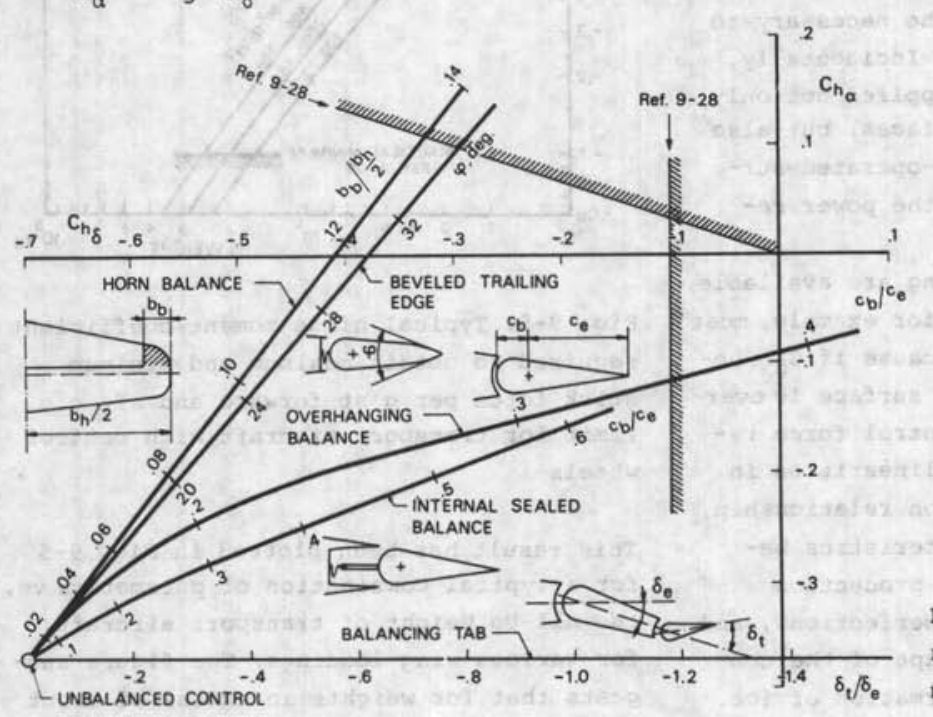

Fig. 9-6. Effect of aerodynamic balance on hinge moment coefficient (example) 


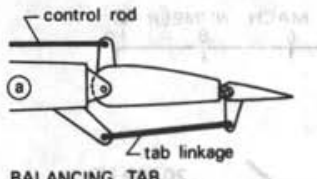

BALANCING TAB

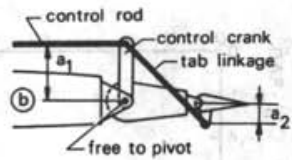

SERVO TAB

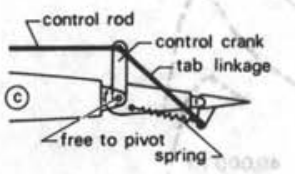

SPRING TABS

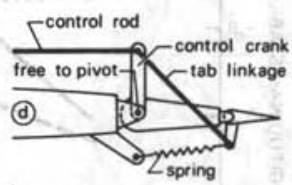

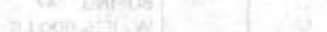

Fig. 9-7. Mechanical principle of tab control systems

the overhang balance, except that nonlinearities do not occur. HORN BALANCE

- Balance weight and aerodynamic loads are not evenly distributed in spanwise direction, and this may give rise to torsion loads in the control surface.

- For an unshielded horn balance the effect of balancing on $\mathrm{C}_{\mathrm{h}_{\alpha}}$ is large. This may give rise to an unfavorable relation between $\mathrm{C}_{\mathrm{h}_{\delta}}$ and $C_{h_{\alpha}}$. This effect is less for a shielded horn balance.

- The formation of ice on an unshielded horn may give rise to control problems. BEVELED TRAILING EDGE BALANCE

- The characteristics are nonlinear, un-

less the control gap is sealed.

- It may be difficult to reproduce the specified trailing-edge angle in the production stage.

BALANCING (OR GEARED) TAB (Fig. 9-7a)

- On deflection of the control surface the tab is geared to move in the opposite direction, thus producing an aerodynamic moment about the hinge line, which assists the pilot in moving the control.

- A balancing tab has the effect of reducing the value of $\mathrm{C}_{\mathrm{h}_{\delta}}$ without appreciably changing $c_{h_{\alpha}}$, since the airfoil shape is not varied, except when the control surface is deflected. This type of balance may thus be used in conjunction with other methods to adjust $c_{h_{\delta}}$ while leaving $c_{h_{\alpha}}$ unchanged.

- A tab chord of 20 percent of the control chord gives the least reduction in control effectiveness due to the action of the tab. - The control forces may, if so desired, be increased by using an anti-balance tab, 1.e. a tab which is geared to move in the same direction as the control surface.

- For a given tab chord/control chord ratio the amount of $c_{h \delta}$ reduction is determined by the fixed ratio of the tab and control deflection angles. This is usually made adjustable in the design stage.

- The balancing tab is frequently used as a trim tab.

THE SERVO TAB (Fig. 9-7b)

- The pilot's control is connected directly to the tab, while there is no rigid connection with the control surface. The control force depends on the hinge moments of both the control surface and the tab to an extent determined by the ratio $a_{1} / a_{2}$. For small $a_{1} / a_{2}$ very low stick forces are obtained.

- For low $a_{1} / a_{2}$ ratios the control is easily overbalanced as the tab is in the boundary layer of the stabilizer and the control.

- Control effectiveness at low speeds may be inadequate and the servo tab is easily overbalanced at the stall. It is mainly for these reasons that the servo tab is not used on many modern aircraft. THE SPRING TAB (Fig. 9-7c and d)

- The principle is basically the same as that of the servo tab, but in addition there is a spring connecting the tab with the fixed airfoil or the main control surface.

- At high speeds the tab carries a large control force in relation to the spring force. The action of the spring tab is then comparable to that of the servo tab. - At low speeds the spring force is large in relation to the 1 ift on the tab and system c. behaves like a plain control, while system d. acts more or less like a geared tab.

- The effective $c_{h_{0}}$ decreases with EAS in a way which is mainly determined by $a_{1} / a_{2}$ and the spring stiffness. This causes the control forces to vary only moderately with 
speed.

- The control forces are relatively independent of the $c_{h_{\alpha}}$ and $c_{h_{\delta}}$ values of both surfaces.

- The spring tab system cannot be overbalanced even at the stall, since the tab deflects only when there is a load on the control surface.

- Spring tabs may be pre-loaded to prevent them from coming into operation for small stick forces, in order to keep the spring tab out of action at low speeds.

- Practical spring tab systems are structurally much more complicated than Fig. 9-7 might suggest.

- The response to control commands is slow in view of the floating action of the main control surface.

\subsubsection{Effects of compressibility and} powerplant operation

When a condition of equilibrium is disturbed, resulting in variations in the airspeed, attention must be paid to the fact that compressibility and engine operation will make the aerodynamic coefficients dependent on the flying speed. Both effects are of a highly complex nature and will be discussed only very briefly. The effects of aeroelasticity on stability will not be dealt with here, but these are quite important in the case of large, highspeed aircraft.

a. Compressibility.

In making an assessment of the influence of the Mach number on the various factors contributing to stability, it will be noted that at subcritical speeds - i.e. where there are no shock waves - the neutral point will generally move forward with increasing M. Fig. $9-8$ shows that $c_{m_{\alpha}}=c_{m} / \partial \alpha$ becomes less negative, which implies that the subcritical compressibility has a destabilizing effect. The available methods of analysis give only a generalized influence of Mach effects on $\mathrm{C}_{\mathrm{m}_{\alpha}}$ and, in addition, a term $\partial \mathrm{C}_{\mathrm{m}} / \partial \mathrm{M}$ must be added to the stability equation.

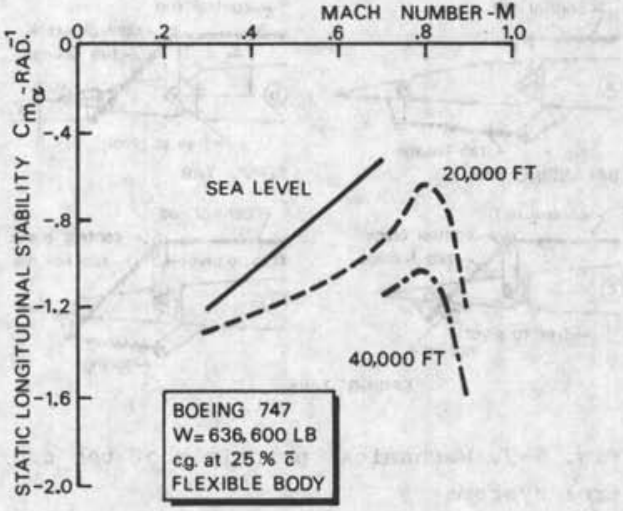

Fig. 9-8. Effects of compressibility and airplane flexibility on static stability

At Mach numbers above the lift-divergence condition, $C_{L_{\alpha}}$ decreases with $M$ and $\partial C_{L} / \partial M$ becomes negative. A shock wave which first appears on the inboard wing causes a rearward shift of the neutral point and the lift loss causes a reduction in the downwash at the tailplane. The result of these phenomena may be a large nose-down pitching moment which will force the aircraft into a high-speed dive from which recovery is very difficult ("tuck under"). Most high-subsonic aircraft have a Mach trimmer incorporated in the autopilot to cure this misbehavior.

b. Engine operation: propeller aircraft. The direct effects are:

1. A force normal to the flow, a component of which is acting in the plane of the propeller if it is at an angle of attack to the flow. For a propeller ahead of the c.g. the effect will be destabilizing. In Appendix E, Section E-8 this contribution is referred to as $\Delta_{p} x_{a c}$.

2. If the c.g. is above the thrustline of the propellers, the thrust will supply a moment which will vary with the airspeed, resulting in a destabilizing effect which will become greater with increasing $C_{L}$. A stabilizing effect will be obtained when the c.g. lies below the thrust-line; an example is shown in Fig. 6-15. 
Indirect effects are:

1. A shift in the a.c. of the wing.

2. An increase in downwash resulting from an increase in the circulation over the wing, which has a destabilizing effect.

3. Increased dynamic pressure behind the propeller. Its influence on stability will depend on the tail load and the location of the tailplane in relation to the propeller slipstream, and may have either a stabilizing or a destabilizing influence. On the whole, engine operation will generally have a destabilizing effect and will be greatest for relatively high power outputs and low flying speeds during takeoff and aborted landings.

Ref. 9-34 gives a relatively simple method for taking account of slipstream effects on the location of the neutral point. This method may be approximated by increasing the downwash term in $(9-5)$ and $(9-6)$ relative to the power-off condition by an amount:

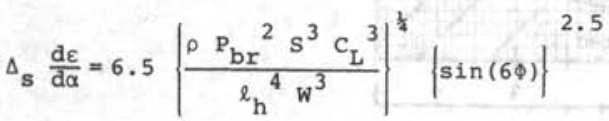

where $\mathrm{P}_{b r}$ is the shaft horsepower of one engine and $\phi$ defines the position of the horizontal tailplane relative to the wing (see Fig. E-13 and Fig. 2-24):

$\Phi=\sin ^{-1} \frac{m}{r}$

Equation 9-27 is valid for $0^{\circ}<\Phi<30^{\circ}$, while $\Delta_{S} d \varepsilon / d \alpha$ may be assumed zero outside this range.

c. Engine operation: jet aircraft. Direct effects:

1. Normal force on the engine inlet which will have a destabilizing effect when it is ahead of the c.g. and a stabilizing effect when it is aft of the c.g.

2. As in the case of propeller aircraft there will be a destabilizing factor when the c.g. lies at a distance $z_{T}$ above the line of thrust. This effect may be regarded as a forward shift of the neutral point in the case of variations in the airspeed with constant thrust:

$\frac{\Delta_{T} x_{a c}}{\bar{c}}=-\sum \frac{T}{w} \frac{z_{T}}{\bar{c}}$

where the summation is made for all operating engines.

An indirect effect of engine operation on jet aircraft is an increase in downwash when the exhaust efflux passes a relatively short distance below the tail.

Engine operation will have a destabilizing effect on aircraft with engines mounted below the wing.

\subsection{SOME ASPECTS OF DYNAMIC BEHAVIOR}

When dealing with large high-speed aircraft, cruising at high altitudes and equipped with effective flaps for takeoff and landing, it will be desirable to know some of the dynamic characteristics at an early design stage, since these will generally be the deciding factors for the size of the tailplane. Only a condensed account of the dynamic stability with fixed controls will be given here. The overall dynamic longitudinal motion of an aircraft can generally be broken down into the short period (SP) oscillation and the phugoid.

\subsubsection{Characteristics of the SP oscilla-} tion

The SP oscillation is a periodic motion which takes place at nearly constant speed and is subject to both angular and normal accelerations. This motion is generally highly damped and stable when the c.g. lies ahead of the stick-fixed neutral point. It is not so much the stability which matters, but rather the character of the oscillation which is defined by the following parameters.

The PERIOD P is generally of the order of a few seconds, but for large aircraft it may amount to some ten seconds. The period increases with a rearward shift of the c.g. 


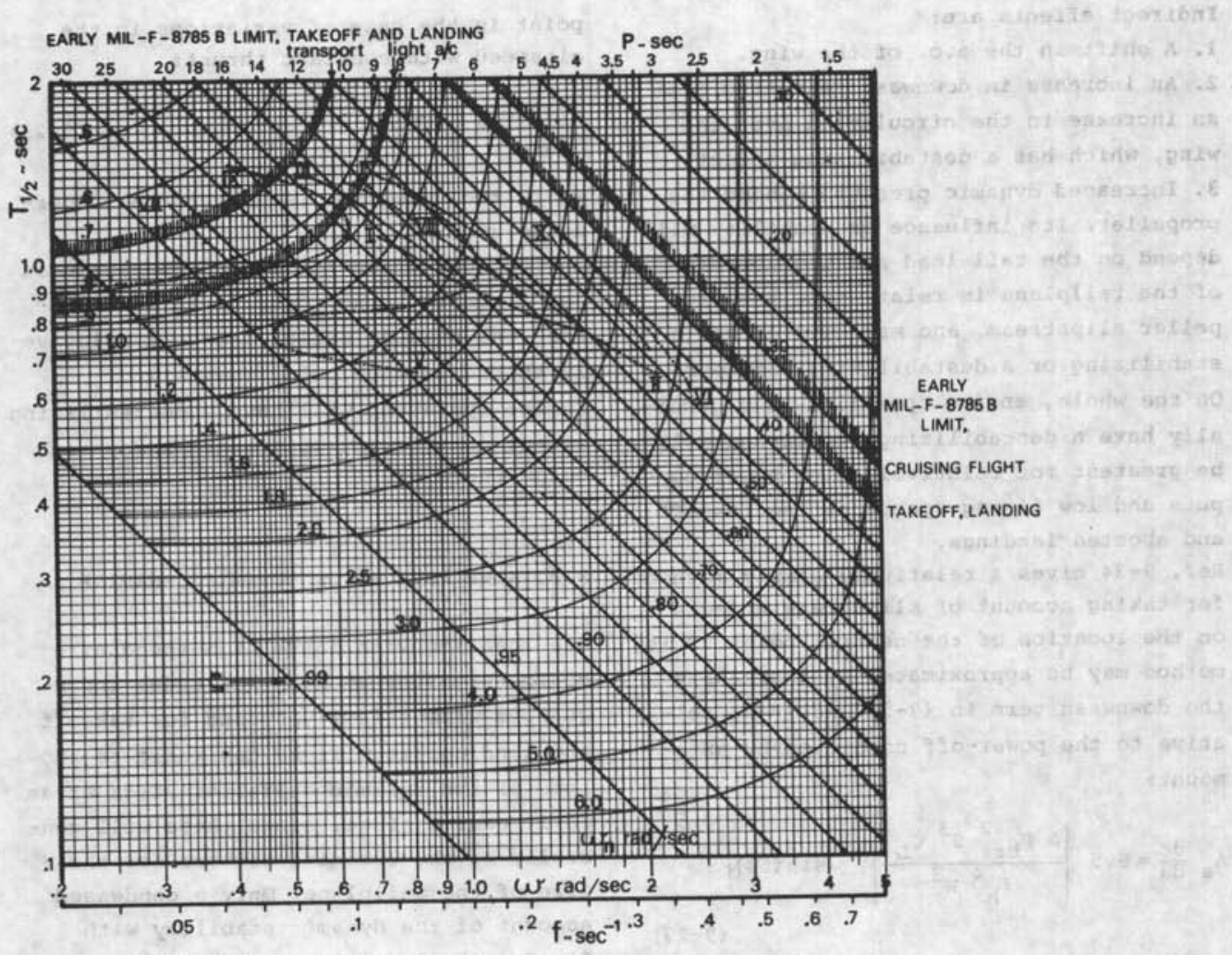

$$
\begin{aligned}
& c_{h_{2}}=\frac{T_{L_{2}}}{P}=\frac{\ln 2}{2 \pi} \frac{\sqrt{1-\zeta^{2}}}{\zeta} \\
& \omega=\frac{2 \pi}{P}=\frac{\ln 2}{T_{h_{2}}} \frac{\sqrt{1-\zeta^{2}}}{\zeta} \\
& \omega_{n}=\frac{\omega}{\sqrt{1-\zeta^{2}}}=\frac{\ln 2}{\zeta T_{4}}
\end{aligned}
$$

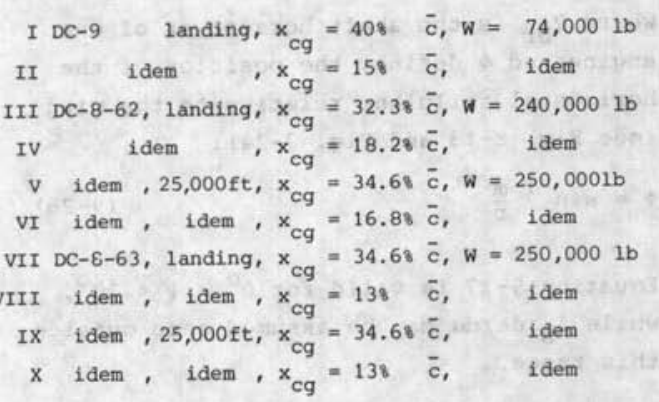

Fig. 9-9. Relationships between the parameters characterizing the short period longitudinal oscillation

and with CRITICAL DAMPING the motion becomes aperiodic ("dead-beat motion"). The TIME TO HALF AMPLITUDE $\mathrm{T}_{\frac{1}{2}}$ is the time which has elapsed when the amplitude of the oscillation has decreased to one half its initial value.

CYCLIC DAMPING is defined as:

$$
C_{\frac{1}{2}}=T_{\frac{1}{2}} / P
$$

RELATIVE DAMPING is the actual damping as a fraction of the critical damping. The FREQUENCY $\omega$ is the angular speed in radians per second: $\omega=2 \pi / P$. Another definition takes the frequency as being 
$\mathrm{f}=1 / \mathrm{P}$.

The UNDAMPED NATURAL FREQUENCY $\omega_{n}$ is the frequency of a short period oscillation in the absence of aerodynamic damping.

Fig. 9-9 shows a pictorial representation of the relationships between the parameters defined above, including data on some high-subsonic aircraft.

The frequency of the short period oscillation should be in the right proportion to the maneuverability, which is of ten expressed in the following parameters:

$\mathrm{L}_{\alpha}=\frac{\text { increase in lift }}{\text { increase in } \alpha \times \text { mass } x \text { speed }}=$

$$
=\frac{\rho g S V C_{L_{\alpha}}}{2 W}
$$

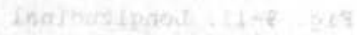

and

$\mathrm{n}_{\mathrm{z}_{\alpha}}=\frac{\text { increase in normal load factor }}{\text { increase in angle of attack }} \approx$

$$
\approx \frac{C_{L_{\alpha}}}{C_{L}}
$$

These parameters appear to have some significance in considerations of the piloting techniques involved in precise flight path control.

9.3.2. Criteria for acceptable SP charac-

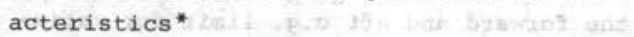

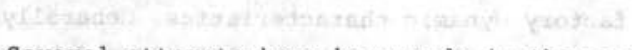
Several attempts have been made in the past to derive criteria which may be useful to designers for ensuring good SP oscillation characteristics. Civil requirements are qualitative in character, but the military authorities have been more specific in setting limits to the undamped natural fre-

*Very recently, at the moment of final production of this chapter, a similar approach has been published for military aircraft in J. of Aircraft, June 1975, entitled: "Use of short period frequency requirements in horizontal tail sizing", by D.J. Moorhouse and W.M. Jenkins quency and the damping ratio in early MILF-8785B requirements (Fig. 9-9). Their present requirements, summarized in Fig. 9-10, may be used for civil aircraft as wel1.

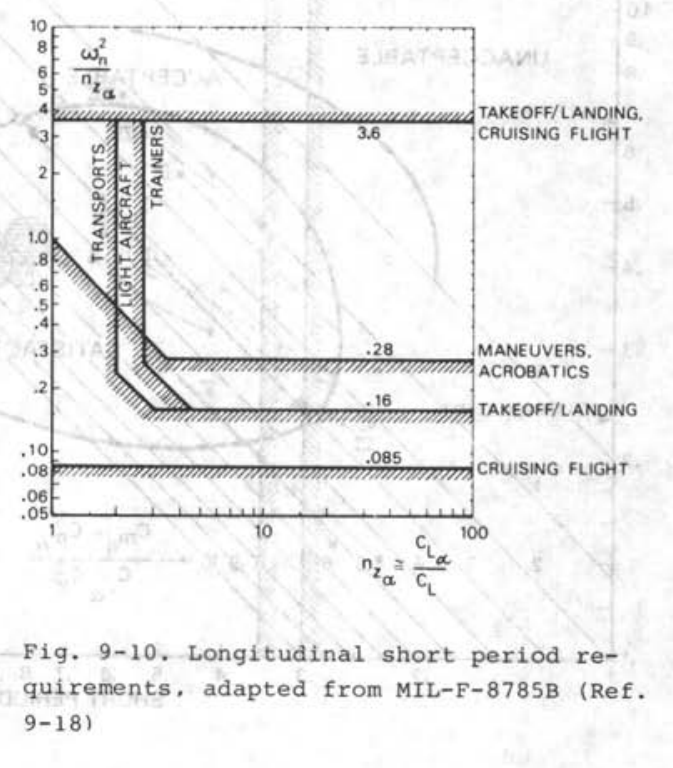

Alternatively, the criteria proposed by Shomber and Gertsen (Ref, 9-15), which are shown in Fig. 9-11, must be considered as an even better starting point for the preliminary design of civil aircraft. These are based on the opinions of pilots who had to perform specific tasks in flight simulators and expressed their views in the form of Cooper Ratings (CR). The characteristics are regarded as satisfactory within a limited region of combinations of $\mathrm{L}_{\alpha} / \omega_{\mathrm{n}}$ and $\zeta$ identified by $\mathrm{CR}<3.5$.

The linearized equations of motion for the longitudinal short period oscillation may be used to find an approximate expression for $L_{\alpha} / \omega_{n}$ in terms of the damping ratio:

$$
\frac{L_{\alpha}}{\omega_{n}}=\frac{4 \zeta}{2-\frac{C_{m_{q}}{ }^{+C}{ }_{m_{\dot{\alpha}}}}{C_{L_{\alpha}} K_{y}{ }^{2}}}
$$

The coefficients $\mathrm{C}_{\mathrm{m}_{\mathrm{q}}}$ and $\mathrm{C}_{\mathrm{m}_{\dot{\alpha}}}$ represent the aerodynamic damping in pitch due to the rate of pitch and change of incidence, and 


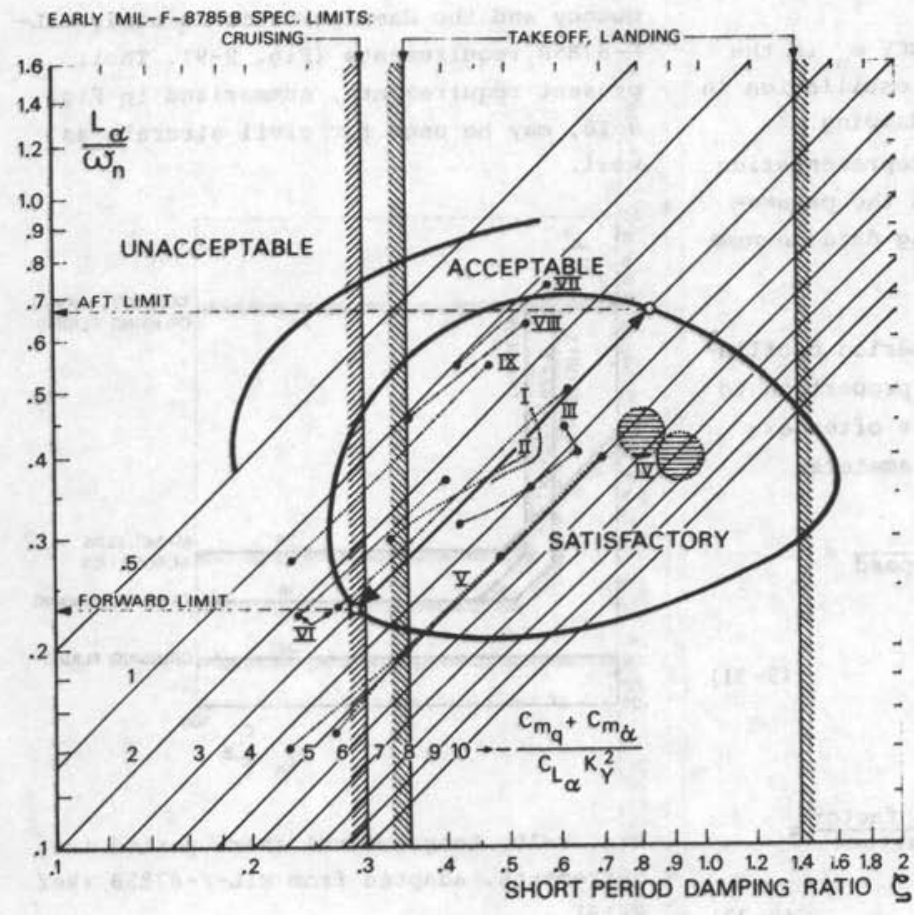

Fig. 9-11. Longitudinal short period oscillation criteria for transport aircraft as proposed by Shomber and Gertsen (Ref. 9-15)

$\mathrm{K}_{\mathrm{y}}$ is the inertia radius about the lateral axis as a fraction of the MAC. For a given type of aircraft $\zeta$ is a function of the stick-fixed maneuver margin, while $k_{y}$ is dependent upon the mass distribution. Lines have been drawn in Fig. 9-11 according to $(9-33)$, which show that aircraft with a given mass distribution at a given altitude approximately follow this relationship when their c.g. location is varied. The figure thus defines acceptable fore-and-aft c.g. limits, which may be expressed as limits to the stick-fixed maneuver margin:

$\frac{x_{m}{ }^{-x_{c g}}}{\bar{c}}=\frac{1}{2} \frac{K_{y}^{2}}{\mu_{c}}\left(\frac{\omega_{n}}{L_{\alpha}}\right)^{2} c_{L_{\alpha}}$

where

$K_{y}^{2}=\frac{I_{y} g}{w \bar{c}^{2}}$

and

$\mu_{c}=\frac{W}{\rho g s \bar{c}}$
Using the approximation

$\frac{C_{m_{g}}+C_{m_{\alpha}}}{C_{L_{\alpha}}}=-2.2 \frac{C_{L_{h}}}{C_{L_{\alpha}}} \frac{S_{h}}{S}\left(\frac{l_{h}}{\bar{c}}\right)^{2}\left(1+\frac{d \varepsilon}{d \alpha}\right)(9-36)$

Fig. 9-11 may be used to find maximum and minimum values of $L_{\alpha} / \omega_{n}$, corresponding to the forward and aft c.g. limit for satisfactory dynamic characteristics. Generally speaking, flight at high altitude and high wing loading will determine the forward c.g. limit, since in this condition damping is poor, while low-speed flight at low weight will determine the aft c.g. limit. An example of acceptable combinations of $\mathrm{s}_{\mathrm{h}} / \mathrm{S}$ and $\mathrm{x}_{\mathrm{cg}} / \overline{\mathrm{c}}$ is shown in Fig. 9-12.

9.3.3. A simple criterion for the tailplane size

The time to half amplitude of the SP oscillation can be approximated by: 


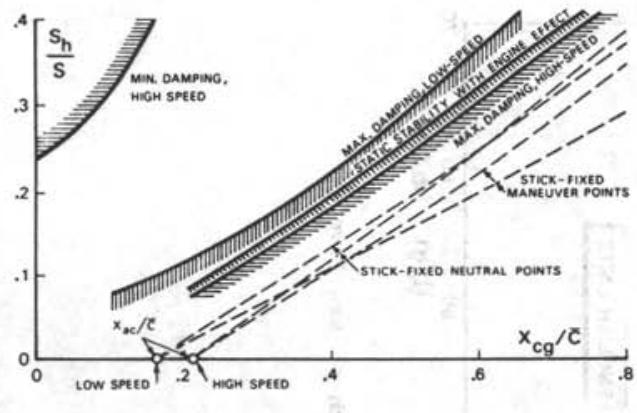

Fig. 9-12. Example of acceptable combinations satisfying SP requirements

$$
T_{\frac{1}{5}}=\frac{4 \ln 2}{L_{\alpha}\left(2-\frac{C_{m_{q}}+C_{m_{\alpha}}}{C_{L_{\alpha}} K_{Y}^{2}}\right)}
$$

This relationship has been plotted in Fig. 9-13, together with values of $T_{\frac{1}{2}}$ for some airplanes of different classes in the landing configuration. The trend which these points seem to indicate suggests

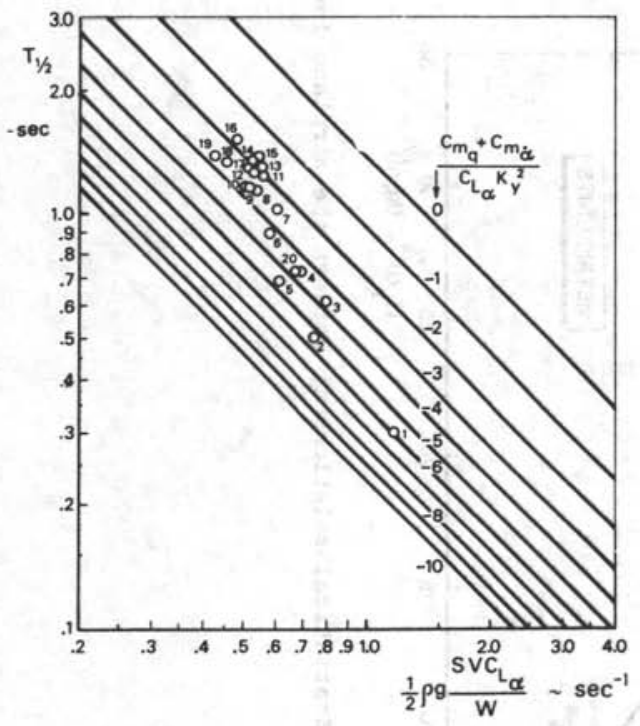

that $T_{\frac{1}{2}}$ may be a useful criterion for sizing the horizontal tailplane. Inspection of $(9-36)$ and $(9-37)$ reveals that $\mathrm{T}_{\frac{1}{2}}$ is primarily determined by the term

$L_{\alpha} \frac{C_{m_{q}}+C_{m_{\alpha}}}{C_{L_{\alpha}} K_{y}{ }^{2}}=-1.1 \frac{\rho V}{I_{y}} S_{h}{ }^{{ }^{2}}{ }^{2} C_{L_{h}}\left(1+\frac{d \varepsilon}{d \alpha}\right)$

which, in fact, represents the aerodynamic damping moment in relation to the polar moment of inertia in pitch. Fig. 9-14 is based on this relation and supplies a simple method for estimating the horizontal tailplane size.* This approach also shows the importance of the tail moment arm.

Analysis of the SP oscillation shows that an inportant part is played by the parameter $I_{Y} /\left(\ell_{h} w\right)$. From Fig. 9-15 it can be seen that the length of the fuselage and the engine location are deter-

* Some data for estimating the horizontal tailplane lift gradient can be obtained from Section E-10.1. of Appendix E

1. Queen Air

2. Beaver

3. Lear Jet 24

4. $\mathrm{F}-27$

5. HS -125

6. $D C-9 / 10$

7. $D C-8 / 21$

8. vC -10

9. $\mathrm{CV}-880$

10. C-141

11. Jetstar

12. B707-120

13. $\mathrm{DC}-8 / 62$

14. B727-100

15. $D C-8 / 63$

16. B727-200

17. B747

18. C- $5 \mathrm{~A}$

19. B737-200

20. MS Paris

Note: the points indicated refer to the approach speed at sea level, at MLW

Fig. 9-13. Time required to damp the short period motion 

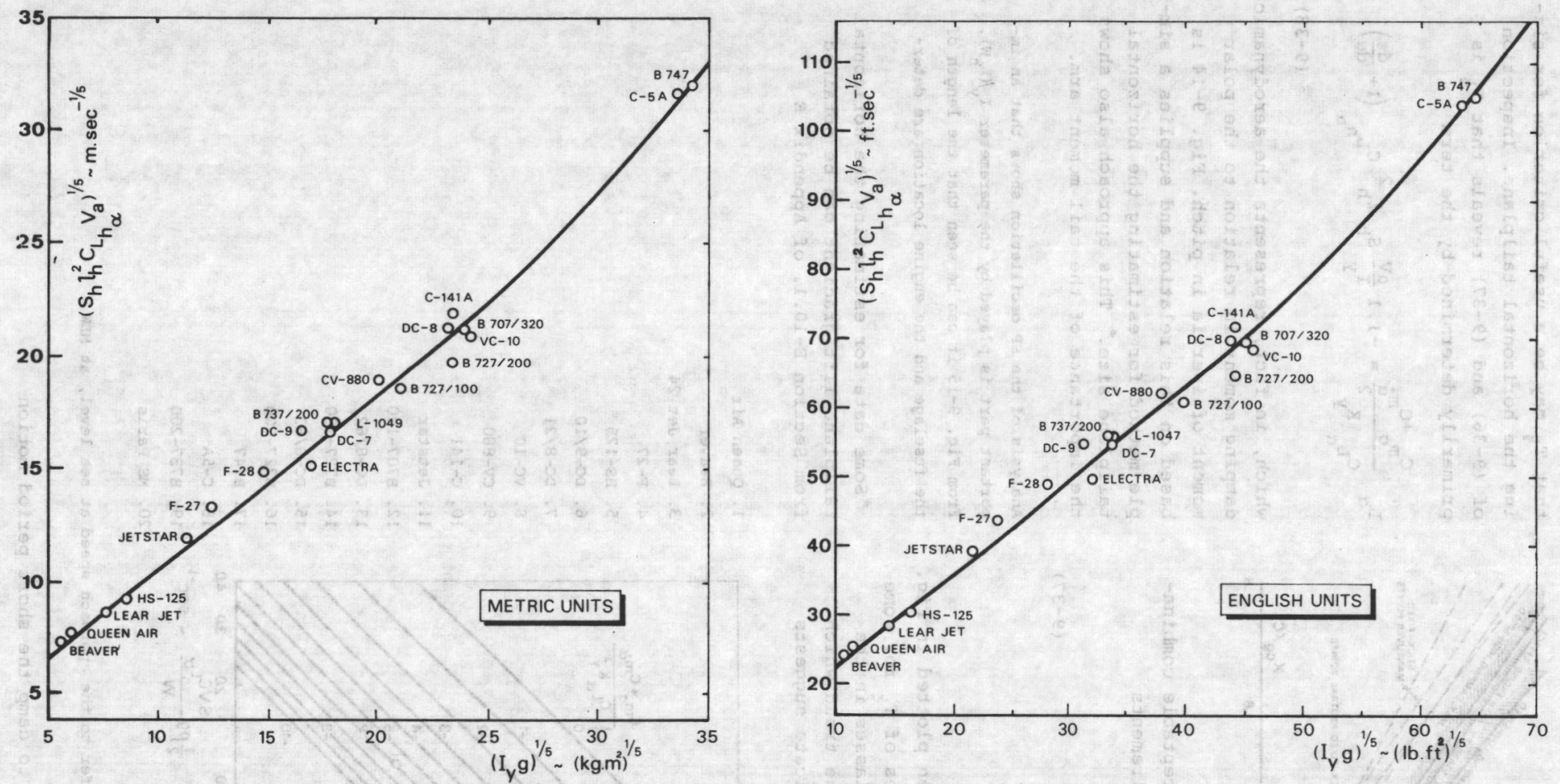

Fig. 9-14. Statistical relationship between the airplane inertia and aerodynamic damping: approach at sea level 


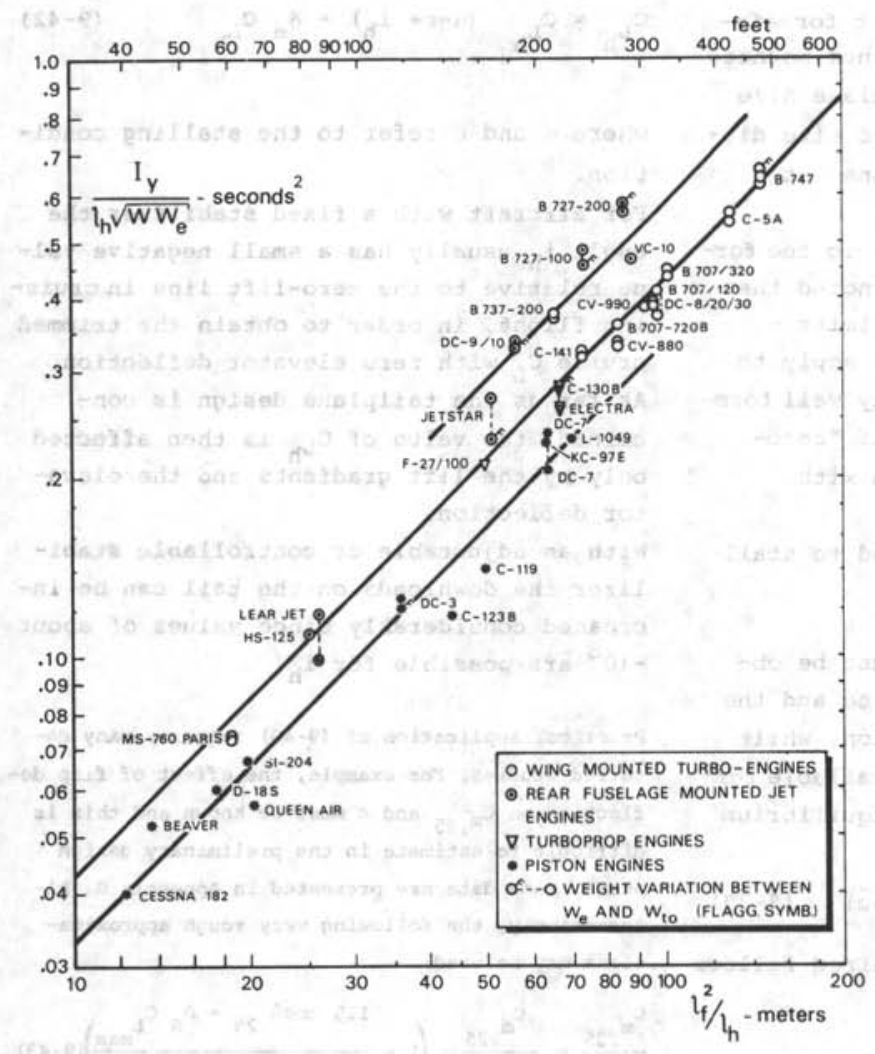

Fig. 9-15. Polar moment of inertia about the lateral axis as affected by airplane size for various categories

mining factors here and problems may therefore be encountered with the SP oscillation in a particular class of high-subsonic jet transports. It is not always possible to solve such problems in the entire range of speeds, weights, and altitudes by good aerodynamic design. It may then become desirable or even necessary to use equipment which supplies artificial stabilization (SAS, Stability Augmentation System).

\section{$9 \cdot 3.4$. The phugoid}

The phugoid is a motion during which speed variations occur which generate tangential and normal forces at an almost constant angle of attack. The aircraft has a constant energy level and oscillates in such a way that an interchange takes place between potential and kinetic energy.

Although the period of the phugoid will generally be of such magnitude that the pilot can still correct any instability by elevator deflections, a stable phugoid is regarded as desirable under normal conditions and particularly during IFR flights. In a preliminary design this may basically be achieved by:

- taking account of power effects as far as possible (see Section 9.2.5.) and ensuring that the static stability remains positive,

- paying due regard to the influence of compressibility in the case of high-subsonic aircraft.

\subsection{LONGITUDINAL CONTROL AT LOW SPEEDS}

For some aircraft the aft c.g. limit may be imposed by the condition that recovery must be possible from a deep stall (see Section 2.4.2c.). From the available 1it- 
erature it can be concluded that for aircraft with T-tails and jet engines mounted on the rear fuselage, the tailplane size may be about 158 larger than the size dictated by stability considerations at a given aft c.g. location.

The criteria given below relate to the forward c.g. location. It will be noted that on aircraft with CCV, similar limits based on control requirements - apply to the aft c.g. location, which may well form the critical design condition if "aerodynamic" stability is dispensed with.

\subsubsection{Control capacity required to stall} the aircraft

The maximum lift coefficient must be obtained with flaps fully deflected and the c.g. in its most forward position, while some reserve will have to be available for maneuvering. The condition of equilibrium in Fig. 9-16 results in:

$L_{h}{ }^{2} h^{\prime \prime}=M_{.25}+L_{A-h}\left(x_{c g}-.25 \bar{c}\right)$

The horizontal tail volume required follows from:

$\frac{s_{h}}{s \bar{c}}={ }_{h}{ }_{n_{h}} \frac{C_{\text {max }}}{C_{L_{h}}}\left(\frac{C_{m} .25}{C_{L_{\max }}}+\frac{x_{c g}}{\bar{c}}-.25\right)$

where

$n_{h}=\frac{l_{h} n}{l_{h}}\left(\frac{V_{h}}{V}\right)^{2}$

The tailplane lift coefficient, which is positive in the upward direction, amounts to:
$c_{L_{h}}=C_{L_{h}}\left(\alpha-\varepsilon+i_{h}\right)+\delta_{e} C_{L_{h_{\delta}}}$

where $\alpha$ and $\varepsilon$ refer to the stalling condition.

For aircraft with a fixed stabilizer the angle $i_{h}$ usually has a small negative value relative to the zero-lift line in cruising flight, in order to obtain the trimmed cruise $C_{L}$ with zero elevator deflection. As far as the tailplane design is concerned, the value of $\mathrm{C}_{\mathrm{L}_{\mathrm{h}}}$ is then affected only by the lift gradients and the elevator deflection.

With an adjustable or controllable stabilizer the downloads on the tail can be increased considerably since values of about $-10^{\circ}$ are possible for $i_{h}$.

Practical application of $(9-40)$ requires many detailed studies. For example, the effect of flap deflection on $c_{\mathrm{m}}, 25$ and $\varepsilon$ must be known and this is difficult to estimate in the preliminary design stage. Some data are presented in Appenaix G. Alternatively, the following very rough approximations may be used:

$\frac{c_{m}{ }_{25}}{c_{L_{\max }}}=\frac{c_{m} .25}{\Delta_{f} c_{L_{\max }}}\left(1-\frac{1.5 \cos \Lambda_{.25}+\Delta_{S} c_{L_{\max }}}{c_{L_{\max }}}\right)(9-43)$

where $\Delta_{f}$ and $\Delta_{S}$ denote the effects of flap and slat deflection on the coefficients to which they are attached. Typical values for $c_{m}, 25 / \Delta_{f} c_{L_{\max }}$ are:

-.18 , split flap, plain flap

-.26 , single slotted flap

- .385, double slotted flap, fixed hinge

- .415, single slotted Fowler flap

- .445, double slotted Fowler flap

-.475 , triple slotted Fowler flap

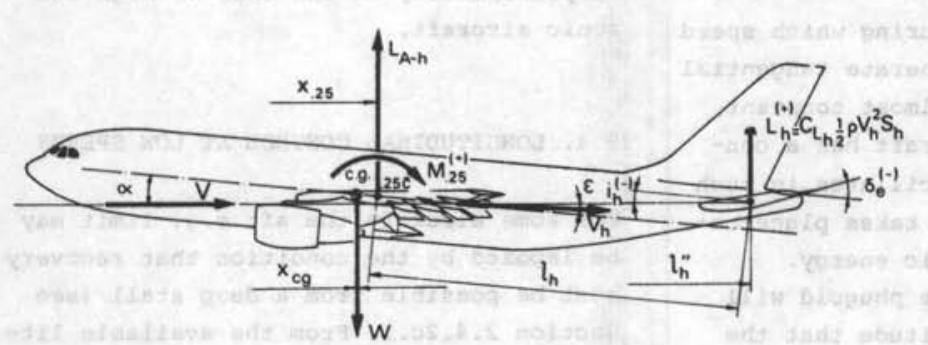

Fig. 9-16. Longitudinal equilibrium at the stall: landing configuration 
The following figures are proposed for $C_{L_{h}}$

$-.35 \cdot A_{h}{ }^{1 / 3}$, fixed stabilizer

-.8 , adjustable stabilizer

- 1.0 , controllable stabilizer.

For $\eta_{h}$ we may assume:

$\begin{array}{ll}.95 & \text {, fin-mounted stabilizer } \\ .85 & \text {, fuselage-mounted stabilizer. }\end{array}$

The condition of adequate control power required to reach $\mathrm{C}_{\mathrm{L}_{\max }}$ in the landing configuration may be depicted in an $\mathrm{s}_{h} / \mathrm{s}$ vs. $\mathrm{x}_{\mathrm{cg}} / \overline{\mathrm{C}}$ diagram; see Fig. 9-18.

9.4.2. Control capacity required for takeoff rotation and landing flareout

It must be possible to generate a sufficiently large tail load to ensure that at the rotation speed the aircraft will have an angular velocity of $\dot{\theta}_{R}$ within a limited period of time, e.g. one second. From Fig. 9-17 we may derive the condition:

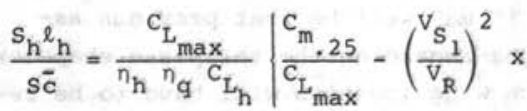

$\frac{x_{g}-z_{T} \sum T / w-x_{C g}}{\bar{c}} \mid+\frac{c_{L_{R}}}{C_{L_{h}}}\left(\frac{x_{g}}{\bar{c}}-.25\right)$ where $\mathrm{v}_{\mathrm{S}_{1}}, \mathrm{C}_{\mathrm{L}_{\max }}$ and $\mathrm{C}_{\mathrm{m}}$, refer to the
takeoff flap setting and

$n_{h}=\frac{x_{h}-x_{g}}{l_{h}}\left(\frac{v_{h}}{v_{R}}\right)^{2}$

and

$n_{q}=1+\frac{c_{L_{h}}}{C_{L_{h}}} \frac{\dot{\theta}_{R}\left(x_{h}-x_{g}\right)}{v_{R}}$
As a minimum for the rotation speed we may take: $V_{R}=1.05 v_{S_{1}}$ for transport aircraft; for light aircraft an explicit requirement will be found in FAR 23.51 regarding the minimum speed at which rotation can take place.

The value for $C_{L_{h}}$ follows from $(9-42)$, where $\alpha$ and $\varepsilon$ relate to the initiation of the rotation and the aircraft is trimmed for $1.3 \mathrm{~V}_{\mathrm{S}_{1}}$. When the stabilizer is adjustable it may be possible to obtain a reasonably high value, such as $C_{L_{h}}=-1.0$. In the case of propeller aircraft, $\mathrm{C}_{\mathrm{L}_{\mathrm{h}}}$ and $n_{h}$ are more difficult to determine because of powerplant operation, ground effect,etc. Various data needed for further evaluation of (9-44) often only isecome available after wind tunnel tests. An example of the resulting $\mathrm{S}_{h} / \mathrm{S}-1$ imit is shown in Fig. 9-18.

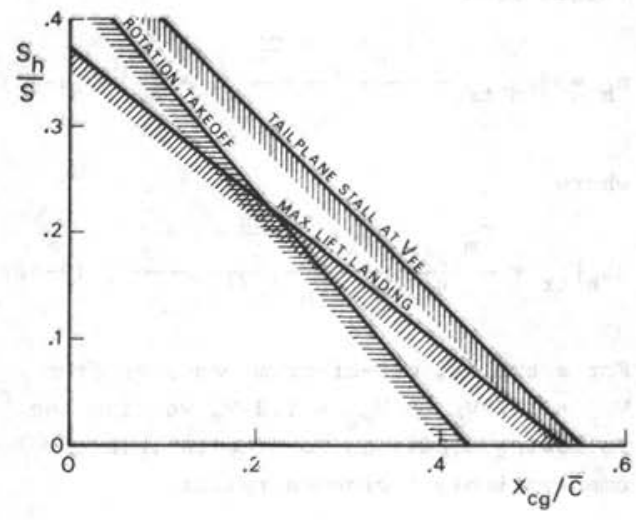

Fig. 9-18. Tailplane size and c.g. limitations based on controllability at low speeds

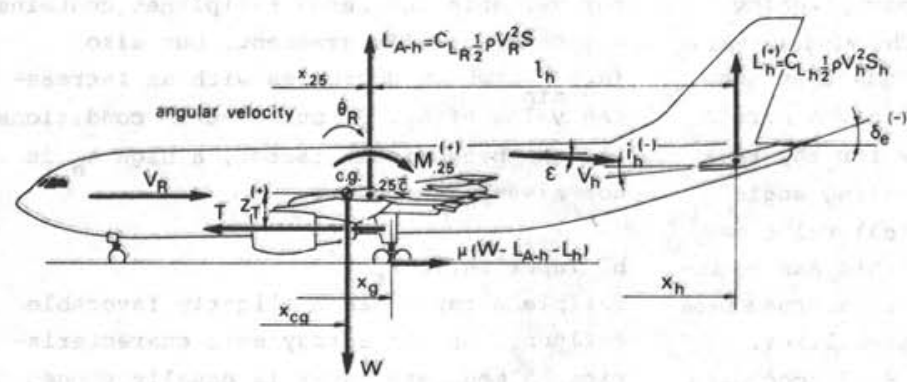

Fig. 9-17. Takeoff rotation 


\subsubsection{Out-of-trim conditions}

When excursions are made from the trimmed conditions, variations in the local flow conditions at the tailplane will occur, but these must not lead to tailplane stall. The critical condition here will generally be experienced in the landing configuration with the most forward c.g. location. Typical maneuvers to be considered are a level speed increase from a trimmed condition at $1.2 \mathrm{~V}_{\mathrm{S}}$ or a push-over maneuver at $\mathrm{n}=.5 \mathrm{~g}$ from a trimmed condition at $1.3 \mathrm{~V}_{\mathrm{S}^{\prime}}$ up to the flap-extended design speed $V_{F E}$ (Ref. 9-32).

Taking, for example, an aircraft with a variable-incidence tallplane, trimmed at 1.2. $v_{S}$, which is accelerated at $n=1$ to $\mathrm{v}_{\mathrm{FE}}$, the tailplane angle of attack is obtained from:

$\alpha_{h}=\left(\alpha_{h}\right)_{t r}-\frac{\left(c_{L}\right)_{t r}-c_{L}}{C_{L}}\left(1-\frac{d \varepsilon}{d \alpha}\right)(9-47)$

where

$\left(\alpha_{h}\right)_{t r}=\frac{c_{m}+\left(c_{L}\right)_{t r}\left(\frac{x_{c g}}{\bar{c}}-.25\right)}{n_{h} c_{L_{h}} s_{h} l_{h} /(s c)}$

For a typical out-of-trim maneuver from $\mathrm{v}_{\mathrm{tr}}=1.2 \mathrm{v}_{\mathrm{S}}$ to $\mathrm{v}_{\mathrm{FE}}=1.8 \mathrm{v}_{\mathrm{S}}$ we find the following condition for the tailplane volume (variable incidence tail):

$$
\frac{s_{h}{ }^{h} h}{s \bar{c}}=\frac{c_{m} / 25 / c_{L_{\max }}+.7\left(x_{c g} / \bar{c}-.25\right)}{\left.n_{h} c_{L_{h}} \mid \frac{\left(\alpha_{h}\right)_{\min }}{C_{L_{\max }}}+.39 \frac{1-d \varepsilon / d \alpha}{C_{L}}\right\}}
$$

where $C_{m}{ }_{25}$ is the aerodynamic pitching moment, tail-off, at $\left(C_{L}\right)_{t r}$ The minimumvalue of $\alpha_{h}$ to be accepted depends upon the detail design of the stabilizer. A margin should be available to allow for the reduction in the tailplane stalling angle due to ice formation. A typical value may be $\left(\alpha_{h}\right)=-.25$ rad., but this can be increased by negative camber or inverse slats on the leading edge of the stabilizer. An example of limitation of $\mathrm{s}_{\mathrm{h}} / \mathrm{s}$ according to $(9-49)$ is plotted in Fig. 9-18. Similar limitations may also be derived for other out-of-trim maneuvers. It will be obvious that a detailed study of flow conditions will be required at a later stage of the design.

\subsection{PRELIMINARY DESIGN OF THE HORIZONTAL} TAILPLANE

The design of the tailplane is always an iterative process. It is tisual to make an initial choice of certain shape parameters such as aspect ratio and thickness ratio, etc. The choice of the type of aerodynamic balance, whether the stabilizer will be fixed or adjustable, and the type of control system is much more difelcult, and more data will generally be required than are available to the preliminary design engineer. Once these decisions have been taken, it may well be that previous assumptions regarding the tailplane shape or even the wing location will have to be revised.

\subsubsection{Tailplane shape and configuration}

a. Aspect ratio $\mathrm{A}_{\mathrm{h}}$.

This factor is of direct influence because of its effect on the lift-curve slope. For manual control systems the c.g. range satisfying the stick-force requirements will be widened, or the required tailplane size may be reduced. For aircraft with a fixed stabilizer the forward c.g. limitation required to cope with the stall is favorably affected with increasing $A_{h}$. Equation 9-49 for variable incidence tailplanes contains not only the lift gradient, but also $\left(\alpha_{h}\right)_{\text {min }}$, which decreases with an increasing value of $A_{h}$. If out-of-trim conditions are the predominant factor, a high $\mathrm{A}_{\mathrm{h}}$ is not always desirable.

b. Taper ratio $\lambda_{h}$.

Tailplane taper has a slightly favorable influence on the aerodynamic characteristics. A moderate taper is usually chosen 
to save structural weight.

c. Angle of sweep $\Lambda_{h}$.

In the case of high-speed aircraft the tailplane angle of sweep, in combination with its thickness ratio, is chosen so that at the design diving Mach number strong shocks are not jet formed. The same procedure as applied to the wing (Section 7.5.2b) will then result in a thinner section and/or larger $A_{h}$ as compared with the wing.

Positive sweepback is occasionally used on low-speed aircraft to increase the tailplane moment arm and the stalling angle of attack, although the result is a decrease in the lift-curve slope. Up to about 25 degrees of sweepback there is still an advantage. The sweepback angle may be determined by the condition of a straight elevator hingeline, which is sometimes imposed in the interest of structural simplicity.

\section{d. Airfoil shape.}

The basic requirements are that the airfoil section should have a high $c_{\ell_{\alpha}}$ and a large range of usable angles of attack. Frequent use is made of approximately symmetrical airfoils with a thickness ratio of 9 to 12 percent and a large nose radius, e.g. NACA 0012. Tailplane stalling - at the lower surface - can be postponed by adopting negative camber (e.g. NACA 23012 upside down), upward nose droop, an increase in the nose radius or by means of a fixed slot.

e. Dihedral.

The position of the tailplane relative to the propeller slipstream or jet efflux may make it desirable to shift it slightly in an upward direction. This may be achieved by using a certain degree of dihedral.

f. Control and trim system.

A variable-incidence (adjustable) tailplane has the advantages that at high-subsonic speeds adjustment of the tailplane is more effective than trimming by means of the elevator - which may cause shock waves - and that the c.g. travel can be extended forward. An all-flying (controllable) tail has the additional advantage that it improves both maneuverability and control in out-of-trim conditions. A geared elevator, deflecting in the same direction, will extend the c.g. range in the forward direction through an increased download. All-flying tails on transport aircraft are usually power-operated. A controllable tailplane is sometimes used on small aircraft and frequently on gliders. An antibalance tab is recommended in order to obtain acceptable control forces and control force stability.

Trim tabs, balance tabs or variable-incidence stabilizers can be used to reduce the control forces to zero. Large trim changes may be induced by the deflection of effective flaps, and in some aircraft there is a coupling between the flap controls and the (trim) tab controls or the stabilizer incidence controls.

g. Elevator area and deflections.

A large elevator area - as a fraction of the tailplane area - promotes good controllability at forward c.g. locations, but for manual control systems the stick forces will increase. Similar arguments apply to the maximum elevator deflection. The danger of tailplane stall and elevator lock-over grows greater with increasing elevator chord and deflection.

\subsubsection{Design procedures}

The objective of preliminary tailplane design is not only to arrive at an acceptable guess with regard to shape and size but, in addition, to ensure a good balance between the wing location in longitudinal direction, the c.g. range required, the c.g. range available and the tailplane design. In view of the many parameters involved, various solutions may be proposed and the design finally selected will be the one which gives a small tailplane and adequate flexibility to suit a variety of cus- 


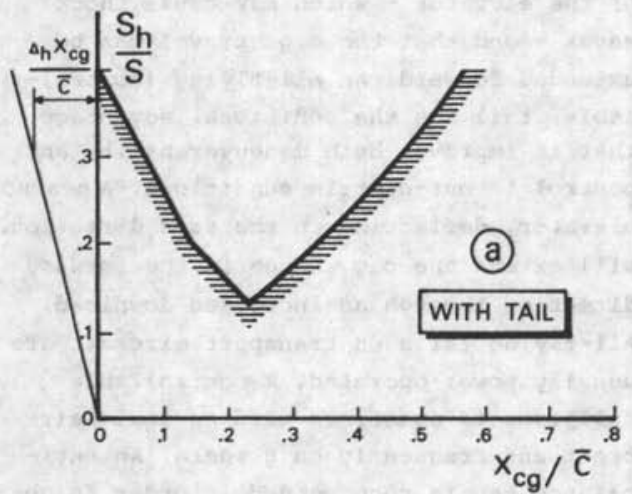

Aerodynamic c.g. limits

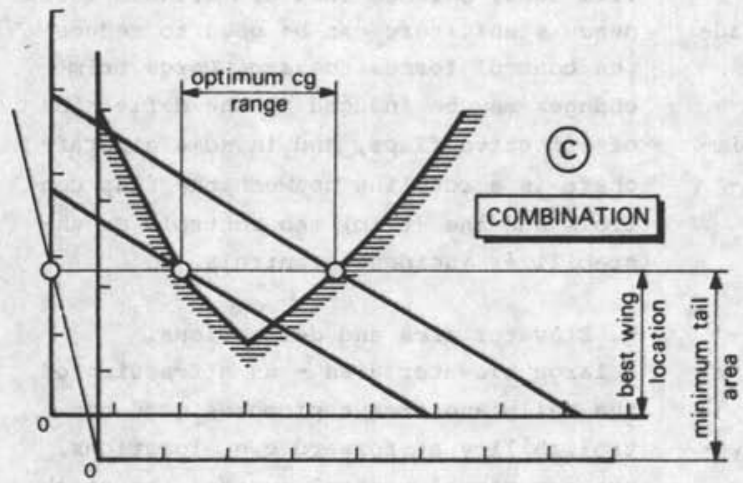

Balancing

Fig. 9-19. Balancing the airplane

tomer requirements and future aircraft growth.

A design procedure will be suggested below. The basic relationships required can be found in this chapter, while methods for predicting aerodynamic characteristics are presented in Appendices $\mathrm{E}$ and $\mathrm{G}$, and in the references. As a considerable amount of work is involved in the detailed procedure, a simple approach will be presented first.

a. Quick method.

It can be argued that longitudinal stability is affected primarily by $s_{h} / S$, whereas the volume $s_{h}{ }^{l} h /\left(s_{\bar{c}}\right)$ is more relevant to controllability. By inspecting the data for

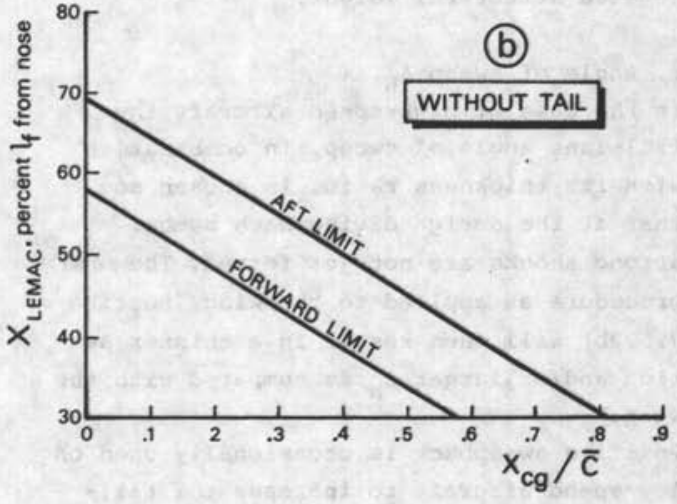

Limits based on loading requirements: aircraft without horizontal tailplane 


\begin{tabular}{|c|c|c|c|c|c|c|c|c|c|c|c|c|c|c|c|c|c|c|c|}
\hline \multirow[b]{2}{*}{ Aircraft type } & \multicolumn{2}{|c|}{\begin{tabular}{|c|} 
Design Dive \\
Speed \\
\end{tabular}} & \multirow[b]{2}{*}{$\begin{array}{l}\frac{s_{h}}{S} \\
-\end{array}$} & \multirow[b]{2}{*}{$\begin{array}{l}A_{h} \\
-\end{array}$} & \multirow[b]{2}{*}{$\lambda_{h}$} & \multirow[b]{2}{*}{$\begin{array}{r}\Lambda_{h} \\
\text { deg. }\end{array}$} & \multirow[b]{2}{*}{$\begin{array}{c}\text { Airfoil Section } \\
\text { root/tip }\end{array}$} & \multirow{2}{*}{$\mid \begin{array}{c}t / c \\
\%\end{array}$} & \multirow{2}{*}{$\begin{array}{c}\text { Tail } \\
\text { Type } \\
*\end{array}$} & \multicolumn{2}{|c|}{$i_{\max }$} & \multirow{2}{*}{$\begin{array}{c}\frac{s_{h}{ }_{h}}{s \bar{c}} \\
-\end{array}$} & \multirow[b]{2}{*}{$\begin{array}{r}r_{h} \\
\text { deg. }\end{array}$} & \multirow{2}{*}{$\begin{array}{c}\frac{s_{e}}{s_{h}} \\
-\end{array}$} & \multirow{2}{*}{$\begin{array}{l}\text { Hinge } \\
\text { Pos. } \\
\% c_{h}\end{array}$} & \multirow{2}{*}{$\begin{array}{c}\text { Balance } \\
\text { Ratio } \\
\% c_{e}\end{array}$} & \multicolumn{2}{|c|}{$\delta_{e_{\max }}$} & \multirow[t]{2}{*}{$\begin{array}{c}\text { Tabs } \\
* *\end{array}$} \\
\hline & \begin{tabular}{|c|}
$v_{D}$ \\
$k t s$ \\
EAS
\end{tabular} & $M_{D}$ & & & & & & & & + & deg. & & & & & & \begin{tabular}{c|}
+ \\
deg. \\
$\cdot$
\end{tabular} & deg. & \\
\hline Wright Flyer (1903) & - & - & .163 & 5.72 & 1 & 0 & curved "plates" & few & M & & & -.357 & 0 & 0 & - & - & - & - & - \\
\hline Scottịsh Aviation Pup & & & .229 & 4.40 & 1 & 0 & & 12 & F & - & - & .730 & 0 & .377 & 60 & - & 25 & 27 & $\mathrm{~T}$ \\
\hline Cessna 177 & & & .202 & 4.00 & 1 & 0 & NACA $0012 / 0009$ & 10.5 & M & & & .600 & 0 & 0 & 25 & - & - & - & $\mathbf{s}$ \\
\hline Aérospatiale $\mathrm{N}-262$ & 260 & - & .285 & 3.84 & .568 & 8.2 & 23015 mod. inv. & 15 & $\mathbf{F}$ & - & - & 1.015 & 0 & .242 & 75 & $31.1 / 36.2$ & & & $\mathrm{~T} / \mathrm{S}$ \\
\hline Fokker-VFW F-27 Mk200 & 288 & .51 & .229 & 6.00 & .400 & 0 & NACA $63 \mathrm{~A}-014$ mod. & 14 & F & - & - & .962 & 6 & .198 & 78 & 42.7 & 22 & 25 & $\mathbf{T}$ \\
\hline Lockh. Hercules $\mathrm{C}-130 \mathrm{~B}$ & 325 & .64 & .313 & 5.20 & .358 & 7.5 & NACA 23012 inv. & 12 & $\mathbf{F}$ & - & - & 1.000 & 0 & .332 & 65 & & 15 & 40 & $\mathrm{~T} / \mathrm{S}$ \\
\hline Lockh. E1ectra $188 \mathrm{~A}$ & & .711 & .246 & 5.25 & .335 & 8.6 & & & $\mathbf{F}$ & - & - & .800 & 8.5 & .247 & 65 & & 15 & 30 & $\mathbf{T}$ \\
\hline Bristol Britannia 310 & 301 & -4 & .282 & 5.14 & .500 & 6 & RAF 30 mod. & 13 & $\mathbf{F}$ & - & - & 1.040 & 0 & .300 & 66 & 30 & 15 & 35 & $T / S$ \\
\hline Cessna Citation 500 & 난 & 8 & .260 & 5.20 & .500 & & NACA $0010 / 0008$ & 9 & $\mathbf{F}$ & - & - & .755 & 9 & .310 & 67 & - & & & \\
\hline Hawker Sidd. HS-125/400B & 370 & .825 & .283 & 4.00 & .580 & 19.6 & $2 y$ & 10 & F & - & - & .677 & 0 & .408 & 58.7 & 25 & 9 & 24 & \\
\hline Aérospat. SN-600 Corvette & $x^{2}$ & & .237 & 4.79 & .523 & 28.6 & neg. cambered & 9 & $\mathrm{v}$ & 2.5 & 7.5 & .640 & 0 & .320 & 64 & - & 15 & 25 & s \\
\hline Yakovlev YAK40 & 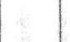 & 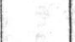 & .207 & 4.33 & .430 & 11.5 & & 10 & $\mathrm{v}$ & 3.0 & 6.0 & .621 & 0 & .377 & 70 & - & 25 & 25 & - \\
\hline VFW-Fokker 614 & 330 & .74 & .281 & 4.50 & .400 & 20 & 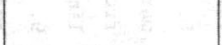 & 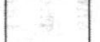 & $\mathrm{v}$ & 1.5 & 12.0 & .834 & 10.5 & .273 & 67 & 8 & 20 & 25 & $\mathbf{T}$ \\
\hline Fokker-VFW F-28 Mk 1000 & 390 & .83 & .255 & 3.83 & .480 & 27.5 & NACA mod. & 10.45 & $\mathrm{v}$ & 2.67 & 8.33 & .972 & 0 & .197 & 78 & 45 & 15 & 25 & $\mathbf{T}$ \\
\hline Aérospat. Caravelle 10-R & 375 & .87 & .204 & 4.03 & .326 & 30 & NACA $65-011$ & 11 & $\mathbf{F}$ & - & - & .995 & 0 & .235 & 75 & - & 12 & 30 & - \\
\hline BAC- $111 / 200-400$ & 410 & .86 & .256 & 3.38 & .600 & 25 & 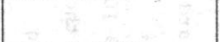 & 10 & $\mathrm{v}$ & & & .908 & 0 & .274 & 65 & 41 & & 25 & $\mathbf{s}$ \\
\hline McDonnell Douglas DC-9 project & & .89 & .242 & 4.21 & .310 & 32 & DSMA; $9.5 \% / 8 \%$ & 8.75 & $\mathrm{v}$ & 2 & 10 & .941 & 0 & .280 & 70 & 35 & 15 & 25 & s \\
\hline McDonnell Doug1as DC- $9 / 10$ & & .89 & .295 & 4.93 & .352 & 31.6 & & 8.7 & $\mathrm{v}$ & 1 & 9 & 1.147 & 0 & .280 & 70 & 35 & 15 & 25 & $\mathbf{s}$ \\
\hline Boeing $737 / 100$ & 398 & .89 & .319 & 4.16 & .380 & 30 & $12 \% / 9 \%$ & 10.5 & v & & & 1.140 & 7 & .224 & 75 & - & 20 & 20 & $\mathbf{s}$ \\
\hline Boeing $727 / 100$ & & .95 & .221 & 3.40 & .400 & 35 & & 8.5 & $\mathrm{v}$ & 4 & 12.5 & .902 & -3 & .230 & 75 & - & 16 & 26 & s \\
\hline Boeing $727 / 200$ & & .95 & .221 & 3.40 & .400 & 35 & 3 & 8.5 & $\mathrm{v}$ & 0 & 12 & 1.062 & -3 & .230 & 75 & - & 16 & 26 & $\mathbf{s}$ \\
\hline Dassault Mercure & & & .275 & 3.79 & .360 & 32.5 & & $8 ?$ & $\mathrm{v}$ & & Ft & 1.050 & 0 & .251 & 75 & - & 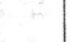 & & - \\
\hline Airbus $A-300 B$ & 420 & .90 & .267 & 4.13 & .500 & 32.5 & & $b$ & M & 3 & 12 & 1.068 & 6 & .295 & 70 & - & - & & - \\
\hline Boeing $707 / 320$ & & .95 & .216 & 3.37 & .421 & 35 & BAC-317 & 11.6 & $\mathbf{v}$ & 0.5 & 14 & .630 & 7 & .251 & & & 15 & 25 & $\mathrm{~T} / \mathrm{S}$ \\
\hline McDonnell Douglas DC-8 proj & . & .95 & .215 & 4.04 & .329 & 35 & DSMA-89/-90 & 8.75 & $\mathbf{v}$ & 2 & 10 & .626 & 10 & .225 & 75 & 35 & 10 & 25 & s \\
\hline McDonnell Douglas DC-8/10 & 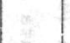 & .95 & .203 & 4.04 & .329 & 35 & DSMA-89/-90 & 8.75 & $\mathbf{v}$ & 2 & 10 & .590 & 10 & .225 & 75 & 35.2 & 16.5 & 27 & s \\
\hline Lockheed L-1011 Tristar & 435 & .95 & .371 & 4.00 & .333 & 35 & & 8 & $\mathbf{v}$ & 0 & 14 & .928 & 3 & .215 & 77 & - & 0 & 25 & - \\
\hline McDonnell Douglas DC- $10 / 10$ & 8 & .95 & .346 & 3.78 & .375 & 35 & 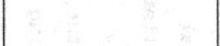 & 9 & v & 5 & 15 & .855 & 10 & .235 & 75 & 30 & 8.5 & 11 & - \\
\hline Boeing $747 / 100-200$ & 445 & .97 & .267 & 3.60 & .264 & 37 & & 9 & v & 3 & 12 & 1.000 & 8.5 & .185 & 77 & 31 & 17 & 23 & $\mathbf{T}$ \\
\hline Lockheed C-5A & 410 & .875 & .156 & 4.89 & .364 & 24.5 & & 10 & v & 4 & 12 & .700 & -4.5 & .268 & 65 & 30 & 10 & 20 & - \\
\hline
\end{tabular}

* $F=$ fixed; $M=$ all-flying; $V=$ variable incidence

** $S=$ servo tab; $T=$ trim tab

Table 9-2. Horizontal tailplane design data 


\section{b. Detailed procedure.}

The results of stability and control considerations are collected in $\mathrm{s}_{\mathrm{h}} / \mathrm{s}$ vs. $\mathrm{x}_{\mathrm{cg}} / \overline{\mathrm{c}}$ plots (Fig. 9-19a), indicating a region where $s_{h} / S$ may be chosen freely. It is advisable to choose the most critical mass (distribution) for each limit.

For aircraft with REVERSIBLE CONTROLS the flying speeds are generally not very high and compressibility effects need not always be taken into account.

1. Calculate the 1ift gradient $\left(\mathrm{C}_{\mathrm{L}_{\alpha}}\right)_{\mathrm{A}-\mathrm{h}}$ and the aerodynamic center $x_{a c}$ for the aircraft without horizontal tailplane.

2. Determine $d \varepsilon / d \alpha$ and the tailplane $11 \mathrm{ft}$-curve slope and calculate $c_{L_{\alpha}}$ and $x_{n}$ as a functicn of $s_{h} / S$, using $(9-5)$ and $(9-6)$.

3. Choose a value for $c_{h \delta}$ - using, for example, Figs. 9-5 and 9-5 - and a (low) value for $C_{h_{\alpha}}$. 4. Calculate the stick-free $11 \mathrm{ft}$-curve slopes of the tailplane and the aircraft, and the stick-free neutral point with (9-9) through $(9-11)$.

5. Choose a value for $G$ and calculate the forward and aft c.g. limits determined by control force stability requirements, using $(9-14)$ through (9-16). 6. Determine the stick-free maneuver points at the maximum cruising altitude and at sea level with (9-21) and $(9-22)$.

7. Calculate the forward and aft c.g. 1imits imposed by the maximum and minimum stick force per $g$ requirements, respectively, using $(9-24)$ and Table 9-1.

8. Use $(9-40)$ through $(9-43)$ to estimate $s_{h} / S$ vs. the c.g. position which permits $C_{L}$-max to be obtained with flaps in the landing position.

9. Limits for $s_{h} / S$ vs. $x_{c g} / \bar{c}$ may also be derived from $(9-44)$ through $(9-46)$ and from $(9-49)$, provided sufficient data are available.

10. If it is found that any of the conditions in relation to the control forces unduly restricts the forward c.g. limit, the value of $c_{h_{\delta}}$ should be revised.

Aircraft flying at high-subsonic speeds or large, high-speed propeller aircraft often have IRREVERSIBLE CONTROLS. Sub-critical compressibility effects on the aerodynamic properties should be taken into account as far as possible.
1. and 2. These steps in the procedure are the same as those above, but all coefficients are calculated for high-speed, high-altitude cruising as well as for low speeds. Typical angles of attack are about 10 degrees without slats and 15 degrees with slats extended.

3. In the case of jet aircraft determine the shift of the neutral point resulting from engine action, using $(9-29)$ for engines placed below the c.g. For propeller aircraft a similar forward shift is obtained from $(9-27)$ and $(9-5)$.

4. Locate the aft c.g. 11mit about $.015 \mathrm{l}_{\mathrm{h}}$ ahead of the stick-fixed neutral point with engines operating at low speeds and high power.

5. Calculate the stick-fixed maneuver points at the maximum cruising altitude and at sea level.

6. Determine $I_{y}$ at the maximum takeoff weight, using Fig. 9-15. Provided the angle of sweep is not excessive, the influence of the fuel can be ignored.

7. Calculate $\left(\mathrm{C}_{\mathrm{m}_{\mathrm{q}}}+\mathrm{C}_{\mathrm{m}_{\dot{\alpha}}}\right) /\left(\mathrm{C}_{\mathrm{L}_{\alpha}} \mathrm{K}_{\mathrm{Y}}{ }^{2}\right)$ with $(9-36)$ and enter this value in Fig. 9-11 (in the example 3.0). The maximum and minimum values of $L_{\alpha} / \omega_{n}$ for satisfactory SP characteristics are then read off.

8. The minimum and maximum stick-fixed maneuver margins are calculated with $(9-34)$ and subtracted from the $x_{m}$ values to obtain aft and forward c.g. limits for each condition.

9. and 10. These steps are identical to steps 8 and 9 for aircraft with reversible controls.

In the case of aircraft with a T-tail and engines pod-mounted on the rear fuselage, the tailplane area for the aft c.g. $11 \mathrm{mit}$ is increased by, say, 15 percent to allow for the deep stall case.

The most critical requirements for $\mathrm{s}_{\mathrm{h}} / \mathrm{s}$ are now selected and plotted in a diagram (Fig. 9-19a) which defines the "aerodynamic" limits to the c.g. range. This diagram must be balanced with the c.g. range required to obtain good loading flexibility (Section $8 \cdot 5.2$.). The following graphical solution is suggested.

1. In a diagram (Fig. $9-19 \mathrm{~b}$ ) on transparent paper the c.g. range of the aircraft without horizontal tailplane is represented as a function of the position of the wing in the longitudinal direction. 2. The magnitude of the c.g. shift as a result of the tailplane weight variation with $s_{h} / s$ is indicated in the $s_{h} / s$ vs. $x_{c g} / \bar{c}$ diagram (Fig. 9-19b): 
$\frac{\Delta_{h} x_{c g}}{\bar{c}}=\frac{s_{h} l_{h}}{s \bar{c}} \frac{\text { specific tailplane weight }}{\text { wing loading }}$

3. Fig. 9-19b is now placed over Fig. 9-19a in such a way that the $\mathrm{X}$ axes are parallel. The aircraft will be balanced (Fig. 9-19c) when the following points of intersection occur at an identical value of $\mathrm{s}_{\mathrm{h}} / \mathrm{s}$ :

- the point of intersection between the acceptable and desired aft positions of the c.g.,

- the point of intersection between the acceptable and desired forward positions of the c.g., and

- the point of intersection of the vertical axis in Fig. 9-19b and the line given by (9-51) in Fig. 9-19a.

4. The optimum values for $s_{h} / S$ and the balanced location of the wing may now be read off (Fig. 9-19c).

If it proves difficult or impossible to obtain a satisfactory balance, the following modifications may be considered:

1. altering the design of the tailplane;

2. changing the position of the tailplane, for example by increasing $l_{h}$, if necessary by extending the length of the fuselage;

3. changing the mass distribution, particularly that of the fixed equipment-fixed ballast is to be avoided - or altering the arrangement of the fuselage interior (see also section 8.5.2.);

4. limiting the c.g. range by imposing loading restrictions.

If none of these measures $y$ ields a satisfactory solution, a complete revision of the general arrangement may be required. In this case it may be found that an alternative disposition of the powerplant is unavoidable.

\subsection{DESIGN OF THE VERTICAL TAILPLANE}

The design of the vertical tailplane is more complicated than that of the horizontal tailplane. It is generally quite difficult to calculate the lateral-directional aerodynamic characteristics, since they are closely connected with a complicated asymmetrical flow field behind the wing/ fuselage combination, which meets the oncoming air at an angle of sideslip.
The following broad design criteria are relevant in this respect:

a. The vertical tailplane must not stall as a result of an oscillation after deflection of the rudder or a sudden engine failure.

b. After failure of the critical engine, multi-engined aircraft must remain controllable to ensure steady flight.

c. It should be possible to land transport aircraft in crosswinds of up to 30 knots (55 km/h).

d. The aircraft must possess positive directional and lateral static stability and the short-period lateral/directional oscillation (Dutch roll) must be well damped. Some degree of spiral instability will be acceptable for aircraft fitted with an automatic pilot.

The effectiveness of the vertical tailplane is difficult to calculate as a result of the large variety in shapes. The NACA has recommended certain definitions which lead to reasonable solutions (Fig. 9-20). For
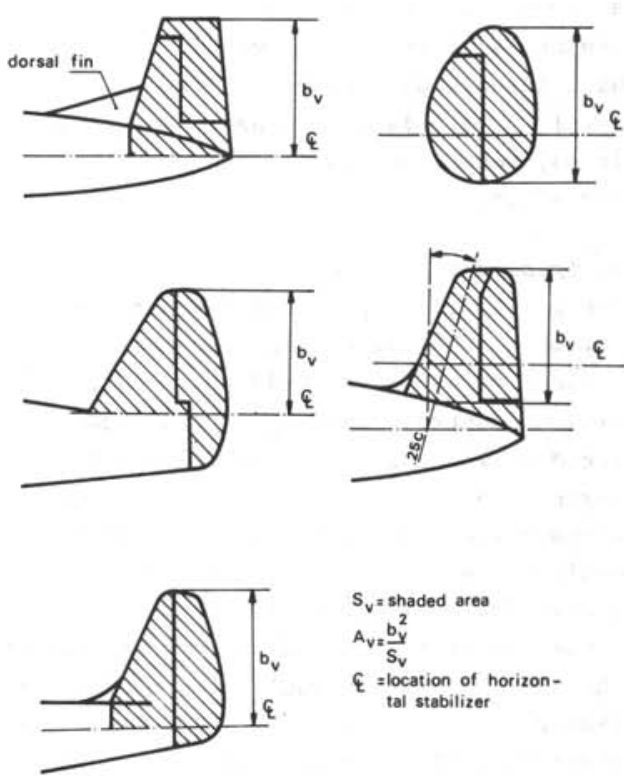

$S_{v}=$ shaded area

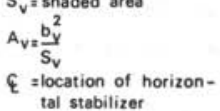

tal stabilizer

Fig. 9-20. Aerodynamic definitions of vertical tailplane area and aspect ratio 
comparison purposes it should be pointed out that the R.Ae.S. Data Sheets use another definition, while some manufacturers base the area on structural considerations. Attention is drawn to the special case of a T-tail (Fig. 2-26), where the aerodynamic aspect ratio is considerably larger than the geometric.

9.6.1. Control after engine failure: multiengine aircraft

Official requirements can be found in FAR 23.149 and 25.149, BCAR Sections $K$ and $D$, Ch. 02-8 Paragraph 4 .

Engine failure must be investigated for multi-engine aircraft in all configurations in a specified range of speeds, while, in addition, failure of two engines in the en route configuration must be catered for in the case of a four-engine aircraft.

Since engine failure generally causes a disturbing yawing moment and - although to a lesser degree - also a rolling moment, deflections of both the rudder and the ailerons will be required. When the dead engine is fitted to the wing, this case will largely determine the size of the vertical tailplane and the rudder capacity. It may sometimes also affect the design of the ailerons.

a. Transient behavior.

The pilot will experience a yawing oscillation which will be more violent at low flying speeds (Refs, 9-49 and 9-50). The minimum control speed $v_{M C}$ is the lowest speed at which control can be established during the takeoff run - by aerodynamic means $-\mathrm{V}_{\mathrm{MC}_{\mathrm{G}}}$ or in flight $\mathrm{V}_{\mathrm{MC}_{\mathrm{A}}}$, with an angle of bank of not more than five degrees. The angle of sideslip involved immediately after the failure must not stall the fin, since that would prevent equilibrium from being re-established. Transient sideslip angles may peak to a value of 1.6 times the angle in steady flight, and angles of up to 20 to 25 degrees are no exception with wing-mounted engines. This "overshoot" may be restricted by such measures as:

- The use of a yaw damper (see Section

7.7.1.) which superimposes rudder deflections on those initiated by the pilot.

- The use of a pneumatic system which derives a control signal from the pressure differential in the compressors of corresponding engines (Hawker Siddeley 125). The following measures will reduce the likelihood of vertical tailplane stall: 1. The choice of a moderate aspect ratio such as $A_{V}<1.8$ - and a fairly large fin. 2. The use of a swept-back leading edge.

3. The addition of a dorsal fin (Fig.

9-20), which does not contribute materially to the directional stability at small angles of sideslip, but will postpone the stall on the lower part of the fin at large angles.

b. Steady flight after engine failure. A good starting point for sizing the vertical tailplane and rudder for aircraft with an outboard engine is formed by the condition of equilibrium which must be established some time from the engine loss. We have to consider the following forces and moments in sideslipping flight. with one engine inoperative (Fig. 9-21):

1. Contribution of the asymmetry in the thrust $\left(\Delta \mathrm{T}_{\mathrm{e}}\right)$ at a distance $\mathrm{y}_{\mathrm{e}}$ from the plane of symmetry. Provided the aircraft is fitted with an automatic feathering system, the drag of the dead engine (and propeller) can be ignored and the disturbing yawing moment will be:

$\mathrm{N}_{\mathrm{e}}=\Delta \mathrm{T}_{\mathrm{e}} \mathrm{Y}_{\mathrm{e}}$

2. Side force and yawing moment on the aircraft without the vertical tailplane (subscript $\mathrm{A}-\mathrm{v}$ ) :

$\mathrm{Y}_{\mathrm{A}-\mathrm{V}}=\frac{\partial \mathrm{Y}_{\mathrm{A}-\mathrm{V}}}{\partial \mathrm{B}}$

$\mathrm{N}_{\mathrm{A}-\mathrm{v}}=\frac{\partial \mathrm{N}_{\mathrm{A}-\mathrm{v}}}{\partial \mathrm{B}}$

3. Contribution of the aircraft weight:

$\mathrm{W} \sin \varphi=\mathrm{L} \tan \varphi \approx \mathrm{L} \varphi$ for $\varphi \leqslant 5^{\circ} \quad(9-55)$ 


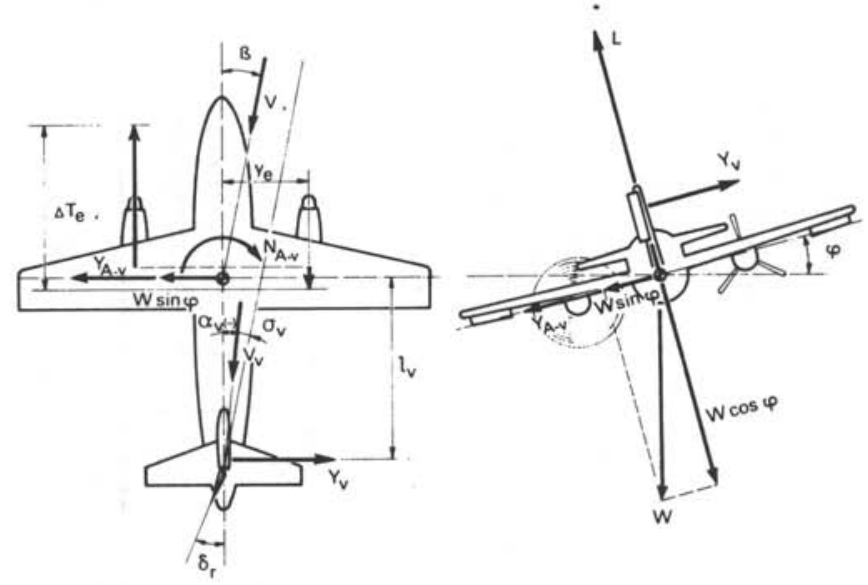

Fig. 9-21. Flight on asym-: metric power

where the difference between the angles of bank and roll has been ignored.

4. Side force and yawing moment due to the vertical tailplane:

$\eta_{v} C_{Y_{v}} \frac{s_{v}}{S}=\frac{C_{L} \frac{y_{e}}{l_{v}} \frac{\Delta T}{w}+B\left(c_{n_{B}}\right)_{A-h} \frac{b}{l_{v}}}{{ }^{\tau} v^{\delta}{ }_{r}-\left(B-\sigma_{v}\right)}$

$\mathrm{Y}_{\mathrm{v}}=\frac{\partial \mathrm{Y}_{\mathrm{v}}}{\partial \alpha_{\mathrm{v}}} \alpha_{\mathrm{v}}+\frac{\partial \mathrm{Y}_{\mathrm{v}}}{\partial \delta_{\mathrm{r}}} \delta_{\mathrm{r}}$

(9-56) where

$\mathrm{N}_{\mathrm{v}}=-\mathrm{Y}_{\mathrm{v}}{ }_{\mathrm{v}}$

$(9-57) \quad n_{v}=\left(\frac{v_{v}}{v}\right)^{2}$

where:

$\alpha_{\mathrm{v}}=-\left(\beta-\sigma_{\mathrm{v}}\right)$

The yawing moment due to aileron deflection has been neglected. Since the position of the airplane c.g. has little influence during flight with engine failure, $\ell_{\mathrm{v}}$ is taken relative to the mean quarter chord point. In the case of a propeller aircraft, as depicted in Fig. 9-21, the sidewash angle $\sigma_{\mathrm{v}}$ will have a negative sign, with the result that the effect of the yawing moment due to engine failure will in fact be increased, particularly in the case of a high-wing aircraft (Ref. 9-54).

The simplest expression for the equilibrium condition is obtained by eliminating $\varphi$. After introduction of the usual nondimensional coefficients (see the Nomenclature) and some algebraic manipulations, we find the following condition for the vertical tailplane area required:

The numerator represents - in nondimensional form - the yawing moments due to engine failure and sideslip on the aircraft, less vertical tailplane. The first term of the denominator is the change in zero-lift angle of the tailplane caused by rudder deflection, and the bracketed term defines the direction of the local airflow, which is dependent on the direction of rotation of the revolving propeller(s). If the operating propeller(s) revolve(s) clockwise - viewed from behind the aircraft - the critical engine will be the outboard port engine. This asymmetry is not present on jet aircraft.

Fig. 9-22 shows that a negative sideslip angle makes it possible to use a smaller tailplane as compared with $B=0$. However, the condition imposed by the angle of bank of five degrees limits the usable sideslip angle, and for $\Delta T_{e} y_{e} C_{L} /\left(W_{l}\right)>.15$ (approximately) this limit will considerably increase the control capacity required. If 


\begin{tabular}{|c|c|c|c|c|c|c|c|c|c|c|c|c|c|c|c|c|}
\hline Aircraft type & \begin{tabular}{|l|}
$V_{D}$ \\
kts \\
EAS
\end{tabular} & $\begin{array}{l}\text { MD } \\
-\end{array}$ & $\begin{array}{l}v_{M C} \\
\text { kts } \\
\text { EAS }\end{array}$ & $\begin{array}{l}\text { Max. } \\
\text { cross- } \\
\text { wind } \\
\text { kts }\end{array}$ & $\frac{\mathrm{S}}{\mathrm{s}}$ & $A_{v}$ & $\begin{array}{l}\Lambda_{\mathrm{v}} \\
\text { deg. }\end{array}$ & $\begin{array}{l}\text { Airfoil Section } \\
\text { root / tip }\end{array}$ & $\begin{array}{c}\text { Average } \\
t / c \\
\%\end{array}$ & $\frac{S_{v}{ }^{1} v}{S b}$ & $\frac{s_{r}}{s_{v}}$ & $\begin{array}{c}\text { Hinge } \\
\text { Position } \\
\text { root/tip } \\
\% c_{v}\end{array}$ & $\begin{array}{c}\text { Balance } \\
\text { Ratio } \\
\% c_{r} \\
\end{array}$ & $\begin{array}{l}\stackrel{+}{\max } \mathrm{r}_{\max } \\
\operatorname{deg} .\end{array}$ & $\begin{array}{c}\text { Tabs } \\
*\end{array}$ & Remarks \\
\hline Wright Flyer (1903) & & & & & .045 & 2.91 & 0 & "flat plates" & - & .0133 & 1 & 30 & 42.8 & & & biplane \\
\hline Scottish Aviation Pup & & है. & & 30 & .182 & .89 & 33 & & 12 & .0750 & .350 & 64 & & 25 & $\mathrm{~T}$ & Horn bal \\
\hline Cessna 177 & & & & 20 & .107 & 1.41 & 35 & NACA $0009 / 0006$ & 7.5 & .0411 & .368 & 60 & & & & \\
\hline Scottish Av. Jetstream & & .60 & 85 & - & .212 & 1.44 & 43 & & & .0820 & .350 & 65 & & & & Horn balance \\
\hline Aérospatiale N262 & 260 & & 90 & 30 & .184 & 1.60 & 9.5 & NACA 0012 mod. & 12 & .0763 & .270 & 68 & 31.2 & & $\mathrm{~T} / \mathrm{C}$ & \\
\hline Fokker-VFW F 27 Mk 200 & 288 & .51 & 78 & 30 & .203 & 1.55 & 3.3 & NACA $63 \mathrm{~A}-015 \mathrm{mod}$. & 15 & .0765 & .218 & 76 & 44.6 & 20 & $\mathrm{~T} / \mathrm{C}$ & \\
\hline Lockheed Hercules C- $130 \mathrm{E}$ & 325 & .64 & 93.5 & & .180 & 1.84 & 18.8 & NACA $64 A-015$ & 15 & .0575 & .239 & 75 & & 35 & $\mathrm{~T} / \mathrm{C}$ & \\
\hline Lockheed Electra $188 \mathrm{~A}$ & & .711 & 110 & & .145 & 1.93 & 14.0 & NACA 0012 & 12 & .0707 & .250 & 69 & 25.8 & $\begin{array}{l}+23.5 \\
-30\end{array}$ & $\mathrm{~T}$ & \\
\hline Bristol Britannia 310 & 300 & 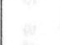 & & $\&$ & .197 & 1.65 & 10 & RAF30 mod. & 13 & .0774 & .225 & $60 / 67$ & 30 & 25 & $\mathrm{~T} / \mathrm{S}$ & $\delta_{r} \pm 16^{\circ}$ for $\mathrm{CL}-44$ \\
\hline Aérospat. Corvette $\mathrm{SN}-600$ & & & 90 & te & .177 & .88 & 50.3 & & 9 & .0720 & .250 & 72 & 25 & 30 & $\mathrm{~T}$ & 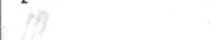 \\
\hline Cessna Citation 500 & & 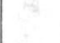 & & & .191 & 1.58 & 33.0 & NACA $0012 / 0008$ & 10 & .0806 & .220 & 75 & & & $\mathrm{~T}$ & \\
\hline Hawker Sidd eley HS- $125 / 400$ & 370 & .825 & 90 & & .161 & 1.19 & 52 & & 11.5 & .0548 & .197 & 72 & 25 & 28.5 & $\mathrm{~T}$ & Approx. T-tail \\
\hline Yakovlev YAK-40 & & & t. & & .162 & .81 & 47.5 & & 10 & .0442 & .222 & 78 & & 30 & $\mathrm{~T}$ & T-tail \\
\hline VFW-Fokker 614 & 330 & .74 & & & .174 & 1.28 & 32.4 & 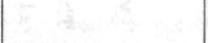 & & .0682 & .321 & & & 35 & $\mathrm{~T} / \mathrm{S}$ & \\
\hline Fokker-VFW F 28 Mk 1000 & 390 & .83 & 71 & 25 & .203 & 1.00 & 40 & NACA mod. & 10.15 & .0910 & .187 & 78 & 30 & 33 & 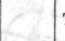 & T-tail \\
\hline Aérospat. Carave11e 10.R & 375 & .87 & & & .106 & 1.24 & 37.9 & NACA $65-011$ & 11 & .0379 & .258 & $76 / 40$ & & 24 & & $=1$ \\
\hline BAC- $111 / 200,400$ & 410 & .86 & & 30 & .132 & .91 & 41.0 & & 12.5 & .0482 & .254 & 70 & 30 & & & T-tail \\
\hline McDonnell Doug1 as DC-9/10 & & .89 & & & .192 & .95 & 43.5 & DSMA & 11 & .0810 & .270 & 68 & 35 & 30 & $\mathrm{~T} / \mathrm{C}$ & T-tail \\
\hline Boeing $737 / 100$ & 400 & .89 & & & .268 & 1.88 & 35 & & 12 & .1117 & .250 & 75 & & & $\mathrm{~T}$ & \\
\hline Dassault Mercure & & & & & .232 & 1.96 & 35 & & 9 & .1025 & .221 & 71.5 & & & & Split rudder \\
\hline Boeing $727 / 100$ & & .95 & 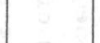 & & .238 & .78 & 55 & & 9 & .0905 & .168 & 80 & & & & T-tail \\
\hline Airbus $A-300 B$ & 420 & .90 & 103 & & .204 & 1.62 & 40 & & 12.5 & .1020 & .248 & 70 & & & & \\
\hline Boeing KC- 135 & & & 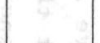 & 100 & .143 & 1.49 & 31 & & 10 & .0628 & .250 & 65 & & 20 & $\mathrm{~s}$ & \\
\hline Boeing $707 / 120$ & & & 107.5 & & .148 & 1.62 & 31 & & 10 & .0656 & .282 & 65 & & 25 & $\mathrm{~s}$ & \\
\hline Boeing $707 / 320 \mathrm{~B}$ & & .95 & 122 & & .144 & 1.81 & 31 & & 10 & .0626 & .242 & 65 & & 25 & s & \\
\hline McDonnel1 Douglas DC- $8 / 10,50$ & 405 & .95 & $<v_{\text {LOF }}$ & 34 & .122 & 1.91 & 35 & DSMA-111/-112 & 9.85 & .0494 & .269 & 65 & 37.2 & 32.5 & c & \\
\hline BAC vC-10/1101 & 380 & .94 & 200 & a & .142 & 1.10 & 38.5 & & & .0453 & .251 & & & & & T-tail \\
\hline Lockh. Tristar L-1011/1 & 435 & .95 & & 30 & .231 & 1.83 & 35 & & 10 & .0830 & .161 & 70 & & & & \\
\hline McDonnel1 Doug1as DC- $10 / 10$ & & .95 & & 30 & .221 & 1.92 & 40 & $\sim 12 \% / \sim 10 \%$ & 11 & .0811 & .145 & 62 & & $23 / 46.5$ & & Tandem rudder \\
\hline Boeing $747 / 100,200$ & 445 & .97 & $103 / 138$ & 30 & .196 & 1.38 & 44 & & & .0990 & .173 & 77 & 42 & 25 & $\mathrm{~T}$ & Split rudder \\
\hline Lockheed C-5A & 410 & .875 & 3 & 43 & .191 & .84 & 34.9 & & 5 & .0951 & .191 & & & & & T-tail \\
\hline
\end{tabular}

${ }^{*} \mathrm{C}=$ control tab; $\mathrm{S}=$ servo tab; $\mathrm{T}=$ trim tab

Table 9-3. Vertical tailplane design data 


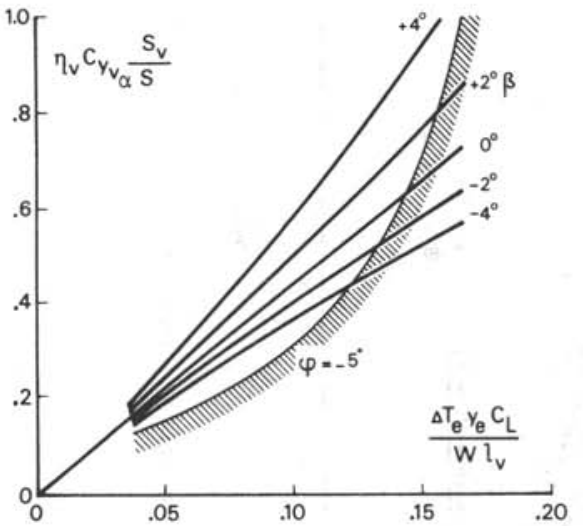

Fig. 9-22. Effect of sideslip on vertical tailplane area required to cope with engine failure

this criterion is exceeded to any great extent, a method of reducing the yawing moment will have to be found. The possibility of interconnecting the propeller shafts (Bréguet 941) and/or adopting an unconventional method of directional control - such as cyclic pitch of the propellers, control by means of air jets or a propeller at the tail (as in helicopters) will have to be investigated in this case.

Practical application of (9-59) is hampered by the difficulty that $\sigma_{\mathrm{v}}$ cannot be predicted with any accuracy for propeller aircraft. In addition, a combination of $W$ and $C_{L}$ has to be substituted, which means, in effect, that a choice of $v_{M C}$ must be made. The statistics in Fig. 9-23 and Table 9-3 are intended to replace a detailed study by a rapid estimation of the vertical tail area required, or rather the tailplane area on which the rudder is fitted. Large tailplanes can be avoided in the following ways:

1. Ensuring that the critical engine is not located further outboard than is strictly necessary. Another way of reducing $\mathrm{y}_{\mathrm{e}}$ is to adjust the angle of the jet efflux (see Fig. 6-18).

2. Choosing a favorable location for the vertical tailplane, or possibly by using twin tails.

3. Employing a special rudder design, e.g. one consisting of two segments (double-hinged or varicam rudder, see the De Havilland Canada DHC-7 and the
DC-10) or a rudder provided with guide vanes (Fokker P 301 sToL project).

4. Choosing an aspect ratio which is not too low. A T-tail will increase the effective aspect ratio, but for structural reasons the geometric aspect ratio will generally not exceed 1.2 .

5. Increasing the tail moment arm (longer fuselage, swept-back $\mathrm{fin}$ ).

An approximate limit would be a rudder chord of 30 to 35 percent of the tailplane chord with a maximum rudder deflection of 25 to 30 degrees. In the case of manual control the rudder deflection may be limited by the requirement that the pressure on the rudder pedals should not exceed $180 \mathrm{lb}(81.6 \mathrm{~kg})$. In connection with Fig. 9-23 it should be noted that the choice of $\mathrm{S}_{\mathrm{v}}$ may have been decided by other criteria in the case of several aircraft. In the absence of relevant data, the rudder deflection of some aircraft has been assumed at 25 degrees. These factors may explain the fairly wide disparities in the lower limit values for a number of types.

c. Drag caused by an inoperative engine.

A detailed account of this drag increment is given in Appendix G, Section G-8. Only one contribution will be mentioned here: the vortex-induced drag resulting from the side force on the vertical tailplane, which may be expressed as a fraction of the total vortex-induced drag:

$\frac{\Delta C_{D}}{C_{L}{ }^{2} /(\pi A)}=\left(\frac{\Delta T_{e} y_{e} b}{W l_{v} b_{v}}\right)^{2}$

This expression shows that $\Delta C_{D}$ is proportional to $\left(\mathrm{y}_{\mathrm{e}} / \mathrm{l}_{\mathrm{v}} \mathrm{b}_{\mathrm{v}}\right)^{2}$, which proves that the recommendations set out above deserve special attention.

\subsubsection{Lateral stability}

\section{a. Directional stability.}

Every aircraft must possess positive stickfixed and stick-free directional stability in normal operational conditions, for all aircraft configurations. The following applies to the stick-fixed directional stability, which may be used as a preliminary design tool: 


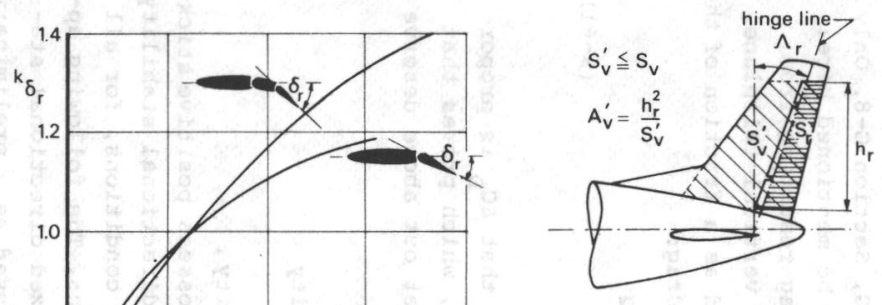

$k_{v}=1.1, T$ tail

$=1.0$, all other configu-

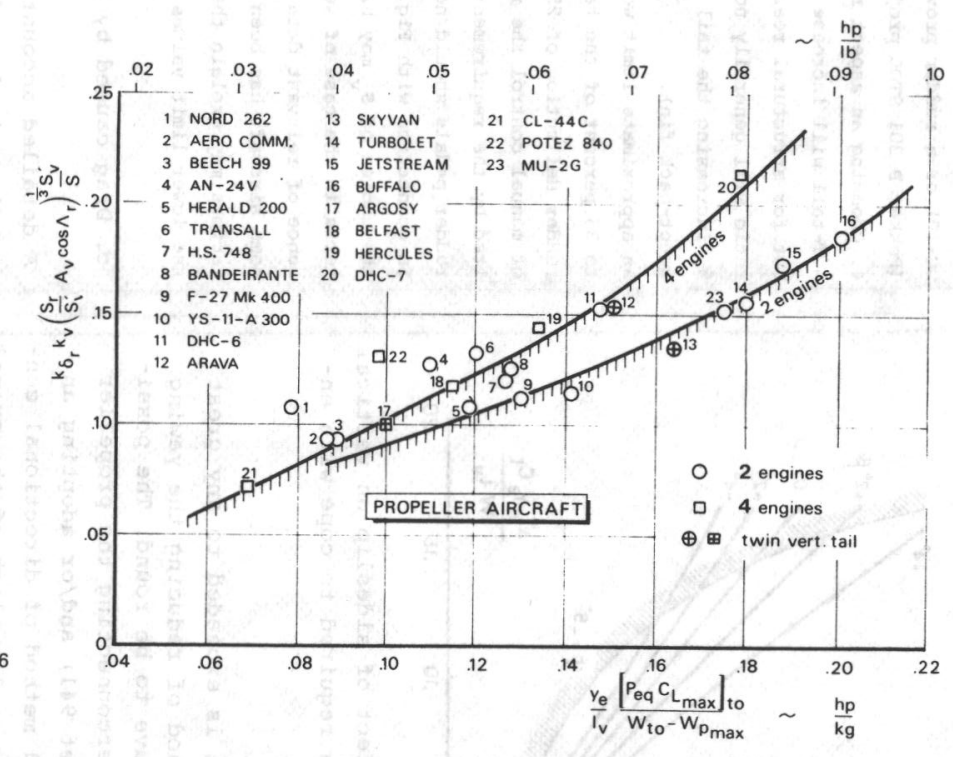

Fig. 9-23. Rapid estimation of vertical tailplane size for multi-engined aircraft with wing-mounted engines 
$c_{n_{B}}=\left(c_{n_{B}}\right)_{A-h}+n v c_{y_{\alpha}} \frac{s_{v} v^{l} v}{s b}\left(1-\frac{d \sigma}{d B}\right)\left(\frac{v_{v}}{v}\right)^{2}>0$

where $n_{v}$ is defined by $(9-60)$. The contribution of engine nacelles to the directional stability is generally small and may be ignored. The same applies to the wing contribution, provided the sweep angle is not too large. Wing/fuselage interference is manifest both in $\left(c_{n_{B}}\right)_{A-h}$ and $\sigma_{v} ;$ these effects will be combined in a single coefficient $\Delta_{i} C_{n_{B}}$, which is mainly dependent on the location of the wing in relation to the fuselage in $\mathrm{z}$-direction. The vertical tail volume required to obtain a specified $c_{n_{\beta}}$ is obtained from:

$\frac{s_{v} v_{v}}{s b}=\frac{c_{n_{B}}-\left(c_{n_{B}}+c_{n_{B}}+\Delta_{i} c_{n_{B}}\right)}{c_{y_{v_{\alpha}}}\left(v_{v} / v\right)^{2}}$

The degree of directional stability cannot be deduced in a straightforward manner from existing requirements. A minimum value of $c_{n_{\beta}}=.03$ is sometimes recommended, but this is generally inadequate for ensuring a well-damped Dutch roll. In the case of single-engine subsonic aircraft $\mathrm{c}_{\mathrm{n}_{B}}$ is often found to 1 ie between .04 and .10, while for transport aircraft values generally range from .10 to .25. For aircraft with swept wings $c_{n_{\beta}}$ is a function of $C_{L}$, while a part is also played by deflection of the flaps.

An estimate of the tail volume required may be made with the help of Fig. 9-24, which applies to aircraft with engines either mounted close to or buried in the fuselage. Aircraft with wing-mounted engines are not mentioned here, since flight with engine failure will mostly be the deciding case.

The following aerodynamic data* have been used to derive the contributions to $c_{n_{B}}$ in Fig. 9-24:

*derived from Ref. 9-1.

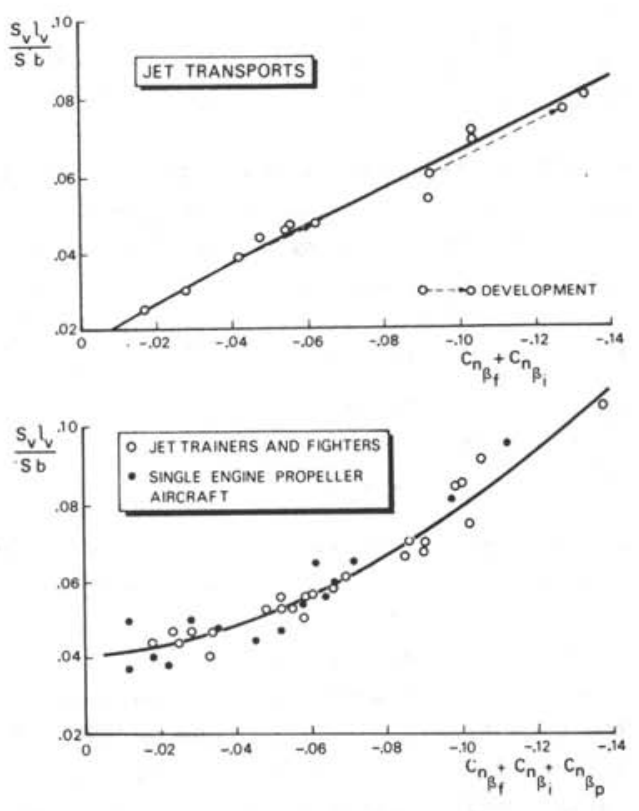

Fig. 9-24. Rapid estimation of vertical tail volume for aircraft with fuselagemounted engines

$c_{n_{f}}=-K_{B} \frac{S_{f s} l_{f}}{S b}\left(\frac{h_{f_{1}}}{h_{f_{2}}}\right)^{1 / 2}\left(\frac{b_{f_{2}}}{b_{f_{1}}}\right)^{1 / 3}$

where, for $l_{f} / h_{f_{\max }} \geqslant 3.5$,

$K_{B}=.3 \frac{l_{\mathrm{cg}}}{l_{f}}+.75 \frac{h_{f_{\max }}}{l_{f}}-.105$

The various dimensions are defined in Fig. 9-25

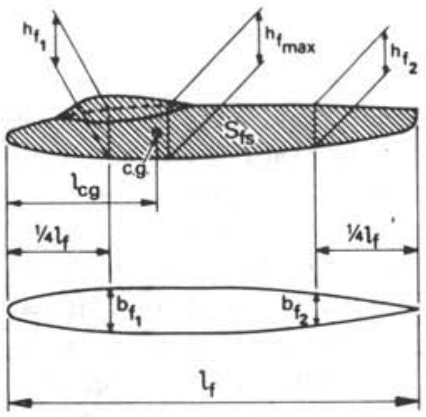

Fig. 9-25. Fuselage geometry in relation to the yawing moment due to sideslip. 
$c_{n_{p}}=-.053 B_{p} \sum \frac{l_{p} D_{p}^{2}}{S b}$

where $l_{p}$ is the distance from the (tractor) propeller plane to the c.g., while $B_{p}$ and $D_{p}$ denote the number of blades per propeller and the propeller diameter, respectively. The summation is carried out for all operating propellers.

Finally we have approximately:

$$
\begin{aligned}
\Delta_{i} c_{n_{B}} & =-.017, & \text { high wing } \\
& =+.012, & \text { mid wing } \\
& =+.024, & \text { low wing }
\end{aligned}
$$

If the design provides for twin vertical tailplanes mounted on the horizontal stabilizer, use $\Delta_{1} C_{n_{B}}$

b. Lateral dynamic stability.

This subject is generally not included in the preliminary design process because of a lack of data and the absence of quantitative design criteria. Some design objectives are laid down in Ref. 9-13 with respect to the damping of the Dutch roll oscillation and the amount of spiral stability required. It should be noted that the dihedral of the wing has a great influence on lateral stability, and since this parameter can be varied without greatly affecting the general arrangement, some measure of freedom is available to the designer, even at a later stage of the project. As explained in Section 7.7.1. most highspeed aircraft with swept wings are equipped with a yaw damper to obtain an adequately damped Dutch roll mode.

\subsubsection{Crosswind landings}

In order to ensure regularity in air transport operations, landings may have to be carried out at crosswind velocities of up to, say, 30 knots $(15.5 \mathrm{~m} / \mathrm{s})$. The associated angle of sideslip to be dealt with will be of the order of 12 to 15 degrees for conventional transport, but may rise to 20 degrees for STOL aircraft. Equation (9-59) may be used to find a lower limit to the vertical tail size by taking $\Delta \mathrm{T}_{\mathrm{e}}=0$ : $n_{v} C_{v_{\alpha}} \frac{s_{v}}{s}=\frac{\beta\left(C_{n_{B}}\right)_{A-v} \frac{b}{{ }^{\ell} v}}{\tau_{v}{ }^{\delta}{ }^{-\left(\beta-\sigma_{v}\right)}}$

where $\tau_{v}$ takes account of the nonlinearity in the $c_{Y}$ vs. $\delta_{r}$ curve when the rudder deflection is large. Although this criterion looks quite simple, a detailed knowledge of the various factors is required to make it usable, since small variations in the terms in the denominator have a great effect on the result. Accurate windtunnel data are therefore required. Ensuring control over the airplane on a wet runway in a crosswind is another important design problem. It should be noted that the operation of thrust reversers may have an adverse effect.

\subsubsection{The spin}

Aircraft to be certified in the Aerobatic Category and some types in the Utility Category must have safe spinning characteristics.

The loading of the airplane, which determines the inertial moments about the three axes, dictates what controls have to be used for recovery from a spin. Particularly in the case of light aircraft, the rudder is usually the most effective control since it provides the yawing moment required to oppose the spin rotation. Additional elevator deflection may be required to apply an antispin yawing moment on aircraft where the mass is heavily concentrated in the wing (engines, fuel, tip tanks).

The shielding effect of the horizontal tail is responsible for a dead air region over much of the vertical tail (see Fig. 2-27). As a rule, it can be said that at least one-third of the rudder area should remain outside this wake, assuming that for an angle of attack of 45 degrees the forward and aft boundaries of the wake form an angle of 60 and 30 degrees respectively with the horizontal tail. In addition, there should be a substantial amount of fixed 
area beneath the horizontal tail to provide damping of the spinning motion. More accurate design criteria have been developed by the NACA; these involve the polar moments of inertia about the longitudinal and lateral axes. A detailed account of this is given in the references on airplane spinning. Spin recovery characteristics can also be affected to a large extent by antispin fillets and dorsal fins (Ref. 9-56).

\subsubsection{Preliminary design of the vertical tailplane}

\section{a. Aspect ratio $\mathrm{A}_{\mathrm{V}}$.}

The aspect ratio, which is defined in Fig. 9-20, has a direct effect on the tailplane contribution to $\mathrm{C}_{\mathrm{n}_{\beta}}$, which is approximate1y proportional to $A_{V}^{1 / 3}$. A high aspect ratio tailplane is effective at small angles of sideslip, but it has a small stalling angle of attack. A low $\mathrm{A}_{\mathrm{v}}$ is required with a high-mounted horizontal tailplane to provide adequate rigidity of the $f$ in without an excessive weight penalty.

\section{b. Taper ratio $\lambda_{\mathrm{v}}$.}

The tailplane contribution to lateral stability is only slightly affected by taper, which is applied mainly to reduce weight and/or increase the rigidity of the fin. Little taper is possible on T-tails.

\section{c. Angle of sweep $\Lambda_{\mathrm{V}}$.}

Design considerations are similar to those for the horizontal tailplane (Section

9.5.1c) but in addition it should be noted that in the case of a fin-mounted horizontal tailplane the moment arm of both tailplanes is increased. Most designers of private aircraft seem to prefer using a swept vertical tailplane in order to please their customers.

\section{d. Airfoil section .}

The usual sections for a vertical tailplane are symmetrical, have thickness ratios of about 12 percent and a relatively large nose radius to permit a large range of angles of attack.

e. Design procedures.

A simple approach is to choose the tailplane volume $s_{v^{\ell}} v^{/(S b)}$ by comparing design data of aircraft with a similar general layout in the same airworthiness category (Table 9-3). The location of the engines (wing- or fuselage-mounted), the wing (low, high or mid-wing), and the vertical position of the horizontal stabilizer are decisive factors. The process is iterative, in view of the fact that the moment arm $\ell_{v}$ cannot be determined accurately before the layout of the tailplane has been chosen. A more detailed procedure may be started if the designer is prepared to make assumptions regarding the (relative) rudder area and maximum deflection.

For multi-engine aircraft with wing-mounted engines a lower limit for $\mathrm{S}_{\mathrm{v}}$ ' can be derived from Fig. 9-23. A subsequent check should be made to ascertain that the volume is not less than the value dictated by Fig. 9-24* If sufficient data are available, the crosswind criterion given by (9-68) should also be checked.

For aircraft with engines mounted on or buried inside the fuselage Fig. 9-24* can be used. to estimate the volume parameter, and the crosswind criterion given by (9-68) may be checked as soon as the required aerodynamic data are available.

Light aircraft, which must demonstrate good spinning characteristics, should satisfy the criteria laid down in Section 9.6.4. It is advisable to apply the NACA criteria summarized in Refs. 9-57 and 9-58.

* The various contributions to $c_{n_{B}}$ are given by $(9-64)$ through $(9-67)$. 



\section{Chapter 10. The undercarriage layout}

\section{SUMMARY}

The basic requirements for the design of an undercarriage are that it must be capable of absorbing a certain amount of energy, both vertically and horizontally, and that during taxying, liftoff and touchdown no other part of the aircraft will touch the ground. No instabilities must occur, particularly during maximum braking effort, crosswind landings and high-speed taxying. In addition, the undercarriage characteristics must be adapted to the load-carrying capacity of the airfields from which the aircraft is intended to operate. This chapter indicates how these requirements can be translated into an acceptable initial choice of the undercarriage layout, without going into the details of its structural design. 
NOMENCLATURE

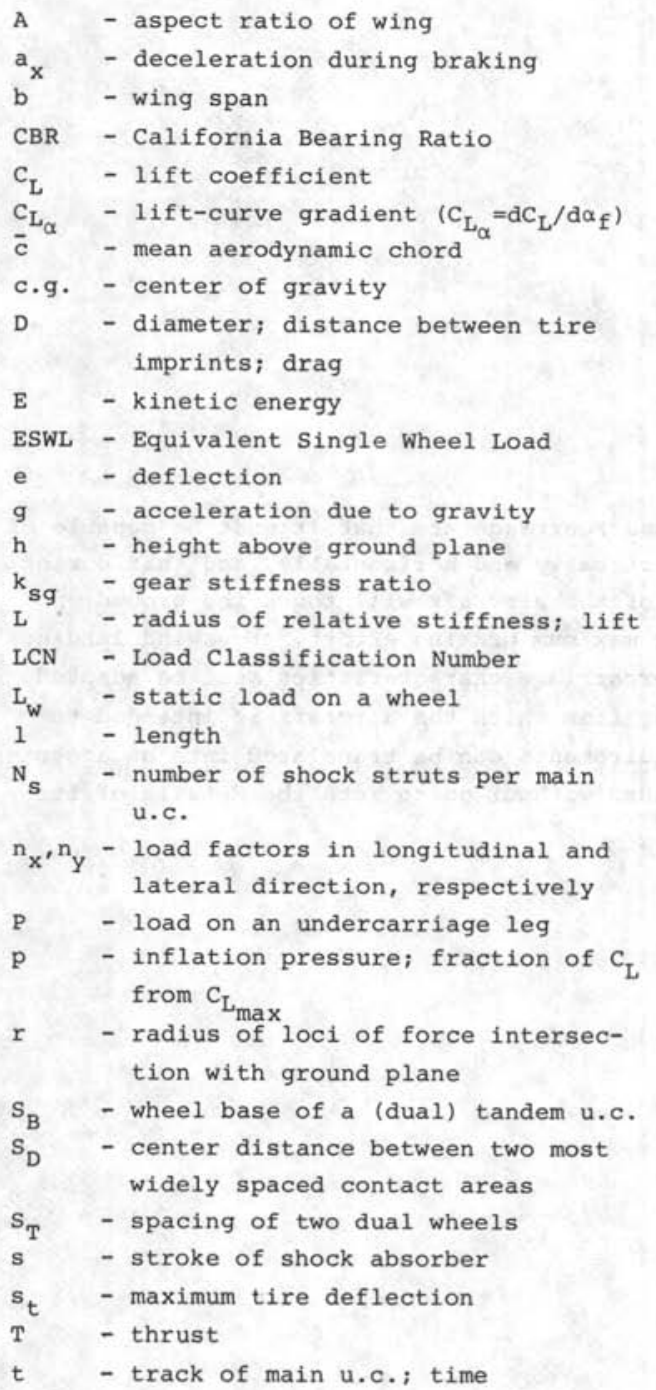

10.1. INTRODUCTION

Although the dimensions of the undercarriage are modest as compared to those of the wing or fuselage, it must not be regarded merely as an accessory, particularly since its function is much more that of an integral part of the structure. Its

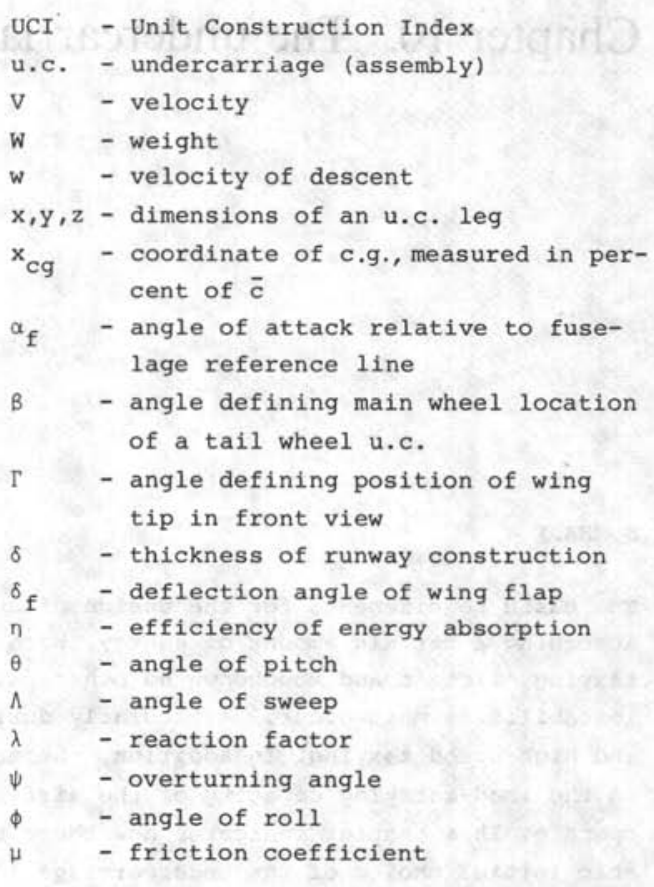

Subscripts

$\begin{array}{ll}\text { cg } & \text { - center of gravity } \\ \text { crit } & \text { - critical (stalling) } \\ \text { cr } & \text { - cruising } \\ \text { g } & - \text { (landing) gear } \\ \text { LOF } & - \text { liftoff } \\ \text { m } & \text { - main u.c. } \\ n & - \text { nose u.c. } \\ \text { s } & - \text { shock absorber; static } \\ \text { TD } & - \text { touchdown } \\ \text { t } & - \text { tire } \\ \text { to } & - \text { takeoff } \\ \text { uc } & - \text { undercarriage } \\ \text { w } & - \text { wheel } \\ .25 & - \text { quarter-chord line }\end{array}$

weight constitutes some 3 to 58 of the Maximum Takeoff Weight, which is equivalent to about one-third to half the structural weight of the wing.

The maintenance costs associated with the undercarriage, such as the inspection and replacement of tires and brakes, represent a considerable item in the total mainte- 
nance bill. This is particularly hard to accept because the undercarriage contributes virtually nothing to the flying and economic capabilities of the aircraft, so it is not surprising that many - unsuccessful - attempts have been made to eliminate it, such as takeoff carts, skid landing gear, aircushions and the like. At present it is unlikely that there will be a breakthrough in this direction. In the basic design costs may be reduced by aiming at simplicity and compactness, such as a simple retraction system, devoid of complicated kinematics and duplication of retracting jacks. It is not the job of the preliminary aircraft design engineer to investigate the details of the undercarriage, nor will he design the detalls of the hydraulic equipment and air conditioning system. Unlike this equipment, which can be stowed in irregularly shaped and widely dispersed volumes, the relationship between the wheels, the points of attachment of the gear to the airframe and the principle of the kinematics of retraction must be carefully tailored to the general arrangement and layout of the aircraft. The following functional requirements have a bearing on undercarriage layout in the preliminary design phase:

1. During the phases of takeoff rotation and liftoff and landing flare-out and touchdown, only the wheels should be in contact with the ground. There should be adequate clearance between the runway and all other parts of the aircraft, such as the rear fuselage, the wingtips and the tips of propellers or engine pods.

2. The inflation pressure of the tires and the configuration of the landing gear should be chosen in accordance with the bearing capacity of the airfields from which the aircraft is designed to operate. 3. The landing gear should be able to absorb the normal landing impact loads and possess good damping characteristics. When taxying over rough ground no excessive shocks should be transmitted by the landing gear.

4. Braking should be efficient, the maximum braking force allowed by the condition off the runway being the limiting factor. During crosswind landings and high-speed taxying there should be no tendency to instabilities such as canting of the aircraft or groundlooping.

5. Suitable structural elements should be provided in the aircraft to serve as attachment points for the landing gear, and there should be sufficient internal space for retraction.

In Section 2.5., which deals with the general arrangement of the undercarriage, the choice between nosewheel (tricycle), tailwheel or tandem (bicycle) landing gear has been discussed. The general conclusion is that the tricycle gear has superseded the tailwheel type almost completely, mainly for reasons of improved stability on the ground, braking and steering. On specialized designs, however, there may be indications that a tailwheel or a bicycle undercarriage will be the best choice.

The information presented in this chapter is mainly a summary of data from References 10-1 through 10-4. For more detailed considerations with regard to the structural design of landing gear, the reader should consult Ref. 10-1, which is a standard textbook on landing gear.

\subsection{TAILORING THE UNDERCARRIAGE TO THE BEARING CAPACITY OF AIRFIELDS}

\subsubsection{Runway classification}

For light aircraft operating from grass or unsurfaced runways, the tire inflation pressure is generally limited to some 60 lb/sq.in. $\left(4 \mathrm{~kg} / \mathrm{cm}^{2}\right)$ for grass, as indicated in Table 10-1. When the load per tire exceeds approximately $10,000 \mathrm{lb}(4,500 \mathrm{~kg})$, and the tire pressure is more than about 60 $\mathrm{lb} / \mathrm{sq}$. in., consideration must be given to the limited bearing capacity of runways. The stresses in the runway or the possibility of damage caused by the undercarriage, are dependent upon the undercarriage con- 


\begin{tabular}{|l|lc|}
\hline \multicolumn{1}{|c|}{ TYPE OF LANDING SURFACE } & \multicolumn{3}{|c|}{ Maximum tire pressure } \\
& $\mathrm{kg} / \mathrm{cm}^{2}$ & $\mathrm{1b} / \mathrm{sq} \cdot \mathrm{in}$. \\
\hline $\begin{array}{l}\text { Large, proper1y maintained } \\
\text { airports (concrete runway) }\end{array}$ & $8.5-14$ & $120-200$ \\
$\begin{array}{l}\text { Sma11 tarmac runway, good } \\
\text { foundation }\end{array}$ & $5-6.3$ & $70-90$ \\
$\begin{array}{l}\text { Sma11 tarmac runway, poor } \\
\text { foundation }\end{array}$ & $3.5-5$ & $50-70$ \\
$\begin{array}{l}\text { Hard grass, depending on } \\
\text { soil }\end{array}$ & $3.2-4.2$ & $45-60$ \\
$\begin{array}{l}\text { Wet, boggy grass } \\
\text { Hard desert sand } \\
\text { Soft, loose desert sand }\end{array}$ & $2.1-3.2$ & $30-45$ \\
\hline
\end{tabular}

Table 10-1. Tire pressure recommendations (Ref. 10-1)

figuration and the type of runway structure. Two classes of runway are generally distinguished:

a. Runways with rigid pavement, consisting of discrete slabs of concrete, laid on a relatively soft subsoil. Failure occurs by fracture across the corner, especially when the subsoil is bad, e.g. clay. A sandy subsoil provides a good foundation.

b. Runways with a flexible pavement, consisting of a relatively thick layer of asphalt or tarmacadam on a base of gravel or sand, often with an intermediate layer of crushed stone. The total thickness is about twice that of a rigid pavement. Failure occurs because of local indentation.

Apart from these airfields, which are most commonly used in civil aviation, airfields with a simply prepared surface of, for example, fine gravel are becoming increasingly important for aircraft designed for operation in less accessible regions (Ref. 10-2).

In order to avoid damage to runways airfields have been classified according to various characteristic parameters:

CBR: California Bearing Ratio

UCI: Unit Construction Index

RLI : Runway Loading Index

LCN: Load Classification Number

On some airfields the bearing capacity of the runways is simply given as the maximum allowable gross weight of the aircraft.

The CBR index gives us the bearing capacity of the runway, expressed as a percentage of the bearing capacity of a surface consisting of a hard type of stone. This index, which is fully explained in Ref. 10-2, is used to characterize the substructure of runways with a flexible pavement. Airfields without a hard upper layer are also classified according to the CBR index.

The UCI is an index used in the United States and shows affinity to the LCN, which will be discussed later. A method of determining the UCI graphically is given in Ref. $10-2$. The RLI also shows some affinity to the LCN, but will not be discussed here. The LCN rating method has been introduced by the ICAO on the basis of much theoretical and experimental work, and is now widely accepted in many countries. Permissible values of the $L C N$ have been assigned to all major runways, and aircraft have to be designed in such a way that the undercarriage will not exceed the lowest $\mathrm{LCN}$ value of the airfields from which the aircraft is likely to operate. Typical values of LCN for various aircraft categories are given in Table 10-2.

\begin{tabular}{|l|r|r|l|}
\hline AIRCRAFT TYPE & $\begin{array}{r}\text { MTOW } \\
\text { (LB) }\end{array}$ & $\begin{array}{r}\text { TIRE } \\
\text { PRESS. } \\
\text { (PSI) }\end{array}$ & LCN \\
\hline Fokker F-27 Mk 500 & 45,000 & 80 & 19 \\
Fokker F-28 Mk 2000 & 65,000 & 100 & 27 \\
McDonne11 D. DC-9/10 & 90,700 & 129 & 39 \\
Boeing 707/320 & 300,000 & 135 & 58 \\
McDonne11 D. DC-10/10 & 410,000 & 175 & 88 \\
\hline
\end{tabular}

Table 10-2. Load Classification Number of several airliners - main landing gear

A large number of tests on both rigid and flexible pavements have shown that the bearing capacity of a runway is dependent upon the wheel load and the inflation pressure of the tire(s). The relationship is reproduced in Fig. 10-1, which can be used directly for single wheels to find the limiting values of the wheel load for given values of the inflation pressure and LCN. For multiwheel undercarriages the concept 

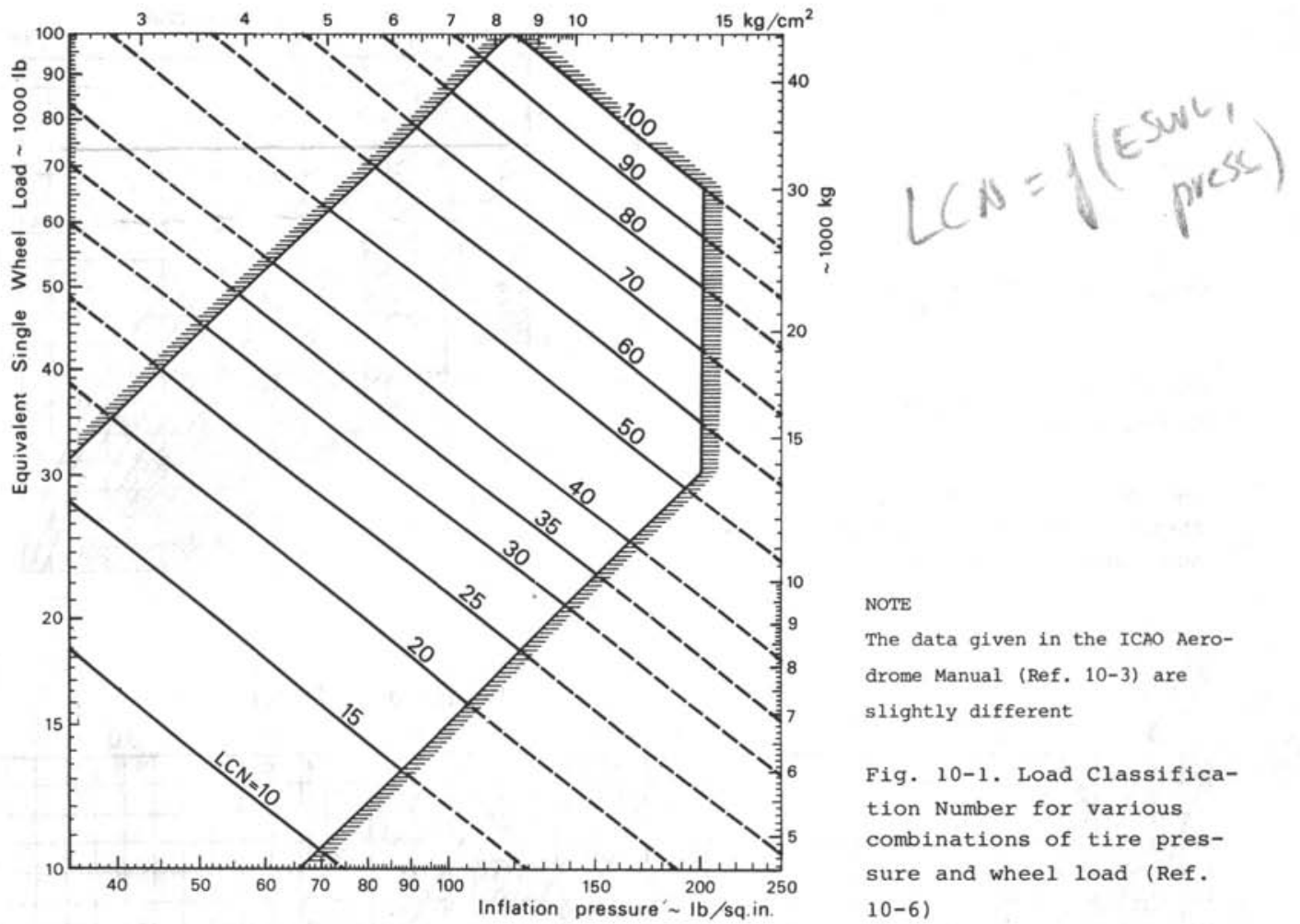

NOTE

The data given in the ICAO Aerodrome Manual (Ref. 10-3) are slightly different

Fig. 10-1. Load Classification Number for various combinations of tire pressure and wheel load (Ref. 10-6)

of the ESWL, as explained in the following section, can be employed in using this diagram.

\subsubsection{The Equivalent Single Wheel Load} (ESWL)

The ESWL of a group of two or more wheels which are relatively close together, is equal to the load on an isolated wheel, having the same inflation pressure, and causing the same stresses in the runway material as those due to the group of wheels. This equivalent wheel load accounts for the fact that a given loading, spread over a number of contact areas, causes lower stresses in the runway material than would be the case when the same load is concentrated on a single wheel. Typical reduction factors - i.e. the actual static load on the leg divided by the ESWL - are $4 / 3$ for dual wheel layouts and 2 for twin tandem (bogie) layouts (Ref. 10-4). Various methods have been developed for calculating the ESWL, depending on the mechanical characteristics of the runway and the undercarriage layout. The methods described below for rigid and flexible pavements and a method for graval runways can be found in Refs. 10-3 and 10-4.

\section{a. Rigid pavements.}

Runway characteristics are generally represented by the radius of relative stiffness $L$ of the concrete. In the absence of more adequate information the following approximation may be used:

$L=$ constant $\times \delta^{3 / 4}$

where $\delta$ denotes the thickness of the runway construction. The constant takes account of the stiffness of the substructure; typical values are:

constant $=8.0$ when $\mathrm{L}$ and $\delta$ are in inches, 
CONTACT AREA = TOTAL CONTACT AREA OF ALL WHEELS OF ONE UNDERCARRIAGE ASSEMBLY

EQUIVALENT SINGLE TOTAL LOAD ON ONE U.C. ASSEMBLY REDUCTION FACTOR

Fig. 10-2. Equivalent Single Wheel Load assessment curves - rigid pavements - dual wheel undercarriages (Ref. 10-3)

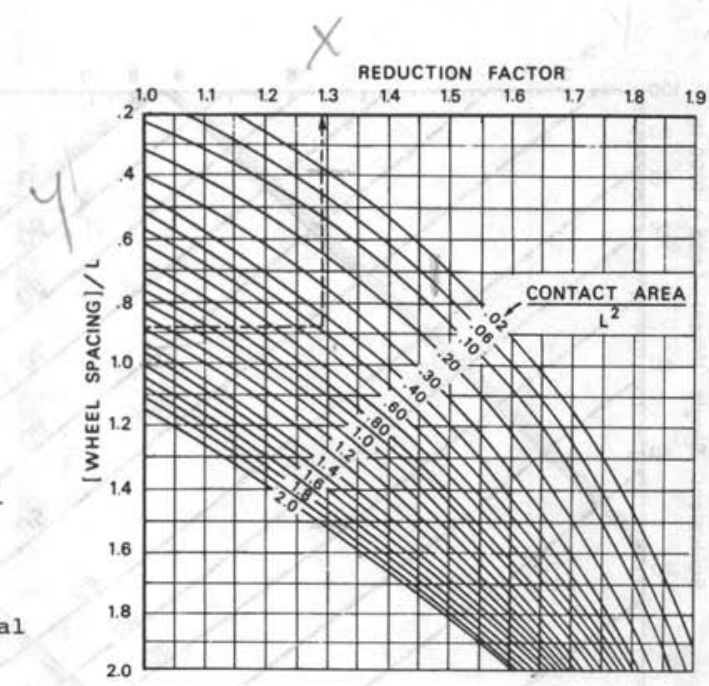

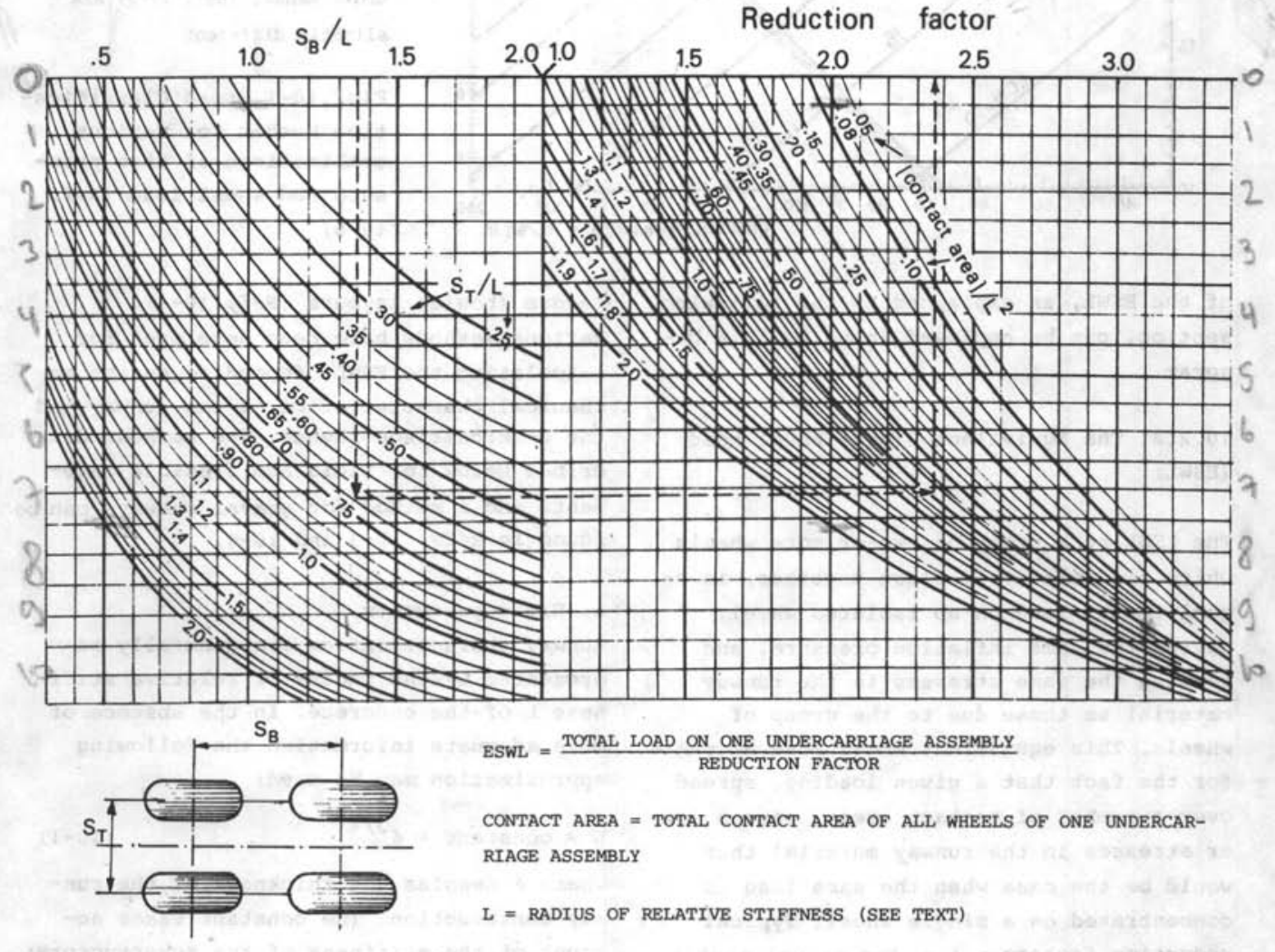

Fig. 10-3. Equivalent Single Wheel Load assessment curves - rigid pavements - dual and tandem undercarriages (Ref. 10-3) 
or $1 Q .1$ when $\mathrm{L}$ and $\delta$ are in $\mathrm{cm}$, for a bad, soft'substructure;

constant $=6.1$ when $L$ and $\delta$ are in inches, or 7.7 when $\mathrm{L}$ and $\delta$ are in $\mathrm{cm}$, for a good, hard substructure.

When the thickness of the rigid pavement is not specified, L is usually assumed equal to $45 \mathrm{in}$. (115 cm), which is equivalent to $\delta=10 \mathrm{in.}(25 \mathrm{~cm})$ on a bad substructure. These assumptions will keep the designer on the conservative side. In the case of large aircraft the method may lead to excessive requirements where the undercarriage assembly is concerned, and more precise data should be collected in this case.

Using the calculated or chosen value for $L$ and the geometrical data of the landing gear, the ESWL may now be calculated on the basis of Fig. 10-2 or 10-3. Strictly speaking the ESWL of the nosewheel leg should also be determined, but this is seldom a critical case. For dual tandem assemblies with a pair of wheels at each end of the axle, each pair of wheels is replaced by one equivalent wheel with the same ESWL as the pair of wheels, using Fig. 10-2. The ESWL of the four replacing wheels is then computed on the basis of Fig. 10-3. b. Flexible pavements.

The graphical method of ESWL determination for flexible pavements is shown in Fig. $10-4$.

The ESWL is considered to be equal to the wheel load from one tire when the pavement thickness is equal to or less than half the distance between the closest contact area edges, $D / 2$. The contact area for each tire is equal to the wheel load divided by the tire inflation pressure. Assuming the tire imprint to be an ellipse with the major axis equal to 1.4 times the minor axis, we can derive:

$\frac { \mathrm { D } } { 2 } = \frac { \mathrm { S } _ { \mathrm { T } } } { 2 } - 1 \longdiv { \frac { \mathrm { L } _ { \mathrm { w } } } { 1 . 4 \pi \mathrm { p } } }$

where $S_{T}$ is the distance between the imprint centers, $L_{w}$ the load per tire and $p$ the inflation pressure. When the pavement thickness equals or exceeds twice the center distance of the two most widely spaced contact areas (i.e. $\mathrm{S}_{\mathrm{T}}$ for dual wheel assemblies or $S_{D}$ for twin tandem wheel assemblies), the ESWL is considered to be equal to the load from one undercarriage assembly.

Between the two limits for $\delta$ it is assumed that $\log$ ESWL varies linearly with $\log \delta$.

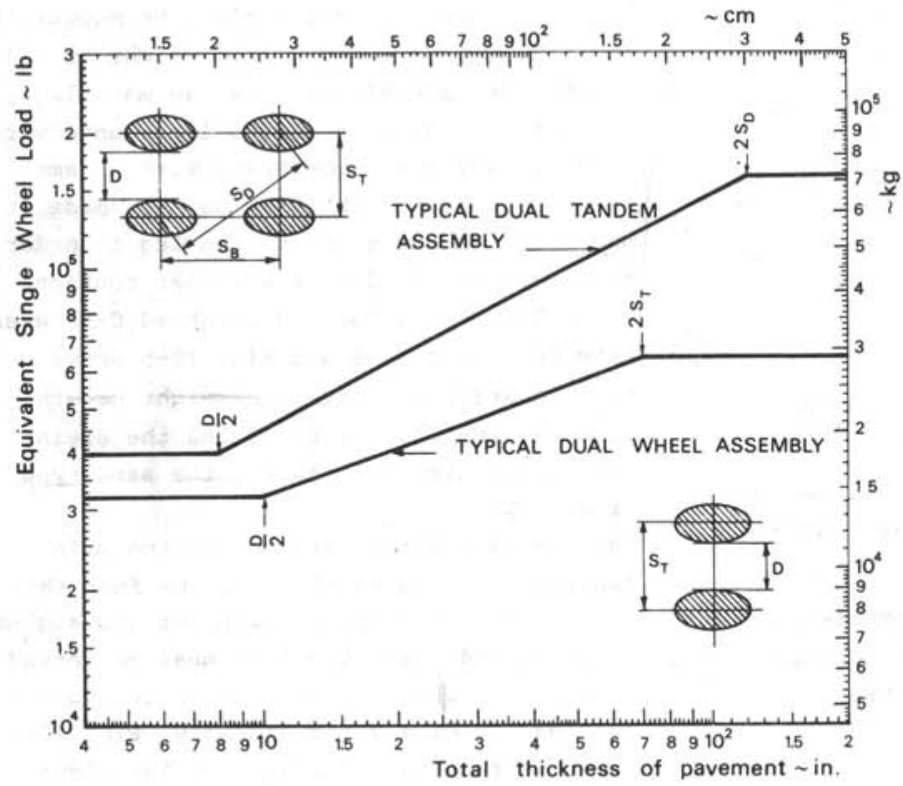

Fig. 10-4. Multiple wheeled undercarriages on flexible pavement-Equivalent Single Wheel Loads at varying depths 
Instead of the graphical construction, one of the following expressions may be used:

dual wheel assemblies:

$\log \mathrm{ESWL}=\log \mathrm{L}_{\mathrm{W}}+.3 \frac{\log \delta-\log \mathrm{D} / 2}{\log 2 \mathrm{~S}_{\mathrm{T}^{-1}}}(10-3)$

twin tandem wheel assemblies:

$\log \mathrm{ESWL}=\log \mathrm{L}_{\mathrm{w}}+.6 \frac{\log \delta-\log \mathrm{D} / 2}{\log 2 \mathrm{~S}_{\mathrm{D}}-\log \mathrm{D} / 2}$

An example of the determination of LCN values will be given for the McDonnell Douglas $D C-10 / 10$. For an all-up weight of $388,000 \mathrm{lb}(176,000 \mathrm{~kg})$ it is assumed that the load on one main gear is equal to 468 of the weight, or $178,500 \mathrm{lb}(81,000 \mathrm{~kg})$. Tire pressure is $170 \mathrm{lb} / \mathrm{sq}$. in. $\left(12 \mathrm{~kg} / \mathrm{cm}^{2}\right)$.

For a rigid pavement of 12 in. $(30.4 \mathrm{~cm})$ thickness, the radius of relative stiffness for a good subsoll is $L=6.1(12)^{3 / 4}=39.6$ in. $(100 \mathrm{~cm})$. For $S_{B}=64$ in. $(163 \mathrm{~cm})$ and $S_{T}=54$ in. $(137 \mathrm{~cm})$ we find $S_{B} / L=1.63$ and $S_{T} / L=1.37$. The total contact area per undercarriage assembly is $178,500 /$ $170=1050$ sq.in. $\left(6774 \mathrm{~cm}^{2}\right.$ ), hence (contact area)/ $L^{2}=.677$. Using Fig. 10-3, we obtain a reduction factor of 3.40 , resulting in an ESWL of 178,500 / $3.4=52,500 \mathrm{lb}(23,800 \mathrm{~kg})$. Fig. $10-1$ is then used to read $L C N=76$.

For a flexible pavement of $\delta=43.3 \mathrm{in}$. $(110 \mathrm{~cm})$ thickness, the distance $S_{D}=\left(64^{2}+54^{2}\right)^{\frac{1}{2}}=83.74$ in. $(213 \mathrm{~cm})$ - see Fig. $10-4$. Hence, we find $D / 2=$

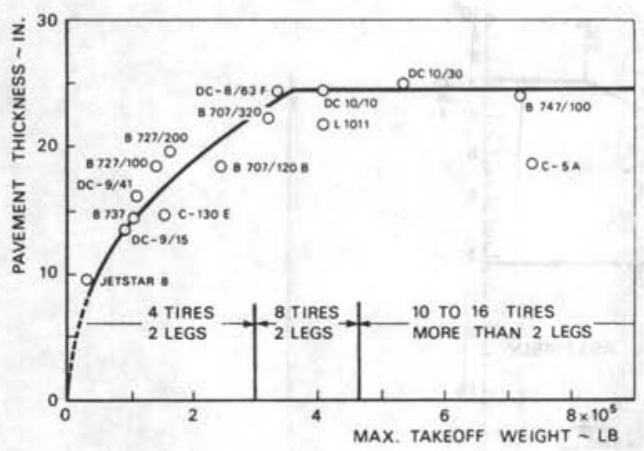

Fig. 10-5. Thickness of a flexible pavement required for several transport aircraft types - $\mathrm{CBR}$ of sublayer $=15$ (Ref. 10-23)

$54 / 2-\{178,500 /(4 \times 1.4 \pi \times 170)\}^{\frac{1}{2}}=19.3$ in. $(49 \mathrm{~cm})$, for a load per tire of $44,625 \mathrm{lb}(20,250 \mathrm{~kg})$. Using the logarithmic relationship of ESWL vs. $\delta$, we find with Fig. 10-4: ESWL $=74,800 \mathrm{lb}(33,930 \mathrm{~kg})$ and using Fig. 10-1, we find $\mathrm{LCN}=100$.

These results are in fair agreement with the data quoted by the airplane manufacturer.

10.2.3. Multiple wheel undercarriage configurations

The ability to utilize runways of moderate quality and the ever-increasing all-up weight of transport aircraft have resulted in the use of twin, tandem, dual tandem and dual twin tandem wheel patterns. The application of multiple wheel undercarriages also results in a gain in safety, a flat tire being of little or no consequence. In addition, tandem or twin tandem gears are superior in taxying over obstacles in that the fore and aft wheels meet these obstacles at different times, thus only raising the airplane about half the total obstacle height.

Fig. 10-5 shows that for takeoff weights of up to about $450,000 \mathrm{lb}(200,000 \mathrm{~kg})$ the use of two main undercarriage legs appears to be adequate; for higher weights radical changes in the undercarriage configuration are unavoidable. For example, the McDonnell Douglas DC-10/10 with a ramp weight of $443,000 \mathrm{lb}(200,940 \mathrm{~kg})$ has two main legs, but the introduction of the long-range versions $D C-10 / 30$ and $D C-10 / 40$, with a ramp weight of $558,000 \mathrm{lb}(253,105 \mathrm{~kg})$, made it necessary to add a third main leg in order to spread the load over a larger contact area. The Boeing 747 and Lockheed C-5A even have four main legs and Fig. 10-5 shows that in spite of the large weight growth from the $D C-8$ to the $D C-10$ and the Boeing 747 , these aircraft can use the same type of runways.

The use of multiple struts for the main landing gear has highlighted the fact that on uneven pavements, or with certain angles of pitch and roll, the load must be spread evenly over these legs in order to avoid overloading of any one assembly. Fig. 10-6 explains the main functions of the oleo- 


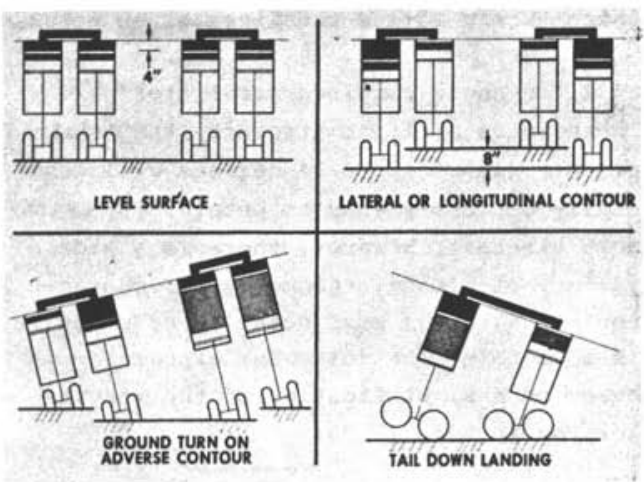

Fig. 10-6. Load equalizing system of the Boeing 747 main undercarriage system

pneumatic load equalizing system of the Boeing 747. An oil pipe connects the shock struts of the pair on each side, so that uneven loads are balanced out.

The Lockheed C-5A, having four main gear units (Ref. 10-29), each comprising a sixwheel bogie, illustrates an extreme in flotation capability. At reduced takeoff weights this aircraft may even be operable from unprepared runways.

The multiple gear develops higher ground turning torques, particularly if the aircraft is pivoted about one main gear.

Steerable main landing gear systems may be employed to improve the turn radius, to avoid excessive side loads and to reduce tire wear from scrubbing. Detailed design considerations of these specialized designs, however, are considered to be outside the scope of this book.

\subsection{DISPOSITION OF THE WHEELS}

The following sections will deal with the choice of the wheel location. Boundaries will be derived for the intersection of the wheel load with the ground, based on conditions of stability during taxying, liftoff and touchdown. Assuming that for a given undercarriage assembly the center of area of the tire imprints coincides with these intersections, conditions will thus be obtained for the location of the tires. In most cases it is fair to approximate the resulting ground contact points by the wheel axis or the pivot point of a bogie assembly.

10.3.1. Angles of pitch and roll during takeoff and landing

The landing gear legs should be sufficient1y long to allow for any combination of the pitch angle $(\theta)$ and roll angle $(\phi)$ which may occur in normal operational use, without the risk that parts of the aircraft will come into contact with the ground. It is generally acknowledged that the avajlable angle of pitch on liftoff and touchdown should at least be equal to, or preferably exceed, the limits imposed by performance or flight characteristics. A geometric limitation to the pitch angle will be detrimental to the liftoff speed and hence to the takeoff distance. A geometrical roll angle limitation may result in an undesirable operational limit in the case of crosswind landings.

The geometric limits may be reproduced in a $\phi-\theta$ diagram (Fig, 10-7). The various

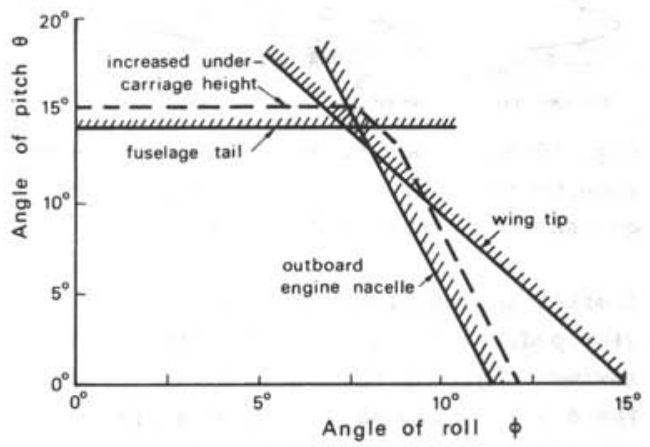

Fig. 10-7. Limitations on the angles of pitch and bank determined by the airplane geometry

boundaries define the point where the rear fuselage tail (bumper, if present), the wingtip, engines suspended below the wing, trailing-edge flaps, or any other part of the aircraft, just touches the ground plane. For a given aircraft geometry and 
length of the main undercarriage, the limit for $\theta$ will follow directly from the side elevation. The condition that the tip of the wing just touches the ground (Fig. 10-8*) will be:

$\tan \phi=\tan \Gamma+\frac{2 \mathrm{~h} g}{b-t}-\tan \theta \tan \Lambda$

$(10-5)$

Similar conditions may be deduced for other parts of the aircraft. Fig. $10-7$ shows a
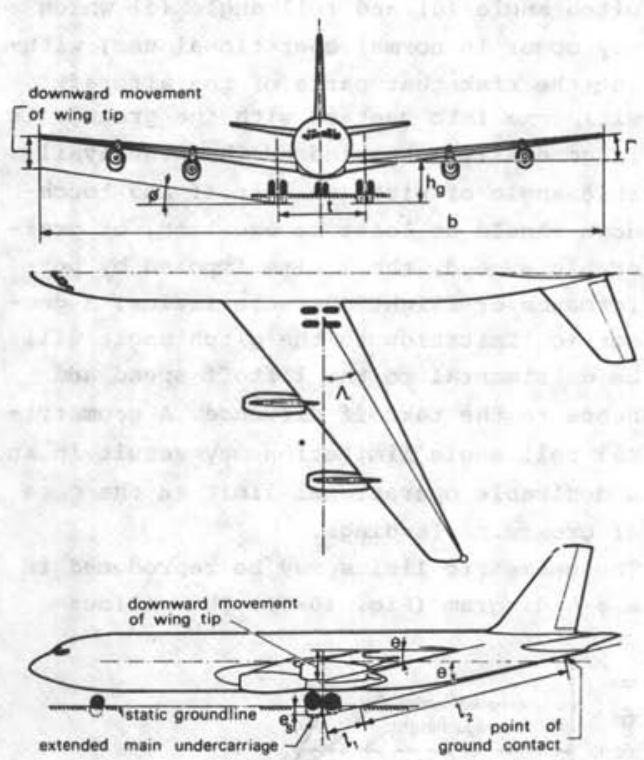

Fig. 10-8. Geometric definitions in relation to the angles of bank and pitch required for takeoff and landing

limitation associated with the outer engine pod, which in this case limits the maximum angle of roll over a large region. The diagram also shows that the limits may be modified by lengthening the main gear leg.

Accurate determination of the desired values for $\phi$ and $\theta$ can only be carried out when the performance calculations of the design have been completed. A provisional

* The difference between the angle of roll and angle of bank has been ignored here which is acceptable for small angles of pitch. estimate may follow the lines given below.

a. Pitch angle required for liftoff.

In the case of light aircraft, the required pitch angle of 12 to 14 degrees will generally be fairly easy to obtain. For transport aircraft, however, there is a wide variety of aircraft geometry and characteristics, and a more detailed calculation is advisable. The following expression is based on a simplification of the results derived in Ref. 10-25:

$\theta_{L O F}=\alpha_{L O F}+\frac{d \theta}{d t}\left(\frac{2 l_{1}}{v_{L O F}}+\sqrt{\frac{l_{2} C_{L_{L O F}}}{g d C_{L} / d \alpha}}\right)$

where $\ell_{1}$ and $\ell_{2}$ are dimensions defined in Fig. $10-8$, and $\theta_{\text {LOF }}$ refers to the condition where the undercarriage is fully extended.

In $(10-6)$ the value to be used for $\alpha_{\text {LOF }}$ is the highest angle of attack to be anticipated for normal operational use. The second term is a correction to allow for the climb angle of the center of gravity associated with the extending undercarriage. The last term represents an increment to the pitch angle which will be needed, since immediately after rotation the rear of the fuselage will still be moving in the direction of the runway (Ref. 10-25), an effect which is particularly noticeable on large aircraft. Typical values to be used for the rate of rotation are:

$\mathrm{d} \theta / \mathrm{dt}=3$ to 4 degrees $/ \mathrm{sec}$ for large transports (DC-8, B 707, 747 class)

$\mathrm{d} \theta / \mathrm{dt}=4$ to 5 degrees $/ \mathrm{sec}$ for small transports (F-28, BAC $1-11$ class).

Prediction methods for the aerodynamic data required to use $(10-6)$ can be found in Appendices E, $G$ and $K$. The amount of work involved in applying these methods is not always justified at the undercarriage design stage and the simplified approximation given below may be acceptable instead. In the case of propeller aircraft, the effect of the propeller slipstream on 1 ift will be quite appreciable. Assuming that slats are not present, a reasonable guess is obtained from: 
$\theta_{\text {LOF }}=7\left(1+\frac{3}{A}\right)$

(deg.)

$(10-7)$

For jet transports the liftoff condition can be derived from the stalling angle of attack (see Fig. 10-9). Ignoring tailplane trim and ground

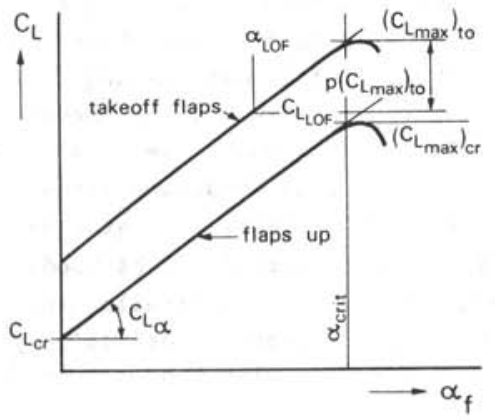

Fig. 10-9. Determination of the liftoff angle of attack from the lift-curves for cruising flight and takeoff

effect, we may obtain:

$\alpha_{\text {LOF }}=\frac{1}{c_{L_{\alpha}}}\left[\left(c_{L_{\max }}\right)_{\delta_{f}=0}-c_{L_{C r}}-p\left(c_{L_{\max }}\right)_{\text {to }}\right]$

where it has been assumed that in the takeoff configuration flap deflection has no appreciable effect on the critical angle of attack, and that the fuselage is horizontal during cruising flight. Introducing an approximation for the lift-curve slope of high aspect ratio wings,

$\mathrm{C}_{\mathrm{L}_{\alpha}}=\frac{2 \pi \cos \Lambda_{.25}}{1+2 / \mathrm{A}}$

and allowing for ground effect, we find:

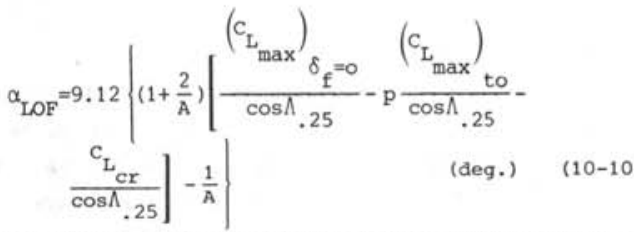

The factor $p$ allows for the margin relative to $\mathrm{C}_{\mathrm{L}}{ }^{-}$ max which should be adhered to in any case during liftoff. It is dependent on the aerodynamic characteristics, and may be assumed equal to .15 to .20. In the absence of better information, the designer may use: $\frac{\left({ }^{C} L_{\max }\right)_{\delta_{f}=0}}{\cos \Lambda_{.25}}=1.50-$ no leading-edge devices

$=2.10-$ with leading-edge devices

b. Pitch and roll angles during landing . In the case of jet aircraft, the largest angle during landing is generally less than that during takeoff, because for fully deflected flaps the critical angle of attack of the wing will be smaller by some degrees than in takeoff. For propeller aircraft, we may take almost the same values in landing and takeoff.

For the desirable angle of roll we may assume:

$8^{\circ}$ - transport aircraft

$15^{\circ}$ - light aircraft

10.3.2. Stability at touchdown and during taxying: tricycle undercarriages

Although attention should be given to various types of static and dynamic instability during the design stage, only the measures which have to be taken to prevent the aircraft from canting over will be mentioned here. We shall use the plan view of the aircraft and assume a statically compressed landing gear (Fig. 10-11). The aircraft will cant over about any of the lines connecting the tire contact areas, if the resultant of air and mass forces intersects the ground at a point which lies outside the triangle formed by these connecting lines (Fig. 10-10). In that case the ground is unable to exert a reaction force which opposes the tendency to cant over.

a. Condition at touchdown .

The most unfavorable condition will be a landing with the centre of gravity in its most rearward and highest location. When there are no retarding forces (spin-up load), only a vertical force will be present which intersects the ground plane at 


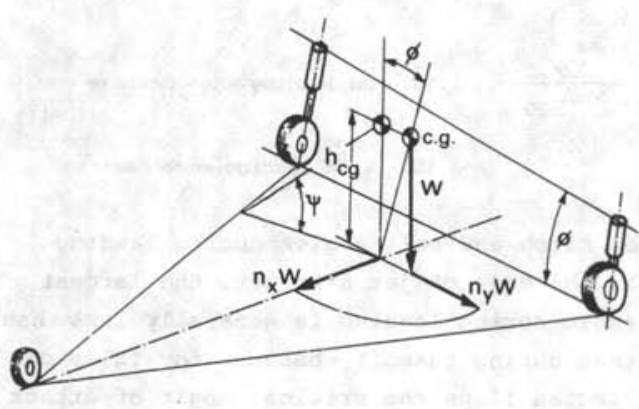

Fig. 10-10. Condition for stability at touchdown and during taxying

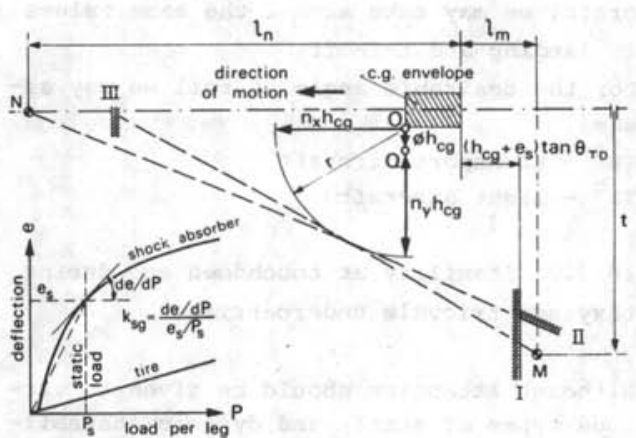

$M$ : chosen position of main u.c. leg

$N$ : chosen position of nose u.c. leg

I : limit of main leg position determined by touchdown angle of pitch

II : limit for the main leg position for given $\mathrm{N}$, to attain stability against turnover

III : limit for the nosegear location for given $M$, to attain stability against turnover

Fig. 10-11. Limits for the undercarriage disposition based on stability considerations

a certain distance behind the projection of the center of gravity on the ground. The main gear must be placed at least this distance behind the aft c.g. position, nence:

$\ell_{m} \geqslant\left(h_{c g}+e_{s}\right) \tan \theta_{T D}$

where $e_{s}$ is the static deflection of the tire plus shock absorber and $\mathrm{h}_{\mathrm{cg}}$ the c.g. height during taxying.

\section{b. Avoiding sideways turnover of the air-}

craft.

Forces acting sideways on the airplane may be the result of a crosswind, an angle of yaw relative to the runway or a high-speed turn during taxying. In addition, taxying over uneven surfaces creates the danger of turnover. A simple rule of thumb states that the angle $\psi$ (Fig. 10-10), determining the tendency to overturn sideways, must never exceed $60^{\circ}, 55^{\circ}$ to $57^{\circ}$ probably being a maximum safe limit. This condition fixes a lower limit for the track. This statistical working rule cannot always be used, however, and a somewhat more detailed investigation may be desirable, particularly in the case of aircraft with fuselagemounted main gear.

Fig. 10-11 shows a case with an unfavorable c.g. location, that is, the extreme forward and lateral position with asymmetrical loading (point 0). As a result of the force $n_{y} w$, directed sideways, the aircraft will assume an angle of roll which is mainly dependent on the stiffness of the landing gear and the track. The angle of roll may be deduced from the balance of forces, with the following result:

$\tan \phi=4 k_{s g} \frac{e_{s}}{t} \frac{h_{c g}}{t} n_{y}$

where $k_{s g}$ is an undercarriage stiffness parameter defined in Fig. 10-11, while

$e_{s}=$ static deflection (tire plus shock strut)

$s$ = track

$h_{c g}=$ height of c.g. above the ground during taxying and

$n_{y}=1$ ateral 1oad/weight

As a result of the angle of bank the c.g. will be displaced sideways over a distance $\mathrm{h}_{\mathrm{cg}} \operatorname{tan\phi }$ (point Q). If the weight is represented by a vector having a length $\mathrm{h}_{\mathrm{cg}}$ from the c.g:, then with a sideways loading $n_{y} w$ the intersection of the resultant force with the ground will lie at a distance $\mathrm{n}_{\mathrm{y}} \mathrm{h} \mathrm{cg}$ towards the side from point $Q$. When there is only an inertia force in the longitudinal direction $n_{x} w$ during braking, the point of intersection will lie at a distance $n_{x} h_{c g}$ ahead of 0 .

In view of the danger of turning over the most unfavorable case will be on a dry hard surface when the adhesion forces between the tire and the ground 
will be maximum. In such a case, values of .5 to .6 may be found for $n_{x}$, while $n_{y}=.5$. For a dry grass surface we may take $n_{x}=n_{y}=.35$ as the limit. Since the loads in all directions will be of the same order of magnitude, a circle may be taken as the extreme limit of the points of intersection mentioned above. This circle will have 0 as its centre, while the radius is given by:

$r=n_{y} h_{c g}\left(1+4 k_{s g} \frac{e_{s}}{t} \frac{h_{c g}}{t}\right)$

When the line interconnecting the mainwheel with the nosewheel contact areas touches this circle, a condition is found that defines the lower limit of the track of the landing gear, provided the nosewheel location is fixed.

Equation 10-13 demonstrates that under a given lateral force the angular roll is inversely proportional to the square of the track. In a condition where a narrow track is dictated by the general arrangement, the aircraft becomes liable to lateral "wallowing" and a landing gear with high stiffness (i.e. small value of $\mathrm{k}_{\mathrm{sg}}$ ) and a short stroke (i.e. small value of $e_{s}$ ) is desirable.

When using $(10-13)$ and $(10-14)$ it may be assumed that the static deflection of the tire is about $1 / 3$ of the maximum, while that of a conventional shock absorber will be about 3/4. The factor $k_{\text {sg }}$ will be 1 for a landing gear where the compression has a linear relationship with the wheel load (spring without pre-tensioning, pneumatic tire), a typical range of values for a strut with hydraulic shock absorber will be $k_{\text {sg }}=1 / 3$ to $1 / 2$.

10.3.3. Gear length, wheelbase and track: tricycle undercarriages

The most favorable location of the wheels can be determined by indicating limits in the three-view drawing of the aircraft (Fig. 10-12).

a. Disposition in elevation.

1. During takeoff and landing the rear of the fuselage should remain clear of the ground by an amount equal to at least the maximum tire deflection, or about 28 of $\imath_{1}+\imath_{2}$ (see Fig. 10-8). The attitudes of the aircraft are known from performance and aerodynamic data or may be estimated with the approximations given in Section 10.3.1. In this case the landing gear is completely extended.

2. During taxying (landing gear statically compressed) there should be a propeller clearance of at least $7 \mathrm{in.}(18 \mathrm{~cm})$. In the case of an inflated tire and a fully deflected shock absorber, the propeller(s) or any other part of the aircraft must remain clear of the ground*.

3. To avoid a tail slam on touchdown, the center of the tire contact areas must be located just behind the intersection of the normal from the rear center of gravity to the ground. In the absence of adequate information, the pitch angle on touchdown $\theta_{\text {TD }}$ may be assumed equal to $\theta_{\text {LOF }}$.

b. Disposition in plan.

1. When the load on the nosewheel is less than about 88 of the MTOW, controllability on the ground and stability during taxying will suffer, particularly in crosswind conditions. When the static load on the nosewheel exceeds about 158 of the MTOW, the load during heavy braking may become excessive, braking may be less efficient, and too much effort may be required for steering. Although the margin between these $1 \mathrm{im}-$ its seems to be generous, it should be remembered that variations in the location of the center of gravity have a great effect on the nosewheel load. Fig. 10-12 relates the limits for the nosewheel disposition to the airplane load and balance diagram.

The figures stated above should be considered as recommendations and not as requirements. Structural considerations may be conclusive in deciding where to place the nose gear.

2 . Once the location of the nosewheel has been chosen, the maximum overturning angle of $57^{\circ}$ - or alternatively the graphical method given in Section 10.3.2. - will provide a lower limit for the track of the main gear. If the location of the main

\footnotetext{
* see also Section 6.4.1.
} 


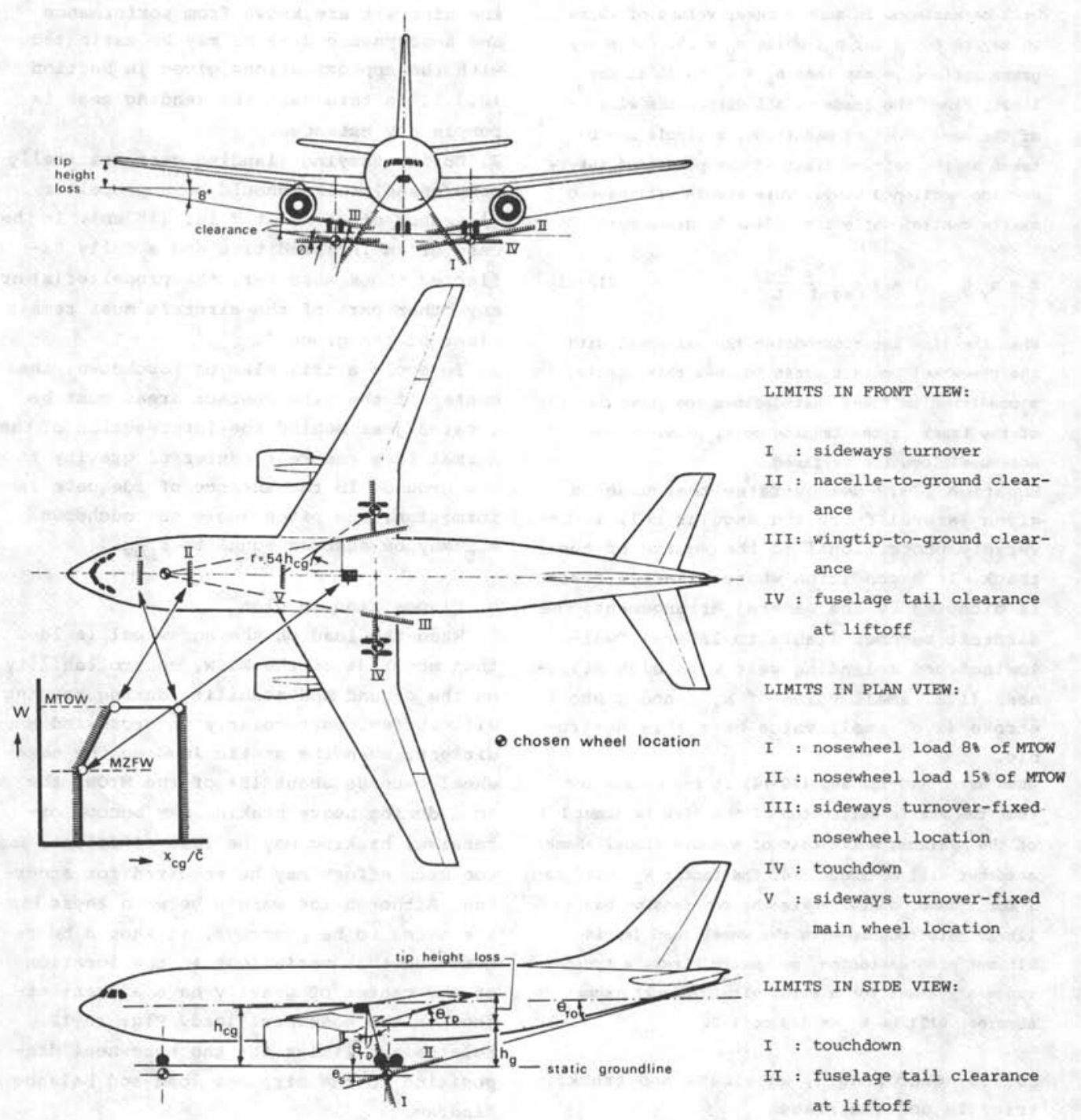

Fig. 10-12. Disposition of the wheels in the three-view drawing

gear, and thus the track, have been fixed, a lower limit for the wheelbase will follow, although this is less sharply defined.

3. Occasionally there may be arguments in favor of shifting the main wheels further back than required for stability during landing. Examples are:

- freighters with the loading door in the rear of the fuselage,
- aircraft with engines at the rear which have a backward c.g. when empty. The object in these two cases is to ensure safe loading without having to place ballast in the nose or fit a support at the tail of the fuselage. However, there are serious objections to such a backward shift:

- Rotation in the takeoff will be mare dif- 
ficult, with the result that the aircraft will unstick later, while accurate longitudinal control will be more difficult for the pilot, unless the elevator power is considerably increased (Ref. 10-14).

- The load on the nosewheel will be increased and it may not be possible to stop efficiently unless brakes are also fitted to the nosewheel.

\section{c. Disposition in front view.}

The main factors to be considered in determining the landing gear height follow from the considerations in Section 10.3.1. with regard to the available angles of roll and pitch. The minimum track of the main wheels follows from the required stability against overturning sideways.

Once all limits have been established, the designer may choose the shortest landing gear which satisfies every requirement. There may, however, be good reasons for departing from this rule:

- With the track chosen a main leg of minimum length may be too short to ensure that the wheels will lie in the desired location when retracted.

- The leg will be too short to accommodate the shock absorber (see Section 10.5.2.). - There may be reason to anticipate fuselage stretch, requiring a higher landing gear for the same rotation angle (see Fig. 2-8).

If the main gear is unacceptably high, possibilities of modifying the design of the aircraft must be investigated. Depending on the critical limitations, this may involve:

- changing the contour of the rear fuse-

lage,

- increasing the angle of incidence of the wing relative to the fuselage,

- increasing the dihedral of the wings and/ or

- re-locating an engine.

The length of the nosewheel leg will sometimes follow directly from the required clearance of a propeller in the nose of the aircraft (see section 6.4.1.). In other cases the length is generally based on the requirement that the fuselage should be horizontal or slightly tilted nosedown when the aircraft is on the ground.

10.3.4. Disposition of a tailwheel undercarriage

The governing factor here will be the landing, since the aircraft touches down near the condition of stalling. Assuming the fuselage reference line to be horizontal during cruising, an approximation for the critical angle of attack is:

$\alpha_{\text {crit }}=\left(12-10 \mathrm{C}_{\mathrm{L}_{\mathrm{Cr}}}\right)\left(1+\frac{3}{\mathrm{~A}}\right) \quad$ (deg.)

With the landing gear fully extended, at least this angle should be available with he aircraft tail down.

The declding factor for the location of the main wheels will be the stability attainable when full braking is applied during landing. The resultant force of the load acting on the wheels perpendicular tu the ground and the friction with the ground must therefore create a tail-down moment. The condition for the angle $\beta$ in Fig. 10-13

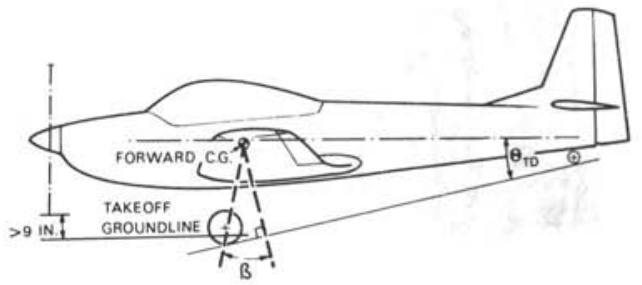

Fig. 10-13. Main wheel disposition for tailwheel undercarriages

will then be:

$\tan \beta>\mu$

$(10-16)$

Assuming $\mu$ to be limited to .25 to .35 as a result of the runway condition (e.g. grass) or the limited braking torque, $B$ should be at least $14^{\circ}$ to $17^{\circ}$, the latter value generally being taken as the lower limit. When the main gear is placed too far for- 
ward, the aircraft cannot be brought into a favorable attitude for taking of $f$. The clearance of the propeller to the ground in the takeoff attitude must be at least 9 in. $(23 \mathrm{~cm}$.$) - see also Section 6.4.1.$

10.4. TYPE, SIZE AND INFLATION PRESSURE OF THE TIRES

Fig. 10-14 shows some examples of tire

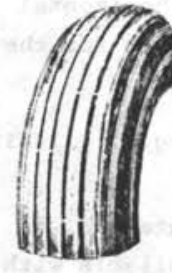

Normal, grooved tire, wearresistant and having good characteristics on wet runways

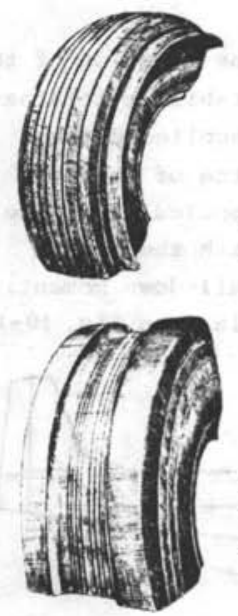

Chined tires, used on aircraft with rear-mounted engines

Anti-shimmy tire, used on castoring nosewheels of light aircraft

Fig. 10-14. Tire tread patterns

treads, the choice of which will depend on the application envisaged. The grooved pattern normally used is provided with ribs. This has been found necessary in order to obtain good adhesion on wet runways and to minimise the effects of the cutting action of stones or flints in the runway surface. Aircraft with engines located in the wing roots or at the sides of the rear fuselage use tires provided with a chine, which serves to direct the water on a wet runway sideways.

Treads with two contact areas are intended to counteract the tendency to shimmy; they are mainly used on light aircraft with a single, castoring nosewheel.

Shimmy is a violent, self-excited oscillation about the swivel axis, caused by positive trail in the case of a castoring wheel. The problem can be overcome by incorporating friction or hydraulic damping, or heavy self-centering. Alternatively, twin wheels may be employed to achieve the same effect as a single wheel with two contact areas.

Manufacturers of tires issue catalogs in American, British and French standard sizes. Definitions of tire sizes and a survey of applications in aircraft can be found in Ref. 10-30.

The choice of the size of tire is simplified by using Fig. 10-15 which gives representative values for the static loading. Since the characteristics of tires are continually being improved, the data in Fig. 10-15 should be checked against the latest tire manufacturer's data to make sure that a certain type is still in production and its characteristics have not been changed.

\subsubsection{Main wheel tires}

The choice of the main wheel tires is generally made on the basis of the static loading case. The load is determined by the weight of the aircraft, the number of main wheel legs, the number of wheels per leg and the location of the leg in relation to the center of gravity. The aircraft is considered to be taxying without braking, at low speed, and hence the wheel load follows from the static equilibrium. From Fig. 10-15 we may derive the following expression for the total main gear load:

$\mathrm{P}_{\mathrm{m}}=\frac{l_{\mathrm{n}}}{l_{\mathrm{m}}+\ell_{\mathrm{n}}} \mathrm{W}$

The critical condition is the aft location of the center of gravity at the Maximum Takeoff Weight*. If the data required to carry out a calculation are not available,

*More precisely: the Maximum Ramp Weight 
it may be assumed that when two main legs are used, each of them will have to carry 468 of the MTOW.

When the characteristics of the shock absorber in the landing gear leg are known, it will be possible to determine the energy absorption required for the impact during landing. The maximum dynamic load can then be calculated. Alternatively, for a preliminary design it is justifiable to assume that the design of the shock absorber will be adapted to the energy which the tire is able to absorb at maximum deflection. In that case the choice of the tire may be based on the static load it can carry.

For undercarriage legs with a single axis the total load on the leg is divided equally over the tires. In bogie assemblies the load per wheel depends on the position of the bogie pivot point. If the pivot point is midway between the front and rear wheel axles the wheel loads are equal in the static case, but the front wheels will

Ib / sq. in.

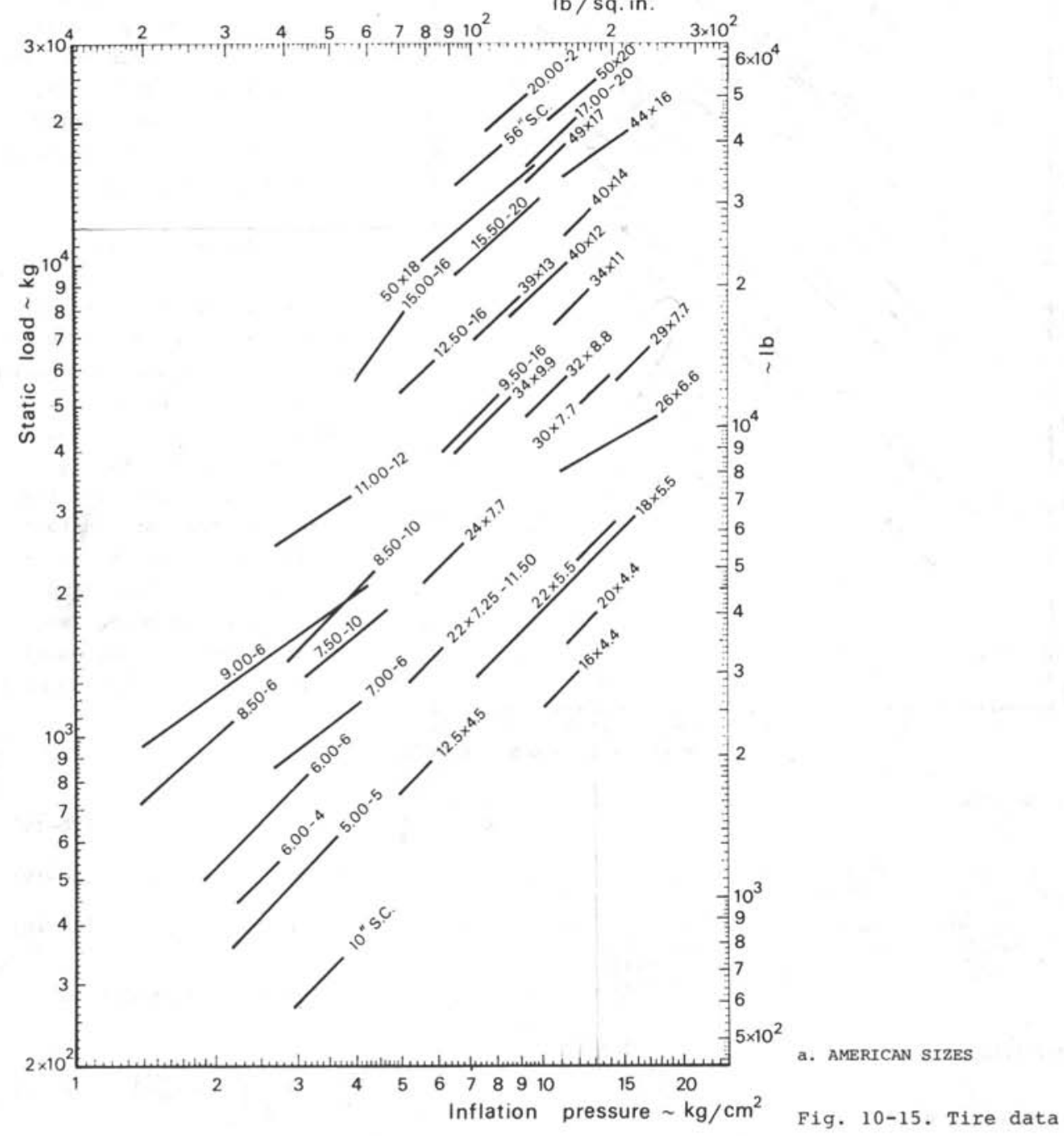


$\sim$ lb $/$ sq. in.

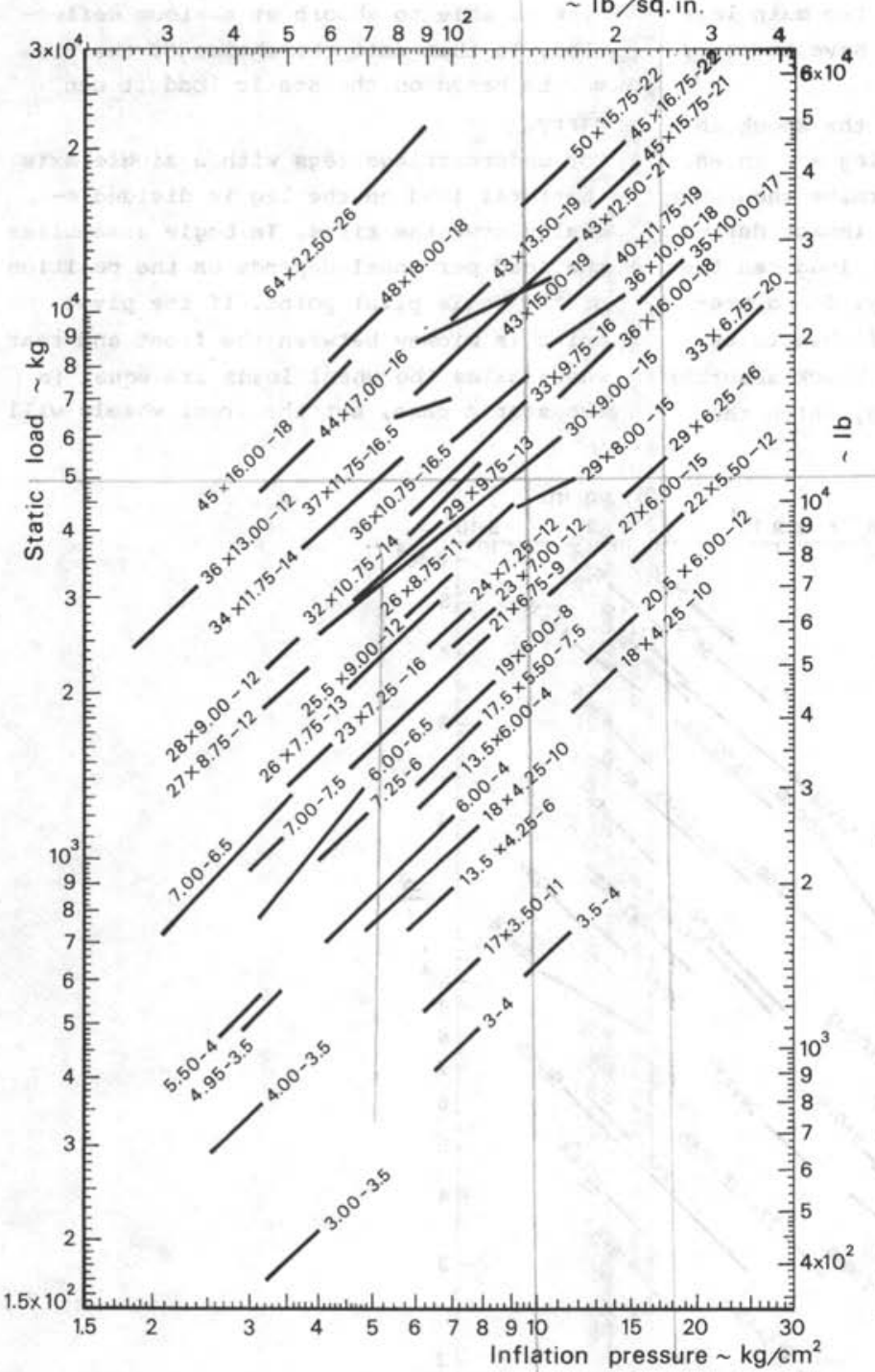

be overloaded during braking. This overloading can be reduced by

- a low pivot point position - a rearward shift of the pivot point, and/or

- the use of compensating linkages.

The bogie pivot is usually placed so that the distance between it and the front and rear wheel axles is about 55 and 458 of the bogie arm, respectively. The static and dynamic overload on any of the wheels will then be limited to approximately 108 , and the load to be carried by the tires may be adapted accordingly (Ref, 10-1).

\subsubsection{Nosewheel tires}

The choice of the tire size is generally based on the nosewheel load during braking at maximum effort - the steady braked load. Using the symbols shown in Fig. 10-16, we may calculate the nosewheel load for constant deceleration from the equations of motion. Ignoring the aerodynamic moment and assuming that the nosewheel has no brakes, we have during the braked roll: b. BRITISH SIZES

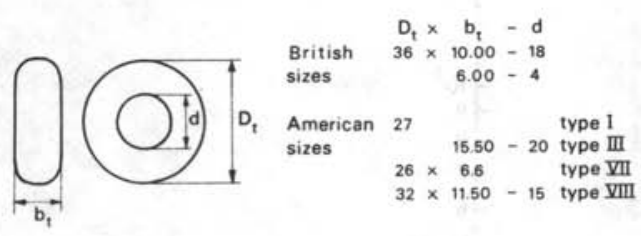

c. EXPLANATION OF THE TIRE CODE

Fig. 10-15 (continued)

$$
\begin{aligned}
\frac{a_{x}}{g} W & =\mu P_{m}+D-T \\
0 & =W-L-P_{m}-P_{n} \\
0 & =P_{m}^{l}{ }_{m}+\mu P_{m} h_{c g}-P_{n}^{l} n
\end{aligned}
$$

The nosewheel load can be derived from this:

$$
\frac{P_{n}}{W}=\frac{l_{m}}{l_{m}^{+l}}\left(1-\frac{L}{W}\right)+\frac{{ }^{h} c g}{l_{m}+l_{n}}\left(\frac{{ }^{a}}{g}-\frac{D-T}{W}\right)
$$




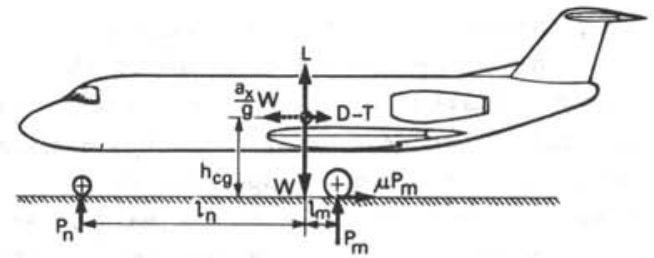

Fig. 10-16. Forces acting on the aircraft during a braked roll

As both $D$ and $L$ are positive, the maximum nosewheel load occurs at low speed. Reverse thrust decreases the nosewheel load and hence the condition $\mathrm{T}=0$ results in the maximum load:

$\frac{\mathrm{P}_{\mathrm{n}}}{\mathrm{W}}=\frac{l_{\mathrm{m}}+\frac{\mathrm{a}_{\mathrm{x}}}{\mathrm{g}} \mathrm{h}_{\mathrm{cg}}}{l_{\mathrm{m}}+l_{\mathrm{n}}}$

or:

$\frac{P_{n}}{W}=\frac{l_{m}+\mu h_{c g}}{\ell_{m}+l_{n}+\mu h_{c g}}$

Provided the necessary data are available to calculate the friction coefficient (see Appendix K), equation 10-23 can be used, whereas $(10-22)$ is more suitable when these data are not available. Typical values are $a_{x^{\prime}} g=.35-$ dry concrete, simple brake system

$a_{x} / g=.45$ - dry concrete, automatic brake pressure control.

The design condition is the forward c.g. limit at a high takeoff weight.

\subsubsection{Inflation pressure}

When the size of the tire is to be determined there will be a limited choice regarding the inflation pressure. Assuming that the wheel load and configuration of the landing gear are constant, the weight and volume of the tires will decrease with an increase in pressure. The wheels will take up less space when retracted while the drag of the extended undercarriage will be reduced as a result of the smaller frontal area. There will, however, be secondary factors which the designer should note:

a. With increasing inflation pressure the contact area with the runway will decrease. As explained in Section 10.2. the bearing capacity of the runway will therefore impose a limit on the inflation pressure. b. There must be sufficient space for fitting internal brakes inside the wheel rims and this will set a lower limit to the diameter of the hub as well as the wheel flange width. The total brake energy to be absorbed must therefore be determined, and for transport aircraft this may4 be assumed at approximately 600 'ib.ft per lb (183 kgm per $\mathrm{kg}$ ) of Maximum Landing Weight for jet aircraft and $450 \mathrm{lb} . \mathrm{ft}$ per lb (137 kgm per $\mathrm{kg}$ ) for propeller aircraft*. A brake manufacturer should be consulted to insure that this amount can be accommodated within the tire and wheel size. The choice of a lower inflation pressure will result in a larger tire size and hence more space will be available for the brakes. If an acceptable size for the tires and wheels still cannot be arrived at after this, a non-symmetrical wheel hub arrangement may be required.

c. The contact area of the tires with the ground is inversely proportional to the inflation pressure, and braking will be less effective with high inflation pressures. For example, Ref. 10-27 quotes for the tire/runway friction coefficient at zero rolling velocity, on dry concrete:

$\mu_{\text {static }}=.93-$ constant. $p$

where the constant is equal to $.0011 \mathrm{sq}$. in. per $1 \mathrm{~b}$ or $.0155 \mathrm{~cm}^{2}$ per $\mathrm{kg}$.

d. The aircraft will frequently have to be operated from wet runways. Assuming a tire with a suitable tread pattern, the aquaplaning velocity is found to depend almost entirely on the inflation pressure alone (Ref. 10-27):

* These data are reasonably accurate, provided the landing speed of the design has a normal value as compared with existing aircraft in the category considered. 
$\mathrm{v}_{\mathrm{aqua}}=$ constant $\sqrt{\mathrm{P}}$

where the constant is equal to 9 if $p$ is in $1 \mathrm{~b} / \mathrm{sq}$. in. and $\mathrm{V}$ in knots, or 17.5 if $\mathrm{p}$ is in $\mathrm{kg} / \mathrm{cm}^{2}$ and $\mathrm{V}$ in $\mathrm{m} / \mathrm{s}$.

The tire pressure also has an influence on the drag caused by water or slush on the runway. Although the performance of jet transport aircraft with their relatively high power loadings and rolling speeds is sensitive to this effect, it is no design practice to adapt the tire pressure accordingly.

\subsection{GEAR GEOMETRY AND RETRACTION}

Having chosen the disposition of the undercarriage and the tire dimensions, the airplane project design engineer must pay attention to the shock strut configuration and dimensions and, in the case of retractable gear, to the basic solution for the kinematics of the retraction and the space required for wheelbays.

\subsubsection{Energy absorption on touchdown}

The maximum kinetic energy of the aircraft normal to the runway to be absorbed when touching down is:

$$
E=\frac{W}{2 g} w^{2}
$$

where $w$ is the ultimate velocity of descent. Assuming conservatively that this energy will have to be absorbed completely by the main undercarriage, thus ignoring the energy transmitted to the atmosphere, the required stroke of each shock absorber is derived from:

$E=N_{s} P_{s} \lambda\left(n_{t} s_{t}+n_{s} s\right)$

where

$\mathrm{N}_{\mathbf{S}}$ is the number of main gear shock absorbers

$\mathrm{P}_{\mathbf{S}}$ is the static load per leg

$\lambda$ is a reaction factor, or ratio of maximum load to static load per leg

$s_{t}$ is the maximum tire deflection
$S$ is the stroke of the shock absorber $n$ is an efficiency factor, equal to the energy absorbed by the tire or the absorber divided by the product of $P$ and the maximum deflection or stroke, respectively.

Assuming the static load on the main under.* carriage equal to 928 of $\mathrm{W}$, we find for the required shock absorber stroke*:

$s=\frac{1}{n_{s}}\left(\frac{w^{2}}{1.84 g \lambda}-n_{t} s_{t}\right)$

It is recommended to add one inch to this value to allow for inaccuracies in the method and miscellaneous factors. The ultimate descent velocity $w$ is specified in the airworthiness regulations: FAR 23.473 and 25.473, BCAR Section D Chapter D3-5 Par. 4 and Section K Chapter K3-5 Par. 2 . For light aircraft:

$w=$ constant $\times(w / s) \cdot 25$

where the constant is 4.4 if the wing loading is in $\mathrm{lb} / \mathrm{sq} . \mathrm{ft}$ and $\mathrm{w}$ in $\mathrm{ft} / \mathrm{s}$, or . 9 if the wing loading is in $\mathrm{kg} / \mathrm{m}^{2}$ and $w$ in $\mathrm{m} / \mathrm{s}$. For transport aircraft $w$ is generally 12 $\mathrm{ft} / \mathrm{s}(3.66 \mathrm{~m} / \mathrm{s})$, although in the BCAR requirements it is dependent on the stalling speed.

The reaction factor $\lambda$ may be assumed equal to 2 to 2.5 for transport aircraft and 3.0 for light aircraft.

The efficiency data to be used in $(10-28)$ may be assumed as follows:

tires: $n_{t}=.47$

air springs: $n_{s}=.60$ to .65

metal springs with oil damping: $n_{s}=.70$ liquid springs: $n_{\mathrm{s}}=.75-.85$ oleo-pneumatic absorbers: $\eta_{s}=.80$. The maximum tire deflection can be obtained from the tire handbook, or alternatively from the approximation:

$s_{t}=$ constant $\frac{\lambda L_{w}}{p \sqrt{D_{t} b_{t}}}$

where $L_{w}$ is the static load per wheel and $D_{t}$ and $b_{t}$ are the tire diameter and maximum width. The

*more precisely: the projection of the stroke normal to the ground. 
constant of proportionality in $(10-30)$ is equal to .5. A simpler assumption is to take $s_{t}$ equal to three times the static deflection of the tire.

\subsubsection{Dimensions of the gear}

Useful statistical data can be found in Refs. 10-4 and 10-30, which are summarized as follows.

a. The diameter of the cylinder of a telescopic main gear unit may be taken as

$D=.5+.03 \sqrt{\mathrm{P}_{\mathrm{S}}}$ in., for $\mathrm{P}_{\mathrm{s}}$ in $1 \mathrm{~b}$

$\mathrm{D}=1.3+.11 \sqrt{\mathrm{P}_{\mathrm{S}}} \mathrm{cm}$, for $\mathrm{P}_{\mathrm{S}}$ in $\mathrm{kg}$

where $\mathrm{P}_{\mathrm{S}}$ is the maximum unfactored vertical load per leg.

b. Clearance allowances between the tire and the adjacent parts of the aircraft are based on

- the maximum dimensions of the inflated tire

- a growth allowance due to service

- the effect of centrifugal forces at highspeed rolling, which increase the diameter. Although the growth of tires depends to some extent on the type used, a 48 growth in maximum width and $10 \%$ in diameter during use will be good average values. The clearance around the tire required in connection with centrifugal forces may be taken from Fig. 10-17.

c. The distance between the tire center lines of twin tires $\mathrm{S}_{\mathrm{T}}$ shall be at least 1.18 times the maximum grown width of the tire. The minimum distance between the axle centers of tires in tandem shall be equal to the maximum grown tire diameter plus twice the radial clearance allowance according to Fig. 10-17. As the wheelbase of the bogie should be as short as possible in order to minimize tire scrubbing and bending moments, a value of 1.2 times the tire grown diameter can be taken as a workable ratio.

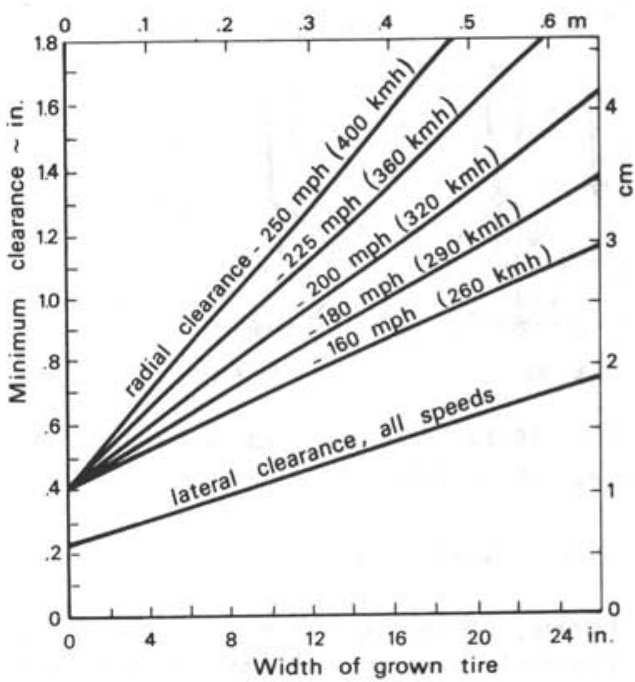

Fig. 10-17. Minimum clearance between tires and parts of the aircraft

d. The wheel spacing on a landing gear unit, with the wheels located on each side of the shock absorber strut, may be derived from (10-31) and Fig. 10-17 by adding twice the lateral clearance to the cylinder diameter. This dimension determines the total width of a dual wheel assembly.

e. In the case of a dual tandem bogie assembly, there should be a lateral spacing between the wheel center lines $\mathrm{S}_{\mathrm{T}}$ of 1.8 times the maximum tire width.

f. The length of the leg is determined mainly by the shock absorber stroke (see Section 10.5.1.) and the amount of overlap of the sliding assembly. Minimum values for this length are indicated in Fig. 10-18 for two types of simple telescopic gears.

Ref. 10-4 presents statistical data for determining the dimensions $X, Y$ and $z$. It is generally found that the undercarriage leg length required is about 3 times the shock absorber stroke for dual and multiwheel assemblies, plus a tire radius for the single wheel layout. 
SINGLE WHEEL

\section{TWIN \& MULTI-WHEEL}

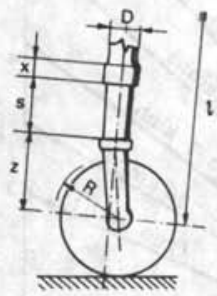

$l>2.6(s+x)+2$

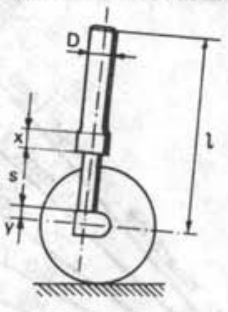

$1>2.5(5+x+y)$

Fig. 10-18. Dimensions of undercarriage legs (Ref. 10-4)

\subsubsection{Gear retraction}

Nowadays fixed landing gear is almost exclusively used on many small private aircraft at the lower end of the speed scale, i.e. cruising speeds of less than 135 to 160 knots $(250$ to $300 \mathrm{~km} / \mathrm{h})$. It is relatively easy to design a robust fixed landing gear and this may be the right choice for aircraft which have to use rough airfields. It may sometimes be attractive to fit a semi-retractable landing gear. Table 10-3 shows that even at speeds around

\begin{tabular}{|c|c|c|}
\hline & $\begin{array}{l}\text { Curdinal RC } \\
\text { (Ketractable } \\
\text { Gear) }\end{array}$ & $\begin{array}{l}\text { Cardinal in? } \\
\text { (Fixed Gear) }\end{array}$ \\
\hline Gross weight & $2,800 \mathrm{ib}$ & $2,500 \mathrm{lb}$ \\
\hline Empty weight & 1.63016 & $1,480 \mathrm{tb}$ \\
\hline Baggage & $120 \mathrm{tb}$ & $120 \mathrm{lb}$ \\
\hline Fuel capaeity & S1 gal & $30 \mathrm{gat}$ \\
\hline Top speed at sea level & $176 \mathrm{mpth}$ & $153 \mathrm{mph}$ \\
\hline $\begin{array}{l}\text { Cruise upeed ( } 753 \text { power at } 7,000 \mathrm{ft}^{\circ} \text { ) } \\
\text { Kange at } 752 \text { power at } 7,000 \mathrm{ft} \mathrm{t}^{\circ} \text {. }\end{array}$ & $166 \mathrm{mph}$ & 162 mph \\
\hline no reserve) & $765 \mathrm{sm}$ & $690 \mathrm{~nm}$ \\
\hline kate of eliab at sea level & $860 \mathrm{fpm}$ & $840 \mathrm{fpm}$ \\
\hline Service ceiting & $16,900 \mathrm{ft}$ & $14,600 \mathrm{ft}=$ \\
\hline Takeoff over So-ft obstacle & $1.585 \mathrm{ft}$ & $1,400 \mathrm{ft}$ \\
\hline Land ing over so-ft obstacle & $1,350 \mathrm{ft}$ & $1,220 \mathrm{ft}$ \\
\hline Stall speed, flaps up, pover off & $66 \mathrm{mph}$ & $63 \mathrm{mph}$ \\
\hline Stall speed, flaps down, power off & $57 \mathrm{mph}$ & $53 \mathrm{mph}$ \\
\hline \multicolumn{3}{|l|}{ Power: } \\
\hline \multicolumn{3}{|l|}{ - Cardinal RC: 200 hp Lycoming t0-360als6 } \\
\hline \multicolumn{3}{|l|}{ OCardinal 177:180 hp Lycoming 0-360azP } \\
\hline
\end{tabular}

Table 10-3. Performance comparison of Cessna Cardinals

160 knots $(300 \mathrm{~km} / \mathrm{h})$ there is a considerable improvement in cruising performance with the use of a retractable landing gear. Al-

though the two versions of the Cessna Cardinal shown are also slightly different in other respects, the gain in cruising speed is caused primarily by gear retraction. Incidentally, the manufacturer appears to stress the improved appeal of the RG version more than the actual gain in performance.

There are almost as many gear retraction schemes as there are different aircraft designs. A review of known retraction solutions should be made before the actual geometric design is started; Refs. 10-1 and 10-13 are valuable contributions in this field.

Most retraction mechanisms are derived from the four-bar linkage and the designer must have very good reasons to deviate from this. A suitable pivot point must be chosen for the leg which, at the same time, gives the required wheel positions and allows adequate length of the leg. A retraction mechanism, generally consisting of a folding stay member and a retraction jack, is then required. Provided a reasonable looking mechanism has been found, a retraction curve should be constructed, indicating the retraction load on the jack and a number of checks will have to be made. For example:

a. Make sure that the jack has adequate dead length.

b. The efficiency of the retraction geometry - i.e. the work done by the jack divided by the maximum jack force times the total jack travel - should be at least about 508 . Excessive variations in the jack force during retraction must be avoided. c. The points where the undercarriage is to be suspended must be arranged as close as possible to the wing spars, major frames or ribs or any other strong major structural member.

d. There must be adequate clearance between the members of the retraction mechanism. e. The leg must be adequately supported against forces in a plane normal to the plane of retraction.

A solution for various retraction problems 
may sometimes be found by arranging the swivel axis so that the wheel goes up a spiral path, with the result that it is rotated during retraction. Shock absorber contraction during retraction can be used to facilitate stowage, particularly when an articulated landing gear is used. The bogie undercarriage may have an extra degree of freedom available in that the bogie unit can swivel with respect to the main member, thus requiring a minimum of

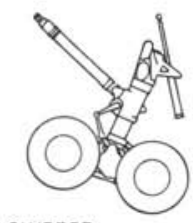

LOWERED

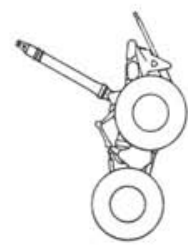

PARTIALLY RETRACTED

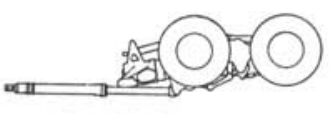

RETRACTED
Fig. 10-19. Retraction sequence of a typical bogie undercarriage

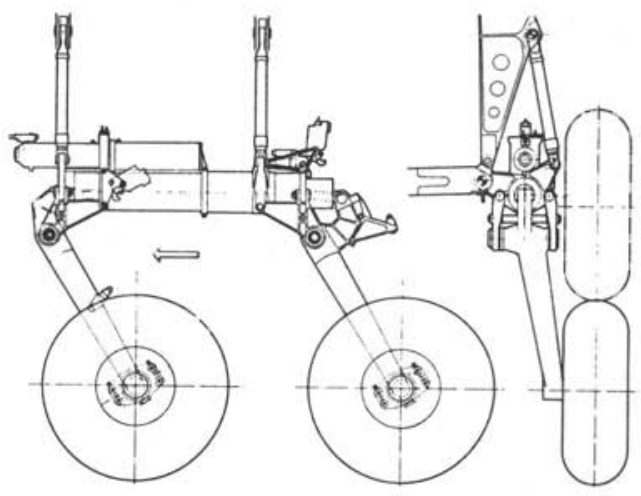

Fig. 10-20. The jockey undercarriage of the Breguet 941

space when retracted (Fig. 10-19). Jockey undercarriages (Fig. 10-20) are sometimes used on rough-fiela landing gear; they require only a small streamline fairing when retracted and may therefore be particularly suitable for fuselage-mounted gears.

Omission of a main wheel door may be considered on aircraft where the drag penalty thus incurred is of less importance than the gain in weight and retraction space (e.g. the Boeing 737, Yak 40). 



\section{Chapter 11. Analysis of aerodynamic and operational characteristics}

\section{SUMMARY}

The object of the design synthesis process dealt with in the previous chapters is to achieve the goals laid down in the design specification. The first cycle of the iterative design process will be concluded with an analysis of the operational characteristics for the purpose of investigating to what extent the design requirements have been met.

Some general comments on the prediction of aerodynamic characteristics are made in this chapter. Definitions and subdivisions of the drag according to several schemes are discussed. The choice of operational limit speeds and the determination of $n-v$ diagrams are then briefly reviewed. A procedure to analyze the flight profile, reserve fuel quantity and payload-range characteristics is given, followed by some general aspects of climb and field performance.

The chapter concludes with certain aspects of operating economy and some critical notes on the use of standard formulas for estimating direct operating costs. 


\section{NOMENCLATURE}

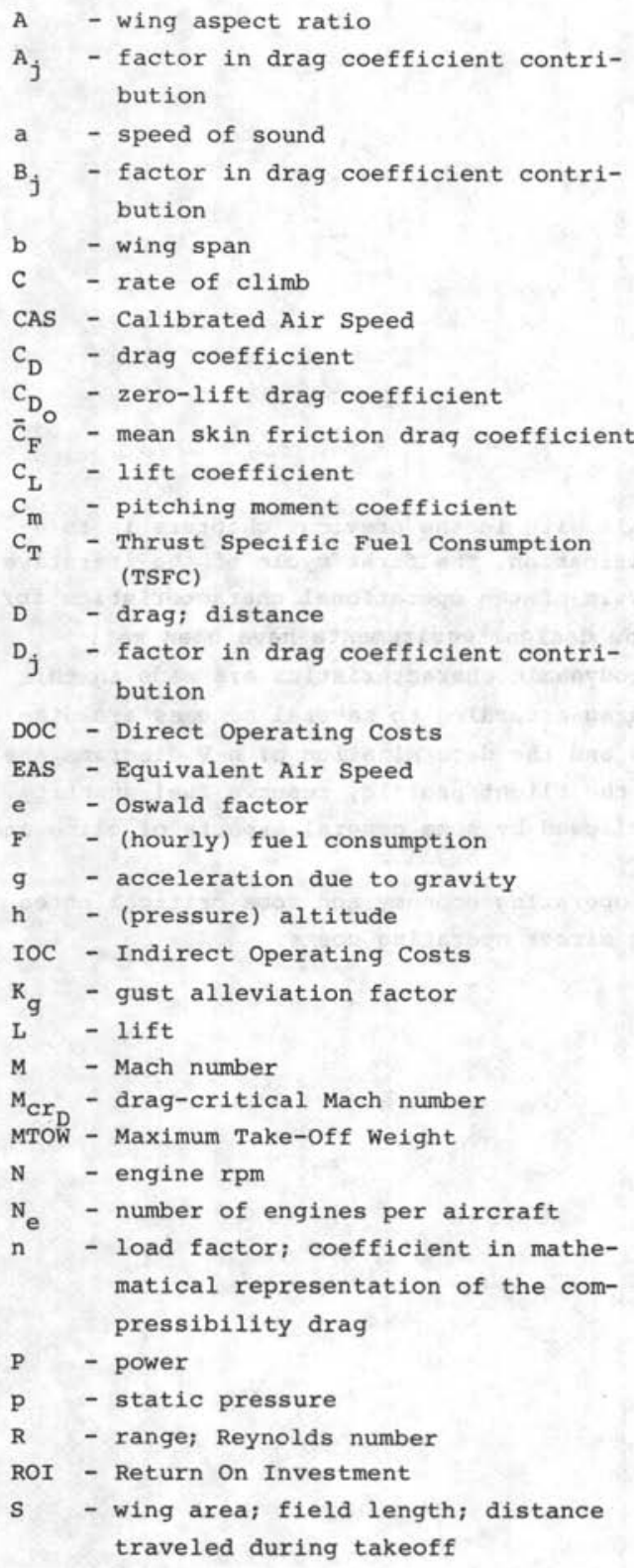

11.1. INTRODUCTION

The previous chapters were intended to provide the designer with background in-
- thrust; temperature

- True Air speed

- time

- airspeed

- equivalent airspeed

- weight

- gust velocity (EAS)

- angle of attack

- factor of proportionality in the drag coefficient

- ratio of specific heats; climb angle

- relative atmospheric pressure

- elevator deflection

- increment

- relative atmospheric temperature

- atmospheric density

- coefficient of frictional retardation

\section{Subscripts}

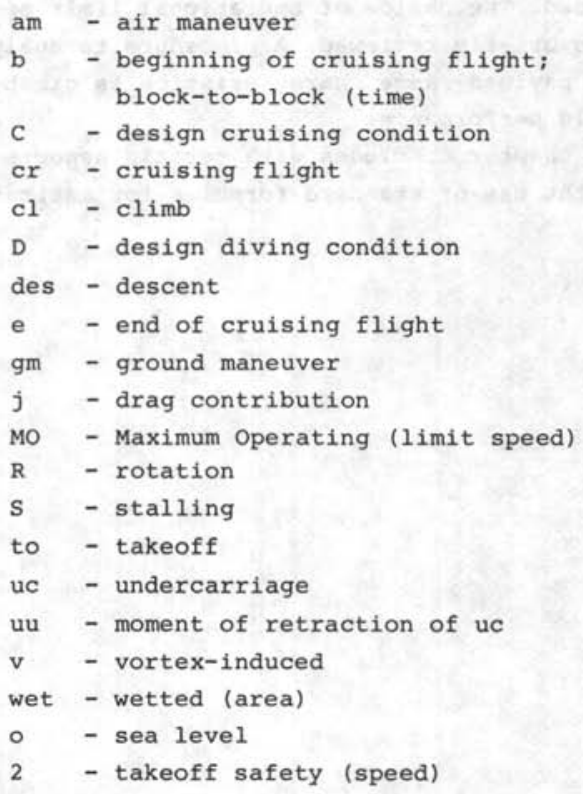

formation and basic (semi-) statistical methods and procedures for sizing the major parts of the airplane and allocating and disposing them in relation to each 
other. These considerations are intended to be of some help in obtaining a reasonable insight into the design process and in finalizing the first design iteration in a reasonable space of time.

Referring back to section 1.5 of the first chapter and in particular to Figs. 1-7 and $1-9$, we find that the designer's next step is to analyze the design by estimating some of the aerodynamic characteristics and determining major performances and certain flying qualities. The primary aim of this step is to verify whether the design meets the initial specification. Generally speaking, this comparison will reveal that design improvements are required but experience gained during the design stage may well lead to the conclusion that the design specification should be changed instead of or in combination with the aircraft. In any case, the design analysis and evaluation will finally result in an initial baseline design which takes into account the most recent views on the design requirements.

Although many aspects can only be considered after detailed study and testing, estimates are usually male of several performance aspects, such as cruising performance, airfield performance, design speeds and Direct Operating Costs (DOC). A report on the background information for a design study might contain the following elements. a. A prediction of the lift curve, the drag polar, and the pitching moment curve for representative cruising conditions.

b. Calculations of the cruising speed and/ or maximum flight speed and the specific range (i.e. distance traveled per unit weight of fuel) for several flight speeds and/or cruising altitudes.

c. Considerations for choosing the most relevant structural design speeds $\left(\mathrm{V}_{\mathrm{C}}\right.$ and $V_{D}$ ) and determining the limitations of operational speeds vs. pressure altitude, i.e. the flight envelope.

d. Calculations of $v-n$ diagrams for maneu- vering and gust loads for several representative operational conditions.

e. Computations of the maximum rate of climb and ceilings with all engines operating and with a failed engine (as appropriate).

f. A prediction of the $c_{L}{ }^{-\alpha}$ curves and the airplane drag polar for several positions of the high-lift devices, with undercarriage up and down.

g. Limits for the takeoff and landing weight, based on the airworthiness requirements in relation to climb performance, i.e. WAT curves.

h. Calculations of the takeoff and landing field length required in accordance with the airworthiness rules appropriate to the particular airplane category.

j. Payload-range diagrams for several cruising conditions, due allowances being made for reserve fuel.

$k$. An estimate of the operating costs as a function of the stage length, using a suitable standard method.

Many other questions can be raised and the designer must make a decision on the basis of what he considers necessary and appropriate in the preliminary design stage. For example, a detailed study of some principal stability and control characteristics can be carried out, particularly if there are sound reasons for doubting the adequacy of the empennage and control surface design. However, the designer should also realize that many assumptions have to be made which must be verified later on and decide whether much time should be spent in studies the conclusions of which are subject to considerable inaccuracy. Several topics in this chapter will be touched upon in a rather superficial manner since a thorough treatment might necessitate as many chapters as there are sections in this chapter. More detailed information can be obtained from the publications listed in the references and also in Appendices E, F, G and $\mathrm{K}$. 
11.2. TERMINOLOGY RELATING TO THE DETERMINATION OF DRAG

One difficulty that arises when drag prediction methods are compared or drag measurements interpreted is that the terminology often creates confusion. Several schemes (Fig. 11-1) are possible for sub-

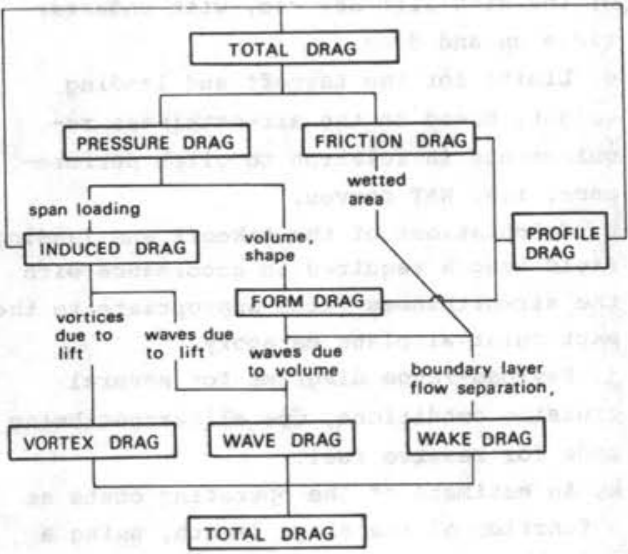

Fig. 11-1. Drag breakdown of a body without internal flow

dividing the drag of an airplane, as explained in Refs. 11-1 and $11-2$ and the following sections.

\subsubsection{Pressure drag and skin friction drag}

The total action that a fluid exerts on a body can be considered as the resultant of the elementary forces exerted on all points of the surface, both normally and tangentially. Considering the drag on a closed body (i.e. one with no internal flow due to powerplant installation or internal systems), the SKIN FRICTION or SURFACE DRAG is generated by tangential forces, while PRESSURE DRAG is caused by the normal forces. The lift can be subdivided in a similar manner, but it is usually considered a component of only pressure forces. The pressure distribution is also affected by the boundary layer and regions of separated flow. In wind tunnel experiments the pressure forces can be obtained directly by measuring the distribution of the pressure at several small holes, situated at the surface of the body, while friction drag is obtained by subtracting the pressure drag from the total drag. Assuming the mean friction drag coefficient based on the wetted areas of the various aircraft parts to be denoted $\overline{\mathrm{C}}_{\mathrm{F}^{\prime}}$. the skin friction drag can be written:

$D_{F}=\bar{C}_{F} \frac{1}{2} \rho V^{2} s_{\text {wet }}$

where $\bar{C}_{F}$ is typically of the order of .003 to .005 for most subsonic aircraft. The wetted area of a body or wing is therefore the main geometric parameter determining the skin friction drag.

11.2.2. Wake drag, vortex-induced drag, and wave drag

According to the law of conservation of energy, the work produced in overcoming the aerodynamic drag of a body moving at constant speed is equal to the energy increase in the surrounding fluid. The forms of energy transmitted to the fluid are vortex-induced energy, wake energy, and shock wave energy. The aerodynamic drag can be subdivided accordingly (Fig. 11-1). VORTEX-INDUCED DRAG is part of the pressure drag, corresponding to the kinetic energy - distributed throughout the fluid associated with the trailing vortices shed by a lifting wing of finite aspect ratio. For high aspect ratio wings with relative1y thick airfoil sections, the vortex-induced drag can be computed accurately with lifting-line or lifting-surface theories. However, when section leading edges are sharp, angles of sweepback large and/or aspect ratios low, the leading-edge suction force begins to break down at some critical lift coefficient and the vortexinduced drag increases considerably (see Fig. 7-22 and Ref. 11-12).

For elliptical wings of high aspect ratio, the vortex-induced arag is given by: 
$\frac{D_{V}}{W}=\frac{C_{L}^{2} \frac{1}{2} \rho V^{2} S}{\pi A W}=\frac{1}{\pi} \frac{W / b^{2}}{\frac{1}{2} \rho V^{2}}$

This equation illustrates that, for given flight conditions, the span loading $\mathrm{w} / \mathrm{b}^{2}$ determines the induced drag.

WAKE DRAG is caused by the boundary layer and region:s of separated flow. The main source $c f$ drag generated by the boundary layer is shear action, resulting in skin friction drag; the pressure drag is generally an order of magnitude less. Separation drag, however, is predominantly caused by forces normal to the surface. for a well-streamlined body at small angles of attack, the skin friction drag is the dominant part of the wake drag. SEPARATION DRAG increases sharply when the stall is approached and is also manifest in areas near ill-shaped wing body junctions, blunt bases, sharp corners, etc. Flow separation can be predicted and analyzed theoretically in only a few simple cases and drag prediction is therefore of an empirical nature. It cannot be estimated with good accuracy for project design.

WAVE DRAG is another part of the pressure drag, associated with the work produced by compression of the fluid at high (local) flow velocities, which manifests itself in the form of shock waves. A complicating factor is that strong shock waves may induce flow separation, resulting in an increase in both the wake drag and the vortex-induced drag. At supersonic speeds, assuming linear theory, wave drag can be subdivided into wave drag due to the body volume and wave drag due to lift, but at transonic speeds, when mixed flow is present, this subdivision is less evident.

It will be clear from the foregoing that the subdivision of drag into vortex-induced, wake and wave drag components is not very well defined in cases where appreciable interactions between the various flow fields occur. These interference effects result in drag increments, frequently referred to as INTERFERENCE DRAG.
11.2.3. Form drag, profile drag and induced drag

The pressure drag component of the wake drag and the wave drag due to volume (if present) may be combined into the FORM DRAG. For a specified angle of attack this contribution depends upon the shape or form of the body. For a nonlifting body, shedding no vcrtices, the form drag is equal to the pressure drag on the body. When a two-dimensional wing is placed in a wind tunnel, it experiences skin friction plus form drag, a combination called the PROFILE DRAG or section drag. This term is also used to define the skin friction plus form drag of three-dimensional bodies, with or without lift.

INDUCED DRAG is the resultant of vortexinduced drag and wave drag due to lift. In the absence of shock waves, profile drag is equal to wake drag and for a wind tunnel model it can be derived from static and total pressure distribution measurements in the wake of the body. The induced drag is found by subtracting the wake drag from the total drag.

\subsubsection{Zero-lift drag and 1ift-dependent} drag

The subdivision shown in Fig. 11-1 is useful for the purpose of theoretical and semi-empirical drag computation and for the interpretation of wind tunnel measurements. When it comes to the analysis of flight test results, only the total lift and drag are available, as these are derived directly from measurements of the aircraft motion (cf. Ref. 11-13). In view of the fact that the vortex-induced drag coefficient is essentially proportional to $C_{L}{ }^{2}$, it is usual to plot measured $C_{D}$ values versus $C_{L}{ }^{2}$ (Fig. 5-4b). In the range of normal operational values of $C_{L}$ a straightline approximation is found to be acceptable. The subdivision of drag into zerolift drag and lift-dependent drag,

$C_{D}=c_{D_{0}}+\frac{C_{L}{ }^{2}}{\pi \AA e}$ 
originates from this concept. The meaning of the Oswald factor e is explained in Section 5.3 .1 .

It is regrettable that the term $C_{L}{ }^{2} / \pi A e$ is frequently referred to as "induced drag coefficient", which makes confusion with the previous scheme unavoidable. Liftdependent drag comprises not only vortexinduced drag and lift-induced wave drag but also the variation of the profile drag with the angle of attack.

According to $(5-9)$ the condition of minimum drag is $C_{L}=0$, while this should in

fact be some positive value of $C_{L}$, referred to as $C_{L_{\text {ref }}}$ in Fig. $11-2$. The fol-

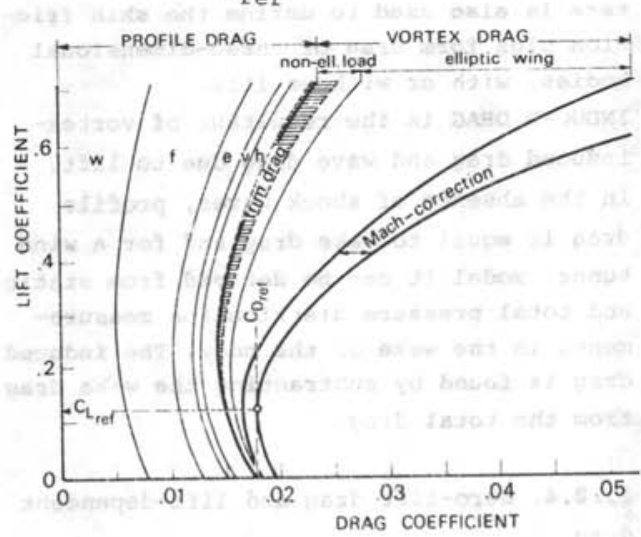

Fig. 11-2. Drag buildup by analysis ( $w=$ wing; $f=$ fuselage $e=$ engine installation; $\mathrm{v}=$ vertical tailplane; $\mathrm{h}=$ horizontal tailplane)

lowing representation of the drag polar yields a more accurate approximation, particularly for low values of $C_{L}$ :

$C_{D}=C_{D_{\text {ref }}}+\beta\left(C_{L}-C_{L_{\text {ref }}}\right)^{2}$

The drag for $C_{L}=C_{L}$ is referred to as the reference drag or basic drag.

\subsubsection{Breakdown for drag analysis}

In the preliminary design stage, when no wind tunnel data are available, traditional drag prediction methods are used, based on the following procedure:
1. Calculate the drag of each major part separately, assuming that they are isolated from each other.

2. Add all contributions.

3. Make corrections for the interaction of the flow fields.

The last term is sometimes called "interference drag", but the term "interference corrections" is more justifiable. A classical subdivision of the total drag is as follows :

- (minimum) wing profile drag,

- (minimum) parasite drag*, i.e. the drag of all other airplane items plus corrections for interference,

- lift-dependent drag.

Most individual drag contributions can be approximated as follows:

$C_{D_{j}}=A_{j}+B_{j} C_{L}+D_{j} C_{L}{ }^{2}$

Fic. 11-2 shows the variation of several contributions with $\mathrm{C}_{\mathrm{L}}$. The minimum value of the wing profile drag - the basic profile drag - corresponds to the design lift coefficient. Similarly, a flow condition can be defined for minimum profile drag for other airplane parts; this is usually at a positive value of $C_{L}$ which is representative of cruising conditions. TRIM DRAG is defined as the drag due to the horizontal tailplane load required to ensure longitudinal equilibrium at a specified center of gravity position. This drag component is composed of vortex-induced drag due to tail load and a profile drag increment due to elevator deflection. It takes into account the wing lift increment or reduction needed to obtain a given $C_{L}$, resulting from the horizontal tail download or upload.

Compressibility correction is not a pure drag contribution, as compressibility affects both the vortex-induced and profile drag. Most drag predictions are based on a low speed drag polar $(M<.5)$ and a sep-

*The term "parasite drag" is not uniquely defined in the literature and is too arbitrary to serve any useful purpose nowadays 
arate correction is made for compressibility effects at $M>.5$.

A detailed subdivision, useful for the estimation of a drag polar, is presented in Appendix F, Table F-1.

\subsubsection{Bodies with internal flow}

All subdivisions treated previously are strictly applicable only to closed bodies without internal flow. The effects of engine power on the aerodynamic properties can be fairly complicated, as explained in some detail in Appendix F, Section F-5.6. If the effects of powerplant operation on lift and drag are appreciable, lift curves and drag polars must be established for various engine operational conditions. In view of the limited scope of this chapter no details will be given here. The interested reader is referred to Ref. $11-6$ and the references mentioned in Appendix F.

\subsection{DETERMINATION OF AERODYNAMIC CHARAC-} TERISTICS

In order to analyze the performance and flight characteristics of an aircraft in the preliminary design stage, several aerodynamic characteristics must be determined:

$C_{L}$ vs. $\alpha$ : the lift curve

$C_{D}$ vs. $C_{L}$ : the drag polar

$C_{m}$ vs. $C_{L}$ or $C_{m}$ vs. $\alpha$ (for $\delta_{e}=$ constant): pitching moment curves.

These relationships must be determined for the cruise configuration (en route) and for various flap deflection angles. In the case of high-subsonic speeds the Mach number must also be treated as a separate variable.

The abovementioned relationships are adequate for performance calculation and determination of the neutral point, with the stick fixed. Prediction methods and data can be found in Appendices E, F and G. For the calculation of the stick-free longitudinal stability the elevator hinge moments must be known and additional stability derivates are required for an assessment of dynamic longitudinal stability, lateral stability, and control properties. In view of the limited space available in this book no attempt has been made to present calculation methods for these properties. Valuable standard methods are available in the form of Data Sheets issued by the Engineering Sciences Data Unit (ESDU) and the USAF Stability and Control DATCOM (Ref. 11-4).

The designer may be faced with a number of difficulties when he tries-to predict the aerodynamic properties of his project: a. The external shape is not yet fully defined in the preliminary design stage; hence pressure distributions cannot be calculated and semi-empirical prediction methods must be developed. The effects of surface imperfections, excrescences, etc., must be estimated on a statistical basis. b. Wind tunnel measurements are sometimes available, but their interpretation may be difficult and their applicability limited due to the appreciable differences in Reynolds number compared with free flight conditions.

c. Theoretical prediction methods generally yield good results for conventional shapes, but drag due to flow separations and shock waves is very difficult to predict and much remains to be done in this field for aerodynamicists.

\subsubsection{Reynolds number effects}

For each airplane configuration the operational variation in airspeed and altitude results in Reynolds number variations. In the preliminary design stage only one polar is usually generated for each configuration. A representative value for $R$ is therefore chosen for each polar curve, but a simple refinement on this particular point is also possible, as discussed in Appendix F, Section F-3.2. 
11.3.2. Mach number effects

A more fundamental difficulty is how compressibility effects on drag have to be taken into account. The critical Mach number $\mathrm{M}_{\mathrm{Cr}}$, at which a sharp increase in the drag occurs (drag rise), as defined in Section $7.5 .1 \mathrm{~b}$ may be used to characterize the drag due to compressibility. For Mach numbers up to this value the drag increment is referred to as the drag creep. An arbitrary mathematical representation may take the following form:

$$
\begin{aligned}
\Delta C_{D}=.002\{1+ & \left.n \frac{\mathrm{Cr}_{D}-M^{-1}}{\Delta M}\right\}^{\text {for } M \leqslant M_{C r_{D}}}
\end{aligned}
$$

while the drag rise can be represented as follows:

$$
\begin{aligned}
& \Delta C_{D}=.002\left\{1+\frac{M-M_{C r_{D}}}{\Delta M}\right\}^{n} \\
& \text { for } M \geqslant M_{C r_{D}}
\end{aligned}
$$

These expressions, like the symbols $\mathrm{n}$ and $\Delta M$, have no physical significance, but they can be used to approximate the actual shape of the drag curve (Fig. 11-3). A suitable combination of $\Delta M$ and $n$ can be derived from experimental data, if available. Assuming, for example, $\Delta M=.05$ and $\mathrm{n}=2.5$, it is found that $\mathrm{dC}_{\mathrm{D}} / \mathrm{dM}=.10$ for $\mathrm{M}=\mathrm{M}_{\mathrm{Cr}}$. In this case the two definitions of ${ }^{M_{C r}}$ given in Section 7.5 are identi-

11.3.3. Low speed polars

The aerodynamic characteristics for the configuration with high-lift devices in various positions and undercarriage up or down must be available before we can analyze the low-speed performance. Estimation of these characteristics on a basis of theoretical analysis is usually not possible in the conceptual design stage and the designer must rely on semi-empirical procedures. A fairly complete survey of this subject is presented in Ref. 11-15, while some prediction methods have been summarized in Appendix G. The designer must be prepared to accept considerable inaccuracy in the results of these handbook-type methods and it is very desirable that wind tunnel experiments are made available as early as possible in the development stage.

Calculations of low-speed polars (undercarriage up) for a hypothetical design presented in Fig. 12-1 have resulted in Fig. 11-4. Drag due to engine failure is not included in this figure but it is important for takeoff and approach climb performance in the case of an inoperative outboard engine. Some information on this subject is presented in section G-8 of Appendix G. It has been found convenient

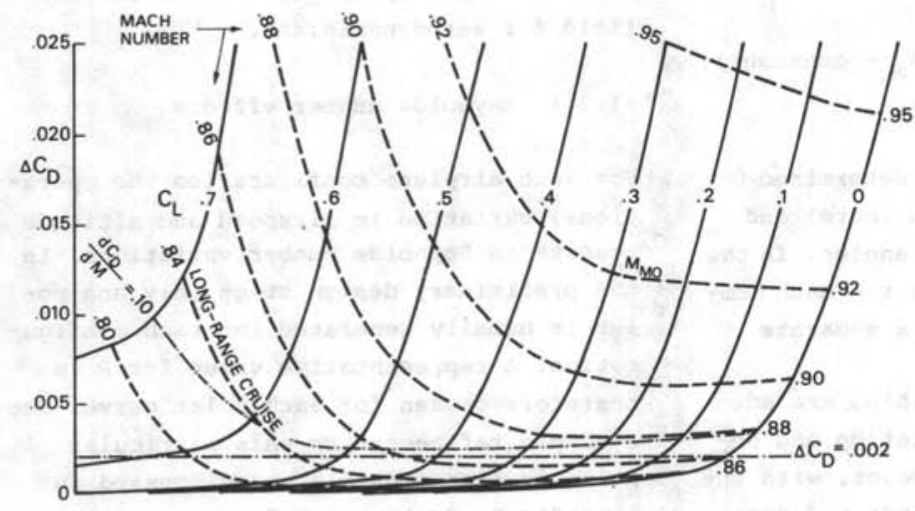

Fig. 11-3. Drag rise of a long-range aircraft 


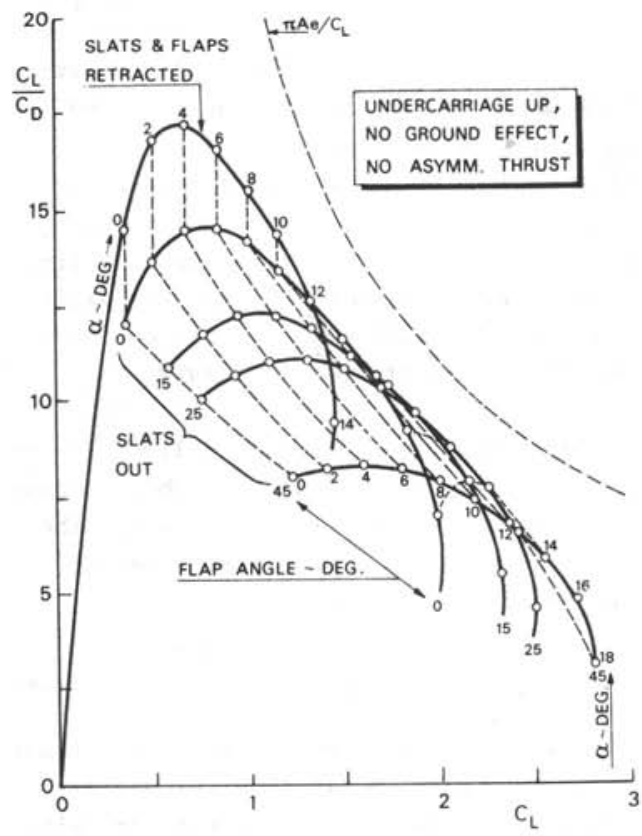

Fig. 11-4. Lift-to-drag ratio for the design in Fig. 12-1.

to consider the induced drag of the vertical tail due to the associated side force to be equivalent to a loss of thrust, by multiplying the thrust available by a factor:

$\frac{\text { thrust }-\Delta \text { drag }}{\text { thrust }}=1-$ constant $\cdot \frac{T}{W} C_{L} \quad(11-7)$

For the project shown in Fig. 12-1 a typical value is .92 at $V_{2}$. Hence, the asymmetric drag is equal to 88 of the total thrust in this condition.

\subsection{THE FLIGHT ENVELOPE}

The main background to this subject is found in:

FAR 23.335, 23.1505, BCAR Section K Ch. K3-2 and $\mathrm{K} 7-2$ for light aircraft; FAR 25.335, 25.1505, BCAR Section D Ch. D3-2 and D7-2 for transport aircraft. The determination of performance capabil- ities is preceded by a proper choice of the operational variation of flight speeds and altitude - an example is given in Fig. 11-5 - referred to as th flight envelope. The following definitions refer to jet transports.

a. Maximum Operating Limit Speed $\left(V_{M O}\right)$ or Mach Number $\left(\mathrm{M}_{\mathrm{MO}}\right)$ is the EAS, CAS or Mach number (whichever is applicable to the altitude) which should not be exceeded in any flight regime. It is so selected that the aircraft remains free from buffeting or undesirable flying qualities associated with compressibility up to this speed. $\mathrm{V}_{\mathrm{MO}}$ must not exceed $\mathrm{V}_{\mathrm{C}}$.

b. The Design Cruising speed $\left(V_{C}\right)$ is the maximum EAS in level flight at which the structure is designed to withstand particular loads specified in the airworthiness regulations, e.g. a gust load at $50 \mathrm{ft} / \mathrm{s}$ $(15.3 \mathrm{~m} / \mathrm{s})$ vertical gust velocity. $v_{C}$ must be high enough to permit economic climb and cruise performance, but low enough to avoid excessive structure weight penalties in order to cater for gust loads. At high altitude $v_{C}$ becomes related to the

\section{ALTITUDE $(\mathrm{ft})$}

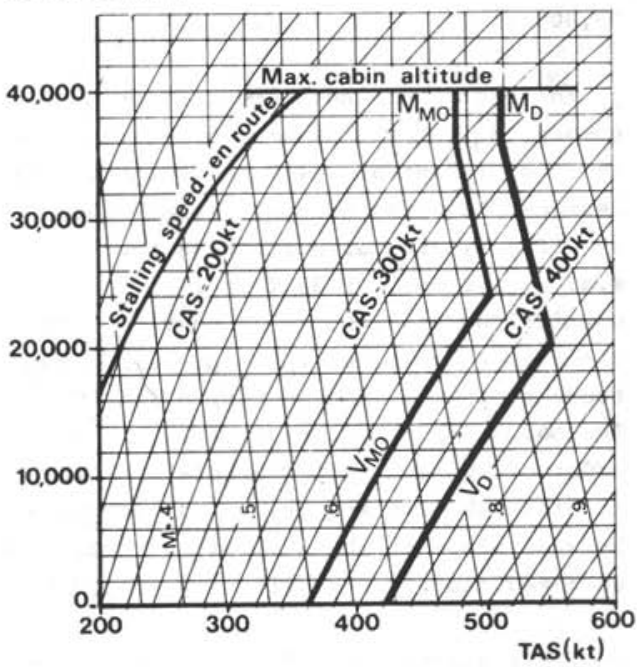

Fig. 11-5. Example of a flight envelope. 
design cruising Mach number for optimum long-range cruise capability. A calculation should be made of the maximum speed in cruising flight, using a performance diagram (Fig. 11-6), for various atmos-

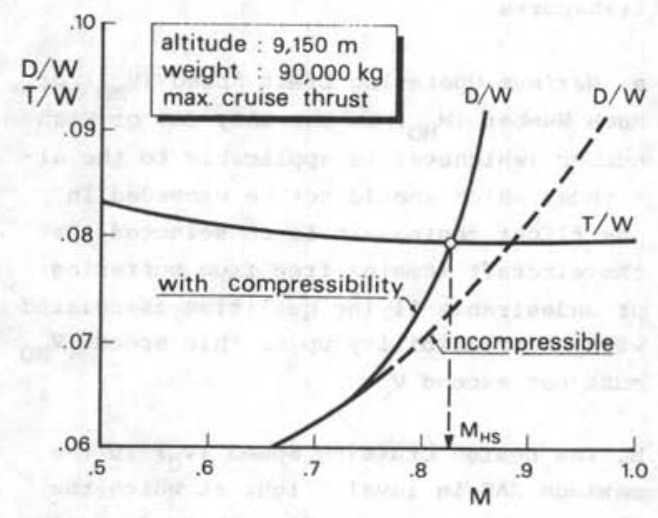

Fig. 11-6. Determination of the cruise speed

pheric temperatures and altitudes - see also Fig. 5-10. The choice of $\mathrm{V}_{\mathrm{MO}} / \mathrm{M}_{\mathrm{MO}}$ at cruising altitude must be such that highspeed cruise conditions are not unduly limited by this placard speed. For highsubsonic jet aircraft $V_{M O}$ usually becomes limiting at altitudes below 20,000 to $25,000 \mathrm{ft}(6,000$ to $7,500 \mathrm{~m})$.

c. The Design Diving Speed $\left(V_{D}\right)$ or Mach number $\left(M_{D}\right)$ is the maximum speed (EAS, CAS) in level flight at which the structure is designed to withstand particular loads specified in the airworthiness regulations, e.g. a gust load at $25 \mathrm{ft} / \mathrm{s}(7.55 \mathrm{~m} / \mathrm{s})$ vertical gust velocity. It must be sufficiently greater than $v_{C}$ to provide for safe recovery from inadvertent upsets at $v_{C}$. The margin $M_{D}-M_{M O}$ is generally of the order of .05 to .08 for aircraft cruising at high-subsonic speeds. It has to be shown by analysis that the aircraft is free from undamped flutter vibrations up to $1.2 \mathrm{~V}_{\mathrm{D}}$.

d. The stalling speed $v_{S}$ is the minimum speed with power off, encountered in the stalling maneuver referred to in section
5.4.4. (flaps up or down). It is acceptable to assume $v_{S}$ to be about 948 of the l-g stalling speed, if a significant reduction in the stalling speed relative to $\mathrm{V}_{\mathrm{S}^{-1 \mathrm{~g}}}$ is*anticipated.

It should be noted that $v_{S}$ increases with pressure altitude. Due to the increasing Mach number at the stall, compressibility effects will reduce $C_{L}-\max$ at high altitudes and therefore $v_{S}$ is not a constant EAS for most high-speed aircraft.

e. For pressurized aircraft a limit is imposed on the cruising altitude by the maximum pressure differential for which the cabin structure and the pressurization system are designed:

$\Delta \mathrm{p}_{\mathrm{C}}=$ ambient static pressure cabin pressure

This quantity is normally chosen such that at normal cruising altitude the cabin pressure is equivalent to a pressure altitude of approximately $6,000 \mathrm{ft}(1,830 \mathrm{~m})$, i.e. $\delta=.80$ in the ISA. Hence,

$\Delta \mathrm{p}_{\mathrm{c}}=\left(.80-\delta_{\mathrm{cr}}\right) \mathrm{p}_{\mathrm{o}}$

The maximum allowable cabin pressure altitude is $8,000 \mathrm{ft}(2,440 \mathrm{~m})$, i.e. $\delta=.67$ in the ISA. Thus the maximum altitude limited by the cabin design is defined by

$\delta=.67-\frac{\Delta \mathrm{p}_{\mathrm{c}}}{\mathrm{p}_{\mathrm{o}}}=\delta_{\mathrm{cr}}-.13$

f. Diagrams for maneuver and gust loads vs. EAS, referred to as $q-v$ or $n-v$ diagrams, can be drawn once the design airspeeds have been determined. The conditions for defining the gust speeds, design speeds, and normal load factors are defined in the airworthiness regulations, FAR 23.321-341, 25.321-341, BCAR Ch. D3-2 and Ch. K3-2. The maneuver load factor is a simple straightforward function of the aircraft All-Up Weight for the appropriate airworthiness category. The gust load is determined by:

$\mathrm{n}=1 \pm \mathrm{K}_{\mathrm{g}} \frac{\mathrm{dC} \mathrm{L}_{\mathrm{L}}}{\mathrm{d \alpha}} \frac{1}{2} \rho_{\mathrm{o}} \mathrm{w} \mathrm{v}_{\mathrm{E}} \frac{\mathrm{S}}{\mathrm{w}}$ 
The lift-curve slope $\mathrm{dc}_{L} / \mathrm{d} \alpha$ can be obtained from Appendix E, Section E-4.1. It is affected by the compressibility of the air. The gust alleviation factor $\mathrm{K}_{g}$ can be taken from the rules if better methods are not available to the designer. Critical conditions for the gust loads are frequently met at an altitude of about $20,000 \mathrm{ft}$ $(6,000 \mathrm{~m})$ with approximately 508 of the fuel load burnt off.

\subsection{FLIGHT PROFILE ANALYSIS AND PAYLOAD- RANGE DIAGRAMS}

Range performance has a direct effect on the transportation costs through the aerodynamic quality of the aircraft and its engine characteristics. The payload-range diagram - explained in Section 8.2.3. must be drawn to check whether the specified performance aims can be achieved with the MTOW assumed previously.

Fig. 11-7 shows a typical breakdown of the

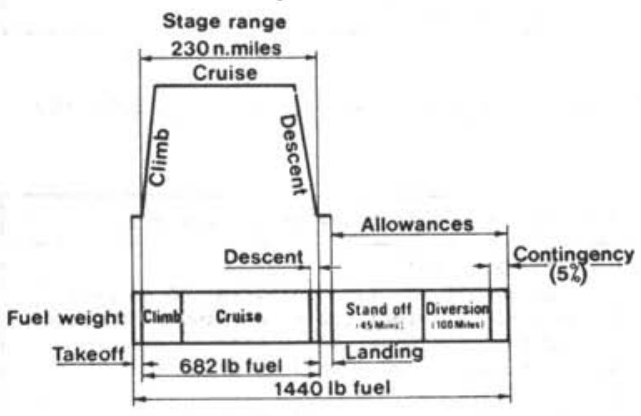

Fig. 11-7. Typical fuel breakdown for an executive aircraft (Ref. SAWE Paper No. 996)

total fuel load for an executive aircraft. For civil operations the basic mission profile analysis for a specified stage length can be carried out with the data of Table 11-1. Minimum fuel reserve loads are specified in FAR 91.23 (IFR operations) and 91.207 (VFR operations), but in airline practice the quantity of reserve fuel is usually larger. Typical policies are given in Table 11-2.
In the next part of this section it is assumed that the principles of flight mechanics and performance calculation are known or at least available to the reader. A selected list of publications on this subject can be found in the references.

11.5.1. Operational climb

In view of the limited equivalent cabin rate of climb and the general desirability of obtaining a high block speed, the operational climb speed is usually taken greater than the speed for maximum rate of climb. A climb at constant EAS results in a steadily increasing TAS and $M$ with increasing altitude. Above a certain altitude it will be necessary to continue the climb with constant $\mathrm{M}$ in order to avoid undesirable compressibility effects. For small climb angles the rate of climb is given by:

$C=\frac{(T-D) V}{W\left(1+\frac{V}{g} \frac{d V}{d h}\right)}$

The correction term for accelerated flight depends on the flight procedure. For flight in the ISA, it can be shown that:

$$
\begin{aligned}
& \frac{\mathrm{V}}{\mathrm{g}} \frac{\mathrm{dV}}{\mathrm{dh}}=.5668 \mathrm{M}^{2}-\text { EAS constant } \mid \text { tropo- } \\
& =-.1332 \mathrm{M}^{2}-\mathrm{M} \text { constant sphere } \\
& =.7 \mathrm{M}^{2}-\text { EAS constant strato- } \\
& =\begin{array}{lll}
0 & -M & \text { constant }
\end{array} \text { sphere }
\end{aligned}
$$

Similar corrections for flight at constant CAS can be obtained from Ref. 11-23. The operational climb is computed by means of a step-by-step calculation procedure, for an appropriate engine rating which is usually specified by the engine manufacturer.

\subsubsection{Cruise performance}

In view of the large number of variables associated with cruising in various operational conditions (weight, altitude, speed, engine rating) it is desirable to carry out the analysis using nondimensional parameters. This will be shown here for jet aircraft, but the reader may work it out for propeller aircraft, if desired. 


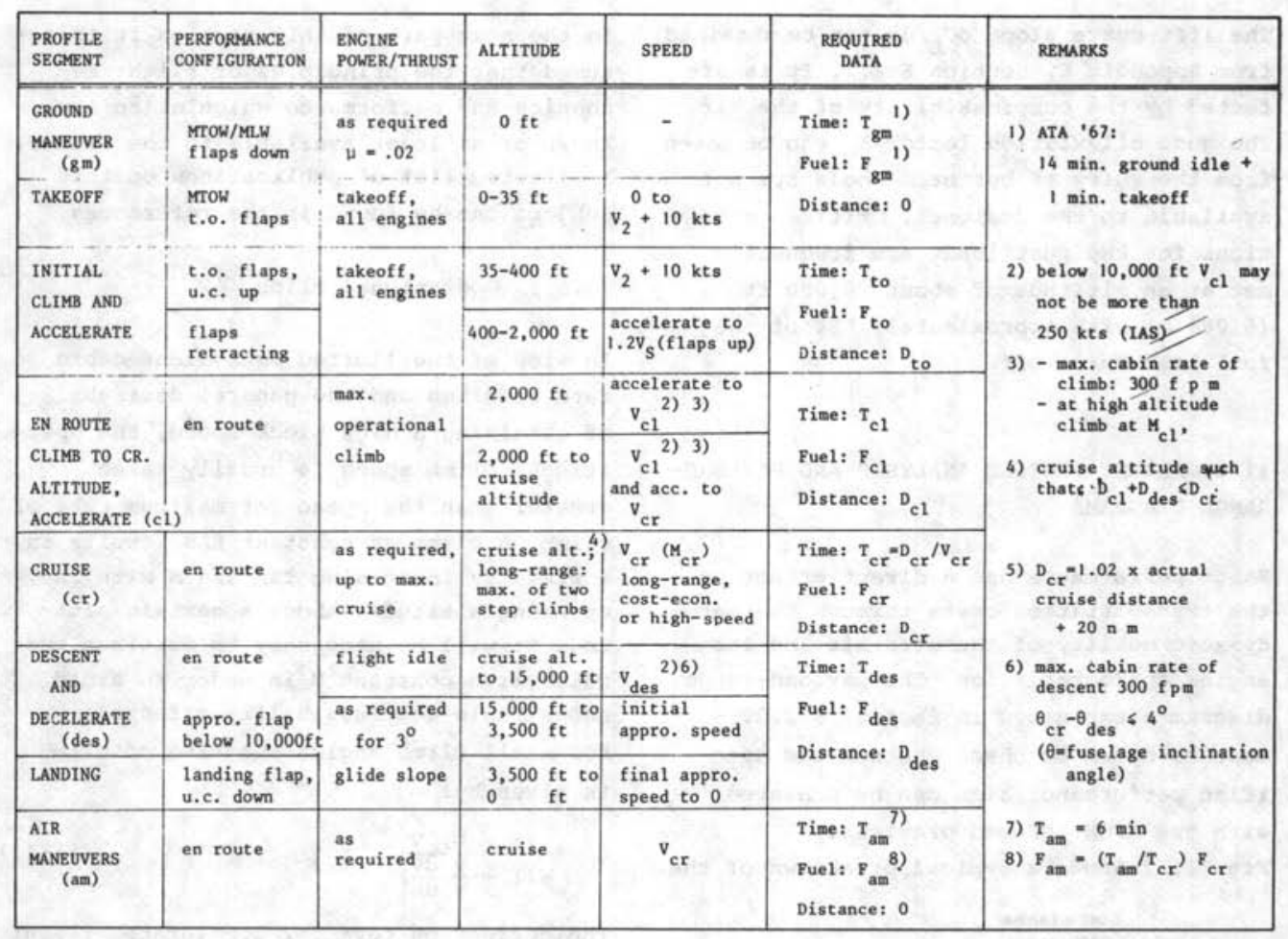

Table 11-1. Typical basic flight profile analysis, transport aircraft (Refs.: ATA '67 method, SAWE Technical Report 619)

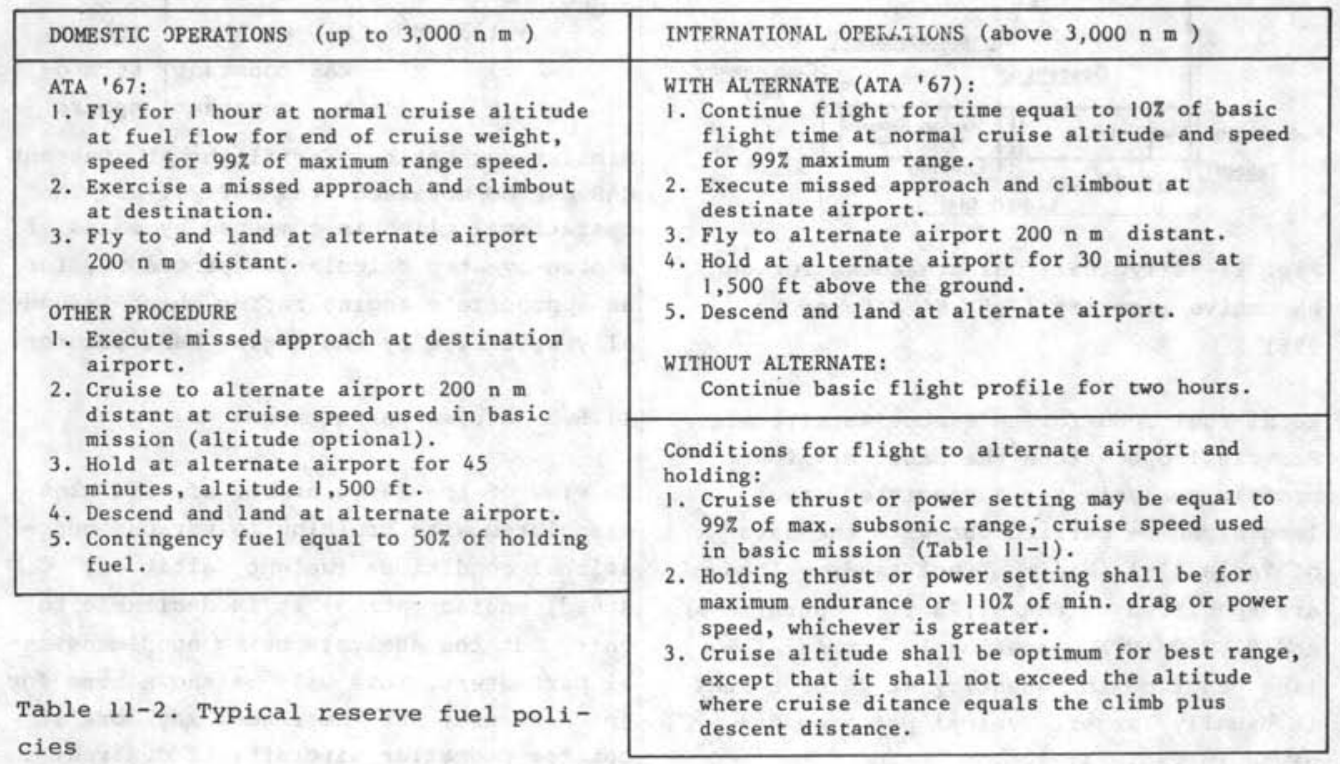


Assuming steady horizontal flight, the lift coefficient is

$\mathrm{C}_{\mathrm{L}}=\frac{\mathrm{W} / \mathrm{S}}{\frac{1}{2} \gamma \mathrm{pM}^{2}}=\frac{\mathrm{W}}{\delta \mathrm{W}_{\text {to }}} \frac{1}{\mathrm{M}^{2}} \frac{\mathrm{W}_{\mathrm{to}} / \mathrm{S}}{\frac{1}{2} \gamma \mathrm{p}_{\mathrm{O}}}=\frac{\mathrm{W}_{\mathrm{to}} / \mathrm{S}}{\frac{1}{2} \gamma \mathrm{p}_{\mathrm{O}}} \mathrm{f}_{1}\left(\frac{\mathrm{W}}{\delta \mathrm{W}_{\text {to }}}, \mathrm{M}\right)$

A general expression for the drag polar (Fig. 5-6) is:

$C_{D}=f\left(C_{L}, M\right)=f_{2}\left(\frac{W}{\delta W_{\text {to }}}, M\right)$

The thrust required is obtained from the condition $T=D$, hence

$\frac{\mathrm{T}}{\delta \mathrm{T}_{\text {to }}}=\frac{\mathrm{C}_{\mathrm{D}}}{\mathrm{C}_{\mathrm{L}}} \frac{\mathrm{W}}{\delta \mathrm{W}_{\text {to }}} \frac{\mathrm{W}_{\text {to }}}{\mathrm{T}_{\text {to }}}=\frac{\mathrm{W}_{\text {to }}}{\mathrm{T}_{\text {to }}} \mathrm{f}_{3}\left(\frac{\mathrm{W}}{\delta \mathrm{W}_{\text {to }}}, \mathrm{M}\right)$

For a given engine the nondimensional performance can be specified as follows:

$\frac{T}{\delta T_{\text {to }}}=f_{T}\left(\frac{N}{\sqrt{\theta}}, M\right)$

$\frac{C_{T}}{\sqrt{\theta}}=f_{C}\left(\frac{N}{\sqrt{\theta}}, M\right)$

and the specific fuel consumption is obtained from these equations by eliminating $\mathrm{N} / \sqrt{\theta}$ :

$\frac{C_{T}}{\sqrt{\theta}}=f\left(\frac{T}{\delta T_{\text {to }}}, M\right)=f_{4}\left(\frac{W}{\delta W_{\text {to }}}, M\right)$

The effects of intake losses, bleed air takeoff, and power extraction are ignored in this elementary analysis, which is probably not acceptable in practice. Finally, the range parameter $V / F$ is found by combining $(11-12)$ through $(11-17)$ :

$w \frac{V}{F}=\frac{W V}{C_{T} T}=a_{0} \frac{M}{C_{T} / \sqrt{\theta}} \frac{C_{L}}{C_{D}}=a_{0} f_{5}\left(\frac{W}{\delta W_{t o}}, M\right)(11-18)$

It is thus possible to represent the specific range for all cruise conditions in the form of a set of curves, as exemplified by Fig. 11-8.

The distance flown in cruising flight is:

$R=\int_{w_{e}}^{w_{b}} \frac{V}{F} d w=\int_{w_{b} / w_{t o}}^{w_{e} / w_{t o}} w \frac{V}{F} d\left(-\ell n w / w_{t o}\right)$

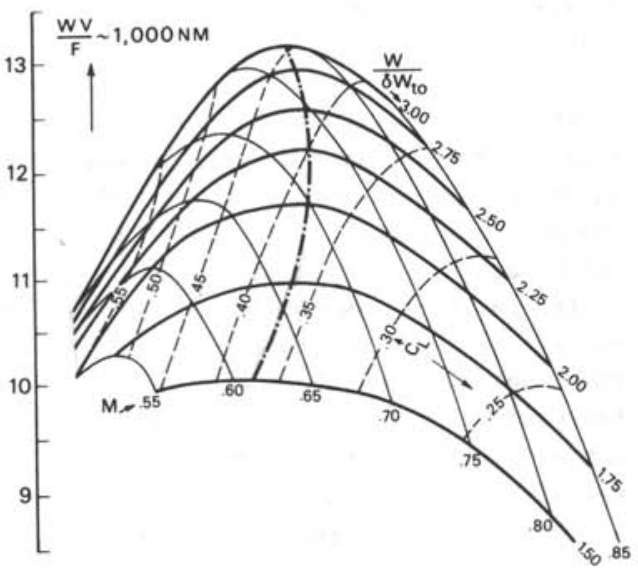

Fig. 11-8. Generalized range performance of a long-range jet transport

where WV/F is given by (11-18). Different cruise procedures can be assumed, e.g. flight at constant Mach number, constant engine rating, or constant lift coefficient, resulting in slightly different ranges for a given fuel weight. With the initial conditions known, the range can be computed numerically for several values of $w_{e} / w_{\text {to }}$ or, alternatively, the cruise fuel required for various distances can be determined.

\subsubsection{Descent}

The procedure for analyzing the descent (Table 11-1) is based on several assumptions regarding the descent speed, the use of airbrakes, and engine ratings. It may be argued that in the pre-design stage several assumptions cannot be substantiated and a simpler approach may be acceptable. A good approximation is usually found by assuming that the amount of fuel used in a descent over a given distance is equal to the fuel used during an extended cruising flight over the same distance. The time to descend is derived directly from the assumed cabin rate of descent, taking into account the permitted slope of the cabin floor. 
11.5.4. Payload-range diagram and block time

If the cruise distance is varied, the block time and total trip fuel are obtained as a function of the trip distance by adding of the contributing items in Table $11-1$. The weight of reserve fuel may now be computed on the basis of assumptions concerning the reserve fuel policy (Table 11-2).

The total fuel weight is subtracted from the Useful Load* to obtain the payloadvs. range diagram, which is frequently presented both for long-range and for highspeed cruise conditions (Fig. 11-9). Pay-

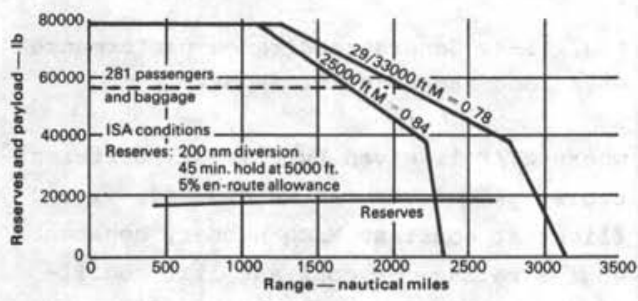

Fig. 11-9. Payload-range diagram for the Airbus $\mathrm{A}-300 \mathrm{~B}$

load-range diagrams (example in Fig. 11-9) must be compared with the design requirements and a decision made whether to alter the MTOW or not. Any such corrective action will have farreaching consequences on all calculations carried out previously, particularly the structural weight prediction and the loading and balance of the complete airplane.

\subsection{CLIMB PERFORMANCE}

11.6.1. Maximum rate of climb, time to climb, and ceilings

For most conventional subsonic aircraft the angle of climb is limited to roughly $10^{\circ}-15^{\circ}$ and $(11-11)$ can be used to compute the rate of climb as a function of the flight speed. The usual assumption is made

\footnotetext{
* See Section 8.2 .
}

that climb performance is defined for constant CAS; for low-subsonic speeds this is approximately equivalent to constant EAS. The maximum rate of climb must be determined by a numerical process as the simplifying analytical process, assuming thrust or power available to be invariable with speed, usually leads to considerable errors.

The minimum time to climb to a specified altitude can be approximated by

$t_{c l}=\int_{0}^{h} \frac{d h}{c_{\max }}$

where $C_{\max }$ is the maximum rate of climb for the particular altitude. However, $C_{\max }$ is frequently defined for a constant CAS (or EAS) and this does not necessarily result in the optimum climb procedure. The energy method in Ref. $11-23$ is the most practical procedure for obtaining accurate results.

Absolute and service ceilings are defined as those altitude where $c_{\max }=0$ and 100 $\mathrm{ft} / \mathrm{min}(.5 \mathrm{~m} / \mathrm{s})$, respectively. The ceiling is found by computing $c_{\max }$ for several altitudes and plotting this result vs. the altitude. For multi-engine aircraft the ceilings are determined with all engines operating and with one engine inoperative. The results must be compared with airworthiness and operational requirements.

\subsubsection{Takeoff and landing climb}

As stated in Section 5.4.3., airworthiness requirements for each airplane category specify the minimum permissible climb performance, with and without engine failure. A summary for turbine-powered transport aircraft is presented in Table 11-3. Although these data pertain to the FAR 25 regulations, the equivalent BCAR Section D requirements are very similar.

The takeoff climb is divided into a number of nominally distinct segments, all with engine failed (Fig. $\mathrm{K}-1$ of Appendix $\mathrm{K}$ ): a. A first segment, virtually defining a climb potential immediately after liftoff, 


\begin{tabular}{|c|c|c|c|c|c|c|c|c|c|c|}
\hline \multirow{2}{*}{\multicolumn{2}{|c|}{ PHASE OF FLIGHT }} & \multicolumn{6}{|c|}{ AIRPLANE CONFIGURATION } & \multicolumn{3}{|c|}{ MINIMUM CLIMB GRADIENT } \\
\hline & & \multirow{2}{*}{$\begin{array}{c}\begin{array}{c}\text { flap } \\
\text { setting }\end{array} \\
\text { t.o. }\end{array}$} & u.c. & \multicolumn{2}{|c|}{\begin{tabular}{|l} 
engine \\
thrust (power)
\end{tabular}} & speed & \multirow{2}{*}{$\begin{array}{l}\text { altitude } \\
\left.0 \rightarrow \mathrm{h}_{\mathrm{uu}} 1\right)\end{array}$} & \multirow{2}{*}{$\frac{\mathrm{N}_{\mathrm{e}}=2}{0}$} & \multirow{2}{*}{$\begin{array}{c}\mathrm{N}_{\mathrm{e}}=3 \\
.3\end{array}$} & \multirow{2}{*}{$\begin{array}{c}\mathrm{N}_{\mathrm{e}}=4 \\
.5\end{array}$} \\
\hline $\begin{array}{r}\text { TAKE OFF CL } \\
\text { ("first }\end{array}$ & $\begin{array}{l}\text { IMB POTENTIAL } \\
\text { segment") }\end{array}$ & & $\downarrow$ & \multirow{4}{*}{ 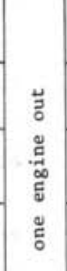 } & t.o. & $\mathrm{v}_{\text {LOF }}$ & & & & \\
\hline \multirow{2}{*}{$\begin{array}{l}\text { TAKEOFF } \\
\text { FLIGHT PATH }\end{array}$} & "second segment" & t.o. & $\uparrow$ & & t.o. & $v_{2}{ }^{2)}$ & $h_{u u} \rightarrow 400 \mathrm{ft}$ & 2.4 & 2.7 & 3.0 \\
\hline & $\begin{array}{c}\text { final takeoff } \\
\text { ("third segment") }\end{array}$ & $\begin{array}{c}\text { en } \\
\text { route }\end{array}$ & $\uparrow$ & & $\begin{array}{l}\max . \\
\text { cont. }\end{array}$ & $v \geqslant 1.25 v_{s}$ & $400 \rightarrow 1,500 f t$ & 1,2 & 1.5 & 1.7 \\
\hline \multicolumn{2}{|c|}{ APPROACH CLIMB POTENTIAL } & approach & $\uparrow$ & & t.o. & $\mathrm{V} \leqslant 1.5 \mathrm{~V}_{\mathrm{S}}$ & $0^{1)}$ & 2.1 & 2.4 & 2.7 \\
\hline \multicolumn{2}{|c|}{ LANDING CLIMB POTENTIAL } & landing & $\downarrow$ & \multicolumn{2}{|c|}{$\begin{array}{l}\text { all engines } \\
\text { take of } f^{4)}\end{array}$} & $\mathrm{v} \leqslant 1.3 \mathrm{v}_{\mathrm{S}}$ & $0^{1)}$ & 3.2 & 3.2 & 3.2 \\
\hline \multicolumn{3}{|c|}{ 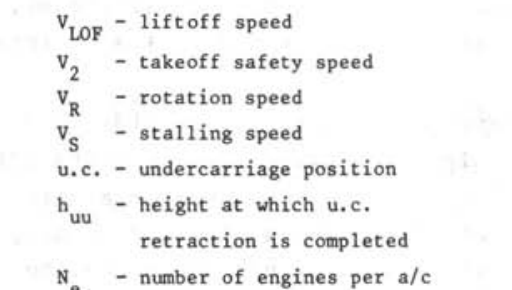 } & $\begin{array}{l}\text { 1) } \\
\text { 2) } \\
\text { 3) } \\
\text { 4) }\end{array}$ & $\begin{array}{l}\text { out } \\
\text { def } \\
\mathrm{f} 1 \mathrm{a} \\
\text { mor } \\
8 \mathrm{~s} \\
\text { tak } \\
\text { req }\end{array}$ & $\begin{array}{l}\text { of ground } \\
\text { ined in Se } \\
\text { setting } \\
\text { econds aft } \\
\text { eoff requi } \\
\text { uirements }\end{array}$ & $\begin{array}{l}\text { d effect } \\
\text { such that } \\
\text { 1y: the et } \\
\text { ter throt } \\
\text { irements } \\
\text { at landi }\end{array}$ & $\begin{array}{l}\text { of Appendix } \mathrm{K} \\
\mathrm{t} \mathrm{v}_{\mathrm{S}}<1.10 \mathrm{v}_{\mathrm{S}} \\
\text { ngine power ( } \mathrm{t} \\
\text { tle opening to } \\
\text { are at actual } \\
\text { ng (touchdown) }\end{array}$ & $\begin{array}{l}\text { for } \\
\text { thrust) } \\
\text { take } \\
\text { weight } \\
\text { weigt }\end{array}$ & $\begin{array}{l}\text { anding } \\
\text { availat } \\
\text { ff rati } \\
\text { other } \\
\end{array}$ & \\
\hline
\end{tabular}

Table 11-3. Summary of climb requirements for turbine-powered transport category aircraft (FAR 25)

while the undercarriage is still extended and high-lift devices are in the takeoff position. The generally favorable effects of ground proximity on climb performance are disregarded.

b. A second segment, extending from the point where the undercarriage is retracted up to $400 \mathrm{ft}(120 \mathrm{~m})$. During this phase the high-lift devices are in the takeoff position, the engine(s) operate(s) at takeoff rating.

c. A third segment, extending from $400 \mathrm{ft}$ $(120 \mathrm{~m})$ to a height of at least $1500 \mathrm{ft}$ $(450 \mathrm{~m})$, during which the airplane is accelerated, flaps retracted and engine power (thrust) reduced to the maximum continuous rating.

Minimum permissible climb performance is also specified for the approach and landing configuration (Table 11-3):

a. The approach climb potential, applying to the approach configuration with one engine inoperative. For a specified landing weight this requirement may limit the flap deflection angle in the approach, and as a result of this the landing performance will be affected since there is a relation between the stalling speeds in the approach and landing configurations.

b. The landing climb potential is intended to ensure safe wave-off and climbout after a baulked landing. In view of the rapid response of modern turbo engines, this requirement is usually not critical.

The available angle of climb is determined from the relationship:

$\tan \gamma=\frac{T}{W}-\frac{C_{D}}{C_{L}}$

assuming that the effect of acceleration due to flight with constant CAS is negligible. For a specified position of the 
high-lift devices and ambient conditions, the available $\gamma$ may be computed for various values of the takeoff weight, taking into account the variation of stalling speed with weight. For each gradient requirement a weight limit is thus obtained, defining the condition in which the available climb gradient equals the minimum required value. The results are usually presented in the form of Weight limits for variable Altitude and Temperature, usually referred to as WAT curves (Fig. 11-10).

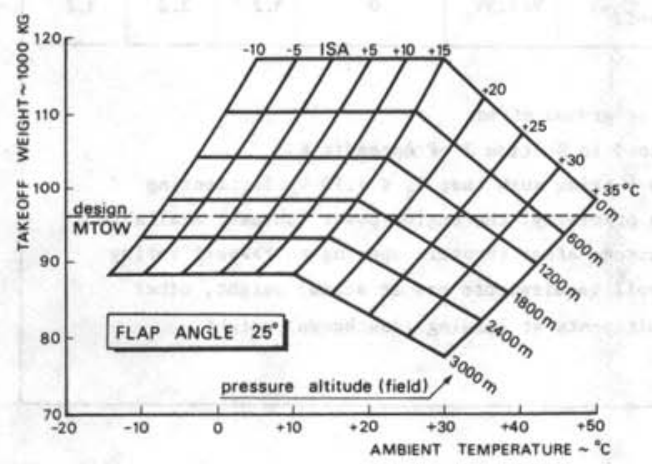

Fig. 11-10. WAT curves for the aircraft shown in Fig. 12-1

The following additional points may be mentioned.

a. Appropriate drag penalties must be accounted for in the engine-failure case (Appendix G, Section $\mathrm{G}-8$ ).

b. In the case of a takeoff climb gradient limit the flight speed may be increased above $v_{2}-m i n$ (Appendix K); this will usually improve the climb gradient, particularly for jet aircraft. However, owing to the connection between $v_{2}$ and the takeoff performance, the required field length will progressively increase.

c. In cases where the takeoff weight is unduely limited by the WAT requirements - on high and hot airfields - it will be appropriate to investigate the possibility of several intermediate positions of the high-1ift devices.

\subsection{AIRFIELD PERFORMANCE}

\subsubsection{Takeoff field length}

Analysis of the takeoff characteristics is the most complicated item of performance; this applies particularly to transport aircraft, where the possibility of engine failure must be considered. Detailed consideration is given to this subject in Appendix $\mathrm{K}$. For small aircraft the matter is much simpler and it is left to the reader to translate the requirements into a practical process for computating the field length required. The main differences in relation to transport category airplanes are as follows:

- the takeoff height is $50 \mathrm{ft}(15,24 \mathrm{~m})$, - the takeoff safety speed is $1.3 \mathrm{v}_{\mathrm{S}}$ (FAR 23),

- emergency braking is not considered (FAR 23) or only to a limited extent (SFAR 23). In order to examine the operational flexibility and to optimize the performance, the effect of several variables must be considered.

a. Position of high-lift devices. Increasing the flap deflection angle results in increased values of $\mathrm{C}_{\mathrm{L}}$ for stalling and liftoff; $v_{S}$ and $v_{2}$ will consequently decrease and the takeoff run will be shortened. However, the L/D ratio and the climb gradient deteriorate, and the airborne distance increases. For a given $T / W$ ratio, an optimum flap angle can be defined, resulting in a minimum field length. The flap deflection is limited by the climb requirements with one engine failed (Section 11.6.2.) and for low $\mathrm{T} / \mathrm{W}$ the optimum flap angle is not feasible (Fig. 11-11a).

b. Takeoff weight .

The takeoff reference speed variation in relation to the stalling speed for varying $\mathrm{T} / \mathrm{W}$-ratio is discussed in Section $\mathrm{K}-2$ of Appendix K. For a specified flap angle three regimes can be distinguished in the takeoff distance vs. weight relationship (Fig. 11-11b) : 

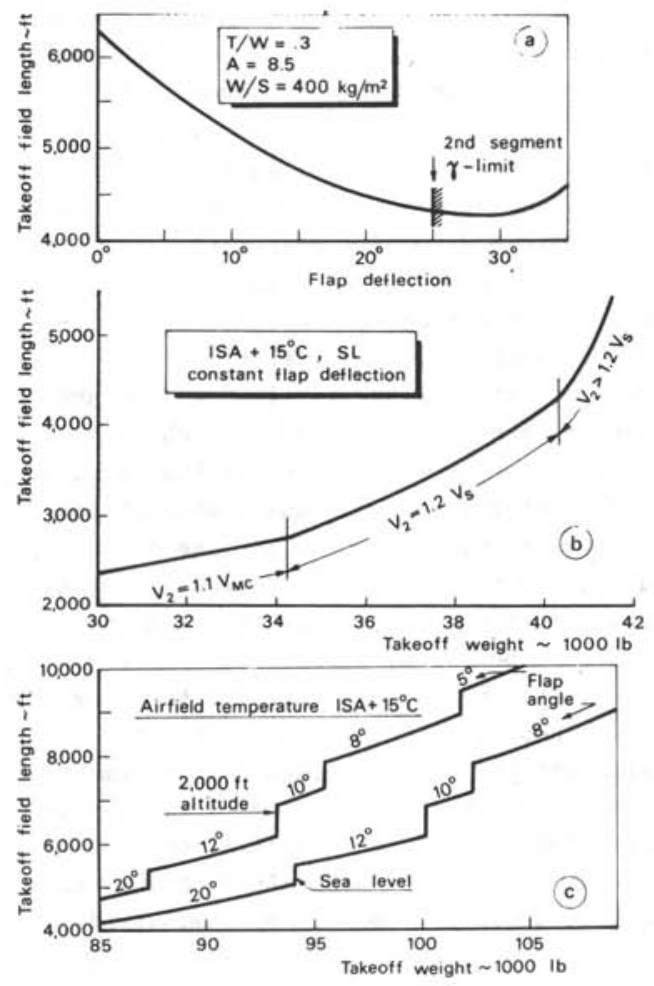

Fig. 11-11. Effect of operational variations on takeoff performance ( $a, b$ and $c$ apply to different aircraft)

- For moderate $\mathrm{P} / \mathrm{W}$ or $\mathrm{T} / \mathrm{W}$ ratios $\mathrm{V}_{2}=1.2$ $\mathrm{V}_{\mathrm{S}}$ and the field length is proportional to $\mathrm{v}_{\mathrm{S}}{ }^{2}$ - and hence to $\mathrm{w} / \mathrm{S}$ - and to the thrust or power loading. Hence $S_{\text {to }}$ is approximately proportional to $\mathrm{w}^{2}$.

- For high T/W (low takeoff weight) the margin relative to $V_{M C}$ forms a lower limit to $v_{R}$ and $v_{2}$. In this region it is mainly the $\mathrm{P} / \mathrm{W}$ or $\mathrm{T} / \mathrm{W}$ ratio that governs the field length and $S_{\text {to }}$ is approximately a linear function of the weight.

- For low P/W or T/W ratios the secondsegment climb gradient requirement cannot be satisfied; $V_{R}$ and $V_{2}$ must be increased relative to $V_{S}$ and the field length increases rapidly with $W$. For a given flap angle the takeoff weight cannot be increased beyond a value where the maximum climb gradients only just satisfy the min- imum required values, e.g. for $v_{2}$ equal to the speed for maximum climb gradient. In the case of a variable flap setting, low $\mathrm{T} / \mathrm{W}$ values are possible by gradually decreasing the flap angle.

\section{c. Temperature and altitude variation.} Increasing ambient temperatures reduce the air density and thrust (power)*; the field length is thus increased. For similar reasons, the field length increases with altitude.

The complete picture of field length vs. weight is shown in Fig. 11-11c, illustrating the flexibility of the design to variations in operational conditions.

\subsubsection{Landing field length}

Some basic principles of landing performance estimation have been explained in Section 5.4.6. The designer should consult the airworthiness requirements to define the operational conditions for which the analysis must be made. For transport aircraft the "arbitrary" landing distance is generally used, i.e. the shortest possible landing distance, to be demonstrated in ideal conditions on a dry runway, multiplied by a factor 5/3. British requirements additionally specify a "reference" landing distance, both for dry and for wet runways, in conditions that are representative of day-to-day operation. The following refinements on the simple analysis in Section 5.4.6. may be introduced.

a. The landing weight limitation must be established, using WAT curves, or the maximum flap deflection determined for a given landing weight.

b. The flare-out may be analyzed by a step-by-step type of analysis (cf. Ref. 11-60), assuming any suitable control law, e.g. a linear increase of the pitch angle with time from the steady approach condition to the touchdown. Analytical solu-

*depending upon the possibility of flat rating or water injection (cf. Section 4.4.6). 
tions may be acceptable if the accuracy required is not very high (Refs. 11-58, 11-59).

c. The flight path angle at touchdown is of the order of $\frac{1}{2}$ degree, corresponding to a rate of descent of 1 to $2 \mathrm{ft} / \mathrm{s}(.3$ to $.6 \mathrm{~m} / \mathrm{s})$.

d. The braked run after touchdown can be analyzed in much the same way as in the case of the aborted takeoff (Section 4 of Appendix K). According to Ref. 11-52, typical time delays are:

2 seconds from touchdown to brake operation,

$3 \frac{1}{2}$ seconds from touchdown to spoilers effective,

7 seconds from touchdown to reverse thrust effective, with a cancellation speed of 45 - 50 kts.

The use of reverse thrust is not permitted in the case of FAR 25 requirements. The BCAR allow the use of reverse thrust, but the reference factor is higher. in this case.

\subsection{SOME ASPECTS OF OPERATING ECONOMY}

\subsubsection{Economy criteria}

The operating costs of airlines are generally divided into Direct operating Costs (DOC) and Indirect Operating costs (IOC). The DOC are broadly defined as the costs that are associated with flying operations, and the maintenance and depreciation of the flying material while the IOC include the operator's other costs associated with maintenance and depreciation of ground properties and equipment, servicing, administration, and sales.

The revenues are usually referred to the passenger-mile or ton-mile production. For a given type of operation the ratio of the revenues to the operating costs is affected primarily by the load factor, the actual number of seats sold as a percentage of the seats available. For most scheduled airlines the average load factor is of the order of 50-60\%. The question arises as to what criterion should be used in the preliminary design stage to compare different designs on an economic basis.

a. We can use the DOC per aircraft mile, which is of particular importance when traffic is scarce. This criterion is important for a private aircraft.

b. The DOC per seat-mile and per ton-mile, on the other hand, are of particular interest when traffic is dense.

c. Alternatively, we may look at the number of passengers needed to break even (i.e. $D O C+I O C=$ Revenue), as the ratio of this factor to the maximum number of passengers should be as low as possible. d. Taking the comparison between aircraft still further, the total costs and the revenues per annum define the annual profitability and relating this to the investment associated with the purchase of the aircraft yields the Return on Investment (ROI). This type of cost analysis is typical for an operator's evaluation of a particular type of aircraft on his network, but it is seldom used in preliminary design.

e. Sometimes the profitability is approximated by the "profit-potential", defined as the area in the payload range diagram in which the operation yields a profit (Fig. 11-12).

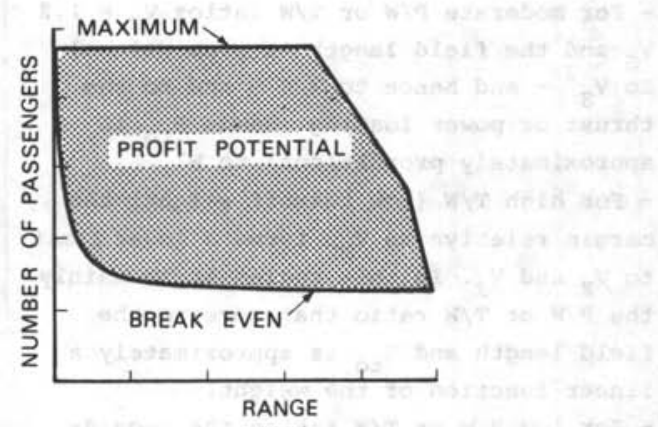

Fig. 11-12. Definition of profit potential

Although the DOC constitute only one aspect of the economic profitability of an airliner or private aircraft, most attention will have to be paid to this aspect, for the reason that several factors con- 
tributing to the DOC are directly related to the technical conception and operational characteristics of the airplane and as such are under the direct control of the design team. Nevertheless the economic aspects of aircraft operation will not be dealt with fully here. We will confine ourselves to the methods available to the designer to determine the position of his design relative to other designs or types of aircraft.

A breakdown of DOC into several cost items can be found on Table 11-4, while Fig. 11-13 shows the relative magnitude of the DOC contributions.

It is surprising that very little generalized information has been published on the subject of Indirect Operating Costs. There may be various reasons for this situation: a. The IOC are not under the control of the airplane designer, although he may incidentally wish to estimate IOC in order to assess the profitability of an airplane design.

b. IOC will be affected by the operator's type of organisation and managerial policy, while considerable differences in passen-

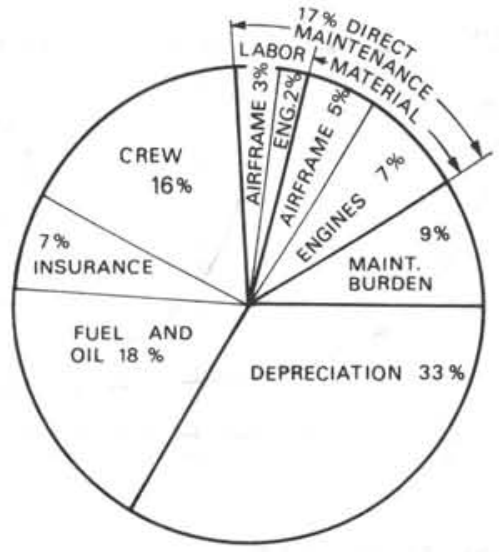

Fig. 11-13. Typical DOC breakdown (1967 ATA method, at design range $=2880 \mathrm{~nm}$, from Ref. 11-92)

ger servicing can also be observed between operators.

c. Published data are very scarce and available essentially only for aviation in the United States.

A subdivision of IOC is presented in Table 11-4; a statistical estimation method is published in Ref. 11-82.

\begin{tabular}{|c|c|}
\hline DIRECT OPERATING COSTS & I) \\
\hline FLYING OPERATIONS & MAINTENANCE - GROUND PROPERTY AND EQUIPMENT \\
flight crew costs & direct maintenance \\
fuel and oil & maintenance burden \\
hull insurance costs & SERVICING - FLIGHT OPERATIONS \\
DIRECT MAINTENANCE - FLIGHT EQUIPMENT & passenger service \\
labor - airplane & aircraft servicing \\
material - airplane & traffic servicing \\
labor - engine & ADMINISTRATION AND SALES \\
material - engine & servicing administration \\
DEPRECIATION - FLIGHT EQUIPMENT & reservation and sales \\
& advertising and publicity \\
& general administration \\
& DEPRECIATION - GROUND PROPERTY AND EQUIPMENT \\
\hline
\end{tabular}

NOTES

1) Subdivision according to ATA-method

2) Subdivision according to the Aeroplane, Dec. 22, 1966, page 14

3) Including landing fees; in other subdivisions this item is considered as part of the DOC

Table 11-4. Subdivision of operating costs 


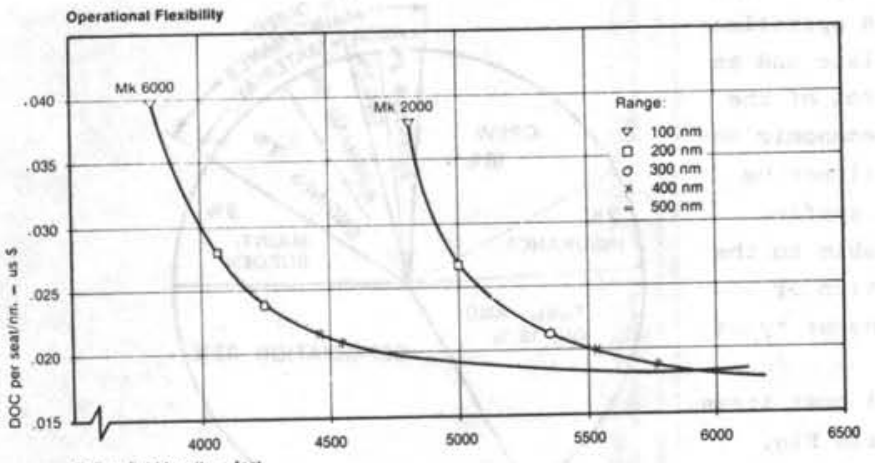

FAP to field length - teet

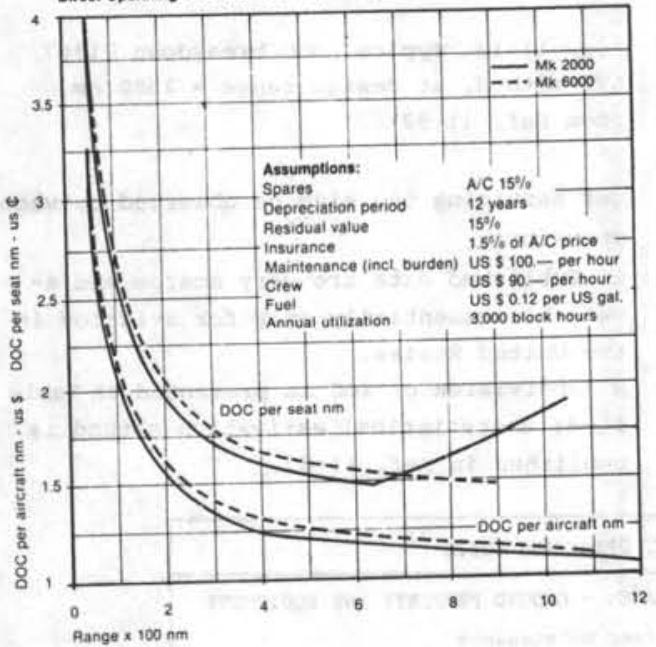

11.8.2 Estimation of DOC for preliminary design

The objectives of a standardized method for the estimation of aircraft operating costs are:

a. to provide a ready means for comparing the operating economics of competitive aircraft and/or aircraft designs under a standard set of conditions, and b. to assist airlines and the aircraft manufacturer in assessing the economic suitability of an airplane for operation on a given route.

Standardized methods have been developed by the Society of British Aircraft Con-
Direct Operating Cost based on 79 passengers (max)
Fig. 11-14. Direct Operating Costs of two versions of the Fokker F-28 (1973 data) structors (Ref. 11-68) and the Air Transport Association of America (Ref. 11-83). The 1967 ATA Direct Operating Cost prediction method is frequently employed as the basis for the presentation of cost data. Such design and economic criteria are useful principally for establishing firstorder trends and will be of little help in designing the aircraft. An example of an economic comparison between two versions of the Fokker F-28 Fellowship, based on the ATA formula, is presented in Fig. 11-14. The DOC is usually expressed as a function of the stage length, primarily because the block speed is a function of range. For short ranges the block speed and the productivity decrease, while the increased ground maneuver time has an unfavorable effect on utilization. The cost per aircraft mile and the number of passengers rise sharply at stage distances below approximately $200 \mathrm{~nm}$.

CREW cosTs form a substantial part of the DOC, but are essentially outside the control of the designer.

FUEL AND OIL are directly affected by the aircraft and engine performance, but also by the fuel price, which varies significantly geographically and with time. It is not necessary to dwell here on the recent (1974) fuel cost explosion, which has entailed considerable difficulties for many operators. 
MAINTENANCE COSTS have been based traditionally on such parameters as airplane empty weight, engine thrust and airframe or engine initial cost. It should be noted that the correlations used in the ATA method are purely statistical. There is no direct relationship, for example, between the aircraft weight and ease and cost of maintenance. On the contrary, provisions intended to simplify maintenance by means of improved accessibility will tend to increase the empty weight, but this will result, wrongly, in increased maintenance costs if the ATA method is used incorrect1y.

Ideally, the maintenance cost item should be broken down into various components, as systems vary widely in their maintenance costs, partly due to their difference in complexity and sensitivity to hourly and cyclical effects. A typical example is engine maintenance, which appears to be very sensitive to the complexity of the engine and the TET (Ref. 11-95), while the introduction of a new category of engine will also entail higher maintenance.

DEPRECIATION, like insurance, is in reality an annual cost. In the ATA-67 method the appreciation period is taken as 12 years. The lifetime of the aircraft, in terms of both flying hours and flight cycles, must obviously exceed this period.

UTILIZATION is principally affected by the elapsed time during which the airplane is on the ground due to traffic requirements, loading, unloading and refueling, and regular maintenance, and due to delays caused by the weather and unscheduled maintenance. Utilization is also affected by the airline's network and flight planning. The general trend given in the ATA method is a curve of annual utilization vs. block time, showing a variation between $3,000 \mathrm{hr} /$ year for a block time of one hour, to $4,500 \mathrm{hr} /$ year for a block time of 8 hours. If the number of flights per day is fixed, however, the utilization is proportional to the block time. It should be noted that measures to improve the utilization, e.g. by reducing the air maneuver time, transition time between two flights, etc., are not reflected in the statistical correlation. To appreciate potential improvements in utilization, a detailed assessment must be made of the effects involved.

AIRPLANE COSTS (engines included) are the other primary factor governing the depreciation costs, which must be estimated in the preliminary design stage. As in the case of weight estimation, various degrees of detail may be aimed at, varying from a first-order statistical relationship of the type given in Fig. 11-15, to a detailed initial cost breakdown drawn up by the factory's cost accounting department. A decision must be made on the number of

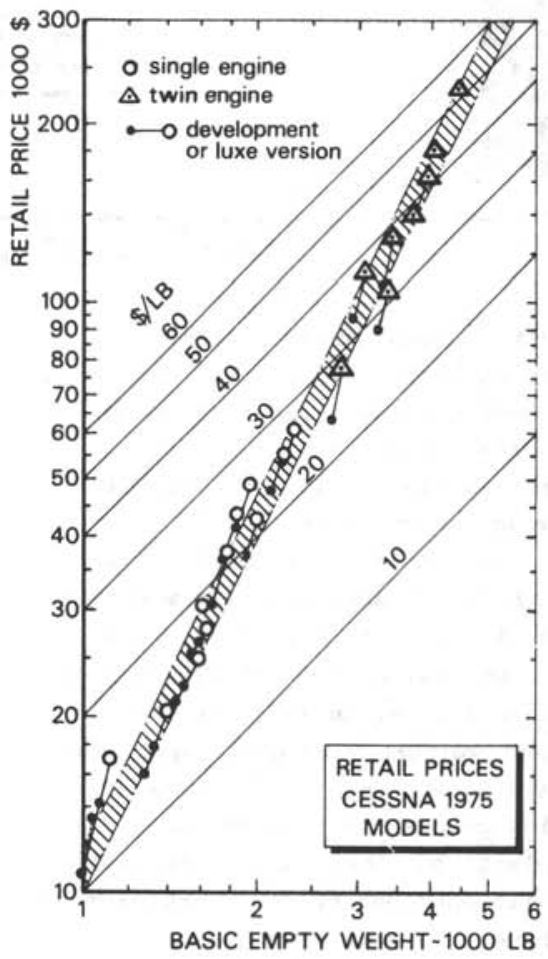

a. Retail prices of light aircraft with reciprocating engines

Fig. 11-15. Aircraft prices 


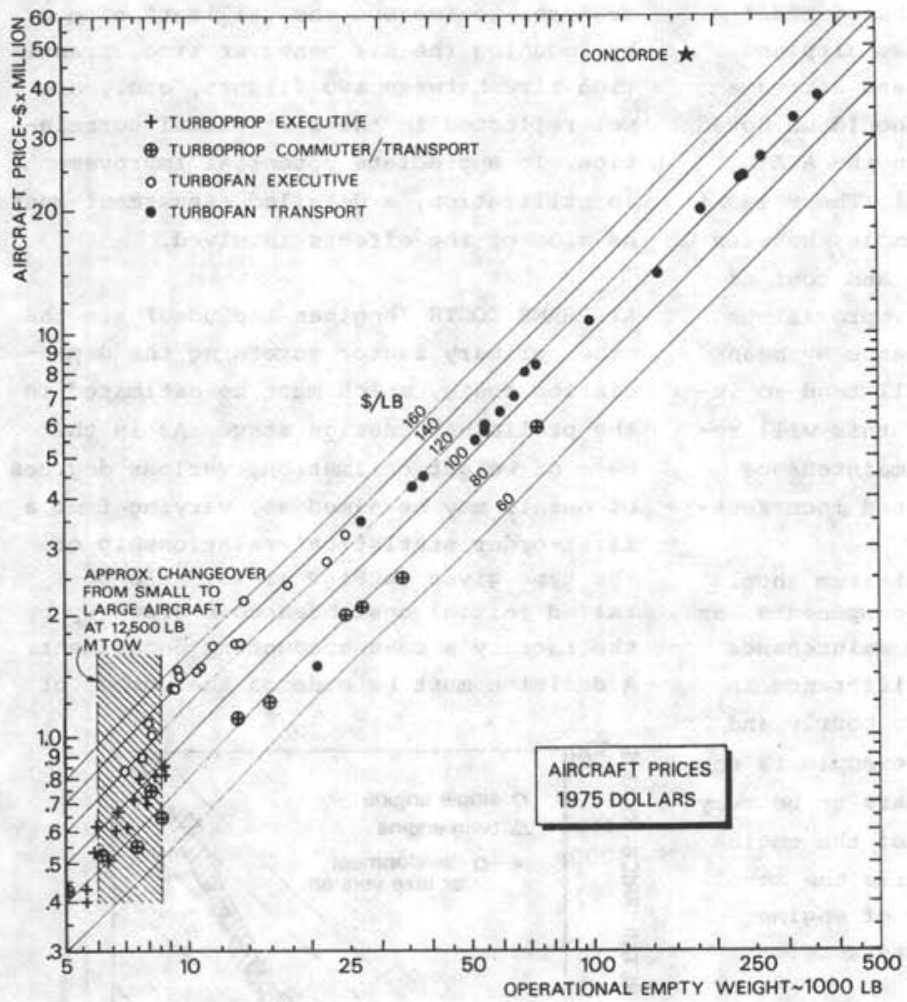

b. Prices of transport category aircraft

Fig. 11-15. (continued)

aircraft to be produced. Attempts to generalize on (military) aircraft and engine first cost have been reported in Refs. 11-71, 11-76 and 11-79.

The data in Fig. $11-15^{*}$ give rise to the following observations.

a. The cost of light piston-powered aircraft increases approximately with the square of the empty weight from $\$ 10$ per 1b $(\$ 22$ per $\mathrm{kg})$ at 1000 lb $(450 \mathrm{~kg})$ empty weight to $\$ 50$ per $1 \mathrm{~b}(\$ 110$ per $\mathrm{kg})$ at 4,500 Ib $(2,000 \mathrm{~kg})$ empty weight, approximately. The main reason for this trend is the increasing complexity of structural design, powerplant, equipment and accommodation. The fully equipped price is approximately 208 above the basic price.

b. Jet-propelled transport aircraft cost between approximately $\$ 120$ per $1 \mathrm{~b} \quad(\$ 265$ per $\mathrm{kg}$ ) of empty weight for the smaller

* Price levels are for the 1973-1975 period. types to $\$ 100$ per $1 \mathrm{~b}(\$ 220$ per $\mathrm{kg})$ for large transports. This level is fairly constant due to the comparable degree of complication and sophistication of these aircraft, irrespective of their size.

c. Jet-propelled business aircraft form the costliest category; their price depends particularly on the type of interior and completeness of the equipment and may go as high as $\$ 160$ per $1 \mathrm{~b}$ ( $\$ 350$ per $\mathrm{kg})$. d. Turboprop transport aircraft cost approximately $\$ 80$ per $1 \mathrm{~b}$ ( $\$ 176$ per $\mathrm{kg}$ ) of empty weight.

A word of warning is appropriate when Fig. $11-15$ is used. There is no explicit functional relationship between aircraft weight and first cost. Structure weight, for example, may be reduced by the use of special manufacturing methods or materials, but this will generally raise the costs instead of reducing them, contrary to what 
might be concluded from the figure. On the other hand, putting more sophisticated equipment into an aircraft will raise both its empty weight and its cost, but the cost will increase more rapidly than the weight.

For transport-type aircraft profitability is a major economic factor and it can be argued that the price level will be related not only to the costs of the technical development, but also to the productivity. Hence, instead of plotting the aircraft first costs vs. the empty weight, attempts can be made to correlate first costs with the product of payload and cruising speed, as this factor affects revenue to a considerable extent. Other refinements may also be justified, such as breaking down the aircraft first cost into airframe costs and engine costs. In any case the cost level expressed in $\$$ per unit of empty weight must be used with great care.

The designer should be reluctant to compare the results of the ATA formula with actual airline costs as reported to the CAB. Standardized methods do not take account of factors such as fleet size, fleet mix, route structure, actual winds encountered, variations in labor rate and fuel costs with time*, etc., all of which can have a significant effect on costs and will vary from one airline to another. In view of the limited applicability of standard methods in general a number of restrictions must be placed on their use:

*At the time of writing this chapter (1975) the general situation is that the ATA-67 formula underestimates crew costs by about $40 \%$, and fuel prices have more than doubled since 1967, while maintenance costs are overestimated by 308 , depreciation by 258 and insurance by 1008 (approximate figures). a. If absolute levels of $D O C$ are required for coli. paring study airplanes with operational airplanes, or for distinguishing between airplanes with different numbers of engines, generalized methods are generally inadequate.

b. Misleading information may be produced as to the relative value of improvements in specific fuel consumption and engine specific weight when the ATA-67 method is used.

Where incremental DOC are of main importance, standard methods may have some utility by providing a simplified tool to evaluate the relative importance of aircraft and engine first costs, fuel costs and maintenance costs. Thus the DOC analysis yields weighting factors for several types of studies:

a. Certain categories of parametric investigations where a decision is sought concerning the relative desirability of airplane design and sizing options - an example is given in Section 5.5.5. These cases are restricted to airplane comparisons made on a consistent basis of technology level and airframe and engine price level.

b. Optimization studies of the cruising speed. Cruise fuel costs are minimum for the airspeed for maximum distance traveled per $1 \mathrm{~b}$ of fuel (Fig. 11-8); increasing the airspeed results in increased cruise fuel cost. However, due to a reduced block time, the depreciation per flight can be reduced, provided the yearly utilization remains at least constant. At some cruise speed intermediate between the long-range and high-speed cruise conditions a minimum DOC is reached. For longrange aircraft the cost-economical cruise speed is close to the long-range cruise condition, for short-haul aircraft it is close to the high-speed cruise condition. 


\begin{tabular}{|c|c|c|c|c|c|c|c|c|c|c|c|}
\hline & AIRPLANE TYPE & Mrow & $\begin{array}{l}\text { A.P.U.- } \\
\text { GROUP }\end{array}$ & $\begin{array}{c}\text { INSTR. } \\
\text { NAV.EQPT. }\end{array}$ & $\begin{array}{l}\text { HYDR. } \\
\text { PNEUM. }\end{array}$ & $\begin{array}{l}\text { ELEC- } \\
\text { TRICAL }\end{array}$ & $\begin{array}{l}\text { ELEC- } \\
\text { TRONICS }\end{array}$ & $\begin{array}{l}\text { FURNISH. } \\
\text { EQPT. }\end{array}$ & $\begin{array}{l}\text { AIRCOND. } \\
\text { ANTI-ICE }\end{array}$ & MISc. & TOTAL. \\
\hline \multirow{17}{*}{ 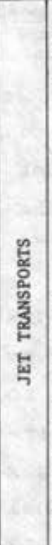 } & At las Airbus A-300 B2 & 302,000 & 983 & 377 & 3,701 & 4,923 & 1,726 & 13,161 & 3,642 & 732 & 29,245 \\
\hline & BAC $1-11$ Srs 300 & 87,000 & 457 & 182 & 997 & 2,317 & 1,005 & 4,933 & 1,579 & - & 11,465 \\
\hline & Boeing $707 / 320 \mathrm{c}$ & 330,000 & 151 & 515 & 1,086 & 4,179 & 2,338 & 9,527 & 3,608 & -389 & 21,015 \\
\hline & $707 / 321$ & 301,000 & - & 561 & 498 & 3,959 & 1,716 & 14,854 & 3,290 & - & 24,878 \\
\hline & $720 / 022$ & 203,000 & - & 555 & 505 & 4,070 & 1,200 & 13,055 & 2,890 & - & 22,275 \\
\hline & $727 / 100$ & 160,000 & 60 & 756 & 1,418 & 2,142 & 1,591 & 10,257 & 1,976 & 85 & 18,285 \\
\hline & $727 / 100 \mathrm{C}$ & 160,000 & 52 & 802 & 843 & 3,617 & 1,559 & 6,729 & 2,401 & is & 16,078 \\
\hline & $737 / 200$ & 100,400 & 836 & 625 & 873 & 1,066 & 956 & 6,643 & 1,416 & 124 & 13,539 \\
\hline & $747 / 100$ & 710,000 & 1,130 & 1,909 & 4,471 & 3,348 & 4,429 & 37.245 & 3,969 & -421 & 54,380 \\
\hline & Fokker VFW F-28 Mk 1000 & 65,000 & 346 & 302 & 364 & 1,023 & 869 & 4,030 & 1,074 & - & 8,008 \\
\hline & Mk 2000 & 65,000 & 353 & 309 & 366 & 1,045 & 869 & 4,614 & 1,111 & - & 8,667 \\
\hline & Lockheed Jetstar & 30,680 & - & 153 & 262 & 973 & 318 & 1,521 & 510 & 560 & 4,297 \\
\hline & McDonnell Douglas $D C-8 / 55$ & 328,000 & - & 1,271 & 2,196 & 2,398 & 1,551 & 14,335 & 3,144 & 57 & 24,952 \\
\hline & $\mathrm{DC}-9 / 10 \mathrm{RC}$ & 91,500 & 818 & 719 & 714 & 1,663 & 914 & 7,408 & 1,476 & 24 & 13,736 \\
\hline & North Am. T-39A Sabreliner & 16,700 & - & 122 & 116 & 720 & 407 & 857 & 333 & - & 2,555 \\
\hline & Aerospatiale Caravelle VI R & 114,640 & - & 236 & 1,376 & 2,846 & 1,187 & 6,481 & 1,752 & - & 13,878 \\
\hline & VFW Fokker - 614 & 40,981 & 305 & 215 & 403 & 1,054 & 436 & 2,655 & 719 & 49 & 5,836 \\
\hline \multirow{10}{*}{ 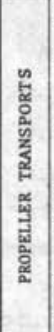 } & Bristol Britannia $300 \mathrm{~A}$ & 155,000 & - & 505 & 650 & 1,800 & 1,040 & 6,866 & 3,000 & - & 13,861 \\
\hline & Canadair CL- $44 \mathrm{C}$ & 205,000 & - & 858 & 630 & 3,040 & 1,229 & 12,349 & 2,536 & - & 20,662 \\
\hline & $\mathrm{CL}-44 \mathrm{D}$ & 205,000 & - & 783 & 640 & 2,875 & 1,046 & 3,155 & 4,090 & - & 12,589 \\
\hline & Fokker VFW F-27 Mk 100 & 39,000 & - & 81 & 242 & 835 & 386 & 2,291 & 1,225 & - & 5,060 \\
\hline & Mk 500 & 45,000 & - & 126 & 256 & 840 & 329 & 3,035 & 1,257 & - & 5,843 \\
\hline & Grumman Gulfstream I & 33,600 & 355 & 97 & 235 & 966 & 99 & 415 & 755 & 6 & 2,929 \\
\hline & Lockheed C-130 E & 151,522 & 466 & 665 & 671 & 2,300 & 2,432 & 4,765 & 2,126 & 62 & 13,487 \\
\hline & L- $1049 \mathrm{E}$ & 133,000 & - & 503 & 654 & 1,505 & 1,371 & 7,405 & 3,298 & - & 14,736 \\
\hline & Nord 262 & 23,050 & - & 133 & 76 & & 238 & 1,324 & 527 & 33 & 3,020 \\
\hline & Vickers Viscount 702 & 50,044 & - & 154 & 331 & 2,048 & 447 & 2,519 & 1,516 & - & 7,015 \\
\hline \multirow{3}{*}{ 䔎竞 } & Beechcraft MS 760 & 7,650 & - & 70 & - & 284 & 158 & 169 & 48 & 30 & 759 \\
\hline & Cessna $T-37$ & 6,436 & - & 132 & 56 & 194 & 86 & 256 & 69 & 3 & 796 \\
\hline & Northrop T-38A Talon & 11,651 & - & 211 & 154 & 296 & 246 & 460 & 142 & 24 & 1,539 \\
\hline \multirow{4}{*}{ 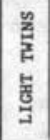 } & Beechcraft 95 Travel Air & 2,900 & - & 49 & - & 96 & 26 & 194 & 48 & 25 & 438 \\
\hline & G-50 & 7,150 & - & 80 & - & 184 & 9 & 333 & 81 & 27 & 834 \\
\hline & $\mathrm{E}-18 \mathrm{~s}$ & 9,700 & - & 100 & - & 295 & 63 & 524 & 144 & 58 & 1,184 \\
\hline & Cessna $310 \mathrm{C}$ & 4,830 & - & 46 & - & 121 & - & 154 & 46 & 65 & 498 \\
\hline \multirow{5}{*}{ 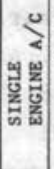 } & Beechcraft Bonanza J-35 & 2,900 & - & 16 & - & 72 & - & 174 & 12 & 7 & 281 \\
\hline & Cessna $150 \mathrm{~A}$ & 1,500 & - & 7 & 2 & 41 & - & 42 & 4 & - & 96 \\
\hline & $172 \mathrm{~B}$ & 2,200 & - & 7 & 3 & 41 & - & 99 & 4 & - & 154 \\
\hline & $180 \mathrm{D}$ & 2,650 & - & 8 & 3 & 59 & - & 105 & 6 & - & 181 \\
\hline & $210 \mathrm{~A}$ & 2,900 & - & 16 & 4 & 60 & - & 116 & 12 & 20 & 228 \\
\hline
\end{tabular}

AL.L WEIGHTS IN LB

Table 8-11. Airframe services and equipment group weight breakdown

Table 8-11. Several items will be discussed in greater detail in the paragraphs below.

a. APU group.

An APU is installed in most modern transport aircraft and also in some jet execu- tives. The installed weight may be based on the dry weight of the APU:

Weight $=\mathrm{k}_{\mathrm{APU}} \mathrm{W}_{\mathrm{APU}}$

The installation factor accounts for the inlet and exhaust ducting mounting frames, 


\section{Chapter 12. Evaluation and presentation of a preliminary design}

\section{SUMMARY}

The presentation of a preliminary design comprises layout drawings, a summary of the principal characteristics and geometry, and the results of a preliminary estimate of performance, flight characteristics and operating costs.

This concluding chapter gives a framework for an initial qualitative assessment of the structural integrity and a design philosophy in the interest of low structural weight. A checklist is presented for completing the layout drawings and mention is made of points to be considered when initial conclusions have to be drawn regarding the feasibility and further development of a project. 


\subsection{PRESENTATION OF THE DESIGN}

The initial baseline design was characterized in Section 1.2. as a feasible configuration which the designer anticipates will satisfy the design requirements. The major part of this textbook is devoted to providing the design engineer with directives for obtaining such a feasible preliminary design. As already indicated in Fig. 1-3, the subsequent elaboration of the design may require parametric design studies and the investigation of alternative design solutions. The design activities will subsequently be intensified in the configuration development phase, when a large engineering effort is required to arrive at a final detalled project definition.

The initial baseline design thus being the starting point for further studies, it is essential that all results of the design activities, together with the relevant background information, are summarized and incorporated in a status report. The following documents are usually produced by a preliminary design department and incorporated in the report in which a new design is presented:

a. Layout drawings (example in Fig. 12-1) intended to give an impression of the detailed arrangement of both the interior and the external shape, as well as some of the major elements of systems and installations. The location of major structural elements will have to be indicated to make it unlikely that major problems will arise during the subsequent stage of structural design.

b. A summary of the principal characteristics and geometry, including a three-view drawing. This summary is comparable to the short descriptions given in, for example, Jane's All the World's Aircraft.

c. Results of a preliminary estimate of performance, flight characteristics and operating costs.

The initial baseline design is frequently used during a period of discussions with interested potential users of the aircraft.
For this reason and in order to compare a transport aircraft design with existing and competitive new designs, the designer may add to the presentation a survey of the major characteristics of comparable designs. Finally, he will arrive at conclusions as regards the suitability and feasibility of the design and state his view on how to proceed with the project. The presentation of a project is a stage in the project when the designer is inclined to reflect on the suitability of the general arrangement and the overall integrity of the aircraft. The final sections of this book are therefore intended to give a framework for an overall structural assessment.

\subsection{EXTERNAL GEOMETRY AND STRUCTURAL ARRANGEMENT}

The preliminary design engineer or design team is not directly responsible for the detailed structural features of the project. However, there is a definite interface between the external geometry, i.e. the outline or outside contours of the airplane, and the structural arrangement. Both have a significant effect on weight and weight distribution. In the early stage of preliminary design the basic principles of the structural layout must be laid down in order to ensure that a light structure can be fitted inside the external lines. Only the major structural elements need be considered; Fig. 12-2 gives an example of the type of drawing defining an initial structural arrangement. The design is still sufficiently flexible to allow certain modifications in the interest of a good general design philosophy, to be formulated as follows.

a. Structural integrity of the airframe must be insured in the event of failure of a single primary structural element or the occurrence of partial damage in extensive structures, such as skin panels. Adequate residual strength and stiffness and a slow rate of crack propagation must be ensured. 


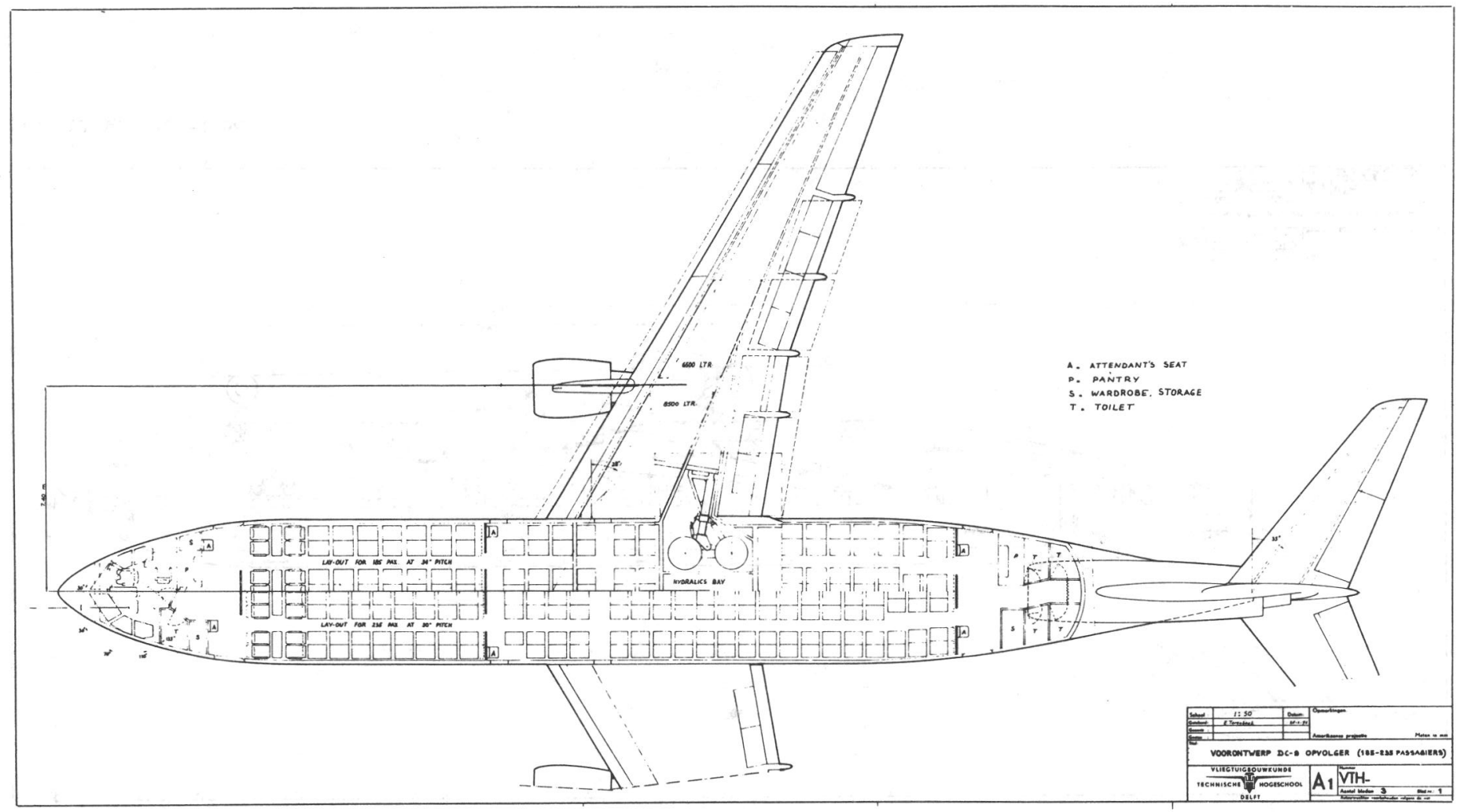

Fig. 12-1. Layout drawings of a preliminary design of a hypothetical short-haul airliner 


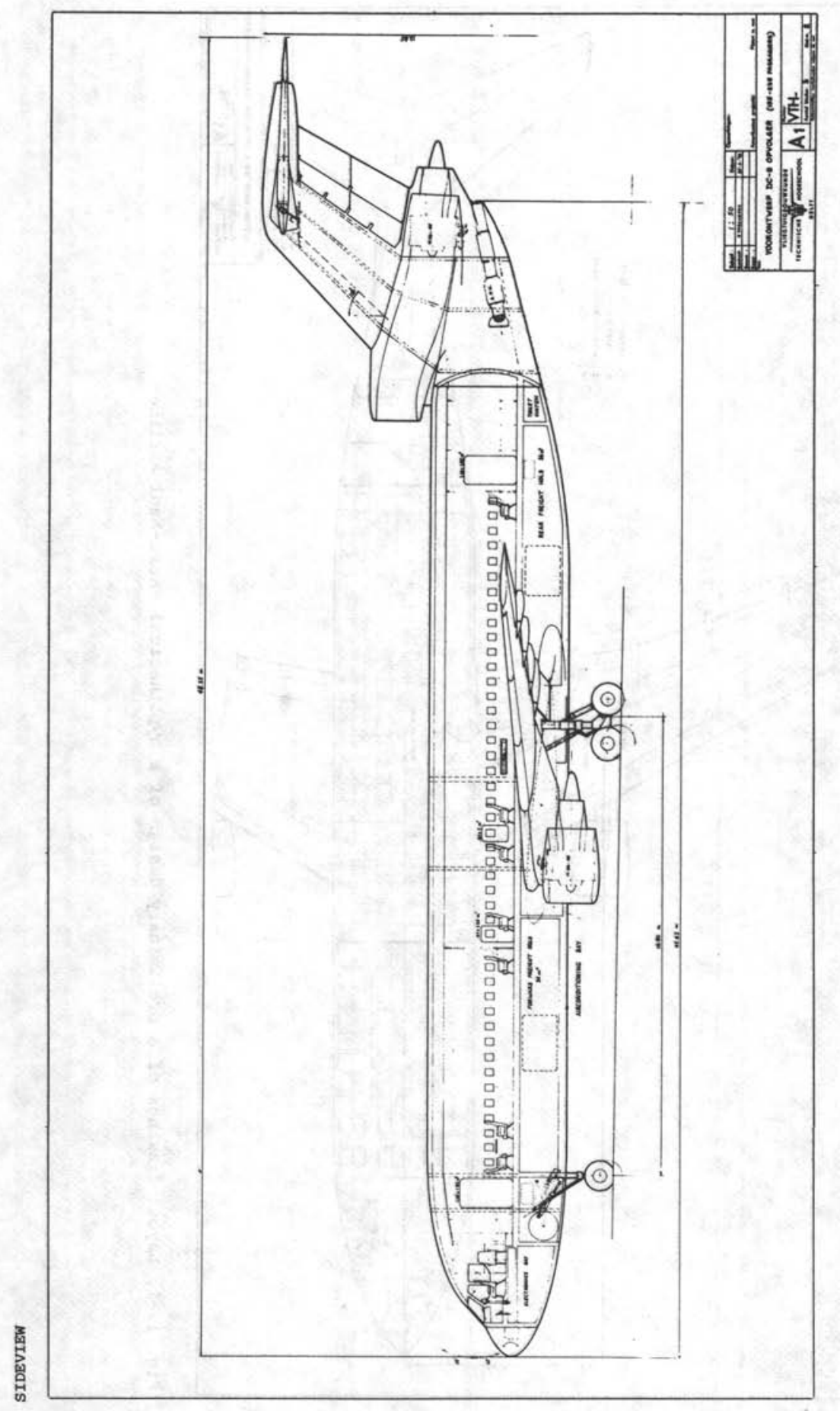

वृ 


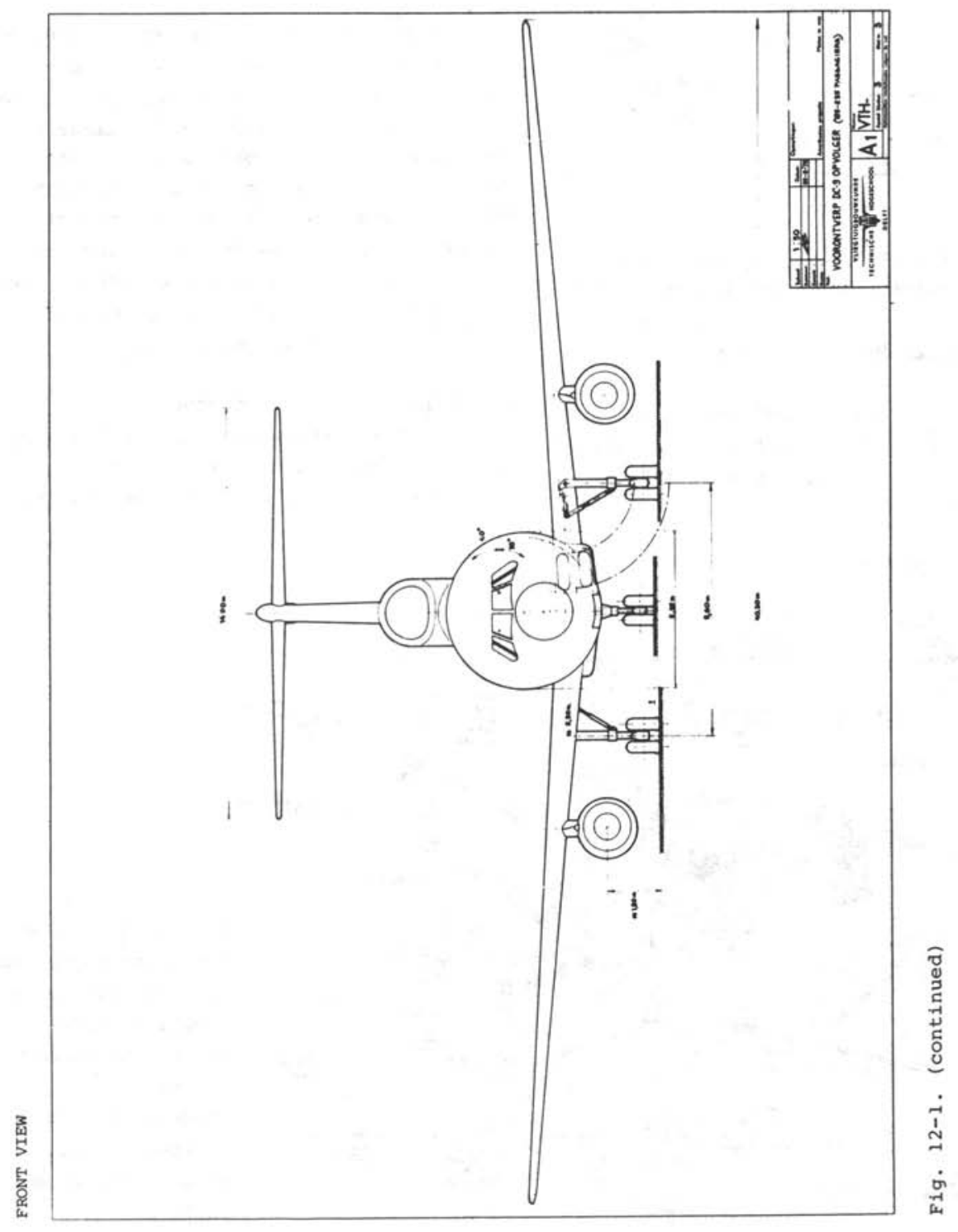




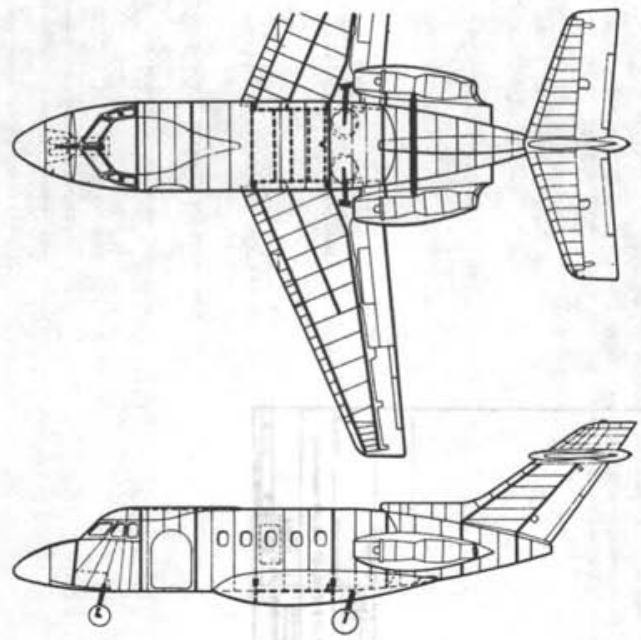

Fig. 12-2. Structural arrangement of an initial baseline design and indication of the basic principles (the example is a Hawker Siddeley HS 125 Dominie)

b. Ease of maintenance and inspection, not only of the powerplant and other installations, but also of the structure itself (Fig. 12-3). c. Minimum structural weight, involving an accurate assessment of loading and strength requirements, a functional and simple structural arrangement and appropriate choice of materials.

d. Design for a specified life of the structure, in terms of operational hours, (e.g. $30,000-60,000)$, numbers of flight cycles or takeoffs and landings (e.g. $30,000)$.

Most of the considerations mentioned below will be general statements, which may in some cases be incompatible with other, considerations. In every design "a number of special structural problems will arise, but it is not an intention to deal with detailed problems here. Basic material properties will likewise not be treated in any detail, although the choice of the type of material and production methods becomes increasingly important.

REDUCE LOADS ON THE STRUCTURE

a. Place fixed items outboard on the wing to reduce the wing load in flight.

b. If the landing gear is mounted to the

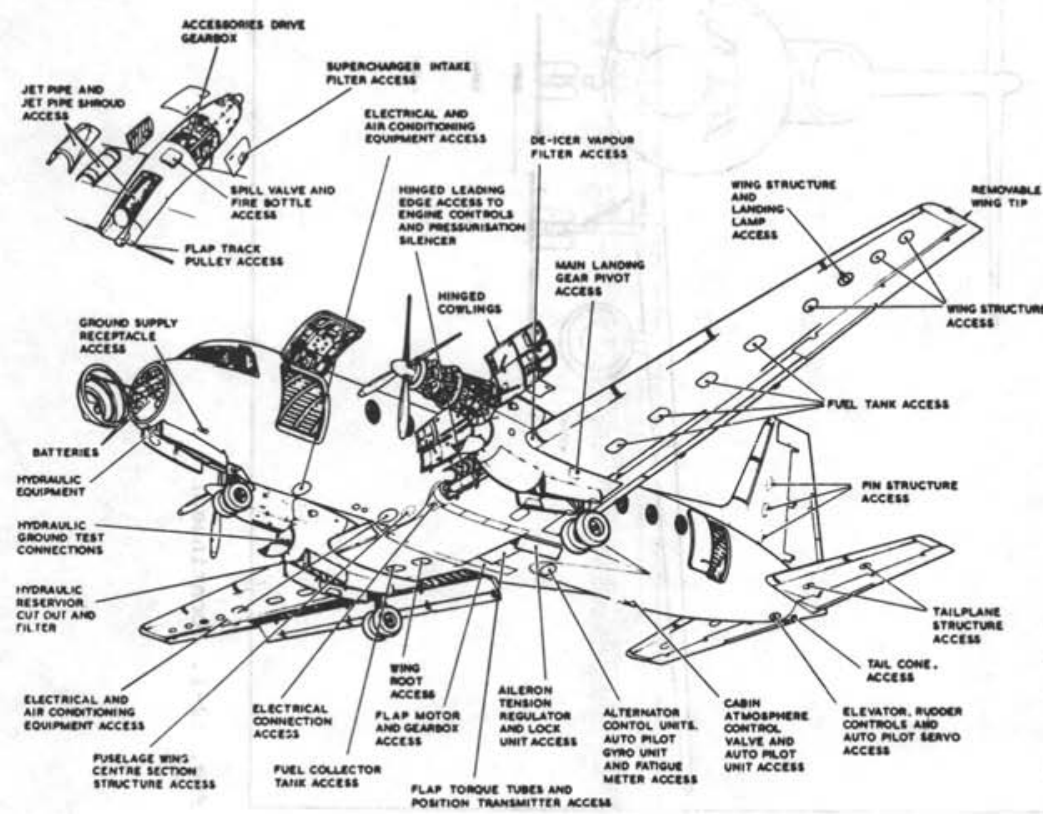

Fig. 12-3. Design for maintenance: accessibility for inspection, maintenance, and repair on the Hawker Siddeley HS 748 (Aircraft Eng., Sept. 1961, page 249) 
wing, keep it in a position such that landing-impact bending and shear loads are not more critical than flight loads for most of the wing structure.

c. Avoid mounting heavy masses to the wing behind the elastic axis as these may lead to aeroelastic problems.

d. Place wing tanks outboard to reduce the bending load in level flight, but keep about $3 \mathrm{ft}(.9 \mathrm{~m})$ of wing tip structure free from fuel, in view of the fire risk caused by electrostatic loads.

e. Locate heavy masses in the fuselage near the c.g. to reduce the inertia loads. f. Tailplane loads can be reduced by keeping the tail arm sufficiently long.

g. Use undercarriage legs of minimum length.

h. Avoid large nosewheel loads by properly locating the nosewheel leg.

\section{KEEP LOAD PATHS AS SHORT AS POSSIBLE}

a. Limit the sweep angle of the wing to the minimum value required. Use straight wing center sections and avoid any reduction in wing thickness in the plane of symmetry, unless simplification and weight reduction can be achieved elsewhere (Fig. 12-4).

b. Keep the support structure for landing

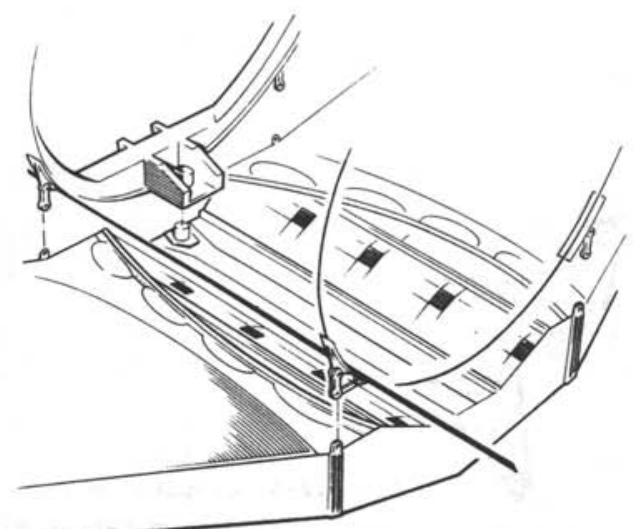

Fig. 12-4. Reduction in wing thickness in the plane of symmetry makes it possible to avoid interruption of the fuselage structure of the HS 125 (see also Fig. 12-2) gear and engine nacelles close to the primary structure.

c. Provide a continuous wing structure through the fuselage and avoid ring bulkheads if other solutions are possible. d. To maintain structural continuity where the (low) wing is connected to the fuselage, a central keel member, extending over the length of the center section, may be used.

e. Locate the wing root so that the loads from the wing shear webs can be easily distributed over the fuselage shell. Make sure that major frames do not interfere with openings such as emergency exits, windows, etc.

f. Heavy masses or gear attachment structures should not be cantilevered frombulkheads.

\section{CUTOUTS AND DISCONTINUITIES}

a. Large cutouts should be kept out of highly stressed regions, e.g. the wing root or the fuselage structure immediately behind the wing fuselage connection.

b. Sharp corners in cutouts must be avoided altogether, especially in pressurized fuselages.

c. The height of windows in pressure cabins should preferably be larger than the width.

d. Locate parts of the flight control system (cables, push rods), fuel lines, air ducts, etc. outside the primary wing structure to simplify the provision of inspection facilities.

e. Avoid cutouts in the primary wing structure for engine intake ducts and exhaust pipes.

f. Special attention must be paid to buried engines in view of the need to inspect and remove them.

g. Avoid abrupt changes in the load path of wing, tail and landing gear attachment to the fuselage.

h. Try to locate wheelbays outside the primary wing box, particularly in the case of a stressed wing skin.

j. Reduce the number of joints to a bare minimum, compatible with manufacturing re- 
quirements and maintenance considerations.

MAKE THE STRUCTURE FUNCTIONAL AND AVOID COMPLEXITY

a. Avoid complicated mechanisms employing tracks, rollers, etc. This applies particularly to flap operating and undercarriage retraction mechanisms. Fig. $7-25 e$ is a good example of simplicity in design of a highly effective flap system. An example in which structural complexity cannot be avoided is shown in Fig. 12-5.

b. Reduce regions of compound curvature to a minimum and avoid doors with appreciable double curvature.

c. Use configurations with low noise exposure to primary structural members. High acoustical loads on skin panels will result from noise levels above $145 \mathrm{~dB}$. Bonded honeycomb structure is suitable in regions of high acoustic loads due to the absence of rivet holes and favorable damping properties.

MAKE MEMBERS PERFORM MULTIPLE FUNCTIONS a. Combine wing ribs supporting the engine(s), landing gear, and flaps.

b. Ensure that bending material also provides torsional stiffness.

c. Use common fuselage bulkheads to support the horizontal and vertical tailplane carry-through. d. Use existing bulkheads to divide fuel tank bays and for attachment of items of equipment, undercarriage legs, etc.

e. Avoid an extreme number of keels, sloors, frames, etc.

\section{SAFETY}

a. Multipath and safe-life structure must be provided throughout in the primary structure of transport category aircraft. b. Provide adequate safeguard against shedding propeller or turbine blades or fragments of blades. Avoid primary structural elements in regions that are likely to be struck in the event of engine or propeller disintegration.

c. Main gear or support structure must not damage fuel tanks in the event of a crash landing.

d. Sufficient clearance must be provided between the loaded and deformed fixed structure and moving parts of, for example, surface controls.

e. Flaps and elevators on both sides of the plane of symmetry must be interconnected.

f. The engine suspension system must be capable of absorbing a certain percentage of the limit loads even after failure of any one link.

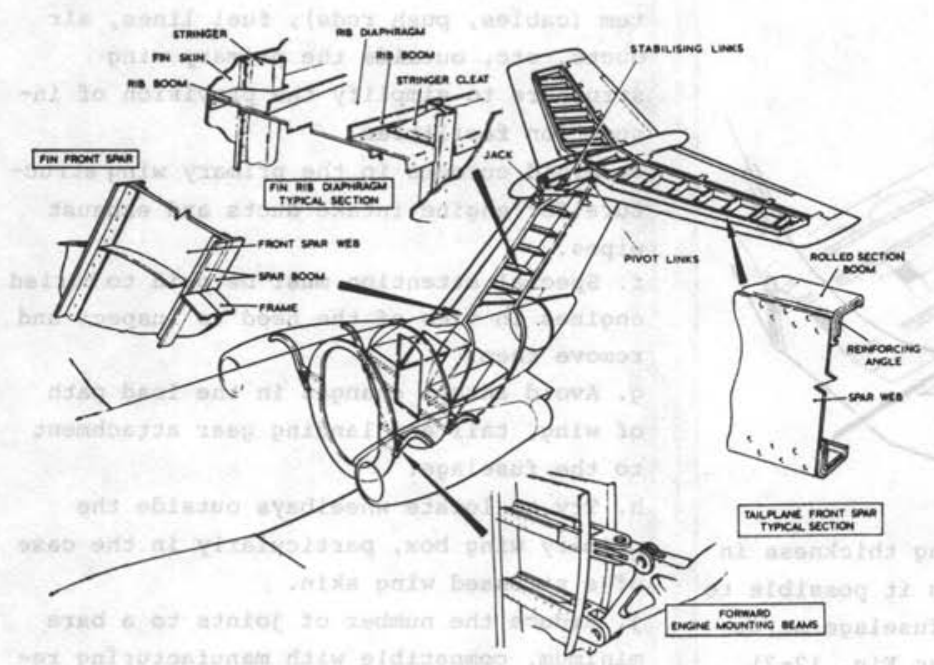

Fig. 12-5. Complexity in structural design cannot always be avolded altogether rear fuselage and empennage structure of the BAC 1-11 (Aircraft Eng. May 1962) 


\subsection{LAYOUT DRAWINGS}

Detailed drawings have to be produced on a sufficiently large scale and to embody both the internal arrangement and the most relevant contour lines (Fig. 12-1). Several cross-sections are usually also drawn (Fig. 12-6). Attention must be paid to the following items.

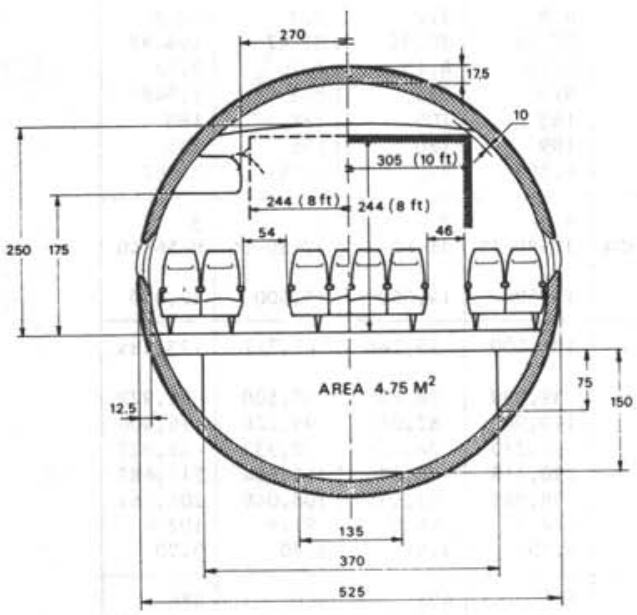

A11 dimensions in centimeters, except where indicated.

Fig. 12-6. Fuselage cross-section for tourist (left) and economy (right) layout for the design shown in Fig. 12-1

\section{FUSELAGE}

Passenger cabin: arrangement of seats, layout of cross-section, windows, entrance doors, emergency exits, lavatories, pantries, cloakrooms, baggage holds, overhead racks, cabin separations, and pressure bulkheads.

Freight and cargo holds: dimensions, hatches and freight doors.

Flight deck: location of the pilot's seat and instrument panel, location and arrangement of the windscreen, primary flight controls (schematic), accessibility. Structural layout: location of major frames, longerons, major bulkheads, and keels.

\section{POWERPLANT AND NACELLES}

Engines and nacelles: external lines of the nacelles, propeller(s), intake and exhaust ducts, engine mounting frames, pylons, panels and hinged doors for engine maintenance, firewalls, thrust reversers, cooling system. Fuel tanks: location, dimensions, volume, accessibility.

\section{WING}

Root and tip section, leading and trailing edges, mean aerodynamic chord, high-lift devices in retracted and extended positions, ailerons, spoilers, speedbrakes. Structural layout: provisions for engine and undercarriage mounting, flap and aileron controls, wing spars and heavily loaded ribs, bracing elements (if used), flap tracks, hinges and supports.

\section{TAILPLANE AND FLIGHT CONTROL SYSTEM}

Root and tip section, leading and trailing edges, mean chord, elevator and rudder deflection angles, hinge location, incidence (range for adjustable tails). Major elements of the structure and the flight control system.

\section{UNDERCARRIAGE}

Location of the gear, wheel positions fully extended, fully deflected and statically loaded by the MTOW. Principle of the retraction system; retracted position, wheelbays.

\section{SYSTEMS}

Although systems design takes place in a fairly advanced stage of the design, space must be provided for some primary elements of these systems, notably those of the environmental control system and the APU, while bays must be provided for the hydraulic and/or pneumatic system and NAV/ com equipment.

Layout drawings for a hypothetical shorthaul airliner are presented in Fig. 12-1 to illustrate the result of a preliminary 


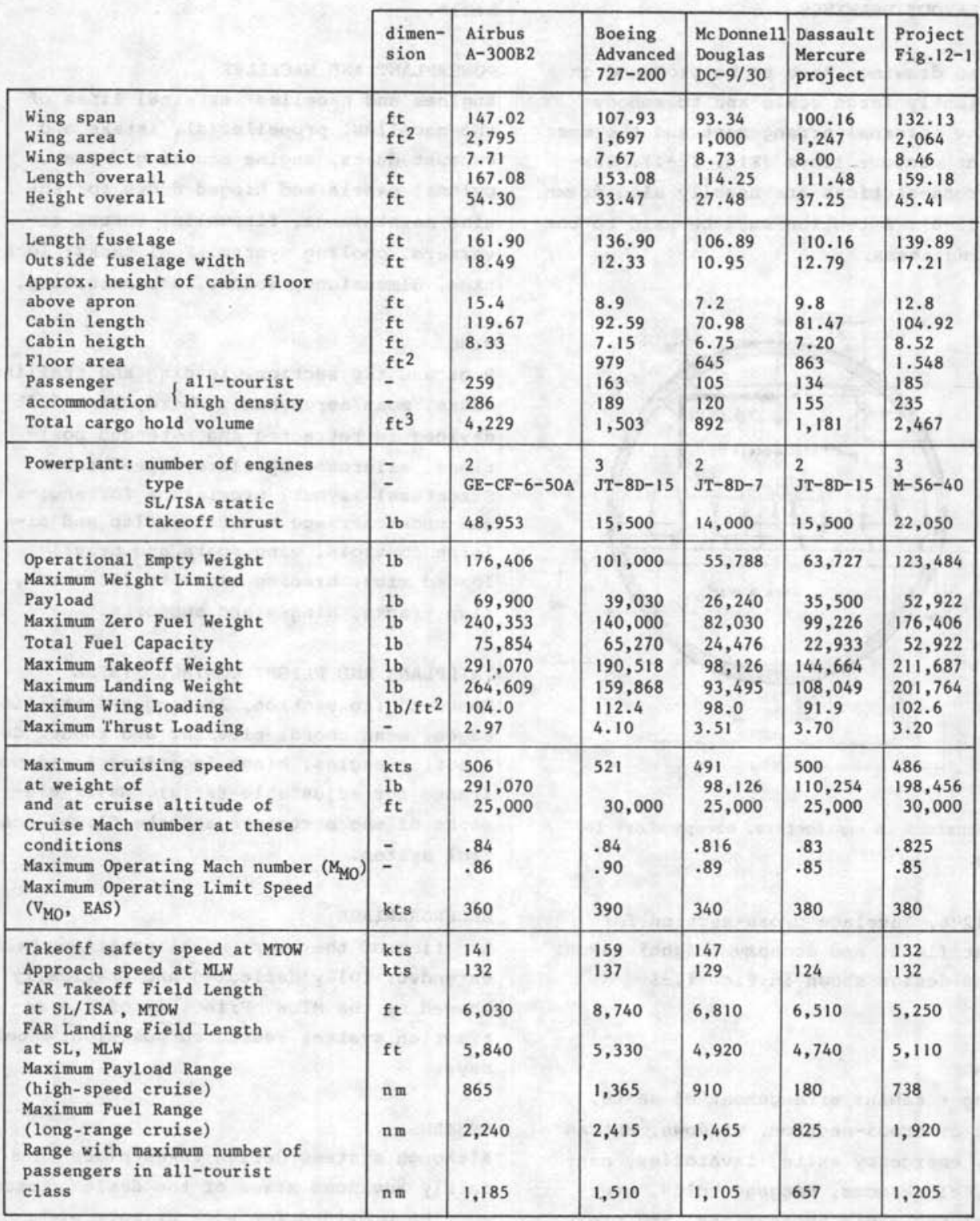

including normal reserves

Table 12-1. Principal characteristics of the design in Fig. 12-1 compared with presentday aircraft types

design exercise. The design specification of this aircraft is summarized in Fig. 1-5, while a three-view drawing can be found in Fig. 2-4.

\subsection{CONCLUSION}

Any assessment of the qualities and characteristics of a preliminary design should 
be accompanied by an appreciation of other design projects and/or design specifications of aircraft types, considered as competitors or types to be replaced by the new design. These comparisons must be made on the basis of a well-defined set of ground rules and similar methods of analysis. The results of such an assessment constitute an essential part of the basis on which further decisions have to be made. Table 12-1 summarizes the major items to be considered.

In reporting his activities, the designer will not only describe the most relevant characteristics of the design, but he will also pay attention to those aspects which he thinks are of vital importance to the successful further development of the project, particularly if there are reasons for doubts about the major predictions and assumptions on which the design characteristics are based. The designer may also anticipate certain design modifications, worth considering in the subsequent "parametric design phase" (cf. Fig. 1-3), for example:

- Is the general arrangement suitable, so that no unsatisfactory characteristics have to be anticipated? The various aspects pointed out in Chapter 2 may be considered here once more.

- Is the engine type to be installed matched to the airplane? In the case of performance deficiencies in certain areas, investigate whether the engine manufacturer is prepared to accept modifications.

- Is it necessary to modify the main wing proportions, such as area, aspect ratio or taper ratio? Critical performance items may be improved in this way, without unduly penalizing other characteristics.
- Is the choice of the high-lift system satisfactory? Only after performance analysis it is possible to be conclude whether the flap system should be more sophisticated or whether alternatively, a simplification may be acceptable.

- Do opportunities exist to reduce the drag by improving the aerodynamic design or the wetted area?

-How satisfactory is the weight breakdown? A final weight analysis, drawn up after the performance estimation has been made, may indicate significant differences compared with other aircraft. If so, an explanation must be sought.

- Can any measures be indicated to reduce the noise production? This point will be of particular concern to the designer of an airplane type, the airworthiness standards for which can be expected to be revised in the near future (cf. Section 5.5.6.).

Practically all decisions to be made in the preliminary design stage have implications with respect to the operating costs and benefits and although a quantitative evaluation is not always possible, the designer should be attentive to improving the economic potential of the design. It is only fairly recently that much emphasis has been placed on the environmental aspects. Every new civil airplane type must now satisfy the broad requirement that it will be acceptable not only to the passenger or the owner, but to society as a whole. A creative approach to any aspect tending to reduce noise, pollution and energy, space and land requirements will be most welcome. 


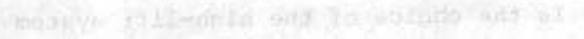

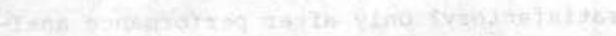

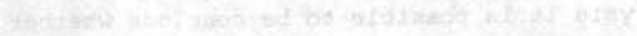

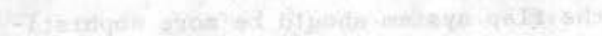

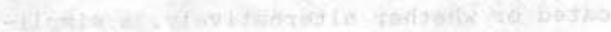

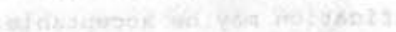

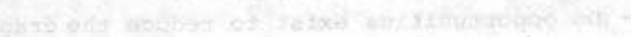

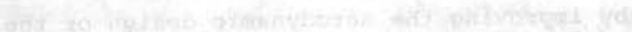

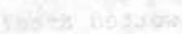

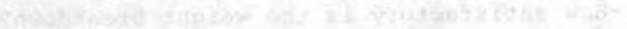

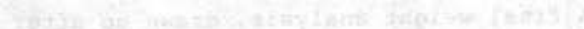

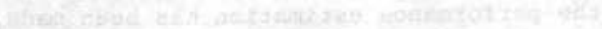

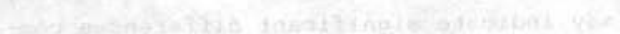

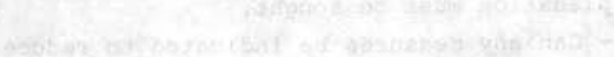

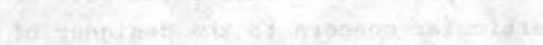

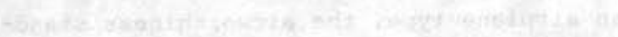

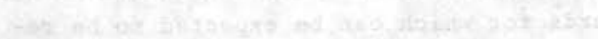

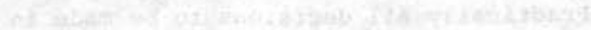

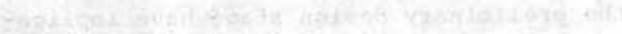

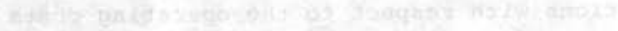

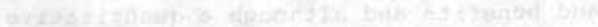

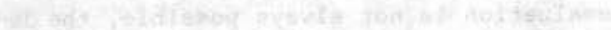

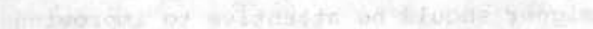

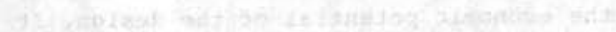

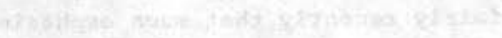

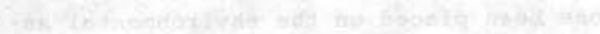

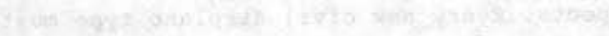

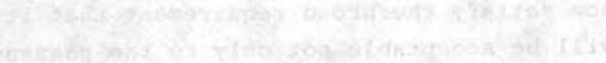

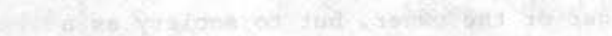

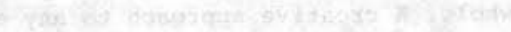

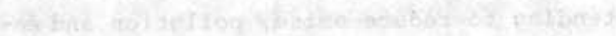

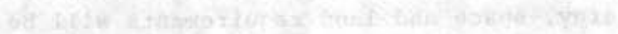

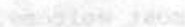

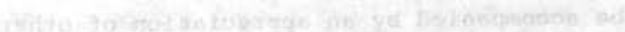
a1)

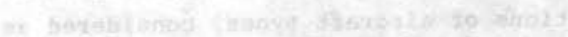

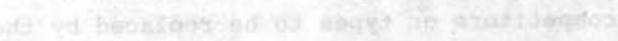

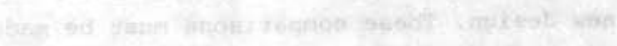

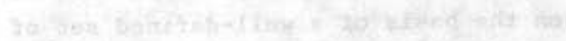

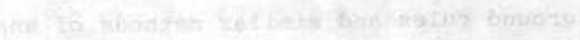

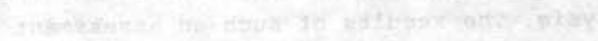

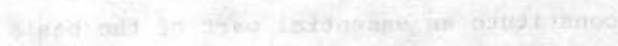

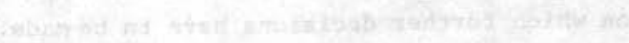

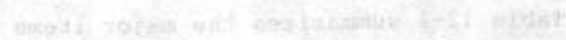

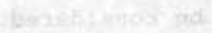

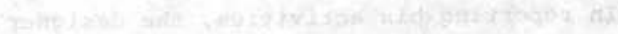

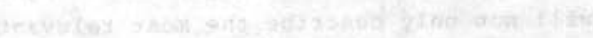

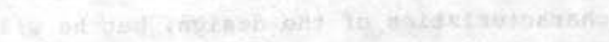

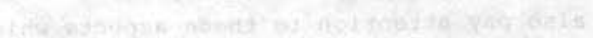

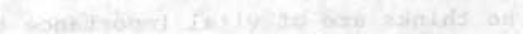

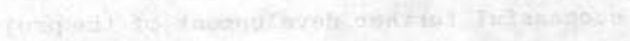

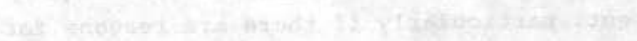

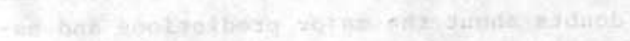

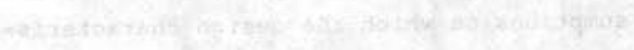

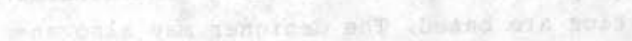

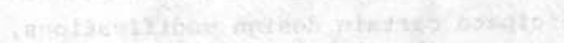

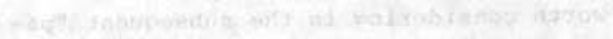

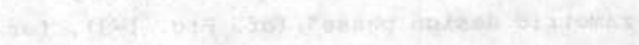

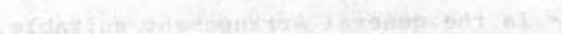

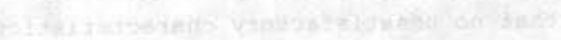

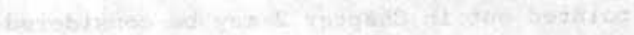

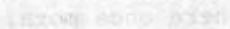

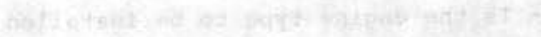

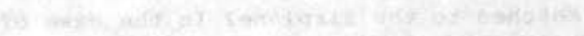

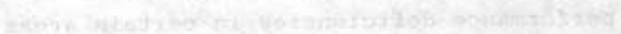

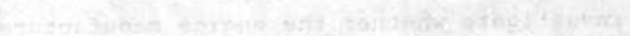

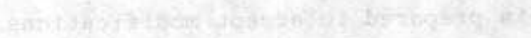

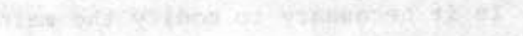

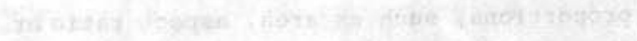

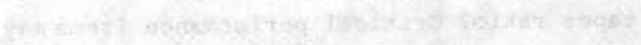

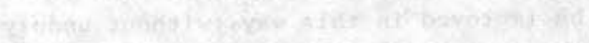

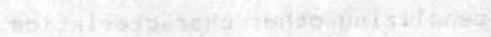




\section{References}

\section{CHAPTER 1.}

Textbooks and general literature on aircraft design

1-1 F.K. Teichmann: "Airplane design manual". Pitman Publishing Corp., Third Edition, 1950.

1-2 G. Corning: "Supersonic and subsonic airplane design". Published by the author, 1953.

1-3 J. Hay Stevens: "The shape of the aeroplane". Hutchinson \& Co. Ltd., London, 1953.

1-4 G.S. Schairer: "Economic considerations of a jet transport airplane". SAB Paper No. 426, Jan. 1955.

1-5 R.B. Morrison, and M.J. Ingle: "Design data for aeronautics and astronautics". John Wiley and Sons, Inc., New York and London, 1961.

1-6 E.E. Sechler, and L.G. Dunn: "Airplane structural analysis and design". Dover Publications Inc., New York, 1963.

1-7 A.C. Kermode: "The aeroplane structure". Second Edition, Pitman \& Sons Ltd., London, 1964.

1-8 K.D. Wood: "Aerospace vehicle design". Volume I: Aircraft design. Johnson Publishing Company, Boulder, Colorado, Second Edition, 1966.

1-9 R. Miller and D. Sawers: "The technical development of modern aviation". New York, Prager, 1968.

1-10 F. Maccabee (ed.): "Light aircraft design handbook". Loughborough University of Technology, Dept. of Transport Technology, TT 6801, Second Edition, Feb. 1969.

1-11 J.M. Swihart (ed.): "Jet transport design". AIAA Selected Reprint Series, Vol. 8, 1969.

1-12 M.O. Wilmer: "Some aspects of optimisation in the design of civil aircraft". Two-day convention on economic factors in aviation. The R.Ae.S., 13-14th May, 1970.

1-13 E.V. Krick: "An introduction to engineering and engineering design". J. Wiley \& Sons, Inc., New York, Second Edition 1969.

1-14 D.D. Hufford, J.A. Ross and K.W. Hoefs: "The economics of subsonic transport airplane design, evaluation and operation". SAE Paper No. 710423.

1-15 L.M. Nicolai: "Design of airlift vehicles". USAF Academy, Dept. of Aeronautics, Aero 464, July 1972.

Trends in aircraft design and technological forecast

1-16 J.C. Floyd: "Some current problems facing the aircraft designer". J.R.Ae.S. 1961, pp. 613-631,

1-17 G. Schairer: "Aircraft design, present and future". ICAS Paper No. 64-533.

1-18 various contributors: "Cheap short-range air transport". A Symposium, J.R.Ae.S., Nov. 1965, pp. 737758.

1-19 M.B. Morgan: "Some aspects of aircraft evolution". 5th ICAS Congress, Paper 66-1, 1966. (See also Aerospace Proceedings 1966 Vol. 1, MacMillan London, 1967).

1-20 P.G. Masefield: "Light aviation problems, prospects and performance". Journal of the Royal Aero. Soc., 
Nov. 1966.

1-21 F.W. Kolle and D.R. Blundell: "Evolution and revolution with the jumbo trijets". Astronautics and Aeronautics, Oct. 1968, pp. 64-69.

1-22 J.C. Brizendine and C.R. Strang: "The future transport world". J. of Aircraft, Nov.-Dec. 1967, pp. 481-486.

1-23 Handel Davies: "Some thoughts about the future of European aeronautics". The Aeronautical Journal, May 1968 , pp. 385-395.

1-24 D. Küchemann and J. Weber: "Analysis of some performance aspects of various types of aircraft, designed to $\mathrm{fly}$ at different ranges and speeds". Progress in Aeronautical Sciences, Vol. 9, 1968, pp. 329-456.

1-25 E. Ufer: "Das Reiseflugzeug von Morgen". Deutscher Aerokurier, Jan. 1970, pp. 8-10.

1-26 M.B. Morgan: "The impact of research and development programmes in various technical areas on the economics of future aircraft design". Two-day convention on economic factors in aviation, R.Ae.S., 13 th - 14th May 1970.

1-27 F.A. Cleveland: "Size effects in conventional aircraft design". AIAA Paper No. 70-940.

1-28 J.E. Steiner: "Aircraft development and world aviation". The Aeronautical Journal, June 1970, pp. 433-443.

1-29 Various contributors: "Vehicle technology for civil aviation, the seventies and beyond". NASA SP-292, Nov. 1971.

Airplane design and development

1-30 G.R. Edwards: "Problems in the development of a new aeroplane". Journal of the Royal Aero. Soc. Vol. 53, pp. $197-252,1949$

1-31 E. Weining: "Design factors in the development of 11ght aircraft". Aero. Eng. Review, July 1950, pp. 18-19.

1-32 Anon.: "Design of aircraft for commercial use". SAE Preprint, 1959.

1-33 Anon.: "Design for the future". An outline of the research, design and development organisation of Bristol Aircraft Limited, undated.

1-34 в. Mansfield: "Billion Dollar Battle". David McKay Comp. Inc., New York.

1-35 J.E. Steiner: "Planning a new commercial aircraft". Astronautics and Aeronautics, Sept. 1967, pp. 52-60.

1-36 W.M. Magruder: "Development of requirement, configuration and design for the Lockheed 1011". SAE Paper No. 680688 .

1-37 J. Spintzyk: "Ueber Methoden der Systemtechnik beim Flugzeugentwurf". Zeitschrift für Flugwissenschaften, June 1968, pp. 206-212.

1-38 D.M. Ryle: "Parametric design studies". AGARD Lecture Series No. 16, April 1969.

1-39 J.T. Stamper: "Management in design". The Aeronautical Journal, Vol. 73, March 1969, pp. $174-185$.

1-40 E.S. Bradley, W.M. Johnston and G.H. von Keszycki: "Passenger transport at low supersonic speeds" . AIAA Paper No. 69-776.

1-41 R.H. Lange: "Parametric analysis of ATT configurations". AIAA Paper No. $72-757$.

1-42 D.L.I. Kirkpatrick and D.H. Peckham: "Multivariate analysis applied to aircraft optimisation - some effects of research advances on the design of future subsonic transport aircraft". R.A.E. Techn. Memo Aero 1448 , Sept. 1972.

1-43 A.W. Bishop: "Optimisation in aircraft design - the whole aircraft". Symposium of the Royal Aeron. Soc. on "Optimisation in aircraft design". 15th November 1972.

1-44 W.M. Eldridge et al: "Conceptual design studies of candidate V/STOL lift fan commercial short haul transport for 1980-85 V/STOL lift fan study". NASA CR-2183, Feb. 1973.

1-45 R.G. Knight, w.V. Powell Jr. and J.A. Prizlow: "Conceptual design study of a V/STOL lift fan commercial short haul transport". NASA CR-2185, April 1973. 
1-46 R.R. Heldenfels: "Integrated, computer-aided design of aircraft". AGARD CP-147, Vol. 1, Oct. 1973.

Broad design requirements and market analysis

1-47 B.S. Shenstone: "Why airlines are hard to please". J. of the Royal Aero. Soc., May 1958, p. $319-336$.

1-48 w.P. Kennedy: "Short-haul air transportation". Aerospace Engineering, Dec. 1961, pp. $24,25,72-74$.

1-49 R. Nivet: "Airlines' approach to aircraft selection". J. of the Royal Aeron. Soc., Vol. 66, Dec. 1962, pp. 751-759.

1-50 B.P.G. de Bray: "Aerodynamic effects of top dressing operations". J. of the Royal Aeron. Soc., Oct. 1962, pp. 631-636.

1-51 J.M. McMahon: "Agricultural aircraft for the future - the fixed wing aircraft". J. of the Royal Aeron. Soc., Dec. 1962, pp. 776-777.

1-52 R.D. Fitzsimmons: "Design and economic suitability of present and future conventional aircraft in Boston - to - Washington service". AIAA Paper No. 66-945.

1-53 K.M. Trûtzscher: "Marktforschung für das Europăische Airbus Projekt". Jahrbuch der wGLR 1967, pp. 59-67.

1-54 Anon.: "The short-haul market". The Aeroplane, July 31, 1968, pp. 4-11.

1-55 D.J. Lloyd-Jones: "Airline equipment planning". J. of Aircraft, Jan./Feb. 1968, pp. 60-63.

1-56 н.M. Drake, G.C. Kenyon and T.L. Galloway: "Mission analysis for general aviation in the 1970's". AIAA Paper No. 69-818.

1-57 T. Oakes: "Airline technical requirements for 1975 STOL and V/STOL systems". SAE Paper No. 700312.

1-58 B.A.M. Botting: "Market research - an economic necessity". Two-day convention on economic factors in aviation, Royal Aero. Soc., 13-14th May 1970.

1-59 G.C. Kenyon and H.M. Drake: "Technological factors in short haul air transportation". AIAA Paper No. 70-1287.

1-60 L. Pazmany: "Future trends in general aviation". AIAA Paper No. 70-1220.

1-61 R.E. Black, D.G. Murphy and J.A. Stern: "The crystal ball focuses on the next generation transport aircraft". SAE Paper No. 710750 .

1-62 Various contributors: "Two day convention on aviation's place in transport, 12/13th May 1971, Royal Aero. Soc.

1-63 L.K. Loftin Jr.: "Aeronautical Vehicles - 1970 and beyond". J. of Aircraft, Vol. 8, No. 12, Dec. 1971, pp. 939-951.

1-64 E. Torenbeek and G.H. Berenschot: "Preliminary design of a next-generation short-haul airliner". Delft University of Technology, Dept. of Aeron. Eng., Memorandum M-184, July 1972.

\section{Airworthiness requirements}

1-65 Anon.: "Federal Aviation Regulations Parts 1-189". Department of Transportation: Federal Aviation Administration.

1-66 Anon.: British Civil Airworthiness Requirements, Sections D and $\mathrm{K}$.

1-67 w. Tye: "Influence of Recent Civil Airworthiness Requirements on Aircraft Design". Journal of the Royal Aero. Soc. Vol. 52, No. 452, Aug. 1948, pp. 513-522.

1-68 w. Tye: "Modern Trends in Civil Airworthiness Requirements". Journal of the Royal Aero. Soc. Vol. 56, Feb. 1952, pp. 73-108.

1-69 w. Tye: "The Arithmetic of Airworthiness". Journal of the Royal Aero. Soc. Vol. 58, March 1954, pp. 195-200.

1-70 w. Tye: "Philosophy of Airworthiness". AGARD Report 58, Aug. 1956.

1-71 в. Caplan: "The Investigation of Aircraft Accidents and Incidents". Journal of the Royal Aero. Soc. Vol. 59, Jan. 1955, pp. 45-60.

1-72 н. Caplan: "Aircraft Design Philosophy". Journal of the Royal Aero. Soc. Vol. 60, May 1956, pp. $301-312$. 
1-73 P.P. Baker, S.G. Corps, Capt. D.F. Redup and S.M. Harris: "All-Day Symposium on Flight Testing for the Certification of Civil Transport Aircraft". Journal of the Royal Aero. Soc. Vol. 71, Nov. 1967, pp. 745-772.

1-74 Anon.: "Provisional Acceptable Means of Compliance - Aeroplane Performance". ICAO Circular 58 -AN/53, July-Aug. 1959.

1-75 Anon.: "International Standards - Airworthiness of Aircraft". ICAO-ANNEX 8 to the Convention on International Civil Aviation. Fifth Edition, April 1962, amended up to Dec. 7th, 1972.

1-76 Anon.: Basic Glider Criteria Handbook - 1962 Revision. Federal Aviation Agency - Flight Standards Service, Washington D.C.

1-77 c.C. Jackson: " $v_{1}, v_{2}$ and all that ....". Flight Int. CCJ's Column, 1964-1966.

1-78 H. Slaughter and N.S. Dobi: "Changing Airworthiness Requirements for Air Taxi Operators and their Effect on Manufacturers of small Airplanes". SAE Paper No. 690320 .

1-79 J.D. Harris: "Airworthiness Regulations - National and International". The Aeronautical Journal, Vol. 73, May 1969, pp. 453-459.

1-80 J.н.н. Grover: "Beechcraft 99 Performance". Flight Int. 25 Sept. 1969, p. 487.

1-81 w. Tye: "Airworthiness and the Air Registration Board". The Aeronautical Journal Vol. 74 , Nov. 1970, pp. 873-887.

1-82 D.J. Wodraska: "The Gates Learjet 24C". Shell Aviation News. 389, 1970, pp. 14-19.

1-83 Anon.: Economic Regulations Part 298. Civil Aeronautics Board.

1-84 D.A. Kier: "Flight Comparison of Several Techniques for Determining the minimum Flying Speed for a Large, Subsonic Jet Transport". NASA TN D-5806, June 1970.

1-85 Anon.: OSTIV Airworthiness Requirements for Sailplanes. Organisation Scientifique et Technique Internationale du Vol à Voile - Sept. 1971 .

1-86 Anon.: "Part 298 Contenders". Flight Int., Vo1. 103, No. 3352, 7 June 1973, p. 898.

1-87 Anon.: "FAA Orders Noise Limits". Business \& Commercial Aviation, Dec. 1973, pp. 80-82.

1-88 Anon.: "FAA Airworthiness Regulation Review Set". Aviation Week \& Space Technology, Vol. 100, No. 8, Feb. 25, 1974 , p. 28.

\section{CHAPTER 2.}

General arrangement and configuration development

2-1 G.R. Edwards: "Problems in the development of a new aeroplane". J.R.Ae.S., Vol. 53, 1949, pp. 197-252.

2-2 M.L. Penne11: "Evolution of the Boeing jet tanker transport". Aeronautical Eng. Review, Aug. 1954, pp. $32-36$.

2-3 Anon.: "Cessna studying radical business aircraft designs". Aviation Week and Space Technology, Nov. 6, 1961 .

2-4 н. Wocke: "Veberlegungen zur Entwicklung eines Geschăftsflugzeugs mit Strahlturbinenantrieb". Jahrbuch 1965 der WGLR, pp. 55-63.

2-5 D. Stinton: "The anatomy of the aeroplane". London, G.T. Foulis Co. Ltd., 1966.

2-6 K. Kens and H.J. Nowarra: "Die deutschen Flugzeuge 1933 - 1945". J.F. Lehmann Verlag, Munich.

2-7 S.D. Davies: "The history of the Avro Vulcan". The Aeronautical Journal, May 1970, pp. $350-364$.

2-8 L.T. Goodmanson: "Transonic transports". 12th Anglo-American Aeronautical Conference, 7th-9th July, 1971, Calgary.

2-9 W.C. Dietz: "Preliminary design aspects of design-to-cost for the YF-16 prototype fighter". AGARD CP No. 147, Vol 1, Oct. 1973.

2-10 W.C. Swan: "Design evolution of the Boeing 2707-300 supersonic transport". Part 1 - configuration development, aerodynamics, propulsion and structures. AGARD CP No. 147, Vo1. 1, Oct. 1973. 
2-11 G.S. Schairer: "Pod mounting of jet engines". Fourth Anglo-American Aeronautical Conference, London, 1953, pp. 29-46.

2-12 B.T. Salmon: "High speed transport turbojet installation considerations". SAE Paper No. 85, April 1953.

2-13 E.S. Allwright: "Engineering features of rear engine installations in transport aircraft". Lecture for the R.Ae.S., Dec. 17, 1959, London.

2-14 D.J. Lambert: "Design of jet transports with rear-mounted engines". Aerospace Eng., Oct. 1960, pp. $30-35$ and $72-74$.

2-15 C. Dawson: "British push up performance of rear-engine airliners". Space-Aeronautics, Sept. 1960, pp. 52-54.

2-16 Anon.: "The design of modern pure jet transports with rear-mounted engines. Reviewed from engineering and operational aspects". ESSO World 60/61, page 35 .

2-17 Anon.: "The Vickers VC-10". Aircraft Eng., Vol. 34, No. 400, June 1962.

2-18 R. Cabiac: "Voici pourquoi notre Caravelle est unique". Aviation Magazine, Feb. 1963, pp. 20-23.

2-19 J.E. Steiner: "The development of the Boeing 727". J.R.Ae.S., Vol. 67, Feb. 1963, pp. $103-110$.

2-20 Anon.: "The Bac 1-11". Aircraft Eng., Vol. 35, No. 5, May 1963.

2-21 G.F. Mahony: "Balance of power". A slight dissertation how many engines and where to put them.

Flight Int., June 29, 1967, pp. 1046-1047.

2-22 Laser: "How many engines, and where?". Flight Int., Aug. 8, 1968, pp. 206-207.

2-23 F.S. Hunter: "L-1011 S-duct versus DC-10's straight-through engine inlet" American Aviation, September 2, 1968, pp. 39-40.

2-24 w.c. Swan and A. Sigalla: "The problem of installing a modern high bypass engine on a twinjet transport aircraft". AGARD CP-124, April 1973.

Tailplane configuration

2-25 M. Duguet: "L'Aérođynamique des fuseaux réacteurs à l'arrière du fuselage". Proceedings of the Second European Aeronautical Congress, 1956.

2-26 G.M. Moss: "Some aerodynamic aspects of rear-mounted engines". J. of the Royal Aero. Soc., Dec. 1964, pp. $837-842$.

2-27 Anon.: "Erfahrungen mit dem Veberziehverhaiten von Flugzeugen mit T-Leitwerk". Luftfahrttechnik Raumfahrttechnik, Jan. 1965, pp. 19-22.

2-28 A.L. Byrnes: "Effect of horizontal stabilizer vertical location on the design of large transport aircraft". AIAA Paper No. 65-331.

2-29 D.J. Lambert: "A systematic study of the factors contributing to post-stall longitudinal stability of T-tail transport configuration". AIAA Paper No. 65-737.

2-30 R.S. Shevell and R.D. Schaufele: "Aerodynamic design features of the DC-9". AIAA Paper No. 65-738.

2-31 R.T. Taylor and E.J. Ray: "Factors affecting the stability of T-tail transports". Journal of Aircraft, Vol. 3, No. 4, July-Aug. 1966, pp. 359-364.

2-32 E.B. Trubshaw: "Low speed handling with special reference to the super stall". J. of the Royal Aero. Soc., Vol. 70, №. 667, July 1966, pp. 695-704.

2-33 D.P. Davies: "Handling the big jets". Published by the A.R.B., Second Ed., 1968. "The superstall", pp. $115-128$.

2-34 D.A. Lovell: "A low speed wind-tunnel investigation of the tailplane effectiveness of a model representing the airbus type of aircraft". ARC R and M No. 3642, April 1969.

The undercarriage

2-35 R.M. Robbins: "Flight characteristics of the Boeing B-47 Stratojet" Aviation Week, 30 April 1951, 
pp. 25-31.

2-36 H.G. Conway: "Landing gear design". Textbook Royal Aeronautical Society, Chapmann \& Hall Ltd., London, 1958.

\section{Unconventional aircraft configurations}

2-37 Anon.: "Konstruktionsbeispiele aus dem Flugzeugbau", Vol. 4: Fahrwerk. Fachbücher für Luft- und Raumfahrt. Luftfahrtverlag Walter Zuerl.

2-38 A. Cameron-Johnson: "The undercarriage in aeroplane project design". Aircraft Eng., Feb. 1969, pp. 6-11.

2-39 J.K. Northrop: "The development of all-wing aircraft". J. of the Royal Aero. Soc. 1947, pp. 481-510.

2-40 G.H. Lee: "Tailless aircraft design problems". J. of the Royal Aero. Soc. 1947, pp. $109-131$.

2-41 I.L. Ashkenas: "Range performance of turbojet aircraft". J. Aero. Sciences, Vol. 15, Feb. 1948,

2-42 J.H. Stevens: "The shape of the aeroplane", Chapter 13, Hutchinson, London, 1953.

2-43 н. Wocke: "Einige Ueberlegungen über die Anwendbarkeit der Entenanordnung bei Unterschallverkehrsflugzeugen". Jahrbuch 1959 der WGLR, pp. 129-137.

2-44 D. Küchemann: "Aircraft shapes and their aerodynamics for flight at supersonic speeds". Proc. 2nd Int. Aeron. Congress, ICAS, Zürich, 1960.

2-45 G.H. Lee: "The possibilities of cost reduction with all-wing aircraft". J. of the Royal Aero. Soc., 1965, pp. 744-749.

2-46 月. Behrbohm: "Basic low speed aerodynamics of the short-coupled canard configuration of small aspect ratio". SAAB TN No. 60, July 1965.

CHAPTER 3.

3-1 J. Morris and D.M. Ashford: "Fuselage configuration studies". SAE Paper No. 670370, April 1967.

3-2 E.S. Krauss: "Die Formgebung von Rümpfen neuerer Verkehrsflugzeuge und ihr Einfluss auf die Wirtschaftlichkeit im Flugbetrieb". Luft- und Raumfahrttechnik, May 1970, pp. $127-132$.

3-3 A.A. Badiagin: "Concerning an efficient slenderness ratio for the fuselage of civilian aircraft" . In: Methods of selection and approximate calculation of air design parameters. Trudy Inst., Moscow, 1961 .

3-4 M.O. Wilmer: "Some aspects of optimisation in the design of civil aircraft". R.Ae.S. Two-day convention on economic factors in aviation, May 1970.

3-5 R.A. McFarland: "Human factors in air transport design". McGraw-Hill Book Company, Inc. 1946.

3-6 B.S. Shenstone: "Why airlines are hard to please". J. of the Royal Aero. Soc., May 1958, pp. 319-336.

3-7 R.A. McFarland: "Buman body size and passenger vehicle design". SAE Paper No. $142 \mathrm{~A}$, Oct. 1962.

3-8 G. Nason: "Interior design and the airliner". The Architectural Review 140 (1966), pp. $413-422$.

3-9 R.G. Mitchell: "Evaluation of economics of passenger comfort standards", SAWE Paper No. 338 , 1962.

3-10 R. Maccabee: "Light aircraft design handbook". Loughborough University of Technology, TT 6801 Feb. 1970.

3-11 E.D. Keen: "Freighters - a general survey". J. of the Royal Aero. Soc., March 1959, pp. $135-152$.

3-12 Anon.: "New aspects of air freight". Interavia, 1961, pp. 645-650.

3-13 C.A. Hangoe: "World-wide survey of cargo densities". SAWE Technical Paper No. $339,1962$.

3-14 A.H. Stratford: "Air cargo operational and economic problems". Chapter 4 of "Air transport ecor.omics in the supersonic era". MacMillan, London 1967.

3-15 M. Heinemann and M.A. Hiatt: "Quick-Change (QC) airplane systems: a prospective". J. of Aircraft. Jan.-Feb. 1967, pp. 42-47.

3-16 Several contributors: "Air logistics". Proceedings of the IAS National Midwestern Meeting, Tulsa, Oaklahoma U.S.A., Oct. 3-5, 1960.

3-17 J. Doetsch: "Neue Verfahren zum Verladen von Luftfrachtgütern". Luftfahrttechnik-Raumfahrttechnik, Feb. 1968 , pp. 51-56. 
3-18 J.E. Nichols and R.L. Meyers: "Design for quick turnaroud - payload system considerations". ASME Conf. Proc. of the Annual Aviation and Space Conference, June 1968, pp. 373-377.

3-19 R.C. Hornbug, W.A. Alden and M. Newman: "Preliminary design of all-cargo aircraft". Ref. 3-18, pp. 365-372.

3-20 S.M. Levin: "Uncompromised cargo - the Mach 0.9 box". Space/Aeronautics, Oct. 1969, pp. 34-44.

3-21 J.B. Teeple, В.J. Bond and R.B. Sleight: "How to design a cockpit: from the man out". Aviation Age, Jan. 1956.

3-22 D.W. Conover: "Cockpit landing visibility". SAE Paper No. 920 B, Oct. 1964.

3-23 Anon.: "Pilot visibility from the flight deck; design objectives for commercial aircraft". SAE AS $580 \mathrm{~A}$.

3-24 Anon.: "Location and actuation of flight deck controls for commercial transport type aircraft". SAE ARP 268 C, 1952, Rev. 1962.

3-25 T.R. Nettleton: "Bandling qualities research in the development of a STOL utility transport aircraft". Canadian Aero. and Space Journal, March 1966, pp. 93-104.

3-26 w.J. Rainbird, R.S. Crabbe, D.J. Peake and R.F. Meyer: "Some examples of separation in three-dimensional flows". Can. Aero. and Space Journal, Dec. 1966, pp. 409-423.

3-27 D.J. Peake: "Three-dimensional flow separations on up-swept rear fuselages". Can. Aero. and Space Journal, Dec. 1969 , pp. 399-408.

\section{CHAPTER 4.}

\section{General literature and textbooks}

4-1 Anon.: "Powerplant Review". A survey of the world's turbine engines. The Aeroplane, March 15, 1957, pp. 360-365.

4-2 W.R. Hawthorne and W.T. Olson: "Design and performance of gas turbine power plants". High speed aerodynamics and jet propulsion, Vol. XI, Princeton Univ. Press, Princeton, 1959.

4-3 O.E. Lancaster (ed.): "Jet propulsion engines". High speed aerodynamics and jet propulsion, Vol. XII, Princeton Univ. Press, Princeton, 1959.

4-4 M.J. Zucrow: "Aircraft and missile propulsion" (Vol. 1: Thermodynamics of fluid flow and application to thermal engines; Vol. 2: The gas turbine power plants, turboprop, turbojet, ramjet and rocket engine). Wiley and Sons, New York, 1958.

4-5 H. Cohen, G.F.C. Rogers and H.I.H. Saravanamuttoo: "Gas turbine theory". Second Edition, Longman, London, 1974.

4-6 W.J. Hesse and N.V.S. Mumford Jr.: "Jet propulsion for aerospace applications". Pitman Publishing Corporation, 1964 .

4-7 P.G. Hill and C.R. Peterson: "Mechanics and thermodynamics of propulsion". Addison-Wesley Publ. Cy., Reading, Massachusetts, 1965.

4-8 w. Thomson: "Thrust for flight". Pitman Publishing Cy., 1969.

4-9 I.E. Treager: "Aircraft gas turbine engine technology. McGraw-Hill Book CY., 1970.

4-10 P.J. McMahon: "Aircraft Propulsion". Pitman Aeronautical Engineering Series, 1971.

\section{RECIPROCATING ENGINES}

4-11 A.W. Morley: "Performance of a piston-type aero-engine". London, Pitman and Sons Ltd., 1951.

4-12 J. Liston: "Powerplants for aircraft". McGraw-Hill Book Company, Inc. 1953.

4-13 F. Eisfeld: "Der Kolbenmotor als Antrieb für Leichtflugzeuge und Möglichkeiten seiner Verbesserung". Jahrbuch 1965 der WGLR, pp. 327-336.

4-14 L. von Bonin and $\mathrm{K}$. Grasmann: "Zukunftaussichten der Kolbenmotoren und Turbotriebwerke für Leichtflugzeuge". Jahrbuch 1965 der WGLR, pp. 337-352. 
4-15 L.M. Yanda: "Correction of turbocharged engine performance to standard conditions and prediction of non-standard day performance". SAE Paper No. 690309, March 1969.

4-16 W.A. Wiseman: "Altitude performance with turbosupercharged light aircraft engines". SAE Paper No. 622A, Jan. 1963.

4-17 W.A. Wiseman and E.M. Ounsted: "Tiara light aircraft engines - A new generation". SAE Paper No. 700205 .

4-18 J.L. Dooley: "Two-stroke light-aircraft engine potential". SAE Paper No. 670238.

4-19 D.L. Satchwe11: "Performance characteristics of aircraft with turbo-supercharged engine and cabin 1968". SAE Paper No. 680266.

4-20 D.E. Cole: "The Wankel engine". Scientific American, Aug. 1972, pp. 14-23.

4-21 C. Jones and $\mathrm{H}$. Lamping: "Curtiss-Wright's development status of the stratified charge rotating combustion engine". SAE Paper No. 710582.

\section{Turboprop engines}

4-22 B. Pinkel and I.M. Karp: "A thermodynamic study of the turbine-propeller engine". NACA TN 2653, March 1952.

4-23 J. Szydlowski: "Bedeutung der Propellerturbine für das leichte 4- bis 6-sitzige Mehrzweckflugzeug". WGLR Jahrbuch 1960 , pp. 289-296.

4-24 R.M. Sachs: "Small propeller turbines compared with other power plants in low speed flight applications". AIAA Paper No. 64-799, Oct. 1964.

4-25 E.P. Cockshutt and C.R. Sharp: "Gas turbine cycle calculations: design-point performance of turbopropeller and turboshaft engines". NRC Report LR-449, Nov. 1965.

4-26 E.P. Cockshutt: "Cycle calculations for turboprop engines". Can. Aeron. and Space Journal, Feb. 1968, Vo1. 14, pp. $61-71$.

4-27 E.J. Kordik: "Kleine Fluggasturbinen für Hubschrauber, Propeller- und Strahlflugzeuge". Jahrbuch 1969 der DGLR, pp. 194-234.

4-28 E.P. Neate and J.J. Petraits: "The Allison Model 250 engine: a case for the small turboprop". SAE Paper No. 7002-6.

Turbojet engines

4-29 M.S. Chape11 and E.P. Cockshutt: "A comprehensive method for calculating turbojet and turbofan design-point performance". Can. Aeron. and Space Journal, June 1964, Vol. 10 No. 6.

4-30 S. Boudigues: "Défense et illustration des propulseurs pour avions rapides". Technique et Sciences Aéron. et Spatiales, July-Aug. 1964, pp. 271-285.

4-31 L.R. L'Anson: "The application of the high bypass turbofan for business and executive aircraft". SAB Paper No. 660221, April 1966.

4-32 C.L. Bagby and w.L. Andersen: "Effect of turbofan cycle variables on aircraft cruise performance". Journal of Aircraft, Sept.-Oct. 1966.

4-33 R.E. Neitzel and M.C. Hemsworth: "High-bypass turbofan cycles for long-range subsonic transports". Journal of Aircraft, Vol. 3, No. 4, July-Aug. 1966, pp. 354-358.

4-34 J.P. Armstrong and A.T. Jones: "The advantages of three-shaft turbofan engines for civil transport operation". The Aeronautical Journal, Vol. 73, Jan. 1969, pp. 25-33.

4-35 พ.H. Sens and R.M. Meyer: "New generation engines - the engine manufacturers' outlook". SAE Paper No. 680278, April-May 1968.

4-36 E. Torenbeek: "Analytical method for computing turbo-engine performance at design and off-design conditions". Memorandum M-188, Delft University of Technology, Department of Aeronautical Engineering, Delft, Jan. 1973.

4-37 J.H. Horlock and J.F. Norbury: "The aero-thermodynamics of subsonic propulsion". Int. congress on 
subsonic aerodynamics, New York Acad. of Sciences, 22nd Nov. 1968, Vo1. 154, No. 2, pp. 549-575. 4-38 B. Wrigley: "Engine performance considerations for the large subsonic transport". Von Karman Institute for Fluid Dynamics, Lecture Series 16, April 1969.

4-39 w. Dettmering and F. Fett: "Methoden der Schuberhohung und ihre Bewertung". Zeitschrift für Flugwiss., Aug. 1969, Vol. 19, No. 8, pp. 257-267.

4-40 R.H. Weir: "Propulsion prospects". The Aeronautical Journal, Vol. 73, Nov. 1969, pp. 923-934.

4-41 J. Wotton: "Engine development - where now?". Flight Int., Dec. 4, 1969, pp. 888-890.

4-42 R.M. Denning and J.A. Hooper: "Prospects for improvement in efficiency of flight-propulsion systems". AIAA Paper No. 70-873.

4-43 R.L. Cummings and H. Gold: "Low-cost engines for Aircraft". In: NASA SP-259, VII, Nov. 1970.

4-44 L.G. Dawson: "Propulsion". In: "The future of aeronautics", edited by J.E. Allen \& J. Bruce, Hutchinson of London, 1970.

4-45 E.S. Taylor: "Evolution of the jet engine". Astronautics and Aeronautics, Nov. 1970, pp. 64-72.

4-46 в. Gevelhoff: "Ein Beitrag zur optimalen Auslegung von Installierten, Luftansangenden Strahltriebwerken". Dissertation, Dec. 1970, T.H. Aachen.

4-47 G. Rosen: "Prop-fan. A high thrust, low noise propulsor". SAE Paper No. 710470.

4-48 M.A. Beheim a.O.: "Subsonic and supersonic propulsion". In: Vehicle technology for civil aviation, NASA SP-292, Nov. 1971, pp. 107-156.

4-49 P. Alesi: "Des hautes températures devant turbine sur turboréacteurs et turbines à gaz". 1'Aéronautique et 1'Astronautique, No. 33, 1972-1, pp. 69-78.

4-50 R.W. Koenig and L.H. Fischback: "GENENG - A program for calculating design and off-design performance for turbojet and turbofan engines". NASA TN D-6552 and D-6553, Feb. 1972.

4-51 J.F. Dugan Jr.: "Engine selection for transport and combat aircraft". Von Karman Institute of Fluid Dynamics Lecture Series 49, April 1972 (NASA TM-X-68009).

4-52 D.G.M. Davies: "Variable pitch fans". Interavia, March 1972, pp. 241-243.

4-53 w.L. McIntire: "Engine and airplane - will it be a happy marriage?" SAWE Paper No. 910 , May 1972.

4-54 M.J. Banzakein and S.B. Kazin: "The NASA/GE Quiet engine A". AIAA Paper No. 72-659.

4-55 E.A. White and G.L. Wilde: "Engines for civil V/STOL". The Aeronautical Journal, Oct. 1972, pp. 627632 .

\section{CHAPTER 5 .}

General weight and performance "guesstimates"

5-1 I.H. Driggs: "Aircraft design analysis". J. of the Royal Aero. Soc., 1950, Vo1. 54, pp. 65-116.

5-2 H.K. Millicer: "The design study". Flight, 17 Aug. 1951, pp. 201-205.

5-3 G. Backhaus: "Grundbeziehungen für den Entwurf optimaler Verkehrsflugzeuge". Jahrbuch der wGLR, 1958, pp. 201-213.

5-4 D. Kūchemann and J. Weber: "Analysis of some performance aspects of various types of aircraft, designed to fly at different ranges and speeds". Progress in Aeronautical Sciences, Vol. 9, 1968, Pp. $329-456$.

5-5 R.A. Werner and G.F. Wislecinus: "Analysis of airplane design by similarity considerations". AIAA Paper No. 68-1017.

5-6 A. Krauss: "Vorhersage des Abfluggewichtes von Verkehrsflugzeugen". Luftfahrttechnik-Raumfahrttechnik, Feb. 1966 , pp. 54.58 .

5-7 E. Vallerani: "Evaluation of aircraft weight by the method of partial growth factors - case of passenger transport aircraft". Rivista di Ingegneria, Dec. 1967, pp. 989-1002 (in Italian) .

5-8 W.E. Caddell: "On the use of aircraft density in preliminary design". SAWE Paper No. 813, May 1969.

5-9 W. Richter et al.: "Luftfahrzeuge". Das Fachwissen des Ingenieurs. Carl Hansen Verlag, München, 1970, section 4.2 .4 
5-10 D. Howe: "The prediction of empty weight ratio and cruise performance of very large subsonic jet transport aircraft". Cranfield Report, Aero No. 3; 1971.

\section{Initial drag prediction}

5-11 в.G. Sheridan: "Aircraft preliminary design methods used in the weapon systems analysis division". NAVWEPS Report No. R-5-62-13, June 1962.

5-12 S.F. Hoerner: "Fluid-dynamic drag". Published by the author, 1965.

5-13 J.E. Linden and F.J. O'Brinski: "Some procedures for use in performance prediction of proposed aircraft designs". SAE Paper No. 650800 .

5-14 B. McCluney and J. Marshall: "Drag development of the Belfast". Aircraft Engineering, Oct. 1967, pp. $33-37$.

5-15 A.B. Haines: "Subsonic aircraft drag: an appreciation of present standards". The Aeronautical Journal, Vo1. 72, No. 687, March 1968 , pp. 253-266.

5-16 D.A. Norton: "Airplane drag prediction". Annals of the New York Academy of Sciences, Nov. 1968 , Vol. 154, Art. 2, pp. 306-328.

\section{Evaluation of performance requirements}

5-17 B. Göthert: "Einflusz von Flächenbelastung, Flügelstreckung und Spannweitenbelastung auf die Flugleistungen". Luftfahrtforschung, Vol. 6, 1939, pp. 229-250.

5-18 w.B. Oswald: "Designing to the new CAA transport category performance requirements". SAE Quarterly Transactions, Vo1. 1, No. 2, April 1947, pp. 321-333.

5-19 н.В. Cherry and A.B. Croshere Jr.: "An approach to the analytical design of aircraft". SAE Quarterly Transactions, Jan. 1948, Vol. 2, No. 1, pp. 12-18.

5-20 I.L. Ashkenas: "Range performance of turbojet airplanes". J. of the Aeronautical Sciences, Vol. 15, Feb. 1948, pp. 97-101.

5-21 D.в. Perry: "Exchange rates between some design variables for an aircraft just satisfying take-off distance and climb requirements". RAE Technical Report No. 69167, Aug. 1969.

5-22 в. Slaughter and N.S. Dobi: "Changing airworthiness requirements for air taxi operators and their effect on manufacturers of small airplanes". SAE Paper No. 690320.

5-23 J. Roskam and D.L. Kohlman: "An assessment of performance, stability and control improvements for general aviation aircraft". SAE Paper No. 700240 .

5-24 D.o. Carpenter and P. Gotlieb: "The physics of short take-off and landing". AIAA Paper No. 70-1238. 5-25 R.H. Wild: "Format for flight". Flight Int., 11 March 1971, pp. 338-340, 350.

5-26 J.D. Raisbeck: "Consideration of application of currently available transport-category aerodynamic technology in the optimization of general aviation propeller-driven twin engine design". SAE Paper No. 720337 .

5-27 E. Torenbeek: "An analytical expression for the balanced field length". AGARD Lecture Series No. 56, April 1972.

5-28 M.L. Gallay: "Plight of aircraft with partial and unbalanced thrust". NASA TT F-734, April 1973.

\section{Aircraft synthesis and optimization}

5-29 K.L. Sanders: "The optimum design of long-range aircraft". Aircraft Eng., April 1957, Vol. 29 , No. 338, pp. $98-106$.

5-30 в. Kimura, S. Kikuhura and J. Kondo: "Operations research in the basic design of the YS-11 transport airplane". Advances in Aeronautical Sciences, Vol. 3, pp. 557-574.

5-31 K.L. Sanders: "Aircraft Optimization". SAWE Paper No. 289, May 1961.

5-32 J.K. Wimpress and J.M. Swihart: "Influence of aerodynamic research on the performance of supersonic 
airplanes". J. of Aircraft, Vol. 1, No. 2, March-April 1964.

5-33 н. Pakendorf: "Zur Optimierung der Triebwerksanlage eines Kurzstreckenflugzeuges, unter besonderer Berücksichtigung hoher by-pass Verhältnisse". WGLR Jahrbuch 1966, pp. 261-270.

5-34 C.L. Bagly and W.L. Andersen: "Effect of turbofan cycle variables on aircraft cruise performance". J. of Aircraft, Sept.-Oct. 1966, Vol. 3, No. 5, pp. 385-389 (also AIAA Paper No. 65-796).

5-35 M.L. Olason and D.A. Norton: "Aerodynamic design philosophy of the Boeing 737". Journal of Aircraft, Nov.-Dec. 1966, Vol. 3, No. 6, pp. 524-528.

5-36 G.J. Schott Jr.: "Analytical propulsion research". Luchtvaarttechniek, J. of the Dutch Society of Engineers, Jan. 20, 1967.

5-37 G.H. Lee: "The aerodynamic design philosophy of the Handley Page Jetstream". Aircraft Engineering, Sept. 1967, pp. 10-21.

5-38 V.A. Lee, H.G. Ball and E.A. Wadsworth: "Computerized aircraft synthesis". J. of Aircraft, Sept.-Oct. 1967, Vol. 4, No. 5, pp. 402-408.

5-39 z.M. v. Krzywoblocki and w.z. Stepniewski: "Application of optimization techniques to the design and operation of V/STOL aircraft". International Congress on Subsonic Aeronautics, Nov. 1968, Annals of the New York Academy of Sciences, Vol. 154, Art. 2, pp. 982-1013.

5-40 D. Ryle: "Parametric studies in aircraft design", AGARD Lecture Series No. 16, Apri1 1969.

5-41 B.H. Little: "Advanced computer technology". AGARD Lecture Series No. 16, April 1969.

5-42 D.D. Hufford, J.A. Ross and K.W. Hoefs: "The economics of subsonic transport airplane design, evaluation and operation". SAE Paper No. 710423.

5-43 R.E. Wallace: "Parametric and optimisation techniques for airplane design synthesis". AGARD Lecture Series No. 56, April 1972.

5-44 D.L.I. Kirkpatrick: "Review of two methods of optimising aircraft design". AGARD Lecture Series No. 56, Apri1 1972.

5-45 R.E. Wallace: "A computerized system for the preliminary design of commercial airplanes". Document of the Boeing Company, Commercial Airplane Group, Seattle, Washington.

5-46 R.R. Heldenfels: "Integrated, computer-aided design of aircraft". 43rd AGARD Flight Mech. Panel Symposium, Florence, Oct. 1973.

5-47 D.L.I. Kirkpatrick and M.J. Larcombe: "Initial design optimization on civil and military aircraft". 43rd AGARD Flight Mechanics Panel Symposium, Florence, Oct. 1973.

Community noise considerations

5-48 Anon.: "Definitions and procedures for computing the perceived noise leve 1 of aircraft noise". Aerospace Recommended Practice 865, SAE, 15th Oct. 1964.

5-49 F.B. Greatrex and R. Bridge: "The evaluation of the engine noise problem". Aircraft Engineering, Feb. 1967, pp. 6-10.

5-50 B.J. Richards: "Aircraft noise-mitigating the nuisance". Aircraft Eng., Feb. 1967, pp. 11-18.

5-51 н. Drell: "Impact of noise on subsonic transport design". SAE Paper No. 700806.

5-52 R.E. Russe11 and J.D. Kester: "Aircraft noise, its source and reduction". SAE Paper No. 710308.

5-53 J.F. Dugan: "Engine selection for transport and combat aircraft". AGARD Lecture Series No. 56, April 1972 (NASA TM-X-68009).

5-54 L.G. Dawson and T.D. Sills: "An end to aircraft noise?". The Aeronautical Journal, May 1972, pp. 286-297.

5-55 Anon.: "The future of short-haul air transport within Western Europe". Report of the Netherlands V/STOL Working Group, June 1973, NLR, Amsterdam.

5-56 D. Howe: "The weight, economic and noise penalties of short haul transport aircraft resulting from the reduction of balanced field length". Cranfield Institute of Technology Report Aero. No. 24, Jan. 1974. 


\section{CHAPTER 6 .}

\section{Choice of the number and type of engine(s)}

6-1 s.J. Moyes and W.A. Pennington: "The influence of size on the performance of turbojet engines". Third Anglo-American Aeronautical Conference, Brighton, 1951, pp. 545-562.

6-2 A.E. Russell: "The choice of power units for civil aeroplanes". Journal of the Royal Aero. Soc., August 1954, pp. 523-539.

6-3 F.H. Keast: "Big engines or small?". The Aeroplane, August 10, 1956, pp. 189-191.

6-4 w.A. Pennington: "Choice of engines for aircraft". Shell Aviation News, Jan. 1959, pp. 14-19.

6-5 N. Scholz and G. Preininger: "Einige Gesichtspunkte zur Frage der günstigsten Schubklasse von TLTriewerken". Luftfahrttechnik 8, No. 12, December 1962, pp. 327-332.

6-6 D.R. Newman: "The De Havilland 121 Trident". Aircraft Engng., May 1962, pp. 149-152.

6-7 R.W. Higgins: "The choice between one engine or two". Aircraft Engng., Nov. 1962, pp. 19-21.

6-8 J. Karran: "Airbus, 2, 3 or 4 engines?". The Aeroplane, Nov. 3, 1966, pp. 4-5.

6-9 G.F. Mahoney: "Balance of power". Flight Int., June 29, 1967, pp. 1046-1047.

6-10 Laser: "How many engines and where?". Flight Int., August 8, 1968, pp. 206-207.

6-11 Anon.: "Two or three engines?". Flight Int., Sept. 1969, pp. 446-447.

6-12 N.L. Gallay: "Flight of aircraft with partial and unbalanced thrust". NASA TT F-734, April 1973.

\section{Propellers}

6-13 н. Glauert: "The elements of aerofoil and airscrew theory". McMillan, New York, 1943.

6-14 G.C.I. Gardiner and J. Mullin: "The design of propellers". Journal of the Royal Aero. Soc., Vol. 53 , 1949, pp. 745-762.

6-15 J.L. Crigler and R.E. Jaquis: "Propeller-efficiency charts for light airplanes". NACA TN $1338,1947$.

6-16 Anon.: "SBAC Standard method of propeller performance estimation". Society of British Aircraft Constructors Ltd., April 1950.

6-17 J. Gilman Jr.: "Propeller performance charts for transport airplanes". NACA TN 2966, 1953.

6-18 Anon.: "Aircraft Propeller Handbook". ANC-9 Bulletin, Sept. 1956.

6-19 Anon.: "Choice of propellers for turbine engines in the medium power range". Rotol Ltd. Performance office Report No. 1104, Issue II, July 1959.

6-20 G. Rosen: "New problem areas in aircraft propeller design". Can. Aero. Journal, June 1960, pp. 213-220.

6-21 Anon.: "Generalized method of propeller performance estimation". Hamilton Standard Publication PDB 6101A, 1963.

6-22 K.D. Wood: "Aerospace vehicle design". Vol. 1: Aircraft Design, 2nd. edition. Johnson Publishing Company, Boulder, Colorado, 1966.

6-23 D.P. Currie: "propeller design considerations for turbine powered aircraft". SAE Paper No. 680227.

6-24 G. Rosen and W.M. Adamson: "Next generation V/STOL propellers". SAE Paper No. 680281.

6-25 G. Rosen and C. Rohrbach: "The quiet propeller - A new potential". AIAA Paper No. 69-1038.

6-26 R. Worobel: "Computer program user's manual for advanced general aviation propeller study". NASA CR2066, May 1972.

Powerplant installation

6-27 J. Seddon: "Air intakes for gas turbines". J. Royal Aero. Soc., Oct. 1952, pp. 747-781.

6-28 D. Kũchemann and J. Weber: "Aerodynamics of propulsion". McGraw-Bill Book CY., 1953, New York.

6-29 G.S. Schairer: "Pod mounting of jet engines". Fourth Anglo-American Aeronautical Conference, London, 1953, pp. 29-46.

6-30 G. Schulz: "Aerodynamische Regeln für den Einbau von Strahltriebwerksgondeln". z. für Flugwiss. 3 
(1955), Vol. 5, pp. 119-129.

6-31 J.T. Kutney and S.P. Piszkin: "Reduction of drag rise on the Convair 990 aircraft". J. of Aircraft, January 1964, Vol. 1, p. 8.

6-32 R.S. Shevell and R.D. Schaufele: "Aerodynamic design features of the DC-9". AIAA Paper No. 65-738. 6-33 M.L. Olason and D.A. Norton: "Aerodynamic design philosophy of the Boeing 737". AIAA Paper No. 65-739. 6-34 R.T. Taylor and E.J. Ray: "Factors affecting the stability of T-tail transports". J. of Aircraft, Vol. 3 no. 4, July/August 1966, pp. 359-364.

6-35 w.M. Magruder: "Development of requirement, configuration and design for the Lockheed 1011 jet transport". SAE Paper No. 680688.

6-36 K.S. Lawson: "The influence of the engine on aircraft design". Royal Aero. Soc., Symposium lecture on V/STOL design, London, 1969.

6-37 J.A. Bagley: "Some aerodynamica problems of powerplant installation on swept-winged aircraft". Royal Aeron. Soc., Symposium lecture on V/STOL design, London, 1969.

6-38 D.D. Hufford, J.A. Ross and K.W. Hoefs: "The economics of subsonic transport airplane design, evaluation and operation". SAE Paper No. 710423.

6-39 P.G.R. Williams and D.J. Stewart: "The zomplex aerodynamic interference pattern due to rear fuselage mounted powerplants". AGARD Conference Proceedings CP-71.

6-40 W.C. Swan and A. Sigalla: "The problem of installing a modern high bypass engine on a twinjet transport aircraft". AGARD CP-124, April 1973.

6-41 J. Barche: "Beitrag zum Interferenzproblem von über den Tragflügeln angeordneten Triebwerken". DGLR Annual Conference, 1969.

6-42 н. Griem, J. Barche, H.J. Beisenherz and G. Krenz: "Some low speed aspects of the twin-engine short haul aircraft VFW 614". AGARD-CP-160, Takeoff and landing, April 1974.

Thrust reversal

6-43 J.C. Pickard: "Thrust reversers for jet aircraft". Aerospace Engineering, Jan. 1961.

6-44 M.J. Green: "Rolls-Royce thrust reversers-compatibility". SAE Paper No. 69040.

6-45 J.S Mount and D.W.R. Lawson: "Developing, qualifying and operating business jet thrust reversers". SAE Paper No. 690311.

6-46 S.K. Wood and P.E. Mc.Coy: "Design and control of the 747 exhaust reverser system". SAE Paper No. 690409.

6-47 D.J. Lennard: "Design features of the CF-6 engine thrust reverser and spoiler". SAE Paper No. 690411.

6-48 Anon.: "Approximate estimation of braking thrust of propellers (piston engines)." R.Ae.S. Data Sheets "Performance", Sheet ED 1/2, Feb. 1963.

Auxiliary Power Units

6-49 J. Wotton: "Integrated auxiliary power". Flight Int., 8 August 1968, pp. 210-212.

6-50 B.H. Nicholls and A.D. Meshew: "Auxiliary power systems for 1975 fighter aircraft". SAE Paper No. 680311.

6-51 W.E. Arndt: "Secondary power requirements for large transport aircraft". SAE Paper No. 680708.

6-52 н.S. Keltto: "Installation of the APU in the 747 airplane". SAE Paper No. 680709.

6-53.P.G. Stein: "The Hamilton Standard APU for Lockheed's Tri Star Airliner". SAE Paper No. 700814.

6-54 D.A. Malohn: "TSCP 700 Auxiliary Power Unit for the DC-10 Aircraft". SAE Paper No. 700815.

6-55 L.B. Allen: "APU selection - An airline viewpoint". SAE Paper No. 700816.

6-56 W.P. Frey and E. Schne11: "Auxiliary power units for secondary power systems". AGARD-CP-104, June 1972.

6-57 C.H. Paul: "The development of small gas turbines for aircraft auxiliary power". The Aeronautical Journal, Oct. 1970, pp. 797-805. 
CHAPTER 7.

Textbooks

7-1 A.F. Donovan and H.R. Lawrence: "Aerodynamic components of aircraft at high speeds". Volume vII of "High speed aerodynamics and jet propulsion". Princeton University Press, 1957.

7-2 F.W. Riegels: "Aerodynamische Profile". Oldenburg, 1958.

7-3 н. Schlichting and E. Truckenbrodt: "Aerodynamik des Flugzeuges". (Part II). Springer-Verlag, Berlin, 1960.

7-4 G. Corning: "Supersonic and subsonic airplane design". Published by the author. Second Edition, 1964.

7-5 I.H. Abbott and A.E. von Doenhoff: "Theory of wing sections". Dover Publications Inc., New York, 1958.

7-6 A.C. Kermode: "The aeroplane structure". Pitman Publishing Cy., Second edition, 1964.

7-7 D.O. Dommasch, S.S. Sherbey and T.F. Connolly: "Airplane aerodynamics". Pitman Publishing Corp., New York, Fourth Edition, 1967.

7-8 D.P. Davies: "Handling the big jets". Air Registration Board, Second edition, May 1968.

Wing design philosophy and development

7-9 W.S. Farren: "The aerodynamic art". J. of the Royal Aeron. Society, Vol. 60, July 1956, pp. $431-449$.

7-10 J.A. May: "Aerodynamic design of the Vickers VC-10". Aircraft Eng., June 1962, pp. 158-164.

7-11 B.J. Prior: "Aerodynamic design of the BAC 1-11". Aircraft Eng., May 1963, pp. 149-152.

7-12 J.K. Wimpress and J.M. Swihart: "Influence of aerodynamic research on the performance of supersonic airplanes". J. of Aircraft, Vo1. 1, No. 2, March-April, 1964.

7-13 R.S. Shevell and R.D. Schaufele: "Aerodynamic design features of the DC-9". AIAA Paper No. 65-738.

7-14 M.L. Olason and D.A. Norton: "Aerodynamic design philosophy of the Boeing 737". AIAA Paper No. 65-739.

7-15 J.H.D. Blom: "Fokker F-28 evolution and design philosophy-aerodynamic design and aeroelasticity". Aircraft Eng., June 1967, pp. 17-21.

7-16 R.D. Schaufele and A.W. Ebeling: "Aerodynamic design of the DC-9 wing and high lift system". SAE Paper No. $670846,1967$.

7-17 в. Herb: "Zur Entwicklungsgeschichte der Flugmechanik". DLR Forschungsbericht 67-44, July 1967.

7-18 G.H. Lee: "Aerodynamic considerations of a medium Mach business aeroplane". SAE Paper No. 670244, April 1967.

7-19 J.H. Paterson: "Aerodynamic design features of the C-5A". SAE Paper No. 670847.

7-20 D. Ryle: "Wing design, body design, high lift systems and flying qualities". AGARD-VKI Lecture Series 16, April 21-25, 1969.

7-21 с.н. Hurkamp, w.M. Johnston and J.H. Wilson: "Technology assessment of advanced general aviation aircraft". NASA CR-114339, June 1971.

7-22 D. Küchemann: "Aerodynamic design". The Aeron. Journal, Feb. 1969, pp. 101-110.

7-23 J.M. Swihart (ed.): "Jet transport design". AIAA Selected Reprints/Volume VIII, Nov. 1969.

7-24 R.E. Bates: "Progress on the DC-10 development program". AIAA Paper No. 69-830.

7-25 T.I. Ligum: "Aerodynamics and flight dynamics of turbojet aircraft". NASA Technical Translation TT F542.

7-26 J.D. Raisbeck: "Consideration of application of currently available transport-category aerodynamic technology in the optimization of general aviation propeller-driven twin design". SAE Paper No. 720337.

7-27 A.L. Byrnes Jr.: "Aerodynamic design and development of the Lockheed S-3A Viking". AIAA Paper No. 72746.

7-28 D.M. McRae : "The aerodynamic development of the wing of the A-300B". The Aero. Journal, July 1973, pp. 367-379. 
7-29 н. Wittenberg: "Some considerations on the design of very large aircraft". Jaarboek 1974 of the Netherlands Association of Aeronautical Engineers (paper No. 3).

\section{Wings for high-speed aircraft}

7-30 W.A. Waterton: "Some aspects of high performance jet aircraft". J. of the Royal Aeron. Soc., June 1953, pp. 375-390.

7-31 K.E. Van Every: "An engineering comparison using straight, swept, and delta wings". Interavia, Vol. 8, No. 1, 1953, pp. 23-27. Followed by various other contributions on pp. 27-35.

7-32 C.L. Johnson: "Airplane configurations for high speed flight". Interavia, Vo1. 9, No. 1, 1954, pp. 47-51.

7-33 E.W.E. Rogers and J.M. Hall: "An introduction to the flow about plane swept-back wings at transonic speeds". J. of the Royal Aeron. Soc., Vol. 64, August 1960.

7-34 R.C. Lock and E.W.E. Rogers: "Aerodynamic design of swept wings and bodies". Advances in Aeron. Sciences, Vol. 3. Pergamon Press, London.

7-35 J.A. Bagley: "Aerodynamic principals for the design of swept wings". Progress in Aeronautical Sciences, Vol. 3. Pergamon Press, London.

7-36 R.C. Lock: "The aerodynamic design of swept winged aircraft at transonic and supersonic speeds". J. of the Royal Aeron. Soc., Vol. 67, No. 6, June 1963, pp. 325-337.

7-37 K.G. Hecks: "The high-speed shape". Flight Int., Jan. 2 1964, pp. 13-18.

7-38 A.B. Haines: "Recent research into some aerodynamic design problems of subsonic transport aircraft", ICAS Paper No. 68-10, Sept. 1968.

7-39 T.G. Ayers: "Supercritical aerodynamics worthwhile over a range of speeds". Astronautics and Aeronautics, August 1972 , pp. 32-36.

Discussions of shape parameters

7-40 в.н. Cherry and A.B. Croshere Jr.: "An approach to the analytical design of aircraft". SAE Quarterly Transactions, Vol. 2, No. 1, Jan. 1948, pp. 12-18.

7-41 I.L. Ashkenas: "Range performance of turbojet airplanes". J. of the Aeron. Sciences, Vol. 15, Feb. 1948, pp. 97-101.

7-42 G. Gabrielli: "A method for determining the wing area and its aspect ratio in aircraft design". Monografie del Laboratorio di Aeron. Politecnico di Torino, No. 294.

7-43 G. Backhaus: "Grunbeziehungen für den Entwurf optimaler Verkehrsflugzeuge". Jahrbuch 1958 der WGL , pp. 201-213.

7-44 w. Lehmann: "Wahl der Profildicke und Flügelpfeilung bei Verkehrsflugzeugen". Luftfahrttechnik, Nov. 1961 , pp. 323-326.

7-45 K.L. Sanders: "Aircraft optimization". SAWE Paper No. 289, 1961.

7-46 D. Küchemann and J. Weber: "An analysis of some performance aspects of various types of aircraft designed to fly over different ranges at different speeds". Progress in Aeron. Sciences, Vol. 9, pp. $324-456,1968$.

7-47 J. Roskam and D.L. Kohlmann: "An assessment of performance, stability and control improvements for general aviation aircraft". SAE Paper No. 700240.

7-48 R.A. Cole: "Exploiting AR". Shell Aviation News, 1970, No. 387, pp. 10-15.

7-49 K.H. Bergley: "Debating A.R. (aspect ratio)". Shel1 Aviation News, 1971, No. 398, pp. 14-18.

7-50 D.L.I. Kirkpatrick: "Review of two methods of optimising aircraft design". AGARD Lecture Series No. 56 (Paper 12), April 1972. 
7-51 I.H. Abbott, A.E. von Doenhoff and L.S. Stivers: "Summary of airfoil data". NACA Repcrt 824, 1945.

7-52 L.K. Loftin: "Theoretical and experimental data for a number of NACA 6A-Series airfoil sections". NACA TR Nंo. $983,1948$.

7-53 Anon.: "Critical Mach number for high-speed aerofoil sections". Royal Aero. Soc. Data Sheets, Aerodynamics Sub. Series, Vol. 2 "Wings", Sheet 00.03.01, April 1953.

7-54 B.N. Daley and R.S. Dick: "Bffect of thickness, camber and thickness distribution on airfoil characteristics at Mach numbers up to 1.0". NACA TN 3607, 1956.

7-55 A.B. Haines: "Wing section design for sweptback wings at transonic speeds". J. of the Royal Aero. Soc., Vol. 61, April 1957, pp. 238-244.

7-56 в.н. Pearcy: "The aerodynamic design of section shapes for swept wings". Second Int. Congress of the Aeron. Sciences, Zürich, 1960. Advances in Aeron. Sciences, Vol. 3, pp. 277-322. Pergamon Press.

7-57 Anon.: "A method of estimating the drag-rise Mach number for two-dimensional airfoil sections". Transonic Data Memorandum 6407, Royal Aero. Soc., 1965.

7-58 D.W. Holder: "Transonic flow past two-dimensional aerofoils". J.R.Ae.S. Vol. 68, August 1964, pp. 501-516.

7-59 J.W. Boerstoel: "A survey of symmetrical transonic potential flow around quasi-elliptical airfoil sections". NLR-TR 136, Jan. 1967.

7-60 L.R. Wootton: "Effect of compressibility on the maximum lift coefficient of airfoils at subsonic speeds". J. of the Royal Aero. Soc., Vol. 71, July 1967.

7-61 G.Y. Nieuwland: "Transonic potential flow around a family of quasi-elliptical airfoil sections". NLR Report TRT 172, 1967.

7-62 E.C. Polhamus: "Subsonic and transonic aerodynamic research". NASA SP-292, 1971.

7-63 R.H. Liebeck and A.I. Ormsbee: "Optimization of airfoils for maximum 1ift". J. of Aircraft, Vol. 5, Sept./Oct. 1970, pp. 409-415.

7-64 G.J. Bingham and K.W. Noonan: "Low-speed aerodynamic characteristics of NACA 6716 and NACA 4416 airfoils with 35-percent-chord single-slotted flaps". NASA TN X-2623, May 1974.

7-65 R.T. Whitcomb and L.R. Clark: "An airfoil shape for efficient flight at supercritical Mach numbers". NASA TM X-1109, July 1965.

7-66 R.J. McGhee and W.D. Beasly: "Low-speed characteristics of a 17-percent-thick airfoil section designed for general aviation application". NASA TN D-7428.

7-67 ш.н. Wentz, Jr.: "New airfoil sections for general aviation aircraft". SAE Paper No. 730876.

7-68 J.J. Kacprzynski: "Drag of supercritical airfoils in transonic flow". AGARD Conference Proceedings No. CP-124, April 1973.

7-69 W.E. Palmer: "Thick-wing flight demonstrations". SAE Paper No. 720320.

\section{Low-speed stalling}

7-70 R.F. Anderson: "Determination of the characteristics of tapered wings". NACA Report $572,1936$.

7-71 B.A. Soulé and R.F. Anderson: "Design charts relating to the stalling of tapered wings". NACA Report 703,1940 .

7-72 н.н. Sweberg and R.C. Dingeldein: "Summary of measurements in Langley full-scale tunnel of maximum lift coefficients and stalling characteristics of airplanes". NACA Report No. 829.

7-73 พ.H. Philips: "Appreciation and prediction of flying qualities". NACA Report 927, 1949.

7-74 G.B. McCullough and D.E. Gault: "Examples of three representative types of airfoil-section stall at low speed". NACA Technical Note 2502, 1951.

7-75 J.A. Zalovchick: "Summary of stall warning devices". NACA TN 2676, 1952.

7-76 D. Küchemann: "Types of flow on swept wings". J. Roy. Aero. Soc., Vol. 57, pp. 683-699, Nov. 1953.

7-77 A.D. Young and B.B. Squire: "A review of some stalling research", with an appendix on "Wing sections and their stalling characteristics". ARC R and M 2609, 1951.

7-78 в.O. Palme: "Summary of stalling characteristics and maximum lift of wings at low speeds". SAAB TN 15, 
Apri1 1953.

7-79 G.G. Brebner: "The design of swept wing planforms to improve tip-stalling characteristics". RAE Report Aero 2520 (ARC 17624), July 1954.

7-80 J. Black: "Flow studies of the leading edge stall on a swept-back wing at high incidence". J. of the Royal Aeron. Soc., Vol. 60, Jan. 1956, pp. 51-60.

7-81 D.E. Gault: "A correlation of low-speed, airfoil-section stalling characteristics with Reynolds number and airfoil geometry". NACA TN 3963, 1957.

7-82 G.C. Furlong and J.G. McHugh: "A summary and analysis of the low speed longitudinal characteristics of swept wings at high Reynolds number". NACA Report 1339, 1957.

7-83 R.L. Maki: "The use of two-dimensional data to estimate the low-speed wing lift coefficient at which section stall first appears on a swept wing". NACA RM A51E15, 1951.

7-84 K.P. Spreeman: "Design guide for pitch-up evaluation and investigation at high-subsonic speeds of possible limitations due to wing-aspect-ratio variations". NACA TM X-26, 1959.

7-85 J.G. Wimpenny: "Low speed stalling characteristics". AGARD Report 356, 1961.

7-86 A. Spence and D. Lean: "Some low-speed problems of high speed aircraft". J. of the Royal Aeron. Soc., Vol. 66 No. 616, April 1962, pp. 211-225.

7-87 J. Fletcher: "The stall game". Shell Aviation News, 1966, Number 341, pp. 16-19.

7-88 C.L. Bore and A.T. Boyd: "Estimation of maximum lift of swept wings at low Mach numbers". J. of the Royal Aeron. Soc., Vol. 67, April 1963, pp. 227-239.

7-89 C.W. Harper and R.L. Maki: "A review of the stall characteristics of swept wings". NASA TN D-2373, July 1964.

7-90 Ph. Poisson-Quinton and E. Erlich: "Analyse de la stabilité et du controle d'un avion au dela de sa portance maximale". AGARD Conference Proceedings No. CP-17, 1966.

7-91 H.н.B.M. Thomas: "A study of the longitudinal behaviour of an aircraft at near-stall and post-stall conditions". AGARD Conference Proceedings No. CP-17, 1966.

7-92 P.D. Chappe11: "Flow separations and stall characteristics of plane, constant-section wings in subcritical flow". J. of the Royal Aeron. Soc., Vol. 72, 1968, pp. 82-90.

7-93 D. Isaacs: "Wind tunnel measurements of the low speed stalling characteristics of a model of the Hawker-Siddeley Trident 1C". ARC R and M Report No. 3608, May 1968.

7-94 B. van den Berg: "Reynolds number and Mach number effects on the maximum lift and the stalling characteristics of wings at low speeds". National Aerospace Laboratory NLR TR 69025 U.

7-95 G.J. Hancock: "Problems of aircraft behaviour at high angles of attack". AGARDograph $136,1969$.

7-96 M.A. McVeigh and E. Kisielowski: "A design summary of stall characteristics of straight wing aircraft". NACA CR-1646, June 1971.

7-97 D.N. Foster: "The low-speed stalling of wings with high-1ift devices". AGARD Conference Proceedings CP-102 (Paper 11), April 1972.

7-98 J.K. Wimpress: "Predicting the low speed stall characteristics of the Boeing 747". AGARD Conference Proceedings CP-102 (Paper No. 21), Nov. 1972.

7-99 T. Schuringa: "Aerodynamics of the wing stall of the Fokker F-28". AGARD Conference Proceedings CP-102 (Paper 20), Nov. 1972.

7-100 W.D. Horsfield and G.P. Wilson: "Flight development of the stalling characteristics of a military trainer aircraft". AGARD Conference Proceedings CP-102, Nov. 1972.

7-101 V.E. Lockwood: "Effect of Reynolds number and engine nacelles on the stalling characteristics of a twin-engine light airplane". NASA TN D-7109, Dec. 1972.

7-102 н. Griem, J. Barche, н.J. Beisenherz and G. Krenz: "Some low-speed aspects of the twin-engine short haul transport VFW 614". AGARD CP No. 160 (Paper 11).

7-103 C.R. Taylor: "Aircraft stalling and buffeting - introduction and overview". AGARD Lecture Series No. LS-74, March 1975.

7-104 W. McIntosh and J.K. Wimpress: "Prediction and analysis of the low speed stall characteristics of the Boeing 747". AGARD Lecuture Series No. LS-74 (Paper 3), March 1975. 
7-105 M.A. Garbe11: "Wing flaps in 1ight aircraft design". J. of the Aeron. Sciences, Jan. 1945, pp. 1420 .

7-106 н.о. Palme: "Summary of wind tunnel data for high-lift devices on swept wings". SAAB T. Note 16 , April 1953.

7-107 J.F. Cahill: "Summary of section data on trailing edge high-lift devices". NACA TR 938, 1949.

7-108 R. Duddy: "High lift devices and their uses". J. Roy. Aero. Soc., Oct. 1949, Vol. 53, pp. 859-900.

7-109 A.D. Young: "The aerodynamic characteristics of flaps". ARC R and M 2622, 1953.

7-110 A.D. Young: "Flaps for landing and take-off". Chapter 14 of: "The principles of the control and stability of aircraft" edited by w.J. Duncan. Cambridge Aeronautical Series.

7-111 G.H. Lee: "High Maximum Lift". The Aeroplane, Oct. 30, 1953.

7-112 T.R.F. Nonweiler: "Flaps, slots and other high-lift aids". Aircraft Engineering, Sept. 1955.

7-113 K.L. Sanders: "High-1ift devices, a weight and performance trade-off methodology". SAWE Technical Paper No. 761, May 1963 .

7-114 S.T. Harvey and D.A. Norton: "Development of the Model 727 Airplane high lift system". Society of Automotive Engineers S 408, April 1964.

7-115 Anon.: "High lift devices for short field performance". Interavia No. 4/1964, pp. 569-572.

7-116 J.K. Wimpress: "Shortening the take-off and landing distances of high speed aircraft". AGARD Report 501, June 1965.

7-117 R.D. Schaufele and A.W. Ebeling: "Aerodynamic design of the DC-9 wing and high-1ift system". SAE Paper No. 670846 .

7-118 J.H. Paterson: "Aerodynamic design features of the C-5A". Aircraft Engineering, June $1968, p p .8-15$. 7-119 J.C. Wimpenny: "The design and application of high-lift devices". Annals of the New York Academy of Sciences, Vol. 154, Art. 2, pp. 329-366.

7-120 J.K. Wimpress: "Aerodynamic technology applied to takeoff and landing". Annals of the New York Academy of Sciences, Vol. 154, Art. 2, pp. 962-981.

7-121 A.D. Hammond: "High-1ift aerodynamics". Proceedings of Conference on vehicle technology for civil aviation, NASA SP-292, 1971, pp. 15-26.

7-122 M.A. McVeigh and E. Kisielowski: "A design summary of stall characteristics of straight wing aircraft". NACA CR-1646, June 1971 .

7-123 J.A. Thain: "Reynolds number effects at low speeds on the maximum lift of two-dimensional aerofoilsections equipped with mechanical high lift devices". Nat. Res. Council of Can. Quart. Bull. of the Div. of Mech. Eng. and the N.A.E., 30 Sept. 1973, pp. 1-24.

7-124 R.J. Margason and B.L. Morgan Jr.: "High-1ift aerodynamics - trends, trades and options". AGARD Conference on Takeoff and Landing, CP No. 160, April 1974.

7-125 A.M.O. Smith: "High-11ft aerodynamics". 37th Wright Brothers lecture, AIAA Paper No. 74-939.

Buffeting and high-speed stalling

7-126 C.J. Wood: "Transonic buffeting on aerofoils". J. of the Royal Aeron. Soc., Vol. 64 No. 599 , Nov. 1960, pp. 683-686.

7-127 W.R. Burris and D.E. Butchins: "Effect of wing leading edge geometry on maneuvering boundaries and stall departure". AIAA Paper No. 70-904.

7-128 E.J. Ray and L.W. McKinney: "Maneuver and buffet characteristics of fighter aircraft". AGARD Conference Proceedings CP-102, Nov. 1972 (Paper 24).

7-129 G.F. Moss, A.B. Haines and R. Jordan: "The effect of leading edge geometry on high speed stalling". AGARD Conference Proceedings CP-102, Nov. 1972.

7-130 н.H.B.M. Thomas: "On problems of flight over an extended angle of attack range". The Aero. Journal, Vo1. 77, Aug. 1973, pp. 412-423. 
7-131 D.G. Mabey: "Beyond the buffet boundary". The Aero. Journal, Apri1 1973, pp. $201-215$.

7-132 н. John: "Critical review of methods to predict the buffet penetration capability of aircraft". AGARD Lecture Series No. 74, March 1975.

\section{Wing structure}

7-133 S.J. Pipitone: "Modern wing structures". Aircraft Production, Jan. 1950, pp. 27-32.

7-134 в.н. Gardner: "Structural problems of advanced aircraft". Journal of the Royal Aeron. Soc., April 1952, pp. 221-250.

7-135 E.D. Keen: "Integral construction". J. of the Royal Aeron. Soc., Apri1 1953, pp. $215-227$.

7-136 0. Ljungström: "Wing structures of future aircraft". Aircraft Eng., May 1953, pp. 128-132.

7-137 G.H. Lee: "The aerodynamic and aeroelastic characteristics of the crescent wing". J. of the Royal Aeron. Soc., Vol. 59 No. 529, Jan. 1955, pp. 37-44.

7-138 E.G. Broadbent: "Aeroelastic problems in connection with high-speed flight". J. of the Royal Aeron. Soc., Vol. 60 , July 1956 , pp. 459-475.

7-139 A.F. Newe11: "Impressions of the structural design of American civil aircraft". The Aeroplane, August 15, 1958, pp. 229-231.

7-140 D.M. McElhinney: "Structural design of the VC-10". Aircraft Eng., June 1962, pp. 165-171, 178.

7-141 K. Bentley: "BAC One-eleven structural design". Aircraft Eng., May 1963, pp. $142-147$.

7-142 Anon.: "Wing design-civil transport aircraft". Cranfield Institute of Technology, Sheet DES 430 (unpublished).

7-143 Anon.: "Potential structural materials and design concepts for 1ight aircraft". NASA CR-1285, March 1969 .

7-144 W.W. Williams: "An advanced extensible wing flap system for modern aeroplanes". AIAA Paper No. 70911.

\section{CHAPTER 8.}

Weight subdivision and limitations

8-1 United States Department of Defence: "Weight and balance data reporting forms for aircraft". Military Standard, MIL-STD-254 (ASG), Aug. 26, 1954.

8-2 Direction Technique et Industrielle de 1'Aéronautique: "Devis de poids (avions)". AIR-2001/C, Edition No. 4, Dec. 15, 1959.

8-3 Society of British Aircraft Constructors: "Standard method for the estimation of Direct Operating Costs of aircraft". Dec. 1959.

8-4 Bundesminister für Verteidigung: "Normstelle der Luftfahrt". No. LN 9020, Nov. 1962.

8-5 J.R. McCarthy: "Definition and equipment list and standard form for presentation of weight and balance data". SAWE Technical Paper No. 354, 1962.

8-6 (British) Ministry of Aviation: "Weight, geometric and design data". AVMIN Form 2492, 1964.

8-7 "Glossary of standard weight terminology for commercial aircraft". Society of Aeronautical Weight Engineers, Revision 1964.

8-8 "Proposed Glossary of Standard Weights Terminology". International Air Traffic Association, Specification No. 100 .

8-9 C. Payton Autry, P.J. Baumgaertner: "The design importance of airplane mile costs versus seat mile costs". SAE Paper No. 660277.

8-10 "Standard method of estimating comparative Direct Operating Costs of turbine-powered transport airplanes". Air Transport Association of America, Dec. 1967.

8-11 "Recommended standard data format of transport airplane characteristics for aircraft planning". National Aerospace Standard NAS 3601", 1968/1970. 
General weight considerations and prediction methods

8-12 N.S. Currey: "Structure weight". Interavia, Vo1. 4, pp. 89-92, Feb. 1949.

8-13 F. Grinsted: "Aircraft structural weight and design efficiency". Aircraft Eng., July 1949, pp. 214217.

8-14 L.W. Rosenthal: "The weight aspect in aircraft design". Journal of the Royal Aeron. Soc., Vol. 54, March 1950, pp. 187-210.

8-15 E. Weining: "Design factors in the development of light aircraft". Aeron. Eng. Review, July 1950, pp. 18-19.

8-16 A. Schritt, W. Buckley: "A realistic approach to structural weight estimation". SAWE Paper No. 73 , May 1952.

8-17 J. Taylor: "Structure weight". J. of the Royal Aero. Soc., Vol. 57, pp. 646-652, Oct. 1953.

8-18 K. Thalau: "Geschwindigkeit, Konstruktionsgewicht und Nutzlast moderner Verkehrsflugzeuge". Jahrbuch der WGLR, 1953, pp. 110-123.

8-19 F.C. Hopton-Jones: "A practical approach to the problem of structural weight estimation for preliminary design". SAWE Paper No. 127, May 1955.

8-20 R.M. Simonds: "A generalized graphical nethod of minimum gross weight estimation". SAwE Paper No. 135, May 1956.

8-21 M.G. Heal: "Problems in estimating structure weight". Aeron. Eng. Review, March 1957, pp. 52-56.

8-22 W.E. Caddell: "The development of generalized weight estimating methods". SAWE Paper No. 219, May 1959.

8-23 Anon.: "An introduction into aeronautical weight engineering". SAWE, 1959.

8-24 C.R. Liebermann: "The unity equation and growth factor". SAWE Paper No. 267, May 1960.

8-25 M.E. Burt: "Effects of design speed and normal acceleration on aircraft structure weight", ARC CP 490, 1960.

8-26 в. Hertel: "Grundlagenforschung für Entwurf und Konstruktion von Flugzeugen". Arbeitsgemeinschaft für Forschung, Nordrhein-Westfalen, Vo1. 102, 1961.

8-27 M.E. Burt: "Structural weight estimation for novel configurations". J. of the Royal Aeron. Soc., Vol. 66, Jan. 1962, pp. 15-30.

8-28 E.E. Sechler and L.G. Dunn: "Airplane structural analysis and design". Dover Publications New York, 1963.

8-29 J.J. Pugliese: "Gross weight estimation of an attack airplane by generalized graphical solution". SAWE Paper No. $364,1963$.

8-30 R.J. Atkinson: "Structural design". J. of the Royal Aero. Soc., Vol. 67, pp. 692-695, Nov. 1963 (also: RAE TN No. Structures 333).

8-31 A.C. Kermode: "The aeroplane structure". Second Edition, Pitman and Sons, London, 1964.

8-32 w.J. Strickler: "Application of regression and correlation techniques in mass properties engineering". SAWE Paper No. 422, May 1964.

8-33 A.C. Kermode: "The aeroplane structure", Chapter 6: "Weight". Pitman and Sons Ltd., London, Second Edition, 1964.

8-34 R. Riccius: "Untersuchungen uber die Gewichte vertikalstartender Flugzeuge'. ILTUB Jahrbuch 1965/ 1966.

8-35 J.A. Neilson: "Value of a pound". SAWE Paper No. 586, May 1966.

8-36 M.A. Kochegura: "Determination of the weight of an empty aircraft by methods of mathematical statistics". USAF Foreign Technology Division FTD-MT-24-224-68, Aug. 1968.

8-37 H.L. Roland: "General approach to preliminary design weight analysis and structural weight prediction". Short course in modern theory and practice of weight optimization and control for advanced aeronautical systems, University of Tennessee, Nov. 1968.

8-38 พ.н. Ahl: "Rational weight estimation based on statistical data". SAWE Paper No. 791, May 1969.

8-39 W.E. Caddell: "On the use of aircraft density in preliminary design". SAWE Paper No. 813, May 1969. 
8-40 R.S. St. John: "The derivation of analytical-statistical weight prediction techniques". SAWE Paper No. 810, May 1969.

8-41 R.N. Staton: "Constrained regression analysis - A new approach to statistical equation development". SAWE Paper No. 762, May 1969.

8-42 C. Vivier and P. Cormier: "Masse d'un avion". AGARD Lecture Series No. 56 on "Aircraft performance prediction and optimization". April 1972.

8-43 D.P. Marsh: "Post-design analysis for structural weight estimation". SAWE Paper No. 936, May 1972.

8-44 w. Schneider: "Die Entwicklung und Bewertung von Gewichtsabschătzungsformeln für Flugzeugentwürfe unter Zuhilfnahme von Methoden der Mathematischen Statistik und Warscheinlichkeitsrechnung". Dissertation, Technical University of Berlin, Feb. 1973.

8-45 A.A. Blythe: The hub of the wheel - A project designer's view of weight". SAwE Paper No. 996, June 1973.

8-46 w. Schneider: "Project weight prediction based on advanced statistical methods". Paper presented at the 43rd AGAPD Flight Mechanics Panel Meeting, Symposium on "Aircraft design integration and optimization". Florence, oct. 1-4, 1973.

\section{Complete weight prediction methods}

8-47 I.H. Driggs: "Aircraft design analysis". J. of the Royal Aero. Soc., Vol. 54, Feb. 1950, pp. 65-116. 8-48 M. Vautier and M. Dieudonné: "Le probleme des poíds dans 1'aviation" (2 Parts), Service de Documentation et d'Information Technique de 1'Aéronautique, 1950.

8-49 0. Kơhler: "Gewichtsunterlagen für den Flugzeugentwurf". Luftfahrttechnik, Dec. 1955, pp. 134-139 and Jan. 1956, pp. 15-18.

8-50 D. Howe: "Initial aircraft weight prediction". College of Aeronautics Note 77, 1957.

8-51 F.K. Teichman: "Airplane design manual". Chapter 8: "Preliminary weight estimate". Pitman Publishing Co., New York, fourth edition, 1958.

8-52 H.G. Sheridan: "Aircraft preliminary design methods used in the weapon systems analysis divisior". US Navy BUWEPS Report No. R-5-62-13, June 1962.

8-53 E. Sechler and L.G. Dunn: "Airplane structural analysis and design", Chapter 1: "The airplane layout" Dover Publications Inc., New York, Jan. 1963.

8-54 พ.н. Marr: "Basic weight trends for bomber and transport aircraft". SAWE Paper No. 434, May 1964.

8-55 K.D. Wood: "Aircraft Design". Vol. 1 of "Aerospace vehicle design", second edition, 1966, Johnson Publishing $\mathrm{Cy}$.

8-56 H.L. Roland: "Parametric weight-sizing methods - structure, propulsion, fixed equipment - fighters (USAF \& USN)". June 30, 1965, General Dynamics Fort Worth Division, MR-S5-040, Revision, Sept. 30, 1966.

8-57 G. Corning: "Subsonic and supersonic aircraft design". Pp. 2:27 to 2:35: "Weight estimation". College Park, Maryland, second edition, 1966.

8-58 w. Richter et al: "Luftfahrzeuge". Das Fachwissen des Ingenieurs. Carl Hanser Verlag, MOnchen, 1970.

8-59 D. Howe: "Structural weight prediction". Cranfield Institute of Technology DES903, 1971 (unpublished).

8-60 D. Howe: "Empty weight and cruise performance of very large subsonic jet transports". Cranfield Institute of Technology, Report Aero No. 3, 1972.

8-61 R.N. Staton: "Weight estimation methods". SAWE Journal, April-May 1972, pp. 7-11.

8-62 H.F. Kooy and H. Rekersdrees: "Weight estimation method for subsonic transport aircraft". Fokker Report H-0-15, June 1972 (unpublished).

8-63 L.M. Nicolai: "Design of airlift vehicles". USAF Academy, Dept. of Aeronautics, Aero $464,1972$.

General wing structure weight considerations

8-64 R.J. Lutz: "Applications of optimum design principles to structural weight estimation". SAWE Paper 
No. 205, April 1951 .

8-65 D.J. Farrar: "The design of compression structures for minimum weight". J. of the Royal Aero. Soc., Nov. 1949, pp. 1041-1052.

8-66 F. Shanley: "Weight-strength analysis of aircraft structures", McGraw-Hill Book Cy. Inc., New York, 1952.

8-67 o. Ljungstrōm: "Wing structures of future aircraft". Aircraft Eng., May 1953, pp. 128-132.

8-68 A.L. Kolom: "Optimum design considerations for aircraft wing structures". Aero. Eng. Review, Cct. 1953, pp. 31-41.

8-69 C.R. McWorther: "Considerations of bending and torsional stiffness in the design of wings for minimum weight". SAWE Paper No. 84, 1953.

8-70 L.D. Green and J. Mudar: "Estimating structural box weight", Aeron. Eng. Review, Feb, 1958, pp. 4851.

8-71 S. Sichveland, F.M. de Graan and R.H. Trelease: "The weight engineer's approach to the problem of fatigue in aircraft structures". SAWE Paper No. 172, 1958.

8-72 N.N. Fadeev: "A theoretical formula for the weight of a tapered wing". In: "Methods of selection and approximate calculation of aircraft design parameters". Trudy Institute No. 138, Moscow 1961, pp. $28-52$.

8-73 R.J. Taylor: "Weight prediction techniques and trends for composite material structure". SAWE Paper No. 887 .

8-74 B. Sealman: "Multitapered wings". J. of Aircraft, July-Aug. 1965, pp. 348-349.

8-75 B. Sealman: "Effect of wing geometry on volume and weight". J. of Aircraft, Vol. 1 No. 5, Sept.-Oct. 1964 , p. 305.

8-76 D.H. Emero and L. Spunt: "Wing box optimization under combined shear and bending". J. of Aircraft, Vol. 3, No. 2, March-April 1966, pp. 130-141.

8-77 C.A. Garrocq and J.T. Jackson: "Estimation of wing box weights required to preclude aeroelastic instabilities". SAWE Paper No. 500, May 1966.

8-78 K.L. Sanders: "A review and summary of wing torsional stiffness criteria for predesign and weight estimations". SAWE Paper No. 632, May 1967.

8-79 D.J. Lamorte: "Non-optimum factor and preliminary weight estimation of a boron composite wing structure". SAWE Paper No. 891, May 1971.

Weight prediction of wing and tailplane structure

8-80 w. Tye and P.E. Montangnon: "The estimation of wing structure weight". ARC R and M 2080, 1941.

8-81 F. Grinsted: "Simple formulae for predicting the weights of wing, fuselage and tail unit structures". RAE Report Structures No. 24, 1948.

8-82 F. Grinsted: "Prediction of wing structure weight". RAE Report Structures No. 15, 1948.

8-83 G.K. Gates: "Weight estimation of metal wings". Aircraft Eng.,April 1949, p. 116.

8-84 J.F. Carreyette: "Aircraft wing weight estimation". Aircraft Eng., Jan. 1950, pp. 8-11 and April 1950 , p. 119.

8-85 E.L. Ripley: "A simple method of tail unit structure weight estimation". RAE Report Structures No. 94, Nov. 1950.

8-86 J. Kelley Jr.: "Wing weight estimation". AAI Technical Report 5161.

8-87 I.H. Driggs: "Aircraft design analysis". J. of the Royal Aero. Soc., Vol. 54, Feb. 1950.

8-88 J. Solvey: "The estimation of wing weight". Aircraft Eng., May 1951, pp. 143-144.

8-89 E.L. Ripley: "A method of wing weight prediction". RAE Report structures No. 109, May 1951.

8-90 M.E. Burt: "Weight prediction of ailerons and landing flaps". RAE Report Structures No. 116, Sept. 1951.

8-91 A. Hyatt: "A method for estimating wing weight's. J. of the Aero. Sciences, Vo1. 21 No. 6, June 1954, pp. 363-372. 
8-92 M.E. Burt: "Weight prediction for wings of box construction". RAE Report Structures No. $186,1955$.

8-93 w. v. Nes and O. Köhler: "Das Gewichtsanteil der tragenden Teile am Flügelgewicht". Luftfahrttechnik, Nov. 1056, pp. 206-210.

8-94 K.L. Sanders: "Abschătzung des Flügelgewichtes". Luftfahrttechnik, Oct. 1957, p. 224.

8-95 D. Howe: "Initial aircraft weight prediction". College of Aeronautics Note No. 77, 1957.

8-96 M. Schwartzberg: "Blown flap system for STOL performance - weight considerations". Aerospace Eng., March 1959, pp. 48-52.

8-97 C.R. Ritter: "Rib weight estimation by structural analysis". SAWE Paper No. 259, 1960.

8-98 K.L. Sanders: "High-lift devices; a weight and performance trade-off technology". SAWE Paper No. 761, May 1969.

8-99 w.w. Williams: "An advanced extensible wing flap system for modern aeroplanes". AIAA Paper No. 70911, July 1970.

8-100 R.L. Gielow: "Performance prediction and evaluation of propulsion-augmented high lift systems". AIAA Paper No. 71-990, Oct. 1971 .

8-101 E. Torenbeek: "Prediction of wing group weight for preliminary design". Aircraft Eng., July 1971, pp. 16-21. Summary in Aircraft Eng., Feb. 1972, pp. 18-19.

8-102 F.o. Smetana: "A design study for a simple to fly, constant attitude 1ight aircraft. NASA CR-2208, March 1973.

Wings for high-speed aircraft

8-103 R.E. Lowry: "Problems and solutions of delta wings". SAWE Paper No. 77, 1952.

8-104 W.J. Conway: "Factors affecting the design of thin wings". SAE Preprint No. 357, Oct. 1954.

8-105 R.L. Hammitt: "Structural weight estimation by the weight penalty concept for preliminary design". SAWE Paper No. 141, 1956.

8-106 M.G. Heal: "Structural weights on supersonic aircraft with low aspect ratio unswept wings". SAWE Paper No. 193, 1956.

8-107 A.C. Robinson: "Problems associated with weight estimation and optimization of supersonic aircraft". SAWE Paper No. 234, 1959.

8-108 R.A. Anderson: "Weight-efficiency analysis of thin-wing construction". Transactions of the ASME, Vol. 79, July 1957 (II), pp. 974-979.

8-109 D.J. Johns: "Optimum design of a multicell box to a given bending moment and temperature distribution". College of Aeronautics Note No. 82, April 1958.

8-110 M.E. Burt: "Structural weight estimation for novel configurations". J. of the Royal Aero. Soc., Vol. 66, Jan. 1962, pp. 15-30.

Fuselage structure

8-111 L.w. Rosenthal: "The influence of aircraft gross weight upon the size and weight of hulls and fuselages". J. of the Royal Aero. Soc., Vol. 51, Nov. 1947, pp. 874-883.

8-112 F. Grinsted: "Simple formulae for predicting the weights of wing, fuselage and tail unit structures". RAE Report Structures No. 24, May 1948.

8-113 E.L. Ripley: "A method of fuselage structure weight prediction". RAE Report Structures No. 93, 1950. 8-114 W.R. Micks: "Structural weight analysis. Fuselage and shell structures". The Rand Corporation, Report No. R-172. 1950.

8-115 M.E. Burt and J. Philips: "Prediction of fuselage and hull structure weight". RAE Report Structures No. 122, April 1952 .

8-116 L.D. Green: "Fuselage weight prediction". SAWE Paper No. 126, May 1955.

8-117 R.L. Hammitt: "Structural weight estimation by the weight penalty concept for preliminary design". SAWE Paper No. 141, May 1956. 
8-118 E.W. Tobin Jr.: "A method for estimating optimum fuselage structural weight". SAWE Paper No. 152, May 1957.

8-119 A.A. Badiagin: "Concerning an efficient slenderness ratio for the fuselage of civilian aircraft". In: "Methods of selection and approximate calculation of air design parameters". Trudy Institute No.

138, Moscow, 1961, pp. 19-27.

8-120 J. Morris and D.M. Ashford: "Fuselage configuration studies". SAE Paper No. 670370, April 1967.

8-121 A.R. Di Pierro: "Minimum weight analysis of fuselage frames". SAWE Paper No. 826, May 1970.

8-122 D.E. Poggio: "Theoretical and real weight of she11 fuselages". Ingegneria, Jan. 1971, pp. 1-12.

8-123 D.M. Simpson: "Fuselage structure weight prediction". SAWE Paper No. 981, June 1973.

\section{Alighting gear}

8-124 J. Philips: "A method of undercarriage weight estimation". RAE Report structures No. 198, March 1956.

8-125: C.R. Liebermann: "Rolling type alighting gear weight estimation". SAwE Paper No. 210 , May. 1959.

8-126: M.E. Burt and E.L. Ripley: "Prediction of undercarriage weights". RAE Report Structures No. 80, June 1950 .

8-127 P.R. Kraus: "An analytical approach to landing gear weight estimation". SAWE Paper No. 829, May 1970.

The powerplant

8-129 G.R. Holzmeier: "A rational method for estimating fuel system weight in preliminary design". SAWE Paper No. $147,1957$.

8-130 w.C. Crooker: "Aircraft fuel system weight estimation for the tri-sonic era". SAWE Paper No. 232, May 1959.

8-131 G. Rosen: "New problem areas in aircraft propeller design". Canadian Aero. Journal, Vol. 6, June 1960 , p. 219.

8-132 Anon.: "Hamilton Standard propeller and gear box weight generalization". Figs. 1, 2 and 3 of Publication PDB 6101, 1963.

8-133 R.C. Engle: "Jet engine weight and thrust trends including future development promises by the engine manufacturers". SAWE Paper No. 682, May 1968.

8-128 I.H. Driggs and O.E. Lancaster: "Engine weights". In: "Gasturbines for aircraft". Section 8.9, 1955, Ronald Press, New York.

8-134 M.L. Yaffee: "propeller research gains emphasis". Aviation Week and Space Technology, Nov. 1969, pp. 56-65.

8-135 R.P. Gerend and J.P. Roundhill: "Correlation of gasturbine engine weights and dimensions". AIAA Paper No. 70-669.

8-136 J.F. Dugan Jr.: "Bngine selection for transport and combat aircraft". NASA TMX-68009, April 1972. (Also in AGARD Lecture Series 49).

Airframe services and equipment, operational items, payload

8-137 C.K. McBaine: "Weight estimation of aircraft hydraulic systems". SAWE Paper No. 128, 1955.

8-138 J.R. McCarty: "A review and revised approach to the average passenger weight". SAWE Paper No. 22,3, May 1959.

8-139 C.A. Hangoe: "Comparison of passenger service equipment". SAWE Paper No. 220, May 1959.

8-140 G.R. Williams: "Optimization of fluid lines". SAWE Paper. No. 291, May 1961.

8-141 Anon.: "The use of standard baggage weights". European Civil Aviation Conference, Strassbourg, July 1961, Vol. II, Section 2, Doc. 8185, ECAC/4-2, pp. 355-356. 
8-142 R.G. Mitchel1: "Evaluation of economics of passenger comfort standards". SAWE Paper No. 338, May 1962.

8-143 C.A. Hangoe: "World-wide survey of cargo densities". SAWE Paper No. 339, May 1962.

8-144 J.R. McCarty: "Airline new aircraft evaluations". SAWE Paper No. 619, May 1967.

8-145 J. Morris and D.M. Ashford: "The use of standard baggage weights". SAWE Preprint.

8-146 R.J. Taylor and K. Smith: "Advanced aircraft parametric weight analysis". SAWE Paper No. 637, May 1967.

8-147 B.H. Nicholls and A.D. Meshew: "Auxiliary power systems for 1975 fighter aircraft". SAE Paper No. 680311, April-May 1968.

8-148 T.P. Clemmons: "Systems design for weight optimization". SAWE Paper No. 757, May 1969.

8-149 н.L. Roland: "Advanced design weight analysis and systems and equipment weight prediction". SAWE Paper No. 790, May 1969.

8-150 D.M. Cate: "A parametric approach to estimate weights of surface control systems of combat and transport aircraft". SAWE Paper No. 812, May 1969.

8-151 P.A. Ward and W.G. Lydiard: "Aircraft auxiliary power system and their influence on power plant design". Lecture presented at Symposium of the Royal Aero. Soc., London, 1969.

8-152 R.S. Kaneshiro: "Weight estimation of hydraulic secondary power system". SAWE Paper No. 935, May 1972 .

Some recent publications on weight prediction

8-153 J. Banks: "Preliminary weight estimation of canard configured aircraft". SAWE Paper No. 1015 , May 1974.

8-154 A. Krzyzanowski: "A method for weight/cost trade-offs in preliminary vehicle design". SAWE Paper No. 1017, May 1974.

8-155 R.N. Staton: "Fuselage basic shell weight prediction". SAWE Paper No. 1019 , May 1974.

8-156 w. Schneider: "A procedure for calculating the weight of wing structures with increased service 1ife". SAWE Paper No. 1021, May 1974 (summary in SAWE Journal), Vol. 34 No. 1, Jan. 1975, pp. 1-12 and $40-41$.

8-157 J.L. Anderson: "A parametric analysis of transport aircraft system weights and costs". SAWE Paper No. 1024, May 1974 .

8-158 C.R. Glatt: "WAATS - a computer program for weights analysis of advanced transportation systems" NASA CR-2420, Sept. 1974.

8-159 B. Saelman: "Methods for better prediction of gross weight". SAWE Paper No. 1041, May 1975.

8-160 R. St. John: "Weight effects of structural material variation". SAWE Paper No. 1044, May 1975.

Balance and loadability

8-161 K.L. Sanders: "Simpler wing location for a specified longitudinal stability". Space/Aeronautics, March 1960, page 67-70.

8-162 J.R. McCarty: "Passenger seating pattern. A statistically based cabin load or passenger seating assumption applicable to airline operation". SAWE Paper No. 250, May 1960.

8-163 G.H. Hopper: "The influence of balance and loadability on the design of commercial passenger transports". SAWE Paper No. 269, May 1960.

8-164 D.J. Lambert: "Design of jet transport with rear-mounted engines". Aerospace Engineering, Oct. 1960, page 30-35, 72, 74 .

8-165 G.W. Benedict: "Methods of evaluating aircraft loadability". SAWE Paper No. 334, May 1962.

8-166 K.L. Sanders and D.O. Nevinger: "Balancing options in aircraft configuration design". SAWE Paper No. 840, May 1970.

8-167 н. Waldon: "Theoretical vs. actual seating patterns variations in wide body airvraft". SAWE Paper 
No. 1080, May 1975 .

8-168 R. Maswell: "A loadability comparison: L1011/DC-10-10". SAWE Paper No. 1094, May 1975.

\section{CHAPTER 9.}

General aspects of airplanes stability and control

9-1 C.D. Perkins and R.E. Hage: "Airplane performance, stability and control", New York, John wiley and Sons, Inc., 1949.

9-2 C.F. Joy: "Power controls for aircraft". J. of the Royal Aero. Soc., Jan. 1952, pp. 7-24.

9-3 C.S. Draper: "Flight control". J. of the Royal Aero. Soc., Vol. 59, July 1955, pp.451-478.

9-4 A.W. Babister: "Aircraft stability and control". International Series of Monographs in Aeronautics and Astronautics, Pergamon Press, 1961.

9-5 О.H. Gerlach: "Vliegeigenschappen I". Lecture notes VTH-D10, Delft University of Technology, Dept. of Aeron. Eng., Dec. 1967 (in Dutch).

9-6 S.B. Dickinson: "Aircraft stability and control for pilots and engineers". Pitman and Sons Ltd., 1968.

9-7 R.B. Holloway, P.M. Burris and R.P. Johannes: "Aircraft performance benefits from modern control systems technology". J. of Aircraft, Vol. 7 No. 6, Nov.-Dec. 1970, pp. 550-553.

9-8 F. O'Hara: "Stability augmentation in aircraft design". The Aeron. Journal of the Royal Aeron. Soc., Vol. 75 No. 724, April 1971, pp. 293-304.

9-9 D.P. Davies: "Handling the big jets". Air Registration Board, third edition, Dec. 1971.

9-10 B. Etkin: "Dynamics of atmospheric flight". J. Wiley Publ. Corp., New York, 1972.

9-11 F.O. Smetana, D.C. Summey and w.D. Johnson: "Riding and handling qualities of light aircraft - a review and analysis". NASA CR-1975, March 1972.

9-12 J. Roskam: "Flight dynamics of rigid and elastic airplanes". University of Kansas, 1972.

Flying qualities criteria

9-13 Anon.: "Design objections for flying qualities of civil transport aircraft", SAE ARP 842 B.

9-14 C. Leyman and E.R. Nuttall: "A survey of aircraft handling criteria". ARC Current Paper No. 833.

9-15 H.A. Shomber and w.M. Gertsen: "Longitudinal handling qualities criteria: an evaluation". AIAA Paper No. $65-780$.

9-16 F. O'Hara: "Handling criteria". J. of the Royal Aeron. Soc., Vol. 71, April 1967, pp. $271-291$.

9-17 E.E. Larrabee and J.P. Tymczyszyn: "The effect of flying qualities requirements on the design of general aviation aircraft in the 1980's". AIAA Paper No. 68-189.

9-18 Anon.: "Flying qualities of piloted airplanes". MIL-F-8785 B (ASG), August 1969.

9-19 C.R. Chalk et.al.: "Background information and user guide for MIL-F-8786 B (ASG), Military specification - flying qualities of piloted airplanes". T. Report AFFDL-TR-69-72, August 1969.

9-20 Anon.: "Flying qualities of piloted V/STOL aircraft". MIL-F-83300, Dec. 1970.

Tailplane and control surface design

9-21 w.S. Brown: "Spring tab controls". ARC R and M 1979, 1941.

9-22 W.H. Philips: "Application of spring tabs to elevator controls". NACA TR 797, 1944.

9-23 M.B. Morgan and н.н.B.M. Thomas: "Control surface design". J. of the Royal Aeron. Soc., Vol. 49, 1945, pp. 431-510.

9-24 L.E. Root: "The effective use of aerodynamic balance on control surfaces". J. of the Aeron. Sciences, April 1945, pp. 149-163.

9-25 w.M. Phillips: "Appreciation and prediction of flying qualities". NACA TR 927, 1949. 
9-26 F.B. Baker: "Choice of fin area and dihedral". Aircraft Eng., March 1948, pp. 87-88.

9-27 O.R. Dunn: "Aerodynamically boosted surface controls and their application to the D.C. 6 transport". Second International Aeronautical Conference, New York, 1949.

9-28 D.E. Morris: "Designing to avoid dangerous behaviour of an aircraft due to the effects on control hinge moments of ice on the leading edge of the fixed surface". ARC Current Paper 66, 1952.

9-29 J.C. Wimpenny: "Stability and Control in Aircraft Design". J. of the Royal Aeron. Soc., May 1954, pp. 329-360.

9-30 B.R.A. Burns: "Design considerations for the satisfactory stability and control of military aircraft". AGARD :P-119 stability and Control.

9-31 W.D. Thompson: "Improvements in flying qualities of modern 1ight planes". SAE Paper No. 622D, Jan. 1963.

9-32 E. Obert: "Low-speed stability and control characteristics of transport aircraft with particular reference to tailplane design". AGARD CP-160 (Paper 10), April 1974.

Determination of aerodynamic characteristics

9-33 D.J. Lyons and P.L. Bisgood: "An analysis of the lift slope of aerofoils of small aspect ratio wings including fins, with design charts for aerofoils and control surfaces". ARC R and M 2308, 1945. Also: Aircraft Eng., Sept. 1947, pp. 278-286.

9-34 D.E. Morris and J.C. Morrall: "Effect of slipstream on longitudinal stability of multi-engined aircraft". ARC R and M 2701, Nov. 1948.

9-35 A. Silverstein and $\mathrm{S}$. Katzoff: "Design charts for predicting downwash angles and wake characteristics behind plain flaps an flapped wings". NACA TR 648 .

9-36 J. Weil and w.C. Sleeman Jr.: "Prediction of the effects of propeller operation on the static longitudinal stability of single-engine tractor monoplanes with flaps retracted". NACA TR 941.

9-37 S. Katzoff and H. Sweberg: "Ground effect on downwash angle and wake 10cation". NACA TR 738.

9-38 D.E. Hoak: "USAF Stability and Control Datcom". Wright Patterson Air Force Base. Oct. 1960 (Revision August 1968).

9-39 D.E. Ellison and D.E. Hoak: "Stability derivative estimation at subsonic speeds". Annals of the New York Academy of Sciences, Vol. 154, Part 2, pp. 367-396.

9-40 G.M. Moss: "Some aerodynamic aspects of rear mounted engines". J. of the Royal Aeron. Soc., Vol. 68, Dec. 1964, pp. 837-842.

9-41 Anon.: Data Sheets, Aerodynamics, Vols. I, II and III, Royal Aeronautical Society.

9-42 J. Roskam: "Methods of estimating stability and control derivatives of conventional subsonic airplanes". The University of Kansas, 1971.

9-43 С.н. Wolowicz: "Longitudinal aerodynamic characteristics of light, twin-engine, propeller driven airplanes". NASA TN D-6800, 1972.

9-44 C.H. Wolowicz and R.B. Yancey: "Lateral-directional aerodynamic characteristics of light, twinengine, propeller-driven airplanes". NASA TN D-6946, Oct. 1972.

9-45 R.K. Heffly and W.F. Jewell: "Aircraft handling qualities data". NASA CR-2144, Dec. 1972.

9-46 C.G.B. Mitchell: "A computer programme to predict the stability and control characteristics of subsonic aircraft". RAE Technical Report No. 73079, Sept. 1973.

9-47 M.I. Goldhammer and N.F. Wasson: "Methods for predicting the aerodynamic and stability and control characteristics of STOL aircraft". Technical Report AFFDL-TR-73-146, Dec. 1973.

9-48 F.O. Smetana, D.C. Summey, N.S. Smith and R.K. Carden: "Light aircraft 1ift, drag and pitching moment prediction - a review and analysis". NASA CR-2523, May 1975.

Engine failure considerations 
9-49 A.н. Yates: "Control in flight under asymmetric power". Aircraft Eng., Sept. 1947, pp. 287-290.

9-50 L.E. Wright: "Flight on asymmetric engine power". Aircraft Eng., Dec. 1950, pp. 350-355.

9-51 A. Hammer: "Die analytische und experimentelle Ermittlung der Mindestkontrollgeschwindigkeiten von Flugzeugen (minimum control speeds) unter Berūcksichtigung von Flugeigenschaftsforderungen". Dissertation Berlin, 1971.

9-52 E.J.N. Archbold and K.T. McKenzie: "Response in yaw". J. of the Royal Aeron. Soc., Vol. 50, 1946, pp. $275-285$.

9-53 M.E. Kirchner: "Turboprop airplane control problems associated with engine failure considerations". SAE Paper No. 613, 1955.

9-54 J. Mannée: "Windtunnelinvestigation of the influence of the aircraft configuration on the yawing and rolling moment of a twin-engined, propeller-driven aircraft with one engine inoperative". NLL Report A 1508 B, 1963.

\section{Airplane spinning}

9-55 A.I. Neihouse: "Tail-design requirements for satisfactory spin recovery for personal-owner-type 1ight airplanes". NACA TN-1329, June 1947.

9-56 L.J. Gale and I.P. Jones Jr.: "effects of antispin fillets and dorsal fins on the spin recovery characteristics of airplanes as determined from free-spinning-tunnel tests". NACA TN-1779, Dec. 1948.

9-57 J.S. Bowman: "Airplane spinning". NASA SP-83, May 1965.

9-58 A.I. Neihouse, I. Anshal, W.J. Klinar and S.H. Scher: "Status of spin research for recent airplane designs". NASA TR R-57, 1960.

\section{CHAPTER 10.}

10-1 B.G. Conway: "Landing gear design". Textbook Royal Aeronautical Society, Chapman \& Hall Ltd., London, 1958.

10-2 G. Bock: "Operations from unprepared and semiprepared airfields". Agardograph 45, Sept. 1960.

-10-3 Anon.: "ICAO Aerodrome Manual, Part 2". Doc. 7920-AN/865/2, Second edition, 1965.

10-4 A. Cameron-Johnson: "The undercarriage in aeroplane project design". Aircraft Eng., Feb. 1969, pp. 6-11.

10-5 R.C. Cussons: "Bogie undercarriages", J. Royal Aeron. Soc., July 1952.

10-6 J.A. Skinner: "Testing runway foundations and pavements". Airport Paper No. 17, Proc. of the Institution of Civil Engineers, 1951.

10-7 L.S. Bialkowski: "The basic problem of undercarriage geometry". Aircraft Eng., August 1953.

10-8 E.G. Collinson: "A note on the load transference on multi-wheel bogie undercarriages". J. of the Royal Aero. Soc., Oct. 1949.

10-9 Anon.: "Konstruktionsbeispiele aus dem Flugzeugbau". Band 4 Fahrwerk, Fachbücher für Luft- und Raumfahrt. Luftfahrtverlag Walter zuerl.

10-10 R. Hadekel: "The mechanical characteristics of pneumatic tyres". S \& T, Memo No. 10/52, Ministry of Supply, Nov, 1952 .

-10-11 R.O. Dickinson: "A fresh approach to aircraft landing gear design". ASME Paper No. 57-SA-30.

-10-12 W.E. Eldred: "Landing gear design as applied to modern aircraft". SAE Paper No. 146, Sept./Oct. 1953.

10-13 E. Schumacher: "Die Fahrwerke der heutigen Flugzeuge". Luftfahrttechnik, Nov./Dec. 1958, pp. 295306 and 320-338, Jan. 1959, pp. 22-30.

10-14 E. Overesch: "The problems of exact calculation of takeoff and landing characteristics of conventional transport aircraft ". AGARD Report 417, Jan. 1963.

10-15 V.K. Karrask: "The trim of an aircraft with a landing gear of the tricycle type in a crosswind". Trudy Institute, Moscow, 1961. 
10-16 R. Lucien: "Military requirements and undercarriage design". Interavia 6/1961, pp. 839-842.

10-17 G. Bruner: "L'attérrisseur à amortisseur horizontal "jockey" de la société Messier pour avions cargos". DOCAERO, No. 66, Jan. 1961, pp. 5-16.

10-18 E. Schumacher: "Die Fahrwerke der heutigen Flugzeuge". Luftfahrttechnik 5 (1959) No. 1, Jan 15, pp. 22-30, No. 4, March, pp. 89-92.

10-19 W.W. Williams, G.K. Williams and W.C.J. Garrard: "Soft and rough field landing gears". SAE Paper No. 650844 , Oct. 1965 .

10-20 K.S. Carter: "The landing gear of the Lockheed SST". SAE Paper No. 650224, April 1965.

10-21 Anon.: "Some current types of landing gear". Aircraft Engineering, Jan. 1968, pp. 26-31.

10-22 S.W.H. Wood: "Problems of undercarriage design for V/STOL aircraft". The Aeronautical Journal, Feb. 1969, pp. $157-168$.

10-23 R.A. Werner and G.F. Wislecinus: "Analysis of airplane design by similarity considerations". AIAAPaper No. 68-1017, Oct. 1968.

10-24 L.G. Hoare: "Aircraft landing gear". Aircraft Eng., Jan. 1968, pp. 6-8.

10-25 w.J.G. Pinsker: "The dynamics of aircraft rotation and liftoff and its implication for tail clearance requirements, especially with large aircraft". ARC R and M No. 3560, 1969.

10-26 J.F. O'Hara: "Aircraft crosswind performance". AGARD Report 492, Oct. 1964.

10-27 W.B. Horne, T.J. Yager and G.R. Taylor: "Recent research on ways to improve tire traction on water, slush or ice". Luchtvaarttechniek 5, Sept. 1966.

10-28 J.W.H. Thomas: "Design for runway conditions". J. of the Royal Aeron. Soc., Sept. 1965, pp. 571-576.

10-29 H.S.D. Yang: "C-5A main landing gear bogie pitch control". AIAA Paper No. $70-914$.

10-30 Anon.: Draft International Standard ISO/DIS 3324/1: "Aircraft tyres and rims". 1974/1975.

CHAPTER 11.

\section{Prediction of aerodynamic characteristics}

11-1 Anon.: "Report of the definitions panel on definitions to be used in the description and analysis of drag". ARC CP No. 369, 1957.

11-2 G. Gabrielli: "On the subdivision in different "forms" of the aircraft drag at the maximum speed". Troisième Congrès Aéronautique Européen, 1958, pp. 398-407 and 979-984.

11-3 H. Schlichting and E. Truckenbrodt: "Aerodynamik des Flugzeuges", Band 1 und 2 , Springer Verlag, Berlin, 1962 .

11-4 D.E. Hoak and D.E. Ellison: "USAF Stability and Control DATCOM". McDonnell Douglas Airplane Cy., Oct. 1960, Revised Aug. 1968.

11-5 Anon.: "Subsonic lift-dependent drag due to wing trailing vortices". R.Ae.S. Data Sheets, "Wings" $02.01 .02,1965$

11-6 B.W. McCormick: "Aerodynamics of V/STOL flight". Academic Press, New York, London, 1967.

11-7 J. Williams and A.J. Ross: "Some airframe aerodynamic problems at low speeds". Annals of the New York Academy of Sciences, Vo1. 154, Art. 2, Nov. 22, 1968, pp. 264-305.

11-8 D.A. Norton: "Airplane drag prediction". Annals of the New York Academy of Sciences, Vol. 154, Part 2. pp. 306-328, 1968.

11-9 J.C. Wimpenny: "The design and application of high lift devices". Annals of the New York Academy of Sciences, Vol. 154, Art. 2, Nov. 22, 1968, pp. 329-366.

11-10 D.E. Ellison and D.E. Hoak: "Stability derivative estimation at subsonic speeds". Annals of the New York Academy of Sciences, Vol. 154, Part 2, Nov. 22, 1968, pp. 367-396.

11-11 B.H. Little: "Scaling effects on shock-induced separations". AGARD-VKI Lecture Series No. 16, April $21-25,1969$.

11-12 G.J. Hancock: "Problems of aircraft behaviour at high angles of attack". AGARDograph 136 , 1969.

11-13 J.H. Patterson: "A survey of drag prediction techniques applicable to subsonic transport aircraft 
design". AGARD Conference on aerodynamic arag, CP-124, 1973.

11-14 S.F.J. Butler: "Aircraft drag prediction for project appraisal and performance estimation". AGARD Conference Proc. No. 124, April 1973.

11-15 J.G. Callaghan: "Aerodynamic prediction methods for aircraft at low speeds with mechanical high lift devices". AGARD Lecture Series No. 67, May 1974.

11-16 G.M. Bowes: "Aircraft lift and drag prediction and measurement". AGARD Lecture Series No. 67, May 1974.

11-17 J.K. Wimpress: "Predicting the low speed stall characteristics of the Boeing 747". AGARD CP-102 (Paper 21), April 1972 .

\section{Performance prediction - general literature}

11-18 C.D. Perkins and R.E. Hage: "Airplane performance, stability and control". John wiley, 1949.

11-19 J. Rotta: "Ueber die Flugleistungsmechanik des Flugzeuges mit Turbinenstrahltriebwerk". Zeitschrift für Flugwiss., Vol. 4, 1953.

11-20 Various contributors: "F1ight Test Manual". Vol. I, Performance, AGARD, 1954.

11-21 G.S. Schairer and M.L. Olason: "Some performance considerations of a jet transport airplane". First Turbine Powered Air Transportation Meeting, IAS, August 1954.

11-22 R. Ludwig: "Ein Beitrag zur dimensionslosen Methode der Leistungsberechnungen von Flugzeugen mit Strahlantrieb". Zeitschrift für Flugwissenschaften, vol. 6, 1955.

11-23 Anon.: Data Sheets "Performance". Vols. 1, 2 and 3. Royal Aeronautical Society and Engineering Sciences Data Unit.

11-24 A. Miele: "Flight mechanics". Vol. 1, Theory of flight paths, Pergamon Press, London, 1962.

11-25 D. Fiecke: "Stationăr betrachtete Flugleistungen". Luftfahrttechnik, October 1962, pp. $266-283$.

11-26 D.o. Dommasch, S.S. Sherby and T.F. Connally: "Airplane aerodynamics". Pitman Publishing Corp., New York, 4th Edition, 1967.

11-27 Boeing Cy.: "Jet transport performance methods". The Boeing Company, Commercial Airplane Group, Seattle, Washington, Boeing Document No. D6-1420, 6th Edition, May 1969.

11-28 R.L. Schultz and N.R. Zagalsky: "Aircraft performance optimization". J. of Aircraft, Vol. 9, Feb. 1972, pp. 108-114.

11-29 в. Wittenberg: "Prestatieleer van v1iegtuigen". Dictaat VTH-D14, Delft University of Technology, Dept. of Aeron. Eng., July 1971 (in Dutch).

11-30 R.F. Creasy: "Propulsion/aircraft matching experience". AGARD Lecture Series No. 65, May 1974.

11-31 F.o. Smetana, D.C. Summery and W.D. Johnson: "Point and path performance of 1ight aircraft". NASA CR-2272, June 1973.

11-32 W.J. Moran: "Performance methods for aircraft synthesis". SAWE Paper No. 909, May 1972.

Climb, cruise and descent performance

11-33 k.J. Lush: "Total energy methods". AGARD Flight Test Manual, Vol. I (Performance), 1954.

11-34 Anon.: "Estimation of cruise range and endurance". ESDU Data Sheets 73018 and 73019, Oct. 1973.

11-35 в. Friedel: "Flight-manoeuvre and climb-performance prediction". AGARD Lecture Series No. 56, April 1972.

11-36 R.K. Page: "Range and radius-of-action performance prediction for transport and combat aircraft". AGARD Lecture Series 56, April 1972.

11-37 J.J. Spillman: "Climb and descent techniques". Short course: "Aircraft performance estimation". Cranfield Institute of Technology, Feb, 1973.

11-38 J.J. Spillman: "Cruise characteristics". Short course: "Aircraft performance estimation". Cranfield Institute of Technology, Feb. 1973. 
11-39 F. Handley Page: "Towards slower and safer flying, improved take-off and landing and cheaper airports". J.R.Ae.s., Dec. 1950, pp. 721-739.

11-40 R.E. Gillman: "Performance standards in practice". The Aeroplane, May 13, 1955, pp. 634-638.

11-41 G.S. Alias: "Notes on the ground-run of jet-propelled aircraft during take-off and landing". AGARD Report 82, 1956.

11-42 G. Mathias: "On the optimum utilization of an airplane high-1ift device for minimum take-off run and climb distance". Zeitschrift für Flugwissenschaften, 1961, pp. 276-284.

11-43 E. Overesch: "The problems of exact calculation of take-off and landing characteristics of conventional transport aircraft". AGARD Report 417, 1963.

11-44 B.N. Tomlinson and M. Judd: "Some calculations of the take-off behaviour of a slender wing SST design constrained to follow a specific pitch-attitude time history". ARC R \& M 3493, RAE TR 65174, 1965.

11-45 J.K. Wimpress: "Shortening the take-off and landing for high-speed aircraft". AGARD Report 501 and Aircraft Engineering, June 1966, pp. 14-19.

11-46 w.J.G. Pinsker: "The dynamics of aircraft rotation and lift-off and its implication for tail clearance requirements, especially with large aircraft". ARC R \& M 3560, 1967.

11-47 D.P. Davies: "Handling the big jets". Second edition, Air Registration Board, 1968.

11-48 J.K. Wimpress: "Technology of take-off and landing". Annals of the New York Academy of Sciences, Vo1. 154, Part 2, Nov. 22, 1968, pp. 962-981.

11-49 T.G. Foxworth and H.F. Mathinsen: "Another look at accelerate-stop criteria". AIAA Paper No. 69-772.

11-50 D.H. Perry: "An analysis of some major factors involved in normal take-off performance". ARC CP No. $1034,1969$.

11-51 D.н. Perry: "Exchange rates between some design variables for an aircraft just satisfying take-off distance and climb requirements". RAE Technical Report 69197, 1969.

11-52 J. Williams: "Airfield performance prediction methods for transport and combat aircraft". AGARD Lecture Series No. 56, April 1972.

\section{Landing performance}

11-53 G.S. Alias: "Notes on the ground-run of jet-propelled aircraft during landing and take-off". AGARD Flight Test Panel Meeting, August 1956.

11-54 Anon.: " A first approximation to the landing distance from $50 \mathrm{ft}$ ". R.Ae.S. Data Sheet "Performance" EG $6 / 1,1960$ (See also Sheets EG $6 / 3$ and $6 / 4$ ).

11-55 R. Staubenfiel: "Computation of the shortest landing distance". DVL Bericht 130, July 1960.

11-56 J.M.N. Willis: "Effects of water and ice on landing". Shell Aviation News, Number 296, 1963, pp. 16-20.

11-57 w.J.G. Pinsker: "The landing flare of large transport aircraft". ARC R \& M No. 3602, Nov. 1967.

11-58 M.D. White: "Proposed analytical model for the final stages of a landing of a transport airplane". NASA TN D-4438, 1968.

11-59 D.H. Perry: "A first-order analysis of landing performance based on current British Civil Airworthiness Requirements". RAE Technical Memorandum Aero 132 (1970).

11-60 A.J. Walton: "Landing performance of conventional aircraft". Short course: "Aircraft performance estimation". Cranfield Institute of Technolgy, Feb, 1933.

Economics

11-61 พ.C. Mentzer and B.E. Nourse: "Some economic aspects of transport airplane performance". J. of the Aeron. Sciences, April-May 1940. 
11-62 P.G. Masefield: "Some problems and prospects in civil air transport". J.R.Ae.S., April 1955, pp. 235-248.

11-63 R.G. Thorne: "The influence of range, speed and lift-drag ratio on the operating costs of a civil aircraft ". RAE Tech. Note Aero 2487, Nov. 1956.

11-64 L.B. Aschenbeck: "Passenger air-1ine economics". Aeron. Eng. Review, Dec. 1966, pp. 39-43.

11-65 R.G. Thorne: "The estimation of civil aircraft direct operating costs". Aircraft Eng., Feb. 1957, pp. 56-57.

11-66 F.H. Robertsen: "Note on an improved short-cut method for estimating aircraft d.o.c.". J. of the Royal Aeron. Soc., Jan. 1957, pp. 52-53.

11-67 N.E. Rowe: "Complexity and progress in transport aircraft". J. of the Royal Aeron. Soc., Nov. 1958, pp. 787-795.

11-68 Anon.: "Standard method for the estimation of direct operating costs of transport airplanes" . Society of British Aircraft Constructors, Issue No. 4, 1959.

11-69 G. Backhaus: "Einflusz der ökonomischen Forderungen auf die Verkehrsflugzeugentwicklung". Deutsche Flugtechnik, 1961, Vol. 2, pp. 48-57.

11-70 M.H. Smith and H.P. Schmidt: "A rational method for selecting business aircraft". SAE Paper No. 797c, 1964.

11-71 A.F. Watts: "Aircraft turbine engines - development and procurement costs". The Rand Corporation, Memorandum RM-4670-PR, Nov. 1965.

11-72 E.L. Courtiney: "Some factors affecting fares". J. of the Royal Aeron. Soc., Nov. 1965, pp. 727-732.

11-73 R.B. Whitberg: "An airline view on cheap short-range air transport". J. of the Royal Aeron. Soc., Nov. 1965 , pp. $732-739$.

11-74 C.J. Hamshaw Thomas: "Steps towards lower operational costs with conventional jet transport". J. of the Royal Aeron. Soc., Nov. 1965, pp. 737-743.

11-75 A.H. Stratford: "The prospects of lower airline and airport costs". J. of the Royal Aeron. Soc., Nov. 1965 , pp. $749-755$.

11-76 E.H. Yates: "Cost analysis as an aid to aircraft design". J. of Aircraft, March-April 1965, pp. 100-107 (also: AIAA Paper No. 64-178).

11-77 E.C. Wells: "Some economic aspects of air transport design". Fifth Dr. Albert Plesman Memorial Lecture, Delft, September 5, 1966.

11-78 E.L. Thomas: "ATA Direct Operating Cost formula for transport aircraft". SAE Paper No. 660280.

11-79 G.S. Levenson and S.M. Barro: "Cost-estimating relationships for aircraft airframes". The Rand Corporation, Memorandum RM-4845-PR, May 1966.

11-80 C. Peyton Autry and P.J. Baumgaertner: "The design importance of airplane mile costs versus seat mile costs". SAE Paper No. 660277.

11-81 w.G. Kaldahl: "Factors affecting utilization". SAE Paper No. 660279.

11-82 Anon.: "Operating costs, the intractable other half". The Aeroplane, Dec. 22, 1966, pp. 14-15.

11-83 Anon.: "Standard method of estimating direct operating costs of turbine powered transport airplanes" Air Transport Association of America, Dec. 1967.

11-84 A.M. Jackes: "The influence of performance characteristics on the economic effectiveness of transport vehicles". AIAA Paper No. 67-802, Oct. 1967.

11-85 M. Besinger: "Einige Wirtschaftliche Aspekte beim Entwurf von Verkehrsflugzeuge". WGLR Jahrbuch 1967, pp. 68-77.

11-86 D.J. Lloyd-Jones: "Airline equipment planning". AIAA Paper No. 67-392.

11-87 A.H. Stratford: "Air transport economics in the supersonic era". Macmillan/London, Melbourne, Toronto, St. Martin's Press/New York, 1967.

11-88 R.B. Ormsby: "Total airline profit program". SAE Paper No. 690413.

11-89 J.E. Gorham: "Long term trends in airlines economics". SAE Paper No. 690414.

11-90 H.D. Kysoz: "A value analysis approach to evaluating business aircraft". Bus. \& Comm. Av., May 1969, pp. $90-94,162$. 
11-91 н. Wittenberg: "De vervoersprestatie en de exploitatiekosten van subsone en supersone verkeersvliegtuigen voor de lange afstand". Tijdschrift voor vervoerswetenschap (Magazine for transport science), 1970, Nos. 1, 2 (in Dutch).

11-92 D.D. Hufford, J.A. Ross and K.W. Hoefs: "The economics of subsonic transport airplane design, evaluation and operation". SAE Paper No. 710423.

11-93 R.H. Wild: "The state of the art in light aircraft design". Interavia, April 1973, pp. 346-348.

11-94 R. Jensen: "The weight/performance interface - an argument for weight control". SAWE Technical Paper No. 967, June 1973.

11-95 G.P. Sallee: "Aircraft economics and its effects on propulsion system design". AIAA Paper No. 73-808.

\section{CHAPTER 12 .}

Structural design of aircraft

12-1 A.E. Russe11: "Some factors affecting large transport airplanes with turboprop engines". J.R.Ae.S. Feb. 1950, pp. 67-106.

12-2 G. de Vries: "Safeguards against flutter of airplanes". NACA TM1423, Translated from La Recherche Aéronautique, No. 12, 1949 and No. 13, 1950.

12-3 E.D. Keen: "Integral construction, application to aircraft design and effect on production methods". J.R.Ae.S., Vol. 57, April 1953, pp. 215-227.

12-4 o. Ljungstrő: "Wing structures of future aircraft". Aircraft Eng., Vo1. 25, No. 251, May 1953, pp. 128-132.

12-5 E.F. Bruhn and A.F. Schmitt: "Analysis and design of aircraft structures". Tri-State Offset Company, Ohio, 1958.

12-6 A.F. Newe11: "Impressions of the structural design of American civil aircraft". The Aeroplane, Aug. 15 and $22,1958$.

12-7 A.J. Troughton: "Relationship between theory and practice in aircraft structural problems". J.R.Ae.S., Nov. 1960 , pp. 653-667.

12-8 A.F. Newe11: "Recent British progress in aeronautics. Part One: Structural design". Aircraft Eng., Sept. 1961, pp. 248-254.

12-9 A.F. Newel1 and D. Howe: "Aircraft design trends". Aircraft Eng.,Vo1. 34, No. 399, May 1962, pp. 131139.

12-10 D.M. Mc.Elhinney: "Structural design of the Vickers VC-10". Aircraft Eng., June 1962, pp. 165-171, 178.

12-11 K. Bentley: "Structural design of the BAC 1-11". Aircraft Eng., May 1963, pp. 142-147.

12-12 E.E. Sechler and L.G. Dunn: "Airplane structural analysis and design". Dover Publications, New York, 1963.

12-13 A.C. Kermode: "The aeroplane structure". Pitman \& Sons, London, second edition, 1964.

12-14 Anon.: "Trident structural design". Aircraft Eng., June 1964, pp. 166-171.

12-15 P.L. Sandoz: "Structural considerations for long-haul transport aircraft". AIAA Paper No. 66-882.

12-16 พ.T. Shuler: "Large cargo airplane structural considerations". SAE Paper No. 660669.

12-17 G. Gerard: "Structural guidelines for materials development; some vehicle performance and design generalizations". AIAA Paper No. 68-331.

12-18 K.L. Sanders: "High-lift devices, a weight and performance trade-off methodology". SAWE Paper No. 761, May 1969.

12-19 Anon.: "Potential structural materials and design concepts for 1ight aircraft". NASA CR-1285, March 1969.

12-20 W.M. Laurence: "Special structural considerations for wide-body commercial jet transports". AIAA Paper No. 70-845. 
12-21 D.P. Marsh: "Post-design analysis for structural weight estimation". SAWE Technical Paper No. 936, May 1972.

12-22 P.L. Sandoz: "Structural design of future commercial transports". AIAA Paper No. 73-20.

12-23 A.I. Gudkov and P.S. Leshakov: "External loads and aircraft strength". NASA TT F-753, July 1973.

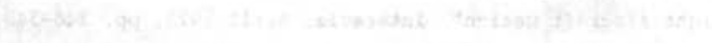

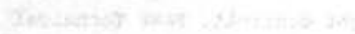




\section{Appendix A. Definitions relating to the geometry and aerodynamic characteristics of airfoils}

SUMMARY

This appendix deals with the most common definitions of some major geometric and aerodynamic characteristics of wings with rounded noses and sharp trailing edges. Formulas and graphs are presented for calculating the Mean Aerodynamic Chord and the mean quarterchord point for straight-tapered wings, with or without prismoidal center section. 
Nomenclature

A - aspect ratio

a.c. - aerodynamic center

b - span

c - chord

$\bar{c}$ - chord length of the MAC

$c_{g}$ - chord length of the SMC

c.p. - center of pressure

e - fraction of the chord

1 - wing angle of incidence

MAC - Mean Aerodynamic Chord

0 - origin of system of axis (wing apex)

$S$ - gross wing area

$\mathrm{S}_{\text {net }}$ - net wing area

$\mathrm{S}_{\text {wet }}$ - wetted (exposed) wing area

SMC - Standard Mean Chord

t - maximum airfoil section thickress

$x$ - wing axis, coinciding with root chord

$x \quad$ - coordinate in $\mathrm{X}$-direction

$\bar{x} \quad-x$-coordinate of mean quarter-chord point

Y - lateral axis

y - ordinate of section, measured from chord line; lateral coordinate of

A-1. GENERAL

This appendix deals with definitions of the geometry and some aerodynamic properties of symmetrical wings. Only wing sections with rounded noses and sharp trailing edges are considered. Slender wings such as low aspect ratio delta wings are not treated. Most definitions are also applicable to horizontal tailplanes and subscripts w or $\mathrm{h}$ have therefore been omitted.

Various formulas will be presented without proof. More details and background can be found in the publications mentioned in the list of references.

\section{A-2. WING SECTIONS}

A-2.1. Geometric definitions

A WING SECTION is formed by the external a wing section, measured from $\mathrm{X}$ axis

- y-coordinate of mean quarter-chord point of a half wing

$y_{C}$ - mean-line ordinate of a section

$\Delta y$ - leading edge sharpness parameter

$\mathrm{Z}$ - axis perpendicular to XOY-plane

z - coordinate in z-direction

$\bar{z} \quad$ - z-coordinate of mean quarter-chord point

a - angle of attack

$a_{L}$ - wing zero-lift angle

$\alpha_{\ell}{ }^{\circ}$ - section zero-lift angle

$\Gamma^{\ell}$ - dihedral angle

$\varepsilon \quad$ - twist angle

$\varepsilon_{\text {a }}$ - aerodynamic twist angle

$\varepsilon_{\mathrm{g}}$ - geometric twist angle

$n$ - non-dimensional lateral coordinate

$\Lambda \quad$ - angle of wing sweep

$\lambda$ - taper ratio

- semi-span (non-dimensional) of prismoidal center section; tail angle

Subscripts

$\begin{array}{ll}r & - \text { root } \\ t & - \text { tip }\end{array}$

contour of a wing cross section with a plane parallel to the plane of symmetry of the wing, or a plane perpendicular to the quarter-chord line. The cambered wing sections of most conventional families of airfoils are obtained by the combination of a MEAN LINE and a THICKNESS DISTRIBUTION (FIg. A-1).

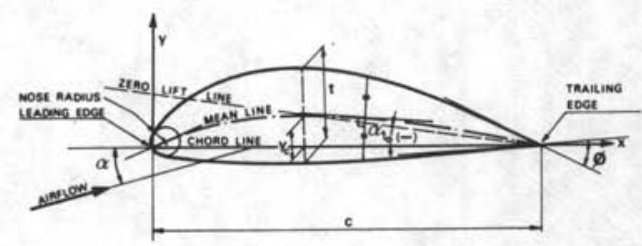

Fig. A-1. Geometric definitions of a wing section

The LEADING and TRAILING EDGE are defined, respectively, as the forward and rearward extremities of the mean line. 
The CHORD LINE is defined as the straight line connecting the leading and trailing edges. Its length is defined as the CHORD LENGTH or simply THE CHORD (c).

SECTION THICKNESS $(t)$ is the maximum distance between corresponding points on the upper and lower section surface (Fig. A-1). It is usually expressed as a thickness/ chord ratio $(t / c)$.

The NOSE RADIUS defines the sharpness of the section at the leading edge. Its center is located on the tangent to the mean line, drawn at $.5 \%$ of the chord.

CAMBER is the distance between corresponding points on the mean line and the chord line; mean line ordinate: $\mathrm{y}_{\mathrm{C}}$. The maximum camber is frequently referred to as "the camber".

The following geometric parameters have also proved to be useful in the prediction of certain aerodynamic characteristics of wing sections:

LEADING EDGE SHARPNESS PARAMETER:

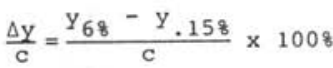

Values for $\triangle \mathrm{y}$ for several NACA sections are presented in Fig. A-2.

TAIL ANGLE ( $\phi)$ is the angle between the tangents to the upper and lower surfaces at the trailing edge (Figs. $\mathrm{A}-1$ and $\mathrm{A}-3$ ).

\section{A-2.2. Aerodynamic definitions}

ANGLE OF ATTACK $(\alpha)$ : angle between the chord line and the direction of the undisturbed flow. (Positive $\alpha$ : nose-up, see Fig. A-1).

THE ZERO LIFT ANGLE $\left(\alpha_{\ell}\right)$ : angle of attack for zero lift. Positive $\alpha_{\ell}$ : nose up; for sections with positive camber $\alpha_{\ell}$ is negative, as in Fig. A-1.

ZERO-LIFT LINE: line through the trailing edge parallel to the direction of the undisturbed airflow at $\alpha_{\ell}$.

CENTER OF PRESSURE (c.p.): the intersection of the vector, representing the resulting aerodynamic force, with the chord line. The
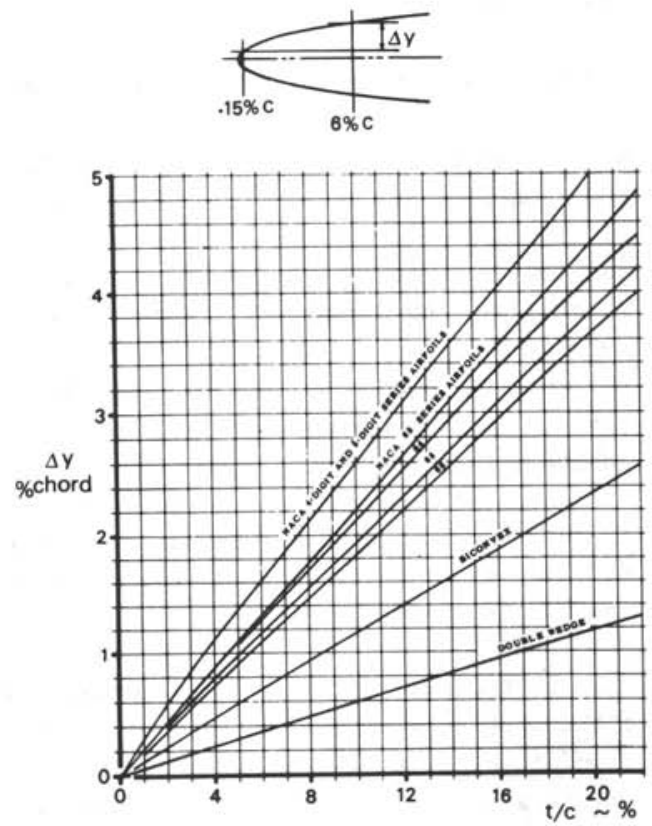

Fig. A-2. Leading edge sharpness parameter of NACA sections (Ref. A-6)

c.p. varies with $\alpha$ for cambered sections. AERODYNAMIC CENTER: a point about which the pitching moment for given dynamic pressure is essentially independent of the angle of attack up to maximum lift in subcritical flow. For design purposes it is generally acceptable to assume the a.c. as $.25 \mathrm{c}$

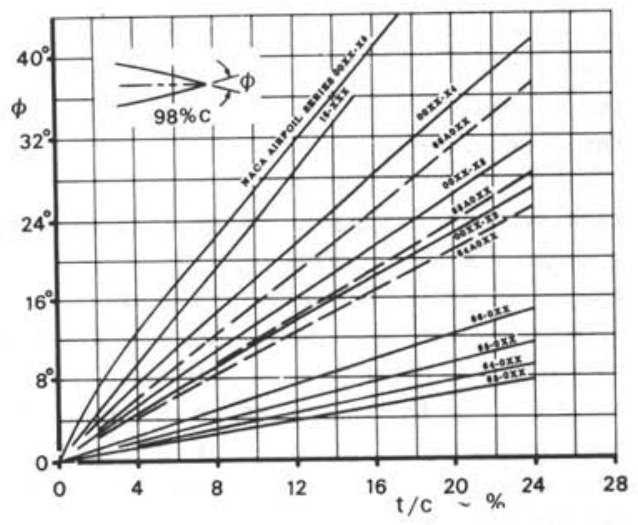

Fig. A-3. Tail angle of NACA sections (Ref. A-6) 
(low-subsonic speeds).

A-2.3. Nomenclature for some NACA sections

NACA FOUR-DIGIT SERIES (example: NACA 2415). The numbering system is based on the section geometry. The first integer indicates the maximum value of the mean-line ordinate $y_{c}$ as a percentage of the chord. The second integer indicates the distance from the leading edge to the location of the maximum camber in tenths of the chord. The last two integers indicate the section thickness as a percentage of the chord. Hence, the NACA 2415 section has 28 camber at . 4 of the chord from the leading edge and is 158 thick.

NACA FIVE-DIGIT SERIES (example: NACA 23012) The numbering system is based on a combination of theoretical aerodynamic characteristics and geometric characteristics. The first integer indicates the amount of camber in terms of the relative magnitude of the design lift coefficient*; the design lift coefficient is three halves of the first integer. The second and third integers together indicate the distance from the leading edge to the location of the maximum camber; this distance as a percentage of the chord is one-half the number represented by these integers. The last two integers indicate the section thickness as a percentage of the chord.

Hence, the NACA 23012 wing section has a design lift coefficient of . 3 , has its maximum camber at .15 of the chord, and has a thickness ratio of 128 .

NACA 6-SERIES OR LAMINAR-FLOW WING SECTIONS (example: NACA $65_{3}-218$ )

The first digit is the series designation of a family of low-drag airfoils, designed on the basis of theoretical methods. The second digit is the chordwise position of minimum pressure in tenths of the chord behind the leading edge for the basic symmetrical section at zero lift. The digit

*Definition in Ref. A-1. following the comma or written as a subscript gives the range of lift coefficient in tenths above and below the design lift coefficient in which pressure gradients favorable for obtaining low drag exist on both surfaces. The digit following the dash gives the design lift coefficient in tenths. The last two digits represent the thickness of the wing section as a percentage of the chord.

Hence, NACA $65_{3}-218$ is a 6 -series section. The point of minimum pressure at zero lift for the 65-018 section is at .50 of the chord. The favorable range of lift coefficients is between -.1 and .5 , the design lift coefficient is .2 and the maximum thickness 188 of the chord.

Some modifications of the 6-series sections are designated by replacing the dash by a capital A. These sections are substantially straight on both surfaces from about .8 of the chord to the trailing edge. The 6series airfoils without this designation have cusped trailing edges.

A-3. WINGS

A-3.1. Wing planform

A system of axes is generally used as indicated in Fig. A-4. The origin is located

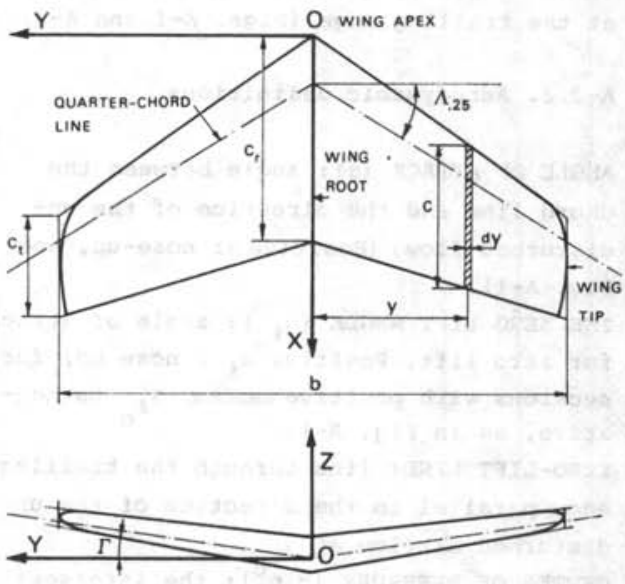

Fig. A-4. System of axis in relation to the wing planform 
at the WING APEX 0 , which is the intersection of the leading edges of both wing halves. The $x$ axis coincides with the chord of the root section, positive backwards. The $\mathrm{Y}$ axis is perpendicular to the plane of symmetry, positive to port side. The $z$ axis is positive upwards.

LEADING and TRAILING EDGE: the lines through the leading and trailing edges of the wing chords.

ROOT CHORD and TIP CHORD $\left(c_{r}, c_{t}\right)$ : the chord length of the wing sections in the plane of symmetry and at the outer extremity of the wing, respectively. For rounded tips the definition of the tip chord is given in Fig. A-4.

TAPER RATIO $(\lambda)$ is the ratio of the tip chord to the root chord:

$$
\lambda=c_{t} / c_{r}
$$

Straight-tapered wings have straight leading and trailing edges.

GROSS or DESIGN WING AREA (S): the area enclosed by the wing outline, including wing flaps in the retracted position and ailerons, but excluding fillets or fairings, projected on the XOY plane. The leading and trailing edge are assumed to be extended through the nacelles and fuselage to the XOZ plane in any reasonable manner (example in Fig. A-5)

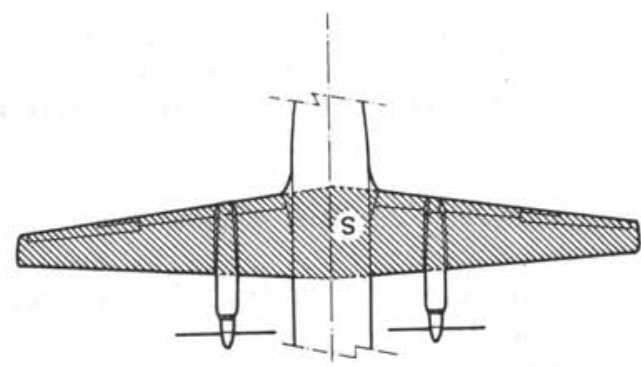

Fig. A-5. Definition of gross wing area

$s=2 \int_{0}^{b / 2} c d y$

NET WING AREA $\left(\mathrm{S}_{\text {net }}\right)$ : the gross wing area minus the projection of the central wing part, inside the fuselage.
EXPOSED or WETTED WING AREA * $\left(S_{\text {wet }}\right)$ : the net external wing surface area, which is exposed to the airflow.

SPAN (b): the distance between the wing tips, measured perpendicular to the $\mathrm{xOz}$ plane, navigation lights excluded.

GEOMETRIC Or STANDARD MEAN CHORD (SMC) : the length of this chord $\left(c_{g}\right)$ is equal to the gross area, divided by the span:

$c_{g}=s / b$

For straight-tapered wings:

$c_{g}=c_{r} \frac{1+\lambda}{2}$

ASPECT RATIO (A): the span divided by the geometric mean chord:

$A=b / c_{g}=b^{2} / S$.

For a straight tapered wing the root chord can be calculated for given gross area, aspect ratio and taper ratio from:

$c_{r}=\frac{2}{1+\lambda} \sqrt{S / A}$

QUARTER-CHORD LINE: the line through all points at $.25 \mathrm{c}$ of the sections.

(ANGLE OF) DIHEDRAL $(\Gamma, 25)$ : the angle between the projection of the quarter-chord line on the Yoz plane and the $\mathrm{Y}$ axis (positive upwards, Fig. A-4). Negative angle: ANHEDRAL.

SWEEP ANGLE of the quarter-chord line $(\Lambda .25)$ : the angle between the projection of the quarter-chord line on the XOY plane and the $Y$ axis. Positive angle backwards (sweepback), negative forwards (sweep forward).

Sweep angles of other characteristic lines (trailing edge, leading edge, mid-chord line) have a similar definition and can be calculated for a straight-tapered wing from the following relationship:

*In some publications the term "exposed wing area" has the same meaning as the net wing area defined above, i.e. a projected area. 


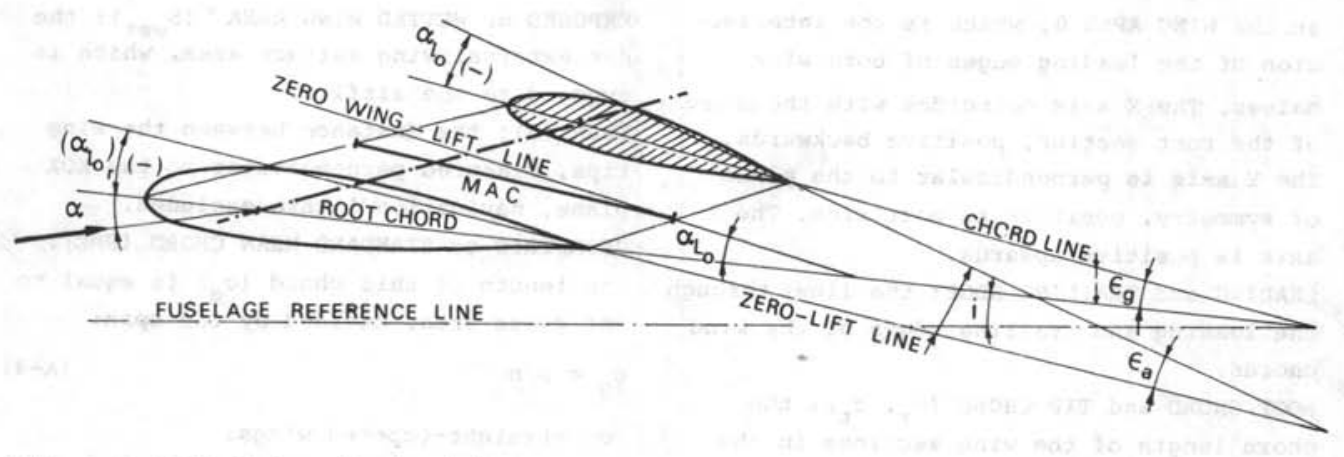

Fig. A-6. Definition of wing twist

$\tan \Lambda_{2}=\tan \Lambda_{1}+\frac{4}{A} \frac{1-\lambda}{1+\lambda}\left(e_{1}-e_{2}\right)$

where $e_{1}$ and $e_{2}$ denote the fraction of the chord according to the definition of the sweep angles $\Lambda_{1}$ and $\Lambda_{2}$, respectively. For example, the trailing edge sweep angle can be calculated from $\Lambda_{.25}$ by substitution of $e_{1}=.25$ and $e_{2}=1.0$. For a wing (or tailplane) with straight trailing edge $\left(\Lambda_{2}=0\right)$ we have:

$\tan \Lambda_{.25}=\frac{3(1-\lambda)}{A(1+\lambda)}$

\section{A-3.2. (Wing) twist and incidence}

(WING) TWIST $(\varepsilon)$ is the angle of incidence of a wing section relative to that of the root section, measured in a plane parallel to the XOz plane (Fig. A-6). Positive twist: nose rotated upwards, WASH-IN. Negative twist: nose rotated downwards, WASHOUT.

Geometric twist $\left(\varepsilon_{g}\right)$ is the twist of the chord line of a section relative to the chord line of the root section.

Aerodynamic twist $\left(\varepsilon_{a}\right)$ is the twist of the zero-lift line of a section relative to the zero-lift line of the root section. For an arbitrary section the geometric and aerodynamic twist are interrelated as follows: $\varepsilon_{a}=\varepsilon_{g}+\left(\alpha_{\ell_{0}}\right)-\alpha_{r}$

The WING TWIST ANGLE usually refers to the twist angle at the tip chord:

$$
\left(\varepsilon_{\mathrm{a}}\right)_{t}=\left(\varepsilon_{\mathrm{g}}\right)_{t}+\left(\alpha_{\ell_{0}}\right)_{\mathrm{r}}-\left(\alpha_{\ell_{0}}\right)_{t}
$$

For a wing with LINEAR TWIST, the aerodynamic twist angle increases in proportion to the lateral coordinate:

$\varepsilon_{a}=n\left(\varepsilon_{a}\right)_{t}$

where

$n=\frac{y}{b / 2}$

A LINEAR LOFTED (GEOMETRIC) TWIST is obtained on a wing where the intermediate sections are formed by linear lofting between the root and tip sections:

$\varepsilon_{g}=\left(\varepsilon_{g}\right)_{t} \frac{\lambda \eta}{1-(1-\lambda) \eta}$

WING ANGLE OF INCIDENCE (angle of wing setting) i: the angle between the root chord line and the airplane reference axis, e.g. the fuselage center line.

\section{A-3.3. Aerodynamic definitions}

MEAN AERODYNAMIC CHORD (MAC, $\bar{c}$ ): the chord of an equivalent untwisted, unswept and non-tapered wing, for which the total lift and pitching moment are essentially equal to the lift and pitching moment on the actual wing. The derivation of $\bar{c}$ can se found in Ref. A-9:

$\bar{c}=\frac{2}{s} \int_{0}^{b / 2} c^{2} d y$ 


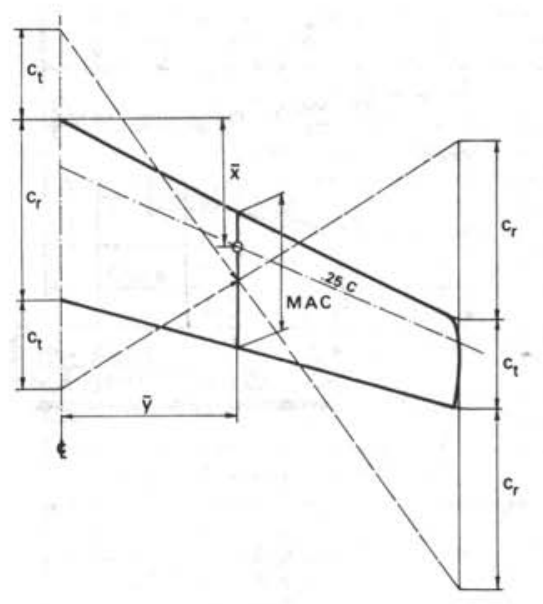

Fig. A-7. Graphical construction of the MAC
Alternatively, the simple graphic construction depicted in Fig. A-7 can be used to find the length and location of the MAC of a wing half. For wings with prismoidal inboard sections and tapered outboard sections, Fig. A-8 may be used.

AERODYNAMIC CENTER (a.c.): a point in the XOZ plane about which the aerodynamic pitching moment coefficient of the wing is essentially constant up to maximum lift in subcritical flow.

For moderate sweep angle a reasonable approximation for the a.c. is the MEAN QUARTER-CHORD POINT. For a half wing, this point is located at $.25 \bar{c}$, with coordinates:

$\bar{x}=.25 c_{r}+\frac{2 \tan \Lambda .25}{S} \int_{0}^{b / 2}$ cy dy $(A-16)$ $\bar{y}=\frac{2}{s} \int_{0}^{b / 2}$ cy dy (half wing)

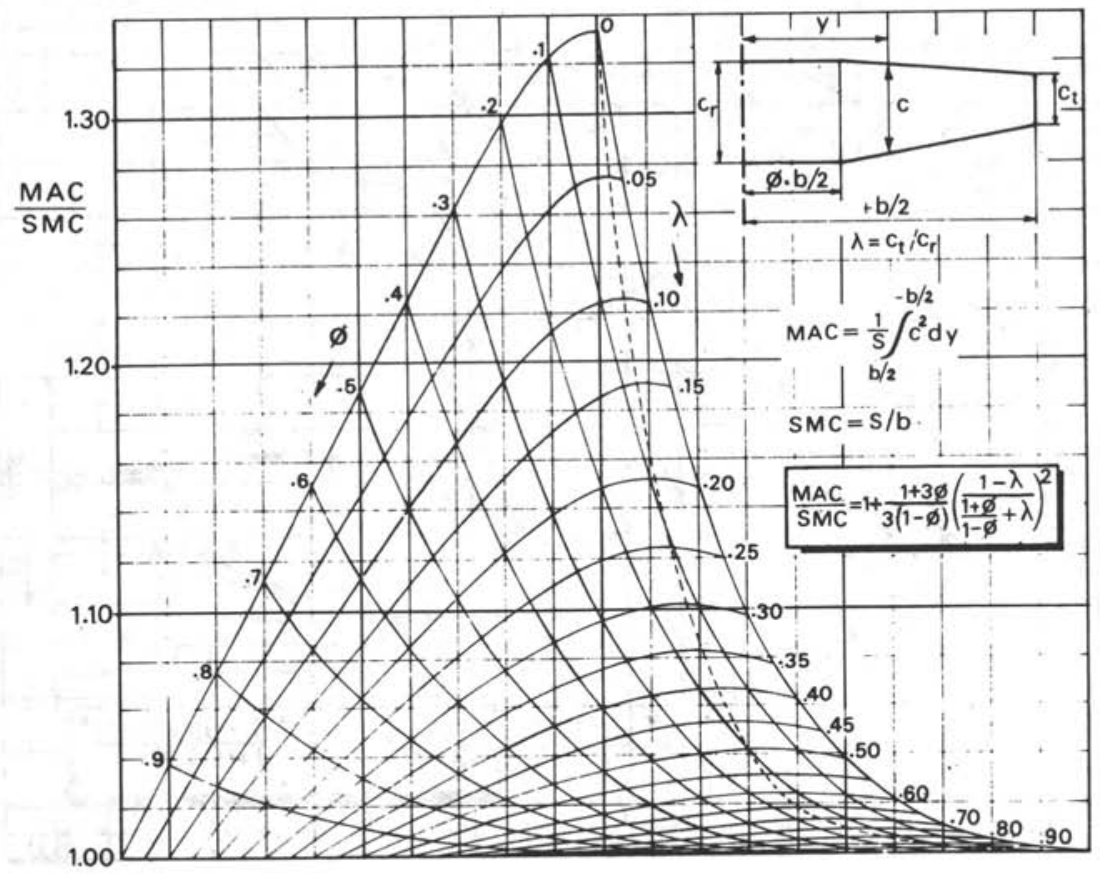

Fig. A-8. Diagram for the Mean Aerodynamic Chord of straight-tapered wings with or without prismoidal inboard section 


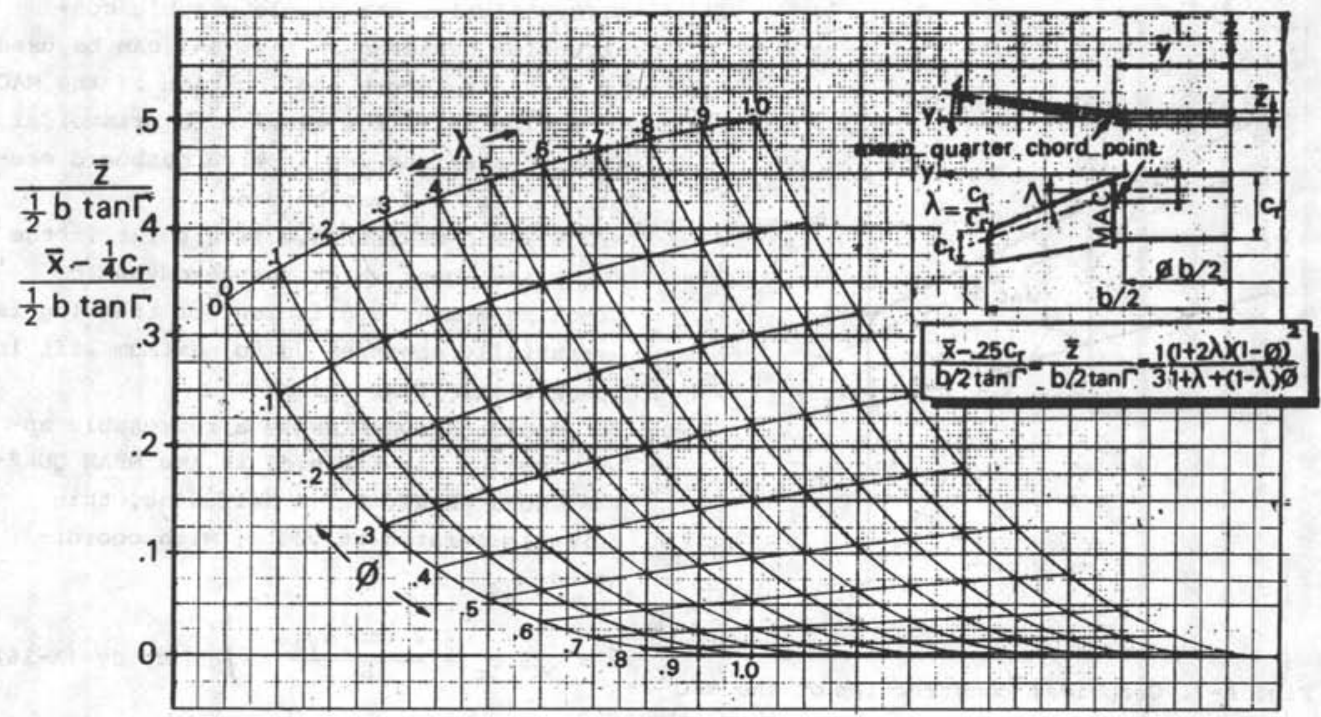

Fig. A-9. Diagram for the mean quarter-chord point for straight-tapered wings with or without prismoidal center section

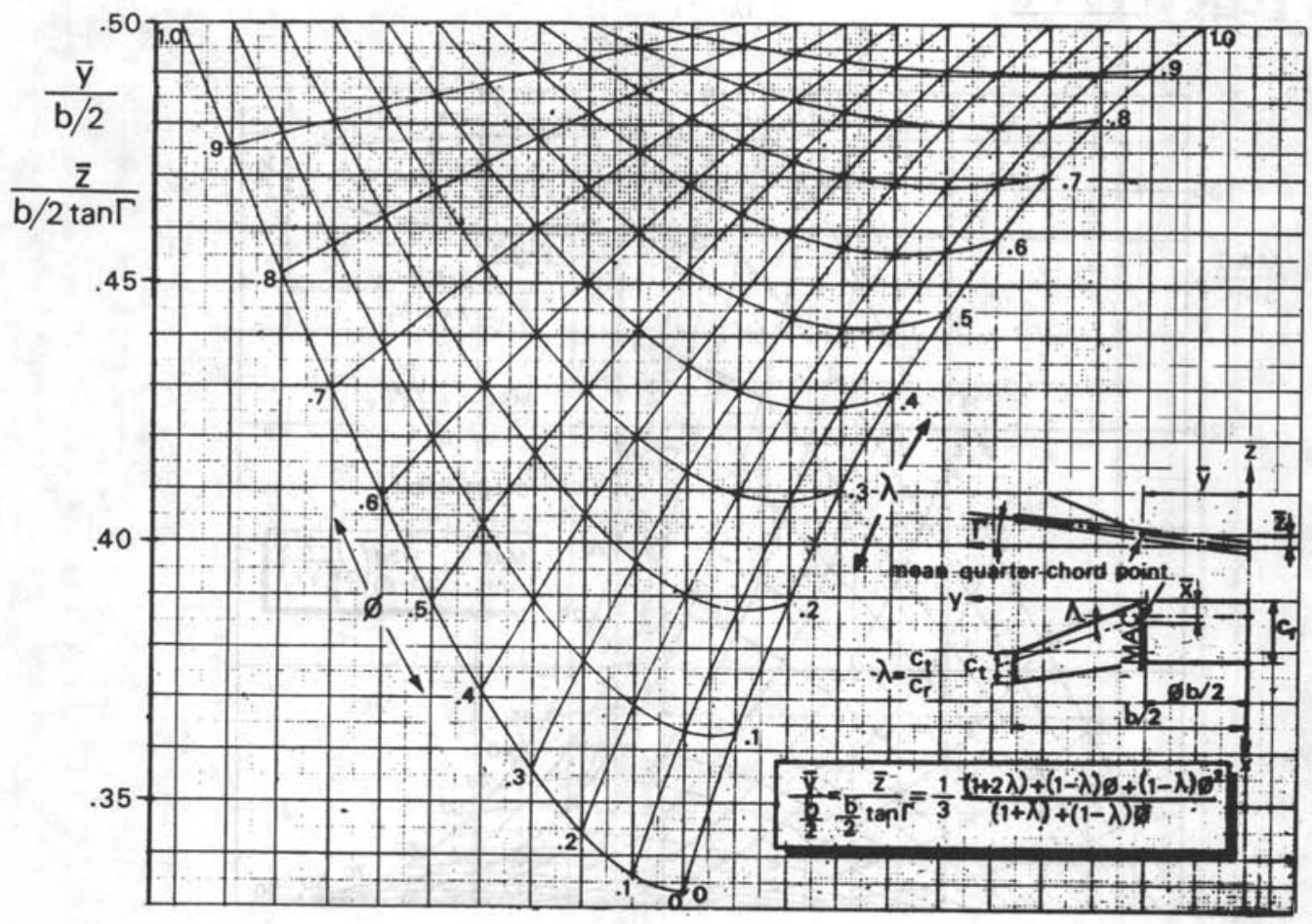

Fig. A-10. Diagram for the mean quarter-chord point of straight-tapered wings with or without prismoidal center section 
$\bar{z}=\frac{2 \tan \Lambda .25}{S} \int_{0}^{b / 2} c y d y$

For straight-tapered wings the MAC is 10cated at the lateral coordinate:

$\bar{y}=\frac{b}{2} \frac{1+2 \lambda}{3(1+\lambda)} \quad$ (half wing)

$(A-19)$

while the quarter-chord point of this chord is identical to the mean quarter-chord point. Figs. A-9 and A-10 can be used for more complex wings shapes. WING ANGLE OF ATTACK $(\alpha)$ : the angle between the root chord and the direction of the undisturbed airflow (Fig. A-6). THE ZERO-LIFT LINE is drawn through the trailing edge of the MAC, parallel to the undisturbed airflow, in a position at which the wing lift is zero (Fig. A-6). ZERO-LIFT ANGLE $\left(\alpha_{L}\right)$ is the angle between

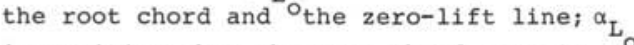
is positive when the root chord nose is directed upwards relative to the airflow (Fig. A-6).

\section{REFERENCES}

A-1. I.H. Abbott, A.E. von Doenhoff: "Theory of Wing Sections". Dover Publications, Inc., New York, 1958.

A-2. F.W. Riegels: "Aerodynamische Profile". Published by R. Oldenbourg, Munich, 1958.

A-3. R.C. Pankhurst: NPL Aerofoil Catalogue and Bibliography ARC R \& M No. 3311, 1963.

A-4. T. Nonweiler: "The Design of Wing Sections". Aircraft Engng., July 1956, pp. 216-227.

A-5. Anon.: R.Ae.S. Engineering Sciences DATA Sheet WINGS 01.01.05, oct. 1958.

A-6. D.E. Hoak and J.W. Carlson (red): "USAF Stability and Control Handbook", prepared by Douglas Aircraft Cy., Oct. 1960. Rev. 1968.

A-7. Anon.: "Dictionary of Technical Terms for Aerospace Use", NASA SP-7, First Edition, 1965.

A-8. w.S. Diehl: "The Mean Aerodynamic Chord and the Aerodynamic Centre of a Tapered wing". NACA Report 751, June 1942 .

A-9. A.H. Yates: "Notes on the Mean Aerodynamic Chord and the Mean Aerodynamic Centre of a Wing". Journal of the Royal Aeron. Soc., June 1952, pp. 461-474. 


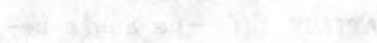

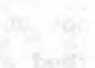

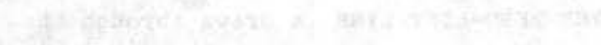

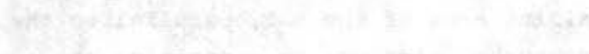

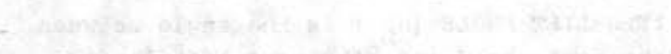

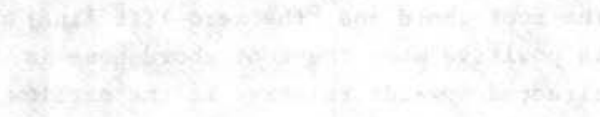

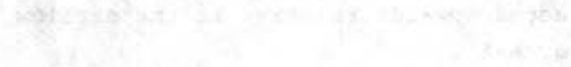

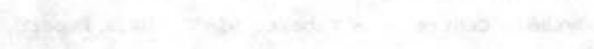




\section{Appendix B. The computation of circumferences, areas and volumes of curves, sections and bodies}

SUMMARY

Methods are presented for the calculation of circumferences, projected areas, wetted areas and volumes of sections, fuselages, wings and tailplanes, fuel tanks and engine nacelles.

The methods presented are simplified results derived from Ref. B-1; they can be readily applied in the preliminary stage of an aircraft design in cases where the shape and dimensions are not known in great detail. 


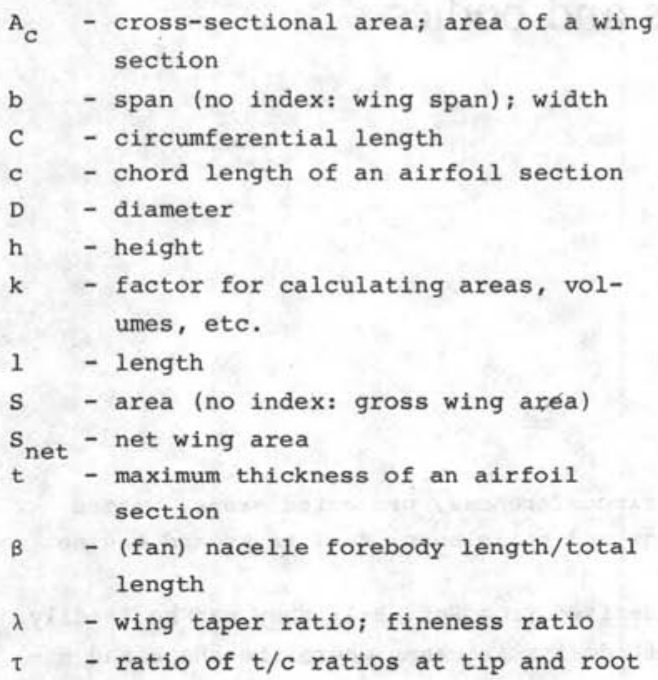

Subscripts

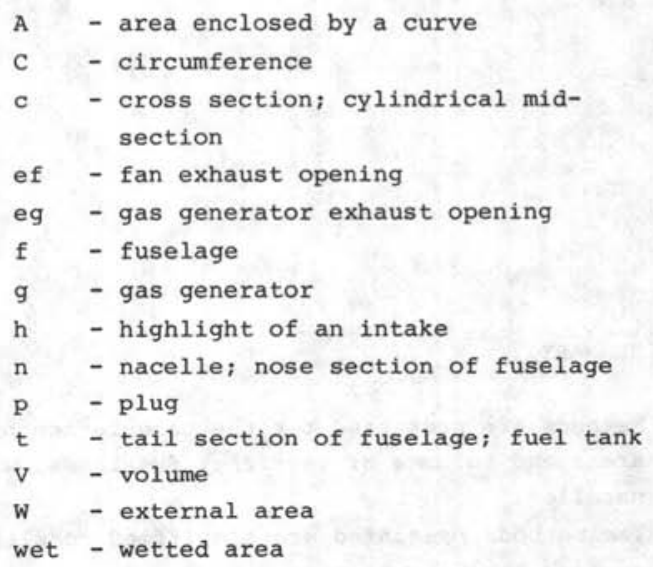

B-1. FUSELAGE

The present methods refer to the gross wetted area and volume of streamline bodies by which most fuselages can be approximated. Cockpit hoods, air scoops, fillets and the like, as well as non-exposed parts at the junction of wing and empennage, must be calculated separately, e.g. with the data on wing sections (Section $\mathrm{B}-2$ ), and subtracted.

B-1.1. General method

The generalized curves in Fig. B-1 are derived from approximations of the external lines by polynomials with fractional exponents (Ref. B-1). In most cases the fuselage can be divided into a nose section, a cylindrical mid-section and a tail section. The shape parameter $\phi$ is measured from the plan view and the factors $k_{A^{\prime}}, k_{C^{\prime}}$ $\mathrm{k}_{\mathrm{V}}$ and $\mathrm{k}_{\mathrm{W}}$ are then determined in order to calculate the characteristic areas and volume as follows (symbol in Fig. B-2):
Cross-sectional area* (frontal area):

$A_{c}=k_{A} b_{f_{\max }} h_{f_{\max }}$

Circumferential length of the cross section:

$c_{f}=2 k_{C}\left(b_{f_{\text {max }}}+h_{f_{\text {max }}}\right)$

where $k_{C}$ is obtained from Fig. B-1. If necessary, the cross section is subdivided into several parts.

Fuselage volume:

$v_{f}=A_{c} \quad\left(1_{c}+k_{v_{n}} 1_{n}+k_{v_{t}} 1_{t}\right)$

where $\mathrm{k}_{\mathrm{V}}$ is found by measuring $\Phi$ in the plan view for the nose section ( $n$ ) and the tail section $(t)$ and using Fig. $B-1$. Fuselage wetted area:

$s_{f_{\text {wet }}}=c_{f}\left(1_{c}+k_{w_{n}} 1_{n}+k_{w_{t}} 1_{t}\right)$

* at the fuselage station where the width and height are maximum 


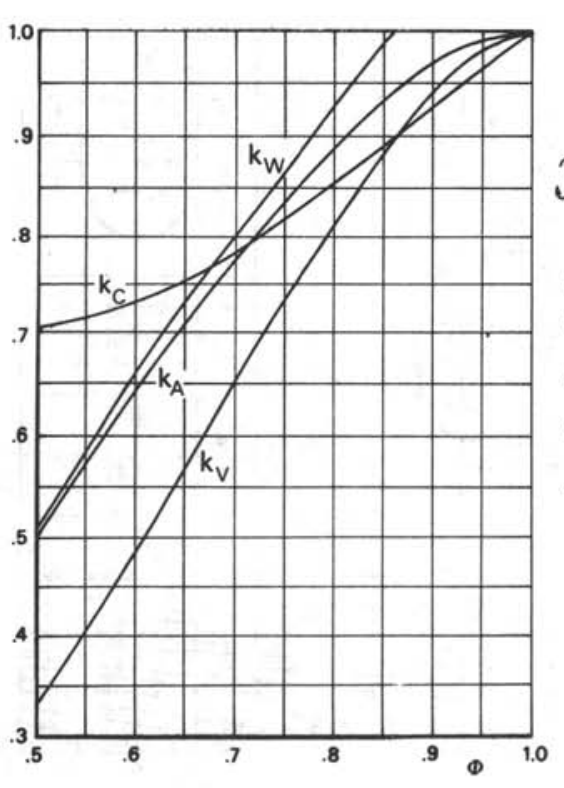

where $C_{f}$ is given by $(B-2)$ and the factor $k_{W}$ is obtained from Fig. B-1.

Projected areas of plan view and side view can be calculated in the same way as crosssectional areas by subdividing them into several parts and using the appropriate $k_{A}$ factors given in Fig. B-1.

For a cross section built up from two circular segments, the frontal area, volume and wetted area are first calculated from the plan view, assuming the fuselage to be a body of revolution. Correction factors given in Fig. B-3 are then applied.

B-1.2. Quick method for bodies of revolution

The following approximations apply to fuselages with cylindrical mid-sections:

volume $=\frac{\pi}{4} D_{f}^{2} 1_{f}\left(1-\frac{2}{\lambda_{f}}\right)$
$\begin{aligned} & \text { wetted } \\ & \text { area }=\pi D l_{f}\left(1-\frac{2}{\lambda_{f}}\right)^{2 / 3}\left(1+\frac{1}{\lambda_{f}^{2}}\right)\end{aligned} \mid \lambda_{f} \geqslant 4.5$
Fig. B-1. Factors for calculating the area, circumference, volume and wetted area of sections and bodies

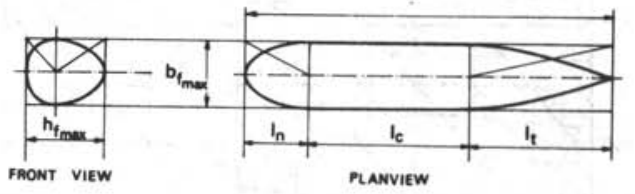

Fig. B-2. Definition of streamline body geometry

where $\lambda_{f}$ is the fuselage fineness ratio: $\lambda_{f}=1_{f} / D_{f}$.

For fully streamlined shapes without cylindrical mid-section the following expressions apply:

volume $=\frac{\pi}{4} D_{f}{ }^{2} 1_{f}\left(.50+.135 \frac{1_{n}}{1_{f}}\right)$

wetted area $=\pi D 1_{f}\left(.50+.135 \frac{1_{n}}{1_{f}}\right)^{2 / 3} \times$

$$
\left(1.015+\frac{.3}{\lambda_{f}^{1.5}}\right)
$$

where $1_{n}$ is the length of the nose section in front of the maximum cross section.

\section{B-2. WINGS AND TAILPLANES}

For most subsonic airfoil sections the following simple expressions are reasonably accurate:

$c=2 c(1+.25 t / c)$ 


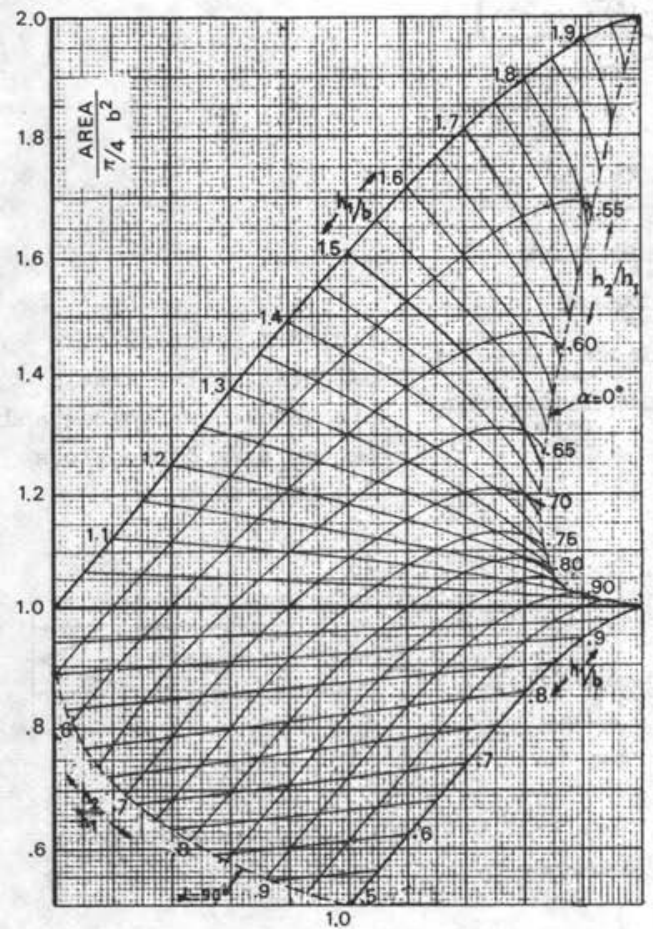

$\mathrm{A}=.68 \mathrm{tc}$

$(\mathrm{B}-10)$

A more accurate result can be obtained with Fig. B-1.

The wetted area of a linear lofted wing without nacelles is derived by integration of the circumferential length in a spanwise direction. A simple approximation for the result of this procedure is:

$\mathrm{S}_{\text {wet }}=2 \mathrm{~S}_{\text {net }}\left\{1+.25(\mathrm{t} / \mathrm{c}) \mathrm{r} \frac{1+\tau \lambda}{1+\lambda} \mid\right.$

where $\lambda=c_{t} / c_{r}$ and $\tau=(t / c)_{t} /(t / c)_{r}$. In this case the rcot section is not taken at the wing centerline, as usual, but at the wing-fuselage intersection. For a wing with nacelles the wetted area should be reduced by an amount equal to the total area of the wing inside the nacelle structure.

\section{B-3. FUEL TANK VOLUME}

a. Most bladder tanks can be compared with a geometric body having parallel end faces.

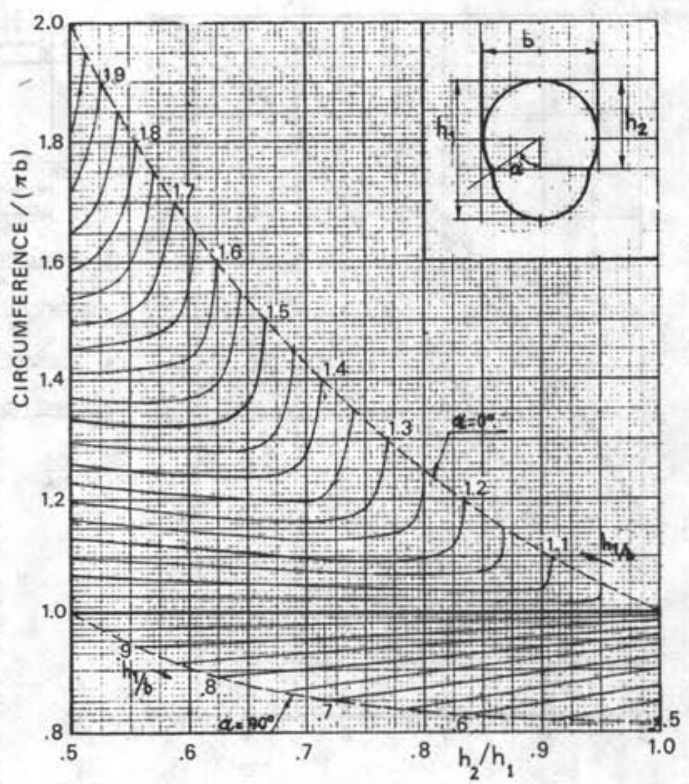

Fig. B-3. Area and circumference of doublebubble and flattened fuselage cross sections

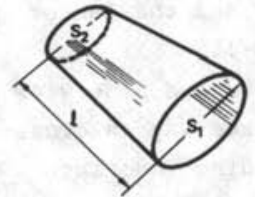

TRUNCATED EYRAMIO VOLUME $=\frac{1}{3}\left(s_{1}+s_{2}+\sqrt{s_{1} s_{2}}\right)$
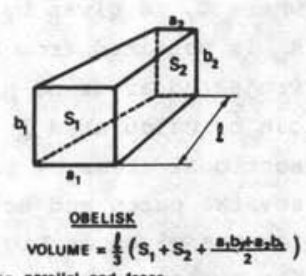

Fig. B-4. Volume of bodies with parallel end faces

Their volume is obtained from the appropriate formula given in Fig. $\mathrm{B}-4$.

b. For integral tanks the external shape of the structure enclosing the tank may be used to apply Fig. B-4. In this case the volume of the structure, which is equivalent to about 48 of the tank volume, should be subtracted to obtain the net tank volume.

c. External streamlined fuel tanks can be treated in the same way as the fuselage. The net tank volume is 48 less than the 
volume enclosed by the skin.

In project design a check on the available tank capacity is usually made in order to check the wing size or thickness/chord ratio. A first guess for the total tank volume available in a wing with linear lofted intermediate airfoils is:

$v_{t}=.54 \frac{s^{2}}{b}(t / c)_{r} \frac{1+\lambda \sqrt{\tau}+\lambda^{2} \tau}{(1+\lambda)^{2}}$

where

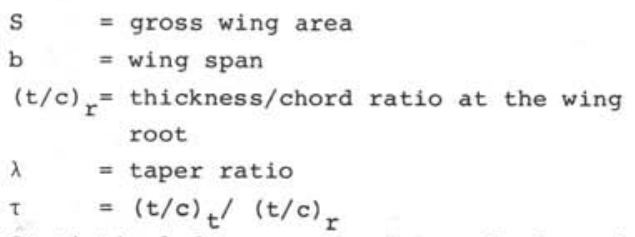
Statistical data were used to calculate the constant .54 in $(B-12)$ but the accuracy is not very high. Thus, if the available tank volume appears to be critical, a more precise calculation is necessary to account for the actual section shape and the wing structural layout.

Equation B-12 may also be used for calculating the tank capacity of a part of the wing. In that case the geometric definitions of $\mathrm{S}, \mathrm{b}$, etc. apply only to the wing part containing fuel.

Note that the Usable Fuel Capacity* is some 58 less than the tank volume to allow for

REFERENCES expansion of the fuel.

\section{B-4. ENGINE NACELLES AND AIR DUCTS}

In the most general case, the engine nacelle group may consist of a fan cowling, a gas generator cowling and a plug in the hot flow (Fig. B-5). The wetted areas of these components may be computed as follows :

External wetted area of fan cowling:

$$
1_{n} D_{n}\left\{2+.35 \beta+.8 \beta \frac{D_{h}}{D_{n}}+1.15(1-\beta) \frac{D_{e f}}{D_{n}}\right\}
$$

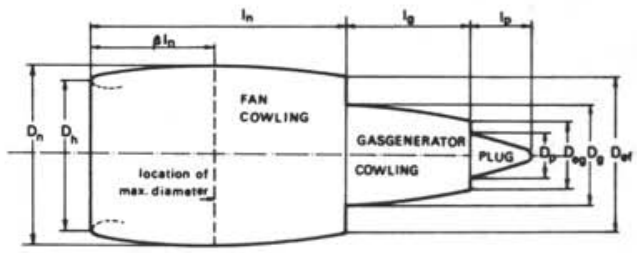

Fig. B-5. Geometry of a turbofan engine pod

Wetted area of gas generator cowling:

$$
\pi 1_{g} D_{g}\left[1-\frac{1}{3}\left(1-\frac{D_{e g}}{D_{g}}\right)\left\{1-.18\left(\frac{D_{g}}{1_{g}}\right)^{\frac{5}{3}}\right\}\right]
$$

Wetted area of plug:

$$
.7 \pi 1_{p} D_{p}
$$

B-1. E. Torenbeek: "The computation of characteristic areas and volumes of major aircraft components in project design". Delft University of Technology, Dept. of Aeron. Engng., Memorandum M-188, Feb. 1973. B-2. A.H. Schmidt: "A simplified method for estimating the wetted area of aircraft fuselages". SAWE Technical Paper No. 308, 1962.

B-3. R.T. Bullis: "Geometric analysis". SAWE Technical Paper No. 1025, May 1974.

\footnotetext{
*defined in Section 8.2.2.
} 


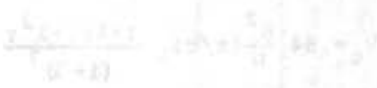

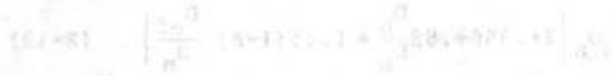

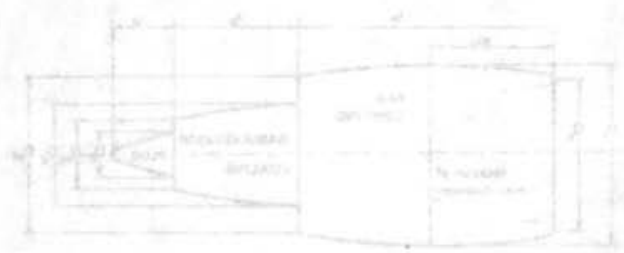

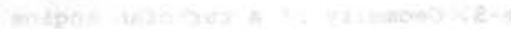

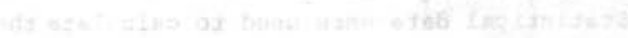

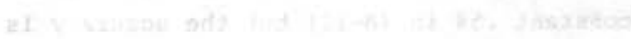

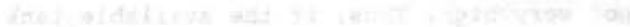

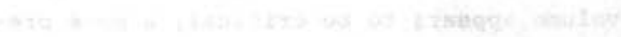

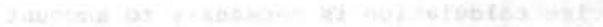

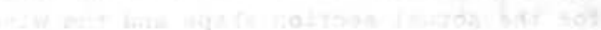

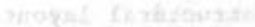

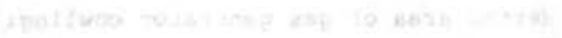

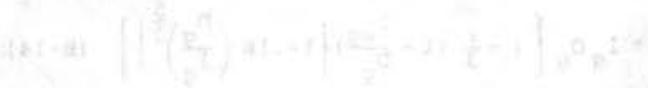

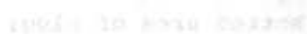

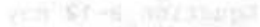

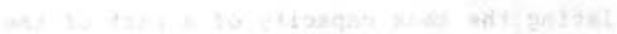

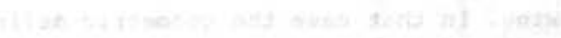

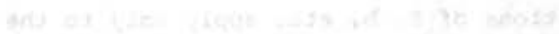

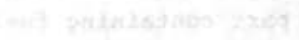

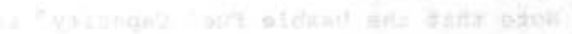

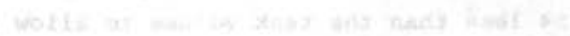

$\operatorname{sen}(-1)$

ans

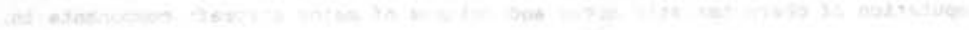

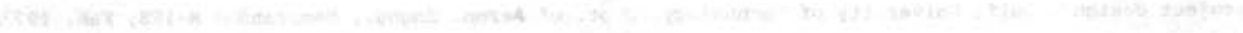

antan whe 


\section{Appendix C. Prediction of wing structural weight}

\section{SUMMARY}

The derivation of the present method (Ref. C-1) is based on a generalized expression for the material required to resist the root bending moment due to wing lift in a specific operational condition. The average stress is related to the loading index of the compression structure. A correction is made for the extra weight to provide the torsional stiffness required to withstand wing flutter. Separate contributions for high-lift devices, spoilers and speed brakes are given. Statistical analysis of the wing weight of many aircraft types actually built has yielded the necessary factors of proportionality. The method is applicable to light and transport category aircraft with wing-mounted engines in front of the elastic axis or engines not mounted to the wing. 
NOMENCLATURE

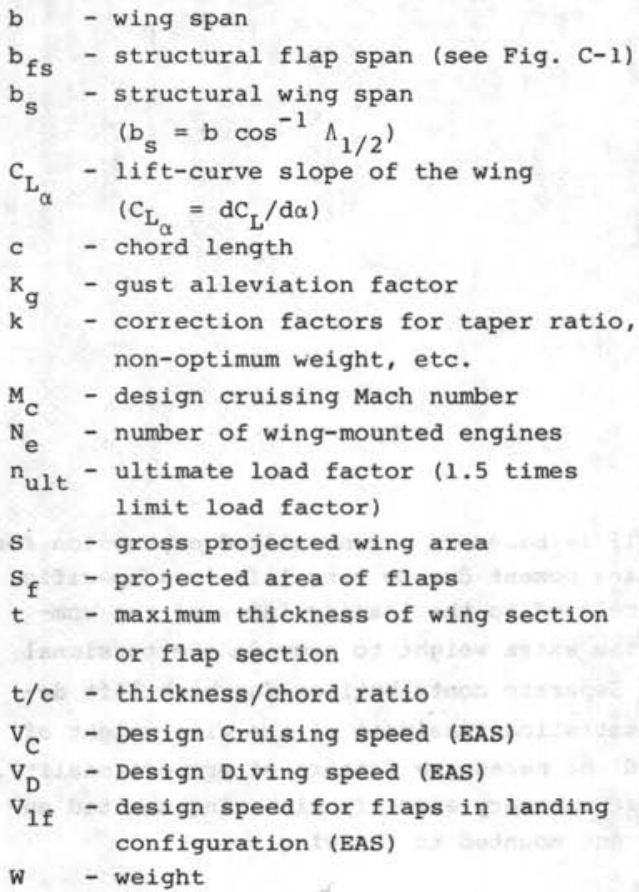

\section{C-1 INTRODUCTION}

The method presented in this Appendix is based on Ref. C-1, where the primary wing structure weight is derived from the requirement that in a specified critical flight condition the bending moment due to wing lift must be resisted. The weight of high-lift devices is based on a critical loading condition at the flap design speed. Application of the method to several highsubsonic short-haul airliners led to the conclusion that for this category the original method in Ref. C-1 results in an underestimation of the wing weight. It was assumed that the main reasons for this are the extra weight required to provide adequate stiffness against wing flutter and the weight penalty due to the long service life required, resulting in a rather low level flight stress level. Only the first of these aspects has resulted in a modifi- $w_{\text {des }}$ - design All-Up Weight of aircraft

$w_{w}$ - wing group weight as defined in AN 9103-D

$\Lambda_{1 / 2}$ - sweep angle of mid-chord line (Fig. C-1)

$\Lambda_{f} \quad$ - average sweep angle of flap structure (Fig. $\mathrm{C}-1$ )

$\delta_{f}$ - maximum flap deflection angle at $v_{1 f}$, measured streamwise

ns - distance of the strut mounting on a braced wing from the wing root, divided by the wing semi-span ( $n$ defined by equation $\mathrm{A}-12$ )

$\lambda$

- taper ratio $\left(\lambda=c_{t} / c_{r}\right)$

\section{SUBSCRIPTS}

b - basic

e - engines

f - flap

hld - high-lift devices

lef - leading-edge devices

$r$ - wing root

sp - spoilers; speed brakes

t - wing tip

tef - trailing-edge devices

w - wing

cation of the original formula. Other minor modifications have been introduced in the engine relief factor and in the weight of the high-lift devices. A weight penalty for spoilers and speed brakes has been introduced.

\section{C-2. BASIC WING STRUCTURE WEIGHT}

The weight of the basic wing structure (i.e. the wing group weight less the weight of high-lift devices, spoilers and speed brakes) is given by the equation:

$$
\begin{gathered}
w_{w_{\text {basic }}}=\text { constant } \times k_{n o} k_{\lambda} k_{e} k_{u c} k_{s t} x \\
{\left[k_{b} n_{u l t}\left(w_{d e s}-.8 w_{w}\right)\right]^{.55} x} \\
b^{1.675}(t / c)_{r}^{-.45}\left(\cos \Lambda_{1 / 2}\right)^{-1.325}
\end{gathered}
$$


where the constant is:

$8.94 \times 10^{-4}, w_{w}$ and $w_{\text {des }}$ in $1 b, b$ in $f t$, or $4.58 \times 10^{-3}, w_{w}$ and $w_{\text {des }}$ in $\mathrm{kg}, \mathrm{b}$ in $\mathrm{m}$.

For geometrical definitions see Fig. C-1.

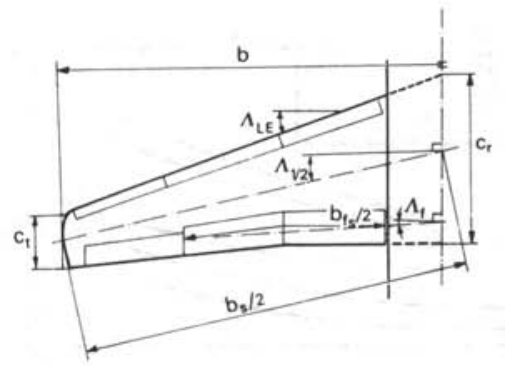

Fig. C-1. Geometric definitions

The correction factors are defined as follows :

$k_{\text {no }}=1+\sqrt{\frac{b_{\text {ref }}}{b_{s}}}$

where $b_{\text {ref }}=6.25 \mathrm{ft}(1.905 \mathrm{~m})$. The factor $k_{\text {no }}$ represents the weight penalties due to skin joints, non-tapered skin, minimum gauge, etc.

$k_{\lambda}=(1+\lambda) \cdot 4$

where $\lambda$ is the wing taper ratio, $\lambda=\mathrm{c}_{\mathrm{t}} / \mathrm{c}_{\mathrm{r}}$. The bending moment relief factor due to the engine and nacelle installation is:

$k_{e}=1.0$ engines not wing-mounted

.95 two wing-mounted engines in front of the elastic axis

.90 four wing-mounted engines in front of the elastic axis

The correction factor for undercarriage suspension is:

$\mathrm{k}_{\mathrm{uc}}=1.0$ wing-mounted undercarriage .95 undercarriage not mounted towing

For the extra weight required to provide stiffness against flutter the following correction is proposed for high-subsonic jet aircraft with engines not mounted to the wing or two engines wing-mounted in front of the elastic axis of the wing: $k_{s t}=1+\operatorname{constant} x \frac{\left(b \cos \Lambda_{L E}\right)^{3}}{w_{\text {des }}}\left\{\frac{V_{D} / 100}{(t / c)_{r}}\right\}^{2} \cos \Lambda_{1 / 2}$

where the constant is $1.50 \times 10^{-5}$ when $b$ is in $\mathrm{ft}, \mathrm{W}_{\text {des }}$ in $1 \mathrm{~b}$ and $V_{D}$ in $k t s$, or $9.06 \mathrm{x}$ $10^{-4}$ when $\mathrm{b}$ is in $\mathrm{m}, \mathrm{w}_{\text {des }}$ in $\mathrm{kg}$ and $\mathrm{V}_{\mathrm{D}}$ in $\mathrm{m} / \mathrm{s}$. For low-subsonic aircraft and highsubsonic aircraft with four wing-mounted jet engines $k_{\text {st }}=1.0$.

The correction factor for strut location on braced wings $\left(k_{b}=1.0\right.$ for cantilever wings) is:

$k_{b}=1-n_{s}^{2}$

The maximum weight with wing tanks empty should be used for the design weight $w_{\text {des }}$ in eq. C-1, although the Maximum Zero Fuel Weight is acceptable for most preliminary designs.

The ultimate load factor 'n 1.5 times the limit load factor, is the higher of the gust and the maneuver load factor. The gust load is based on the design cruising speed $V_{c}$ at an altitude of $20,000 \mathrm{ft}$ for pressurized cabins and sea level for non-pressurized cabins.

The load factors are determined in accordance with the airworthiness regulations FAR 23.341, FAR 25.341 or BCAR Section D ch. 3.1.4., as appropriate. The original formula in Ref. $\mathrm{C}-1$ is based on the simplified gust alleviation factor found in earlier regulations:

$\left.\begin{array}{l}K_{g}=.8-\frac{1.6}{\left(W_{\text {des }} / S\right)^{3 / 4}} \text { for } W_{\text {des }} / S>161 \mathrm{~b} / \mathrm{sq} \cdot \mathrm{ft} \\ K_{g}=.3 \times\left(w_{\text {des }} / \mathrm{s}\right)^{1 / 4} \text { for } w_{\text {des }} / \mathrm{S} \leqslant 161 \mathrm{~b} / \mathrm{sq} \cdot \mathrm{ft}\end{array}\right\}$

In this equation $W_{\text {des }} / \mathrm{S}$ is in $1 \mathrm{~b} / \mathrm{sq} . \mathrm{ft}$. The gust load is determined from the following expression for the wing liftcurve slope:

$C_{L_{\alpha}}=\frac{2 \pi}{2 / A+\sqrt{\frac{1}{\cos ^{2} \Lambda_{1 / 2}}-M_{C}^{2}+(2 / A)^{2}}}\left(\operatorname{rad}^{-1}\right)(C-7)$ 
where $\mathrm{M}_{\mathrm{c}}$ corresponds to $\mathrm{V}_{\mathrm{c}}$ in the operational condition mentioned previously.

C-3. HIGH-LIFT DEVICES, SPOILERS AND SPEED BRAKES

The weight of high-lift devices can be broken down as follows:

$\mathrm{w}_{\text {hld }}=\mathrm{w}_{\text {tef }}+\mathrm{w}_{\text {lef }}$

The trailing-edge flap weight is calculated from:

$\frac{W_{\text {tef }}}{S_{f}}=$ constant $x k_{f}\left(s_{f} b_{f s}\right)^{3 / 16} x$

$$
\left[\left(\frac{v_{1 f}}{100}\right)^{2} \frac{\sin \delta_{f} \cos \Lambda_{f}}{(t / c)_{f}}\right]^{3 / 4}
$$

where the constant is equal to .105 when $w_{\text {tef }}$ is in $1 b, s_{f}$ in sq. $f t, b_{f s}$ in $\mathrm{ft}$ and $v_{1 f}$ in $\mathrm{kts}$, or 2.706 when $w_{\text {tef }}$ is in $\mathrm{kg}$, $\mathrm{s}_{\mathrm{f}}$ in $\mathrm{m}^{2}, \mathrm{~b}_{\mathrm{fs}}$ in $\mathrm{m}$ and $\mathrm{v}_{1 \mathrm{f}}$ in $\mathrm{m} / \mathrm{s}$. The flap deflection angle $\delta_{f}$ and the flap thickness/chord ratio $(t / c)_{f}$ are measured streamwise. Other geometric definitions are given in Fig. C-1.

The factor $k_{f}$ represents the effect of the flap configuration:

$k_{f}=k_{f 1} k_{f 2}$

where

$k_{f 1}=1.0$ : single slotted; double slotted, fixed hinge

1.15: double slotted, 4-bar movement; single slotted Fowler

1.30: double slotted Fowler

1.45: triple slotted Fowler

$k_{f 2}=1.0:$ slotted flaps with fixed vane 1.25: double slotted flaps with

"variable geometry", i.e. extending flaps with separately moving vanes or auxiliary flaps

For variable geometry flaps the $(t / c)_{f}$ ratio in (C-9) refers to the retracted position. If the flap speed $v_{1 f}$ is not known, 1.8 times the stalling speed in the landing configuration may be assumed as a first guess.

In the absence of the various data required to compute the flap weight according to $(C-9)$, the data of Fig. $C-2$ may be

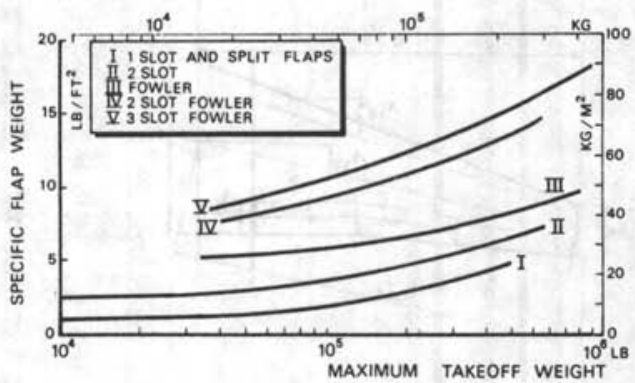

Fig. C-2. Specific weight of trailing-edge high-lift devices (Ref. C-2)

used. The specific weight of leading edge high lift devices can be read from Fig. c-3.

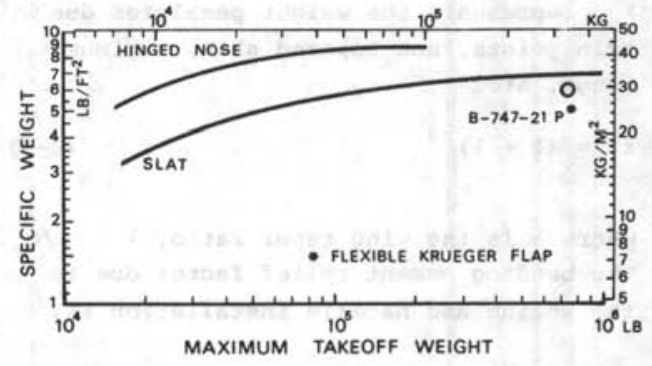

NOTE

The specific weight of Krueger flaps is approximately equal to that of slats

Fig. C-3. Specific weight of leading-edge high-1ift devices (Ref. C-2)

The weight of spoilers and speed brakes $\mathrm{w}_{\mathrm{sp}}$ may be taken either as $2.5 \mathrm{lb}$ (sq.ft (12.2 $\mathrm{kg} / \mathrm{m}^{2}$ ) or 1.5 of of the wing weight.

\section{C-4. WING GROUP WEIGHT}

The structural weight of the wing group, as defined in accordance with AN-9103D, is 
given by:

$w_{w}=w_{w_{\text {basic }}}+1.2\left(w_{h 1 d}+w_{s p}\right)$

where the basic wing weignt is defined by $(\mathrm{C}-1), \mathrm{w}_{\mathrm{hld}}$ by $(\mathrm{C}-8)$ and the spoiler and speed brake weight by the data y+ven in Section $\mathrm{C}-3$.

A first estimate of the wing weight must be available for substitution in $(C-1)$. Equation 8-12 in Section 8.4.1 is sufficiently accurate to make a second iteration superfluous.

The standard error of prediction of the present method is $9.64 \%$, but this figure can be considerably improved by adaptation of the constants of proportionality of $(C-1)$ and $(C-9)$ to accurately known weights of similar airplane wings. In this case the formula obviously applies to a very restricted category of aircraft only.

\section{REFERENCES}

C-1. E. Torenbeek: "Prediction of wing group weight for preliminary design". Aircraft Engng., July 1971, pp. $16-21$.

C-2. W. Schneider: "Die Entwicklung und Bewertung von Gewichtsabschătzungsformeln für den Flugzeugentwurf unter Zuhilfnahme von Methoden der mathematischen Statistik und Wahrscheinlichkeitsrechnung". Dissertation, University of Berlin, 23-2-1973. 


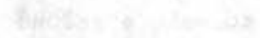

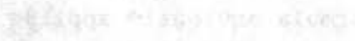

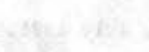




\section{Appendix D. The weight penalty method for fuselage structural weight prediction}

SUMMARY

The basic weight penalty method for fuselage structural weight prediction was introduced for the first time by Burt (Ref. D-1) and a number of other publications based on the same approach have been published since (Refs. D-2 through D-5). The present method represents a synthesis of these publications, updated for modern pressurized fuselages with or without engines mounted to the side of the rear fuselage section. 


\section{NOMENCLATURE}

$\mathrm{b}_{\mathrm{f}}$ - maximum width of fuselage

$c_{r}$ - theoretical wing root chord (see Fig. C-1)

$D_{f}$ - diameter of main fuselage lobe

$f$ - design hoop stress in fuselage skin in level flight

$\mathrm{h}_{\mathrm{f}}$ - maximum depth of fuselage

$\mathrm{K}_{\mathrm{f}} \quad$ - ratio of fuselage structure weight to gross shell weight

$k_{\lambda}$ - correction factor on shell weight for fuselage slenderness

$\mathrm{k}_{\mathrm{bh}}$ - proportionality factor for bulkhead weight

$k_{f 1}$ - proportionality factor for floor weight

$1_{e}$ - distance from engine support to the rear wing center section spar

$1_{t}$ - distance between quarter-chord points of wing root and horizontal tail root (Fig. D-2)

MTOW - Maximum Takeoff Weight

$\mathrm{n}_{\text {ult }}$ - ultimate load factor corresponding to $\mathrm{w}_{\text {to }}(1.5$ times $1 \mathrm{mit}$ load factor)

$P_{f 1}$ - maximum floor loading

$\Delta \mathrm{p} \quad$ - maximum operational differential pressure in cabin or pressurized fuselage section

$\mathrm{S}_{\mathrm{G}}$ - gross shell area (all openings faired over, no excrescences, fairings or blisters)

$\mathrm{S}_{\mathrm{bh}}$ - bulkhead area

$V_{D}$ - Design Diving speed (EAS)

$w_{f}$ - fuselage structure weight

$w_{f 1}$ - floor weight

$W_{f r}$ - gross frame weight

$W_{G}$ - gross shell weight

$\mathrm{w}_{\text {sk }}$ - gross skin weight

$\mathrm{W}_{\text {str }}$ - gross stringer and longeron weight

$\mathrm{w}_{\text {to }}$ - Maximum Takeoff Weight

\section{D-2. GROSS SHELL WEIGHT}

The weight of the fuselage shell structure is divided into the amounts contributed by the skin, stringers and frames:

$w_{G}=w_{s k}+w_{s t r}+w_{f r}$

D-2.1. Gross skin weight

The gross skin weight $\mathrm{w}_{\mathrm{sk}}$ is the greatest of the values given by $(D-2),(D-4)$ and (D-5).

$\mathrm{w}_{\mathrm{sk}}=$ constant $\times \mathrm{k}_{\lambda} \mathrm{s}_{\mathrm{G}}{ }^{1.07} \mathrm{v}_{\mathrm{D}} \cdot 743 \quad(\mathrm{D}-2)$

where the constant is equal to .00575 when $\mathrm{w}_{\mathrm{sk}}$ is in $1 \mathrm{~b}, \mathrm{~s}_{\mathrm{G}}$ in sq.ft and $\mathrm{v}_{\mathrm{D}}$ in knots, or .05428 when $w_{s k}$ is in $\mathrm{kg}, \mathrm{s}_{\mathrm{G}}$ is in $\mathrm{m}^{2}$ and $v_{D}$ in $\mathrm{m} / \mathrm{s}$. Allowing for the influence of the fuselage slenderness ratio, the factor $k_{\lambda}$ is approximated as follows:

$k_{\lambda}=.56\left(\frac{1_{t}}{b_{f}+h_{f}}\right)^{3 / 4}$

are added for floors, bulkheads, support structure and various additional items, resulting in the total fuselage group weight. for $1_{t} /\left(b_{f}+h_{f}\right)$ up to 2.61 , while for higher 


\begin{tabular}{|c|c|c|}
\hline \multicolumn{2}{|l|}{ chosS SHELL } & \\
\hline 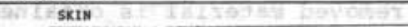 & 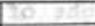 & \multirow{3}{*}{ 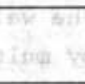 } \\
\hline STRINGERS AND LONCERONS & & \\
\hline Funes & & \\
\hline \multicolumn{2}{|l|}{ CROSS SHELL MOOIYICATTONS } & \\
\hline MATERUL RDNOVED & & \multirow{10}{*}{ 20. } \\
\hline \multirow{4}{*}{\multicolumn{2}{|c|}{\begin{tabular}{l|l|} 
PASSENGER AND CREW DOORS & \\
CARCO HOLD DOORS & \\
(LARCE) FREICAT HOLD DOORS \& RWTS & \\
ESCAFE HATCHES &
\end{tabular}}} & \\
\hline & & \\
\hline & & \\
\hline & & \\
\hline ENCLOSURES NAD WTIDSAIELDS & 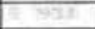 & \\
\hline VINDOUS A PONTS & & \\
\hline LANDING GEAR DOORS & & \\
\hline 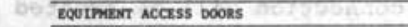 & 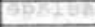 & \\
\hline SPEEDBRUXES & +3 & \\
\hline \multicolumn{3}{|l|}{ MODIFIED SAEL. } \\
\hline \multicolumn{2}{|l|}{ FLORING } & +28 \\
\hline \multicolumn{2}{|l|}{ PASSEMCER CABIN FLOOR, BENAS AND RATLS } & \multirow{5}{*}{ 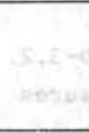 } \\
\hline \multicolumn{2}{|l|}{ FRETGAT COAPARTEEST FLOOR A LONDING SYST. } & \\
\hline \multicolumn{2}{|l|}{ CARCO/MCGAGE HOLD FLOOR } & \\
\hline \multicolumn{2}{|l|}{ FLIGIT DECK FLOR } & \\
\hline \multicolumn{2}{|l|}{ EQUIMENT BAY TLOOR } & \\
\hline \multicolumn{2}{|l|}{ BULKHEADS AND PRESSURE FLOORS } & \\
\hline \multicolumn{2}{|l|}{ PROST PAESSURE GULKHED } & \multirow{5}{*}{ 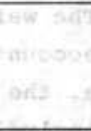 } \\
\hline \multicolumn{2}{|l|}{ REAR PRESSURE SULKGEAD } & \\
\hline \multicolumn{2}{|l|}{ LASOTNC CEAR WREELAAYS } & \\
\hline \multicolumn{2}{|l|}{ COCKPIT MOLOIEAD } & \\
\hline \multicolumn{2}{|l|}{ SPECLAL (MOUNTINC) FRMESS } & \\
\hline \multicolumn{2}{|l|}{ SUPPOKT STRUCTURE } & \multirow{6}{*}{ 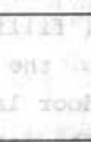 } \\
\hline \multicolumn{2}{|l|}{ MINC-FUSELAGE IMTERCONNECTION } & \\
\hline \multicolumn{2}{|l|}{ TAIUPLANE SUPPORT } & \\
\hline ENGINE(s) SUPFORT & & \\
\hline LANDING GEAR SUPPORT & arian 50 & \\
\hline PUSELACE TANKS SUPPORT & & \\
\hline ADDITIONAL, WEICHT TrEAS & & \\
\hline FAIRTES \& FILLETS & rasige & $7 x_{3} 3$ \\
\hline AIRSCOOPS & & \\
\hline STAIRS & & \\
\hline PATMT, SEALIMG, REDUX & Whang & 9ut? \\
\hline FASTENERS, JOINTS & & \\
\hline MTSCELLANEOUS & & \\
\hline 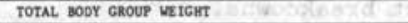 & IP $15 \times$ & \\
\hline
\end{tabular}

Table D-1: Weight breakdown of the fuselage group (applicable to tail booms as well)

values $k_{\lambda}=1.15$. The definition of $1_{t}$ is slightly modified as compared with that given in Ref. D-1, in order to account for the effect of wing sweep on the fuselage bending moment (Fig. D-2).

The gross skin weight based on cabin pressure, for constant skin thickness over the complete fuselage shell, is: aliall wish $\mathrm{w}_{\mathrm{sk}}=$ constant $\times \Delta \mathrm{p} \mathrm{D}_{\mathrm{f}} \mathrm{S}_{\mathrm{G}} \frac{\mathrm{f}_{\text {ref }}}{\mathrm{f}} \quad(\mathrm{D}-4)$ where the constant of proportionality is .007 when $w_{s k}$ is in $1 b, \Delta p$ in $1 b / s q . i n$. and $s_{G}$ in sq.ft., or 1.595 when $w_{s k}$ is in $\mathrm{kg}, \Delta \mathrm{p}$ in $\mathrm{kg} / \mathrm{cm}^{2}$ and $\mathrm{s}_{\mathrm{G}}$ in $\mathrm{m}^{2}$. Equation D-4 represents the skin weight required to resist the design cabin pressure differen- tial $\Delta p$, based on a mean hoop stress level of $f_{\text {ref }}=12,000 \mathrm{lb} / \mathrm{sq} \cdot \mathrm{in} .\left(843.7 \mathrm{~kg} / \mathrm{cm}^{2}\right)$. The actual value for $f$ to be anticipated may be substituted in (D-4), but in the absence of this information assume $f_{\text {ref }} / f=1$.

The minimum value for $\mathrm{w}_{\mathrm{sk}}$, based on a minimum gauge of $.8 \mathrm{~mm}(.315 \mathrm{in.})$, is equal to:

$w_{s k}=\frac{w_{s k}}{S_{G}} s_{G}$

where $\mathrm{w}_{\mathrm{sk}} / \mathrm{S}_{\mathrm{G}}=.445 \mathrm{lb} / \mathrm{sq}$. ft $\left(2.173 \mathrm{~kg} / \mathrm{m}^{2}\right)$.

In all equations the gross shell area $\mathrm{S}_{\mathrm{G}}$ is defined as the area of the entire outer surface of the fuselage, assuming that all holes for doors, windows, cutouts, etc. are faired over, and all local excrescences such as blisters, wheel fairings and canopies are removed and faired over. The gross shell area can be estimated with the methods given in Appendix B.

\section{D-2.2. Gross stringer and longeron weight}

The graphs presented in Ref. $D-1$ can be approximated as follows:

$w_{\text {str }}=$ constant $\times k_{\lambda} S_{G}^{1.45} v_{D} \cdot 39 n_{u l t} \cdot 316(D-6)$

where the constant of proportionality is .000635 when $\mathrm{w}_{\text {str }}$ is in $1 \mathrm{~b}, \mathrm{~s}_{\mathrm{G}}$ in sq. $\mathrm{ft}$ and $v_{D}$ in knots, or .0117 when $w_{s t r}$ is in $\mathrm{kg}, \mathrm{s}_{\mathrm{G}}$ in $\mathrm{m}^{2}$ and $\mathrm{V}_{\mathrm{D}}$ in $\mathrm{m} / \mathrm{s}$.

\section{D-2.3. Gross standard frame weight}

The curves in Ref. $\mathrm{D}-1^{1}$ are approximated as follows:

Freighters :

$w_{f r}=.32\left(w_{s k}+w_{s t r}\right)$

All other types:

$\mathrm{w}_{\mathrm{sk}}{ }^{+\mathrm{W}_{\mathrm{str}}}>630 \mathrm{lb}(286 \mathrm{~kg}): \mathrm{w}_{\mathrm{fr}}=19\left(\mathrm{w}_{\mathrm{sk}}+\mathrm{w}_{\mathrm{str}}\right)$

$\mathrm{w}_{\mathrm{sk}}{ }^{+\mathrm{W}_{\mathrm{str}}}<630 \mathrm{lb}(286 \mathrm{~kg}):$

$w_{f r}=$ const $\times\left(w_{s k}+w_{s t r}\right)^{1.13}$ 
where the constant is .0822 when $\mathrm{W}_{\mathrm{fr}}, \mathrm{W}_{\mathrm{sk}}$ and $w_{\text {str }}$ are in $1 b$, or .0911 when all weights are in $\mathrm{kg}$.

\section{D-3. GROSS SHELL MODIFICATIONS}

Computation of the total net. weight penalty relative to the gross shell weight is a lengthy task. In the conceptual design phase the required information is not always present and available data may be inaccurate and/or 111 defined. The alternative way of allowing for the departure of the fuselage design from the ideal is to use a simple overall correction factor for the gross shell weight, as suggested in Ref. D-1. This can be done with reasonable accuracy where the design is conventional. Defining a correction factor $\mathrm{K}_{f}$ as the ratio of the total fuselage group weight to the gross shell weight, we find:

$w_{f}=K_{f} w_{G}$

Values of $\mathrm{K}_{\mathrm{f}}$ have been calculated for several aircraft types, with the following result:

Hawker Siddeley HS-125: $K_{f}=2.40$

Fokker $\mathrm{F}-27 / 100$

$: \mathrm{K}_{\mathrm{f}}=1.82$

Fokker $\mathrm{F}-28 / 1000$

$: \mathrm{K}_{\mathrm{f}}=1.83$

McDonnell Douglas DC-9/10: $K_{f}=2.20$

McDonnell Douglas DC-8/55: $K_{f}=1.88$

Lockheed C-5A (freighter): $K_{f}=2.51$

Except in the case of the C-5A, a heavy freighter with fuselage-mounted undercarriage, a systematic explanation of the variation in $\mathrm{K}_{f}$ cannot be given. Instead of the rough estimate given by $(D-10)$, the more detailed computation method can be followed, using Table D-1 for collecting the various contributions.

\section{D-3.1. Removed material}

Fig. D-1 lists the openings to be considered for a typical passenger transport. Allowance must be made for cutouts of appreciable size, while for small openings it is reasonable to assume that the weight of skin removed is equal to the reinforce- ment added.

The weight of removed material is obtained by multiplying the specific gross shell weight $\left(\mathrm{W}_{G} / \mathrm{S}_{G}\right)$ by the "wetted" area of each opening. This procedure is performed for all 1tems listed in Table D-1 under the heading "gross skin modifications", as far as they are applicable to the airplane under consideration. The bay required at the wing-fuselage connection will be treated in a different way by equation $\mathrm{D}-21$ or D-22.

D-3.2. Doors, hatches, windows and enclosures

The weight of these items is taken into account by addition of

a. the actual weight of the doors, etc., including any operating mechanism ("fillings"), and

b. the surround structure weight, i.e. door landings, frames, etc.

The data presented in Table D-2 are not applicable to the (large) freighthold doors of freighter airplanes. They have been obtained by comparing the results of various references and those of detailed fuselage weight breakdowns.

If data relating to the number and dimensions of access doors are not available, their net weight penalty (i.e. removed material included) may be assumed at 1.5 to 28 of the gross shell weight. Note that landing gear wheelbays are generally not pressurized and that the "unpressurized" data must be used for landing gear doors. Nose landing gear doors are included in the weight penalty given in Section D-5.2 and may therefore be omitted here. Main landing gear doors are frequentIy regarded as a wing weight contribution. For freighter airplanes the following net weight penalty (i.e. removed material and surround structure are taken into account) is suggested for (large) freighthold dours: Side doors - Weight $=4 \sqrt{\Delta \mathrm{p}} \mathrm{lb} / \mathrm{sq} \cdot \mathrm{ft}$ of height $\times$ width, with $\Delta p$ in $1 b / s q . i n$.

$$
\text { - Weight }=73.65 \sqrt{\Delta \mathrm{p}} \mathrm{kg} / \mathrm{m}^{2} \text { of }
$$
height $x$ width, with $\Delta \mathrm{p}$ in $\mathrm{kg} / \mathrm{cm}^{2}$. 


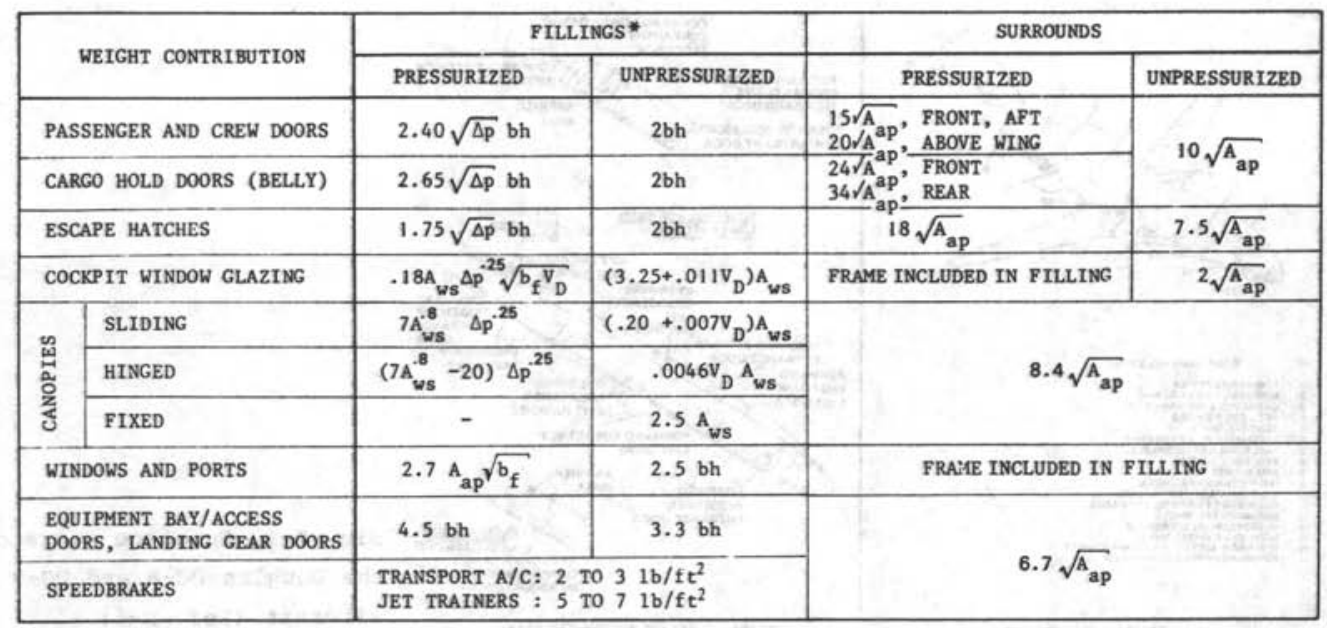

WEIGHT IN LB

\begin{tabular}{|c|c|c|c|c|c|}
\hline \multirow{2}{*}{\multicolumn{2}{|c|}{ WEIGHT CONTRIBUTION }} & \multicolumn{2}{|c|}{ FILLINGS * } & \multicolumn{2}{|c|}{ SURROUNDS } \\
\hline & & PRESSURIZED & UNPRESSURIZED & PRESSURIZED & UNPRESSURIZED \\
\hline \multicolumn{2}{|c|}{ PASSENGER AND CREW DOORS } & $44.2 \sqrt{\Delta \mathrm{p}}$ bh & $9.765 \mathrm{bh}$ & $\begin{array}{l}22.3 \sqrt{\mathrm{A}_{\text {ap }}} \text {-FRONT, AFT } \\
29.8 \sqrt{\mathrm{A}_{\text {ap }}} \text {-ABOVE WING }\end{array}$ & \multirow{2}{*}{$14.9 \sqrt{\mathrm{A}_{\text {ap }}}$} \\
\hline \multicolumn{2}{|c|}{ CARGO HOLD DOORS (BELLY) } & $48.8 \sqrt{\Delta \mathrm{p}}$ bh & 9.765 bh & $\begin{array}{l}35.7 \sqrt{\mathrm{A}_{\text {ap }}}-\text { FRONT } \\
50.6 \sqrt{\mathrm{A}_{\text {ap }}}-\text { REAR }\end{array}$ & \\
\hline \multicolumn{2}{|c|}{ ESCAPE HATCHES } & $32.2 \sqrt{\Delta \mathrm{p}} \mathrm{bh}$ & 9.765 bh & $26.8 \sqrt{\mathrm{A}_{\text {ap }}}$ & $11.2 \sqrt{\mathrm{A}_{\text {ap }}}$ \\
\hline \multicolumn{2}{|c|}{ COCKPIT WINDOW GLAZING } & $4.31 \mathrm{~A}_{\mathrm{vss}} \Delta \mathrm{p} \cdot \sqrt{\mathrm{b}_{\mathrm{f}} \mathrm{v}_{\mathrm{D}}}$ & $\left(15.9+.104 \mathrm{~V}_{\mathrm{D}}\right) \mathrm{A}_{\mathrm{ws}}$ & $\begin{array}{l}\text { FRAME INCLUDED } \\
\text { IN FILLING }\end{array}$ & $2.98 \sqrt{\mathrm{A}_{\text {ap }}}$ \\
\hline \multirow{3}{*}{$\frac{\frac{w}{2}}{\frac{0}{0}}$} & SLIDING & $41.3 \mathrm{~A}_{\mathrm{ws}}^{.8} \Delta \mathrm{p}^{.25}$ & $\left(.98+.0664 \mathrm{v}_{\mathrm{D}}\right) \mathrm{A}_{\mathrm{ws}}$ & \multirow{3}{*}{\multicolumn{2}{|c|}{$12.5 \sqrt{\mathrm{A}_{\text {ap }}}$}} \\
\hline & HINGED & $\left(41.3 \mathrm{~A}_{w s} \cdot{ }^{-8}-17.6\right) \Delta \mathrm{p}^{.25}$ & $.0436 \mathrm{~V}_{\mathrm{D}} \mathrm{A}_{\text {ws }}$ & & \\
\hline & FIXED & 18. nota & $12.2 \mathrm{~A}_{\mathrm{ws}}$ & & \\
\hline \multirow{2}{*}{\multicolumn{2}{|c|}{$\begin{array}{l}\text { WINDOWS AND PORTS } \\
\text { EQUIPMENT BAY/ACCESS } \\
\text { DOORS, LANDING GEAR DOORS }\end{array}$}} & $23.9 \mathrm{~A}_{\mathrm{ap}} \sqrt{\mathrm{b}_{\mathrm{f}}}$ & $12.2 \mathrm{bh}$ & \multicolumn{2}{|c|}{ FRAME INCLUDED IN FILLING } \\
\hline & & 22 bh & $16.1 \mathrm{bh}$ & \multirow{2}{*}{\multicolumn{2}{|c|}{$9.97 \sqrt{\mathrm{A}_{\text {ap }}}$}} \\
\hline \multicolumn{2}{|c|}{ SPEEDBRAKES } & $\begin{array}{l}\text { TRANSPORT A/C: } 10 \\
\text { JET TRAINERS : } 25\end{array}$ & $\begin{array}{l}\text { TO } 15 \mathrm{KG} / \mathrm{M}^{2} \\
\text { To } 35 \mathrm{KG} / \mathrm{M}^{2}\end{array}$ & & \\
\hline
\end{tabular}

WEIGHT IN KG

* ALL ITEMS QUOTED INCLUDE OPERATING MECHANISM

$A_{w s}=$ windshield wetted area, frame included (sq.ft or $\mathrm{m}^{2}$ ): $A_{a p}=$ "wetted" area of aperture or opening (sq.ft or $\mathrm{m}^{2}$ ) $; b=$ wiath ( $\mathrm{ft}$ or $\mathrm{m}$ ); $b_{\mathrm{f}}=$ fuselage width (ft or $\mathrm{m}$ ); $\mathrm{v}_{\mathrm{D}}=$ Design Diving speed (knots or $\mathrm{m} / \mathrm{s}$ EAS); $\Delta \mathrm{p}=$ maximum operational pressure differential of relevant fuselage section (lb/sq. inch or $\mathrm{kg} / \mathrm{cm}^{2}$ )

Table D-2. Gross weight penalties of fillings and surrounds (References D-1 through D-7 and various detail weight data) 


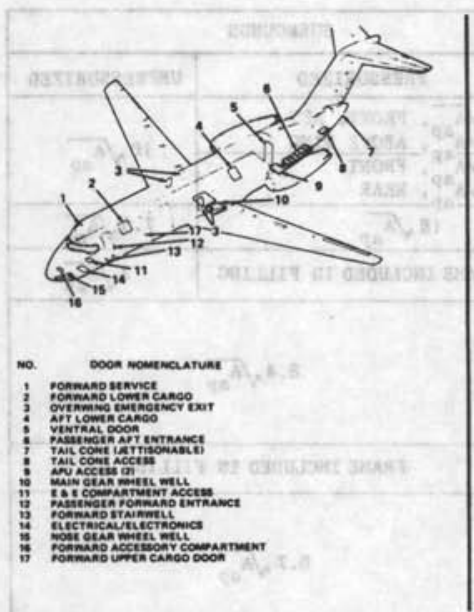

Senvice ocon Locatrons

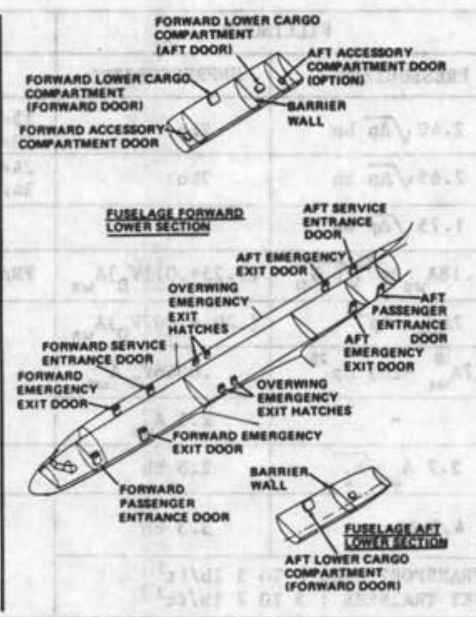

Doons - Fustuat messunized NaEA

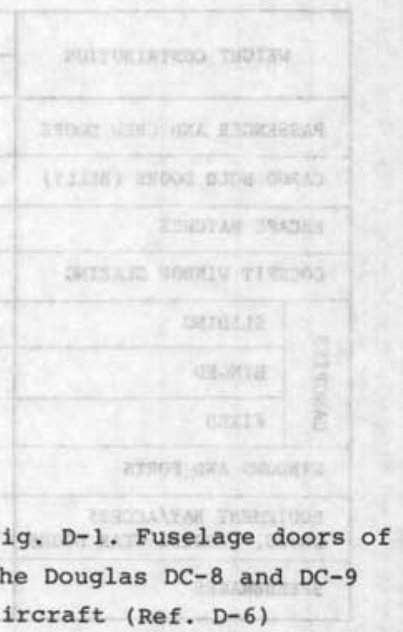

aircraft (Ref. D-6)
Rear loading doors, ramp and headroom door included -

Weight $=3 \sqrt{\Delta \mathrm{p}} \mathrm{lb} / \mathrm{sq} . \mathrm{ft}$ of total wetted area, with $\Delta p$ in $1 b / s q .1 n$.

Weight $=55,24 \sqrt{\Delta \mathrm{p}} \mathrm{kg} / \mathrm{m}^{2}$ of wetted area, with $\Delta \mathrm{p}$ in $\mathrm{kg} / \mathrm{cm}^{2}$.

Nose loading door weight - $15 \mathrm{lb} / \mathrm{sq} . \mathrm{ft}$ $\left(73.236 \mathrm{~kg} / \mathrm{m}^{2}\right)$ of frontal area.

D-4. FLOORING

Typical floor weights are:

for passenger transports - 1 to $2 \mathrm{lb} / \mathrm{sq}$. ft ( 5 to $10 \mathrm{~kg} / \mathrm{m}^{2}$ )

for heavy freighters $\quad-4$ to $5 \mathrm{lb} / \mathrm{sq}$. $\mathrm{ft}$

(20 to $25 \mathrm{~kg} / \mathrm{m}^{2}$ )

For a more detailed calculation it is necessary to allow for the floor support configuration and the floor loading.

\section{D-4.1. Passenger cabin and freight hold} floors

The diagram in Ref. D- 1 is approximated as follows :

$w_{f 1}=k_{f 1}\left(s_{f 1}\right)^{1.045}$

Floors are categorized as follows:
TYPE A: Passenger floors with beams resting directly on the fuselage frames, normally used in flat-bottomed fuselages: $k_{f l}=.85$ when $w_{f 1}$ is in $1 b$ and $s_{f 1}$ in sq. $\mathrm{ft}$, or 4.62 when $W_{f l}$ is in $\mathrm{kg}$ and $\mathrm{S}_{\mathrm{fl}}$ in $\mathrm{m}^{2}$.

TYPE B: Passenger floors with beams attached to the fuselage sides and possibly braced to the bottom by struts, normally used in pressurized fuselages with underfloor cargo holds:

$k_{f 1}=$ const $\times \sqrt{P_{f 1}}$

where the constant of proportionality is equal to .125 when $W_{f l}$ is in $1 b, S_{f l}$ in sq.ft and $P_{f l}$ in $1 \mathrm{~b} / \mathrm{sq} . \mathrm{ft}$, or .3074 when $W_{f l}$ is in $\mathrm{kg}, \mathrm{S}_{\mathrm{fl}}$ in $\mathrm{m}^{2}$ and $\mathrm{P}_{\mathrm{fl}}$ in $\mathrm{kg} / \mathrm{m}^{2}$. The factor $k_{f l}$ includes floor panels, lateral and longitudinal stiffeners, support struts and seat tracks.

TYPE C: Freight compartment floors designed for the carriage of containerized freight $k_{f 1}=2.0$ when $w_{f 1}$ is in $1 b$ and $s_{f l}$ in sq. $f t$, or 10.867 when $w_{f l}$ is in $k g$ and $s_{f l}$ in $\mathrm{m}^{2}$.

TYPE D: Freight compartment floors designed for the carriage of bulk freight $-k_{f l}=$ 2.70 when $w_{f 1}$ is in $1 b$ and $s_{f 1}$ in sq. $f t$, or 14.67 when $w_{f 1}$ is in $k g$ and $s_{f 1}$ in $\mathrm{m}^{2}$. TYPE E: Freight compartment floors for 
heavy freighters, intended for all-vehicle operation $-k_{f l}=3.85$ when $w_{f l}$ is in $1 b$ and $S_{f l}$ in sq. $f t$, or 20.92 when $w_{f 1}$ is in $\mathrm{kg}$ and $\mathrm{s}_{\mathrm{fl}}$ in $\mathrm{m}^{2}$.

\section{D-4.2. Various other floors}

The weight of floors in (belly) cargo and baggage holds is related to the total cargo or baggage hold volume and the permissible floor loading as follows:

$\mathrm{W}_{\mathrm{fl}}=$ constant $\times($ volume $) \cdot 7 \sqrt{\mathrm{P}_{\mathrm{fl}}}$

where the constant is .4 when the volume is in cu.ft and the floor loading in lb/ sq.ft, or 1.0 when the volume is in $\mathrm{m}^{3}$ and the floor loading in $\mathrm{kg} / \mathrm{m}^{2}$.

Flight deck floors have a specific weight of approximately $80 \%$ of a passenger cabin floor weight per unit of area.

Equipment bay floors have to be considered only if a separate equipment bay is provided. Their weight is approximately 1.58 of the gross shell weight for transport aircraft and $.5 \mathrm{lb} / \mathrm{cu} . \mathrm{ft}\left(8 \mathrm{~kg} / \mathrm{m}^{3}\right)$ of $\mathrm{fu}-$ selage volume (Ref. D-3) for other types.

\section{D-5. PRESSURE BULKHEADS AND FRAMES}

\section{D-5.1. Pressure cabin bulkheads}

Flat bulkheads in front or at the rear of the pressurized section or cabin have a weight of:

$\mathrm{w}_{\mathrm{bh}}=20+.19 \Delta \mathrm{p}^{\cdot 8} \mathrm{~s}_{\mathrm{bh}} 1.2$ (1b)

$\mathrm{w}_{\mathrm{bh}}=9.1+12.48 \Delta \mathrm{p}^{.8} \mathrm{~s}_{\mathrm{bh}}{ }^{1.2}(\mathrm{~kg})$

where $\mathrm{S}_{\mathrm{bh}}$ is the actual area of each bulkhead in sq. $\mathrm{ft}\left(\mathrm{m}^{2}\right)$ and $\Delta \mathrm{p}$ the design cabin pressure differential in $1 \mathrm{~b} / \mathrm{sq}$. in $\left(\mathrm{kg} / \mathrm{cm}^{2}\right)$. Due to their more favorable loading condition, spherical bulkheads are considerably lighter (Ref. D-6). The following approximations may be used:

$\mathrm{w}_{\mathrm{bh}}=20+.11 \Delta \mathrm{p}^{.8} \mathrm{~s}_{\mathrm{bh}}^{1.2}$ $\mathrm{w}_{\mathrm{bh}}=9.1+7.225 \Delta \mathrm{p}^{.8} \mathrm{~s}_{\mathrm{bh}}^{1.2}(\mathrm{~kg}) \quad(\mathrm{D}-15)$ where $\mathrm{S}_{\mathrm{bh}}$ is $\frac{\pi}{4}$ (diameter) ${ }^{2}$ in sq.ft or $\mathrm{m}^{2}$ and $\Delta \mathrm{p}$ is in $1 \mathrm{~b} / \mathrm{sq}$.in or $\mathrm{kg} / \mathrm{cm}^{2}$ respectively.

D-5.2. Wheelbays for retractable undercarriages

If the bays are surrounded by a pressurized section, the sidewalls, frames and roofs are pressure bulkheads; $(D-14)$ may be used for weight estimation. Alternative method (Ref. D-5):

Nose landing gear bay weight - .2828 of the MTOW plus $7 \mathrm{lb}(3.18 \mathrm{~kg})$. Nosewheel bay doors are included in this figure and should be subtracted if they have been counted previously as a "gross shell modification" (Section D-3.2).

For main landing gear wheelbays inside a pressurized fuselage (section), we have: Weight $=.015 \Delta \mathrm{p}^{.8} \mathrm{~W}_{\mathrm{G}}(\Delta \mathrm{p}$ in $\mathrm{lb} / \mathrm{sq} . \mathrm{in})$

Weight $=.125 \Delta \mathrm{p}^{.8} \mathrm{w}_{\mathrm{G}}\left(\Delta \mathrm{p}\right.$ in $\left.\mathrm{kg} / \mathrm{cm}^{2}\right)$

For other aircraft types - generally with non-pressurized fuselages - Ref. D- 3 gives:

nose landing gear bay weight $=$

$.26 \times 10^{-3} \mathrm{n}_{\text {ult }} \mathrm{w}_{\text {to }}$

main landing gear bay weight =

$10^{-3} \mathrm{n}_{\text {ult }} \mathrm{w}_{\text {to }}$

On several designs, for example high-wing airplanes, the main landing gear is suspended from the fuselage and retracts into a separate fairing outside the fuselage external lines. The weight per fairing is approximately:

Weight $=.03 \sqrt{V_{D}}$ (wetted area) 1.2 (1b)

Weight $=.328 \sqrt{V_{D}}(\text { wetted area })^{1.2}(\mathrm{~kg})$

Landing gear doors are included. The wetted area is in $\mathrm{ft}^{2}\left(\mathrm{~m}^{2}\right)$ and the Design Diving speed in $\mathrm{kts}(\mathrm{m} / \mathrm{s})$ respectively.

Cockpit bulkheads typically have a specific 
weight of $.751 \mathrm{~b} / \mathrm{sq} . \mathrm{ft}\left(3.66 \mathrm{~kg} / \mathrm{m}^{2}\right)$.

Special (mounting) frames are required at any abrupt change in cross-section where loads have to be redistributed - e.g. near intake or exhaust openings - and for the support of engine pods mounted to the sides of the rear fuselage section:

Weight $(1 \mathrm{~b})=1.2$ (area) $)^{1.2}$ - area in sq. $\mathrm{ft}$

Weight $(\mathrm{kg})=9.42$ (area) 1.2 - area in $\mathrm{m}^{2}$

\section{D-6. SUPPORT STRUCTURE}

\section{D-6.1. Wing/fuselage connection}

For low-wing transport aircraft with a continuous torque box across the fuselage, Ref. D-5 states:

Weight $=45+.907 \times 10^{-3} n_{u 1 t} w_{t o}(1 b)$

Weight $=20.4+.907 \times 10^{-3} \mathrm{n}_{\text {ult }} \mathrm{W}_{\text {to }}(\mathrm{kg})$

The expression given in Ref. D-1 generally results in a rather lower weight and is probably based on data for unpressurized fuselages:

Weight $(1 \mathrm{~b})=.345 \times 10^{-3}\left(\mathrm{n}_{\mathrm{ult}} \mathrm{w}_{t o}\right)^{1.185}-$ $w_{\text {to }}$ in $1 b$

Weight $(\mathrm{kg})=.4 \times 10^{-3}\left(\mathrm{n}_{\mathrm{ult}} \mathrm{w}_{\mathrm{to}}\right)^{1.185^{-}}$ $w_{\text {to }}$ in $\mathrm{kg}$

For high-wing configurations with continuous torque box across the fuselage, the penalty is roughly two-thirds of these values.

If the wing torsion box is not continuous, the weight penalty may be three times greater than that given by $(D-22)$. However, the wing weight is reduced accordingly (Ref. D-1).

\section{D-6.2. Engine support structure*}

The direct weight penalty is approximately

*For fuselage-mounted engines only'
2 to 38 of the bare engine weight. An indirect, but by no means negligible effect is caused by the increased fuselage bending moment on touchdown. A calculation method is not available to the author; the following expression applying to rear fuselage-mounted engines is therefore presented as a tentative suggestion.

Weight $=$ constant $\times \frac{1 e^{2}}{b_{f}} w_{t o}$

where the constant of proportionality is equal to $2 \times 10^{-4}$ when $1_{e}$ and $b_{f}$ are in $f t$, or $6.56 \times 10^{-4}$ when $1_{e}$ and $b_{f}$ are in m. Definitions of $1_{e}$ and $b_{f}$ are given in Fig. D-2.

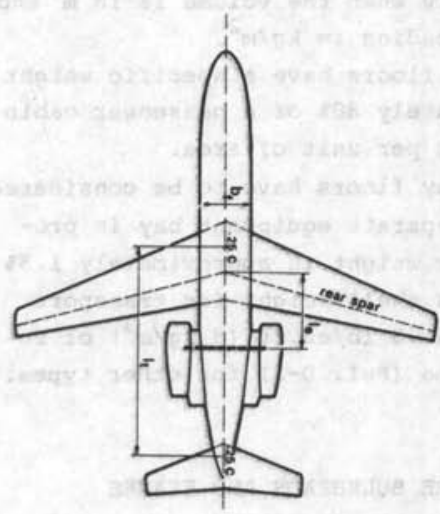

Fig. D-2. Geometrical definitions

\section{D-6.3. Other support structures}

The tailplane support structure weighs approximately 10 of of the tailplane structure group weight, or .258 of the MTOW in the case of central fin tailplane configurations.

For nose landing gears the support structure weight is included in the figure given in section D-5.2.

For wing-mounted main landing gears, but fuselage-mounted sidestays, the fuselage weight penalty is typically 58 of the main landing gear weight, or .158 of the MTOW. For fuselage-mounted main landing gears: 
Weight $=3 \times 10^{-3} \mathrm{n}_{\text {ult }} \mathrm{w}_{\text {to }}$

The tank support structure weighs approximately .2 lb per 0.S. gal. (.024 kg/liter) of tank volume for fuselage-mounted tanks.

\section{D-7. ADDITIONAL WEIGHT ITEMS}

Wing/fuselage fairings may be quite heavy sometimes of the order of 5 of of the wing structure weight. They are usually considered as part of the wing group weight.
Fairings for a double-bubble fuselage weigh about 1.58 of the gross shell weight.

For air intakes (buried engines) the duct skin is regarded as part of the engine section weight. An allowance may be required for the modification of frames and holes in the shell.

Passenger stairs weigh approximately $20 \mathrm{lb}$ per ft $(30 \mathrm{~kg} / \mathrm{m})$ extended length.

For paint, sealing and redux a weight penalty of 1 to 28 of $w_{G}$ may be assumed, while for production joints in the fuselage of a transport aircraft the weight penalty is about 2 to 38 of $\mathrm{W}_{\mathrm{G}}$.

REFERENCES

D-1. M.E. Burt and J. Philips: "Prediction of fuselage and hull structure weight". RAE Report Structures 122. April 1952.

D-2. D. Howe: "Structural weight prediction". Cranfield Institute of Technology, DES 903.

D-3. R.L. Hammit: "Structural weight estimation by the weight penalty concept for preliminary design". SAWE Technical Paper No. 141, 1956.

D-4. B.B. Coker: "Problems in airframe development associated with weight and balance control in heavy logistics transport vehicles such as the C-5A transport". Short Course, University of Tennessee, Nov. 1968.

D-5. D.M. Simpson: "Fuselage structure weight prediction". SAWE Paper No. 481, June 1973.

D-6. D.P. Marsh: "Post-design analysis for structural weight estimation". SAwE Paper No. 936, May 1972.

D-7. R.J. Atkinson: "Structural design", RAE Tech. Note Structures 133, May 1963. 



\section{Appendix E. Prediction methods for lift and pitching moment of aircraft in the en route configuration}

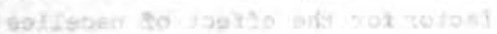

SUMMARY

A concise collection of formulas, generalized data and methods is presented for estimating the lift and pitching moment coefficients at subcritical flight speeds in the cruise configuration. All methods are readily applicable in the preliminary design stage of conventional aircraft.

Some effects of wing-fuselage interference and the trim load on the tailplane are included and conditions are derived for choosing the wing and horizontal stabilizer angles of incidence relative to the fuselage. 
- aspect $\operatorname{ratio} ; \mathrm{A}=\mathrm{b}^{2} / \mathrm{S}$ (no index: wing aspect ratio)

a.c. - aerodynamic center

B $\quad$ - number of blades per propeller

b - span; width (no index: wing span)

$c_{1} / C_{4}$ - coefficients in Diederich's method

$C_{L} \quad$ - 3-dimensional lift coefficient; $C_{\mathrm{L}}=\mathrm{L} / \mathrm{qS}$

$c_{L_{\alpha}} \quad$ - lift-curve slope; $c_{L_{\alpha}}=d c_{L} / d \alpha_{f}$

$c_{L}-c_{L}$ when the fuselage is horizontal $\left(\alpha_{f}=0\right)$

$c_{m} \quad-3$-dimensional pitching moment coefficient; $c_{m}=M / q s \bar{c}$

$c_{m a c}-c_{m}$ about an axis through the aerodynamic center

- chord

- mean aerodynamic chord (MAC) defined in Appendix A, Section A-3.3

c g mean geometric chord (SMC), defined in Appendix A, Section A-3.1

c.g. - center of gravity

$c_{\ell} \quad$ - 2-dimensional lift coefficient; $c_{\ell}=11 \mathrm{ft} /$ (unit span $x \mathrm{qc}$ )

- lift-curve slope of an airfoil section

- "additional" lift coefficient

- "basic" lift coefficient

- design lift coefficient

- 2-dimensional pitching moment coefficient; $c_{m}=$ pitching moment/ (unit span $\times \mathrm{qc}^{2}$ )

$c_{m}-c_{m}$ about the airfoil aerodynamic center

$c_{m_{1 / 4}}-c_{m}$ about the airfoll quarterchord point

$D_{p} \quad$ - propeller diameter

E - Jone's edge-velocity correction; $\mathrm{E}$ = semiperimeter/span

f - Anderson's correction factor for $\mathrm{C}_{\mathrm{L}_{\alpha}}$; Diederich's lift distribution function

G

- Anderson's factor for computing

\section{$h_{f}$ \\ 1

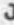

$$
\begin{aligned}
& k_{I} \\
& k \\
& k_{n} \\
& k_{s} \\
& L \\
& L_{a} \\
& 1 \\
& M \\
& m
\end{aligned}
$$$$
k_{n}, k_{p} \text { - factor for the effect of nacelles }
$$
and propellers on $x_{a c}$

$k_{s} \quad$ - correction factor for c

L $\quad-11 \mathrm{ft}$

$L_{a}, L_{b}$ - Anderson's lift functions$$
a_{\mathrm{L}}
$$$$
\mathrm{K}_{I}, \mathrm{~K}_{\mathrm{II}} \text { - factors for calculating the }
$$$$
\text { lift on the wing plus body }
$$

$k_{n}, k_{p}$ - factor for the effect of nacelles

- length; moment arm

- pitching moment; Mach number

- geometric parameter defining the vertical position of the horizontal stabilizer

q - dynamic pressure; $q=\frac{1}{2} \rho v_{0}{ }^{2}$

R - Reynolds number ; $R=V 1 / \mathrm{v}$

r - geometric parameter defining the horizontal stabilizer longitudinal position

s - area (no index: gross wing area)

$t$ - airfoil thickness, defined in Ap pendix A, Section A-3.1

$v_{0} \quad$ - flight speed

$x$ - coordinate measured from the MAC. leading edge, measured in the direction of zero lift, positive to the rear

y - spanwise coordinate, measured from the airplane centerline, positive to port

$\alpha$ - angle of attack (no index: measured relative to the wing zerolift line)

$\left(\alpha_{L_{0}}\right)_{f}$ - zero-1ift angle of attack of the wing relative to the fuselage datum liné

$\alpha_{\ell} \quad$ - zero-lift angle of an airfoil section, defined in Appendix A, Section A-2.2
- Prandtl's compressibility correction: $B=\sqrt{1-M^{2}}$

- increment; for example: $\Delta_{h} \ldots$. 
increment due to horizontal tail

- leading edge sharpness parameter of a section, defined in Fig. $\mathrm{A}-2$ of Appendix A

- aerodynamic twist; angle of downwash

- non-dimensional spanwise station; $n=y / \frac{b}{2}$

$\Lambda_{1 / 4}$ - sweep angle of quarter-chord line, defined in Appendix A, Section A-3.1

$\Lambda_{B} \quad-$ corrected sweep angle; $\tan \Lambda_{B}=$ $\tan \Lambda_{1 / 4} / B$

$\lambda$ - taper ratio (no index: wing taper ratio, defined in Appendix A, Section $\mathrm{A}-3.1$ ).

- kinematic viscosity

- air density

$\phi_{\text {TE }}^{\prime}$ - airfoil section trailing-edge angle, defined in Appendix A, Section $\mathrm{A}-2.1$, and $\mathrm{Fig} . \mathrm{A}-3$

\section{E-1. APPLICABILITY OF THE METHODS}

Apart from the simplifications occasionally made in the derivation of the methods presented, some general restrictions on the validity of the methods must be mentioned.

a. Flight speeds are subcritical, $1 . e$. shock waves are absent and compressibility effects are restricted to those that can be analyzed with subsonic potential flow theory.

b. Angles of attack are relatively small, so that the flow is predominantly nonseparated.

c. Wing aspect ratios exceed $4 / \cos \Lambda_{1 / 4}$ and wing sweep angles are less than $35^{\circ}$, approximately.

d. Only power-off conditions are considered. e. The effects of aero-elasticity are ignored.

f. Ground effect is not considered; see Appendix G, Section G-7.

The above conditions are generally met by "conventional" subsonic aircraft in most flight conditions. Other types of aircraft

\section{INDICES}

$\begin{array}{ll}\text { A-h } & \text { - aircraft minus horizontal tail } \\ \text { a } & \text { - additional lift } \\ \text { ac } & \text { - aerodynamic center } \\ \text { b } & \text { - basic lift } \\ \text { cg } & \text { - center of gravity } \\ \text { ex } & \text { - exposed } \\ \text { f } & \text { - fuselage } \\ \text { fn } & \text { - nose section of the fuselage } \\ \text { h } & \text { - horizontal tailplane } \\ \text { LE } & \text { - leading edge } \\ n & \text { - nacelle(s) } \\ \text { net } & \text { - net wing area } \\ \text { n } & \text { - neutral point } \\ \text { P } & \text { - propeller } \\ \text { r } & \text { - root } \\ \text { TE } & \text { - trailing edge } \\ \text { t } & \text { - tip } \\ \text { z } & \text { - vertical displacement of wing }\end{array}$

and types of flow can be analyzed with the USAF Stability and Control DATCOM (Ref.E-3) and the R.Ae.S. Data Sheets (Ref. E-5). In the sections which follow it is assumed that a copy of Abbott and Von Doenhoff's textbook, "Theory of wing sections" (Ref. E-14), is available to the reader.

\section{E-2. CONTRIBUTIONS TO THE LIFT}

The airplane lift is thought of as being composed of wing lift, fuselage lift, horizontal tailplane lift, nacelle lift and lift due to the powerplant installation. In this Appendix only the "clean" configuration will be considered, i.e. flaps, undercarriage, spoilers, etc. are assumed retracted.

In the preliminary design stage the airplane lift is frequently approximated by the lift on the isolated gross wing ${ }^{*}$.

*) Definition in Appendix A, Section A-3.1, and Fig. A-5. 
It is therefore assumed that the fuselage lift is roughly equal to the lift on the wing center section in the absence of the fuselage. Other contributions are ignored. However, if the fuselage width is relatively large, wing/fuselage interference effects on lift should be taken into account. Particularly in the case of a forward c.g. location the horizontal tail download may be appreciable. Methods for predicting these effects are therefore included in the present survey. The effect of nacelles on lift is generally small and difficult to predict and is frequently ignored, but the shift of the aerodynamic center due to nacelles should be taken into account. Power effects on $11 \mathrm{ft}$ and pitching moment are of vital importance to the performance and operation of propeller-driven V/STOL aircraft, but the subject is considered to be outside the scope of this appendix. It is covered thoroughly in the DATCOM method and in Refs. E-39 through E-43.

For a given Mach number the lift is a linear function of the angle of attack (see Fig. $\mathrm{E}-1$ ):

$\left.c_{L}=c_{L_{\alpha}} \mid \alpha_{f}-\left(\alpha_{L_{o}}\right)_{f}\right\}$

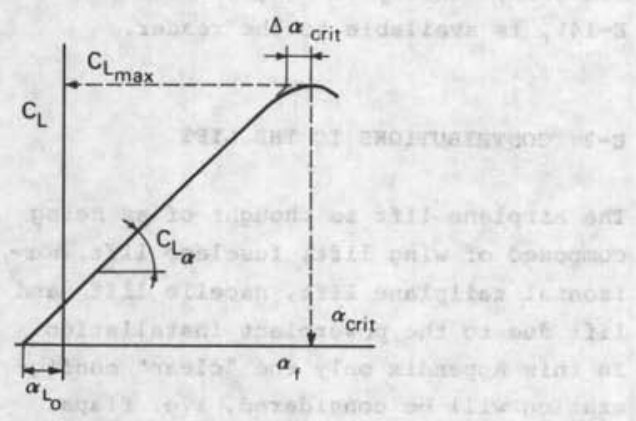

Fig. E-1. General shape of the lift curve

This expression is valid up to angles of attack approaching the critical angle of attack. Except insofar as it may give him some idea of certain stalling properties (Ref. $\mathrm{E}-7$ ), the $\mathrm{C}_{\mathrm{L}}$ vs. $\alpha$ curve in the stalling region is generally of little concern to the designer in the pre-design stage. It is usually found that $\Delta \alpha_{\text {crit }}$ is between $1^{\circ}$ and $3^{\circ}$ (see Fig. E-1). The power-off airplane lift is

$c_{L}=\left(c_{L}\right)_{A-h}+c_{L_{h}} \frac{s_{h}}{s} \frac{q_{h}}{q}$

where the first contribution is due to the wing, fuselage, nacelles and (windmilling or feathered) propeller(s), i.e. the aircraft minus the horizontal tallplane. The second term is a correction for the tailplane $11 \mathrm{ft}$.

E-3. LIFTING PROPERTIES OF AIRFOIL SECTIONS

Section properties may form a basis for estimating the wing characteristics with a reasonable degree of accuracy. For several standard airfoil sections these characteristics may be obtained from Refs. E-12 through E-16 and many other NACA publications. An example is given in Fig. $\mathrm{B}-2$. For non-standard airfoils the DATCOM presents a generalized method based on the leading edge sharpness parameter $\Delta y$ defined in Appendix A, Fig. A-2.

\section{E-3.1. The zero-lift angle}

The zero-lift angle may be computed from potential flow methods or empirical data: four-digit NACA airfoils: $\alpha_{\ell}=-(8$ wing camber) (deg.)

five-digit NACA airfoils: $\alpha_{\ell_{0}}=-4 c_{\ell_{1}}$ (deg.) six -digit NACA airfoils: $\alpha_{\ell_{0}}=-6.6 c_{l_{1}}$
(deg.)

\section{E-3.2. Lift-curve slope}

According to the DATCOM method, the $11 \mathrm{ft}-$ curve slope is given by:

$c_{l_{\alpha}}=\frac{1.05}{\beta}\left[\frac{{ }_{l_{\alpha_{1}}}^{c_{1}}}{\left(c_{l_{\alpha_{2}}}\right)_{\text {theory }}}\left(c_{l_{\alpha}}\right)_{\text {theory }}\right.$ 


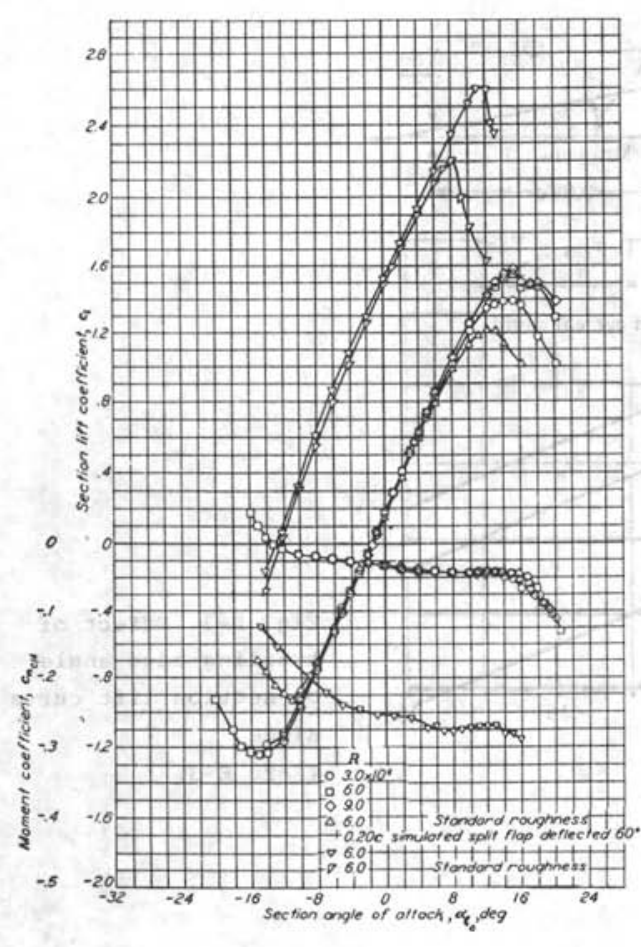

Fig. E-2. Experimental data on the NACA 64215 airfoil section (Ref. E-14)

$$
\begin{array}{r}
\left(c_{\ell}\right)_{\alpha_{\text {theory }}}=2 \pi+4.7 t / c\left(1+. .00375 \phi_{\mathrm{TE}}^{\prime}\right) \\
\left(\operatorname{rad}^{-1}\right)
\end{array}
$$

data. Instead of presenting a generalized method, Fig. E-4 is proposed as a guideline to the designer who is interested in the highest value of $c_{\ell}$-max that can pos sibly be achieved for a given $t / c$ and chord Reynolds number, provided the section is suitably cambered and an optimum nose shape is chosen. An envelope of $c_{\ell}$-max values for NACA sections is shown on which the following comments may be made:

a. For $t / c<108$ the $c_{\ell}$-max envelope is not very sensitive to Reynolds effects.

b. For $t / c<12$ \& leading edge stall predominates. The leading edge sharpness parameter $\Delta y$, defined in Fig. A-2 of Appendix A, may be used to predict $c_{\ell}$-max using the DATCOM method.

c. For $t / c>128$ trailing edge stall is predominant and $c_{\ell}$-max is sensitive to Reynolds number effects.

d. High $c_{l}-\max$ values are obtained with 5digit series airfoils. Laminar flow air foils with $c_{\ell_{1}}{ }^{2} .4$ and the maximum thickness not too far aft have $c_{l}$-max values some $.08-.12$ lower.

e. The ultimate $c_{\ell}-\max$ for airfoils of the standard NACA series appears to be approx. 1.8 for the NACA 23012 airfoil at $R=9 \times 10^{6}$. Higher values are possible with special airfoil designs, e.g. recent experiments with a 178 thick supercritical rear loading section indicate that even values slightly above 2.0 are possible (Ref. E-18).

$\phi_{\mathrm{TE}}^{\prime}=$ section trailing-edge angle (degrees), defined in Fig. E-3.

E-4. WING LIFT AND LIFT DISTRIBUTION

See also Fig. A-3 of Appendix A.

For the ratio of experimental to theoretical $c_{\ell_{\alpha}}$ for boundary layer transition near the leading edge, see Fig. E-3. For thickness/chord ratios between $10 \%$ and 208 , $c_{\ell}=6.1$ per radian is a representative value. Ref. E-5 gives a method for transition at 508 chord.

E-3.3. Maximum lift

\section{E-4.1. Lift-curve slope}

a. For straight wings $\left(\Lambda_{1 / 4}=0^{\circ}\right)$ in incompressible flow, according to Ref. E-20: $C_{L_{w_{\alpha}}}=f \frac{c_{\ell_{\alpha}}}{E+C_{\ell_{\alpha}} / \pi A}$

where $f$ is a correction factor for wing taper, shown in Fig. 8 of Ref. E-14. For $.2<\lambda<1.0 \mathrm{f}$ may be assumed equal to .995 . Jone's edge velocity factor $\mathrm{E}$ is equal to

The best approach is to use experimental 


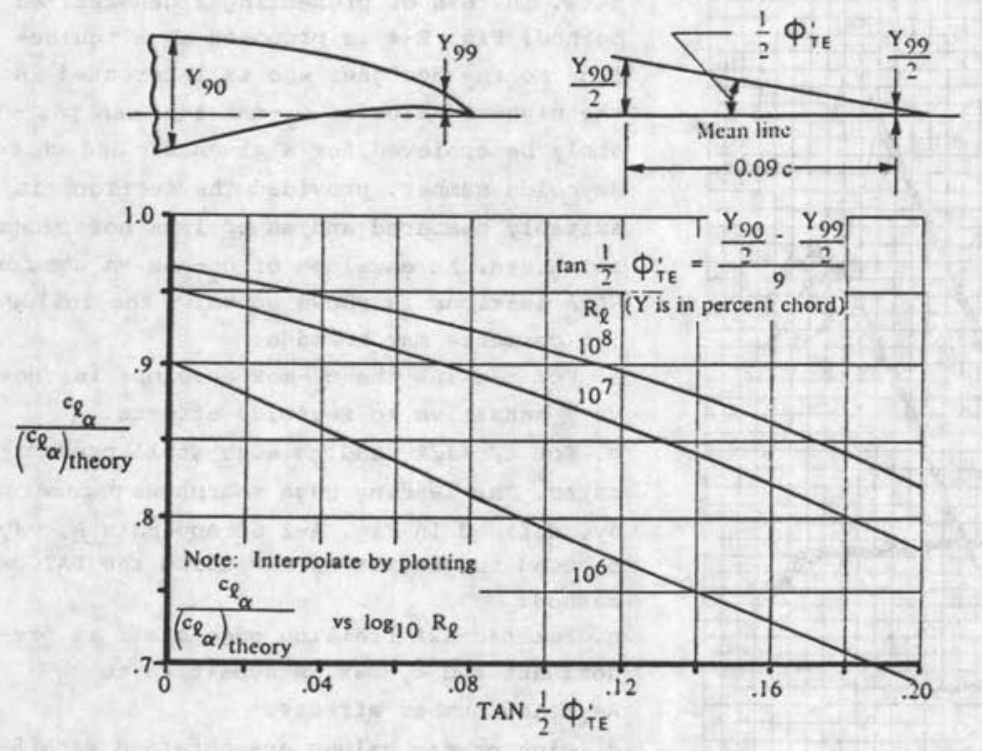

Fig. E-3. Effect of trailing-edge angle on section lift curve slope

(Ref. B-3)
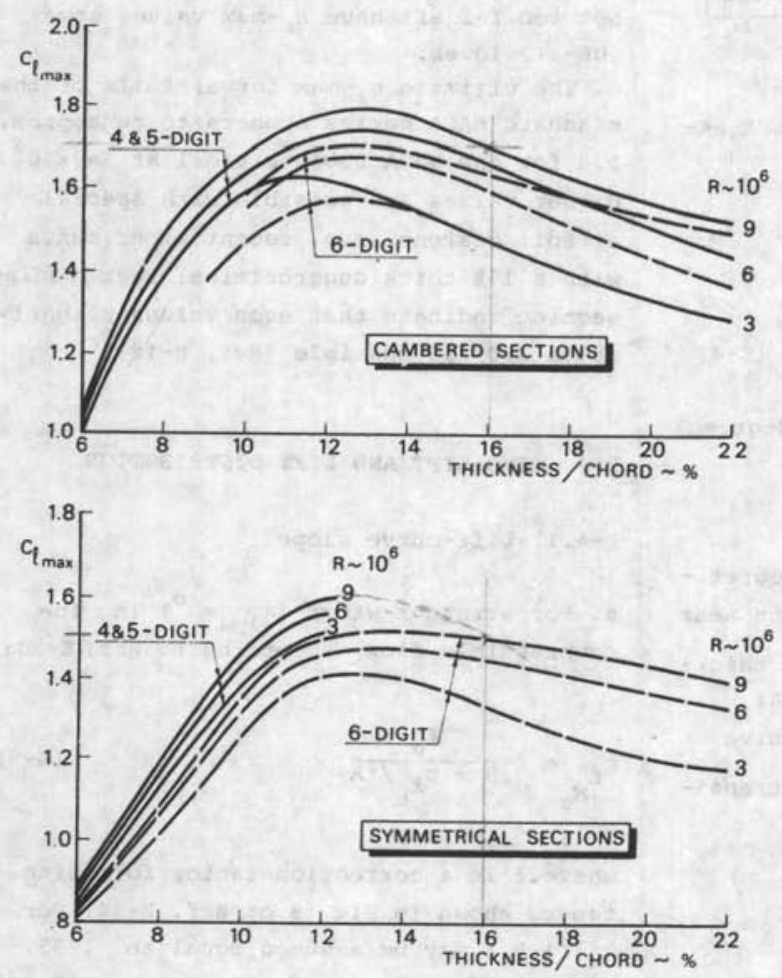

Fig. E-4. Envelopes of $c_{\ell}$-max values obtainable with NACA standard airfoils

(composed from experimental data in Ref. $\mathrm{E}-14$ ) 
the ratio of the planform semiperimeter to the span of the wing. For straight-tapered wings a reasonably accurate approximation is:

$E=1+\frac{2 \lambda}{A(1+\lambda)}$

For a 2-dimensional lift-curve slope of $2 \pi /$ radian:

$C_{L_{w_{\alpha}}}=\frac{2 \pi}{1+\frac{2}{A} \frac{1+2 \lambda}{1+\lambda}}$

$$
\left(\operatorname{rad}^{-1}\right)
$$

The effect of compressibility may be incorporated by replacing $A$ bij $B A$ and $C_{L_{w_{\alpha}}}$ by $B C_{L_{w_{\alpha}}}$.

b. Swept wings, compressible flow (DATCOM method).

$$
B C_{L_{w_{\alpha}}}=\frac{2 \pi}{\frac{2}{B A}+\sqrt{\frac{1}{k^{2} \cos ^{2} \Lambda_{\beta}}+\left(\frac{2}{B A}\right)^{2}}}\left(\operatorname{rad}^{-1}\right)(E-8)
$$

where $\tan \Lambda_{\beta}=\frac{\tan \Lambda_{\frac{1}{2}}}{\beta}$

and

$$
k=\frac{B c_{\ell}}{2 \pi} \quad\left(c_{\ell} \operatorname{rad}^{-1}\right)
$$

As compared to results from lifting surface theory (Ref. E-31), (E-8) yields good results for $\Lambda_{B}>30^{\circ} ; C_{L_{w_{\alpha}}}$ is overestimated by $\sim 48$ for $\Lambda_{B}=0^{\circ}$ and by $\sim 28$ for $\Lambda_{B}=20^{\circ}$.

\section{E-4.2 Spanwise lift distribution}

The lift may be divided into additional and basic lift:

$$
c_{\ell}=c_{\ell}+c_{\ell}
$$

In terms of the non-dimensional parameters
$\mathrm{L}_{\mathrm{a}}$ and $\mathrm{L}_{\mathrm{b}}$ used by Anderson:

$c_{\ell} \frac{c}{c_{g}}=L_{a} c_{L}+\frac{\varepsilon_{t} c_{\ell}}{E} L_{b}$

$$
L_{a}=\frac{c_{l_{a}} c}{C_{L} c_{g}}
$$

$$
L_{b}=\frac{c_{l_{b}}}{c_{g}} \frac{E}{\varepsilon_{t} c_{\ell_{\alpha}}}
$$

The definition of wing twist $\varepsilon_{t}$ is explained in Appendix A, Section A-3.2. Anderson presents tables for $L_{a}$ and $L_{b}$ for straight-tapered wings with linear twist in incompressible flow. The following more general semi-empirical method by Diederich (Ref. E-21) yields satisfactory results for pre-design purposes. It is valid for wings with arbitrary planform and lift distribution, provided the quarter-chord line of a wing half is approximately straight. This method can thus be used for straight and swept wings in compressible, subcritical flow.

a. Additional lift distribution.

$L_{a}=c_{1} \frac{c}{c_{g}}+c_{2} \frac{4}{\pi} \sqrt{1-n^{2}}+c_{3} f$

For coefficients $C_{1}, c_{2}$ and $c_{3}$, see Fig. $\mathrm{E}-5$; for lift distribution function $\mathrm{f}$, see Fig. E-6. For straight wings $f$ is elliptical and (E-13) can be simplified to:

$$
\begin{aligned}
L_{a}=C_{1} \frac{c}{c_{g}}+\left(C_{2}+C_{3}\right) \frac{4}{\pi} \sqrt{1-n^{2}} \\
\text { for } \Lambda_{1 / 4}=0^{\circ}
\end{aligned}
$$

This result is similar to the well-known approximation by Schrenk (Ref. E-19), provided $c_{1}=C_{2}+c_{3}=.5$. 


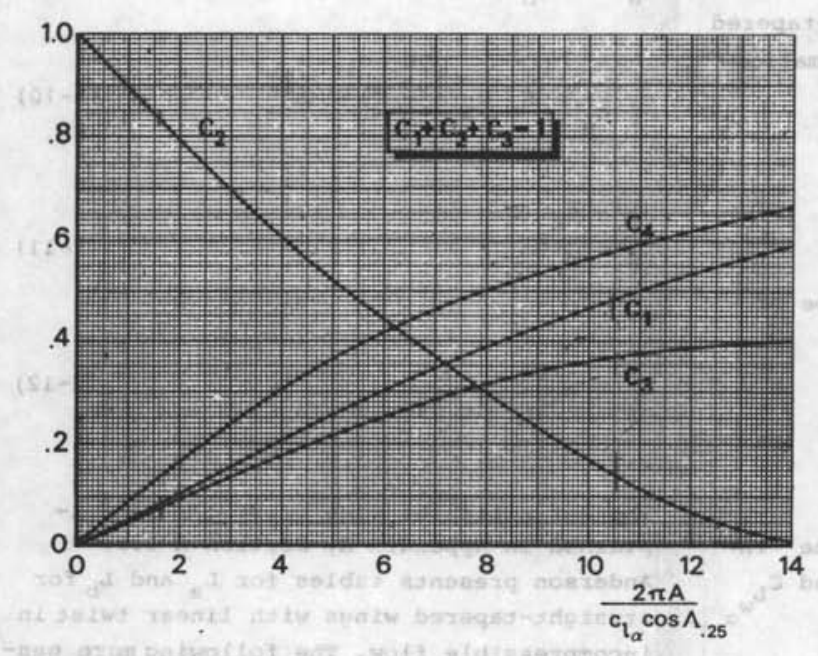

Fig. E-5. Factors in

Diederich's method (Ref. E-21)

b. Basic lift distribution.

The formula derived by Diederich in Ref. E-21 may be modified to a form similar to that used by Anderson:

$\frac{L_{b}}{B E}=L_{a} C_{4} \cos \Lambda_{B}\left(\frac{\varepsilon}{\varepsilon_{t}}+\alpha_{o_{1}}\right)$

where $\alpha_{o_{1}}=-\int_{0}^{1} \frac{\varepsilon}{\varepsilon_{t}} L_{a} d \eta$

and the factor $\mathrm{C}_{4}$ (Fig. E-5) is identical to the product of $k_{0}$ and $k_{1}$ used in Ref. $E-21$. The factor $\alpha_{0}$ is equal to the local aerodynamic twist at the spanwise station for which $c_{\ell_{b}}=0$, assuming a wingtip twist angle of one degree relative to the root. For the case of a linear twist*) distribution $\left(\varepsilon=n \varepsilon_{t}\right)$ and elliptic $L_{a}, \alpha_{0_{1}}=4 / 3 \pi$. For straight-tapered unswept wings with linear twist distribution, (E-16) can be evaluated with $L_{a}$ in $(E-14)$ to yield:

$-\alpha_{o_{1}}=c_{1} \frac{1+2 \lambda}{3(1+\lambda)}+\left(c_{2}+c_{3}\right) \frac{4}{3 \pi}$

$(\mathrm{E}-17)$

*) Definition in Section A-3.2 of Appendix A.

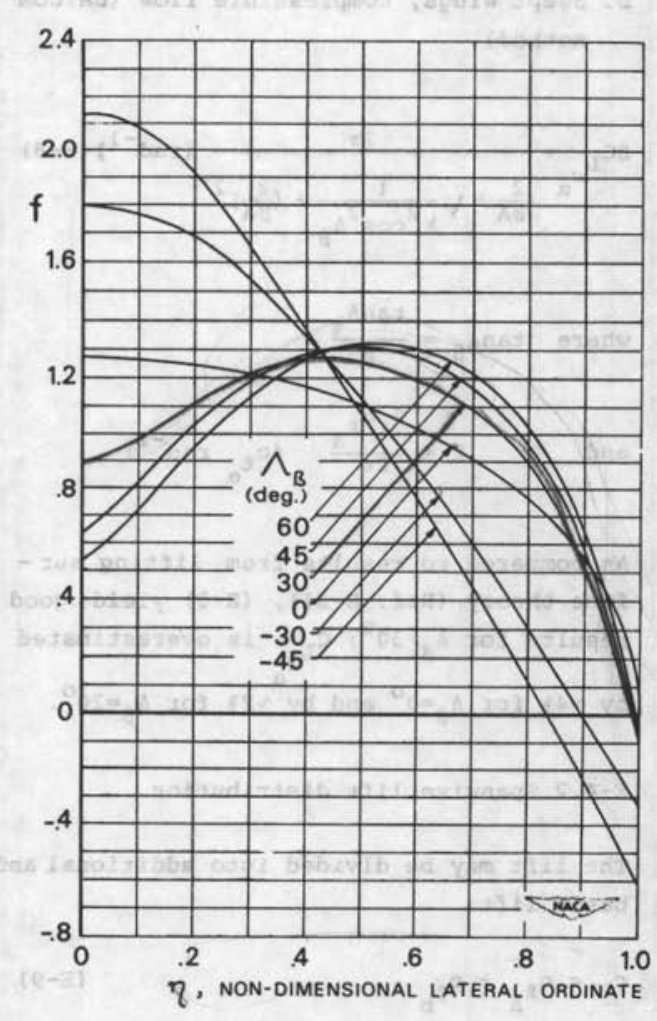

Fig. E-6. The lift distribution function $f$ (Ref. E-21) 
The factor $\alpha_{0}$ is very similar to Anderson's factor $J$ for straight wings with rounded tips (Ref. $E-20$, but see also Ref. E-14).

For straight wings with linear lofted twist*) Fig. E-7 can be used. This diagram is based on results of lifting surface theory, reported in Ref. $\mathrm{E}-31$, from which it can also be concluded that relative to Fig. $\mathrm{E}-7, \alpha_{0_{1}}$ for swept wings should be reduced by approximately .0006 per degree of $\Lambda_{\beta}$. For arbitrary twist distributions $\alpha_{0}$ must be computed numerically.

E-4.3. Zero-1ift angle

The lift coefficient in the linear range is $c_{L_{w}}=C_{L_{w}}\left\{\alpha_{r}-\left(\alpha_{\ell_{0}}\right)-\alpha_{o_{1}} \varepsilon_{t}\right\}$

The angle of attack $\alpha_{r}$ is defined relative to the root chord, while the zero-lift angle of attack is :

*) Definition in Section A-3.2 of Appendix A.
$\left(\alpha_{L_{0}}\right)=\left(\alpha_{\ell_{0}}\right)+\alpha_{o_{1}} \varepsilon_{t}$

For the root section $\alpha_{\ell_{0}}$ can be obtained from airfoil characteristics (Section E-3). Note that for washout $\varepsilon_{t}$ is negative.

E-4.4. Maximum 1ift

The basic procedure for estimating the maximum lift of high aspect-ratio straight wings is explained in, for example, Refs. E-14 and E-32. Abbott and Von Doenhoff's summary is quoted here:

"The maximum lift coefficient of the wing may be estimated from the assumption that this coefficient is reached when the local section lift coefficient at any position along the span is equal to the local $c_{\ell}$ max for the corresponding section. This value may be found conveniently by the process indicated in Fig. B-8. Spanwise variations of the local $c_{\ell}-\max$ and of the additional $c_{\ell_{a}}$ for $c_{L}=1$ and $c_{\ell_{b}}$ distributions are plotted. The spanwise variation of $c_{\ell}$-max minus $c_{\ell_{b}}$ is plotted and

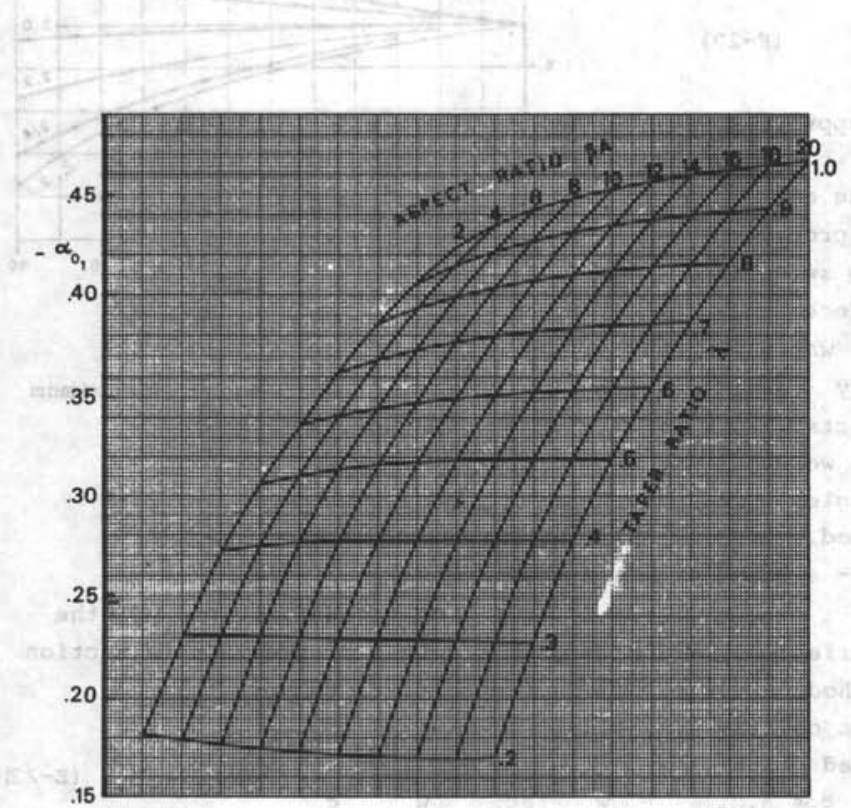

Fig. E-7. Zero-lift angle of attack per unit of twist for straight wings (Ref, E-31) 


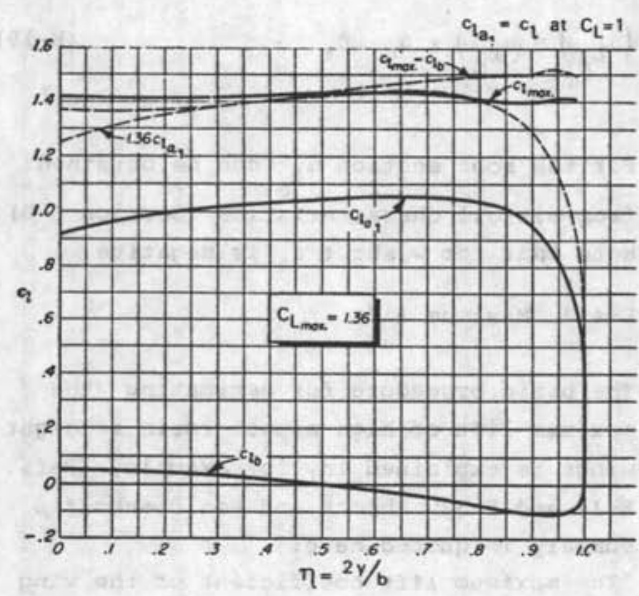

Fig. E-8. Example of lift distribution for a straight wing (Ref. E-14)

the minimum value of the ratio of $\left(c_{l_{\max }}\right.$ $c_{l_{b}}$ ) to $c_{l_{a}}$ at $c_{L}=1$ is then found.

This ratio is considered to be the maximum lift coefficient $C_{L}$-max of the wing."

A simpler but less accurate approach is:

$c_{L_{\max }}=k_{s} \frac{\left(c_{\ell_{\max }}\right)+\left(c_{\ell_{\max }}\right)}{2}$

where $k_{s}=.88$ for $\lambda=1$ and .95, approxi mately, for tapered wings.

This expression does not take the effects of wing twist into account. The procedure described loses validity as wing sweep is increased, mainly due to the effects of the spanwise pressure gradients, which cause crossflows in the boundary layer. In spite of this, Callaghan reports in Ref. E-11 that the same approach works quite well for moderate sweep angles. The following procedures are suggested.

a. Twisted swept wings, airfoil - section variation along the span.

The basic and additional lift variations are computed by any suitable method, e.g. $(E-13)$ and $(E-15)$. The total lift coefficient for each section is compared with the corresponding value of $c_{\ell} \cos \Lambda_{1 / 4}$ and the procedure described previously for straight wings is applied to estimate $C_{L}$-. max. Alternatively, $(E-20)$ may be corrected by multiplication by $\cos \Lambda_{1 / 4^{*}}$.

b. Untwisted, constant airfoil-section swept wings.

$C_{L}$-max is derived from the section $c_{l}$-max with Fig. E-9. The parameter $\Delta y$ may be obtained from Fig. A-2 of Appendix A. Alternatively:

$c_{L_{\text {max }}}=0.9 c_{l_{\text {max }}} \cos \Lambda_{1 / 4}$ for $t / c>0.12$

Compressibility affects $C_{L}-\max$ at Mach numbers above .2 approximately. The DATCOM method (Fig. 4.1.3.4-15) or Ref. E-29 can be used to estimate the order of magnitude of this effect.

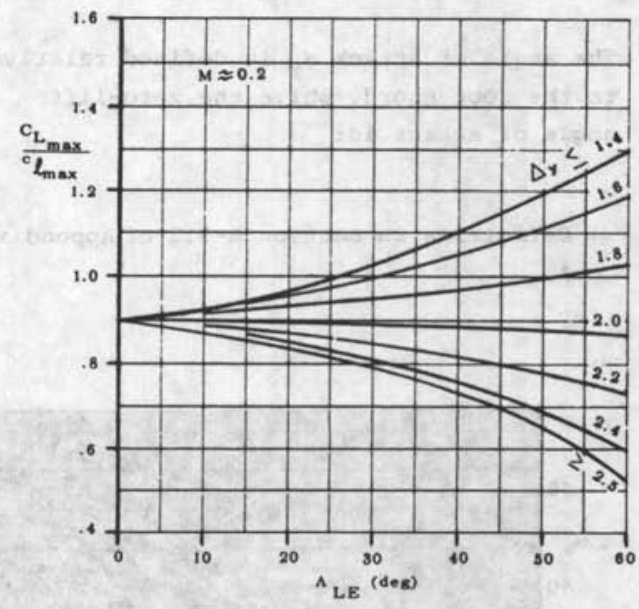

Fig. E-9. Effect of sweep angle on maximum lift (DATCOM method, Ref. E-3)

E-5. PITCHING MOMENT OF THE WING

It follows from the definition of the aerodynamic center of the wing in section A-3.3 of Appendix A that:

$c_{m_{w}}=\left(c_{m_{a c}}\right)+c_{L_{w}} \frac{x_{c g}-\left(x_{a c}\right)_{w}}{\bar{c}}$ 


\section{E-5.1. Aerodynamic center}

Location of the aerodynamic center, relative to the MAC leading edge: see Fig.E-10. This diagram has been compiled from the results of lifting surface theory. The MAC can be computed with the data of Appendix A, Section A-3.3.

E-5.2. Pitching moment $\left(\mathrm{C}_{\mathrm{mac}_{\mathrm{w}}}\right)$

$\left(c_{m_{a c}}\right)_{w}=\left(c_{m_{a c}}\right)_{\text {basic }}+\Delta_{\varepsilon} c_{m_{a c}}$

The first term is the contribution of the spanwise airfoil-section camber distri bution:

$$
\left(c_{m_{a c}}\right)_{\text {basic }}=\frac{2}{s \bar{c}} \int_{0}^{b / 2} c_{m_{a c}} c^{2} d y
$$

The value of $c_{m_{a c}}$ can be obtained from potential flow theory or from experimental data on airfoils. It is primarily sensitive to the shape of the mean line. For a constant airfoil-section wing $\left(\mathrm{c}_{\mathrm{m}_{\mathrm{ac}}}\right)$-basic $=\mathrm{c}_{\mathrm{mac}}$.

The last term in $(\mathrm{E}-23)$ is the contri bution of the basic lift distribution due to twist:

$$
\Delta_{\varepsilon} c_{m a c}=-\frac{2}{s \bar{c}} \int_{0}^{b / 2} c_{l_{b}} c y \tan \Lambda_{a c} d y
$$

Assuming that all section aerodynamic centers are located on a straight quarter chord line:

$\Delta_{\varepsilon} c_{m_{a c}}=-\frac{1}{2} A \frac{c_{g}}{\bar{c}} \tan \Lambda_{1 / 4} \int_{0}^{1} c_{l_{b}} \frac{c}{c_{g}} n d n$

The ratio $c_{g} / \bar{c}=S M C / M A C$ may be obtained from Fig. A-8 of Appendix A.

a. For arbitrary twist distribution, the integral must be solved numerically, using the basic lift distribution obtained from any suitable method, e.g. Diederich's. b. For linear twist distribution, straighttapered wings with rounded tips in incom pressible flow, Anderson has evolved (E-25) to:

$\Delta_{\varepsilon} C_{m a c}=-G \frac{c_{g}}{\bar{c}} \frac{{ }^{\varepsilon_{t} c_{l}}}{E} A \tan \Lambda_{1 / 4}$

A diagram to compute $\mathrm{G}$ can be found on page 20 of Ref. E-14. This result applies to small sweep angles only, since the effect of wing sweep on the lift distribution ist not considered.

c. For linear lofted straight-tapered wings Kapteyn presents diagrams in Ref. B-31, based on lifting-surface theory (Ref. E-30). The result can be generalized as follows:

$$
\frac{-\beta\left(\Delta_{\varepsilon} C_{m_{a c}}\right)}{\varepsilon_{t} C_{L_{w_{\alpha}}}(B A) \tan \Lambda_{B}}=
$$

$$
.0066+.029 \lambda-.03 \lambda^{2}+.00273(\lambda-.095)(B A)
$$

E-6. WING/FUSELAGE INTERFERENCE EFFECTS ON LIFT

The major aerodynamic interference effects between the wing and the non-cambered fuselage are (Fig. E-11):

a. In inviscid flow the resultant force on a closed body is zero. In viscous flow and in the presence of the lifting wing, how ever, the lift on the body nose, which is in the upwash field, is counteracted only partly by the download on the rear fuselage, which is in the downwash behind the wing. b. The flow component normal to the fuselage axis (crossflow) induces increased effective angles of attack of the wing sections, particularly near the wing/fuselage junction.

c. There is a wing lift carry-over into the body, although this lift component is less than the lift that would be produced by the wing section replacing the fuselage if the latter were absent.

d. Vertical displacement of the wing relative to the fuselage centerline alters the 


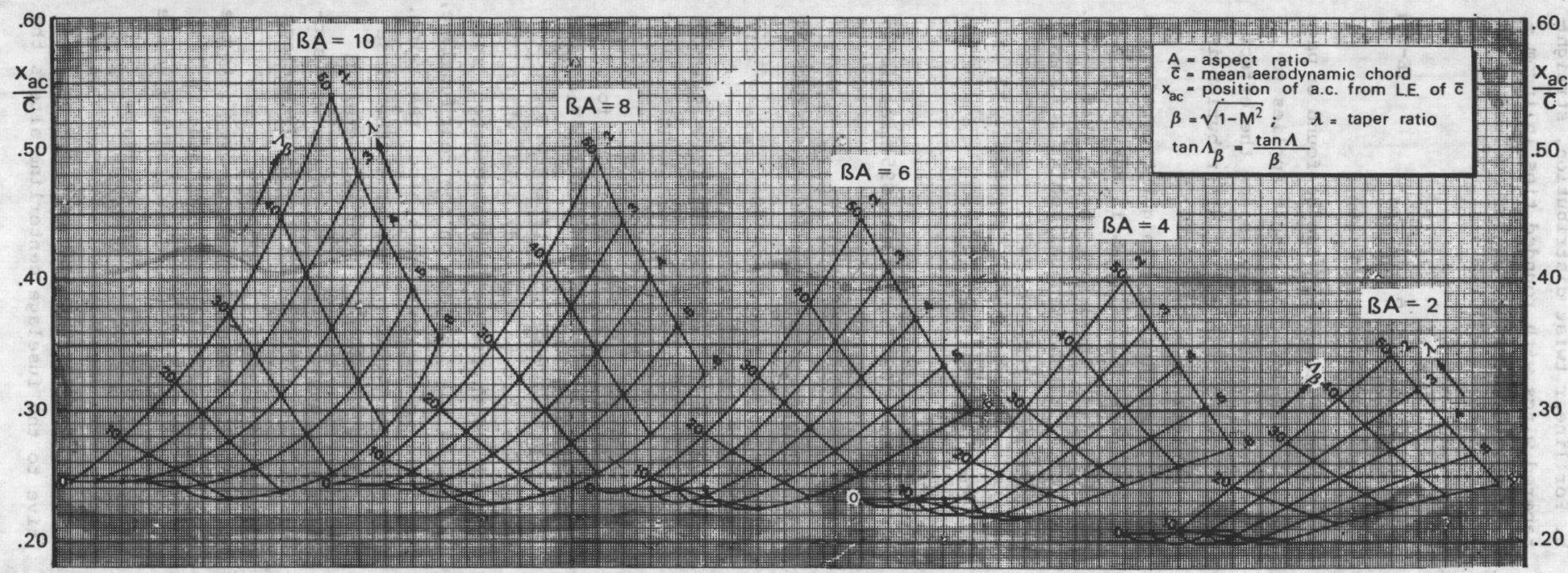

Fig. E-10. Aerodynamic center of lifting surfaces at subsonic speeds (Ref. E-31).

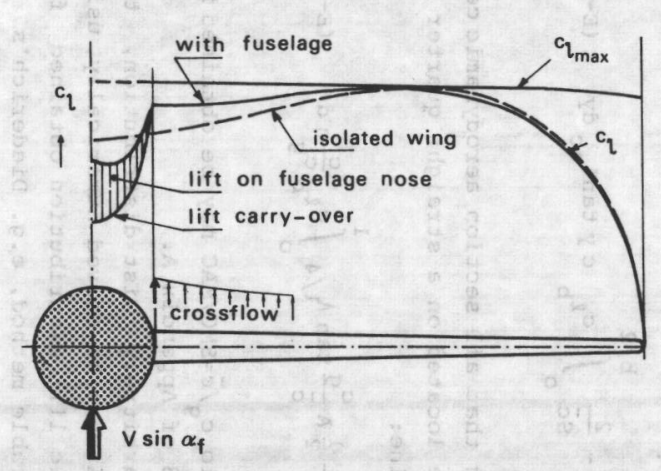

Fig. E-11. Fuselage/wing interference effects on the spanwise lift distribution.

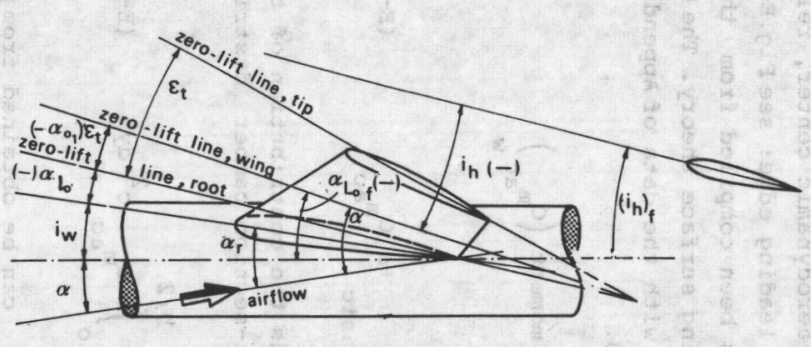

Fig. E-12.Geometric definitions of the wing/body/tail combination. 
flow pattern, as explained by Hoerner (Ref. E-38, p. 8-17). This results in a lift reduction for high-wing and a lift increment for low-wing configurations $\left(\Delta_{z} C_{L}\right)$.

The lift components can be added together to obtain the total wing fuselage lift:

$c_{L_{w f}}=\left(c_{L_{\alpha}}\right)\left[\left(\alpha_{f f}-\alpha_{o_{1}} \varepsilon_{t}\right)+\frac{K_{I I}}{K_{I}}\left\{i_{w}-\left(\alpha_{\ell}\right)\right\}\right]+$

$$
+\Delta_{z} C_{L}
$$

The various angles are defined in Fig. $\mathrm{E}-12$. The wing/body incidence $i_{w}$ is treated in Section E-9, while the lift-curve slope of the wing; body combination is:

$$
\left(c_{L_{\alpha}}\right)_{\text {wf }}=K_{I} c_{L_{w_{\alpha}}}
$$

$K_{I}=\frac{\left(\partial C_{L} / \partial_{\alpha}\right)_{w f} \text { for } 1_{w}=\text { const. }}{C_{L_{w_{\alpha}}}}$

$$
K_{I I}=\frac{\left(\partial C_{L} / \partial i_{w f}\right)_{w f} \text { for } \alpha_{f}=\text { const. }}{C_{L_{w}}}
$$

For $\Delta_{z} C_{L}$ Hoerner states:

$$
\begin{aligned}
\Delta_{\mathrm{z}} \mathrm{C}_{\mathrm{L}} \mathrm{S} / \mathrm{c}_{\mathrm{r}} \mathrm{b}_{\mathrm{f}}= & -.1: \text { high wing } \\
& 0: \text { mid wing } \\
& +.1: \text { low wing }
\end{aligned}
$$

The net wing area is defined in

Appendix A, Section A-3.1. A method to estimate $K_{I}$ and $K_{I I}$ can be found in Ref.E-37. For fuselages with near-circular cross section, with $b_{f} / b<.2$, this method can be approximated by:

$K_{I}=\left(1+2 \cdot 15 \frac{b_{f}}{b}\right) \frac{S_{n e t}}{S}+\frac{\pi}{2 C_{L_{w}}} \frac{b_{f}^{2}}{S}$

and

$K_{I I}=\left(1+\cdot 7 \frac{b_{f}}{b}\right) \frac{S_{\text {net }}}{S}$

The net wing area $\mathrm{s}_{\text {net }}$

is defined as the projection of the part of the wing outside the fuselage, assuming a mid-wing configuration with the same gross area (cf. Appendix A, Section A-3.1). For fuselages with swept-up tails, the flow pattern is considerably more complicated (cf. Section 3.5.1) and the previous methods have very limited value.

All interference effects treated thus far are potential-flow effects and must therefore be considered as "minimum effects" valid for small angles of attack. At high angles of attack separation near the wing/ fuselage junction may result in a reduction of $\iota_{L}{ }^{-m a x}$. These effects may be minimized by suitable filleting, adaptation of the planform shape (e.g. a locally extended root chord), suitable twist and airfoil section modification. From Fig. E-11 it can be concluded that fuselage effects may be small, provided the separation on the isolated wing occurs on the outer wing first. The presence of the fuselage may improve the stalling characteristics by shifting the separation more to the inboard wing. No general rule for estimating the body effect on $C_{L}-\max$ can be given. In preliminary design, therefore, the wing/fuselage $C_{L}-\max$ is frequently assumed equal to that of the wing. The critical angle of attack is modified accordingly. This assumption may be somewhat conservative in the case of a high-wing location and optimistic for mid-wing configurations.

\section{E-7. WING/FUSELAGE PITCHING MOMENT}

The pitching moment equation for the wing/ fuselage combination is written as follows: $\left(c_{m}\right)_{w f}=\left(c_{m_{a c}}\right)_{w f}+c_{L_{w f}} \frac{x_{c g}-\left(x_{a c}\right)_{w f}}{\bar{c}} \quad(E-35)$

\section{E-7.1. Aerodynamic center}

The a.c. location of the wing/body combination is sensitive to the pressure distribution and not to the integrated lift 


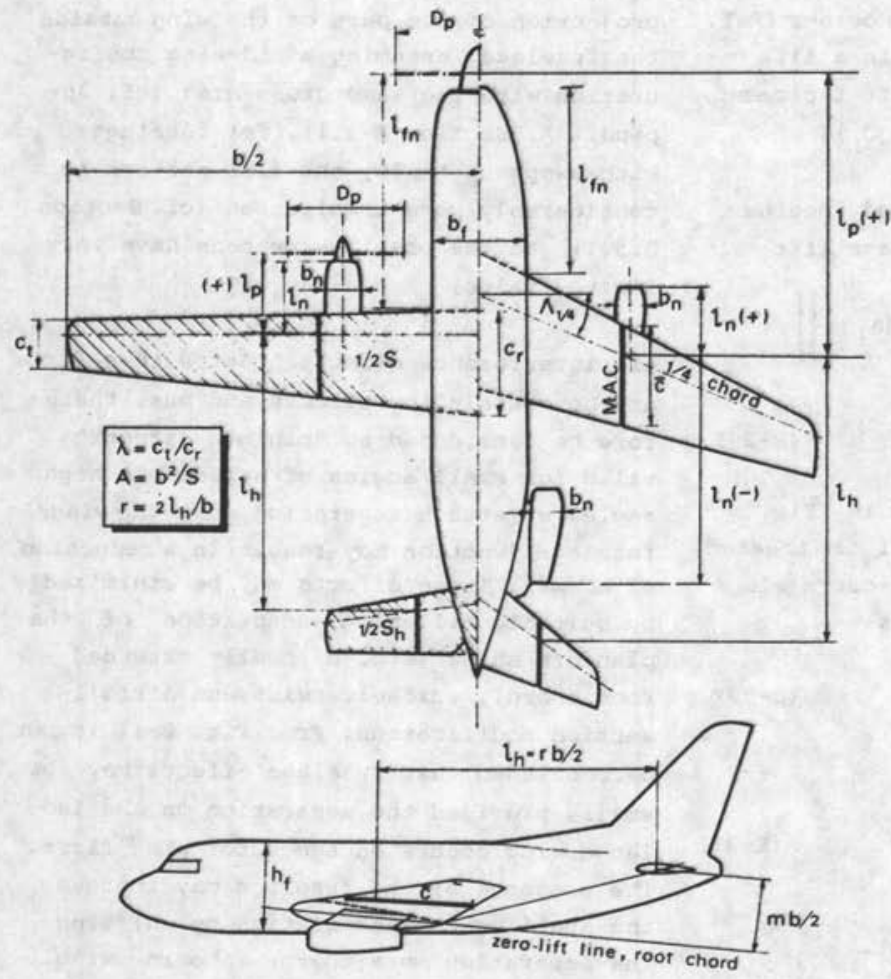

F1g. E-13. Wing/body/tail geometry

force and it is therefore difficult to predict accurately. Only two corrections to the wing a.c. location will be considered here:

$\left(\frac{x_{a c}}{\bar{c}}\right)_{w f}=\left(\frac{x_{a c}}{\bar{c}}\right)_{w}+\frac{\Delta_{f 1} x_{a c}}{\bar{c}}+\frac{\Delta_{f 2} x_{a c}}{\bar{c}}$

The correction $\Delta_{f 1} x_{\text {ac }}$ represents the forward shift due to the fuselage sections forward and aft of the wing. The nose in particular contributes to this shift. Based on experimental data in Ref. E-6:

$\frac{\Delta_{f 1} x_{a c}}{\bar{c}}=-\frac{1.8}{\left(C_{L_{\alpha}}\right)_{w f}} \frac{b_{f} h_{f}{ }^{1} f n}{s \bar{c}}$

For geometric definitions see Fig. E-13. The correction $\Delta_{f 2} x_{a c}$ accounts for the lift loss in the region where the wing/fuselage lift carry-over is concentrated and is derived from the DATCOM method:

$$
\begin{gathered}
\frac{\Delta f_{2} x_{a c}}{\bar{c}}=\frac{.273}{1+\lambda} \frac{b_{f} c_{g}\left(b-b_{f}\right)}{(\bar{c})^{2}\left(b+2.15 b_{f}\right)} \tan \Lambda_{1 / 4} \\
\left(\text { for } \frac{b_{f}}{b}<.2\right)
\end{gathered}
$$

E-7.2 Pitching moment $\left(\mathrm{c}_{\mathrm{m}_{\mathrm{ac}}}\right)_{\mathrm{wf}}$

$$
\left(c_{m_{a c}}\right)_{w f}=\left(c_{m_{a c}}\right)+\Delta_{f} c_{m}
$$

The fuselage contribution $\Delta_{f} c_{m a c}$ can be obtained from Munk's theory (Ref. E-33). For fuselages with near-circular cross-sections: $\Delta_{f} C_{m_{a c}}=-1.8\left(1-\frac{2.5 b_{f}}{I_{f}}\right) \cdot \frac{\pi b_{f} h_{f}{ }_{f}}{4 s \bar{c}} \frac{C_{L_{o}}}{\left(C_{L_{\alpha}}\right)_{w f}}$

where $C_{L_{0}}$ is $C_{L}$ for $\alpha_{f}=0$ (cf. Section E-9.3) 
and $C_{L}$ is per radian. If the cross-section is not ${ }^{\alpha}$ ear-circular, $\Delta_{f} c_{m_{a c}}$ should be multiplied by the ratio of the actual crosssection area to $(\pi / 4) \mathrm{b}_{\mathrm{f}} \mathrm{h}_{\mathrm{f}}$.

\section{E-8. NACELLE AND PROPELLER CONTRIBUTIONS}

Some aerodynamic effects of engine nacelle installation are discussed qualitatively in Section 6.5. Accurate prediction of these effects is generally not possible in the preliminary design stage. It is therefore suggested that only the effects in the aerodynamic center location be estimated for each nacelle separately and then added:

$\Delta_{n} \frac{x_{a c}}{\bar{c}}=\sum k_{n} \frac{b_{n}^{2}{ }^{1} n}{s \bar{c}\left(c_{L_{\alpha}}\right)_{w f}} \quad\left(c_{L_{\alpha}}\right.$ in $\left.\operatorname{rad}^{-1}\right)$

where $k_{n}{ }^{2}-4.0$ for nacelles mounted in front of the LE of the wing

$$
\begin{aligned}
k_{n} \sim-2.5 & \text { for jet engine pods mounted } \\
& \text { to the sides of the rear } \\
& \text { fuselage. }
\end{aligned}
$$

The geometry is defined in Fig. E-13. The distance $1_{n}$ is positive when the nacelle is in front of, negative when behind 1/4 MAC.

Propeller effects can be very considerable in the power-on configuration; power-off effects on lift are small. Huwever, lift on a windmilling propeller at incidence causes a shift in the a.c. For windmilling tractor propellers the data given by Perkins and Hage (Ref. E-1) are approximated by:

$\Delta \frac{x_{a c}}{\bar{c}}=-.05 \sum \frac{{ }_{p} p_{p}^{2}{ }^{1} p}{s \bar{c}\left(c_{L_{\alpha}}\right)} \quad\left(c_{w f}\right.$ in $\left.\mathrm{rad}^{-1}\right)$

where $B_{p}$ is the number of blades per propeller. Other definitions: see Fig. E-13. The effects of nacelles and propellers on $x_{a c}$ are appreciable in the case of wing mounted engines with horizontally opposed cylinders, relatively high-powered airplanes with a single tractor propeller in the fuselage nose and jet airplanes with podded engines mounted to the sides of the rear fuselage.

\section{E-9. LIFT OF THE COMPLETE AIRCRAFT}

In this paragraph the location of the aerodynamic center and moment about the a.c. of the complete aircraft minus horizontal tail, as obtained from the previous sections, will be referred to as $x_{a c}$ and $c_{m_{a c}}$. The moment coefficient is:

$c_{m_{A-h}}=c_{m_{a c}}+c_{L} \frac{x_{\mathrm{cg}^{-x}}{ }^{-x}}{\bar{c}}$

E-9.1. Tailplane lift

The horizontal tailplane lift to trim is obtained from the conditions of longitudinal equilibrium and lift = weight:

$\Delta_{h} c_{L}=c_{L_{h}} \frac{s_{h}}{s} \frac{q_{h}}{q}=\frac{\bar{c}}{1_{h}}\left(c_{m_{a c}}+c_{L} \frac{x_{c g^{-x}} \bar{c}^{-}}{\bar{c}}\right)$

E-9.2. Total trimmed airplane lift

By combining $(E-2),(E-29)$ and $(E-44)$, neglecting nacelles and lift due to the propulsion system, we find:

$C_{L}=C_{L_{w f}}\left(1+\frac{x_{c g^{-x} a c}}{1_{h}}\right)+\frac{\bar{c}}{1_{h}} c_{m_{a c}}$

\section{E-9.3. Wing/body incidence}

The wing incidence (relative to the fuselage reference line), required to obtain a specified $C_{L_{0}}$ with the fuselage reference axis ${ }^{*)}$ horizontal $\left(\alpha_{f}=0\right)$, is obtained from $(E-29),(E-30)$ and $(E-45)$ :

$i_{w}=\frac{C_{w f}^{*}-\Delta_{z} C_{L}}{K_{I I} C_{L_{w}}}+\frac{K_{I}}{K_{I I}} \alpha_{\sigma_{1}} \varepsilon_{t}+\left(\alpha_{\ell_{0}}\right)_{r}$

*) Strictly speaking, the fuselage floor line should be used as the reference for defining $\alpha_{\mathrm{f}}=0$. 
where

$c_{L_{w f}}^{*}=\left(c_{L_{o}}-\frac{\bar{c}}{l_{h}} c_{m_{a c}}\right) /\left(1+\frac{x_{c g}{ }^{-x} a c}{1_{h}}\right)$

The values of $\mathrm{C}_{L_{0}}$ and $x_{c g}$ frequently cosrespond to mean values of $C_{L}$ and airplane weight*) at the design cruising altitude, but there may also be other factors to consider (cf. Section 7.7.2).

E-9.4. Trimmed lift curve

The data generated may now be used to obtain the lift curve of the trimmed airplane in the linear range, with the angle attack defined relative to the fuselage datum line:

$c_{L}=c_{L_{\alpha}}\left\{\alpha_{f}-\left(\alpha_{L_{0}}\right)_{f}\right\}$

where

$c_{L_{\alpha}}=K_{I}\left(1+\frac{x_{c g^{-x} a c}}{I_{h}}\right) c_{L_{w_{\alpha}}}$

and

$\left(\alpha_{L_{0}}\right)_{f}=-\frac{c_{L_{0}}}{c_{L_{\alpha}}}$

provided $i_{w}$ is chosen in accordance with $(\mathrm{E}-46)$ and $(\mathrm{E}-47)$.

The tailplane effect on $C_{L}$-max can be found from the $C_{L}-\alpha$ curve, assuming that the critical angle of attack is not affected by the presence of the horizontal tailplane.

E-10. AIRPLANE PITCHING MOMENT AND NEUTRAL POINT (STICK FIXED)

E-10.1. The stick-fixed neutral point

According to Section 9.2 .1 of Chapter 9:

*) e.g. a payload equal to 50-608 of the maximum payload and half the trip fuel burnt. $\frac{x_{n}}{\bar{c}}=\frac{x_{a c}}{\bar{c}}+\frac{c_{L_{h}}}{c_{L_{\alpha}}}\left(1-\frac{d \varepsilon_{h}}{d \alpha}\right) \frac{s_{h}{ }^{1} h}{s \bar{c}} \frac{q_{h}}{q}$

Contrary to the previous section, $\mathrm{C}_{\mathrm{L}_{\alpha}}$ in this formula refers to the untrimmed (stick fixed) condition:

$c_{L_{\alpha}}=\left(c_{L_{\alpha}}\right)_{w f}+c_{L_{h}}\left(1-\frac{d \varepsilon h}{d \alpha}\right) \frac{s_{h}}{s} \frac{q_{h}}{q}$

and $x_{a c}$ refers to the airplane less horizontal tail.

In the absence of better information, $q_{h} / q$ may be assumed equal to .85 for a fuselagemounted stabilizer and .95 for a finmounted stabilizer, except in the case of a T-tail (with $q_{h} / q^{2} 1$ ).

The lift-curve slope of the tailplane can be computed with the method presented for wings in Sections $\mathrm{E}-3$ and $\mathrm{E}-4$, with the following typical corrections (where applicable):

- a reduction of 88 for an unsealed fullspan gap between the elevator and the stabilizer,

- a reduction of 58 if the elevator is beveled over its full root chord to accommodate rudder deflection.

These data refer to conventional, fuselagemounted fins (class A in Fig. 2-23); for other configurations Ref. E-48 may be consulted. In the case of twin fins mounted as endplates to the stabilizer, the aerodynamic aspect ratio of the horizontal tailplane is approximately 1.5 times the geometric aspect ratio.

The downwash gradient in unpowered flight is approximately:

$\frac{d \varepsilon_{h}}{d \alpha}=1.75 \frac{C_{L_{w_{\alpha}}}}{\pi A(\lambda r)^{.25}(1+|m|)}$

The geometric parameters $r$ and $m$ are defined in Fig. E-13. Pylon-mounted jet engine nacelles at the sides of the rear fuselage reduce the factor $\left(1-d \varepsilon_{h} / d \alpha\right)$ by ap- 
proximately 108 . Due to the propeller-fin effect at zero thrust $\left(1-d \varepsilon_{h} / d \alpha\right)$ is decreased by approximately .012 B (approximation of data in Ref. E-1).

\section{E-10.2. Horizontal stabilizer incidence}

For zero elevator and trim tab deflection, the tailplane lift for a symmetrical section is:

$C_{L_{h}}=C_{L_{h}}\left|\alpha\left(1-\frac{d \varepsilon h}{d \alpha}\right)+i_{h}\right|$

where $\alpha$ and $i_{h}$ are measured relative to the wing zero-lift line (Fig. E-12). For a

fixed stabilizer the condition may be imposed that the airplane is trimmed at.

$C_{L}=C_{L}$ with the elevator neutral in order to minimize the tailplane drag.

Provided that:

$\varepsilon_{h}=0$ for $C_{L}=0$,

$\varepsilon_{h}$ is a linear function of $\alpha$, and $\mathrm{C}_{\mathrm{L}_{\mathrm{w}}}=\mathrm{C}_{\mathrm{L}_{\mathrm{o}}}$,

the required incidence relative to the

fuselage datum line is:

$\left(i_{h}\right)_{f}=\frac{c_{m_{a c}}+c_{L_{o}} \frac{x_{c g}-x_{a c}}{\bar{c}}}{c_{L_{h}} \frac{s_{h}{ }^{1} h}{s \bar{c}} \frac{q_{h}}{q}}+\frac{{ }_{h} / d \alpha}{C_{L_{w}}} C_{L_{o}}$

and that relative to the wing zero-lift

line is:

$i_{h}=\left(i_{h}\right)_{f}-\frac{C_{L_{o}}}{C_{L_{\alpha}}}$
$\mathrm{C}_{L_{0}}$ being the lift coefficient when the fuselaqe is at zero angle of attack (Section $\mathrm{E}-9.3)$.

E-10.3. Pitching moment curve

For zero elevator deflection and angles of attack in the linear range:

$c_{m}=c_{m_{0}}+\frac{d c_{m}}{d C_{L}} c_{L}$

where

$\frac{\mathrm{dc}_{\mathrm{m}}}{\mathrm{dC}_{\mathrm{L}}}=-\frac{\mathrm{x}_{\mathrm{n}}-\mathrm{x}_{\mathrm{cg}}}{\overline{\mathrm{c}}}$

and

$c_{m_{0}}=c_{m_{a c}}-c_{L_{h}} i_{h} \frac{s_{h}{ }^{1} h}{s \bar{c}} \frac{q_{h}}{q}$

A summary of the various contributions to $\mathrm{C}_{\mathrm{m}}$ is given in Fig. $\mathrm{E}-14$.

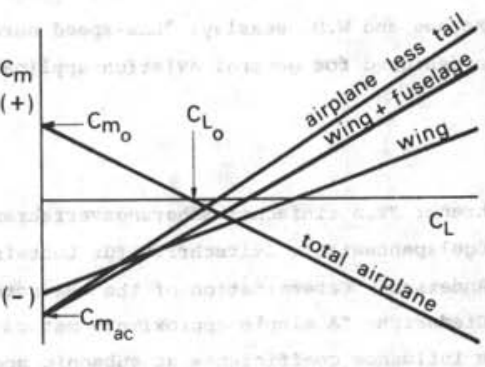

Fig. E-14. Pitching moment curve in the linear angle of attack range for zero elevator deflection.

\section{REFERENCES}

E-1. C.D. Perkins and R.E. Hage: "Airplane performance, stability and control". New York, John Wiley and Sons, Inc., 1949.

E-2. в. Schlichting and E. Truckenbrodt: "Aerodynamik des Flugzeuges". Vo1. II. Springer Verlag, 1960.

E-3. D.E. Hoak and D.E. Ellison: "USAF Stability and Control Datcom". Wright Patterson Air Force Base, October 1960 (rev. August 1968).

E-4. D.E. Ellison and D.E. Hoak: "Stability derivative es' mation at sussonic speeds". Annals of the New York Academy of Sciences, Vol. 154, Part. 2, pp. 367-396.

E-5. Anon.: Data Sheets, Aerodynamics, Vols. I, II and III, Royal Aeronautical Society. 
E-6. w. Just: "Flugmechanik, Steuerung und Stabilităt von Flugzeugen". Verlag Flugtechnik, Stuttgart, 1966.

E-7. G.J. Hancock: "Problems of aircraft behaviour at high angles of attack". AGARDograph 136, Apri1 1969.

E-8. J. Roskam: "Methods of estimating stability and control derivatives of conventional subsonic airplanes". The University of Kansas, 1971.

E-9. С.H. Wolowicz: "Longitudinal aerodynamic characteristics of 1ight, twin-engine, propeller driven airplanes". NASA TN D-6800, 1972.

E-10. R.K. Heffley and W.F. Jewell: "Aircraft handling qualities data". NASA CR-2144, December 1972.

E-11. J.G. Callaghan: "Aerodynamic prediction methods at low speeds with mechaninal high lift devices". AGARD Lecture Series No. 67, May 1974.

Airfoil sections

E-12. I.H. Abbott, A.E. Von Doenhoff and L.S. Stivers: "Summary of Airfoil Data". NACA Report 824 , 1945.

E-13. F.W. Riegels: "Aerodynamische Profile". R. Oldenburg, Munich, 1958.

E-14. I.H. Abbott and A.E. Von Doenhoff: "Theory of wing sections". McGraw-Hil1 Book Comp. Inc., New York; also: Dover Publications Inc., New York, 1959.

E-15. L.K. Loftin: "The effects of variations in Reynolds number between $3.0 \times 10^{6}$ and $25.0 \times 10^{6}$ upon the aerodynamic characteristics of NACA 6-series airfoil sections". NACA TN $1773,1948$.

E-16. L.K. Loftin: "Theoretical and experimental data for a number of NACA $6 \mathrm{~A}-\mathrm{series}$ airfoil sections". NACA Report $903,1948$.

E-17. D.M. McRae: "Aerodynamics of mechanical high-1ift devices". AGARD Lecture Series No. 43, April 2024. 1970.

E-18. R.J. McGhee and W.D. Beasley: "Low-speed aerodynamic characteristics of a 17 percent-thick airfoil section designed for general aviation applications", NASA TN D-7428, December 1973.

Wings

E-19. O. Schrenk: "Bin einfaches Naherungsverfahren zur Ermittlung von Auftriebsverteilingen längs der Tragflügelspannweite". Zeitschrift für Luftwissenschaften, Vol. 7, No. 4, pp. $118-120$.

E-20. R.F. Anderson: "Determination of the characteristics of tapered wings". NACA Rep. 572, 1936 .

E-21. F.W. Diederich: "A simple approximate method for calculating spanwise lift distributions and aerodynamic influence coefficients at subsonic speeds". NACA TN $2751,1952$.

E-22. J. de Young and C.W. Harper: "Theoretical symmetric spain loading at subsonic speeds for wings having arbitrary planform". NACA Rep. 921, 1948.

E-23. R. Stanton Jones: "An empirical method for rapidly determining the loading distribution on swept back wings". College of Aeronautics Report No. 32, 1950.

E-24. G.H. Lee: "High maximum 11ft". The Aeroplane, October 30 and November 6, 1953.

E-25. V. Holmboe: "Charts for the position of the aerodynamic centre at low speeds and small angles of attack for a large family of tapered wings". SAAB TN 27, 1954 (reproduced in "Airplane Design", by K.D. Wood).

E-26. Anon.: "Method for the rapid estimation of theoretical spanwise loading due to a change of incidence" R.Ae.S. Transonic Data Memorandum 6208, 1962.

E-27. Anon.: "Graphical method for estimating the spanwise distribution of aerodynamic centre on wings in subsonic flow". R.Ae.S. Transonic Data Memorandum 6309, Sept. 1963.

E-28. C.L. Bore and A.T. Boyd: "Estimation of maximum lift of swept wings at low Mach numbers", J. of the R.Ae.S., Vol. 67, April 1963, pp. 227-239.

E-29, L.R. Wootton: "The effect of compressibility on the maximum lift coefficient of aerofoils at subsonic airspeeds". J. of the R.Ae.S., Vol. 71, July 1967. 
E-30. T.E. Labruyere and J.G. Wouters: "Computer application of subsonic lifting surface theory". NLR Report TR 70088.

E-31. P. Kapteyn: "Design charts for the aerodynamic characteristics of straight and swept, tapered, twisted wings at subsonic speeds". Delft University of Technology, Dept. of Aeronautical Engineering, M-180, May 1972 .

E-32. M.A. McVeigh and E. Kisielowski: "A design summary of stall characteristics of straight wing aircraft". NASA CR-1646, June 1971 .

Bodies and wing/body combinations

E-33. M.M. Munk; "The aerodynamic forces on airship hulls". NACA T. Rep. 184, 1924,

E-34. J.H. Allen: "Estimation of the forces and moments acting on inclined bodies of revolution". NACA RM A $9126,1949$.

E-35. H. Schlichting: "Calculation of the influence of a body on the position of the aerodynamic centre of aircraft with sweptback wangs". ARC R and M 2582, 1947.

E-36. A,H. Flax and B.R. Lawrence: "The aerodynamics of low aspect -ratio wings and wing-body combinations". Proceedings of the Third Anglo-American Aeronautical Conference 1951, pp. 363-398.

E-37. W.C. Pitts, J.N. Nielsen and G.E. Kaattari; "Lift and center of pressure of wing-body-tail combinations at subsonic, transonic and supersonic speeds". NACA T. Rep. 1307, 1957.

E-38. S.F. Hoerner: "Fluid dynamic drag". Published by the author, 1965.

Propeller effects

E-39. R. Smelt and $\mathrm{H}$. Davies: "Estimation of increase in lift due to slipstream". ARC R and $M 1788$, 1937.

E-40. D.E. Morris and J.C. Morrall; "Effect of slipstream on longitudinal stability of multi-engined aircraft", ARC $R$ and M 2701, November 1948.

B-41. J. Weil and W.C. Sleeman, Jr.: "Prediction of the effects of propeller operation on the static longitudinal stability of single-engine tractor monoplanes with flaps retracted", NACA T. Rep. 941.

E-42. R.E. Kuhn: "Semi-empirical procedure for estimating lift and drag characteristics of propellerwing-flap configurations for vertical and short take-off and landing aeroplanes", NASA Memorandum 1-16-59L, 1959.

E-43. B,W. McCormick: "Aerodynamics of v/STOL Flight". Academic Press, New York/London, 1967 ,

Downwash

E-44. A. Silverstein and S. Katzoff: "Design charts for predicting downwash angles and wake characteristics behind plain and flapped wings". NACA Rep. 648, 1939.

E-45. J. De Young and W.H. Barling: "Prediction of downwash behind swept-wing airplanes at subsonic speeds". NACA TN 3346.

E-46. F.W. Diederich: "Charts and tables for use in calculations of downwash of wings of arbitrary plan form". NACA TN 2353, 1951.

E-47. J.L. Decker; "Prediction of downwash at various angles of attack for arbitrary tail locations". Aero, Bng, Review, August 1956, pp. 22-61.

Tailplane characteristics

E-48. D.J. Lyons and P.L. Bisgood: "An analysis of the lift slope of aerofoils of small aspect ratio wings including fins, with design charts for aerofoils and control surfaces". $R$ and $M \quad 2308$, 1945 . Also in Aircraft Eng., September 1947, pp. 278-286. 


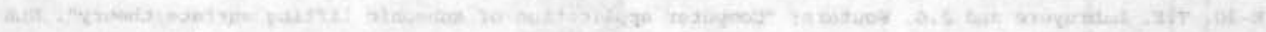

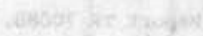

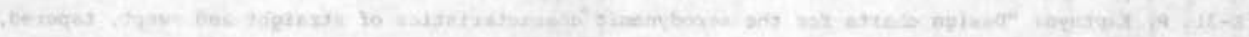

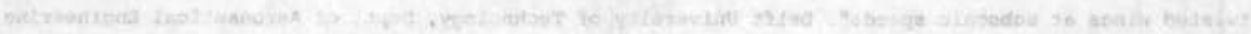

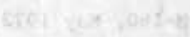

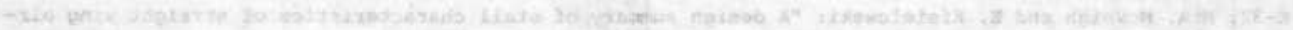

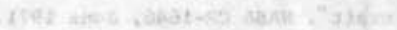

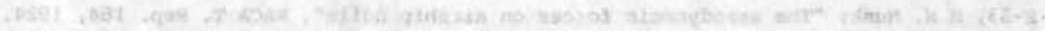

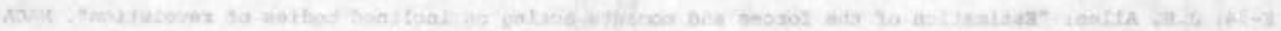
topes wistit in ka

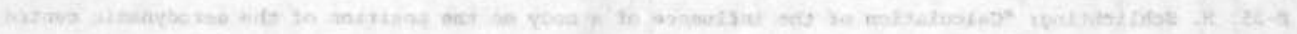

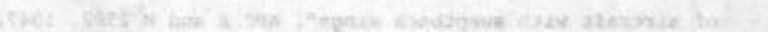

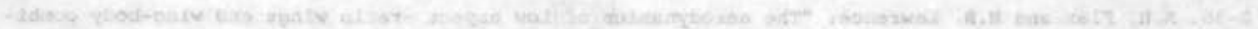

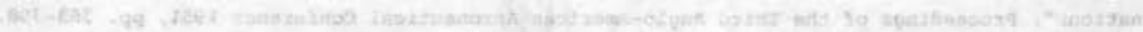

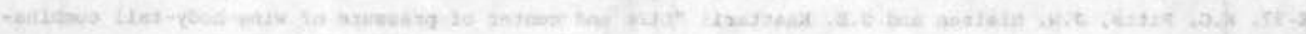

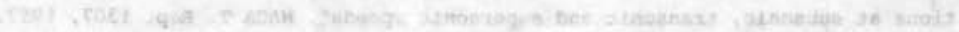

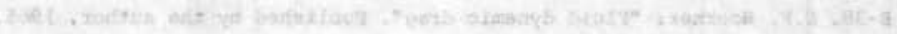

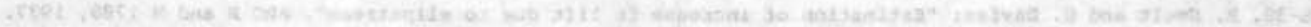

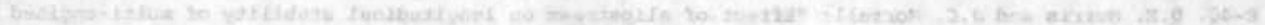

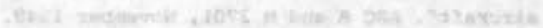

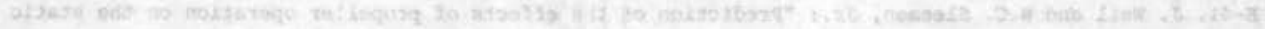

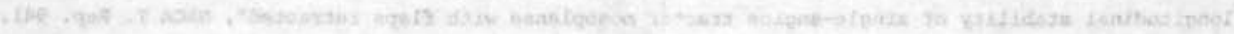

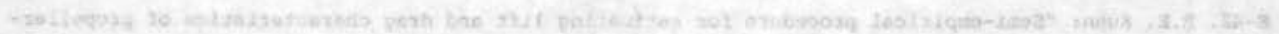

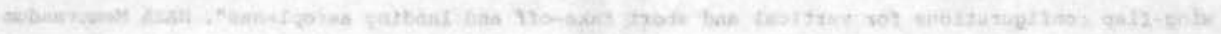

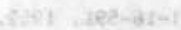

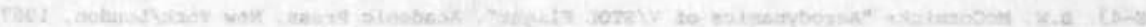

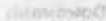

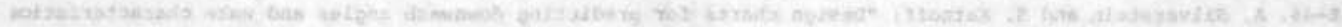

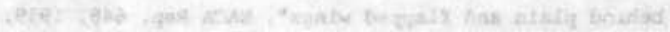

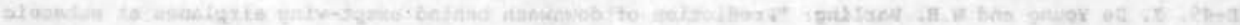

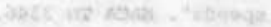

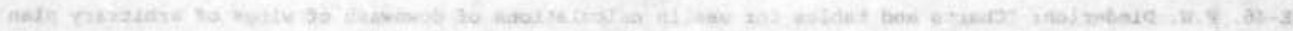

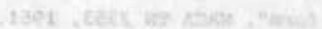

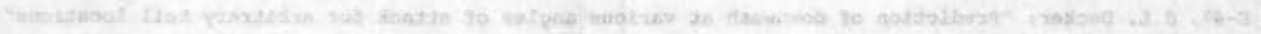

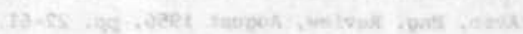

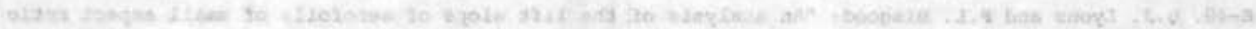

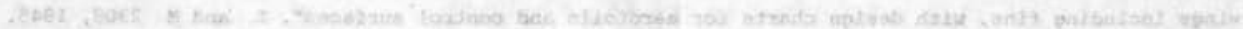

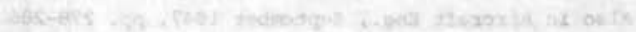




\section{Appendix F. Prediction of the airplane polar at subcritical speeds in the en route configuration}

SUMMARY

A concise collection of formulas, generalized data and methods is presented for estimating the airplane arag at subcritical speeds in the en route configuration. All methods are applicable in the preliminary design stage of conventional aircraft.

Dray associated with the trailing vortices and profile drag are computed for the isolated, smooth airplane parts. Some corrections are given to account for the interaction of the flow fields of parts placed in close proximity. Finally, a number of data and methods are given to account for the effects of surface imperfections, powerplant installation, protuberances and other extras. 


\section{NOMENCLATURE}

A

- aspect ratio (no index: wing aspect ratio); constant in drag equation; planform area

$A_{c} \quad$ - cross-sectional area of a streamline body

B

b

c

$\mathrm{c}_{\mathrm{c}}$

c

$\bar{c}$

$c_{1}, c_{2}, c_{3}$ - coefficients in Diederich's method (cf. Appendix E)

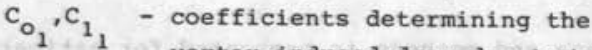
vortex-induced arag due to twist

$c_{c i}$ - total circumferential length of wing/fuselage intersection

$C_{d}, C_{D}$ - two- and three-dimensional drag coefficient

$C_{D_{\pi}}-C_{D}$ based on frontal area

$C_{D_{0}}^{\pi} \quad$ - zero-1ift drag coefficient

${ }_{d_{p}}, c_{D_{p}}$ - two- and three-dimensional profile drag coefficient

$C_{D_{v}} \quad$ - vortex-induced drag coefficient

$C_{D_{\text {ref }}}$ - reference drag coefficient at $C_{L}=C_{L_{\text {ref }}}$

C $\quad$ - skin friction drag coefficient of smooth flat plate

c - geometric mean chord

$c_{\ell}, C_{L} \quad-$ two- and three-dimensional lift coefficient

$\mathrm{C}_{\mathrm{L}_{0}} \quad-\mathrm{c}_{\mathrm{L}}$ at $\alpha_{\mathrm{f}}=0$

$c_{\ell_{\alpha}}{ }^{0} \mathrm{C}_{L_{\alpha}}$ - lift-curve slope of section and aircraft respectively

$c_{\ell_{i}}, C_{L_{i}}$ - design lift coefficient of section and wing respectively

$C_{L_{\text {ref }}}-C_{L}$ for minimum (reference) $C_{D}$

- aerodynamic pitching moment about the aerodynamic center of aircraft less tail

D

- arag; diameter; factor of $C_{L}{ }^{2}$ term in drag equation

Do - diameter of capture area of engine air

$D_{1}, D_{2} \quad$ - factors in fuselage drag equation

$D_{c} \quad-$ drag due to cross flow component of $V_{\infty}$
- diameter of cylindrical forebody of boat tail

E

e

$\overline{\text { FR }}$

f

h

k

L

1

$\mathrm{M}_{\infty}$

$\mathrm{M}_{\mathrm{f}}, \mathrm{M}_{\mathrm{g}}$

m̂n $_{\mathrm{a}}, \mathrm{m}_{\mathrm{c}}, \mathrm{h}_{j}$

NPR

$/ p_{\infty}$

$\mathrm{N}_{\mathrm{e}} \quad$ number of engines

$\mathrm{P}_{\mathrm{b}} \quad$ - brake power

$\mathrm{p}_{\infty} \quad$ - ambient pressure (static)

$q_{\infty}$

R

R

$\mathbf{r}$

S

$s_{\text {net }}$

T

$T_{\infty}$

$T_{c}$

$t$

$u, v, w$

V

$v_{\infty}$

$v_{e}, v_{i}$

$\dot{\mathrm{w}}_{\mathrm{a}}, \dot{\mathrm{w}}_{j}$

x

$\mathrm{x}_{\mathrm{T}}$

- dynamic head $\left(q_{\infty}=\frac{1}{2} \rho_{\infty} v_{\infty}{ }^{2}=\frac{1}{2} \gamma p_{\infty} M_{\infty}{ }^{2}\right)$

- Reynolds number

- radius of curvature

- area; no subscript: gross wing area

- net wing area (cf. Appendix A, Section $\mathrm{A}-3.1$.

- thrust

- ambient temperature (static)

- propeller thrust coefficient.

- thickness; canopy nose or tail s?ction length

- Anderson's factors in vortexinduced drag coefficient of wing

- volume; velocity of flow

- flight speed

- exhaust and inlet velocity respectively

- weight flow per second of engine air and jet flow respectively

- longitudinal coordinate

- distance of transition region downstream from the nose or leading adge 
y - spanwise coordinate, measured from

wing centerline

$\alpha$ - angle of attack

$\alpha_{f}$ - angle of attack relative to fuselage centerline

$\alpha_{f}^{\prime}-\alpha_{f}$ for minimum fuselage drag coefficient

$a_{i}$ - induced angle of attack

$B^{2}$ - Prandt1-Glauert compressibility factor $\left(\beta=\sqrt{1-M^{2}}\right)$; fuselage tail upsweep angle; length of cowl forebody/total cowl length; boat tail angle

$\gamma$ - ratio of specific heats (for air: $\gamma=1.4$ )

$\Delta$ - increment; e.g. $\Delta_{i} C_{D}=$ increment in $C_{D}$ due to interference

$\delta$ - increment of plane wing vortex-irduced drag coefficient due to additional 1 ift

$\varepsilon$ - angle of twist; downwash angle

$n$ - non-dimensional spanwise coordinate $(n=2 y / b)$

$n_{p}$ - propeller efficiency

$\theta^{\mathrm{P}}$ - relative atmospheric temperature $\mathrm{T}_{\infty} /$ ( $T_{\infty}$ at sea level, standard conditions)

$\theta_{j}$ - deflection angle of jet flow relative to free stream

A - sweepback angle of quarter-chord line (unless otherwise stated); no index: wing sweepback angle

$\Lambda_{B}$ - corrected sweepback angle $\left(\tan \Lambda_{B}=\right.$ $(\tan A) / B)$

$\lambda$ - taper ratio; slenderness ratio

$v$ - coefficient of kinematic viscosity

$P_{\infty}$ - density of ambient air

$\sigma \quad$ - relative density $=\rho_{\infty} /\left(\rho_{\infty}\right.$ at sea level, standard conditions)

$\varphi$ - shape factor in profile drag coefficient equation

\section{P-1. DRAG COMPONENTS}

Airplane drag can be subdivided in a number of ways. The terminology used in several schemes is discussed in section 11.2 of Chapter 11. The usual breakdown for the purpose of performance analysis is as follows :
Subscripts

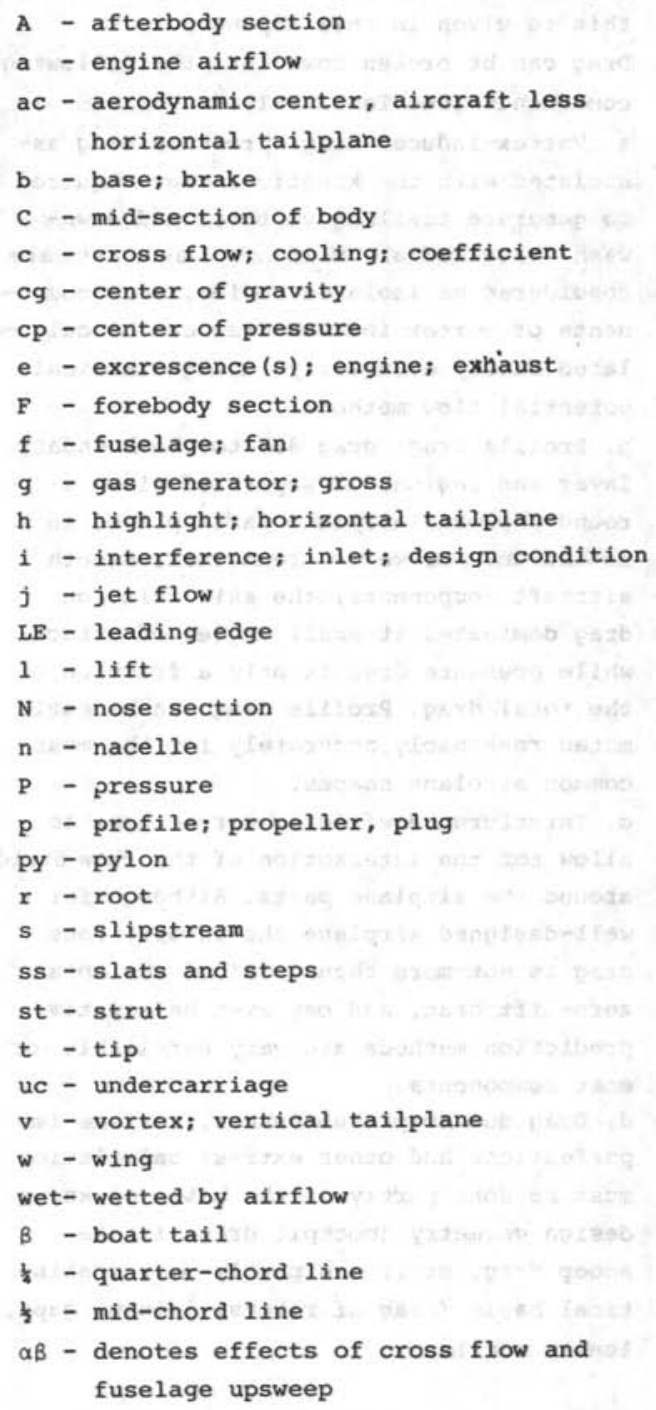

$C_{D}=C_{D_{0}}+\frac{C_{L}{ }^{2}}{\pi A e}$

Section 5.3.1. gives an explanation of these terms and an initial estimation method for $\mathrm{C}_{\mathrm{D}_{\mathrm{O}}}$ and the Oswald factor e. At the stage where the layout and principal geometric properties have already been de- 
fined, a more detailed analysis becomes appropriate. The information required for this is given in this Appendix.

Drag can be broken down into the following components (see Table F-1):

a. Vortex-induced drag: pressure drag associated with the kinetic energy required to generate trailing vortices and down-

wash. Provided all main airplane parts are considered as isolated bodies, most components of vortex-induced drag can be calculated fairly accurately, using classical potential flow methods.

b. Profile drag: drag due to the boundary layer and regions of separated flows around the main airplane parts placed in isolation. For well-streamlined, smooth aircraft components, the skin friction drag dominates at small angles of attack, while pressure drag is only a fraction of the total drag. Profile drag can be estimated reasonably accurately for the most common airplane shapes.

c. Interference effects: corrections to allow for the interaction of the flow field around the airplane parts. Although for a well-designed airplane the interference drag is not more than 5-108 of the total zero-lift drag, and may even be negative, prediction methods are very unreliable for most components.

d. Drag due to protuberances, surface-imperfections and other extras: calculation must be done partly on the basis of known design geometry (cockpit drag, intake scoop drag, etc.) and partly on a statistical basis (drag of rivets, joints, gaps, leaks, etc.).

The vortex-induced drag of a plane wing (1.e. the wing is not twisted) is zero for $C_{L}=0$, but profile drag, interference drag and several vortex drag components are minimum at some positive reference (datum) value for $C_{L}, e . g$. in cruising flight. Hence, the condition for minimum $C_{D}$ will be found for $0<C_{L}<C_{L}$ and $(\mathrm{F}-1)$ therefore approximates the ${ }^{\mathrm{cr}}$ actual polar curve only in a limited region of $\mathrm{C}_{\mathrm{L}}$ (cf. Fig. 5-4).
The drag polar at subcritical speeds can be obtained by computation of all items mentioned in Tables $\mathrm{F}-1$. The drag components are presented in the following form:

$C_{D_{j}}=A_{j}+B_{j} C_{L}+D_{j} C_{L}^{2}$

To avoid confusion as to the reference area to which these components are related, most data wi11 be presented in the form of a drag area $f=\left(C_{D} S\right)_{j}^{*}$. Components can be related to the gross wing area as follows: $C_{D_{j}}=\frac{\left(C_{D} S\right)_{j}}{S}$

All drag areas can be added and the total drag coefficient is:

$C_{D}=\frac{\sum\left(C_{D} S\right)_{j}}{S}$

Drag components are very often expressed as "counts"; 1 count is $\Delta C_{D}=.0001$, based on the wing area. Addition of all components in Table $\mathrm{F}^{*} 1^{* *}$ results in the final expression:

$C_{D}=A+B \quad C_{L}+D C_{L}{ }^{2}$

Alternatively, the arag polar may be expressed as follows:

$C_{D}=C_{D_{\text {ref }}}+B\left(C_{L}-C_{L_{\text {ref }}}\right)^{2}$

where

$C_{L_{\text {ref }}}=-B / 2 D ; C_{D_{\text {ref }}}=A-D C_{L_{\text {ref }}}^{2} ; B=D$

A polar of the shape given by $(\mathrm{F}-1)$ can be obtained by plotting calculated values of $C_{D}$ vs. $C_{L}{ }^{2}$ and making a straight line approximation in the $C_{L}$ region between the high-speed cruise and approximately 70 of of

* An exception is made for wing drag components, related to the gross wing area $s$. * "To avoid mistakes it is useful to fill out Table F-1 in counts, by multiplying all components by $10^{4}$. The values of $A, B$ and $D$ are then divided by $10^{4}$. 


\begin{tabular}{|c|c|c|c|c|c|c|}
\hline \multirow{2}{*}{\multicolumn{2}{|c|}{ A. VORTEX DRAG }} & A & \multicolumn{2}{|r|}{ B } & \multicolumn{2}{|c|}{ D } \\
\hline & & $\left(\Sigma \mathrm{A}_{\mathrm{v}}\right)$ & $(\Sigma \mathrm{B}$ & $\mathrm{B}_{\mathrm{v}}$ ) & $\left(\Sigma D_{\mathrm{v}}\right.$ & \\
\hline & DRAG OF PLANE WING & & & & & \\
\hline & DRAG DUE TO WING TWIST & & & & & \\
\hline & WING TIP CORRECTION & & & & & \\
\hline & DRAG DUE TO FUSELAGE LIFT & & & & & \\
\hline & HORIZONTAL TAILPLANE DRAG (TRIM) & & & & & \\
\hline B. & PROFILE DRAG (SMOOTH CONDITION) & $\left(\Sigma A_{p}\right)$ & & $\left.\mathrm{B}_{\mathrm{p}}\right)$ & $\left(\Sigma D_{p}\right.$ & \\
\hline & - MINIMUM DRAG & & & & & \\
\hline & - INCREMENTAL DRAG & & & & & \\
\hline & FUSELAGE - BASIC DRAG & & & & & \\
\hline & - BOATTAIL AND BASE DRAG & & & & & \\
\hline & - UPSWEEP AND INCIDENCE EFFECTS & & & & & \\
\hline & TAILBOOMS & & & & & \\
\hline & NACELLES - EXTERNAL (FAN) COWLING & & & & & \\
\hline & - GAS GENERATOR & & & & & \\
\hline & - PLUG & & & & & \\
\hline & - PYLONS & & & & & \\
\hline & HORIZONTAL TAILPLANE & ( & ( & ) & 6 & ) \\
\hline & - MINIMUM DRAG & & & & & \\
\hline & - DRAG DUE TO $\delta_{e}$ AND $\alpha_{h}$ & & & & & \\
\hline & VERTICAL TAILPLANE & & & & & \\
\hline c. & INTERFERENCE CORRECTIONS & $\left(\Sigma A_{i}\right)$ & $(\Sigma \mathrm{B}$ & $\left.B_{i}\right)$ & & i) \\
\hline & WING/FUSELAGE & $(\quad)$ & ( & ) & ( & ) \\
\hline & - VORTEX DRAG & & & & & \\
\hline & - VISCOUS INTERFERENCE DRAG & & & & & \\
\hline & - LIFT EFFECT ON FUSELAGE DRAG & & & & & \\
\hline & NACELLE/AIRFRAME & ( & ( & ) & 6 & ) \\
\hline & - VORTEX DRAG & & & & & \\
\hline & - PROFILE DRAG & & & & & \\
\hline & TAILPLANE/TAILPLANE & & & & & \\
\hline & TAILPLANE/AIRFRAME & & & & & \\
\hline & SUB-TOTAL & & & & & \\
\hline
\end{tabular}

NOTE: $C_{D}=\Sigma A+\Sigma B \cdot C_{L}+\sum D \cdot C_{L}{ }^{2}$

\begin{tabular}{|c|c|c|c|}
\hline & A & B & D \\
\hline D. PROTUBERANCES, SURFACE IMPERFECTIONS, ETC. & $\left(\Sigma \mathrm{A}_{\mathrm{e}}\right)$ & $\left(\Sigma B_{e}\right)$ & $\left(\Sigma \mathrm{D}_{\mathrm{e}}\right)$ \\
\hline FIXED UNDERCARRIAGE & & ( & 6 \\
\hline \multicolumn{4}{|l|}{ MAIN UNDERCARRIAGE } \\
\hline \multicolumn{4}{|l|}{ NOSE UNDERCARRIAGE } \\
\hline \multicolumn{4}{|l|}{ TAILWHEEL/TAILSKID } \\
\hline \multicolumn{4}{|l|}{ PARTLY RETRACTED LANDING GEARS } \\
\hline CANOPIES AND CABIN WINDOWS & ( & ( & ( \\
\hline \multicolumn{4}{|l|}{ PROTRUDING COCKPIT ENCLOSURES } \\
\hline \multicolumn{4}{|l|}{ FLIGHT DECK WINDOWS } \\
\hline FAIRINGS AND BLISTERS & ( & ( & ( \\
\hline \multicolumn{4}{|l|}{ WHEEL-WELL FAIRINGS } \\
\hline \multicolumn{4}{|l|}{ BLISTERS } \\
\hline EXTERNAL FUEL TANKS & ( & ( & ( \\
\hline \multicolumn{4}{|l|}{ BASIC PROFILE DRAG } \\
\hline \multicolumn{4}{|l|}{ INSTALLATION AND INTERFERENCE CORRECTIONS } \\
\hline \multicolumn{4}{|l|}{ STRUTS AND WIRES } \\
\hline ENGINE INSTALLATION & ( & ( & ( \\
\hline \multicolumn{4}{|l|}{ - ENGINE AIR INTAKE(S)/SPILLAGE } \\
\hline - ENGINE AIR OUTLET(S)/REVERSERS & & & \\
\hline - OIL COOLER(S)/RADIATOR(S) & & & \\
\hline \multicolumn{4}{|l|}{ - ENGINE COOLING } \\
\hline - OIL COOLER(S)/RADIATOR(S) & & & \\
\hline \multicolumn{4}{|l|}{ SLIPSTREAM EFFECTS - PROFILE DRAG } \\
\hline - VORTEX-INDUCED DRAG & & & \\
\hline \multicolumn{4}{|l|}{ JET INTERFERENCE } \\
\hline EXCRESCENCES, SURFACE IMPERFECTIONS, OTHER EXTRAS & ( & ( & 6 \\
\hline \multicolumn{4}{|l|}{ SURFACE IMPERFECTIONS } \\
\hline \multicolumn{4}{|l|}{ HIGH-LIFT SYSTEM } \\
\hline \multicolumn{4}{|l|}{ FLIGHT CONTROL SYSTEM } \\
\hline \multicolumn{4}{|l|}{ VORTEX GENERATORS, FENCES, STRAKES } \\
\hline \multicolumn{4}{|l|}{ AIRFRAME SYSTEMS INSTALLATION } \\
\hline \multicolumn{4}{|l|}{ MISCELLANEOUS, UNACCOUNTABLE ALLOWANCE } \\
\hline TOTAL & $(\Sigma \mathrm{A})$ & $(\Sigma$ B $)$ & $(\Sigma \mathrm{D})$ \\
\hline
\end{tabular}

Table F-1. Breakdown of the drag for the purpose of drag estimation 
$\mathrm{C}_{\mathrm{L}}$

The most important restrictions to most of the methods presented are the same as those stated in Appendix E for the $11 \mathrm{ft}$ prediction. In terms of wing geometry:

$$
\begin{aligned}
& t / c>98 \\
& A>4 / \cos \Lambda_{\frac{1}{4}} \\
& \Lambda_{\frac{1}{4}}<35^{\circ}
\end{aligned}
$$

These approximate limits generally define wings with attached flow at small angles of attack and full leading edge suction, provided they have adequate camber and washout and the angle of attack is in the normal operating regime. Flight speeds are $1 i$ mited to subcritical Mach numbers. An estimation of the drag-critical Mach number can be found in section 7.5.2.

The data presented in this Appendix are sufficiently complete to make a drag analysis for the most common aircraft shapes. However, the designer should be on the alert for special drag components to be anticipated in his particular design. The well-known book by Hoerner (Ref. F-18) and other publications listed in the references can be consulted in such cases, and if a more detailed analysis is desirable.

\section{F-2. PRIMARY COMPONENTS OF VORTEX-INDUCED DRAG}

The lift-dependent drag due to trailing vortices is associated with the kinetic energy lost in the system of trailing vortices behind the aircraft. For the wing it is given by the equation

$C_{D_{v}}=\int_{0}^{1} \frac{c_{\ell} c}{c_{g}} \alpha_{i} d n$

where

$a_{i}=\frac{1}{4 \pi \AA} \int_{-1}^{+1} \frac{d}{d n^{\prime}}\left(\frac{c_{\ell} c}{c_{g}}\right) \frac{d n^{\prime}}{n-n^{\prime}}$

These equations are valid only for symmetrical lift distributions.
F-2.1. Untwisted plane wings

Assuming for the moment* that all lift of the airplane is generated by an untwisted plane wing, the vortex drag coefficient, based on $S$, can be defined as:

$C_{D_{v}}=(1+\delta) \frac{C_{L}{ }^{2}}{\pi A}$

Equations $\mathrm{F}-7$ and $\mathrm{F}-9$ can be combined:

$1+\delta=\pi \mathrm{A} \frac{\int_{0}^{1} \frac{c_{\ell} c}{c_{g}} \alpha_{i} d n}{\left[\int_{0}^{1} \frac{c_{l} c}{c_{g}} d \eta\right]^{2}}$

with $\delta=0$ for an elliptical lift distribution.

The solution of $(F-8)$ and $(F-10)$ requires a knowledge of the lift distribution along the span, which may be obtained for potential flow using any classical lifting line or lifting surface theory. Although in many institutes these are available in the form of standard computer programs, it may be desirable to use a simpler approach in the preliminary design stage. Several possibilities will be given from which a choice can be made:

METHOD A. Garner has demonstrated (Ref. F-39) that there is a correlation between $\delta$ and the spanwise center of pressure $n_{\mathrm{cp}}$ :

$\delta=46.264\left(\eta_{c p}-\frac{4}{3 \pi}\right)^{2}$

where

$n_{c p}=\int_{0}^{1} \frac{c_{\ell} c}{c_{L} c_{g}} n d n$

For a known lift distribution this integral is simpler to evolve than $(F-8)$ and $(\mathrm{F}-10)$. For example, the expression for the

* Corrections will be dealt with in Section $\mathrm{F}-4$. 
additional lift distribution given by Diederich (Section 4.2. of Appendix E) can be used:

$n_{c p}=c_{1} \int_{0}^{1} \frac{c}{c_{g}} n d \eta+c_{2} \frac{4}{3 \pi}+c_{3} \int_{0}^{1} f n d n$

Although Diederich's approximation for the lift distribution is not very accurate, it has the advantage that arbitrary wing planforms can be dealt with. For the case of a straight-tapered wing, we have

$\int_{0}^{1} \frac{c}{c_{g}} n d n=\frac{1+2 \lambda}{3(1+\lambda)}$

while the last integral in $(\mathrm{F}-13)$ can be approximated by:

$\int_{0}^{1} f \eta d n=\frac{4}{3 \pi}+.001 \Lambda_{B}\left(\Lambda_{\beta}\right.$ deg. $) \quad(F-15)$

Hence, for straight-tapered wings:

$n_{c p}=c_{1} \frac{1+2 \lambda}{3(1+\lambda)}+\left(c_{2}+c_{3}\right) \frac{4}{3 \pi}+.001 \Lambda_{\beta} c_{3}(F-16)$

where $C_{1}, C_{2}$ and $c_{3}$ are factors given in Fig. E-5 of Appendix $E$ and $\Lambda_{\beta}$ is in degrees. The author's experience is that (F-11) gives accurate results for corrected aspect ratios (BA) up to 10 when compared with lifting surface theory.

METHOD B. Anderson has generalized the results of Prandtl's lifting line theory for straight-tapered wings with rounded tips in incompressible flow (Ref. F-29). A summary is given in Fig. 10 of Ref. F-48 in the form of a diagram for $u$,

$u \equiv \frac{1}{1+\delta}$

Lifting surface theory (e.g. Refs. F-40 and F-41) gives very similar results. The results in Ref. F-4l can be represented by: $\delta=\left\{.0015+.016(\lambda-.4)^{2}\right\}(B A-4.5)$

for $6<\beta A<30 ; .3<\lambda<1.0 ; \Lambda_{\frac{1}{4}}=0$
METHOD C. Results of an NLR computer program (Refs. $F-40$ and $F-41$ ) are presented in Fig. F-1, for . $2<\lambda<.5$ and $\Lambda_{B}$ up to $60^{\circ}$. Similar results may also be obtained with Ref. F-36.

F-2.2. Drag due to twist

The shape of the lift distribution of twisted wings is no longer similar for different values of $C_{L}$. For zero lift the induced drag of a plane wing equals zero, but the basic lift distribution of a twisted wing causes a vortex-induced drag at zero lift. For straight wings with linear twist (low speeds) Anderson (Ref. F-29) represents the drag coefficient due to twist, related to the gross wing area, as follows:

$\Delta_{\varepsilon} C_{D_{v}}=C_{L}\left(\frac{\varepsilon_{t} c_{\ell_{\alpha}}}{E}\right) v+\left(\frac{{ }_{t}{ }^{C_{\ell}}{ }_{\alpha}}{E}\right)^{2} w \quad(F-19)$

Diagrams for determining $v$ and $w$ can be found in Ref. F-48, Figs. 11 and 12. For a near-elliptical additional lift distribution a first approximation is:

$\Delta_{\varepsilon} C_{D_{v}}=3.7 \times 10^{-5} \varepsilon_{t}^{2} \quad\left(\varepsilon_{t}\right.$ deg. $)(F-20)$

For straight and swept wings with linear lofted twist (subcritical flow) Ref. F-41 states:

$\Delta_{\varepsilon} C_{D_{v}}=\varepsilon_{t}{ }^{2} C_{O_{1}}+\varepsilon_{t} C_{L} C_{1}$

The vortex drag factors are computed from lifting surface theory and depicted in Figs. F-2 and $\mathrm{F}-3$.

Some comments can be made on the results of the methods summarized above:

1. For a representative straight wing $(A=8$,

$\lambda=.4$ ) the induced drag due to a linear washout of 4 degrees at the tip is roughly twice as high when compared with a linear lofted * wing with the same twist angle.

2. An "optimum twist angle" can be defined for which $\Delta_{E} C_{D_{V}}$ reaches an extreme value. In terms of

\footnotetext{
* 1 inear and linear lofted twist are defined in
} Appendix A, Section A-3.2. 

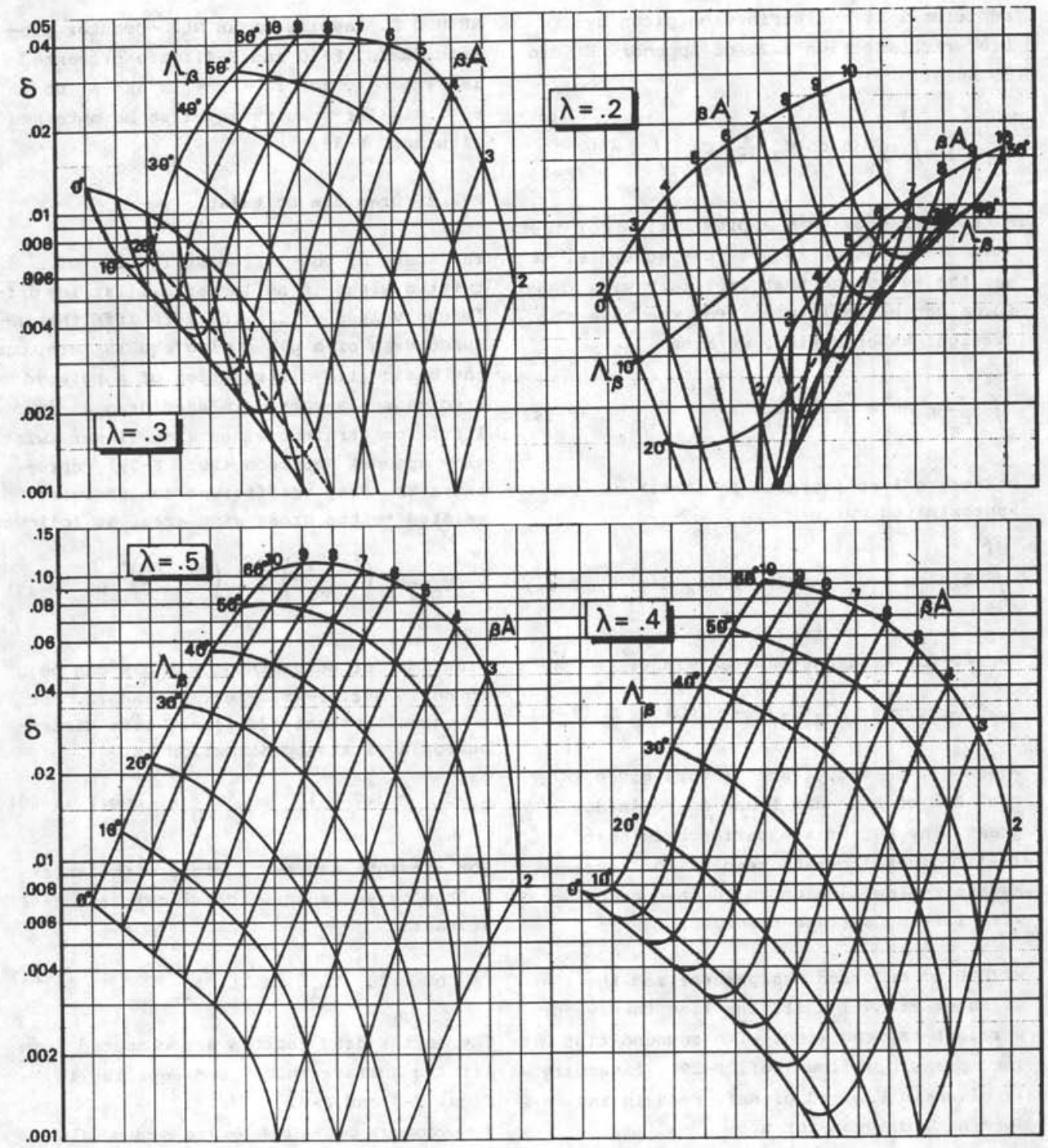

Fig. F-1. The vortex-induced drag factor $\delta$ (Ref. F-41)

Anderson's factors:

$\varepsilon_{t_{\text {opt }}}=-\frac{1}{2}\left(\frac{E C_{L}}{c_{1}}\right) \frac{v}{w}$

corresponding to:

$\left(\Delta_{\varepsilon} c_{D_{v}}\right)_{\text {opt }}=-\frac{1}{4} c_{L}^{2} \frac{v^{2}}{w}$

( $\mathrm{F}-23)$
Since $w$ is positive, the induced drag is reduced when the twist is chosen according to $(\mathrm{F}-22)$ as compared with the plane, untwisted wing. This subject is treated more thoroughly in Ref. F-38 for swept wings. It should be noted, however, that for sweptback wings more aerodynamic twist than this optimum is usually required in order to lessen the tendency towards tip stalling. 


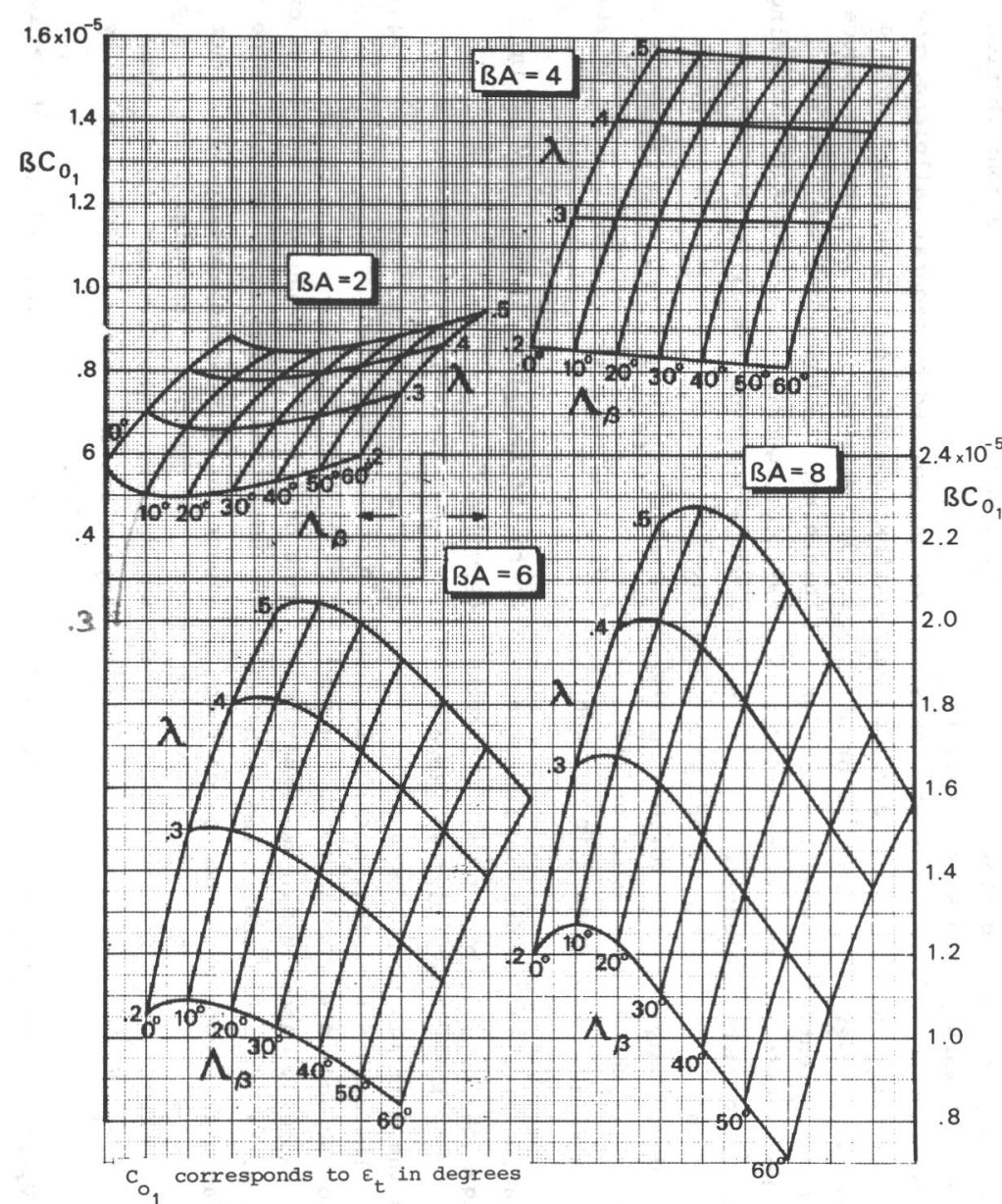

总

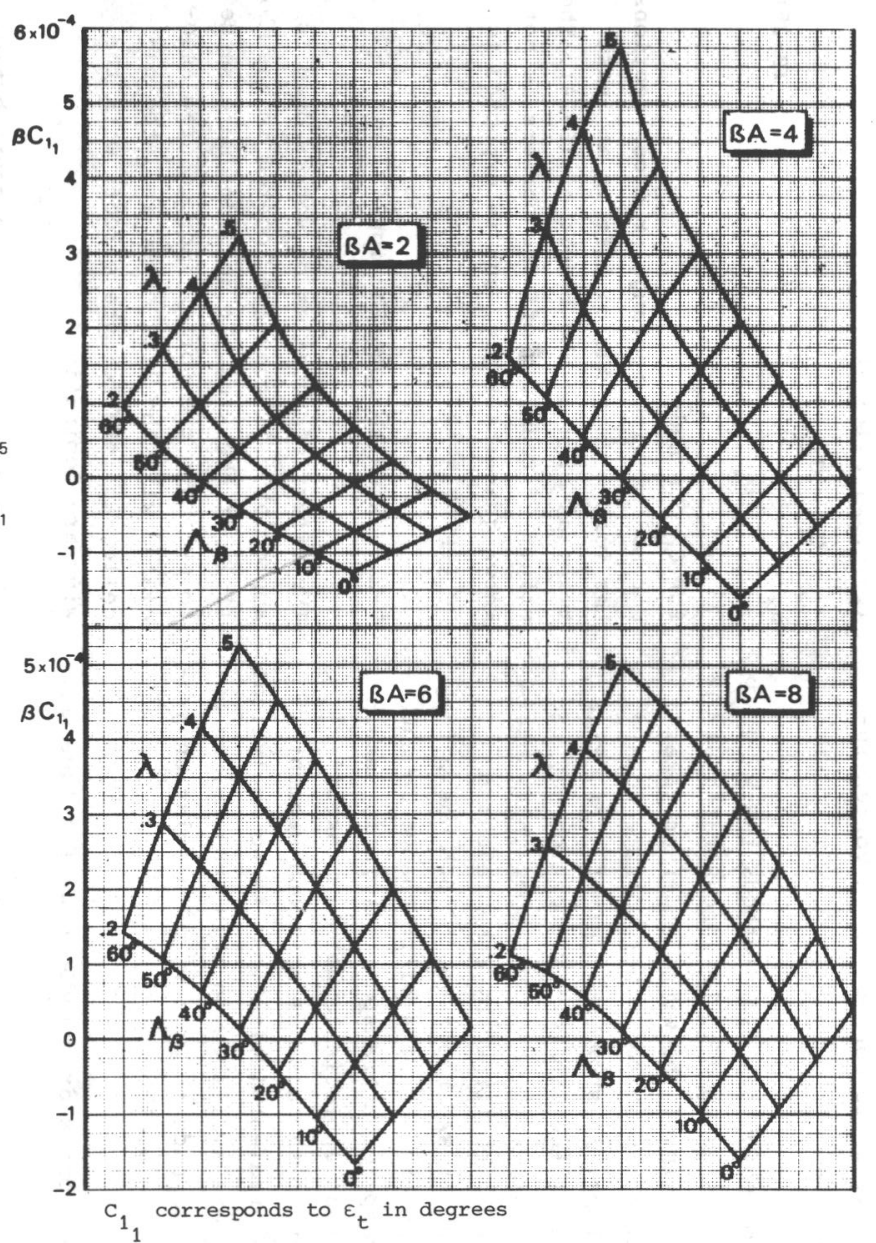

Fig. F-3. The factor $C_{1}$ for the computation of vortexinduced drag due to twist (Ref. F-4l) 
F-2.3. Wing tip correction on vortex-induced drag

In some potential flow methods the wing tip vortex is assumed to be concentrated at the extreme tip of the wing. Anderson takes a circular tip shape, others assume square wing tips. As shown by Hoerner (Ref. F-18, page 7-5) the tip vortex location may vary noticeably with the tip shape. This observation is confirmed by Schaufele and Ebeling in SAE Paper No. 670846 where they give an example of a minor change in the tip shape of a transport aircraft, resulting in an appreciable gain in takeoff weight in the case of a second segment climb gradient limitation. As no analytical tools are available on this subject, no attempt is made to present a calculation procedure here.

F-2.4. Vortex drag induced by fuselage lift

A closed body in inviscid flow does not generate lift when placed at an angle to the flow. In viscous flow, however, the situation is different due to the development of a boundary layer and subsequent breakdown of the potential flow at the rear end of the body. Lift and vortex-induced drag of bodies are much more sensitive to viscous effects than those of a wing. This section deals only with the vortex-induced drag which is the direct result of lift; a second component is discussed in Section $\mathrm{F}-3.4$.

Calculation of lift and drag on isolated fuselages may be carried out with the methods given in Refs. F-57 and F-60. However, due to the interference effects caused by the airflow around the wing, the result will not be accurate and the following simple expression, based on various experimental data, is therefore considered to be an acceptable alternative for bodies of revolution:

$C_{D} S=.15 \alpha_{f}^{2} v_{f}^{2 / 3}$

where $v_{f}$ is the volume of the fuselage and $\alpha_{f}$ is, in radians :

$$
\alpha_{\mathrm{f}}=\frac{\mathrm{C}_{\mathrm{L}}-\mathrm{C}_{\mathrm{L}_{\mathrm{o}}}}{\mathrm{C}_{\mathrm{L}_{\alpha}}}
$$

for $\mathrm{C}_{\mathrm{L}_{\alpha}}$ in $\mathrm{rad}^{-1}$.

For bodies with rectangular cross-sections the vortex drag due to $1 \mathrm{fft}$ is at least twice as high as the result of $(F-24)$. The effect of rounding off the corners is not known quantitatively, but is expected to be appreciable.

\section{F-2.5. Nacelle contribution}

The effect of nacelles on vortex drag is appreciable if they are located in the flow field of the wing. It does not make sense to estimate the vortex-induced drag associated with the lift on an isolated nacelle, as interference effects with the wing are dominating.

\section{F-2.6. Horizontal tailplane contribution}

For an isolated horizontal tailplane, the vortex-induced drag can be obtained in a similar manner as for the wing. To reduce this drag, the tailplane may also be twisted. Ignoring this effect and assuming a deviation from the elliptical drag we have:

$C_{D} S=1.02 \frac{C_{L_{h}}{ }^{2} S_{h}}{\pi A_{h}}$

where the tailplane lift is derived in Appendix $\mathrm{E}$ as :

$c_{L_{h}}=\frac{c_{m_{a c}}+c_{L}\left(x_{c g}{ }^{-x_{a c}}\right) / \bar{c}}{s_{h}^{l}{ }_{h} /(s \bar{c})}$

When dual fins are mounted as endplates on the horizontal stabilizer, the effective aspect ratio is approximately 508 higher than the geometric (see Fig. 2-26). A gap between the stabilizer and the elevator may reduce the effective aspect ratio by 15 to 208 .

It is noted that ( $F-26)$ gives only part of the trim 
drag; profile drag due to elevator deflection (Section F-3.6.) and interference with the wing (Section F-4.4.) alter the result appreciably. Data required to compute $\mathrm{C}_{\mathrm{L}}$ are given in Appendix E. Alternatively, a simple approximation can be obtained by assuming the center of gravity to coincide with the aerodynamic center of the aircraft less tail. Hence,

$\Delta C_{D}=1.02 \frac{c_{m a c}{ }^{2}}{\pi A_{h} s_{h} / s\left(\ell_{h} / \bar{c}\right)^{2}}$

F-3. PROFILE DRAG OF SMOOTH, ISOLATED MAJOR COMPONENTS

Skin friction and pressure drag due to the boundary layer and limited regions of separation are referred to as profile drag. The wing, fuselage, nacelles and empennage are considered to be smooth streamline bodies; corrections are made to allow for the interaction of the flow fields, protuberances and surface roughness, etc. There are two exceptions to this:

a. Roughness is only taken into account insofar it affects boundary layer transition.

b. The wetted area does not include those parts of the wing and fuselage, etc. that are not actually exposed to the flow (cf. Section E-4.1.).

\section{F-3.1. The flat plate analogy}

Provided the pressure distribution in potential flow over the airplane part considered is known, the boundary layer may be analyzed, at least theoretically. The pressure distribution must then be corrected for the effect of the boundary layer and a second iteration may be started. In practice, this leads to considerable difficulties, but the reader who is in a position to avail himself of such methods may use them to advantage.

In most pre-design applications, the airplane shape is not yet fully defined and for this reason the "flat plate analogy" is frequently used. This method assumes that the actual shape of the major parts can be compared to smooth streamline bodies, and excrescences and surface irregularities are ignored by fairing over the body to achieve smooth contours. Provided that the component is well streamlined, a close relation is found between the body profile drag and that of a flat plate. As the skin friction drag of a flat plate (Fig. $\mathrm{F}-4)^{*}$ can be computed very accurate1y, this provides a good basis for estimating the profile drag of the component considered.

The practical application of this flat plate analogy is as follows:

1. The wetted area of an airfoil section, wing or body is computed (Appendix B). Corrections are applied to account for the interconnection of the parts, e.g. the wing/ body junction (cf. Section F-4.1.).

2. The skin friction coefficient $C_{F}$ is determined for a smooth flat plate (Fig. F-4) with the same wetted area and projected length, the transition being located at the same position downstream from the leading edge or nose. The Reynolds number is based on the (chord) length.

3. A shape correction factor $\varphi$ is determined to account for the following factors: a. The boundary layer around a body or airfoil section develops in a different way from the flat plate flow. Boundary layer velocity profiles and local skin friction coefficients are therefore different.

b. Due to the thickness of the body, the average velocıty just outside the boundary layer is higher than the free stream velocity. Local values of the dynamic head and the skin friction per unit area are therefore increased.

c. Unlike a flat plate, a body has a frontal area and undergoes a noticeable pressure drag, particularly at high thickness/ chord or diameter/length ratios. This pressure drag is generally of the order of 5 to

* The curves shown in this figure are valid for incompressible flow. At subsonic speeds the effect of compressibility is small. 


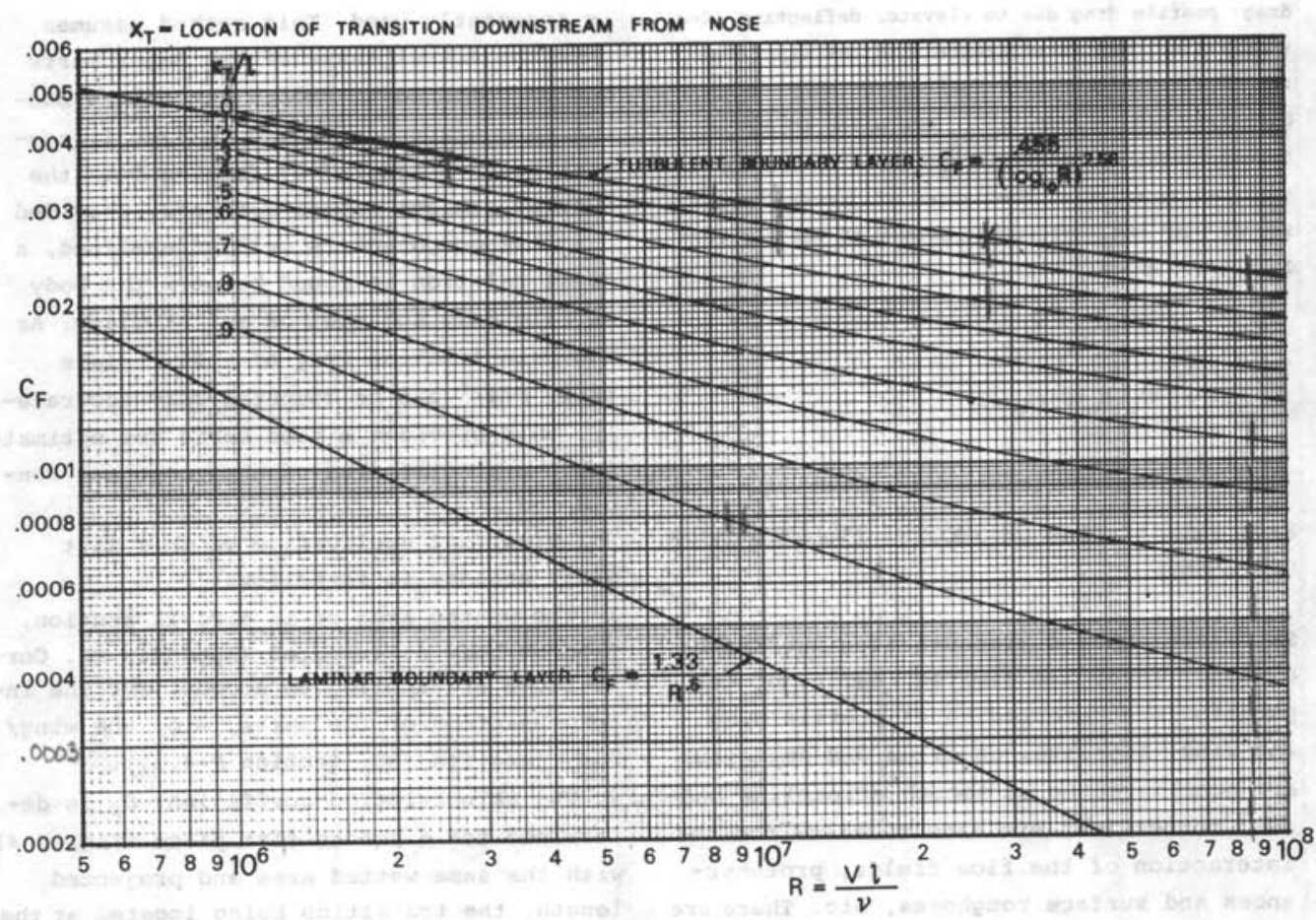

Fig. F-4. Smooth flat plate skin friction coefficient (incompressible)

$10 \%$ of the skin friction drag.

4. The drag area of the body is finally computed as:

$C_{D} S=C_{F}(1+\varphi) S_{\text {wet }}$

The validity of the flat plate analogy is subject to a number of restrictions:

a. Airfoil sections should have a thickness/chord ratio of not more than approximately 258 and body diameter/length ratios should not be more than .25 to .35 .

$$
i^{x} \mathrm{C}^{3}, 4
$$

b. The parts should have gmooth surface contours, free from kinks or steps. Conical or cylindrical body sections are acceptable, provided the transitions to the adjacent sections are gradual.

c. Lift, or angle of incidence, is zero or small. Experimental data (e.g. in Ref. F-44) are usually consulted wherever these conditions are not fulfilled and appropriate corrections are applied for lifting airfoils.

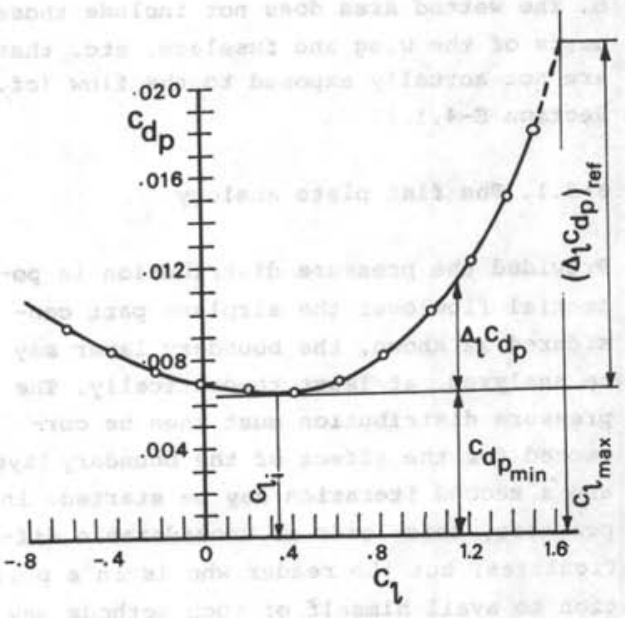

Fig. F-5. Profile drag of an airfoil section in two-dimensional flow 
F-3.2. Wing sections

\section{Poriel}

Fig. F-5 depicts a typical experimentally determined drag polar of a wing section. The profile drag coefficient, related to the chord, is split for the purpose of drag estimation as follows:

$c_{d_{p}}=c_{d_{\text {min }}}+\Delta_{\ell} c_{d_{p}}$

For most sections the condition for minimum profile drag is the design lift coefficient $c_{\ell_{1}}$, while $\Delta_{\ell} c_{d}$ is the drag increment for lift coefficients different from $c_{\ell_{i}}$. For most sections $c_{\ell_{i}}$ is approximately equal to $1 / 10$ of the camber as a percentage of the chord.

a. The minimum profile drag coefficient is: $c_{\mathrm{d}_{\text {min }}}=2 c_{F}\left(1+\varphi_{w}\right)$

The factor 2 is necessary because $c_{d_{p}}$ is based on the chord length and $\mathrm{C}_{\mathrm{F}}$ on the wetted area, both sides being exposed to the flow. The thickness correction factor $\varphi_{w^{\prime}}$ given in Fig. F-6, not only accounts for the aerodynamic effect of thickness, but also for the difference between the

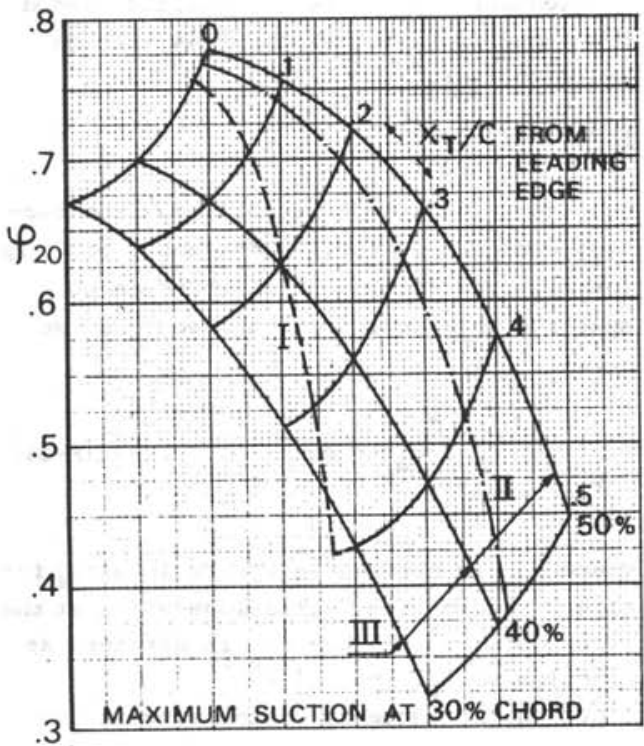

circumference and twice the projected chord length. It is found that moderate section camber has only a very slight effect on the profile drag at $c_{\ell_{j}}$. If only the thickness/ chord ratio is known, the following approximation may be used:

$\varphi_{\mathrm{w}}=2.7 t / c+100(t / c)^{4}$ for $t / c<.21(F-30)$

b. Boundary layer transition is frequently assumed to occur at the leading edge, but for straight high aspect ratio wings the result is conservative, particularly at Reynolds numbers less than $10^{7}$. On the other hand, wind tunnel measurements with free transition yield erroneous results due to scale effects and to the effects of the unavoidable surface irregularities of practical wing constructions. In particular, the low-drag "bucket" on laminar flow sections will seldom be realized in practice, except on carefully polished high performance sailplanes. Wind tunnel experiments are therefore usually carried out with roughness strips at the position where the transition is likely to occur on the aircraft in flight For Reynolds numbers up to $10^{7}$ a transition region 15 to $208^{*}$ of the chord from

* Mean value for the upper and lower surface.

I: "Conventional" airfoils (NACA 4- and 5- digit series):

$\varphi_{\mathrm{w}}=\left(\frac{\mathrm{t} / \mathrm{c}}{.20}\right)^{1.38} \varphi_{20}$

II: Cusped trailing edge, max. thickness at 408 chord (NACA 6-digit series):

$\varphi_{w}=\left(\frac{t / c}{.20}\right)^{1.10} \varphi_{20}$

III: NACA $6 \mathrm{~A}-$ and similar series with straight trailing edge:

$P_{w}=\frac{t / c}{.20} \varphi_{20}$

NOTE: these curves have been derived by approximating the various curves in R.Ae.S. Data Sheets "Wings" 02.04 .02 and 02.04.03.

Fig. F-6. Shape factor for some airfoils 
the leading edge will be representative of good average straight wing constructions. Near the wing/fuselage and wing/nacelle junctions and in the propeller slipscream, however, transition to turbulent flow is to be expected at about 5 to $10 \%$ of the chord. This is also generally the case with sweptback wings, where spanwise pressure gradients cause cross flows at the leading edge, provoking early transition. At Reynolds numbers above $3 \times 10^{7}$ the transition can be assumed to occur at $5 \%$ of the chord for all section shapes.

c. The lift correction to the profile drag can be related to the lift increment relative to $\mathrm{c}_{\ell_{1}}$ :

$\frac{\Delta_{\ell} c_{d_{p}}}{\left(\Delta_{\ell} c_{d_{p}}\right)_{\text {ref }}}=f\left(\frac{c_{\ell}-c_{\ell}}{c_{\ell}-c_{l_{1}}}\right)^{2}$

where $\left(\Delta_{\ell} c_{d_{p}}\right)_{\text {ref }}$ is representative of the (extrapolated) profile drag increment at the stalling angle of attack (cf. Fig. F-5). The following expressions have been derived from experimental data on NACA 4 and 5 digit sections:

$$
\begin{aligned}
& \text { for } R_{6} 10^{7}:\left(\Delta_{\ell} c_{d_{p}}\right)_{\text {ref }}=\frac{67 c_{\ell}}{\left(\log _{10} R\right)^{4.5}}- \\
& -.0046(1+2.75 t / c)
\end{aligned}
$$

$$
\text { for } \begin{array}{r}
\mathrm{R}>10^{7}:\left(\begin{array}{lll}
\Delta_{\ell} & c_{d_{p}}
\end{array}\right)_{\text {ref }}=.01 c_{\ell \max } \\
-.0046(1+2.75 \mathrm{t} / \mathrm{c})
\end{array}
$$

The Reynolds number in this expression should be based on a condition at high lift, for example at 708 to 808 of $c_{l}$-max, as the flight speed is lower than the condition for which the minimum profile drag is computed. The ratio $\Delta_{\ell} c_{d_{p}}\left(\Delta_{\ell} c_{d_{p}}\right)_{\text {ref }}$ is plotted in Fig. $\mathrm{F}-7$ as a mean line; most conventional sections are within +108 from this line. The figure suggests that for $c_{\ell}-c_{\ell_{1}}$ up to 85 of of $c_{\ell}-c_{\ell_{1}}$ the following straight line approximation is acceptable:

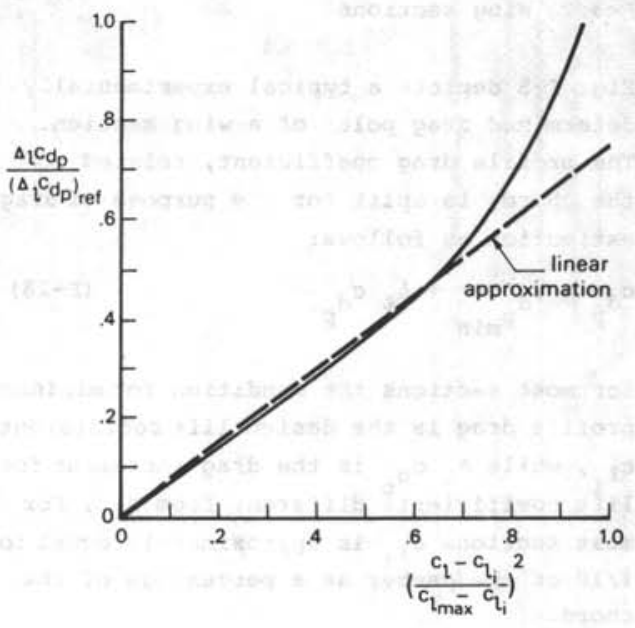

Fig. F-7. Generalized profile drag increment with lift

$\Delta_{\ell} c_{d_{p}}=.75\left(\Delta_{\ell} c_{d_{p}}\right)_{\text {ref }}\left(\frac{\varepsilon_{\ell}-c_{\ell}}{c_{\ell}{ }_{\max }^{-c_{\ell}}}\right)^{2}$

This expression may also be used for laminar flow sections, where the region of low drag is not obtained for practical constructions. If low drag can be achieved, experimental data with free transition must be used instead of the generalized method presented above; cf. Refs. F-44 through $\mathrm{F}-46$ and $\mathrm{F}-48$.

F-3.3. Wings $3^{-1}$

The drag coefficient is obtained from section characteristics. On the basis of strip theory, i.e. assuming that there is no spanwise interaction of the sections, we have:

$C_{D_{p}}=\frac{2}{s} \int_{b_{f} / 2}^{b / 2} \int_{d_{p}}^{b} c d y$

where $c_{d_{p}}$ is determined by the local $c_{\ell}$ for each $C_{L}$ and $b_{f}$ is the fuselage width at the wing root. In this way $C_{D_{p}}$ is obtained as a function of $\mathrm{C}_{\mathrm{L}}$. provided the geometry of the wing is de- 
fined and the sectional shape is known for a number of spanwise stations, the profile drag coefficient can be obtained from

(F-29), corrected for the sweepback angle:

$c_{\mathrm{p}_{\text {min }}}=2 \mathrm{c}_{\mathrm{F}}\left(1+\varphi_{\mathrm{W}} \cos ^{2} \Lambda_{\frac{1}{2}}\right)$

The shape factor $\varphi_{w}$, based on the streamwise section, is given by Fig. F-6 or $(\mathrm{F}-30)$.

Any variation in operational conditions results in variations of $C_{L}$ and the Reynolds number. As it is not customary to calculate a drag polar for each operational value of $R$, the minimum profile drag is determined for an average cruise condition. However, the profile drag increment for $\mathrm{C}_{\mathrm{L}}>\mathrm{C}_{\text {. }}$. should be estimated for a representative value of $R$ at the lower end of the operational flight envelope (flaps up), for example, at a Reynolds number equal to half the value in cruising flight.

The integral in $(F-34)$ may be evaluated as follows: a. Assume a combination of $R$ per unit length and $\mathrm{C}_{\mathrm{L}}$, corresponding to the flight condition to be considered.

b. Compute the spanwise lift distribution using any suitable method available, for example, Diederich's method (Section E-4.2. of Appendix E).

c. Compute the local value of $\mathrm{R}$, related to the chord, at a number of spanwise stations and calculate the minimum profile drag coefficient with $(\mathrm{F}-35)$.

d. Estimate $c_{\ell}$-max from experimental data, or Section E-3.3. of Appendix E, for each spanwise station.

e. Compute $\Delta_{\ell} c_{d_{p}}$ for each station using experimental data or $(F-32)$ and $(F-33)$.

f. For each station plot $c_{d}, c$ versus $y$, draw a line through the calculated points and integrate numerically according to $(\mathrm{F}-34)$.

g. Repeat the process for several values of $C_{L^{\prime}}$, to obtain $C_{D_{p}}=f\left(C_{L}\right)$.

For straight wings this procedure, based on strip theory, yields good results, but for sweptback wings the method is open to criticism in view of the effects of crossflows in the boundary layer.

Instead of the method explained previously, a much simpler procedure will usually be acceptable:

a. For a representative station midway between the wing/fuselage junction and the wing tip, the local section properties are estimated: $\bar{R}, \bar{c}_{\ell_{i}}, \bar{c}_{\ell_{\text {max }}}, \bar{c}_{d_{p_{\min }}}$ with $(\mathrm{F}-35)$ and $\Delta_{\ell}\left(\bar{c}_{\mathrm{d}_{\mathrm{p}}}\right)_{\text {ref }}$ with $(\mathrm{F}-32)$.

b. The three-dimensional profile drag coefficient is derived from these data:

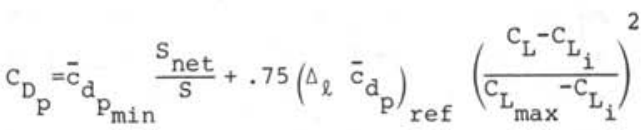

where $\mathrm{C}_{\mathrm{L}_{i}}$ is assumed equal to $\overline{\mathrm{c}}_{\ell_{1}}$ and $\mathrm{C}_{\mathrm{L}_{\max }}$ can be estimated with section E-4.2. of Appendix E. The net wing area, defined in Appendix A, Section A-3.1., is obtained from the gross wing area by subtracting the projected area of the wing section covered by the fuselage.

Equation F-36 can readily be rewritten as an equation expressing $C_{D_{p}}$ in terms of a constant, a term proportional to $C_{L}$ and a term proportional to $\mathrm{C}_{L}{ }^{2}$. Typical minimum profile drag coefficients range between .006 and .010 .

\section{F-3.4. Fuselages and tail booms}

The aerodynamic ideal of a smooth streamlined shape, as defined in Section F-3.1., is not approximated with certain low-speed aircraft, such as agricultural aircraft, light private aircraft and small transports of simple construction. In those cases the designer must rely on experimental and statistical data of similar shapes, as presented in Ref $\mathrm{F}-20$, for example

An impression of the drag coefficient of streamlined fuselages can be gained from Fig. F-8. As these data are valid for rather outdated fuselages, measured at low Reynolds number, a more detailed calculation is usually made, using the flat plate analogy. The outer contour is considered to be a streamlined shape, assuming that 


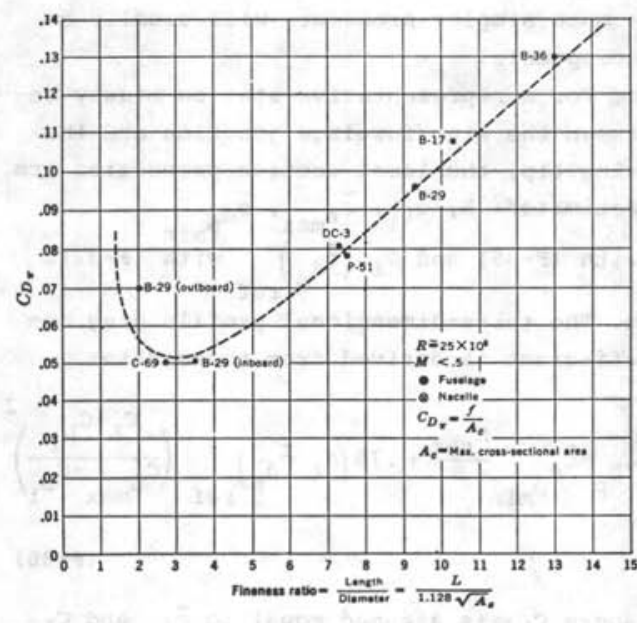

Fig. F-8. Effect of fineness ratio on fuselage and nacelle frontal drag coefficient (Ref. F-4)

all excrescences such as wheel well fairings, canopy enclosures and airscoops, etc. have been removed or faired over. For streamlined fuselages with pointed tail sections with a length/diameter ratio of at least 2 and without a jet efflux, we may write:

$C_{D} S=\left(C_{D} S\right)_{\text {basic }}+\Delta_{\alpha \beta}\left(C_{D} S\right)$

a. Streamlined fuselages without blunt nose or tail sections, no jet efflux.

The basic fuselage drag is the profiledrag of an uncambered, smooth streamline body at zero angle of attack:

$\left(C_{D}\right)_{\text {basic }}=C_{F} S_{f_{\text {wet }}}\left(1+\varphi_{f}\right)$

$(\mathrm{F}-38)$

The wetted area is the gross wetted area, reduced by those areas of the fuselage that are not exposed to the flow (cf. Section $F-4.1$.). The value of $C_{F}$ is read off from Fig. F-4 for $R$ based on the fuselage length Transition is assumed to occur at or very near the nose. The shape factor $\varphi_{f}$ is mainly a function of the effective fuselage slenderness ratio: $\lambda_{\text {eff }}=\frac{l_{f}}{D_{f}}$

or

$\lambda_{\text {eff }}=\frac{\ell_{N}+l_{A}}{D_{\text {eff }}}+2$

whichever is less $(\mathrm{F}-39)$

where

$D_{f_{\text {eff }}}=\sqrt{\frac{4}{\pi} A_{c}}$

$(\mathrm{F}-40)$

and the fuselage nose and tail section

lengths $\ell_{N}$ and $\ell_{A}$ are defined in Fig. F-10. Fig. F-9 shows the shape factor $\varphi_{f}$ vs. the reciprocal value of the slenderness ratio, according to several prediction methods. Experiments are also shown, although several points refer to fuselages with swept-up tail sections, having approximately 5 more drag than the axisymmetric fuselage.

The following relation is suggested for fuselages without a blunt nose and with an optimum pointed tail section:

$\varphi_{f}=\frac{2.2}{\lambda_{\text {eff }} 1.5}+\frac{3.8}{\lambda_{\text {eff }}^{3}} \quad$ for $\frac{l_{A}}{D_{f_{\text {eff }}}} \geq 2 \quad(F-41)$

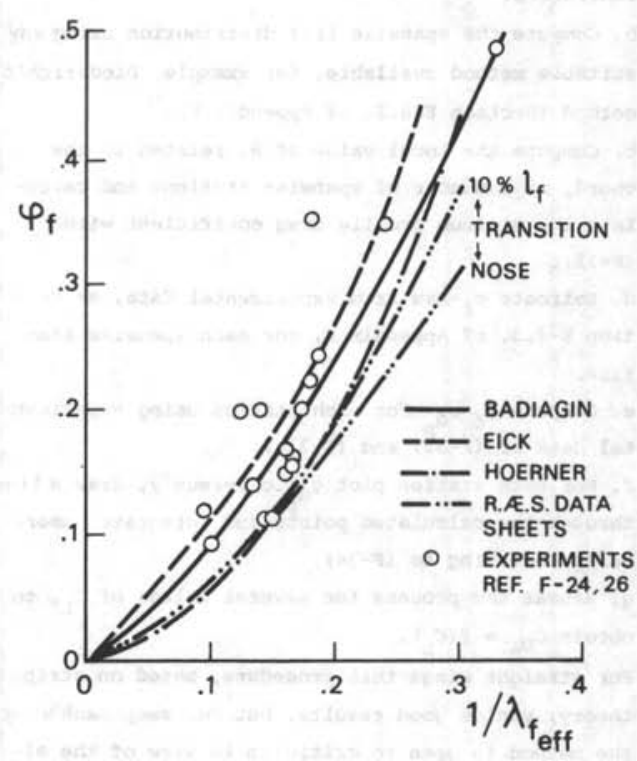

Fig. F-9. Fuselage shape factor 


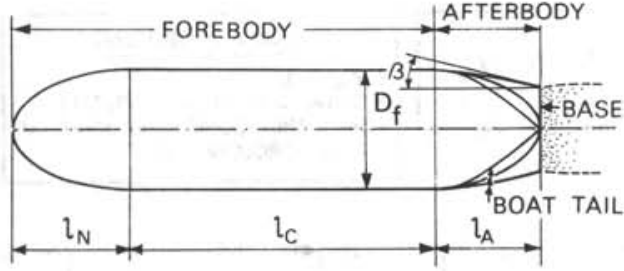

Fig. F-10. Nomenclature for determining the drag of fuselages with short afterbody or a jet efflux

b. Fuselages with blunt and/or short afterbodies, no jet efflux.

Small areas normal to the flow at the rear end of the fuselage may be present, e.g. in the case of an APU installation or a blunt tail fairing. Provided the base area is less than approximately 108 of the fuselage cross-sectional area and there is no jet efflux, it is sufficiently accurate to increase the basic fuselage drag area by an amount which can be written as:

$\Delta C_{D} S=.13 \times$ base projected area

$(\mathrm{F}-42)$

No method is available to give an accurate prediction of the drag due to a large base area and/or a short afterbody, such as is used on some freighter aircraft (Figs . $3-3 b, 3-4$ and 3-2ld). The following procedure will at least yield a qualitatively correct result for short afterbodies and boat tails, with or without jet efflux.

1. Calculate the basic fuselage drag coefficient with the method given in section a above, assuming the afterbody length/diameter ratio equal to 2 , hence:

$\lambda_{\text {eff }}=\frac{l_{\mathrm{N}}{ }^{+\ell} \mathrm{c}}{\mathrm{D}_{\mathrm{f}}}+2$

or whichever is less $(\mathrm{F}-43)$

$\lambda_{\text {eff }}=4+\frac{\ell_{N}}{D_{\text {eff }}}$

2. Calculate the base drag that would occur in the absence of an afterbody, i.e. with the fuselage truncated immediately behind the cylindrical section. Using Hoerner's data (Ref. F-58):

$\left(C_{D_{b}}\right)_{\text {ref }}=.015 \sqrt{\frac{D_{f}}{C_{F}^{\left(l_{N f} N^{+\ell}{ }^{\prime}\right)}}}$

Alternatively, this coefficient may be assumed equal to .13 .

3. A pressure drag increment is added to the basic fuselage drag coefficient to account for actual fuselage afterbody length and shape:

$\Delta\left(C_{D} S\right)_{A}=\left(C_{D_{b}}\right)_{\text {ref }} \frac{\Delta C_{D_{A}}}{\left(C_{D_{b}}\right)_{\text {ref }}} \frac{\pi}{4} D_{f_{\text {eff }}}^{2}(F-45)$ The afterbody pressure drag increment is given in Fig. F-11 as a fraction of the reference base drag.

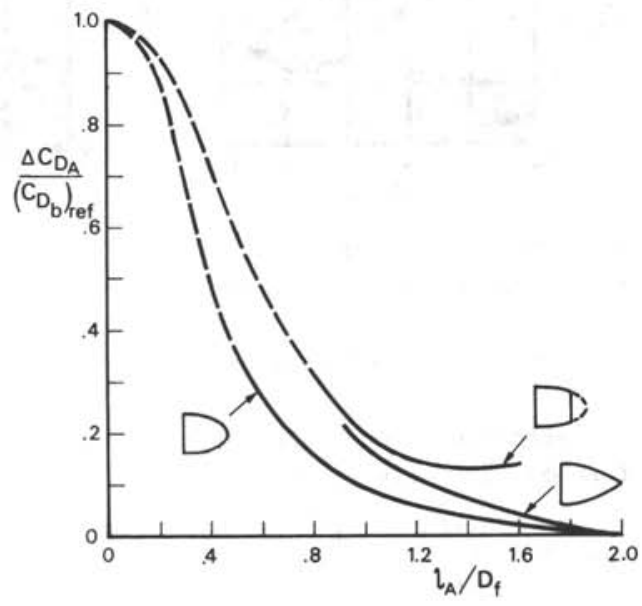

NOTE: $l_{\mathrm{A}}$ and $\mathrm{D}_{\mathrm{f}}$ are defined in Fig. $\mathrm{F}-10$

Fig. F-11. Pressure drag increment vs. fineness ratio of short afterbodies without jet efflux (Refs.: F-18, F-65 and F-98)

c. Fuselages with propulsive jets.

The basic fuselage drag is subdivided into contributions of the forebody and the afterbody boat tail drag:

$\left(C_{D} s\right)_{\text {basic }}=\left(C_{D} s\right)_{F}+C_{D_{B}} \frac{\pi}{4} D_{f_{\text {eff }}}{ }^{2}$ 

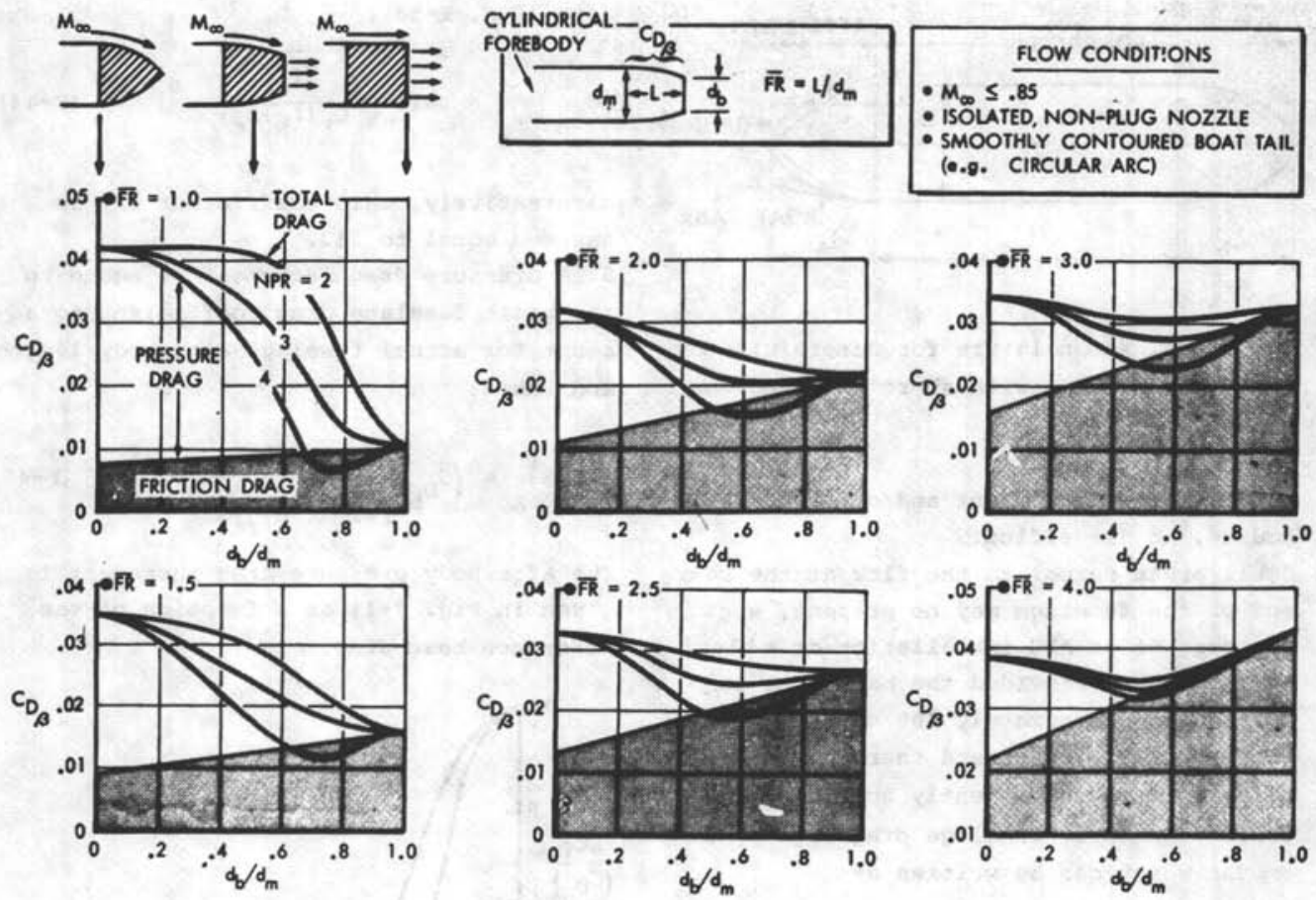

NOTE: The author suggests to analyze alternative boat tail shapes (e.g. parabolic) by taking an equivalent $\overline{F R}$ such that the boat tail angle $\beta$ (Fig. F-10) is equal to $\beta$ for the comparable circular arc.

Fig. r-12. Drag correlation for circular arc boat tails (Ref. F-98)

The effective slenderness ratio for the forebody is defined as follows:

$\lambda_{\text {eff }}=\frac{2 \ell_{N}+l_{C}}{D_{\text {eff }}}$

or

$\lambda_{\text {eff }}=2\left(1+\frac{l_{N}}{D_{f}}\right)$

The forebody drag area $\left(C_{D} S\right)$ is calculated with $(\mathrm{F}-38)$, using the forebody wetted area as the reference and taking the shape correction factor equal to half the value for normal fuselages:

$\varphi_{\mathrm{f}}=\frac{1.1}{\lambda_{\mathrm{eff}}{ }^{1.5}}+\frac{1.9}{\lambda_{\mathrm{eff}}{ }^{3}}$

The boat tail drag coefficient $C_{D_{B}}$ is read off from Fig. F-12 for the appropriate
Nozzle Pressure Ratio (NPR) which should be obtained from the engine type specification. Fig. F-12 does not apply to boat tails with a base area; the reader is referred to Ref. F-86 for this case.

d. Correction for angle of attack and upsweep effects.

A generally accepted method is not available, vut a reasonable approximation can be made on the basis of the cross flow concept (Ref. F-62). For an isolated fuselage in uniform flow the drag on a fuselage element due to the cross flow (Fig. $\mathrm{F}-13$ ) is:

$d D_{c}=c_{d_{c}} \cdot \frac{1}{2} \rho_{\infty}\left\{v_{\infty} \sin \left(\alpha_{f}-\beta\right)\right\}^{2} \frac{b d x}{\cos \beta} \sin \left|\alpha_{f}-\beta\right|$

The effective drag coefficient $c_{d_{c}}$ is that of an infinitely long cylinder, with cross 


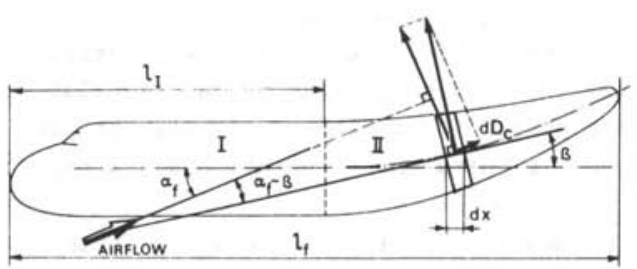

Fig. F-13. Derivation of fuselage cross flow drag

section equal to the local fuselage cross section, perpendicular to an airflow. This coefficient must be determined for a Reynolds number, based on $\operatorname{Vsin}\left(\alpha_{f}-\beta\right)$, and corrected for the finite length of the fuselage. It can be obtained from Ref. F-18 or F-57. For fuselages with a circular cross section $\mathrm{c}_{\mathrm{d}_{\mathrm{c}}} \approx 1.0$ and for a rectangular cross section with rounded corners $c_{d_{c}}=1.5$ to 2.0 .

Integration of $(\mathrm{F}-49)$ yields:

$\frac{D_{C}}{\frac{1}{2} \rho_{\infty} v_{\infty}{ }^{2}}=\Delta_{\alpha \beta}\left(C_{D} s\right)=\int_{0}^{l_{f}}\left|\sin ^{3}\left(\alpha_{f}-\beta\right)\right| c_{d_{c}} x$ $\frac{b d x}{\cos B}$

This integral can be computed numerically as a function of $\alpha_{f}$. For the simplified case of an axisymmetrical forebody (Section I: $B=0$ ) and a constant upsweep angle of the afterbody (Section II) and assuming $c_{d_{c}}=1.0$ : $\Delta_{\alpha \beta}\left(C_{D} s\right)=A_{I}\left|\sin ^{3} \alpha_{f}\right|+A_{I I} \frac{\left|\sin ^{3}\left(\alpha_{f}-\beta\right)\right|}{\cos \beta}$

where $A_{I}$ and $A_{I I}$ are the planform areas of sections I and II (Fig. F-13). By way of example, the result is plotted in Fig. $\mathrm{F}-14$. In spite of the crude assumptions made, the result appears to be realistic when compared with the experimental data reported in Ref. F-62. For $\alpha_{f}$ or $B$ in excess of $5^{\circ}$ the extra drag is a significant fraction of the basic fuselage drag coefficient. The minimum of the curves can be shown to occur at: $\alpha_{f}^{\prime}=\beta \frac{\sqrt{A_{I} / A_{I I}}-1}{A_{I} / A_{I I}-1}$

In order to fit the curves given by $(\mathrm{F}-50)$ or $(F-51)$ into the usual concept of a parabolic polar, they may be approximated by

$\Delta_{\alpha \beta}\left(C_{D} s\right)=D_{1}+D_{2}\left(\alpha_{f}-\alpha_{f}^{\prime}\right)^{2}$

where the constants $D_{1}$ and $D_{2}$ are sbtained by plotting $\Delta_{\alpha \beta}\left(C_{D} S\right)_{f}$ versus $\left(\alpha_{f}-\alpha_{f}^{\prime}\right)^{2}$, and replacing the result by a straight line. In order to express $\Delta_{\alpha \beta}\left(C_{D} S\right)_{f}$ in terms of $C_{L}$, it is noted that:

$\alpha_{f}=\frac{\mathrm{C}_{L}-\mathrm{C}_{L_{0}}}{\mathrm{C}_{L_{\alpha}}}$

See Section E-9.4. of Appendix E.

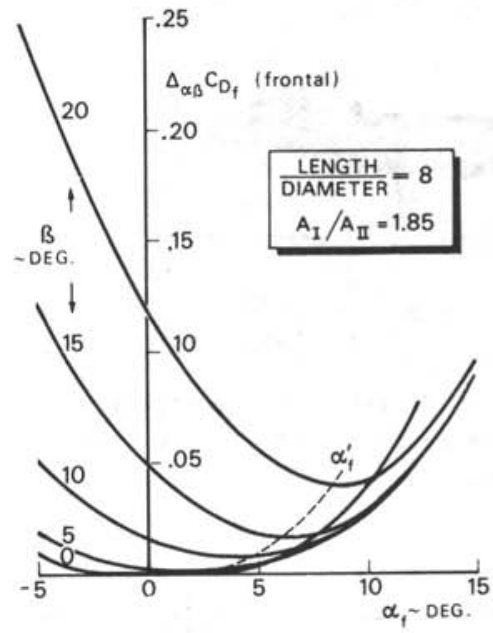

Fig. F-14. Estimated fuselage profile drag increment due to angle of attack and rear fuselage upsweep

F-3.5. Engine nacelles

Although in preliminary design nacelle drag is frequently assumed to be independent of $C_{L}$, interference effects with the wing, for example, can be quite appreciable (cf. Section $\mathrm{F}-4.3$.$) .$ 
a. Propeller aircraft.

The calculation procedure is analogous to that for the fuselage basic drag, explained in section F-3.4. The nacelle (spinner included) is considered to be a smooth streamline body, generally with low slenderness ratio and fully turbulent boundary layer (Fig. F-15). The drag area per na-

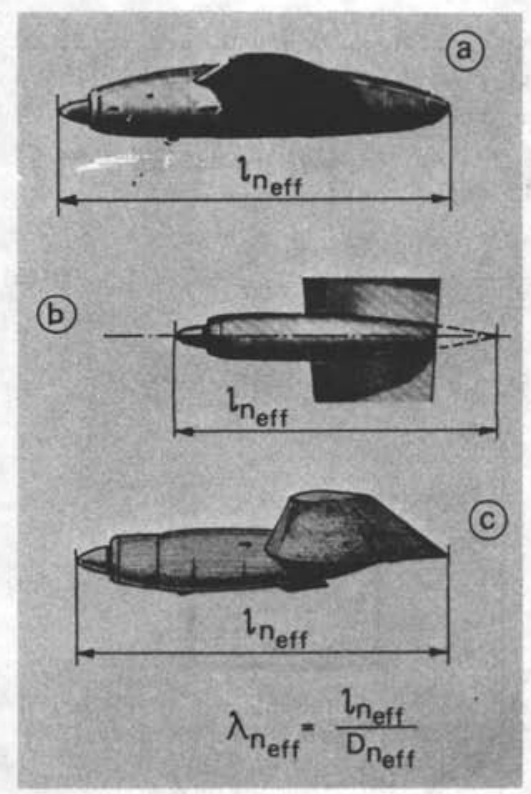

Fig. F-15. Definition of the effective slenderness ratio of nacelles for propeller engines

celle is:

$C_{D} S=C_{F}\left(1+\frac{2.2}{\lambda_{n_{\text {eff }}} 1.5}+\frac{3.8}{\lambda_{n_{\text {eff }}}{ }^{3}}\right) S_{n_{\text {wet }}}$

In order to define $\lambda_{n_{e f f}}$ a rational cnoice of the nacelle length must be made. If the centerline of the nacelle is approximately lined up with the chord, as in Fig. F-15c, the presence of the wing will lead to a reduction in pressure drag. The effective nacelle slenderness ratio should be increased accordingly. Instead of, or in addition to, using the method of $(F-54), a$ rough figure can be obtained from Fig. F-8. b. Jet engine nacelles.

Fig. F-16 shows a typical nacelle arrangement for a high bypass engine installation. A separate fan cowl is generally not present for straight turbojets and low bypass ratio engines.

The general terminology relating to the subdivision of a powered jet engine nacelle drag can be found in the literature, e.g. Ref. F-78. Engine thrust is assumed to be defined as "standard gross" and "standard net thrust", as in the ESDU Data Sheet 69006 . Internal friction losses of the inlet diffuser are not considered; these are accounted for in the (installed) engine thrust determination.

The total drag per nacelle less interference is subdivided into drag contributions of the fan and gas generator cowlings, the plug in the primary flow and the pylon. Reference will be made to the geometric definitions of Fig. B-5 of Appendix B.

The (fan) cowling drag area is written as the sum of a friction drag and an afterbody pressure drag component:

$C_{D} S=C_{F}\left\{\beta\left(1+\varphi_{n}\right)^{5 / 3}+(1-\beta)\right\} S_{n_{\text {wet }}}+\left(C_{D_{B}}\right) \frac{\pi}{4} D_{n}{ }^{2}$

where $\beta$ denotes the cowling forebody length divided by the cowling length.

The flat plate skin friction coefficient $C_{F}$ (Fig. $F-4$ ) is determined for $R$ based on the length of the cowl or nacelle and full$y$ turbulent flow. The (fan) cowling wetted area can be calculated with Section B-4 of

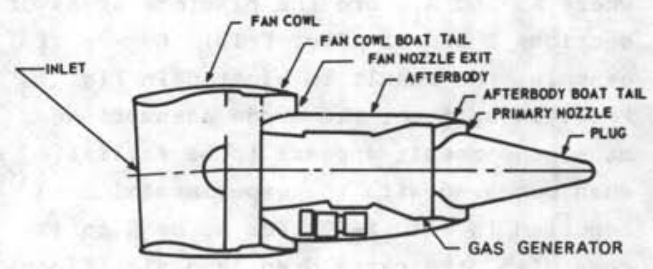

Fig. F-16. Typical high bypass engine nacelle 
Appendix B .

In $(\mathrm{F}-55)$ the terms in the brackets represent the skin friction drag of the forebody, corrected by means of $\varphi_{n}$ for excess velocities associated with the cowl curvature and angle of attack and the skin friction drag of the afterbody (boat tail). According to Ref. F-95 the following correlation applies to the NACA-1 Series of inlets:

$\varphi_{n}=.33 \frac{D_{n}-D_{h}}{B l_{n}}\left(1+1.75 \frac{D_{h}{ }^{2}-D_{o}{ }^{2}}{D_{n}{ }^{2}-D_{h}^{2}}\right)$

where $D_{0}$ is the diameter of the capture area, i.e. the internal stream tube of the engine flow at large distance in front of the engine. It can be shown that:

$\frac{\mathrm{D}_{\mathrm{h}}{ }^{2}-\mathrm{D}_{\mathrm{o}}{ }^{2}}{\mathrm{D}_{\mathrm{n}}{ }^{2}-\mathrm{D}_{\mathrm{h}}{ }^{2}}=\frac{1-31.55 \dot{\mathrm{w}}_{\mathrm{a}} \sqrt{\theta} /\left(\mathrm{p}_{\infty} \mathrm{M}_{\infty} \mathrm{D}_{\mathrm{h}}{ }^{2}\right)}{\left(\mathrm{D}_{\mathrm{n}} / \mathrm{D}_{\mathrm{h}}\right)^{2}-1}$

The last term of $(\mathrm{F}-55)$ is the boat tail pressure drag area. The pressure drag coefficient $\left(C_{D_{B_{p}}}\right)$ can be obtained from Fig. F-12 by subtracting the skin friction drag from the total boat tail drag, as indicated, or from Ref. F-86.

The fan cowl drag is sensitive to the geometries of the cowling and the fan nozzle, and to the engine thrust setting. If (F-56) cannot be used because the cowl design is not yet completely defined, a typical figure may be assumed for a well-designed medium to high bypass ratio turbofan cowling, in cruising flight:

$\mathrm{C}_{\mathrm{D}} \mathrm{S} \approx 1.25 \mathrm{C}_{\mathrm{F}} \mathrm{s}_{\mathrm{n}_{\text {wet }}}$

The expressions given for the engine cowl drag are valid only if the intake lip is well designed and operates at approximately the datum engine mass flow. In that case there is no appreciable loss in lip suction and the pre-entry drag is fully balanced by the suction forces on the front cowl. No additive drag is present in this condition. The validity of this assumption must be verified in later aerodynamic development.

The gas generator cowling drag component is frequently quoted by the manufacturer in the engine brochure as it is usually considered to constitute an effective thrust loss. In the absence of this information the following expression can be used:

$$
\begin{gathered}
C_{D} S=C_{F}\left(\frac{M_{f}}{M_{\infty}}\right)^{11 / 6}\left(\frac{1+.116 M_{\infty}^{2}}{1+.116 M_{f}^{2}}\right)^{2 / 3} s_{g_{\text {wet }}}+ \\
+\left(C_{D_{B}}\right)_{p} \frac{\pi}{4} D_{g}{ }^{2}
\end{gathered}
$$

The first term is the scrubbing drag, associated with the high velocity jet to which the gas generator cowling is exposed (Ref. F-93). The skin friction drag coefficient for fully turbulent flow is related to free stream conditions and the length of the gas generator cowling plus the fan nozzle length. The Mach number of the fully expanded fan flow $M_{f}$ can be obtained from the engine manufacturer's brochure or $(\mathrm{H}-21)$ in Appendix $\mathrm{H}$. The gas generator cowling wetted area can be computed with Section B-4 of Appendix B. The part of the pylon which is immersed in the fan exhaust flow must be included in this wetted area.

The second term of $(\mathrm{F}-59)$ is the gas generator boat tail pressure drag area. Generalized data for calculating $\left(C_{D_{\beta}}\right)_{p}$ are not available, but Fig. F-17 is thought to be fairly representative.

Scrubbing drag of the plug is usually considered as a loss in engine gross thrust. If no data are quoted in the engine brochure its order of magnitude can be estimated as follows:

$$
C_{D} S=C_{F} S_{p_{\text {wet }}}\left(\frac{M_{g}}{M_{\infty}}\right)^{11 / 6}\left(\frac{1+.116 M_{\infty}^{2}}{1+.116 M_{g}^{2}}\right)^{2 / 3}
$$

The plug wetted area is given in Section B-4 of Appendix B, the fully expanded hot flow Mach number $\mathrm{M}_{\mathrm{g}}$ being derived from the engine type specification or $(\mathrm{H}-20)$ of Appendix $\mathrm{H}$. The plug drag amounts to roughly 58 of the total engine pod drag in cruising flight. 


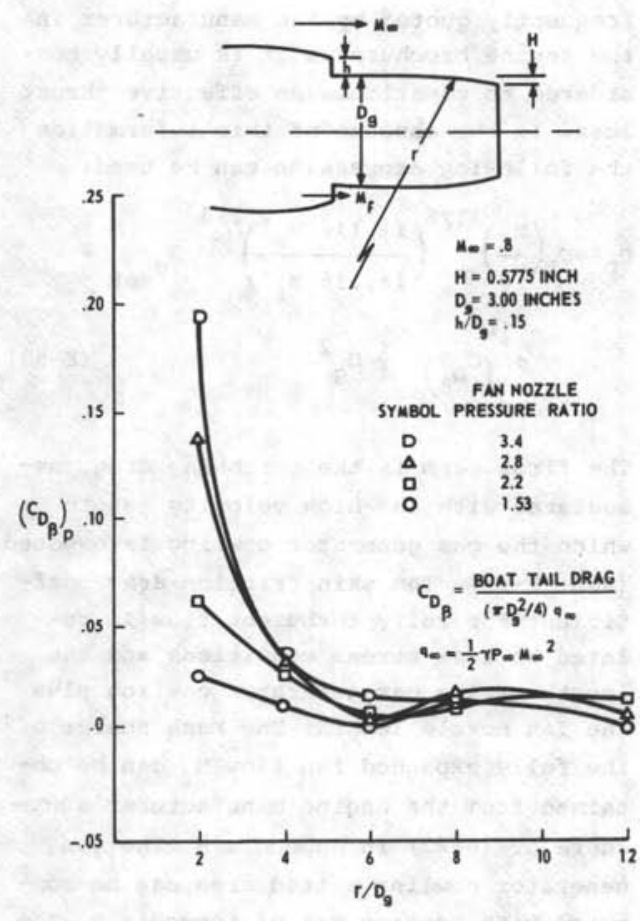

Fig. F-17. Boat tail pressure drag coefficient vs. radius of curvature (Ref. F-89)

Pylon drag is similar to wing profile drag and is determined from:

$C_{D} S=C_{F}\left\{1+2.75(t / c)_{p y} \cos ^{2} \Lambda_{p y}\right\} S_{p y}$ wet

The relative thickness of the pylon is defined in streamwise direction and the sweepback angle applies to the quarterchord line of the pylon.

\section{F-3.6. Tailplane profile drag}

For the horizontal tailplane the profile drag can be subdivided into basic profile drag and profile drag due to lift associated with incidence and elevator deflection:

$C_{D} S=\left(C_{D} S\right)_{h_{\text {basic }}}+\Delta_{\ell}\left(C_{D} S\right)_{h}$

The basic or minimum profile drag is sim- ilar to the wing profile drag:

$$
\left(C_{D} S\right)_{h_{\text {basic }}}=2 C_{F}\left\{1+2.75(t / c)_{h} \cos ^{2} \Lambda_{\frac{1}{2}}\right\}
$$

where $C_{F}$ can be read off from Fig. $F-4$. The reference length for $R$ and $t / c$ is the mean geometric tailplane chord, and it is usual to assume transition at the leading edge. To account for interference with the airplane, $s_{h}$ may be assumed as the gross horizontal tailplane area, including parts covered by the fuselage or vertical tailplane (cf. Section F-4.4.).

Drag increment due to incidence and elevator deflection is difficult to compute accurately. From the very scanty data available it was concluded that this term is mainly dependent upon $\mathrm{C}_{\mathrm{L}_{\mathrm{h}}}$ irrespective of the tailplane angle of attack and the elevator deflection. This drag contribution can therefore be related to the vortex-induced drag of the tailplane by introducing an effective Oswald factor of the order of $.75 \cos ^{2} \Lambda_{h}$ for the horizontal tailplane. Hence:

$\Delta_{\ell}\left(C_{D} s\right)_{h}=\frac{.33 C_{L_{h}}^{2}}{\cos ^{2} \Lambda_{h} \pi A_{h}} s_{h}$

Some comments on the calculation of $\mathrm{C}_{\mathrm{L}_{\mathrm{h}}}$ are given in Section $\mathrm{F}-2.6$.

Vertical tailplane drag is given by:

$$
\left(C_{D} S\right)_{V}=2 C_{F}\left\{1+2.75(t / c)_{v} \cos ^{2} \Lambda_{\frac{1}{2}}\right\} s_{V}
$$

where $C_{F}$ is read off from Fig. F-4. The mean geometric chord of the vertical tailplane is the reference length for $R$ and $t / c$. It is usual to assume transition at the leading edge. The gross vertical tailplane area is used to account for interference (cf. Section F-4.4.). Drag due to sideload on the vertical tailplane is not present in symmetrical flight. The case of engine failure will be treated in Appendix G, Section G-8.3. 
F-4. SUBCRITICAL INTERFERENCE EFFECTS AND CORRECTIONS

For a well designed configuration the effects of interference will be limited to a bare minimum. This can be obtained by suitable filleting, fairings and wing/fuselage blending, etc. If insufficient care is taken in the aerodynamic design, however, interference effects will be considerable, especially at high angles cf attack and high-subsonic speeds.

Most interference effects cannot be calculated accurately in preliminary design, and the approximations presented here must be considered as "minimum effects".

\section{F-4.1. Wetted area corrections}

Viscous interference drag is sometimes approximated by first calculating the drag of the isolated gross wing and fuselage, etc., and then simply adding these components. It is thus assumed that interference may be accounted for simply by ignoring the fact that the intersections of airframe components are not actually exposed to the flow, which implies that all wetted areas mentioned in the previous section are gross areas. For example: the wing wetted area includes the center section and is therefore equal to twice the gross area. The only justification for such a procedure is its simplicity, which may be acceptable for small contributions such as tailplane/fuselage interference, but for larger contributions, like wing/fuselage interference, an alternative method is desirable. For other components - e.g. nacelle/airframe interference - the isolated drag will simply be factored.

A comprehensive survey of the physical aspects of interference and many experimental data are given by Hoerner (Ref. F-18).

\section{F-4.2. Wing/fuselage interference}

a. Vortex-induced drag

The lifting wing induces an upwash in front and a downwash aft of the wing/fuselage junction. Hence, the body nose will experience lift increment and the afterbody a reduction in lift. In addition, the fuselage cross flow component at angle of attack leads to increased effective angles of attack near the wing/body junction. Combination of both effects will generally result in an increment of the lift coefficient for a given angle of attack and of the vortex-induced drag for a given lift. on the other hand, for a given lift of the complete configuration, the fuselage lift reduces the wing lift and consequently vortex-induced drag. It seems fair to assume that for a well designed configuration these effects are of the same order of magnitude. The only interference correction suggested here is the effect of the wing lift carry-over by the fuselage at zero fuselage angle of attack.

The results obtained by Lennertz (Ref. F100) for constant lift along the span and by Marx (Ref. F-101) for an elliptical lift distribution can be brought into reasonable agreement by the following expression for mid-wing configurations with circular fuselage cross-sections:

$\Delta_{i} C_{D_{v}}=\frac{.55 \eta_{f}}{1+\lambda}\left(2-\pi \eta_{f}\right) \frac{C_{L_{o}}{ }^{2}}{\pi A}$

where $\eta_{f}=$ fuselage diameter/wing span. For $\eta_{f}$ up to .15 this result compares reasonably well with the method based on experimental data given by Hoerner (Ref. F18 , page $8-18$ ). No attempt is made to present corrections for the effect of low-wing or high-wing positions.

\section{b. Viscous interference}

Near the intersections of the wing and fuselage contours there is a thickening of the boundary layers and an increase in the local flow velocity; both effects increase the profile drag. At small angles of actack the result is approximately:

$\Delta_{i}\left(C_{D} s\right)_{p}=1.5 c_{F} t_{r} c_{c i} \cos ^{2} \Lambda_{\frac{t}{2}}$

where $c_{c i}$ is the total circumferential 
length for both wing halves of the wing/ fuselage intersection line at which the boundary layers interact. For a mid-wing configuration $\mathrm{C}_{\mathrm{ci}}$ is approximately $4 \frac{1}{2}$ times the root chord. Equation F-66 is based on an interpretation of various results in Ref. F-18.

Due to lift effects, the pressure gradients and section forces increase at the upper side of the wing and decrease at the lower side. This may lead to premature separation near the wing/fuselage junction, especially with low-wing configurations, but this tendency may be suppressed by suitable filleting. However, the profile drag increment of the fuselage section above the wing due to increased velocities cannot be avoided, while on high-wing configurations the fuselage drag is reduced at high lift. A simple correction based on a theoretical analysis of typical configurations is suggested here:

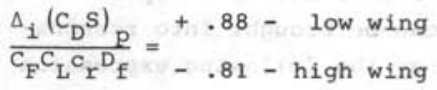

where $C_{F}$ is the local skin friction coefficient of the fuselage near the wing/body interconnection. No correction is required for mid-wing configurations.

Finally, the cross flow effects on fuselage drag (cf. Section F-3.4.) are altered as a consequence of the circulation, inducing a change in the effective angle of attack along the fuselage. Equation F-50 may therefore be modified into:

$\Delta_{\alpha \beta}\left(C_{D} s\right)=\int_{0}^{l_{f}}\left|\sin ^{3}\left(\alpha_{f}-\varepsilon-\beta\right)\right| c_{d_{c}} b \frac{d x}{\cos \beta}$

Note that in front of the wing the downwash angle $\varepsilon$ is negative (i.e. upwash) while at the wing/fuselage intersection there is no cross flow component due to the straightening effect of the wing. Equation F- 60 may be solved if detailed data are available for calculating the flow field around the wing, but in view of the very approximate character of the cross flow component the labor involved may not be justified. It is suggested that this form of interference should be taken into account by reducing the angle $\alpha_{f}^{\prime}$ in $(\mathrm{F}-52)$ by an amount equal to $\beta \cos \Lambda / A$, while $D_{2}$ in $(F-53)$ remains unchanged. Realizing that there is no induced upwash or downwash for $C_{L}=0$, we can derive:

$\Delta_{i}\left(C_{D} S\right)_{p}=\frac{2 \beta \cos \Lambda}{A} D_{2} \frac{C_{L}}{C_{L_{\alpha}}}$

where $B$ is the rear fuselage upsweep angle.

F-4.3. Nacelle/airframe interference

a. Propeller aircraft.

The interference effects due to nacelle installation are comparable to the wing/ fuselage interference effects, but they are more difficult to calculate. A thorough but outdated investigation can be found in Ref. F-71. The drag appears to be a function of the relative fore- and aft-position, the height and angle of inclination of the nacelle centerline relative to the wing chord, the shape of the rear end and the amount of filleting. The most important contribution at low angles of attack is the vortex-induced drag caused by the local change in wing lift due to the nacelle. Typical figures (from Ref. F-18, page $8-18$ ) are:

low wing: $\Delta_{i} C_{D} s=.004 \times$

nacelle frontal area

high wing: $\Delta_{i} C_{D} S=.008 \times\left\{\begin{array}{l}\text { nacelle } \\ \text { ner }\end{array}\right.$

nacelle frontal area

Negligible drag is experienced if the nacelle centerline coincides with the local chord.

Drag due to interference from the boundary layers of the nacelle and the wing can be taken into account by ignoring the wetted area correction required according to Section $\mathrm{F}-4.1$. and by adding the profile drag of the wing area that is not actually exposed to the flow (Ref. F-18, page 8-18). 
At high angles of attack the interference drag may increase considerably when vortices are formed at the nacelle/wing junctions, especially when the nacelle is high on the wing. No method of analysis is available for this effect.

b. Jet aircraft.

Jet engine pods and the jet efflux interact with the flow around the airframe in a number of complex ways that are still the subject of investigation (ci. Section

6.5.2.). For wing-mounted nacelles the determining factors are the nacelle foreand aft position, the distance between the nacelle centerline and the wing chord and jet efflux effects. It is generally found that an interference drag penalty of approximately 208 of the nacelle-plus-pylon drag must be accepted for low bypass engines with long ducts. High bypass engines with short fan ducts exhibit a favorable interference drag (Refs. F-109 and F-24) which is of the order of 5 to 10 counts in cruise conditions. For fuselage-mounted nacelles the shape of the pylon and the fuselage contour are important. The inter- ference drag penalty for straight or low bypass jet engines is of the order of 508 of the profile drag of the isolated nacelle plus pylon. No data for high bypass engine pods are available to the author.

\section{F-4.4. Tailplane/airframe interference}

a. Wing/tailplane interference, vortex-induced drag.

The tailplane lift acts normal to the local airflow. Due to the downwash behind the wing a positive tail load (up-load) has a drag component. On the other hand, this tail load partly offsets the wing lift for a given total lift and hence the vortexinduced drag of the wing is reduced. It can readily be shown that these effects result in the following contribution, ignoring a second order term:

$\Delta_{i}\left(C_{D} S\right)_{v}=C_{L_{h}} C_{L}\left(\frac{d \varepsilon}{d C_{L}}-\frac{2}{\pi A}\right) s_{h}$

where $C_{L_{h}}$ is lefined by $(E-44)$, see Section $F-2.6$. An estimate of $d \varepsilon / d_{L}$ can be made with the data in Section E-10,1. of Appendix E. It is noted that $(\mathrm{F}-71)$ may

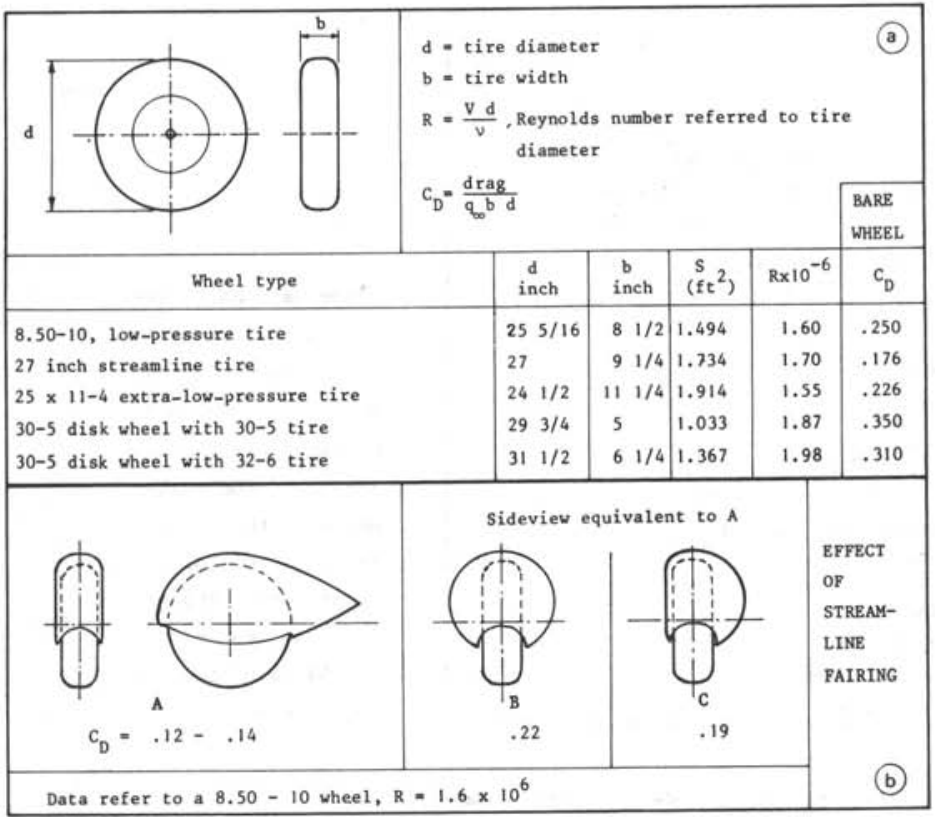

Fig. F-18. Drag of wheels with tires, with and without streamlined fairings (fenders) (Refs. F-110, F-111) 
well represent a negative term, i.e. a drag reduction.

\section{b. Viscous interference (tailplane/tail-} plane and tailplane/airframe).

As the effect of filleting is usually not known and this drag is a small term anyhow - a few percent of the tailplane profile drag - it is justifiable to assume that no extra drag need be accounted for, provided the gross area of the tailplanes is used in Section F-3.6. instead of their net wetted area.
F-5. PROTUBERANCES, SURFACE IMPERFECTIONS AND OTHER EXTRA'S

A large collection of detailed information such as that given in Hoerner's book (Ref. F-18) is required to analyze a particular design in detail, but in project design all required details are usually not yet known. What follows is therefore partly a statistical indication of the most relevant drag penalties that can be foreseen, provided close attention will be paid to detail design.

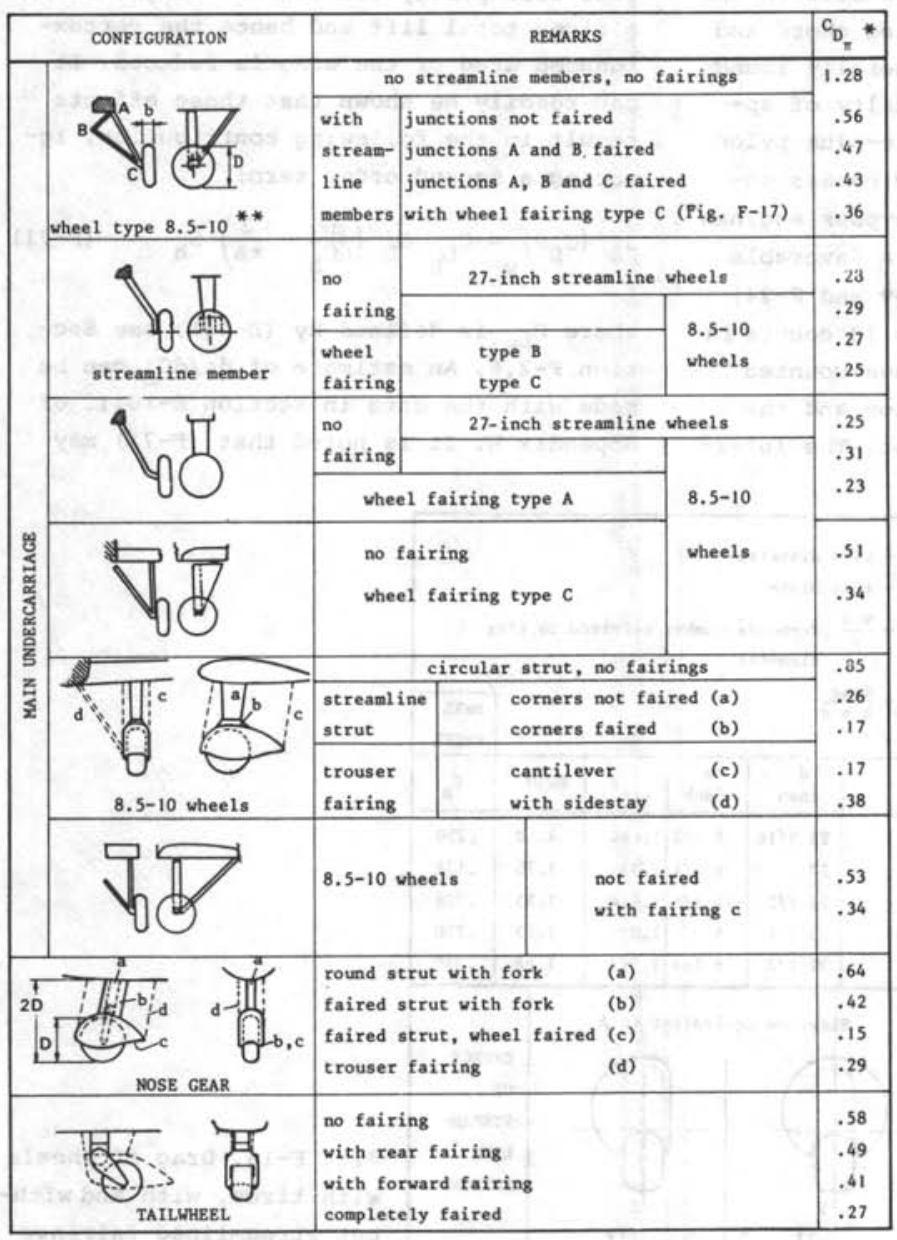

* for main undercarriages $\mathrm{C}_{\mathrm{D}_{\pi}}$ is referred to the circumscribed frontal area of two tires $(2 \mathrm{bD})$, for nose- and tailgears to that of a single tire (bD) **with other types the drag can be up to 158 above the values given

Fig. F-19. Fixed undercarriage drag (Refs. F-18, F-111 and F-112) 


\section{F-5.1. Fixed undercarriages}

Fig. F-18a presents the drag coefficients of several types of isolated wheels. As shown by Fig. F-18b, drag can be appreciably reduced by various types of streamlined fairings.

The drag coefficient of complete main undercarriages is usually related to the circumferential area of two wheels. Fig. F-19 indicates that the effect of fairings and streamlining is noticeable. Data are also given for nose gears and tail wheels, both with and without streamline caps. Tail skids have a drag area between .043 and $.12 \mathrm{sq.ft}\left(.004\right.$ and $\left..011 \mathrm{~m}^{2}\right)$ typically. Al1 data on main undercarriages in Fig. F-19 refer to wing- or fuselage-mounted gears. The drag of nacelle-mounted cantilever fixed undercarriages is approximately comparable to the data of the fifth configuration. The drag of a partly retracted landing gear can be found in Ref. F-111.

\section{F-5.2. Canopies and windshields}

\section{a. Protruding cockpit enclosures.}

Systematic drag measurements on a variety of shapes have been reported in Ref. F-115. Some results are summarized in Figs. F-20 and $\mathrm{F}-21$.

The following observations are made.

1. A minimum value of $C_{D_{\pi}}=.04$ can be achieved for a well-streamlined windshield without cylindrical mid-section, and approximately .045 with cylindrical mid-section, provided optimum nose and tail sections are used.

2. Below a length/diameter ratio of $2 \frac{1}{2}$ to 3 for the nose and tail sections, drag increases sharply.

3. For a short conical nose section $(t \approx h)$ and a long tail section, drag can be appreciably decreased by properly rounding the windshield/hood junction.

4. Rounding the hood-tail section junction for a short tail $(t \approx 2 h)$ is not very effective.

5. In order to obtain a low windshield drag it appears imperative to have a radius at the windshield/hood junction of at least 20 and a tail length of at least 3 h times the windshield height.

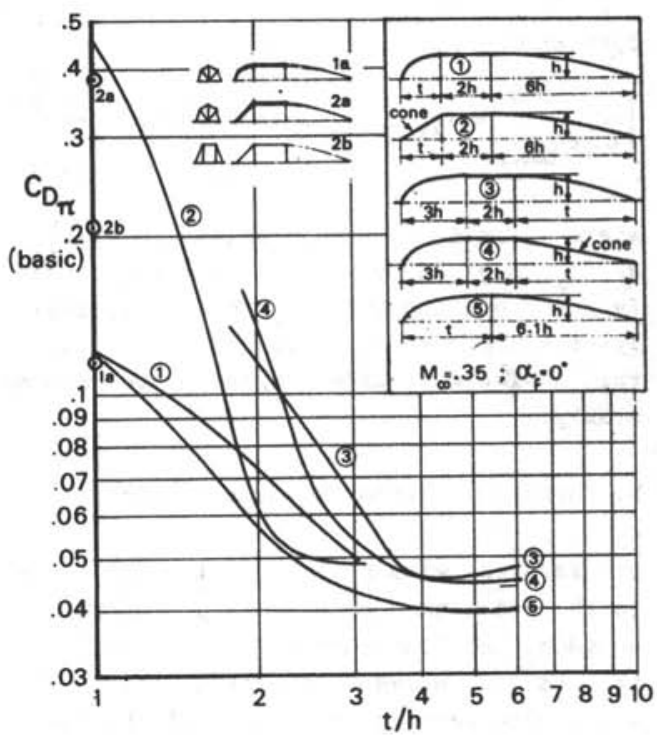

Fig. F-20. Effect of nose and tail length on the drag of windshields (derived from Ref. F-115)

A similar conclusion is found in Ref. F-66 for helicopter canopies.

6. The drag coefficient may amount to approximately .47 for the aerodynamically unfavorable shapes that are used on some agricultural aircraft.

The data of Ref. F-115 have been used to derive the following expression:

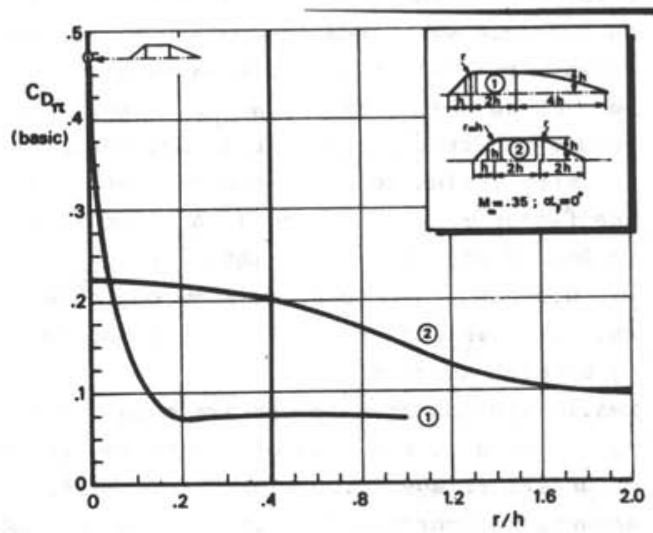

Fig. F-21. Effect of radius at windshield/ hood and hood/tail junctions on windshield drag (derived from Ref. F-115) 
$C_{D} S=.85\left(1+\varphi_{f}\right)\left(1+4.5 \alpha_{f}\right) \times$

$\left\{\left(C_{D_{\pi}}\right)_{\text {basic }}+\Delta_{S S} C_{D_{\pi}} \mid\right.$ xcanopy frontal area

where $\alpha_{f}$ is in radians, defined by $(F-25)$, and $\varphi_{f}$ is given by $(F-41)$. The drag penalty for steps and slots $\Delta_{s s} C_{D_{\pi}}$ is typically of the order of .01 to .02, though detail design will affect this figure considerably.

b. Cockpit windshields for transport aircraft

Provided the windshield is well faired into the fuselage nose and sharp corners are avoided, the drag coefficient can be limited to .02 , based on the frontal area of the window panels, or 2 to 38 of the fuselage drag. For a small unpressurized aircraft with large, flat window panels this coefficient may be as large as .08; more details can be found in Ref. F-115.

F-5.3. Wheel-well fairings* and blisters

Two methods are suggested to calculate the drag of wheel-well fairings.

Method A: use the data on cockpit canopies in $\mathrm{Fig} . \mathrm{F}-20$.

Method B: reduce each fairing to a body of revolution with equivalent diameter based on a circle with area equal to the maximum cross-sectional area of the fairing. Compute $C_{D}$ in a similar way as for fuselages. Assume the interference drag increment due to installation to be of the same order as the factor $\varphi_{f}$ relative to 1 . As discussed in Ref. F-24 unfavorable interference with the wing and rear fuselage flow may show up, but this effect can largely be avoided by careful shaping.

Small fairings or blisters are occasionally used to reduce the drag of protruding parts of undercarriages. Their drag coefficient amounts to approximately .045 , based on the

*Examples: Transa11 C-160, Lockheed C-130, $C-141$ and C-5A, Nord 262, Bréguet 941, Short Belfast and also the design in Fig. 1-4. frontal area. For more details see Ref. $\mathrm{F}-18$.

F-5.4. External fuel tanks

The basic profile drag of the isolated tank can be calculated in the same way as the fuselage drag. According to Fig. 3-2, a slenderness ratio of 4 to 5 results in minimum drag for a given volume. The corresponding drag coefficient is .025 , based on (volume) $2 / 3$.

Interference effects with the airframe are usually accounted for by multiplying the basic profile drag by an installation factor. Typical factors are:

1.20 - mounted symmetrically to the wing tip

1.30 - mounted fairly close to the wing, e.g. on a pylon

1.50 - mounted flush to the wing or fuselage.

Wing tip tanks will also affect the induced drag of the wing. This can be taken into account by assuming a percentage increment of the effective wing aspect ratio which is equal to half the percentage increment in span caused by the addition of the tanks (Ref. F-18, page 7-7).

\section{F-5.5. Streamlined struts}

According to Ref. F-18 (page 6-5) the profile drag coefficient based on the chord length is:

$c_{d_{s t}}=.015(1+t / c)+(t / c)^{2}$

This equation is valid for subcritical Reynolds numbers i.e. $R$ up to $7 \times 10^{4}$ for $t / c=.4$ and $3 \times 10^{4}$ for $t / c=.3$. At high Reynolds numbers the method used for wing sections (Section F-3.2.) can be employed. The usual $t / c$ ratio is approximately .30 . Ignoring interference, the strut drag area is:

$\frac{D_{\text {st }}}{q_{\infty}}=c_{d_{\text {st }}} \times$ chord $\times$ total length

The interference drag with the aircraft can 
be limited to a typical penalty of 108 of the profile drag by proper filleting.

\section{F-5.6. Powerplant installation drag}

The forces exerted on the airframe, both externally and internally, by the airflow passing through a jet engine, are included in the definitions of thrust and nacelle drag and do not appear under this heading. other engine installation drag components may generally be classified as follows.

a. External drag: the sum of all streamwise forces associated with the external flow around the installed powerplant, e.g. the cooling system intake scoops.

b. Internal drag: the forces exerted by the internal flow on the surface which bounds it, e.g. the drag due to pressure losses in the oil cooling systems.

c. Slipstream effects for propeller aircraft: the increase in profile drag of aircraft parts in the slipstream and the increment of vortex-induced drag due to the change in lift distribution.

d. Jet interference, of importance with wing-mounted engines (cf. Section F-4.3.b.). In the case of propeller engines the effects of slipstream interference are directly related to the engine operating condition. As the drag polar is usually defined in the power-off or low power condition, this contribution can be considered as an effective reduction in propeller efficiency (cf. Section 6.3.2.).

a. Reciprocating engines.

For air cooled engines the sources of installation drag are engine cooling drag, air intake and exhaust external drag, oil cooler internal and external drag and engine air intake momentum drag. cooling drag is given by:

$D_{c}=m_{c}\left(v_{\infty}-v_{e}\right)$

For a given engine type the cooling air mass flow $\hat{t}_{c}$ is determined by the ambient temperature, the engine rating and the cylinder head temperature. The exit velocity is mainly determined by the pressure loss in the inlet and the cylinder baffles; the exhaust opening is normally designed to accommodate the desired airflow in cruising flight. These characteristics should be decided in cooperation with the engine manufacturer.

Cooling drag can be considered as an equivalent loss in shaft power:

$\Delta \mathrm{P}_{\mathrm{b}}=\mathrm{D}_{\mathrm{c}} \mathrm{v}_{\infty} / n_{\mathrm{p}}$

This term can be dealt with as a separate contribution in performance calculations for each engine working condition and flight speed. If, on the other hand, it is desirable to include a first estimate in the drag polar for cruising flight, it may be assumed that:

$$
\left(C_{D} S\right)_{C}=C_{C} \times \frac{P_{b} T_{\infty}^{2}}{\sigma V_{\infty}}
$$

$C_{C}=4.9 \times 10^{-7} ; C_{D} S$ in sq. $f t, P_{b}$ in $h p$, $T_{\infty}$ in $R$ and $V_{\infty}$ in $\mathrm{ft} / \mathrm{s}$ $C_{C}=5.9 \times 10^{-10} ; C_{D} S$ in $\mathrm{m}^{2}, P_{b}$ in $\mathrm{kgm} / \mathrm{s}$, $\mathrm{T}_{\infty}$ in $\mathrm{K}$ and $\mathrm{V}_{\infty}$ in $\mathrm{m} / \mathrm{s}$ The increase in wing lift due to slipstream entails an increase in vortex-induced drag, which is less than the vortex-induced drag increment of the wing for the same lift increment. From Ref. F-18 the following expression can be derived for the apparent increase in the wing aspect ratio to which this effect is equivalent:

$\frac{\Delta \mathrm{A}}{\mathrm{A}}=.5 \frac{8}{\pi} \mathrm{T}_{\mathrm{c}} \frac{\mathrm{S}_{\mathrm{S}}}{\mathrm{S}}$

where

$T_{c}=\frac{\text { thrust per propeller }}{\rho \mathrm{V}_{\infty}{ }^{2} \mathrm{D}_{\mathrm{p}}{ }^{2}}$

and $s_{s}$ is the total projected area of the wing part immersed in the propeller slipstreams.

For steady cruising flight $(\mathrm{F}-76)$ can be modified to represent an effective increment in engine power:

$\frac{\Delta \mathrm{P}_{\mathrm{b}}}{{ }_{\mathrm{P}_{\mathrm{P}} \mathrm{P}_{\mathrm{b}}}}=\frac{-\Delta \mathrm{C}_{\mathrm{D}}}{\mathrm{C}_{\mathrm{D}}}=.5 \frac{\mathrm{S}_{\mathrm{S}} / \mathrm{N}_{\mathrm{e}}}{\frac{\pi}{4} \mathrm{D}_{\mathrm{p}}{ }^{2}} \frac{\mathrm{C}_{\mathrm{L}}{ }^{2}}{\pi \mathrm{A}} \quad($ for $\mathrm{T}=\mathrm{D})(\mathrm{F}-78)$ 
The effect of the slipstream on profile drag is accounted for in the form of a reduction in effective propeller efficiency (cf. Section 6.3.2.).

Other installation drag increments are not amenable to analysis before the inlet, exhaust and oil cooling systems have been designed in detail. In preliminary design it may be assumed that $C_{D} S=.02 \mathrm{sq} . \mathrm{ft}$ $\left(.0019 \mathrm{~m}^{2}\right)$ per 100 takeoff bhp.

b. Turboprop engines.

The external drag of the engine air inlets is included in the nacelle drag - see section F-3.5. - unless protruding intake scoops are used; in this case assume $C_{D} S=.10 \times$ the scoop frontal area at datum airflow. The oil cooler drag area is approximately .20 x the cooler inlet scoop frontal area, or $.0065 \mathrm{sq} . \mathrm{ft}\left(.0006 \mathrm{~m}^{2}\right)$ per 100 takeoff eshp. This figure includes internal drag.

Occasionally, protruding exhaust pipes are used, with their axis at a large angle to the flow. A drag coefficient of .5 may be assumed on the basis of their projected frontal area.

Engine air intake momentum drag is accounted for in the calculation of the net thrust of the engine gas flow:

$T_{\text {net }}=\mathrm{T}_{g} \cos \theta_{j}-\mathrm{m}_{j} \mathrm{~V}_{\infty}$

where $\theta_{j}$ is the mean deflection angle of the exhaust gases relative to the airflow. Note that even without deflection $\left(\theta_{j}=0\right) T_{\text {net }}$ need not be positive, and furthermore that for

$\theta_{j}>\cos ^{-1} \frac{\text { m }_{j} v_{\infty}}{T_{g}}$

there can be no positive net thrust component. In both cases there is, in effect, a momentum drag. In the extreme case of $\theta_{j}=90^{\circ}$ :

$c_{D} s=c_{j} \times \frac{\dot{w}_{j}}{\sigma v_{\infty}}$

where $c_{j}=24 ; c_{D} s$ in sq. $\mathrm{ft}, \dot{w}_{j}$ in $\mathrm{lb} / \mathrm{s}, v_{\infty}$ in $\mathrm{ft} / \mathrm{s}$ or $\quad C_{j}=1.6 ; C_{D} s$ in $\mathrm{m}^{2}, \dot{w}_{j}$ in $\mathrm{kg} / \mathrm{s}, v_{\infty}$ in $\mathrm{m} / \mathrm{s}$ This contribution is. of the order of 158 of the total airplane zero lift arag, which is generally unacceptable.
Slipstream effects are similar to those occurring with reciprocating engines.

c. Turbojet engines.

For a daisy-type ejector nozzle/noise suppressor, assume $C_{D} S=.025 \times$ nozzle area. The drag of a thrust reverser on turbofan engines amounts to between 3 and 58 of the nacelle drag, although this figure depends on the type of installation.

Cooling drag is caused by the ventilation/ cooling of the space between the hot engine sections and the surrounding structure; it amounts to about 58 of the nacelle drag.

The drag of air inlets for buried engines may be included in the fuselage or wing drag in the case of a fully integrated inlet, by properly accounting for the increase in wetted area. Scoop type inlets (cf. Fig. 2-2lc) must be treated separately. Their drag is not only dependent upon the shape of the scoop, but also on the inlet velocity ratio $v_{i} / v_{\infty}$. The external drag coefficient is as low as $C_{D_{\pi}}=.06-.07$ for $v_{1} / v_{\infty}=1$, but for a typical cruising value of $v_{1} / v_{\infty}=.6$ to .8 , a figure of $C_{D_{\pi}}=.10$ may be obtained. Assume $C_{D_{\pi}}=.25$ for boundary layer diverters.

\section{F-5.7. Excrescences, surface imperfections} and other extras

A representative breakdown of this drag contribution for a subsonic jet transport is depicted in Fig. F-22. For this aircraft the profile drag penalties are summarized as follows:

wing

: 68 of the wing profile arag

fuselage + empen-

nage : 78 of the fuselage drag engine installa-

tion : 158 of the nacelle drag systems : 38 of the zero-lift drag For project development a detailed assessment can be made of all contributions, using the data given in Ref. F-18, for example. A target can then be set to provide a guideline for detail design, aerodynamic 


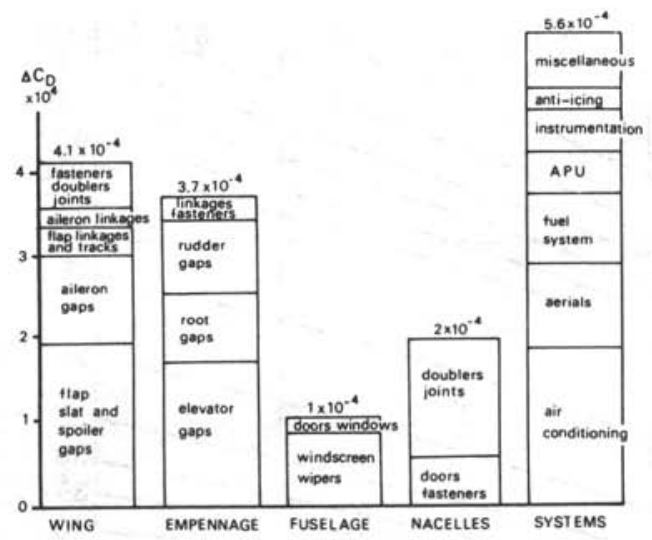

Fig. F-22. Typical breakdown of excrescence drag at cruise (Ref. F-25)

development and manufacturing tolerances. In this survey a statistical approach will be presented instead.

a. Surface imperfections must be accepted in the manufacturing process: doublers, skin joints, steps, gaps, fasteners, rivets, screws, holes, doors, scratches, indentations, waviness. If the size of these roughnesses is less than a critical size, the wing or body can be regarded as aerodynamically smooth and there is no drag penalty. For a roughness immersed in a turbulent boundary layer, this condition is derived from Ref. F-128:

$\left(\frac{\mathrm{k}}{\mathrm{l}}\right)_{\text {crit }}=\frac{39.5}{\mathrm{R}^{.94}}$

where $k$ is the equivalent sand grain size of the roughness, $\ell$ the body or chord length, and $R$ is based on $l$.

For transport aircraft the surface imperfections appear to be equivalent to a sand grain size of .001 inch ( 25 microns) for. average surfaces and about half this value for very carefully treated and smooth surfaces. Using this figure, the profile drag increment for all individual major items can be computed from Fig. F-23. Light aircraft have a roughness drag of the order of 10-15 counts or more.

Painted surfaces can be-treated in similar way, using Fig. F-23, provided the equivalent grain size is known. There is a considerable variation in this quantity (cf. Ref. F-129).

b. Retracted high-lift systems and flight control surfaces.

Surface imperfections such as steps, gaps and discontinuities are unavoidable with retracted flaps and slats, ailerons, elevators, rudder and spoilers. Exposed flap hinges, linkages and tracks are usually present. Some examples are presented in Fig. F-24. For slotted flaps the drag penalty may easily amount to 10-12 counts if no attempt is made to cover the gap properly. The penalty may be reduced to $2-3$ counts, based on the projected area of the wing or tailplane with flaps or controls. For light aircraft the control surfaces entail an appreciable gap drag of the order of $25 \%$ of the tailplane profile drag. Leadirig-edge devices such as slats or plain leading-edge flaps not only provoke boundary layer transition - to be accounted for in the profile drag estimation - but also cause. pressure drag at the various discontinuities and steps. The drag area is roughly $.007 \mathrm{sq} . \mathrm{ft}$ per $\mathrm{ft}$ of total slat span in front view $\left(.002 \mathrm{~m}^{2}\right.$. per $\left.\mathrm{m}\right)$.

c. Airframe installations contribute to the drag. These include air conditioning system inlets and outlets and momentum drag, antennas, lights, fuselage skin waves and leaks due to pressurization, fuel system (dumping provisions), APU installation, instrumentation, anti-icing devices (rubber boots) and windscreen wipers. This drag is of the order of 6 to 88 of the fuselage drag.

d. Vortex generators, wing fences, fuselage strakes: the drag is obviously very much dependent upon the installation.* The or-

* Strictly speaking, their drag will be negative, relative to the condition where these devices are not present, for flight conditions where they improve the flow. 


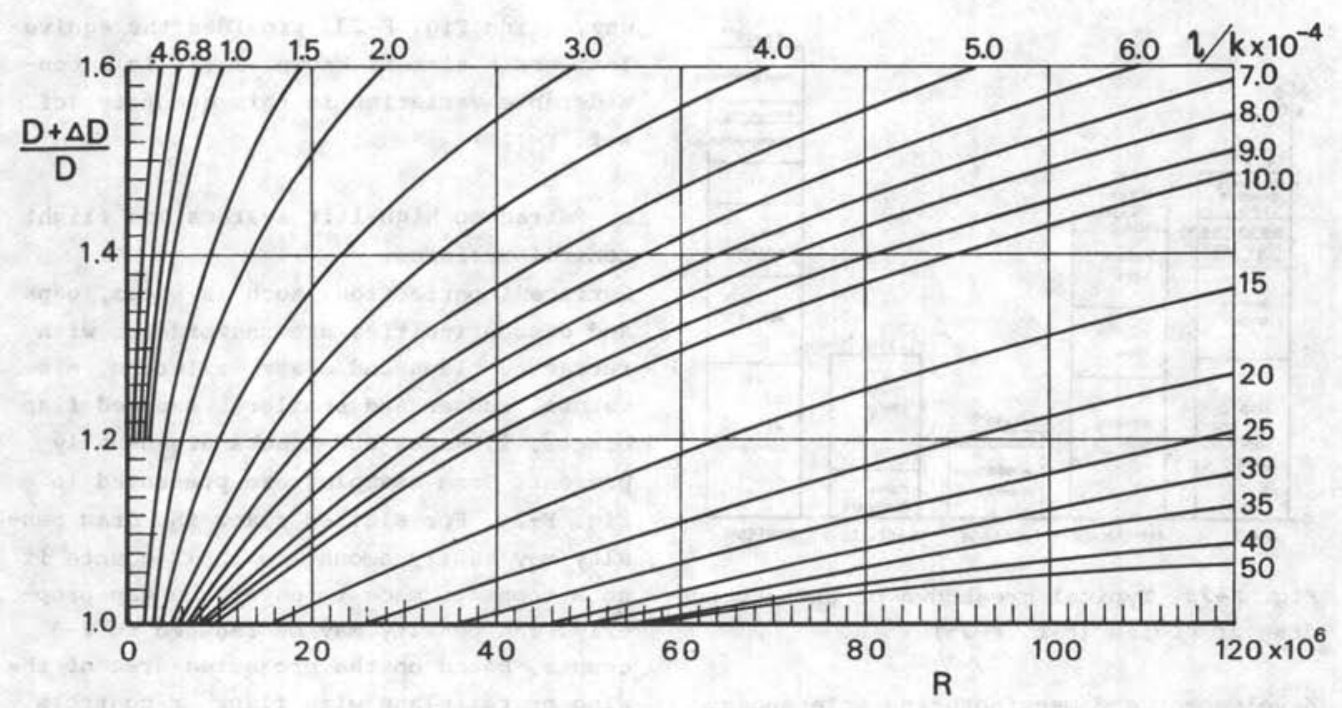

$D=$ profile drag of an aerodynamically smooth surface

$\Delta \mathrm{D}=$ drag increment due to surface roughness

$\ell=$ characteristic length, wing chord or body length

$k=$ equivalent sand grain size

$\mathbf{R}=$ Reynolds number

More complete information for higher Reynolds number, taking into account compressibility effects, can be found in ESDU Data Sheet Item No. 73016, dtd. July 1973

Fig. F-23. Wing or body drag due to surface roughness (Ref. F-128)

der of magnitude is typically a few counts (e.g. Ref. F-134).

e. Miscellaneous sources of lift-dependent drag.

For a number of components and drag items such as engine nacelle drag and various interference effects, only the drag in cruising conditions has been considered. In addition, the major sources of profile arag increment with lift have been given for a smooth surface, whereas it is recognized that the profile drag of a rough body will increase more rapidly with incidence. Published methods for estimating this contribution are not available and the author therefore suggests that 208 of the profile drag increment should be assumed for $\mathrm{C}_{\mathrm{L}} \neq \mathrm{C}_{\mathrm{L}_{\mathrm{O}}}$, or approximately:

$$
C_{b} \times 10^{4}
$$
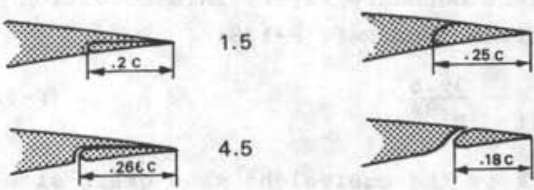

4.5

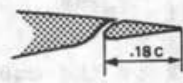

$8.5-10$

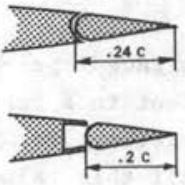

$2.0-3.6$

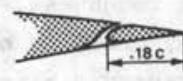

2.6

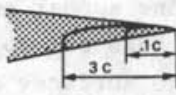

2.0

$C_{D}$ referred to projected area of wing or tailplane with flaps or controls

Fig. F-24. Drag due to gaps caused by retracted flaps and control surfaces (Ref.F-8) 
$\Delta C_{D}=\frac{.0015}{\cos ^{2} \Lambda}\left(C_{L}-C_{L_{O}}\right)^{2}$

It is, of course, obvious that this assump- tion is open to criticism. The best method is to derive the required value from the difference between the actual and estimated drag polars of existing aircraft.

REFERENCES

Drag prediction methodology and general considerations

F-1. B.M. Jones: "The streamline aeroplane". J.R.Ae.S., May 1929.

F-2. W.B. Oswald: "General formulas and charts for the calculation of airplane performance". NACA Report No. 408,1932 .

F-3. н. Eick: "Dex Mindestwiderstand von Schnellflugzeugen". Luftfahrtforschung 1938, Band XV, pp. $445-461$.

F-4. C.D. Perkins and R.E. Hage: "Airplane performance, stability and control". Wiley, New York, 1949. Chapter 2: "Drag estimation".

F-5. I.H. Driggs: "Aircraft design analysis". J.R.Ae.S., Vol. 54, Feb. 1950, pp. 65-116.

F-6. E.J. Richards: "A review of aerodynamic cleannes". J.R.Ae.S., Vol. 54, 1950, pp. 137-186.

F-7. н.K. Millicer: "The design study". Flight, Aug. 17, 1951.

F-8. D. Fiecke: "Die·Bestimmung der Flugzeugpolaren für Entwurfszwecke". I. Teil: "Unterlagen". Deutsche Versuchsanstalt für Luftfahrt E.V., June 1956.

F-9. Anonymus: "The estimation of the drag of an aeroplane". Cranfield Institute of Technology, Aerodynamics Lecture Supplement No. 11, April 1956.

F-10. Anonymus: "Report of the definitions panel on definitions to be used in the description and analysis of drag". ARC C.P. 369, 1957.

F-11. D.D. Dommasch: "Airplane aerodynamics", Pitman Publishing Corp., 1958.

F-12. G. Gabrielli: "On the subdivision in different "forms" of drag of the aircraft at the maximum speed". Troisième Congrès Aéronautique Européen, 1958. Vol. II and III, pp. 398, 407, 981-984.

F-13. D. Fiecke: "Der Einfluss des Widerstandes auf die Flugzeugentwicklung, Teil II". Flug-Revue, June 1962, pp. $40-42,44,46,48$.

F-14. D.E. Hoak and D.E. Ellison: "USAF Stability and Control Datcom". Wright Patterson Air Force Base, Oct. 1960, Rev. 1968 .

F-15. н.G. Sheridan: "Aircraft preliminary design methods used in the weapon systems analysis". U.S. Navy BuWeps. Report R-5-62-13, June 1962.

F-16. พ.H. Cook, C.S. Howel1 and J.K. Wimpress: "Aerodynamic performance". Air, Space and Instruments, Edited by S. Lees, Draper Anniversary Volume, 1963, pp. 238-256.

F-17. J.E. Linden and F.J. O'Brimski: "Some procedures for use in performance prediction of proposed aircraft designs". SAE Preprint No. 650800, Oct. 1965.

F-18. S.F. Hoerner: "Fluid-dynamic drag". Published by the author, 1965.

F-19. A.K. Martynov: "Practical aerodynamics". Pergamon Press, 1965, Chapter 10: "The aerodynamic characteristics of aircraft".

F-20. K.D. Wood: "Airplane design". Second edition, 1966. Johnson Publishing Company, Boulder, Colorado.

F-21. A.B. Haines: "Subsonic aircraft drag". The Aeron. Journal of the R.Ae.S. Vol. 72, No. 687, March 1968, pp. 253-266.

F-22. D.A. Norton: "Airplane drag prediction". Annals of the New York Academy of Sciences, Vo1. 154 part 2, pp. 306-328, 1968 .

F-23. D.C. Leyland and B.R.A. Burns: "Methods of estimating the drag characteristics, manoeuvrability and performance of strike/fighter aircraft". Short Course on Aircraft Performance estimation, Cranfield Inst. of Techn., Feb. 1973.

F-24. J.H. Paterson, D.G. MacWilkinson and w.T. Blackerby: "A survey of drag prediction techniques applicable to subsonic and transport aircraft design". AGARD Conference on aerodynamic drag CCP-124, 1973. 
F-25. S.F. Butler: "Aircraft drag prediction for project appraisal and performance estimation". AGARD CP$124,1973$.

F-26. D.G. MacWilkinson, W.T. Blackerby and J.H. Paterson: "Correlation of full-scale drag predictions with flight measurements on the C-141 aircraft". NASA CR-2333 and CR-2334, Feb. 1974.

F-27. J.G. Callaghan: "Aerodynamic prediction methods for aircraft at low speeds with mechanical high-1ift devices". AGARD Lecture Series No. 67, May 1974.

F-28. G.M. Bowes: "Aircraft 1 fft and drag prediction and measurement". AGARD Lecture Series No. 67, May 1974 .

Lift-induced drag of wings

F-29. R.F. Andersen: "Determination of the characteristics of tapered wings". NACA Report No. 572 , 1936.

F-30. J. De Young and C.W. Harper: "Theoretical symmetrical span loading at subsonic speeds for wings having arbitrary planforms". NACA Report No. 921, 1948.

F-31. T.R.F. Nonweiler: "Lift curve slope and induced arag factors of large aspect ratio straight-tapered wings". J.R.Ae.S. Vol. 64 No. 592, April 1960, pp. 224-225.

F-32. K.L. Sanders: "Subsonic induced drag". J. Aircraft, Vol. 2 No. 4, July-Aug. 1965, pp. 347-348.

F-33. R.C. Frost and R. Rutherford: "Subsonic wing span efficiency". AIAA Journal April 1963 , pp. $931-933$.

F-34. D. Gardner and J. Weir: "The drag due to 1 ift of plane wings at subsonic speeds". J.R.Ae.S., May 1966.

F-35. D. Gardner: "On the value of $\mathrm{C}_{\mathrm{L}}$-crit associated with the drag due to lift of plane wings in subsonic, subcritical flow". British Aircraft Corporation (Preston) Report Ae. 250, 1966.

F-36. Anonymus: "Subsonic 1 ift-dependent drag due to wing trailing vortices". R.Ae.S. Data Sheets Wings 02.01 .02 .

F-37. J.L. Lundry: "Minimum swept-wing induced arag with constraints on lift and pitching moment". J.Aircraft, Vol. 4, No. 1, Jan.-Feb. 1967, pp. 73-74.

F-38. B.G. Gilman and K.P. Burdges: "Rapid estimation of wing aerodynamic characteristics for minimum induced drag". J. Aircraft, Vol. 4 No. 6, Nov.-Dec. 1967, pp. 563-565.

F-39. H.C. Garner: "Some remarks on vortex drag and its spanwise distribution in incompressible flow". The Aeron. Journal of the R.Ae.S., July 1968 , pp. 623-625.

F-40. T.E. Labrujere and J.G. Wouters: "Computer application of subsonic lifting surface theory". N.L.R. Technical Report No. 70088.

F-41. P. Kapteijn: "Design charts for the aerodynamic characteristics of straight and swept, tapered, twisted wings at subsonic speeds". Delft University of Technology, Dept. of Aeron. Engng., Memorandum M-180, May 1972 .

Profile drag of wings and airfoils

F-42. H.B. Squire and A.D. Young: "The calculation of the profile drag of airfoils". A.R.C. R \& M 1818, Nov. 1937.

F-43. Anonymus: "Profile drag of smooth wings". R.Ae.S. Data Sheets wings 02.04.02/03.

F-44. I.H. Abbott, A.E. von Doenhoff and L.S. Stivers Jr.: "Summary of airfoil data". NACA Report No. 824, 1945 .

F-45. L.K. Loftin Jr.: "Theoretical and experimental data for a number of NACA 6A-series airfoil sections". NACA Report No. $903,1948$.

F-46. L.K. Loftin Jr. and w.J. Bursnall: "The effects of variations in Reynolds number between $3.0 \times 10^{6}$ and $25.0 \times 10^{6}$ upon the aerodynamic characteristics of a number of NACA 6-series airfoil sections". NACA Report No. $964,1950$.

F-47. J. Weber and G.G. Brebner: "A simple estimate of the profile drag of swept wings". RAE TN Aero 2168, June 1952 . 
F-48. I.H. Abbott and A.E. von Doenhoff: "Theory of wing sections". Dover Publications 1960.

F-49. J.F. Nash, T.H. Moulden and J. Osborne: "On the variation of profile drag coefficient below the critical Mach number". ARC CP No. 758, Nov. 1963.

F-50. J.C. Cooke: "The drag of infinite swept wings with an addendum". ARC CP No. $1040,3 u n e ~ 1964$.

F-51. H.H. Pearcey and J. Osborne: "On estimating two-dimensional section drag". ARC 27872, 28th. March 1966.

F-52. T. Cebeci and A.M.O. Smith: "On the calculation of profile drag of aerofoils at low Mach numbers". J. Aircraft, Vol. 5, No. 6, Nov.-Dec. 1968.

F-53. A. Barkhem: "Skin-friction formula for tapered and delta wings". J. Aircraft, Vol. 6, No. 3, 1969, page 284 .

\section{Fuselages}

F-54. M.M. Munk: "The aerodynamic forces on airship hulls". NACA Report No. $184,1928$.

F-55. A.D. Young: "The calculation of the total and skin friction drags of bodies of revolution at zero incidence". ARC R \& M 1874, April 1939.

F-56. Anonymus: "Drag of streamline bodies". R.Ae.S. Data Sheets Bodies 02.04.01/02.

F-57. H.J. Allen: "Estimation of the forces and moments acting on inclined bodies of revolution of high fineness ratio". NACA R \& M A9r26, Nov. 1949.

F-58. S.F. Hoerner: "Base drag and thick trailing edges". J. Aeron. Sciences, Oct. 1950.

F-59. H.R. Kelly: "The estimation of normal force, drag and pitching moment coefficients for blunt-based bodies at large indidences". J. Aeron. Sciences Vol. 21, A.ug. 1954, pp. 549-555.

F-60. E.J. Hopkins: "A semi-empirical method for calculating the pitching moment of bodies of revolution at low Mach numbers". NACA RM A51 C14, 1955.

F-61. A.A. Badiagin: "Concerning an efficient slenderness-ratio for the fuselage of civilian aircraft". In: "Methods of selection and approximate calculation of air design parameters". Moscow Aviation Institute Transactions, Sept. 27th, 1962.

F-62. T.R. Nettleton: "A method of estimating the effects of rear fuselage up sweep and fuselage cross sectional shape on fuselage drag". Unpublished Report of De Havilland Canada, Nov. 1964.

F-63. H. Hertel: "Full integration of VTOL power plants in the aircraft fuselage" AGARD CP-9, Vol. I, 1966.

F-64. D. Gyorgyfalvy: "Effect of pressurization on airplane fuselage drag". J. of Aircraft, Vol. II, Nov.Dec. 1965, pp. 531-537.

F-65. W.A. Mair: "Reduction of base drag by boat-tailed afterbodies". Aeron. Quarterly, Vol. 20, Nov. 1969, pp. 307-320.

F-66. C.N. Keys and R. Wiesner: "Guidelines for reducing helicopter parasite drag". J. of the American Helicopter Soc., Jan. 1975, pp. 31-40.

Nacelles and engine installations, propeller aircraft

F-67. D.H. Wood: "Tests of nacelle-propeller combinations in various positions with reference to the wing". NACA Reports No. $415,436,462,1932 / 1933$.

F-68. J.G. Lee: "Air-cooled vs. liquid cooled aircraft". Journal of the Aeron. Sciences, Vol. 8, No. 6, April 1941, pp. 219-229.

F-69. J.V. Becker: "High speed tests of radial engine nacelles". NACA Wartime Report No. L-229.

F-70. Davies: "A review of windtunnel tests at R.A.E. on typical engine nacelle installations". RAE Report BA 1475 .

F-71. R. Smelt, A.G. Smith and B. Davison: "The installation of an engine nacelle on a wing". ARC R. \& M. 2406,1950

F-72. A.L. Courtney: "The estimation of powerplant drag of radial air-cooled engine installations at high speed and in temperature conditions". RAE Aero TN 1776. 
F-73. Squire: "Calculation of the effect of slipstream on lift and drag". Part. I, II and III R.A.E. Reports Aero $2083 \mathrm{~A}, \mathrm{~B}$ and C.

F-74. J.V. Becker: "Windtunnel investigation of air inlet and outlet openings on a streamline body". NACA Report No. 1038, 1951 .

Turbojet engine nacelles and installation drag

F-75. J. Seddon: "Air intakes for gasturbines". J.R.Ae.S. Oct. 1952, pp. 747-781.

F-76. D. Küchemann and J. Weber: "Aerodynamics of propulsion". McGraw-Hill Book Comp., Inc., New York, 1953.

F-77. M. Sibulkin: "Theoretical and experimental investigations of additive drag". NACA Report No. 1187, 1954.

F-78. A.R.C. Panel: "Definitions to be used in the description and analysis of drag". J.R.Ae.S. Nov. 1958, pp. 796-801. See also: J.R.Ae.S. Aug. 1955.

F-79. G. Schulz: "Aerodynamische Regeln für den Einbau von strahltriebwerksgondeln". Zeitschrift für Flugwissenschaften, Vol. 3, 1955, pp. 119-129.

F-80. J.S. Dennard: "The total pressure recovery and drag characteristics of several auxiliary inlets at transonic speeds". NASA Memo 12-21-58 :.

F-81. G. Schulz: "Der Kraftangriff bei Strahltriebwerken und ihren Verkleidungen". Z. Flugwissenschaften, Vol. 9, Sept. 1956, pp. 285-290.

F-82. E.E. Honeywe11: "Compilation of power-off base drag data and empirical methods for predicting poweroff base drag". Convair Report TM 334-337, 1959.

F-83. J.E. Steiner: "The development of the Boeing 727". J.R.Ae.S. Vol. 67, Feb. 1963, pp. 103-110.

F-84. J. Bogdanovic: "A method for determining propulsion system requirements for long-range, long-endurance aircraft". AIAA Paper No. 64-783.

F-85. C.R. Palmer: "Engine pod drag". Rolls-Royce (Hucknall) Brochure HK 15, March, 1964.

F-86. H.Mc. Donald and P.F. Hughes: "A correlation of high subsonic afterbody drag in the presence of a propulsive jet or support sting". J. of Aircraft, Vol. 2, No. 3, May-June 1965, pp. $202-207$.

F-87. R.L. Lawrence: "Afterbody flow fields and skin friction on short duct fan nacelles". J. Aircraft, Vol. 2, No. 4, July-Aug. 1965.

F-88. J.S. Mount: "Effect of inlet additive drag on aircraft performance". J. Aircraft, Vol. 2, No. 5, Sept.-Oct. 1965, pp. 374-378.

F-89. W.C. Swan: "A discussion of selected aerodynamic problems on integration of propulsion systems with airframe on transport aircraft". AGARDograph 103, Oct. 1965.

F-90. L.R. I'Anson: "The application of the high bypass turbofan for business and executive aircraft". SAE Preprint No. 660221.

F-91. J.T. Kutney: "High-bypass vs. low-bypass engine installation considerations". SAE Paper No. 660775 .

F-92. W.S. Viall: "Aerodynamic considerations for engine inlet design for subsonic high-bypass fan engines". SAB Paper No. 660733.

F-93. J.E. Green: "Short cowl front fan turbojets; friction drag and wall-jet effects on cylindrical afterbodies". RAB Technical Report, No. 67144.

F-94. P. Taylor: "Inlet and fan aerodynamics". SAE Paper No. 680711.

F-95. R. Hetherington: "Engine component design problems associated with large subsonic transports". Lecture given at the Von Karman Institute, Brussels, 23rd. Apri1, 1969.

F-96. W. Tabakoff and $\mathrm{H}$. Sowers: "Drag analysis of powered nacelle fan jet engine model tests". Zeitschrift für Flugwiss., Vol. 4, April 1969, pp. 134-144.

F-97. C.E. Swavely: "Twin jet aircraft aft-fuselage performance prediction". Proceedings of the Airforce airframe compatability symposium 24/26th. June, 1969. T. Report AFAPL-69-103.

F-98. D. Bergman: "Implementing the design of airplane engine exhaust systems". AIAA Paper No. $72-112$. F-99. W. Swann and A. Sigalla: "The problem of installing a modern high by-pass engine on a twin fet 
transport aircraft". AGARD Conference on aerodynamic drag, CP-124, 1973.

Interference effects

F-100. J. Lennertz: "Beitrag zum theoretischen Behandlung des gegenseitigen Einflusses von Tragfläche und Rumpf". Abhandlungen aus dem Institut der Technische Hochschule, Aachen, Heft 8, 1928, pp. 1-30.

F-101. A.J. Marx: "Korte beschouwing over de invloed van de romp op de geinduceerde weerstand van de vleuge1". NLL Report V 1299, 1943 (in Dutch).

F-102. A.A. Nikolski: "On lift properties and induced drag of wing-fuselage combinations". NASA RE 5-I59 .

F-103. н.R. Lawrence and A.H. Flax: "Wing-body interference at subsonic and supersonic speeds, survey and new developments". J. Aeron. Sciences, Vol. 21 No. 5, May 1954.

F-104. J.T. Keetney and S.P. Piszkin: "Reduction of drag rise on the Convair 990 airplane". J. of Aircraft, Jan.-Feb. 1964, (AIAA Preprint No. 63-276).

F-105. S. Neumark: "Lift due to interference between an aerofoil and an external non-1ifting boay". ARC R \& M No. 3411, May 1964 .

F-106. J. Seddon: "Factors determining engine installation drag of subsonic and supersonic long-range aircraft". AGARD CP-9, Part I, 1966.

F-107. D.J. Raney, A.G. Kurn and J.A. Bagley: "Windtunnel investigation of jet interference for underwing installation of high bypass ratio engines". ARC CP No. 1044, March 1968.

F-108. P. Williams and D. Stewart: "The complex aerodynamic interference pattern due to rear fuselage mounted power-plants". AGARD CP-71.

F-109. J.C. Patterson Jr.: "A wind-tunnel investigation of jet-wake effect of a high-bypass engine on wing-nacelle interference drag of a subsonic transport". NACA TN No. D-4693, 1968 (see also NACA TN No. D-6067, 1970).

Undercarriages

F-110. F.B. Bradfield: "Wheels, fairings and mudguards". ARC R \& M 1479, 1932.

F-111. Herrnstein and Biermann: "The drag of airplane wheels, wheel fairings and landing gears". NACA Reports No. 485, 1934 and 518, 522, 1935 (Parts I, II and III).

F-112. H. Harmon: "Drag determination of the forward component of a tricycle landing gear". NACA TN No. $788,1940$.

F-113. P.A. Hufton and J.R. Bdwards: "Note on a method of calculating the take-off aistance". RAE Departmental Note B.A. Performance No. 20, Aug. 1940.

Canopies and windshields

F-114. J.B. Hartley, D. Cameron and W.H. Curtis: "Note on windtunnel tests on the design of cabins". ARC R \& M No. $1811,1937$.

F-115. R.G. Robinson and J.B. Delano: "An investigation of the drag of windshields in the 8-foot highspeed windtunne1". NACA Report No. $730,1942$.

F-116. T.V. Somerville and N. Sharp: "Note on the drag and pressure distribution on two types of cabin". RAE Report No. B.A. 1654.

F-117. Anonymus: "The drag of fighter-type canopies at subcritical Mach-numbers". Engineering Sciences Data Sheets Item No. 67041 .

External stores

F-118. J.B. Berry: "Examples of airframe-stores interference". AGARD CP-71, Sept. 1970. 
F-119. P.G. Pugh and P.G. Button: "The drag of externally carried stores - its prediction and alleviation". AGARD CP-124, 1973.

F-120. J.B. Berry: "External store aerodynamics for aircraft performance prediction". AGARD LS-67, 1974.

Trim drag

F-121. C.B. Naylor: "Notes on the induced drag of a wing-tail combination". ARC R \& M 2528, 1954.

F-122. H. Behrbohm: "Basic low-speed aerodynamics of short-coupled canard configurations of small aspect ratio". SAAB TN No. 60, July 1966.

F-123. V.L. Marshal1: "Aircraft trim drag". Thesis, Cranfield Institute of Technology, 1970.

F-124. L.W. MCKinney and S.M. Dollyhigh: "Some trim drag considerations for manoeuvring aircraft". AIAA Paper No. 70-932.

Roughness

F-125. A.D. Young: "Surface finish and performance". Aircraft Engng. Sept. 1939.

F-126. M.J. Wood: "The effect of some common surface irregularities on a wing". NACA TN No. 695 , March 1939.

F-127. J.H. Quin Jr.: "Summary of drag characteristics of practical-construction wing sections". NACA Report No. $910,1948$.

F-128. Anonymus: "wing or body drag due to surface roughness". R.Ae.S. Data Sheets Wings 02.04 .08 . and 02.04 .10 .

F-129. A.D. Young: "The drag effects of roughness at high subcritical speeds". J.R.Ae.S. Vol. 54, 1950, Pp. 534-540.

F-130. E.A. Horton and N. Tetervin: "Measured surface defects on typical transonic airplanes and analysis of their drag contribution". NASA TN D-1024, Oct. 1962.

F-131. J.F. Nash and P. Bradshaw: "The magnification of roughness drag by pressure gradients". J.R.Ae.S., Vol. 71, No. 673, Jan. 1967.

F-132. T.A. Cook: "The effects of ridge excrescences and trailing-edge control gaps on twodimensional aerofo11 characteristics". RAE Technical Report 71080, Apri1 1971.

F-133. J.I. Simper and P.G. Hutton: "Results of a series of wind-tunnel model breakdown tests on the Trident 1 aircraft and a comparison with drag estimates and full scale flight data". ARC CP No. 1170 , 1971.

F-134. J.I. Simper: "Results of a series of wind tunnel tests on the Victor B.Mk. 2 aircraft and a comparison with drag estimates and full scale flight". ARC CP No. 1283, 1974. 


\section{Appendix G. Prediction of lift and drag in the low-speed configuration}

\section{SUMMARY}

A collection of generalized data and methods for estimating the lift curve and drag polar in the configuration for low-speed flight is presented. Passive* trailing-edge and leading-edge high-lift devices are considered. Glauert's linear theory for thin airfoils with deflected flaps forms the basis for most prediction methods; correction factors are given for taking nonlinearity and flow separation into account.

Prediction methods are also added for estimating the drag due to extension of a retractable undercarriage, the effects of ground proximity on lift and drag, and the increase in drag associated with the failure of an outboard engine.

*no action is taken to augment the external flow by means of blowing or suction. 
NOMENCLATURE

Symbols with a' (prime) refer to quantities defined based on the extended chord or to the lift-curve slope with flaps deflected.

A

- aspect ratio (no index: wing aspect ratio)

$\mathrm{A}_{\mathrm{N}} \quad$ - area of a nozzle

a.c. - aerodynamic center

$B_{p} \quad-$ number of blades per propeller

$\mathrm{b}^{\mathrm{p}} \quad$ - span; width

c - chord

$\bar{c}$ - mean aerodynamic chord (MAC)

$c_{g} \quad-$ mean geometric chord $\left(c_{g}=S / b\right)$

$c_{d}, C_{D}$ - two- and three-dimensional drag coefficient, respectively

$\mathrm{C}_{\mathrm{D}_{\mathrm{O}}} \quad-$ zero-1ift arag coefficient

$\mathrm{c}_{\mathrm{d}}, \mathrm{C}_{\mathrm{D}_{\mathrm{p}}}$ - two- and three-dimensional profile drag coefficient, respectively

$C_{D_{v}} \quad$ - vortex-induced arag coefficient

$\mathrm{C}_{\mathrm{D}_{\text {trim }}}$ - trim arag coefficient

$c_{\ell}, C_{L}$ - two- and three-dimensional $11 \mathrm{ft}$ coefficient, respectively

$c_{\ell_{0}}, \mathrm{C}_{\mathrm{L}_{0}}$ - lift coefficient at zero wing angle of attack

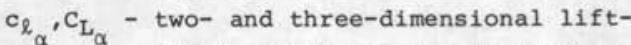
curve slopes, for constant $\delta_{f}$

$c_{\ell} \quad-\partial c_{\ell} / \partial \delta_{f}$ for constant $\alpha$

$\mathrm{C}_{\mathrm{L}_{\infty}} \quad$ - lift coefficient out of ground effect

$c_{m}, c_{m}$ - two- and three-dimensional pitching moment coefficient, respectively

$c_{y_{y}} \quad$ - side force coefficient of vertical tailplane $\left(\mathrm{C}_{\mathrm{y}_{\mathrm{v}}}=\mathrm{Y} \mathrm{y}_{\mathrm{v}} / \mathrm{q}_{\mathrm{v}} \mathrm{S}_{\mathrm{v}}\right)$

$\mathrm{D}_{1} \quad$ - engine inlet diameter

$D_{p} \quad$ - propeller diameter

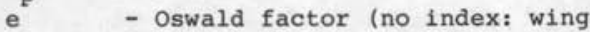
Oswald factor)

$F(\delta)$ - function for determining the profile drag increment due to split flaps

$F_{\text {uc }} \quad$ - function defining lift effect on undercarriage drag

h - height of aerodynamic center above ground plane

heff - effective height of MAC above ground plane
$\mathrm{K}_{\text {as }}, \mathrm{K}_{\sigma}$ - factors for determining drag due to asymmetric flight condition

$\mathrm{K}_{\mathrm{b}} \quad$ - flap-span factor on lift

$K_{c} \quad$ - flap-chord factor on lift

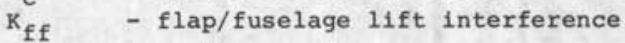
factor

$\mathrm{K}_{2-3}$ - correction factor for profile drag due to flap

$k_{C} \quad-$ flap-chord factor for split flap

$k_{\text {d }} \quad$ - profile arag factor due to flap

$k_{\ell} \quad$ - factor characterizing profile drag increment with lift

$k_{s} \quad$ - factor allowing for nonlinearity in lift curve

k flap angle correction factor for split flap

$\ell_{h}, l_{\mathrm{y}}$ - moment arm of horizontal and vertical tailplane, respectively (see Figs. E-13 and 9-21)

luc - length of undercarriage

M - Mach number

m $\quad$ - engine air mass flow (windmilling engine)

- dynamic head $\left(q=\frac{1}{2} \rho v^{2}\right)$

q

$R_{c} \quad-$ Reynolds number based on chord

$s$ - area (no index: gross wing area)

T - thrust

$\Delta T \quad$ - net thrust loss of inoperative engine plus windmilling and propeller drag

t - absolute profile thickness

$\mathrm{V}$ - flight velocity

$\mathrm{V}_{\mathrm{N}} \quad$ - average velocity of engine nozzle flow

u.c. - undercarriage

$\mathrm{v}, \mathrm{w} \quad$ - induced drag factors

$w_{\text {to }} \quad$ - Maximum Takeoff Weight

$x$ - longitudinal coordinate (origin: MAC leading edge)

$\mathrm{Y}_{\mathrm{v}} \quad$ - sideforce on vertical tailplane

y - lateral coordinate

$\mathrm{y}_{\mathrm{e}} \quad-$ yawing moment arm of inoperative engine

$\Delta \mathrm{y} \quad$ - leading edge sharpness parameter

z - vortex-induced drag factor for fuselage lift carry-over effect

$z_{h} \quad-$ distance from flap hinge to chord

a - angle of attack

$\alpha_{1} \quad$ - induced angle due to trailing vortices 


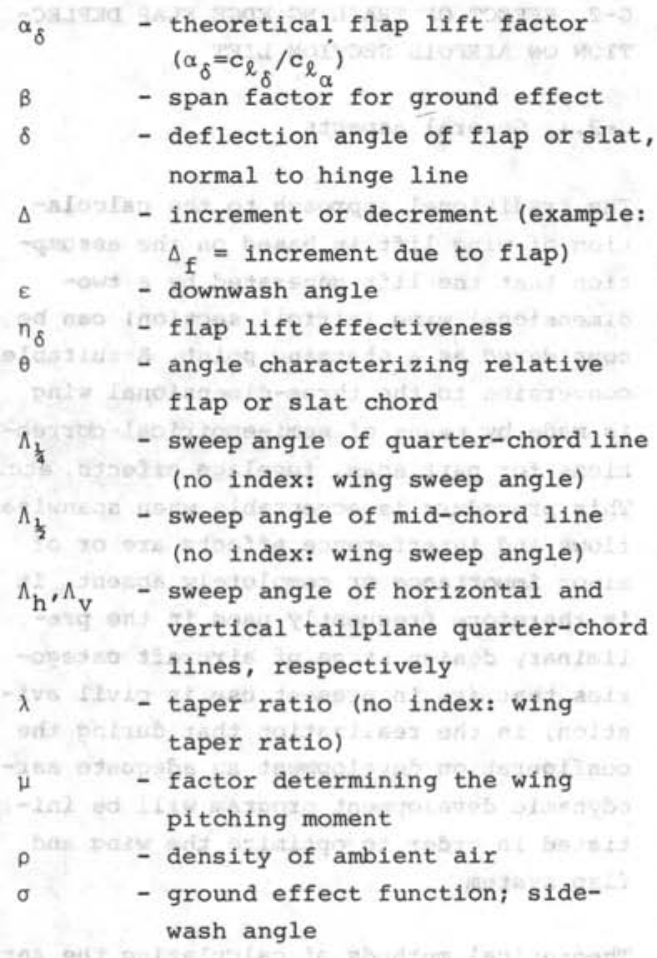

subscripts

A - auxiliary flap section

a.c. - aerodynamic center

as - asymetric flight condition

crit - critical (stalling) condition

e - engine

G-1. INTRODUCTION

The low-speed performance of aircraft, especially for transport aircraft, has received considerable added emphasis during recent years. It is therefore essential in the preliminary design stage to be able to predict the aerodynamic characteristics (lift and drag, trimmed condition) which can be used with a certain amount of confidence to compute low-speed performance and handing qualities and to provide a realistic goal for further aerodynamic development.

The present compilation of methods and da-

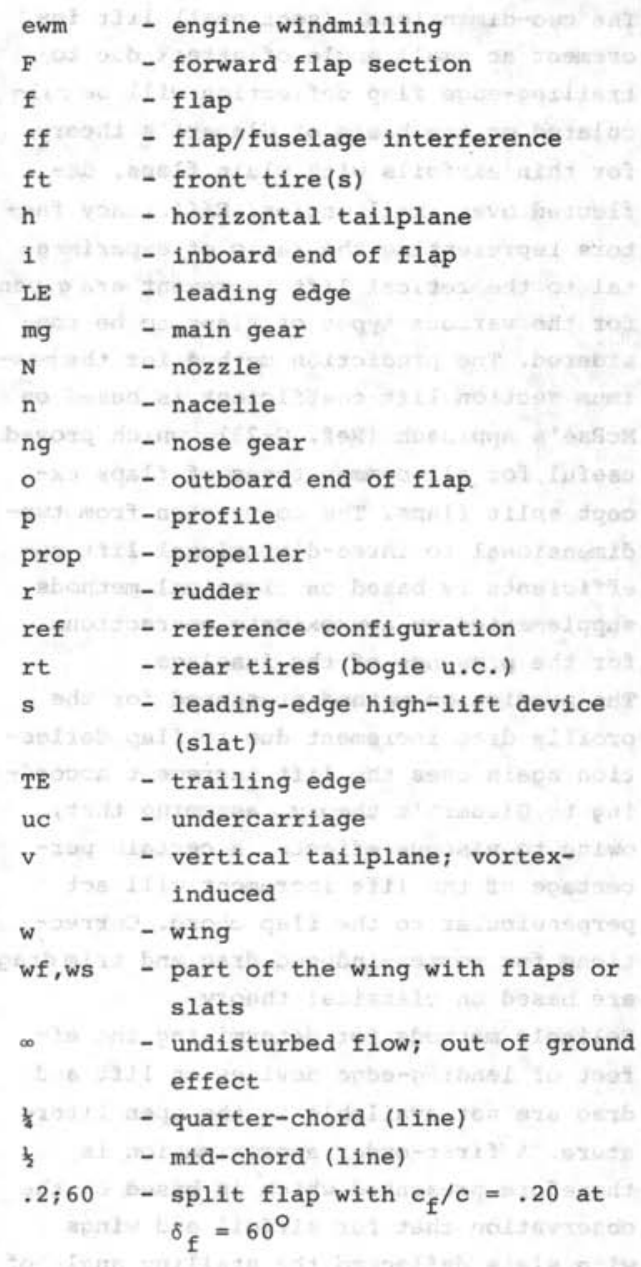

ta will enable the designer to make a good estimate of the lift curve and the drag polar for configurations with leading- and trailing-edge high-lift devices deflected. Split flaps, plain flaps, and single slotted, double slotted, triple slotted and Fowler flaps are the most commonly used trailing-edge devices to be dealt with, and in addition some data are also given on plain leading-edge flaps, slats and Krueger flaps. A method for estimating the pitching moment change due to flap deflection is presented for the purpose of calculating the tailplane load required to trim the aircraft. 
The two-dimensional (sectional) lift increment at small angle of attack due to trailing-edge flap deflection will be calculated on the basis of Glauert's theory for thin airfoils with plain flaps, deflected over small angles. Efficiency factors representing the ratio of experimental to theoretical $11 \mathrm{ft}$ increment are given for the various types of flaps to be considered. The prediction method for the maximum section lift coefficient is based on McRae's approach (Ref. G-23), which proved useful for all common types of flaps except split flaps. The conversion from twodimensional to three-dimensional lift coefficients is based on classical methods, supplemented by approximate corrections for the presence of the fuselage. The prediction method presented for the profile drag increment due to flap deflection again uses the lift increment according to Glauert's theory, assuming that, owing to viscous effects, a certain percentage of the lift increment will act perpendicular to the flap chord. Corrections for vortex-induced drag and trim drag are based on classical theory.

Reliable methods for determining the effect of leading-edge devices on lift and drag are not available in the open literature. A first-order approximation is therefore presented which is based on the observation that for airfoil and wings with slats deflected the stalling angle of attack is the most characteristic parameter.

A separate paragraph is devoted to the effects of undercarriage extension, ground proximity, and engine failure on lift and drag. Although these items are of vital importance to the prediction of transport aircraft performance, in particular takeoff (climb) and landing (climb) performance, little useful information is available in the 1iterature and the methods proposed must be considered only as firstorder approximations.
G-2. EFFECT OF TRAILING-EDGE FLAP DEFLECTION ON AIRFOIL SECTION LIFT

\section{G-2.1. General aspects}

The traditional approach to the calculation of wing lift is based on the assumption that the lift generated by a twodimensional wing (airfoil section) can be considered as a starting point. A suitable conversion to the three-dimensional wing is made by means of semi-empirical cofrrections for part span, fuselage effects, etc. This procedure is acceptable when spanwise flows and interference effects are or of minor importance or completely absent. It is therefore frequently used in the preliminary design stage of aircraft categories that are in present use in civil aviation, in the realization that during the configuration development an adequate aerodynamic development program will be initiated in order to optimize the wing and flap system.

Theoretical methods of calculating the aerodynamic characteristics of flapped sections have recently come to a stage of development where lift and pitching moment can be predicted with reasonable accuracy. A survey of the state of the art is given in Ref. G-31. Particularly for the most effective flap systems (multiple element flaps, slats), the flow is very sensitive to the details of the configuration: the flap and slat shape, their relative position, shroud shape, etc. This would require an optimization program which is a complex subject in itself. Instead of this, we will present generalized semi-empirical methods based on thin airfoll theory and experimental data. Where possible, an indication will be given of the sensitivity of flap performance to design details (average "good" design, best design, poor design). The basic effect of trailing-edge flap deflection on the lift curve of an airfoil 


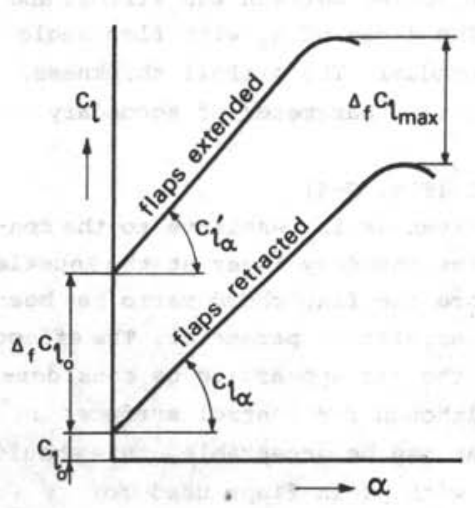

Fig. G-1. Effect of trailing-edge flap deflection on section lift

section is depicted in Fig. G-1. It will be assumed that for conventional wing shapes the lift curves are linear for moderate angles of attack. It can be observed that even if the lift curve of the basic section is fairly nonlinear due to trailing edge stall, the flapped section exhibits a more linear behaviour when the flow near the trailing edge is cleaned up by the flap.

The determination of the $11 \mathrm{ft}$ curve will be subdivided into prediction of the increment in lift at zero angle of attack $\left(\Delta_{f} c_{\ell_{0}}\right)$, the (increment in) maximum lift coefficient and the lift-curve slope at small angles of attack, $c_{\ell_{\alpha}}{ }^{\prime}$

G-2.2. Lift increment at zero angle of attack

For small flap deflections the rate of change of lift with flap deflection at constant angle of attack is frequently used as a measure of flap effectiveness:

$c_{\ell_{\delta}}=\left(\frac{\partial c_{\ell}}{\partial \delta_{f}}\right)_{\alpha}$

Another convention used is the rate of change of zero-lift angle of attack with flap deflection: $\frac{\partial \alpha}{\partial \delta_{f}}=\alpha_{\delta}=\frac{\left(c_{\ell_{\delta}}\right)_{\alpha}}{\left(c_{\ell_{\alpha}}\right)_{\delta}}$

The basis for several estimation methods is formed by Glauert's linearized theory for thin airfoils with flaps (Ref. G-57). A result obtained from this theory for the lift due to flap deflection is as follows:

$\alpha_{\delta}=1-\frac{\theta_{f}-\sin \theta_{f}}{\pi}$

where

$\theta_{f}=\cos ^{-1}\left(2 \frac{c_{f}}{c}-1\right)$

Fig. G-2 gives a plot of $\alpha_{\delta}$ as a function

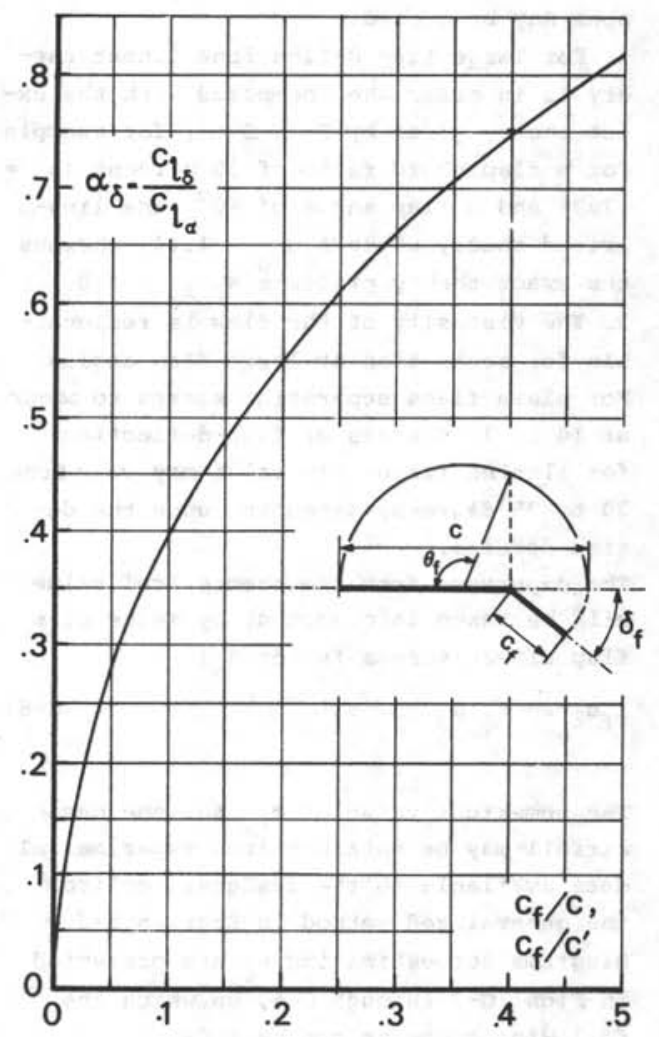

Fig. G-2. Theoretical flap lift factor 
of the relative flap chord. The theoretical $\Delta_{f} c_{l_{0}}$ is thus:

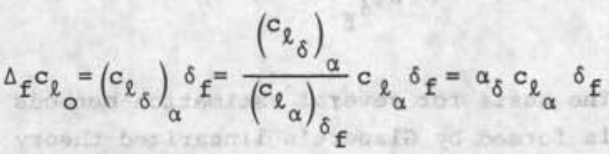

It is found that the theoretical lift effectiveness cannot be realized in practice. For small flap deflections approximately 70 to 858 of the theoretical value is possible, depending upon the type of flap system, while for large flap angles, e.g. those used in the landing configuration, the $11 \mathrm{ft}$ effectiveness may go down to approximately 50 percent of the theoretical value given by $(G-5)$. The following reasons may be quoted:

1. For large flap deflections linear theory is in error when compared with the exact theory given by Ref. G-66, for example. For a flap chord ratio of 35 percent $\left(\alpha_{\delta}=\right.$ $.707)$ and a flap angle of $60^{\circ}$, the linearized theory gives $\Delta_{f} c_{l_{0}}=4.44$, whereas the exact theory predicts $\Delta_{f} c_{l_{0}}=4.0$. 2. The viscosity of the flow is responsible for separation at large flap angles. For plain flaps separation starts to occur at 10 to 15 degrees of flap deflection; for slotted flaps this value may vary from 20 to 35 degrees, depending upon the design details.

The departure from the theoretical value will be taken into account by means of a flap effectiveness factor $n_{\delta}$ :

$\Delta_{f} c_{\ell_{0}}=n_{\delta} \alpha_{\delta} c_{\ell_{\alpha}} \delta_{f}$

The numerical value of $c_{\ell_{\alpha}}$ for the basic airfoil may be obtained from experimental data availaole to the designer, or from the generalized method in Section $\mathrm{B}-3.2$. Diagrams for estimating $n_{\delta}$ are presented in Figs. G-3 through G-6, on which the following comments can be made.

SPLIT FLAPS (Fig. G-3)

The effectiveness factor does not exceed 70 percent, even at small deflections, due to the wake formed between the airfoil and the flap. The decay of $n_{\delta}$ with flap angle is fairly gradual. The airfoil thickness/ chord ratio is a parameter of secondary importance.

PLAIN FLAPS (Fig. G-4)

Flap effectiveness is sensitive to the condition of the boundary layer at the knuckle and therefore the flap chord ratio has been used as a correlation parameter. The effect of sealing the gap appears to be considerable and although for control surfaces an unsealed gap may be acceptable, this should be avoided with plain flaps used for increasing the lift.

SINGLE SLOTTED FLAPS (fig. G-5)

For efficiently designed slotted flaps the lift increment due to flap deflection is not affected by the boundary layer of the basic airfoil, since a new boundary layer forms over the flap surface. The lift effectiveness, however, is very sensitive to the flap and slot geometry. Most of the early systematic measurements by NACA were made on flaps with optimum slot shape for each deflection, although for reasons of structural simplicity a single slotted. flap is frequently supported by means of a fixed hinge. In this latter case performance is sensitive to the hinge location. Generally speaking, the lower the hinge, the better the lift effectiveness will be, provided a good shroud and gap shape are present.

Slotted flaps require a rearward flap motion in order to ensure a good slot. The airfoil chord is thus extended effectively and this in itself contributes to the lift. This can be taken into account by referring the section lift to the extended chord, as defined in Fig. G-7, and then converting the result to the original chord as follows:

$c_{\ell}=\left(c_{\ell_{0}}^{\prime}+\Delta_{f} c_{\ell_{0}}{ }^{\prime}\right) \frac{c^{\prime}}{c}$

where $c_{\ell_{0}}{ }^{\prime}$ and $\Delta_{f} c_{l_{0}}$ ' are based on the extended chord $c^{\prime}$. Assuming that for the basic section $c_{\ell}$ is not altered after chord extension $\left\{c_{\ell_{0}}{ }^{\prime}=c_{\ell_{0}}\right.$ ', we have: 


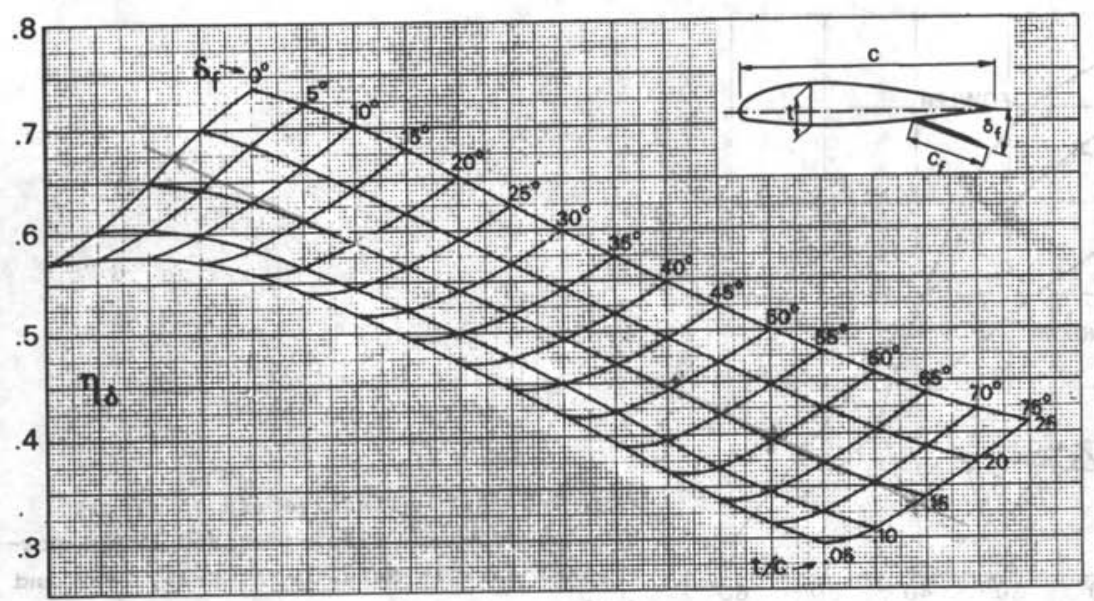

Fig. G-3. Lift-effectiveness factor for split flaps (derived from experimental data in Ref. G-34 and the USAF Datcom, Table 6.1.1.1.-24)

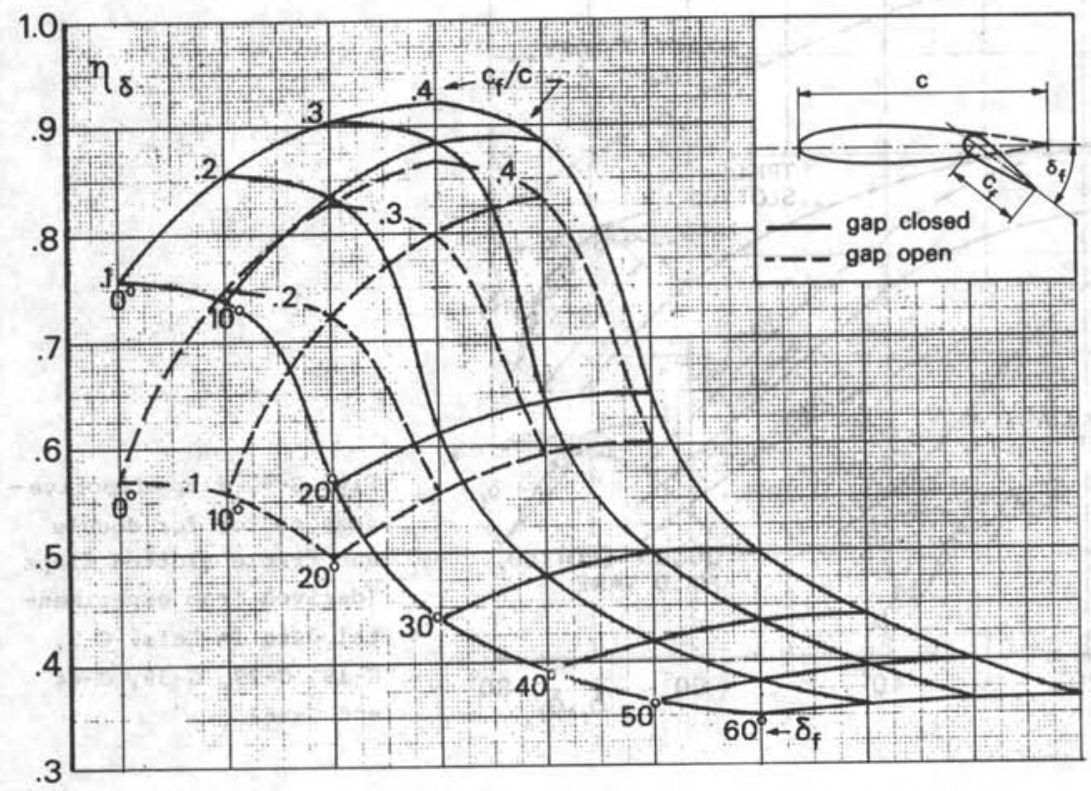

Fig. G-4. Lift effectiveness factor for plain flaps (derived from experimental data in the USAF Datcom, Table 6.1.1.1.-A and Ref. G-64) 


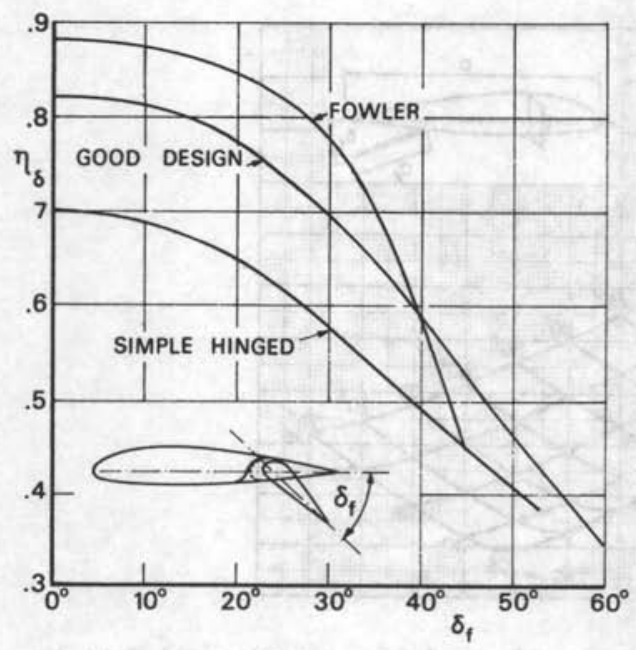

Fig. G-5. Lift effectiveness of single slotted (Fowler) flaps (derived from experimental data in Refs. G-33, G-35, G-37 and G-19)

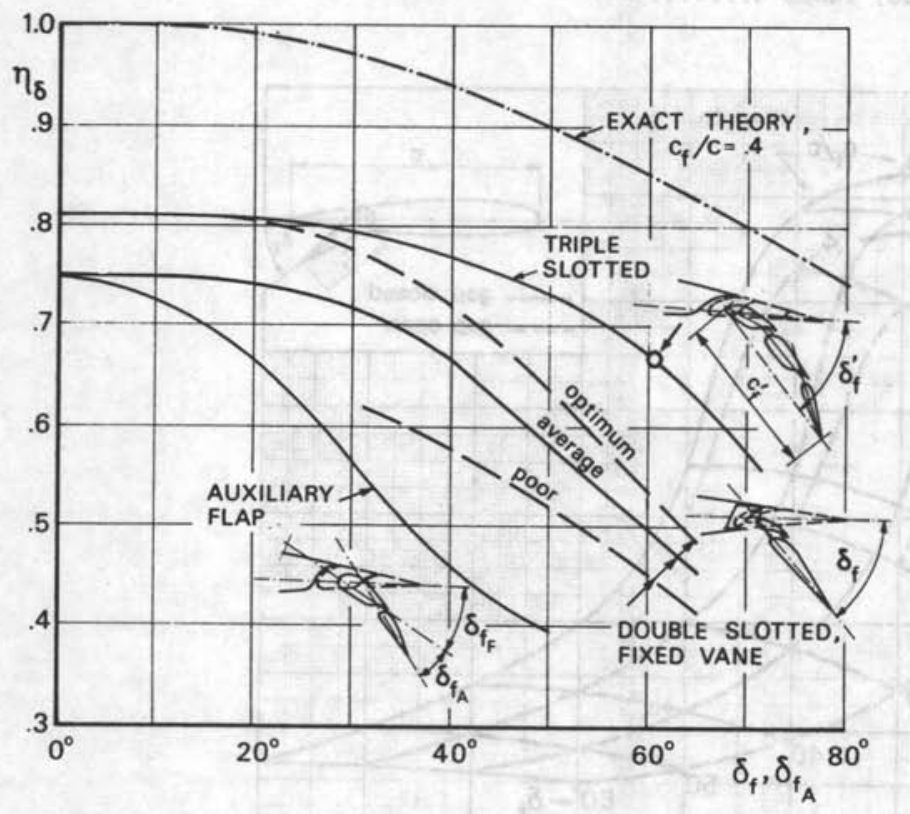

Fig. G-6. Flap effectiveness factor for double and triple slotted flaps (derived from experimental data in Refs. G-3, G-19, G-29, G-39, G-44 and $\mathrm{G}-48$ ) where $\Delta_{f} c_{l}{ }^{\prime}$ is defined by $(G-6)$, noting that $\alpha_{\delta}$ is also based on the extended chord $\left(c_{f} / c^{\prime}\right)$. Replacing the basic section lift gradient by $2 \pi \mathrm{rad}^{-1}$, we have:
$\Delta_{f} c_{\ell}=\Delta_{f} c_{\ell_{0}} \cdot \frac{c^{\prime}}{c}+c_{\ell_{0}}\left(\frac{c^{\prime}}{c}-1\right) \quad(G-8) \quad \Delta_{f} c_{\ell_{0}}^{\prime}=2 \pi n_{\delta} \alpha_{\delta}^{\prime} \delta_{f}$

where $\delta_{f}$ is in radians and $\alpha_{\delta}$, can be obtained from $(G-3)$ and $(G-4)$ or from Fig. G-2.

For a given flap geometry and type of support, the amount of chord extension can be 


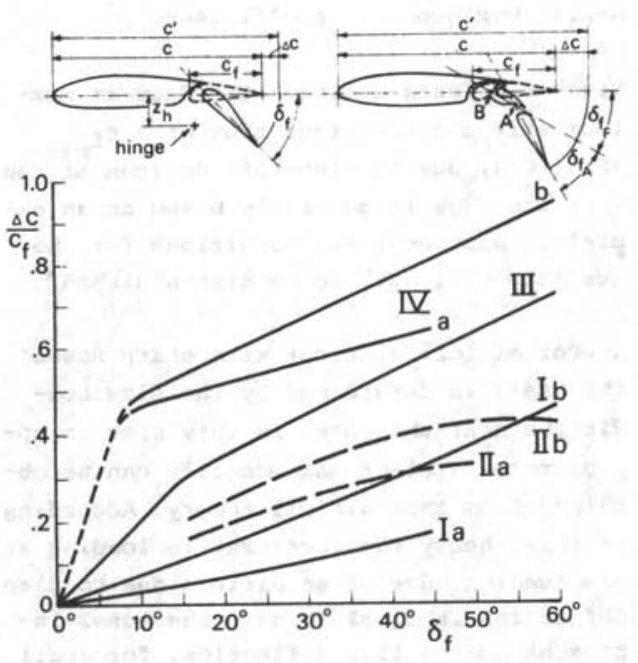

I: Fixed hinge - a: $z_{h} / c_{f}=.2$

$$
\text { b: } z_{h} / c_{f}=.4
$$

II: Typical optimum flap position

$$
\begin{aligned}
& \text { a: single slotted } \\
& \text { b: double slotted, fixed vane }
\end{aligned}
$$

III: Double slotted, variable geometry, with flap extension

IV: Fowler

- a: single slotted, double

slotted with fixed vane

b: double and triple slotted, with flap extension

Fig. G-7. The extended chord - its definition and some typical values for practical configurations

calculated; in the absence of information of this type Fig. G-7 may be used. In the case of a fixed hinge position the chord extension is given by:

$\frac{c^{\prime}}{c}=1+2 \frac{z_{h}}{c} \tan \frac{1}{2} \delta_{f}$

\section{FOWLER FLAPS (Fig. G-5)}

Aerodynamically, the Fowler flap acts in a similar manner to a single slotted flap and the same basic method can be used for both. The effect of chord extension is much larger (Fig. G-7), however, although this is partly offset by a reduction in $c_{f} / c^{\prime}$ and a lower value of $\alpha_{\delta}{ }^{\prime}$. For flap angles up to 30 - 35 degrees the flap ef- fectiveness is found to be superior to that for single slotted flaps. DOUBLE SLOTTED TRAILING-EDGE FLAPS The single slotted flap loses its lift effectiveness when the deflection angle exceeds about 40 degrees. The double slotted flap may be considered as a single slotted flap with a turning vane in the slot to recover the flow turning effectiveness. Consequently, the important parameters for the 2-slot flap are more complicated than for the 1-slot flap and so is the prediction of its lift effectiveness. Two main categories will be distinguished here. a. The flap with fixed vane. The prediction method for $\Delta_{f} c_{\ell}$ is similar to that for the single slotted flap, except that the factor $n_{\delta}$ is plotted in Fig. G-6. Typical curves are given for a good, average design with fixed hinge, together with a poor design and an indication of what can be expected when the flap support mechanism is such that an optimum gap is realized for each deflection. The maximum useful deflection is about 50 to 55 degrees. b. The double slotted flap with variable geometry. The forward flap is deflected to a maximum angle of 30 degrees, typically, and the aft section up to 30 or 40 degrees relative to the forward flap. There may also be a backward movement of the auxiliary flap. The total lift increment of this configuration may be calculated as follows: $\Delta_{f} c_{l_{0}}=\Delta_{1} c_{l_{0}}+\Delta_{2} \dot{c}_{l_{0}}$

where $\Delta_{1} c_{\ell_{0}}$ is the increment in $c_{\ell_{0}}$ due to the combined flap at a deflection equal to that of the forward flap section, assuming that the flap chords of the aft and forward flap coincide and no second slot is present. This contribution can be calculated with the method given above for single slotted flaps (Fig. G-5). $\Delta_{2} c_{\ell_{0}}$ is the increment in $c_{\ell_{0}}$ due to deflection of the auxiliary flap relative to the forward section. Again the method for single slotted flaps is used, except that a reduced lift effectiveness factor $n_{\delta}$ must be substituted in (G-9), given in 
Fig. G-6, because the presence of the forward flap reduces the effectiveness of the auxiliary flap.

The factor $\alpha_{\delta}{ }^{\prime}$ in (G-9) must be related to the ratio of the flap chord to the extended chord, which is defined in Fig. G-7 and obtained as follows:

a. The auxiliary flap is rotated from its deflected position about point $A$, until the two flap chords coincide.

b. Both flap sections are then rotated from the deflected position of the forward flap about point B until they coincide with the wing chord.

c. The distance from the leading edge of the airfoil to the trailing edge thus obtained is the extended airfoil chord c'. Using the typical figures given in Fig. G-7, the extended chord is calculated as follows:

$\frac{c^{\prime}}{c}=1+\frac{\Delta c}{c_{f}} \frac{c_{f}}{c}$

It should be noted that larger values of $\Delta \mathrm{c} / \mathrm{c}_{\mathrm{f}}$ than those given in Fig. G-7 are feasible, although at the expense of more structural complications and weight. TRIPLE SLOTTED TRAILING EDGE FLAPS

Insufficient data have been published on triple slotted flaps to form the basis of a generalized prediction method. The data given in Ref. G-29 have been transformed into a single point for $n_{\delta}$ in Fig. G-6. An equivalent flap deflection angle $\delta_{f}$ ' was defined to characterize the combined effect of the complex flap system, while the factor $\alpha_{\delta}$ ' was determined on the basis of the equivalent flap chord ratio $c_{f}^{\prime} / c^{\prime}$ to derive $a_{\delta}{ }^{\prime}$ from $(G-3)$ and $(G-4)$. In spite of this simplified approach the point is in fair agreement with the statement in Ref. G-29 that 81 percent of the potential flow value can be obtained at a total flap angle of 60 degrees. The suggested curve for $n_{\delta}$ is therefore assumed at 81 percent of the potential flow value (exact theory of Ref. (G-66) up to 50 degrees of flap angle.

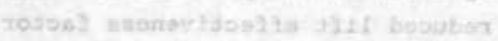

\section{G-2,3. Maximum lift coefficient}

Viscous effects dominate the flow at maximum lift and the prediction of $\Delta_{f} c_{l}$ (Fig. G-1) due to high-lift devices at the trailing edge is generally based on an empirical approach. Two conditions for the stalling will have to be distinguished*.

a. For airfoll sections with sharp noses the stall is determined by the flow conditions near the nose. In this case an approximate level of maximum lift can be obtained from thin airfoil theory. According to this theory the increment in loading at the leading edge of an airfoil due to flap deflection is equal to half the total increment due to flap deflection, for small flap chord ratios. For airfoils whose maximum lift is controlled by leading edge stall, the maximum lift increment due to flap deflection can be shown (Ref. G-16) to be equal to:

$\Delta_{f} c_{l_{\max }}=\Delta_{f} c_{l_{0}} \frac{\sin \theta_{f}}{\pi-\left(\theta_{f}-\sin \theta_{f}\right)} \quad(G-13)$ where $\theta_{f}$ is defined by $(G-4)$. The theoretical ratio of $\Delta_{f} c_{l_{\max }} / \Delta_{f} c_{l_{0}}$ varies between .4 and .5 for practical flap chord ratios. Combination of (G-13) with (G-3) and (G-5) yields a simple expression for the theoretical $\Delta_{f} c_{l}$ of sections with sharp leading edges. Assuming $c_{\ell_{\alpha}}=2 \pi$, we find: $\Delta_{f} c_{l_{\text {max }}}=2 \delta_{f} \sin \theta_{f}$.

b. When the basic section displays a separation associated with the pressure gradient at the rear part of the airfoil, this trailing edge stall will be delayed by the local suction produced by deflection of an effective trailing-edge flap. In this case the gain in maximum lift will be of the same magnitude as the lift increment at small angles of attack or may even be

*A more complete explanation of this subject is given in Ref. G-23. 

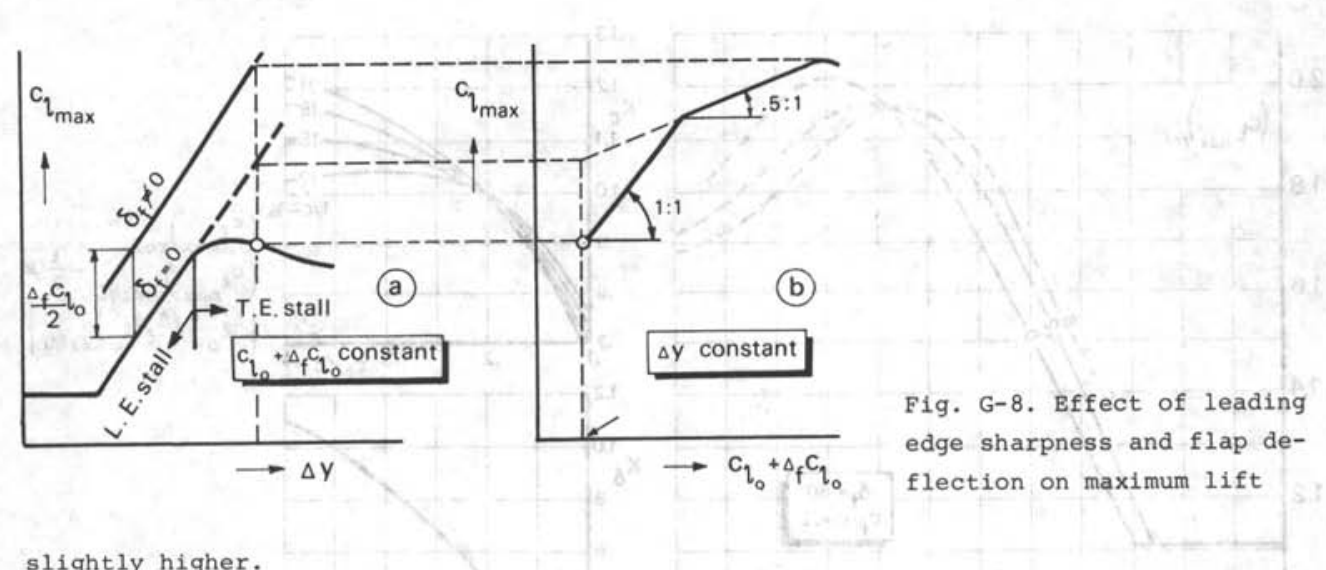

Fig. G-8. Bffect of leading edge sharpness and flap deflection on maximum lift

slightly higher.

Conclusions that can be drawn from the above are illustrated in Fig. G-8. In Fig. 8a the sharpness of the airfoil nose is characterized by the leading-edge sharpness parameter $\Delta y$ (see Appendix $A$, Fig. A-2), which has proved to be a useful correlation parameter for predicting the maximum lift associated with stall at the leading edge. For $\Delta y$ up to 1.2 - 1.5 percent chord, the maximum lift associated with leading edge stall (long bubble) is roughly constant. For larger $\Delta y$ values $c_{l}$ increases approximately proportionally with $\Delta \mathrm{y}$ (short bubble) up to a point where trailing edge stall begins to dominate. Thus for relatively thin airfoils, deflection of a trailing-edge flap results in a maximum lift increment which is preaicted theoretically by (G-13), while thicker airfoils with a trailing edge stall on the basic airfoll have a maximum lift increment of the order of 50 to 100 percent of the lift increment at small angles of attack*.

Consider now an airfoil with a trailing edge stall when the flap is retracted (Fig. G-8b). Small flap deflections clean up the flow near the trafling edge, resulting in a maximum lift increment which is of the same order of magnitude as the lift increment at small angles of attack. At a certain flap angle the load induced at the airfoil nose will increase up to a point at which leading edge stall will occur first, as dictated by the criterion in Fig. G-8a. The slope of the $c^{c} l_{\max }$ vs. $\Delta_{f} c_{l}$ curve will be approximately .5: 1 for this region

${ }^{*}$ Ref. G-16 suggests an average of $\Delta_{f} c_{\ell}$ max $=\frac{2}{3} \Delta_{f} c_{\ell_{0}}$ of flap angles,

The above observations form the basis of the following simple, unified prediction method for the maximum lift of flapped airfoils.

a. Plain and slotted (Fowler) trailingedge flaps.

The lowest value of the following items determines the maximum lift coefficient:

$c_{l_{\max }}{ }^{\prime}=.533 \frac{\Delta y}{c}\left(\frac{R_{c}}{3 \times 10^{6}}\right)^{.08}+\frac{1}{2}\left(c_{l_{0}}+\Delta_{f} c_{l_{0}}{ }^{\prime}\right)$

for $\Delta y / c($ in 8$) \geqslant 1.5$, or:

$c_{\ell_{\max }}=\left(c_{l_{\max }}\right)_{\delta_{f}=0}+\Delta_{f} c_{l_{0}} \cdot$

where $R_{C}$ is the Reynolds number based on the original chord length, and the maximum lift coefficient based on this chord is given by:

$$
c_{l_{\max }}=\frac{c^{\prime}}{c} c_{l_{\max }}
$$

Equation G-15 has been derived from experimental data in the literature quoted in the references. The method gives acceptable results not only for plain and single slotted flaps, but also for multiple-element flaps with or without Fowler motion.

\section{b. Split flaps.}

Airfoil sections with split flaps cannot be dealt with by the previous method, prob- 

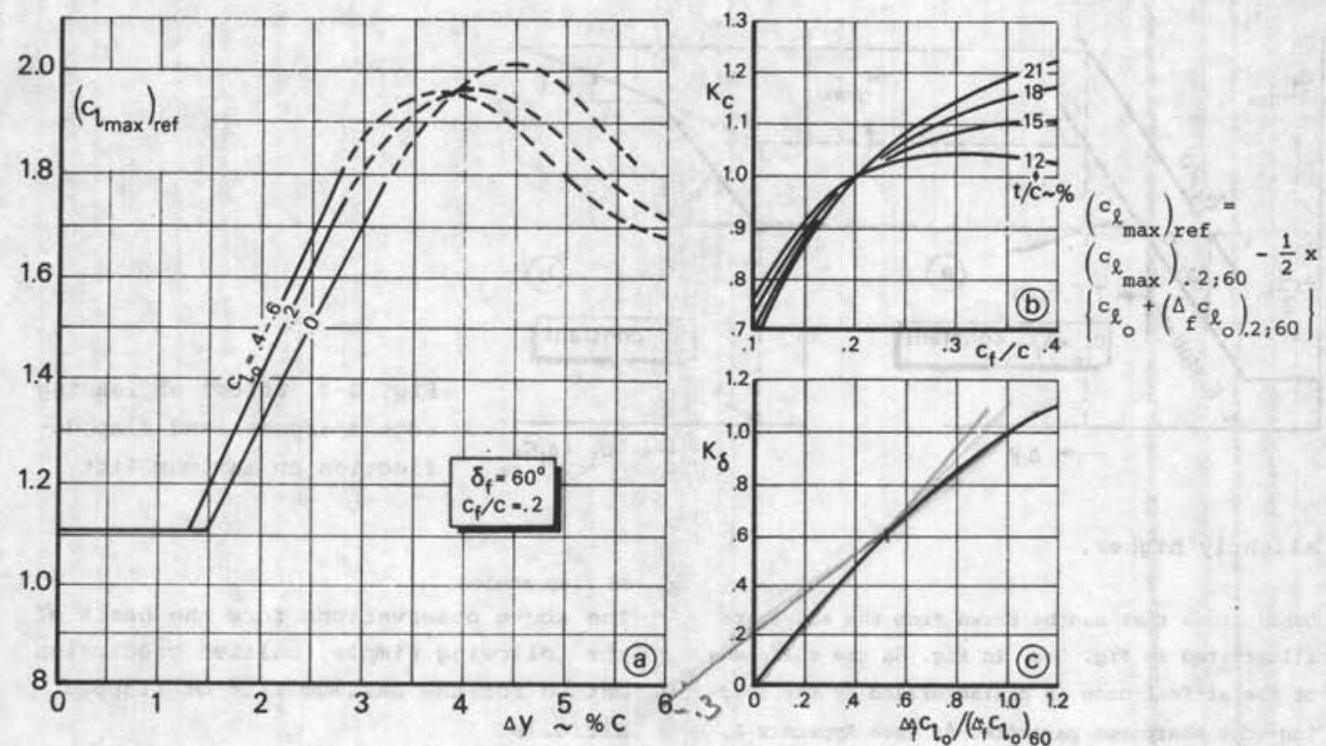

Fig. G-9. Charts for estimating the increment in maximum lift due to split flaps. For $\Delta \mathrm{y}$ : see Fig. A-1 of Appendix A. (Cerived from Ref. G-34)

ably because there is always a wake at the trailing edge when the flap is deflected, and leading edge and trailing edge stall will occur simultaneously. For split flaps the curve of Fig. G-8b has, typically, a mean slope of $.75: 1$ and is slightly curved. A different preaiction method has therefore been devised, consisting of the following steps:

1. Calculate $\Delta_{f} c_{\ell}$ vs. $\delta_{f}$ with the method given in Section G-2.2. for split flaps (Fig. $\mathrm{G}-3$ ).

2. Calculate $\Delta_{f} c_{l}$ for a flap chord ratio $c_{f} / c=.20$ and a flap angle $\delta_{f}=60^{\circ}$. This is denoted by $\left(\Delta_{f} c_{\ell}\right) .2$;

3. Determine $c_{l_{\max }}$ for $\dot{\mathrm{r}}^{2} ; 6 q_{\mathrm{f}}=.2$ and $\delta_{f}=$ $60^{\circ}$, as follows:

$\left(\Delta c_{\ell}\right)_{\max }=\left(c_{\ell_{\max }}\right)_{\text {ref }}-\left(c_{\ell_{\max }}\right)_{\delta_{\mathrm{f}}=0}+$

$+.5\left|c_{l_{0}}+\left(\Delta_{f} c_{\ell_{0}}\right) .2 ; 60\right|$

where $\left(c_{\ell_{\max }}\right)_{\text {ref }}$ is obtained from Fig.

4. Correct for flap chord ratio and flap angle as follows:
$\Delta c_{\ell_{\max }}=k_{c} k_{\delta}\left(\Delta_{f} c_{\ell_{\text {max }}}\right)_{.2 ; 60}$

$k_{c}$ and $k_{\delta}$ are correction factors for the flap chord ratio and flap angle given in Figs. $\mathrm{G}-9 \mathrm{~b}$ and $\mathrm{G}-9 \mathrm{c}$, respectively. The values for $\Delta_{f} c_{\ell_{0}}$ to be used here have been calculated in step 1 .

\section{G-2.4. Lift-curve slope}

The lift gradient is affected by flap deflection in a number of ways:

1. The chord extension increases $c_{\ell_{\alpha}}$. This can be allowed for by the multiplication factor c'/c.

2. The potential flow effect of flap deflection on the lift-curve slope given by the exact theory in Ref. G-66 indicates that $\Delta_{f} c_{\ell}$ is reduced with increasing $\alpha$. Hence $c_{\ell}{ }_{\alpha}^{\prime}$ (based on the extended chord) decreases not only with the flap deflection angle, but also with the angle of attack, and the result is a nonlinearity in the lift curve which is particularly pronounced for large flap angles.

3. The effect of viscosity on the lift ef- 
fectiveness of a flap increases with the angle of attack, thus reducing $\Delta_{f} c_{\ell}$ with increasing $\alpha$.

Fig. G-10 shows that for small flap angles

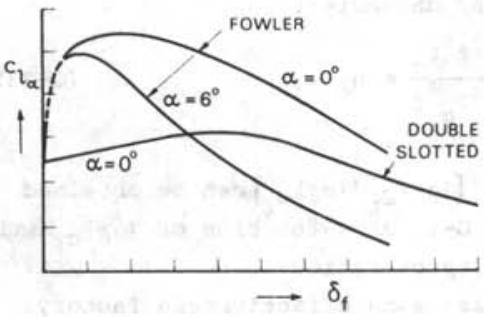

Fig. G-10. Variation of section lift-curve slope with flap angle

the effect of the chord extension dominates, but that this effect is cancelled at large flap angles by the other effects. The reduction in lift-curve slope is more pronounced at angles of attack approaching the stall.

No method for calculating the lift-curve slope is available in the literature. The relationship presented here,

$\frac{c_{\ell_{\alpha}}{ }^{\prime} \text { (flaps down) }}{c_{\ell_{\alpha}} \text { (flaps up) }}=\frac{c^{\prime}}{c}\left(1-\frac{c_{f}}{c^{\prime}} \sin ^{2} \delta_{f}\right)$

approximates the results of the exact theory fairly accurately and is in qualitative agreement with experimental data. In individual cases the reduction of $c_{\ell_{\alpha}}$ with $\delta_{f}$ may be considerably more than that indicated by $(G-19)$, particularly when the shape of the slot is not optimized, and when the stalling condition occurs at small angles of attack.

\section{G-3. LIFT OF AIRCRAFT WITH DEFLECTED TRAIL-} ING-EDGE FLAPS

The prediction of aircraft lift is based on a build-up of various contributing components: the aircraft in the clean configuration (see Appendix E), trailing-edge flaps, the horizontal tailplane and corrections for the presence of the fuselage, powerplant installation, etc. The contribution of leading-edge devices will be dealt with separately in section G-5. The lift curve may thus be expressed in the form of a lift increment relative to the en route configuration:

$\Delta C_{L}=\Delta_{f} C_{L}+\sum \Delta C_{L}+\Delta_{h} C_{L}$

The direct contribution of the trailingedge flap $\Delta_{f} C_{L}$ is dealt with in section G-3.1. , the various corrections $\sum \Delta C_{L}$ are discussed briefly in section G-3.2., and the tailplane contribution $\Delta_{h} C_{L}$ is finally given in Section G-3.3.

G-3.1. Wing lift

In much the same way as was done for airfoil sections, the wing lift curve will be computed from the following characteristics:

$\Delta_{f} C_{L_{0}}$ : the lift increment at zero angle of attack;

$\Delta_{f} C_{L_{\text {max }}}$ : the increment in maximum lift coefficient:

$\mathrm{C}_{\mathrm{L}}{ }^{\prime}$ : the lift-curve slope with flaps deflected.

A result similar to Fig. G-1 is found, except that $c_{\ell}$ is replaced by $C_{L}$.

The reader's attention is drawn to the fact that the maximum lift defined in this appendix refers to the top of the $11 \mathrm{ft}$ curve. As explained in section 5.4.4., this figure cannot be used directly to determine the stalling speed (see also Ref. G-32).

a. Increment in lift for $\alpha=0^{\circ}$. Lifting surface theory can be employed to determine $\Delta_{f} c_{L}$ for moderate flap deflections. The method of Ref. G-64 relies on a knowledge of the section flap effectiveness and is summarized as follows:

$\Delta_{f} c_{L_{0}}=\Delta_{f} c_{\ell}\left(\frac{C_{L_{\alpha}}}{c_{\ell}}\right)\left[\frac{\left(\alpha_{\delta}\right) C_{L}}{\left(\alpha_{\delta}\right) c_{\ell}}\right] K_{b}$

$\Delta_{f} c_{\ell}$ is the section lift increment for 


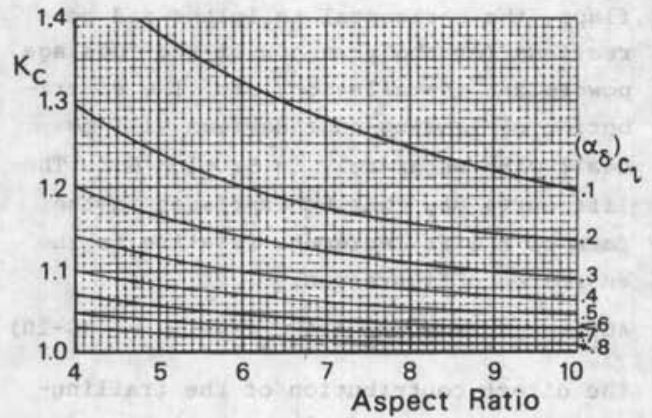

Fig. G-11. Variation of flap chord factor with aspect ratio (Ref. G-64)

$\alpha=0$ due to flap deflection for a representative section, e.g. halfway along the semi-flap span. Experimental results should preferably be used, but when these are not available, the method of Section G-2.2. or any other suitable method may be used (e.g. Ref. G-19 or Ref. G-65). $\mathrm{C}_{\mathrm{L}_{\alpha}}$ and $\mathrm{c}_{\ell_{\alpha}}$ are the lift-curve slopes of the basic wing (see Section E-4.1.) and the basic airfoil (see Section E-3.2.), respectively, while $\left(\alpha_{\delta}\right)_{C_{L}}$ is the three-dimensional flap effectiveness parameter:

$$
\left(a_{\delta}\right)_{C_{L}}=\frac{c_{L_{\delta}}}{c_{L_{\alpha}}}
$$

and $\left(\alpha_{\delta}\right)_{c_{\ell}}$ is the two-dimensional flap effectiveness parameter:

$$
\left(\alpha_{\delta}\right)_{c_{\ell}}=\frac{\Delta_{f} c_{\ell}}{c_{\ell}{ }_{\alpha}{ }_{f}}=n_{\delta} \alpha_{\delta}
$$

The ratio $\left[\left(\alpha_{\delta}\right) \mathrm{C}_{\mathrm{L}} /\left(\alpha_{\delta}\right) \mathrm{c}_{\ell}\right]$ can be obtained from Fig. G-11 as a function of $\left(\alpha_{\delta}\right)_{c_{\ell}}$ and the wing aspect ratio.

$\mathrm{K}_{\mathrm{b}}$ is a flap span effectiveness factor, defined as follows:

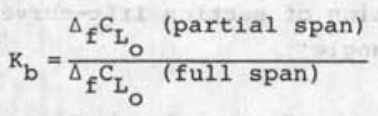

The curve of $\mathrm{K}_{\mathrm{b}}$ versus flap span and taper ratio, as derived from empirical data, is plotted in Fig. G-12. For flaps other than inboard the value of $\mathrm{K}_{\mathrm{b}}$ is obtained by superposition of the flaps, as shown schematically in Fig. G-13 for the case of a flap interrupted by the fuselage.

In the case of inboard flaps which are augmented by ailerons acting as flaps ("flaperons"), the follow-

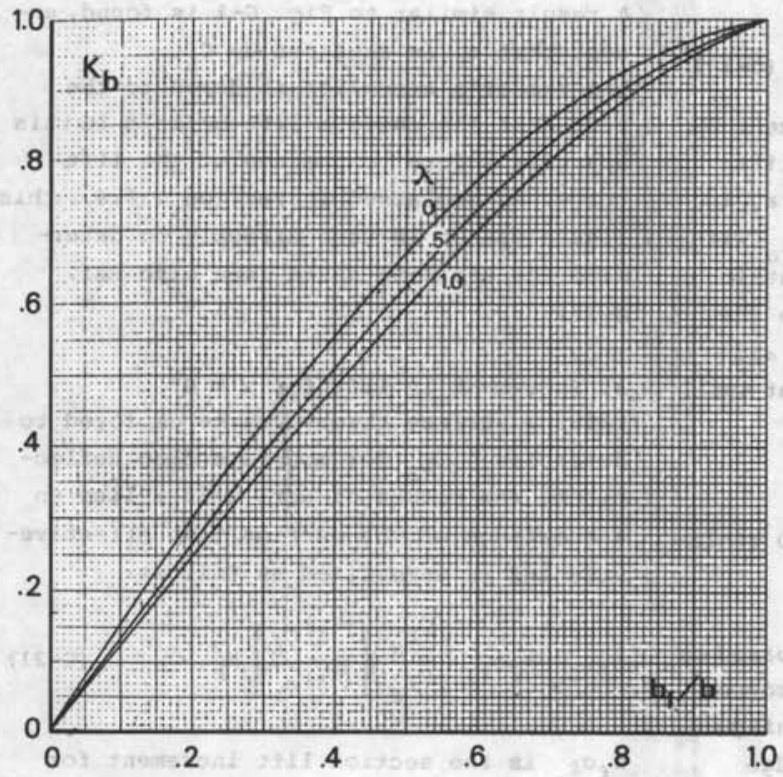

Fig. G-12. Variation of span factor $K_{b}$ with flap span for inboard flaps (Ref. G-64) 


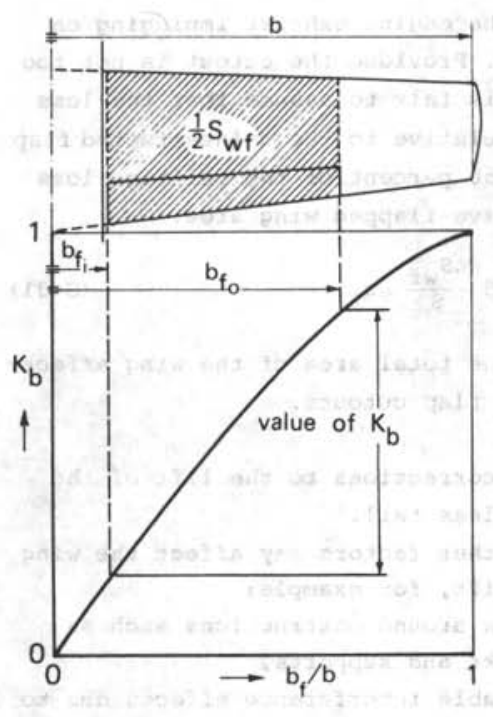

Fig. G-13. Flap span factor for flaps other than inboard flaps and definition of $\mathrm{S}_{\mathrm{wf}}$

Ing procedure is proposed:

1. Estimate the $\Delta_{1} c_{l}$ aue to deflection of the aileron for a representative local section.

2. Compute $\Delta_{1} C_{L_{0}}$, assuming that $\Delta_{1} c_{\ell_{0}}$ acts fullspan of the combined inboard flaps and the deflected ailerons.

3. Estimate the $\Delta_{2} c_{l}$ due to inboard flap deflection for a representative inboard section.

4. Compute $\Delta_{2} C_{L_{0}}$ due to $\Delta_{2} c_{\ell}-\Delta_{1} c_{\ell}$ over the inboard flap span with (G-21).

5. Add $\Delta_{1} C_{L_{0}}$ and $\Delta_{2} C_{L_{0}}$ to obtain $\Delta_{f} C_{L_{0}}$.

b. Maximum lift increment.

The maximum lift increment of a representative section is used for predicting

$\Delta_{f} C_{L_{\max }}$. Section data should preferably be derived from experimental data; if these are not available, the method explained in Section G-2.3. may be used.

The two-dimensional data for efficient slotted flap configurations* are converted into a three-dimensional lift increment as

* For plain flaps the effect of sweepback is much more pronounced, e.g. proportional to $\cos ^{3} \Lambda$ (Ref. G-22). follows :

$\Delta_{f} C_{L_{\text {max }}}=.92 \Delta_{f} C_{l} \frac{S_{\text {max }}}{S} \cos \Lambda_{\frac{l}{2}}$

$\mathrm{S}_{\mathrm{wf}} / \mathrm{S}$ is the ratio of the wing area affected by the trailing-edge flaps to the total wing area. For straight-tapered wings we have:

$\frac{S_{w f}}{S}=\frac{b_{f_{o}}^{-b_{f}}}{b}\left\{1+\frac{1-\lambda}{1+\lambda}\left(1-\frac{b_{f_{o}}^{+b_{f}}}{b}\right)\right\}(G-26)$

The nomenclature is defined in Figs. G-13 and G-14. For moderately tapered wings the numerical value of $\mathrm{S}_{\mathrm{wf}} / \mathrm{S}$ is approximately equal to the factor $\mathrm{K}_{\mathrm{b}}$ defined previously. The factor .92 in (G-25) takes into account the loss of lift near the flap tips, as illustrated by Fig. G-14, where the shaded area represents the increment in maximum lift in the hypothetical case that all sections of the flapped part of the wing stall simultaneously.

c. Lift-curve slope.

The lift-curve slope is corrected for the effects referred to in section G-2.4. above, as follows:

$\frac{C_{L_{\alpha}} \text { (flaps down) }}{C_{L_{\alpha}} \text { (flaps up) }}=1+\frac{\Delta_{f} C_{L_{o}}}{\Delta_{f} C_{l_{o}}} \times$

$\left\{\frac{c^{\prime}}{c}\left(1-\frac{c_{f}}{c^{\prime}} \sin ^{2} \delta_{f}\right)-1\right\}$

where $\Delta_{f} C_{L_{o}} / \Delta_{f} c_{l_{0}}$ is the ratio of the three-dimensional to two-dimensional lift increments at $\alpha=0$, given by $(G-21)$.

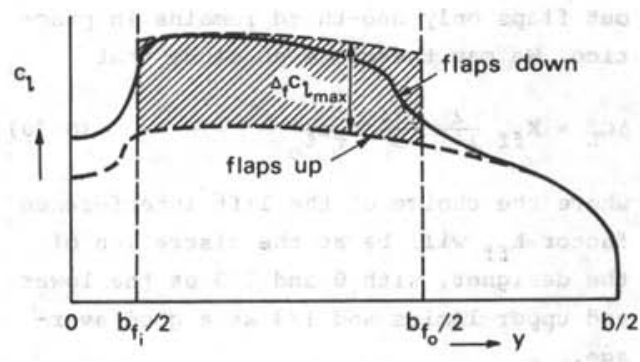

Fig. G-14. Lift distribution of a wingfuselage combination with deflected flaps. 


\section{G-3.2. Various contributions}

The corrections on the lift curve $\left(\Sigma \Delta C_{L}\right)$ to be discussed will simply be expressed in the form of $\Delta C_{L}$ values, as the absence of reliable methods precludes systematic treatment of these effects on $\Delta_{f} C_{L_{o}}, \Delta_{f} C_{L_{\max }}$ and $\mathrm{C}_{\mathrm{L}_{\alpha}}$ ' cannot be presented in view. We shall therefore assume that the complete lift curve will be shifted up or down by the stated value of $\Delta C_{L}$.

a. Wing/fuselage interference.

Some interference effects between the flows around the wing and the fuselage have been discussed in section E- 6 of Appendix E. Similar, but more complicated, flow phenomena will be observed on wing/ flap/fuselage combinations. Analytical treatment of this effect being ruled out, the designer must rely on rules of thumb in the preliminary design stage.

The most conservative approach is to completely ignore the lift carry-over by the fuselage. Though this may be justified for large flap deflections, when there is a large gap between the fuselage and the flaps, the carry-over effect cannot be ignored altogether in all cases. Potential flow theory indicates that the lift carryover by the fuselage is between one-half and two-thirds of the lift generated by the wing center section in the absence of the fuselage, assuming the flaps to be extended to the centerline. The experiments reported by Hoerner (Ref. G-10, page 8-18) indicate that in the case of wings without flaps only one-third remains in practice. We may therefore conclude that

$\Delta C_{L}=K_{f f} \frac{2}{1+\lambda} \frac{b_{f i}}{b} \Delta_{f} c_{\ell}$

where the choice of the lift interference factor $\mathrm{K}_{\mathrm{ff}}$ will be at the discretion of the designer, with 0 and $2 / 3$ as the lower and upper limits and $1 / 3$ as a good average.

b. Flap cutouts.

Flap cutouts are sometimes required to prevent the engine exhaust impinging on the flaps. Provided the cutout is not too wide, it is fair to assume that the loss in lift relative to the uninterrupted flap is about 50 percent of the relative loss in effective flapped wing area:

$\Delta \mathrm{C}_{\mathrm{L}}=-.5 \frac{\Delta \mathrm{S}_{\mathrm{wf}}}{\mathrm{S}} \Delta \mathrm{c}_{\ell}$

$\Delta S_{w f}$ is the total area of the wing affected by the flap cutouts.

c. Other corrections to the lift of the aircraft less tail.

Several other factors may affect the wing maximum lift, for example:

- the flow around obstructions such as flap tracks and supports,

- unfavorable interference effects due to engine nacelles or engine intake scoops,

- losses incurred by devices needed to provide acceptable handing and control at the stall, and

- the effect of aeroelastic deformation on the lift, particularly on large aircraft (Ref. G-32).

Each of these may affect $\mathrm{C}_{\mathrm{L}_{\max }}$ by as much as . 1 to .2, but prediction of their magnitude is generally impossible in the preliminary design stage.

G-3.3. Contribution of the horizontal tailplane

The horizontal tail load required to trim out the nose-down pitching moment due to flap deflection reduces the lift. This contribution can be derived from semiempirical data on pitching moment variations due to flap deflection. A relatively simple approach is thought to be acceptable for the purpose of determining the trim load.

a. Section pitching moment.

When experimental data are not available, the generalized expression quoted in Ref. G-5 may form a useful starting point: 


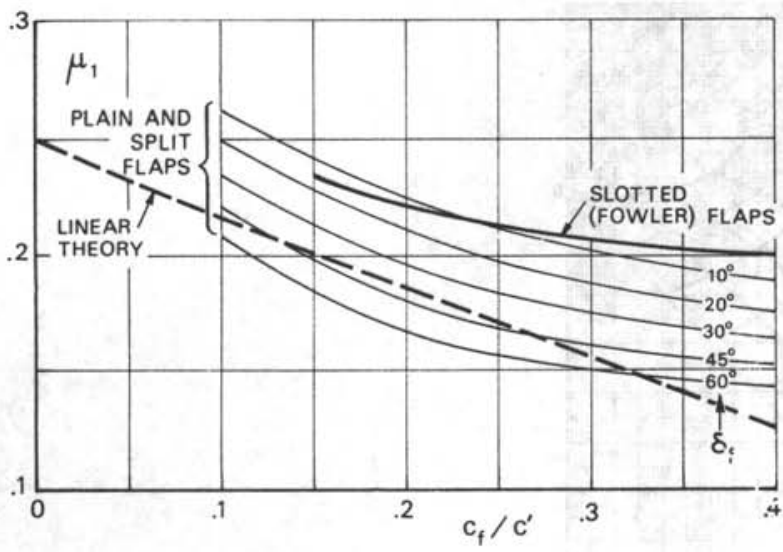

Fig. G-15. The pitching moment function $\mu_{1}$

$$
\begin{aligned}
\Delta_{f^{\prime} m_{\frac{1}{4}}} & =-\mu_{1} \Delta_{f^{\prime}} c_{\ell} \cdot\left(\frac{c^{\prime}}{c}\right)^{2}-\frac{c_{\ell}}{4} \frac{c^{\prime}}{c}\left(\frac{c^{\prime}}{c}-1\right)+ \\
& +\left(c_{m_{\frac{l}{4}}}\right)_{\delta_{f}=0}\left[\left(\frac{c^{\prime}}{c}\right)^{2}-1 \mid\right.
\end{aligned}
$$

The extended chord $c^{\prime}$ (cf. Fig. G-7) allows for the effect of the backward movement of the flap while it is being extended.

The first contribution in (G-32) is due to the increased section camber. The factor $\mu_{1}$ is defined as follows:

$\mu_{1}=-\frac{\Delta_{f} c_{m}^{\prime}}{\Delta_{f} c_{l}}$

According to Glauert's linear theory for small flap deflections, we have

$\mu_{1}=\frac{1}{2}\left(1-\frac{c_{f}}{c}\right) \frac{\sin \theta_{f}}{\pi-\left(\theta_{f}-\sin \theta_{f}\right)}$

where $\theta_{f}$ is defined by $(G-4)$. Fig. G-15 shows that the theoretical value of $\mu_{1}$ generally underpredicts the pitching moment coefficient. It has been found that for slotted flaps, with or without Fowler movement, most data are on a single line, provided the second term in (G-32), representing the theoretical rearward shift of the airfoil aerodynamic center, is halved. In the case of split and plain flaps the flap angle is observed to exert a pronounced influence on $\mu_{1}$. The last term of (G-32), being generally of a low order, can be ignored and the following practical equation is proposed:

$\Delta_{f^{\prime} m_{\frac{l}{2}}}=-\mu_{1} \Delta_{f} c_{\ell} \frac{c^{\prime}}{c}-\frac{c_{\ell}}{8} \frac{c^{\prime}}{c}\left(\frac{c^{\prime}}{c}-1\right)$

where $\mu_{1}$ can be obtained from Fig. G-15.

b. Wing pitching moment.

References G-5 and G-60 convert the twodimensional effect into a three-dimensional pitching moment change approximately in the following manner:

$\Delta_{f} c_{m_{\frac{3}{4}}}=\mu_{2} \Delta_{f} c_{m_{\frac{1}{2}}}+.7 \frac{A}{1+2 / A} \mu_{3} \Delta_{f} c_{\ell} \tan \Lambda_{\frac{1}{4}}(G-36)$

where $\Delta_{f} c_{m_{\frac{1}{2}}}$ can be obtained from (G-35) by substituting:

$c_{\ell}=c_{L}+\Delta_{f} c_{\ell}\left(1-\frac{s_{w f}}{S}\right)$

The correction factor $\mu_{2}$ (Fig. G-16) takes into account the part-span effect on a straight wing, while $\mu_{3}$ is a function determining the effect of wing sweep (Fig. G-17).

c. Pitching moment, aircraft less tail. Most measurements of flapped wing sections have been related to the .25-chord point or to the a.c. of the basic wing section. For this reason we take the aerodynamic pitching moment about the mean quarter chord point as: 


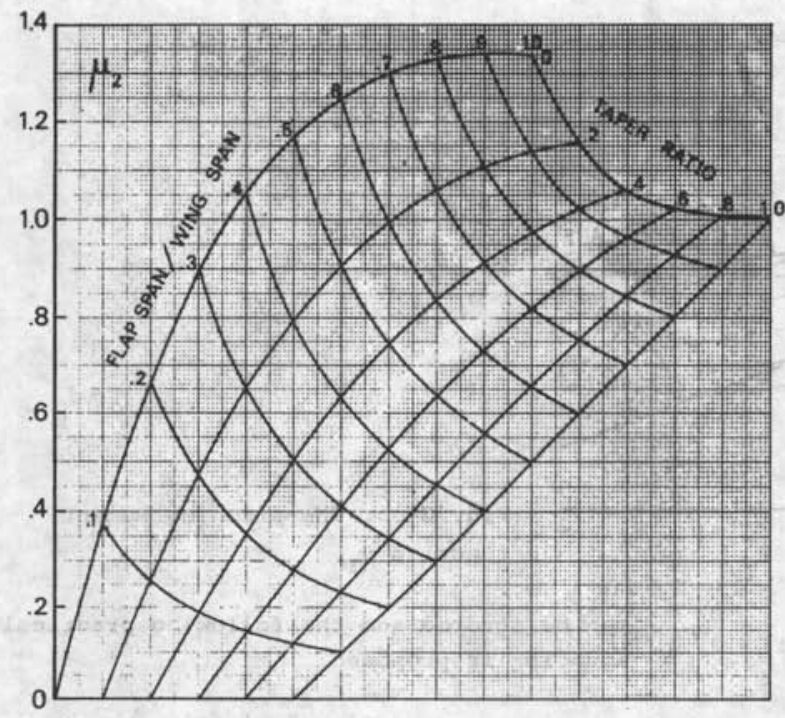

Fig. G-16. Correction factor for part-span effect on pitching moment (derived from Ref. G-5)

$$
c_{m_{\frac{1}{4}}}=\left(c_{m_{\frac{1}{2}}}\right)_{\delta_{f}=0}+\Delta_{f} c_{m_{\frac{1}{4}}}+\sum \Delta c_{m_{\frac{1}{4}}}
$$

The pitching moment coefficient with flaps retracted can be obtained from $(B-35)$ of Appendix B:

$\left(c_{m_{\frac{1}{2}}}\right)_{\delta_{f}=0}=C_{m_{a c}}+\left(c_{L}-\Delta_{f} C_{L}\right)\left(.25-\frac{x_{a c}}{\bar{c}}\right)$

where the aerodynamic center refers to the aircraft less tail with flaps retracted (See Appendix E, Section E-7). The term $\Delta_{f} C_{m_{\frac{1}{4}}}$ in $(\mathrm{G}-38)$ can be calculated as a function of $C_{L}$ using $(G-36)$ and $(G-37)$.

The term $\Sigma \Delta \mathrm{C}_{\mathrm{m}_{\mathrm{k}}}$ is a formal statement for various other effects:

1. The effect of lift carry-over by the fuselage, relative to the theoretical scheme in which the flaps extend to the plane of symmetry.

2. The effect of flap deflection on fuselage forebody and afterbody contributions to the pitching moment.

3. Other effects, e.g. contributions of flap cutouts, nacelle interference, etc.

The first effect may be estimated by using (E-40) of Appendix $\mathrm{E}$, replacing $\mathrm{C}_{\mathrm{L}_{0}}$ by $\mathrm{C}_{\mathrm{L}_{0}}+\Delta_{f} \mathrm{C}_{\mathrm{L}_{0}}$. The second contribution may be approximated by ignoring

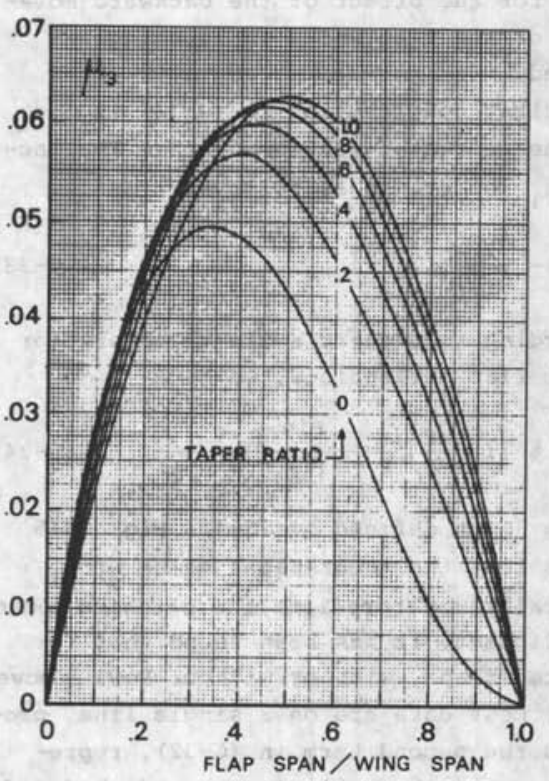

Fig. G-17. The function $\mu_{3}$ for deterinining the pitching moment increment of flaps on sweptback wings. (Ref. G-5). 
altogether the lift carry-over by the fuselage. In view of absence of data a verification for this approach cannot be given. It should be noted that the first two effects mentioned are of opposite sign.

d. Tailplane lift.

The tail load change required to trim the aircraft after flap deflection is, ignoring $\sum \Delta C_{m_{\frac{1}{4}}}$ in $(G-38)$, approximately:

$\Delta_{h} C_{L}=\Delta C_{L_{h}} \quad \frac{s_{h}}{S}=\left\{\Delta_{f} C_{L}\left(\frac{x_{c g}-x_{\frac{1}{2}}}{\bar{c}}\right)+\Delta_{f} C_{m_{\frac{1}{2}}}\right\} \frac{\bar{c}}{l_{h}}$

where $\Delta_{f} C_{L}$ and $\Delta_{f} C_{m 1}$ can be computed by means of the methods presented previously.

G-4. PREDICTION OF THE LOW-SPEED DRAG POLAR

The drag of the aircraft with flaps down (undercarriage retracted) can be obtained from the drag polar in the clean configuration as follows:

$C_{D}=C_{D_{0}}+\frac{C_{L}^{2}}{\pi A e}+\Delta_{f} C_{D_{p}}+\Delta_{f} C_{D_{v}}+\Delta_{\operatorname{trim}} C_{D} \quad(G-41)$

where $\Delta_{f} C_{D_{p}}, \Delta_{f} C_{D_{v}}$, and $\Delta_{t r i m} C_{D}$ are the increments in profile, vortex-induced, and trim drag coefficients. In (G-41) the basic polar is written as a simple parabolic approximation, but other relationships may be used as well. Its derivation is discussed in Appendix F. Although, in principle, all drag contributions discussed in that appendix are slightly altered when the flaps are deflected and the operational conditions are changed, we shall discuss only the most pertinent corrections required.

As illustrated by Fig. G-18, the approach adopted here is artificial in the sense that, for the large values of $c_{L}$ to be achieved with deflected flaps, the basic arag coefficient of the aircraft (flaps up) is assumed to be extrapolated beyond

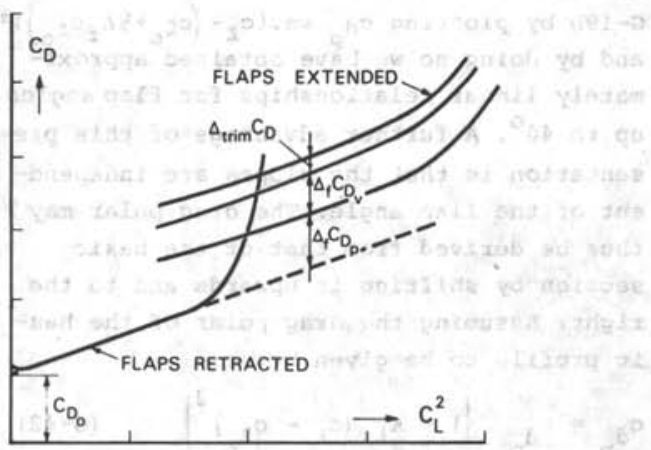

Fig. G-18. Drag polars with trailing-edge flaps retracted and extended

maximum lift values.

G-4.1. Profile drag

a. Drag of airfoil sections.

Fig. G-19a shows an example of drag polars
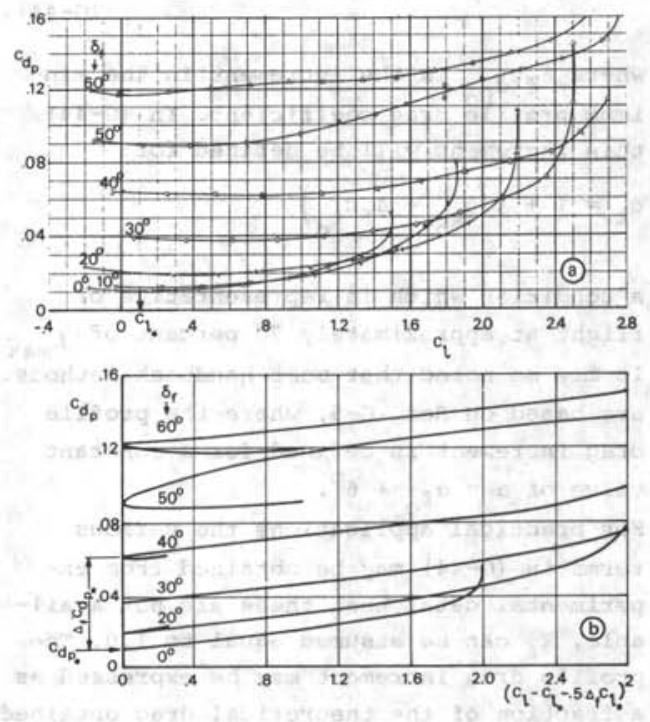

Fig. G-19. Section drag polars of NACA 23012 airfoil with a single slotted flap (Ref. G-33)

for several deflection angles of an airfoil section with a single slotted flap. These polars have been converted in Fig. 
G-19b by plotting $c_{d_{p}}$ vs. $\left\{c_{\ell}-\left(c_{\ell_{0}}+\frac{1}{2} \Delta_{f} c_{\ell_{0}}\right)\right\}^{2}$ and by doing so we have obtained approximately linear relationships for flap angles up to $40^{\circ}$. A further advantage of this presentation is that the slopes are independent of the flap angle. The drag polar may thus be derived from that of the basic section by shifting it upwards and to the right. Assuming the drag polar of the basic profile to be given by:

$c_{a_{p}}=c_{d_{p_{0}}}\left\{1+k_{\ell}\left(c_{\ell}-c_{l_{0}}\right)^{2}\right\}$

we obtain the polar with deflected flap:

$c_{d_{p}}=c_{d_{p_{0}}}+\Delta_{f} c_{d_{p_{0}}}+c_{d_{p_{0}}} k_{\ell}\left\{c_{\ell}-\left(c_{\ell_{0}}+\frac{1}{2} \Delta_{f} c_{\ell}\right)\right\}^{2}$

Consequently, the drag increment due to flap deflection is:

$\Delta_{f} c_{d_{p}}=\Delta_{f} c_{d_{p}}-c_{d_{p_{0}}} k_{\ell} \Delta_{f} c_{l_{0}}\left\{c_{\ell}-\left(c_{l_{0}}^{\left.+\frac{1}{4} \Delta_{f} c_{\ell}\right)}\right\}\right.$

where $\Delta_{f} c^{d}$ is the increment in the minimum profile drag coefficient. In (G-44) this increment will be defined for:

$c_{\ell}=1+\frac{1}{2}\left(c_{l_{0}}+\Delta_{f} c_{l_{0}}\right)$

a condition which is representative of

flight at approximately 70 percent of $\mathrm{C}_{\mathrm{L}_{\max }}$ It may be noted that most handbook methods are based on Ref. G-5, where the profile drag increment is defined for a constant value of $\alpha=\alpha_{\ell_{0}}+6^{\circ}$.

For practical applications the various terms in (G-44) may be obtained from experimental data. When these are not available, $k_{\ell}$ can be assumed equal to 1.0 . The profile drag increment may be expressed as a fraction of the theoretical drag obtained, assuming that $\Delta_{f} c_{l}$ acts normal to the flap chord:

$\Delta_{f} c_{p_{0}}=$ constant $\frac{c_{f}}{c} \Delta_{f} c_{\ell} \sin \delta_{f}$

Taking $\Delta_{f} c_{\ell}$ in accordance with Glauert's linear thin airfoil theory and allowing for chord extension, a generalized result 1s:

$\Delta_{f} c_{d_{p_{0}}}=k_{d} c_{\ell_{\alpha}} \alpha_{\delta} \cdot \frac{c_{f}}{c} \delta_{f} \sin \delta_{f}+c_{d_{p_{0}}}\left(\frac{c^{\prime}}{c}-1\right)$

The factor $\alpha_{\delta}$ ' is based on the ratio $c_{f} / c^{\prime}$, as explained in section $G-2,2$. The factor $k_{d}$ is plotted in Fig. G-20 for various flap

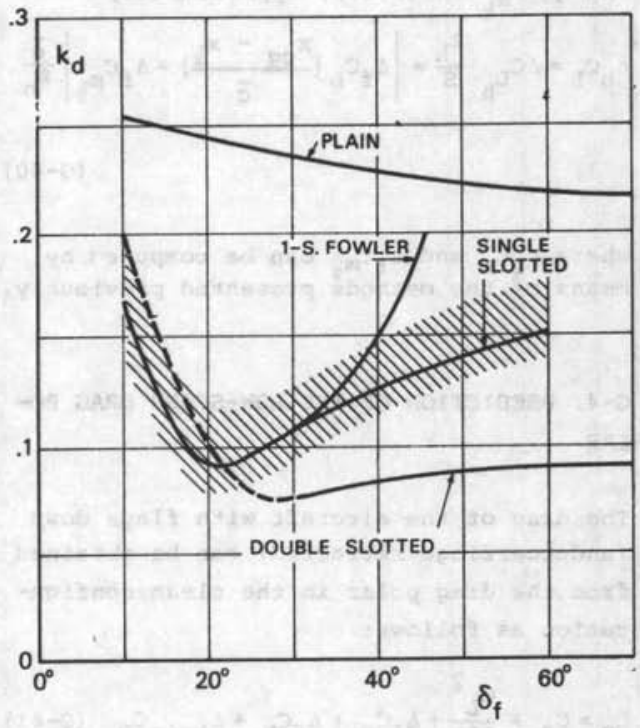

Note: the shaded area indicates a typical variation due to shroud and slot geometry differences for single slotted flaps.

Fig. $G-20$. The factor $k_{d}$ for determining the increment in profile drag caused by flap deflection

configurations. This figure shows that for plain flaps the drag increment is of the order of 20 to 25 percent of the "theoretical" value; for single and double slotted flaps these figures are 10 to 15 and 8 to 10 percent, respectively. The relatively high value of $k_{d}$ for slotted flaps at small deflection angles is caused by unfavorable slot flow and is affected to a large extent by the shape of the shroud.

Split flaps do not lend themselves to this approach. The following relationship has 
been derived from systematic measurements in Ref. G-34:

$\Delta_{f} c_{p_{0}}=.55 \frac{c_{f}}{c}\left\{\frac{c_{f} / c}{(t / c)^{3 / 2}}\right\}^{2 / 9} F(\delta) \quad(G-47)$

where $F(\delta)$ is given in Fig. G-21. Alterna-

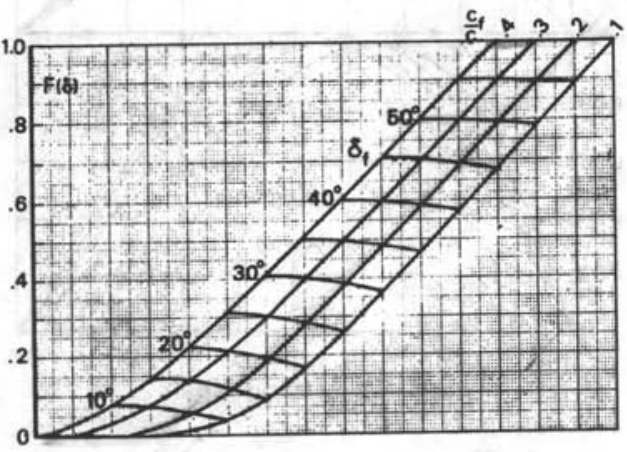

Fig. G-21. Profile drag function $k_{\delta}$ for split flaps (derived from Ref. G-34)

tively, the generalized and more detailed method in Ref. G-71 may be used.

b. Wing profile drag increment.

The profile drag increment of the wing due to flap deflection is obtained from:

$\Delta_{f} c_{D_{p}}=K_{2-3} \frac{s_{w f}}{s} \Delta_{f} c_{d_{p_{0}}} \cos \Lambda_{\frac{l}{2}}-k_{\ell} c_{d_{p_{0}}} \Delta_{f} c_{L_{0}} \times$

$\left\{c_{L}-\left(c_{L_{0}}+\frac{1}{4} \Delta_{f} c_{L_{0}}\right)\right\}$

where $\Delta_{f} c_{\mathrm{d}_{\mathrm{p}}}$ is the two-dimensional drag increment, given by (G-46) or (G-47), and $\mathrm{K}_{2-3}$ is a correction factor allowing for wing/fuselage interference, sweep effects, suspension effects, etc. A figure of $\mathrm{K}_{2-3}=$ 1.15 is proposed, based on the case reported in Ref. G-24.

\section{G-4.2. Vortex-induced drag}

The change in vortex-induced drag is caused by a change in the spanwise lift distribution (see Fig. G-14). The following expression is a synthesis of several existing methods in the literature (Refs. G-5, G-60 and $\mathrm{G}-65$ ) :

$\Delta_{f} C_{D_{v}}=(w+z)\left(\Delta_{f} c_{\ell}\right)^{2}+v C_{L} \Delta_{f} c_{\ell}$

The term $w\left(\Delta_{f} c_{l}\right)^{2}$ represents the induced drag increment due to flaps extending to the wing centerline, for a wing with elliptic loading when the flaps are retracted. The variation of $w$ with wing geometry is shown in Fig. G-22b. The last term is a correction for the nonellipticity of the spanwise lift distribution of the basic wing. The variation of $v$ with wing geometry is shown in Fig. G-22a.

It will be noted that for certain taper ratios the $v$ and $w$ factors are of opposite sign and their contributions counteract each other. In fact, for nontapered wings the elliptic loading may be approached when the flaps are deflected and the induced drag increment may be small or even negative. The term $z\left(\Delta_{f} c_{l}\right)^{2}$ in $(G-49)$ takes into account the effect of the flap cutout and lift carry-over by the fuselage (c.f. Section G-3.2.). The simple drag increment given by Hoerner on page 8-18 of Ref. G-10 is found to be in fair agreement with other data (Refs. G-5 and G-60) and can be converted into:

$z=\frac{.07}{1+\lambda}\left(1-k_{f f}\right)^{2} \frac{b_{f i}}{b} \quad\left(\right.$ for $\left.\frac{b_{f}}{b}<.2\right)(G-50)$

where $b_{f_{i}}$ is defined in Fig. G-13 and the choice of $\mathrm{K}_{\mathrm{ff}}$ was discussed in Section $\mathrm{G}-3.2$. An average value $\mathrm{K}_{\mathrm{ff}}=1 / 3$ appears to be acceptable, provided the flap edge is as close to the fuselage as possible.

G-4.3. Trim drag

On condition that the airplane vortex-induced drag is calculated by assuming that all lift is provided by the aircraft less tail, the trim drag can be taken into account as follows, ignoring a second-order term:

$\Delta_{\operatorname{trim}}\left(C_{D} S\right)=\left(\frac{C_{L_{h}}{ }^{2}}{\pi A_{h} e_{h}}+C_{L_{h}} \sin \varepsilon_{h}-2 \frac{C_{L}}{\pi A} C_{L_{h}}\right) s_{h}$ 


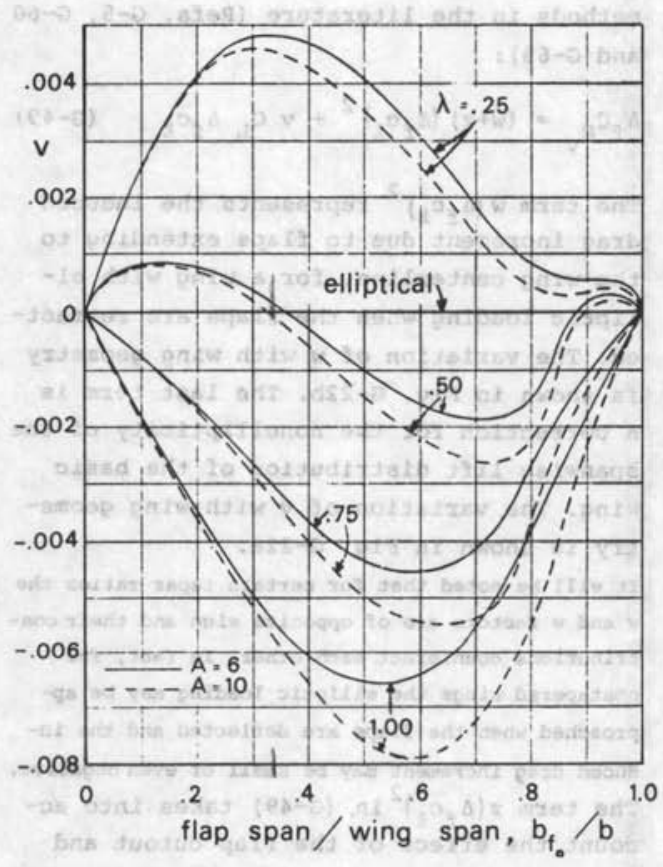

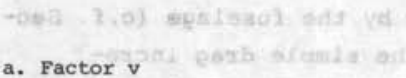

The first contribution to trim drag is the basic vortex-induced drag due to tailplane lift, based on free-stream conditions. The second contribution is the component of the tailplane lift in the direction of the free-stream as a consequence of the local downwash. The last term corrects the vortex-induced drag of the wing to account for the tailplane lift.

The tailplane lift coefficient is defined by the condition that the aircraft will be trimmed, while the downwash angle is given by:

$\varepsilon_{h}=\left(\frac{\partial \varepsilon_{h}}{\partial C_{L}}\right)_{\delta_{f}} C_{L}+\left(\frac{\partial \varepsilon_{h}}{\partial \delta_{f}}\right)_{C_{L}} \delta_{f}$

The estimation of the partial derivatives in this equation is usually very difficult in the preliminary design stage and for this reason we will neglect the net effect of the second and third contribution in.

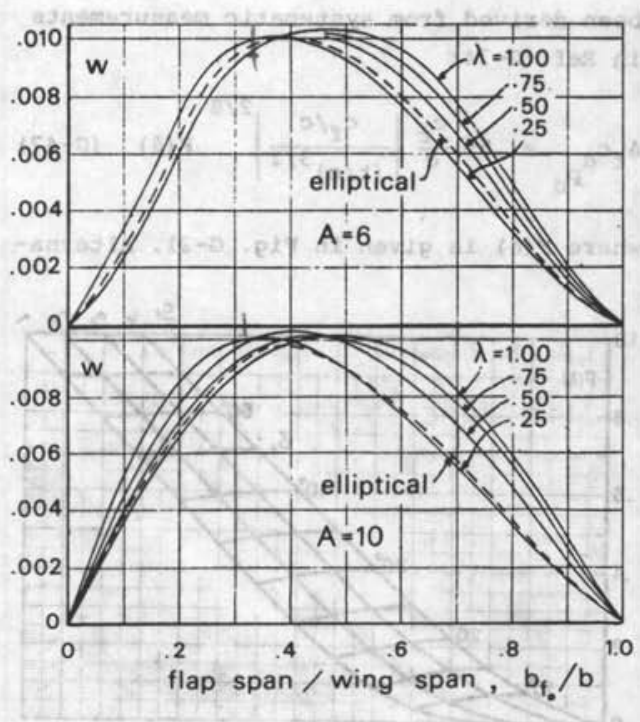

b. Factor w

Fig. G-22. Factors of induced drag of a wing with flaps (Ref. G-60)

$(\mathrm{G}-51)^{*}$. The only term remaining can be evolved with (G-40) into an approximate trim drag coefficient (based on S) relative to the basic polar:

$\Delta_{\text {trim }} C_{D}=\frac{\Delta_{f} c_{m_{\frac{2}{2}}}\left(\Delta_{f} c_{m_{\frac{l}{2}}}+2 c_{m_{a c}}\right)}{\pi A_{h} e_{h}\left(l_{h} / \bar{c}\right)^{2} s_{h} / s}$

where $\Delta_{f} C_{m_{2}}$ is defined by $(G-36)$ and $c_{m_{a c}}$ refers to the aircraft less tail with flaps up (Appendix E, Section E-7.2.). The oswald factor $e_{h}$ is approximated by: $e_{h}=1-\frac{.25}{\cos ^{2} A_{h}}$

and takes into account the profile drag increment due to elevator deflection.** The

* Note that for an elliptic lift distribution $\varepsilon_{h}$ at infinity behind the wing is equal to $2 C_{L} / \pi A$ and the two contributions cancel each other out.

${ }^{* *}$ Cf. Appendix F, Section F-3.6. 
simplified calculation procedure presented here may in some cases lead to considerable errors. A more detailed prediction is required as soon as more accurate data become available, particularly with respect to the downwash at the tail. 10 m nos

\section{G-5. LEADING-EDGE HIGH-LIFT DEVICES}

Leading-edge flaps and slats* level off the high peak suction pressures near the airfoil nose, thereby delaying leading edge separation to a higher angle of attack (Fig. G-23). Since leading-edge flaps do

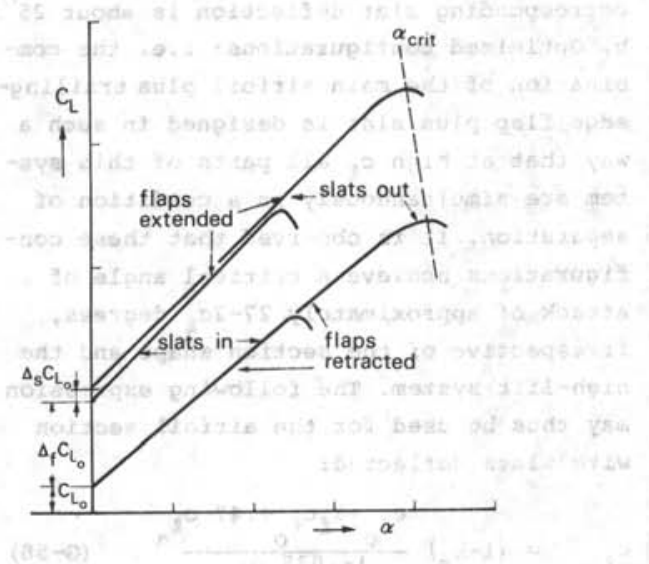

Fig. G-23. The lift curve with and without slats

not significantly alter the trailing edge flow about an unflapped section, they are most effective on thin airfoil sections. Airfoils with a deflected, high-efficiency trailing-edge flap will frequently exhibit a leading edge stall, even on relatively thick sections. Leading-edge slats or flaps suppress this stall, thereby increasing the stalling angle of attack and hence maximum lift. However, a slat wake may cause undesirable interference with the flow around trailing-edge flaps and the increment in $c_{\ell_{\max }}$ due to the slat with trailing-edge

* The terminology is explained in section 7.6.3. flaps deflected is typically some 15 percent lower than the $c_{l_{\max }}$ increment of the unflapped airfoil.

Reliable generalized methods for predicting the effect of leading-edge devices are not known to the author, with the exception of the (maximum) lift increment due to plain leading-edge flaps (Ref. G-19). The following data, indicating an order of magnitude of the effect of leading-edge devices on lift and drag, are the author's provisional conclusions from the very scanty literature on the subject. In view of the many factors involved in the aexodynamic design of leading-edge devices these data must only be considered indicative.

G-5,1. Sections with plain leading-edge tlaps

The leading-edge droop causes a lift loss at zero angle of attack which can be derived from Glauert's linear thin airfoil theory:

$\Delta_{s} c_{\ell_{0}}=-\frac{\theta_{s}-\sin \theta_{s}}{\pi} \delta_{s} c_{\ell_{\alpha}}$

where

$\theta_{\mathrm{s}}=\cos ^{-1}\left(1-2 \frac{\mathrm{c}_{\mathrm{S}}}{\mathrm{c}}\right)$

The increment in $c_{l_{\max }}$ may be estimated on the assumption that this increment is entirely due to the change in the ideal angle of attack. The ideal angle of attack is the angle at which the flow comes smoothly onto the airfoil, causing no singularity at the nose. Using the data from Ref. G-19, we may approximate the result by:

$\frac{\Delta \alpha_{\text {crit }}}{\delta_{\mathrm{s}}}=.58 \mathrm{~V} \frac{\overline{\mathrm{c}}}{\mathrm{c}}$

Experimental data are in fair agreement with this expression for $\delta_{\mathbf{S}}$ up to 20 to 25 degrees. Larger deflection angles cause only a slight increase in $\alpha_{\text {crit }}$ up to $\delta_{s}=$ 30 degrees, further deflection resulting in 
a decreasing $\alpha_{\text {crit }}$.

Leading-edge flap deflection has no effect on $c^{\ell_{\alpha}}$ ' provided the angle of attack is positive and the flap is deflected not much more than 20 degrees. Pronounced nonlinearities in the lift curve will be observed for negative and small positive angles of attack when the flap is deflected more than 25 degrees.

\section{G-5.2. Sections with slats and Krueger flaps}

As in the case of the plain leading-edge flap, extension of a slat results in a slight decrement in lift at zero angle of attack, caused by the nose droop. However, the slat increases the effective chord length and this causes a lift increment when $c_{\ell}$ is based on the original chord. Both effects are generally of the same order of magnitude and it is therefore reasonable to assume that $\Delta_{s} c_{\ell_{0}}=0$, while the lift gradient is not affected to any considerable degree by slat deflection. The estimation of $c_{l_{\max }}$ with a deflected slat is complicated by a number of factors:

1. For a given configuration and deflection of the trailing-edge flap system, the slat will be more effective in terms of $\Delta_{s} c_{\ell_{\max }}$ on an airfoil with a sharp nose than on a well-rounded airfoil nose. For an optimized slat configuration the stall may be of the trailing-edge type whereas without a slat it is of the leading-edge type.

2. The slat position relative to the airfoil (slat deflection and gap between the airfoil and the slat) has a very pronounced effect on the lift and profile drag increment. An elucidating discussion can be found in Ref. G-29.

3. The design of the trailing-edge flap system and the slat should be matched in order to obtain the highest performance. 4. Compressibility effects may set an upper limit to the performance of complex high-lift systems. In fact, Callaghan suggests in Ref. G-31 that the prediction of maximum lift increment due to slats should be based on the condition that sonic flow sets a limit to the pressure peaks.

Refs. G-27 and G-29 demonstrate that the details of a slat design optimization scheme are of paramount importance: for a particular configuration $\Delta_{s} c_{\ell_{\max }}$ due to the slat may vary between .5 and 1.2 . We therefore propose to differentiate between simple (nonoptimized) high-lift systems and fully optimized configurations.

a. Simple slat configurations: the maximum lift increment is about $2.2 \sqrt{c_{s} / c}$ with trailing-edge flaps retracted and 1.9 $\sqrt{c_{s} / c}$ with trailing-edge flaps out. The corresponding slat deflection is about $25^{\circ}$. b. Optimized configurations: i.e. the combination of the main airfoil plus trailingedge flap plus slat is designed in such a way that at high $c_{\ell}$ all parts of this system are simultaneously in a condition of separation. It is observed that these configurations achieve a critical angle of attack of approximately $27-2 c_{\ell}$ degrees, irrespective of the section shape and the high-lift system. The following expression may thus be used for the airfoil section with slats deflected:

$$
c_{\ell_{\max }}=\left(1-k_{s}\right){\frac{c_{\ell_{0}}+\Delta_{f} c_{\ell}+.47 c_{\ell}}{1+.035 c_{\ell}}}_{\alpha}
$$

where $c_{\ell_{\alpha}}$ is in $\mathrm{rad}^{-1}$ and $k_{s}$ is a factor which takes into account the nonlinearity of the $11 \mathrm{ft}$ curve at high $11 \mathrm{ft}$. It is generally between .03 and .15 , with $k_{s}=.07$ as a good average. Factors $c_{\ell_{0}}$ and $c_{\ell_{\alpha}}$ in (G-58) are the lift at zero angle of attack and the $11 \mathrm{ft}$-curve slope for the airfoil with or without flaps deflected, to be obtained from experimental data or from Sections $\mathrm{G}-2,2$. and $\mathrm{G}-2,4$. of this appendix.

The maximum lift capability of Krueger flaps is very similar to that of slats. In view of the pitching moment behavior at large lift coefficients Krueger flaps are generally used inboard only on swept wings, while slats may be used both full span and 
outboard.

G-5.3. Wing lift with leading-edge devices

For plain leading-edge flaps the increment in the critical angle of attack given by (G-57) can be converted into a maximum lift increment:

$\Delta_{s} C_{L_{\max }}=.58 \sqrt{\frac{C_{S}}{c} \delta_{s}} c_{\ell} \frac{S_{W S}}{S} \cos ^{2} \Lambda_{\frac{l}{4}}$

where $S_{w s}$ is the projected wing area equipped with slats.

For full-span optimum slat configurations it was found that $(G-58)$ can be used in a slightly modified form:

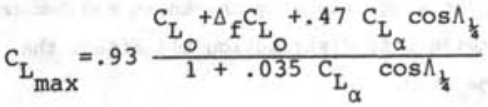

In this expression $\mathrm{C}_{\mathrm{L}_{\mathrm{O}}}$ is defined at $\alpha=0$ for the wing.

For part-span slats the $\mathrm{C}_{\mathrm{L}_{\max }}$ increment is given by:

$\Delta_{s} C_{L_{\max }}=\Delta_{s} c_{\ell} \frac{S_{\text {max }}}{S} \cos ^{2} \Lambda_{\frac{1}{4}}$

where $\Delta_{s} c_{\ell}$ is obtained by subtracting the maximum lift coefficient for the basic airfoil (slats in) from that with slats out. The sweep effect in $(G-61)$ is an approximation of the curve given in Ref. G-31.

\section{G-5.4. Drag due to leading-edge devices}

Leading-edge devices have little influence on the profile camber and when deflected they cause negligible aerodynamic twist. Their effect on the vortex-induced drag may therefore be neglected.

An example of profile drag polars of an airfoil with and without slat is depicted in Fig. G-24, which shows that a. the drag increment at 70 percent of $c_{\ell}{ }_{\max }$ is approximately 70 counts; b. when the polar with slat out is based

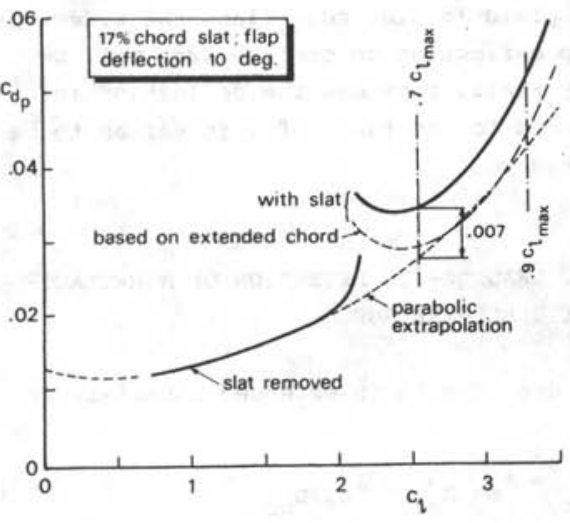

Fig. G-24. Drag polars of an airfoil with single slotted flap, with and without flap (Ref. G-53)

on the extended chord, there is virtually no drag increase between 70 and 90 percent of $c_{\ell_{\text {max }}}$;

c. the profile drag increases sharply at lower lift coefficients, probably due to flow separation on the lower surface of the slat.

The slat has a large influence on the airfoil pressure distribution, resulting in decreased flow velocities near the leading edge of the basic airfoil and consequently a profile drag reduction. On the other hand, the slat itself not only increases the wetted area, but it carries a relatively high load and the local friction. will be high, due to the high flow velocities. slat gap and deflection angle are major parameters contributing to these flow phenomena.

The following provisional rule of thumb may be suggested on the basis of Fig. G-24:

$\Delta_{s} C_{D_{p}}=\left(C_{D_{p}}\right)_{\text {basic }} \frac{s_{\text {ws }}}{s} \frac{c_{s}}{c} \cos \Lambda_{\frac{1}{2}}$

where $\left(C_{D_{p}}\right)_{\text {basic }}$ is the profile drag of the airfoil with slats and trailing edge flaps retracted. The reservation must be made that $(G-62)$ is valid only in the range of lift values where the slat position is optimum. 
For plain leading-edge flaps the effect of flap deflection on profile drag will be very small, provided the deflection is matched to the range of $11 \mathrm{ft}$ values to be considered.

G-6. DRAG DUE TO EXTENSION OF A RETRACTABLE UNDERCARRIAGE

The drag due to an extended undercarriage is:

$C_{D_{u c}}=\Delta_{m g} C_{D_{u c}}+\Delta_{n g} C_{D_{u c}}$

where $\Delta_{m g}$ and $\Delta_{n g}$ denote the contributions of the main and nose gear, respectively. The undercarriage drag is determined not only by its drag area, but also by the 10cal flow conditions. Consequently, the angle of attack (or lift coefficient) and the position of the high-lift devices have to be taken into account:

$C_{D_{u c}}=F_{u c}\left(\alpha, \delta_{f}\right)\left(C_{D_{\text {uc }}}\right)_{\text {basic }}$

The following methods for estimating the basic undercarriage drag coefficient are proposed.

METHOD 1:

$$
\left(\mathrm{C}_{\mathrm{D}_{\mathrm{uc}}}\right)_{\text {basic }}=\frac{1.5 \sum \mathrm{S}_{\mathrm{ft}}+.75 \sum \mathrm{S}_{\mathrm{rt}}}{\mathrm{S}}(\mathrm{G}-65)
$$

where in the case of non-bogie type gears $\Sigma \mathrm{S}_{\mathrm{ft}}$ denotes the total frontal area of all tires, and in the case of bogie-type gears $\Sigma \mathrm{S}_{\mathrm{ft}}$ is the frontal area of the front tires only. $\Sigma s_{\mathrm{rt}}$ is the total frontal area of the rear tires in the case of bogie-type gears and is equal to zero for non-bogie type gears.

Equation G-65 represents the total basic undercarriage drag coefficient and not just the tire drag coefficient.

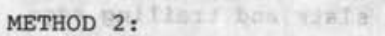

When the tire size and the undercarriage configuration are not (yet) known, a purely statistical expression can be used:
$\left(\mathrm{C}_{\mathrm{D}_{\text {uc }}}\right)_{\text {basic }}=$ constant $\frac{\mathrm{W}_{\text {to }} \cdot .785}{\mathrm{~S}}$

where the constant is equal to $4.05 \times 10^{-3}$ when $w_{\text {to }}$ is in $\mathrm{lb}$ and $\mathrm{S}$ in sq. $\mathrm{ft}$, or $7 \times 10^{-4}$ when $\mathrm{w}_{\text {to }}$ is in $\mathrm{kg}$ and $\mathrm{s}$ in $\mathrm{m}^{2}$.

Factor $F_{\text {uc }}\left(\alpha, \delta_{f}\right)$ depends on various factors.

a. The nose gear drag will not be affected by $\alpha$ or $\delta_{f}$ to any appreciable extent, nor will it affect the airframe drag.

b. The main gear is placed below the wing and will thus be sensitive to the local flow conditions. c. For a given aircraft general arrangement and geometry, the induced effects of wing and flap lift reduce the undercarriage arag.

d. For given flight conditions (incidence and flap angle), the local position of the undercarriage relative to the wing, the wing thickness and camber, and the spanwise $11 \mathrm{ft}$ distribution all affect the undercarriage drag.

e. The undercarriage will have an effect on wing lift and hence on the vortex-induced drag. In the clean configuration the circulation will be slightly increased by the blocking effect of the airflow due to the main gear. When the trailing-edge flaps are deflected, the wake generated by the undercarriage may hit the flaps and in that case the lift will be reduced.

f. Part of the undercarriage of propeller aircraft will be in the slipstream and experiences an effective drag increment, which will depend upon the flight condition.

A theoretical analysis of the function $F_{\text {uc }}\left(\alpha, \delta_{f}\right)$ cannot (and need not) be made in the preliminary design stage. Instead, a reasonable approximation may be used:

$F_{u c}\left(\alpha, \delta_{f}\right)=\left\{1-\left..04 \frac{C_{L}+\Delta_{f} C_{L}\left(1.5 S / S_{w f}-1\right)}{u_{u c} / c_{g}}\right|_{(G-67} ^{2}\right.$

where $\ell_{u c}$ is the length of the main gear legs, i.e. the distance between the local wing chord and the wheel axis. Equation G-67 is based on Helmholtz' theorem, applied to the lift of the basic wing section and the flap separately. 
It should be noted that retraction of the undercarriage during takeoff will initially cause the arag to increase some 20 to 30 percent, mainly due to the extension of the wheelbay doors and the drag of the wheelbays.

G-7. GROUND EFFECTS

The basic nature of the changes in the flow field due to ground effect can be analyzed with simple mathematical models, using the Prandtl lifting-line concept. Wieselsberger has derived some of the results given below (Ref. G-74) in a slightly modified form, which are still satisfactory for present-day conventional wing shapes, provided the distance to the ground is not too small and the lift coefficient is not too high. Wieselsberger's results can be applied readily in preliminary design to wings with flaps retracted, but unfortunately the results are not very good for the condition with flaps deflected at high lift. Some modifications were found to be necessary.

G-7.1. Ground effect on lift

a. In two-dimensional analysis the image vortex of the airfoil below the ground plane induces a velocity distribution at the airfoil in the opposite direction to the free-stream velocity, thus reducing the lift. In addition, the camber and incidence of the airfoil are effectively increased. For low lift coefficients and moderate wing height these opposing effects are approximately equal in magnitude, and the ground effect on lift can be calculated with sufficient accuracy by ignoring them. At low wing heights and high lift coefficients, however, particularly with flaps deflected, the induced horizontal velocity is dominant, causing a decrease in airfoil lift from its freestream value.

When the airfoil section is sufficiently high above the ground, it can be replaced by a single vortex and the velocity in- duced by the image vortex can be derived from Helmholtz' law:

$\frac{\Delta V}{V_{\infty}}=-\frac{c_{\ell}}{8 \pi h / c}$

where $\mathrm{h}$ is the height of the airfoil a.c. above the ground plane. Provided $\Delta \mathrm{V} / \mathrm{V}_{\infty} \ll<1$, the reduction in effective velocity must be regained by increasing the angle of attack in order to maintain $c_{\ell}$ constant:

$\Delta \alpha=\frac{\mathrm{c}_{\ell}{ }^{2}}{4 \pi \mathrm{h} / \mathrm{c}_{\ell_{\alpha}}}$

The effective increase in camber is proportional to $\Delta \mathrm{V} / \mathrm{V}_{\infty}$ and the chord length, and inversely proportional to the a.c. height. Assuming that the average upwash is equal to the upwash at the mid-chord point, we have for constant $c_{\ell}$ :

$\Delta \alpha=-.25 \frac{\Delta V}{V_{\infty}} \frac{c}{2 h}=-\frac{c_{\ell}}{64 \pi(h / c)^{2}}$

For finite wings the effects described above are less due to the finite length of the bound vortex. The correction factor $B$ proposed in Ref. G-95 takes this into account. Thus we find for the total effect due to the bound vortex:

$\Delta_{1} \alpha=\beta \frac{C_{L}}{4 \pi h / c_{g}}\left(\frac{C_{L}}{C_{L_{\alpha}}}-\frac{1}{16 h / c_{g}}\right)$

For several heights of the trailing edge above the ground, $\Delta_{1} \alpha$ is shown in Fig. $\mathrm{G}-25 \mathrm{a}$, and $\beta$ is given in Fig. G-26.

b. The induced upwash due to the images of the trailing vortices was first analyzed by wieselsberger. It is usually expressed as a reduction of the angle of attack required to achieve a given $C_{L}$ :

$\Delta_{2} \alpha=-\sigma \alpha_{1}$

According to the classical lifting line theory for high aspect ratio straight wings with elliptic lift distribution:

$\alpha_{i}=\frac{C_{L}}{\pi A}$ 


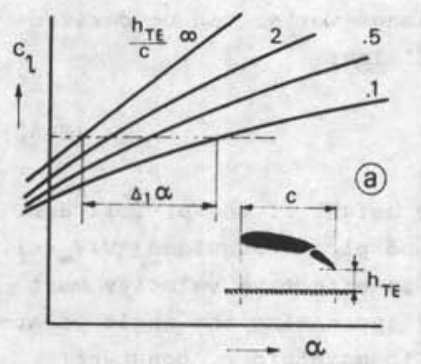

a. Airfoil section with flap; effect of trailing edge height on the lift curve

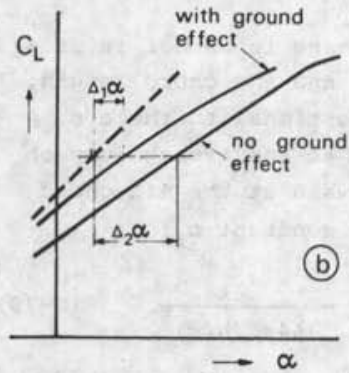

b. Ground effect on the lift curve of a wing

Fig. G-25. Ground effect on airfoil lift

and hence

$\Delta_{2}^{\alpha}=-\frac{\sigma C_{L}}{\pi A}$

A more generally valid expression for swept wings of arbitrary aspect ratio is:

$\Delta_{2} \alpha=-\sigma c_{L}\left(\frac{1}{C_{L}}-\frac{1}{c_{\ell_{\alpha}}}\right)$

The ground effect factor $\sigma$ can be obtained from Fig. G-26.

The combined effects of $\Delta_{1} \alpha$ en $\Delta_{2} \alpha$ on the iift-curve of a wing are shown in Fig. $25 \mathrm{~b}$.

c. The image vortices of the wing trailing vortex system induce an upwash at the horizontal tailplane. For a given angle of attack and tailplane setting the associated lift increment is:

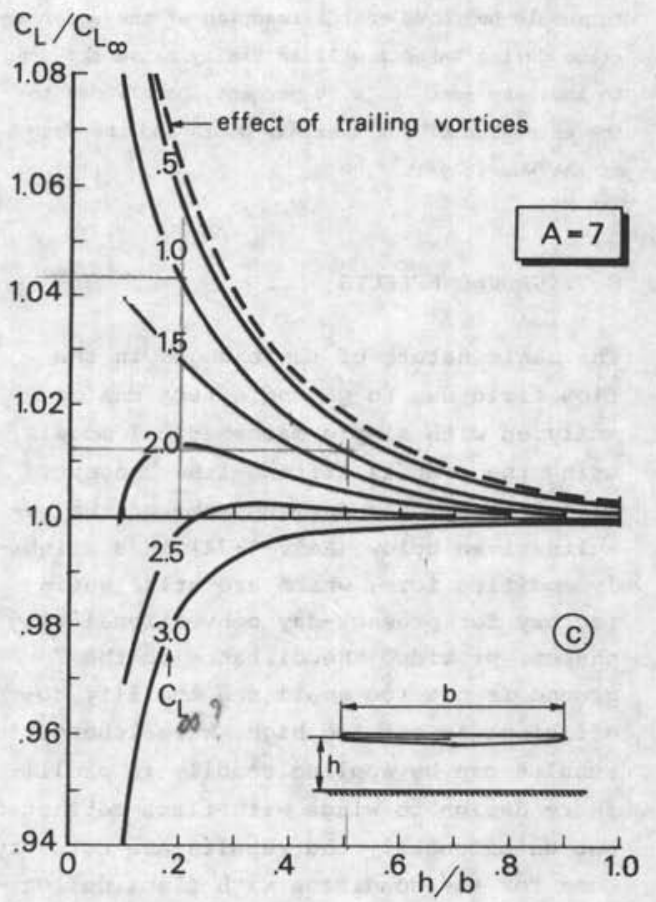

c. Effect of wing height and lift coefficient on ground effect

$\frac{\Delta C_{L}}{C_{L}}=\frac{\Delta \varepsilon_{h}}{\varepsilon_{h}} \frac{d \varepsilon_{h}}{d C_{L}} C_{L_{h}} \frac{s_{h} q_{h}}{s q}$

The position of the tailplane relative to the wing is of paramount importance. Methods for computing $\Delta \varepsilon_{h} / \varepsilon_{h}$ and $d \varepsilon_{h} / d C_{L}$ can be found in Refs. G-19 and G-95.

The induced tailplane lift must be trimmed out by elevator deflection. Moreover, ground effect causes a shift in the wing center of pressure and this effect must also be counteracted by elevator deflection. A secondary effect is the induced downwash or upwash at the horizontal tail due to the image vortices of the tailplane itself.

The various effects of ground proximity on tailplane lift are difficult to calculate and due to their relatively small magni- 
tude they are frequently neglected.

d. The pressure distribution around an airfoil changes considerably when it is placed near the yround, in particular when effective trailing-edge flaps are deflected. A change occurs in the spanwise load distribution, leading to an increased root-stalling tendency on swept wings. The induced adverse pressure gradient at the leading edge may result in early separation, while blocking or even reversal of the flow can occasionally be observed below the wing. As a rule a reduction in $\mathrm{C}_{\mathrm{L}_{\max }}$ will be observed. This effect must not be ignored, particularly for STOL configurations (Ref. G-96), and an assessment by means of wind tunnel experiments must be made in the early stages of aerodynamic development. The effect has an important bearing on the minimum unstick speed $V_{M U}$ (see Appendix K, Section K-2).

The total effect on lift may be found by adding the contributions discussed in a. and b. above. Defining the lift coefficient at a given incidence out of ground effect as $\mathrm{C}_{\mathrm{L}_{\infty}}$, and substituting the expression for $\mathrm{C}_{\mathrm{L}_{\alpha}}$ given in Section $\mathrm{E}-4.1$. of $\mathrm{Ap}-$ pendix $\mathrm{E}$, we find the following approximation for the lift in ground effect at the same angle of attack:

$$
\begin{gathered}
\frac{C_{L}}{C_{L_{\infty}}}=1+\sigma-\frac{\sigma A \cos \Lambda_{L_{2}}}{2 \cos \Lambda_{L_{2}}+\sqrt{A^{2}+\left(2 \cos \Lambda_{L_{2}}\right)^{2}}}-\frac{B}{4 \pi / c_{g}} \times \\
\left(C_{L_{\infty}}-\frac{C_{L_{\alpha}}}{16 h / c_{g}}\right)
\end{gathered}
$$

This expression has been plotted in Fig. G-25c for a straight aspect ratio 7 wing, as a function of wing height for several lift coefficients. The figure shows that for lift coefficients up to two the effect of the induced upwash and camber dominate, while for higher values the effective lift is reduced due to the decrement in effective flow veiocity.

G-7.2. Ground effect on drag

The aerodynamic phenomena referred to in the previous section result in the following expression for the vortex-induced drag in ground effect of the wing:

$$
C_{D_{V}}=\frac{1-\sigma}{1-\frac{\beta C_{L}}{4 \pi h / c_{g}}}\left(C_{D_{v}}\right)_{\infty}
$$

where $\left(C_{D_{v}}\right)_{\infty}$ denotes the vortex-induced drag in the absence of ground effect.

There is also a reduction in the profile drag caused by the reduced effective flow velocity. Assuming that only the wing profile drag is affected, the following expression can be derived for the total arag reduction due to ground effect:

$\Delta C_{D}=-\frac{4 \pi \sigma h / c_{g}-\beta C_{L}}{4 \pi h / C_{g}-\beta C_{L}}\left(C_{D_{v}}\right)_{\infty}-\frac{\beta C_{L}}{4 \pi h / c_{g}}\left(C_{D_{p}}\right)_{w}$

where the vortex-induced and profile drag coefficients of the wing (with or without flaps extended) may be obtained from sections $\mathrm{G}-4.1$. and $\mathrm{G}-4.2$., respectively. The functions $\sigma$ and $\beta$ are plotted in Fig. G-26. The effect of ground proximity on trim drag can be calculated in principle, but the amount of work involved is probably not justified in view of the unavoidable inaccuracy of the calculation.

\section{G-8. DRAG DUE TO ENGINE FAILURE}

The drag increment in steady flight following engine failure is composed of engine windmilling drag, propeller drag, and drag due to the asymmetric flight condition:

$\Delta C_{D}=\Delta C_{D_{\text {ewm }}}+\Delta C_{D_{\text {prop }}}+\Delta C_{D_{\text {as }}}$

\section{G-8.1. Engine windmilling drag}

The drag of a windmilling gas-turbine engine is composed of external drag due to spillage of the inlet and internal drag associated with pressure losses in the flow through the windmilling engine. Very little is known about external arag; 


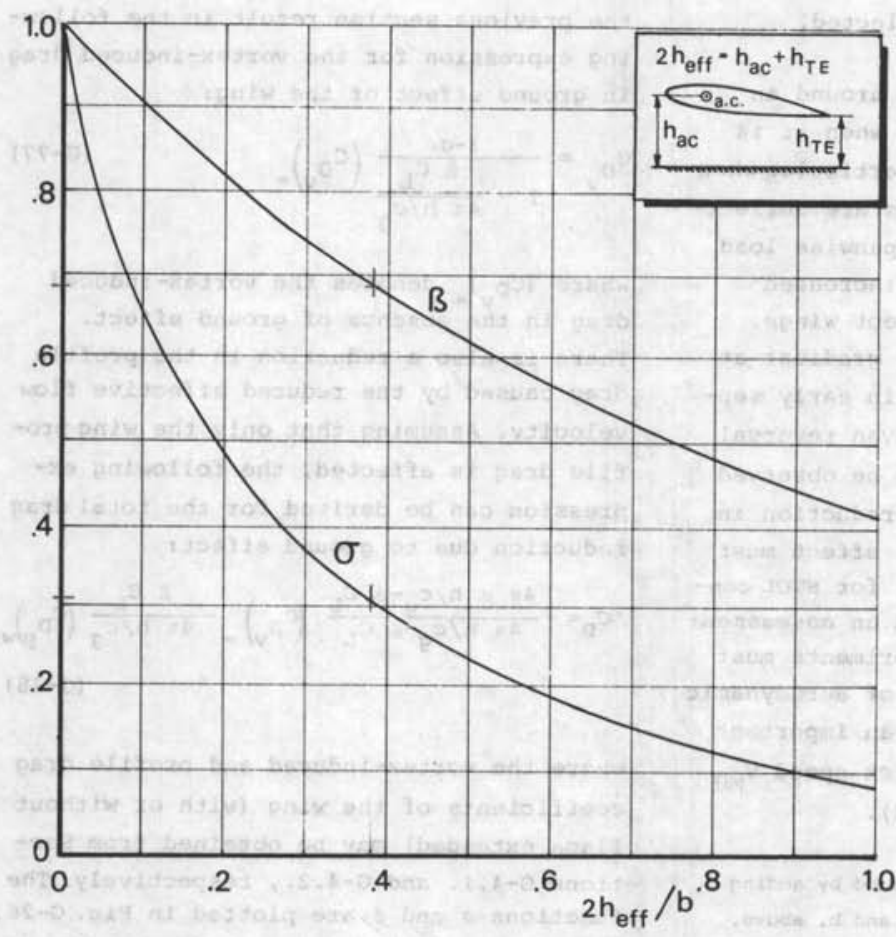

\section{NOTE}

$\sigma=\exp \left\{-2.48\left(2 h_{\text {eff }} / b\right)^{.768}\right\}$

$\beta=\sqrt{1+\left(2 h_{e f f} / b\right)^{2}-2 h_{e f f} / b}$

Fig. G-26. The ground effect functions $\sigma$ and $\beta$ (Ref. G-95)

a reasonable figure is probably:

$C_{D} S=.1 \times \frac{\pi}{4} D_{i}^{2}$

where $D_{i}$ is the engine inlet diameter. The internal drag can be obtained from the momentum theorem:

$\mathrm{D}=\dot{\mathrm{m}}\left(\mathrm{V}-\mathrm{V}_{\mathrm{N}}\right)$

where $v_{N}$ is the mean flow velocity in the nozzle exit and $\dot{m}$ the windmilling mass

flow. Equation G-81 may be further expanded by assuming that the nozzle exit temperature is equal to the static temperature plus 80 percent of the temperature rise due to the stagnation effect. Taking the static pressure of the exit flow equal to the ambient pressure, we obtain for the total drag area due to a windmilling engine:

$\Delta\left(C_{D} S\right)_{W m}=.0785 D_{i}^{2}+\frac{2}{1+.16 M^{2}} A_{N} \frac{V_{N}}{V}\left(1-\frac{V_{N}}{V}\right)$

$(G-82)$
Typical values for $\mathrm{V}_{\mathrm{N}} / \mathrm{V}$ are: straight turbojet and turboprop engines: .25

low bypass ratio engines, mixed flow:

.42

primary airflow of high bypass engines: .12

fan airflow of high bypass engines:

.92

For bypass engines with separate nozzles for the hot and cold flow, the contributions of both flows to the internal drag must be added.

\section{G-8.2. Propeller drag}

A detailed procedure for calculating propeller windmilling drag can be found in Ref. G-100. For a feathered, stopped propeller the following approximation can be derived from Ref. G-98:
$\Delta\left(C_{D}\right)_{\text {prop }}=.00125 \mathrm{~B}_{\mathrm{p}} \mathrm{D}_{\mathrm{p}}{ }^{2}$ 
s

where $B_{p}$ is the number of blades per propeller and $D_{p}$ the propeller diameter.

G-8.3. Drag due to the asymmetric flight condition

Engine failure causes yawing and rolling moments when, as is usual, the inoperative engine is located outside the xOz plane of the aircraft. These moments must be trimmed out by rudder and aileron/ spoiler deflection. The following drag contributions result from this asymmetric condition:

a. induced drag due to the normal force on the vertical tailplane,

b. profile drag increment due to rudảer and aileron (spoiler) deflection,

c. airframe drag due to sideslip,

d. vortex-induced drag due to the change in wing lift distribution, associated with sideslip and the asymmetric slipstream behind propellers, and

e. a change in the profile drag of the parts of the airplane immersed in the propeller slipstream.

The pilot may choose the angle of bank or sideslip after engine failure, within certain limits.* The various drag components are depicted in Fig. 48 of Ref. G-31 as a function of the sideslip angle. It is generally found that the total asymmetric drag after failure of a starboard engine is minimum for a small negative slip angle, i.e. the airplane sideslips in the direction of the operative engine. However, there is very little drag increment in a flight without sideslip. In that condition the most important drag contribution is caused by the vertical tailplane. The lift-induced drag of the vertical tailplane for zero slip is given by:

$\Delta\left(C_{D} s\right)=\frac{C_{y_{v}}^{2} s_{v}}{\pi A_{v} \text { eff }}+C_{y_{v}} s_{v} \sin \sigma_{v}$

where

${ }^{*}$ Cf. Section 9.6.1.
$c_{y_{v}}=\frac{\Delta T}{q_{v} S_{v}} \frac{y_{e}}{l_{v}}$

and $\mathrm{A}_{\mathrm{V}}$ eff is the effective (aerodynamic) aspect ratio of the vertical tailplane (cf. Fig. 2-26).

$\sigma_{v}$ is the mean sidewash angle at the vertical tailplane associated with the asymmetric downwash behind the wing due to the asymmetric lift distribution.

$\Delta T$ is the net thrust loss of the failed engine plus the engine windmilling and propeller drag.

$\mathrm{y}_{\mathrm{e}}$ is the yawing moment arm of the failed engine. Note that in the case of rearmounted engines this distance can be reduced to a certain extent by suitable choice of the direction of the engine exhaust flow (cf. Fig. 6-18).

The profile drag increment due to rudder deflection may be estimated by using the generalized data on plain flap effectiveness and drag increment presented in the relevant sections of this Appendix and Appendix E. The result is approximated by:

$$
\begin{gathered}
\Delta\left(C_{D} S\right)=\frac{2.3}{\pi} \sqrt{S_{r} S_{v}}\left(A_{v_{e f f}}\right)^{-4 / 3}\left(\cos \Lambda_{v}\right)^{1 / 3} \times \\
C_{Y_{v}} 2
\end{gathered}
$$

The drag area due to engine failure is found by adding $(G-84)$ and $(G-86)$ and substituting $(G-85)$. The result can be written in the following form:

$$
\begin{aligned}
& \Delta\left(C_{D} S\right)_{\text {as }}=K_{\text {as }}\left\{\frac{\text { thrust per engine }}{\mathrm{q}}+\Delta\left(C_{D} S\right)_{\text {wm }^{+}}\right. \\
& \left.\Delta\left(C_{D} S\right)_{\text {prop }}\right\}^{2}+K_{\sigma} \frac{\text { thrust per engine }}{W} \quad(G-87)
\end{aligned}
$$

where

$$
\begin{aligned}
& \mathrm{K}_{\mathrm{as}}=\left(\frac{\mathrm{Y}_{\mathrm{e}}}{l_{\mathrm{v}}}\right)^{2} \frac{1}{\mathrm{~S}_{\mathrm{v}}} \frac{1}{\pi \mathrm{A}_{\mathrm{v}_{\text {eff }}}}\left\{1+2 \cdot 3 \sqrt{\frac{\mathrm{S}_{\mathrm{r}}}{\mathrm{S}_{\mathrm{v}}}}\right. \\
& \left({ }^{A} v_{e f f} / \cos \Lambda_{v}\right)^{-1 / 3}
\end{aligned}
$$

$K_{\sigma}=\frac{y_{e}}{l_{v}} \frac{d \sigma_{v}}{d c_{L}} s$

For jet aircraft it is reasonable to as- 
sume $d \sigma_{v} / d c_{L}=0$, hence $K_{\sigma}=0$. For propeller aircraft the order of magnitude of $\mathrm{d} \sigma_{\mathrm{v}} / \mathrm{dC}_{\mathrm{L}}$ is such that the associated asymmetric drag accounts for a very large contribution. The author does not have at his disposal any data for estimating $d \sigma_{v} / d_{L^{\prime}}$, although Ref. G-101 presents a qualitative assessment of the effect. It is found that in some cases, particularly on high-wing aircraft, the effect of $\sigma_{\mathrm{v}}$ is equivalent to an increase of 100 to 200 percent in the yawing moment, resulting in a very large increase in asymmetric drag. It is therefore recommended that the expression presented above be substantiated at the earliest possible moment by appropriate windtunnel experiments.

\section{REFERENCES}

General 1iterature, high-lift devices

G-1. H.A. Wilson and L.K. Loftin: "Landing characteristics of high-speed wings". NACA Res. Mem. L8A28e, 1948.

G-2. R. Duddy: "High lift devices and their uses". J. Royal Aero. Soc., Oct. 1949, Vol. 53, pp. 859-900.

G-3. J.F. Cahi11: "Summary of section data on trailing-edge high-1ift devices". NACA TR 938, 1949.

G-4. F.E. Weick, L.E. Flanagan Jr. and н.H. Cherry: "An analytical investigation of effects of high-1ift flaps on takeoff of light airplanes". NACA TN 2404, 1951.

G-5. A.D. Young: "The aerodynamic characteristics of flaps". ARC R and M 2622, 1953.

G-6. A.D. Young: "Flaps for landing and takeoff". Chapter 14 of: "The principles of the control and stability of aircraft", edited by W.J. Duncan. Cambridge Aeronautical Series.

G-7. G.H. Lee: "High Maximum Lift". The aeroplane, October 30, 1953.

G-8. T.R.F. Nonweiler: "Flaps, slots and other high-1ift aids". Aircraft Eng., Sept. 1955.

G-9. G. Chester Furlong and J.G. Mcliugh: "A summary and analysis of the low-speed longitudinal characteristics of swept wings at high Reynolds number". NACA Report 1339, 1957.

G-10. S.F. Hoerner: "Fluid dynamic drag". Published by the author, 1958.

G-11. I.H. Abbott and A.E. von Doenhoff: "Theory of wing sections". Dover Publications Inc., New York.

G-12. J. Williams and S.F.J. Butler: "Aerodynamic aspects of boundary layer control for high lift at low speeds". AGARD Report $414,1963$.

G-13. W.H. Kuhlman: "The Douglas doubie-slotted flap". Part II of: "Boundary layer and flow control". Volume 1, edited by G.V. Lachmann, Pergamon Press Inc.

G-14. S.T. Harvey and D.A. Norton: "Development of the Model 727 Airplane high 1 ift system". Society of Automotive Engineers S 408, April 1964.

G-15. J.K. Wimpress: "Shortening the takeoff and landing distances of high speed aircraft". AGARD Report 501, June 1965.

G-16. B.W. McCormick: "Aerodynamics of V/STOL Flight". Chapter 6: "Unpowered flaps". Academic Press, New York/London, 1967.

G-17. R.D. Schaufele and A.W. Ebeling: "Aerodynamic design of the DC-9 wing and high-1ift system". SAE Paper No. 670846 .

G-18. J.в. Paterson: "Aerodynamic design features of the C-5A". Aircraft Eng., June 1968, pp. 8-15.

G-19. D.E. Hoak and J.W. Carlson: "USAF stability and control DATCoM". Douglas Aircraft Company, Rev. 1968.

G-20. G.J. Hancock: "Problems of aircraft behaviour at high angles of attack". AGARDograph 136 , April 1969. Appendices AI. 2 and AII.2.

G-21. J.C. Wimpenny: "The design and application of high-lift devices". Annals of the New York Academy of Sciences, Vol. 154, Art. 2, pp. 329-366.

G-22. J.K. Wimpress: "Aerodynamic technology applied to takeoff and landing". Annals of the New York Academy of Sciences, Vol. 154, Art. 2, pp. 962-981.

G-23. D.M. MCRae: "General description and comments on $\mathrm{C}_{\mathrm{L}_{\text {max }}}$ and stalling behaviour". J. Royal Aero. Soc. 
(534), Vo1. 73, June 1969, pp. 535-541. Also in AGARD Lecture Series No. 23.

G-24. D.N. Foster: "Some aspects of the RAE high-1ift research programme". J. Royal Aero. Soc. (534), Vol.

73, June 1969, pp. 541-546.

G-25. K.L. Sanders: "High-lift devices, a weight and performance trade-off methodology". SAWE Technical Paper No. 761, May 1963.

G-26. A.D. Hammond: "High-lift aerodynamics". Proceedings of Conference on vehicle technology for civil aviation, NASA SP-292, 1971, pp. 15-26.

G-27. F. Mavriplis: "Aerodynamic research on high-1ift systems". Canadian Aeronautics and Space Journal, May 1971, pp. 175-183.

G-28. J.A. Thain: "Reynolds number effects at low speeds on the maximum lift of two-dimensional aerofoil sections equipped with mechanical high lift devices". Quarterly Bulletin of the Div. of Mech. Eng. and the NAE, Canada, July 1-Sept. 30, 1973, pp. 1-24.

G-29. B.L.G. Ljungström: "Experimental high lift optimization of multiple element airfoils". AGARD-CP-143, April 1974.

G-30. R.J. Margason: "High-1ift aerodynamics - trends, trades and options". AGARD Conference on Takeoff and Landing, CP-160, Apri1 1974.

G-31. J.G. Callaghan: "Aerodynamic prediction methods for aircraft at low speeds with mechanical high lift aids", AGARD Lecture Series 67, May 1974.

G-32. W.McIntosh: "Prediction and analysis of the low speed stall characteristics of the Boeing 747 " AGARD Lecture Series 74, March 1975.

Experimental results, high-lift devices

G-33. C.J. Wenzinger and T.A. Harris: "Wind-tunnel, investigation of an NACA 23012 airfoil with various arrangements of slotted flaps". NACA TR 664, 1939.

G-34. C.J. Wenzinger and T.A. Harris: "Wind-tunnel investigation of NACA 23012,23021 and 23030 airfoils with various sizes of split flap". NACA TR No. 668, 1939.

G-35. C.J. Wenzinger and T.A. Harris: "Wind-tunnel investigation of an NACA 23021 airfoil with various arrangements of slotted flaps". NACA TR No. 677, 1939.

G-37. J.G. Lowry: "Wind-tunnel investigation of an NACA 23012 airfoil with several arrangements of slotted flaps with extended lips". NACA TN No. 808, May 1941.

G-38. I.H. Abbott, A.E. von Doenhoff and L.S. Stivers, Jr.: "Summary of airfoil data". NACA TR No. 824, 1945.

G-39. A.L. Braslow and L.K. Loftin: "Two-dimensional wind-tunnel investigation of an approximately 14percent-thick NACA 66-series-type airfoil section with a double-slotted flap". NACA TN 1110 , 1946.

G-40. G.M. McCormack and V.I. Stevens: "An investigation of the low-speed stability and control characteristics of swept-forward and swept-back wings in the Ames 40- by 80-foot wind tunne1". NACA RM No. A6K15, 1947.

G-41. F.F. Fullmer, Jr.: "Two-dimensional wind-tunnel investigation of the NACA $64-012$ airfoil equipped with two types of leading-edge flap". NACA TN, No. 1277, May 1947.

G-42. F.F. Fullmer, Jr.: "Two-dimensional wind-tunnel investigation of an NACA $64-009$ airfoil equipment with two types of leading-edge flap". NACA TN No. 1624.

G-43. J.F. Cahill: "Two-dimensional wind-tunnel investigation of four types of high-1ift flap on an NACA 65-210 Airfoil Section". NACA TN 1191, 1947.

G-44. J.F. Cahill and R.M. Racisz: "Wind-tunnel investigations of seven thin NACA airfoil sections to determine optimum double-slotted flap configurations". NACA TN 1545, 1948.

G-45. J.C. Sivells and S.H. Spooner: "Investigation in the Langley 19-foot pressure tunnel of two wings of NACA 65-210 and 64-210 airfoil sections with various type flaps". NACA TR No. 942, 1949.

G-46. R. Hills, R.E.W. Harland and R.H. Whitbey: "Wind tunnel tests on lateral control with high-1ift flaps made on the S24/37". ARC R and M No. 2452, 1950. 
G-47. н. Davies, J.E. Adamson and J. Seddon: "Wind tunnel tests on the Supermarine $5.24 / 37$, a high-wing monoplane with a variable-incidence wing". ARC R and M No. 2451, 1951.

G-48. J.A. Kelly and N.F. Hayter: "Lift and pitching moment at low speeds of the NACA $64 A 010$ airfoil section equipped with various combinations of a leading-edge slat, a leading-edge flap, split flap and double-slotted flap". NACA TN 3007, Sept. 1953.

G-49. H.O. Palme: "Summary of wind tunnel data for high-1ift devices on swept wings". SAAB TN 16 , 1953.

G-50. B.J. Gambucci: "Section characteristics of the NACA 0006 airfoil with leading-edge and trailingedge flaps". NACA TN No. 3797, Dec. 1956.

G-51. G.C. Furlong and J.C. McHugh: "A summary and analysis of the low speed longitudinal characteristics of swept wings at high Reynolds numbers". NACA TR 1339, 1957.

G-52. R.L. Naeseth and E.E. Davenport: "Investigation of double slotted flaps on a swept-wing transport model", NACA TN D-103, Oct. 1959.

G-53. D.N. Foster, H.P.A.H. Irwin and B.R. Williams: "The two-dimensional flow around a slotted flap". ARC $R$ and $M$ No. 3681, 1971.

G-54. W.H. Wentz, Jr.: "New airfoll sections for general aviation aircraft". SAE Paper No. 730876 .

G-55. G.J. Bingham and K.W. Nooman: "Low-speed aerodynamic characteristics of NACA 6716 and NACA 4416 airfolls with 35-percent-chord single-slotted flaps". NASA TM X-2623, May 1974.

G-56. C.J. Wenzinger and W.E. Gauvain: "Wind-tunnel investigation of an NACA airfoil with a slotted flap and three types of auxiliary flap". NACA TR No. $679,1939$.

Calculation of aerodynamic characteristics with high-lift devices

G-57. H. Glauert: "Theoretical relationships for an airfoil with hinged flap". ARC R and M No. $1095,1927$.

G-58. H.A. Pearson: "Span load distribution for tapered wings with partial-span flaps". NACA Report 585, 1936.

G-59. A. Silverstein and S. Katzoff: "Design charts for predicting downwash angles and wake characteristics behind plain and flapped wings". NACA Report 648.

G-60. H.A. Pearson and R.F. Anderson: "Calculation of the aerodynamic characteristics of tapered wings with partial-span flaps". NACA Report 665, 1939.

G-61. J. de Young: "Theoretical symmetric loading due to flap deflection for wings of arbitrary planform at subsonic speeds". NACA TR No. 1071, 1952.

G-62. D. Küchemann: "A simple method of calculating the span and chordwise loading on straight and swept wings of any given aspect ratio at subsonic speeds". ARC R and M 2935, 1952.

G-63. D. Fiecke: "Die Bestimmung der Flugzeugpolaren für Entwurfszwecke". I. Teil: Unterlagen. Deutsche Versuchsanstalt für Luftfahrt, Bericht Nr. 16, 1956.

G-64. J.G. Lawry and E.C. Polhamus: "A method for predicting lift increment due to flap deflection at low angles of attack". NACA TN 3911, 1957.

G-65. Anon.: Royal Aeronautical Society DATA Sheets, "Aerodynamics", Volume 2 "Wings" and Vol. 4 "Flaps".

G-66. J.A. Hay and w.J. Eggington: "An exact theory of a thin aerofoil with large flap deflection". J. Royal Aero. Soc. Vol. 60 (551), Nov. 1956, pp. 753-757.

G-67. A. Roshko: "Computation of the increment of maximum lift due to flaps". Douglas Aircraft Rep. SM$23626,1959$.

G-68. C.L. Bore and A.T. Boyd: "Estimation of maximum lift of swept wings at low Mach numbers". J. Royal Aero. Soc., April 1963.

G-69. J. Mc.Kie: "The estimation of the loading on swept wings with extending chord flaps at subsonic speeds". ARC CP No. $1110,1970$.

G-70. Anon,: "Lift coefficient increment at low speeds due to full-span split flaps". EsDU Item Number 74009, May 1974.

G-71. Anon.: "Low-speed drag coefficient increment at zero lift due to full-span split flaps". EsDU Item Number 74010, July 1974. 
G-72. Anon.: "Rate of change of lift coefficient with control deflection for full-span plain controls". ESDU Item Number 74011, July 1974 .

G-73. Anon.: "Conversion of lift coefficient increment due to flaps from full span to part span". EsDU Item Number 74012, July 1974.

Aerodynamic effects of ground proximity

G-74. C. Wieselsberger: "Uber den Flügelwiderstand in der Năhe des Bodens". z. Flugtechn. u. Motorluftschiffahrt 12, 1921, pp. 145-154 (English translation: NACA TM 77, 1922).

G-75. I. Tani, M. Taima and S. Simidu: "The effect cf ground on the aerodynamic characteristics of a monoplane wing". Rep. Aero. Res. Inst., Tokyo Imperial University No. 156, 1937 (Also: ARC R and M 3376,1938 ).

G-76. I. Tani, H. Itokawa and M. Taima: "Further studies of the ground effect". Rep. Aero. Res:" Inst., Tokyo Imperial University No. 158, 1937.

G-77. W.S. Brown: "Windtunnel corrections on ground effect". ARC R and M No. 1865, July 1938.

G-78. J. Wetmore and L. Turner: "Determination of ground effect from tests of a glider in towed flight". NACA Report No. 695, 1940.

G-79. S. Katzoff and H.N. Sweberg: "Ground effect on downwash angles and wake location". NACA Report 738, 1943.

G-80. Y. Hamal: "Modification des propriétés aérodynamiques d'une aile au voisinage đu sol". Centre National d'Etudes et de Recherches Aéronautiques (Bruxelles). Mémoire No. 4, 1935.

G-81. G. Chester Furlong and T.V. Bollech: "Effect of ground interference on the aerodynamic and flow characteristics of a $42^{\circ}$ sweptback wing at Reynolds numbers up to $6.8 \times 10^{6}$ ". NACA Report 1218 , 1955 .

G-82. R.M. Licher: "Increase in lift for two- and three-dimensional wings near the ground". Douglas, Santa Monica Division, Rep. SM-22615, 1956.

G-83. K. Gersten: "Uber die Berechnung des induzierten Geschwindigkeitsfeldes von Tragflügeln". Jahrbuch 1957 der WGL, pp. 172-190.

G-84. D. Kohlman: "A theoretical method of determining the ground effect on lift and pitching moment for wings of arbitrary planform". Boeing Document D3-1861, 1958.

G-85. F. Thomas: "Aerodynamische Eigenschaften von Pfeil- und Deltaflügeln in Bodennăhe". Jahrbuch 1958 der WGL, pp. 53-61.

G-86. K. Gersten: "Berechnung der aerodynamischen Beiwerte von Tragflügeln endlicher Spannweite in Bodennähe". Abhandlungen der Braunschweigischen Wissenschaftlichen Gesellschaft, Vol. XII, 1960, pp. 95-115.

G-87. J.A. Bagley: "The pressure distribution on two-dimensional wings near the ground". ARC R and M No. $3238,1960$.

G-88. M.P. Fink and J.L. Lastinger: "Aerodynamic characteristics of low-aspect-ratio wings in close proximity to the ground". NASA TN D-926, July 1961.

G-89. U. Ackermann: "Ein Doppeltraglinienverfahren zur Untersuchung des Flügels in Bodennăhe". Jahrbuch 1962 der WGLR, pp. 104-109.

G-90. P.R. Owen and H. Hogg: "Ground effect on downwash with slipstream". ARC R and M No. 2449 , 1952.

G-91. G.H. Saunders: "Aerodynamic characteristics of wings in ground proximity". Canadian Aeronautic and Space Journal, June 1965, pp. 185-192.

G-92. U. Ackermann: "Zur Berechnung der aerodynamischen Beiwerte und des Strömungsfeldes von Tragflügeln in Bodennăhe unter Berücksichtigung von Nichtlinearitäten". Doctor's Thesis, Technological University of Darmstadt, 1966.

G-93. D.J. Willig: "A method of computing Indicated Airspeed in ground effect". J. of Aircraft, Vol. 5 No. 4, July/August 1968 , pp. 412-414.

G-94. K. Gersten and J. von der Decken: "Aerodynamische Eigenschaften schlanker Flūgel in Bodennăhe". Jahrbuch 1966 der WGLR, Pp. 108-125. 
G-95. Anon.: "Low-speed longitudinal aerodynamic characteristics of aircraft in ground effect". ESDU Item Number 72023.

G-96. L.B. Gratzer and A.S. Mahal: "Ground effects in STOL Operations". AIAA Paper No. 71-579 (also: J. of Aircraft, Vol. 9 No. 3, March 1972, pp. 236-242).

G-97. D. Bummel: "Nichtlineare Tragflûgeltheorie in Bodennăhe". z. für Flugwiss., Dec. 1973, pp. 425-442.

Effects of engine failure on aerodynamic characteristics

G-98. н.K. Millicer: "The design study". Flight, August 17, 1951, pp. 201-205.

G-99. B. Wrigley: "Engine performance considerations for the large subsonic transport". Lecture given at the Von Karman Institute, Brussels, 23rd April 1969.

G-100. Anon.: "Approximate estimation of drag of wind-milling propellers". Royal Aero. Soc. Data Sheets "Performance", Sheet ED 1/1, April 1962.

G-101. J. Mannée: "Wind tunnel investigation of the influence of the aircraft configuration on the yawing and rolling moment of a twin-engined, propeller-driven aircraft with one engine inoperative". NLI Report A 1508 B, 1963. 


\section{Appendix H. Procedures for computing turbo-engine performance for aircraft project design work}

SUMMARY

This appendix contains a survey of analytical equations for computing the aerothermodynamic performance of gas turbine engines: straight turbojets, bypass engines (turbofans) and turboprop engines. Both the gas generator and the overall engine performance are dealt with, on the assumption that the engine operates under its design conditions. An approximate expression is also presented for relating the cruise and climb thrust (at altitude) for a turbojet engine to the takeoff thrust.

The method can be used to perform parametric design studies of gas turbine engines for the purpose of investigating the optimum aircraft/engine combination. For this reason the Turbine Entry Temperature, the overall Pressure Ratio and the Bypass Ratio can be dealt with as explicit variables. 


\section{NOMENCLATURE}

$A_{d}$ - fan inlet duct cross-sectional area

$A_{e} \quad$ - total exhaust nozzle area of fan and gas generator flows

$A_{1}$ - intake area of total engine flow

$a_{o 0}$ - speed of sound at sea level ISA

$C_{F}$ - skin friction coefficient for smooth duct flow, turbulent b. 1 .

$C_{P} \quad$ - specific fuel consumption of turboprop engine

$C_{T} \quad$ - specific fuel consumption of turbojet engine

cp - specific heat of engine air at constant pressure

- gas generator power function

- heating value of fuel

- duct pressure loss factor

- flight Mach number

- Mach number at engine inlet face

- design flight Mach number for turboprop engine

- mass flow per unit time (no index: total engine mass flow)

- engine h.p. compressor rpm

$\mathrm{P}_{\mathrm{br}}$

Peq

$\mathrm{P}_{\mathrm{gis}}$

- brake horsepower

- equivalent horsepower

- convertible energy generated by gasifier

p - static pressure

$\mathrm{p}_{\mathrm{L}} \quad$ - ambient pressure

$\mathrm{p}_{\mathrm{oo}}-\mathrm{p}_{\mathrm{o}}$ at sea level

$P_{t}$ - total (stagnation) pressure

$\mathrm{R}_{\mathrm{e}} \quad$ - Reynolds number

T - thrust; (static) temperature

To - ambient temperature

$\mathrm{T}_{\text {oo }} \quad \mathrm{T}_{\mathrm{o}}$ at sea level

$\mathrm{T}_{j} \quad$ - net jet thrust of turboprop engine

$\mathrm{T}_{t}$ - total (stagnation) temperature

$\mathrm{T}_{\text {to }}$ - takeoff thrust (static, sea level)

$v_{0}$ - flight speed

$\mathrm{v}$

- velocity of fully expanded exhaust flow

$\dot{\text { พ }}$

- weight flow per unit time of engine air

$\dot{\mathrm{W}}_{\mathrm{F}} \quad$ - fuel weight flow per unit time

$\gamma$ - ratio of specific heats (for ambient air: $\gamma=1.4$ )

$\delta$ - relative ambient pressure $(\delta=$ $\left.\mathrm{p}^{\circ} / \mathrm{p}_{\mathrm{oo}}\right)$
- total pressure ratio of compressor or fan

- efficiency

- combustion efficiency

- isentropic compressor efficiency

- isentropic fan intake duct efficiency

- isentropic fan efficiency

- gas generator intake stagnation pressure ratio

- efficiency of mechanical transmission (gearbox)

- isentropic efficiency of expansion process in nozzle

- propulsive efficiency

${ }^{n} p$ - propeller efficiency

prop - isentropic turbine efficiency

$n_{t f}$ - product of $n_{f}$ and $n_{t}$

- thermal efficiency of gas generator

th - overall engine efficiency

tot

$\theta$ - relative ambient temperature $(\theta=$ $\mathrm{T}_{0} / \mathrm{T}_{\mathrm{Oo}}$ )

- temperature function of compression process

- bypass ratio

- ratio of stagnation to static temperature of ambient air

- jet velocity coefficient

- nondimensional Turbine Entry Temperature

- gross thrust parameter

- corrected specific thrust

Subscripts

- combustion chamber

- high-pressure compressor

- fan intake duct

- nozzle exhaust

- fuel

- fan

- gas generator

- intake of gas generator

- exhaust jet of turboprop engine

- nozzle

- turbine

tf - combination of turbine and fan

to - takeoff, sea level static condition 
The station numbering system used is in accordance with Fig. 4-16: 0 - ambient; 1 - nacelle leading edge; 2 -(1.p.) com-

\section{H-1. SCOPE OF THE METHOD}

Most of the equations presented in this appendix have been derived in Ref. H-1 within the framework of propulsion system analysis for aircraft with Laminar Flow Control (LFC). They are presented here in a slightly modified form to make them readily usable for takeoff, climb and cruise conditions for normal subsonic engines without special facilities for LFC. In addition, the result of Ref. H- 6 has been reproduced, yielding a simple method for computing the jet engine thrust lapse with altitude.

The classical procedure for analyzing engine performance is to carry out a cycle analysis for the various thermodynamic processes in the engine. Many examples of this procedure can be found in the literature, e.g. Ref. H-7. In Ref. $\mathrm{H}-1$ it is shown that engine performance can be written in closed form if the following simplifications are considered acceptable:

a. The thrust is referred to fully expanded conditions. Thus instead of the usual standard net thrust definition, we use the ideal thrust:

$\mathrm{T}=\dot{\mathrm{m}}_{\mathrm{g}}\left(\mathrm{v}_{\mathrm{g}}-\mathrm{v}_{\mathrm{o}}\right)+\dot{\mathrm{m}}_{\mathrm{f}}\left(\mathrm{v}_{\mathrm{f}}-\mathrm{v}_{\mathrm{o}}\right)$

for fully expanded exhaust flows.

b. The fuel mass flow is neglected relative to the engine air mass flow:

$\dot{\mathrm{m}}_{\mathrm{F}} \ll \dot{\mathrm{m}}_{\mathrm{g}}+\dot{\mathrm{m}}_{\mathrm{f}}$

c. The ratio of specific heats is assumed constant throughout all engine processes $(\gamma=1.4)$.

d. The fan and exhaust flows are unmixed. e. Power extraction and/or bleed air takeoff for airframe services are ignored.

Although assumptions b. and c. will result in considerable errors in individual thermodynamic processes, it is experienced that pressor entry; 3 - (h.p.) compressor exit; 4 - turbine entry; 5 - turbine exit

several effects cancel each other out when the overall engine performance is considered and for most conventional engine configurations the results are found to be reasonably accurate.

Several equations similar to those derived in Ref. $\mathrm{H}-1$ can also be found in Refs. H-2 thicugh H-4. The background to the cycle analysis and the various assumptions and approximations from which the simple analytical expressions have been derived are discussed in Refs. $\mathrm{H}-1, \mathrm{H}-4$ and $\mathrm{H}-6$. The present method is useful for application in aircraft project design work, in which errors of a few percent are acceptable. The results of the present method are not, however, accurate for low engine ratings, particularly when the hot flow nozzle is not choked. An alternative method for turbojets can be found in Ref. H- 8 .

Finally, it should be noted that various definitions used in this appendix are dealt with in greater detail in Section 4.3 .

\section{H-2. THE GAS GENERATOR}

The following major engine parameters are used in the analysis. TURBINE ENTRY TEMPERATURE $\mathrm{T}_{\mathrm{t}_{4}}$, a quantity which particularly affects the (specific) thrust, as discussed in Section 4.4.2. High values of specific thrust are obtainable when turbine blade cooling is used, but the effect of the cooling on engine performance will not be taken into account here. Instead, the data in Ref. H-5 suggest that a correction for cooling can be made by substituting in the present equations an equivalent value of $\mathrm{T}_{\mathrm{t}_{4}}$ which is about $30 \mathrm{~K}$ (54R) below the actual $\mathrm{T}_{\mathrm{t}_{4}}$ of the (cooled) blades.

OVERALL PRESSURE RATIO $\varepsilon_{c^{\prime}}$ an engine characteristic which is of vital importance to the engine (specific) fuel consumption, as 
discussed in Section 4,4.1. For the usual engine configuration with a fan in front of the gas generator, $\varepsilon_{c}$ is equal to the combined effect of the fan and the remaining portion of the engine compressor.

The analysis is simplified by introducing the following characteristic functions: the non-dimensional Turbine Entry Temperature:

$\phi=\frac{T_{t_{4}}}{T_{0}}$

and the temperature function of the compression process:

$\kappa=\mu\left(\varepsilon_{c} e^{\frac{\gamma-1}{\gamma}}-1\right)=\mu\left(\varepsilon_{c} e^{.2857}-1\right)$

where

$\mu=1+\frac{\gamma-1}{2} M_{0}^{2}=1+.2 M_{0}^{2}$

Gas generator performance may be expressed in nondimensional form in terms of a GAS GENERATOR FUNCTION,

$G=\frac{P_{g_{1 s}}}{\dot{m}_{g} c_{p} T_{0}}$

and a THERMAL EFFICIENCY,

$\eta_{t h}=\frac{P_{g_{i s}}-\frac{1}{2} \dot{m}_{g} v_{o}^{2}}{\dot{m}_{g} c_{p}\left(T_{t_{4}}-T_{t_{3}}\right)}$

where $\mathrm{P}_{\mathrm{g}_{\mathrm{S}}}$ is the gas generator CONVERTIBLE ENERGY, defined as the portion of the gas generator hot-gas energy that can be converted into useful propulsive power. The convertible energy is equivalent to the kinetic energy of the gases for the hypothetical case that they would expand to ambient pressure in an isentropic process. The rest of the energy is lost in the form of heat added to the atmosphere.

The gas generator function $\mathrm{G}$ can be calculated as follows:
$G=\left(\phi-\frac{\kappa}{\eta_{c}}\right)\left\{1-\frac{1.01}{\eta_{1} \cdot 2857(\kappa+\mu)\left(1-\frac{\kappa / \phi}{\eta_{c} \eta_{t}}\right)} \mid(H-8)\right.$

where a combustion chamber pressure loss of 3.5 percent has been assumed. The gas generator intake stagnation pressure ratio is defined as follows:

$n_{1}=p_{t_{2}} / p_{t_{0}}$

The isentropic compressor efficiency $n_{c}$ is the ratio of the total temperature rise in an isentropic compression to the actual temperature rise in a (polytropic) compression with the same pressure ratio. The isentropic turbine efficiency $\eta_{t}$ is the ratio of the actual total temperature arop in the (polytropic) expansion process to the temperature drop in an isentropic expansion with the same pressure ratio. Some guidelines with respect to the choice of $n_{i}, n_{c}$ and $n_{t}$ are given in section $\mathrm{H}-7$.

The thermal efficiency is computed as follows:

$n_{\text {th }}=\frac{G-.2 M_{o}^{2}}{\phi-\mu-\frac{K}{n_{c}}}$

Instead of $G$, a velocity coefficient $\sigma$ may be used to characterize the gas generator performance:

$\sigma=\left(\frac{\frac{L}{2} \dot{\mathrm{m}}_{g} v_{0}{ }^{2}}{\mathrm{P}_{g_{\text {is }}}}\right)^{\frac{1}{2}}=\frac{\mathrm{M}_{0}}{\sqrt{\frac{2}{\gamma-1} \mathrm{G}}}=\frac{\mathrm{M}_{0}}{\sqrt{5 G}}$

Unlike G, the velocity ratio is characterized by its large variation with flight speed.

H-3. SPECIFIC PERFORMANCE OF STRAIGHT JET ENGINES

The PROPULSIVE EFFICIENCY is defined as follows:

$n_{p}=\frac{T v_{0}}{P_{g_{1 s}}-\frac{1}{2} \dot{m}_{g} v_{0}^{2}}$ 
and can be computed from:

$n_{p}=\frac{2 \sigma\left(\sqrt{n_{n}}-\sigma\right)}{1-\sigma^{2}}$

where $\sigma$ is given by $(\mathrm{H}-11)$ and the isentropic nozzle efficiency is defined in a manner similar to the turbine efficiency $n_{t}$.

The OVERALL EFFICIENCY is defined as follows :

$\eta_{\text {tot }}=\frac{T v_{0}}{\dot{W}_{F} H}$

and can be computed from:

$n_{\text {tot }}=n_{B} n_{\text {th }} n_{p}=\frac{.4 n_{B} M_{o}^{2}}{\phi-\mu-\frac{\kappa}{n_{C}}}\left(\frac{1}{\sigma} \sqrt{n_{n}}-1\right)$

The SPECIFIC FUEL CONSUMPTION is related to the overall efficiency by:

$\mathrm{C}_{\mathrm{T}}=\frac{\dot{\mathrm{W}}_{\mathrm{F}}}{\mathrm{T}}=.2788 \frac{\mathrm{M}_{\mathrm{O}} \sqrt{\theta}}{n_{\text {tot }}} \quad\left(\mathrm{h}^{-1}\right)$

This quantity may be obtained from the CORRECTED SPECIFIC FUEL CONSUMPTION:

$\frac{C_{T}}{\sqrt{\theta}}=.711 \frac{\phi-\mu-\kappa / \eta_{c}}{\sqrt{5 n_{n} G}-M_{0}}$

for a combustion efficiency of 98 percent. The SPECIFIC THRUST is the engine thrust divided by the intake mass flow per unit time. The following applies to the CORRECTED SPECIFIC THRUST:

$\psi=\frac{T}{\dot{W} \sqrt{\theta}}=34.714 \quad\left(\sqrt{5 n_{n} G}-M_{0}\right) \quad(\mathrm{sec}) \quad(H-18)$

H-4. SPECIFIC PERFORMANCE OF TURBOFAN ENGINES

The BYPASS RATIO $\lambda$, defined as the ratio of the mass flows per unit time through the fan and the gas generator, respectively, is a fundamental parameter of turbofan engines, affecting engine layout, specific thrust, specific fuel consumption, weight, drag and noise. Its significance is dealt with thoroughly in section 4 .
An "optimum" FAN PRESSURE RATIO may be defined, resulting in maximum propulsive efficiency and thrust and minimum $C_{T}$ for a given gas generator. Its value can be obtained from:

$\varepsilon_{f_{o p t}}=\left\{1+\frac{n_{t f}{ }^{2} G-.2 n_{i} M_{o}^{2}}{\mu\left(1+n_{t f}{ }^{\lambda}\right)}\right\}^{3.5}$

This pressure ratio corresponds to the "optimum" jet velocities of the fully expanded hot and cold flows:

$\frac{v_{\text {opt }}}{\sqrt{\theta}}=a_{o \circ} \sqrt{\frac{5 n_{n} G+\lambda m_{o}{ }^{2} n_{i} / n_{t f}}{1+n_{t f}{ }^{\lambda}}} \quad(H-20)$.

where $a_{0 o}$ is the speed of sound at sea level ISA $(1116.9 \mathrm{ft} / \mathrm{s}=340.4 \mathrm{~m} / \mathrm{s})$.

$v_{f_{\text {opt }}}=n_{t} n_{f} v_{g_{\text {opt }}}=n_{t f} v_{g_{\text {opt }}}$

The following equations are valid on condition that the fan pressure ratio is optimized according to $(\mathrm{H}-19)$.

Propulsive efficiency, defined by (H-12):

$n_{p}=\frac{2 \sigma^{2}}{1-\sigma^{2}}\left\{\sqrt{n_{n}\left(1+n_{t f} \lambda\right)\left(\frac{1}{\sigma^{2}}+\frac{n_{d}}{n_{t f}} \lambda\right)}-(1+\lambda)\right\}$

overall efficiency, defined by $(\mathrm{H}-14)$ :

$n_{\text {tot }}=.4 \frac{n_{B} M_{0}^{2}}{\phi-\mu-k / n_{C}}\left\{\sqrt{n_{n}\left(1+n_{t f} \lambda\right)\left(\frac{1}{\sigma^{2}}+\frac{n_{d}}{n_{t f}} \lambda\right)}-\right.$

$$
(1+\lambda) \mid
$$

corrected specific fuel consumption, for a combustion efficiency of 98 percent:

$$
\frac{C_{T}}{\sqrt{\theta}}=\frac{.711\left(\phi-\mu-k / n_{c}\right)}{\sqrt{5 n_{n}\left(1+n_{t f} \lambda\right)\left(G+.2 M_{0}{ }^{2} \frac{n_{d}}{n_{t f}} \lambda\right)}-(1+\lambda) M_{0}}
$$

$$
\left(h^{-1}\right)
$$

and the corrected specific thrust:

$$
\begin{aligned}
\psi=\frac{T}{\dot{w} \sqrt{\theta}}= & 34.714\left\{\frac{1}{1+\lambda} \sqrt{5 n_{n}\left(1+n_{t f} \lambda\right)} \times\right. \\
& \left.\sqrt{\left(G+.2 M_{0}^{2} \frac{n_{d}}{n_{t f}} \lambda\right)}-M_{0}\right\} \text { (sec) }
\end{aligned}
$$


The ISENTROPIC FAN INLET DUCT EFFICIENCY

$n_{\mathrm{d}}$ in these equations is the total temperature rise resulting from an isentropic compression from ambient to fan total intake pressure, divided by the actual temperature rise in an adiabatic compression to the same inlet pressure. Provided the intake of the gas generator and the fan have similar performance, the relationship between $n_{d}$ and $n_{i}$ is as follows:

$n_{i} \frac{\gamma-1}{\gamma}=\frac{1+n_{d} \frac{\gamma-1}{2} M_{0}^{2}}{1+\frac{\gamma-1}{2} M_{0}^{2}}$

or:

$n_{i}=\left(\frac{1+.2 n_{d} M_{o}^{2}}{1+.2 M_{o}^{2}}\right)^{3.5}$

A convenient approximation is:

$1-n_{i}=\left(1-n_{d}\right) \frac{.7 M_{o}{ }^{2}}{1+.2 M_{o}^{2}}$

H-5. THRUST LAPSE RATES, INTAKE AND EXHAUST AREAS OF TURBOJET AND TURBOFAN ENGINES

The engine thrust can be derived from the specific thrust, provided the engine mass flow is known. To this end an estimation must be made of the total nozzle area $\mathrm{A}_{e}$ ' which is assumed to be fixed.

A correlation of $\mathrm{T}_{\text {to }} /\left(\mathrm{A}_{e} \mathrm{P}_{o o}\right)$ vs. the specific thrust in static takeoff conditions is presented in Fig. $\mathrm{H}-1$. The same figure can also be used to find the fan intake area $\mathrm{A}_{1}$ :

$\frac{\mathrm{A}_{1} \mathrm{p}_{\text {OO }}}{\mathrm{T}_{\text {to }}}=\frac{1+.6 \mathrm{M}_{1}{ }^{2}}{.04 \mathrm{M}_{1} \psi_{\text {to }}}$

where $M_{i}$ is the mean Mach number at the fan face and $\psi_{\text {to }}$ the corrected specific static takeoff thrust at sea level. An average value $\mathrm{M}_{1}=.5$ appears to be representative for sizing the fan inlet area.

THE GROSS THRUST PARAMETER $\varphi$ is nOw defined as: $\varphi=\frac{1+\frac{\text { gross thrust }}{\mathrm{A}_{e} \mathrm{P}_{0}}}{\mu^{3.5}}$

This parameter is frequently used in the presentation of nondimensional engine performance. Its meaning can be demonstrated by taking the sealevel static condition for a straight turbojet with choked nozzle. For that condition the following relation can be derived:

$\varphi_{\text {to }}=\left(1+\gamma_{e}\right) \frac{\text { static exhaust nozzle pressure }}{\text { ambient pressure }}$

where $\gamma_{e} \approx 1.33$

The general case of a turbofan engine (static, takeoff) yields:

$\varphi_{\text {to }}=1+\frac{T_{\text {to }}}{\mathrm{A}_{\mathrm{e}} \mathrm{P}_{\text {Oo }}}$

The corrected intake weight flow is $\dot{\mathrm{w}} \sqrt{\mathrm{T}_{\mathrm{t}_{2}}} / \mathrm{p}_{\mathrm{t}_{2}}$, where $\mathrm{T}_{\mathrm{t}_{2}}$ and $\mathrm{p}_{\mathrm{t}_{2}}$ are the total intake temperature and pressure, respectively. Both the gross thrust parameter and the nondimensional weight flow are related to the corrected engine $\mathrm{rpm}\left(\mathrm{N} / \sqrt{\mathrm{T}_{\mathrm{t}_{2}}}\right)$.

The compressor speed is not a convenient parameter for a generalized method as it is greatly dependent upon the particular compressor design, and should therefore be eliminated. The following approximate relationship can be deduced from consideration of several nondimensional graphs obtained from engine performance brochures:

$\frac{\dot{\mathrm{w}} \sqrt{\theta}}{\delta \dot{\mathrm{W}}_{\text {to }}}=\mu^{3} \sqrt{\frac{\varphi}{\varphi_{\text {to }}}}$

The square-root relationship has no physical meaning, but it gives a reasonable approximation (see Fig. H-2) and is convenient for solving the equations analytically. For normal operational conditions in takeoff, climb and cruising flight, the nondimensional nass flow is within \pm 58 of the value at takeoff and the approximation given by $(\mathrm{H}-32)$ is acceptable.

Equation $\mathrm{H}-32$ can be combined with: 


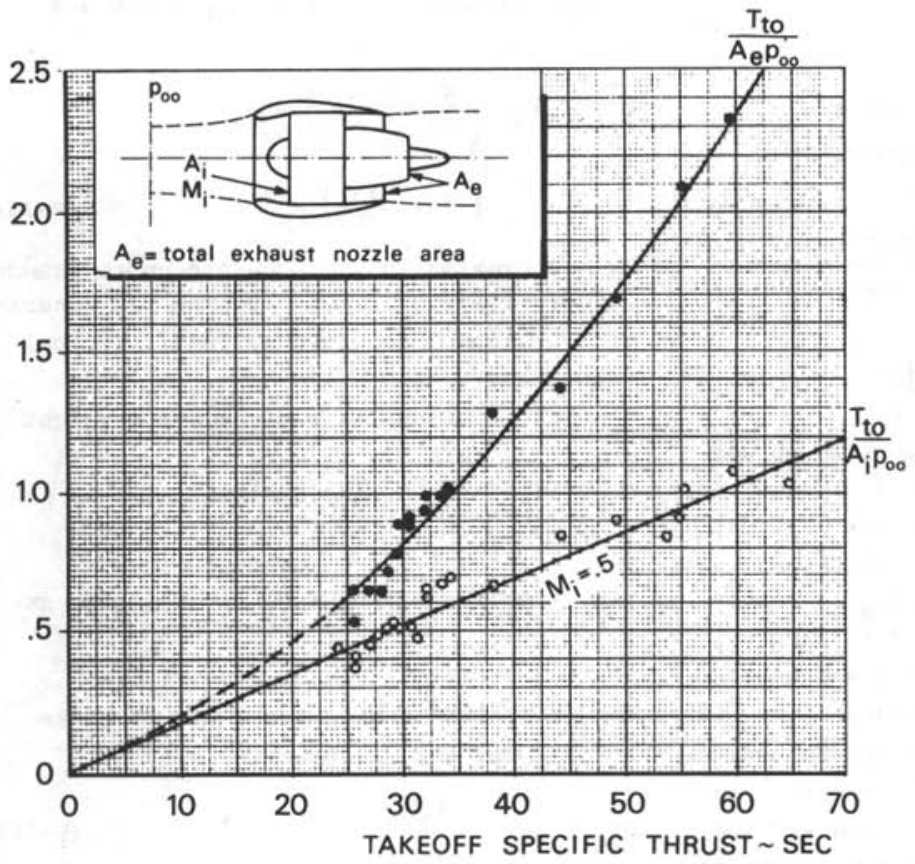

Fig. H-1. Intake and exhaust areas as determined by the takeoff specific thrust (civil jet engines, fixed nozzle)

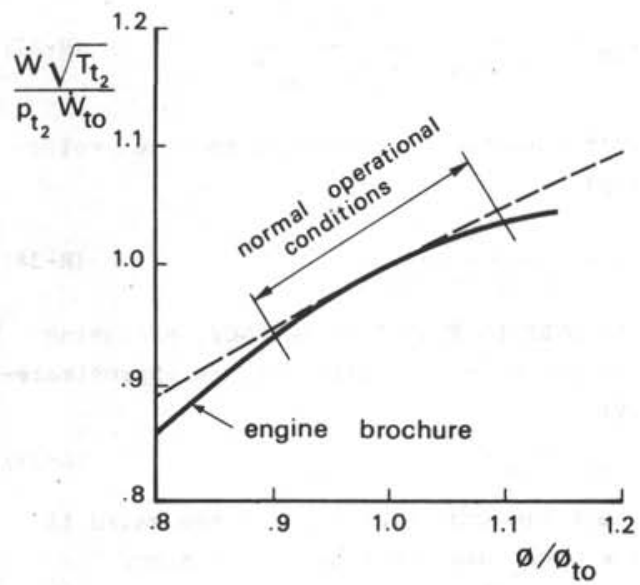

$$
\begin{aligned}
& \text { approximation: } \\
& \frac{\dot{w} \sqrt{\mathrm{T}_{t_{2}}}}{\mathrm{pt}_{2} \dot{\boldsymbol{\omega}}_{\text {to }}}=\sqrt{\frac{\phi}{\phi t_{0}}} \\
& \mathrm{P}_{\mathrm{t}_{2}}=\text { total intake pressure } \\
& \mathrm{T}_{\mathrm{t}_{2}}=\text { total intake temperature } \\
& \dot{\mathrm{w}}=\text { engine weight flow per unit time } \\
& \dot{\mathrm{w}}_{\text {to }}=\dot{\mathrm{w}} \text { at SLS, ISA } \\
& \varphi \quad=\text { gross thrust parameter }
\end{aligned}
$$

Fig. H-2. Nondimensional engine air weight flow and gross thrust parameter for the Rolls-Royce RB183 turbofan engine 
$\frac{\dot{\mathrm{W}} \sqrt{\theta}}{\delta \dot{\mathrm{W}}_{\text {to }}}=\frac{T}{\delta \mathrm{T}_{\text {to }}} \cdot \frac{\psi_{\text {to }}}{\psi}$

to yield the THRUST LAPSE RATE $T / T_{\text {to }}$. The solution of the resulting quadratic equation is approximated by:

$$
\begin{gathered}
\frac{T}{\delta T_{\text {to }}}=\frac{\psi}{\psi_{\text {to }}} \mu^{1.5} \mid \frac{1}{\sqrt{\varphi_{\text {to }}}+.6 \frac{\varphi_{\text {to }}}{\varphi_{\text {to }}}} \mu^{1.5} \times \\
\left(\frac{\psi}{\psi_{\text {to }}}+\frac{34.714 \mathrm{M}_{\mathrm{o}}}{\psi_{\text {to }}}\right) \mid
\end{gathered}
$$

where $\psi$ is the corrected specific thrust for the condition for which $\mathrm{T}$ is required, and $\psi_{\text {to }}$ is the corrected specific thrust in the takeoff (sealevel, static). The gross thrust parameter $\varphi_{\text {to }}$ is calculated with $(\mathrm{H}-31)$ and $\mathrm{Fig} . \mathrm{H}-1$. Expressions for the specific thrust are given by $(\mathrm{H}-18)$ and $(\mathrm{H}-25)$ for straight jet and turbofan engines, respectively. The engine parameters $\lambda, \mathrm{T}_{\mathrm{t}_{4}}, \varepsilon_{\mathrm{c}}$ and $\varepsilon_{\mathrm{f}}$ are in principle not constant under various working conditions. The following assumptions can be made.

Bypass Ratio $\lambda$ : assumed constant and equal to the value in the takeoff condition. For low engine ratings the bypass ratio will increase considerably and the method is no longer valid.

Turbine Entry Temperature $\mathrm{T}_{\mathrm{t}_{4}}$ : the difference in $T_{t}$ between takeoff and maximum cruise rating is of the order of 150 to $200 \mathrm{~K}$ ( $: 70$ to $360 \mathrm{R})$ for low bypass ratios and 50 to $100 \mathrm{~K}$ ( 90 to $180 \mathrm{R}$ ) for high bypass engines. The long-range cruise rating can be optimized with respect to $\mathrm{T}_{\mathrm{t}_{4}}$ (see Section 4.4.2.), while for maximum climb conditions $\mathrm{T}_{\mathrm{t}_{4}}$ is between the values for takeoff and maximum cruise rating. Overall Pressure Ratio $\varepsilon_{c}$ : in cruising flight at altitude $\varepsilon_{c}$ is generally about 5 to 108 above the value for takeoff. For all conditions the fan pressure ratio $\varepsilon_{f}$ is assumed optimum for minimum fuel consumption.

A simple approximation can be derived for the thrust during takeoff from series expansion of $(\mathrm{H}-25)$ and $(\mathrm{H}-32)$, assuming ip to be constant, $\eta_{d}=.98, \eta_{t f}=.75$ and $n_{n}=.97$, at sea level

$$
\begin{aligned}
\frac{\mathrm{T}}{\mathrm{T}_{\text {to }}}=1- & \frac{.454(1+\lambda)}{\sqrt{(1+.75 \lambda) \mathrm{G}}} \mathrm{M}_{\mathrm{O}}+ \\
& \left(.6+\frac{.13 \lambda}{\mathrm{G}}\right) \mathrm{M}_{0}{ }^{2}
\end{aligned}
$$

This approximation is accurate up to a Mach number of approximately .3. The gas generator function $G$ is given by $(H-8)$. Typical values are:

$G=.9$, low-bypass engines and straight jets

$\mathrm{G}=1.1$, high-bypass engines

H-6. SPECIFIC PERFORMANCE OF TURBOPROP ENGINES

The EQUIVALENT POWER is the sum of brake horse power and jet power:

$P_{\text {eq }}=P_{b r}+\frac{T_{j} V_{o}}{n_{\text {prop }}}$

Power distribution between the propeller shaft and exhaust gases is "optimum" (for maximum overall efficiency) if:

$P_{b r}=n_{t} P_{g_{i s}}-\frac{\frac{1}{2} \dot{m} v_{c}{ }^{2}}{n_{t}\left(n_{\text {prop }}\right)^{2}}$

This condition corresponds to a jet velocity:

$v_{j}=\frac{v_{o}}{n_{\text {prop }} n_{t} n_{\text {mech }}}$

THE OVERALL ENGINE EFFICIENCY, excluding the propeller contribution, is approximate1y:

$n_{\text {tot }}=n_{B} n_{t h} n_{t} n_{\text {mech }}$

The following relationships are valid if the power distribution is optimized for $n_{\text {prop }}=.85$ and $n_{B} n_{\text {mech }}=.95$, at a flight Mach number equal to $\mathrm{M}_{0}^{*}$.

(BRAKE) SPECIFIC FUEL CONSUMPTION:

$C_{p}=\frac{\text { fuel consumption per hour }}{\text { brake horsepower }}$ 
$C_{P}=$ constant $\frac{\phi-\mu-k / n_{c}}{n_{t} G-.28\left(M_{0}^{*}\right)^{2} / \eta_{t}}$

where the constant is equal to .1426 if $\mathrm{C}_{\mathrm{P}}$ is in $1 \mathrm{~b} / \mathrm{hp} / \mathrm{h}$, or .0647 if $\mathrm{C}_{\mathrm{p}}$ is in $\mathrm{kg} / \mathrm{hp} /$ h.

SPECIFIC SHAFT POWER:

$\frac{\mathrm{P}_{\mathrm{br}}}{\dot{\mathrm{w} \theta}}=$ conștant $\left\{n_{\mathrm{t}} \mathrm{G}-.28\left(\mathrm{M}_{0}^{*}\right)^{2} / n_{\mathrm{t}}\right\}$

\section{SPECIFIC EQUIVALENT POWER:}

$\frac{P_{\mathrm{eq}}}{\dot{\mathrm{w}} \theta}=$ constant $\left\{n_{\mathrm{t}} \mathrm{G}-\left(\mathrm{M}_{\mathrm{o}}^{*}\right)^{2}\left(.4-.193 / n_{\mathrm{t}}\right)\right\}$

where the constant in $(\mathrm{H}-41)$ and $(\mathrm{H}-42)$ is equal to 173 if $\mathrm{P}_{\mathrm{br}}$ is in hp and $\dot{\mathrm{w}}$ in $1 \mathrm{~b} / \mathrm{s}$, or 382 if $\dot{\mathrm{W}}$ is in $\mathrm{kg} / \mathrm{s}$.

The Turbine Entry Temperature in cruising conditions is about 50 to $100 \mathrm{~K}$ (90 to 180 R) below the value for takeoff rating.

\section{H-7. CYCLE EFFICIENCIES AND PRESSURE LOSSES}

The gas generator intake and fan inlet duct efficiencies $\eta_{i}$ and $n_{d}$ may be calculate with the method in Ref. H-9. A simpler approach will be permissible in most cases if the inlet duct is of regular shape, without sharp bends or divergences. In that case we may use the following equation, derived from Ref. H-10:

$1-n_{d}=4 K_{d} C_{F}\left(\frac{M_{d}}{M_{0}}\right)^{2} \frac{\text { duct length }}{\text { inlet diameter }}$

$\mathrm{K}_{\mathrm{d}}$ is the ratio of the total duct pressure loss to that due to skin friction only, in a constant-area duct. For a good design $\mathrm{K}_{\mathrm{d}}=1.3 . \mathrm{C}_{\mathrm{F}}$ is the skin friction coefficient in a straight duct, approximated by

$C_{F}=\frac{.05}{\left(R_{e}\right)^{1 / 5}}$

where the Reynolds number is based on the average conditions in the duct and the mean duct diameter.

$\mathrm{M}_{\mathrm{d}}=$ mean Mach number in the intake duct:

$\mathrm{M}_{\mathrm{d}}=\frac{24.77}{\mathrm{~A}_{\mathrm{d}} \mathrm{p}_{\mathrm{oO}}} \frac{\dot{\mathrm{W}} \sqrt{\theta}}{\delta}$

The air weight flow $\dot{w}$ is determined from the specific thrust and the thrust. For a generalized study of engines for high-subsonic aircraft it is reasonable to assume $\mathrm{M}_{\mathrm{d}}=.5$.

The stagnation pressure ratio $\eta_{1}$ is obtained from $(\mathrm{H}-27)$.

Other engine component efficiency factors may be assumed as follows:

$n_{f}=.85$ to .87 - takeoff .82 to .85 - cruising

$n_{c}=.84$ to .86

$n_{t}=.87$ to .89

$n_{n}=.96$ to .98

The lower values are applicable to small engines, the higher ones to large civil aircraft engines. Engines can be designed in such a way that high fan efficiencies are obtained in cruising flight and lower values under takeoff conditions. This may be desirable for high-bypass engines in view of their unfavorable thrust lapse with altitude and flight speed.

REFERENCES

H-1. E. Torenbeek: "The propulsion of aircraft with laminar flow control". Report VTH-150, Delft University of Technology, Dept. of Aeron. Eng., 1968.

H-2. S. Boudiques: "Défense et illustration des propulseurs pour avions rapides". Techniques et Science Aéronautiques et Spatiales, July - Aug. 1964 pp. 271-285.

B-3. W. Dettmering and F. Fett: "Methoden der Schuberhöhung und ihre Bewertung". Zeitschrift für Flugwissenschaften. August 1969, pp. 257-267.

H-4. D.G. Shepherd: "Aerospace propulsion". New York, American E1sevier, 1972.

H-5. R.E. Neitzel and H.C. Hensworth: "High-bypass turbofan cycles for long-range subsonic transports", Journal of Aircraft, Vo1. 3 No. 4, July - August 1966, pp. 354-358. 
H-6. B. Torenbeek: "Analytical method for computing turbo engine performance at design and off-design conditions". Memorandum M-188, Delft University of Technology, Dept. of Aeron. Eng. Jan. 1973.

H-7. H. Cohen, G.F.C. Rogers and H.I.H. Saravanamuttoo: "Gas turbine theory". Longman Group Lta., London, Second Edition, 1974.

B-8. B. Ahren: "General performance investigation of turbojet engines". SAAB Technical Note No. 52.

H-9. E.W. Dunlap: "Flight Test Handbook - Performance". Chapter 1, Part 1. AFFTC-TR-59-47, Jan. 1960.

H-10. W.J. Hesse and N.V.S. Mumford Jr.: "Jet propulsion for aerospace applications". Second Edition, 1964, Pitman Publ. Corp., New York. 


\section{Appendix J. Principal data of the US and ICAO standard atmospheres}

SUMMARY

The Standard Atmosphere presented in this appendix is the US Standard Atmosphere 1962, prepared under the sponsorship of the NASA, USAF and USWB (United States Weather Bureau). This atmosphere is in agreement with the ICAO Standard Atmosphere over their common altitude range. For quick reference standard data for sea level are given, together with some of relative values at various practical flying altitudes. 


\begin{tabular}{|c|c|c|c|c|c|c|c|c|c|c|c|}
\hline \multicolumn{6}{|c|}{ METRIC UNITS } & \multicolumn{6}{|c|}{ ENGLISH UNITS } \\
\hline $\begin{array}{c}\text { Altitude } \\
=\end{array}$ & $\begin{array}{c}\text { Pressure } \\
\text { ratio } \\
\delta\end{array}$ & $\begin{array}{c}\text { Temperature } \\
\text { ratio } \\
\theta\end{array}$ & $\sqrt{\theta}$ & $\begin{array}{c}\text { Density } \\
\text { ratio } \\
\sigma\end{array}$ & $\begin{array}{c}\text { Kin.Viscosity } \\
\text { ratio } \\
v / v_{0}\end{array}$ & $\begin{array}{c}\text { Altitude } \\
\mathrm{ft}\end{array}$ & $\begin{array}{c}\text { Pressure } \\
\text { ratio } \\
\delta\end{array}$ & $\begin{array}{c}\text { Temperature } \\
\text { ratio } \\
\theta\end{array}$ & $\sqrt{\theta}$ & $\begin{array}{c}\text { Density } \\
\text { ratio } \\
a\end{array}$ & $\begin{array}{c}\text { Kin.Viscosity } \\
\text { ratio } \\
v / v_{0}\end{array}$ \\
\hline 0 & 1 & 1 & 1 & 1 & 1 & 0 & 1 & 1 & 1 & 1 & 1 \\
\hline 1,500 & .83450 & .96616 & .98294 & .86373 & 1.1271 & 5,000 & .83205 & .96562 & .98266 & .86167 & 1.1293 \\
\hline 3,000 & .69192 & .93233 & .96557 & .74214 & 1.2754 & 10,000 & .68770 & .93124 & .96501 & .73848 & 1.2806 \\
\hline 4,500 & .56973 & .89849 & .94789 & .63410 & 1.4495 & 15,000 & .56434 & .89687 & .94703 & .62924 & 1.4586 \\
\hline 6,000 & .46564 & .86465 & .92987 & .53853 & 1.6549 & 20,000 & .45954 & .86249 & .92870 & .53281 & 1.6693 \\
\hline 7,500 & .37751 & .83082 & .91149 & .45439 & 1.8989 & 25,000 & .37109 & .82811 & .91001 & .44812 & 1.9203 \\
\hline 9,000 & .30340 & .79698 & .89274 & .38069 & 2.1905 & 30,000 & .29696 & .79373 & .89092 & .37413 & 2.2214 \\
\hline 10,500 & .24154 & .76314 & .87358 & .31651 & 2.5415 & 35,000 & .23531 & .75935 & .87141 & .30987 & 2.5852 \\
\hline $11,000 *$ & .22336 & .75187 & .86710 & .29708 & 2.6743 & 36,089 • & .22336 & .75187 & .86710 & .29708 & 2.6743 \\
\hline 12,000 & .19078 & .75187 & .86710 & .25374 & 3.1311 & 40,000 & .18509 & .75187 & .86710 & .24617 & 3.2273 \\
\hline 13,500 & .15059 & .75187 & .86710 & .20029 & 3.9666 & 45,000 & .14555 & .75187 & .86710 & .19358 & 4.1040 \\
\hline 15,000 & .11887 & .75187 & .86710 & .15810 & 5.0251 & 50,000 & .11446 & .75187 & .86710 & .15223 & 5.2189 \\
\hline 16,500 & .09383 & .75187 & .86710 & .12480 & 6.3660 & 55,000 & .09001 & .75187 & .86710 & .11971 & 6.6367 \\
\hline 18,000 & .07407 & .75187 & .86710 & .09851 & 8.0648 & 60,000 & .07078 & .75187 & .86710 & .09414 & 8.4395 \\
\hline 19,500 & .05847 & .75187 & .86710 & .07776 & 10.2169 & 65,000 & .05566 & .75187 & .86710 & .07403 & 10.7321 \\
\hline
\end{tabular}

* tropopause

Table J-1. Atmospheric properties for several geopotential altitudes

\begin{tabular}{|c|c|c|c|c|}
\hline \multirow{2}{*}{$\begin{array}{l}\text { STANDARD SEA } \\
\text { LEVEL DATA }\end{array}$} & \multirow{2}{*}{$\begin{array}{l}\frac{n}{0} \\
\text { है } \\
\text { है }\end{array}$} & \multicolumn{2}{|c|}{ TECHNICAL UNITS } & \multirow{2}{*}{\begin{tabular}{|c|} 
SI UNITS \\
METRIC
\end{tabular}} \\
\hline & & ENGLISH & METRIC & \\
\hline PRESSURE & $P_{0}$ & $\begin{array}{l}2116.221 b_{f} / \mathrm{sq} . \mathrm{ft} \\
\left(14.6961 \mathrm{~b}_{\mathrm{f}} / \mathrm{sq} \cdot \mathrm{in}\right)\end{array}$ & $10332.27 \mathrm{~kg}_{\mathrm{f}} / \mathrm{m}^{2}$ & $\begin{array}{l}101325.0 \text { Newtons } / \mathrm{m}^{2} \\
(1013.25 \mathrm{mill} \text { ibar })\end{array}$ \\
\hline TEMPERATURE & $\mathrm{t}_{0}$ & $\begin{array}{l}59 \mathrm{~F} \\
518.67 \mathrm{R}\end{array}$ & $\begin{array}{l}15 \mathrm{C} \\
288.15 \mathrm{~K}\end{array}$ & $\begin{array}{l}15 \mathrm{C} \\
288.15 \mathrm{~K}\end{array}$ \\
\hline DENSITY & $P_{0}$ & .0023769 slugs $/ \mathrm{ft}^{3}$ & $.12492 \mathrm{~kg}_{\mathrm{f}} \mathrm{s}^{2} / \mathrm{m}^{4}$ & $1.2250 \mathrm{~kg} / \mathrm{m}^{3}$ \\
\hline $\begin{array}{l}\text { VELOCITY OF } \\
\text { SOUND }\end{array}$ & $a_{0}$ & $\begin{array}{l}1116.45 \mathrm{ft} / \mathrm{s} \\
661.48 \mathrm{knots}\end{array}$ & $\begin{array}{l}340.294 \mathrm{~m} / \mathrm{s} \\
1225.06 \mathrm{~km} / \mathrm{h}\end{array}$ & $340.294 \mathrm{~m} / \mathrm{s}$ \\
\hline $\begin{array}{l}\text { KINEMATIC } \\
\text { VISCOSITY }\end{array}$ & $v_{0}$ & $1.5723 \times 10^{-4} \mathrm{sq} . \mathrm{ft} / \mathrm{s}$ & $1.4607 \times 10^{-5} \mathrm{~m}^{2} / \mathrm{s}$ & $1.4607 \times 10^{-5} \mathrm{~m}^{2} / \mathrm{s}$ \\
\hline $\begin{array}{l}\text { ACCELERATION DUE } \\
\text { TO GRAVITY }\end{array}$ & $g_{0}$ & $32.1741 \mathrm{ft} / \mathrm{s}^{2}$ & $9.80665 \mathrm{~m} / \mathrm{s}^{2}$ & $9.80665 \mathrm{~m} / \mathrm{s}^{2}$ \\
\hline
\end{tabular}

- Contrary to the convention used in this book, the subscript $f$ for "force" has been retained here in order to avoid confusion with the SI system.

Table J-2. Standard sea level data 


\section{Appendix K. The definition and calculation of the takeoff field length required for civil transport aircraft}

SUMMARY

A summary and explanation are given of the various definitions associated with the takeoff of civil transport aircraft, taking into account the event of engine failure. The schedule to be set up for determining the takeoff reference speeds is discussed and a suggested generalized procedure for analyzing the takeoff performance is presented. A survey of the methods and data required to actually perform the calculations for zero wind and a level runway concludes this appendix, which is intended to give adequate information for evaluating the takeoff performance in the preliminary design stage.

The principles of takeoff field length determination apply to civil transport aircraft (weight $\geqslant 12,500 \mathrm{lb}=5,670 \mathrm{~kg}$ ) only, but the methods of analysis and data presented may well be used for other aircraft categories, e.g. light aircraft. 


\section{NOMENCLATURE}

A - aspect ratio; factor in the deceleration

a - acceleration or deceleration

BFL - Balanced Field Length

$C_{D}$ - (aircraft) drag coefficient

$C_{D_{0}}$ - zero-lift drag coefficient

$C_{L}$ - (aircraft) lift coefficient

C.g. - center of gravity

D - drag

- Oswald factor

$F(h)$ - non-dimensional height function

$F(\gamma)$ - non-dimensional climb angle function

g - acceleration due to gravity

h - height

L $\quad$ - lift

$1_{b}$ - wheelbase

$\mathrm{N} \quad$ - normal ground reaction force

n - load factor

$\mathrm{n}_{\alpha}$ - rate of change of load factor with $\alpha$

$Q_{\max }$ - total maximum torque of the wheelbrakes

$r_{t} \quad-$ (deflected) tire radius

$S$ - wing area; distance

$\mathrm{T}, \overline{\mathrm{T}}$ - thrust, mean thrust respectively

$t \quad$ - time

u.c. - undercarriage

$\mathrm{V}$ - speed (relative to the ground and the air)

w - weight

a - angle of attack

\section{K-1. REFERENCE DISTANCE DEFINITIONS}

Although the FAR and BCAR codes differ in detail, both attempt to ensure a similar level of safety in the takeoff with all engines operating, and in the event of an engine failure accurring at any point. Consequently, the basic procedures for takeoff calculations are identical. Definitions associated with the takeoff are introduced by reference to Fig. $\mathrm{K}-1$. Although the example applies to a twin-engine airplane, the requirements for three- and four-engine airplanes differ only in detail. The follow-
- angle of climb

- increment

- braking efficiency

- angle of pitch

- friction coefficient

- air density

Subscripts

A - airborne phase

b - braking

cg - center of gravity

cp - center of pressure

eff - effective

G - ground roll

LOF - 1iftoff

MC - Minimum Control (Speed)

MU - Minimum Unstick (Speed)

$\mathrm{N} \quad$ - al1 engines operating

$\mathrm{N}-1$ - one engine inoperative

ROT - rotation

S - stalling

STOP - stopping

$T$ - thrust line

to - takeoff

uc - undercarriage

$x \quad$ - moment of engine failure

- - standstill at beginning of runway

- decision point; main u.c.

- $35 \mathrm{ft}$ height point (one engine inoperative); nose u.c.

- $35 \mathrm{ft}$ height point (all engines operating)

ing principal distances* determine the takeoff field length required for a fixed weight, altitude, temperature and airplane configuration (e.g. flap setting).

a. The TAKEOFF RUN required is derived from calculations by taking the distance needed to accelerate to the moment of $11 \mathrm{ft}-$ off, plus a proportion of the airborne dis-

* The definitions given here are interpretations of the formal definitions and procedures in FAR 25.103 through 25.121 and BCAR Section D Ch. D2-3. 


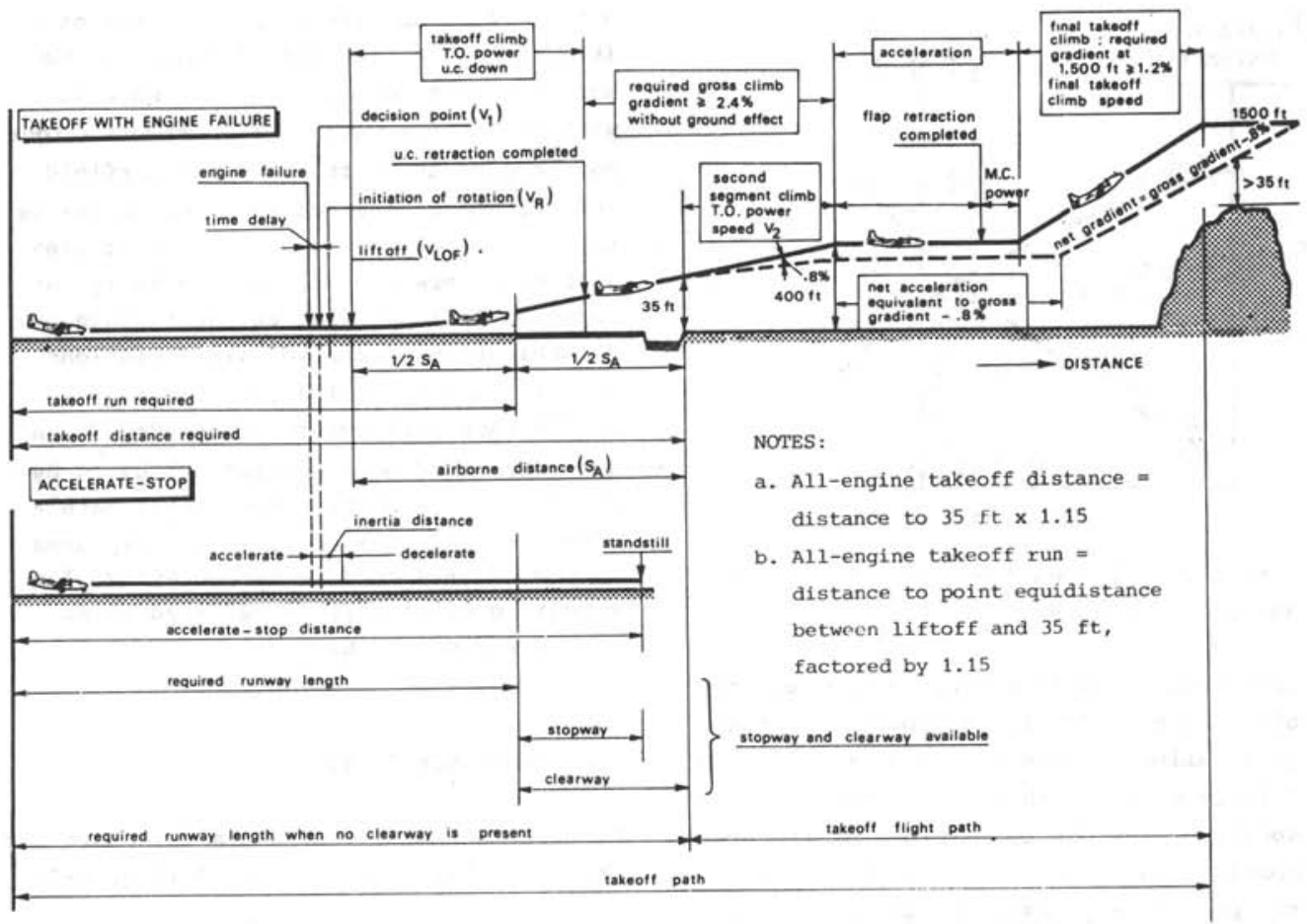

Fig. K-1. Takeoff procedures and requirements for a twin-engine civil transport aircraft

tance to a height of $35 \mathrm{ft}$ (FAR: half, BCAR: a third). In the all-engine case, a safety margin of 158 is added. The greater of these distances must not exceed the takeoff ground run available - i.e. the length of the runway, having a hard prepared surface compatible with the aircraft weight and undercarriage design.

\section{b. The EMERGENCY or ACCELERATE-STOP DIS-} TANCE required is the distance to accelerate to the speed at which the critical engine fails $\left(V_{x}\right)$, plus the distance to come to a standstill by means of braking, when the pilot decides to abort the takeoff. This distance must not exceed the length of the takeoff ground run plus the stopway (if present). The latter has a surface capable of supporting the aircraft with little damage and is suitable for braking.

c. The TAKEOFF DISTANCE required is the distance needed to accelerate, rotate to flying attitude and climb to a height of $35 \mathrm{ft}$. In the all-engines case a safety margin of 158 is added. The greater of these distances must not exceed the length of the takeoff runway plus clearway* (if present). The latter is essentially free of large protruding obstacles, but does not necessarily have a solid surface. For example, water may form a clearway.

d. The BALANCED FIELD LENGTH (BFL) is the distance required in the situation where the emergency distance becomes identical with the takeoff distance to $35 \mathrm{ft}$ with engine failure. The corresponding value of $\mathrm{V}_{\mathrm{x}}$ is the CRITICAL POWER FAILURE SPEED (Fig. K-2). Engine failure prior to this

*Fig. K-1 assumes the stopway to be included in the clearway, but there is no unanimity about this. 


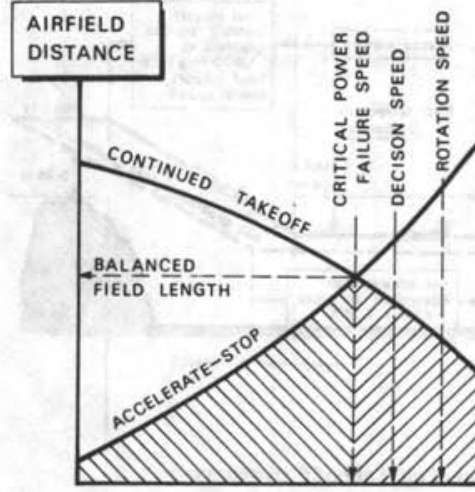

POWER FAILURE SPEED

Fig. K-2. Balanced field length concept and decision speed

speed must be followed by an aborted takeoff; the takeoff will be continued if engine failure occurs at the critical power failure speed or thereafter. Generally speaking, the BFL is obtained at the worst condition at which an engine can fail. In the preliminary design stage the BFL is usually determined without allowing for the existence of a stopway or clearway.

e. The takeoff field length required is the greatest of the emergency distance, the takeoff distance to $35 \mathrm{ft}$ with engine failure and the all-engine takeoff distance to $35 \mathrm{ft}$, in the last case multiplied by 1.15 .

Contrary to the all-engine case, the unfactored BFL is accepted by the airworthiness authorities as the reference engine failure case, the reason being that engine failure at the critical power failure speed is considered as an extremely unlikely combination of unfavorable conditions, to which no extra safety margin need be added.

f. The TAKEOFF PATH from rest to at least $1,500 \mathrm{ft}(450 \mathrm{~m})^{*}$ is divided into the take-

* More precisely: the altitude where the flaps are retracted and the airspeed is increased to the operational climb speed. off distance and the takeoff flight path (Fig. $\mathrm{K}-1$ ). The takeoff distance is the more complicated case and will be dealt with in greater detail. The takeoff flight path begins at $35 \mathrm{ft}$ above the airfield and must be determined in order to ensure that, for a particular flight, obstacles will be cleared by at least $35 \mathrm{ft}$ in adverse conditions, with engine failure and a combination of unfavorable deviations from the GROSS (nominal, calculated) FLIGHT PATH performance. These deviations are determined on a statistical basis by subtracting from the gross flight path a margin of $.88, .98$ or 1.08 for airplanes with 2,3 or 4 engines respectively. The resulting trajectory is referred to as THE NET FLIGHT PATH.

\section{K-2. REFERENCE SPEEDS}

Certain minimum criteria are laid down for the takeoff reference speeds* (Fig. K-3)

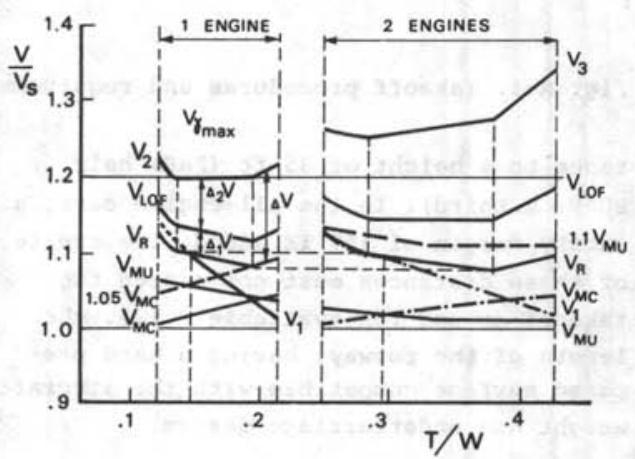

Fig. K-3. Example of a takeoff reference speed schedule for a twin-engine aircraft

to ensure adequate safety and handling, particularly in the case of engine failure, minor mis-handling on the part of the pilot and atmospheric turbulence.

* The nomenclature of several reference airspeeds in this section is simplified; for full details the reader should refer to the airworthiness rules, particularly as regards $\mathrm{v}_{\mathrm{S}}$. 
As already mentioned in Section 5.4.4., THE STALLING SPEED $\mathrm{v}_{\mathrm{S}}$ can be approximated as follows:

$v_{S}=\sqrt{\frac{W}{S} \frac{2}{\rho} \frac{1}{(1.13) C_{L_{\text {max }}}}}$

where $C_{L}-\max$ is the "physical" maximum lift coefficient, i.e. $C_{L}$ at the top of the $C_{L}$ vs. $\alpha$ curve, while the statistical factor 1.13 takes account of the speed loss in the FAA stall maneuver. THE MINIMUM CONTROL SPEED (in the air) $v_{M C}$ is the lowest airspeed at which it has proved to be possible to recover control of the airplane after engine failure with a bank angle of up to five deg. and takeoff thrust (power). The broad requirement is that $\mathrm{V}_{\mathrm{MC}}$ must not exceed $1.2 \mathrm{~V}_{\mathrm{S}}$ with MTOW. For a specified pressure altitude and flap angle there is only one $V_{M C}$, regardless of the weight. Hence, the ratio $\mathrm{V}_{\mathrm{MC}} / \mathrm{V}_{\mathrm{S}}$ increases when the $\mathrm{T} / \mathrm{W}$ ratio increases because of decreasing takeoff weight.

In the project design phase an estimate of $\mathrm{V}_{\mathrm{MC}}$ may be based on wind tunnel measurements or statistical evidence (cf. Section 9.6.1. and Table 9-3).

There is also a minimum control speed on the ground below which the takeoff must always be aborted when engine failure occurs. This characteristic speed is generally of minor importance for performance estimates in the preliminary design stage, provided the vertical tailplane size and rudder capacity are adequate.

THE MINIMUM UNSTICK SPEED $V_{M U}$ is the airspeed at and above which it can be demonstrated by means of flight tests that the aircraft can safely leave the ground and continue the takeoff; $V_{M U}$ is usually very close to $\mathrm{v}_{\mathrm{S}}$. In view of the required positive gradient it may be a function of the $\mathrm{T} / \mathrm{W}$ ratio.

Alternatively, $\mathrm{V}_{\mathrm{MU}}$ may be determined by the geometry of the aircraft as the airspeed for which the fuselage tail scrapes the ground prior to liftoff. In view of the great influence of the ground proximity effects, $V_{M U}$ is very difficult to es- timate in the preliminary design stage. An attempt may be made to estimate the geometry-limited $\mathrm{V}_{\mathrm{MU}}$, using the $\mathrm{C}_{L^{-\alpha}}{ }^{-\alpha}$ curve with ground effect included.

THE TAKEOFF SAFETY SPEED $v_{2}$ is the airspeed obtained at the $35 \mathrm{ft}$ height point. The broad requirement stipulates:

$\mathrm{v}_{2} \geqslant \mathrm{v}_{2 \min }$

$\mathrm{v}_{2} \geqslant 1.1 \mathrm{v}_{\mathrm{MC}}$

where $\mathrm{V}_{2}$ min must not be less than $1.2 \mathrm{~V}_{\mathrm{S}}$ ' except in the case of aircraft with four power units where the application of power results in a significant reduction of the one-engine-inoperative power-on stalling speed (in this case: $\mathrm{v}_{2}$ min $=1.15 \mathrm{v}_{\mathrm{S}}$ ). This requirement is intended to ensure an adequate safe climbout with the critical engine inoperative. $v_{2}$ may be increased relative to the minimum values in order to improve the climb gradient with a failed engine ("overspeed"). It should be noted that during the airborne phase $\mathrm{dV} / \mathrm{dt}$ must not be negative at any point.

THE ALL-ENGINES SCREEN SPEED $v_{3}$ is the airspeed attained at the $35 \mathrm{ft}$ height point with all engines operating. Since rotation of the airplane is initiated at $V_{R^{\prime}}$ generally determined by the engineout case, $v_{3}$ is greater than $v_{2}$ by an amount depending on the $\mathrm{T} / \mathrm{W}$ ratio. For instance, $v_{3}=v_{2}+10 \mathrm{kts}\left(\mathrm{v}_{2}+5 \mathrm{~m} / \mathrm{s}\right)$ is a typical value (not a requirement). THE LIFTOFF SPEED (or touchoff speed) $v_{\text {LOF }}$ is the airspeed at which the aircraft

first becomes airborne. This speed is determined by the rotation speed $V_{R}$ and the piloting technique during rotation. A positive climb gradient potential (out of ground effect) must be present at $V_{L O F}$. THE ROTATION SPEED $V_{R}$ is the reference speed for the pilot at which he raises the nosewheel. The broad requirement is:

$v_{R} \geqslant v_{1}$

$\mathrm{v}_{\mathrm{R}} \geqslant 1.05 \mathrm{v}_{\mathrm{MC}}$

In addition, $V_{R}$ must be chosen such that 
$\mathrm{v}_{2}$ is reached at $35 \mathrm{ft}$, taking into account the speed increment between $V_{R}$ and $v_{2}(\Delta V)$. If the airplane is rotated at its maximum practicable rate of rotation, $v_{\text {LOF }}$ must not be less than $1.10 \mathrm{~V}_{\mathrm{MU}}$ in the allengine operating condition, or $1.05 \mathrm{~V}_{\mathrm{MU}}$ in the one-engine-out case.

At very low $T / W$ ratios, $V_{R}$ and $v_{L O F}$ may be increased in order to obtain a positive climb gradient potential at $\mathrm{V}_{\text {LOF }}{ }^{*}$. However, when we have

$\Delta \mathrm{C}_{\mathrm{uc}}<\mathrm{r}_{\mathrm{I}_{\min }} \mathrm{C}_{\mathrm{L}_{2}}$ (approximately)

it can be shown that the second segment climb requirement* is more critical. This is the usual condition.

THE DECISION SPEED $v_{1}$. The procedure explained in Section $\mathrm{K}-1$, leading to the BFL, results in a critical power failure speed $V_{x}$. The pilot will need time to recognize the failure and to decide whether to abort or continue the takeoff. During this decision time (typically 1 second for a test pilot) the airplane accelerates to a speed, called the decision speed $v_{1}$. However, once the rotation is initiated, the pilot must continue the takeoff when an engine fails, hence: $v_{1} \leqslant v_{R^{\prime}}$, while $v_{1}$ must also be at or above $V_{M C}$ on the ground.

For the aircraft in Fig. $\mathrm{K}-3$ the interrelation between the various characteristic speeds is depicted as a function of the $\mathrm{T} / \mathrm{W}$ ratio, both for the all-engine case and the one-engine-out case. The following observations apply fairly generally to aircraft with adequate one-engine-out directional control properties:

a. For moderate $T / W, V_{2}$ is usually equal to $\mathrm{V}_{2}$ min . The margin relative to $\mathrm{V}_{\mathrm{MC}}$ is generally not critical, provided the vertical tailplane design is adequate.

b. For high $T / W$ ratios (low weight) $V_{2}$ is determined by the required margin relative to $\mathrm{V}_{\mathrm{MC}}$, and consequently $\mathrm{v}_{2} / \mathrm{V}_{\mathrm{S}}, \mathrm{v}_{3} / \mathrm{V}_{\mathrm{S}}$ and the field length increase. This situation

* An explanation and summary of the climb requirements can be found in Section 11.6.2. is typical for a twin-engine aircraft with wing-mounted engines.

c. For very low $T / W$ ratios (hot and high airfields) $v_{R}, v_{L O F}$ and $v_{2}$ may be increased up to the point where $V_{2}$ equals the speed for maximum climb gradient, in order to meet the climb gradients required. d. At a particular $\mathrm{T} / \mathrm{W}$ ratio $\mathrm{v}_{1}$ becomes equal to $V_{R}$; for $T / W$ ratios below this value, the one-engine-out takeoff is always critical and thus the field length is not balanced.

e. In practical takeoff calculations the engine-out case determines the reference speeds, particularly $v_{R}$ which is equal for both the all-engines and the one-engineout case. It is therefore appropriate to analyze the engine failure case prior to the all-engines takeoff.

\section{K-3. PROCEDURE FOR DETERMINING THE TAKEOFF} FIELD LENGTH

In what follows it is assumed that the ambient conditions (temperature, pressure altitude), aircraft weight and position of the high-lift devices are specified. Aerodynamic data (lift curve, drag polar) are assumed to be known, as well as the effects of ground proximity on these characteristics. Runway gradients and wind effects are usually ignored in pre-design calculations.

Steps 1 through 17 of the following procedure apply to the engine failure case, except when otherwise specified. Various methods of analysis are given in section $\mathrm{K}-4$. 1. Calculate $\mathrm{V}_{S^{\prime}}, \mathrm{V}_{2}$ min
minimum allowable $\mathrm{V}_{2}$.

2. Check the second segment climb gradient for $V=v_{2_{\text {min }}}$ by means of WAT curves (Section 11.6 .2$.$) . If necessary: increase v_{2}$ to the airspeed at which this requirement is satisfied.

3. If even the climb gradient at the optimum speed for maximum climb gradient $\mathrm{V}_{\gamma_{\max }}$ is too low, reduce the flap deflection angle, if possible, or the takeoff 
weight.

4. Calculate the airborne distance $\left(\mathrm{S}_{\mathrm{A}}\right)_{\mathrm{N}-1}$ and speed increment $\left(\Delta_{2} \mathrm{~V}\right)_{\mathrm{N}-1}$ for several values of $\mathrm{V}_{\text {LOF }}$. Ground effect, undercarriage retraction initiated 3 seconds after liftoff, and extra drag due to engine failure must be taken into account (see Appendix G) .

5. Determine $v_{\text {LOF }}$ for which $v_{\text {LOF }}+\left(\Delta_{2} V_{N-1}\right.$ $=\mathrm{V}_{2}$ and the corresponding air distance.

6. Check the first segment climb potential (Section 11.6.2.) and increase $\mathrm{V}_{\text {LOF }}$ and $\mathrm{v}_{2}$ if necessary. Alternatively, the flap angle may be reduced or the takeoff weight decreased, and the procedure is then started afresh.

7. Calculate the speed increment $\left(\Delta_{1} \mathrm{~V}\right)_{\mathrm{N}-1}$ and distance travelled $\left(\mathrm{S}_{\mathrm{R}}\right)_{\mathrm{N}-1}$ during rotation prior to liftoff and $V_{R}=V_{L O F}{ }^{-\left(\Delta_{1} V / N-1\right.}$ A normal rate of rotation is assumed. Make sure that $V_{R}$ has an adequate margin to $\mathrm{V}_{\mathrm{MC}}$. Increase $\mathrm{v}_{\mathrm{R}}$ to 1.05 times $\mathrm{V}_{\mathrm{MC}}$ if necessary.

8. Analyze the rotation phase for the maximum practicable rate of rotation, both for the all-engines-operating and the engine failure case and make sure that $V_{\text {LOF }}$ has an adequate margin relative to $\mathrm{V}_{\mathrm{MU}}$. If this is not so, increase $V_{R}$.

9. After the final choice of $V_{R^{\prime}}$ the (normal) rotation phase and airborne distances may be analyzed, resulting in final values of $\left(\mathrm{S}_{\mathrm{R}}\right)_{\mathrm{N}-1}, \mathrm{v}_{\mathrm{LOF}},\left(\mathrm{S}_{\mathrm{A}}\right)_{\mathrm{N}-1}$ and $\mathrm{v}_{2}$.

10. Choose several values for $v_{x} \geqslant V_{M C}$ on the ground, e.g. $V_{x}=908,958$ and 1008 of $\mathrm{V}_{\mathrm{R}}$.

11. Determine the ground run from standstill to $\mathrm{v}_{\mathrm{x}}\left(\mathrm{S}_{\mathrm{O}-\mathrm{x}}\right)$ for all values of $\mathrm{v}_{\mathrm{x}}$ with all engines operating.

12. Determine the ground run from $v_{x}$ to $v_{R}\left(S_{X-R}\right)$ for all values of $v_{x}$ with one engine inoperative. A realistic assumption should be made concerning the decay of thrust with time after engine failure. It is preferable to use the engine manufacturer's data, if available.

13. Determine the takeoff distance with engine failure at $\mathrm{v}_{\mathrm{x}}$ :

$\mathrm{S}_{\mathrm{N}-1}=\mathrm{S}_{\mathrm{O}-\mathrm{x}}+\mathrm{S}_{\mathrm{X}-\mathrm{R}}+\left(\mathrm{S}_{\mathrm{R}}\right)_{\mathrm{N}-1}+\left(\mathrm{S}_{\mathrm{A}}\right)_{\mathrm{N}-1}$ for the chosen values of $\mathrm{V}_{\mathrm{X}}$ and plot $\mathrm{S}_{\mathrm{N}-1}$ vs. $\left(v_{x} / v_{S}\right)^{2 *}$.

14. Determine the distance to come to a standstill from $v_{x}$ : $S_{\text {STOP }}$.

15. The accelerate-stop (emergency) distance,

$\mathrm{s}_{\mathrm{AS}}=\mathrm{s}_{\mathrm{O}-\mathrm{x}}+\mathrm{s}_{\mathrm{STOP}}$

is computed for the chosen values of $\mathrm{V}_{\mathrm{x}}$ and plotted vs. $\left(\mathrm{v}_{\mathrm{x}} / \mathrm{v}_{\mathrm{S}}\right)^{2 *}$.

16. The intersection of $\mathrm{S}_{\mathrm{N}-1}$ and $\mathrm{S}_{\mathrm{AS}}$ defines the critical power failure speed and the BFL (Fig. $\mathrm{K}-2$ ).

17. Calculate $\mathrm{V}_{1}$ and make sure that $\mathrm{V}_{1} \leqslant \mathrm{~V}_{\mathrm{R}}$. If this is not so, the condition of engine failure one second prior to $V_{R}$ determines the field length required with engine failure.

18. Analyse the rotation phase in the allengine takeoff, assuming $v_{R}$ equal to the one-engine-out case. The distance travelled is $\left(S_{R}\right)_{N}$, and $v_{\text {LOF }}$ follows from the speed increment $\left(\Delta_{1} \mathrm{~V}\right)_{\mathrm{N}}$.

19. Analyze the airborne phase in the allengine takeoff. The distance travelled is $\left(\mathrm{S}_{\mathrm{A}}\right)_{\mathrm{N}}$ and the speed increment $\left(\Delta_{2} \mathrm{~V}\right)_{\mathrm{N}}$. 20. The factored takeoff distance is:

$\mathrm{S}_{\mathrm{N}}=1.15\left[\mathrm{~S}_{\mathrm{O}-\mathrm{R}}+\left(\mathrm{S}_{\mathrm{R}}\right)_{\mathrm{N}}+\left(\mathrm{S}_{\mathrm{A}}\right)_{\mathrm{N}}\right]$

21. The takeoff field length required is the greater of the BFL and $\mathrm{S}_{\mathrm{N}}$.

K-4. METHODS AND DATA FOR THE ANALYSIS OF THE TAKEOFF

The following methods are generally used in the preliminary design stage, when the data required are not sufficiently complete to warrant a detailed analysis.

$\mathrm{K}-4 \cdot 1$. The ground run from standstill to $\mathrm{v}_{\mathrm{x}}$

The distance required to accelerate the aircraft from standstill to $\mathrm{v}_{\mathrm{x}}$ is:

${ }^{*} \mathrm{~S}_{\mathrm{N}-1}$ and $\mathrm{S}_{\mathrm{AS}}$ vs. $\left(\mathrm{V}_{\mathrm{x}} / \mathrm{V}_{\mathrm{S}}\right)^{2}$ are almost linear relationships 
$s_{0-x}=\int_{0}^{v_{x}} \frac{v}{a} d v=\frac{1}{2 g} \int_{0}^{v^{2}} \frac{d v^{2}}{a / g}$

Assuming the thrust vector and the runway to be horizontal, it can be shown that:

$\frac{a}{g}=\frac{d V / d t}{g}=\frac{T}{w}-\mu-\left(C_{D_{G}}-\mu C_{L_{G}}\right) \frac{\frac{1}{3} \rho v^{2} S}{W}$

For an arbitrary relationship of engine thrust with speed, the integral in $(\mathrm{K}-8)$ may be solved numerically. Numerous methods have been developed in the literature to simplify this process (e.g. Refs. K-1 through $\mathrm{K}-13$ ). A well-known approximation is: $\mathrm{T}=$ constant $=\overrightarrow{\mathrm{T}}$. In that case substitution of $(K-9)$ into $(K-8)$ results in an integral, which can be solved analytically:

$S_{0-x}=\frac{v_{x}{ }^{2}}{2 g(\bar{T} / w-\mu)} \cdot \frac{1}{a} \ln \left(\frac{1}{1-a}\right)$

where $a=\frac{C_{D_{G}}-\mu C_{L_{G}}}{(1.13) C_{L_{\max }}(\bar{T} / W-\mu)}\left(\frac{V_{x}}{V_{S}}\right)^{2}$

Coefficients of rolling friction are fundamentally a function of tire pressure and ground speed; good average values are: hard runways (concrete, tarmac): $\mu=.02$ hard turf, gravel $: \mu=.04$ short, dry grass long grass soft ground $: \mu=.05$ : $\mu=.10$ $: \mu=.10$ to $\cdot 30$

The mean thrust $\overline{\mathrm{T}}$ is defined at a mean velocity given by $\mathrm{Fig} . \mathrm{K}-4$. When the numerical procedure is used instead, the thrust lapse with speed is obtained from the engine manufacturer's brochure supplemented by a propeller diagram in the case of propeller-powered aircraft (cf. Section 6.3.2.). Alternatively, Fig. 4-35 may be used for jet engines as a first approximation. The angle of incidence is assumed invariable during the ground roll for nosewheel aircraft and is specified by the three-point ground attitude. Note that during the roll $C_{L}$ and $C_{D}$ are affected by the ground effect. For tailwheel aircraft an optimum attitude is defined by the condition that the accel-

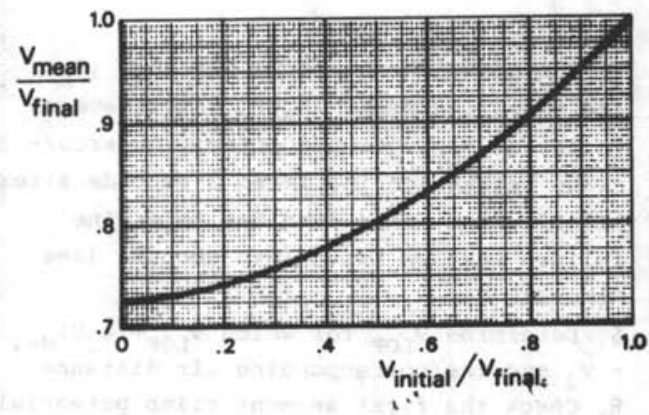

NOTE

$\mathrm{V}_{\text {mean }}=$ speed at which the mean thrust should be calculated for distance estimation using constant thrust during the takeoff run

Fig. K-4. Mean speed for estimating the distance required to accelerate from a given initial speed to a given final speed

eration has a maximum when:

$C_{D_{G}}-\mu C_{L_{G}}=C_{D_{0}}-\frac{1}{4} \mu^{2} \pi A e$

for $C_{L_{G}}=\frac{1}{2} \mu \pi A e$

The ground effect can be estimated from the data given in Appendix G, Section G-7.

$\mathrm{K}-4.2$. The ground run from $\mathrm{V}_{\mathbf{x}}$ to $\mathrm{V}_{\mathbf{R}}$

A precise analysis of the motion of the aircraft after engine failure is complicated by several factors which affect the variation of external forces with time (see Fig. $\mathrm{K}-5$ ).

a. Immediately after engine failure, the thrust decays in a finite time (typically 4 seconds) to zero or idling thrust.

b. Engine failure causes windmilling drag of the dead engine and extra drag due to the asymmetric flight condition. Additional drag is also created in the rotation and flare maneuvers, and this is considerably affected by the piloting technique. The download on the tailplane must be compensated for by extra wing lift, resulting in increased induced drag. The ground effect gradually decreases after liftoff; hence the induced drag increases. Retraction of 


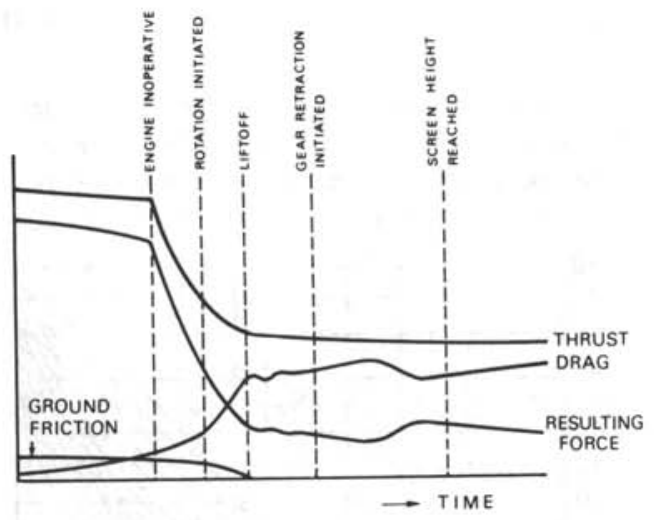

Fig. K-5. Forces on the airplane during the takeoff with engine failure

the undercarriage, initiated 3 seconds after liftoff, results in another drag variation.

c. Ground friction drag is related to the lift and vanishes at liftoff. The calculated total force component in the direction of the flight path has an irregular shape, but the variation may not be observed in practice due to the dynamic character of the motion.

d. Another complication is that, especially on large aircraft, the motion of the lowest point, and not just the center of gravity, must be observed. During the rotation phase prior to liftoff, and immediately after, a check should be made to see whether the fuselage tail has adequate clearance to the ground. When the screen is passed, the total height gain of the c.g. during the airborne phase may be considerably more than $35 \mathrm{ft}$ for large aircraft.

Equation (K-8) applies to the acceleration phase from $V_{X}$ to $V_{R^{\prime}}$ provided the integration is carried out from $V_{x}$ to $V_{R}$. The following analytical expression can be used instead of a numerical procedure:

$S_{x-R}=\frac{v_{x}^{2}}{2 g(\bar{T} / W-\mu)} \cdot \frac{1}{a} \ln \left[\frac{1-a}{1-a\left(v_{R} / v_{x}\right)^{2}}\right]$
It is noted that $\overline{\mathrm{T}}$ and "a" have different values from the previous case, due to the thrust reduction and drag increment after engine failure. The velocity for which $\bar{T}$ is to be determined can be obtained from Fig. K-4.

\section{$\mathrm{K}-4.3$. The rotation phase}

Assuming the mean rate of rotation about the lateral axis equal to $(d \theta / d t)_{R}$ and the mean acceleration along the runway conservatively equal to the value at liftoff, we have:

$S_{R}=\frac{1}{2}\left(v_{R}+v_{L O F}\right) \frac{\alpha_{L O F}-\alpha_{G}}{(d \theta / d t)_{R}}$

and

$V_{L O F}=V_{R}+\Delta_{1} V=V_{R}+g\left(\frac{T-D}{W}\right)_{L O F} \frac{{ }^{\alpha}{ }_{L O F}{ }^{-\alpha}{ }_{G}}{(d \theta / d t)_{R}}$

In view of the short duration of the rotation phase ( 2 to 4 seconds), this simple approximation will be acceptable in the pre-design stage. Typical values for $(d \theta / d t)_{R}$ are quoted in Section 10.3.1. The liftoff angle of attack is found from the $\mathrm{C}_{\mathrm{L}}-\alpha$ curve in proximity to the ground.

\section{$\mathrm{K}-4,4$. The airborne phase}

A detailed study of a particular takeoff problem will usually involve a numerical calculation of the step-by-step type, assuming a control law for the lift coefficient, the angle of attack or pitch, or the elevator deflection. The problem with these methods is to define an adequate control law, which usually has to be based on previous experience with similar types of aircraft.

Many attempts have been made to develop analytical methods for simple control laws, such as taking $C_{L}=$ constant or assuming the flare-up to be a circular arc with $\mathrm{n}=\mathrm{L} / \mathrm{W}=$ constant. We shall quote two representative examples here:

a. The AGARD Flight Test Handbook method (Ref. K-9), where the equations of motion are linearized and solved analytically for 
$C_{L}=C_{L-L O F}+\Delta C_{L}=$ constant and $(T-D)=$ constant. The flare becomes a sector of a phugoid for this particular case. The method gives the height, speed and distance travelled vs. time after liftoff. However, the guidelines for choosing $\Delta C_{L}$ are not very practical for civil aircraft. The author's experience is that the airborne distance is reasonably well predicted for $\Delta \mathrm{C}_{\mathrm{L}} / \mathrm{C}_{\mathrm{L}-\mathrm{LOF}}=0$ to .05 for the $\mathrm{N}-1$ takeoff, and for $\Delta C_{L} / C_{L-L O F}=.10$ to .15 when all engines are operating.

b. The method developed by Perry (Ref. K12) uses a constant rate of pitch after liftoff: $(d \theta / d t)_{A}$. The equations of motion are linearized by assuming $\mathrm{V}=$ constant and $(T-D)=$ constant. The advantage of this method is that the control law is representative for practical civil aircraft piloting techniques, while - contrary to $C_{L}$ - the pitch angle can be directly observed. As the speed increment after liftoff is ignored in solving the equation of motion normal to the flight path, the shape of the flare-up may not be realistic for high $\mathrm{T} / \mathrm{W}$ ratios.

The method devised by Perry may be summarized as follows:

1. The airborne distance is composed of a flare-up, during which the flight path angle $\gamma$ is increased from zero* to $\gamma_{2}$ at $v_{2}$ (or $\gamma_{3}$ at $V_{3}$ ) and a phase with constant climb angle $\gamma_{2}$ or $\gamma_{3}$.

2. The height gain after liftoff is given by :

$h=\frac{V_{L O F}{ }^{2}}{g} \frac{T-D}{W} F(\dot{\theta}) F(h)$

where

$F(\dot{\theta})=1+\frac{V_{L O F}}{2 g} \frac{W}{T-D} n_{\alpha}\left(\frac{d \theta}{d t}\right)_{A}$

and

* The actual climb angle of the c.g. at liftoff is of the order of a half to one degree, due to the extension of the undercarriage and rotation.
$\mathrm{n}_{\alpha}=\frac{\mathrm{dc}_{\mathrm{L}} / \mathrm{d} \alpha}{\mathrm{C}_{\mathrm{L}_{\mathrm{LOF}}}}$

In these equations $(d \theta / d t)_{A}$ is in $\mathrm{rad} / \mathrm{sec}$ and, $\mathrm{dC}_{L} / \mathrm{d} \alpha$ is in $\mathrm{rad}^{-1} . \mathrm{F}(\mathrm{h})$ is a non-dimensional height function, reproduced in Fig. $K-6$. The mean value of $(T-D)$ may be

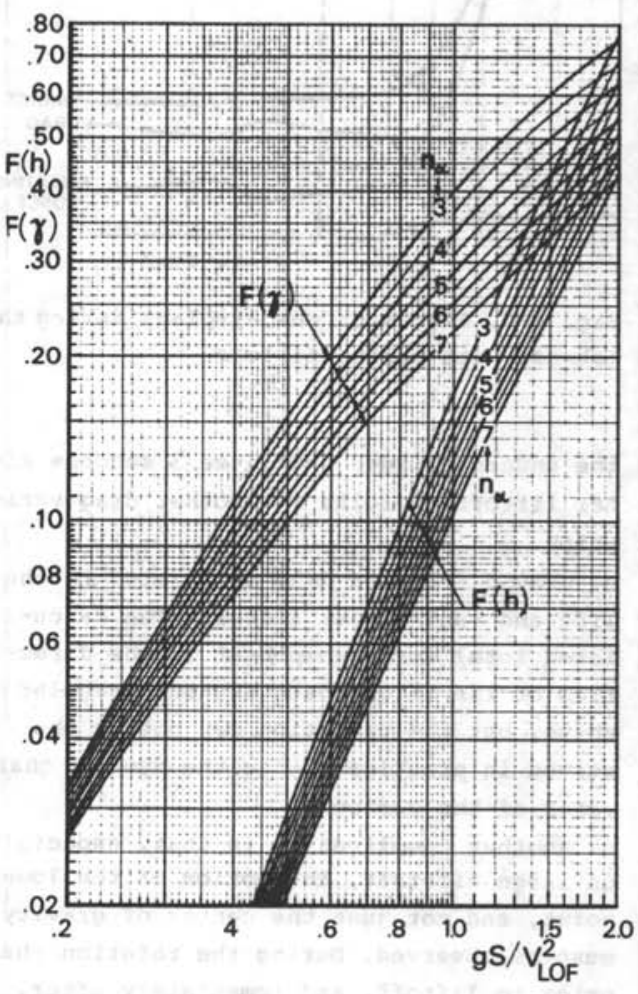

Fig. $\mathrm{K}-6$. The functions $F(h)$ and $F(\gamma)$ used in Perry's method for the analysis of the airborne path (Derived from Ref. K-12)

taken as being halfway the airborne distance. A provisional assumption may be made for $(d \theta / d t)_{A^{\prime}}$ for instance $1 \% / \sec (d \theta / d t=$ .018 ) for the case with engine failure, or $2 \% / \mathrm{sec}(\mathrm{d} \theta / \mathrm{dt}=.035)$ for the all-engine case.

3. The climb angle during the transition is given by:

$\gamma=\frac{d h}{d S}=\frac{T-D}{W} F(\dot{\theta}) F(\gamma)$

$(\mathrm{K}-19)$

where $F(\gamma)$ is a non-dimensional flight path 
angle function depicted in Fig. $\mathrm{K}-6$.

4. The end of the transition is defined by

$F(\gamma)=\frac{\gamma_{2}}{\frac{T-D}{W} F(\dot{\theta})}$

In the all-engine case $\gamma_{3}$ is used instead of $\gamma_{2}$. Using Fig. $\mathrm{K}-6$ both the height and distance travelled at the end of the flareup are found from this condition. If this height is less than $h_{\text {to }}=35 \mathrm{ft}(10.7 \mathrm{~m})$, an additional phase must be added with climb angle $\gamma_{2}$ or $\gamma_{3}$. If the takeoff height is reached before the end of the transition, $F(h)$ is found by substitution of $h_{\text {to }}$ into $(\mathrm{K}-16)$ and the airborne distance is obtained from Fig. $\mathrm{K}-6$.

5. A check on $(d \theta / d t)_{A}$ can be made by assuming that during the flare-up the pitch angle increases linearly from $\alpha_{\text {LOF }}$ to the pitch angle at $v_{2}$ in stationary flight:

$\left(\frac{\mathrm{d} \theta}{\mathrm{dt}}\right)_{\mathrm{A}}=\frac{\mathrm{v}_{\text {LOF }}\left[\alpha_{\mathrm{V}_{2}}{ }^{-\alpha_{\text {LOF }}}+\gamma_{2}\right]}{\text { transition distance }}$

For the all-engine case $v_{3}$ and $\gamma_{3}$ are used instead of $v_{2}$ and $\gamma_{2}$.

6. The speed increment after liftoff is obtained from the eneryy equation:

$\Delta_{2} V=\frac{g S}{V_{L O F}}\left(\frac{T-D}{W}-\frac{h}{S}\right)$

where $\mathrm{h}$ and $\mathrm{S}$ are the height and distance travelled after liftoff at the end of the flare-up, or at the $35 \mathrm{ft}(10.7 \mathrm{~m})$ height point, whichever is less. It should be noted that $\gamma_{2}$ and $\gamma_{3}$ can only be calculated when $v_{2}$ and $v_{3}$ are known. The calculation is therefore iterative.

\section{K-4.5. The stopping distance}

The velocity after engine failure is depicted in Fig. $\mathrm{K}-7$ as a function of time. Initially an appreciable overshoot is observed due to the still considerable thrust of the inoperative engine immediately after failure. Time delays are necessary to allow for failure recognition and decision (1 second) and subsequent operation of the

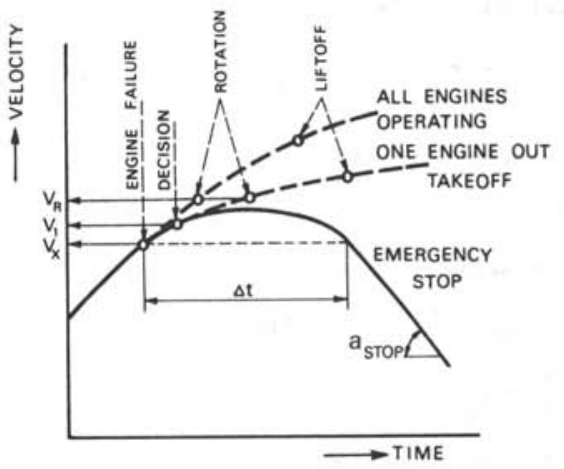

Fig. K-7. Time history of the airspeed during the takeoff

wheelbrakes, throttle closure, lift dumpers and airbrakes.

Integration of the velocity yields the emergency brake distance

$\mathrm{S}_{\text {STOP }}=\mathrm{v}_{\mathrm{x}} \Delta \mathrm{t}+\frac{1}{2 \mathrm{~g}} \int_{\mathrm{v}_{\mathrm{x}}}^{\circ} \frac{\mathrm{dV}}{\mathrm{a} / \mathrm{g}}$

where $\Delta t$ is approximately 3 to 4 seconds. In Fig. $K-1 v_{x} \Delta t$ is referred to as the inertia distance.

In principle, the equation of motion during braking is similar to $(\mathrm{K}-9)$ for the acceleration phase. However, consideration must be given to the fact that the retarding ground force mainly acts at the main undercarriage, as the nosewheel is usually not provided with brakes.

The deceleration during the steady braked roll can be derived from the conditions of equilibrium (Fig. K-8) :

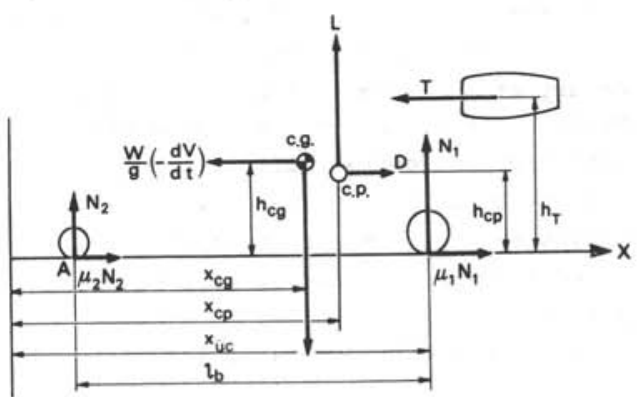

Fig. K-8. Forces on the aircraft during the braked roll 
horizontal forces:

$\frac{W}{g} \frac{d V}{d t}+D-T+\mu_{1} N_{1}+\mu_{2} N_{2}=0$

forces normal to the runway:

$L-W+N_{1}+N_{2}=0$

and the moment about point $\mathrm{A}$ :

w $\left\{1_{b}-\left(x_{u c}-x_{c g}\right)\right\}-L\left\{1_{b}-\left(x_{u c}-x_{c p}\right)\right\}-$

$\frac{\mathrm{W}}{\mathrm{g}}\left(-\frac{\mathrm{dV}}{\mathrm{dt}}\right) \mathrm{h}_{\mathrm{cg}}+\mathrm{Dh} \mathrm{h}_{\mathrm{cp}}-\mathrm{T} \mathrm{h}_{\mathrm{T}}-\mathrm{N}_{1} \mathrm{I}_{\mathrm{b}}=0$

In the absence of nosewheel braking we take $\mu_{1}=\mu$ and $\mu_{2}=0$, resulting in the following expression for the deceleration: $\frac{\mathrm{a}}{\mathrm{g}}=\frac{-\mathrm{dV} / \mathrm{dt}}{\mathrm{g}}=\mathrm{A}_{1} \mu+\frac{\mathrm{A}_{2} \mathrm{C}_{\mathrm{D}_{\mathrm{G}}}-\mathrm{A}_{3} \mu \mathrm{C}_{\mathrm{L}_{\mathrm{G}}}}{(1.13) \mathrm{C}_{\mathrm{L}_{\max }}}\left(\frac{\mathrm{V}}{\mathrm{V}_{\mathrm{S}}}\right)^{2}-\mathrm{A}_{4} \frac{\mathrm{T}}{\mathrm{W}}$

where

$A_{1}=\frac{1_{b}-\left(x_{u c}-x_{c g}\right)}{1_{b}+\mu h_{c g}} ; A_{2}=\frac{1_{b}+\mu h_{c p}}{1_{b}+\mu h_{c g}} \approx 1 ;$

$A_{3}=\frac{1_{b}-\left(x_{u c}-x_{c p}\right)}{1_{b}+\mu h_{c g}} ; A_{4}=\frac{1_{b}+\mu h_{T}}{1_{b}+\mu h_{c g}}$

If brakes are also present on the nosewheel, having the same braking effectiveness as those on the main u.c., $A_{1}$ through $\mathrm{A}_{4}$ are equal to 1 . Some data for calculating the stopping distance are given below.

a. The friction coefficient $\mu$.

1. The tyre-to-runway friction during braking is a function of the slip ratio, i.e. the relative velocity of the tire relative to the ground*. The maximum friction occurs at an optimum slip ratio and is dependent mainly upon the runway surface condition and contamination, tire pressure and the type of tread design. Typical curves for $\mu_{\max }$ vs. ground speed are presented in Fig. K-9a for several runway conditions.

2. The average pilot can achieve only 30 to

*A precise definition and background information can be found in Ref. K-31

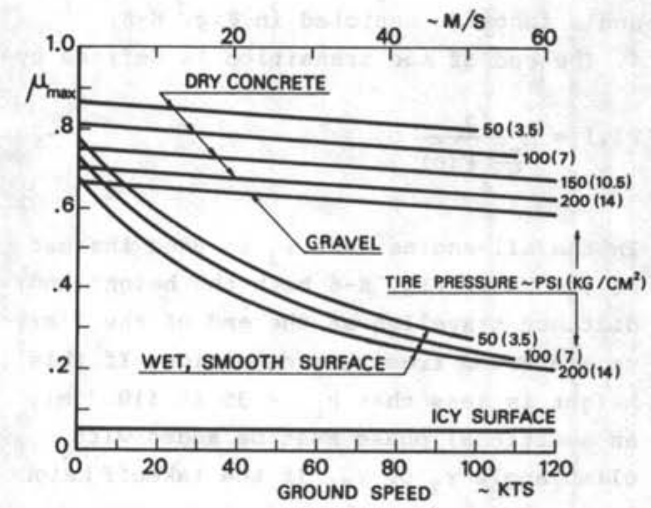

a. Typical braking coefficients of friction

Fig. K-9. Some data on braking performance

50 percent of $\mu_{\max }$ (hence $\mu \approx .35$ ) in view of his inability to maintain the optimum slip ratio. For this reason, anti-skid devices based on mechanical or electronic pressure control systems have been developed. Their effectiveness may be expressed in the form of an efficiency factor:

$n_{b}=\frac{\mu}{\mu_{\max }}$

Fig. K-9b presents typical values for $n_{b}$ '

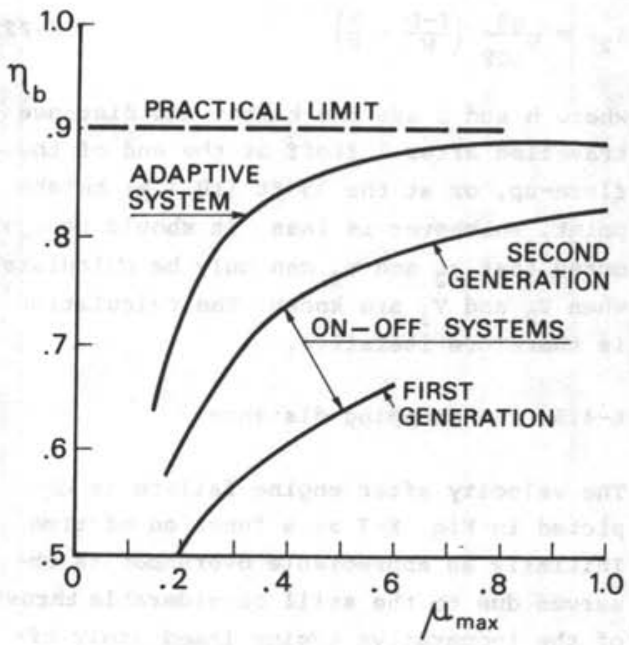

b. Typical anti-skid system efficiency

Fig. K-9 (continued) 
although in practice wide variations will be found. The simple, first-generation anti-skid systems based on the on-off principle achieve low values of $\eta_{b}$ ' of the order of .5 to .6 at low $\mu_{\max }$ (wet runways). Adaptive brake pressure control systems boost this performance considerably, and the most recent systems have a value of up to $n_{b}=.9$, almost independent of the runway condition.

3. Brake effectiveness is reduced by various dynamic effects such as normal load variation, undercarriage vibration and suspension effects. These may reduce $n_{b}$ by up to 208 (Ref. K-31).

4. The total torque obtainable from all brakes $\left(Q_{\max }\right)$ forms a limiting factor at low ground speeds on dry runways, when $\mu$ is very high. To ensure passenger comfort during normal braking the maximum torque is usually so designed that decelerations will not exceed .5 to $.6 \mathrm{~g}$ during a maximum braking effort. On light aircraft and small passenger transports a typical value for $2_{\max }$ results in a wheel braking force of $.35 \mathrm{~W}$ at zero ground speed.

5. A very large amount of heat is developed during braking and the brakes and tires may become very hot. A limit is set to the maximum heat sink capacity, which may have different values for various operating conditions (e.g. normal braking or emergency braking).

The effective friction coefficient may be obtaineu by taking the least of the following:

$\mu=.85 n_{b} \mu_{\max }$

and

$\mu=\frac{Q_{\max }}{\mathrm{N}_{1} \mathrm{r}_{t}}$

where $r_{t}$ is the radius of the deflected tire, which is a function of the tire load. In view of these characteristics, it may be generally observed that at low ground speed $\mu$ increases with speed $-\mathrm{N}_{1}$ decreases and $r_{t}$ increases, but less rapidly - while at high ground speeds the available friction is critical and decreases with speed, particularly on wet runways.

b. Engine thrust during braking. The operational program for manipulating the thrust must be chosen in accordance with the airworthiness rules. The FAR 25 requirements do not allow thrust reversal, and idle forward thrust is assumed in this case. British rules allow the use of reverse thrust under certain conditions, but the failed engine will cause asymmetry and in the pre-design stage it is wise to be conservative and neglect the reverse thrust in performance estimation.

c. Aerodynamic properties.

The deflection and effectiveness of the high-lift devices, the effect of ground proximity, the design and operation of lift dumpers (ground spoilers) and reverse thrust - particularly for propeller aircraft - are all factors affecting the lift and drag coefficients. In the absence of aerodynamic data on spoilers, their effect may be assumed to be equivalent to an increment in the mean deceleration of .08 to $.10 \mathrm{~g}$.

A simple solution of $(\mathrm{K}-23)$ can be obtained by taking $\mu=\mu_{\text {eff }}=$ constant and $\mathrm{T}=0$. The result is as follows:

$\mathrm{S}_{\text {STOP }}=\frac{\mathrm{v}_{\mathrm{x}}{ }^{2}}{2 \mathrm{~g} \mathrm{A_{1 } \mu _ { \mathrm { eff } }}} \cdot \frac{1}{\mathrm{a}} \ln \left(\frac{1}{1-\mathrm{a}}\right)+\mathrm{v}_{\mathrm{x}} \Delta t$

where $a=\frac{A_{3} \mu_{\text {eff }} C_{L_{G}}-A_{2} C_{D_{G}}}{(1.13) A_{1} \mu_{\text {eff }} C_{L_{\max }}}\left(\frac{V_{x}}{V_{S}}\right)^{2}$

An even simpler solution is found when the deceleration during braking is assumed constant:

$\mathrm{S}_{\text {STOP }}=\frac{\mathrm{v}_{\mathrm{x}}{ }^{2}}{2 \mathrm{a}_{\mathrm{STOP}}}+\mathrm{v}_{\mathrm{x}} \Delta \mathrm{t}$

The highest values obtainable for a $_{\text {STOP }}$ are typically:

$.55 \mathrm{~g}$ - dry runway, maximum effort, ignoring passenger tolerance,

$.35 \mathrm{~g}$ - wet runway, modern braking with anti-skid, lift dumpers, reverse thrust,

$.15 \mathrm{~g}$ - wet runway, simple braking; or flooded run- 
way, reverse thrust,

while $\Delta t$ is approximately $3 h$ seconds.

REFERENCES

Analysis of takeoff performance

K-1. W.S. Dreh1: "The calculation of takeoff run". NACA Technical Report No. $450,1932$.

K-2. B. Göthert: "Der Abflug von Landflugzeugen mit besonderer Berücksichtigung des Uebergangsbogens". Jahrbuch 1937 der Deutschen Luftfahrtforschung.

K-3. J.R. Ewans and P.A. Hufton: "Note on a method of calculating takeoff distances". RAE Technical Note Aero 880 (ARC 4783), 1940.

K-4. W.R. Buckingham: "A theoretical analysis of the airborne path during takeoff". Aircraft Eng., Jan. 1958, pp. 5-8.

K-5. D.J. Kettle: "Ground performance at takeoff and landing". Aircraft Eng., Jan. 1958, pp. 2-4.

K-6. A.D. Edwards: "Performance estimation of civil jet aircraft". Aircraft Eng. 1950 , pp. $70-75$.

K-7. Anon.: "Estimation of takeoff distance". R.Ae.S. Data Sheet "Performance" EG $5 / 1,1952$.

K-8. G.E. Rogerson: "Estimation of takeoff and landing airborne paths". Aircraft Eng., Nov. 1960, Pp. 328-330.

K-9. F.E. Douwes Dekker and D. Lean: "Takeoff and landing performance". AGARD Flight Test Manual, Vol. 1, Chapter 8. Pergamon Press, 1962.

K-10. L. Bournet: "Bstimation de la longueur de roulement au décollage, un problème simple parfois méconnu Technique et Science Aéronautique, 1967, pp. 213-222.

K-11. A.R. Krenkel and A. Salzmann: "Takeoff performance of jet-propelled conventional and vectored-thrust aircraft". J. of Aircraft, 1968, pp. 429-436.

K-12. D.H. Perry: "The airborne path during takeoff for constant rate-of-pitch manoeuvres". ARC CP No. $1042,1969$.

K-13. Anon.: "Estimation of takeoff distance". ESDU Data Sheets "Performance" EG 5/1, $1971 / 1972$.

K-14. J. Collingbourne: "A digital computer program (EMA) for estimating aircraft takeoff and acceleratestop distances". RAE Tech. Memo Aero, 1252, 1970.

K-15. D.H. Perry: "A review of methods for estimating the airfield performance of conventional fixed wing aircraft". RAE Tech. Memo Aero 1264, 1970.

K-16. J. Williams: "Airfield performance prediction methods for transport and combat aircraft". AGARD Lecture Series No. 56, April 1972.

K-17. R.N. Harrison: "Takeoff and climb characteristics". Short course on aircraft performance estimation, Cranfield Institute of Technology, Feb. 1973.

Braking friction, water, snow, slush and ice on runways

K-18. J.W. Wetmore: "The rolling friction of several airplane wheels and tires and the effect of rolling friction on takeoff". NACA Technical Report No. 583, 1973.

K-19. E.C. Pike: "Coefficients of friction". J. of the Royal Aero. Soc., December 1949.

K-20. M.N. Gough, R.H. Sawyer and J.P. Trant: "Tire-runway braking coefficients". AGARD Report 51, Feb. 1956.

K-21. W.B. Horne, U.T. Joyner and T.J.W. Leland: "Studies of retardation force developed on an aircraft tire rolling in slush or water". NASA TN D-552, Sept. 1960.

K-22. J.A. Zabovchik: "Ground deceleration and stopping of large aircraft". AGARD Report 231 , Oct. 1958.

K-23. W.B. Horne and J.W.L. Tafford: "Influence of tire tread pattern and runway surface conditions on braking friction and rolling resistance". NASA TN D-1376, Sept. 1962.

K-24. G. Ciampolini: "A method of evaluating runway friction for the prediction of actual takeoff runs". 
AGARD Report $418,1963$.

K-25. H,R. Herb: "Problems associated with the presence of water, slush, snow and ice on runways". AGARD Report $500,1966$.

K-26. R.L. Maltby and H.W. Chinn: "Effects of slush on takeoff". Shell Aviation News, No. 296-1963, pp. 8-11.

K-27. T.G. Foxworth and H.F. Marthinsen: "Another look at accelerate-stop criteria". AIAA Paper No. 69-772.

K-28. L.S. McBee: "Effective braking - a key to air transportation progress". SAE Paper No. 640376 .

K-29. E.G. Wilkinson: "Lift-dump system shortens landing roll on ice runways". Space/Aeronautics, Oct. 1969, pp. 73-75.

K-30. J.T. Yager: "A comparison of aircraft and ground vehicle stopfing performance on dry, wet, flooded, slush, snow and ice-covered runways". NASA TN D-6098, 1970.

K-31. Anon.: "Frictional and retardation forces on aircraft tyres". ESDU Data Sheets No. 71025 , 71026 and 72008.

K-32. J.T. Yager, W. Pelham Phillips and P.L. Deal: "Evaluation of braking performance of a light, twinengine airplane on grooved and ungrooved pavements". NASA TN D-6444, 1971.

K-33. J.L. McCarthy: "Wear and related characteristics of an aircraft tire during braking". NASA TN D6963, Nov. 1972 .

STOL - takeoff performance

K-34. P.L. Sutcliffe, V.K. Merrick and A.R. Howell: "Aerodynamics and propulsion of minimum field aircraft". Proc. 8th Anglo-American Aeron. Conf., 181-232, London, 1961.

K-35. J. Hamann: "Contribution à la définition d'un avion léger SToL". Jahrbuch 1965 der WGLR, pp. $133-140$.

K-36. F.H. Schmitz: "Takeoff trajectory optimization of a theoretical model of a STOL aircraft". AIAA Paper No. 69-935.

K-37. D.O. Carpenter and P. Gotlieb: "The physics of short takeoff and landing (STOL)", AIAA Paper No. $70-1238$.

K-38. R.K. Ransone: "STOL definition and field length criteria". AIAA Paper No. 70-1240. 



\section{Index}

Accelerate-stop distance, 167, 168, 575, 579

Accelerated climb, 375, 379

Accommodation, 63,68

Acoustic

fatigue, 51

lining, 179

treatment, 130

Acrobatic (category), 20, 271

Activity Factor, 192, 202, 203, 204

Adaptive brake pressure control, 585

Additional lift (distribution), 473, 493

Advance ratio, 191, 194

Aerodynamic center, 57, 208, 232, 317, 437, 441, $476,477,479,480,481,542$

Aeroelastic effect, 218, 251, 306, 316, 469, 540

Aft-fan, 116

Afterbody drag, 66, 503

Agricultural aircraft, 25, 197

Aileron, $234,236,257$

control, 232

control power, 231

deflection, 332

design, 257, 332

high-speed, 257

reversal, 218,257

Air (borne) distance, 167, 170, 575, 579, 582 phase, 581

Air

maneuver time, 385

mass flow, 563, 565, 568

Navigation Regulations, 22

spring, 360

supply, 213

Taxi Operator, 22

Traffic Control, 159

Airbrake, 258, 377

Airconditioning, $62,67,69,93,194$

group weight, 292

system, $110,126,213,343$

Aircraft

category, 20

design and development, 3

growth, 186, 226

Prepared for Service, Items, 269

project design, 2

Weight, 270

Aircushion, 343

Airduct, 449

Airfield performance, 14, 172, 173, 217, 219, 233, 380,381

Airflow capacity, 289

Airfoil, 206, 231, 251, 438

chord, 437, 530
Airfoil (continued)

(nose) geometry, 231, 250, 257, 436, 437, 471, 548

section, $143,218,228,229,237,241,243,249$, $327,339,436,450,499,543$

selection, 237

shape, 191, 236, 327

Airframe

services and equipment, 9, 16, 268, 269, 286, 297,300

installations, drag due to, 417

structure, 268, 269, 277, 278

Airfreight, $80,81,82,84$

Airworthiness regulations/requirements, 6, 19, 81, $160,169,227,268,275,373,378$

Aisle, $38,67,71,76$

Alighting gear group, 282

All-engines screen speed, 577

All flying tail, 327

A11-Up Weight, 174, 219, 223, 225, 226, 271, 293

Anderson's method, 238, 473, 474, 493

Angle of attack, 191, 192, 205, 208, 228, 229, 231, $232,242,247,350,440,443,450,477,547$

Angle of attack

critical, $228,229,231,355,450,459,547,549$

for maximum 1ift, 228, 229, 231, 252, 256

for zero lift, $239,437,443,470,475,529$

Anhedral (angle of), 258, 259, 439

Anti-balance tab, 315, 327

Anti-icing, 194, 262, 293

group weight, 292

Anti-shock body, 241

Anti-skid system, 584,585

Anti-spin fillet, 339

Approach

climb, 379

configuration, 155,379

glide, $234,381,179$

speed, $15,170,381$

Aquaplaning, 359, 360

Area of vision, 89

Area rule, 6, 67

A-series airfoil section, 239

Aspect ratio, 53, 56, 57, 143, 148, 155, 160, 171, $180,208,217,218,222,225,229,232,233$, $234,235,236,240,251,326,332,339,439$, 555

Asymmetry, thrust/power, 44, 184, 333, 555, 556

ATA-method, 383,384

Atmospheric properties, 223, 571, 572

Autopilot, 259, 283, 331

Auxiliary

flap, 532, 533, 534 
Auxiliary (continued)

inlet doors, 207

Power Unit, $62,183,213,288,289,397$

Avionics, 289, 397

Balanced Field Length, 167, 169, 176, 183, 575

Balancing

aerodynamic, 313,314

tab, 307, 315, 327

Ballast, 354

Bank, angle of, $332,350,352$

Base drag, 150, 491, 503

Baseline configuration, 18, 145

design, 7,8

development, $5,6,8,18$

Basic

Empty Weight, 267, 269, 270

lift (distribution), 473, 474, 493

Operational Weight, 270

Weight, 270

Baulked landing, 379

BCAR, $19,22,23,25$

Beaver tail, 87,93

Belly, 82

flattening, $37,38,67,71,73$

freighthold, 79

Bernoulli's equation, 242, 246

Beta control, 137, 190, 191, 195, 198, 199

Birdproof windshield, 25

Bladder tank, 448

Blade angle control, see Beta control

Bleed air, 126, 190, 194, 289, 377, 563

Blister drag, 514

Block

speed, $12,13,375,384$

time, $158,378,385$

Boattail drag, 504, 507, 508

Booster engine, 130

Boundary layer, $66,88,149,208,255,368,369$, $490,497,498$

control, 252

laminar, 229, 239

separation, $153,208,231,368,369,490$

transition, 451, 499

turbulent, $145,229,231$

velocity profile, 497

Bound vortex, 551, 552

Box beam wing structure, 260, 261, 395

Braced wing, 36, 233, 281, 453

Brake, 54, 210, 342, 358, 359, 538

energy, 359

Horse Power, 103

Mean Effective Pressure, 103

pressure control, 169, 585

Braking, $68,343,382$

coefficient of friction, 579, 584, 585

thrust of propeller, 213

Brayton cycle, 113

Break even load factor, 382

Bréguet's range equation, 145, 157, 159

Bubble, 229, 230, 231, 535

Buffet, $158,218,225,228,232,243,245,249$, 373

boundary, 225, 245, 246, 249

margin, 219, 246, 249

onset, 225

penetration, 225,245

Bulkhead, 74, 87, 395, 396

pressure, weight of, 462

Bullet (weight), 281

Buried engine, 42, 46, 48, 57, 151, 206, 395, 516

Business aircraft, 76

Butterfly tail, 51
Bypass

engine, 99, 112, 116, 133

ratio, $117,120,125,129,132,133,134,171$, $183,207,565,566$

Cabin

arrangement, $15,80,390$

attendant, 81,92

design, 69

dimension, $71,74,76,77$

door, $76,78,87,205$

floor, $37,68,71,73,377$

furnishing, 71, 75

layout, $74,297,300$

noise, 68,204

passenger, $67,68,71,79,80,81,82,84,397$

pressure (differential), 6, 68, 71, 78, 87, 374, 459

rate of climb/descent, 375,377

volume, 69

wall thickness, 68,71

California Bearing Ratio, 344

Camber, $87,248,436,437,471,541$

rear fuselage, 87, 94, 479, 504, 505

tailplane, 326, 327

wing, 222, 238, 239, 244, 250, 252, 492, 549

Canard aircraft, 56, 58, 306

Cancellation speed, 210,382

Canopy drag, 513

Cantilever

ratio, 235, 236, 251, 281

wing, $233,236,280,453$

Capacity payload, $12,17,266,272$

Carbureted engine, 106

Cargo, 67, 78, 82, 84, 298, 299

aircraft, 67,81

compartment/hold, 69, 72, 73, 78, 79, 205, 299, 300,397

loading, $67,78,298,382$

weight/density, 79

- Category II operation, 22

Ceiling, 87,378

absolute, 378

service, $162,185,378$

Center of gravity

determination, 275, 296

iimit, 294, 296, 298, 299, 311, 312, 313, 320, 326,330

location, $59,68,79,184,209,213,227,232$, $258,268,272,275,294,296,297,299,300$, $306,309,320,326,351,356,468,581$

travel (shift, variation), 44, 45, 79, 207, 297, $299,300,302,313,326,327$

Center of pressure, 231, 236, 437, 492

Certification, $4,21,52,213,227,245,271$

Chord (length), 206, 237, 436, 437, 439, 475, 499, $537,544,548$

extension, $256,530,532,533,534,541$

Circumference, 446

Clearway, 575

climb, 67, 191, 237, 375, 378

angle of $, 378,379,582$

gradient, $154,155,160,164,169,176,251,252$, $380,381,577,578$

rate of, $160,375,378,379$

requirements, $160,162,163,233,251,252,378$, $379,380,381,578,579$

Cockpit, $62,64,67,71,84,87,88,205,300,513$ bulkhead weight, 462

controls weight, 283

window, 89

Combined passenger/freight transport, 68, 297

Comfort, $63,68,69,73$ 
Commuter aircraft, 10, 73, 75

Compound engine, 105, 110

Compressibility, 237, 241, 242, 316, 373

drag, due to, 152,249

effects, 99, 152, 192, 226, 241, 247, 251, 306, $316,370,372,374,469,476$

Compression (ratio), 102, 108, 243, 244, 564, 566

Compressor, 114, 129, 194, 207, 564

axial, 122, 137

centrifugal, 104, 122, 137

entry (face), 46, 563

exit, 134,563

pressure ratio, 114, 117, 122, 125, 133, 135

Conceptual design phase, 4, 5, 6, 241, 265

Configuration

aircraft, $4,5,16,28,30,68,71,217$

development, $4,5,25,390,528$

selection method, 144, 145, 275

Constant speed propeller, 134, 191, 195, 196, 197, 198

Container, $79,82,84,85,87,291$

Control

capacity, 324,325

feel, 307

force, $311,313,314,315,316,327$

law, 381, 581, 582

manual, $307,310,313,326$

surfaces, 228, 517

system, 205, 228, 229, 236, 305, 307, 313, 315, 327,330

wheel, 89,258

Control Configured Vehicle, 307, 324

ontrollable

pitch propeller, 195

stabilizer, 324,327

Convertible energy, 114, 125, 564

Cooling

air, 81,205

drag, 515,516

Cost, $82,375,382,383,385$

Direct Operating, $15,82,158,178,180,185$,

$219,382,383,384,387,399$

- economical cruise, 158,387

engine, $100,120,135,185,187,387$

fuel $82,158,384$

Indirect Operating, $178,382,383$

Ma1ntenance, $342,382,383,384,385$

Operating, $12,15,63,69,265$

Count, 148,490

Cowling drag, 506, 507

Crescent wing, 249

Crest, 243, 244

Crew, 81, 82, 87, 93, 205

Critical

engine, 331, 333

Mach number, 153, 236, 237, 239, 241, 242, 247, 372

Power Failure Speed, 575, 579

Crossflow (drag), 476, 501, 504, 505, 510

Cross-section, $34,63,66,67,68,69,71,74,75$, $78,79,87,94,397,446,479$

Crosswind landing, $338,343,349,352$

Cruise, 66, 106, 120, 121, 191, 219, 224, 234, 237

altitude, $158,201,219,222,223,224,125,245$, 246,482

configuration, 148

distance, 378

fuel (consumption), 153, 158, 174, 219, 377

performance, $153,219,224,246,373,375,377$

power (rating), 104, 158, 234

procedure, 157,377

speed, $66,67,158,197,219,234,241,245,374$, 378,387

Cycle, 119, 120, 186, 563, 568
Cylinder, 107, 110, 515

Damping, 234, 311, 318, 321, 322, 343, 396

critical, 318

cyclic, 318

(moment) in pitch, 319, 321

ratio, 319

of spinning motion, 339

Decision speed, $168,184,576,578$

Deep stall, 45, 53, 208, 227

De-icing, 194, 272, 293

Delivery Bmpty Weight, 270

Density of load, 69, 79, 82, 83, 84, 275

Descent, 68, 377

Design

Cruising Mach number, $76,373,374$

Cruising Speed, 13, 131, 176, 373, 374, 453

Living Mach number, 232, 237, 245,.327, 374

Diving Speed, 25, 78, 281, 374

initial baseline, 5, 7, 18, 367, 390, 394

point (performance), 120, 122

process, 16,19

project, 399

Takeoff Weight, 271

Weight (Minimum), 271, 272, 453

Detail design, 4, 5, 266

Diederich's method, 473, 474, 493, 501

Dihedral (angle of), 39, 258, 259, 327, 338, 355, 439

Direct

drive engine, 106

Lift Control, 258

Directional (static) stability, 331, 335

Disposable load, 271.

Domestic flight operations, $75,81,376$

Door, 74, 87, 291, 395, 460

freight, $64,67,79,84,86,87,299$

passenger (cabin), 76, 78

(rear) loading, 63, 299

service, 78

weight, $87,460,461,462$

Dorsal fin, 332, 339

Double

bubble fuselage, $67,68,79,448$

hinged rudder, 335

slotted flap, 253, 254, 255, 527, 532, 533, 541 544

Downwash, 40, 53, 208, 209, 232, 317, 477, 482, $490,509,511,546,547$

Drag, $63,64,65,66,68,87,95,206,234,237$, 370,399

area, 148, 241, 490, 498

breakdown, 148,368

coefficient, $63,64,222,270,278,368,377$, $490,500,502$

component, 489

creep, 153,372

- critical Mach number, 153, 158, 208, 242, 246, 492

- divergence Mach number, 242, 243, 244, 246, $248,249,251$

minimum, $62,63,71,73,160,222,223,370$

polar, $148,153,370,371,372,377,490,499$, 527,544

rise, $153,243,244,245,372$

Dutch roll, 258, 331, 338

Dynamic pressure, 62, 128, 224, 317, 497

Economic

performance, 271, 373, 399

Regulations, 22

Economy, $68,74,241,399$ 
Economy (continued)

class, 73

criteria, 382

operating, 68,382

Effective Perceived Noise Decibel, 180

Efficiency, engine (component), 117, 120, 568

Blectrical system, 67,213 weight, 289

Electronic system, $67,267,289$

Elevator, $38,294,324,327$

control, 227, 228, 308

deflection, $310,313,315,327,371$

Elliptical wing lift, $368,492,493,509,545$

Emergency

braking, 380,585

distance, 575, 579

equipment, 291

exit, $38,67,73,76,77,78,93,273,395$

power supply, 213

Empennage, 50, 63, 66, 173, 217, 306

Empty

Tank Weight, 270

weight, $45,57,68,147,184,226,251,267,270$ 275,276

Energy absorption, 357, 360

Engine

accessories, $194,285,286$

configuration, 123,155

failure, $40,167,168,169,184,185,191,209$, $210,227,228,331,332,378,379,380,528$, 576,578

failure, drag due to, 155, 335, 372, 553, 555, 556,579

growth, 186, 204

location, $23,39,41,42,45,183,206,228$, 299,397

performance, $133,155,190,194,564,565,567$

rating, $127,158,190,223,224,227,228,377$, $379,563,567$

selection, $158,162,167,178,183,186,187$

weight, $99,100,103,109,110,120,123,124$,

$129,130,137,147,158,186,210,285,565$

Engines, number of, 155, 182, 183, 184, 190

En route

configuration, 148, 133, 185, 537

Weight, 271

Equivalent

Shaft Horse Power, 112, 118, 567, 568

Single Wheel Load, 344, 345

Estimated Normal Payload, 270

Excrescences, drag due to, 391, 516

Exhaust, $47,48,49,126,133,206,516,540$

area, 566

gases, 51, 132, 205, 206, 209, 563

nozzle, 116, 117, 221, 504, 563, 565, 566

velocity, $101,125,130,132,135,169,170$, $205,206,565,567$

Exposed wing area, 149, 439

Extended overwater operation, 185, 186, 291

External fuel tank, 152, 274, 448, 514

Fail-safe design, 15, 260, 390

Fairing, 38, 241, 249, 464, 513

Fan, 114, 116, 125, 133, 563, 565

adjustable rotor blade, 133

cowling, 449, 506

diameter, $126,127,131,184$

efficiency, 568

pressure ratio, 120, 125, 134, 565, 567

tip speed, 133

variable pitch, 130

FAR, 19, 20, 21, 22, 23, 24, 25, 166

Fatigue, 63, 268
Feathering propeller, 165, 198, 470

Feederliner, 20, 75, 123

Fillet, $38,94,479,510$

Fin, see Vertical tailplane

Firewa11, 213, 284

First class, $73,75,80,81$

Fixed equipment and services, 269

Flap (see also High-1ift device), 183, 206, 234, $251,252,396,532,534,535,537,538,539$

deflection (angle), 154, 253, 351, 379, 380, 533,578

design speed, $326,452,454$

effectiveness, 529, 530, 531, 532, 533, 536, 537

fixed hinge, $255,530,533$

motion, 255, 256, 530, 535, 541

operation mechanism, 284, 396

setting, $227,228,379,380$

tracks and supports, 255, 256, 517, 532, 540

Flaperon, 257, 538

Flare maneuver, $169 ; 170,234,325,343,381,581$, 582,583

Flat

plate analogy, 149,497

rating, 134,381

Fleet Empty Weight, 268

Flight

control system, 9, $87,205,252,257,289,294$, $330,395,397$

deck accommodations weight, 291

deck design, 85, 87, 92, 205, 397

envelope, 99, 373

profile analysis, 375,376

Rules, 22, 289, 323, 375

Floor, $68,73,79,80,82,84,377,397$

loading, $73,87,273,297$

level, 37,84

weight, 461,462

Flutter, 48, 218, 280, 374, 452, 453

Flying

qualities, $9,38,45,186,218,227,273$

wing, 56,57

Form drag, 368, 369

Four stroke engine, 101, 112

Fowler flap, 253, 255, 256, 527, 532, 533, 535, 541,544

Frame, 67, 78, 395, 459, 462

Freighter, $63,64,65,68,71,81,82,83,298$

Freight hold, $63,65,67,73,79,83,84,87,95$, 397

Friction (coefficient), 359, 579, 580, 581, 584

Frontal area, $63,71,78,110,122,131,137,151$, 446,447

Fuel, 63, 108, 269, 270, 273, 297, 301, 375 consumption, $63,87,99,106,118,119,121,125$, $126,135,136,155,186,190,217,219,377$. $563,565,567$

injection, 108,111

reserve, $22,80,157,159,173,270,375,376$, 378

specific heat, $118,119,293,563$

system, 195, 198, 214, 275, 285, 395

tank, 145, 159, 219, 225, 251, 262, 269, 270, $395,448,449$

unusable, 269, 273, 292

usable, capacity, 270, 271, 273, 449

weight, $82,145,158,293$

Furnishing and equipment, 82, 291

Fuselage

afterbody (tail), 62, 64, 65, 66, 67, 86, 87, 93,503

configuration, $63,64,65,66,67,149$

dimension, $37,63,67,69,71,73,76,79,87$

drag, 63, 66, 150, 159, 501, 502, 503, 504, 510

lift, 469,540 
Fuselage (continued)

- mounted engines, $41,42,44,45,46,47,48$, $205,206,207,208$

nose, $67,79,84,87,93$

slenderness (fineness), 63, 64, 65, 66, 458, 502, 504

strakes, drag of, 517

structure, $62,63,68,96,397$

(structure) weight, 64, 87, 282, 458, 459, 460, 463

volume, $63,64,65,446$

Galley, 79, 80, 291, 292

Gap, 255, 314, 315, 482, 517, 530

Gas generator, 113,114, 118, 125, 563, 565

cowling, 285, 449, 506

function, $114,128,564,567$

plug, 131, 449, 506

Gas producer/gasifier, see Gas generator

Geared fan, 123, 127, 130, 178

Gearing, reduction, 107, 108, 117, 123, 127, 134, $178,191,199$

General arrangement, 29, 37, 62, 79, 132, 179, 217, $222,296,390,393$

Glauert's thin airfoil theory, 528, 529, 541, 544, 547

Gross Weight, 271, 297

Ground effect, 38, 469, 528, 551, 552, 553, 580, 585

Ground run, 204, 342, 579, 580

Group weight (statement), 268, 275

Gust

allevation factor, 225, 227, 375, 453

load (factor), 227, 251, 373, 374, 375, 453

speed, 225, 227, 373, 374

High density class, 73, 74, 75, 275

High-1ift device, 143, 165, 217, 218, 219, 231 $234,252,380,399,452,454$

drag due to, $373,517,543,544,545,546,549$

leading-edge, 231, 250, 251, 252, 255, 256, 527 , 547

trailing-edge, 231, 235, 236, 251, 252, 253, 454, $527,528,537,548$

weight, $281,452,454$

High speed, 155, 183, 218, 219, 224, 241, 244, 245, $248,249,374$

Bigh-wing aircraft, $30,34,36,38,71,205,479$, 510

Binge moment, $304,310,313,314,315,330,37$

Horizontally opposed cylinder engine, 110

Horizontal stabilizer, 51, 52, 53, 281, 282, 324, $325,326,483$

Horizontal tailplane, 208, 209, 219, 228, 231, 250, $258,294,326,397,537,540$

arrangement, $48,63,326$

design, $305,324,325,326,327,330,339$

drag, 151, 496, 508

lift, $310,324,328,469,470,481,482,483$

load, 228, 239, 253, 297, 370, 395, 470, 580

sta11, 326

variable incidence, $281,326,327$

Horn balance, 315

Hydraulic system, 289, 343

Incidence, angle of, 191, 206, 259, 355, 440

Incompressible flow, see Subcritical flow

Indicated Mean Effective Pressure (Power), 102

Induced drag (see also Vortex-induced drag), 39,

$148,233,235,240,368,369,370,373,492$,

$493,545,555$

\section{Inertia}

airplane, 306, 320, 322, 323

distance/time, $169,170,583$

In-Flight Weight, 271

Initial baseline design, 5, 7, 18, 367, 390, 394

Inlet/intake, $47,48,207,516,540$

area/diameter, 119, 158, 186, 566

duct (efficiency), 46, 113, 206,506, 566, 568

drag due to, 515, 516

pressure (1oss), 46, 103, 106, 133, 194, 206, $377,564,568$

velocity, $206,516,568$

weight flow, 566

In-line engine, 11

Instruments group weight, 289

Integrated design lift coefficient , 203

Interference

effects, 58, 232, 240, 337, 369, 370, 470, 477, $490,497,509,515,540$

arag, i31, 208, 209, 369, 370, 510, 511

viscous, 509, 512

Interior design, 37, 67, 68, 71

Internal layout, 64, 67, 71, 397

International flight (operation), 75, 81, 376

Jet engine, 99, 112, 116, 119, 121, 132

Krueger flap, 256, 527, 528 weight, 454

Laminar

airfoil section, $66,239,438,471,499$

Flow Control, 44, 563

Landing

configuration, 155, 234, 379

gear (see also Undercarriage), 38, 67, 184, 204, $205,363,394$

impact load, 272, 283, 343, 395

performance, 22, 38, 170, 171, 176, 225, 379, 381

Weight (Maximum), 225, 271, 272, 273, 275, 381

Large aircraft, $20,23,185$

Lateral stability, 218, 259, 331, 335, 338

Lavatory, $68,73,79,291$

Layout design, $64,143,148,390,397$

Leading edge, 229, 249, 250, 301, 436, 439, 471, 500

droop, 250, 547, 548

shape, $231,250,437,470,471,534,535$

stal1, 229, 230, 231, 235, 249, 252, 256, 471,

$534,536,547,548$

suction, 368,492

Lift

carry-over, 477, 479, 480, 540, 542, 545

coefficient, $156,223,237,240,242,245,247$, 368

coefficient, design, 203, 222, 239, 245, 246, $247,438,499$

curve, $231,253,371,453,470,471,527,528$, $529,537,540$

- curve slope, 225, 229, 231, 242, 251, 453, $470,479,536,537,539$

drag due to, 369,370

distribution (spanwise), 51, 236, 240, 471, 473, $492,539,545$

divergence, 242, 243, 245, 316

/arag ratio, 43, 56, 154, 219, 222, 223, 224, 234,373

dumper, 169, 238, 585

engine, 130

increment due to flap, 529, 530, 533, 537

maximum, see Maximum lift

trimmed, 481, 482 


\section{Lifting}

line theory, 368, 492, 551

surface theory, $368,473,492,493,537$

Liftoff, $167,343,349,351,577,581$

Light aircraft, $20,23,87,275,281,298$

Load, $63,64,87,259,260,271,349,395$

and Balance diagram, 296, 298, 301

Classification Number, 15,344

factor, 12, 47, 84, 161, 170, 227, 272, 277, 312, $374,382,453$

on structure, 23, 394, 395

Useful, 65, 154, 226, 271, 293, 378

Loading, $63,64,84,86,87,206,297,394$

flexibility, 45, 58, 79, 296, 297

procedure, 297, 298

restriction, 58, 272, 296, 297, 298, 300, 302

Lofting, wing, $237,249,448$

Long-haul, $76,79,126,183,274$

Long-range

aircraft, $28,75,119,123,126,127,173,219$

cruise, $157,158,222,234,274,378,387$

Longeron weight, 459

Longitudinal

control, 323

stability, 43, 50, 53, 205, 218, 251, 256, 308,

$310,313,317,319,321,323,328,371$

Low-wing aircraft, $30,37,38,206,479,510$

Luggage, $68,69,71,79,81$

Mach number effect, see Compressibility effect

Mach trimmer, 218, 243, 316

Main gear, $34,36,38,54,217,283$

Maintenance, $15,82,120,213,342,382,385$

Maneuver

load, 235, 245, 374, 453

margin, 297, 312, 320

point, $311,312,313$

stability, 311

Maneuverability, 234, 319

Manifold pressure, 103

Manufacturer's Empty Weight, 370

Market analysis and research, 4, 6, 9, 10, 83

Mass boom wing structure, 259

Maximum 1ift, $38,45,232,235,236,251,471,475$ coefficient, 14, 57, 59, 155, 217, 218, 229, 234, $235,237,238,239,240,251,253,279,324$, $374,475,476,479,492,534,547$

\section{Maximum}

Weight, 271, 272

Zero Fuel Weight, $79,82,270,272,275,453$

Mean

Aerodynamic Chord, 296, 301, 309, 440, 441

Geometric Chord, 233, 439

line, 239, 436

quarter-chord point, 441

Medium-haul, 76, 82, 183

Mid-wing aircraft, $30,36,479,510$

Minimum

Control Speed, 332, 335, 381, 577

Drag, 158

Speed, 165, 227, 374

Stall Speed, 165, 170

Unstick Speed, 553, 577

Weight, 271,272

Mission (performance), 143, 144, 172

Monospar wing structure, 259, 260

Multispar wing Structure, 260, 261

Munk's airship theory, 480

NACA airfoil section, $235,238,239,240,242,243$, 438,499

Nacelle, 65, 79, 131, 205, 206, 232, 233, 397, 449,
Nacelle (continued)

$506,540,563$

drag, 131, 137, 151, 184, 496, 505, 506

(group) weight, 283

Naturally aspirated engine, 103,106, 108, 165

NAV/COM equipment, 289,397

Neutral point, $51,297,308,309,310,312,316$, 317,482

Noise, $15,16,68,120,126,132,133,173,178$, $179,190,200,213,396,399,565$

approach, 179

engine component, 51, 101, 131, 132, 192, 565

propeller, 101, 190, 195, 199, 202

reduction, $117,132,179,180,285,399,516$

regulations, $11,20,144,273$

Normal Rated Power, 104, 109

Nose

Aroop, 250, 257, 327, 547, 548

gear (nosewheel) $54,55,93,169,205,343,353$, 358,395

Nozzle, see Exhaust nozzle

Off-design condition, $120,241,243,244,246$

Oil cooler, 205, 515, 516

Operating Weight, 271,273

Operational

Empty Weight, 79, 82, 145, 270, 296

Items, 269, 293, 297

limitations, $21,23,165,174,232,373,374$

rules, $19,22,30,160,273$

Weight (1imitations), 271, 273

Optimization, $17,63,100,125,158,171,178,180$, 387,563

Oswald's span efficiency factor, 148, 165, 370 , $486,508,546$

Otto-type engine, 101

Out-of-trim condition, 326

overall

engine efficiency, $118,565,567$

Pressure Ratio, $118,120,121,133,136,171$, $186,563,567$

Overhanging balance, 307, 314

Overspeed, $160,169,380,577$

Overturning, 353

Oxygen system, $81,159,291$

Pallet, 84, 291

Pantry, 69, 73, 79, 80, 291

Parametric design study, 5, 7, 144, 171, 390, 399

Parasite drag, 2, 255, 370

Passenger, 68, 69, 77, 78, 79, 80, 81, 84, 207

transport (aircraft), 75, 76, 84, 87, 297

service, 73, 79, 293

weight, $76,79,293$

Payload, $62,65,67,68,69,83,84,145,174,206$, $266,270,272,301$

Maximum (Structural) Weight limited, 79, 270, 272,273

- range performance, 217, 270, 271, 272, 294, $375,378,382$

Volumetric Capacity, 79, 84, 270, 272, 273, 275 weight, 293

Peaky airfoil, 244, 246

Performance, $38,45,144,153,179,217,268,273$. 373,374

group, 22,25

low-speed, $165,219,224,227$

requirement, $110,143,155,217,219$

Perry's method, 582

Phugoid, 57, 317, 323, 582

Piston

engine, 2, 39, 99, 101, 134, 205 
Piston (continued)

displacement, 106, 107

Pitch, angle of, 256, 349, 350, 351

pitching moment, $53,57,63,229,231,237,239$, $242,250,308,324,371,476,477,480,482$, $483,540,541,542$

behavior at stall, 227, 232, 244, 249, 540, 548 camber effect on, 477

flap effect on, 540,541, 542

fuselage effect on, $479,480,542$

nacelle effect on, 481

powerplant effect on, 51, 481

wing twist effect on, 477

Pitch-up, 52, 249, 250, 251, 256

Plain flap

leading-edge, $256,527,547,549,550$

trailing-edge, $253,254,255,527,530,531,535$, 541,544

Plain control surface, 314

Pneumatic system, 290, 332

Pod-mounted engine, $42,43,44,45,46,151,206$, 207,209

Point design, 7

Post-stall behavior, 52, 227

Potential flow, 241, 479, 490, 492, 534, 536

Power

Coefficient, 192, 195

extraction (offtake), 126, 190, 194, 375, 563

lapse ratio, $120,127,155,165$

loading, $14,155,156,225,381$

rating, $103,162,227,379$

specific, $106,110,118,119,135,136,568$

takeoff, 103,109, 110, 155, 162

Powerplant

effect on drag, 120,124, 131, 137, 151, 165,

$506,515,516,565$

effect on lift, $316,371,469,515,537$

Preliminary design, 4, 5, 9, 18, 25, 66, 68

Pressure

coefficient, 242

distribution, 229, 231, 241, 242, 244, 245, 246, $248,368,369,371,438,479,549,553$ drag, $64,368,490,497$

Pressurization, 2, 65, 67, 68, 374

Price, aircraft, $385,386,387$

Productivity, 2, 12, 81, 83, 266, 382, 387

Profile drag, 194, 219, 222, 235, 237, 242, 545

control surface deflection effect on, 497, 546, 555

lift effect on, 500

minimum, 239, 242, 499, 544

Profit, 69, 382, 387

Project evaluation, 9, 28, 143, 155, 172, 399

Projected area, 447

Propeller, 99, 117, 202

adjustable, 195

blade angle, 191, 195, 210

blade camber, 202, 203

blade planform, 191, 196, 202

coefficient, 191

control system, 191, 197, 213, 335

design, 183, 186, 197, 201, 202

diameter, 107, 191, 195, 197, 199, 200, 201

disk loading, 168, 200

drag, 137, 553, 554

efficiency, 135, 136, 165, 191, 194, 199, 516

fixed-pitch, 168, 190, 195, 196, 197

geometry, 183,192, 199

installation/location, $41,80,95,186,190,191$, $199,204,205,206,277$

number of blades, 191, 202, 203, 204

performance diagrams, 191, 194, 195, 199.

reversing, $197,198,213$

slipstream, see slipstream effect
Propeller (continued)

thrust determination, 194, 195

thrust line tilt-down, 205, 206, 316

(tip) speed, 101, 107, 123, 126, 137, 192, 195, 199,200

vibration, 190, 204

weight, $100,130,199,286$

Propulsion group weight, 268, 269, 285, 286

Propulsive

device, $113,114,116,117,118,130$

efficiency, 99, 114, 116, 118, 125, 135, 564, 565

Protuberances, drag due to, 490, 512

Pylon, 206, 250

drag, 131, 506, 508

Quarter-chord line, 232, 436, 439, 473

Q-V diagram, 374

Ram drag, 118

Ramp Weight, 271, 272, 356

Range, $62,63,80,82,157,160,274,377,384$

design (max. payload), 13, 130, 274

performance, 157, 158, 160, 225, 234, 375, 377

Reaction factor at touchdown, 360

Rear loading (airfoil), 244, 248

Reference

drag, 370

eye point, 89

Regenerative engine, 137

Regular Weight, 273

Removable equipment and services, 269

Return on Investment, 382

Revenue, 266, 382

Reverse

flow principle, 139

thrust, 171,382

Reynolds number, 64, 149, 152, 186, 192, 228, 231, $250,253,371,471,497,499,535$

Ride quality, 174,227

Rolamat system, 84,86

Ro11

angle of, $227,349,351$

control, 257, 258

Rolling moment, $50,184,231,257,258,332$

Roof-top (pressure distribution), 243, 244

Rotary Combustion engine, 111, 112

Rotation

angle (1imit), 67, 354, 355

phase, $343,579,581$

rate of $350,578,579,581,582$

Speed, 325, 576, 577

Roughness, 497, 499, 517

Route structure, 13, 387

Rubberizing of engine, 186, 190

Rudder

control effectiveness, 53

deflection, $51,332,335$

varicam, 335

Runway

bearing capacity, 343, 349, 359

classification, 343

flexible pavement, 344,347

gravel, 342, 345

Loading Index, 344

loading restriction, 273

rigid pavement, 344,345

performance (wet, dry), 15, 25, 210, 338, 356 359,381

unprepared, 344, 349 
Safe-1ife design, 15, 396

Safety, 2, 12, 23, 25, 67, 165, 184, 185, 396

Sailplane, 56, 65, 195

Sand grain size, equivalent, 517

Schrenk's method, 473

Scrubbing drag, 131, 507

Sealed internal balance, 314

Seat

crew, 81, 87, 92, 291

dimension, $67,71,73,75,76$

load, 76

number abreast, $67,69,71,74$

pitch, $67,73,75,76,77,78,80$

passenger, 75, 76, 269, 291

weight, 76

Seating arrangement, $62,63,68,71,77,78,272$, 275,297

Sensitivity study, 172

Separation of airflow, 228, 229, 231, 245, 249, $369,371,549$

Service

life, $15,120,128$

passenger, $68,69,73$

Servicing, aircraft, $68,78,80,382,383$

Servo tab, 314, 315

Shel1 weight, $282,458,459$

Shimmy, 356

Shock

absorber, $349,352,356,357,360,361$

wave, $153,208,242,243,244,245,247,369$, 371,469

Short

period, longitudinal oscillation, $317,318,319$, 320,321

Takeoff and Landing, 179, 210

Short-haul, 13, 69, 76, 79, 127, 158, 159, 183, 218,273

Short-range aircraft, $82,123,225$

Sideslip, 331, 332, 555

Sidewash, $51,333,555$

Single slotted flap, 253, 254, 255, 527, 530, 532, Skin

friction drag (coefficient), 64, 149, 368, 490, $497,498,507,568$

structure (weight), 63, 259, 260, 261, 458

slat, leading-edge, 256, 326, 527, 547, 548, 549

Slipstream, 40, 205, 232, 241

effect on drag, 515, 516, 555

effect on neutral point, 317

effect on propeller thrust, 194, 201

slot, 255, 256, 257, 327, 530, 537

slotted flap, 255, 530, 535, 539, 541

Small aircraft, $20,22,23,185$

Sound insulation, $69,147,213,291$

Span, 219, 233, 235, 251, 280, 281, 438, 439, 473

loading, $155,218,222,223,252,368,369$

Spar, 47, 251, 258, 259, 260, 261

Special FAR Part 23, 23

Specification, design, $6,10,29,69,145,217$, $367,398,399$

Specific

aircraft density, 277

Excess Power, 160

heats, ratio of, 563

Speed stability, 137, 138

Speedbrake, 258, 281, 452, 454, 455

Speed/Power Coefficient, 194, 197

Spin, 53, 338, 339

Spiral stability, 331, 338

Split flap, 253, 254, 255, 527, 530, 531, 535, 541, Spoiler, 255, 257, 258, 382, 585

weight, $281,452,454$

spring tab, 307, 314, 315

Square-cube 1 aw, $186,226,281$
Stability, 51, 294, 297, 308, 351, 371

Augmentation System, 307, 323

Stal1, 51, 52, 227, 231, 232, 250

characteristics, 227, 228, 229, 230, 231, 233, $239,240,249,251,528$

progression, 228, 231, 232, 236, 237, 240, 553 proofing, 228

test, $166,227,228,241,374$

warning and protection, 227, 228, 229

Stalling speed, $78,111,121,122,126,185,218$, $227,228,233,234,374,379,537$

standara

error of prediction, 277, 281, 455

Fuel Capacity, 273

Item (Variations) , 269, 270, 292

Mean Cr.ord, 296, 301, 439

Static margin, 309

Steering, landing gear, 343, 349, 353

stick

control, 87

displacement, 311

force, $307,310,311,312,315,327$

gearing, 311

pusher, 53, 218, 229

shaker, 53, 228

Stiffness, $57,64,66,268,390$

Stopway, 575

Streamline body, $63,64,65,369,446,490,497$, 502

Stress level, 63, 226, 261, 268, 276, 459

Stressed skin, 260, 395

Stretching, 4, 37, 64

Stringer, gross weight, 459

Structural

design, 9, 39, 64, 217, 219, 224, 225, 390, 394, 395,397

design speed, 367,373

requirement, 228, 232, 272

Structure, $79,80,83,226,373$

fuselage, $62,95,282$

multipath, 396

primary, 23, 218, 262, 390, 395, 396, 452

weight, $184,225,226,227,233,235,236,265$,

$277,394,451$

wing, $218,259,275,395$

Strut, $36,281,453,514$

Subcritical, 236, 241, 242, 243, 316, 469, 473, 490

Suction, 229, 256, 547

Supercharger, 103, 104, 108, 110, 165

Supercritical airfoil (section), 67, 242, 243, 244, 246,471

Supersonic flow, 242, 243, 244

Superstal1, 44

Supervelocity, 239, 243, 245, 246

Support structure, 395,396

weight, $275,463,464$

Surface

control system, 283, 307

imperfections (drag), 371, 490, 512, 516

Sweep, 153, 219, 232, 241, 246, 247, 251, 438, 439, 469

angle of, 37, 69, 225, 248, 250, 251, 327, 339, 395,439

simple theory, 246, 251

Swept-up rear fuselage, 479

System

controls weight, 283,284

design, $62,82,194,397$

Tail

angle, 37,437

boom, $50,62,65,66,328,329$

- first aircraft, see Canard aircraft 
Tail (continued)

surface design, 50, 51, 62, 63, 64, 308

group weight, 281

Tailless aircraft, 56, 58, 306

Tailwheel undercarriage, 54, 343, 355, 513

Takeoff, 167, 180, 183, 191, 237, 381, 574, 575, 576,579

distance, $14,15,38,167,184,349,380,381$, 575,577

field length, 14, 15, 167, 183, 574, 579

flight path, 576,582, 583

height, $168,224,380$

speed, $154,168,184,185,380,577$

weight, $69,145,147,155,224,226,271,380$

Weight, Maximum, 82, 154, 271, 272, 274, 275, 293

Taper (ratio), 219, 233, 236, 240, 251, 326, 339, $439,440,471$

Tare Weight, 270

Taxying, 204, 209, 349 Weight, 271

Thermal efficiency, 108, 114, 136, 564

Thermodynamic cycle, 112, 125, 132

Thickness

distribution, $238,239,248,436$

ratio (of section), 47, 149, 153, 219, 229, 231 $232,235,236,237,239,241,246,247,248$, $249,395,437,438,439,477,530$

Three-shaft engine, 178

Thrust

ambient temperature effect on, 134

coefficient, 192,515

ideal, 563

idling, 580,585

gross (standard) , 116, 128, 135, 506

lapse (rate), 120, 127, 155, 161, 224, 563, 566, $567,579,580$

line, 206,316

loading, 14, 155, 156, 161, 162, 168, 171, 174, $180,183,224,578$

installation loss, $47,155,206,210$

net (standard), 116, 117, 187, 506, 563

specific, $118,119,120,121,124,126,186,563$, $565,566,567,568$

reversal (system), 126, 183, 210, 295, 338, 516, 585

takeoff (static), 128, 129, 130, 155, 565, 566, 567

/weight ratio, see Thrust loading

Time Between Overhauls, 101, 107, 190

Tip tank drag, 152

Tire

American size, 356, 357

anti-shimmy, 356

British size, 356,358

characteristics, $342,343,356$

clearance, 205, 361

dimension, 359, 361

inflation pressure, $343,344,345,356,357,359$, 360,580

load, $343,356,357,358$

size. 356

tread pattern, 356

Toilet facilities, 69, 79, 80, 205

Torsion, 236, 237, 280, 396

Total

Activity Factor, 202, 203, 204

Pressure Ratio, see Overall Pressure Ratio

Tourist class, $73,74,75,80,81$

Touchdown, 56, 126, 343, 349, 381, 382

Tractor engine, 39, 152, 156, 205

Tradeoff study, 13, 172

Traffic, 3, 11, 75, 270, 382

Trailing edge, 436, 438, 439, 499

angle, 471
Trailing edge (continued)

beveled, 315

stal1, 229, 230, 231, 235, 249, 471, 529, 534, 536,548

Trailing vortex, 192, 368, 490, 492, 551, 552, 553

Trainer, 65, 76, 206

Transition distance, 583

Transport(-category) aircraft, 22, 23 Trim

drag, 237, 239, 244, 297, 370, 496, 528, 543, 545,546

tab, 315, 327

Triple slotted flap, 253, 254, 255, 527, 532, 534

T-tail, 45, 50, 53

Tuck under, 243, 316

Turbine, 104, 114, 117, 125, 207, 396

blade cooling, 124,563

efficiency, 564

Entry (Inlet) Temperature, 114, 120, 123, 124, $133,134,135,136,171,186,199,385,563$, 564,567

free, 137

Turbocharger, 103,104, 105, 110

Turbofan engine, 2, 99, 116, 121, 565

Turboprop engine, 39, 99, 112, 117, 119, 137, 198, 205,206

weight, 130

Turboshaft engine, 112

Turning flight, 161,245

Twist, $192,218,222,228,239,240,248,251,440$, 493, 549

aerodynamic, 440,474

geometric, 440

linear, $440,473,474,477,493$

linear lofted, $248,440,473,475,477$

Two stroke engine, 111, 112

Undercarriage

bogie, 345, 367

design, 56, 71, 207, 249, 268, 342, 343, 397

drag, $152,165,359,492,493,528,550$

fixed, $362,512,513$

Jockey, 363

geometry, 37, 184, 251, 296, 348, 353, 355, 360 , 361,395

layout, $227,343,345$

retraction, $55,62,67,68,236,249,297,360$, $362,396,579,580$

tandem, 55,343

track, 255, 352, 353, 354

tricycle, $54,343,351$

weight, 282,283

Unit Construction Index, 344

Upwash, 256, 477, 509, 551, 553

Utilization, aircraft, 3, 12, 63, 84, 267, 384, 385

Vane, fixed, 255, 454, 532, 533

Vee engine, 111

Vertical tailplane, 207, 209, 331, 332

area, 39, 184, 331, 332

drag, 373, 508

geometry, $331,332,335,337,338,339$

stal1, 327, 332

twin, $50,335,338,482$

Viscous flow effect, $192,477,496,530,534,546$ Visibility from the cockpit, 89, 91, 92, 205, 256 V-n diagram, 367, 373, 374

Volume, 222, 225, 226, 238, 277, 368, 446, 447

Vortex generator, 250, 517

Vortex-induced drag (coefficient), 38, 222, 233, $237,368,370,490,492,493,496,508,509$, $510,511,528,543$ 
Vortex-induced arag (continued)

effect of flap deflection on, 545

effect of fuselage lift on, 496

effect of interference on, 509, 511

effect of tailplane lift on, 546

effect of wing lift on, 492, 493,494, 495, 496, 555

Vortilons, 250

v/sToL aircraft, 203

$v-\operatorname{ta11}, 51,53$

Wake, $47,53,208,231,232,530,536$ drag, 368,369

Wankel engine, 112

Wararobe, $73,79,80$

Wash-in, 440

Wash-out, $57,240,248,250,440,475,492$

Water injection system, 134

weight of, 269,292

Wave drag, $66,67,368,369,371$

Weight

Altitude Temperature diagram, 273, 380, 381

breakdown, 25, 145, 266, 268, 269, 399

control, 9, 144, 275, 276, 395

distribution, 159, 217, 296

Empty Equipped, 270

growth, $63,186,265,266$

imitation, 268, 271, 274

prediction method, $144,145,268,277$

Wetted area, 42, 131, 149, 151, 222, 368, 446, 447, $448,497,509,549$

engine nacelle, 449

fuselage, $63,64,66,80,87,446,447$

gas generator, 449

tailplane, 447,448

wing, $439,447,448$

Wheel

contact area, $346,347,359$

door, 363,460

load, 344,356

location, $349,353,354,356,361$

multiple, $344,345,348$

- well fairing drag, 514

Wheelbay, 395, 462, 551

Whitcomb's area rule, 67,208

Wide-body aircraft, $67,73,75,79,80$

windmilling engine, 470, 533, 553, 554, 580

window, $67,76,78,87,460$

seating procedure, 298,301
Windshield drag, 513, 514

wing

apex, 438, 439

area, $143,219,222,225,226,232,233,252$, $256,439,479,501$

bending, $209,218,235,236,259,260,276,280$, $395,396,452,453$

center-section, $67,206,395,470$

design, $68,69,158,217,218,227,232,241$, 246

fences, 250,517

loading, $2,14,15,69,155,156,168,171,174$, $176,180,183,217,218,219,222,223,224$,

$225,226,227,228,233,234,245,252$

- mounted engines, $156,233,332,336,453$

planform, 218, 228, 232, 233, 237, 240, 248, 249, $251,438,473$

position (setting), 30, 37, 258, 259, 294, 300, $477,479,481$

root, $36,43,68,231,235,236,240,395,438$, 439,449

section, see Airfoll section

shape, 218, 225, 227, 229, 232, 233, 236, 237, $240,441,442$

size, $173,218,219,227$

straight, $232,233,471$

straight-tapered, $232,233,439,441,442,443$, 473,493

sweptback, 6, 209, 229, 232, 247, 249, 473, 548 untapered, 232, 233

structure weight, $45,184,226,227,235,276$, $280,452,454$

tip, 231, 236, 237, 247, 250, 259, 395, 438, 439

(tank) volume, $174,222,225,238,448$

Yaw

angle of, 227

damper, 218, 258, 259, 332, 338

Yawing

moment, $51,184,209,331,332,333,337,555$

oscillation, 258,332

Zero

Fuel Weight (Maximum), 79, 82, 270, 271

- 11ft drag, $56,63,87,148,149,161,369,370$, 490,526

- lift line, $324,436,437,443,483$

Payload Weight (Maximum), 271, 273 



